\title{
Greenfield Alternative Study \\ LEU-Mo Fuel Fabrication Facility
}

July 2008

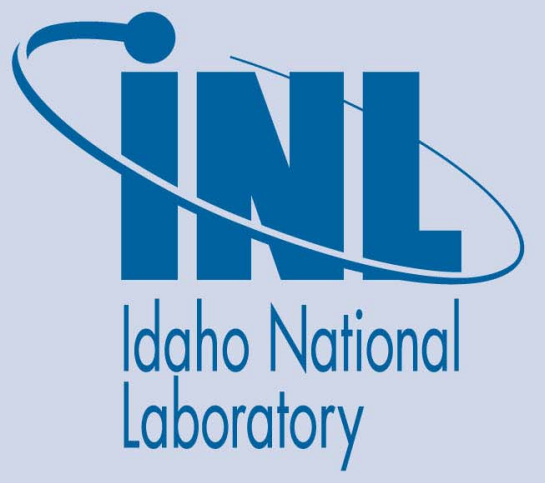

The INL is a U.S. Department of Energy National Laboratory operated by Battelle Energy Alliance 
INL/EXT-08-14577

URS WD Project No. 17989-325

\title{
Greenfield Alternative Study LEU-Mo Fuel Fabrication Facility
}

\author{
July 2008 \\ Prepared by: \\ Washington Division of URS \\ 7800 E. Union Avenue, Suite 100 \\ Denver CO 80237
}

For:

Idaho National Laboratory
Idaho Falls, Idaho 83415

http://www.inl.gov

Prepared for the

U.S. Department of Energy

Office of National Nuclear Security Administration

Under DOE Idaho Operations Office

Contract DE-AC07-05ID14517 


\section{TABLE OF CONTENTS}

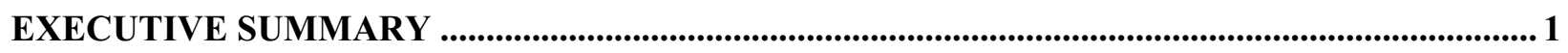

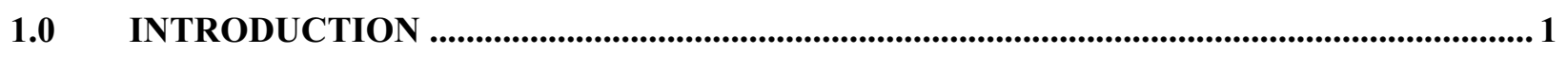

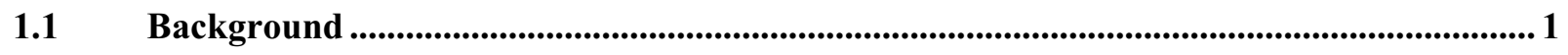

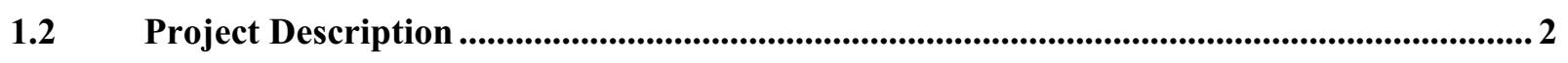

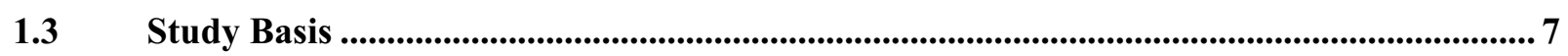

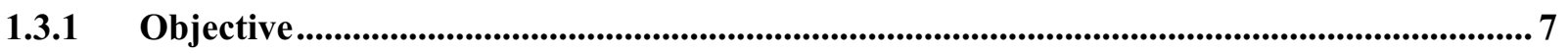

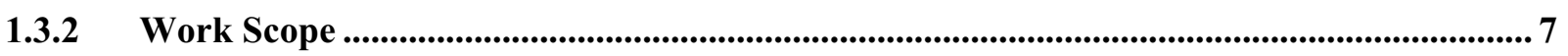

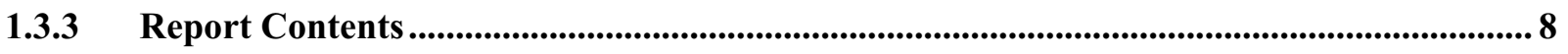

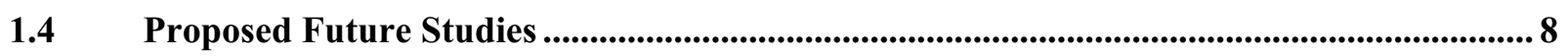

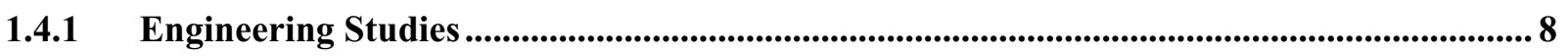

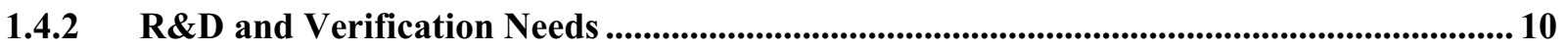

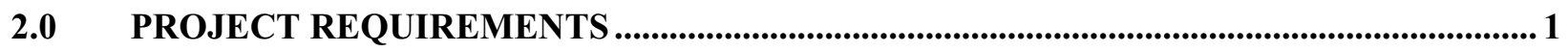

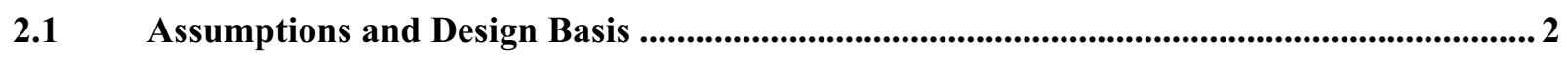

2.2 High Level Functions and Requirements ..................................................................... 7

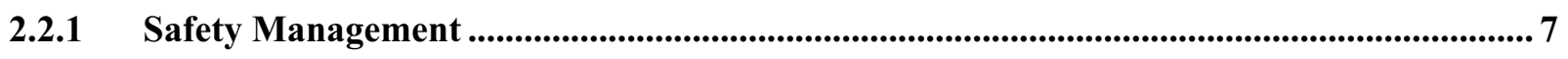

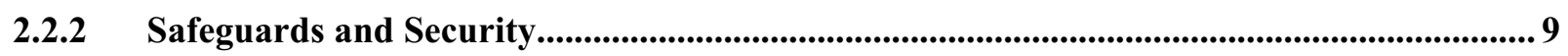

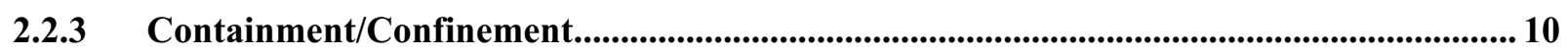

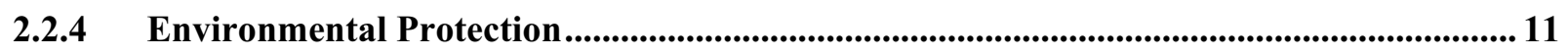

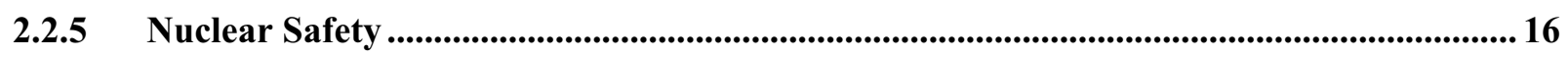

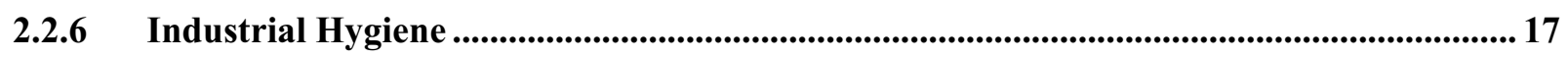

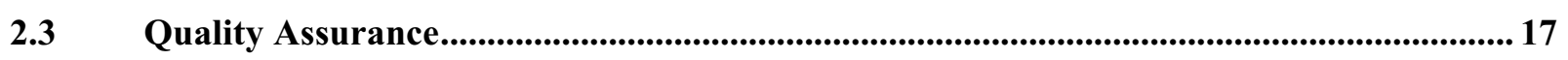

2.4 Pollution Prevention and Waste Minimization ................................................................... 18

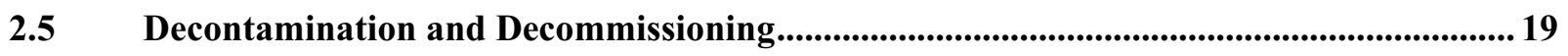

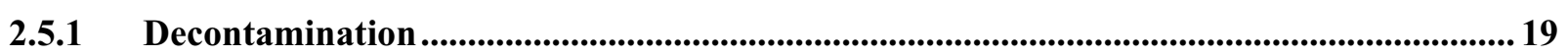

2.5.2 Decommissioning ............................................................................................................................. 20

2.6 Maintenance Philosophy ................................................................................................................. 21

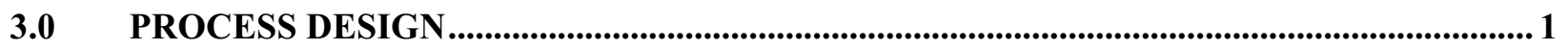

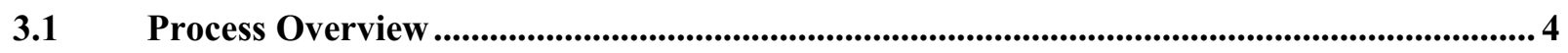

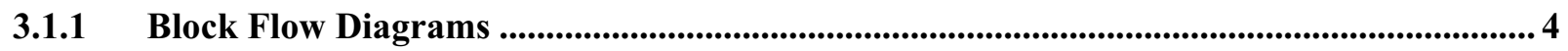

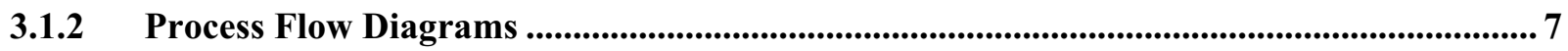




\begin{tabular}{|c|c|}
\hline 3.1.3 & Material Balance \\
\hline 3.2 & Facility Throughput Basis............................... \\
\hline 3.3 & Process Design Basis and Description \\
\hline 3.3.1 & 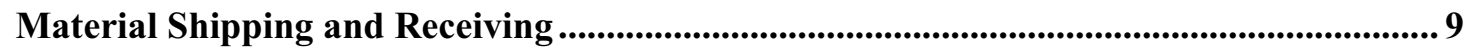 \\
\hline 3.3.2 & Ingot Casting ................................. \\
\hline 3.3.3 & 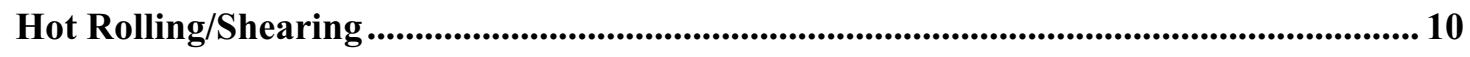 \\
\hline 3.3.4 & 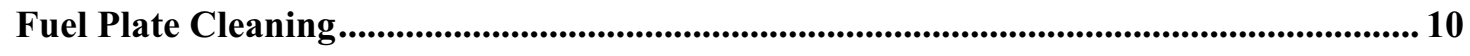 \\
\hline 3.3.5 & Zr Cladding and Final Rolling ......................... \\
\hline 3.3.6 & 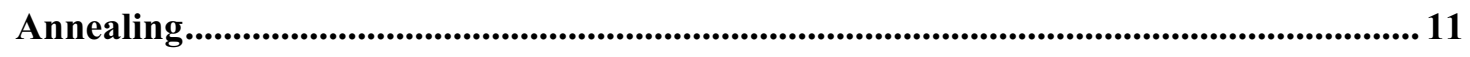 \\
\hline 3.3.7 & Foil Cleaning ................................................. \\
\hline 3.3.8 & Friction Bonding ................. \\
\hline 3.3.9 & Alternate HIP Process .......... \\
\hline 3.3.10 & 13 \\
\hline 3.3.11 & Plate Finishing......... \\
\hline 3.3.12 & Plate Forming (curved plates only) 15 \\
\hline 3.3.13 & Sub-Assembly Fabrication \\
\hline 3.4 & 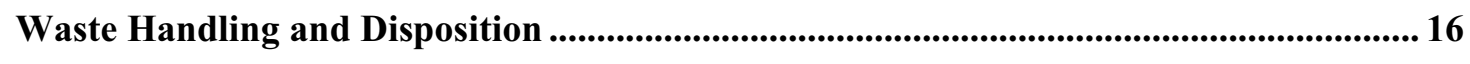 \\
\hline 3.4.1 & Liquid Waste \\
\hline 3.4.2 & Solid Waste \\
\hline 3.4.3 & 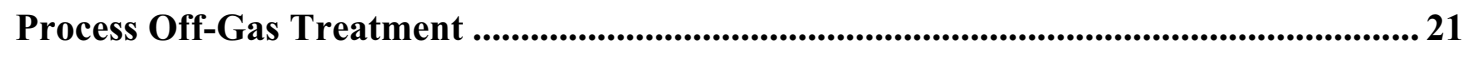 \\
\hline 3.4.4 & Scrap Management ...................... \\
\hline 3.5 & 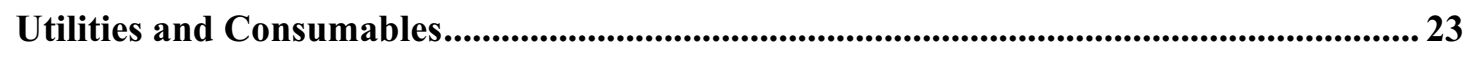 \\
\hline 3.6 & Project Equipment List ........................ \\
\hline
\end{tabular}

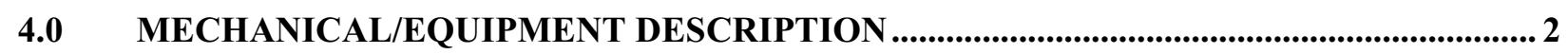

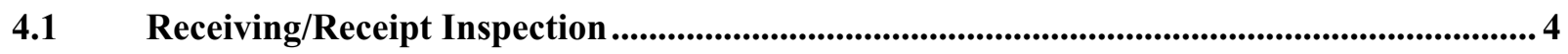

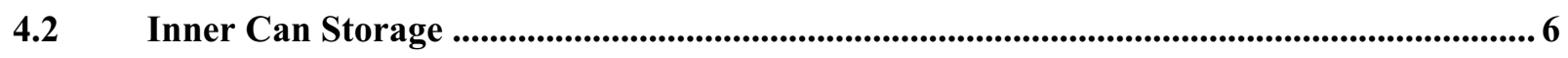

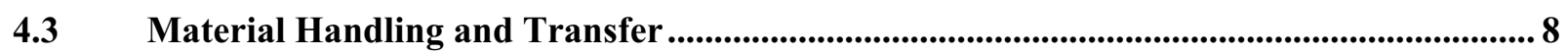

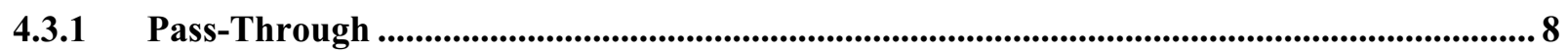

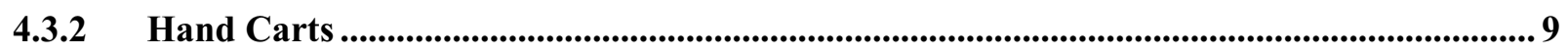

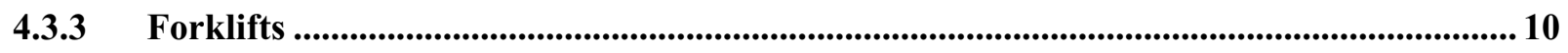

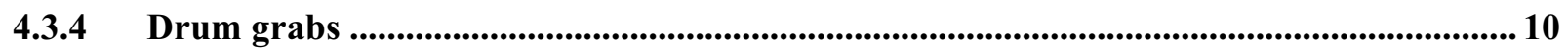

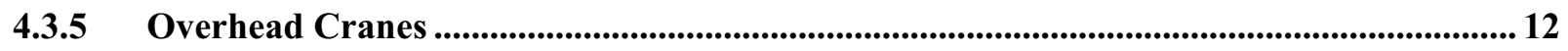

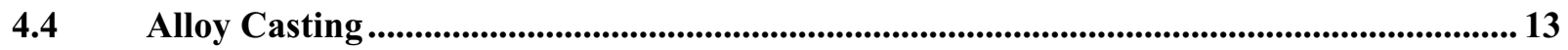




\begin{tabular}{|c|c|}
\hline 4.5 & Hot Roll to Intermediate Thickness \\
\hline 4.6 & 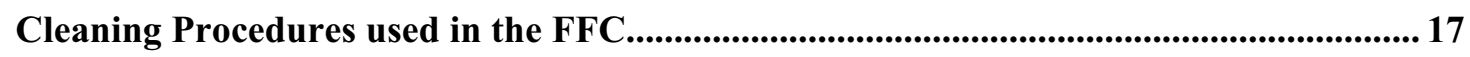 \\
\hline 4.7 & 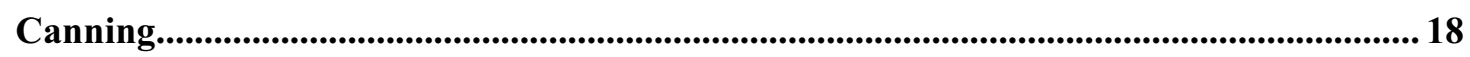 \\
\hline 4.8 & oll to Final Thickness 19 \\
\hline 4.9 & 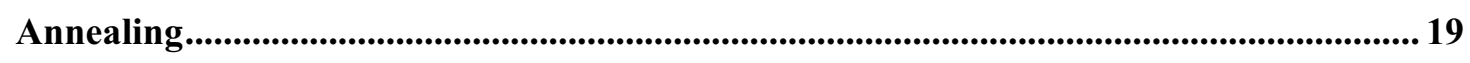 \\
\hline 4.10 & Shear Foil to Dimension ..................................... \\
\hline 4.11 & 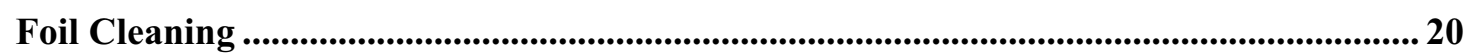 \\
\hline 4.12 & 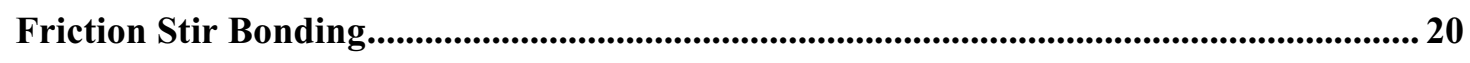 \\
\hline 4.13 & Alternate HIP Process .......... \\
\hline 4.14 & 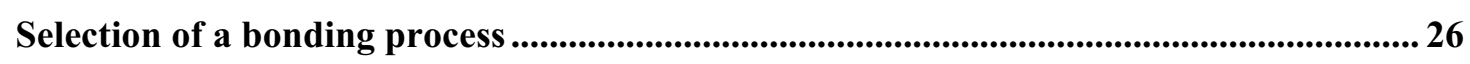 \\
\hline 4.14.1 & Friction Bonding Pros \\
\hline 4.14.2 & Friction Bonding Cons ............................. \\
\hline 4.14.3 & HIP Pros .............................. \\
\hline 4.14.4 & HIP Cons ....... \\
\hline 4.14.5 & 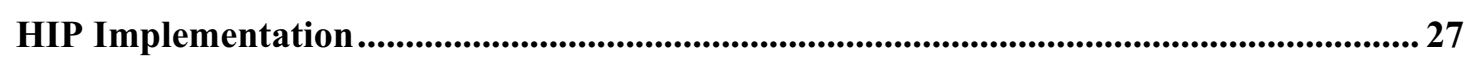 \\
\hline 4.15 & QA Inspection ......................... \\
\hline 4.16 & 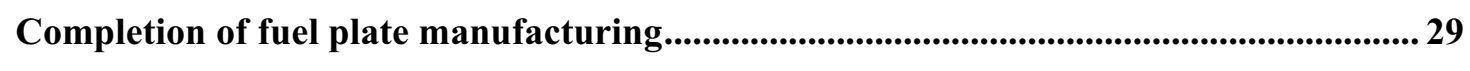 \\
\hline 4.17 & 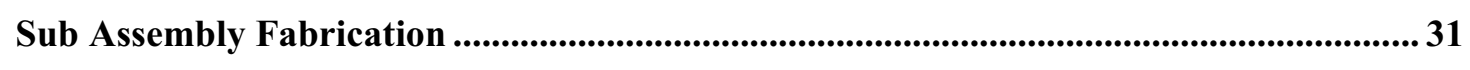 \\
\hline 4.18 & 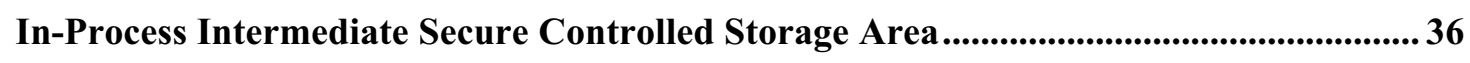 \\
\hline 4.19 & 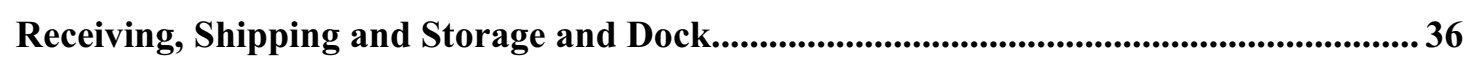 \\
\hline 4.19.1 & 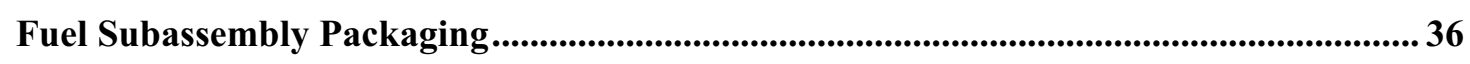 \\
\hline 4.19.2 & Product Storage Area \\
\hline 4.19.3 & Waste Storage Area ..... \\
\hline 4.19.4 & Fuel Material Storage Area \\
\hline 4.19.5 & Dock \\
\hline 4.19.6 & 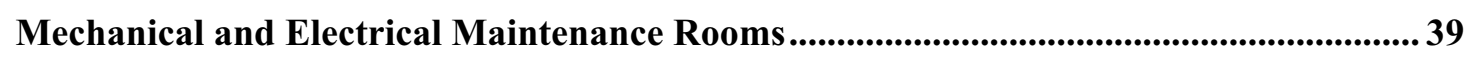 \\
\hline 4.19.7 & 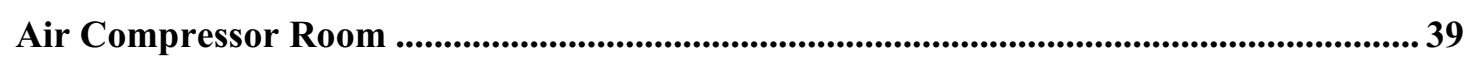 \\
\hline 4.19.8 & 39 \\
\hline 4.19.9 & 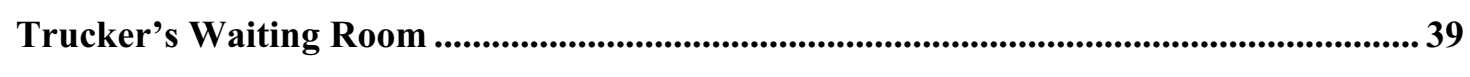 \\
\hline 4.19.10 & CTV \\
\hline
\end{tabular}

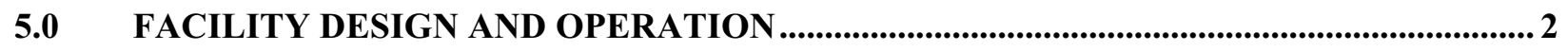

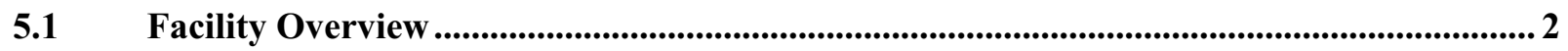

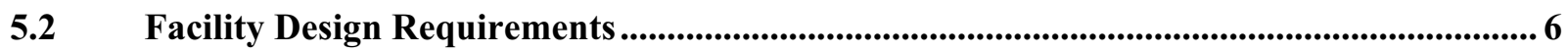




\begin{tabular}{|c|c|}
\hline 5.3 & $\ldots 7$ \\
\hline 5.4 & 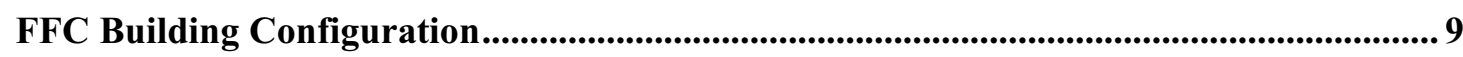 \\
\hline 5.5 & tility Connections \\
\hline 5.6 & 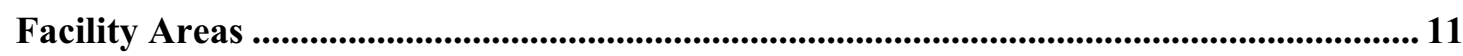 \\
\hline 5.6.1 & 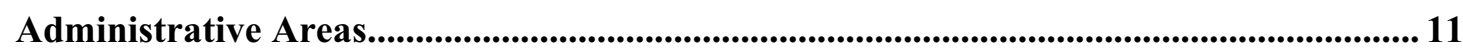 \\
\hline 5.6.2 & Production Areas \\
\hline 5.6.3 & Storage Areas (including safeguards and security) \\
\hline 5.6.4 & 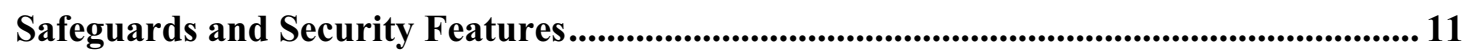 \\
\hline 5.6.5 & Safety and Support Areas \\
\hline 5.6.6 & Personnel Amenities \\
\hline 5.7 & Ventilation ............................................. \\
\hline 5.7.1 & 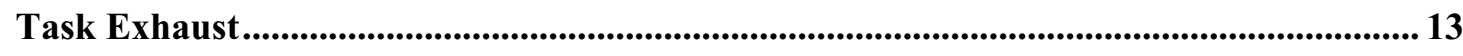 \\
\hline 5.7 .2 & Shop Area \\
\hline 5.7.3 & Office/clean areas............... \\
\hline 5.7 .4 & Design features of the HVAC system \\
\hline 5.8 & 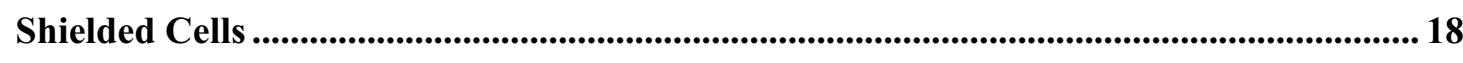 \\
\hline 5.9 & Fire Protection ..................................... \\
\hline 5.9 .1 & Basic Criteria \\
\hline 5.9 .2 & Fire Protection Design Features \\
\hline 5.9 .3 & Waste Drains \\
\hline 5.9 .4 & Floor Finish ........................................ \\
\hline 5.10 & Warehouse \\
\hline 5.11 & FFC Staff \\
\hline 5.11.1 & Operational Staff ............................... \\
\hline 5.11 .2 & Office Staff....................................... \\
\hline 5.12 & Personnel Protective Equipment \\
\hline 5.13 & 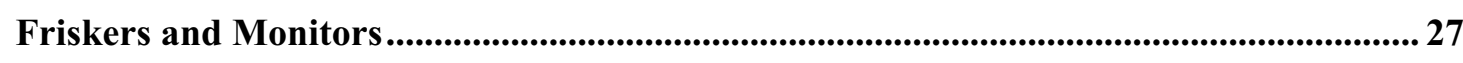 \\
\hline 5.14 & Facility Operation \\
\hline 5.15 & Control Room \\
\hline 5.16 & Utilities \\
\hline 5.17 & 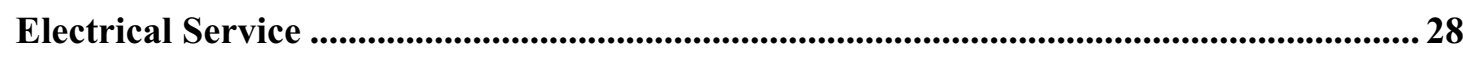 \\
\hline 5.18 & 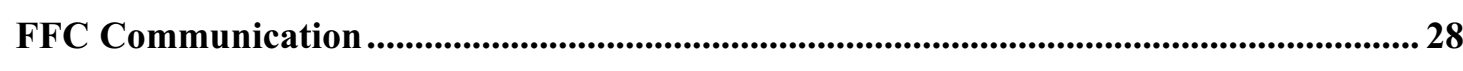 \\
\hline 5.19 & FFC Facility Operation \\
\hline 5.19 .1 & Management and Labor \\
\hline 5.19 .2 & pnoblo \\
\hline
\end{tabular}


5.19.3 Maintenance and upkeep of the building and process systems ........................................ 31

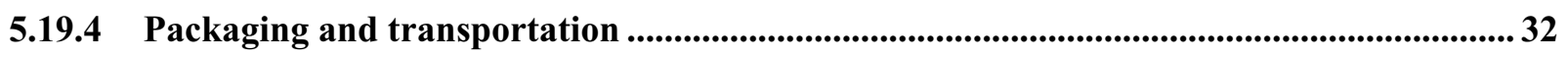

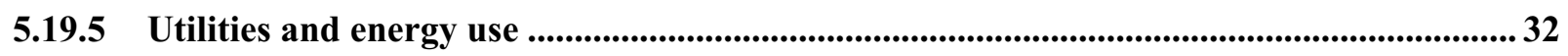

5.19.6 Contracts or subcontracts provided by suppliers and service providers ......................... 32

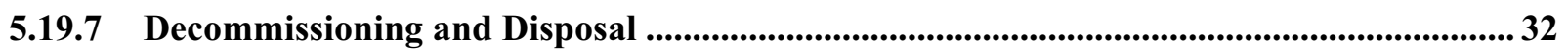

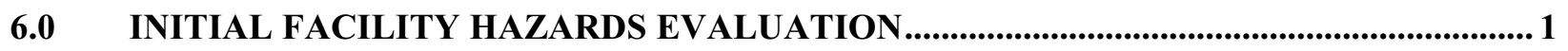

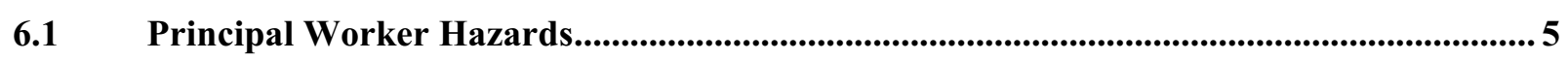

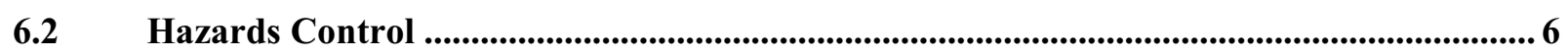

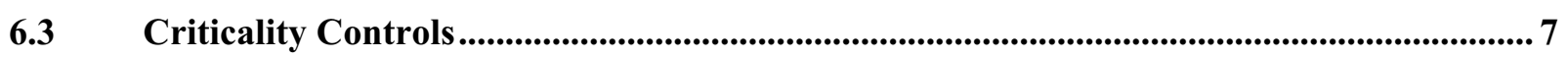

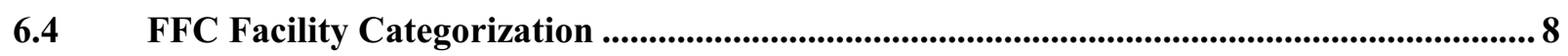

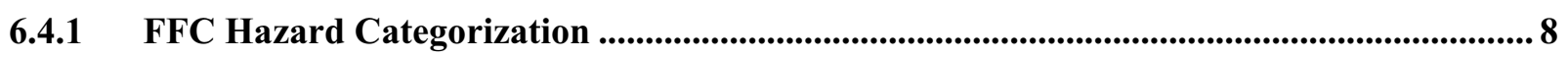

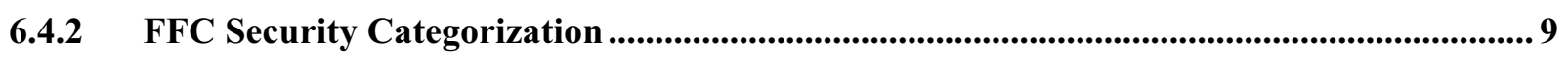

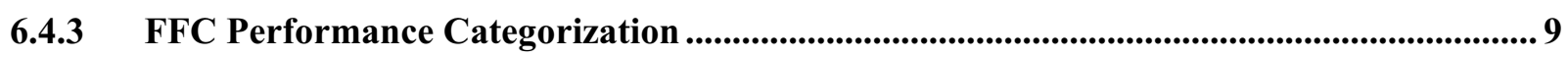

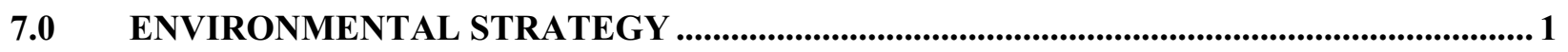

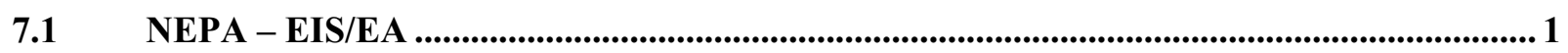

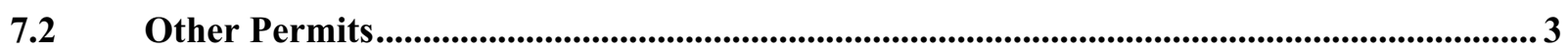

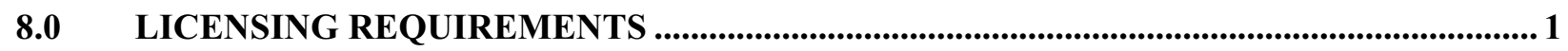

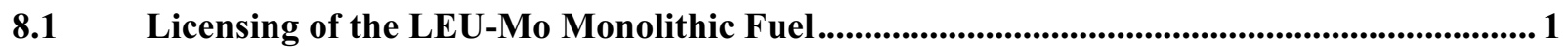

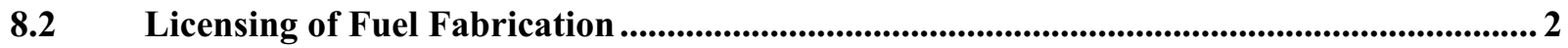

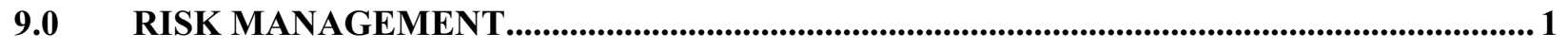

9.1 Programmatic Risks ............................................................................................................ 1

9.2 Technical Risks .................................................................................................................... 4

9.3 Risk Management Plan ....................................................................................................... 8

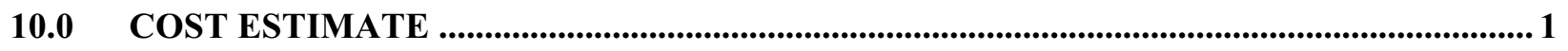

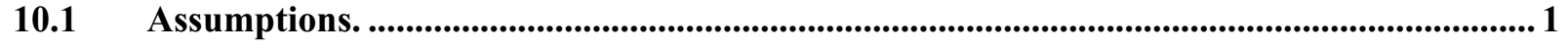

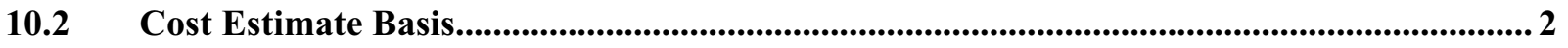




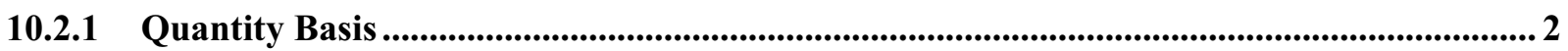

10.2.2 Pricing Basis........................................................................................................................................ 3

10.3 Contingency .................................................................................................................................... 4

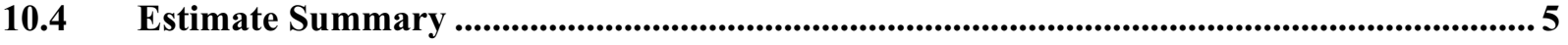

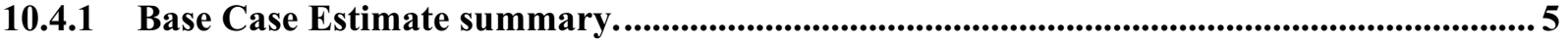

10.4.2 HIP case (HIP bonding) estimate summary ............................................................................... 6

10.5 Operations Cost .......................................................................................................................... 7

10.6 Startup \& Testing .................................................................................................................... 7

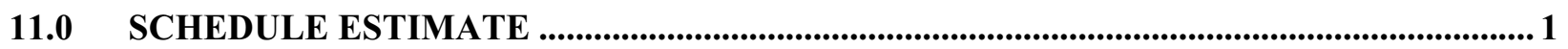

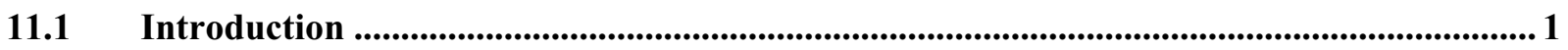

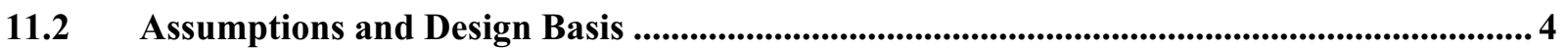

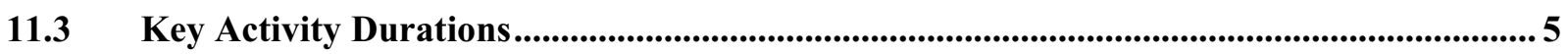

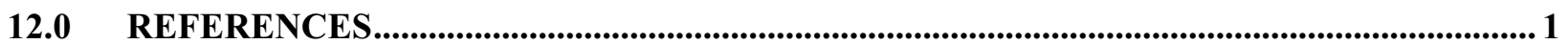

\section{APPENDIX A - DRAWINGS AND DATA}

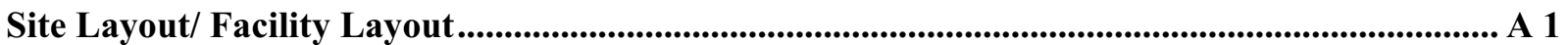

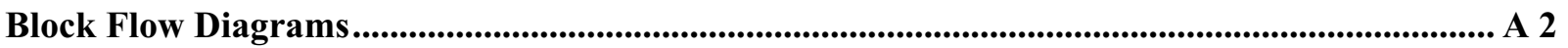

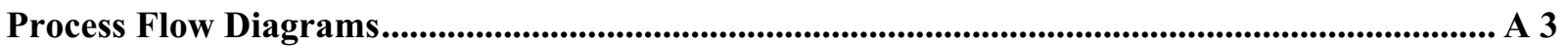

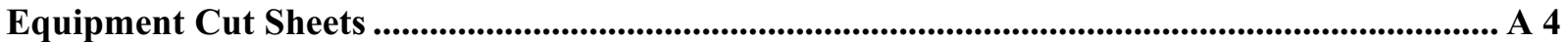

\section{APPENDIX B - ESTIMATE DETAILS}

Base Case (Friction Bonding): Estimate Summary ............................................................................ 1

Base Case (Friction Bonding): Estimate Details ......................................................................................B 2

HIP Alternate: Estimate Summary ........................................................................................................... 3

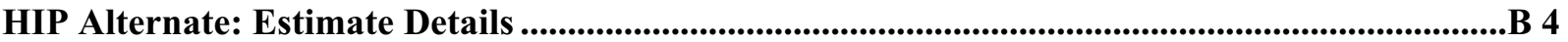




\section{EXECUTIVE SUMMARY}

This report provides the initial "first look" of the design of the Greenfield Alternative of the Fuel Fabrication Capability (FFC); a facility to be built at a Greenfield DOE National Laboratory site. The FFC is designed to fabricate LEU-Mo monolithic fuel for the 5 US High Performance Research Reactors (HPRRs). This report provides a pre-conceptual design of the site, facility, process and equipment systems of the FFC; along with a preliminary hazards evaluation, risk assessment as well as the ROM cost and schedule estimate.

The FFC is an engineered steel framed structure, with insulated metal wall and roof panels. The FFC has a footprint area of approximately $75,000 \mathrm{sq}$. $\mathrm{ft}$. and includes a high bay for the primary process operations area and an adjoining low bay for the element assembly and storage functions. The FFC site occupies an area of 12.5 acres; the site also has a warehouse and an electric substation along with an entry control facility. A 6' high barbed wire fence encloses the site area. The site is assumed to be adjoining a national laboratory site in-order to take advantage of the inplace capabilities of security, emergency services, utilities, low level waste treatment, packaging and disposal services and established transportation corridors.

The feed for the FFC comprising of acorns and broken pieces of the LEU-Mo alloy is transported to the FFC in ES-3100 shipping containers from Y-12. The feed material is receipt inspected and stored in critically safe storage racks in a secured controlled storage room. The fuel fabrication process encompasses the following unit operations.

\section{- Casting \\ - Initial Hot Rolling \\ - Canning \\ - $\quad$ Final Hot Rolling \\ - Annealing \\ - $\quad$ De-Canning and Trimming}

- $\quad$ Cleaning

- $\quad$ Bonding

- $\quad$ Final trimming, finishing and inspection

- $\quad$ Assembly, packaging, and shipping

The FFC is designed to produce up to 17,000 foils (rolled LEU-Mo) a year sufficient to meet the annual needs of the US HPRRs. The FFC is provided with a cascade ventilation system, and a wet pipe sprinkler system. The primary facility hazards are radiological, criticality, fire and industrial hazards. Overall the hazards are low and pose minimal risk to the public. The primary hazards are to the facility worker. The FFC is characterized preliminarily as nuclear hazards category 2, performance category 2 and a security category IV facility. The FFC will have 55 personnel on the shop floor (operators, inspection personnel) and 17 personnel in the office providing management and administrative services.

An integrated timeline has been prepared for the design, licensing, permitting, construction and fuel qualification activities. Initial Critical path activities include licensing and design, which require an early start of conceptual design (10/1/08) in-order to support follow-on critical path activities leading to the hot ops of the FFC by the end of 2012 and conversion of the US HPRRs by the end of 2014 as planned by the NNSA. A DOE 413.3A compliant critical decision approval process has been utilized for the integrated planning timeline.

Based on the facility and process design, a total project cost range for the base case (utilizing friction bonding) FFC is $\$ 62 \mathrm{M}-\$ 73 \mathrm{M}$. An alternate HIP bonding process design has also been prepared. The total project cost of the FFC with the alternate HIP process is $\$ 81 \mathrm{M}-\$ 93 \mathrm{M}$. A Monte Carlo type risk assessment was performed to determine the project contingency, which has been included in the Total Project Cost. A facility annual operations cost has been estimated at $\$ 15 \mathrm{M}-\$ 20 \mathrm{M}$. 


\subsection{INTRODUCTION}

This report comprises the Greenfield Alternative Study for the LEU-Mo Fuel Fabrication Capability (FFC). The study was developed at a pre-conceptual/scoping level to provide a "first look" at the facility footprint, the design of the key process and equipment systems as well as the preliminary process hazards and their mitigation. A Rough Order magnitude cost and schedule estimate range has been provided for the capital and life cycle cost of the facility.

The Greenfield Alternative study is a precursor to the follow-on Conceptual Design, and provides a basis for conceptual project planning and the development of the conceptual functions and operations requirements of the FFC. Additional design development and rigor is required in the following key areas to meet the CDR requirements of DOE O 413.3A:

- Alternatives Evaluation - Facility, Process

- Safety Design Documentation

- Cost, Schedule and Risk

- Management Planning and Control documentation

- Scope/technical development of Balance of Plant Systems.

\subsection{Background}

Since 1978, the US has pursued a policy aimed at reducing the threats posed by civilian commerce in HEU. Since HEU fueled research reactors provide essential services to society, it was recognized that new and advanced fuel technology would be required to replace existing HEU fuels and to accomplish our non-proliferation goals while maintaining the flow of products and services provided by these research reactors.

The National Nuclear Security Agency's (NNSA) Global Threat Reduction Initiative (GTRI) is tasked with enabling the conversion of research reactor fuels from High Enriched Uranium (HEU) to Low Enriched Uranium (LEU) Fuels (The LEU/HEU threshold is defined as material containing $20 \%$ of the ${ }^{235} \mathrm{U}$ isotope). The GTRI supports the DOE's Fuel development program known as the Reduced Enrichment for Research and Test Reactors (RERTR), for developing the technical means for converting these research reactors including the development of new LEU fuels.

The RERTR program cooperates with the research reactor operators to achieve this goal of HEU to LEU conversion while maintaining the reactor performance. The goal of this program is to complete the conversion of the civilian domestic research reactors by 2014 . These reactors include the five domestic high performance research reactors (HPRRs) namely: The High Flux Isotope Research Reactor (HFIR) at the Oak ridge National Laboratory (ORNL); the Advanced Test Reactor (ATR) at the Idaho National laboratory (INL); The National Institute of Standards and Technology (NIST) research reactor; The Missouri University research reactor (MURR) at the University of Missouri, Columbia; and the MIT reactor II (MITR-II) at the Massachusetts Institute of Technology (MIT).

The US HPRR fuel elements currently consist of HEU based dispersion fuel meat clad in aluminum plates. The HEU is used due to the high fissile loadings needed to meet HPRR performance requirements. Existing LEU dispersion fuels do not provide the necessary HPRR uranium loadings for LEU fuels, thus forcing development of new ultra high uranium density fuels. The monolithic fuel form has been identified as a promising ultra high density fuel type (U density $15.3 \mathrm{~g}-\mathrm{u} / \mathrm{cm} 3)$ that is appropriate for research reactor application. This fuel design 
consists of a monolithic U-Mo alloy foil encapsulated (clad) in aluminum plates (Al-6061). The monolithic fuel design provides the maximum in-reactor fuel uranium loading. The result is a uranium loading density that should allow the US HPRRs to maintain their existing performance upon conversion without major modifications. The unique aspects of the monolithic fuel design, necessitates development and implementation of new fabrication techniques and establishment of the Fuel Fabrication Capability (FFC) to meet the fuel needs of the US HPRRs

The development, testing and qualification of monolithic LEU fuel fabrication technology is being aggressively pursued by the national laboratories (INL, ANL and others) in-order to meet the goal of the program to achieve the generic qualification (NRC Approval) of the U-Mo monolithic fuel design by the end of 2012 .

In order to meet the programmatic mandate to complete conversion of all civilian domestic HPRR reactors by 2014, the construction of the necessary Fuel Fabrication Capability (FFC) for the fabrication of the U-Mo monolithic fuel must occur concurrently with the completion of the fuel qualification effort. A U-Mo monolithic Fuel Fabrication Facility is therefore required to be on line by the end of 2012, to provide the required capability to manufacture the new LEU fuel for the HPRRs.

\subsection{Project Description}

The FFC is designed to be a state-of-the-art fuel fabrication facility with a design life of 40 years. It is proposed to be built at a Greenfield location within a DOE National Laboratory site or a NNSA complex site. The FFC is designed to fabricate up-to 17,000 monolithic foils of LEU-Mo alloy per year to meet the needs of the US HPRRs. INL and other national laboratories have developed the monolithic fuel fabrication process, which will be adapted for use in the production scale fuel fabrication capability facility.

The FFC is classified as a Nuclear Hazard Category 2 facility on account of criticality hazards, and will be designed to Performance category 2 requirements for the NPH design. FFC handles/processes LEU which is classified as Category 4, Attractiveness level E material for safeguards and security requirements.

The FFC is housed in a steel framed structure with insulated metal panels for the walls and the roof. The FFC has a high bay process area for the fuel plate fabrication processes and an adjoining low bay area for the fabrication of the fuel assembly, secure controlled storage areas and the shipping and receiving operations. The plate fabrication and fuel assembly areas include a central isle for material handling and transfers, with the process equipment placed on either side of the isle. Overhead cranes (5 Tons) are provided to service operations on each side of the isle.

The fuel fabrication process includes the following key operations:

- Casting: LEU-Mo acorns and broken pieces received from Y-12 are melted using induction furnaces and cast into book molds. Each mold is $1 / 4$ " 3.5 " x 16 " long.

- Initial hot rolling: The casting are hot rolled to intermediate thickness of 0.1 " thick x 40" long. These intermediate U-Mo foils are sectioned into 10 pieces each 4" long.

- Canning: The rolled foils are sandwiched between $\mathrm{Zr}$ sheets and sealed inside a welded steel can. The can is coated on the inside with Yttria.

- Final Hot Rolling: The steel can is hot rolled to final foil thickness.

- Annealing: The clad foil is annealed to reduce residual stresses from rolling, reduce waviness and improve flatness 
- De-canning and trimming: The can is opened. The 0.010 " to 0.015 " thick rolled foil is then sheared to final size.

- Cleaning: The foil is chemically cleaned in a solution containing Nitric acid, DI water and HF followed by a drying step (blowing filtered air or nitrogen and wiping the outside)

- Bonding: The cleaned foil is then clad in aluminum plates, and the fuel meat (U-Mo foil) is bonded to the aluminum cladding by either friction stir wedding or by using the Hot Isostatic Press process. For the purpose of this study Friction stir welding is the primary process, the HIP process is considered as an alternate.

- Final trimming, surface finishing, inspection, cold forming and final cleaning: The fuel plates are flattened, surface finished, marked and cold formed to match the required fuel configuration. The aluminum clad fuel plates are next subject to final chemical cleaning. Quality inspections are performed using a number of NDE techniques such as radiographyfluoroscopy, UT and Gamma scanning.

- Final Assembly, Packaging and Shipping: Multiple fuel plates are retrieved from interim storage, and fitted within side and end plates to form the fuel element (sub-assembly) for the specific HPRR. The sub-assemblies are machined and cleaned and or autoclaved and dried (blowing filtered air or nitrogen and wiping the outside). The sub-assemblies are inspected, packaged and shipped to the specific HPRR

Offices and support areas (Lunch room, Change rooms) are provided as well as secured controlled storage areas for the storage of incoming feed material from Y-12, and storage of inprocess foils and plates as well as finished plates. Accounting areas are provided within the facility for accountability controls. Zoned cascade HEPA filtered ventilation is provided for ventilation of process areas, local HEPA filtered task exhaust is provided at work stations, and recirculating ventilation system is provided for clean areas. A wet pipe sprinkler system is provided throughout the facility. Provision of fire water collection and disposition is included in the facility design.

Criticality control requirements relative to the storage of the incoming cans of U-Mo as well as storage of in process foils, plates and final assemblies have been addressed in the design of the storage racks and cabinets.

The FFC site is enclosed within a fenced boundary. An entry control facility is provided for access control for personnel and materials. A standalone warehouse is provided for the storage of non SNM materials and consumables for the fuel fabrication process. An electrical sub-station may be required to support the power needs for the project.

As the FFC is to be sited at a DOE National Laboratory or a NNSA complex site, the supporting infrastructure for the FFC is assumed to be in place. The following services are assumed to be provided by the host site: Utilities (water, power, sewer); waste treatment and disposal; emergency management; Site security and established access to transportation corridors and trained nuclear facility operations personnel

An integrated design, construction and NRC licensing strategy have been proposed for the acquisition of the FFC. The strategy conforms to the DOE 413.3A Project Acquisition Order, as well as provides realistic timeframes for these activities and yet meeting the hot ops date for the FFC operations in January of 2013.

A facility layout plan showing the arrangement of the equipment and support areas is shown in Fig. 1-1. An overall facility perspective showing the FFC site and the Fuel Fabrication Facility is shown in Fig 1-2. Detail descriptions of the facility and equipment are provided in sections 4 and 5 of the study report. 


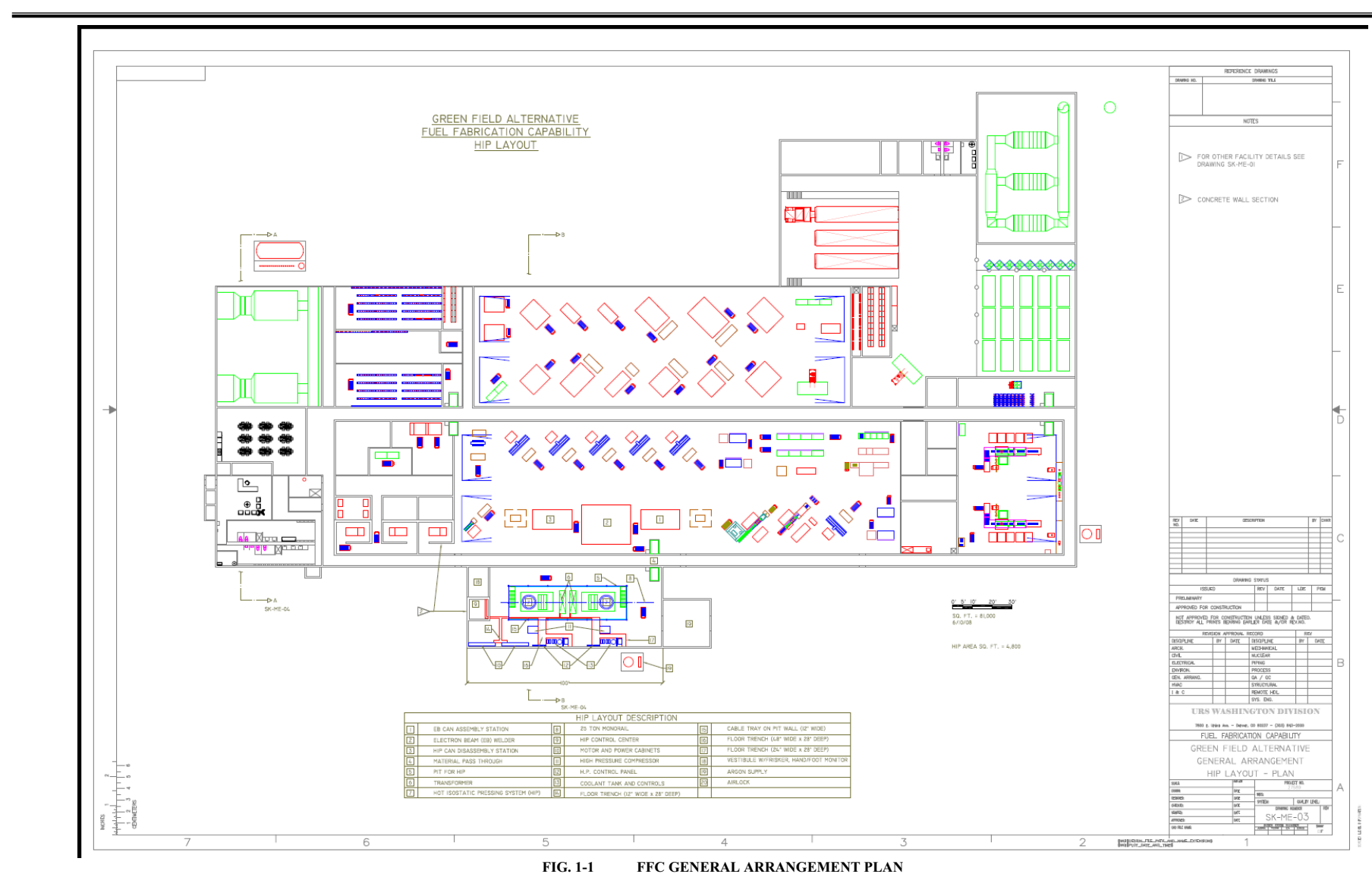




\section{GREEN FIELD ALTERNATIVE FFC}

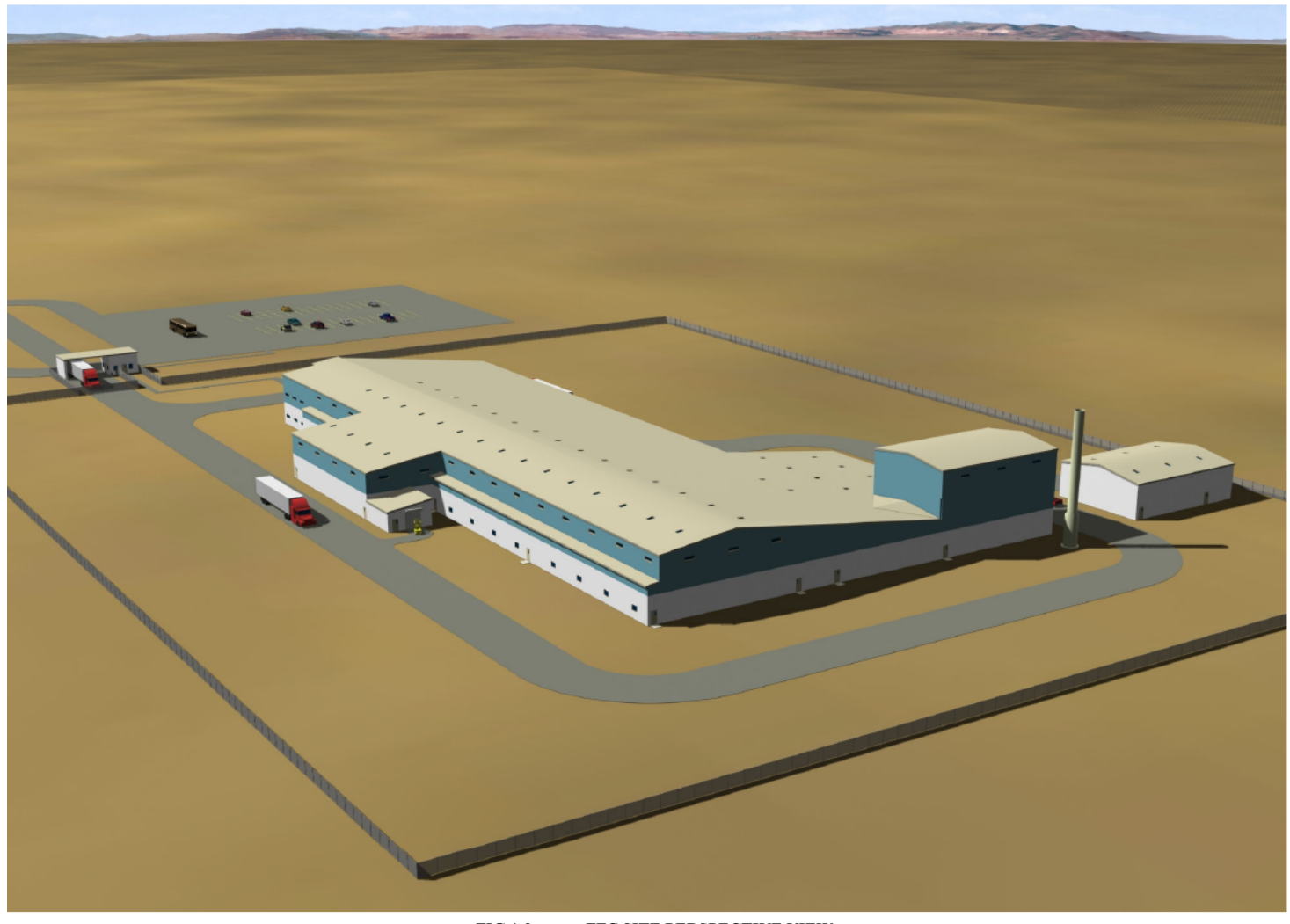

FIG 1-2 FFC SITE PERSPECTIVE VIEW 


\subsection{Study Basis}

This section provides an overview of the study objectives, work scope and the study report contents.

\subsubsection{Objective}

The key objective of the study is to provide an initial scoping level design of the process, facility and equipment required to fabricate the monolithic LEU-Mo fuel for the HPRR. The study also provides a Rough Order Magnitude Cost and schedule for acquiring the FFC.

The study provides an initial concept for the required LEU-Mo monolithic fuel fabrication process, the layout/general arrangement of the facility showing equipment arrangement and support areas, the description of the process equipment, as well as an initial look at the hazards and their mitigation; and Rough Order magnitude cost and schedule estimate of the Total project and Life Cycle cost.

\subsubsection{Work Scope}

The work scope for this Greenfield Alternative Study includes the preparation of this Greenfield Alternatives Study Report which includes the following:

- Prepare preliminary arrangement, sections and elevations drawings for the FFC, which indicate salient features, access and egress characteristics, major equipment, and major utility routings.

○ Develop facility functional arrangements for the Greenfield FFC.

- Establish rough space allocations for general occupancy and for equipment installation, operation and maintenance.

- Establish and quantify type of construction, significant design features, preliminary hazard classifications and occupancy ratings, and required building systems and/or utilities.

- Identify sufficient project features to develop the cost estimate. The high-level design description shall describe details not specifically delineated on the drawings.

- Identify preliminary hazards and process conditions and recommend mitigations for control of hazardous material and conditions. Recommend preliminary hazards classification for the facility or, by using a graded approach, portions of the facility.

- Identify requirements for security, containment, environmental protection, safety and industrial hygiene.

- Establish preliminary flowsheets, which include waste, recycle, utility, mechanical, off-gas, and HVAC requirements. Leverage the production/throughput evaluations provide in the Conceptual Process Description to provide an integrated process throughput that will meet the demand requirements for the 5 domestic HPRRs. Consider process efficiency and estimated maintenance downtimes. Determine the availability and interface locations of existing process and utility systems, Determine preliminary system requirements and provide descriptions of the recommended systems.

- Some heat applications and machining processes will require ventilation over and above normal HVAC for habitation. Assume minimal shielding and construction options, which meet DOE ALARA requirements. 
- Provide an early NRC licensing strategy recommendation and basis for the recommendation.

- Provide drawings for the FFC plant. The drawings should include a generic site plan, facility and equipment layout and process flow diagrams.

- Provide a process and mechanical equipment list and identify Long Lead time equipment.

- Identify initial high level technical and programmatic risks.

- Provide an initial Rough Order Magnitude type estimate for the Total Project Cost (TPC) range as well as yearly operations cost.

- Prepare an overall project schedule addressing design and construction activities addressing the requirements of DOE O $413.3 \mathrm{~A}$ as well as NRC licensing.

\subsubsection{Report Contents}

The Greenfield Alternatives Study is presented in one volume, and provides a preliminary understanding of the LEU-Mo monolithic fuel manufacturing process commencing from the receipt of the LEU-Mo feedstock from Y-12 to the final assembly of the fuel elements and shipment for insertion in the 5 US HPRR's; the facility required to house the process equipment and systems as well as the required facility support. The report covers the following key technical areas:

- Basis and Assumptions

○ Process Systems Design

○ Mechanical/Process Equipment Description

○ Facility Design

○ Initial Hazards Evaluation

- Environmental Strategy

$\bigcirc \quad$ Licensing Strategy

○ Risk Management

○ ROM Cost Estimate

- Schedule Estimate

○ Supporting Process Flow Diagrams, Site and facility Layout Drawings

\subsection{Proposed Future Studies}

This section lists the key engineering studies as well as R\&D needs to support the design engineering studies

\subsubsection{Engineering Studies}

The Greenfield Alternatives study provides an initial look at the site, facility, process and equipment systems required for the FFC. A number of alternative and value engineering studies are proposed below to optimize the initial concept included in this report. These studies may be performed as part of the "Alternatives Analysis" at the start of the conceptual design effort. 
- Address principles of "Lean manufacturing" in the process operations/facility layout. Perform Discrete Element modeling/Time and Motion modeling to identify any bottlenecks and assure the required throughput can be achieved. In addition the study will address if the requirements for multiple stations, spare parts and redundancy have been established to meet the FFC plant throughput of 17,000 plates per year.

- Perform trade study to evaluate if the casting operations should be conducted in the FFC Vs casting at Y-12. This evaluation will be based on the existing shipping container limitations, but will allow variation of the inner "convenience can" geometry.

- Perform trade study in conjunction with developmental work to define the most effective means of fuel plate assembly and bonding by using either the friction bonding process or the Hot Isostatic Press (HIP) Process.

In addition, for the HIP process two development tracks have been identified for isolating the fuel from the HIP environment. These two methods are use of a HIP can and the other method is the use of a perimeter weld with either vacuum welding or ultrasonic welding.

With the HIP process, a steel can is built around a stack comprising of a number of plates (Fuel foil sandwiched between aluminum cladding). The plates are sandwiched between rigid strong-backs to keep the fuel plates flat during the HIP process.

The Perimeter welding method eliminates the need for a HIP can. In the perimeter welding method the welded fuel plates are stacked between strong-backs to provide rigidity during the HIP process and to maintain the flatness of the plates.

In the Vacuum perimeter welding method the fuel plate is welded under vacuum to hermetically seal the fuel plate from the HIP media using an electron beam welder.

In the ultrasonic perimeter welding method, a contact probe vibrates the cladding material causing friction and localized welding. When used in a seam welding configuration a hermetic seal is achieved under a vacuum.

- Evaluate the cost and benefit of the waste management strategy of providing limited waste management capability in the FFC of using an evaporator to minimize the volume of liquid waste, as well as perform the required waste stabilization prior to shipping the waste to an on-site Treatment, Storage and Disposal (TSD) facility. The objective is to reduce the operations cost by reducing the waste volumes. In addition ways to minimize $\mathrm{Zr}$ clad U-Mo and other mixed alloy waste should be addressed.

- Evaluate the facility expansion required to provide LEU-MO monolithic fuel to 5 reactors in Western Europe in addition to the five US HPRRs.

- Study the cost/benefit of additional automation in the facility

- Perform a trade study to identify the cost effective method of either fabrication of the sub-assembly end-pieces and or cladding plates in house or to out-source the same.

- Perform a source survey to confirm availability of appropriate quality level suppliers of pre-fabricated Al-6061 aluminum cladding plates, end and side pieces for use in the FFC.

- Perform an optimization of the facility layout considering evaluation of the flow of materials and personnel for accomplishing the process operations. 
- Perform a source survey to identify quality manufacturers of furnaces, milling and rolling machines as well as HIP, stir welding and ultrasonic inspection machines, as well as the lead to time for supply of these specialized equipment items.

- Evaluate storage requirements for the in coming feed (LEU-Mo) and the final sub assemblies, based on a system study of the HPRR fuel demand and efficient inventory of materials required to meet demand.

- Perform a trade-study to address the need for the filter blow-back system and filter leaching Vs use of the filter bag-out system.

- Perform a study using a workable casting line concept for casting plates of LEU-Mo using preheated graphite book molds that function under an inert atmosphere. Study whether microwave or induction melting of alloy within the crucible should be implemented. Microwave melting is an emerging technology that requires a special two part crucible that is more efficient than induction melting and all water cooling takes place outside the inert atmosphere enclosure. Induction melting is the industry standard, however the induction coils that closely surround the crucible containing the melt inside the enclosure require water cooling that poses a safety hazard.

- Conduct vulnerability assessment to define the security requirements considering the proposed FFC operations and inventory

- Conduct RAMI analysis of the process equipment train to confirm proposed facility throughput rates can be achieved

\subsubsection{R\&D and Verification Needs}

The following lists the R\&D as well as process validation needs in support of the design of the FFC

- Conduct performance testing to down-select the fuel plate bonding process between either friction bonding or HIP.

- Conduct performance testing for down-selecting the diffusion barrier material of either Zirconium, Molybdenum or Silicon.

- Perform required malleability testing of the cast LEU-Mo ingot, to provide the required foil size upon rolling, thereby reducing scrap

- Perform testing to determine the "rollability" parameters and material properties of U-Mo and canned U-Mo foils at room and elevated temperature

- Perform testing to evaluate the feasibility of the use of microwave melting for the casting operations, which has the potential benefits of eliminating water cooling from the inert gas enclosure surrounding the crucible and reducing energy usage.

- Number of in process non-destructive examination processes (i.e. UT, radiography, gamma scanning and fluoroscopy) have been proposed for the current fuel fabrication process. Some of these methods (e.g. ultrasonic de-bond measurement) need to be qualified on the higher density monolithic fuel form. Work also needs to be done to validate updated measurement processes (i.e. real-time radiography as opposed to the film process that is now used) to make the analysis process more efficient.

- Perform testing to validate the need for an in-can annealing process in a constrained or an un-constrained state. 


\subsection{PROJECT REQUIREMENTS}

The following INL documents provide the High Level Basis for this Greenfield Alternative study:

- Statement of Work for An Engineering Alternative Study for a Greenfield LEU Molybdenum Fuel Fabrication Capability - April 2008

- Conceptual process description for the Manufacture of Low Enriched Uranium Molybdenum Fuel; Wachs Et al; INL/EXT-08-13840; February 2008

Additional guidance and specific project design basis and requirements were provided by cognizant INL and Y-12 personnel involved with the development of the monolithic LEU-Mo fuel. Notes of meetings and Notes of weekly teleconference document the programmatic and technical direction relative to the process and equipment requirements. In addition a 35\% complete design review was conducted by the cognizant INL and Y-12 personnel, comments and directions resulting from the design review were recorded in the Notes of the $35 \%$ complete design review meeting.

The directions, comments received from the cognizant INL and Y-12 personnel during the weekly teleconference, as well as at the design review and Kick-off meetings were considered as design basis requirements and incorporated into the facility and process design.

The Applicable Codes and Standards that provide design basis requirements appropriate for this Study are as follows:

- National Environmental Policy Act (NEPA), 40 CFR 1500

- Resource Conservation and Recovery Act (RCRA)

- Clean Air Act

- Clean Water Act

- Applicable DOE Orders including DOE Order 413.3A

- Federal Regulation 10 CFR 830, Nuclear Safety Management

- Nuclear Waste Policy Act of 1982

- 10 CFR 70, Domestic Licensing of Special Nuclear Materials

- DOE-ID Architectural and Engineering Standards

- ANSI/ANS 8.1 and ANSI/ANS 8.10, Criticality Control

- DOE O 420.1B, Facility Safety

\subsection{Assumptions and Design Basis}

Table 2-1 provides the listing of Assumptions and Design Basis used for the preparation of this Greenfield alternatives study, including the development of the process and mechanical equipment designs as well as the layout of the fuel fabrication facility.

The Assumptions and design basis are grouped into the following categories:

- Programmatic

- Process

- Facility 
- Safeguards and Security, and

- Quality Inspection

TABLE 2-1 - LIST OF ASSUMPTIONS AND DESIGN BASIS

\begin{tabular}{|c|c|}
\hline A & PROGRAMMATIC \\
\hline 1 & $\begin{array}{l}\text { The FFC will be a Government owned and contractor operated facility. The DOE will } \\
\text { provide the necessary safety oversight with guidance from the NRC as appropriate }\end{array}$ \\
\hline 2 & The Fuel Fabrication Facility (FFC) will be designed with a 40 year life \\
\hline 3 & $\begin{array}{l}\text { The FFC will be designed to provide LEU-Mo fuel for the five currently operating } \\
\text { domestic High Performance Research Reactors }\end{array}$ \\
\hline 4 & $\begin{array}{l}\text { The FFC will be built at a Greenfield site located at a DOE National Laboratory. All site } \\
\text { services (Fire and emergency response, waste treatment and disposal, site security etc.), } \\
\text { utility services (power, water, sewer etc.) and infrastructure are available at the selected } \\
\text { site. }\end{array}$ \\
\hline 5 & $\begin{array}{l}\text { The site selection will be completed by } 4 / 09 \text { in time to support preparation of the } \\
\text { Environmental report. The selected site will be free of contamination and will be well } \\
\text { characterized. }\end{array}$ \\
\hline 6 & $\begin{array}{l}\text { The Fuel Qualification effort and the facility design and construction shall be conducted in } \\
\text { parallel, with completion by the end of } 2012 \text {. }\end{array}$ \\
\hline 7 & $\begin{array}{l}\text { The FFC will be licensed by the NRC, and will meet all applicable state and federal } \\
\text { regulations (DOE, NRC, EPA), as well as National Consensus Codes and Standards. }\end{array}$ \\
\hline 8 & $\begin{array}{l}\text { The developmental LEU-Mo fuel fabrication line at INL for the R\&D program will } \\
\text { produce the LTAs for the qualification of the fuel for the first converting reactor while the } \\
\text { FFC becomes operational. }\end{array}$ \\
\hline \multirow[t]{2}{*}{9.} & $\begin{array}{l}\text { The Fuel Qualification Report will be submitted to the NRC -December 2010. Anticipated } \\
\text { NRC approval of the Fuel Qualification Report - December } 2011 .\end{array}$ \\
\hline & $\begin{array}{l}\text { Down selection of the interlayer fuel design and the bonding process will be made by } \\
\text { December } 2009 \text {. }\end{array}$ \\
\hline \multirow[t]{2}{*}{10.} & $\begin{array}{l}\text { The NRC has sufficient staff as well as in-place regulations for the licensing of the FFC as } \\
\text { planned. A dual track licensing strategy is proposed for the licensing of the FFC } \\
\text { (construction permit and operations license for the facility) and for the LEU-Mo fuel (Fuel } \\
\text { Qualification reports containing results obtained from the pre-irradiation and post- } \\
\text { irradiation tests on miniature plates and full sized fuel elements of monolithic LEU-Mo } \\
\text { fuel). A NUREG } 1313 \text { type licensing approach is proposed for the licensing of the LEU- } \\
\text { Mo fuel. }\end{array}$ \\
\hline & $\begin{array}{l}\text { Early involvement of the NRC during the design phase of the project is crucial to the } \\
\text { overall timely approval of the construction permit. }\end{array}$ \\
\hline 11. & $\begin{array}{l}\text { The Fuel fabrication capability acquisition strategy will be approved prior to } 9 / 30 / 08 \text {. } \\
\text { Conceptual design effort will commence } 10-1-08 \text {, in-order to meet the end date for facility } \\
\text { hot ops by } 1 / 2013 \text {. Site selection needed by April } 2009 \text {. }\end{array}$ \\
\hline
\end{tabular}


12 CD-1 approval documentation per PDRI, comprising of Scope/ Technical, Cost, Schedule, Management Planning and Control, and External factors will be prepared during the CDR effort. DOE's CD-1 approval will be received by 7/1/2009

12. Sufficient funding as planned will be provided on an annual basis to allow for uninterrupted completion of the design, construction, test and start-up as well as fuel development work required for the completion of the fuel qualification data package submittal to the NRC.

13 NNSA will fund the design, construction and start-up of the FFC. DOE/NE will provide the funding for the operations and maintenance of the FFC.

\section{B Process}

1. FFC will receive LEU-Mo cast Feedstock (acorns or broken pieces) from Y-12 in ES-3100 shipping containers.

MC\&A will meet the requirements of DOE (DOE O 470.4, DOE M 470.4-6, and DOE M 474.1-2) or the NRC (NUREG/BR-0006)

Induction melting and coupon casting will be performed at the FFC for the purpose of this study. As an alternative a future trade study will be performed to address if the coupon casting work should be performed at Y-12.

Process operations will be conducted in a batch or campaign mode.

2. Up to one core storage of fabricated fuel for each of the five HPRRs must be provided, in addition to storage of up-to a one year supply of LEU-Mo cast feedstock.

Waste will not be allowed to accumulate in the facility for more than 90 days.

3. Waste will be packaged and shipped to other facilities on or off the DOE site for treatment and disposal.

4. The Al-6061 aluminum cladding machine work (flattening, sizing and pocketing) will not be performed in the FFC. These items as well as fuel assembly side and end pieces will be supplied commercially from an off-site source, with dedicated production and suitable quality assurance capabilities. Configuration of supplied items will be controlled by the FFC.

A future trade study should be performed to evaluate if this task can be performed more cost effectively by outsourcing to an external machine shop or if it might be incorporated into the process line of the FFC.

5. The diffusion barrier layer will be applied by hot rolling a thin layer of Zirconium or other metal on the top and bottom of the fuel coupon in a sealed can at atmospheric pressure that has been evacuated and backfilled with Argon.

A future trade study and performance tests should be performed to evaluate from an engineering and process efficiency perspective if other materials or a thermal spray technique applying an aluminum-silicon alloy would be better. 
6. The fuel plate assembly bonding shall use the friction bonding process. The alternate HIP process will be evaluated and the required floor space to accommodate the HIP process will be determined.

A future trade study performed in conjunction with the miniplate fuel fabrication development process will evaluate, from engineering and process requirements standpoint; the appropriate fuel plate bonding process (friction bonding or the HIP process).

7. Fuel plate pre-assembly shall be manually performed.

A future trade study will be performed to determine if plate pre-assembly (prior to bonding) can be practically automated or a manual operation is required.

8. The FFC is designed to provide LEU-Mo fuel for the five US High Performance Research Reactors. No provision for FFC expansion will be made to accommodate the fuel needs of additional foreign research reactors.

A future trade study will be performed to identify the additional facility footprint and cost schedule implications as a result of the facility expansion to accommodate foreign research reactors.

9. Clean scrap LEU-Mo alloy may be returned to Y-12 or reused within the FFC.

10. Aluminum plates of the required size, configuration and thickness, and cans used during rolling of $\mathrm{Zr}$ onto the fuel meat will be purchased as outsourced materials.

11. Equipment will have a $75 \%$ availability to allow for maintenance and scheduled operations shut-down.

C Facility

1. The Facility is classified as Nuclear Hazard Category 2 and Performance Category 2.

2. A secure but not hardened controlled storage area is required for the storage of convenience cans containing LEU-Mo acorn/broken pieces received from Y-12 as well as cast ingots, fuel foils, and complete fuel assemblies. Cabinets/racks will be used for the in-process storage of foils.

3. A receipt inspection area is required for receiving and un-packaging of the shipping containers, performing the required receipt inspections, and completing the required receiving documentation.

4. A 6' high corrugated wire fence will enclose the site. A manned Entry Control Facility (ECF) will be provided at the site boundary for security and access control.

5. A warehouse for the storage of materials/consumables, tooling/fixtures etc. and a work shop for minor repairs shall be provided within the site.

6. Hard walled offices and bull pen type cubicles shall be provided for office personnel including rest rooms and a lockable records storage area.

7. Shop floor personnel will wear PPE during performance of their work within the radiological control area. Facility operation will normally be single shift; however multiple shifts may be required to address bottle necks and to meet production schedule. Provide change rooms and lunch room for the staff. 
8. The Casting area will be segregated from the balance of the production area by a ( $2 \mathrm{hr})$ fire-rated barrier to reduce the potential consequences of a fire in the casting area. This barrier shall not be degraded by activation of any active fire suppression system.

9. A high bay shall be provided in the main processing area, including overhead crane/s for material handling as well as for equipment change out. At some point in the life of the plant, any or all stations involved in monolithic fuel manufacturing may require change out.

10. The shop floor will be equipped with parts cleaning baths including nitric acid and caustic cleaning solutions. Molten salt baths for pre-heating, prior to rolling, are also incorporated in the plan.

Such baths, and other designated stations that have the possibility to be sources of contamination, will be equipped with pre-filtered exhaust ventilation ducts that induce a draft away from the operator. Face velocity at the opening to the task exhaust duct will be 250 feet per minute.

11. A cascaded negative air pressure (relative to the atmosphere) will be established and maintained within the entire shop floor volume of the FFC at all times. The air flow will be from areas with low or no contamination toward areas of increasing probability of contamination, where it is exhausted.

Air circulation shall be provided to maintain the airborne concentration of uranium below $0.25 \mathrm{mg} / \mathrm{m} 3$ which is the permissible exposure limit ( 8 hour time weighted average) for chemical toxicity for insoluble compounds of uranium (29 CFR 1910.1000 Table Z-1). If soluble uranium compounds are present, the permissible exposure limit is $0.05 \mathrm{mg} / \mathrm{m} 3$.

HEPA filtered exhaust from the shop floor area will be released to the atmosphere through a stack outside the building. Redundant HEPA filtration will be provided before release to the atmosphere.

Filtered and conditioned air will be supplied to all areas of the plant.

The FFC is arranged so that material or personnel cannot exit the confinement area to the outside directly without passing through a ventilation buffer area first.

12 The non process areas of the FFC shall be designed to provide handicapped personnel access in accordance to the requirements of ADA.

\section{Safeguards and Security}

1. The FFC will store and process LEU-Mo material categorized as Attractiveness level E. The SNM inventory for the facility will exceed the reportable quantity, making the FCC a Security Category 4 facility. Facility hardening is not required.

2. Material Balance areas shall be established within the facility. Material accountability shall be performed in accordance with DOE O 470.4 and DOE M 470.4-6 unless NRC accountability requirements supersede the DOE requirements.

3. Traceability of lot numbers of the LEU-Mo during the casting and foil/sub-assembly fabrication process shall be maintained.

4. Partitioned, geometrically safe secure storage racks shall be used for the storage of LEUMo shipping cans shipped from Y-12, as well as storage of in-process foils. 
1. An approximately $30^{\prime} \times 30^{\prime}$ area shall be provided adjacent to the process area for a quality/inspection laboratory. Besides dimensional inspection, ultrasonic testing, radiography and fluoroscopy, metallographic, chemical analysis, and microscopic inspection (including sectioning, polishing and etching) capabilities shall be available in the FFC lab.

2. Y-12 will provide the certified chemical and isotopic analysis of the incoming LEU-Mo feed to the FFC. Alloys cast in the FFC will be certified to meet reactor requirements by the FFC. Chemical and isotopic analysis will need to be redone

3. During the foil fabrication process, four QA hold points for inspection will be required: Inspection of foil thickness and aluminum thickness (with recess)

Inspection of the pre-assembly of the aluminum clad plate

Inspection of the assembly prior to bonding of the aluminum clad plates.

Inspection of the sub-assembly prior to packaging and storage

\subsection{High Level Functions and Requirements}

The LEU-Mo Fuel Fabrication Facility (FFC) will fabricate nuclear fuels using lowenriched uranium (LEU) (less than $20 \% \mathrm{U}^{235}$ ) that is alloyed with $10 \%$ molybdenum. Per Title I of the Atomic Energy Act of 1954, uranium enriched in the isotope $U^{235}$ constitutes special nuclear material (SNM) that is subject to nuclear materials safeguards. Possession and use of the feedstock material as well as the fuel assemblies fabricated and the scrap materials generated during the fabrication process are tightly regulated by DOE and/or the NRC.

The nature and amount of nuclear material in the fuel fabrication facility means that nuclear criticality cannot be excluded from the set of accidents that could occur within the facility. The potential for nuclear criticality drives the facility hazard characterization, and therefore, impacts the overall facility design, including cost.

These and other requirements place design and operating constraints on the overall facility that must be integrated into the facility design at the conceptual design stage. Early identification of requirements and integration of these requirements into the facility design helps ensure acceptability of the design for licensing as well as the ultimate operability of the final facility.

This section identifies key topics that define the functions and requirements that must be addressed by the FFC design. These high-level topics are related to permits or licenses required for start-up and operation of the facility and include safeguards and security for protection, control and accountability of the nuclear material; design of the facility for containment and/or confinement of nuclear (and other hazardous) materials; protection of the environment; nuclear safety of the facility; and industrial hygiene/worker safety.

\subsubsection{Safety Management}

DOE has implemented policy regarding integration of safety management in all phases of its work. This policy, defined in DOE P 450.4, Safety Management System Policy, requires that principles of Integrated Safety Management be implemented in all work performed by DOE or its subcontractors. DOE implements this integrated safety management policy in its acquisition of new facilities through DOE O 413.3A, Program 
and Project Management for the Acquisition of Capital Assets. This order mandates compliance with DOE STD-1189, Integration of Safety into the Design Process. STD1189 provides implementation guidance for Hazard Category 1, 2, and 3 nuclear facility safety requirements.

This study has been performed at a preconceptual or scoping level, and where possible, has implemented requirements of DOE STD-1189 at an "alternative study level" only. Safety goals and strategy are based on assumptions for the study. Areas of compliance to guide safety in design for this alternative study are as follows:

- Although project functions and requirements have not been formally documented it is expected they will be for Conceptual Design and will mature as the project progresses through the Critical Decision Phases. For this study initial requirements were developed in INL/EXT-6456, Statement of Work for a Greenfield LEU Molybdenum Fuel Fabrication Capability, INL/EXT-08-13840, Conceptual Process Description for the Manufacture of Low-Enriched Uranium-Molybdenum Fuel and through assumptions documented in this study that have been agreed to by the technical subject matter experts from INL and Y-12.

- Based on estimated LEU material quantities, criticality potential and attractiveness levels, the facility has been initially assumed to be a Hazard Category 2, Security Category IV nuclear facility with a natural phenomena hazard performance category of PC-2.

- An initial qualitative assessment of hazards was completed, including identification of principal hazards, and initial control strategies that may have a significant influence on the facility design were identified.

- Initial risks have been identified and obvious mitigation strategies have been considered for the processes and project.

- Initial qualitative evaluations for nuclear safety, nuclear criticality safety, and fire protection have been considered in study design process.

- Developed initial waste management process assumptions

- "In process" (informal) design reviews have been conducted by various DOE Laboratory subject matter experts (SMEs) for criticality safety, nuclear safety, fire protection, safeguards and security, and technical process to help ensure that significant safety elements have been conservatively considered in the preconceptual study.

Full implementation of the DOE requirements for the Preconceptual Phase, Conceptual Design Phase, etc., is assumed to be implemented at the project level. Items not included with this preconceptual alternative study are as follows:

- Formal programmatic High-level Functions and Requirements

- Documented programmatic safety goals and strategy

- Integration of safety for other acquisition alternatives

At the beginning of the Conceptual Design Phase, it is assumed that detailed acquisition and process alternatives analysis will be conducted along with hazards evaluations. Safety related requirements that will need to be implemented for the Conceptual Design Phase include: 
- Development of a Safety Design Strategy (SDS)

- Hazards analysis for project alternatives, and process alternatives

- Identification of Safety Systems, Structures and Components (SSCs)

- A formal Preliminary Hazards Analysis and control strategies for the selected Alternative

- Natural Phenomena Hazards (NPH) performance categories

- Site specific hazards analysis

- Risk Management Plan

- Risk Assessment

- Conceptual Safety Design Report (CSDR)

- Integration of safety elements into the Concept Design Report

\subsubsection{Safeguards and Security}

Nuclear material control and accountability (MC\&A) is the joint responsibility of the US Nuclear Regulatory Commission (NRC) and the US Department of Energy (DOE). This responsibility is implemented by the Nuclear Materials Management \& Safeguards System (NMMSS) that is administered by NAC International under contract with DOE HS-1.22 (Office of Information Management) that is managed by the DOE Savannah River Operations Office. This program tracks nuclear material flows between and among various governmental, commercial, and educational entities as well as foreign entities to ensure that nuclear materials are not improperly used or diverted for improper use. Although the LEU-Mo materials used in fabricating nuclear fuels for high performance research reactors have low attractiveness for misuse or diversion, they still are subject to the $\mathrm{MC} \& \mathrm{~A}$ requirements.

The DOE MC\&A requirements are defined in DOE M 470.4-6 ${ }^{1}$. This manual sets forth the methodology for determining the security category for the facility based on the attractiveness level of the SNM and the amount of SNM contained. The security categorization is used to define additional requirements for physical protection (DOE M 470.4- $2^{2}$ ), protective force (DOE M 470.4-3 ${ }^{3}$ ), information security (DOE M 470.4-4 $4^{4}$ ), and personnel security (DOE M 470.4-5 $5^{5}$ ), as required.

Enriched uranium (including LEU) is reportable at the one gram level (reference 1, Table I-1). LEU is classified as Attractiveness Level E material (reference 1, Table I-4), making the FFC a security category IV (SC IV) facility. This categorization defines the requirements for the FFC MC\&A program as well as additional requirements (e.g., physical protection) from other sections of DOE M 470.4.

Under NRC regulation, the inventory of $\mathrm{U}^{235}$ that would be present in FFC causes the facility to be categorized as processing "special nuclear material of moderate strategic

${ }^{1}$ DOE M 470.4-6, Chg.1, Nuclear Material Control and Accountability, August 14, 2006, 168 pp.

2 DOE M 470.4-2, Chg.1, Physical Protection, August 26, 2005.

${ }^{3}$ DOE M 470.4-3, Chg.1, Protective Force, August 26, 2005.

${ }^{4}$ DOE M 470.4-4, Chg.1, Information Security, August 26, 2005.

${ }^{5}$ DOE M 470.4-5, Personnel Security, August 26, 2005. 
significance"6. Physical protection requirements for this facility are defined in 10 CFR 73.67. MC\&A requirements for this facility are defined in 10 CFR 74.41.

A security category IV facility would have the following basic components for the material control and accountability program (Ref: DOE M 470.4-6; Personal

Communication with Roger Haga, INL Safeguards, 12 June 2008)

- Accounting System - There will need to be in place a method for tracking and accounting for the nuclear material including

$\circ$ shipping and receiving records,

0 transfer records, and

- procedures for tracking and controlling material movement.

- Inventory - Periodic inventory of the nuclear material will be required. They will

○ be performed annually for security category IV facilities,

○ include safeguards measurements (typically nondestructive assay) of selected materials

0 include inspections of the storage and processing areas to verify material locations, quantities, identification numbers, etc.

- Access Control - Access to the nuclear material will need to be restricted to authorized personnel.

To facilitate implementation, of the material control and accountability requirements the FFC is divided into Material Balance Areas (MBAs) as shown in Figure 2-1. These MBAs are preliminary.

\section{Eight Mass Balance Areas within the FFC}

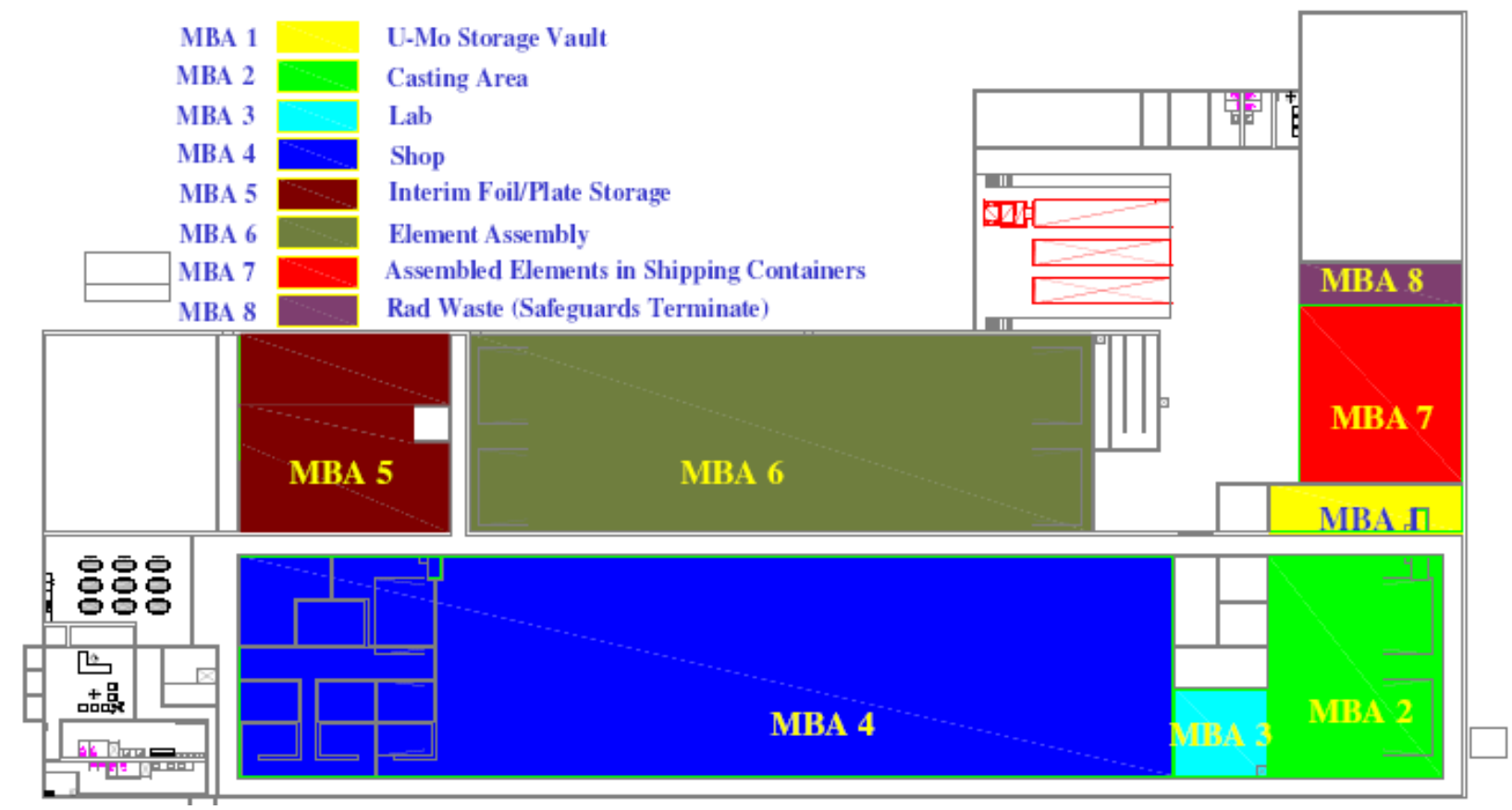

FIGURE 2-1 MASS BALANCE AREAS (MBAS)

\footnotetext{
${ }^{6}$ The definition in 10CFR70.4 includes: "(2) 10,000 grams or more of uranium-235 (contained in uranium enriched to 10 percent or more but less than 20 percent in the U-235 isotope)". At $20 \%$ enrichment, a total uranium mass of $50 \mathrm{~kg}$ meets the definition for "moderate" strategic significance.
} 


\subsubsection{Containment/Confinement}

Fuel fabrication processes include steps involving unconfined nuclear material. For the most part, these fabrication processes involve LEU in non-dispersible, bulk alloy form, and much of the subsequent processing involves this same material with an aluminum cladding. In these forms, the LEU does not require special containment or confinement to protect workers or the public from routine exposures. However, work areas will include local HEPA-filtered ventilation (task exhaust) to capture minor airborne contamination that might be generated from the processes.

Certain processes (especially casting) do have potential for generating airborne particulates. Those processes having increased potential for airborne releases will be conducted within enclosures that contain any airborne materials. These enclosures include appropriate filtration to capture particulates before the filtered air enters the exhaust ventilation system.

The FFC ventilation system for the processing area is designed for cascading air flows from areas of low potential contamination to areas of higher potential contamination. Filtered, conditioned supply air is provided to the processing area via a supply plenum. The supply system is designed to maintain the air supply to the processing area without pressurizing that area with respect to normal atmospheric pressure. Thus, the supply ventilation system is controlled by sensors that maintain a negative differential pressure between the processing area and the atmospheric pressure outside the building. The ventilation supply system includes dampers that close in the event of a fire to prevent backflow of hot exhaust out the air supply system.

The FFC exhaust ventilation system has multiple stages of HEPA filtration. The first stage of exhaust ventilation filtration is located at work areas where the potential exists to generate particulate materials that might become airborne. Examples include machining operations, chemical cleaning operations, and casting operations. Task exhaust systems include local HEPA filters that remove particulates from the exhaust gas before it enters the facility exhaust ventilation system upstream of the building HEPA filters. Task ventilation systems may also include mist eliminators to remove moisture from specific exhaust streams. Future studies will evaluate the need for additional off-gas treatment and/or heating before the HEPA filtration.

The main building exhaust ventilation system includes two-stages of HEPA filtration upstream of the building exhaust blowers. To protect the HEPA filters from accident conditions, especially fires, the exhaust ventilation system will include a deluge spray system, mist eliminators, fire screens, and roughing filters upstream of the first stage of HEPA filtration.

\subsubsection{Environmental Protection}

The US Environmental Protection Agency is responsible for protecting the environment through regulation of pollutant release to the environment. The EPA administers compliance with key environmental legislation that is applicable to the FFC, including: the National Environmental Policy Act (NEPA), the Clean Air Act (CAA), the Clean Water Act (CWA), the Toxic Substances Control Act (TSCA), the Resource Conservation and Recovery Act (RCRA), and the Superfund Amendments and Reauthorization Act (SARA). 
National Environmental Policy Act Requirements

NEPA requirements applicable to the FFC are defined in 40 CFR parts 1500 through 1508. The DOE, as the federal agency responsible for the FFC, is required to follow the DOE NEPA Implementing Procedures defined in 10 CFR 1021 in its planning and decision-making processes. DOE implements the requirements of these regulations following DOE O $451.1 \mathrm{~B}^{7}$. The NEPA process requires that DOE evaluate whether a major action requires an environmental assessment (EA), an environmental impact statement (EIS), or is categorically excluded from the requirement to prepare either of these documents. The decision to convert high performance research reactors to low enriched fuel might constitute a major action subject to the NEPA process. Siting a new facility for fabricating such fuel will almost certainly require an EA. Preparation of an EA or an EIS will result in additional activity that will increase cost and delay the schedule for construction and startup of the FFC.

If the FFC is to be a commercial fuel fabrication facility licensed by the NRC, the responsibility for NEPA compliance lies with the NRC. NRC implementation of NEPA requirements is defined in $10 \mathrm{CFR}$ 51, and the NEPA compliance process is integrated into the licensing process.

\section{Clean Air Act}

CAA requirements that are potentially applicable to the $\mathrm{FFC}$ are defined in the following Parts of 40 CFR:

- Part 50 (National primary and secondary ambient air quality standards),

- Part 61 (National emission standards for hazardous air pollutants)

- Part 68 (Chemical accident prevention provisions),

- Part 70 (State operating permit programs),

- Part 71 (Federal operating permit programs).

10 CFR 50 establishes ambient air quality standards for a specific set of air pollutants (sulfur oxides, nitrogen oxides, ozone, carbon monoxide, lead and particulate matter). Except for the construction of a fossil-fuel fired plant to provide electricity or steam for process or space heating, the FFC will not emit any of these criteria air pollutants and will therefore be exempt from $10 \mathrm{CFR} 50$ requirements.

10 CFR 61 sets requirements for permitting the construction, modification and operation of facilities that could be a source of hazardous air pollutants as defined in section 112(b) of the Clean Air Act. This regulation also defines requirements for monitoring and controlling pollutant emissions. Two chemicals identified as being present in the FFC are found on the list of hazardous air pollutants, hydrofluoric acid and radionuclides. The presence of these chemicals potentially requires the FFC to obtain an operating permit under part 70 or part 71 . The low volume of hydrofluoric acid that could be present in the facility could form the basis for waiver of a permitting requirement for this substance. The standard for radionuclides (10 CFR 61.92) sets an effective dose equivalent limit of $10 \mathrm{mrem} / \mathrm{year}$ to any member of the public. Public dose from radioactive air emissions can be estimated using the EPA CAP- 88 model, and this information is required in a permit application. Further study is required to evaluate the applicability of 10 CFR 61.

\footnotetext{
${ }^{7}$ National Environmental Policy Act Compliance Program, DOE O 451.1B, October 26, 2000, 12 pp. http://www.directives.doe.gov/pdfs/doe/doetext/neword/451/o4511bc1.pdf
} 
10 CFR 68 describes the requirements for a risk management plan for hazardous chemicals that exceed threshold quantities defined in the regulation. For hydrofluoric acid, the threshold quantity (40 CFR 68.130) is $1000 \mathrm{lb}$, and radionuclides are not listed in there is no threshold quantity for radionuclides. Because the expected FFC inventory of hydrofluoric acid is far less than the threshold quantity, and there is no threshold quantity for radioactive materials, it seems that the FFC will not be subject to the requirements of this regulation.

10 CFR 70 and 71 are the State and Federal operating permit programs, respectively. Where the state has been granted full or partial approval of its operating permits program, the Part 70 requirements apply. The Part 71 requirements cover those portions of a permit application for which there is no approved Part 70 program. The permits covered by these regulations are those required by other CAA regulations defined above.

\section{Clean Water Act}

The Clean Water Act (CWA) regulates point source discharges of pollutants into surface water and groundwater. Regulations that are potentially applicable to the FFC are defined in the following Parts of 40 CFR:

- Part 116 (Designation of hazardous substances)

- Part 117 (Determination of reportable quantities for hazardous substances)

- Part 122 EPA administered permit programs: The national pollutant discharge elimination system

- Part 125 (Criteria and standards or the national pollutant discharge elimination system)

- Part 131 (Water quality standards)

Several substances used in FFC are listed as hazardous substances in 40 CFR 116.4: These include: hydrofluoric acid, nitric acid, and sodium hydroxide. Reportable quantities for these substances (from 40 CFR 117.3) are $100 \mathrm{lb}$ for hydrofluoric acid and $1000 \mathrm{lb}$ each for nitric acid and sodium hydroxide. 40 CFR 117.21 requires that discharge of a reportable quantity of a hazardous substance within a 24 hour period be reported to the EPA (or other cognizant federal agency). (Note: radioactive materials regulated under the Atomic Energy Act are exempt from the definition of "pollutant" in 40 CFR 122.2). Because the FFC use of these chemicals is low compared to the reportable quantity, the FFC should adopt a strategy of ensuring that on-site inventories of these chemicals are always less than the reportable quantities.

The EPA regulates discharges of pollutants from point sources to surface water or groundwater via the National Pollutant Discharge Elimination System (NPDES) program (40 CFR 122). Under this regulation, an NPDES permit is required to release pollutants that could enter surface water or groundwater. The FFC plans to ship liquid wastes to an off-site treatment facility rather than to treat wastes from its processes. Except for sanitary wastes discharged to a permitted sewage treatment plant and stormwater that can be discharged to the environment through a permitted outfall, the FFC does not intend to discharge liquids from the site. However, the FFC must provide for potential discharges from upset conditions, for example, fire water from a fire in the process area. The FFC is designed to collect any such water and to sample the water before releasing this water. The FFC design concept includes a mechanism for collecting, sampling and analyzing collected fire water. The disposition pathway(s) for such water requires further study to define alternatives. 
In addition to planned discharges (of treated effluent, for example), this regulation requires permitting of storm water runoff from the site, both during construction and operation of the facility. The FFC would be required to have an NPDES permit for storm water discharge. The EPA regulates stormwater discharges from construction sites of one acre or more, thus, the FFC would require a Construction General Permit for the construction activities. Stormwater runoff from the operating facility will be collected in a stormwater collection system that meets the permit requirements.

\section{Toxic Substances Control Act}

The Toxic Substances Control Act (TSCA) empowers the EPA to regulate the use or distribution (including disposal) of any chemical substance deemed to be hazardous to human health or the environment. EPA classifies chemicals as "new" or as "existing". The list of existing chemicals includes about 75,000 chemical substances, and the public portion of this inventory (not including substances identified as proprietary) is available online at http://www.epa.gov/srs/ (the substance registry system).

All commercially-available substances used in the FFC will already have been entered in this inventory by the manufacturer. Per 40 CFR 720.3(e)(4), special nuclear material is exempt from the definition of chemical substance, and is not subject to reporting requirements per 40 CFR 720.30(a). Thus, there are no TSCA reporting requirements for the FFC.

\section{Solid, Hazardous and Radioactive Wastes}

Wastes generated at the FFC include solid ${ }^{8}$, hazardous, and radioactive wastes. Solid and hazardous wastes are regulated by the EPA under the Solid Waste Disposal Act and the Resource Conservation and Recovery Act (RCRA). Solid waste regulations are located in 40 CFR 239 through 259, and hazardous waste regulations are located in 40 CFR 260 through 279.

FFC solid waste streams include normal office trash as well as non-hazardous solid wastes from fuel fabrication activities - non-radioactively-contaminated process wastes from the process area such as metal scrap from the machining and shearing processes; wood, paper and plastic materials; spent equipment and tooling that do not contain hazardous components; etc. The requirements for packaging, storing, and managing these wastes are defined in 40 CFR 243. These wastes would be disposed in permitted municipal solid waste landfills.

FFC waste streams containing RCRA hazardous wastes are listed in Table 2-2. None of the hazardous wastes generated in the FFC are acutely hazardous, one is a listed (toxic) waste, and two wastes are corrosivity characteristic wastes. Waste generators are allowed to store hazardous wastes for periods up to 90 days without a RCRA permit. This storage is subject to the packaging, documentation and other requirements of 40 CFR 262.34.

\footnotetext{
${ }^{8}$ The RCRA definition of solid waste is: "garbage, refuse, sludges, and other discarded solid materials, including solid waste materials resulting from industrial, commercial, and agricultural operations, and from community activities, but does not include solid or dissolved materials in domestic sewage or other significant pollutants in water resources, such as silt, dissolved or suspended solids in industrial wastewater effluents, dissolved materials in irrigation return flows or other common water pollutants. Unless specifically noted otherwise, the term "solid waste" as used in these guidelines shall not include mining, agricultural, and industrial solid wastes; hazardous wastes; sludges; construction and demolition wastes; and infectious wastes"
} 
TABLE 2-2. HAZARDOUS WASTES GENERATED AT FFC

\begin{tabular}{|c|c|c|c|}
\hline Designation & Waste & Process Source & $\begin{array}{l}\text { Estimated Annual } \\
\text { Production }\end{array}$ \\
\hline $\begin{array}{l}\text { Toxic Waste } \\
\text { U-listed } \\
\text { U134 }\end{array}$ & $\begin{array}{l}\text { Hydrofluoric } \\
\text { Acid }\end{array}$ & $\begin{array}{l}\text { Cleaning of } \mathrm{Zr} / \mathrm{U} 10 \mathrm{Mo} \text { foils } \\
\text { to remove yttrium }\end{array}$ & 240 gallons \\
\hline $\begin{array}{l}\text { Characteristic: } \\
\text { Corrosivity } \\
\text { D002 }\end{array}$ & Nitric Acid & $\begin{array}{l}\text { - Cleaning of U10Mo foils } \\
\text { - Cleaning of Zr clad } \\
\text { U10Mo foils } \\
\text { Cleaning of aluminum } \\
\text { clad }\end{array}$ & 6460 gallons \\
\hline $\begin{array}{l}\text { Characteristic: } \\
\text { Corrosivity } \\
\text { D002 }\end{array}$ & \begin{tabular}{|l} 
Sodium \\
Hydroxide \\
Solution
\end{tabular} & Cleaning of aluminum clad & 3120 gallons \\
\hline
\end{tabular}

Treatment, storage (for more than 90 days), or disposal of hazardous wastes requires a RCRA permit to perform that activity. The FFC does not intend to perform any activity that would require a RCRA permit. Hazardous wastes accumulated in the 90 day storage area would be appropriately packaged and shipped off-site to a firm that specializes in hazardous waste treatment, stabilization, and disposal in accordance with the requirements of 40 CFR 262.

Some of the solid wastes generated at the FFC will include radioactive materials such as the scrap from trimming Zr-clad LEU-Mo foils or the heels from the casting operation. Wastes containing radioactive materials will be low level radioactive waste (LLW). 10 CFR 61.55 defines four classes of LLW: Classes A, B, C, and "Greater-Than-Class-C" or GTCC, and this regulation provides thresholds for determining classification of a radioactive waste depending on the radionuclide present in the waste. Uranium is not listed in the tables, and by 10 CFR 61.55(a)(6), the radioactive wastes from FFC would be Class A LLW.

Three LLW disposal facilities are licensed by the NRC (or by agreement states) Barnwell, SC (operated by Chem-Nuclear/Duratek); the Energy Solutions (formerly Envirocare) facility in Clive, Utah; and the US Ecology facility at Hanford, Washington.Barnwell16 and Hanford are licensed to accept classes A-C. The Envirocare facility is only licensed to accept class A LLW. Disposal of DOE-generated LLW is authorized at Hanford, Idaho National Laboratory, Los Alamos National Laboratory, Nevada Test Site, Oak Ridge Reservation and the Savannah River Site. Of these six sites, only Hanford and Nevada Test Site are authorized to receive LLW from off-site (DOE) sources.

The two acid wastes identified in Table 2-2 have the potential of containing uranium, and therefore, these wastes could be mixed low-level wastes that would require treatment of the hazardous components at a RCRA licensed facility before disposal as LLW.

Radioactive waste packaging would require criticality safety evaluation to ensure that there is no potential for a criticality safety event during packaging, storage, transportation or disposal. 
Superfund Amendments and Reauthorization Act

Title III of the Superfund Amendments and Reauthorization Act (SARA) includes provision for emergency planning and for informing local governments and the public about chemical hazards in facilities. This legislation is regulated by the EPA (40 CFR 355). Appendix A and Appendix B to Part 355 of 40 CFR set forth lists of "Extremely Hazardous Substances and Their Threshold Planning Quantities". Two chemicals in FFC are on these lists: hydrofluoric acid and nitric acid. The threshold planning quantities for these substances are $100 \mathrm{lb}$ and $1000 \mathrm{lb}$, respectively. If the FFC inventory of these (or any other chemicals on the lists) exceeds the threshold planning quantities, then the FFC must report the that fact to the state emergency response commission for the state in which the FFC is located.

It is not anticipated that FFC inventories will exceed the threshold planning quantities and therefore, FFC should not be required to report chemical inventories to state officials.

\subsubsection{Nuclear Safety}

Nuclear safety requirements focus on facility design and operation that protect the public and facility workers from exposure to nuclear materials. For DOE facilities, the regulatory driver for nuclear safety is 10 CFR 830 (Nuclear Safety Management). NRC regulates fuel fabrication facilities under 10 CFR 70 (Domestic Licensing of Special Nuclear Material). Specific NRC licensing requirements for fuel fabrication facilities requirements are detailed in NUREG- $1520^{9}$.

Under DOE regulation, the FFC would be categorized as a Hazard Category 2 (HC2) facility ${ }^{10}$. This characterization results from the fact that sufficient SNM is present at several locations within the facility such that a nuclear criticality event cannot be precluded. This categorization means that a Documented Safety Analysis (DSA) and Technical Safety Requirements (TSR) are required for operating the facility. Included in the DSA/TSR are a number of safety management programs that increase the development, operating and oversight expense of the facility.

Additional design requirements for DOE hazard category 1, 2, and 3 nuclear facilities are defined in DOE $\mathrm{O} 420.1 \mathrm{~B}^{11}$ in the areas of nuclear facility design, fire protection, criticality safety, and mitigation of natural phenomena hazards. This order further establishes a requirement for a Systems Engineer program. These additional requirements are implemented at later design stages, but they must be identified early in conceptual design and integrated into the design process.

Under NRC regulation, the FFC would be required to prepare a license application that includes the same basic information as for a DOE DSA/TSR, but this information would be formatted according to NRC requirements in reference 6 .

One significant difference between NRC and DOE processes for review and approval of nuclear safety documents (DSA/TSRs for DOE; license application for NRC) is that the $\mathrm{NRC}$ process requires that the license applicant fund the NRC review.

\footnotetext{
${ }^{9}$ Standard Review Plan for the Review of a License Application for a Fuel Cycle Facility, NUREG-1520, March 2002, 220 pp. http://www.nrc.gov/reading-rm/doc-collections/nuregs/staff/sr1520/sr1520.pdf

${ }^{10}$ Hazard Categorization and Accident Analysis Techniques for Compliance with DOE Order 5480.23, Nuclear Safety Analysis Reports, DOE STD 1027-92, Change Notice \#1, September, 1997, 49 pp. http://www.hss.energy.gov/NuclearSafety/techstds/standard/std1027/s1027cn1.pdf

${ }^{11}$ Facility Safety, DOE O 420.1B, December 22, 2005, 65 pp.
} 


\subsubsection{Industrial Hygiene}

Industrial hygiene involves "anticipating, recognizing, evaluating and controlling workplace conditions that may cause workers injury or illness"12. Federal regulation of workplace safety is the province of the Occupational Safety and Health Administration (OSHA) of the Department of Labor. These safety regulations are defined in the following subparts of 29 CFR 1910:

- $\quad$ Subpart D. Walking \& Working Surfaces

- $\quad$ Subpart E. Means of Egress

- Subpart F. Powered Platforms, Manlifts, and Vehicle-Mounted Work Platforms

- $\quad$ Subpart G. Occupational Health and Environmental Control

- $\quad$ Subpart H. Hazardous Materials

- $\quad$ Subpart I. Personal Protective Equipment

- $\quad$ Subpart J. General Environmental Controls (Sanitation)

- $\quad$ Subpart K. Medical and First Aid

- Subpart L. Fire Protection

- $\quad$ Subpart M. Compressed Gas \& Compressed Air Equipment

- $\quad$ Subpart N. Materials Handling and Storage

- $\quad$ Subpart O. Machinery and Machine Guarding

- $\quad$ Subpart P. Hand and Portable Powered Tools and other Hand-Held Equipment

- $\quad$ Subpart Q. Welding, Cutting and Brazing

- Subpart S. Electrical

A detailed list of all worker safety requirements that are applicable to the FFC is beyond the scope of this report. The FFC generalized design concept automatically incorporates facility design requirements such as are covered in subparts D, E, H, J, and L. Stationary equipment that will be placed in the facility will incorporate the requirements of subpart O. Safety requirements covered by other subparts involve a combination of engineered controls and administrative controls such as detailed operating procedures. Where the preliminary hazards analysis identifies workplace hazards that are subject to OSHA regulation, the appropriate section of 29CFR 1910 will be used in defining the required controls.

\subsection{Quality Assurance}

The Greenfield Alternatives study was performed in accordance with good engineering practice for conducting pre-conceptual design studies. Subsequent conceptual, preliminary and final design, as well as procurement of equipment and services and construction shall be conducted in conformance with an established QA program that complies with ASME ANSI NQA-1, as well as 10 CFR 830.120 and DOE Order 414.1C

A graded approach shall be used to determine the appropriate QA requirements to be implemented throughout the life cycle of the project. The Graded approach shall be defined in the implementing project quality documents. The graded approach will utilize a process by which the level of analysis, extent of documentation, and degree of rigor of process control are applied based on risk commensurate with their regulatory significance; importance to safety, safeguards, security and the environment; programmatic mission and any other factors determined important by management.

\footnotetext{
${ }^{12}$ Industrial Hygiene, OSHA Information Booklet \#3143, 1998
} 
Key requirements pertaining to design control during the conceptual, preliminary and detail design include the following: (1) ensure design inputs are traceable to approved documents and are correctly translated into design documents (2) organizational and physical interfaces are identified and controlled; (3) changes to design are controlled in a manner commensurate with the original design; (4) design is independently verified to be adequate; and (5) documentation and records of the design and design verification processes are maintained in accordance with the QA program.

\subsection{Pollution Prevention and Waste Minimization}

The DOE requires that principles of pollution prevention, waste minimization, sustainable design and energy efficiency be incorporated into the facilities built with federal funds or used to house federally funded operations. The following regulations pertaining to pollution prevention should be considered during the conceptual, preliminary and detail design of the FFC:

- The design and selection of materials shall consider the federal requirement and DOE goal to purchase EPA-designated items with recycled content except when not available competitively at a reasonable price or that do not meet performance standards (FDD, Executive Order13101, Secretary of Energy Memorandum dated November 12, 1999).

- Sustainable building design and energy efficiency principles shall be incorporated into the design of the new facility (Executive Order 13123, Secretary of Energy Memorandum dated November 12, 1999).

- The design shall consider the federal agency requirements to incorporate waste prevention and recycle into daily operations (Executive Order 13101, Executive Order 13148).

- Source reduction techniques shall be preferred over recycling, treatment, and disposal techniques where technically feasible and cost effective (Pollution Prevention Act of 1990).

- Purity of air and water shall be preserved (pollution prevention) and energy efficiency encouraged (S.C. Code of Laws Title 48).

- System design shall minimize the generation of wastes and minimize the mixing of radioactive and non-radioactive wastes. This will minimize the risk of exposure of the public, the worker and the environment - DOE Order 420.1.

- The design shall minimize the generation of Low level Waste - DOE Order 435.1.

To the maximum extent practicable, the FFC project shall be designed to integrate the following fundamental pollution prevention goals during the life cycle of the project:

- Minimize the discharge of radionuclide's and other pollutants to the environment

- Minimize waste through source reduction and recycling,

- Meet all effluent release criteria/regulatory standards; and

- Utilize ALARA principles to minimize exposure to workers, the public and the environment from pollutants 
During each phase of the design i.e. conceptual and Title Design, a pollution prevention assessment shall be performed, utilizing the following guidelines:

(1) Commit to performing an assessment and engage all project staff in pollution prevention activities.

(2) Gather data about key waste generating activities by reviewing existing design

(3) Identify potential pollution prevention opportunities.

(4) Research and analyze pollution prevention opportunities for potential waste reduction, cost avoidance, and return on investment.

(5) Make recommendation for pollution prevention implementation projects based on the waste and cost analysis.

(6) Summarize the findings.

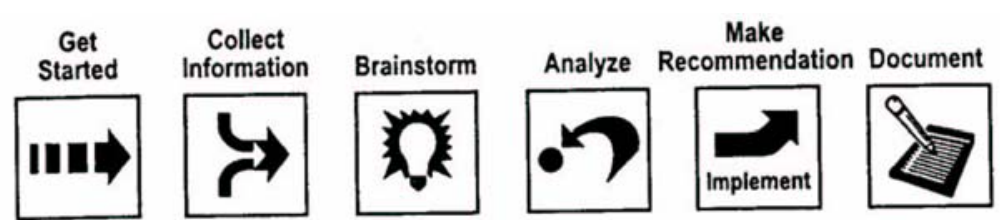

\section{STEPS IN A POLLUTION PREVENTION DESIGN ASSESSMENT (ENGEL-COX 1999). (GRAPHIC REPRODUCED WITH AUTHORS' PERMISSION)}

The following pollution prevention concepts have been included in this Greenfield Alternative Study of the FFC:

- Segregate non-radioactive operations: This minimizes the spread of contamination and the generation of radioactive waste.

- Recycle of U-Mo scrap from the foil fabrication: This minimizes the waste generated and conserves resources.

- Reuse of foil and plate cleaning solution: This reduces the amount of waste

The following trade studies have been proposed to be performed during the conceptual design phase to evaluate potential for meeting DOE's pollution prevention objectives:

- Incorporating an evaporator in the process to minimize the amount of liquid waste to be shipped off-site for treatment and disposal.

- Minimize the amount of scrap resulting from the foil and plate shearing process.

\subsection{Decontamination and Decommissioning}

The facility shall be designed to facilitate decontamination of equipment for maintenance repair and replacement, and to minimize costs of the eventual decontamination and decommissioning.

\subsubsection{Decontamination}

The FFC facility shall include a decontamination area within the process or operating area. This area shall be furnished with all necessary cleaning equipment, radioactivity monitors, waste handling capability, and safety features to safely perform equipment cleaning tasks. 
Air cleaning devices shall be located as close to the source of contamination as practicable to capture and remove contaminates to avoid the unnecessary spreading of the contamination into ducts, conveyors, or other process areas. This would include the filtration of task exhaust ducts, hoods and glovebox exhaust air prior to the exhaust air entering a duct leading to the exhaust plenum.

Protection shall be provided for bare floors, walls, and ceilings, particularly for structurally important parts of the building. Protection shall be in the form of liners or durable coatings for which effective cleaning methods have been developed. The liners and coatings will selected to be compatible with the process liquids including waste and reagents used.

Surfaces in operating or process areas shall have no seams, cracks, or rough or absorbent surfaces. In some areas, metal liners shall be required. If necessary all cracks, crevices, and joints shall be caulked or sealed and finished smooth to prevent contaminated material accumulation in inaccessible areas.

In areas that are most likely to become contaminated, adequate access shall be provided, such as crawl spaces, piping tunnels, and hatches into ductwork, to facilitate decontamination.

The design of equipment shall include features and characteristics to avoid build-up of materials, so as to minimize its contamination and facilitate decontamination.

In addition, the design shall incorporate as low as reasonably achievable (ALARA) concepts to minimize impacts on the operators, the public, and the environment.

\subsubsection{Decommissioning}

The facility design shall include features that will facilitate decontamination for future decommissioning. The following design principles shall be considered for FFC facilities:

(1) Use of modular, separable confinements for radioactive and other hazardous materials to preclude contamination of fixed portions of the structure;

(2) Location of exhaust filtration components of the ventilation systems at or near individual enclosures so as to minimize long runs of internally contaminated ductwork;

(3) Use of equipment, including effluent decontamination equipment, that minimizes, the accumulation of radioactive or other hazardous materials in relatively inaccessible areas including curves and turns in piping and ductwork. Accessible, removable inspection covers are encouraged to allow visual inspection;

(4) Use of materials that are easily decontaminated and reduce the amount of radioactive and other hazardous materials requiring disposal;

(5) Use of designs that ease cut-up, dismantlement, removal, and packaging of contaminated equipment from the facility (e.g., removal and dismantlement of gloveboxes, air filtration equipment, large tanks, vessels, equipment and ductwork);

(6) Use of lifting lugs on large equipment; and

(7) Use of fully drainable piping systems that carry contaminated or potentially contaminated liquids. This is a requirement for facility analytical laboratories. 


\subsection{Maintenance Philosophy}

The FFC availability of $75 \%$, allows an annual, one month long scheduled plant outage period for major equipment maintenance and refurbishing activities.

The maintenance philosophy of the FFC facility design is guided by the following three considerations listed in order of priority:

a. Maximizing the predictive capability as well as the use of regularly scheduled preventive maintenance (instead of the after-failure corrective maintenance) by adopting conservative system designs and state-of-the-art process condition monitoring techniques.

b. Maximizing the use of modular designs (i.e., replaceable units for off-line maintenance) of process systems and equipment for quick change out of failed system or equipment during plant operation. The objective is to enhance the on-line availability of the FFC process plant. This approach assumes maximized off-line repair capabilities and appropriate spare inventory of process systems and equipment at the FFC facility.

c. For process systems and equipment that are impractical to be designed for quick change-out, the systems will be conservatively designed with phased preventative overhaul requirements that can be cost-effectively scheduled during annual, one-month long plant outage period. These systems will also be designed with appropriate redundancy provisions for any unscheduled failure and outage.

The FFC facility design will contain necessary process equipment bypass/isolation provisions and manual control capabilities in addition to automated programmable control to facilitate the maintenance activities required at the plant. Requirements for the equipment redundancy should be evaluated during the conceptual design

The operations of the FFC will be conducted on a campaign basis. Up to 2 campaigns may be conducted concurrently. Adequate in process material storage to allow for equipment change over and re-calibration for the second campaign is provided.

The FFC fabrication process incorporates surge storage between process unit operations, such that if there is any down-steam unit operation outage, upstream processes can be performed normally. When the affected down stream outage is corrected, extra shifts can be worked to resolve the backlog.

All maintenance of the process systems in the FFC facility will be conducted hands-on with stateof-the-art condition monitoring and testing capabilities. 


\subsection{PROCESS DESIGN}

This section describes the design of the Process Systems included in the FFC. The description includes listing of key process assumptions, defining overall throughput as well as the throughput through the various process operations, descriptions of each of the key unit operations, waste estimations and treatment pathways as well as utility and consumable uses through the facility. Included in Appendix A-3 are the Block Flow Diagrams and Appendix A-4 includes the Process Flow Diagrams.

The FFC facility is designed to meet the fuel assembly production requirements for the five U.S. high performance research reactors (HPRR) using low-enriched uranium (LEU) foils instead of highly enriched uranium compacts. The LEU is alloyed with $10 \%$ molybdenum (Mo) in order to stabilize the fuel into the gamma-phase. The gamma-phase is crucial to obtain a high density fuel that behaves acceptably during irradiation. The LEU/Mo fuel is coated with zirconium ( $\mathrm{Zr}$ ) to avoid fuel-cladding reaction between the LEU/Mo fuel layer with the aluminum cladding during fuel plate irradiation. This process is described in detail in Section 3.3.

The major processing areas of the FFC are:

- Casting the LEU/Mo into ingots

- Hot rolling to intermediate thickness

- Sectioning

- Cleaning of the fuel ingots

- Canning ingots to apply $\mathrm{Zr}$

- Rolling to final thickness

- Annealing to flatten plates

- De-can and shear foils to size

- Cleaning of the Zr-clad fuel foil

- Friction Stir Welding to clad plates with aluminum (HIP is an alternative process)

- Rough Shear

- Plate Flattening

- Surface Machining

- Final shearing to final plate size

- Final Assembly of the sub-elements

- Autoclave (for ATR sub-assemblies only)

- Final Inspection

- Element Shipping

Storage is provided in locked areas for in-process foil/plates, which provide intermediate processing breaks to accommodate schedule, upstream, or downstream process disruptions, processing strategy such as forming multiple pieces of each size before changing the shear operations to cut different sizes, etc. 
Various assumptions have been made in order to design the fuel fabrication capability (FFC) facility. Table 3-1 shows the process assumptions that exist and were used in the design and subsequent cost estimation.

TABLE 3-1 PROCESS ASSUMPTIONS FOR THE FFC

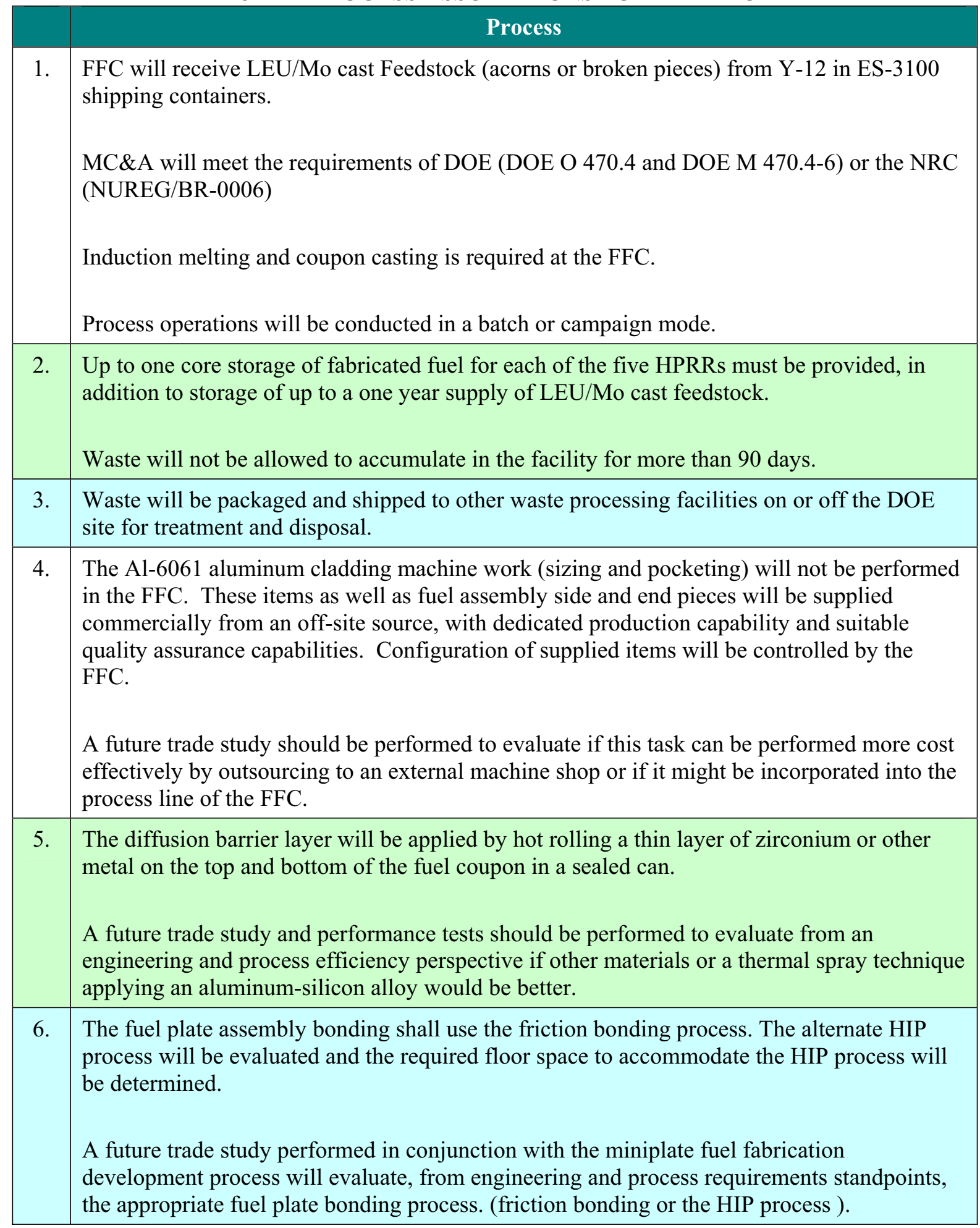




\begin{tabular}{|c|c|}
\hline 7. & $\begin{array}{l}\text { Fuel plate pre-assembly shall be manually performed. } \\
\text { A future trade study will be performed to determine if plate pre-assembly (prior to bonding) } \\
\text { can be practically automated or a manual operation is required. }\end{array}$ \\
\hline 8. & $\begin{array}{l}\text { The FFC is designed to provide LEU/Mo fuel for the five US High Performance Research } \\
\text { Reactors. No provision for FFC expansion will be made to accommodate the fuel needs of } \\
\text { additional foreign research reactors. }\end{array}$ \\
\hline & $\begin{array}{l}\text { A future trade study will be performed to identify the additional facility footprint and cost } \\
\text { schedule implications as a result of the facility expansion to accommodate foreign research } \\
\text { reactors. }\end{array}$ \\
\hline 9. & Scrap fuel may be returned to Y 12 or reused within the FFC. \\
\hline 10. & Cans used for rolling of $\mathrm{Zr}$ to the fuel meat will be purchased as outsourced materials. \\
\hline 11. & $\begin{array}{l}\text { Equipment will have a } 75 \% \text { availability to allow for maintenance and scheduled operations } \\
\text { shut-down. }\end{array}$ \\
\hline
\end{tabular}

\subsection{Process Overview}

\subsubsection{Block Flow Diagrams}

Block flow diagrams (BFDs) show a very basic outline of the flow through the processing facility. Each major process is shown as a block with inputs and outputs as well as connection streams for recycles and inputs to the next process. The BFD for FFC is shown as BF-1 in Appendix A-3. Figure 3-1 also shows the BFD for the FFC facility for ease in viewing as the various processing areas are discussed in section 3.3. 

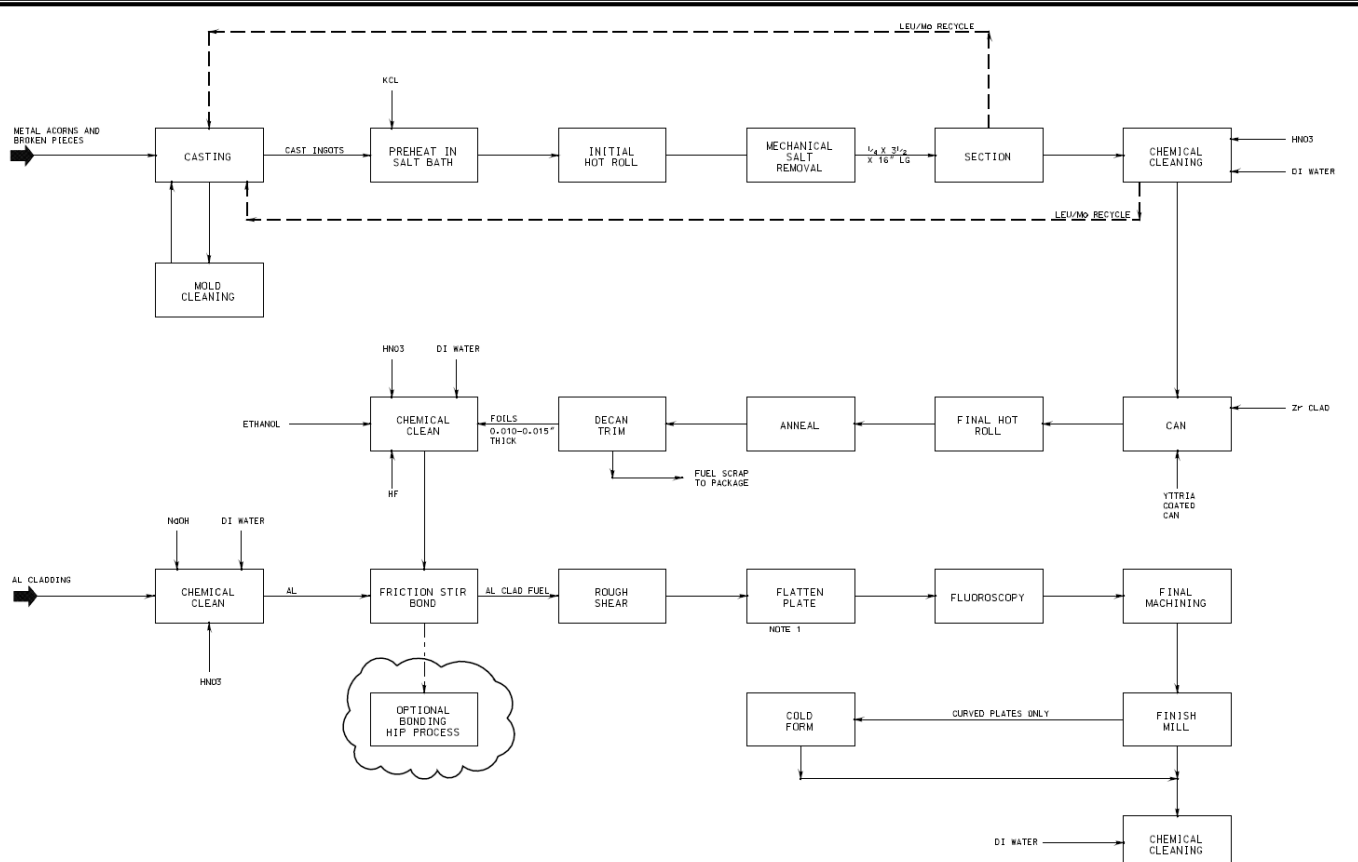

HNos

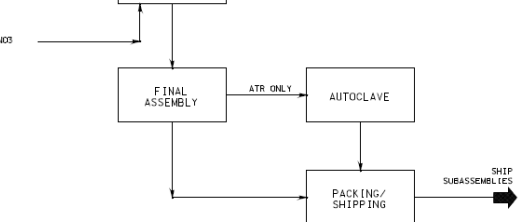

FIGURE 3-1 OVERALL BLOCK DIAGRAM OF THE FFC FACILITY PROCESSES 
The major steps in fuel fabrication are as follows:

- Casting: Broken metal feedstock and recycled fuel are melted and cast into ingots for further processing. The ingots are analyzed to ensure the correct isotopic mixture and chemical composition. Each mold is $1 / 4$ " x 3.5 " x 16 "

- Hot Roll: The cast ingots are preheated using a salt bath at $650^{\circ} \mathrm{C}$ and rolled to an intermediate thickness of 0.1 " causing the ingot to lengthen to 40 ". The rolled ingots are sectioned into 10 pieces.

- Salt Removal: The salt bath material adhering to the ingots is removed using mechanical brushing.

- Cleaning: The rolled ingots are cleaned in a nitric acid bath and rinsed with DI water to promote bonding without voids.

- Canning: Zr cladding is added to each side of the LEU/Mo rolled section and this "sandwich" is welded inside a steel can. The interior of the can is coated with yttria $\left(\mathrm{Y}_{2} \mathrm{O}_{3}\right)$ to prevent the $\mathrm{Zr}$ from bonding to the steel can, allowing for easy removal.

- Final Hot Roll: The steel can is heated in a tunnel furnace and rolled to the final (foil) thickness of 0.010 to 0.015 ".

- Anneal: The steel can with the Zr-clad LEU/Mo fuel foil inside is placed in an annealing furnace to reduce residual stresses from rolling and flatten the foils. Constraints are placed on top of the foil to help flatten the foils.

- Decan and Trim: The foils are removed from the can and are trimmed to the final size required for each fuel plate.

- Cleaning: Fuel foils are cleaned using a nitric acid/ hydrofluoric acid mixture to remove the yttria and are rinsed with DI water. Ethanol is used as a final wipe.

- Aluminum Hardware Cleaning: Aluminum cladding is cleaned using sodium hydroxide, nitric acid, and a DI water rinse prior to assembly with the fuel foil.

- Friction Stir Welding: The foil is sandwiched between the aluminum plates. A friction stir welder is used to bond the fuel foil to the aluminum. An alternative method of bonding uses a hot isostatic press (HIP).

- Final Preparation: The fuel plates are trimmed to final size, flattened, and prepared for final assembly. Each plate is marked with a unique identifier to meet $\mathrm{QA} / \mathrm{QC}$ requirements. The plates are degreased following machining steps using nitric acid, soapy water, and hot and cold DI water rinses.

- Plate Inspection: The plates are inspected using various inspections to ensure compliance for reactor specifications such as UT, gamma scanning, and radiography.

- Plate Forming (curved plates only): The plates are pressed over a curved mandrel to the proper curvature.

- Final Plate cleaning: Prior to assembly, the plates are cleaned using nitric acid and a DI water rinse.

- Final Assembly: Multiple fuel plates are collected and secured into sub-assembly hardware for each of 5 US reactors. Sub-assemblies are swaged, welded, and 
machined to final tolerance. Following fabrication, the assemblies are cleaned using hot and cold DI water washes (the HFIR plates are assembled using a different method).

- Autoclaving (ATR only): ATR sub-assemblies are processed in an immersion autoclave to form a stable oxide layer on the surfaces of the element.

- Final Inspection: The sub-assemblies are thoroughly examined to ensure compliance with reactor requirements. This includes dimensional, visual, radiological and gap thickness checks.

- Loading and Shipping: Completed sub-assemblies are loaded into shipping containers and stored onsite until they are shipped to the individual reactors.

\subsubsection{Process Flow Diagrams}

Process flow diagrams (PFDs) define the major pieces of equipment, the process flows, and utilities used within the process. Equipment is tagged and shown in PFDs. This is helpful in constructing the facility equipment list. PFDs show equipment such as filters, pumps, furnaces, heat exchanges, tanks, etc. as well as waste pathways, utilities required, and chemicals consumed. The PFDs are shown in Appendix A-4. The PFDs list is shown below in Table 3-1:

\section{TABLE 3-1 LIST OF PROCESS FLOW DIAGRAMS}

\begin{tabular}{|ll|}
\hline Process Flow Diagrams & \\
\hline Symbols and Legend & SK-1 \\
\hline Front End Processing & SK-2 \\
\hline Fuel Zr Cladding & SK-3 \\
\hline Fuel Al Cladding & SK-4 \\
\hline Final Assembly & SK-5 \\
\hline Inspection & SK-6 \\
\hline Off-Gas Treatment & SK-7 \\
\hline Waste Characterization and Packaging & SK-8 \\
\hline Shipping, Receiving and Storage & SK-9 \\
\hline Alternate HIP Bonding Process & SK-10 \\
\hline Ar Purification and DI Water Supply & SK-11 \\
\hline Final Assembly Machining & SK-12 \\
\hline
\end{tabular}

\subsubsection{Material Balance}

The material balance was calculated based on the production requirements to meet HPRR refueling schedules shown in Table 3-2. A key process assumption is that the facility will be available $75 \%$ of the year. The values shown in Table 3-2 allow for an additional 15\% excess capacity for future increases in reactor orders and for process yields throughout the facility. A detailed discussion of the material balance is provided in Section 3.2. 


\subsection{Facility Throughput Basis}

The FFC facility must be capable of providing the required yearly core replacements for each of the five U.S. research reactors. These reactors include the MIT Reactor-II (MITR-II), the National Bureau of Standards Reactor (NBSR), the Missouri University Research Reactor (MURR), the Advanced Test Reactor at INL (ATR), and the High Flux Isotope Reactor (HFIR). The yearly requirements for these five reactors are included in Table 3-2.

TABLE 3-2 FUEL REQUIREMENTS FOR THE FFC FACILITY

\begin{tabular}{|c|c|c|c|c|c|c|}
\hline Reactor & Cores/yr & Elements/yr & Plates/yr & Eplates & Foils/yr & $\Sigma$ foils \\
\hline MITR & 1 & 38 & 625 & \multirow{5}{*}{15236} & 694 & \multirow{5}{*}{16929} \\
\hline MURR & 4 & 44 & 1185 & & 1317 & \\
\hline NBSR & 2 & 83 & 1574 & & 1749 & \\
\hline HFIR & 10 & 14 & 8333 & & 9259 & \\
\hline ATR & 3 & 167 & 3519 & & 3909 & \\
\hline
\end{tabular}

According to Table 3-2, nearly 17,000 foils are required each year to provide the required output. The facility is assumed to have $75 \%$ availability. This allows for approximately 265 operating days per year. This conservative estimate requires the FFC facility to produce 64 foils per day. The capacity has been increased to 67 foils per day to allow for unscheduled shutdown of the facility especially during start-up. The foil production is shown for each major area on the process flow diagrams. All equipment is designed to produce 67 foils per day but the normal operating rate may be lower given the necessary production and storage limitations. These values are based on the process yields for each area as found in the Conceptual Process Description for the Manufacture of LEU/Molybdenum Fuel (INL report INL/EXT-08-13840). Each major process function allows for scrap and reject material. For instance, Table 3-2 shows that the five research reactors require 16,929 foils but only 15,236 plates. This shows that the plate production step has a process yield of 0.90 , indicating that up to $10 \%$ of the material may be scrapped and will not continue downstream for further processing. The outputs provided in Table 3-2 also provide an additional 15\% output incase reactor demand increases in the future. The FFC will have storage space to allow for excess sub-assemblies until the next campaign for each reactor design.

\subsection{Process Design Basis and Description}

The process flow diagrams (PFDs) included in Appendix A-4 show the following processes along with flows into and out of the process as well as any utility or consumable that is associated with the process. The facility equipment is described in Section 4 and the facility layout is described in Section 5. Figure 3-2 shows how the material moves through the various stages and changes shape as it progresses downstream.

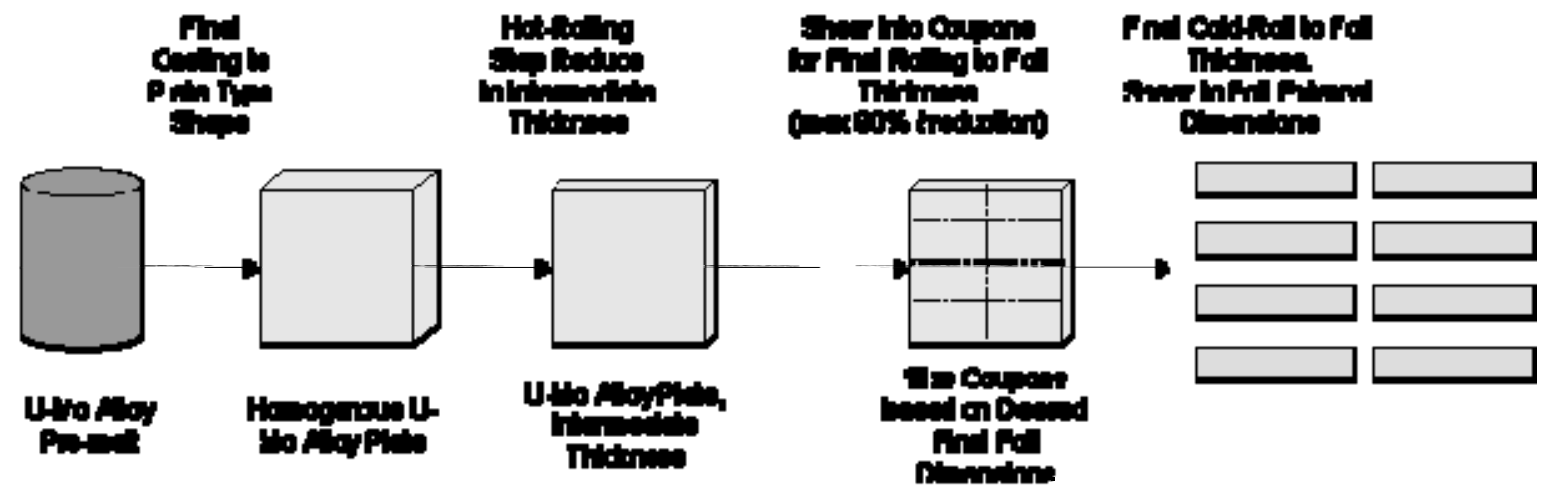

FIGURE 3-2 CONCEPTUAL FFC PROCESSING DIAGRAM 


\subsubsection{Material Shipping and Receiving}

Shipping and Receiving is shown on PFD SK-9. Broken fuel pieces or "acorns" are trucked in from Y-12 using ES-3100 shipping containers. It may be possible for Y-12 to ship cast ingots rather than broken metal pieces. The ingots would still be shipped in cans packaged within ES-3100 shipping containers. This is part of a future study to determine if Y-12 will increase the scope of their delivery method. This report has assumed that the FFC will receive broken pieces so as to bound the facility and the functional requirements. The shipping crane, $\mathrm{CRN}-001$, unloads the shipping container and moves material throughout the shipping and receiving area. Each container from Y12 contains 3 shipping cans, which each contain LEU/Mo fuel pieces in the proper composition and enrichment. After the cans are unloaded from the shipping container, testing and inspection will be conducted on the cans to make sure that the received material meets the appropriate specifications. A separate, representative sample will be included with each casting batch for chemical and isotopic sampling. Any rejected material will be shipped back to Y-12 while approved material will be placed into a locked storage area, ST-001 until processing. Final sub-assemblies are also packaged in this area to be shipped to the reactor site. The sub-assemblies are packaged in PK-001 packaging area in the appropriate shipping containers.

\subsubsection{Ingot Casting}

After the representative fuel piece is inspected and approved for processing, the can is opened and may be moved to the casting area for processing. The first step in the process in shown on SK-2 and is cleaning of the LEU/Mo feedstock (fuel pieces require a nitric acid wash prior to casting to remove gross oxide from the surface of the metal) in wash station, WS-015. After cleaning, the material is placed into one of the casting furnaces. The casting furnaces, HT-001 A/B, are located in one of two gloveboxes, GB-001 A/B, to maintain an inert atmosphere (there are two casting furnaces and two gloveboxes, hence the A/B designation). The glovebox maintains an argon atmosphere with a purification system discussed in section 3.5 below. Cast molds are also introduced into the glovebox and are placed on a heater, HT-003 A/B, to help the cast LEU/Mo mixture form to the cast mold and overcome surface tension and limited flow concerns. The cast molds are moved throughout the glovebox on a conveyor belt, CV-001 A/B. The cast fuel is removed from the mold where a filter, FIL-009, and ventilation hood is located to capture any particles that may be released during the mold removal process. The crucible sits within the casting furnace and holds the broken pieces. The crucible will also contain any dross material or heel that may be left after the casting process. Both the crucible and the cast molds are recycled with a cleaning in X-001 and a coating in CT-001 with yttria to allow for easy removal of the LEU/Mo ingot from the casting surface. A ventilation hood and pre-filter, FIL-017, are connected to the mold and crucible cleaning station to collect any chemical fumes and also collect any yttria particles in the air. The off-gas from the casting furnace goes through a roughing filter, FIL-027, which is composed of sintered metal capable of collecting the uranium oxide formed during the casting process. This roughing filter will prolong the life of the HEPA filters downstream. Y-12 has experience with these roughing filters, which do not require a blow-back system. The filters will be replaced when material buildup begins to impact the casting process. Any waste material that is introduced during the casting process will be collected in a geometrically safe collection area and packaged for waste disposal. After the ingots are cast, they are chemically inspected using an ICP-AES-MS to ensure the proper LEU/Mo mix. Once the results prove that the fuel is acceptable for reactor design, the cast ingots can move into further processing. 


\subsubsection{Hot Rolling/Shearing}

After the cast ingots are approved for processing, the next step is to roll the fuel pieces into the proper sizes for fuel foils as shown on SK-2. The cast ingot is approximately $1 / 4$ " thick by 3.5 " wide and 16 " long. The first step of the rolling process is to heat the cast ingot in a salt bath, HT-002, using a mixture of salts including potassium chloride and lithium chloride salts. The bath is heated to between $600^{\circ} \mathrm{C}$ and $650^{\circ} \mathrm{C}$. A local ventilation hood and filter, FIL-001, collect the hot fumes from this furnace (both for waste heat removal and for radiological contamination control). After the ingot is heated, it is rolled through a rolling mill, RL-001, with filter FL-002 collecting any off-gases from this process. The rolled ingot is repeatedly rolled and reheated in the salt until it achieves a thickness of between 0.09 " and 0.1 ". Once the fuel piece has achieved the desired thickness, it is cleaned of the salt using a mechanical finishing mill, FM-001. FIL-018 is a localized ventilation hood and filter to collect any dust particles that occur as the salt is removed from the fuel. After the salt removal station, the fuel is washed in WS-016 to remove any salt particles or scrap material and sheared into 4.5" long pieces using SR-001. The off-gas and fine particles from the shear are collected in FIL-003. The edges are also trimmed to ensure the proper width for further processing. Any trim pieces from this stage are recycled back to the casting furnace to incorporate into future castings. The intermediate rolled foils may be transported to storage or continue on to plate cleaning.

\subsubsection{Fuel Plate Cleaning}

The fuel plate cleaning process is shown on PFD SK-3. The clean sheared foil at approximately 0.1 " thick is cleaned using a $30 \%$ nitric acid bath, WS-001. The bath will be sized to hold about 10 plates in each fuel cleaning. The bath will recirculate the nitric acid to promote mixing and agitation of the acid against the plate. The recirculating stream will also have a filter on it to clean out any dirt or other small particles that may be cleaned off the fuel plate. The $\mathrm{pH}$ of the bath will be tested daily to ensure the integrity of the acid. Once the acid is below the normal operating $\mathrm{pH}$ level, the acid will be packaged for waste and fresh nitric acid will be placed in the bath. A demister pad, FIL-010 is placed above the bath to ensure acid fumes are collected. Any liquid that condenses and collects in the bottom part of the demister will be returned to the bath. Following the nitric acid bath, the fuel ingots are inspected for pass/fail based on dimensions. Any failed pieces are sent back to the casting furnace for recycling and recasting. Accepted fuel pieces are sent to the canning station to receive the Zirconium (Zr) clad material.

\subsubsection{Zr Cladding and Final Rolling}

Acceptable washed fuel foils are transferred to the $\mathrm{Zr}$ cladding station as shown on SK-3. The LEU/Mo fuel plate is placed inside an enclosure consisting of zirconium foil tack welded to both sides of a steel can with a layer of yttria between the $\mathrm{Zr}$ foil and the steel can to ensure that the $\mathrm{Zr}$ will not adhere to the steel can (the cans, complete with $\mathrm{Zr}$ and yttria are procured from an outside vendor). This placement of the foil in the can occurs in the canning station, CS-001. A local ventilation hood is placed at the canning station along with a filter, FIL-011. The can has a "pig tail" associated with it that is first backfilled with argon and then connected to a vacuum pump, P-001, to remove any air from the can. The vacuum off-gas is passed through FIL-025 to clean any particles that may be removed from the can through the vacuum process. The air evacuated can then passes through a tunnel furnace, HT-004, before being rolled through RL-002 to a foil thickness of between 0.010 and 0.015 ”. A filter, FIL-016, removes any hot off-gasses 
from the furnace while filter FIL-004 collects any particles and off-gas from the rolling mill.

\subsubsection{Annealing}

Once the foil has been rolled, SK-3 shows that the next step is to anneal the fuel and $\mathrm{Zr}$ plates. This process is done to flatten the foil. The process occurs in the can previously used to roll the fuel plate and the $\mathrm{Zr}$ clad together. This can remains intact during the rolling process; therefore, the annealing furnace, HT-005, can be in an atmospheric pressure environment. A constraint is recommended to keep the foil flat during the heating process. The carbon steel can forms a eutectic with the fuel foils at temperatures higher than $725^{\circ} \mathrm{C}$. Therefore, the annealing process must be regulated to occur between $600^{\circ} \mathrm{C}$ and $675^{\circ} \mathrm{C}$. A ventilation hood and filter, FIL-026, is used to collect the heated off-gas from the annealing furnace. After the annealing process, the can is removed from the fuel foil using SR-002, the can removal shear. FIL-005 filters the off-gas and collects any fines from the can removal process. The can waste is sent to waste packaging for final disposal. The fuel plate is then cleaned to remove any yttria coating and contamination.

\subsubsection{Foil Cleaning}

Foil cleaning is accomplished after the annealing process to remove contaminants such as oxide and yttria coating from the canning process. The first step is to put the fuel plate in a wash station, WS-002, with a mixture of $50 \%$ water $\left(\mathrm{H}_{2} \mathrm{O}\right), 45 \%$ nitric acid $\left(\mathrm{HNO}_{3}\right)$ and $5 \%$ hydrofluoric acid (HF). The HF is essential for etching the $\mathrm{Zr}$ cladding to allow the yttria particles to release. This wash station is similar to the fuel plate wash in that it holds multiple plates at one time and uses a recirculating flow within the wash bath to agitate and ensure a thorough cleaning. The recirculation flow is filtered to remove the yttria particles and any $\mathrm{Zr}$ cladding that is also etched off the fuel foil. The wash bath off-gas is filtered using FIL-012 in order to remove any acidic fumes from the processing area. The filter contains a demister pad that allows the condensed vapors to flow back into the wash station. The off-gas that passes through the demister will combine with the other ventilation off-gas flows for further processing. A pH meter is used to test the wash solution daily. Once the wash solution is no longer at an operating level for thorough cleaning, the wash solution will be replaced. For waste estimation purposes, the wash solution is assumed to be exchanged once a week.

The second wash process occurs in WS-003 and is a DI water rinse. The FFC facility will have a DI water filtration unit with a storage tank capable of providing the DI water usages for this process. The DI water rinse will remove any residual acids and contaminants from the fuel foil before further processing. The water rinse will be a large basin capable of holding multiple fuel foils with a recirculation pump and filter. A conductivity measurement will be used daily to ensure that the water is an effective cleaning agent. Once the water conductivity is outside of normal operating limits, the water will be removed and replaced with clean DI water. Since this water has contact with LEU/Mo, the water cannot be recycled back to the DI filtration unit, but is rather bottled and packaged for waste disposal.

The third and last step in the fuel foil cleaning is an ethanol cleaning wash using WS-004. The ethanol is used to remove the residual rinse water from the fuel plate. The ethanol vapors are collected using FIL-013. This is important since alcohol vapors are a fire hazard especially in a machine shop area where metal processing could potentially create sparks. The current philosophy for doing an ethanol wash, is to soak a wipe with ethanol and wipe both side of the fuel foil (this serves to both coat the foil with a thin layer of 
ethanol and to give mechanical action to help release the yttria particles). However, given the throughput rate of the FFC facility, a more automated way may be preferred. The waste (either from a wipe or from an automated wash basin) is sent to packaging for final disposition. The wipes would be compressed and packaged with other Ucontaminated LLW. The liquid would be bottled and packaged for disposal to a liquid treatment facility. This will be resolved by a future study.

\subsubsection{Friction Bonding}

The cleaned fuel foils are now ready to be bonded with the Al cladding. Prior to the bonding, the aluminum is cleaned using a three step process. First, the aluminum is placed in a heated sodium hydroxide solution $(\mathrm{NaOH})$ bath using WS-005. The heated bath has a demister pad, FIL-014, to absorb any chemical fumes that are released from the wash bath. The bath is designed to hold 20 aluminum pieces- enough to support the 10 fuel foils that are washed in each process as explained above. The wash bath uses a recirculating pump and filter to remove any contamination particles. The solution is tested periodically using a $\mathrm{pH}$ meter to ensure efficiency of the cleaning solution. Once the solution is exhausted, it is packaged for shipment to a liquid waste treatment facility. It may be possible in future studies to determine if this sodium hydroxide waste can be combined with other wash nitric acid waste and the water evaporated to condense the liquid waste generated from the facility. Following the $\mathrm{NaOH}$ wash, the plates are rinsed with DI water using WS-017.

The second wash step that the $\mathrm{Al}$ cladding receives is a nitric acid wash with an ultrasonic probe in WS-006. FIL-015 is a demister pad that absorbs any chemical fumes that are off-gassed from the wash bath. The nitric acid bath tank is also sized to hold 20 pieces and is cleaned with a filter in the recirculating line. A pH meter is used to test the $\mathrm{pH}$ of the solution daily to ensure proper cleaning. Once the $\mathrm{pH}$ is in a level no longer acceptable to the process, the nitric acid is drained and replaced. The spent acid is bottled and packaged for shipment to a waste processing facility.

The third and final wash step is a cold, and then hot DI water rinse to remove any residual nitric acid. The DI water is provided to cold wash station WS-007 and the hot wash station, WS-018 from the facility DI water filtration system. Each DI water wash is capable of holding $20 \mathrm{Al}$ clad pieces and is recirculated through the wash basin with a pump and filter to remove any contaminants. The DI water is tested daily for conductivity. When the conductivity of the water falls out of normal operating range, the water is removed from the wash basin and replaced. Since this water does not have direct contact with the uranium or molybdenum fuel, the water can be recycled back to the DI water filtration unit. The clean aluminum plates are then ready to be bonded with the clean fuel foils.

One section of the aluminum cladding has a pocket machined into one surface sized to fit the fuel foil; the other is a flat sheet. The thickness of the two plates is such that the fuel foil will be centered in the final product. The fuel foil is placed in the pocket of the first sheet and the flat sheet is placed on top. This assembly is then placed in the friction stir welder, FSW-001 A/B/C/D. There are four friction stir welders in order to complete the daily throughput in one shift. The friction stir welder uses pressure and friction to heat the fuel foil and aluminum to a temperature hot enough to plasticize the aluminum cladding, but low enough that the plate does not melt or cause permanent undesired material property changes. The friction stir welding process occurs on both sides of the fuel plate. Figure 3-3 shows a cut-away diagram of the fuel plate after aluminum cladding bonding. 
FIGURE 3-3 ALUMINUM CLAD FUEL PLATE CUT-AWAY DIAGRAM

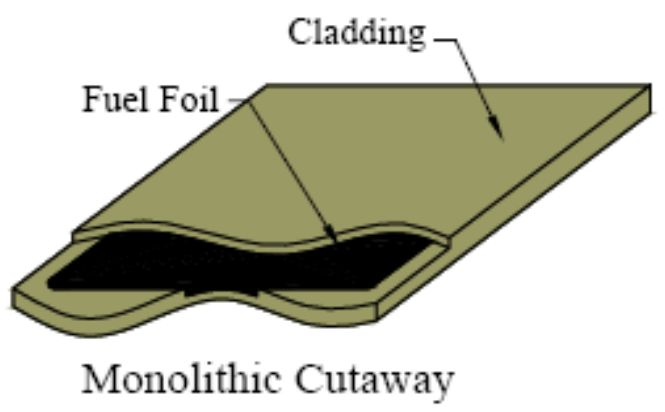

\subsubsection{Alternate HIP Process}

As an alternative to friction bonding the fuel plates, Hot Isostatic Pressing (HIP) is being considered as a method to bond the fuel plates. HIP uses heat and pressure to create a diffusion bond between the aluminum cladding and the fuel foil. This is done by placing the process part in a pressure vessel and applying a specific time/temperature regime. For HIP it is critical that the interior of the plate (the interface between the two cladding sheets and the interface between the cladding and the fuel) be isolated from the processing media. This is done by sealing the perimeter of each plate in a vacuum by using electron beam welding. For processing, a number of plates are bound together separated by rigid slabs (strongbacks) that constrain the plates during the HIP run to keep them from warping. The plates are processed at $100 \mathrm{MPa}$ and $560^{\circ} \mathrm{C}$ for a 30 minute hold time. With the high thermal mass of the HIP system a single cycle (including loading and unloading) takes nearly 24 hours. The FFC facility layout has included an alternative placement for the HIP process and an alternative flow diagram, SK-10 shows the HIP. Future studies will determine which bonding process will be used to bond the fuel plates to the aluminum cladding.

\subsubsection{QA Inspection}

A number of inspections must be accomplished throughout the process to provide quality assurance that the fuel plates are fabricated to the technical standards required for nuclear reactor operation. Some of these inspections are visual, some are physical such as weight and dimensions, and some are chemical such as composition and isotopic analysis using an inductively coupled plasma with atomic emission spectroscopy and a mass spectrometer (ICP-AES-MS). Still other inspections occur on structural aspects such as the ultrasonic tester for bond integrity. SK-6 shows some of the analytical equipment that will be used to perform inspection within the FFC facility. Other inspection requirements include dimensional analysis, gap testing, visual inspections, etc.

The various analyzers listed on the PFD are two ultrasonic testing machines, UT-001 $\mathrm{A} / \mathrm{B}$, a radiography analyzer, RD-001, a fluoroscopy analyzer, FS-001, an optical microscope, OM-001, the ICP-AES-MS, ICP-001, a scale, W-001, and two gamma scanners, GS-001 for the plate scan and GS-002 for the sub-assembly scan. There is a local ventilation area, FIL-019, to collect any off-gasses from the laboratory equipment during analysis.

The ultrasonic testing stations are used to measure two different things, bond integrity and the thickness of the cladding over the fuel. The debond is measured by a through transmission of ultrasound energy (where the signal has been attenuated from the baseline the bond is shown to be of lower quality) involving a transmitter and receiver on opposite 
sides of the plate. The cladding thickness is measured through a time-of-flight indication (the signal bounces back to the transmitter/receiver (this is a separate unit from the debond diction) from any discontinuity in the fuel plate). There are two ultrasonic testers to provide the testing for the daily capacity of production.

Another piece of laboratory equipment is the radiography analyzer. A radiograph uses xray waves to produce a visual image of the test item based on density differences. Radiography is used within the FFC both to examine where the fuel foils lie within the Al cladding (and to see if any fuel lies outside the acceptable region of the plate) and also to measure the fuel zone areal density (regions of high fuel loadings are prone to higher heating and may violate the safety case for the reactor).

Fluoroscopy is a real-time x-ray used to locate the fuel within an in-process fuel plate. This apparatus is coupled with a punch system that will drive index holes into the cladding to allow for machining of the fuel plate to its final dimensions.

Optical microscopy is used to analyze the interior of selected fuel plates. As this is a destructive analysis method it is only done on a random selection of plates or on failed plates to help determine processing problems. The optical metallograph and the related equipment (for sample preparation) are all standard commercial equipment.

Chemical and isotopic analyses are done in a small laboratory located on the FFC site. Even through the LEU/Mo feedstock is fully characterized by Y-12 prior to shipment the casting steps that occur at the FFC require the reanalysis of the material (if the ingot casting is moved from the FFC to Y-12 the need for this analysis goes away). The necessary analysis is done by an Inductively Coupled Plasma Mass Spectrometer (ICPMS), and two "determinators": one for oxygen/nitrogen and the other for carbon and sulphur. The ICP-MS measures the uranium isotopic composition and the amount of molybdenum in the material while the determinators measure the amount of impurities in the material. The analysis equipment is all available as off-the-shelf equipment and will only need to be located inside containment.

Scales are used throughout the facility to weigh the process material (both fuel and scrap), keep accountability records, and also ensure that the fuel plates meet physical specifications. Each scale will have an accuracy of at least $0.1 \mathrm{~g}$.

One last piece of laboratory equipment accounted for in the inspection system are two gamma scanners- one for the plate and one for the element. The gamma scanners are non-destructive tests used to assay the final products to determine correct make-up of the plates and final sub-assemblies.

Inspection areas are shown on the flow diagrams as an A in a circle. However, not all analyzers will be used at each inspection area. Some of the inspections will be done on every plate while other inspections use statistical analysis, especially the inspections such as optimal microscopy, which involve destroying the sample by cutting a cross-sectional sample.

\subsubsection{Plate Finishing}

Once the LEU/Mo and Zr clad foil has been friction bonded to the aluminum cladding, the fuel plate is ready to be cleaned and prepped for final assembly of the sub-assembly elements. The first step after friction stir welding is to do a rough shear using SR-005. Any trim collected from the shear is weighed for accountability and packaged for waste shipment (as potentially contaminated). FIL-024 is a ventilation hood with a pre-filter that collects any fines and particles from the shear process. The next step is to flatten the 
plate using RL-003, the roller leveler. This unit may be used multiple times as needed during the plate finishing process in order to ensure the plates are flat before pressing to the final shape required by the reactor design. After this rolling, the fuel plates can be sent to storage until further processing.

Process flow diagram SK-5 shows the next steps in the process. The first step is to locate the fuel inside the plate using the fluoroscopy analyzer, which will index the location of the fuel by punching reference holes in the plate. From this point, the plates will be processed by a series of finishing mills $(\mathrm{FM}-002 \mathrm{~A} / \mathrm{B} / \mathrm{C} / \mathrm{D} / \mathrm{E})$ on which the plates will be cut to final thickness, have a final surface finish applied and cut to final dimensions (using the reference holes for a datum point). Other special geometry (the 'fins' in the MIT plates) will be added at this point. The machined scrap material from this process is considered suspect contaminated and is packaged for shipment to a waste processing facility. A ventilation hood with filter FIL-008 is used to collect any fines and particles that may result from the shearing operation.

Each plate is marked using the marking station, MK-001, for material accountability purposes. The marking must occur after the friction stir welding, which obliterates any markings on the surfaces that will be on the final plate, but before the final assembly of the sub-assembly element. The marking station puts a permanent mark such as a laser etch or permanent press marking into the plate that can be traced back to the LEU/Mo batch that the plate came from and various processing steps that are controlled by QA/QC.

After the plate is marked, the plate is washed using a two stage process. The first wash step is to use soapy water in WS-008 to degrease the plates from the cutting process. The second stage is to use a hot DI water wash in WS-009. These two wash processes will remove any fines and contaminants from the shearing, marking, and finishing mill processes. Since there is no fuel interaction with the liquids, the liquids can be flushed to the firewater collection tank. The firewater collection tank has a series of pencil tanks that are geometrically configured for criticality control associated with the firewater collection tank. The liquid waste enters the pencil tanks where recirculation occurs to ensure mixing. A sample port is located on the recirculation line. Samples can be taken of these wash liquids and analyzed for contamination. If any contamination is measured, the waste will be extracted from the firewater tank, packaged, and sent to a waste treatment facility. If the is no contamination measured, the waste will be sent to the firewater collection tank until enough is collected to warrant an extraction where the solution is sent to a water treatment facility. Once the plates have been washed, they can either go back to storage or continue on for further processing.

\subsubsection{Plate Forming (curved plates only)}

Following plate fabrication, the plates requiring a curved shape are formed into shape using a mandrel in a hydraulic press.

Each plate must be cleaned before entering the final assembly process. This cleaning involves three steps. The first step occurs in WS-010, which is a $30 \%$ nitric acid wash. A demister filter, FIL-029 is used to collect any acid fumes from the wash station. The second wash is a cold DI water rinse to remove the nitric acid and the third and final wash is a heated DI water rinse, WS-011 and WS-012 respectively. The nitric acid wash is tested daily with a $\mathrm{pH}$ meter to ensure proper cleaning efficiency. When the $\mathrm{pH}$ is outside of normal operating range, the acid is removed from the wash station and packaged for shipment to a waste treatment facility. Even though the nitric acid wash does not have suspect contamination, it is acidic and must be treated properly before 
being safe to dispose of. The water rinses can be disposed of through the firewater collection tank and sample for contamination. All three wash stations use recirculation pumps and filters to recycle fluid through the wash and stimulate agitation for thorough cleaning.

\subsubsection{Sub-Assembly Fabrication}

After washing the plates, the plates are ready for final assembly, as shown on SK-12. This area includes swaging, hydraulic pressing into curved forms according to the reactor design and other mechanical manipulations as described in Sections 4.0 and 5.0 below.

Final sub-assemblies are cleaned prior to being packaged for shipment. The subassemblies are washed in cold water, WS-013, and then hot water, WS-014, before being dried using a dry air spray, DRY-002, over the sub-assemblies. The water from these wash stations can be sent to the firewater collection tank and tested for contamination prior to being packaged for shipment to either a waste treatment facility if contaminated or a water treatment facility if there is no contamination present. The water rinses are designed to remove any contamination and residues from the final assembly process. The dry air will be provided either by compressing and drying atmospheric air or by using pressurized gas cylinders such as nitrogen to remove any of the water particles from the thin spaces in the final assembly.

One additional step that is only pertinent to ATR sub-assemblies is to heat the subassembly in an autoclave, HT-006. The autoclave is a cylindrical pressure vessel with an integrated heater. The vessel is filled with DI water and the elements (up to four elements at one time are placed into the autoclave) are immersed in the water for processing at elevated pressure and temperature to place a prefilm on the surface. After processing, the unit is cooled and the water is drained prior to unloading. A ventilation hood with filter, FIL-022, is used to collect the heated off-gas from the furnace after opening.

The sub-assemblies are ready to be packaged and shipped to the appropriate reactor site after cleaning and autoclaving (for ATR only).

\subsection{Waste Handling and Disposition}

The FFC facility will not have any capabilities for processing waste. For example, sodium hydroxide is used as an aluminum cladding wash step and nitric acid is used to clean the fuel plates. These two solutions would be packaged separately for shipment to a hazardous waste treatment facility. Waste will be packaged in its current state (liquid, solid, scrap trims, etc.) and be shipped either to a treatment or storage and disposal facility. As the FFC is likely to be located at a DOE National Laboratory, it is probable that RCRA-permitted waste treatment facilities exist at the site, and the hazardous wastes could be treated locally. Table 3-3 provides a list of the wastes generated from FFC processing. 
TABLE 3-3 LIST OF WASTE GENERATION, ANNUAL VOLUMES, AND DISPOSAL PATHWAYS

\begin{tabular}{|c|c|c|c|}
\hline & Waste Source & Generation & Disposition \\
\hline \multirow[t]{15}{*}{ Liquids } & $\begin{array}{l}\text { Fuel Receipt Wash } \\
\left(\mathrm{HNO}_{3}\right)\end{array}$ & 240 gallons/ year & $\begin{array}{l}\text { Contaminated (sub-accountable), } \\
\text { mixed LLW to treatment }\end{array}$ \\
\hline & $\begin{array}{l}\text { LEU/Mo Ingot Wash } \\
\left(\mathrm{HNO}_{3}\right)\end{array}$ & 240 gallons / year & $\begin{array}{l}\text { Contaminated (sub-accountable), } \\
\text { mixed LLW to treatment }\end{array}$ \\
\hline & $\begin{array}{l}\mathrm{LEU} / \mathrm{Mo} / \mathrm{Zr} \text { Foil Wash } \\
\left(\mathrm{HNO}_{3} / \mathrm{HF}\right)\end{array}$ & 480 gallons / year & $\begin{array}{l}\text { Contaminated (sub-accountable), } \\
\text { mixed LLW to treatment }\end{array}$ \\
\hline & $\begin{array}{l}\text { LEU/Mo/Zr Foil Wash } \\
\text { (DI Water) }\end{array}$ & 480 gallons / year & LLW to treatment \\
\hline & $\begin{array}{l}\text { LEU/Mo/Zr Foil Wash } \\
\text { (ethanol) }\end{array}$ & 480 gallons / year & LLW to treatment \\
\hline & Al Wash $(\mathrm{NaOH})$ & 3120 gallons / year & $\begin{array}{l}\text { Hazardous, non-contaminated waste } \\
\text { to treatment }\end{array}$ \\
\hline & $\mathrm{Al}$ Wash $\left(\mathrm{HNO}_{3}\right)$ & 3120 gallons / year & $\begin{array}{l}\text { Hazardous, non-contaminated waste } \\
\text { to treatment }\end{array}$ \\
\hline & Al Wash (DI Water) & 3120 gallons / year & $\begin{array}{l}\text { Sent to firewater collection tank for } \\
\text { sampling and disposition }\end{array}$ \\
\hline & Plate Wash (soapy water) & 3120 gallons / year & $\begin{array}{l}\text { Sent to firewater collection tank for } \\
\text { sampling and disposition }\end{array}$ \\
\hline & $\begin{array}{l}\text { Plate Wash (Hot DI } \\
\text { Water) }\end{array}$ & 3120 gallons / year & $\begin{array}{l}\text { Sent to firewater collection tank for } \\
\text { sampling and disposition }\end{array}$ \\
\hline & Final Plate Wash $\left(\mathrm{HNO}_{3}\right)$ & 3120 gallons / year & $\begin{array}{l}\text { Hazardous, non-contaminated waste } \\
\text { to treatment }\end{array}$ \\
\hline & $\begin{array}{l}\text { Final Plate Wash (Cold } \\
\text { DI Water) }\end{array}$ & 3120 gallons / year & $\begin{array}{l}\text { Sent to firewater collection tank for } \\
\text { sampling and disposition }\end{array}$ \\
\hline & $\begin{array}{l}\text { Final Plate Wash (Hot DI } \\
\text { Water) }\end{array}$ & 3120 gallons / year & $\begin{array}{l}\text { Sent to firewater collection tank for } \\
\text { sampling and disposition }\end{array}$ \\
\hline & $\begin{array}{l}\text { Final Assembly Wash } \\
\text { (Cold DI Water) }\end{array}$ & 5000 gallons / year & $\begin{array}{l}\text { Sent to firewater collection tank for } \\
\text { sampling and disposition }\end{array}$ \\
\hline & $\begin{array}{l}\text { Final Assembly Wash } \\
\text { (Hot DI Water) }\end{array}$ & 5000 gallons / year & $\begin{array}{l}\text { Sent to firewater collection tank for } \\
\text { sampling and disposition }\end{array}$ \\
\hline \multirow[t]{3}{*}{ Solids } & $\mathrm{LEU} / \mathrm{Mo} / \mathrm{Zr}$ trim & $\begin{array}{l}523 \mathrm{~kg} / \text { year; } \\
1.5 \mathrm{ft}^{3} / \mathrm{yr}\end{array}$ & LLW to NTS for burial \\
\hline & $\mathrm{LEU} / \mathrm{Mo} / \mathrm{Zr} / \mathrm{Al}$ trim & $\begin{array}{l}15,500 \mathrm{~kg} / \text { year; } \\
210.5 \mathrm{ft}^{3} / \mathrm{yr}\end{array}$ & LLW to NTS for burial \\
\hline & $\begin{array}{l}\text { Failed samples and foils/ } \\
\text { plates }\end{array}$ & $\begin{array}{l}\text { Up to } 5000 \text { pieces } \\
\text { in various forms } \\
\text { (foils, Al clad } \\
\text { plates, etc.) }\end{array}$ & $\begin{array}{l}\text { Possible re-use at Y-12; LLW to } \\
\text { NTS for burial }\end{array}$ \\
\hline
\end{tabular}




\begin{tabular}{|c|c|c|c|}
\hline & Waste Source & Generation & Disposition \\
\hline & Rolling Cans & $\begin{array}{l}6,660 \mathrm{~kg} / \mathrm{yr} \\
50 \mathrm{ft}^{3} / \mathrm{yr}\end{array}$ & LLW to NTS for burial \\
\hline & Spent Equipment & & $\begin{array}{l}\text { Refurbish any useable equipment } \\
\text { (shears, rolls, etc); LLW to NTS for } \\
\text { burial }\end{array}$ \\
\hline & $\begin{array}{l}\text { Filters (HEPA/ } \\
\text { Demisters/ Sintered } \\
\text { Metal Filters/ Pre-Filters) }\end{array}$ & & $\begin{array}{l}\text { Compaction/ shear if possible; LLW } \\
\text { to NTS for burial }\end{array}$ \\
\hline & Spent Molds/ Crucibles & & LLW to NTS for burial \\
\hline & $\begin{array}{l}\text { Job Control Waste/ PPE/ } \\
\text { Misc. Processing Waste }\end{array}$ & & LLW to NTS for burial \\
\hline & $\begin{array}{l}\text { Slag/ Dross/ Heel from } \\
\text { Casting }\end{array}$ & & $\begin{array}{l}\text { Possible re-use at Y-12; LLW to } \\
\text { NTS for burial }\end{array}$ \\
\hline & Spent Salt from Salt Bath & & LLW to NTS for burial \\
\hline Gases & $\begin{array}{l}\text { Dry Air for Final } \\
\text { Assembly Drying }\end{array}$ & & $\begin{array}{l}\text { Tanks are re-filled through vendor; } \\
\text { air goes through ventilation and off- } \\
\text { gas treatment }\end{array}$ \\
\hline & $\begin{array}{l}\text { Argon for Inert Casting } \\
\text { Furnace Glovebox }\end{array}$ & & $\begin{array}{l}\text { Regenerated through filters and } \\
\text { driers; purged to ventilation and off- } \\
\text { gas treatment }\end{array}$ \\
\hline
\end{tabular}

\subsubsection{Liquid Waste}

There are a few sources of liquid waste in the FFC facility. The main source of liquid waste is from the fuel plate and assembly cleaning steps. Some of these steps, such as when the $\mathrm{Zr}$ clad fuel plates are washed in acid and water, will contain radioactive material. Other wash steps, such as the DI water rinse of the final assembly, will not contain radioactive material and can be characterized as non-hazardous and sent to the firewater drain system for clean-up and recycling. Other sources of liquid waste are purges from the DI water system and process water chiller system, liquid effluents from the demister pads, janitorial wastes, spent hydraulic oil, and various other minor sources.

Any waste that has probable radioactive contamination will be packaged in bottles to minimize leak potential. The bottles will then be packaged in cans and stacked in 55-gal drums for shipment to a facility that will treat the liquid waste. Spent hydraulic oil will be collected and discarded through a vendor. The vendor will make regular stops to switch out the dirty 55-gal drum with a clean drum for further disposal. Each 55-gallon drum of contaminated oil will cost about $\$ 3300$ for treatment and an additional $\$ 6000$ $\$ 8000$ for transportation costs, which is usually split over the cost of the load $(>10$ drums). However, it is not estimated that multiple drums of oil will be processed from this facility. Therefore, the maximum cost per drum of oil will be approximately $\$ 11,300$.

Process knowledge of where the liquid waste came from will be used to estimate contamination potential for radioactive material. Liquid waste that may not be 
contaminated will be sent into the fire water collection system and sampled. If the sample returns a negative result for contamination presence, the liquid can be sent to a waste treatment facility for clean-up. This will only be effective for non-hazardous chemicals. This pathway would follow for the soapy water rinse of the plates, any janitorial water such as dirty mop water, and the DI rinse of the aluminum cladding. The sodium hydroxide solution, even though it is non-contaminated, will not go into the firewater collection drain since it has chemical components that the waste treatment facility would not be able to dispose of. The sodium hydroxide will be bottled and packaged for shipment to a waste processing facility.

Liquid waste estimates were calculated based on the assumption that wash solutions will be changed out weekly, through the entire year (365 days). This will allow for solutions that may sit and still lose cleaning efficiency or have natural reduction in acidity/ basicity of the chemical compound. As stated in section 3.3, the wash stations are designed to hold multiple fuel plates at a time. For the LEU/Mo and LEU/Mo/Zr fuel foils, each wash station is designed to hold 10 plates at a common size of 3.5 " x 10 " x 0.1 " before final hot rolling and $3.5 " \mathrm{x} 50$ " $\mathrm{x} 0.015$ ". This equates to the wash bin being approximately 8 " x 12 " x 8 " for the pre-final roll wash, which is assumed to be the same size as the pre-cast wash to clean the acorns and broken pieces, and 8 " x 55 " $\mathrm{x} 4$ " for the 3 post-final roll washes. This equates to a total volume of 3.3 gal and 7.6 gal respectively. Each wash basin has a recirculation loop with a pump and filter, therefore each wash basin is assumed to hold 5 gallons or 10 gallons. There is a 90 day maximum temporary storage for hazardous materials, so if the solutions are changed out every 7 days, after 90 days, there are 60 or 120 gallons of waste for each wash solution. The 90 day temporary storage will be required to be emptied 4 times per year. Therefore, each wash basin produces 240 gallons of solution for two washes and 480 gallons for three washes. This is a total of nearly 2000 gallons. Each 55-gal drum of contaminated waste costs $\$ 600$ for treatment and each load costs less than $\$ 100$ for transportation. Assuming a $75 \%$ fill availability of the 55-gal drum, the 2000 gallons of waste would require approximately 50 drums. If a load consists of 10 drums, the total cost for treatment and transportation of these wash solutions is $\$ 29,300$ per year.

Aluminum fuel plates also receive a five cycle wash prior to being bonded to the fuel foil. Each piece of aluminum is 0.050 " thick and the widest piece is 3.6" thick. Since the fuel wash basins hold 10 foils, the aluminum wash basins are designed to hold 20 pieces. Each wash basin is sized to be 16" x 56" x 14" for a total of 54 gallons. With the recirculation line, pump, and filter, the actual volume of liquid is assumed to be 60 gallons. The general assumption of a full change out every 7 days is used for the aluminum washes also. Therefore, in 90 days, there are 780 gallons of wash produced. This equates to 3120 gallons per year for each of the three wash stations (sodium hydroxide, nitric acid, and DI water). Since the aluminum has not come into contact with the fuel foil, the wash solutions are not anticipated to be contaminated with radioactive elements. Therefore, the three DI water washes can be sent to the DI water system where a water softener removes any metallic contamination and a DI generator removes any ions within the water. If the water is not recyclable, the non-suspect contaminated water can be sent to the firewater collection tank for sampling and removal to a water treatment facility. However, since the other two aluminum washes are with hazardous chemicals, they must be packaged and sent to treatment. Using the assumption that liquid will occupy $75 \%$ of a 55 -gal drum during packaging, each of the two washes will require 76 drums for a total of 152 drums per year. The cost for each drum is $\$ 500$ and transportation of a load (assume 10 drums) is $\$ 30-\$ 40$. The cost for treatment of the 
drums equates to $\$ 76,000$ with an additional $\$ 640$ for transportation costs. Therefore, the aluminum wash liquid wastes will cost approximately $\$ 76,650$ per year.

After the fuel plates are bonded together, there are multiple washes that occur; however, most of these washes are with DI water and soapy water. Since these washes have no interaction with radioactive fuel, these non-hazardous washes can be sent to the firewater collection system and sampled for safety. They will then go down the normal water drains and be treated in a water treatment facility, most likely on the DOE site where the FFC facility is located. There is one additional nitric acid wash that occurs for the fuel plates prior to final assembly. This wash basin will be very similar in size to the aluminum wash basins. Therefore, it is assumed to be 60 gallons worth of material that is replaced every 7 days. This equates to 3120 gallons per year as discussed above. This will be non-radioactive, hazardous waste. It will require 76 drums for packaging at a cost of $\$ 500$ per drum and 8 shipments at $\$ 40$ per shipment for transportation costs. This equates to an additional $\$ 38,320$ in transportation and treatment costs for fuel plate cleaning.

Table 3-4 provides a summary of the liquid waste generation and costs associated with treating the wastes.

TABLE 3-4: LIQUID WASTE SUMMARY

\begin{tabular}{|c|c|c|c|c|c|}
\hline Source & $\begin{array}{l}\text { Yearly } \\
\text { Generation }\end{array}$ & $\begin{array}{l}\text { Packaging } \\
\text { Requirement }\end{array}$ & $\begin{array}{l}\text { Treatment } \\
\text { Cost }\end{array}$ & $\begin{array}{l}\text { Transportation } \\
\text { Cost }\end{array}$ & Total Cost \\
\hline $\begin{array}{l}\text { Receipt Fuel } \\
\text { Wash }\end{array}$ & 240 gallons & 6 drums & $\begin{array}{l}\$ 600 / \text { drums }= \\
\$ 3600\end{array}$ & $\$ 100$ & $\$ 3700$ \\
\hline $\begin{array}{l}\text { LEU/Mo wash } \\
\left(\mathrm{HNO}_{3}\right)\end{array}$ & 240 gallons & 6 drums & $\begin{array}{l}\$ 600 / \text { drum }= \\
\$ 3600\end{array}$ & $\$ 100$ & $\$ 3700$ \\
\hline $\begin{array}{l}\mathrm{LEU} / \mathrm{Mo} / \mathrm{Zr} \\
\text { wash } \\
\left(\mathrm{HNO}_{3} / \mathrm{HF}\right)\end{array}$ & 480 gallons & 12 drums & $\begin{array}{l}\$ 600 / \text { drum }= \\
\$ 7200\end{array}$ & $\$ 200$ & $\$ 7400$ \\
\hline $\begin{array}{l}\mathrm{LEU} / \mathrm{Mo} / \mathrm{Zr} \\
\text { rinse (DI } \\
\text { water) }\end{array}$ & 480 gallons & 12 drums & $\begin{array}{l}\$ 600 / \text { drum }= \\
\$ 7200\end{array}$ & $\$ 200$ & $\$ 7400$ \\
\hline $\begin{array}{l}\mathrm{LEU} / \mathrm{Mo} / \mathrm{Zr} \\
\text { wash (ethanol) }\end{array}$ & 480 gallons & 12 drums & $\begin{array}{l}\$ 600 / \text { drums }= \\
\$ 7200\end{array}$ & $\$ 200$ & $\$ 7400$ \\
\hline $\begin{array}{l}\text { Al wash } \\
(\mathrm{NaOH})\end{array}$ & 3120 gallons & 76 drums & $\begin{array}{l}\$ 500 / \text { drum }= \\
\$ 38,000\end{array}$ & $\$ 320$ & $\$ 38,320$ \\
\hline $\begin{array}{l}\text { Al wash } \\
\left(\mathrm{HNO}_{3}\right)\end{array}$ & 3120 gallons & 76 drums & $\begin{array}{l}\$ 500 / \text { drum }= \\
\$ 38,000\end{array}$ & $\$ 320$ & $\$ 38,320$ \\
\hline $\begin{array}{l}\text { Final plate } \\
\text { wash }\left(\mathrm{HNO}_{3}\right)\end{array}$ & 3120 gallons & 76 drums & $\begin{array}{l}\$ 500 / \text { drum }= \\
\$ 38,000\end{array}$ & $\$ 320$ & $\$ 38,320$ \\
\hline & & & & $\begin{array}{l}\text { Total Yearly } \\
\text { Cost }\end{array}$ & $\$ 144,560$ \\
\hline
\end{tabular}

An annual cost range of $\$ 150 \mathrm{~K}-\$ 500 \mathrm{~K}$ for the treatment, transportation and disposal of liquid waste. 


\subsubsection{Solid Waste}

Solid waste will be generated in the FFC facility in many forms. Solid waste will come from personal protection equipment (PPE), fuel plate wipes for cleaning, spent equipment, process scrap, as well as many other forms. Any spent equipment that can be refurbished such as rollers or dull shear blades will be shipped off-site after decontamination for refurbishment at the supplier or approved vendor.

A large volume source of solid waste will be spent filters. There are nearly 30 local ventilation filters that will require periodic change-out of pre-filters, HEPA filters, and/or demister filters. There are also 3 sets of 4 x 5 HEPA filter banks for process and building ventilation. This equates to 180 filters in the HEPA banks. The pre-filters will be changed out once a year while the HEPA filters can be changed out every five years. The secondary HEPA filter should never require changing as it should only receive small doses of radioactivity during breakthrough of the first HEPA filter. The local ventilation on the hoods should capture most of the radioactive contamination; therefore, the HEPA filter banks should receive little contamination. One other filter is the sintered metal filter on the casting exhaust. This filter will collect the uranium oxide that forms from the dross during casting. These filters will be changed out once a year. There is currently no facility for treatment of filters in FFC; therefore, the filters will be bagged out of the operation and placed in a waste container. Filters could potentially be compacted in CM-001, the compactor, or sheared to smaller volumes in the waste area.

One of the largest volumes of solid waste generated will be the rolling cans from the zirconium cladding process. The dimensions of the can are 6" $\mathrm{x} 7$ " $\mathrm{x} 0.2$ " for a total volume of $8.4 \mathrm{in}^{3}$. Each plate requires a separate can for rolling, so the maximum production will be 17,000 cans (and plates) per year. This equates to a yearly consumption of $142,800 \mathrm{in}^{3} /$ year. The density of steel is $4.9 \mathrm{~g} / \mathrm{cm}^{3}$; therefore, the total weight of can waste is approximately $11,500 \mathrm{~kg}$ per year. This waste will be LLW since it has direct contact with the low enriched uranium fuel plate. This waste can be compacted and placed in a cargo container and sent to NTS, for example, for disposal. Each cargo container holds $1,280 \mathrm{ft}^{3}$ at a cost of $\$ 5000$. The cargo container will hold 17455 -gal drums with a recommended weight limit in each drum of 500 pounds. 11,500 $\mathrm{kg}$ is equivalent to 25,300 pounds, which will require 5155 -gal drums for disposal. This corresponds to nearly $30 \%$ of the cargo containers, which has a dedicated cost of approximately $\$ 1500$.

\subsubsection{Process Off-Gas Treatment}

Process Off-gas is collected throughout the facility using localized ventilation hoods. A hood is placed wherever a process is used that may create acid or basic fumes that are not recommended for personnel inhalation or fines that may contain radioactive particles. Examples of these locations are the shear machines that cut off thin strips of fuel plate and nitric acid washes that would release nitric acid fumes whenever a batch is inserted or removed from the wash area. The assumed flowrate based on common HVAC best practices for hood ventilation is $150 \mathrm{ft} / \mathrm{s}$ at a distance of 3 inches from the face of the hood. Section 5.5 explains that this equates to a face velocity at the hood inlet of $250 \mathrm{ft} / \mathrm{s}$. This will allow for dust particles in the processing areas to be collected by the ventilation hoods and not disperse into the occupied space. Each ventilation hood has a localized HEPA filter for dust suppression. The filtered off-gas along with purge gas from the argon purification system and any other gas supplies will be combined with the building ventilation gas prior to entering the final HEPA banks. SK-7 in the PFDs shows the flow for the off-gas treatment. Three trains of 4 x 5 HEPA filter banks (FIL-020, FIL-021, and 
FIL-023) filter the off-gas before exiting through the stack (STK-001). Three blowers (one for each train) (BL-001, BL-002, and BL-003) overcome any pressure differentials due to filtering as well as maintain a negative pressure relative to atmosphere in the shop. The blowers also allow for excess flow capacity so that any leaks in the ventilation system will force air into the ventilation filtering rather than allow radioactive elements to escape prior to capture. The HEPA filter banks consist of three levels of protection. A pre-filter collects most large particles within the off-gas stream. The second level of filter protection is HEPA filters that collect any radioactive isotopes or dust particles. Continuous monitoring of the off-gas downstream of the HEPA warn of break-through within the HEPA filter, which is captured by the third layer of filtering- a second HEPA filter. If a break-through is detected, the train is shut down until the filter can be replaced. Fire protection exists before each layer of filtering to protect the filters against off-normal process upsets.

\subsubsection{Scrap Management}

Scrap management will be a key feature in the FFC facility due to the large volume of plate production. Any trim scrap of the LEU/Mo fuel that is produced before the $\mathrm{Zr}$ cladding is added to the fuel can be mixed with the incoming fuel pieces for casting. After the $\mathrm{Zr}$ cladding is added to the LEU/Mo plate, the trim pieces are no longer recyclable within the facility and must be sent back to Y-12 for processing or disposed as LLW. Future design efforts could focus on scrap minimization techniques perhaps using optimization and automated cutting lathe or larger castings for multiple fuel pieces per roll. It may also be possible for another facility to remove the $\mathrm{Zr}$ from the LEU/Mo and ship the LEU/Mo back to Y-12 for incorporation into more fuel pieces; however, this is subject to further study and not part of this scope. Any scrap produced from the shear processes must be collected and weighed regularly for accountability purposes. Since this material is special nuclear material (SNM) and 20\% enriched Uranium, it must be tracked to the 1 gram level $+/-0.5$ grams. Therefore, it is imperative for accountancy measures to collect the scrap and weigh the material.

Estimations of the waste generated from scrap removal have been calculated. For the shear that occurs after the LEU/Mo is coated with $\mathrm{Zr}$, the plate has been rolled to a thickness of 0.015 " and is an average of 25 " long and 2.8 " wide with 0.15 " of trim on each long edge and 1" of trim on each short edge. Assuming the maximum of 17,000 plates per year, the scrap produced from this shear is 0.015 " $\mathrm{x}(0.15$ " $\mathrm{x} 25$ " $\mathrm{x} 2+1.0$ " $\mathrm{x}$ $2.8 ” \times 2)=0.1965 \mathrm{in}^{3}$ per plate. This equates to a yearly scrap rate of around $3340 \mathrm{in}^{3} / \mathrm{yr}$ or $54,740 \mathrm{~cm}^{3} / \mathrm{yr}$. Assuming the uranium density of the alloy is $15 \mathrm{~g} / \mathrm{cm}^{3}$, the weight of this waste is $54,740 \mathrm{~cm}^{3} / \mathrm{yr} \times 15 \mathrm{~g} / \mathrm{cm}^{3} \times 1 \mathrm{~kg} / 1000 \mathrm{~g}=821 \mathrm{~kg}$ per year of scrap waste. Information from Idaho National Laboratory shows that storage at NTS can be achieved at no current cost. However, there are transportation costs of approximately $\$ 4000$ $\$ 5000$ per cargo container. Each cargo container is capable of holding 174 55-gal drums with each drum holding 500 pounds. Converting the $821 \mathrm{~kg}$ to pounds results in a yearly waste generation of 1810 pounds. This will require 4 ea 55-gal drums for packaging and equate to only $3 \%$ of the space in a cargo container. The transportation cost directly related to shipping this waste to LLW storage is approximately $\$ 115$ per year.

The 500 pounds per 55-gal drum assumes that the scrap will be combined with other LLW and sent to a shallow burial facility. If the material is to be re-cycled, the scrap cannot be mixed with other LLW, but must remain with the other scrap material. This would require packaging to accommodate criticality concerns. Therefore, each ES-3100 drum would hold three cans with a maximum of $11 \mathrm{~kg}$ of scrap material per can, which is the shipping requirement for receipt to FFC. This would allow each ES-3100 to carry a 
maximum of $33 \mathrm{~kg}$ or approximately 72 pounds. Using the above example of the $\mathrm{LEU} / \mathrm{Mo} / \mathrm{Zr}$ scrap, the shipping containers would increase from 455 -gal drums to 12 ES3100 shipping containers. Each ES-3100 container also costs $\$ 3000$ to refurbish the container as well as replace the seals and leak check the container. Using the ES-3100 to recycle the LEU/Mo scrap material will add significant costs to the operation of the facility, but could potentially lessen the amount of material required to be processed. A future study should be done to determine the disposal pathway for scrap material.

The waste from the shear after the Al cladding is bonded to the fuel plate is also suspected LLW from contamination with the fuel foil. The assumption made for waste estimation is that an additional 3-4" of aluminum is added on each side for the brackets to hold down the fuel plate during friction stir welding. The thickness of the plate is 0.05 " with the widest plate being about 3.5 " wide and 50" long. For conservative estimation purposes, the maximum production of 17,000 fuel plates is assumed. Therefore, the scrap removed from this shear operation is calculated as follows: 0.05 " x (4" x 50" x $2+$ $4 " x 3.5 "$ x 2) $=21.4 \mathrm{in}^{3}$ per plate. $21.4 \mathrm{in}^{3} \times 17,000=363,800 \mathrm{in}^{3} / \mathrm{yr}$ or $5,961,614$ $\mathrm{cm}^{3} / \mathrm{yr}$. The density of this alloy cladding material is nearly identical to aluminum at 2.6 $\mathrm{g} / \mathrm{cm}^{3}$. Therefore, the yearly weight of waste produced from this scrap source is $5,961,614 \mathrm{~cm}^{3} / \mathrm{yr} \times 2.6 \mathrm{~g} / \mathrm{cm}^{3} \times 1 \mathrm{~kg} / 1000 \mathrm{~g}=15,500 \mathrm{~kg}$. Each 55 -gal drum can hold 500 pounds, so this waste will require 31 drums for packaging. The cargo container, which can hold 174 drums based off of the $1280 \mathrm{ft}^{3}$ information, will be nearly $20 \%$ occupied from this waste stream. Since each cargo container costs $\$ 5000$ to ship, this waste corresponds to $\$ 1000$ per year in transportation costs to LLW storage.

These two sources are the largest contributors to scrap management waste. Combined, these two sources of LLW contribute just over $20 \%$ of the capacity of a cargo container. This equates to a shipping cost of approximately $\$ 1000$. Fortunately, the disposal cost of burying the LLW at Nevada Test Site (NTS) is currently free, but if conditions were to change, this could increase the cost of disposing of this waste.

\subsection{Utilities and Consumables}

The fuel production processes in the FFC facility require process utilities and consumables in order to produce the sub-assemblies. The FFC facility has a large list of consumables within the process to create fuel plates and sub-assemblies. Some of the major consumables are carbon steel cans for rolling, crucibles, casting molds, and wash solutions including nitric acid, hydrofluoric acid, and sodium hydroxide. Other consumables within the facility include yttria coating, filters (HEPA, prefilters, demisters, and sintered metal). Still other consumables are included in the final product such as $\mathrm{Zr}$ coating, Al cladding, and non-fuel bearing components such as end pieces and sub-assembly hardware. Table 3-5 shows a list of the chemicals and consumables involved in the FFC processing facility. 


\section{TABLE 3-5 FFC PROCESSING CHEMICALS AND CONSUMABLES}
Component Use
Disposition
Consumption

S

\section{Solids}

\begin{tabular}{|c|c|c|c|}
\hline U10Mo & acorns, ingots, coupons, foil & fuel meat & $5288 \mathrm{~kg} / \mathrm{yr}$ \\
\hline U10Mo & foil trimmings & scrap for recycle & \\
\hline $\mathrm{Zr}$ & foil & diffusion barrier in fuel & 17,000 foils \\
\hline $\mathrm{Zr}$ & foil trimmings & solid or low-level waste & \\
\hline $\mathrm{Nb}$ & foil & diffusion barrier in fuel & 17,000 foils \\
\hline $\mathrm{Nb}$ & foil trimmings & solid or low-level waste & \\
\hline $\mathrm{Al}-6061$ & metal shapes & fuel clad & $\begin{array}{l}17,000 \text { plates } \\
\text { (x2 sides) }\end{array}$ \\
\hline $\mathrm{Al}-6061$ & milling scrap (cuttings \& dust) & solid waste & \\
\hline grafoil & flexible graphite sheeting & consumable & \\
\hline steel & $\begin{array}{l}\text { can for rolling Zr U10Mo } \\
\text { sandwich (consumable) }\end{array}$ & solid or low-level waste & $6660 \mathrm{~kg} /$ year \\
\hline yttria & $\begin{array}{l}\text { coating on inside of steel can } \\
\text { (consumable) }\end{array}$ & solid or low-level waste & \\
\hline $\mathrm{W}$ & $\begin{array}{l}\text { welding electrodes } \\
\text { (consumable) }\end{array}$ & solid or low-level waste & \\
\hline W & $\begin{array}{l}\text { friction bonding tool } \\
\text { (consumable) }\end{array}$ & solid or low-level waste & \\
\hline $\begin{array}{l}\text { paper, } \\
\text { plastic }\end{array}$ & cleaning supplies (consumable) & solid or low-level waste & \\
\hline graphite & crucible (consumable) & solid or low-level waste & \\
\hline yttria & $\begin{array}{l}\text { coating on graphite crucible } \\
\text { (consumable) }\end{array}$ & solid or low-level waste & \\
\hline $\mathrm{KCl}$ & salt for heating U10Mo & process feed & $67 \mathrm{lbs} / \mathrm{yr}$ \\
\hline $\mathrm{KCl}$ & contaminated/spent salt & solid or low-level waste & $67 \mathrm{lbs} / \mathrm{yr}$ \\
\hline $\mathrm{LiCl}$ & salt for heating U10Mo & process feed & 67 lbs/yr \\
\hline $\mathrm{LiCl}$ & contaminated/spent salt & solid or low-level waste & $67 \mathrm{lbs} / \mathrm{yr}$ \\
\hline slag & from melter & solid or low-level waste & \\
\hline steel & convenience cans & solid or low-level waste & \\
\hline $\begin{array}{l}\text { steel (HR- } \\
160)\end{array}$ & Steel molds for casting & reusable & \\
\hline
\end{tabular}




\begin{tabular}{|c|c|c|c|c|}
\hline & $\begin{array}{l}\text { Component } \\
\text { s }\end{array}$ & Use & Disposition & Consumption \\
\hline & & Off-gas absorbers (if required) & solid or low-level waste & \\
\hline & & HEPA filters & solid or low-level waste & \\
\hline & & Job control wastes & solid or low-level waste & \\
\hline & & Spent equipment & solid or low-level waste & \\
\hline \multicolumn{5}{|c|}{ Liquids } \\
\hline & $\mathrm{HNO}_{3}$ & $\begin{array}{l}(30 \%) \text { cleaning UMo alloy, } \\
\text { cleaning Zr sandwich, cleaning } \\
\text { Al } 6061\end{array}$ & $\begin{array}{l}\text { spent solution to } \\
\text { stabilization, off gas }\end{array}$ & $\begin{array}{l}6700 \text { gallons at } \\
30 \%, 240 \text { gallons } \\
\text { mixed with HF }\end{array}$ \\
\hline & $\mathrm{HF}$ & $\begin{array}{l}\text { Hydrofluoric acid cleaning } \\
\text { solution }\end{array}$ & $\begin{array}{l}\text { spent solution to } \\
\text { stabilization, off gas }\end{array}$ & $\begin{array}{l}24 \text { gallons mixed } \\
\text { with HNO3/DI }\end{array}$ \\
\hline & $\mathrm{NaOH}$ & (75 g/lit) cleaning Al 6061 & $\begin{array}{l}\text { spent solution to } \\
\text { stabilization, off gas }\end{array}$ & $\begin{array}{l}3120 \text { gallons per } \\
\text { year }\end{array}$ \\
\hline & $\begin{array}{l}\mathrm{H}_{2} \mathrm{O}, \mathrm{DI} \\
\mathrm{H}_{2} \mathrm{O}\end{array}$ & $\begin{array}{l}\text { cooling, rinsing acid cleaning } \\
\text { solutions }\end{array}$ & $\begin{array}{l}\text { spent solution to } \\
\text { stabilization, off gas }\end{array}$ & $\begin{array}{l}20,000 \text { gallons } \\
\text { per year in } \\
\text { process plus } \\
\text { cleaning/ cooling }\end{array}$ \\
\hline & ethanol & wiping cleaned foil assemblies & $\begin{array}{l}\text { spent solution to } \\
\text { stabilization, off gas }\end{array}$ & 480 gallons/ yr \\
\hline & \multirow[t]{5}{*}{$\mathrm{KCl}$} & $\begin{array}{l}\text { molten salt bath (liquid \& } \\
\text { vapors) }\end{array}$ & off-gas treatment & \\
\hline & & janitorial supplies & solid or low-level waste & \\
\hline & & hydraulic fluids & spent liquid & \\
\hline & & $\begin{array}{l}\text { off-gas scrubber chemicals } \\
\text { (if required) }\end{array}$ & $\begin{array}{l}\text { spent solution to } \\
\text { stabilization }\end{array}$ & \\
\hline & & vacuum pump oil & spent oil & \\
\hline \multicolumn{5}{|l|}{ Gases } \\
\hline & Ar & inerting, inert gas welding & & \\
\hline & $\mathrm{He}$ & $\begin{array}{l}\text { leak testing of shipping } \\
\text { containers }\end{array}$ & & \\
\hline & $\begin{array}{l}\text { process off } \\
\text { gas }\end{array}$ & off-gas treatment & & \\
\hline & $\begin{array}{l}\text { furnace off } \\
\text { gas }\end{array}$ & off-gas treatment & & \\
\hline & dry air & Final assembly drying & off-gas treatment & Lab tanks $<20 / y r$ \\
\hline
\end{tabular}


Some utilities will be provided from the adjacent federal site such as electrical power and fire water. Even though electrical power is provided close to the site, the FFC facility will include a sub-station that will reduce the high-voltage to the $480 \mathrm{~V}, 3$ phase AC electricity outputs appropriate for the fabrication machines. Other utilities such as process chilled water, DI water, argon, dry air, and vacuum will be provided within the FFC facility. The liquid utilities will be recirculated and cleaned within the facility with a purge to the sewer drain or waste packaging as appropriate. Argon will be used in the casting furnace for inert atmosphere control as well as cooling of the cast ingots. Argon gas will be recirculated throughout the glovebox and cleaned using a drier and filter. An argon purge will be used as necessary through the stack to ensure efficient and clean argon. Dry air will be used to dry the sub-assemblies after cleaning and will be collected by the ventilation system and sent out of the FFC stack. No collection or recycling will occur with the dry air.

\subsection{Project Equipment List}

The project equipment list for the FFC facility is included in Table 3-6. Included in the equipment list are all process equipment that are shown in the process flow diagrams as well as mechanical equipment such as cranes, tables, forklifts, etc. Where applicable, the equipment list shows the number of pieces of equipment, the equipment tag number, the sketch that the equipment is found on as well as important notes in determining the size and cost of the equipment. For example, the first piece of equipment in the process equipment list (see Table 36) is CV-001 A/B, the Mold Feed Conveyor. The Area Code refers to the part of the process that this equipment supports and is shown in Table 3-7. Area 1 is the casting of the fuel pieces. The sketch is SK-2 showing that the conveyor is pictured on the PFD SK-2. The A/B designation shows that there are two pieces of equipment that serve the same function. This is also shown in the quantity column. The name is shown along with the description column, which is used to relay any notes that may be helpful in sizing or pricing the equipment. This equipment list was used to estimate the construction cost of the FFC for the majority of the processing and storage areas. Also included is a count on office space, which was used to estimate cost for office furniture, conference room tables, and other functional areas.

\section{TABLE 3-6 FFC EQUIPMENT LIST}

\begin{tabular}{|c|l|l|l|l|c|}
\hline $\begin{array}{c}\text { Area } \\
\text { Code }\end{array}$ & \multicolumn{1}{|c|}{ Sketch } & \multicolumn{1}{|c|}{$\begin{array}{c}\text { Equipment } \\
\text { ID }\end{array}$} & \multicolumn{1}{c|}{ Equipment Name } & \multicolumn{1}{c|}{ Description } & Quantity \\
\hline 1 & SK-2 & CV-001 A/B & Mold Feed Conveyor & Conveyor for casting mold & 2 \\
\hline 1 & SK-2 & GB-001 A/B & Argon Inerted Glovebox & & 2 \\
\hline 1 & SK-2 & HT-001 A/B & Casting Furnace & & 2 \\
\hline 1 & SK-2 & CT-001 & Coating Station & & \\
\hline 1 & SK-2 & X-001 & Recycle Station & & \\
\hline 1 & SK-2 & HT-003 A/B & Mold Heater & & \\
\hline 1 & SK-2 & FIL-009 & Prefilter \#9 & & \\
\hline 1 & SK-2 & FIL-017 & Prefilter \#13 & & \\
\hline 1 & SK-2 & FIL-027 & Sintered Metal Filter & & \\
\hline 1 & SK-2 & WS-015 & Incoming Fuel Wash Station & $\mathrm{HNO}_{3}$ & \\
\hline
\end{tabular}




\begin{tabular}{|c|c|c|c|c|c|}
\hline $\begin{array}{l}\text { Area } \\
\text { Code }\end{array}$ & Sketch & $\begin{array}{l}\text { Equipment } \\
\text { ID }\end{array}$ & Equipment Name & Description & Quantity \\
\hline 1 & SK-2 & FIL-028 & Demister \#6 & & \\
\hline 2 & SK-2 & HТ-002 & Molten Salt Bath & & \\
\hline 2 & SK-2 & RL-001 & Roll Mill & & \\
\hline 2 & SK-2 & FIL-001 & Prefilter \#1 & & \\
\hline 2 & SK-2 & FIL-002 & Prefilter \#2 & & \\
\hline 3 & SK-2 & FM-001 & Salt Removal Finishing Mill & & \\
\hline 3 & SK-2 & WS-016 & Ethanol/Water Wash Station & & \\
\hline 3 & SK-2 & FIL-007 & Prefilter \#7 & & \\
\hline 3 & SK-2 & SR-001 & Shear Machine & $0.1 "$ thick U-Mo & \\
\hline 3 & SK-2 & FIL-003 & Prefilter \#3 & & \\
\hline 3 & SK-2 & FIL-018 & Prefilter \#14 & & \\
\hline 3 & SK-3 & WS-001 & $\begin{array}{l}\mathrm{HNO}_{3} \text { Wash Station for U/Mo } \\
\text { Fuel }\end{array}$ & & \\
\hline 3 & SK-3 & FIL-010 & Demister \#1 & & \\
\hline 4 & SK-3 & CS-001 & Canning Station & $\begin{array}{l}\text { Welder, can motion, } \\
\text { interlock }\end{array}$ & \\
\hline 4 & SK-3 & P-001 & Clad Vacuum Pump & Purge cans with inert gas & \\
\hline 4 & SK-3 & FIL-011 & Prefilter \#10 & & \\
\hline 4 & SK-3 & FIL-025 & Prefilter \#17 & & \\
\hline 5 & SK-3 & HТ-004 & Tunnel Furnace & & \\
\hline 5 & SK-3 & RL-002 & Heated Zr Clad Roller & & \\
\hline 5 & SK-3 & FIL-016 & Prefilter \#12 & & \\
\hline 5 & SK-3 & FIL-004 & Prefilter \#4 & & \\
\hline 6 & SK-3 & SR-002 & Can Removal Shear & & \\
\hline 6 & SK-3 & HТ-005 & Annealing Furnace & Air Atm & \\
\hline 6 & SK-3 & FIL-005 & Prefilter \#5 & & \\
\hline 6 & SK-3 & FIL-026 & Prefilter \#18 & & \\
\hline 7 & SK-3 & WS-002 & $\begin{array}{l}\mathrm{HNO}_{3} \text { Wash Station for } \\
\mathrm{U} / \mathrm{Mo} / \mathrm{Zr}\end{array}$ & & \\
\hline 7 & SK-3 & WS-003 & DI Water Rinse for $\mathrm{U} / \mathrm{Mo} / \mathrm{Zr}$ & & \\
\hline 7 & SK-3 & WS-004 & Ethanol Hand Wipe Station & & \\
\hline 7 & SK-3 & FIL-012 & Demister \#2 & & \\
\hline 7 & SK-3 & FIL-013 & Prefilter \#11 & & \\
\hline
\end{tabular}




\begin{tabular}{|c|c|c|c|c|c|}
\hline $\begin{array}{l}\text { Area } \\
\text { Code }\end{array}$ & Sketch & $\begin{array}{l}\text { Equipment } \\
\text { ID }\end{array}$ & Equipment Name & Description & Quantity \\
\hline 7 & SK-4 & WS-005 & Heated $\mathrm{NaOH}$ Wash Station & & \\
\hline 7 & SK-4 & WS-017 & DI Water Rinse for $\mathrm{NaOH}$ & & \\
\hline 7 & SK-4 & WS-006 & $\mathrm{HNO}_{3}$ Wash Station for Al Clad & & \\
\hline 7 & $\mathrm{SK}-4$ & WS-007 & $\begin{array}{l}\text { DI Water Wash Station for Al } \\
\text { Clad }\end{array}$ & & \\
\hline 7 & SK-4 & WS-018 & Heater DU Rinse for Al Clad & & \\
\hline 7 & $\mathrm{SK}-4$ & FIL-014 & Demister \#3 & & \\
\hline 7 & SK-4 & FIL-015 & Demister \#4 & & \\
\hline 8 & SK-4 & $\begin{array}{l}\text { FSW-001 A- } \\
\text { D }\end{array}$ & Friction Stir Welders & & 4 \\
\hline 3 & $\mathrm{SK}-4$ & SR-003 & Rough Shear & Foil rough shear & \\
\hline 8 & SK-4 & RL-003 & Final Flatten/ Level Mill & Plate flatness & \\
\hline 8 & $\mathrm{SK}-4$ & FIL-006 A-D & Prefilter \#6 A-D & & 4 \\
\hline 8 & SK-4 & FIL-024 & Prefilter \#16 & & \\
\hline 9 & SK-5 & MK-001 & Marking Station & & \\
\hline 9 & $\mathrm{SK}-5$ & FS-001 & Fluoroscopy Analyzer & & \\
\hline 9 & SK-5 & FM-002 A-E & Final Assembly Finishing Mill & $\begin{array}{l}\text { one mill dedicated to } \\
\text { cutting grooves }\end{array}$ & 5 \\
\hline 9 & SK-5 & WS-008 & Soapy water bath for plates & & \\
\hline 9 & SK-5 & WS-009 & Heated DI rinse for plates & & \\
\hline 11 & $\mathrm{SK}-5$ & WS-010 & $\mathrm{HNO}_{3}$ Wash Station for plates & & \\
\hline 11 & $\mathrm{SK}-5$ & WS-011 & DI rinse for plates & & \\
\hline 11 & $\mathrm{SK}-5$ & WS-012 & Heater DI rinse for plates & & \\
\hline 11 & SK-5 & FIL-029 & Demister \#5 & & \\
\hline 10 & SK-6 & OM-001 & Optical Microscopy & etching, polishing, saw & \\
\hline 10 & SK-6 & PT-001 & Pull Tester & & \\
\hline 10 & SK-6 & $\mathrm{UT}-001 \mathrm{~A} / \mathrm{B}$ & Ultrasonic Tester & & 2 \\
\hline 10 & SK-6 & $\mathrm{RD}-001 \mathrm{~A} / \mathrm{B}$ & Radiography Analyzer & & 2 \\
\hline 10 & SK-6 & ICP-001 & ICP AES & & \\
\hline 10 & SK-6 & W-001 & Scale (balance) & & \\
\hline 10 & SK-6 & FIL-019 & Prefilter \#15 & & \\
\hline 10 & SK-6 & GS-001 & gamma scan - plates & & \\
\hline 10 & SK-6 & GS-002 & gamma scan - fuel subassembly & & \\
\hline
\end{tabular}




\begin{tabular}{|c|c|c|c|c|c|}
\hline $\begin{array}{l}\text { Area } \\
\text { Code }\end{array}$ & Sketch & $\begin{array}{l}\text { Equipment } \\
\text { ID }\end{array}$ & Equipment Name & Description & Quantity \\
\hline Exhaust & SK-7 & FIL-020 & HEPA Bank \#1 & $\begin{array}{l}4 \text { X 5, } 3 \text { banks ( } 2 \text { X2 } \\
\text { HEPA) }\end{array}$ & \\
\hline Exhaust & SK-7 & FIL-021 & HEPA Bank \#2 & & \\
\hline Exhaust & SK-7 & FIL-023 & HEPA Bank \#3 & & \\
\hline Exhaust & SK-7 & BL-001 & Off-gas Blower \#1 & $150 \mathrm{HP}$ & \\
\hline Exhaust & SK-7 & BL-002 & Off-gas Blower \#2 & $150 \mathrm{HP}$ & \\
\hline Exhaust & SK-7 & BL-003 & Off-gas Blower \#3 & $150 \mathrm{HP}$ & \\
\hline Exhaust & SK-7 & STK-001 & Stack & $\begin{array}{l}70^{\prime \prime} \text { dia, } 25^{\prime} \text { above building } \\
\text { roof }\end{array}$ & \\
\hline & SK-8 & CM-001 & Compactor & & \\
\hline & SK-8 & CS-003 & Liquid Bottling Station & & \\
\hline & SK-8 & CS-002 & Canning Station for shipping & & \\
\hline & SK-8 & PK-002 & Waste Packaging Station & & \\
\hline 11 & SK-9 & $\mathrm{CRN}-001$ & Overhead Crane & $\begin{array}{l}\text { (2) } 5 \text { Ton (shop); (2) } 7 \text { 1/2 } \\
\text { Ton (Assembly) }\end{array}$ & 4 \\
\hline 11 & SK-9 & ST-001 & Secure Storage Room & & \\
\hline 11 & SK-9 & PK-001 & Final Assembly Packaging & & \\
\hline 8 & SK-10 & HIP-001 A/B & Hot Isotatic Press & Alternate to HIP & 2 \\
\hline 1 & SK-11 & DRY-001 & Ar Drier & & \\
\hline 1 & SK-11 & HT-007 & Ar Heat Exchanger & argon cooler & \\
\hline 1 & SK-11 & FIL-024 A/B & Ar Purification Filter A/B & $\begin{array}{l}\text { recirculation fan, bleed and } \\
\text { feed }\end{array}$ & 2 \\
\hline 1 & SK-11 & WT-001 & DI Water Treatment Unit & & \\
\hline 1 & SK-11 & TK-001 & DI Storage Tank & & \\
\hline 1 & SK-11 & WSF-001 & Water Softener & $\begin{array}{l}\text { prepares water for further } \\
\text { purification }\end{array}$ & \\
\hline 11 & SK-12 & AF-001/-005 & Assembly Fixture & $\begin{array}{l}\text { Includes holding parts in } \\
\text { alignment, final assembly, } \\
\text { and swaging }\end{array}$ & 5 \\
\hline 11 & SK-12 & $\begin{array}{l}\text { WDS-001/- } \\
003\end{array}$ & $\begin{array}{l}\text { Welding Station and Final } \\
\text { Assembly }\end{array}$ & & 3 \\
\hline 11 & SK-12 & MS-001/-003 & Machine Station & & 3 \\
\hline 11 & SK-12 & WS-013 & Element DI Wash & & \\
\hline 11 & SK-12 & WS-014 & Hot Element DI Wash & & \\
\hline
\end{tabular}




\begin{tabular}{|c|c|c|c|c|c|}
\hline $\begin{array}{l}\text { Area } \\
\text { Code }\end{array}$ & Sketch & $\begin{array}{l}\text { Equipment } \\
\text { ID }\end{array}$ & Equipment Name & Description & Quantity \\
\hline 11 & SK-12 & DRY-002 & Element Dryer & & \\
\hline 11 & SK-12 & HТ-006 & Autoclave & & \\
\hline 11 & SK-12 & FIL-022 & Prefilter \#14 & & \\
\hline 11 & & & Channel Probe 1 & & \\
\hline 11 & & & Channel Probe 2 & & \\
\hline 11 & & & Inspection (tables) & & \\
\hline \multirow[t]{5}{*}{11} & & & Inspection (tables) & & \\
\hline & & & Steam Boiler & & 1 \\
\hline & & & Water Chiller & w/ Air cooled condenser & 1 \\
\hline & & & Supply Air Handling Fans & & 2 \\
\hline & & & Supply Filters \& Conditioning & $\begin{array}{l}\text { Pre Filter } 30 \% \text { \& Bag } \\
\text { Filter, steam coil, chilled } \\
\text { water, dehumidification, } \\
\text { drains, radiant heater on } \\
\text { inlet louvers to keep frost } \\
\text { off }\end{array}$ & \\
\hline 1 & & & Casting Molds (set) - Carbon & $\begin{array}{l}2 \text { ea. specific to each fuel } \\
\text { plate type } \& \text { Size }\end{array}$ & 32 \\
\hline 1 & & & Crucible & graphite 6" ID & 20 \\
\hline Bldg & & & Fire Suppression System & $\begin{array}{l}\text { Sprinkler (building) @ 10' } \\
\text { O.C. both ways, and low } \\
\text { flow BETE Type N nozzles } \\
\text { (for HEPAs) }\end{array}$ & \\
\hline Bldg & & & Automatic Fire Alarm System & $\begin{array}{l}\text { FACP, sensors, alarm bells } \\
\& \text { lights, connect to CAS }\end{array}$ & \\
\hline Bldg & & & Emergency Lighting & & \\
\hline \multirow[t]{3}{*}{ Bldg } & & & Signage & $\begin{array}{l}\text { Warehouse, FFC, Guard } \\
\text { shack and Site }\end{array}$ & \\
\hline & & & Public Address System & & \\
\hline & & & $\begin{array}{l}\text { Personnel Accountability } \\
\text { System }\end{array}$ & $\begin{array}{l}\text { ensures all personnel are } \\
\text { accounted for in an } \\
\text { emergency }\end{array}$ & \\
\hline \multirow[t]{3}{*}{ Bldg } & & & Personnel elevator & $\begin{array}{l}\text { Hydraulic two story, with } \\
\text { pit }\end{array}$ & 1 \\
\hline & & & Truck Bay Rollup Doors & $7^{\prime} \times 15^{\prime}$ & 3 \\
\hline & & & Warehouse rollup door & $7^{\prime} \times 15^{\prime}$ & \\
\hline
\end{tabular}




\begin{tabular}{|c|c|c|c|c|c|}
\hline $\begin{array}{l}\text { Area } \\
\text { Code }\end{array}$ & Sketch & $\begin{array}{l}\text { Equipment } \\
\text { ID }\end{array}$ & Equipment Name & Description & Quantity \\
\hline & & & Forklifts - electrically powered & 2 Ton & 4 \\
\hline & & & Forklift Charging Station & 4 station & 4 \\
\hline & & & Forklifts - electrically powered & Warehouse & \\
\hline & & & Forklift Charging Station & Warehouse & \\
\hline \multirow[t]{15}{*}{ Bldg } & & & UPS System & $\begin{array}{l}\text { Battery backup for Crit } \\
\text { alarms and CAMs }\end{array}$ & \\
\hline & & & Network Server & & \\
\hline & & & Material Accountability Server & & \\
\hline & & & $\begin{array}{l}\text { Material Accountability } \\
\text { Stations }\end{array}$ & & \\
\hline & & & Warehouse Inventory Tracking & $\begin{array}{l}\text { Computer, Bar code, } \\
\text { software }\end{array}$ & \\
\hline & & & Analytical balances & & 12 \\
\hline & & & Dock scale & & \\
\hline & & & Hand \& Foot Monitors & Eberline & 5 \\
\hline & & & $\begin{array}{l}\text { Personnel Contamination } \\
\text { Monitors }\end{array}$ & Eberline & 3 \\
\hline & & & $\begin{array}{l}\text { Radiological Surveying } \\
\text { Equipment }\end{array}$ & & Lot \\
\hline & & & $\begin{array}{l}\text { Radiological Surveying } \\
\text { Equipment }\end{array}$ & Storage Area & \\
\hline & & & $\begin{array}{l}\text { Radiological Surveying } \\
\text { Equipment }\end{array}$ & Calibration Area & \\
\hline & & & $\begin{array}{l}\text { Radiological Surveying } \\
\text { Equipment }\end{array}$ & HIP & \\
\hline & & & Counting Equipment & Counter & \\
\hline & & & Office Ventilation System & $\begin{array}{l}\text { Recirculating, offices, } \\
\text { warehouse, inspection }\end{array}$ & \\
\hline 1 & & & Crucible Tilter & & 1 \\
\hline 1 & & & Slag \& Heel Removal & $\begin{array}{l}\text { Glovebox for mold and } \\
\text { crucible cleaning }\end{array}$ & \\
\hline \multirow[t]{3}{*}{1} & & & Waste Drum for Slag \& Heel & & \\
\hline & & & $\begin{array}{l}\text { Material Pass-through to } \\
\text { Storage }\end{array}$ & $\begin{array}{l}\text { Secured controlled storage } \\
\text { to Casting \& Shop with } \\
\text { scissor cart on tracks }\end{array}$ & 2 \\
\hline & & & Material Pass-through to HIP & & 1 \\
\hline
\end{tabular}




\begin{tabular}{|c|c|c|c|c|c|}
\hline $\begin{array}{l}\text { Area } \\
\text { Code }\end{array}$ & Sketch & $\begin{array}{l}\text { Equipment } \\
\text { ID }\end{array}$ & Equipment Name & Description & Quantity \\
\hline 1 & & & Casting Mold Entry Airlock & $\begin{array}{l}\text { for Argon inerted casting } \\
\text { g'box }\end{array}$ & 1 \\
\hline 1 & & & Casting Mold Exit Airlock & $\begin{array}{l}\text { for Argon inerted casting } \\
\text { g'box }\end{array}$ & 1 \\
\hline 1 & & & Glovebox for crucible cleaning & & 1 \\
\hline 1 & & & Jib Crane & $\begin{array}{l}\text { for crucible handling, } \\
\text { furnace bell jar }\end{array}$ & 2 \\
\hline 1 & & & Motion Table & for casting mold filling & 2 \\
\hline 1 & & & Mold Pre-Heater Power Supply & & \\
\hline 1 & & & RF Heating \& Cooling Coils & for induction furnace & 2 \\
\hline 1 & & & RF Generator & for induction furnace & 2 \\
\hline 1 & & & Vacuum System & for induction furnace & 2 \\
\hline \multirow[t]{2}{*}{1} & & & Argon Supply & $\begin{array}{l}\text { Dewar, auto pressurization } \\
\text { system, vaporizer, } \\
\text { regulator, fill, thief }\end{array}$ & 1 \\
\hline & & & Argon Supply & $\begin{array}{l}\text { Dewar, auto pressurization } \\
\text { system, vaporizer, } \\
\text { regulator, fill, thief }\end{array}$ & \\
\hline \multirow[t]{4}{*}{1} & & & Scissor Lift & Access to crucible loading & 1 \\
\hline & & & Scissor Lift & HVAC Room Filter change & 1 \\
\hline & & & Material Carts & & 20 \\
\hline & & & Specialty Rack for alloy storage & geometrically favorable & 20 \\
\hline 1 & & & Day Storage Casting Cabinet & & 20 \\
\hline \multirow[t]{2}{*}{1} & & & Heel Storage Cabinet & & 1 \\
\hline & & & included in AS-001 & Tooling rack for Press & 1 \\
\hline 11 & & & Hydraulic Press: 50 Ton & for coutouring plates & 1 \\
\hline 2 & & & Salt Bath Staging Area & & \\
\hline 2 & & & $\begin{array}{l}\text { Salt Bath Loading/Removal } \\
\text { Equipment }\end{array}$ & $\begin{array}{l}\text { Material handling system: } \\
\text { indexing, racks }\end{array}$ & 1 \\
\hline \multirow[t]{4}{*}{2} & & & $\begin{array}{l}\text { Salt Bath Heater Power Supply } \\
\& \text { Control }\end{array}$ & for HT-002 & 1 \\
\hline & & & Local Task Ventilation Ducts & & 25 \\
\hline & & & Hood, enclosure, with sash & & 5 \\
\hline & & & Plate Scrap Bin & & \\
\hline
\end{tabular}




\begin{tabular}{|c|c|c|c|c|c|}
\hline $\begin{array}{l}\text { Area } \\
\text { Code }\end{array}$ & Sketch & $\begin{array}{l}\text { Equipment } \\
\text { ID }\end{array}$ & Equipment Name & Description & Quantity \\
\hline & & & $\begin{array}{l}\text { Scissor Cart - Rolled Plate } \\
\text { Storage }\end{array}$ & & \\
\hline & & & Fuel Plate Day Storage & cabinet & \\
\hline & & & $\begin{array}{l}\text { Critically Safe runoff Water } \\
\text { Collection System }\end{array}$ & $\begin{array}{l}\text { Geometrically favorable } \\
\text { sumps, } 6 " \text { dia.tube tanks, } \\
\text { recirculating \& transfer } \\
\text { pumps, filters, } 8400 \text { gal } \\
\text { horizontal underground } \\
\text { storage tank w/ } \\
\text { hemispherical heads, } \\
\text { sampling system }\end{array}$ & 1 \\
\hline & & & Mech/Elect Maint. Equip & & lot \\
\hline & & & Fuel Alloy Can storage rack & $\begin{array}{l}5 \text { movable shelves w/ } \\
\text { inserts \& gates, } 5 \mathrm{~h}\end{array}$ & \\
\hline & & & Fuel Subassembly storage rack & & 10 \\
\hline & & & $\begin{array}{l}\text { Motor Control Ctr w/ starters \& } \\
\text { CB }\end{array}$ & & 2 \\
\hline & & & $\begin{array}{l}\text { Electrical Substation w/ } 480 \mathrm{~V} \\
\text { Xmfrs }\end{array}$ & & \\
\hline & & & $\begin{array}{l}\text { Granite Surface Table w/ } \\
\text { parallel }\end{array}$ & & 5 \\
\hline & & & Coordinate Measuring Machine & & 1 \\
\hline & & & Stack Monitor (CEMS) & & 1 \\
\hline & & & $\begin{array}{l}\text { Alpha CAMs and Central } \\
\text { Control }\end{array}$ & Battery backed & \\
\hline & & & Criticality Detectors & $\begin{array}{l}\text { Battery backed, Vacuum } \\
\text { pump and facility wide } \\
\text { distribution system, connect } \\
\text { to CAS }\end{array}$ & \\
\hline & & & Air Compressor & $10 \mathrm{HP}$ & 1 \\
\hline & & & Instrument Air dryer & $25 \mathrm{CFM}$ & 1 \\
\hline & & & Instrument air filter & & 1 \\
\hline & & & $\mathrm{N}_{2}$ Bottle regulator & & 2 \\
\hline & & & Off-Line Programming Station & software and computer & 2 \\
\hline & & & Waste Size Reduction Shear & for aluminum trimmings & 1 \\
\hline
\end{tabular}




\begin{tabular}{|l|l|l|l|l|c|}
\hline $\begin{array}{c}\text { Area } \\
\text { Code }\end{array}$ & Sketch & \multicolumn{1}{|c|}{$\begin{array}{c}\text { Equipment } \\
\text { ID }\end{array}$} & \multicolumn{1}{c|}{ Equipment Name } & \multicolumn{1}{c|}{ Description } & Quantity \\
\hline & & & video surveillance equipment & $\begin{array}{l}\text { Cameras in secured } \\
\text { controlled storage areas and } \\
\text { dock, security monitors, } \\
\text { recording equip }\end{array}$ & lot \\
\hline & & & fenced security areas & dock & 3 \\
\hline & & & drum handling equipment & & 5 \\
\hline
\end{tabular}




\begin{tabular}{|c|c|}
\hline & FACILITY FUNCTIONAL AREA BREAKDOWN \\
\hline $\begin{array}{l}\text { Area } \\
\text { Code }\end{array}$ & Function \\
\hline 1 & Ingot Cleaning, Casting, Mold and Crucible prep \\
\hline 2 & Hot roll unclad U-Mo to $\sim 0.1$ " thick \\
\hline 2 & Heated Salt Bath (prior to rolling cast plates) \\
\hline 3 & Sectioning (four plates cut from each pre-rolled U-Mo strip) \\
\hline 3 & Salt Removal (sanding or media blasting prior to sectioning) \\
\hline 4 & Canning (automatic weld station load / unload) \\
\hline 5 & Hot rolling of Zr Clad U-Mo in purged C'Stl can \\
\hline 6 & De-canning, foil trimming, can scrap management \\
\hline 6 & Anneal \\
\hline 7 & Chemical Cleaning (Al plates and Zr Clad U-Mo are cleaned in separate baths) \\
\hline 8 & Friction Bonding \\
\hline 9 & Fuel Element Trim, flattening and Surface Finish \\
\hline 9 & Fluoroscopy (verify position of foil inside bonded Al cladding) \\
\hline 10 & QA \\
\hline 10 & Rad Tech \\
\hline 10 & Lab - UT \\
\hline 10 & Lab - Radiologist \\
\hline 10 & Lab - Microscopy and sample prep \\
\hline 10 & Lab - Metallography / Chemistry \\
\hline 10 & Dimensional Inspection / Setup \\
\hline 11 & End piece Machining, Element Assembly, swaging, Autoclaving Final Product Assembly \\
\hline 11 & Fuel Element Shape Forming (Press) \\
\hline 12 & Shipping and Receiving (Product packaging + shipping container unloading) \\
\hline 12 & Storage Area, order picking and stocking, Accountancy \\
\hline
\end{tabular}




\subsection{MECHANICAL/EQUIPMENT DESCRIPTION}

The Mechanical Equipment in the FFC is shown on the drawing SK-ME-1 and listed on the FFC equipment List. The fuel manufacturing process is outlined on the Overall Block Flow Diagram BF-1. Equipment for the various steps in the process and outline descriptions of what takes place in the various areas within the plant are described in this section.

The FFC is arranged as a typical machine shop, foundry and assembly area, complete with 21 st century technology for fabrication of LEU-Mo plate fuel subassemblies. The shop area has a central aisle for forklift and hand-cart traffic, the predominant means of production material handling. The shop itself provides primary ventilation confinement, and is surrounded by the personnel corridor. This corridor provides a place for personnel to walk to the vicinity of their work station without conflict with hand-cart or forklift movement. Viewing windows into the shop are present all along the length of the corridor accommodating tour visits without need for entry, and allowing site personnel to observe activity within the shop. The shop is a high bay area with overhead bridge cranes. Suspended shop lighting hangs above the bridges, and the ceiling of the shop is open to the trusses.

Two bridges on parallel runways run the length of the shop including the casting area. This permits heavy maintenance procedures (such as roller replacement or motor or bed removal) to be performed on any piece of shop equipment, and allows spent machinery or replacement items to be moved to or from the dock area. There are no central runway crane support columns, because they would interfere with forklift travel. The inner runway rails are suspended from the overhead roof trusses. The outer runway rails are supported by columns offset from the building columns as shown in Figure 4-1 below. This allows exhaust duct risers and electrical trays to be routed against the shop walls, and below the crane rails, to be brought into the shop floor area horizontally above head height such as for a ventilation hood duct. This arrangement avoids interference with use of the overhead cranes.

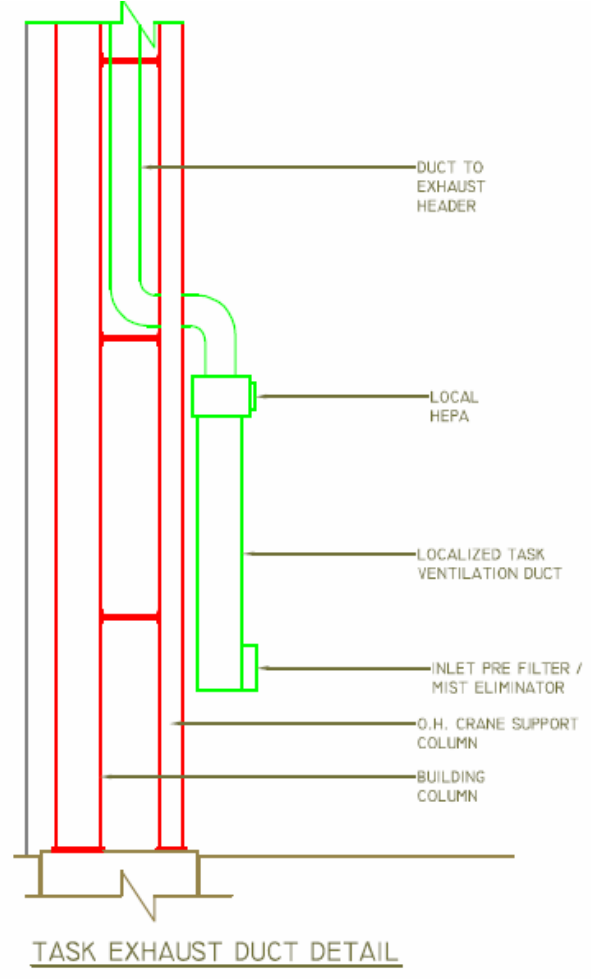

FIGURE 4-1 BRIDGE CRANE RUNWAY OUTER SUPPORT COLUMNS 
Air is exhausted from the shop and carried to the exhaust filters and fans via overhead ducts, which run amongst the roof trusses. The amount of airborne contamination from activities conducted in the FFC is expected to be small. The ventilation system is designed to meet personnel safety requirements and good practices. At the end of the shop closest to the office area, the opposite end from the foundry, all in-process fuel material is clad; hence this is the 'cleanest' area from a contamination standpoint, which is also where the majority of the fresh ventilation supply air flows into the shop from the normally uncontaminated Personnel Corridor. The primary activities expected to produce radiological material contaminates are casting, cutting and rolling operations. Non radiological sources of dust are associated with grinding and media blasting activities. All such locations where these activities are conducted are provided with task exhaust systems, preventing spread of contamination into the work environment. The exhausted air is drawn mainly from the 'back' of the shop, near the foundry, where bare alloy fuel material is being handled, or the 'dirtiest' end, thus air flows from the area of lowest potential for contamination to the areas with a higher likelihood of contamination. All air leaving the shop is filtered via HEPA filters before it is released to the atmosphere. Many hoods and task exhaust ducts are present drawing air from the shop; these constitute more than $20 \%$ of the exhaust air flow.

The foundry area houses two induction furnaces where cast 'acorns' or buttons and broken pieces of LEU-Mo alloy from Y-12 are melted and cast into plates 1/4" thick. Furnace capacity is such that a single batch per day is poured from one of the two furnaces, meeting the needs of production. In the shop, the cast plates are then hot rolled to an intermediate thickness $(\sim 0.1$ "). The plates get longer in the same proportion as are rolled and become thinner. Following the initial roll, the U-Mo plates are sectioned into lengths suitable for canning. The surfaces of the rolled plates are covered with zirconium metal shim stock which serves as a diffusion barrier, and the $\mathrm{Zr}$-fuel-Zr sandwich is canned between steel sheets welded together. The canned fuel plates are hot rolled, and the rolled cans are opened, exposing the $\mathrm{Zr}$ clad U-Mo foils at their final thickness, 0.010 " to 0.015 " thick depending on the final application. The foils are trimmed to the final rectangular dimensions (maximum of 3.5 " wide X 48 " long) then the aluminum cladding is applied. At this point, the monolithic fuel foil is completely confined.

The assembly area is where completed LEU-Mo plate fuel elements are assembled into completed research reactor fuel subassemblies. Extensive machining, welding, swaging and inspection operations are performed here, and modern equipment workstations are provided to suit the varied needs of the different fuel sub-assembly configuration required to support the five domestic reactors serviced by the FFC.

The fuel manufacturing steps and facility features are explained further in the report sections that follow. 


\subsection{Receiving/Receipt Inspection}

LEU-Mo raw material, in the form of 1.5" acorns or broken pieces, is shipped in ES-3100 containers, shown in Figure 4-2, from the foundry at the Y-12 Plant at Oak Ridge in Tennessee. The alloy fuel material is inside sealed stainless steel shipping containers, 43" tall, resembling a 'stretched' 30 gallon drum as shown below. The outer drum assembly houses an inner containment vessel with up to three inner containers, described as paint cans, packaged in the center of the shipping container. The inner containment vessel, Figure 4-3, is 32' tall and 5" diameter and is also stainless steel. The lid is closed with a sealing nut and two o-rings are present beneath the lid to allow the inner container to be purged and sealed.

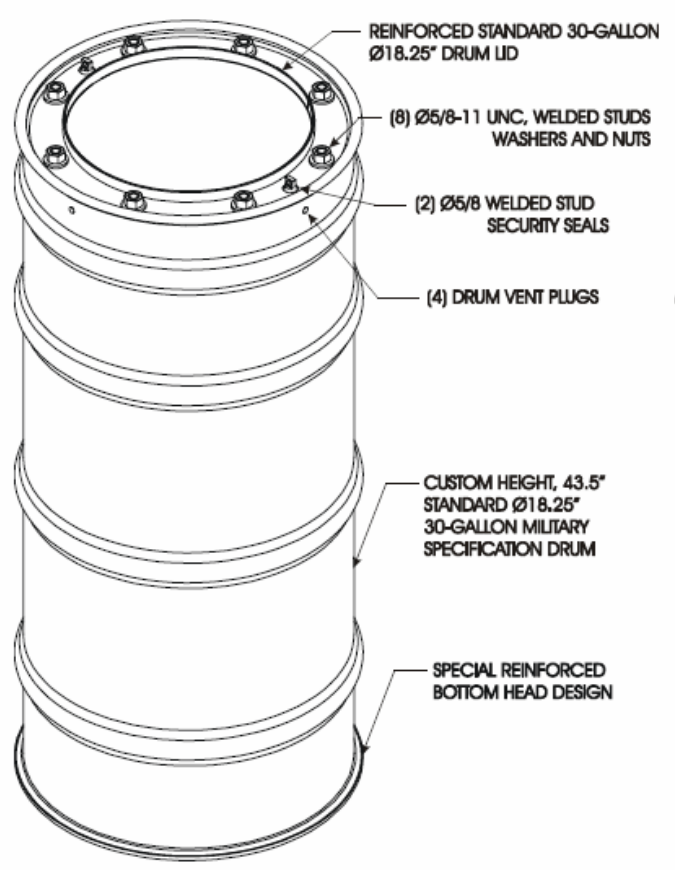

Exterior View of the ES-3100.

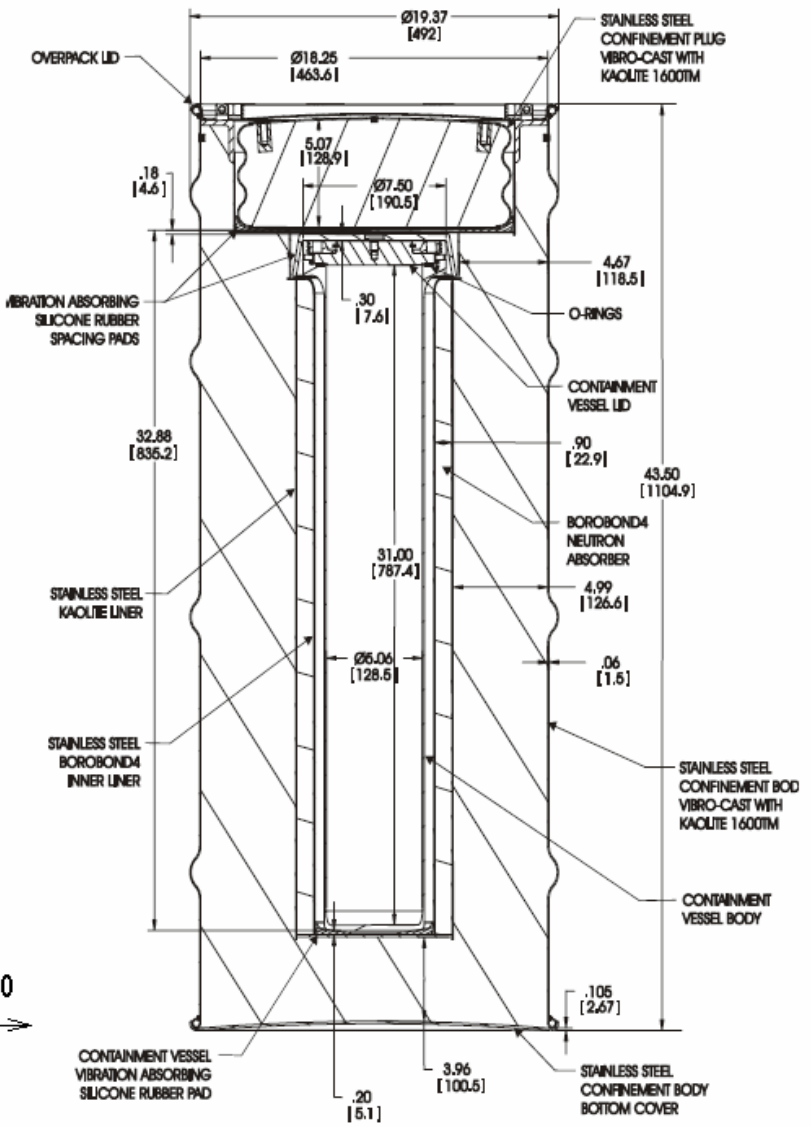

FIGURE 4-2 ES-3100 SHIPPING CONTAINER 


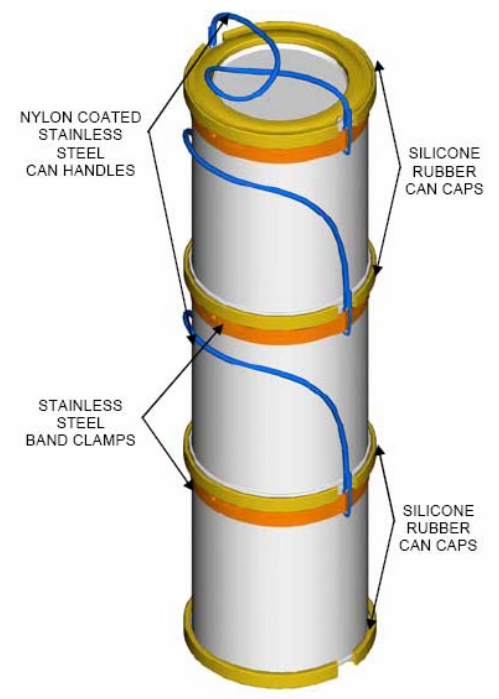

Each of the three 10" tall inner cans contain up to 11 $\mathrm{kg}$, to a maximum of $33 \mathrm{~kg}$ of LEU-Mo per ES-3100 package.

\section{FIGURE 4-3 INNER CAN}

The drums are secured to pallets, and the pallets are shipped across country in covered trucks. They may be shipped by commercial carriers including UPS. There are no criticality constraints on the shipping containers because they are both internally insulated and shielded. The containers are rated for other uses including HEU. The maximum gross weight of the package is 420 pounds including the contents.

Trucks are admitted to the FFC through the entry control facility and shipments are scheduled in advance. The trucks are backed into an indoor dock area of the FFC which provides year-round weather protection. Space heaters are present in the dock, and up to three vehicles may be parked within the enclosed dock area with the rollup doors closed. Door controls are such that vehicle and cargo storage is secure overnight. An elevated dock suitable for forklift passage into the trucks is featured in the FFC arrangement. Dock parking bumpers are present. A tip down ramp is placed between the vehicles and the dock and secured allowing the fork lift vehicles to enter the bed of the truck to off load pallets of drums. A typical shipment of LEU-Mo alloy from Y-12 may consist of 64 drums (16 pallets), which would be about 2 tons of alloy, and must be scheduled a year in advance to get in to the normal production run. Such shipments may be in 4 month deliveries, or otherwise at the convenience of the customer, and some material may be stockpiled at Y-12. The FFC uses about 6 tons of alloy yearly. The fork lift will be equipped with a drum carrier attachment for handling single drums. A sea-land cargo type container (for larger shipments) may also be parked at one stall within the dock, in which case unloading will be at grade with the fork lift elevating drums to the dock. 


\subsection{Inner Can Storage}

While a shipment of raw material is still on the truck at the dock, a receiving inspection is conducted, Figure 4-5. A physical drum count is made to assure agreement with shipping documents, and a scan for gross gamma is made using a hand-held instrument. A pallet of drums at a time is moved with a forklift to the U-Mo secured controlled storage area. Individual container weights are measured as the drums are de-palletized to evaluate shipper/receiver differentials and any differences are tracked. Prior to transfer of the drums to the unloading area, smear tests for contamination are conducted. Contaminated drums are wiped clean. Inner cans are removed from the shipping container in the container unloading area within the U-Mo secured controlled storage area. Lids are removed from the individual drums, and the inner containers (shown above) are individually weighed and stored in the storage rack within the storage area. A chemical check sample is included by Y-12 in a package separate from the inner cans. This allows the FFC to leave the inner cans sealed while the chemistry and isotopic composition is verified. The secured controlled storage area is equipped with specialized moveable shelving units as shown below in Figure 4-4.

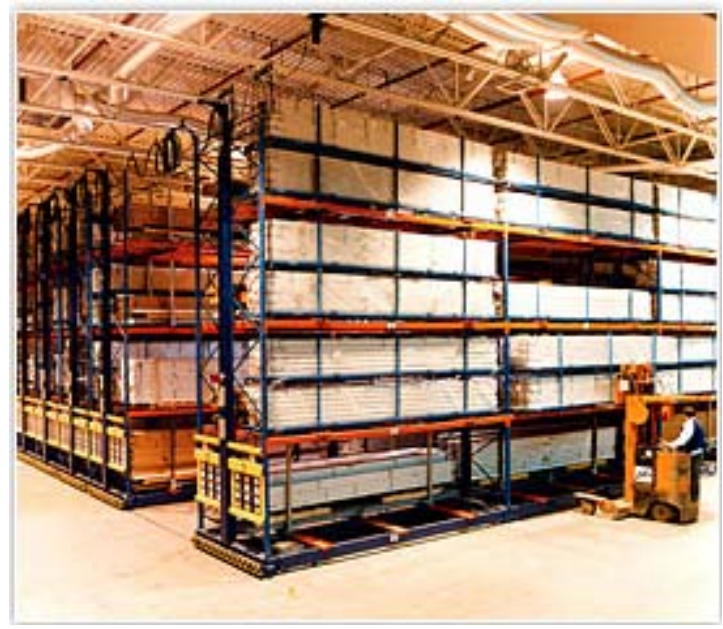

Commercially available moveable storage shelves allow the aisle to be located at the position orders are being placed. The storage area is compact, yet accessible. The shelving racks move on sets of upper and lower tracks. Shelves are seismically rated and occupy $1 / 2$ the area occupied by shelves with aisles between racks.

FIGURE 4-4 MOVEABLE SHELF EXAMPLE

Each area of storage is marked, and each storage area has inserts dedicated to the stated purpose. In addition to the marking, the storage positions are uniquely sized to accommodate the material in the particular in-process state that it is in when it is stored. Due to the large quantity of fissile material stored in the secured controlled storage area, dedicated storage racks ensure that engineering controls are present in the storage area in addition to the administrative controls. Fuel material in cans is stored in criticality-safe stacks such that no stack of cans is closer than 12 " from its neighbors. For raw alloy, a storage 'bin' or pocket in the shelf is contoured to fit a single 10" tall can, and only one can fits in a storage location. A lockable 'gate' is closed over the can, which secures it to the shelf so in a seismic event; the cans do not fall out of the shelves. The 'gate' permits visual identification that the can is present and the tracking number on the can is visible without opening the 'gate'. Individual can identification permits lot and batch tracking. This may be by bar-code, printed and affixed when the cans are received, or by the markings present on the can (if such markings are present when shipped from Y-12). Upon receipt of the material, the FFC accountancy process begins. Custody of the material is transferred from the shipper to the receiver. Later, cans may be opened and material samples analyzed for impurities, 
chemistry and isotopic verification. A contingency plan will be prepared for disposition of any off-spec material received.

The storage racks for raw material (cans) are moveable on tracks in the floor (and ceiling). This allows a single aisle to be located anywhere within the rows of shelves that access is required, thus saving space. When the shelves are in their most compact position, cans are still separated with an 8 " gap between neighboring cans. As shown in the arrangement on SK-ME-02, there are nine double sided shelves, containing cans in columns five high, 12 inches apart for a total of 540 cans; one year's supply of raw LEU-Mo, enough for about 17,000 foils.

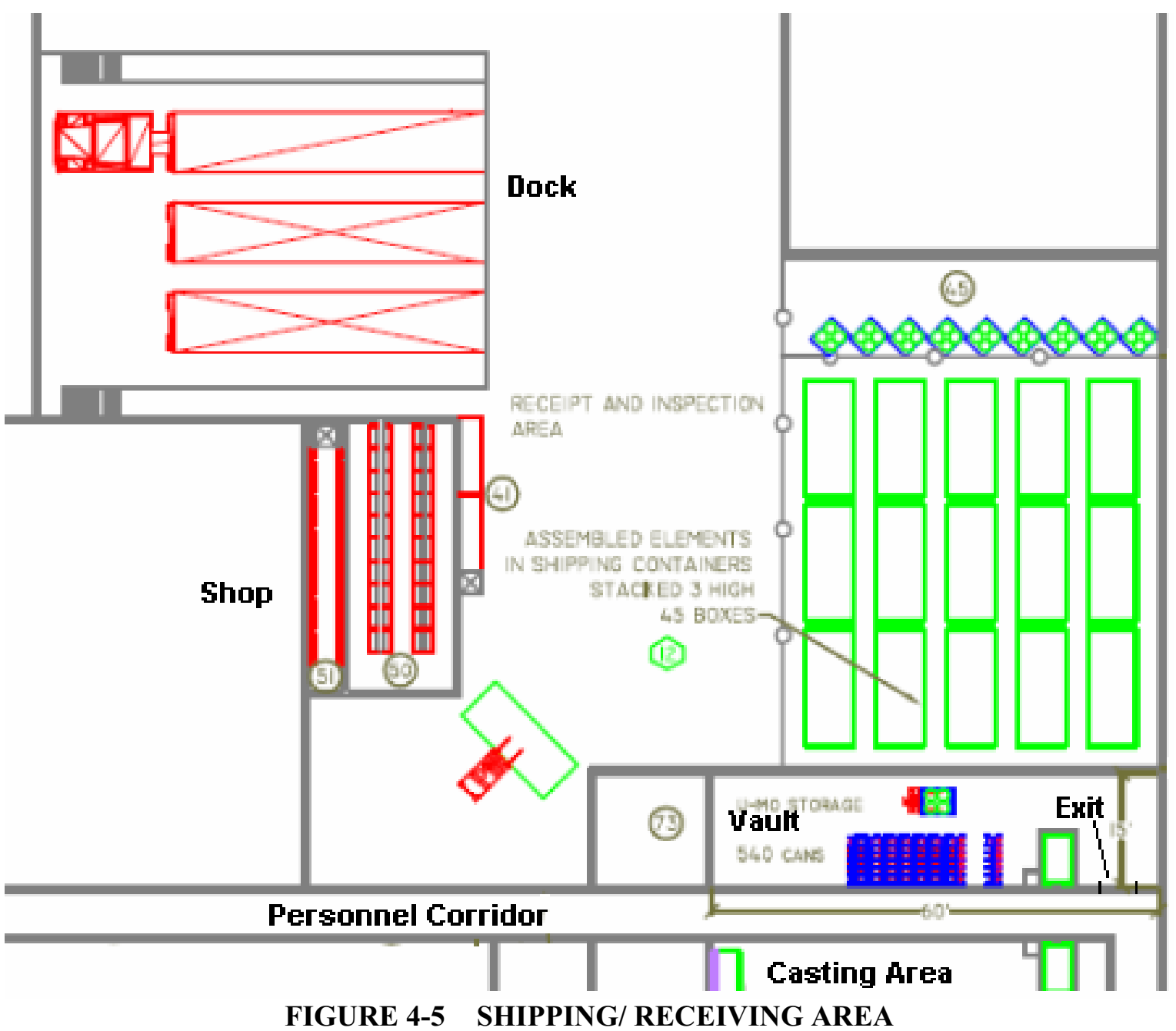

The interior of the U-Mo secured controlled storage area, the entrance vestibule, and the other temporary storage areas around the dock are equipped with CCTV cameras for security. Admittance is controlled by security approved access control methods. The emergency exit door from the secured controlled storage area (to the personnel corridor) has hardware such that it can only be opened from the inside for emergency egress, thus avoiding any 'confined space' implications. When a vulnerability assessment is performed on the FFC (future), some details may be subject to modification or refinement. 


\subsection{Material Handling and Transfer}

Orders made from the floor are picked manually from shelves in the secured controlled storage area, placed on movable carts and passed via the pass through below the personnel corridor to the shop. The weight of the containers moved to casting is tracked for accounting purposes.

\subsubsection{Pass-Through}

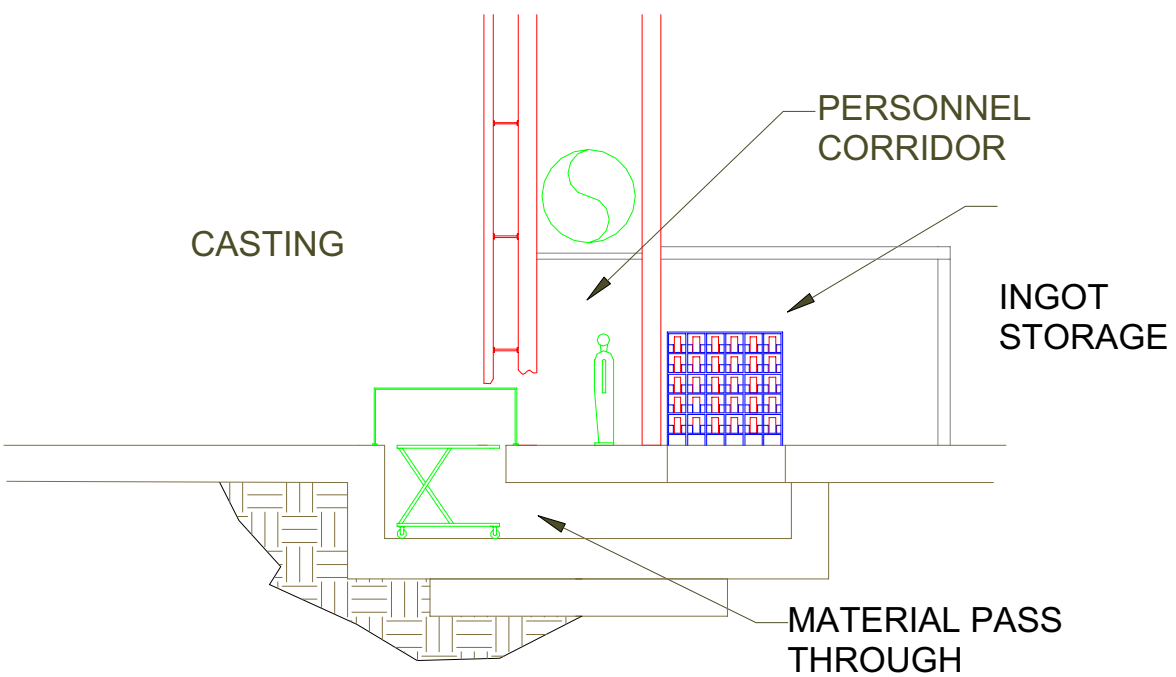

FIGURE 4-6 MATERIAL PASS-THROUGH EXAMPLE

There are two material pass-throughs in the FFC, shown in Figure 4-6 above. The instance shown above is used to pass the raw fuel alloy from the U-Mo secured controlled storage area, on the right, to the casting area, and the second is used to pass material to and from the inspection area or shop to and from the intermediate storage area. A third would be present if the HIP option is invoked, which allows canned work packages, made up in the shop, to be passed to the HIP, and back again for disassembly. The purpose of the Material Pass-Through (MPT) is to be able to pass material from one primary confinement zone to another with out exposing non contaminated zones to 'contaminated' material. Another reason for having a material pass-through is to minimize personnel movement.

The MPT operates within a concrete lined trench which houses a rail mounted transfer cart with a scissor lift. The unit is electrically powered and is equipped with end of travel limit switches and interlocks that allow the lift to function only at endpoints of travel. The system is controlled with a simple control system from which workers will control the transfer of material. The trench has a safety rail around it to keep workers away from the trench. A locally mounted terminal on both sides will be used to place 'orders' for material and account for material moved from one material balance to another area. 


\subsubsection{Hand Carts}

Hand carts, such as in Figure 4-7, will be used through out the FFC for material transfer between different work stations and for general movement throughout the shop. Heavy duty steel platform trucks with a flush platform measuring up to $60 " \times 30$ " x 11 " tall are used. Commercially available flat platform carts will be outfitted with special compartmentalized racks to hold the inprocess fuel material securely, to ensure material is not stacked too deeply (which could cause criticality concerns) and to keep the material being

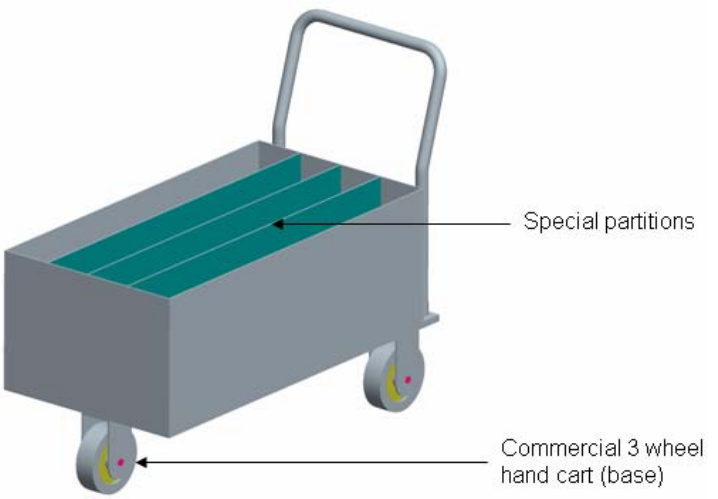

FIGURE 4-7 HAND CART EXAMPLE transferred organized (shown right).

The trucks are manually pushed and roll on two rigid and one or two swiveling rubber tired casters for tight turns and smooth maneuverability. Each hand cart will be equipped with a removable handle and a floor lock to keep the truck stationary when it is parked at a work station.

\subsubsection{Forklifts}

A three-wheel stand-up counterbalanced forklift, shown below in Figure 4-8, with a 2 ton load capacity is featured in the FFC design for material transfer and loading and unloading of fuel or material shipments from trucks. A three-wheel, stand-up forklift has a reduced turning radius for greater maneuverability and easier material handling in tight spaces.

The battery powered forklift emits no exhaust and is equipped with quick tine disconnects to allow for easy and quick change between forks and drum grabs or other attachments as needed.

A powered pallet truck is used where tight spaces may be encountered and the need to stack pallets is not needed; such a place is the LEU-Mo secured controlled storage area. The powered pallet truck is used to move a pallet of LEU-Mo alloy shipping containers to the secured controlled storage area where the drums of alloy resting on a pallet are unloaded from the ES-3100 manually by a worker.

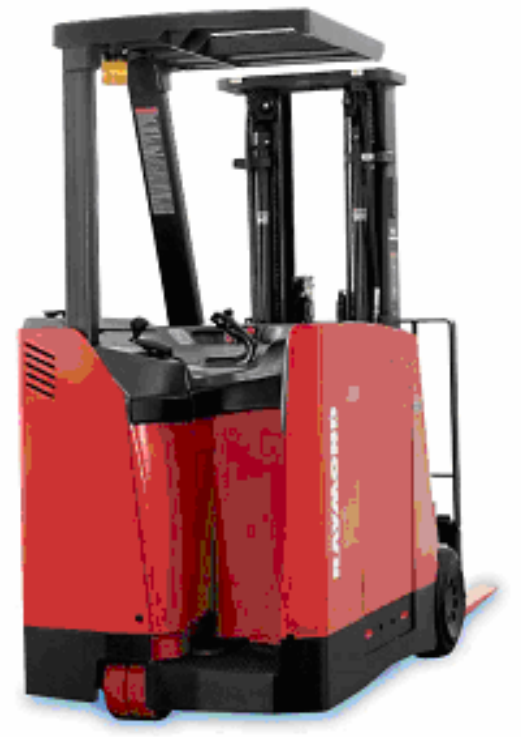

FIGURE 4-8 FORKLIFT 


\subsubsection{Drum grabs}

Forklift mounted mechanical drums grips as shown below, Figure 4-9, are used to move and stack drums in the FFC. Auto-Grip drum handlers allows a single operator to easily slide the drum handler on the fork tines to pick-up, transport and unload empty or full drums up to 1500 pounds.

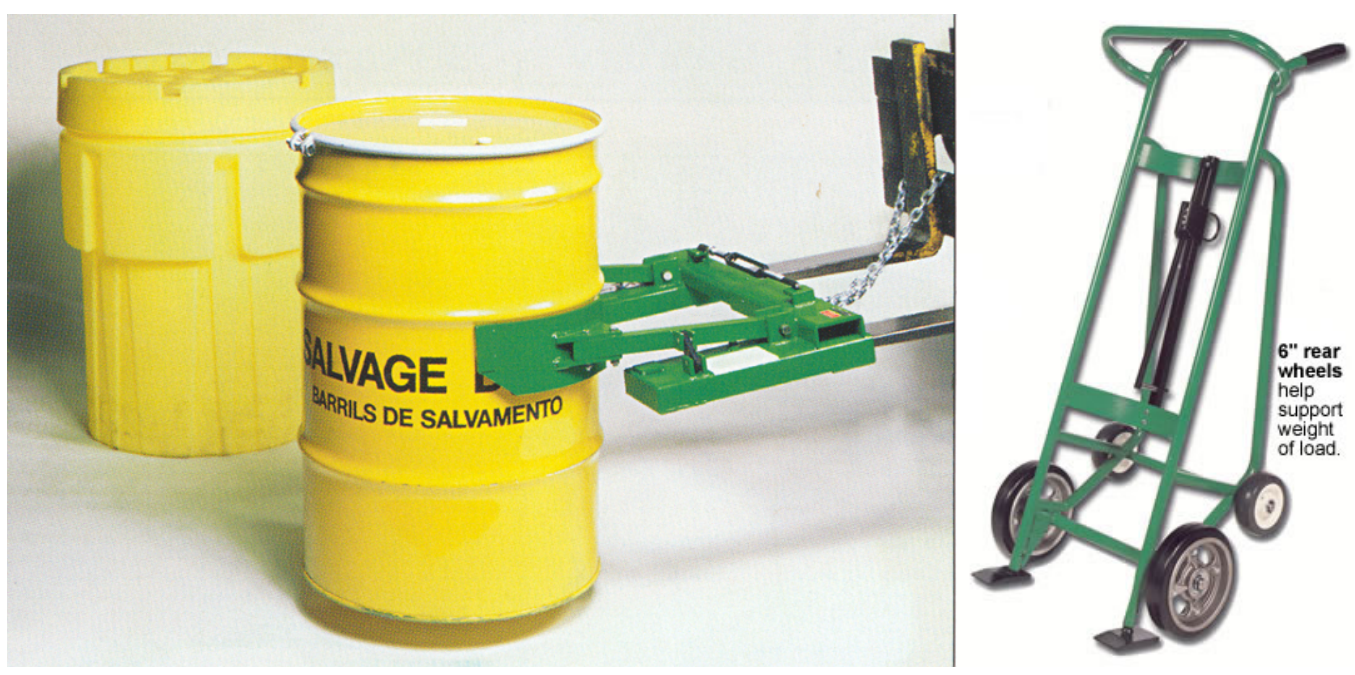

FIGURE 4-9 EXAMPLES OF DRUM HANDLERS

When empty or full drums are moved from one location to another and the need for a forklift is not required, four-wheel drum carts, shown on the right, may be used. 
4.3.5 Overhead Cranes

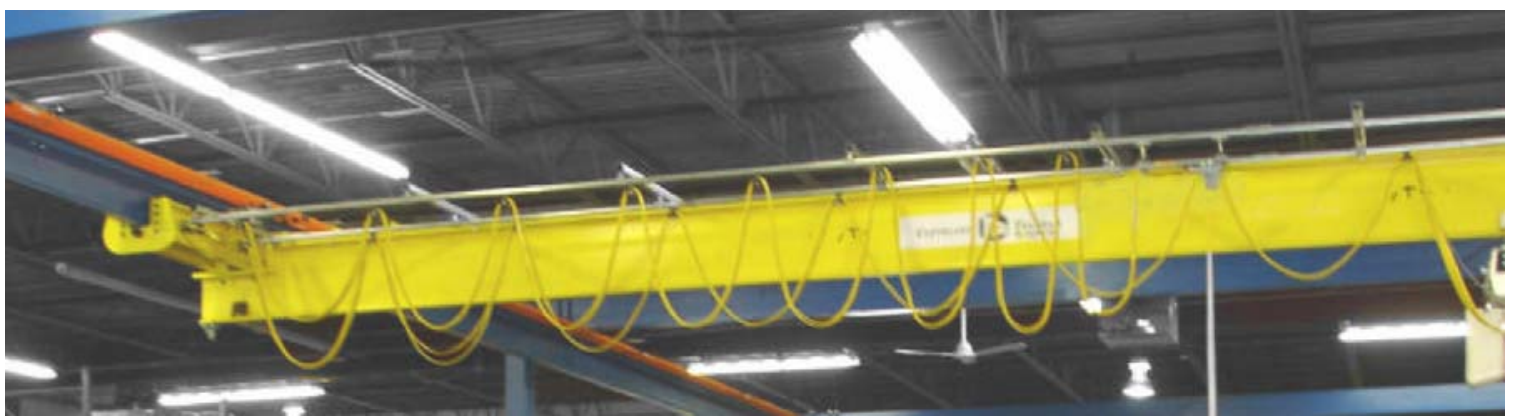

FFC Overhead Cranes

- Two parallel Runways

Entire length of bays

Shop and Assembly Areas

- Rail Supports

Posts on outside (allow duct routing for hoods)

Ceiling truss suspension inside

Unobstructed center fork lift aisle

- Cross-Over from bridge to bridge

Two locations each:

Shop and Assembly Areas

- Hoist can be used for:

Machinery movement

Service and maintenance

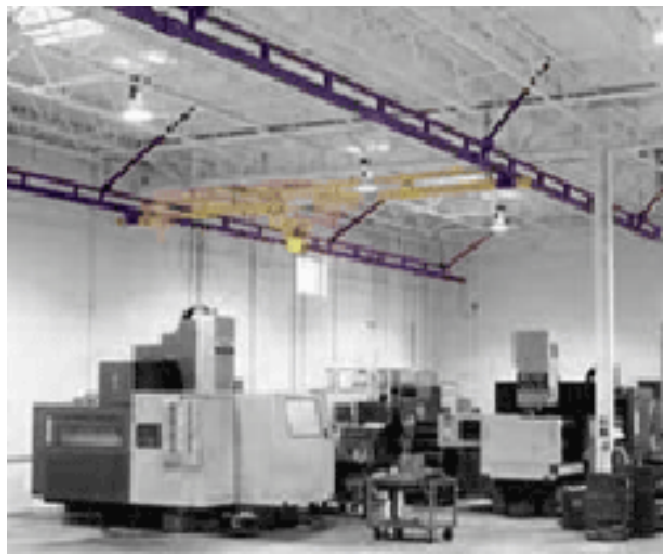

Remove and replace components

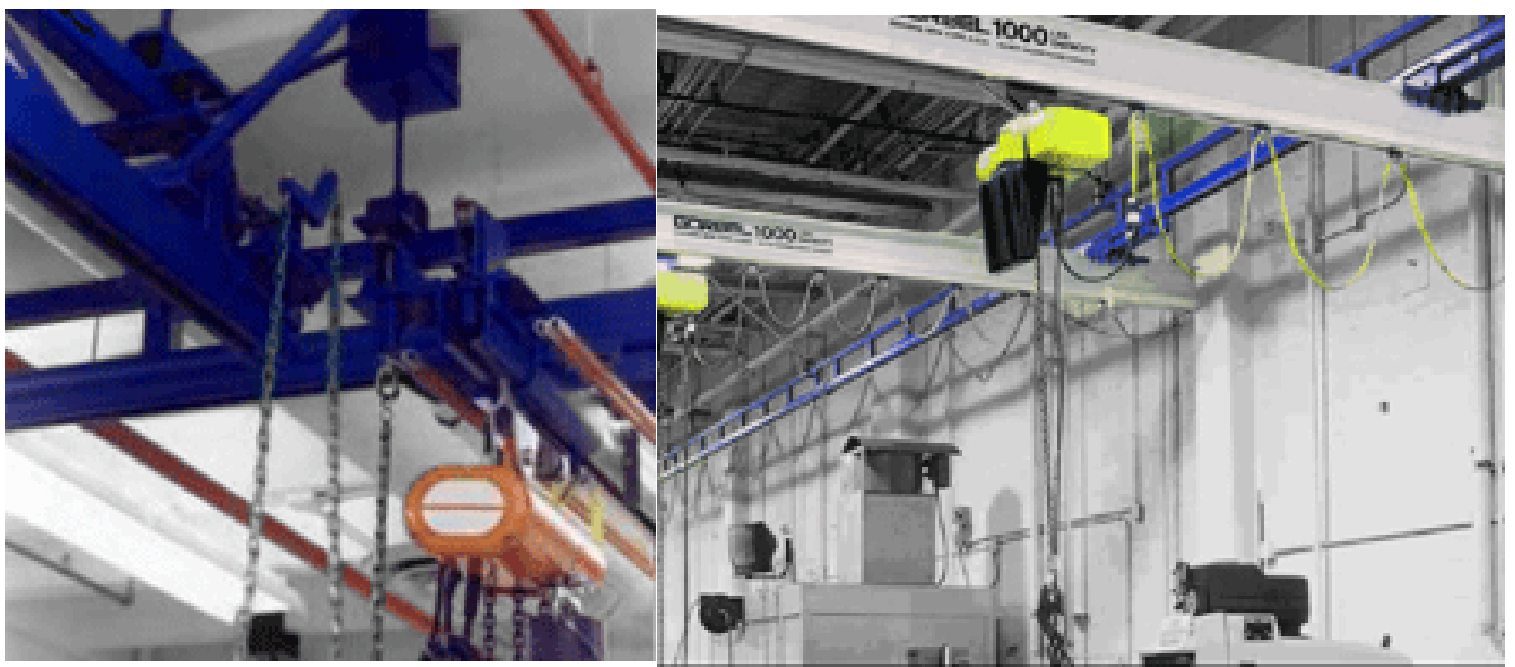

FIGURE 4-10 OVERHEAD CRANES

Overhead cranes, like those shown in figure 4-10, are used in the FFC to facilitate heavy maintenance procedures, and are not generally used for material handling. Cranes fall under a different category for nuclear material handling (ASME NUM-1), and that requirement is not currently being considered for the FFC. The 5 ton (shop) and 7.5 ton (Assembly Area) rating is suitable for support from the building roof trusses. The load 
rating, and distance under the hook shown in the elevation drawings (see Appendix) may be subject to change based on detailed support mission studies (future). Some of the functions that the overhead cranes may be used for in the shop are listed above.

\subsection{Alloy Casting}

The castings are $90 \%$ Uranium and 10\% Molybdenum (eutectic gamma phase) with a melting point of $1150^{\circ} \mathrm{C}$. The alloy is melted within a bottom pour graphite crucible surrounded by water cooled induction coils within a bell jar operating at a vacuum. Temperature is measured using an optical pyrometer through a hole in the cover of the crucible. After a 15 minute dwell at $+50^{\circ} \mathrm{C}$ superheat, the vacuum in the jar is broken with argon then the melt is poured into a preheated book mold inside an inert atmosphere enclosure. Casting molds are moved beneath the furnace using an indexing material handling system. Preheating of the molds utilizes an infrared radiant heating system. After the mold is filled it is moved to the quench area within the enclosure where cooled gas is forced against the mold as the next mold is being filled. A full day's supply of alloy is present in the 6" dia. single batch crucible and a $20 \%$ heel (containing the dross) remains after filling the molds. Seven ingots are poured during this casting run, approximately $1 / 4$ " thick X 3.5" $\mathrm{X} 16$ " long. Following the quench and when the plates are below $\sim 500^{\circ} \mathrm{C}$ the molds cool by natural convection inside the enclosure to a safe handling temperature (below $120^{\circ} \mathrm{F}$ ) in the exit airlock. The molds are manually cracked open and the ingots are inspected, weighed, serialized and sent to the roll mill. A slight draft in the molds facilitates ejection of the cast plates. Book molds (which hinge on the bottom and produce a flat vertical cast plate) are used because the alloy material does not gravity level and uniform ingot dimensions are necessary for rolling such that a minimum of trimming is required. Molds are likely to be carbon to avoid formation of an alloy with the fuel material. Two runners are present on the top of the casting cavity of the mold. Molten material is added to the fill sprue until it overflows the vent assuring the mold is full. Trimmed alloy material and sprue volumes are recycled to a following melt.

One casting line is used one day, and as the plates are stripped from the molds in the A line, the next melt batch is being prepared in the B line. The casting area arrangement may be seen in the plan view below. Fuel alloy material is manually cleaned in nitric acid, then rinsed and dried prior to crucible loading to remove oxides and any organics that may be present. The alloy used to prepare a batch may be from a single lot of acorns and broken pieces from Y-12, or may be mixed from left-over from other lots or mixed with heels and/or pieces retained and accumulated from previous runs. Such mixed batches will be analyzed at the FFC for certification of alloy chemistry and isotopic composition to assure fuel specifications are met. Fuel alloy material is retrieved from the secured controlled storage area via the pass-through in convenience cans which are weighed before and after the fuel is readied for use. Molds and crucibles are refurbished and reused. Within hoods, molds and crucibles are cleaned and any appreciable amounts of residual alloy fuel material is saved and weighed for accountability purposes. Fines are disposed of as LLW following accounting for the material lost. Release compound is applied to clean molds and crucibles prior to re-use. Crucibles have a finite life. Spent crucibles are cleaned prior to disposal as LLW. 


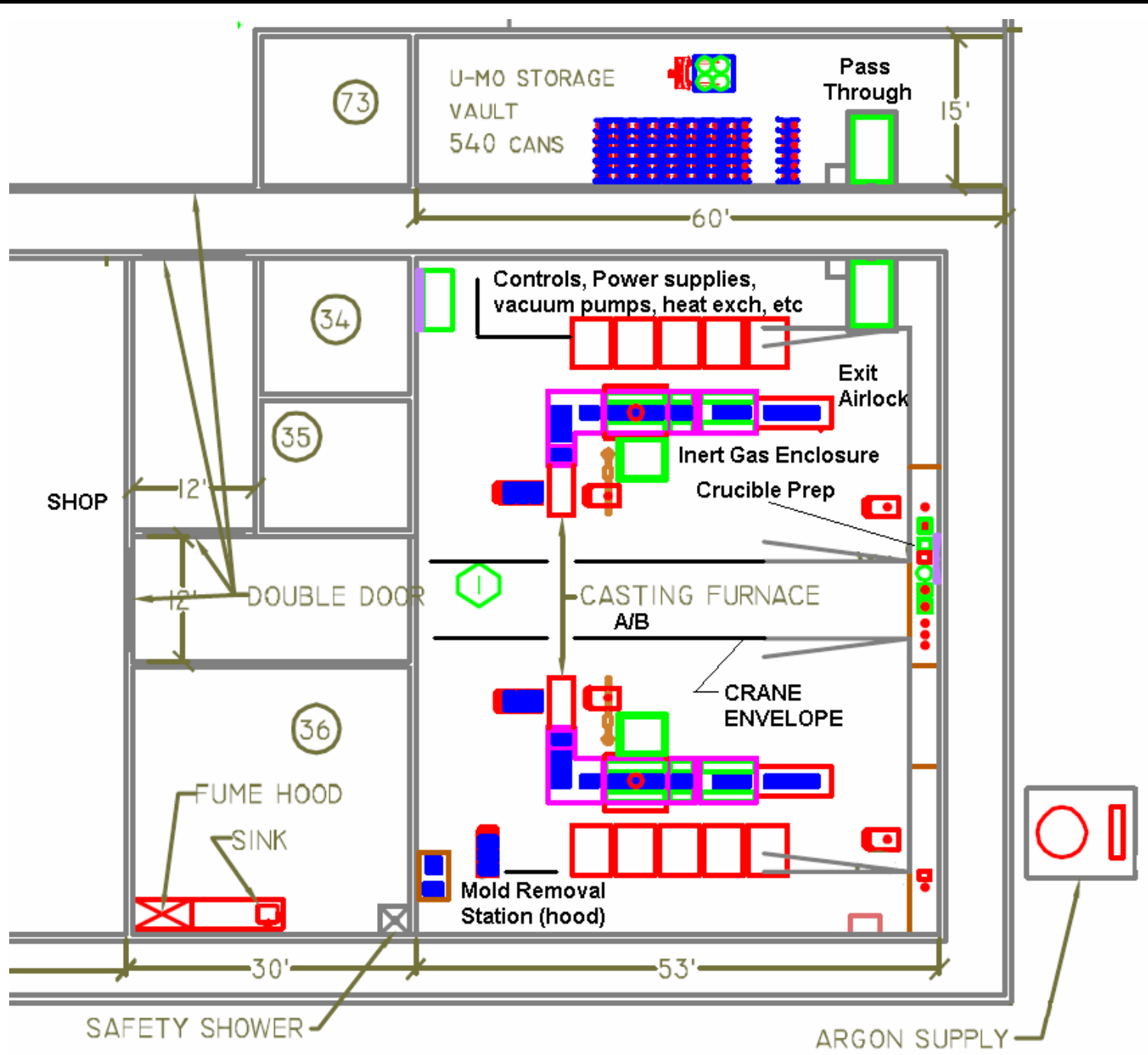

FIGURE 4-11 ALLOY CASTING AREA (PLAN)

The induction furnace featured in the FFC study was selected for use because it is in common production use in similar industrial facilities. Induction casting of thin plates made from U-Mo alloy has been successfully implemented since the early 1960's (Ref: http://www.osti.gov/bridge/servlets/purl/4836788-7KCcrK/). According to existing experience at $\mathrm{Y}-12$, sufficient induction stirring takes place within the melt to ensure adequate homogeneity, with the oxides floating to the top of the melt and remaining in the dross. Due to the presence of oxides in the top layer of the crucible, and the potential for formation of dispersible particulate, a sintered metal filter is present between the vacuum chamber and the vacuum pump. Spent filters, expected to contain LEU oxide, are disposed of as LLW because no filter leach capability exists at the FFC.

The exact casting technology to be used in the production plant is subject to further study (future) and it has been noted that a microwave furnace offers a potential advantage in that the water cooling is contained within the control cabinet and not in the argon enclosure as is the case with the induction furnace. It is also energy efficient compared with other furnace types. It is also possible that cast ingots of the proper size complete with alloy certification may be supplied from $\mathrm{Y}-12$, in which case the casting area and lab instrumentation to certify fuel alloy feedstock for 
impurities, isotope and chemistry would be eliminated from the FFC floor plan. Studies to compare costs, benefits and risks and related value engineering activities will be conducted during the Conceptual Design phase of the project.

The bell jars (for vacuum service surrounding the crucible) are lifted and moved out of the way, for crucible loading and unloading, by a small jib crane located at each furnace. The induction melter, Figure 4-12, has a cooling water system which requires a water to water heat exchanger, and chiller and air cooled condenser (mounted outdoors) for heat removal, a radiofrequency power supply (which is also water cooled), the water cooled induction coils within the vacuum bell jar, a water cooled chill plate, vacuum pump with 5 micron non-woven mesh, sintered metal filter, an operators control and instrument console that executes the pre-programmed recipe, interlocks (including evacuation timer and leak down), an argon venting system with pressure relief, and is constructed on a integrated modular basis allowing for factory testing and field service of components. A schematic of a generic induction heating furnace is shown below for reference.

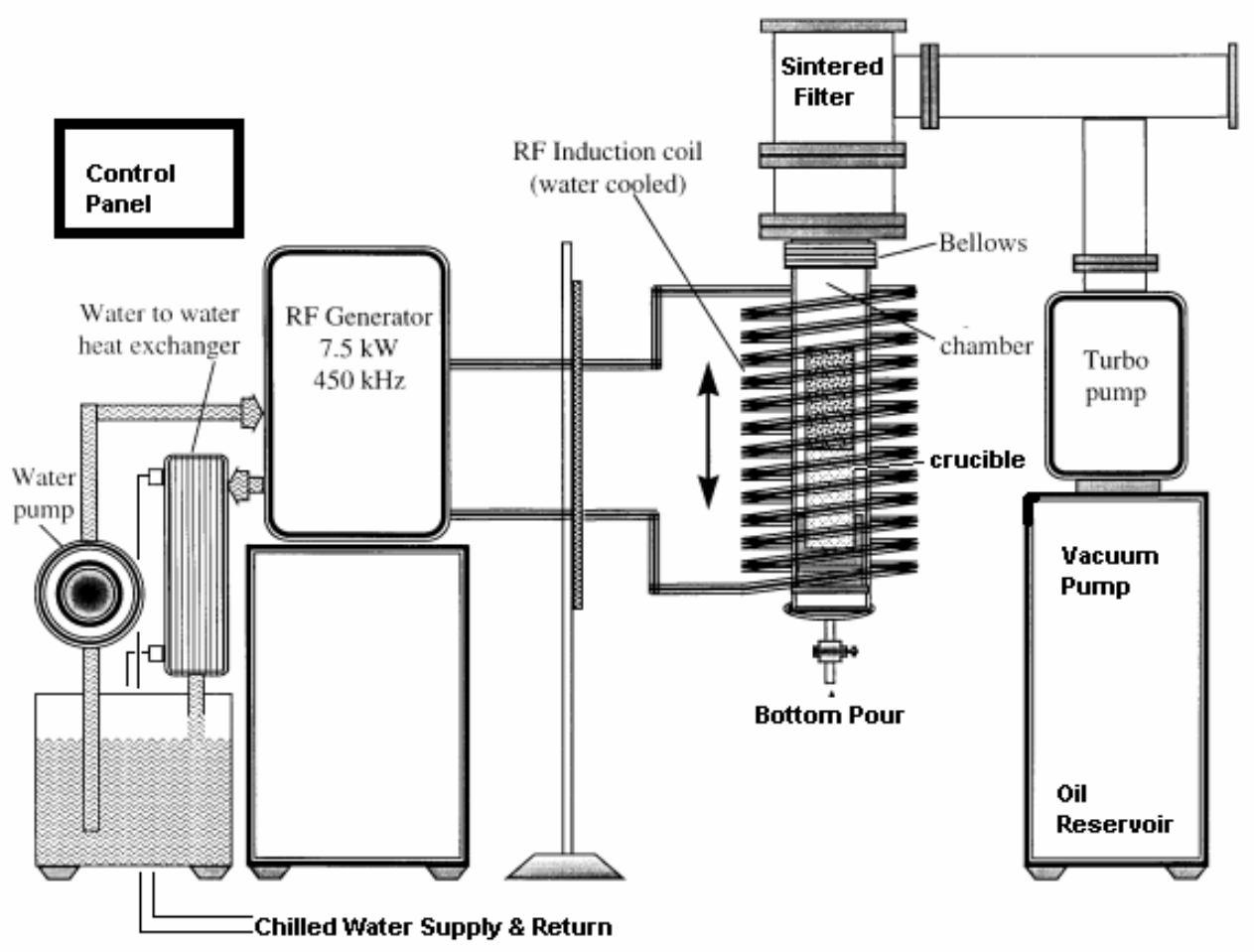

FIGURE 4-12 INDUCTION HEATING FURNACE SCHEMATIC

In addition to the induction furnace, a control console and power supply is present for the high intensity quartz lamp mold preheating system, and for the material handling systems, mold cooling systems and argon filled enclosure and enclosure inlet and outlet airlock doors.

The casting area, Figure 4-11, is in a room by itself and equipment inside may be serviced by the shop bridge cranes. The walls surrounding the lab area, between the shop and casting area, although they do not reach to the roof, provide a measure of fire spread prevention. Spent vacuum pump oils and spent filters from casting are disposed of as contaminated waste. 


\subsection{Hot Roll to Intermediate Thickness}

U-Mo material cold rolls with properties similar to stainless steel and hot rolls similar to Zirconium. On Roll Mill RL-001 hot roll the 1/4" thick X 3.5" X 16" long wide cast ingots to 0.1 " thick. One ingot makes 10 pieces. Approximately seven ingots are cast and rolled each day. The rolled plates are roughly 40" long. Prior to rolling, the cast plates are heated. The heating process used is a molten salt bath at $630 \mathrm{C}$. The salt coating on the parts retards oxidation and provides a protective coating on the plates and transfers to the rolls. A custom material handling technique removes the heated plates from the bath and feeds it to the roll mill. Conceptually, a rack securely holding a 1" thick stack of plates, spaced apart slightly, is used to lower the plates in the salt bath. The rack has long handles that extend above the molten salt surface when the rack with plates is immersed. After suitable retention time, a chain wheel retracts the handles and raises the stack of plates. An indexing feeder pushes the plates one at a time between the rolls. A reversing roll mill is envisioned with the material taking multiple passes through the rollers. It may take multiple passes through the mill rolls to obtain the desired plate thickness with a reheat between passes while the operator resets the rolls to the final nip (gap between rolls).

Following rolling, the 40" long fuel plates are then cut (sheared) into 10 pieces 4" long. The edges perpendicular to the rolled length are also trimmed. The trim material, being pure U-Mo, is recycled back to casting.

Hydraulic force against an adjustable chock is used to control the separating forces between the rolls, and the synchronized rolls are rotated by a variable speed electric motor operating through a gear box. It is desirable to obtain calibrated digital readouts on the roll gap, which is remotely set from the operator's console. An example is shown in figure 4-13. Such equipment will be procured to have a 40 year life. The furnaces for heating the input parts may or may not be part of the vendor's offering, if they are, the furnace controller will be part of the vendor's supply. Rolls (such as on the canning mill that are uncontaminated) may be periodically refurbished via a service contract. When the equipment is purchased representatives of the FFC project will witness a factory test. Roll-ability tests using surrogate material may be performed to determine rolling characteristics. The roll mills will feature an extremely rigid frame and heavy duty bearings to achieve and maintain high accuracy and good surface finish, with less than $\sim .0005 "$ thickness variation across the foil. The drive will be by motorized gear drives or by high torque hydraulic motor. 


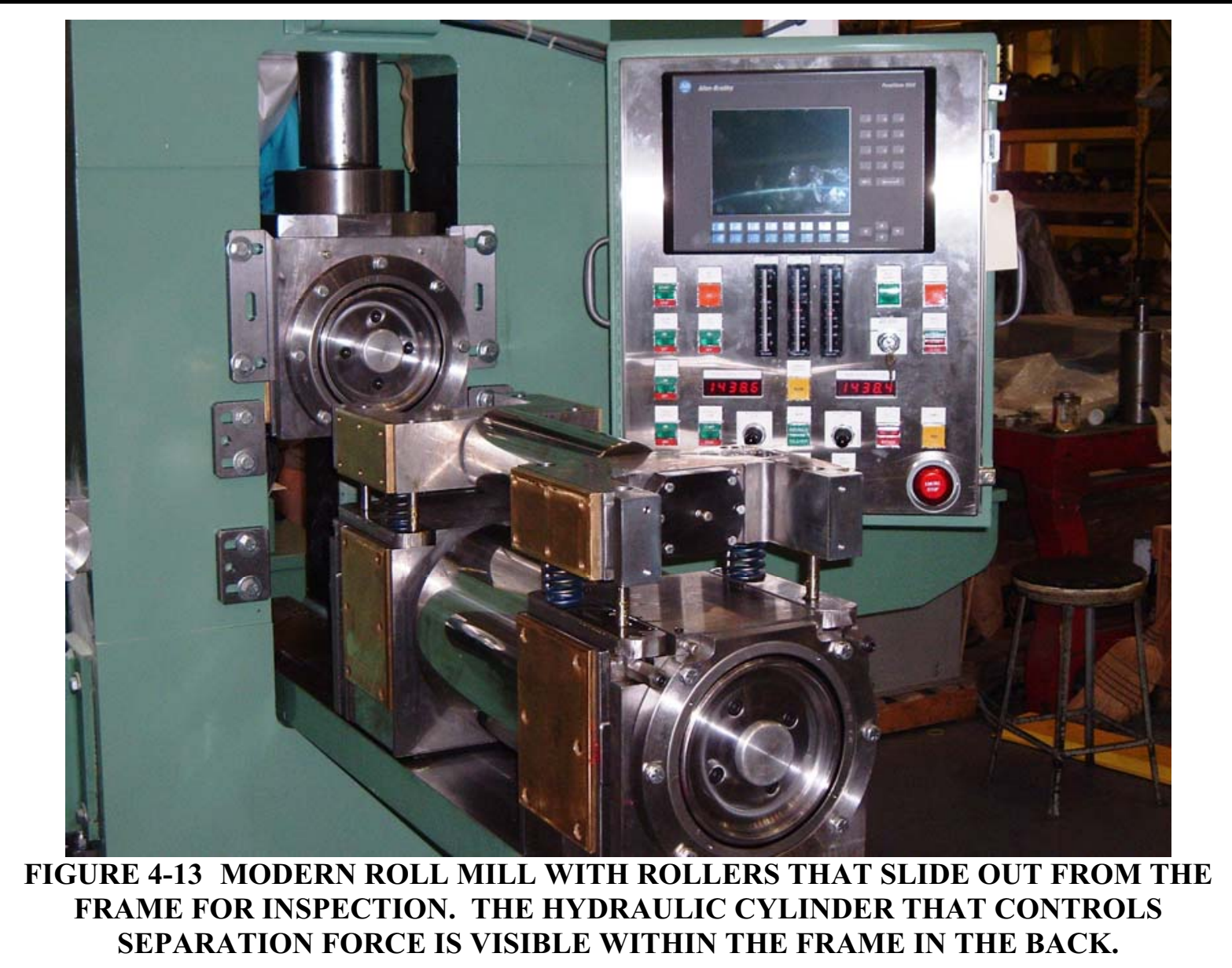

\subsection{Cleaning Procedures used in the FFC}

A number of cleaning procedures are performed as the fuel fabrication process is executed in the FFC. Ingots are cleaned prior to casting, salt from the heating bath is removed from the cast plates following the initial roll, the rolled plates are chemically cleaned prior to application of the zirconium diffusion boundary, and the foils and cladding are chemically cleaned prior to bonding. The bonded plates are cleaned following surface finishing prior to ultrasonic inspection, and the completed fuel subassemblies are cleaned prior to packaging for shipment. Although not a cleaning process per-se, the completed ATR fuel subassemblies are autoclaved to fix an approved oxide layer on the surface of the aluminum.

For cleaning, foils and aluminum cladding plates are typically immersed in sequential cleaning baths. The liquid cleaning solution may be heated or at room temperature and the baths are recirculating to provide agitation and continuously filtered. Parts placed in open racks are immersed, and soaked then removed. Racks drip drain above the bath and are then moved to the next cleaning solution or dried after rinsing. The volume and dimensions of the bath tank and rack is such that the process is geometrically favorable. Racks limit the number of parts that may be immersed at any time, and the potential for 'double batching' can be avoided using techniques such as hinging the rack so that it must be lifted from the tank prior to loading. Design studies (future) shall determine the practical level of automation present for use during the various cleaning procedures.

Space for cleaning stations, convenient to the workstations to which they apply, has been allotted in the arrangement. 


\subsection{Canning}

After cleaning, the 4" long cut and cleaned U-Mo plates are then sandwiched between two pieces of .005" thick Zirconium shim stock that acts as a diffusion barrier material between the fuel alloy and the aluminum cladding to which the fuel material is bonded in a process described later in the text below. The shim stock is tack welded to the inner surfaces of the cans to ensure everything in the stack remains in place during rolling. The sandwich is then enclosed in a flat can made of 1018 carbon steel and the edges of the can are then seal welded using low carbon filler material on an automatic wire feed TIG welding station, in Figure 4-14 below. The can plates are 0.10" thick therefore the can is $\sim 0.3^{\prime \prime}$ thick. The can is evacuated then purged with argon, via a short pigtail attached. The nozzle to which the pigtail is attached is stamped in to the two can halves, and seal welded around the perimeter when the can halves are welded. The pigtail is secured to the nozzle by welding. The inert gas prevents oxidation of the internal parts. Prior to assembly, the inside surfaces of the can are spray coated with Yttria to prevent bonding of the Zirconium with the can.

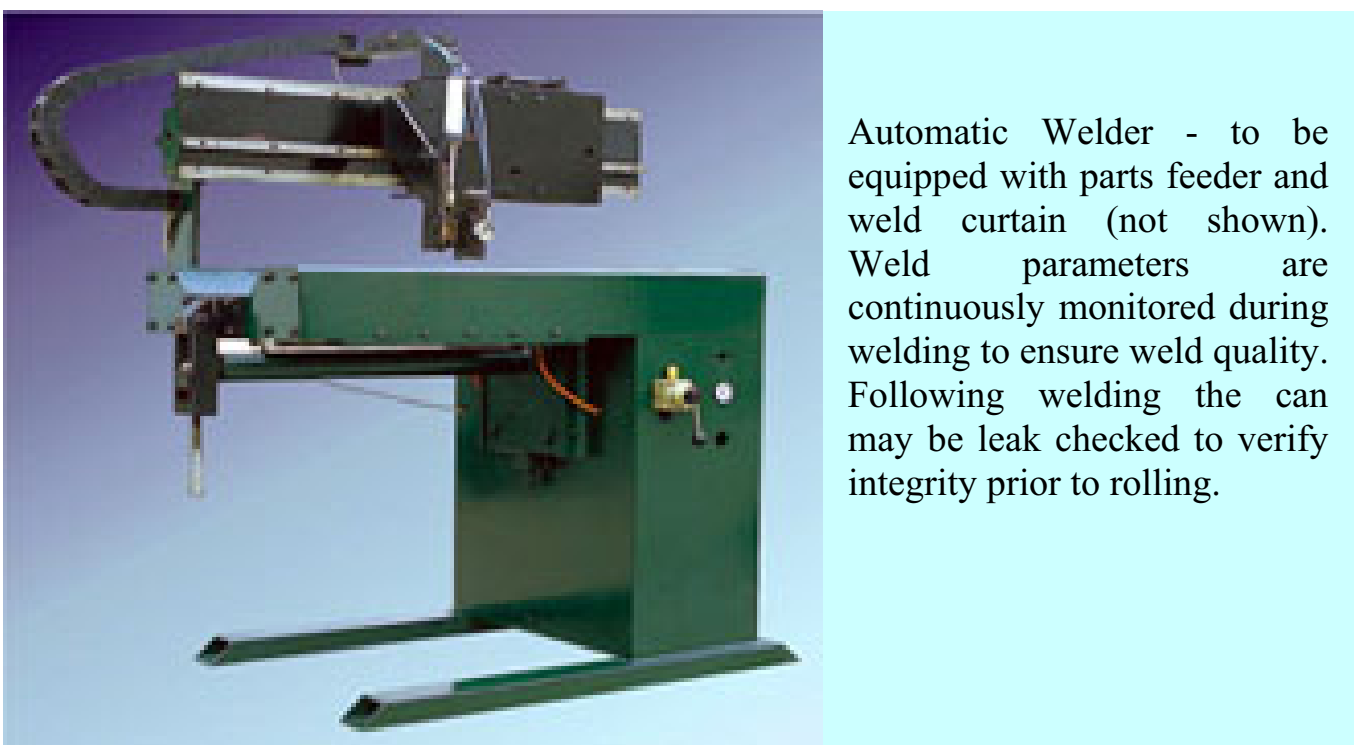

FIGURE 4-14 AUTOMATIC WELDER

At the automatic can welding station, a dual sliding part feeder is envisioned. The operator loads the can stack in a fixture on one side, this then slides to the vicinity of the welder, inside the curtain, which automatically executes the weld path. The just welded part, on the other side, slides out simultaneously, and the operator unloads that side, and re-loads it with another stack. By that time the other part is complete, it slides out and the next one slides in. The operator never enters the curtain enclosure surrounding the welder except when the machine requires service or consumables. Welding capability is locked out during service. 


\subsection{Roll to Final Thickness}

The can produced in the step above is roughly 6" long, 6" wide X 0.3 " thick with fully welded edges. The plate is heated in the can in an air atmosphere tunnel furnace and the hot cans are automatically fed to mill RL-002. Approximately 70 pieces per day are so processed. The $\mathrm{Zr}$ clad U-Mo is then rolled in the sealed can to a can thickness of 0.030 " to $0.45^{\prime \prime}$ thick. A tandem roll mill is envisioned with three pairs of rollers, like those shown in figure 4-15, and it may take multiple passes through the mill train to obtain the canned film thickness with a reheat between passes while the rolls are reset by the operator to the subsequent and final nip. Heat contained by the parts transfers to the rollers during the time of contact. By closely spacing the rolls, the individual stages are self feeding from roll to roll and the heat is retained in the part as it passes between the individual rolls. Threading guides between the roll stages ensure the cans are fed properly to the next stage.

Rollers used on the roll mills have a high surface finish. Over time with repeated use, the surface degrades. Rollers are refurbished as needed, usually by service contract. Contaminated rollers are disposed of as rad-waste.

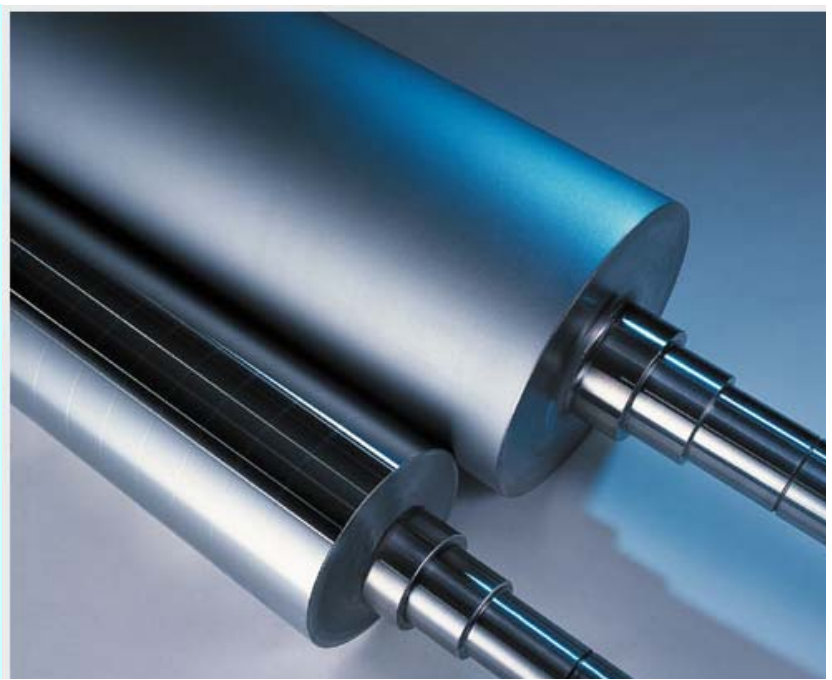

FIGURE 4-15 MILL ROLLERS

\subsection{Annealing}

Annealing of the can containing the rolled fuel foil is performed in an air furnace at $650-675$ (deg C). If the heat is too high, bonding of the fuel to the can may take place. The cans may be accumulated and annealed in a batch that can be processed concurrently. Processing of the fuel material in the FFC is administratively controlled such that quantities within any particular area are below levels where criticality considerations become a concern. The Fe-U eutectic forms at $725^{\circ} \mathrm{C}$ so any canned annealing will be performed below $700^{\circ} \mathrm{C}$. The parts dwell at the annealing temperature for $\sim 30$ minutes. The parts cool slowly in the furnace while temperature is monitored. Following the cooling, the parts are safe to handle (below $120^{\circ} \mathrm{F}$ ) and the furnace may be opened and the parts removed. If necessary a constraint fixture will be present in the furnace. This may be in the form of a heavy weight placed on the stack of parts in the furnace.

Because the can weld seams are subjected to plastic deformation and they must endure high strains (during rolling) while maintaining integrity, failures may occur. Such failed foils oxidize during annealing, and are rejected. 


\subsection{Shear Foil to Dimension}

Shearing is used as a method of opening the rolled can. The can is opened to remove the foil and the can becomes contaminated scrap. The foils, removed from the can, are rough sheared outside the final dimensions. Finally, cans are sheared into pieces suitable for disposal. Therefore, three shearing processes are in use at this point in the fabrication process, and space in the FFC layout has been made to accommodate the shear stations, shown in figure 4-16, and their support systems. To facilitate packaging and minimize waste volume, a scrap shear and a scrap bin are provided to facilitate size reduction and minimize labor input. Waste must be carefully surveyed to ensure alloy fuel material is segregated from can scrap. Cans are thin-wall following rolling and can be easily sheared open. Assuming the steel can rolls like the fuel foil inside, when the can is opened a fuel foil 0.010 " to 0.015 " thick will be inside. The foil size, after roughly trimming oversize, will be on average 40 " long (range from 48 " to 24 ").

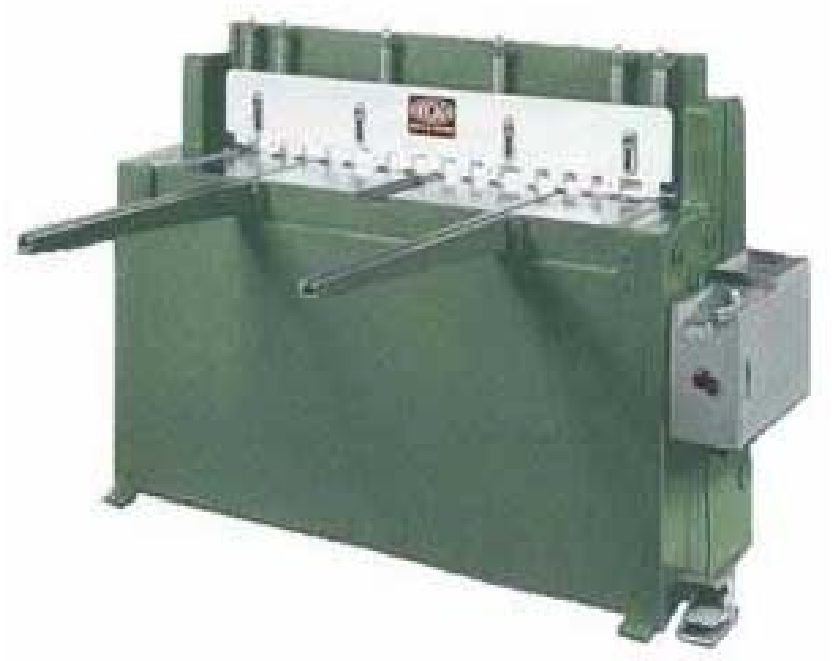

Metal Shear for can removal may be hydraulic actuated. Operator safety is enhanced by requiring use of material hold downs to secure stock prior to cutting.

FIGURE 4-16 HYDRAULIC METAL SHEAR

\subsection{Foil Cleaning}

Yttria, transferred from the can, will be present on the surfaces of the $\mathrm{Zr}$ clad foils that must be removed. Removal of the Yttria is necessary because it would interfere with the bonding of the foil to the Aluminum cladding. Yttria isn't soluble in nitric acid, so within a hood, the foils are wiped with an HF cleaning solution, which slightly dissolves the $\mathrm{Zr}$, freeing the Yttria, allowing it to be wiped off by a nitric acid wipe.

\subsection{Friction Stir Bonding}

Aluminum cladding is bonded to the foil using a friction stir bonding technique using tooling developed and successfully demonstrated at INL FASB. 

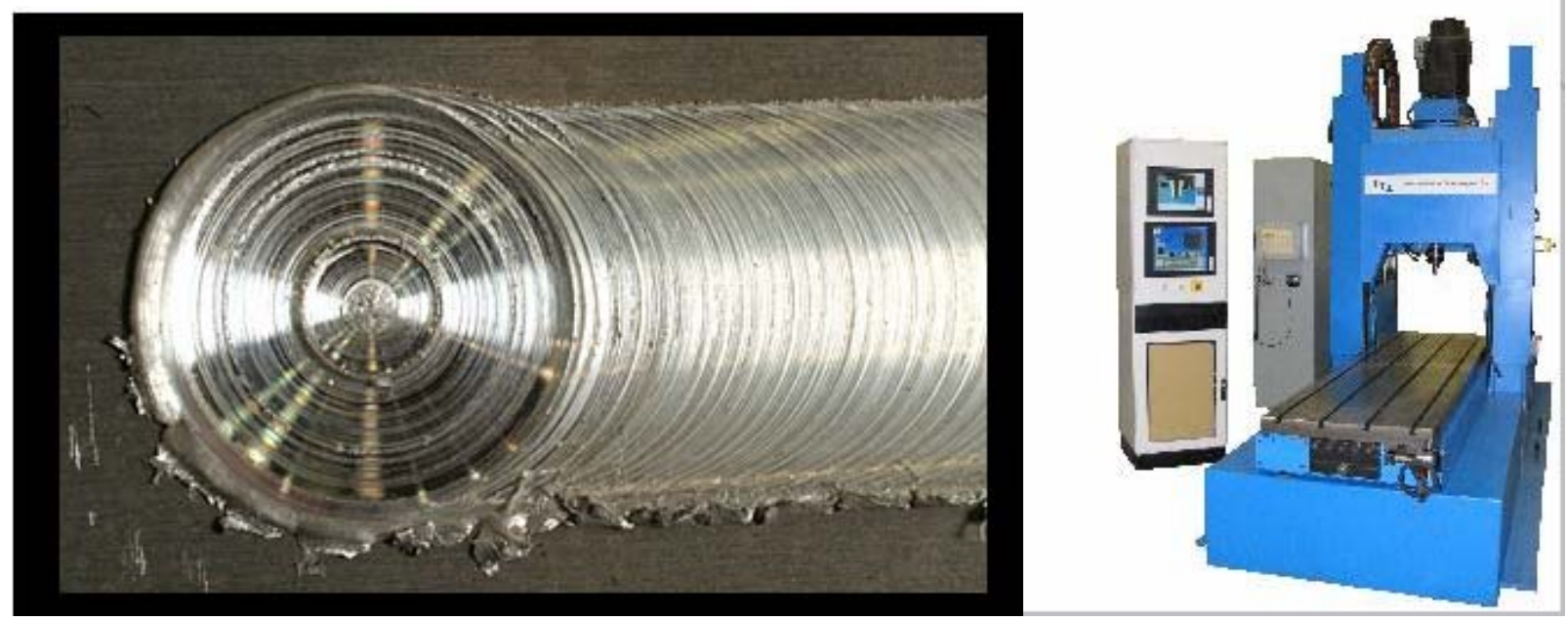

FIGURE 4-17 EXAMPLES OF FRICTION STIR BONDING AND MILL

Pressure applied to the rotating mandrill in the mill similar to the one shown above, Figure 4-17, generates friction on the aluminum cladding plate heating it to $\sim 60 \%$ of its melting point while it is under a high normal force, creating a bond between the cladding plate and the foil. The surface representative of the cladding after bonding is shown close-up on the left. A license from The Welding Corporation (TWC) for friction stir bonding (a \$50,000 allowance is included in the estimate). Formation of a bond throughout the entire surface, on both sides, without any voids is necessary to produce acceptable fuel plates. In order to perform the stir bond, the cladding requires a perimeter margin around the finished part for clamping. Clamps are engaged by the operator to hold the aluminum-fuel-aluminum sandwich securely to the mill table. After the part is bonded on one side, the operator unclamps the part and turns it over, reclamps the part, then bonds the other side. An 'area filling' routine is executed by the mill's numerical control program to ensure the whole foil face area is covered without dependency on operator skill.

The friction bonding technique requires two cooling systems, one for the tool head and one for the anvil. The tool head uses a closed loop chiller and the anvil is cooled via building cooling water. The capability to control both coolant flows is necessary, and an alarm or operator notification is needed if capacity requirements are not being met.

\subsection{Alternate HIP Process}

Use of a Hot Isostatic Press, Figure 4-18, process to produce the bond between the fuel foil and the aluminum cladding is a successfully demonstrated technique for meeting high performance plate fuel fabrication goals. The HIP has the capability to produce a uniform, controllable and completely void free bond on both sides of the plate, in an entire stack of plates at once without a lot of hand work, or need to turn the plates to do the other side. The plates are processed by placing the foil between the aluminum cover plates. This perimeter of this assembly is welded under vacuum in the electron beam welding unit. This forms a hermetically sealed assembly which is placed into the hip unit without an additional over pack. A batch of the assembled plates is bound together with rigid spacers to prevent warping of the plates during the HIP process.

The use of HIP bonding versus the Friction Stir bonding technique will be a future alternative study and value engineering activity performed during the conceptual design phase of the project. One bonding technique or the other will be selected; the deselected method will be deleted 
entirely from the FFC scope. Issues unique to each process and differences between the methods of bonding implementation are described in detail below.

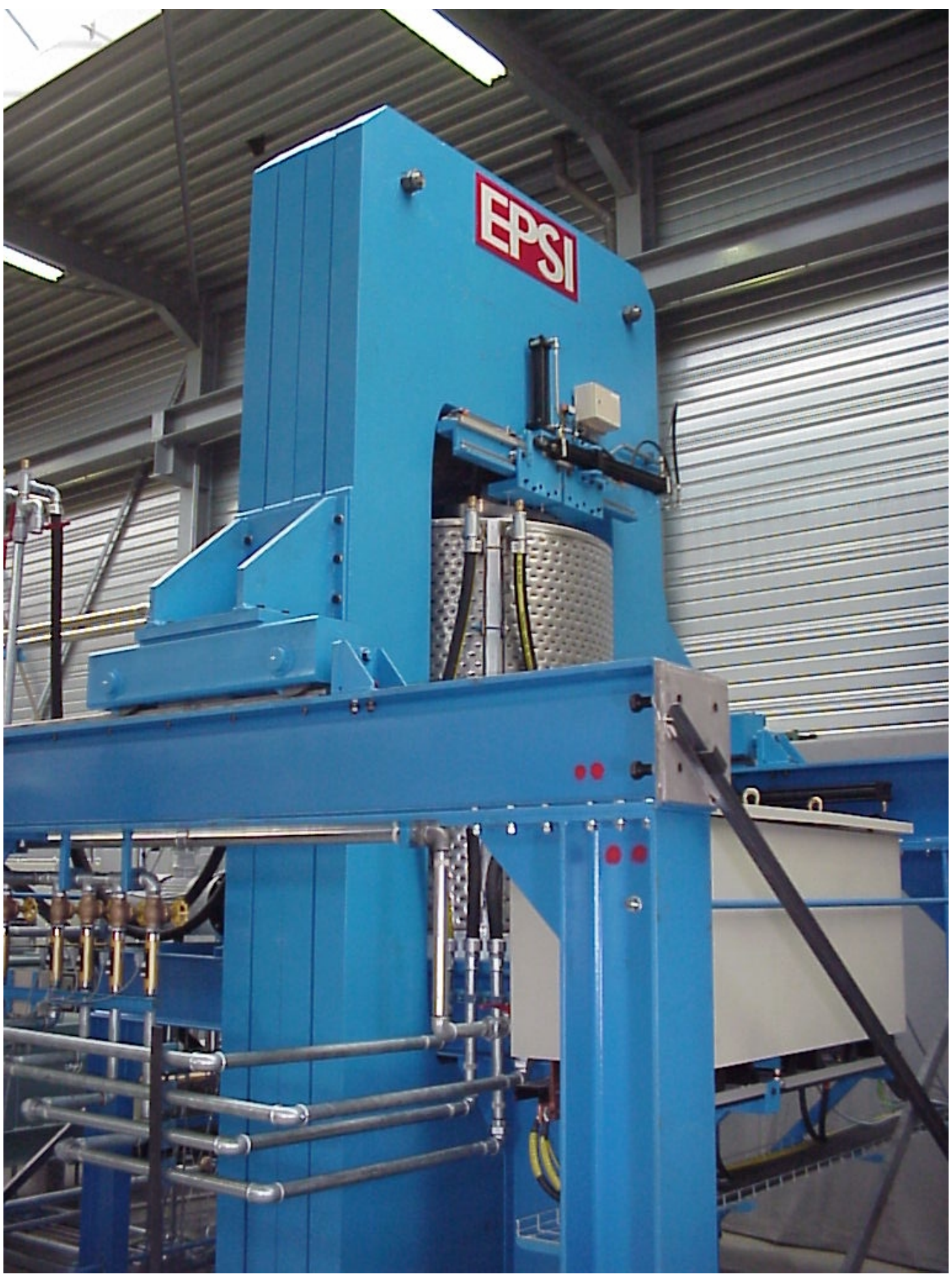

FIGURE 4-18 EXAMPLE OF A HOT ISOSTATIC PRESS

For monolithic fuel fabrication for high performance research reactors, a 20 " diameter X 60" long void work cavity is needed, similar to the unit shown. 
- $\quad$ Pressure Vessel Assembly

- $\quad$ Furnace \& Heatshield Assembly

- $\quad$ Part Support Tooling

- Control/System

- Process Gas Handling \& Compression System

- Vacuum System

- Electrical Power Distribution System
Two HIP units are indicated to achieve the production throughput. Each system includes the subsystems and components shown. In addition to electrical service, and connection to the control system and interlocks, an overhead monorail hoist and trolley is present to load and unload parts. A crane is not expected to be necessary to service the pressure vessel and the moveable external yoke over the 40 year life of the facility.

A pit area is necessary within the FFC to accommodate the HIP units. For this option, the HIP room has been added to the FFC arrangement, as a lean to structure, or wing, adjacent to the Shop, on the opposite side from the Assembly Area. The HIP room is entered from the Personnel Corridor. Material is passed from the shop using the Pass-Through provided, which maintains the primary confinement integrity of the shop and avoids movement of potentially contaminated material through the normally non contaminated corridor. All fuel material is handled within a can in the HIP room. Since bare fuel is not handled the HIP room operates as an uncontaminated area. Contamination would be present only in the event of an off-normal event such as a ruptured can or HIP vessel seal leak. The HIP room is suitably isolated from the environment and the personnel corridor such that any contamination present could be cleaned up without spread to adjacent areas.

Sealed HIP work packages are made up in the shop. The plate assemblies are placed between the strongbacks and the stack is bound together. 


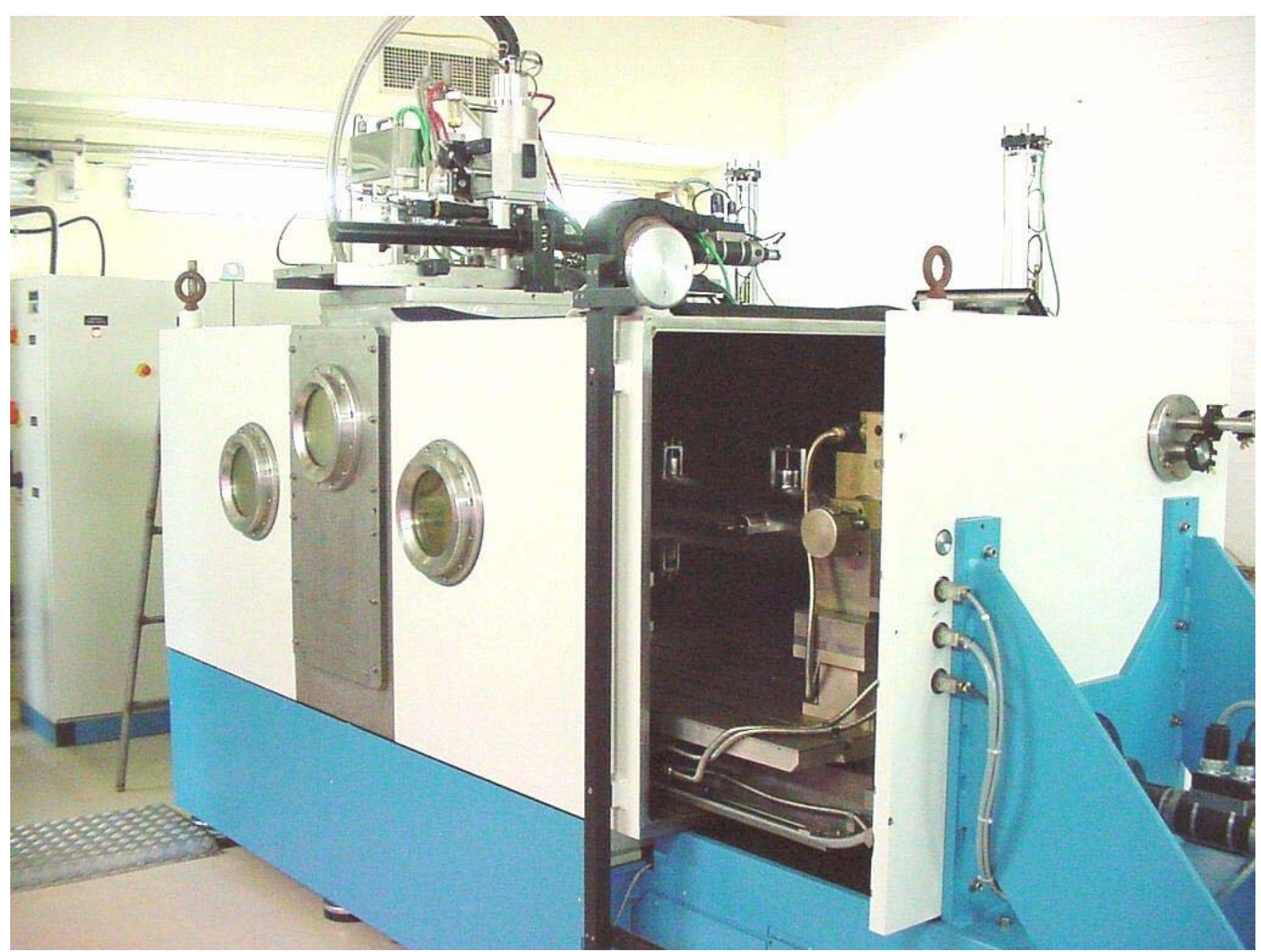

FIGURE 4-19 HIP CAN EXAMPLE

The work package is passed through from the shop to the HIP room, and using the monorail and handling device, it is lowered into the HIP vessel inside the furnace cavity. Chairs inside the furnace position the work package at the proper position and elevation. The heat shield is stacked on top inside the cavity and the vessel lid is positioned. Using the motion control rack, the yoke is moved laterally to constrain the top and bottom vessel covers. Under high heat and pressure, $560^{\circ} \mathrm{C}$ and $15,000 \mathrm{psi}$, the plate assemblies are compressed, bonding the inside faces of the aluminum cladding plates to both sides of the fuel foil. The $\mathrm{Zr}$ coating on the foils acts as a diffusion barrier preventing diffusion of the U-Mo into the aluminum. The HIP process is initiated by the operator and the heating and pressurization sequence is performed automatically. Following the sequence and after cooling and depressurization of the HIP vessel, the can is removed and moved via the transfer port back to the shop where it is opened. During routine operation the HIP room operates in an uncontaminated state. 


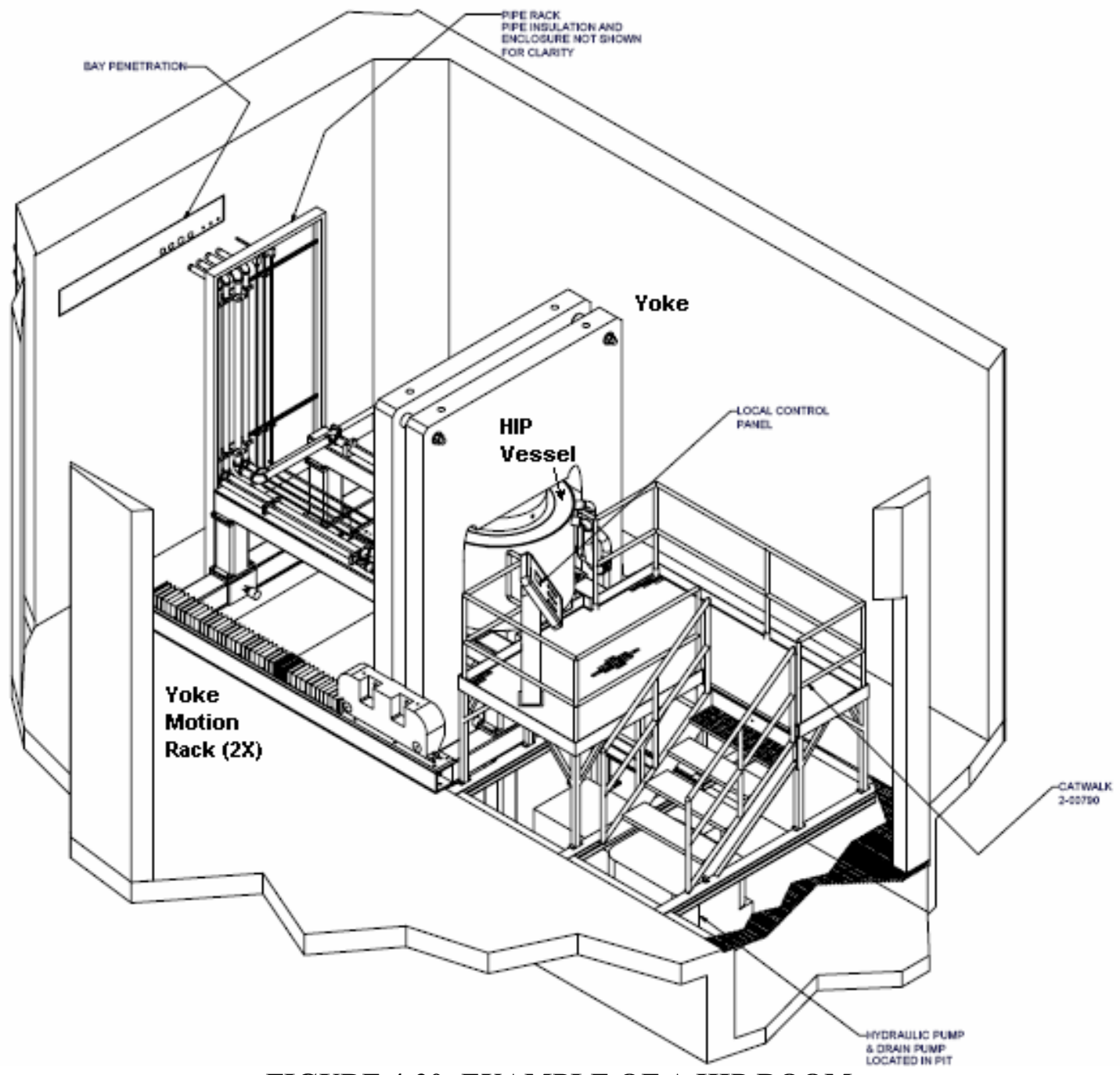

FIGURE 4-20 EXAMPLE OF A HIP ROOM

In the arrangement shown above (from an unrelated project), the yoke is visible as well as the HIP vessel itself. The yoke moves back (on the rack shown) allowing the vessel top cover to be lifted for access to the work zone inside the furnace contained within the vessel. The vessel and its covers, yoke, furnace, heat shield and work package are structurally supported by a steel stand anchored to the floor of the pit. The grade level pit cover is covered with removable floor plates and plate support structure. The 17' wide x 60' long rectangular pit, 13' deep, accommodates both HIP units. Forklifts may be brought through the airlock in from outside, but do not travel over the pit, although shop and maintenance personnel and hand-cart traffic routinely travel across the pit covers. The pit is accessed by stair cases with gates at either end; lighting drainage and ventilation systems are present in the pit. Oxygen monitors with alarms are present in the pit; the HIPs operate with an internal argon atmosphere which is heavier than air. Control panels are located away from the units, in a concrete walled HIP control room and automatic interlocked sequences are remotely engaged or concluded. Lockouts will be in place when servicing the units. A 5 ton overhead monorail, with dual hoists and trolleys, used for work-package removal/placement and routine component removal for periodic inspection testing, is present over the pit area. The hoist may also be used for heat shield removal, furnace removal, work support 
chair, or top or bottom cover removal for maintenance or inspection purposes. The work package size is limited by criticality considerations. The HIP process is supported by utilities including vacuum pumping, compressed air, liquid argon vaporization, gas pressurization and recovery systems, furnace controller, yoke motion and top cover interlock, cooling water and external connections to the facility alarm systems. Many of the supports are routed to the HIP stations via trenches in the floor of the pit. The pit will be equipped with sumps which can be drained with a sump jet.

To reduce facility costs, the need for an overhead crane system suitable for removing the yoke or the vessel has been eliminated from the design following checking with a potential supplier of HIP systems for the FFC. During a chain of correspondence: the following dialog was provided by the Sales Manager for EPSI:

"...to do a valid NDT vessel inspection on an EPSI vessel design there is no need to remove the vessel or yokes. We have qualified procedures to inspect the vessel and yokes with minimal disassembly of the HIP unit. Therefore the overhead crane only needs to be rated to lift the closure assembly or the heaviest items located in the pit or the heaviest item the customer will be lifting. EPSI would suggest a 5 ton crane at this point to be safe, but will confirm crane capacity needed when the design is finalized."

This cost saving recommendation reduces equipment cost (eliminating the need for a 25 to 30 Ton crane) and lowers the roof and ceiling height over the HIP room, which reduces air conditioning costs. In-place NDE will require a service outage for removal of the upper and lower heads, but it will be of a shorter duration then complete disassembly and removal of the yoke or vessel, shipment, turnaround, reinstallation, and re-commissioning. Other suppliers of HIP equipment may not have the inspect-in-place configuration; therefore the final design of the FFC should take this into account.

\subsection{Selection of a bonding process}

The two fabrication processes described above (HIP and friction bonding) targeted to bond monolithic fuel plates are emerging technologies. As such, development in many areas is still ongoing and the best educated opinions available from subject matter specialists were utilized to prepare this study report.

Because the selection of one method over another has significant consequences on the overall design and cost of the fabrication facility, it was decided to (at a fundamental level) leave the option open for either process (a process down select milestone is shown in the project schedule [See Section 11]).

Although both techniques have been successfully demonstrated on mini-plates, for the purposes of this report friction stir bonding has been chosen as the reference method. The HIP option has been broken out as a special case in the project cost estimate. An outline of pros and cons associated with each method is provided below. It must be stated that these are 'as understood at this time', and as with any ongoing R\&D effort the actual progression may move in an unanticipated direction.

\subsubsection{Friction Bonding Pros}

- Lower equipment capital cost

- Fewer utility costs (lower power consumption, no argon usage etc.)

- Less waste generation (with the HIP can requirement) 
- Smaller facility footprint/cost

\subsubsection{Friction Bonding Cons}

- Single-pass process (both sides of each plate must be machined)

- Lower success rate (on test plates fabricated to date)

- High degree of operator attention required

- More operators required/higher personnel costs

\subsubsection{HIP Pros}

- Batch process

- Higher success rate (on test plates fabricated to date)

- Less operator attention required

- Fewer operators required/lower personnel costs

\subsubsection{HIP Cons}

- High equipment capital cost

- High utility costs (power, argon etc.)

- High waste generation (with the HIP can requirement)

- Larger facility footprint/cost

\subsubsection{HIP Implementation}

In addition to the choice between Friction Bonding and HIP there is still ongoing development in how best to perform and implement each process. Specifically the method used to isolate the HIP plates from the processing media (the interface between the cladding in a stack of plates and the interface between the cladding and the fuel foil) must be hermetically sealed, under vacuum to achieve a successful HIP bond. Two methods have been employed:

\section{A. HIP Can Method}

To make the HIP can assembly, a steel can is built around a stack consisting of a number of plate assemblies (two aluminum cladding plates with a fuel foil sandwiched in between). These spacers are in the form of rigid 'strongbacks' that keep the fuel plates flat during processing. This method has the advantages of providing very good isolation from the HIP media and being leak testable but has the disadvantages of being expensive in time and material as well as a tendency to fuse the plates to the strongbacks, necessitating special precautions.

B. Vacuum welding method:

Vacuum welding of the perimeter of the plates themselves is a newer method employed to prepare the plate assemblies for HIP with a focus on lowering production costs during mass production (by eliminating the can). In this method the perimeter of the fuel plate assembly is welded under vacuum to hermetically seal the fuel plate from the HIP media. In this method the welded plate assemblies would be stacked between strongbacks to provide rigidity during the HIP process to keep the plates flat (unlike the hip can method, the strongbacks would be exposed to the HIP media). 


\section{Perimeter Weld with Electron Beam Welder}

The method for achieving the plate perimeter weld is still being investigated. Currently an electron beam welder is the reference method. E-Beam welding uses a tightly focused beam of excited electrons to provide the energy to fuse materials together. Due to scattering of the electron beam in air the process is almost universally performed in a high vacuum. E-beam welding is currently being used in monolithic R\&D (but is admittedly in its early stages) and is the reference method for HIP fabrication. The downside of E-Beam welding is the high capital cost $(\sim 4 \mathrm{M})$ and large footprint of the equipment.

\section{2. $\quad$ Perimeter Weld with Ultrasonic welder}

A second method for achieving the plate perimeter weld that has the potential to emerge as the favored technology is ultrasonic welding where a contact probe vibrates the material causing friction and localized welding. When used in a seam welding configuration a hermetic seal can be made. While UT welding is typically performed in air, vacuum operation is well known in the industry. The advantages of this method are lower cost $(\sim 200 \mathrm{k})$ and a smaller footprint.

\subsection{QA Inspection}

Many in-progress and quality assurance tests and measurements are performed in the FFC. A micro-hardness tester for rolled foils a tensile testing machine (shown below) for testing of swages made during final assembly, and an optical microscope for microstructure inspection are used. Analytical lab instruments are also present to qualify and verify fuel alloy chemistry, isotopic content and purity such as an AES and an ICP-MS. A deep throated micrometer is provided for foil thickness measurements and a vernier is used to verify length. A coordinate measuring machine (CMM), similar to one model shown below, Figure 4-21, is provided to conduct flatness and dimensional inspection of clad fuel plates.

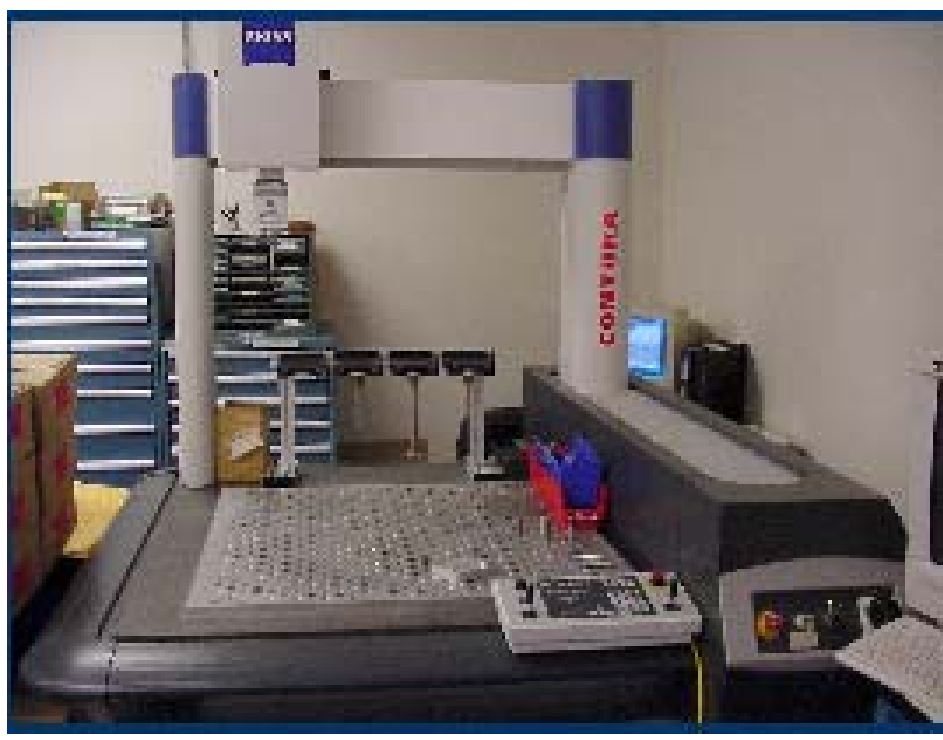

CMM for plate measurements can detect flatness variations and can measure overall part dimensions. Measurements can be automated or manually directed.

FIGURE 4-21 COORDINATE MEASURING MACHINE (CMM) 


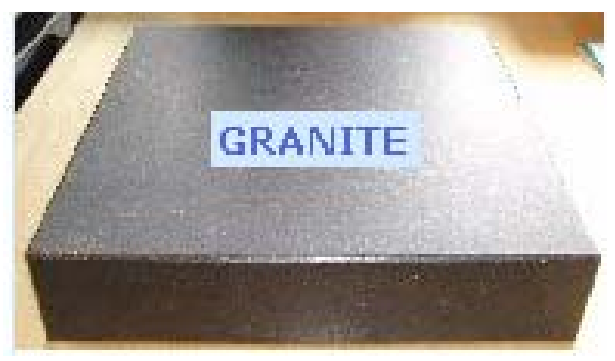

Floor stations exist where in-process measurements and verification of setups can be made. These stations consist of a granite flats on a stand with parallels and measurement instruments.

\section{FIGURE 4-21 EXAMPLE OF A FLOOR STATION}

It is assumed that the FFC will be located on a DOE site where analytical lab capabilities exist, and no highly unusual or specialized lab equipment will be needed. QA holds are interspersed throughout the manufacturing process and include alloy, chemical and isotopic properties, alloy impurities (Oxygen, Nitrogen, Sulfur and Carbon), determination of foil thickness and position, fuel density measurement, and dimensional inspection of the aluminum cladding thickness (including the machined recessed pocket) and fuel subassembly end pieces. Inspection holds are utilized prior to assembly of the fuel foils with the aluminum cladding plates and inspection of the fuel plate following fusing of the Al cladding plates with the foils. Space in the arrangement has been allotted to accommodate instruments, personnel access, support systems and controls. Storage space has been allotted for holding material during pre-and post inspection periods.

\subsection{Completion of fuel plate manufacturing}

The fuel manufacturing process steps following bonding are outlined below.

1. Mark plate ID (using a temporary mark: see note below)

2. Friction Bond (or HIP)

3. Rough-trim (remove clamping boundary)

4. Flatten plate

5. Finish both surfaces of plate (on mill)

6. Flatten plate (as needed) on roller leveler (shown schematically below)

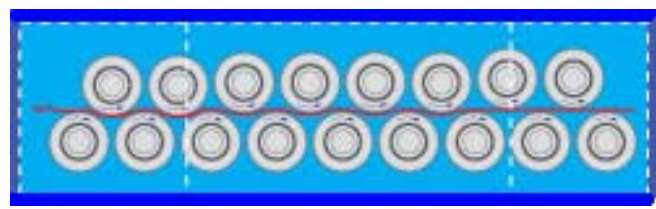

7. Fluoroscope

FIGURE 4-22 ROLLER LEVELER

a. Locate fuel within cladding

b. Punch index holes

c. Move back to shop

8. Final Serial number marking (this mark shall be the final plate marking, see below)

9. Final Trim (on mill) using index holes punched in step 7 above, and Deburr

10. Degrease plate (clean using water based cleaning solution, then rinse and dry)

a. Perform subsequent handling steps using white cotton gloves

11. Perform dimensional and surface inspection 
12. Radiography (on either 130 or $225 \mathrm{kV}$ Real Time Direct Radiography machines, set up to advance part in one direction as scan takes place, producing a $25 \mu$ and $0.5 \mu$ (micron) micro-focused image)

a. Density

b. Fuel location

c. Retain Data (image is stored digitally, large amounts of data are kept throughout life of fuel)

13. Automated Ultrasonic test (UT) - inspect for:

a. Bonding

b. Discontinuities and defects

c. Minimum aluminum cladding thickness

d. Retain data (large amounts of data are kept throughout life of fuel)

e. Part is automatically scanned using 'terrain following' feedback technique, under water (through and echo) and complete coverage is achieved

14. Cold work finished and marked plates using hydraulic press and special tooling dedicated to plate number and fuel type. Plates are cold formed (stamped to a concave or spiral shape as viewed from the end) to meet the fuel configuration requirements of the various reactors. Some types use flat plates and this step is not necessary.

Note that the fuel plate and subassembly configuration varies for each of the five HPRR reactors for which fuel is fabricated at the FFC. The list above is intended to be general, or typical. Specific peculiarities, for example, such as 'grooving' or finning of the 0.080 " thick MIT fuel plates, which requires a dedicated machine, have been accounted for the in the FFC design and layout, even if not mentioned here.

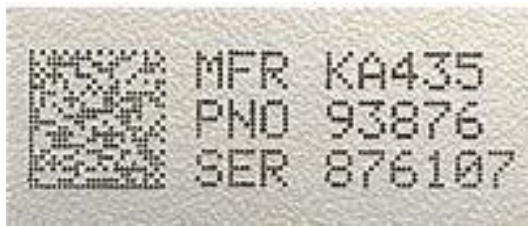

FIGURE 4-23 EXAMPLE PART MARKING 


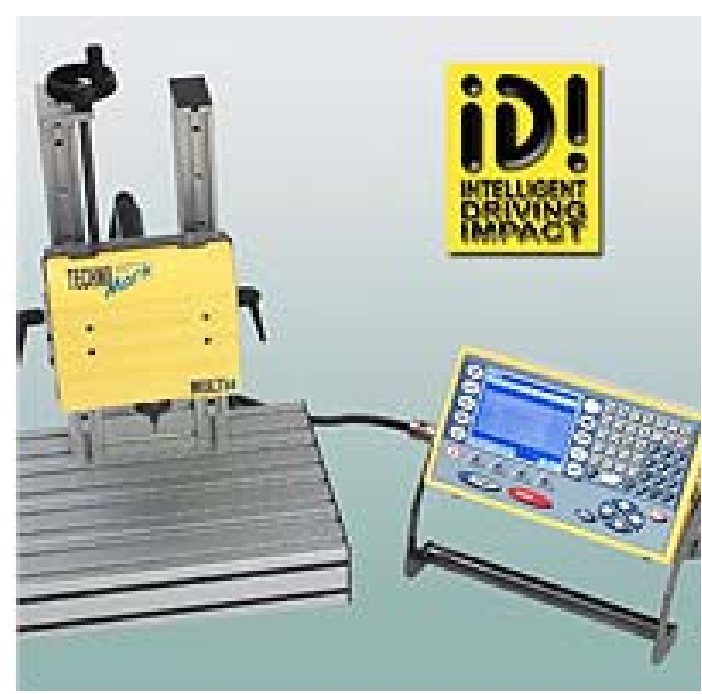

FIGURE 4-24 COMMERCIALLY AVAILABLE MARKING EQUIPMENT

Note on Maintaining plate ID:

An important part of keeping the accountancy record clean and balanced will be tracking of the fuel at all times during the fabrication process at the FFC. Batches of ingots (and even foils) can be tracked as a group of multiple items (so long as the mass can be checked). As soon as an individual foil is split off the main batch, such as for bonding, or for inspection purposes, it must be assigned a unique ID that accompanies the plate during each step that it is subjected to.

While friction bonding will obliterate the processed area, the plate assembly can be marked on the perimeter of the frame and this identifier can be moved inward by re-marking as the outer sections are removed during processing. After fluoroscopy (where the final plate region is defined) the final marking can be made. This procedure will be amended as necessary as the design ensues and process methodology evolves. In any event, following plate surface finishing and edge trimming, the final mark shall be present and easily visible on the finished fuel plate, and this identity shall preserve traceability all the way back to the alloy (melt) from which it originated which captures the enrichment, alloy purity, isotopic and chemical composition. Once the fuel subassembly is completed, its identity shall be comprised of the identities of the individual fuel plates.

\subsection{Sub Assembly Fabrication}

The Sub-Assembly Fabrication area is approximately $12,000 \mathrm{ft}^{\wedge} 2$. It is north of the shop and is located convenient to the secured controlled storage area (where completed plates are stored and accumulated), the inspection area, and the shop. The assembly area is uncontaminated: bare alloy fuel material is never present in the assembly area.

The fuel element subassembly build-up process involving the completed fuel plates are outlined below.

1. Transfer the daily lot of fuel plates from the secured controlled storage area to the Assembly Area. Track accountability of material.

Completed fuel plates of all of the various sizes are stored in the secured controlled storage area and accumulated until all parts are present and a given fuel sub-assembly campaign is executed.

2. Clean plates (using nitric acid followed by cold DI water rinse, followed by hot DI water rinseto be done within 72 hours of swaging)
a. Fuel plates
b. Side plates

3. Move fuel plates from cleaning to assembly station

4. Gamma scan plates (individually)

5. Place fuel subassembly end pieces on dedicated swaging assembly fixture

a. Swage plates into side plates; one at a time, building out from one side. 
b. The swaging process involves a roller that passes along the full length of the fuel plate, closing the gap between the 'comb' or grooved fuel subassembly side piece and the fuel plate. This retains the plate while allowing it to expand thermally along its length during service.

6. Perform quality inspection

a. Dimensional

b. Pull Test: Note: typically a mockup will be built to periodically test swaged joints on surrogate fuel plates and ensure sage machines are properly adjusted. Actual fuel subassemblies will NOT typically be tested.*

c. Inspect Channel gap

d. Clear QA hold [NOTE: it may take some time to complete this inspection (and also the quality inspection below) - unfinished assembly to be placed in interim storage in the assembly area]

* Note that the swages securing the individual plates may be tested with a pull test apparatus such as an Instron testing machine. This is done on a prototypical basis, and the equipment performance is periodically re-verified.

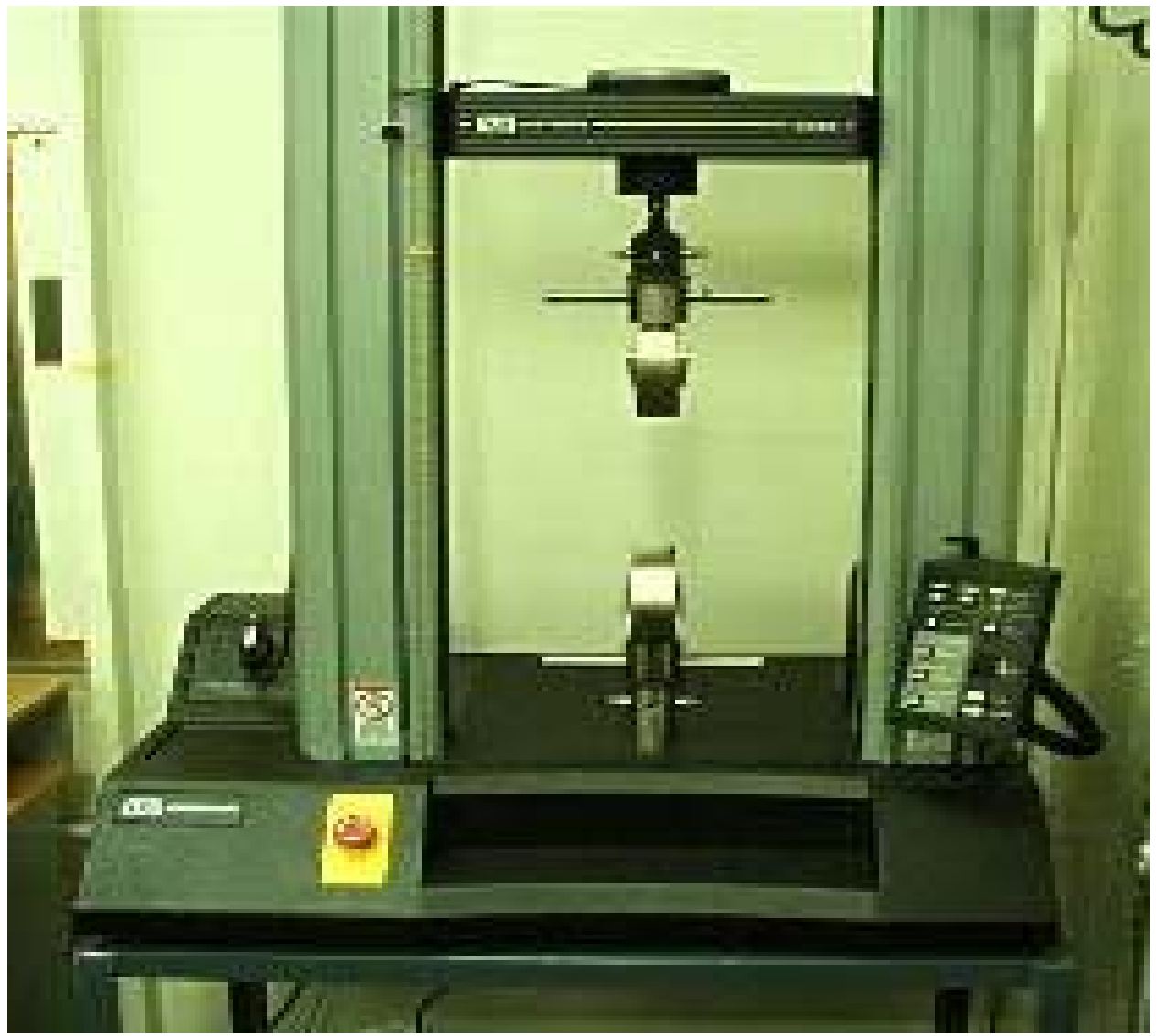

FIGURE 4-25 TENSILE PULL TEST APPARATUS FOR PROTOTYPE FUEL SUBASSEMBLY

7. When assembly is complete (all elements are present) and swaged, weld on end boxes on dedicated welding fixtures

8. Final machining of fuel subassembly (end pieces)

9. Quality inspection of fuel element 

a. Dimensional
b. Channel gap
c. Clear QA hold

10. Final subassembly cleaning
a. Cold Deionized water
b. Hot Deionized water
c. Blow dry with compressed filtered dry instrument air or bottled N2

11. Gamma scan fuel subassembly

a. Part is rotated as scan takes place, and by either translating the part or the instrument, scan the full length of the subassembly throughout $360^{\circ}$ of rotation.

12. Autoclave (ATR Only)

a. The autoclaving process generates the desired oxide film

b. Drain water from autoclave

c. Dispose of spent water

d. Allow element to air dry

13. Place the (dry) element in plastic bag

14. Move back to the secured controlled storage area (Transfer custody to the secured controlled storage area)

15. Store, accumulate sub assemblies

16. Accumulate until shipping quantity is reached

17. Package in shipping container

18. Move to Packaged Fuel Element Storage Area. Store loaded shipping containers within secure storage area. Containers are accumulated until such quantities are available that a full core can be shipped. This relates to 6 months of storage - for example: 20 boxes of four completed element subassemblies.

19. Following the accumulation of packaged completed fuel subassemblies and compilation of the required $60-80$ pages of QA documentation that accompanies the shipment, shipping containers are moved from the accumulation area to the shipping dock and a forklift is used to load the truck. Elements are then shipped to the destination. 


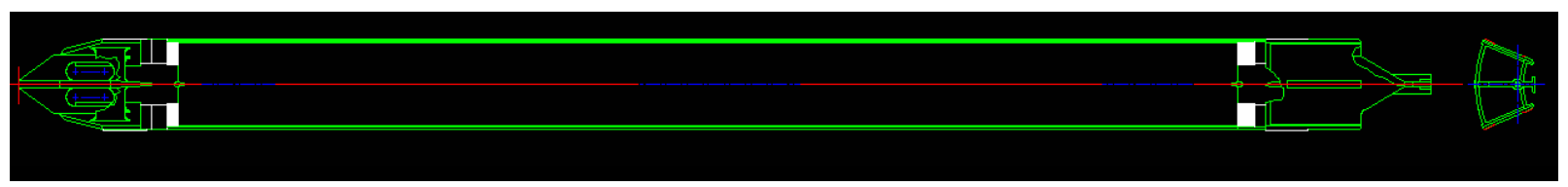

FIGURE 4-26 ATR FUEL SUBASSEMBLY
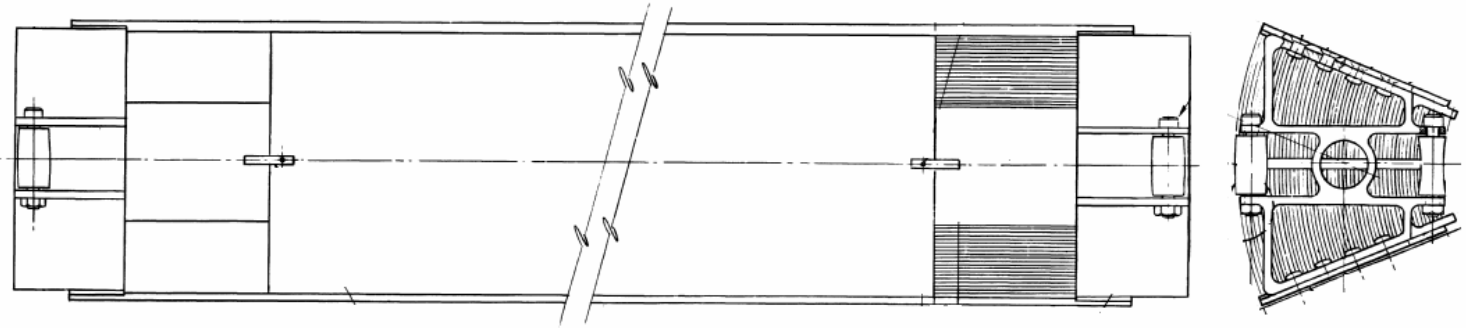

FIGURE 4-27 MURR FUEL SUBASSEMBLY

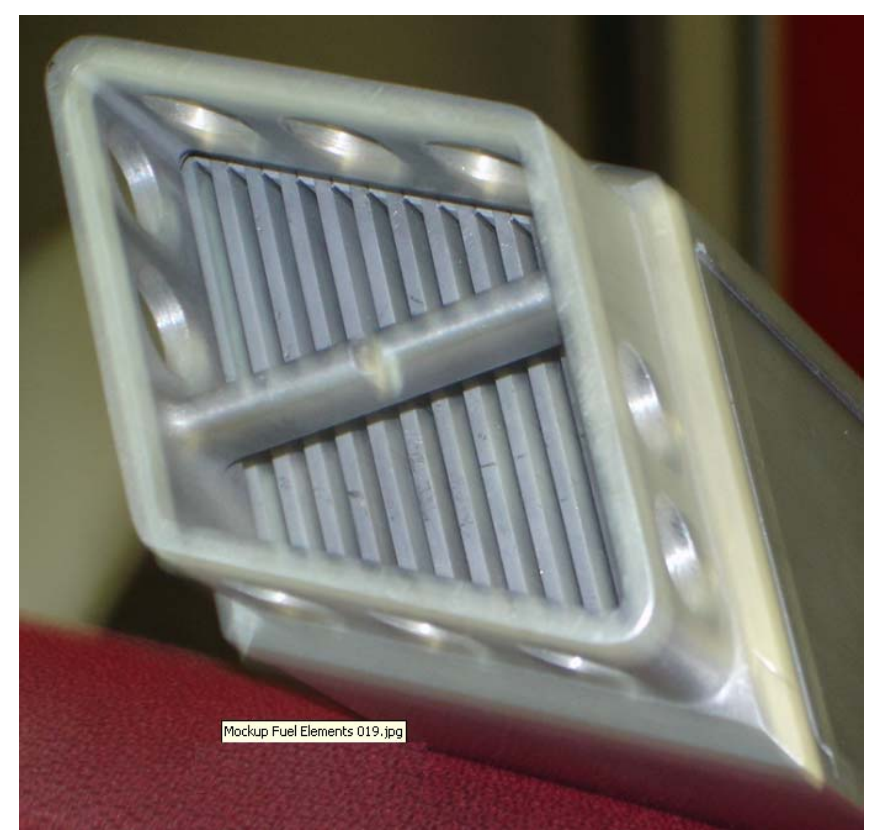

FIGURE 4-28 END OF MIT FUEL ELEMENT SHOWING FUEL PLATES 

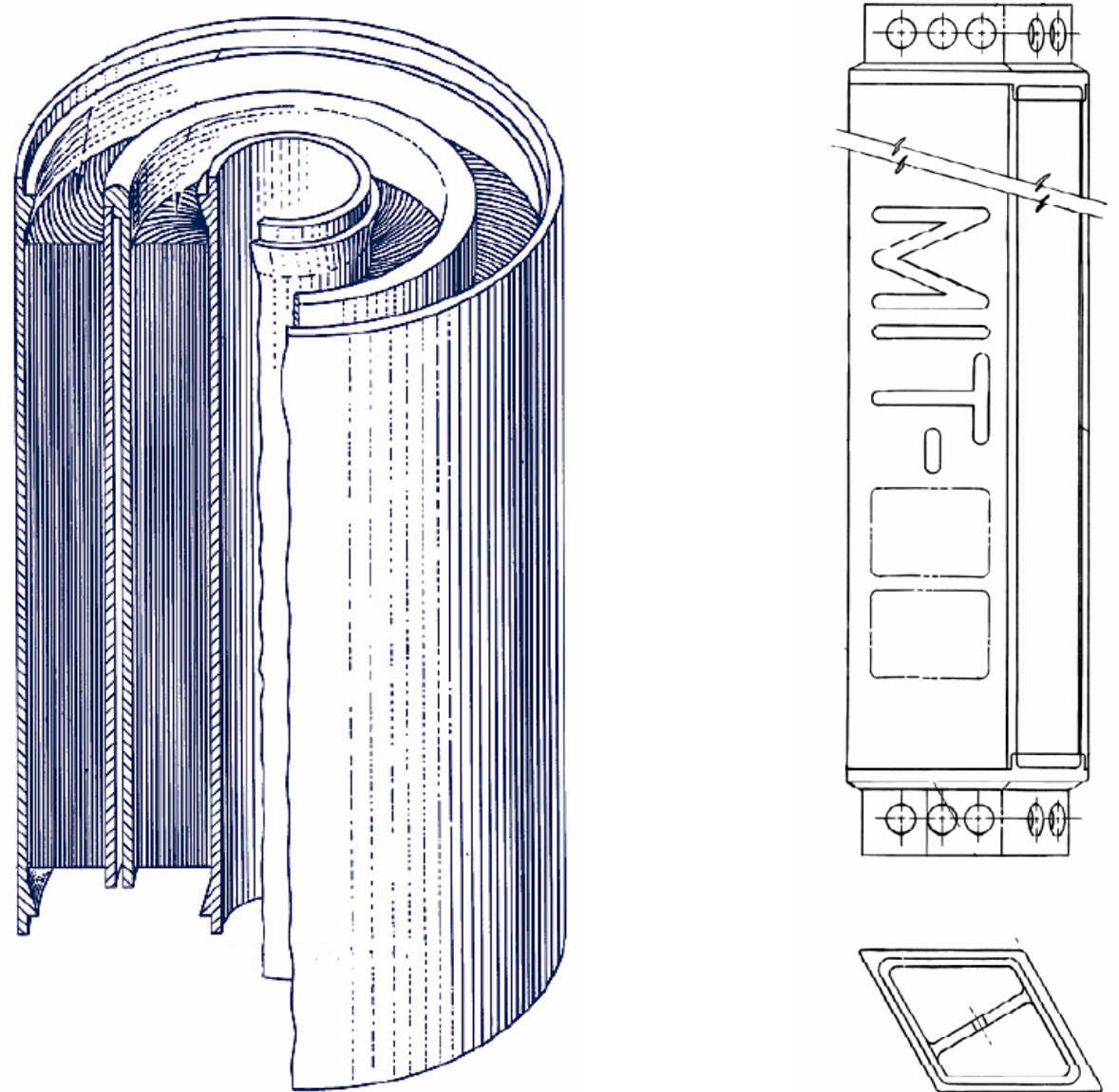

FIGURE 4-29 HFIR (LEFT) AND MIT FUEL SUBASSEMBLIES

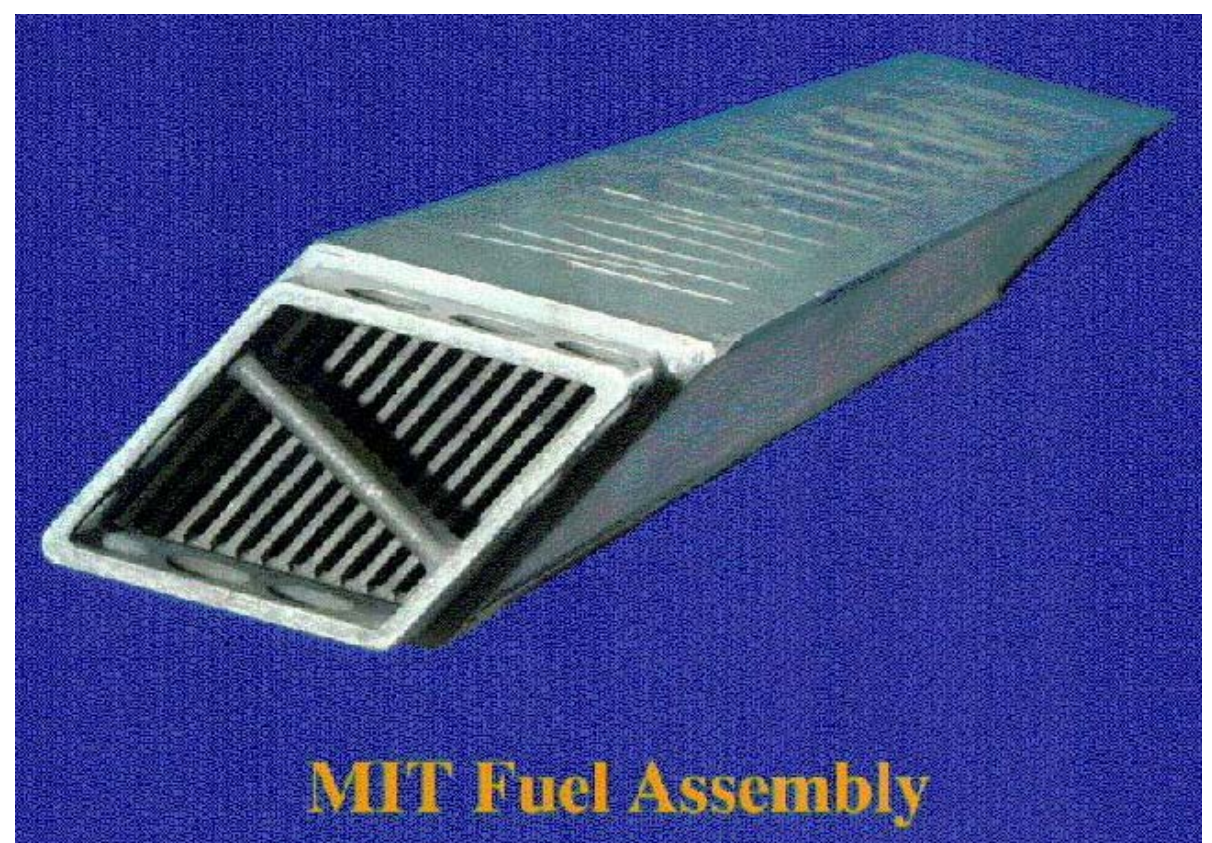

FIGURE 4-30 RENDERING OF MIT FUEL ASSEMBLY 
4.18 In-Process Intermediate Secured controlled storage area

In-process fuel forms are stored in the secured controlled storage area and accumulated and staged for efficient fuel campaign management. The intermediate secured controlled storage area, located adjacent to the shop, is equipped with a custom storage rack system. The secured controlled storage area has a pass-through which passes below the Personnel Corridor for efficient fuel material transfer to and from the shop. The in-process intermediate storage secured controlled storage area is not generally used for storage of raw fuel alloy material. Dedicated storage locations with geometrically favorable configurations exist for up to six days of production of each stage of the fuel manufacturing process. This maintains fuel in all its interim configurations in stacks 1" thick separated from the next stack by at least 12". Sets of racks are present for two fuel types providing storage capacity for each of the intermediate in-progress stages which may be processed concurrently. A QA hold area is also present in the intermediate secured controlled storage area for in-process material awaiting inspection or awaiting completion of certifying documents. Since unclad alloy fuel forms will be present, the intermediate secured controlled storage area will be maintained within primary ventilation confinement. Activities within the storage area are such that appreciable quantities of respirable sized particles will not be generated, and no hoods or task exhaust ducts are present.

\subsection{Receiving, Shipping and Storage and Dock}

Staging areas of three types are present in the receiving, shipping and storage area near the dock. Co-location of these staging areas near the dock is utilized to accelerate loading of outgoing shipments of waste and finished fuel product, and to accelerate unloading of shipments of raw alloy fuel and re-shipment of empty fuel packaging containers which are returned to the fuel alloy supplier Y-12. The temporary storage areas are each enclosed by a locked security fence displaying appropriate signage. The indoor dock has space for unloading of three trucks simultaneously. This may be for incoming or outgoing shipments of equipment, product, provisions, fuel alloy, packaging materials or waste. Housekeeping procedures will be routinely performed on the dock. Compatible packaged and labeled liquid wastes are stored within divided berms with a chemically compatible liner that provides spill protection and prevents commingling of incompatible materials during storage. All accumulation areas are under CCTV surveillance and are inventoried regularly. All personnel access the dock from the Personnel Corridor, and friskers are present to ensure contaminated material does not get passed through to the dock from the confinement area.

On the dock, and in staging areas, swipes and smear samples will be obtained from incoming shipments of radiological material, and for free release (on public roadways) of shipped product, or empty packaging and/or material returned to Y-12. The receiving inspection of alloy fuel shipments (for gross rad count and contamination) will be followed by recording of the container weight which involves de-palletizing drums and weighing each container individually. Any shipper/receiver differential will be evaluated and any differences will be tracked. Empty containers will be surveyed (can, inner packaging, outer container) for presence of any remaining alloy fuel material contents and for removable contamination.

\subsubsection{Fuel Subassembly Packaging}

Completed Fuel Subassemblies are packaged in specialized dedicated containers that suit the physical size of the parts and the quantities that are ship and are suitable for ground travel over long distances and are geometrically favorable such that any number of packages can be stacked together in any arrangement including a tightly stacked cubical 
array without criticality concerns. Shipping container descriptions for the ATR and HFIR fuel (which has an inner and an outer part) are provided below.

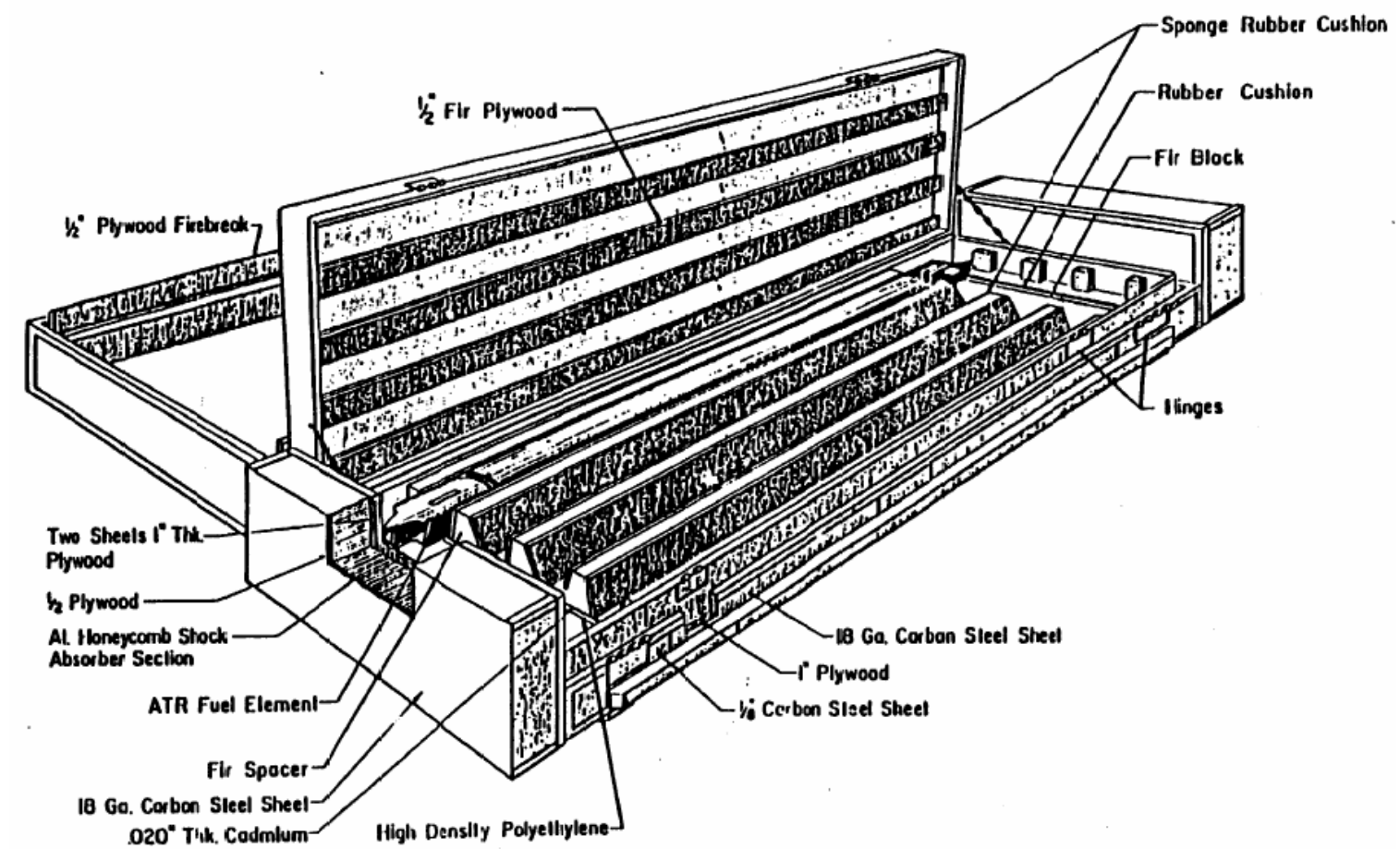

FIGURE 4-31 SKETCH OF ATR FRESH FUEL SHIPPING CONTAINER CAPABLE OF HOLDING FOUR COMPLETE SUBASSEMBLIES

The packaging for the inner HFIR fuel element has overall dimension of 25 inches OD by 45 inches high, a 10-7/8-inch diameter by 30-1/4-inch deep cavity, and a 660 pound gross weight.

The packaging for the outer HFIR fuel element has overall dimensions of 31.5 inches OD by 45.75 inches high, a 17-3/8-inch diameter by 31-1/8-inch deep cavity, and a 1,050 pound gross weight.

HFIR Fuel Element Shipping Container Description

$\begin{array}{lc}\text { Single Container } \\ \text { Dimensions } & 8 \times 8 \times 73 \\ \text { Empty Weight } & 230 \mathrm{lb} \\ \text { Max Weight } & 290 \mathrm{lb} \\ \text { Full Rack } & \\ \text { Dimensions } & 71 \times 36 \times 77 \\ \text { Containers } & 12\end{array}$




\subsubsection{Product Storage Area}

Packaged fuel is stored in shipping containers in 'road-ready' configuration. Space is available for a 6 month supply of each of the five research reactors supported by the FFC. The ATR Fresh Fuel Shipping Container (capacity: four fuel subassemblies) was used as the basis for determining the storage area floor space requirements, and may be subject to revision during FFC detail design, to account for each of the individual reactors' shipping containers, which may decrease the space allotment. The ATR assumption is conservative because the containers are designed for an infinite stack in three directions without criticality concerns.

\subsubsection{Waste Storage Area}

Storage of packaged waste takes place using contamination free, properly labeled 55 gallon drums or similar DOT Spec. 7A Type A containers suitable for temporary storage and transport of hazardous waste. Separate bermed areas which each provide spill containment are provided for storage of liquid wastes that may be incompatible if mixed, such as spent acid and spent caustic. The berm must be lined or painted with a material that is compatible with the material stored within, therefore marked dedicated storage areas are available for each of the waste types expected to be produced as a result of routine operations of the FFC. The drums will be closed, non-vented, and the waste storage area will be completely enclosed by a security fence with a locked gate. A 90 day accumulation of waste is stored in drums secured to pallets which may be stacked. Pallets are handled by forklift. Besides the fence, which allows visibility of the contents without entry, the Waste Storage Area is subject to surveillance by CCTV camera to ensure loss does not occur. Drums are stored in a manner, as shown on the plans, that allows visual inspection of the containers' exterior to ensure degradation is not taking place.

\subsubsection{Fuel Material Storage Area}

The Fuel Material storage area is for receiving and storage of LEU/Mo in the inner containers. Storage racks are described above (Section 4.2), and Y-12 has informed the project that it is not necessary to open containers for assay to verify material characteristics; that lab sized coupons of the material will be shipped separately with each lot. Therefore, open containers will not be present in the secured controlled storage area, therefore primary ventilation confinement is not necessary. Although the Storage Area may be configured as a primary confinement area (such as the Shop is), this allows the room to be ventilated normally during routine operation. A spill could occur, such as by a dropped or ruptured container, but the material inside is usually foil wrapped and it seems reasonable that even a dropped container that came open would not release a quantity of any appreciable size, and housekeeping procedures could be invoked that would clean up any spilled contamination and verify that the area is contamination free. The capacity to store a one year's supply of alloy fuel material is provided, along with an area to open outer containers and inner vessels and to survey containers' exteriors, weigh and mark the containers, and a terminal is present to allow the operator to register the accountable material into the tracking system, or to transfer custody of material via transfers to the Casting Area 
4.19.5 Dock

Space is available for parking three trucks simultaneously, with space along both sides with steps and personnel doors to outside. 48" high dock with bumpers. Tip down ramps for forklift access. Individual roll up doors, 15' high, are present on each bay. Doors roll up into a drum outside the building, in a configuration that does not interfere with dock overhead lighting when doors are raised.

\subsubsection{Mechanical and Electrical Maintenance Rooms}

Space has been provided for these functions in the FFC. Minor equipment maintenance or calibration will be performed in these spaces, and a limited supply of instruments and tools will be available as well as miscellaneous part storage, benches, utility outlets and lighting.

\subsubsection{Air Compressor Room}

A small room has been set aside for an air compressor, reservoir, regulator, air filtration and drying. A piping network will be routed throughout the plant for service air uses which will be throughout the shop and assembly area, and the mechanical room.

\subsubsection{M/F Water Closets}

Water closets for male and female occupants of the FFC are available near the dock. A drinking fountain is also present. The water closets will be connected to the FFC floor drain system.

\subsubsection{Trucker's Waiting Room}

A small lounge has been provided to allow transportation personnel to wait in while shipments are loaded or offloaded from vehicles parked in the dock. This area has a water fountain, coffee machine and chairs and table.

\subsubsection{CCTV Surveillance}

The receiving and storage areas and dock area is under 24 hour surveillance by CCTV cameras. Images from the cameras may be viewed on monitors at the control area (behind the receptionist). Electronic records are kept for an interval that exceeds material accountancy periods, and can be reviewed, if necessary, following any events that involve unauthorized movement of material. CCTV surveillance may be extended to the lot with opposite facing cameras positioned at each corner of the building, the entry control facility, and the lobby. The perimeter fence is equipped with exterior lighting. The intent of the cameras is more oriented towards safety than security, although security purposes, which would include the parking lot, may be achieved to some extent. 


\subsection{FACILITY DESIGN AND OPERATION}

The 76.000 square foot arrangement of FFC for the Greenfield Alternative Study is shown in figure 5-1. The facility layout provides a bounding arrangement of the shop, assembly and storage areas, lab and personnel areas and incorporates the necessary facility requirements needed to operate the building. All known FFC requirements have been accommodated and depicted in a reasonable and realistic configuration.

Drawing SK-ME-01 shows a plan view of the FFC, General Design criteria is provided in section 2, and a brief summary description of the Greenfield Alternative is provided below.

\subsection{Facility Overview}

The FFC is a modern, state of the art facility with a design life of 40 years. Energy conserving practices have been incorporated in the facility design and layout. Located at or adjacent to an existing NNSA Complex or DOE National Lab, it is assumed that infrastructure, security and utilities are available (specifics will be dependant on the actual DOE site chosen). As shown in the rendering below, the FFC is an above ground, two bay (three bays with HIP option) free standing, rigid steel framed structure with insulated metal siding and roofing. The buildings will be finished to conform to the color and appearance theme of the surrounding structures. In addition to the main building a storage warehouse also exists on site to serve the FFC. 


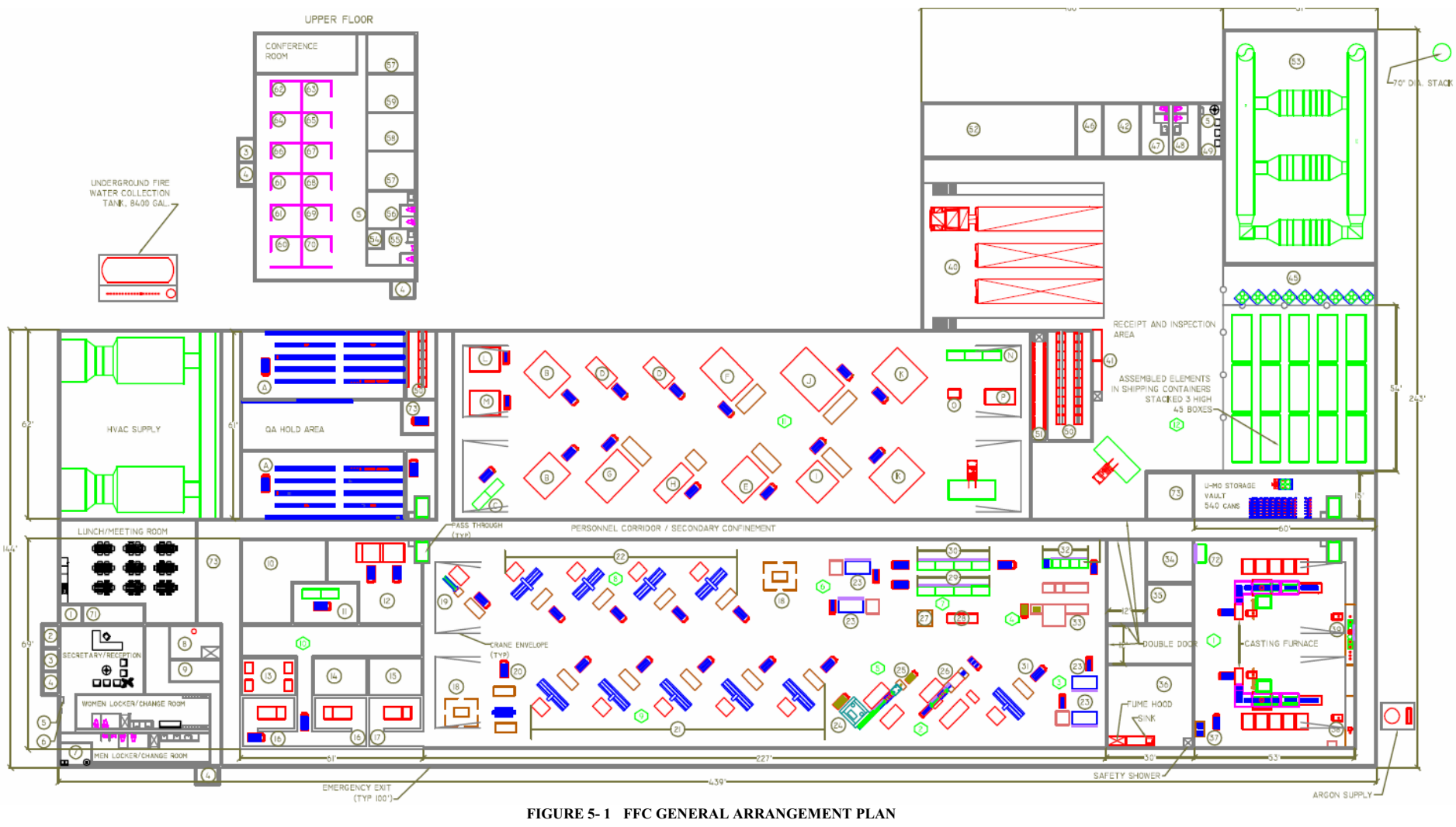




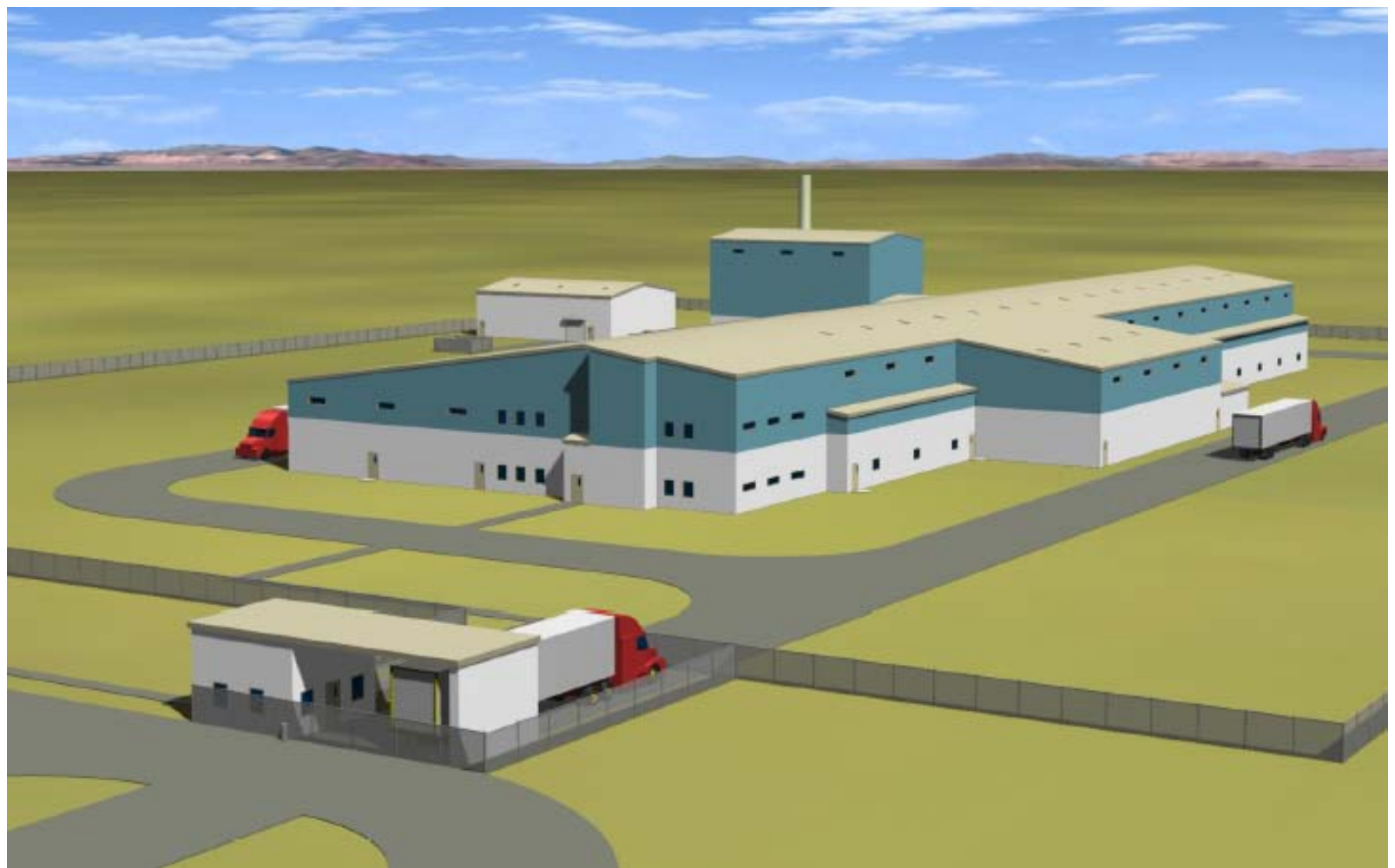

FIGURE 5- 2 FFC GREEN FIELD ALTERNATIVE

A two story administrative area is located in the main building (adjacent to the production area) and serves as the primary entry point for the facility. This area contains the bulk of the offices and has restrooms and meeting/break rooms.

The production area is comprised of a high bay shop, a low bay assembly area, a shipping/receiving area, secured controlled storage areas for storing fuel alloy feed material, inprocess nuclear material and finished fuel subassemblies, labs and inspection areas, mechanical rooms to provide the required utilities and the necessary production control offices.

The high bay shop is where fuel plates are manufactured. This area is serviced by two overhead bridge cranes running the length of the area. The heavy equipment is configured in a compact arrangement and machining stations are located down the sides within the crane envelopes. The central aisle between crane envelopes serves as the route for primary traffic and material handling (personnel, forklifts, carts, etc.). Building construction is such that the central aisle is unencumbered by columns. The casting area is located at the end farthest from the administrative area. This area is separated from the rest of the high bay shop by a production office and lab area (these areas are low ceilinged to allow passage of the overhead cranes). The high bay shop is the primary fabrication floor where the U-Mo foils are rolled and bonded into fuel plates and machined and formed into their final form. Adjacent to this is the inspection area (which lies between the primary fabrication floor and the administrative area and is outside the overhead crane envelope) which houses specialized equipment to non-destructively examine the fuel plates.

The low-bay Assembly Area is located adjacent to the high bay (separated by a personnel corridor) and is serviced by two overhead cranes on parallel runways. This room houses the equipment needed to fit the finished plates into the element hardware and to perform all fuel subassembly fabrication and quality analysis steps. Like the high bay, the heavy equipment is located under the cranes with an aisle down the center. 
Shipping and receiving is located centrally such that the secured controlled storage area for LEUMo fuel alloy material received, and finished fuel elements (product) to be shipped are efficiently located in close proximity to the dock. Likewise, supplies or new machinery unloaded from trucks at the dock can be easily brought in to the shop area or to final assembly. Secured controlled storage areas are present both for locked storage of incoming fuel alloy (one year's supply) and for interim storage of the various configurations of the fuel plates as they proceed throughout the fabrication process. Storage also exists for completed fuel assemblies. As subassemblies are completed in the assembly area, they are packaged and stored 'road-ready' in shipping packages in a locked indoor storage area near the dock. Sheltered space is present for up to three trucks to dock at the same time and ample space is available for receiving and inspection.

Nuclear material not being processed is stored in one of a number of secured controlled storage areas strategically located around the production area. The placement of the secured controlled storage areas allows the material to move through the facility in a logical pattern and serves to keep the amounts of fissile material on the working floor within criticality safety limits. These secured controlled storage areas have a higher fissile material limit than the production floor due to geometric configuration control.

At either end of the main production floor are low ceillinged lab and inspection areas. These are placed to conform to the typical path of the production parts through the laboratory. They are comprised of destructive (wet chemistry and metallography) and non-destructive (ultrasonic and radiography) analysis equipment

Offices and support areas such as a lunch room and change rooms are provided as well as a conference facility and reception area. A central control area (for monitoring ventilation, alarm systems, etc.) is on the main floor inside the front door to the office area. Other areas in the FFC house utilities mechanical rooms and offices in support of the production area (shop supervisor's office, calibration rooms, etc.).

Fuel subassemblies for the five domestic reactors requiring plate fuel serviced by the FFC are manufactured on a campaign basis. A standalone warehouse is featured in the design adjacent to the dock to facilitate campaign change-over between fuel campaigns. This houses supplies (both for the office area and shop operations), non radioactive material stocks, and tooling for off-duty fabrication campaigns.

The building has an engineering confinement ventilation system with cascaded ventilation zones and HEPA filtered exhaust ensuring that nuclear material is not released to the environment. Local HEPA filtered task ventilation and hoods at a number of work stations provide protection to the facility operators wherever necessary. The confinement area (shop) is completely surrounded by a Personnel Corridor which ensures that a ventilation barrier exists at all times between the shop and the environment. A zoned pressure differential approach is featured in the design to ensure airflow is from areas of less potential for contamination to areas of higher potential for contamination. Pressure differential shall be maintained between the zones and atmosphere. The exhaust system shall be designed to withstand anticipated normal, abnormal, and accident system conditions and maintain confinement integrity. Materials of construction should be appropriate for normal, abnormal, and accident conditions. Ventilation system design shall comply with DOE regulations and good practice. Conditioned air is recirculated in clean areas and offices for energy conservation.

The building is completely protected by a wet pipe sprinkler system, and impoundment of fire water runoff is included in the facility design. The FFC, stack, warehouse and substation is enclosed within a fenced boundary, and an entry control facility is provided to control access of personnel and materials. 


\subsection{Facility Design Requirements}

Pertinent AE requirements at the site selected will be followed for facility design requirements as well as the general design criteria specified in section 2. Site and facility layout drawings are provided in Appendix A1. Equipment arrangement concepts are provided in the body of the report and selected cut sheets are provided in Appendix A4.

This section provides a summary of the design basis requirements and initial study level descriptions of the FFC site and facility. The FFC will be designed, licensed, constructed, and operated in compliance with the applicable Federal, state, and local regulations, and will comply with NRC and DOE regulations and national consensus codes and standards.

The programmatic and technical design basis requirements are presented in the Statement of Work for an engineering alternative study for a Greenfield LEU-Mo Fuel Fabrication Capability and the processing requirements are contained in the Conceptual Process Description of LEU-Mo Fuel. Additional key design basis requirements include the following:

- 40-year Facility design life.

- Facility will be built at a Greenfield DOE site in the continental US. The Federal Facility to which the FFC adjoins will provide all support infrastructures.

- Fuel fabrication and product storage areas will be designed to Hazard Category 2 and Security Category IV.

- Facility Availability: $75 \%$ (equivalent 255 full operating days in a calendar year).

- All waste (Hazardous, LLW) will be packaged shipped for treatment and disposal by others at approved disposal sites.

- Facility will be designed to produce 17,000 foils/year. 


\subsection{Site Layout}

The FFC Site is shown on drawing SK-ME-06, and is reproduced below.

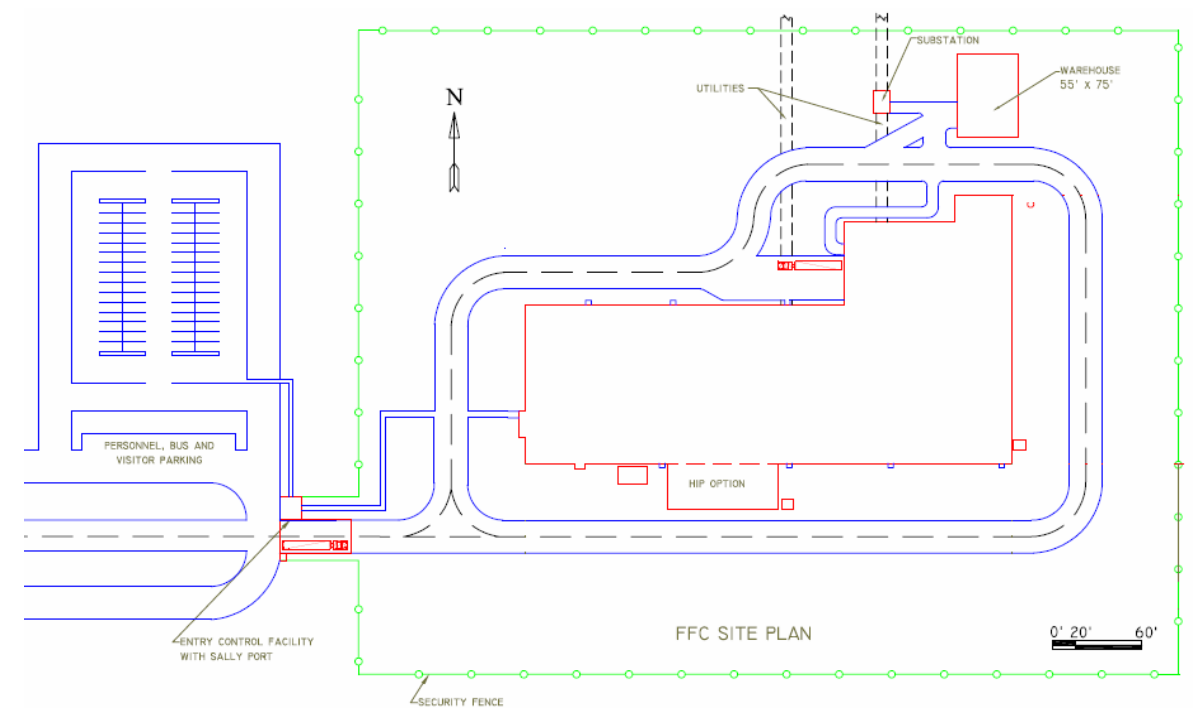

\section{FIGURE 5- 3 FFC SITE ENCLOSED WITH SECURITY FENCE, WITH ECF,} BUILDING, WAREHOUSE, AND SUBSTATION

The following are key requirements for the layout of the FFC:

- The facility layout and equipment arrangement shall provide to the extent practical, efficient and functional support to the operation and management of the FFC. The facility design shall also provide for life safety of occupants and support of LEU-Mo Alloy Fuel processing, environmental control, and routine maintenance functions, including safe storage, shipping, and receiving functions.

- The facility arrangement shall include all necessary floor space and systems, including safeguards and security, material handling equipment, material control and accountability equipment, product handling, packaging and storage facilities, alloy fuel processing equipment and systems, sampling and analysis systems, and waste management systems and equipment to package waste for shipment to waste treatment and disposal sites.

- Processing and storage areas shall be designed to control spread of contamination, and control emissions to acceptable levels and limit the extent of potential contamination in the unlikely event of an accident. The design shall provide for ease of personnel access, material handling, decontamination, process operations, and adequate ventilation. Radioactive materials shall be contained within acceptable packages and handled openly only within engineered confinement zones. Fuel subassemblies shall be packaged for safe storage, handling and transportation. All concrete surfaces within areas expected to be contaminated or have the potential to become contaminated shall have surfaces coated to facilitate decontamination.

- HVAC equipment and auxiliary mechanical and electrical services shall be provided from service areas that facilitate access, efficient space utilization, and low installation and maintenance cost while providing energy efficient building utilities. Limited capabilities shall be provided for routine maintenance and repair services for mechanical, electrical, and instrumentation components. 
The layout of the alloy fuel foil and reactor subassembly fabrication operations in the FFC is guided by the following design considerations:

- Process flow continuity and operational efficiency - Process facility layout facilitates a oncethrough operation, from the fuel receipt through shipment of the fabricated research reactor subassemblies.

- Segregate the 'bare' alloy processing operations from the "clad" fuel processing, fuel fabrication and product storage operations, minimizing spread of contamination and ensuring that the final product and packaging is not contaminated.

- Typically, during high temperature processes, including melting and rolling, the fuel alloy will be exposed only to inert gas atmospheres to minimize oxidation. A noted exception is salt bath heating of cast alloy plates and rolling in air. During canned hot rolling of foils or HIP bonding, the inert atmosphere is contained within the can.

- Facility safety - All work stations that impart mechanical action to exposed alloy surfaces shall be provided with task ventilation that prevents dilution of contamination into the occupied workspace.

- Physical security - Entry control facilities are present for access to the FFC buildings, with a security fenced perimeter around the site.

- Close proximity between the analytical capabilities and the fuel processing operations.

- To the extent practical, Modular equipment systems shall be utilized to facilitate operation as well as factory testing, and removal and replacement of components or whole subsystems for service and minimize downtime for overhaul. Replacement intervals shall be preventative and performed prior to the point that performance degradation or safety implications occur.

- Reliability, Availability, Maintainability and Install-ability (RAMI) considerations will be utilized (during future design studies) to assure necessary production rates can be met and to minimize the facility footprint. All maintenance operations are performed manually.

- Work areas are provided within the FFC for decontamination and maintenance of failed equipment as part of normal operations in the facility. Articles that have been exposed to nuclear material will be decontaminated to the extent practical before disposal as rad-waste.

- Radioactive waste collection, treatment, packaging and shipping systems are provided within the FFC. The facility shall also be designed to minimize hazardous and radioactive waste generation.

The FFC site is to be built on a level lot, without contamination or existing structures, within the boundaries of an existing Federal Facility. The site is well characterized and in close proximity to utilities (water power sewer, phone and internet) and a transportation corridor. A property protection fence surrounds the entire site (parking lot is outside the fence). A roadway encircles the building, and simple gravel landscaping is present between the road and the structure. Outside the road, natural vegetation will be present; no landscaping is included in the estimate. An underground fire water loop will surround the road with hydrants every 300'. A paved parking lot for 60 vehicles plus bus parking is outside the fence. All pedestrians, vehicles and service trucks enter via the entry control facility, and a sally port is present to allow security personnel to screen incoming and outgoing vehicles. A 55' truck turning radius has been incorporated for delivery and tanker trucks. The site plan shows the HIP option because this bounds the depth of the 10 acre site. The warehouse is located convenient to the dock, and an allowance has been made in the paved road to allow trucks to back in to both the dock and the warehouse. 


\subsection{FFC Building Configuration}

The FFC has a central high bay over the shop and low bays on each side for the assembly area and the HIP room (one side lean-to without the HIP option). Standard steel roof trusses are shown below in the building cross-section, from drawing SK-ME-04.

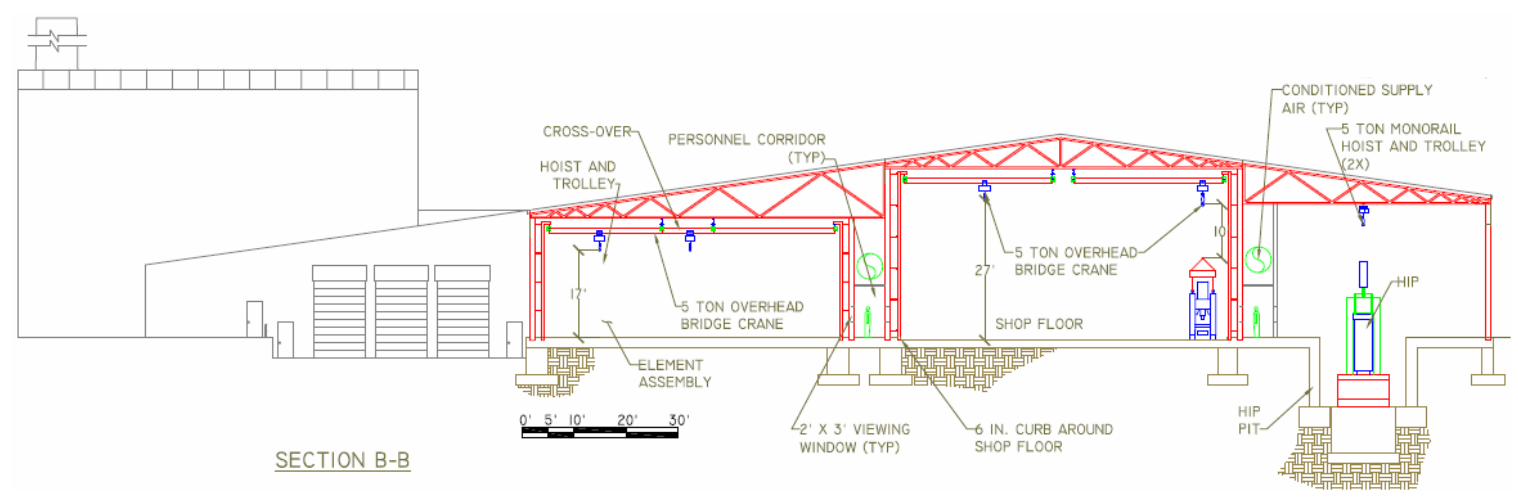

FIGURE 5- 4 HIGH BAY (SHOP) WITH LEAN-TO'S ON EACH SIDE

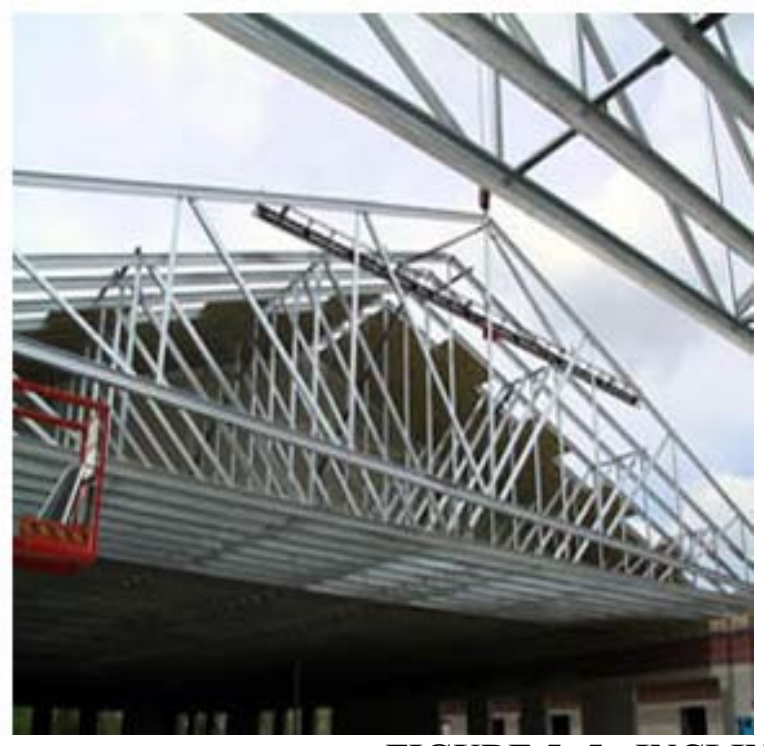

Standard roof trusses have a number of advantages

- Non Combustible, UL rated

- Long Span without central supports

- Design to suit crane runway

- Pre-engineered, lightweight

- Economical, low design costs

- Trusses, connections and bolting purchased as a package

- Prefabricated trusses facilitate installation

- Seismic rated, Commercially available

- Competitive lead times

- Long life

- Ample area for lighting, ducting, electrical trays and sprinklers to meet project needs

FIGURE 5- 5 INCLINED ROOF BENEFITS

The slope of the pitched roof versus a flat roof, using flat roof trusses, is an issue that will be resolved in later phases of the project (future) and will include considerations for the local annual precipitation and climate at the site selected. One possible disadvantage of a flat roof has to do with drainage and snow load considerations, whereas with a pitched roof, the volume of the room increases if the trusses are exposed (as they are in the shop), increasing the ventilation load and 24 hour per day horsepower usage. A pitched roof concept has been prepared in lieu of a flat roof. In the pitched roof concept shown, there is a suspended ceiling in the assembly area, because it is not part of engineering confinement, so the severity of the later disadvantage is lessened (the area above the ceiling is un-heated and only naturally ventilated). The concept shown 'won out' over a concept with three gables (which reduces the area volume enclosed by the 'lean-to' trusses by 50\%), due to the potential for drainage problems in the valley. 


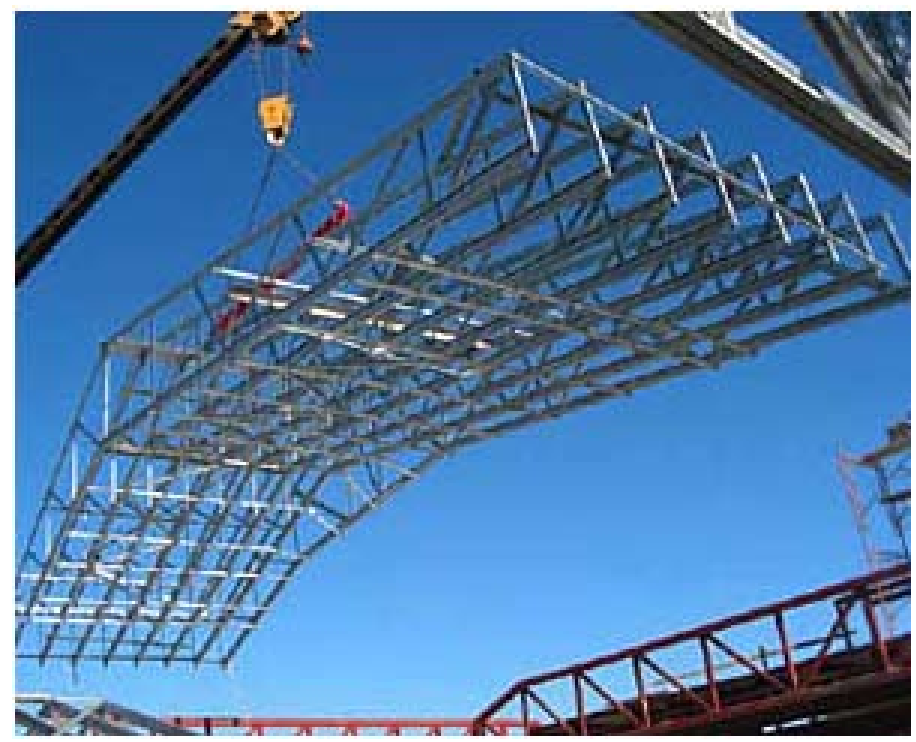

Trusses such as these galvanized steel long span sectional trusses may be used over the Dock and Shipping and Receiving area.

Roof may be pitched or flat.

FIGURE 5- 6 TRUSS SECTION

\subsection{Utility Connections}

The building is erected on a slab on grade on a flat lot graded for precipitation runoff and although there may be a 13' deep pit (in the HIP option) the FFC does not have a basement. Utilities are routed underground after the underlying soil has been prepared and before the slab is poured. From the electrical substation underground power conductors pass under the building floor slab in conduits and penetrate the floor of the two motor control centers. Water and sewer likewise penetrate the floor slab at the appropriate locations, and sumps are spaced throughout the floor of the shop in lieu of floor drains. Utility connections including communications lines (site alarms, emergency notifications, phone and internet) are branched from the adjoining federal facility. Electrical services are routed in trenches independent from water services, and sewer is routed independently from potable water. 


\subsection{Facility Areas}

The FFC is comprised of a number of functional areas that differ by design, arrangement, ventilation barrier criteria, or materials of construction, to accommodate the activities performed within. Areas are broken down by function and footprint below.

5.6.1 Administrative Areas: $6,485 \mathrm{ft}^{\wedge} 2$
A. Reception Area
B. Second floor offices, meeting rooms
C. Elevator, stairs

5.6.2 Production Areas
A. Casting Area: $\underline{3,624 \mathrm{ft}^{\wedge} 2}$
B. Chemical/metallography lab
C. Production offices
D. Shop (Fuel Fabrication, Rolling and Bonding): $\underline{15,438 \mathrm{ft}^{\wedge} 2}$
E. Non-destructive inspection area
F. Assembly area: $\underline{11,771 \mathrm{ft}^{\wedge} 2}$

5.6.3 Storage Areas (including safeguards and security)
A. Dock, Shipping and Receiving Area: $\underline{8,521 \mathrm{ft}^{\wedge} 2}$
B. Secured controlled storage area for U-Mo Alloy feed material: $\underline{892 \mathrm{ft}^{\wedge} 2}$
C. Casting and crucible heel Storage Areas
D. Interim storage areas (for in-progress material): $\underline{2,352 \mathrm{ft}^{\wedge} 2}$
E. Material control areas
F. QA hold area
G. Storage room
H. Assembled element storage
I. Completed Subassembly storage area (fuel elements, road-ready, in shipping packaging): $2,746 \mathrm{ft}^{\wedge} 2$
J. Waste staging area: $\underline{664 \mathrm{ft}^{\wedge} 2}$
K. Warehouse (external to the FFC): $\underline{4,125 \mathrm{ft}^{\wedge} 2}$

5.6.4 Safeguards and Security Features
A. Security Fencing
B. Entry Control Facility (Guard House and Sally Port)
C. Central Control Room
D. Security Cameras 


\subsubsection{Safety and Support Areas}
A. Air Handler room: $\underline{3,259 \mathrm{ft}^{\wedge} 2}$ (also houses packaged boiler)
B. Exhaust Fan and Filter rooms (2 stories): 3,795 X $2=\underline{7,590 \mathrm{ft}^{\wedge} 2}$
C. Safety showers and eye wash stations
D. Decon areas
E. Dosimetry
F. Mechanical and Electrical maintenance areas: $\underline{878 \mathrm{ft}^{\wedge} 2}$
G. Personnel Corridor: allows personnel to move to their workstation without conflicting with work carts or forklifts movement down the central aisle of the shop. Viewing windows allow observation of shop activities prior to entering the shop. Emergency exits are accessed from the observation corridor.

H. Battery room

\subsubsection{Personnel Amenities}
A. Change Rooms, locker room
B. Lunch room, meeting room, conference room
C. Water closets upstairs and downstairs with handicapped access, trucker's lounge/waiting room, WC in shop, drinking fountains

\subsection{Ventilation}

The shop is the primary engineering confinement zone. The air exhausted from the shop is conveyed via large main ducts near the roof above the cranes. Air exits the shop either via inlet registers in the main ducts or via branch ducts carrying air removed from the shop by a number of task exhausts or hoods. The personnel corridor surrounds the shop and is the secondary confinement area. The supply air headers are routed above the personnel corridor and diffusers supply filtered and conditioned air to the corridor which passes through the wall to the shop, via barometric dampers. Personnel exiting the shop must pass through the personnel corridor before exiting the building. To exit the personnel corridor they must monitor for the presence of radioactive contamination. Unclad fuel material, in general, is not passed through the personnel corridors. The personnel corridor is normally non-contaminated but is a radiologically controlled zone and is frequently 'swept' by health physics personnel and maintained clean by housekeeping procedures. The color coded general engineering confinement zone map is shown below. Unshaded areas operate at atmospheric pressure. 


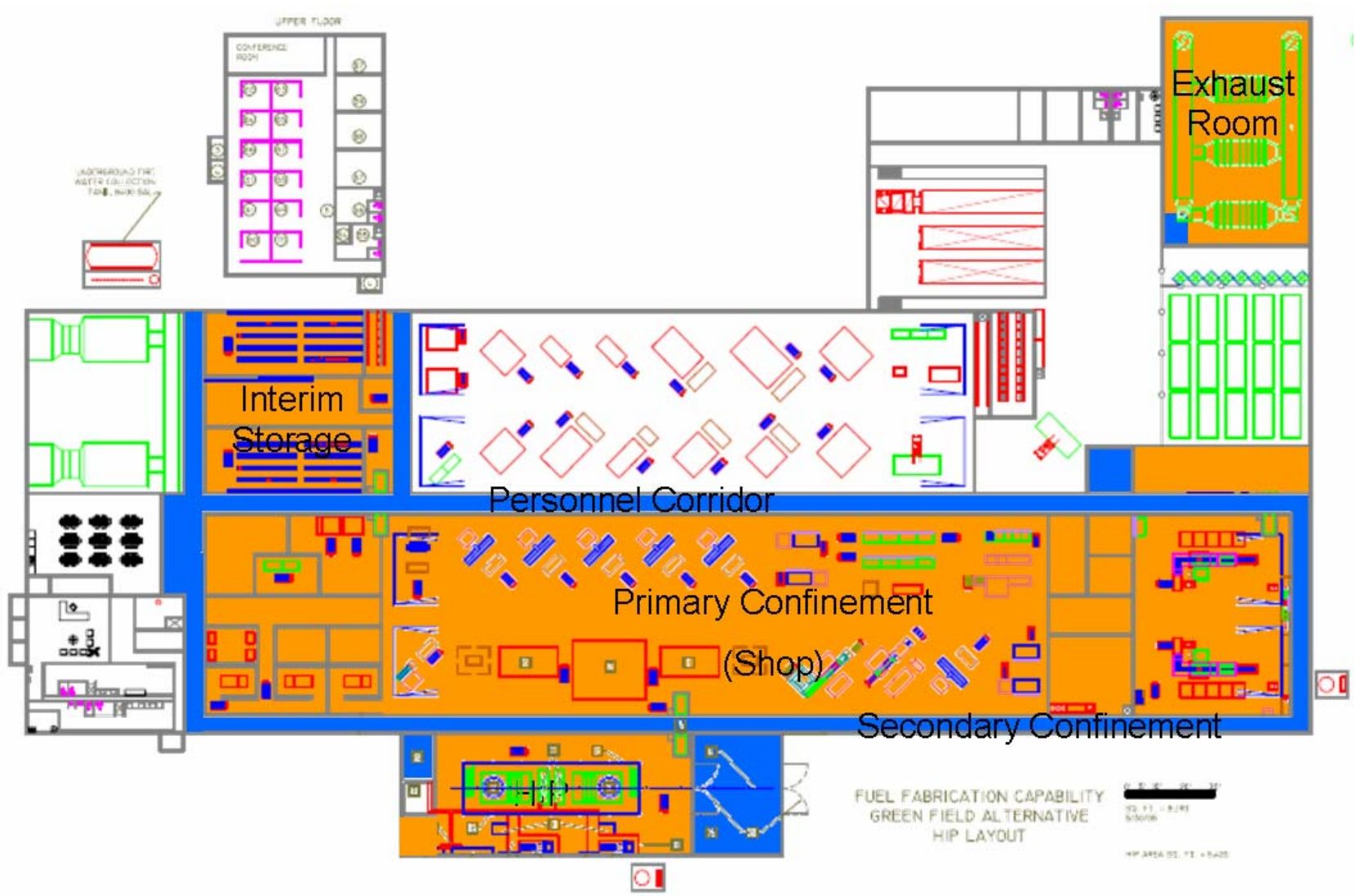

\section{FIGURE 5-7 PERSONNEL CORRIDOR (SECONDARY) SURROUNDS THE PRIMARY CONFINEMENT AREA}

\subsubsection{Task Exhaust}

Task exhaust ducts are present in the AFCF whenever operators are involved in operations involving machining or cold working fuel material such as cutting, shearing, trimming, rolling, that may expose material. The task exhaust duct is shown below. Some operations may require a greater level of isolation of the work environment from the operator, which requires use of negative pressure hoods, with the operator's hands within a moveable sash. Most operations anticipated for the FFC will not generate large volumes of particulate susceptible to respiratory uptake by the operators. Any exceptions, such as cleaning out used molds and crucibles, media blasting, or working with potentially contaminated liquids such as salt baths or cleaning baths, or dissolved fuel solutions will be performed in the FFC within enclosed hoods. Ducts from task exhausts or hoods connect to the main exhaust ventilation headers. Although discharge from hoods and task exhausts are HEPA filtered, the remainder of the air exhausted from the shop area is drawn from inlet registers located in the main branch headers therefore the exhaust headers are equipped with hatches in the duct work to allow cleanout. 


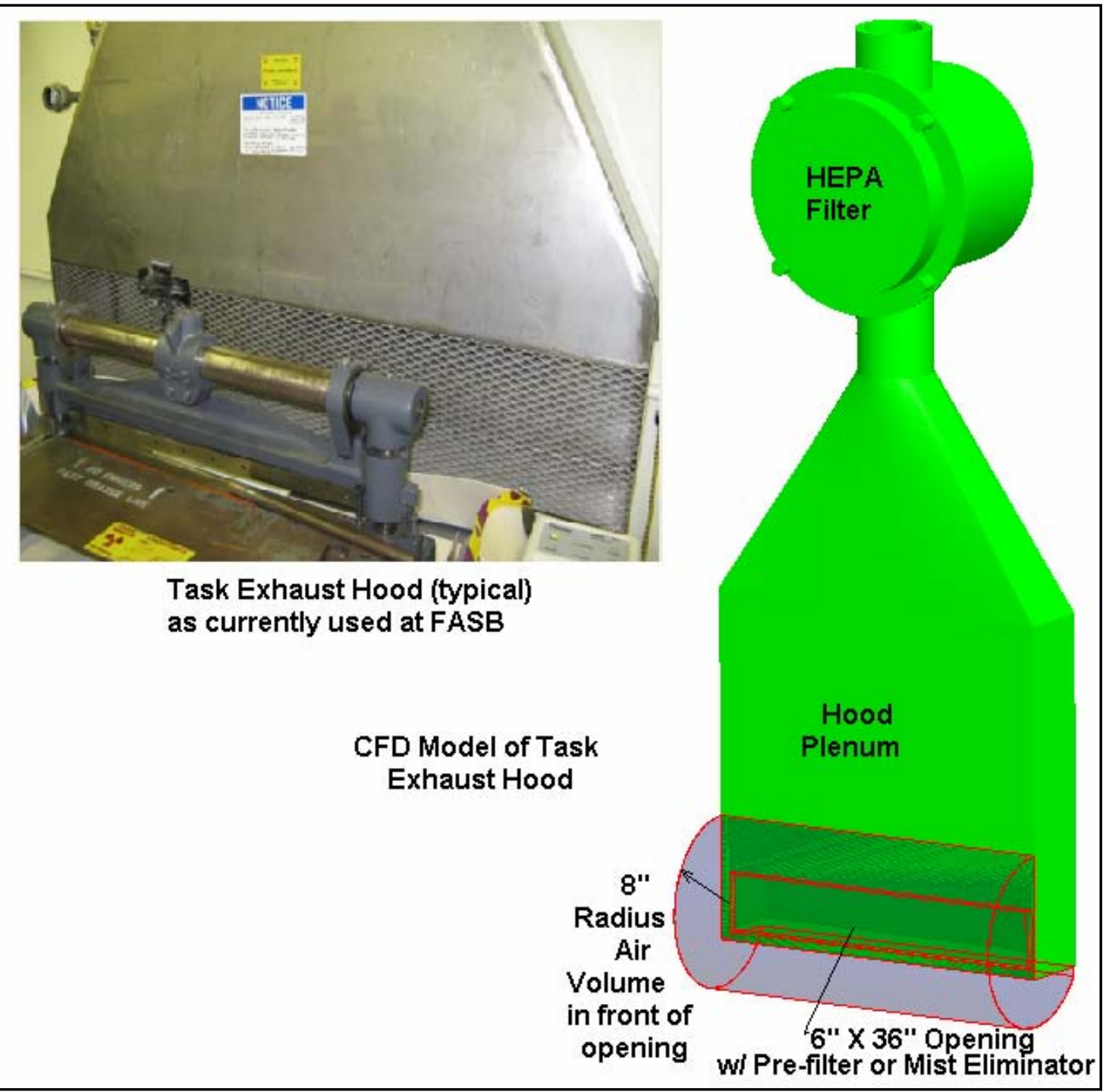

FIGURE 5-8 TASK EXHAUST

The photo above shows a task exhaust duct in FASB, behind a small shear. The CFD model used to simulate the air flow in three dimensions around the duct inlet us shown on the right.

- The criteria for the local task exhaust duct concept follows.

- Local task exhaust system captures and removes emitted contaminants before dilution into the workplace ambient air can occur.

- Basis Airflow criteria: $100 \mathrm{ft} / \mathrm{min}$ 'capture velocity' three inches from the face of the opening.

- Boundary condition is $250 \mathrm{ft} / \mathrm{min}$ across the 6" X 36" opening of the task exhaust duct. 


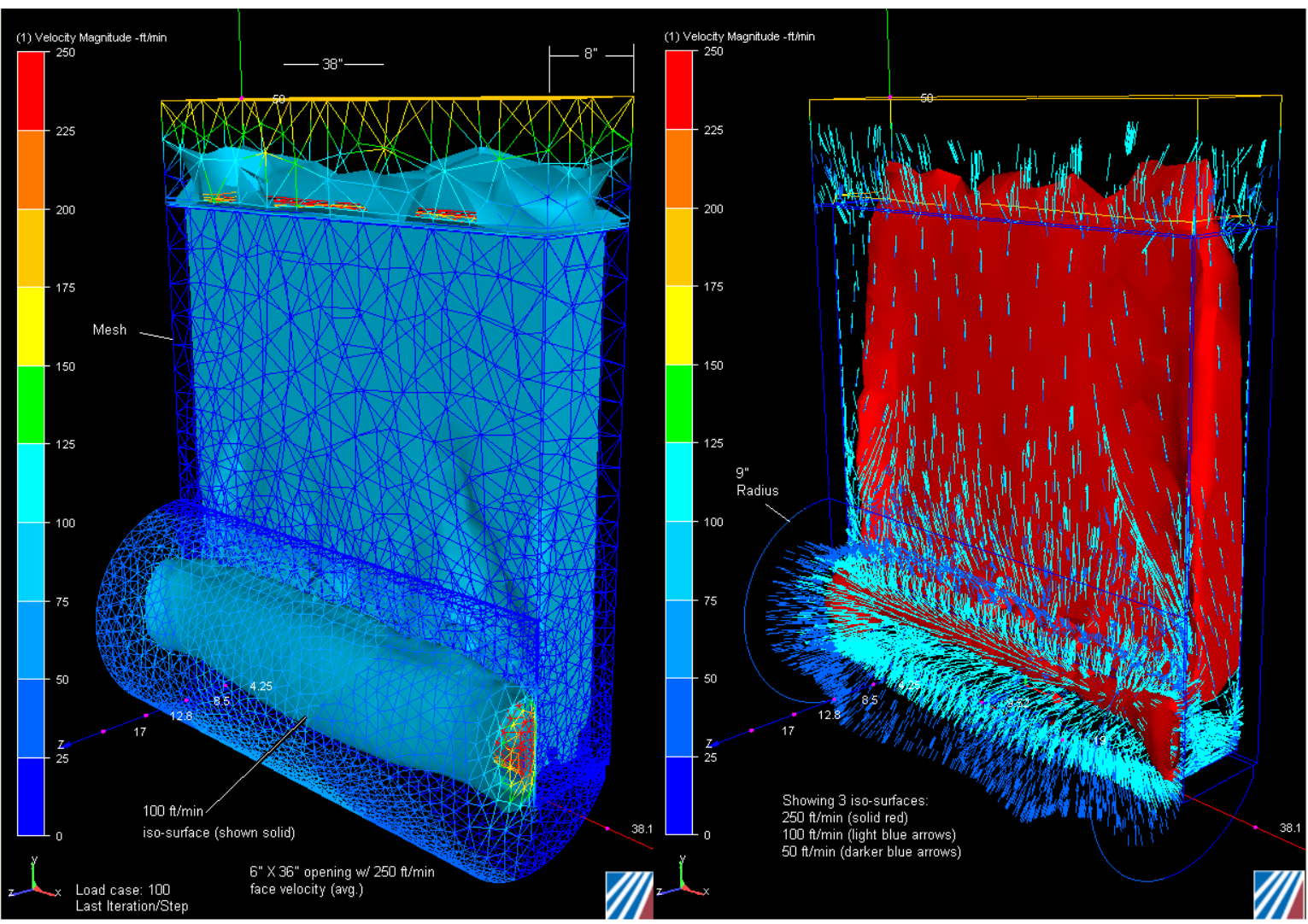

FIGURE 5-9 CFD MODEL - AIRFLOW TASK EXHAUST

The finite element mesh representing the model is shown on the left along with a (solid) $100 \mathrm{ft}$ per minute iso-surface. The left view shows isosurfaces of 250 (solid) fpm, 100 fpm (arrows) and 50 fpm (dark blue arrows).

The CFD model results show that the $250 \mathrm{fpm}$ face velocity boundary condition produces flow rates of $100 \mathrm{ft} . / \mathrm{min}$. approximately 4 " from the face of the opening, and $60 \mathrm{ft} . / \mathrm{min}$. approximately 8 " in front of the opening.

Note that the Occupational Exposure Limit (for $U$ ) is $0.2 \mathrm{mg} / \mathrm{m} 3$. For lead (not present in the FFC), the exposure limit is $133 \mathrm{X}$ tighter $(1.5 \mu \mathrm{g} / \mathrm{m} 3)$.

Task exhaust ducts must be located in direct proximity to the mechanical action imparted to the alloy fuel material (such as from shears and rollers). Off gas, such as from the enclosures on the furnaces, shears, roll mills, milling machines and baths, require HEPA filtration. Task exhaust duct inlets are fitted with a pre-filter to reduce HEPA change outs, and exhausts from baths are equipped with a mist eliminator (in addition to the local HEPAs). $\mathrm{NaOH}$ when exposed to aluminum gives off hydrogen gas, and $\mathrm{HNO}_{3}$ gives off NOX, and this off gas is exhausted through the ventilation system. The heated salt bath generates chlorine gas above the liquid level and also requires exhaust ventilation. There are local vents on the casting furnace vacuum pumps, rolling mills, the cleaning stations shears, and on the friction bonding stations, (which also requires dust removal). 


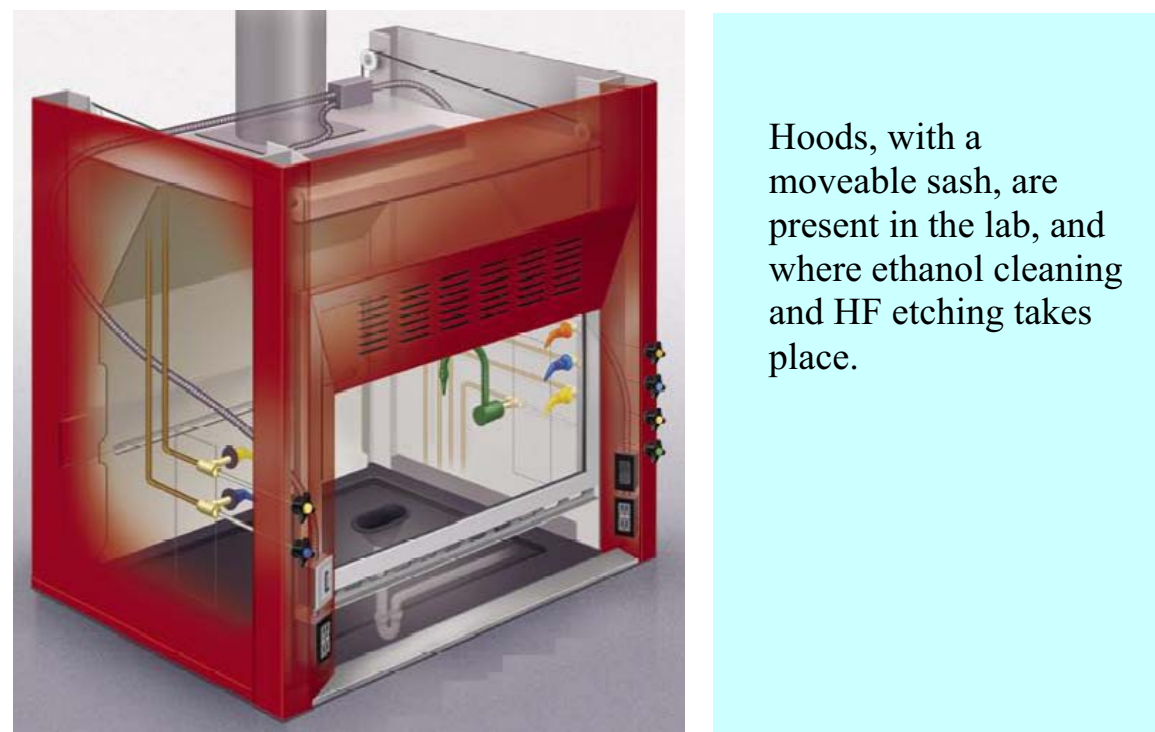

FIGURE 5-10

VENTILATION HOOD

A table documenting the location of the task exhaust ducts and hoods used to estimate the combined flow is provided below.

TABLE 5- 1 AIRFLOW ESTIMATION CHART 


\begin{tabular}{|c|c|c|c|c|c|}
\hline \multicolumn{3}{|c|}{ FFC Ventilation Air Flow Needs } & Face Velocity & 250 & $\mathrm{ft} / \mathrm{min}$ \\
\hline & Ventilation Hood & Size Ht (ft) & Size Lg (ft) & Area $\left(\mathrm{ft}^{\mathrm{n}} \mathrm{2}\right)$ & CFM \\
\hline 1 & Cast plate cooling & 0.5 & 4 & 2 & 500 \\
\hline 2 & Crucible \& mold recycle & 0.5 & 4 & 2 & 500 \\
\hline 3 & Raw alloywash station & 0.5 & 4 & 2 & 500 \\
\hline 4 & Plate Rolling & 0.5 & 3 & 1.5 & 375 \\
\hline 5 & Salt Bath & 0.5 & 2 & 1 & 1000 \\
\hline 6 & Trimming (4 shears) & 0.5 & 3 & 1.5 & 375 \\
\hline 7 & Salt removal & 1 & 3 & 3 & 750 \\
\hline 8 & Cleaning Nitric plate & 0.5 & 3 & 2 & 500 \\
\hline 9 & Can welding & 1 & 3 & 3 & 750 \\
\hline 10 & TunnelFurnace & 2 & 4 & 8 & 2000 \\
\hline 11 & Foil Rolling & 0.5 & 4 & 2 & 500 \\
\hline 12 & Can opening & 0.5 & 5 & 2.5 & 625 \\
\hline 13 & Cleaning Nitric Foil & 0.5 & 4 & 2 & 500 \\
\hline 14 & Cle aning Ingot Nitric & 0.5 & 4 & 2 & 500 \\
\hline 15 & Ethanol Wipe & 0.5 & 4 & 2 & 500 \\
\hline 16 & Cleaning Caustic & 0.5 & 4 & 2 & 500 \\
\hline 17 & Cle aning Nitric Al & 0.5 & 4 & 2 & 500 \\
\hline 18 & Autoclave & 1 & 2 & 2 & 500 \\
\hline 19 & Friction Bonding 1 & 0.5 & 3 & 1.5 & 375 \\
\hline 20 & Friction Bonding 2 & 0.5 & 3 & 1.5 & 375 \\
\hline 21 & Friction Bonding 3 & 0.5 & 3 & 1.5 & 375 \\
\hline 22 & Friction Bonding 4 & 0.5 & 3 & 1.5 & 375 \\
\hline 23 & Friction Bonding 5 & 0.5 & 3 & 1.5 & 375 \\
\hline 24 & Lab hood (3 stations) & 0.5 & 4 & 2 & 1500 \\
\hline & Total: (from hoods) & CFM & & & 14750 \\
\hline
\end{tabular}

\subsubsection{Shop Area}

Since in the shop bare alloy material is present and undergoing processing, the shop is the primary confinement within the radiological material control zone. The secured controlled storage areas and labs, in which unenclosed fuel alloy material is handled, are also confinement zones. The shop area of the FFC is surrounded by the Personnel Corridor which provides secondary ventilation confinement. It serves as an air-lock for personnel entering or exiting the shop. Ventilation supply air is ducted in and distributed throughout the length of the personnel corridor. The shop and other primary areas are operated and maintained at a slight negative pressure (minus $\sim 1 / 2 " \mathrm{H}_{2} \mathrm{O}$ gage) compared to the atmosphere. The Personnel Corridor is at about minus $1 / 4$ ", and the office and clean areas are at atmospheric pressure. This assures that air flows from a vicinity of no or low probability of contamination to an area of higher potential for contamination. Unpackaged alloy fuel material is handled only in the shop, Interim secured controlled storage area, casting and lab areas. A minimum of four fresh air changes per hour pass through the shop. Approximately $20 \%$ of the air is exhausted from the hoods and task exhaust ducts (regions of highest contamination potential), the remainder being exhausted via inlet registers in the main exhaust ducts (headers) that run the length of the shop within the trusses, below the roof.

Filtered supply air, discharged from the air handling units, flowing to the personnel corridors and passing through barometric dampers mounted in the shop walls may become contaminated as it passes through the shop. Shop air is exhausted by two means: by the hoods and task exhaust ducts, and directly into the exhaust headers. Air exhausted 
from the primary confinement area (shop, casting and interim storage area) is released to the atmosphere via the HEPA exhaust filters and fans. The exhaust filters provide secondary and tertiary HEPA filtration, while hoods, enclosures and task exhaust ducts are equipped with local (primary) HEPA filters as well as pre-filters or mist eliminators to minimize duct contamination. A control damper in the supply duct upstream of the branch headers to the personnel corridor prevents 'pressurization' of the shop should the exhaust fans fail to provide the necessary flow to maintain negative pressure in the primary confinement area. (This might occur in the off-normal case wherein the supply fans continue to operate when the exhaust fans do not.) Each of the local filters and prefilters, as well as the main exhaust filters, have 'bag-out' capability. Spent filters are compacted and packaged as rad. waste when removed from service.

\subsubsection{Office/clean areas}

Filtered and conditioned supply air is provided to the office and clean areas of the plant, and this air is returned to the inlet section of the air handling units. Recirculating conditioned air in offices and clean areas provides a measure of energy conservation, decreasing energy usage. Conditioned air passing through the shop is exhausted via the building HEPA Filters and discharged to the atmosphere through the stack; passing through the building only once.

\subsubsection{Design features of the HVAC system}

The air handling room has inlet louvers below the ceiling that are placed in the exterior wall of the building. Inside there are two Supply Air Handlers. The inlet louvers are equipped with radiant heat panels for frost removal in cold climates. The fresh outdoor air passes through pre-filters and bag filters after passing through steam and chilled water heat exchangers for heating and cooling. During the summer air conditioning season the incoming air is sub-cooled and reheated for dehumidification prior to distribution in the building. Drains are present in the inlet section to remove condensed humidity. Recirculated air from the offices and clean areas is mixed with fresh air upstreame of the 1 intake filters. Two large supply air fans, mounted to the floor slab in the room, drawing on a common plenum provide air from the inlet conditioning and intake filter units. Flow from the air handler fan outlet plenum is distributed throughout the building to provide effective circulation, maintain ventilation confinement barriers and ensure there are no dead spaces. Two main supply ducts run the length of the FFC along the length of the personnel corridor above the suspended ceiling and diffusers distribute fresh air throughout the length of the personnel corridor via control valves operated by the central ventilation control unit. Supply and return air registers are also provided in the air handling room and a branch of the supply air duct runs to the Exhaust Fan Room. Supply air is also ducted to uncontaminated areas such as the dock and assembly area.

The two-story Exhaust Fan Room has three exhaust fans which remove air from the shop, casting area, secured controlled storage areas and labs. Two large exhaust headers collect air from branches leaving the shop, from hoods, task exhausts, off-gas systems, labs and secured controlled storage areas. Air is exhausted from primary confinement areas maintaining these areas at below atmospheric pressure at all times. Air drawn from these areas is supplied from inlet valves along the length of the personnel corridor penetrating the wall into the shop. The two main exhaust ducts, below the roof in the high bay, run to a common inlet plenum in the Exhaust Fan Room which discharges via prefilters and fire 
screens to three 4 by 5 HEPA filter units. Each of the three units has 2' X 2' HEPA filter banks in two columns 2 filters wide X 5 high. There are 6 such banks, 5 are capable of handling the whole exhaust flow. When changing HEPA filters, any one of the six banks can be isolated for testing or for filter bag-out. A moveable scissor man lift or portable platform is needed to access the upper bays on the second floor. In addition to pre-filters, two stages (secondary and tertiary) of HEPA filters are provided. The three units lead to a common exhaust plenum which is exhausted by three $150 \mathrm{hp}$ exhaust fans on the ground floor. The outlet flow from the fans leads to a common exhaust duct then to a 70" diameter stack outside the building. The stack ends 25 feet above the peak of the building roof and is equipped with an isokinetic stack monitor.

The facility ventilation air flow is based on four air changes per hour for the entire building. Distribution and exhaust branches are arranged and controlled such that most flow is drawn from the casting and rolling areas, and air in other areas of the shop flows toward the highest areas of contamination probability.

The exhaust HEPA filter banks are equipped with low flow water mist fire suppression nozzles required by DOE regulations and the filter units are equipped with drains that overflow a normally oil filled P-trap. Spent fire suppression water collects on the floor.

\subsection{Shielded Cells}

No radiological shielding is necessary for the fuel material as it LEU only very slightly radioactive. Three reinforced concrete (on walls and ceiling) shielded cells are present in the FFC for radiography and fluoroscopy. These cells are shown with labyrinth entries which are effective at preventing reflected energy from penetrating occupied areas, and a warning light system or locking door will be provided as required to ensure personnel are not present in the cells when exposures are being made. In the direction that the x-rays are emitted from the source, they will directly impinge on the wall which will be made thick enough to prevent exposure to personnel on the opposite side of the wall. For purposes of this study, 12" thick walls are shown.

\subsection{Fire Protection}

\subsubsection{Basic Criteria}

Revision 1

The FFC has a fire protection strategy based upon DOE STD 1066-99, NFPA 101, NFPA 801, Life Safety Code, and the IBC. The building has been determined to be a Special Purpose Industrial Occupancy in accordance with NFPA 101.

In accordance with the IBC, for H-type occupancy, the FFC will be of Type II-B construction.

Safe practices are in use such as to limit flammable and combustible material accumulation, separation of oxidizers from corrosives (by distance or containment), the use of regular fire safety inspections, and through the use of special protocol procedures, unique to the $\mathrm{FFC}$, and techniques that encourage the high levels of fire safety required by DOE STD 1066-99.

\subsubsection{Fire Protection Design Features}

A. Fire Water Supply and Duration:

It is assumed the existing federal facility fire water system, to which the FFC is connected, is capable of providing the required fire water flows, pressures and duration to meet the design criteria for the FFC. 


\section{B. Fire Sprinkler Systems:}

The entire building is protected by a wet-pipe automatic fire sprinkler system as required by DOE STD 1066-99, Chapter 7. The system will be designed and installed in accordance with the requirements of NFPA 13 . The fire sprinkler system will be designed for Ordinary Hazard Group 2 criteria: $0.18 \mathrm{GPM} / \mathrm{ft}^{2}$ over a design area of 2500 $\mathrm{ft}^{2}$, with an outside hose stream allowance of 250 GPM. Although there are no unventilated areas within the FFC, anti-freeze systems will be utilized in any unheated area, if required.

\section{Building HEPA filters:}

The building HEPA filters will be protected in accordance with DOE STD 1066-99, Chapter 14. The deluge fire protection systems shall be designed to deliver 0.25 GPM over the entire filter face areas. In order to minimize system overage, low flow BETE nozzles will be utilized, in additional to the possible use of pressure control valves on the HEPA filter deluge risers.

D. Fire Alarm System:

The building fire alarm system will be compatible with the existing fire alarm systems currently in use at the Lab Site where the FFC is constructed. The fire alarm system will comply with NFPA 72. Manual pull stations, indicating devices, and detection devices will be installed as required by DOE STD 1099-99 and NFPA 101. The fire alarm system will also monitor all fire sprinkler system pressure switches, tamper switches, and solenoid valves for triggering of the HEPA filter deluge systems.

E. Fire Extinguishers:

Fire extinguishers will be of a type required for the specific hazard and located in accordance with DOE STD 1066-99 and NFPA 10.

\section{F. Exiting:}

Building exits will be arranged such that the travel distances, including common paths and dead-end distances, will be in accordance with NFPA 101, Chapter 42.

G. Fire Water Loop:

An 8" diameter underground fire water loop encircles the FFC, and hydrants are present outside the roadway sufficiently spaced (approximately every 300'). The loop is supplied with fire water from the adjoining federal facility.

H. Exit Signage and Emergency Lighting:

Exit signage shall be located in accordance with NFPA 101. Emergency lighting, consisting of self-contained lighting with battery packs will be located as required by NFPA 101. 

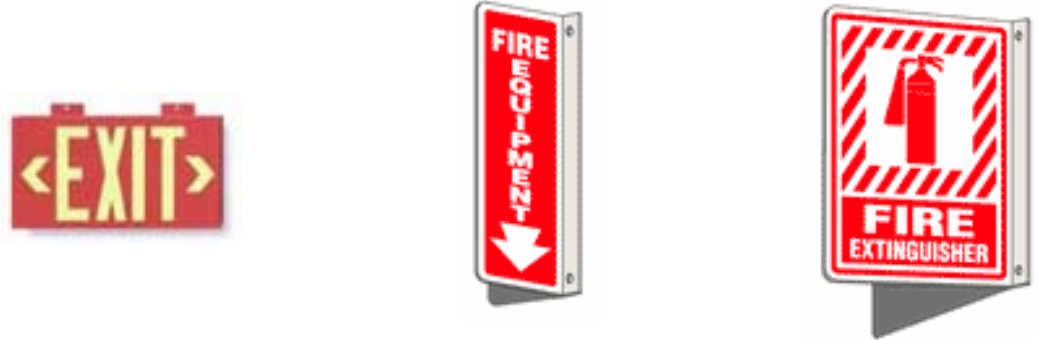

FIGURE 5-11 EXAMPLES OF EMERGENCY SINAGE

\section{Alloy Fuel Fire Control}

Although the alloy fuel material is not pyrophoric, the potential exists for a metal fire within the FFC. If such a fire occurred, the fire would be allowed to burn itself out prior to allowing water to be applied. This may require the automatic fire sprinkler system be turned off in order to prevent application of fire sprinkler discharge over the burning metal. Such a condition would be required to be addressed in the building policy and procedure manual. The FFC will certainly include in its policy a mandate for use of safe practices such as limited flammable material accumulation, isolating oxidizers from corrosives, regular fire safety inspections, and use of protocol procedures and techniques that encourage fire safety.

The Site on which the FFC is located must have a fire water supply capacity capable of delivering water to $2500 \mathrm{ft}^{2}$ at $0.18 \mathrm{gpm} / \mathrm{ft}^{2}$ for 60 minutes $(27,000 \mathrm{gal})$.

J. Fire water and disposition

Fire water dispensed during an event accumulates in the shop area on the floor. The collection depth on the floor of the shop is 6 inches deep, more than sufficient to allow accumulation of the discharge from 15 heads for 30 minutes. This necessitates flat concrete floors, ramps at the doors, and geometrically favorable sumps. Should fissile material 'burn' in a fire event, and become 'smoke particles' which wash down and accumulate on the floor because of the sprinkler discharge, the floor provides a suitably flat geometrically safe plane. Six inch diameter sumps, located throughout the shop, are each equipped with a drain jet which routes spent fire water to tube (or pencil) sampling tanks with an ID of 6" or less. The array of three tube tanks fits vertically in the underground secured controlled storage area. The tanks are fitted with pumps and valves to provide recirculation capability to suspend settled solids in the runoff water allowing a representative sample to be taken of the water drained from the sumps. If fissile material is not present in the sample, the water in the tube tank may be drained into the under ground accumulation tank via a filtration unit. The water in the tank, having been shown to be free of fissile material, is then disposed of as waste water. Any fissile material collected in the filter is disposed of as low level rad. waste. The drain water cRlersion 1 tank is sized to receive 30 minutes discharge from 15 heads, or about 8400 gallons. The size of the collection tank in the underground secured controlled storage area is 8 ' diameter X 24' long. This size is based on the Casting Area in the FFC which is approximately $50^{\prime} \mathrm{X} 50^{\prime}$ or $2500 \mathrm{ft}^{2}$. This area requires 25 Heads at $10^{\prime}$ O.C. both ways. Sprinklers discharge at $18 \mathrm{gal}$ per head $\left(0.18 \mathrm{gal} / \mathrm{ft}^{2}\right)$. Say one of two casting areas are affected by fire ( $>1 / 2$ the casting area), then 15 heads produce $280 \mathrm{gpm}$. For 30 minutes of discharge, the result is 8400 gallons. 


\subsubsection{Waste Drains}

Since sumps will be present, spaced regularly through out the FFC along the shop perimeter, and the sumps are geometrically favorable (less than 6" diameter), then these sumps could be used for convenience-disposal of non-reactive liquids and generally uncontaminated waste water, such as dirty mop water, or spent rinse water used for cleaning lab equipment, or final rinses following a spill cleanup and similar dirty but non hazardous liquids. Any material thus dispatched must be compatible with the drainage and tank material, and acids, bases, salt solutions, organics, radioactively contaminated solutions, volatiles or flammables are expressly excluded. Each sump is equipped with a sump level indicator which triggers an indication on the central plant monitor that liquid is present in the sump. An extensive control system is not envisioned, however, a local operator could energize a local valve allowing an air energized jet to discharge the sump liquid to the tube (sample) tanks, allowing a sample to be taken after recirculating the liquid in the tube tank. When sample analysis shows that the material is free of nuclear material, this would be transferred to the underground fire runoff water collection tank via a filter which removes the solids. If used in this manner, the accumulation tank would require a level control to indicate that sufficient empty volume to capture fire runoff water remains available. Over time, as the level in the collection tank increases with convenience use, the tank will need to be pumped out and the spent water solution transferred to a liquid waste treatment facility (LWTF). Vendor service as needed will be contracted to remove waste liquid, using a tank truck, for transport to the LWTF.

\subsubsection{Floor Finish}

The floor of the shop areas of the FFC is coated with a durable epoxy coating, which will be maintained as needed in high traffic areas. The floor finish and other construction details present in the FFC facilitate decontamination and cleanup.

Revision 1 


\subsection{Warehouse}

The 55' $\mathrm{X} 70$ ' warehouse is used at the FFC to facilitate efficient operation of the plant. The warehouse is used for storing non nuclear material, packaging, provisions, seasonable items, consumables, tools and supplies. Between campaigns, tooling and specialty items related to specific fuel types not being processed are stored in the FFC Warehouse. Empty shipping containers and other packaging materials such as drums are stored here along with cold chemicals (in totes), spare parts, and mechanical and electrical supplies. Pallets on racks are the dominant storage theme. Using a pallet rack two high, in five rows, 7 pallets long with forklift aisles between (two rows are back to back), up to 70 pallets can be accommodated, occupying a $40 \mathrm{X}$ $53^{\prime}$ footprint. A preliminary takeoff shows this warehouse occupies approximately a 55' X 70' footprint, one story, 12' under the trusses, and includes a dock, single rollup door, a small office, and a water closet. The building has a concrete floor, and is a non-combustible metal structure equipped with automatic fire sprinklers, emergency lighting and fire alarm. An electric forklift is used for access to storage and material handling, and an inventory / database system provides minimum quantities, and keeps track of stock on hand. A networked data entry terminal is present in the warehouse.

Tools or fixtures that are used or have been used for fuel manufacture are likely to have traces of fixed contamination. Decontamination of this equipment will be undertaken to reduce levels as approved by health physics. No items possessing accountable material will be stored in the warehouse. Contaminated materials will be packaged under the direction of health physics using acceptable practices (such as in heavy poly bags, and no sharp corners) and will be stored in labeled boxes. Tool decontamination or maintenance procedures are not performed in the warehouse. There is no provision for material control or confinement or ventilation barriers, or accountability.

The insulated metal warehouse building is single story, 12' under the trusses, and includes a dock, single rollup door, two personnel doors, a small (prefabricated) office, and a water closet with a small hot water heater and drinking fountain. The building has a reinforced concrete floor with floor drains, and is a non-combustible structure equipped with automatic fire sprinklers, emergency lighting and fire alarm. Suspended under-truss lighting and task lighting systems are utilized. There will be roof drains that runoff into the surrounding paved lot, and connections will be made to potable water, sanitary sewer, and electrical service. Admission to the warehouse is controlled, and the property is secured and locked and all doors are closed when not occupied. 


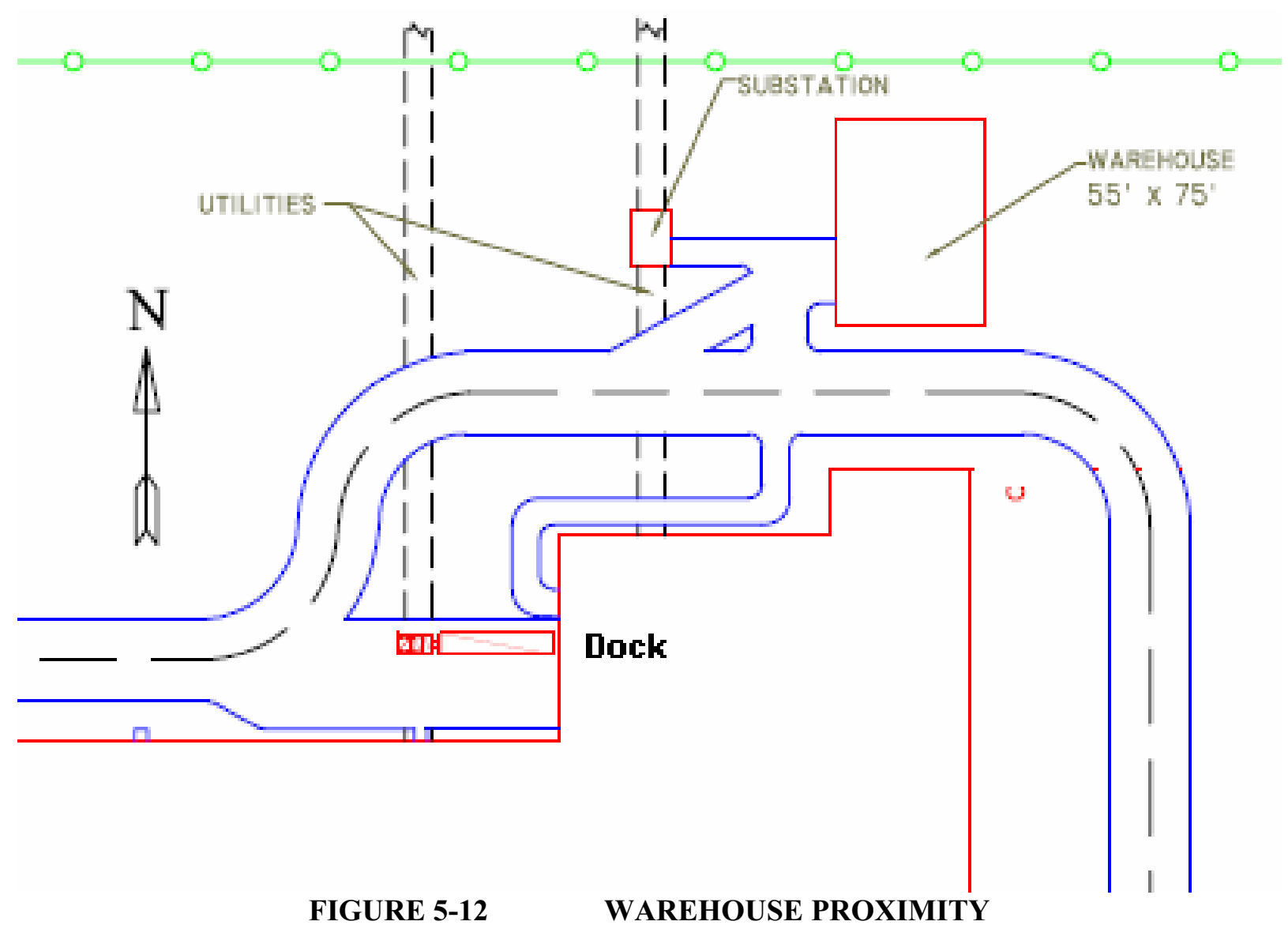

The warehouse is situated close to the FFC dock, because material is frequently moved between these two areas. It is within the fenced perimeter and is accessible by delivery truck and on-site site vehicles. Vehicles must pass through the entry control facility prior to moving to the warehouse dock to make deliveries. A roadway for delivery trucks and fire-truck access exists (outdoors) between the warehouse and the FFC. Entrances to the FFC, the dock, or access portals in the main building for equipment load-out (such as for HIP vessel removal) will not be blocked by the warehouse. A paved walkway is shown between the warehouse and dock for pedestrian traffic, and a grade level personnel door is available next to the dock to allow material moved on a 2 wheeler to be moved back and forth between the buildings.

A material takeoff is provided below to determine space requirements and is factored into the study estimate. 


\section{TABLE 5- 2 ESTIMATION TABLE FOR SPACE REQUIREMENTS}

\begin{tabular}{|c|c|c|c|c|}
\hline & & FFC Wareho & & \\
\hline Type & Contents & Cha racteristic & Campaign Related & Sq Ft. \\
\hline Provisions & Office Iterns & Boxes & & 16 \\
\hline & Signage & & & \\
\hline & Barriers & & & \\
\hline Supplies & Cleaning & Boxes & & 16 \\
\hline & Inventory, Files & Computer & & 80 \\
\hline Material & Cladding & & 5 sets & 80 \\
\hline (fuel related) & Rolling Cans & & 5 sets & 80 \\
\hline & Zr dadding & & & \\
\hline & Subass'y End Pieces & & 5 sets & 40 \\
\hline Consumables & Spray mold release & Cans & & \\
\hline & Cold Chemicals & Totes & & 64 \\
\hline & Salt & Bags & & 16 \\
\hline Tools & Casting Molds & & 5 sets & 80 \\
\hline & \begin{tabular}{|l} 
Crucibles \\
\end{tabular} & & & \\
\hline & Inserts for storage bins & & 5 sets & 80 \\
\hline & Inserts for Vault storage & & 5 sets & 80 \\
\hline & Constraint fixtures & & & \\
\hline & Press dies & 19 sizes & 5 sets & 72 \\
\hline & Swaging tools & & 5 sets & 16 \\
\hline Packaging & Empty Drums & Pallets & & 32 \\
\hline & Shipping Boxes & Pallets & 5 sets & 360 \\
\hline & Bottles & & & \\
\hline & Empty containers & Pallets & & 32 \\
\hline Spares & Rollers & Pallet & & 16 \\
\hline & Hold Downs & & & 16 \\
\hline & Air Hose & & & \\
\hline & Sump Pump & & & \\
\hline & Weld filler & & & \\
\hline & Lifting Equipment & & & 16 \\
\hline & Heating elements & & & \\
\hline & Mechanical & & & 32 \\
\hline & E lectrical & & & 32 \\
\hline & Scales & & & \\
\hline & Stir Welder m andrill & Box & & \\
\hline & Spare Filters & & & \\
\hline & Dessicant cartridge spare & & & \\
\hline & Chucks & Box & & 16 \\
\hline Warehouse & F ork lift, charging station & & & 64 \\
\hline Operation & $P$ allets & & & 16 \\
\hline & Rack Shelving System & $\begin{array}{l}\text { Pallet Rack: } 2 \\
\text { high, } 7 \text { long, } 5 \\
\text { rows }=53^{\prime} \times 40^{\prime}\end{array}$ & & 2120 \\
\hline & P ack aging & & & 400 \\
\hline & Heating Equipm ent & & & \\
\hline & Dock Door & & & \\
\hline & Building Systern & & & $55^{\prime} \times 70^{\prime}$ \\
\hline & Rest-room & & & $\begin{array}{r}80 \\
\end{array}$ \\
\hline & Drinking Fountain & & & 4 \\
\hline & E ye Wash & & & 4 \\
\hline & Shipping/Recv'g office & & & 96 \\
\hline & Lockers & & & 8 \\
\hline & Carts & & & 32 \\
\hline & Dock & & & 600 \\
\hline & Fire Extinguishers & & & \\
\hline & Fire P rotedtion & & & \\
\hline & Receiving Area \& tools & & & 320 \\
\hline & Lighting & & & \\
\hline & Communication & & & 8 \\
\hline
\end{tabular}




\subsection{FFC Staff}

For the purposes of this study, the number of personnel present on a daily basis has been estimated for development of life-cycle costs, rest-room stalls, locker room capacity, etc. The total count consists of operational staff (including machinists, inspection personnel, health and safety specialists and technicians) that work in the shop, and professional or office staff (including security personnel). The personnel and the breakdown of where they work are in the table below. The allocation of offices occupied by the office staff is also shown. The drawing SK-ME-1 shows the office layout, restrooms, and conference or lunch rooms on both floors and the shop layout in which the FFC Staff work.

\subsubsection{Operational Staff}

\section{TABLE 5-3 FFC SHOP PERSONNEL TALLY}

\begin{tabular}{|c|c|c|}
\hline $\begin{array}{l}\text { Area } \\
\text { Code }\end{array}$ & Function & $\begin{array}{l}\text { Number of } \\
\text { personnel }\end{array}$ \\
\hline 1 & Ingot Cleaning, Casting, Mold and Crucible prep & $3 *$ \\
\hline 2 & Hot roll unclad U-Mo to $\sim 0.1$ " thick & 2 \\
\hline 2 & Heated Salt Bath (prior to rolling cast plates) & - \\
\hline 3 & Sectioning (four plates cut from each pre-rolled U-Mo strip) & 1 \\
\hline 3 & Salt Removal (sanding or media blasting prior to sectioning) & - \\
\hline 4 & Canning (automatic weld station load / unload) & 1 \\
\hline 5 & Hot rolling of Zr Clad U-Mo in purged C'Stl can & 2 \\
\hline 6 & De-canning, foil Trimming, can scrap management & 2 \\
\hline 6 & Anneal & - \\
\hline 7 & Chemical Cleaning (Al plates and Zr Clad U-Mo are cleaned in separate baths) & 1 \\
\hline 8 & Friction Bonding & 4 \\
\hline 9 & Fuel Element Trim, flattening and Surface Finish & - \\
\hline 9 & Fluoroscopy (verify position of foil inside bonded $\mathrm{Al}$ cladding) & - \\
\hline 10 & QA & 3 \\
\hline 10 & Rad Tech & 2 \\
\hline 10 & Lab - UT & 1 \\
\hline 10 & Lab - Radiologist & 2 \\
\hline 10 & Lab-Microscopy and sample prep & 1 \\
\hline 10 & Lab - Metallography / Chemistry & 1 \\
\hline 10 & Dimensional Inspection / Setup & 1 \\
\hline 11 & Element swaging, welding, machining, autoclaving and final product assembly & 11 \\
\hline 11 & Fuel Element Shape Forming (Press) & $1 *$ \\
\hline 12 & Shipping and Receiving (Product packaging + shipping container unloading) & 2 \\
\hline \multirow[t]{11}{*}{12} & Storage Area, order picking and stocking, Accountancy & - \\
\hline & Radiation Control & 2 \\
\hline & Runner & 1 \\
\hline & Programmer (shop floor programming area) & $2 *$ \\
\hline & Mechanical Maintenance & $2 *$ \\
\hline & Electrician / Calibration & $1 *$ \\
\hline & Waste Management / SNM Accountability & 2 \\
\hline & Warehouse & 2 \\
\hline & Housekeeping (shop \& warehouse) & 2 \\
\hline & Shop Supervisor & 1 \\
\hline & Total Shop Personnel......................................................... & 55 \\
\hline
\end{tabular}


- Area codes refer to markers on Layout Plan and Flow Diagrams.

\subsubsection{Office Staff}

TABLE 5-4 FFC OFFICE PERSONNEL TALLY

\begin{tabular}{|l|c|}
\hline Function & $\begin{array}{c}\text { Number of } \\
\text { personnel }\end{array}$ \\
\hline Production Manager & 1 \\
\hline QA Manager & 1 \\
\hline Maintenance Manager & 1 \\
\hline Security (2 at gate) & 3 \\
\hline Secretary / Receptionist & 1 \\
\hline Records management & 1 \\
\hline Procurement / Purchasing & 1 \\
\hline Rad Con, ES\&H, Environmental Compliance & 1 \\
\hline Scheduling & 1 \\
\hline Facilities Manager (HW) & 1 \\
\hline Guest & 1 \\
\hline Safety and Environmental Compliance & 1 \\
\hline MC\&A & 1 \\
\hline Total Office Personnel............................................................ & $\mathbf{1 7}$ \\
\hline
\end{tabular}

Notes:

- Total count includes two guards in Entry Control Facility that are part of the assigned staff and will make use of the FFC rest rooms.

- Guests may be representatives or QA personnel from reactors with ongoing fuel manufacturing campaigns.

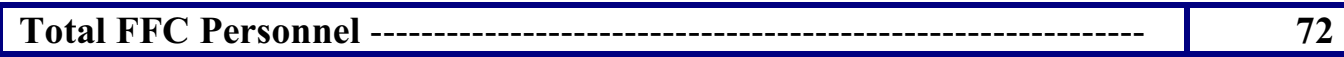

TABLE 5-5 OFFICE SPACE DESIGNATION

\begin{tabular}{|c|c|c|c|c|c|}
\hline & & & Area & $\begin{array}{c}\text { Hard } \\
\text { walled } \\
\text { (HW) } \\
\text { offices }\end{array}$ & $\begin{array}{c}\text { Cubicl } \\
\text { es }\end{array}$ \\
\hline Engineering & (HW) & 2 & QA Manager & & 1 \\
\hline Production Manager & $(\mathrm{HW})$ & 1 & Maintenance Manager & & 1 \\
\hline $\begin{array}{l}\text { Women's Rest Room/ Change } \\
\text { Room }\end{array}$ & (HW) & - & $\begin{array}{l}\text { Men's Rest Room/ Change } \\
\text { Room }\end{array}$ & (HW) & - \\
\hline Upstairs Women's Rest Room & $(\mathrm{HW})$ & - & Upstairs Men's Rest Room & (HW) & - \\
\hline Housekeeping (supply closet) & & - & $\begin{array}{l}\text { Rad Con, ES\&H, Safety \& } \\
\text { Environmental Compliance } \\
\text { manager }\end{array}$ & & 1 \\
\hline $\begin{array}{l}\text { Security, Central Control } \\
\text { (ground floor) }\end{array}$ & $(\mathrm{HW})$ & 1 & Scheduling & & 1 \\
\hline $\begin{array}{l}\text { Secretary / Reception (ground } \\
\text { floor) }\end{array}$ & $(\mathrm{HW})$ & 1 & Facilities Manager & $(\mathrm{HW})$ & 1 \\
\hline Records (lockable) & $(\mathrm{HW})$ & 1 & Guest & & 2 \\
\hline Procurement / Purchasing & & 1 & $\begin{array}{l}\text { Safety and Environmental } \\
\text { Compliance }\end{array}$ & & 1 \\
\hline $\begin{array}{l}\text { Lunch / Vending / Meeting } \\
\text { (ground floor) }\end{array}$ & & - & Copier/ Fax/ Office Supplies & & - \\
\hline
\end{tabular}




\subsection{Personnel Protective Equipment}

PPE for personnel in the FFC includes lab coats, shoe covers over foot wear suitable for the industrial environment, work gloves, cotton gloves and safety glasses. No respiratory protection is in use beyond local task exhaust ducts and hoods, and casual use of nuisance dust filters such as for cleanup or maintenance tasks.

\subsection{Friskers and Monitors}

Full body friskers and hand and foot monitors will be present at entrances/exits to the RBA which are at the Locker-room/Change room exit to the Personnel Corridor, and between the Personnel Corridor and the Receiving, Shipping and Storage Area. Within small alcoves, there is a personnel waiting area, separated by a handrail from the corridor, at each monitoring station. In addition to the stack monitor, there are battery backed criticality alarms present within the shop and fuel accumulation areas, as are continuous air monitors (CAMs). The multiple CAM samplers, which are located at face level near each personnel work station involving working with unclad fuel, make use of a central vacuum and distribution system. The small individual filters in the samplers are replaced and counted periodically by industrial health personnel.

\section{$5.14 \quad$ Facility Operation}

The facility operates normally in a single (day) shift mode. However, the FFC can accommodate up to three shifts per day to resolve production bottlenecks or increase production. Some small personnel presence may be required for certain backshift operations, primarily monitoring long run process steps (such as autoclave or HIP processes). Campaign changeovers are accounted for in the production schedule. The facility operating basis is a six day week and 260 days per year of availability. The FFC is designed for a 40 year facility life, wherein it may be necessary to change out any piece of equipment or control system component during the plant life.

\subsection{Control Room}

The central facility control room is located on the first floor in the common office area behind the receptionist. CCTV monitors display images from cameras in the storage areas, dock, building entrances ECF, warehouse and lot. Recordings are made from the camera signals and stored for a period of time for security reasons. HVAC control, door monitors, stack monitor, sump monitors, central alarm annunciators, and communications are available in the central control room which in addition to the office space needed for the operator, also includes a desk, computer, a small server farm, and I/O. The central control room will be the lead point for the incident command function during operation of the FFC.

\subsection{Utilities}

Utilities present at the FFC include electrical service, potable water, deionized water, fire water and sanitary sewer. No emergency power needs are identified that would require a standby generator; however alarms for criticality and air monitoring are battery backed. An argon dewar provides a supply of gas at atmospheric temperature and pressure for bleed and feed to the furnace enclosures and for the entry and exit airlocks in the casting area. If the HIP option is utilized, a second argon dewar will be provided because the HIP attains pressurization in part by 
vaporizing and heating argon gas Some 400 gallons of liquid argon may be consumed during a HIP run, but with additional equipment, much of this can be recycled..

\subsection{Electrical Service}

High voltage is stepped down to 480 VAC service using transformers at a substation located outside the FFC. The majority of the machines utilize 480VAC, in combination with 120 VAC. Lighting, heating (including steam generation) and ventilation loads dominate the power usage because these are typically 24 hour per day services whereas machine loads are intermittent, however 25HP machine tools are common. The three 150HP exhaust fans used to maintain facility ventilation confinement are the largest attached loads.

\subsection{FFC Communication}

At the time of preparation of this study report, in-plant wireless communication details are insufficiently defined to allow INL security personnel to definitively predict what the rules will be concerning radio communication for the FFC. Crane controls, inventory tracking, material orders made up for production batch use, material packages sent to interim storage, monitoring information and alarm summaries could be made available throughout the plant using a local wireless network. However, if encrypted radio frequency signals are not allowed, hard wired communications are acceptable. Accountability tracking data transfer will be accomplished using a hard wired or local (plant wide) fiber-optic network. Communication connections from the Federal Site, to which the FFC adjoins, will be made to service the connection with the public communication network and site alarms.

\subsection{FFC Facility Operation}

Facility operation and cost to operate the FFC consist of 1) a management and labor component, 2) use of expendable consumables and resources, 3) maintenance and upkeep of the building and process systems, 4) packaging and transportation, 5) utilities and energy use and 6) contracts or subcontracts provided by suppliers and service providers that fulfill various aspects required to meet the needs of daily use and delivery of fuel to the reactors serviced. A cost must also be factored in to account for, at the end of life of the facility, the costs to decommission the plant and dispose of the residuals and return the site to a 'green field' condition (7).

The list that follows divides the operational aspects into various 'accounts' or functional areas, which are intended to represent known usage of consumables, and expendables. The list is inclusive of service and maintenance needs.

\subsubsection{Management and Labor}

As shown above (section 5.11), some 72 personnel are full time staff that work within the on a daily basis. An average annual salary and benefit package including insurance, unemployment and training needs to be budgeted for each individual. In addition, there will be perhaps two full time managers outside the facility and at least two QC personnel that work outside the plant at supplier facilities. 
5.19.2 Expendable consumables and resources

A. Chemicals and Consumables

Process Consumables (solids)

- LEU-10Mo - raw alloy: 7.5 metric tons/yr.

- Zirconium - foil: 11,500 lbs./yr.

- Aluminum pre-machined cladding plates: $164,000 \mathrm{lbs} . / \mathrm{yr}$.

- Aluminum fuel subassembly end and side pieces and hardware: 100,000 lbs./yr. (est.)

- Grafoil - graphite sheeting: 100,000 lbs./yr. (est.)

- Steel - rolling cans; 408,400 lbs./yr.

- HIP cans (if applicable)

- Yttria - aerosol spray for can coating: 1 gross/yr.

- Electrodes - welding electrodes and filler material

- Paper, Plastic - cleaning supplies

- Graphite - crucible (bottom pour), lids

- Graphite -book molds (various types for different fuels and different sizes)

- $\mathrm{KCL}-$ salt bath: 50\#/yr. (est.)

- $\quad \mathrm{LiCl}$ - salt bath : 50\#/yr. (est.)

Process Consumable (liquids)

- $30 \% \mathrm{HNO}_{3}$ - cleaning (U-Mo, $\mathrm{AL}, \mathrm{Zr}$ sandwich): 6,700 gallons/yr.

- $\mathrm{NaOH}$ - cleaning (aluminum cladding): 3,200 gallons/yr.

- HF - Yttria removal: 24 gallons/yr.

- Ethanol - foil cleaning: 480 gallons/yr. (bath)

- Water treatment chemicals for DI water - element cleaning

Process Consumables (Gases)

- Argon-, HIP (if applicable): usage is 400 gallons per cycle, assume 100 gallons per cycle use, with the remainder being recycled; 265 cycles per year

- Argon - Casting furnace: $15 \mathrm{SCFH}: 130,000 \mathrm{ft}^{\wedge} 3 / \mathrm{yr}$, entry/exit Glove Box: $100 \mathrm{ft}^{\wedge} 3 /$ day: $26,500 \mathrm{ft} \wedge 3 / \mathrm{yr}$.

- Bottled Argon for welding: $35 \mathrm{ft}^{\wedge} 3 /$ day: $10,000 \mathrm{ft}^{\wedge} 3 / \mathrm{yr}$.)

- Bottled Helium - can leak test: 20,000 ft^3/yr.

- Desiccant for dry instrument air - and element drying

Equipment Consumables

- Casting Furnace

- Glovebox seals

- Conveyor motor(s)

- Conveyor rolls

- Vacuum seals

○ Vacuum pump oil

- Finishing Mill(s)

○ Chucks

○ Milling bits

- Lubricating oils

○ Hydraulic oils 
- Machining/Cooling oils

- Friction Stir Welder

- Friction stir welding pin (machining tool)

- Lubricating oils

- Hydraulic oils

- Machining/Cooling oils

- Drive motor (spare)

- Spare Chucks

- Spares for recirculating coolant systems

- Hydraulic Shears

○ Shear Blades

○ Hydraulic oil

- Rolling Mill(s)

○ Drive Motor (Spare)

- Roll refurbishing

- Roller bearings

- Roller chains

- Lubricating oils

- Hydraulic oils

- Roller Leveler

- Drive motor(spare)

○ Roll refurbishing

- Roller bearings

- Roller Chains

- Lubricating oils

- Hydraulic oils

- Hydraulic Press

○ Tooling

○ Hydraulic oil

- HIP

○ Cooling system

- Vacuum seals

- Hydraulic oils

○ Vacuum pump oil

- HIP recertification (subcontract)

- Argon dewar spare valve \& gage

- Ultrasonic Tester

- Filters (water recirculation)

- Probes

- Cleaning Baths

- Filters (cleaner liquid recirculation)

- Spare level probes

B. Lab Consumables

- Sample bottles

- Pipettes, spectrometer specimen vials

- Equipment Gas

○ Oxygen

- Nitrogen

- Argon 


\section{○ Helium}

- Crucibles (\#samples per year)

C. Safety Equipment

- PPE (general)

- Safety toe shoes for machine shop and assembly area

- Gloves; leather, 10 pair/yr, 500 count latex, 500 count cotton

- First Aid kit

- Safety Glasses: 100 /yr

- Lab coats, aprons, hair nets or caps: 100 count

- Biohazard spill cleanup kit

- Cardio/pulmonary resesutation

- Film badges: 50 count

- Swipes, kimwipes

- Hand/Foot Monitor calibration coupons

D. Electronic Media

- Manufacturing Record Storage

- Radiography

- Ultrasonic

- Accountability records

- Copy paper (1 pallet / year)

E. Spare Parts, Maintenance

- Switches

- Transducers

- Encoders

- Measurement accessories

- Tools, Testers

- Compressor oil: $5 \mathrm{gal} / \mathrm{yr}$

- (see also spare-parts material takeoff in estimate, and individual callouts above)

F. Other

- Calibration Standards

- Test Gages

- Signage

- Labels

5.19.3 Maintenance and upkeep of the building and process systems

A. Building Consumables

- Battery backup for alarms (3 year replacement interval)

- Lighting (building and lot)

- Waste Packaging Drums and bottles,

- Sorbant

- Shipping containers for assembled elements (400/yr)

- Janitorial supplies 
- Pallets

- Office supplies

- Refrigerant (HVAC system)

- Filters - HEPA filters (building): 60/year and prefilters: 60/yr, inlet filters and bags (1 set per year), Local HEPA filters (30/yr), demisters (15/yr), sintered metal filters (3/yr), pre-filters (20/yr)

- Off-gas absorbers

B. Building Upkeep

- Grounds keeping (subcontract)

- Snow removal (subcontract)

- Painting

- Roof upkeep

- Stack maintenance

C. Forklift(s)

- Battery(s) (3 year replacement interval)

- Wheels, Tines, accessories

- Hand Cart (spare)

5.19.4 Packaging and transportation

5.19.5 Utilities and energy use

5.19.6 Contracts or subcontracts provided by suppliers and service providers

- Copy machine: Maintenance Contract

- Laundry contract

- Radiography: Maintenance Contract

- Ultrasonic Tester: Maintenance Contract

- Fluoroscope: Maintenance Contract

- Roll mill roller maintenance contract

- Waste haulage and disposal subcontract

5.19.7 Decommissioning and Disposal

This one time expense might be paid for by adding to an interest bearing account on a yearly basis, with a future value, 40 years after facility commissioning, suitable for paying the cleanup subcontract and disposal cost. No or very little salvage value is expected to be realized, other than as scrap material. 


\subsection{INITIAL FACILITY HAZARDS EVALUATION}

Consistent with the requirements of DOE STD-1189-2008 ${ }^{1}$, this chapter provides an initial evaluation of the hazards that are expected to be present in the facility based on the current preconceptual stage of development of the design. For those hazards identified in this initial hazards evaluation, potential controls for preventing or mitigating the hazards are also identified. For the purposes of this preliminary hazards evaluation, a "hazard" is "anything that has the potential to cause harm". The two principal "receptors" for hazards at nuclear facilities are the public and facility workers.

\section{Public Consequence Analysis}

The consequence (dose) of an accidental release of LEU-Mo material to the "maximally exposed off-site individual" or MEOI is calculated following the standard methodology detailed in DOE STD 3009-94 ${ }^{2}$ for identifying safety-class equipment (equipment that protects the public from the determination of safety-class equipment. This dose is the "committed effective dose equivalent (CEDE)" from the inhalation pathway. The CEDE is the total dose equivalent deposited in tissue over the 50 year period following intake of the radionuclide. The DOE defines an evaluation guideline of 25 rem to the MEOI as the threshold for defining safety-class systems, structures and components (SSCs). For the purposes of this analysis, we will assume that an MEOI dose greater than 5 rem (consistent with the guidance in DOE STD 1189 will require additional consideration as potentially requiring safety-class SSCs.

The importance of this "safety-class" designation is primarily one of cost. Safety-class SSCs require significant quality assurance documentation, configuration control, documented surveillance to ensure operability within defined margins, etc. Direct and indirect costs for installation, operating, maintenance, and replacement of safety-class SSCs are substantially higher than for other plant equipment.

The following scenario is assumed for this dose calculation:

A total of $5300 \mathrm{~kg}$ of LEU $10 \%$ Mo (one year's throughput) is involved in a fire that converts the material to dispersible oxide form $\left(\mathrm{UO}_{2}\right.$ or $\left.\mathrm{U}_{3} \mathrm{O}_{8}\right)$. It is assumed (consistent with analyses in DOE-HDBK- $3010^{3}$ and DOE-STD- $1027^{4}$ ) that $0.1 \%$ of this material is entrained in the smoke, and that all of the airborne material is respirable (i.e., has a mean aerodynamic diameter less than 10 microns). The entire amount of airborne material is released from the facility at ground level, consistent with the requirements of DOE-STD1189-2008. The MEOI is located at the site boundary $100 \mathrm{~m}$ downwind from the fire, and that the plume is dispersed in the air in accordance with the assumptions provided in DOE-STD-1189-2008. The CEDE is calculated as follows:

$$
\operatorname{CEDE}(\text { rem })=\text { MAR } \times \text { DR } \times \text { ARF } x \text { RF } x \text { LPF } x \text { SA } x \text { DCF } x \text { BR } x \chi / Q
$$

where:

MAR material at risk - inventory available in an accident $\left(5300 \mathrm{~kg}\right.$ of $\mathrm{U}-10 \% \mathrm{Mo}=4.77 \times 10^{6}$ grams uranium @20\% $\mathrm{U}^{235}$ enrichment)

${ }^{1}$ Integration of Safety into the Design Process, DOE-STD-1189-2008, March 2008, 191 pp.

2 Preparation Guide for US Department of Energy Nonreactor Nuclear Facility Documented Safety Analyses, DOESTD-3009-94, Change Notice \#3, March 2006, 144 pp.

${ }^{3}$ Airborne Release Fractions/Rates and Respirable Fractions for Nonreactor Nuclear Facilities, Volume 1 Analysis of Experimental Data, DOE-HDBK-3010-94, December 1994, 359 pp.

${ }^{4}$ Hazard Categorization and Accident Analysis Techniques for Compliance with DOE Order 5480.23, Nuclear Safety Analysis Reports, DOE STD-1027-92, Change Notice \#1, September 1997, 49 pp. 


\begin{tabular}{|c|c|}
\hline DR & damage ratio - fraction of the available inventory affected by the accident (1.0) \\
\hline ARF & $\begin{array}{l}\text { airborne release fraction }- \text { fraction of the material that becomes airborne }\left(1 \times 10^{-3}\right) \text { (from } \\
\text { DOE HDBK } 3010-94, \mathrm{p} 4-42)\end{array}$ \\
\hline $\mathrm{RF}$ & $\begin{array}{l}\text { respirable fraction }- \text { fraction of the airborne material having a mean aerodynamic } \\
\text { diameter }<10 \mu \mathrm{m}(1.0) \text { (from DOE HDBK 3010-94, p 4-42) }\end{array}$ \\
\hline LPF & leak path factor - fraction of the airborne material that leaves the facility (1.0) \\
\hline SA & specific activity - curies/gram for the material $\left(7.12 \times 10^{-7} \mathrm{Ci} / \mathrm{g}\right.$ for $20 \%$ enriched $\left.\mathrm{U}\right)$ \\
\hline DCF & $\begin{array}{l}\text { dose conversion factor }- \text { rem/curie for the material }\left(1.2 \times 10^{8} \text { for } 20 \% \text { enriched)(from }\right. \\
\left.\text { Federal Guidance Report } \# 11^{5} \text {, Table } 2.1\right)\end{array}$ \\
\hline BR & breathing rate $-3.5 \times 10^{-4} \mathrm{~m}^{3} / \mathrm{s}$ \\
\hline$\chi / \mathrm{Q}$ & $\begin{array}{l}\text { atmospheric dispersion factor based on site-specific conditions (recommended value from } \\
\text { DOE STD } 1189-2008 \text { on the order of } 3.5 \times 10^{-3} \mathrm{~s} / \mathrm{m}^{3} \text { ) }\end{array}$ \\
\hline
\end{tabular}

This result is only one-tenth of the DOE-recommended threshold for challenging the evaluation guideline. None of the other identified hazards has a potential consequence to the public that exceeds this result. Therefore, we can conclude that none of the safety systems will be designated as safety-class. The fact that no safety-class SSCs are required will reduce the QA requirements as well as the costs for the design, procurement, installation, operation and maintenance of equipment and structures for the FFC.

\section{Worker Safety}

The remainder of the preliminary hazards evaluation focuses on worker safety issues. As discussed in the requirements section, above, worker safety issues can be categorized as either standard industrial hazards or as radiological hazards. Industrial hazards are typically regulated by OSHA and are covered by the facility industrial hygiene program. Radiological hazards at DOE sites are regulated under 10 CFR 835 and are covered by the facility radiological protection program. Both types of worker hazards must be addressed in the hazards and accident analysis (chapter 3) as well as in the safety management programs (chapters 6-17) of the Documented Safety Analysis. A Hazards Analysis will be performed for the Conceptual Design and a formal Preliminary Documented Safety Analysis (PDSA) will be performed during Preliminary Design.

This preliminary hazards evaluation for the FFC was performed by evaluating the identified process equipment for those radiological and industrial hazards that might be expected to be present for that equipment. For each piece of process equipment, the hazards were evaluated for four phases of the equipment life-cycle: normal operation, maintenance, installation, and removal. The evaluation focused on sources of kinetic and potential energy, including mechanical, thermal, chemical, and radioactive forms. Thus, the equipment evaluation included identification of the chemicals that would be present in the process equipment area. An example of the evaluation sheet used in performing this evaluation is shown in Figure 6-1.

\footnotetext{
${ }^{5}$ Limiting Values of Radionuclide Intake and Air Concentration and Dose Conversion Factors for Inhalation, Submersion, and Ingestion, Federal Guidance Report \# 11, EPA-520/1-66-020, September 1988, 224 pp.
} 


\begin{tabular}{|c|c|c|c|c|c|}
\hline Process Equipment ID: & CV-001 & & & & \\
\hline Process Equipment Name: & \multicolumn{2}{|c|}{ Mold Feed Conveyor } & & & \\
\hline Process Equipment Description: & \multicolumn{2}{|c|}{ Conveyor for casting mold } & & & \\
\hline Sketch: & SK-2 & & & & \\
\hline Area Code: & \multicolumn{2}{|c|}{1} & & & \\
\hline Hazards: & Operation & Maintenance & Installation & Removal & Controls \\
\hline Thermal - Burns & $\mathrm{x}$ & & & & Insulated Gloves, Tools \\
\hline Thermal - Cold & & & & & Insulated Gloves, Tools \\
\hline Mechanical - Pressure & & & & & Pressure Relief Devices \\
\hline Mechanical - Pinching & $x$ & $\mathrm{x}$ & $\mathrm{x}$ & $\mathrm{x}$ & Equipment Guarding \\
\hline Mechanical - Rotating Equipment & $x$ & $\mathrm{x}$ & $x$ & $x$ & Equipment Guarding \\
\hline Mechanical -Falling Objects & & $\mathrm{x}$ & $x$ & $\mathrm{x}$ & \\
\hline Mechanical - Flying Objects & & & & & Eye Protection, Face Shields \\
\hline Mechanical - Lifting & & $x$ & $x$ & $x$ & \\
\hline Mechanical - Sharp Objects & & $x$ & $x$ & $x$ & \\
\hline Mechanical - Cutting & & & & & Equipment Guarding \\
\hline Electrical & & $\mathrm{x}$ & $x$ & $\mathrm{x}$ & Lockout/tagout \\
\hline Chemical - Corrosive & & & & & Eye Protection, Face Shields, Gloves \\
\hline Chemical - Ignitability & & & & & \\
\hline Chemical - Toxic & & & & & Ventilation, Respiratory Protection \\
\hline Chemical - Reactive & $\mathrm{x}$ & & & & \\
\hline Chemical - Fumes & $x$ & & & & Ventilation, Filtration, Respiratory Protection \\
\hline Non-lonizing Radiation & & & & & \\
\hline External lonizing Radiation - Chronic & $\mathrm{x}$ & $\mathrm{x}$ & & $\mathrm{x}$ & $\begin{array}{l}\text { ALARA Program, Time/Distance/Shielding, } \\
\text { Dosimetry }\end{array}$ \\
\hline Nuclear Criticality & & & & & $\begin{array}{l}\text { Geometry, Inventory, and Moderator Control, } \\
\text { Criticality Alarms }\end{array}$ \\
\hline Airborne Radioactive Materials & & & & & Ventilation, Filtration, Respiratory Protection \\
\hline ( & $\mathrm{x}$ & & & & $\begin{array}{l}\text { Combustible Control, Fire Detection/ } \\
\text { Suppression/Alarm System }\end{array}$ \\
\hline Explosion & & & & & \\
\hline Spills & & & & & \\
\hline Confined Space & & & & & \\
\hline Air Displacement/Asphyxiation & $x$ & $x$ & & $\mathrm{x}$ & \\
\hline Noise & & & & & \\
\hline Ergonomics & & & & & \\
\hline Slips, Trips \& Falls & & & & & Non-skid Surfaces, Handrails \\
\hline Chemicals: & & & & & \\
\hline U10Mo acorns, ingots, coupons, foil & $\mathrm{x}$ & & & & \\
\hline U10Mo foil trimmings & & & & & \\
\hline Zr foil & & & & & \\
\hline U10Mo-Zr Clad Plates & & & & & \\
\hline U10Mo-Zr Clad trimmings & & & & & \\
\hline $\mathrm{Nb}$ foil & & & & & \\
\hline $\mathrm{Nb}$ foil trimmings & & & & & \\
\hline $\mathrm{Al}-6061$ metal shapes & & & & & \\
\hline Al - 6061 milling scrap & & & & & \\
\hline Al Clad Plates & & & & & \\
\hline Fuel Assemblies & & & & & \\
\hline steel can & & & & & \\
\hline yttria coating in can & & & & & \\
\hline W welding electrodes & & & & & \\
\hline W friction bonding tool & & & & & \\
\hline paper, plastic cleaning supplies & & & & & \\
\hline Steel Mold (HR-160) & $x$ & & & & \\
\hline graphite crucible & & & & & \\
\hline yttria coating on crucible & $x$ & & & & \\
\hline & & & & & \\
\hline $\mathrm{KCl}$ salt for salt bath & & & & & \\
\hline $\mathrm{KCl}$ contaminated/spent salt & & & & & \\
\hline slag from melter & & & & & \\
\hline steel convenience cans & & & & & \\
\hline & & & & & \\
\hline HNO3 $30 \%$ cleaning solution & & & & & \\
\hline HF cleaning solution & & & & & \\
\hline $\mathrm{NaOH}(75 \mathrm{~g} /$ lit) cleaning solution & & & & & \\
\hline $\mathrm{H} 2 \mathrm{O}$ (cooling, etc) & & & & & \\
\hline $\mathrm{H} 2 \mathrm{O}$ DI rinse & & & & & \\
\hline ethanol & & & & & \\
\hline $\mathrm{KCl}$ molten salt/vapors & & & & & \\
\hline janitorial supplies & & & & & \\
\hline hydraulic fluids & & & & & \\
\hline vacuum pump oil & & & & & \\
\hline
\end{tabular}

FIGURE 6-1 - EXAMPLE EVALUATION SHEET FOR PRELIMINARY HAZARDS ANALYSIS 
The presence of $\mathrm{U}^{235}$ in the FFC introduces radiological hazards associated with fissile radioactive materials (especially nuclear criticality and external ionizing radiation). In addition, this material increases the consequences of other accidents such as fires or explosions because of the airborne radioactive material hazard. Radiological hazards can be subdivided into two categories. The first is worker exposure to ionizing radiation that is largely a chronic condition in the workplace. The second category includes upset conditions that would potentially result in higher doses for short time periods.

To assess the importance of worker chronic exposure to $20 \%$ enriched LEU materials, a simple dose rate calculation was performed using the Uranium Radiation Individual Dose Calculator at http://www.wise-uranium.org/rdcu.html. This estimate assumed that a worker receives external exposure from working $2000 \mathrm{hr} /$ year at a distance of $2 \mathrm{~m}$ from a $20 \mathrm{~kg}$ point source of $20 \%$ enriched uranium. These conditions might be expected in the casting area. The annual dose received by the worker is calculated to be $2.8 \mathrm{mSv}(280 \mathrm{mrem})$ which is approximately $14 \%$ of the administrative dose for occupational radiation exposure at DOE sites $(2 \mathrm{rem} / \mathrm{yr})$. Workers in other areas such as the material storage area would likely receive higher doses from working in the vicinity of larger source terms.

Radiological hazards from upset conditions would include inhalation of airborne radioactive materials, external exposure to inadvertent nuclear criticality events, or external exposure from worker entry into a radiation area, a high-radiation area, or a very-high radiation area such as a radiography cell while the radiography source is exposed. Controls for these hazards are discussed in a later section of this report.

Many of the hazards identified as standard industrial hazards were included in the evaluation even if the equipment is designed to significantly reduce the risk of those hazards. For example, electrically-powered equipment that is designed and built to meet electrical codes and standards typically poses negligible risk from electrical hazards to the workers during normal operation. However, the electrical hazard continues to exist as long as power is supplied to the equipment, even if all the electrical components are contained within the appropriate enclosures. The rationale for identifying these "low-risk" hazards is in defining the importance of the hazard controls (enclosures, operating and maintenance procedures, lock-out/tag-out protocols, etc.). Modifying or removing these controls may increase the risk of the hazard.

In addition to posing industrial-type hazards to workers, process equipment can also serve as initiators of an accident sequences that are unrelated to the function performed by that equipment. For example, electrically-powered equipment could initiate a fire. The fire could suspend and transport radioactive materials (inhalation hazard), and the activation of the fire suppression system could provide moderator for a nuclear criticality event. For this preliminary hazards evaluation, hazards that could be initiated by a piece of equipment were also included as part of the hazards inventory for that equipment.

\subsection{Principal Worker Hazards}

The principal hazards to the worker identified in the preliminary hazards analysis include standard industrial hazards and hazards related to the presence of radioactive materials. These hazards are not listed in order of importance or of consequence.

$\underline{\text { Standard Industrial Hazards }}$

- Fire

- Steam explosion (introduction of water or other liquid into molten salt or molten metal)

- Electrical hazards 
- Mechanical hazards (falling objects; rotating equipment; pinching, cutting, or crushing hazards; sharp objects; pressure)

- Thermal hazards (burns)

- Non-ionizing radiation (welding)

- Chemical hazards (toxic, reactive, or corrosive chemicals; chemical vapors or fumes)

- Spills of hazardous materials

$\underline{\text { Radiological Hazards }}$

- Spills of radioactive materials

- Ionizing radiation (radiography, radioactive materials)

- Inadvertent nuclear criticality

\subsection{Hazards Control}

Prevention and Mitigation of Standard Industrial Hazards

Most of the standard industrial hazards listed above are typical of hazards that would be expected in a machine-shop/foundry environment. Generally, these types of hazards are controlled by equipment design (built-in guarding, shields, and interlocks), operating and maintenance procedures, personal protective equipment (hardhats, face/eye protection, gloves, safety shoes, protective clothing), and personnel training. Additional mitigation for these hazards would result from implementation of nuclear facility safety management/administrative control programs. For example, DOE non-reactor nuclear facilities operate maintenance, change control, and worker safety programs with greater formality than is usual for non-radiological facilities. Application of the increased formality to operation and maintenance (e.g., use of a formal lockout/tagout procedure for equipment maintenance, and use of formal change control processes for equipment modification) should reduce both the likelihood and the severity of standard industrial hazards.

\section{Prevention and Mitigation of Radiological Hazards}

The principal controls for radiological hazards include a radiation protection program, an ALARA program, sensors and alarms for upset conditions (continuous air monitors, personnel contamination monitors, nuclear criticality alarms, personal dosimetry), support from radiation control technicians, periodic dose rate and contamination surveys, critically safe geometries for equipment and storage areas, material accumulation limits based on criticality safety evaluations, maintenance and operating procedures, worker training, and management walk-arounds and selfassessments.

Radiation protection program requirements are defined by 10 CFR 835 and are incorporated into the facility Documented Safety Analysis.

In addition to the hazards specifically identified as radiological hazards, several of the standard industrial hazards could act to disperse radioactive materials or could be initiators for other serious accidents involving radioactive materials. The first two items in the list of standard industrial hazards are examples. For example, the consequences of a fire in a machine shop or foundry would be made much worse if the fire acted to disperse nuclear material.

\section{Prevention and Mitigation of Fires}

Although the fire hazard is listed above as a standard industrial hazard, a fire in a nuclear facility is a much greater hazard than a general fire due to the potential for nuclear criticality from the 
introduction of water into the facility, to increased possibility of dispersal of airborne radioactive material, and to the potential for loss of the safety function of one or more safety systems in a fire. DOE O $420.1 \mathrm{~B}^{6}$ defines fire protection program requirements for DOE nuclear facilities. DOE STD-1066- $97^{7}$ provides design guidance that supplements the requirements of national consensus fire protection design standards. The increased design requirements for fire protection systems in nuclear facilities, coupled with administrative control programs (such as transient combustible control and housekeeping) are important contributors to the prevention and the mitigation of fires.

\subsection{Criticality Controls}

In addition to providing fire protection requirements, DOE O 420.1B defines nuclear criticality safety for Hazard Category 2 nuclear facilities. The primary controls for nuclear criticality hazards are preventive controls which are set by the criticality safety program and validated using criticality safety evaluations (CSEs) of specific equipment, processes or conditions. Likely controls that would be used in the FFC include:

Equipment or container geometry

Fissionable material mass limits

Fissionable material concentration limits

Fissionable material spacing limits

Control of moderators and reflectors

Examples of these controls as proposed for implementation in the FFC include:

- The casting crucible diameter is limited to 6 inches which is expected to be a critically safe geometry for the LEU-10\%Mo material in the solid and in the molten state (CSE needed). The height of the crucible limits the total mass of LEU-10\%Mo alloy, but detailed analysis is required to ensure that this mass is subcritical in any geometry.

- Spills of fissionable material from the casting crucible should be captured in a criticallysafe geometry (CSE needed).

- Fissionable material in or spilled from the casting crucible should not be capable of being moderated by spills of induction coil cooling water (CSE needed) (note: Induction coil cooling water system is a limited volume system).

- Fire water from sprinklers that is collected on the process area floor will not exceed 6 inches in depth at any location (CSE needed). This is expected to be a critically safe geometry for all nuclear material configurations. (note: sumps are 18 inches deep)

- Any equipment, structure, or container that could collect water more than 6" in any dimension shall be evaluated to ensure that a criticality event is not credible even if water collection did occur. (CSE needed)

- Floor sumps for collecting water spills on the process area floor have a diameter of 6" or less to provide geometry control (CSE needed).

- Waste water that potentially contains fissionable material in a dissolved or suspended state will be collected in geometrically safe tanks for sampling and analysis before being transferred to uncontrolled geometries (CSEs needed).

${ }^{6}$ Facility Safety, DOE O 420.1B, December 2005, 65 pp.

${ }^{7}$ Fire Protection Design Criteria, DOE STD-1066-97, March 1997, 85 pp. 
- Thickness of stacks of cast materials, rolled materials, clad materials and encapsulated plates will not exceed 1" in thickness in the narrowest dimension. This is expected to be a critically-safe geometry (CSE needed).

- Spacing between containers of fissionable materials will not be less than 8" in any direction (CSE needed). The spacing will be maintained by physical restraints.

- Mass of fissionable material per container will be limited to $11 \mathrm{~kg}$. This is expected to be a critically safe mass for any container geometry (CSE needed).

In addition to these preventive controls, the consequences of a nuclear criticality event are reduced by the use of mitigative controls, including:

Criticality accident alarm systems to alert workers of the presence and location of a criticality event

Public address system to alert and instruct workers about the presence and location of a criticality event

Emergency response program to ensure proper evacuation of the facility, account for personnel, control access during the incident, and plan recovery actions

Personal nuclear accident dosimeters (PNADs) provide estimates of the doses received by exposed personnel to support their medical treatment.

Worker training in nuclear criticality safety and in emergency response procedures are important elements of both preventive and mitigative controls.

Criticality controls that are not considered for FFC include neutron absorbers/poisons and fixed shielding. These controls are not viewed as cost effective for implementation in FFC.

\subsection{FFC Facility Categorization}

\subsubsection{FFC Hazard Categorization}

DOE non-reactor nuclear facilities are categorized based on inventory according to DOESTD-1027-92 Error! Bookmark not defined. to determine the rigor of the safety analysis required to support licensing of the facility. Attachment 1 of this standard provides a listing of the threshold quantities for hazard categories 2 and 3 for selected isotopes. A portion of this table is reproduced in Figure 6-2. The second and third columns in this attachment show the threshold (minimum) values for a hazard category 2 facility in curies and grams, respectively, for the isotope shown in the first column. The fourth and fifth columns show the threshold (minimum) values for a hazard category 3 facility in curies and grams, respectively, for that isotope. Because the LEU-10\%Mo has the potential for nuclear criticality, the hazard category 2 threshold is given in the note as 700 grams compared to a total facility inventory on the order of 7000 grams.. Thus, the FFC is categorized as a hazard category 2 (HC2) facility. This result means that the facility will require a Documented Safety Analysis with Technical Safety Requirements (DSA/TSR) prepared in accordance with DOE-STD-3009-9 to meet the requirements of 10 CFR 830 Subpart B. 


\begin{tabular}{|c|c|c|c|c|}
\hline \multirow{3}{*}{ Isotope } & \multicolumn{3}{|c|}{$\begin{array}{c}\text { DOE-STD-1027-92 } \\
\text { ATTACHMENT } 1\end{array}$} & \multirow{3}{*}{$\begin{array}{l}\text { Threshold } \\
\text { Grams }\end{array}$} \\
\hline & Category 2 & Threshold & Category 3 & \\
\hline & Curies & Grams & Curies & \\
\hline $\mathrm{U}-233$ & $2.2 \mathrm{E}+02^{* \star *}$ & $2.3 \mathrm{E}+04^{\star * *}$ & $4.2 \mathrm{E}+00$ & $4.4 \mathrm{E}+02$ \\
\hline $\mathrm{U}-234$ & $2.2 \mathrm{E}+02$ & $3.5 \mathrm{E}+04$ & $4.2 \mathrm{E}+00$ & $6.7 \mathrm{E}+02$ \\
\hline U-235 & $2.4 \mathrm{E}+02^{* \star *}$ & $1.1 \mathrm{E}+08^{* * *}$ & $4.2 \mathrm{E}+00$ & $1.9 \mathrm{E}+06$ \\
\hline $\mathrm{U}-238$ & $2.4 \mathrm{E}+02$ & $7.1 \mathrm{E}+08$ & $4.2 \mathrm{E}+00$ & $1.3 \mathrm{E}+07$ \\
\hline \multicolumn{5}{|c|}{$\begin{array}{l}\text { To be used only if segmentation or nature of process precludes potential for criticality. } \\
\text { Otherwise, use the criticality lists for } \mathrm{U}_{233}, \mathrm{U}_{235} \text { and } \mathrm{Pu}_{239} \text { of } 500,700 \text {, and } 450 \text { grams, } \\
\text { respectively. }\end{array}$} \\
\hline
\end{tabular}

FIGURE 6-2. Extract from DOE-STD-1027-92, Attachment 1 showing isotopes of importance to the FFC.

\subsubsection{FFC Security Categorization}

The security category for the FFC is determined from the amount and type of SNM in the facility based on the attractiveness level of the material. The DOE categorization scheme from DOE M 470.4- $6^{8}$ is reproduced in Figure 6-3. Uranium that is enriched in $\mathrm{U}^{235}$ to a level less than $20 \%$ is designated as Attractiveness Level "E". Attractiveness Level E material is not readily converted to use in nuclear weapons and is therefore subject to lower levels of physical security. Quantities of Attractiveness Level E material larger than the reportable quantity of 1 gram cause the facility to be categorized as a Security Category IV (SC IV) facility as shown in the red outline in Figure 6-3.

Physical security requirements for SC IV facilities include a perimeter fence, secured material storage areas, and access control to prevent unauthorized entry into the facility. Security features such as armed guards, hardened material (LEU) storage areas, and motion detectors are not required for protection of SC IV material.

\subsubsection{FFC Performance Categorization}

In addition to other requirements, DOE $\mathrm{O} 420.1 \mathrm{~B}$ defines the requirements for DOE nuclear facility design and construction to withstand Natural Phenomena Hazards (NPH) ${ }^{9}$ and to ensure confinement of hazardous materials, protection of occupants and members of the public, continued operation of essential facilities, and protection of government property. DOE STD-1021-93 ${ }^{10}$ details the process for selecting the performance category for the facility and for safety SSCs consistent with the risks identified for the facility. Initial performance categorization provides a means of identifying, early in the design process, the level of design required consistent with the facility safety basis and the required safety systems. Figure 6-4 shows the guidelines from DOE STD-1021-93 for

\footnotetext{
${ }^{8}$ DOE M 470.4-6, Chg.1, Nuclear Material Control and Accountability, August 14, 2006, 168 pp.

${ }^{9} \mathrm{NPH}$ include earthquakes, wind and wind-driven missiles, flooding, lightning, etc.

${ }^{10}$ Natural Phenomena Hazards Performance Categorization Guidelines for Structures, Systems, and Components, DOE-STD-1021-93, Change Notice \#1, January 1996, 37 pp.
} 
Table I-4. Graded Safeguards

\begin{tabular}{|c|c|c|c|c|c|c|c|c|c|c|}
\hline & \multirow{2}{*}{$\begin{array}{c}\text { Attractiveness } \\
\text { Level }\end{array}$} & \multicolumn{4}{|c|}{$\begin{array}{c}\mathrm{Pu} / \mathrm{U}-233 \text { Category } \\
(\mathrm{kg})\end{array}$} & \multicolumn{4}{|c|}{\begin{tabular}{|c|}
$\begin{array}{c}\text { Contained U-235/Separated Np- } \\
\text { 237/Separated Am-241 and -243 } \\
\text { Category (kg) }\end{array}$ \\
\end{tabular}} & \multirow{2}{*}{$\begin{array}{c}\text { All E } \\
\text { Materials } \\
\text { Category IV }\end{array}$} \\
\hline & & I & II & III & IV $^{1}$ & I & II & III & IV $^{1}$ & \\
\hline $\begin{array}{l}\text { WEAPONS } \\
\text { Assembled weapons and test devices }\end{array}$ & $\mathrm{A}$ & All & $\mathrm{N} / \mathrm{A}$ & N/A & $\mathrm{N} / \mathrm{A}$ & All & $\mathrm{N} / \mathrm{A}$ & $\mathrm{N} / \mathrm{A}$ & $\mathrm{N} / \mathrm{A}$ & $\mathrm{N} / \mathrm{A}$ \\
\hline $\begin{array}{l}\text { PURE PRODUCTS } \\
\text { Pits, major components, button ingots, } \\
\text { recastable metal, directly convertible } \\
\text { materials }\end{array}$ & B & $\geq 2$ & $\begin{array}{c}\geq 0.4< \\
2\end{array}$ & $\geq 0.2<0.4$ & $<0.2$ & $\geq 5$ & $\geq 1<5$ & $\geq 0.4<1$ & $<0.4$ & $\mathrm{~N} / \mathrm{A}$ \\
\hline $\begin{array}{l}\text { HIGH-GRADE MATERIALS } \\
\text { Carbides, oxides, nitrates, solutions } \\
(\geq 25 \mathrm{~g} / \mathrm{L}) \text { etc.; fuel elements and } \\
\text { assemblies; alloys and mixtures; } \mathrm{UF}_{4} \text { or } \\
\mathrm{UF}_{6}(\geq 50 \% \text { enriched) }\end{array}$ & $\mathrm{C}$ & $\geq 6$ & $\geq 2<6$ & $\geq 0.4<2$ & $<0.4$ & $\geq 20$ & $\geq 6<20$ & $\geq 2<6$ & $<2$ & N/A \\
\hline $\begin{array}{l}\text { LOW-GRADE MATERIALS } \\
\text { Solutions ( } 1 \text { to } 25 \mathrm{~g} / \mathrm{L}) \text {, process } \\
\text { residues requiring extensive } \\
\text { reprocessing; moderately irradiated } \\
\text { material; Pu- } 238 \text { (except waste); } \mathrm{UF}_{4} \text { or } \\
\mathrm{UF}_{6}(\geq 20 \%<50 \% \text { enriched) }\end{array}$ & $\mathrm{D}$ & N/A & $\geq 16$ & $\geq 3<16$ & $<3$ & $\mathrm{~N} / \mathrm{A}$ & $\geq 50$ & $\geq 8<50$ & $<8$ & N/A \\
\hline $\begin{array}{l}\text { ALL OTHER MATERIALS } \\
\text { Highly irradiated forms, solutions } \\
(<1 \mathrm{~g} / \mathrm{L}) \text {, uranium containing }<20 \% \mathrm{U}- \\
235 \text { or }<10 \% \mathrm{U}-233^{2} \text { (any form, any } \\
\text { quantity) }\end{array}$ & $E$ & $\mathrm{~N} / \mathrm{A}$ & $\mathrm{N} / \mathrm{A}$ & N/A & $\begin{array}{c}\text { Reportable } \\
\text { Quantities }\end{array}$ & $\mathrm{N} / \mathrm{A}$ & $\mathrm{N} / \mathrm{A}$ & $\mathrm{N} / \mathrm{A}$ & $\begin{array}{l}\text { Reportable } \\
\text { Quantities }\end{array}$ & $\begin{array}{l}\text { Reportable } \\
\text { Quantities }\end{array}$ \\
\hline
\end{tabular}

FIGURE 6-3. - Table I-4 from DOE M 470.4-6. The red outline indicates the security categorization of the FFC.

performance categorization of SSCs. According to this figure, and recalling that the preliminary hazards evaluation did not identify any safety-class SSCs, we conclude that the FFC performance category is PC2. 


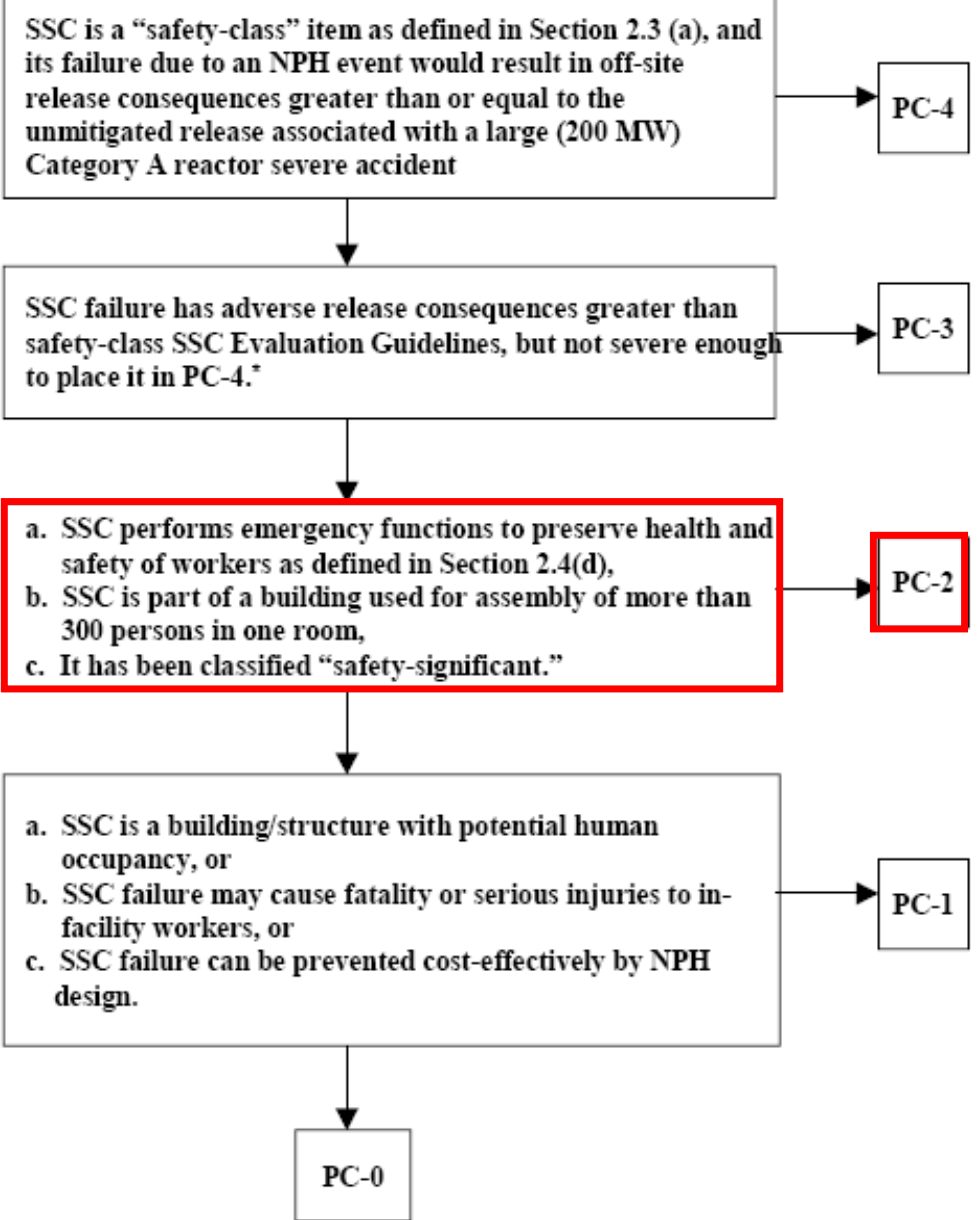

FIGURE 6-4. BASIC GUIDELINES FOR PRELIMINARY NPH PERFORMANCE CATEGORIZATION OF STRUCTURES, SYSTEMS AND COMPONENTS (FIGURE 2-1 FROM DOE STD 1021-93) 


\subsection{ENVIRONMENTAL STRATEGY}

Section 2.2.4 of this report identifies Environmental Protection requirements for the FFC based on Federal environmental protection regulation. This chapter defines the permitting activities that must be completed before the FFC can be constructed and/or operated. In several cases, these permits can take many months and require significant investment to complete. The intent here is to identify those required items that could impact cost and schedule for the FFC.

\subsection{NEPA - EIS/EA}

This study assumes that the FFC will be regulated and licensed by the NRC. As part of the license application process, the licensee prepares an environmental report (ER) that provides the baseline information required for NRC to prepare an Environmental Impact Statement (EIS) or an Environmental Assessment (EA). The ER includes analysis of environmental impacts of construction and operation of the facility, including availability of water and other utility resources, release of pollutants to the environment, transportation impacts, economic costs and benefits, evaluation of alternatives, cumulative impacts (e.g., consideration of impacts from nearby activities), etc. Preparation of the ER requires both financial resources and time for the licensee staff to prepare the report. The NRC preparation of the EIS or EA does not start until the ER is submitted to the NRC. Similarly, the review of the EIS/EA includes receiving and responding to public comments on the proposed licensing of the facility before the NRC can issue a record of decision (ROD). The ROD is one component of the NRC license to begin construction of the facility. The important point here is that the NEPA process can impose a significant schedule constraint on the overall design and construction timeline.

To preclude significant schedule delays due to the NEPA process, the ER must be prepared as early as possible in the design process. This often means that the ER is based on conceptual designs rather on more detailed designs where the design information is more complete and accurate. ERs prepared based on conceptual-level designs adjust for uncertainty in the design by "bounding" the actual impacts with conservative estimates of the impacts.

One option that might be available to accelerate the NEPA process for the FFC would involve the case where the DOE is the licensee and the FFC would be built on a DOE site having existing NEPA documentation such as a site-wide EIS, a programmatic EIS, or a project-specific EIS that covers fuel fabrication or related activities. In this case, the existing NEPA documentation would be evaluated to see if it covers new activities such as construction and operation of a new facility such as the FFC. If the existing NEPA documentation does, in fact, include such new activities, the DOE could determine that the increased activity of the FFC on the DOE site has no significant additional impact over the impacts already approved. This determination could substantially reduce the time and resources required for NEPA compliance. In addition to reducing the time and cost to produce NEPA documentation, this option also has the potential to reduce impacts from site construction activities if the existing site infrastructure includes facilities that could support the FFC.

This option would require a Memorandum of Understanding (MOU) be developed between the NRC (as licensor) and the DOE (as licensee and as the responsible agency for the NEPA compliance activity) detailing the responsibilities of both agencies regarding the NEPA process. However, interdepartmental MOUs that reapportion responsibilities are not uncommon. 


\subsection{Other Permits}

\section{Air Emissions - NESHAPS}

Because of the small number of hazardous chemicals, and the small inventories of those chemicals within the FFC, it is expected that the FFC will have negligible emissions of hazardous air pollutants. However, a dose rate study is needed to ensure that the radioactive content of air emissions from the facility do not exceed the $10 \mathrm{mrem} / \mathrm{year}$ standard for a member of the public (10 CFR 61.92). This study requires use of an EPA-approved model (CAP-88PC or AIRDOSPC) for evaluating the dose from all exposure pathways (inhalation, immersion, ingestion) ${ }^{1}$. The results of the analysis will determine what additional air emission monitoring and control technology, if any, would be required to meet the Clean Air Act standards.

\section{Discharge of Water - NPDES Permit}

As discussed in Chapter 2, the FFC will require NPDES permits for discharging stormwater runoff from the site during construction and during operation of the facility. The permit for the construction period is called the Construction General Permit, and the forms are available on the EPA website at http://cfpub.epa.gov/npdes/stormwater/const.cfm (note: the EPA is in the process of changing CGP forms). EPA is the permitting authority for the states of MA, NH, NM, ID, and $\mathrm{AK}$, for the District of Columbia and in most Indian Country lands. In other states, the permitting authority has been delegated to the state, but some states do not have permitting authority for federal facilities. Timescales for issuance of CGPs under state authority are typically on the order of one month from submission of the Notice of Intent.

The FFC will require an NPDES permit for discharging stormwater runoff from the site's runoff collection structure. The EPA is the permitting authority for the states of MA, NH, ID, NM, and $\mathrm{AK}$, the District of Columbia, and most Indian Country lands. In other states, the permitting authority has been delegated to the state, but some states do not have permitting authority for federal facilities (CO, DE, VT, and WA). In those cases, the EPA retains permitting authority for federal facilities. Timescales for approval of NPDES stormwater permit applications are on the order of one to three months.

\section{Hazardous and Radioactive Wastes}

As a generator of hazardous waste, the FFC will be required to have an EPA identification number (40 CFR 262.12). The FFC will store but not treat or dispose of hazardous, radioactive and mixed wastes. Hazardous and mixed wastes would be stored in a 90 day storage area that does not require a permit. The volumes of hazardous wastes generated at the FFC are small, and the FFC may qualify as a Small Quantity Generator or as a Conditionally Exempt Small Quantity Generator. The requirements and benefits for these designations are presented in Table 7-1.

\footnotetext{
${ }^{1}$ The analysis presented in Chapter 6 included only inhalation and was the cumulative dose over a 50 year period from an acute exposure. The air emissions analysis described here is the annual dose from chronic exposure to low concentrations of radionuclides.
} 


\section{TABLE 7-1. DESCRIPTION OF SMALL QUANTITY GENERATOR AND CONDITIONALLY EXEMPT SMALL QUANTITY GENERATOR DESIGNATIONS}

\begin{tabular}{|c|c|c|}
\hline Designation & Description & Requirements \\
\hline $\begin{array}{l}\text { Small Quantity } \\
\text { Generator }\end{array}$ & $\begin{array}{l}\text { Generate more than } \\
100 \mathrm{~kg} \text { but less than } \\
1000 \mathrm{~kg} \text { of hazardous } \\
\text { waste per month }\end{array}$ & $\begin{array}{l}\text { - May accumulate hazardous waste on site for } 180 \text { days } \\
\text { without a permit (or } 270 \text { days if shipping a distance greater } \\
\text { than } 200 \text { miles). } \\
\text { - On-site quantity of hazardous waste must never exceed } \\
6000 \mathrm{~kg} \\
\text { - At least one employee always available to respond to an } \\
\text { emergency } \\
\text { - Not required to have detailed, written emergency plans }\end{array}$ \\
\hline $\begin{array}{l}\text { Conditionally } \\
\text { Exempt Small } \\
\text { Quantity } \\
\text { Generator }\end{array}$ & $\begin{array}{l}\text { Generate } 100 \mathrm{~kg} \text { or } \\
\text { less per month of } \\
\text { hazardous waste or } 1 \\
\mathrm{~kg} \text { or less per month } \\
\text { of acutely hazardous } \\
\text { waste }\end{array}$ & $\begin{array}{l}\text { - Must identify all the hazardous waste generated } \\
\text { - May not accumulate more than } 1000 \mathrm{~kg} \text { of hazardous } \\
\text { waste at any time } \\
\text { - Must ensure that hazardous waste is delivered to a person } \\
\text { or facility authorized to manage the waste. } \\
\text { - Is exempt from various EPA requirements (see } 40 \mathrm{CFR} \\
\text { 261.5). }\end{array}$ \\
\hline
\end{tabular}

Current estimates of hazardous waste volumes suggest that the FFC would qualify as a Conditionally Exempt Small Quantity Generator.

To ship hazardous wastes to a permitted TSD facility, the FFC must use the EPA's Uniform Hazardous Waste Manifest system (http:/www.epa.gov/epaoswer/hazwaste/gener/manifest).

Low Level Radioactive wastes will be packaged for storage and for shipment to a commercial or a DOE LLW disposal site, as appropriate. 


\subsection{LICENSING REQUIREMENTS}

The Atomic Energy Act requires the NRC to licenses the following activities:

- Construction, operation and decommissioning of commercial reactors and fuel cycle facilities.

- Possession, use, processing, exporting, importing, and certain aspects of transporting nuclear materials and waste.

- Siting, design, construction, operation, and closure of waste disposal sites.

The NRC regulates fuel cycle facilities that mill and enrich uranium and then fabricate it into fuel for use in nuclear reactors. The NRC also regulates the fabrication of nuclear fuel that is a combination of uranium and other materials. (Exclusive DOE use facilities are not regulated by the NRC. However, the FFC will provide fuel for both NRC and DOE regulated reactors and thus, require a NRC license.)

The NRC licenses fuel that is used in nuclear reactors by reviewing and approving the fuel qualification report. (A given fuel type is deemed acceptable for use in nuclear applications as a result of the review and acceptance of the qualification report. A fuel is licensed for use in a given reactor after the review of safety documentation for that reactor which references information in the qualification report and any additional fuel performance data that is required for the specific application.) NRC has adopted a policy of discouraging the use of HEU fuel. This policy is reflected in 10 CFR 50.64.

This chapter provides the NRC regulatory requirements for the licensing of the LEU-Mo monolithic fuel and the licensing of the Fuel Fabrication Facility.

Although the FFC will be licensed by the NRC, applicable DOE requirements for the design, construction and operation will also be considered.

\subsection{Licensing of the LEU-Mo Monolithic Fuel}

A fuel is considered "qualified" within a given envelope of operating conditions after the NRC has reviewed and accepted documentation supplied by the fuel developer which summarizes the fuel performance and properties within that specific envelope. Licensing is the action of the regulatory authority i.e. the NRC to approve the use of the fuel in a specific reactor, since the suitability of the fuel is judged with respect to the conditions that the fuel may experience in that reactor. In order to facilitate licensing of a number of reactors to use the same fuel, the NRC has agreed to review a fuel qualification report on a generic basis and issue approval for it's use within an envelope of conditions covered by the qualification tests, similar to the approach the NRC used in issuing it's approval for the $\mathrm{U}_{3} \mathrm{Si}_{2}$ dispersion fuel: NUREG - 1313 Safety Evaluation Report related to the Evaluation of Low Enriched Uranium Silicide-Aluminum Dispersion Fuel for Use in Non Power Reactors. Such generic approval is not a license to use the fuel; each reactor must show that its intended use fits within the approval envelope. Generic approval of the fuel may be viewed both as the end of fuel qualification and beginning of licensing.

The RERTR program has a well planned fuel qualification program to pursue generic approval of the NRC for the LEU-Mo monolithic fuel. (See Report INL/EXT-05-01017.) The qualification process for the monolithic fuel is based on developing industrial processes and adequate specifications for manufacturing LEU-Mo fuel plates, getting basic data for ex-reactor and inreactor irradiation tests on miniature and full sized plates, obtaining a comprehensive 
understanding of the LEU-Mo fuel performance under reactor operational conditions, developing models to aid in understanding and predicting irradiation behavior, and confirming through the irradiation of full size sub-assemblies that the integrated fuel behavior is consistent with the testing database. The manufacture and irradiation of full sized sub-assemblies is the last stage of the fuel qualification process. During the qualification process one fuel sub-assembly is irradiated to a high burn-up, typically $70-80 \%$ average, in order to demonstrate that adequate margins exists beyond the burn-ups normally reached in reactors that will use this fuel. The generic fuel qualification data is submitted to the NRC in a fuel Qualification Report. The NRC reviews the report and issues a Safety Evaluation Report (SER) signifying its approval of the generic fuel deign. Following issuance of the SER by the NRC that describes the generic behavior of the LEU-Mo fuel, specific analysis and testing required to license individual reactors to use the fuel is initiated. Additional tests may be necessary to support utilization in a specific reactor subsequent to the NRC acceptance and review of the fuel qualification report. In these cases Lead Test Assemblies (LTAs) will be fabricated for the given reactor in order to demonstrate satisfactory behavior in the specific configuration and operating conditions.

In addition to qualifying the fuel itself, the fuel fabricator must be qualified. To be qualified, the fabricator must demonstrate its ability to manufacture the LEU-Mo monolithic fuel plate subassemblies, which consistently meet specifications developed and demonstrated during the fuel qualification process, and that fuel assembly performance is consistent with the fuel performance database. This is normally accomplished by quality tests and inspections during the hot start-up process. In addition this is also accomplished through the manufacture and irradiation of the full sized sub assemblies - Lead Use Assemblies (LUA's). The irradiation of the full sized subassemblies provide better statistics through the irradiation of many plates and demonstrate the ability of the fuel fabricator to produce acceptable plates and sub-assemblies by an industrial scale process.

\subsection{Licensing of Fuel Fabrication}

The NRC regulates fuel cycle facilities through a combination of regulatory requirements; licensing; safety oversight, including inspection, assessment of performance, and enforcement; operational experience evaluation; and regulatory support activities. The purpose of the NRC regulations is to ensure adequate protection of the health and safety of workers and members of the public, safeguards and security and the environment for activities performed at the fuel fabrication facilities

NRC's regulations are found in chapter I of Title 10, "Energy" of the Code of Federal Regulations. Chapter I is divided into Parts 1 through 199. The following lists parts relevant to the licensing and operation of the FFC:

- Part 20 - Standards for the Protection Against Radiation

- Part 40 - Domestic Licensing of Source Materials

- Part 51 - Environmental Protection regulations

- Part 70 - Domestic Licensing of Special Nuclear Materials

- Part 73 - Physical Protection of Plants and Materials

- Part 74 - Material Control and Accounting for Special Nuclear Material

- All current rule making 
NRC has also issued as guidance Regulatory Guides that are issued in 10 divisions and are intended to aid licensees in addressing regulations. The guides that are most applicable to the design and license of the FFC are:

- Division 3 - Fuels and Materials Facilities

- Division 4 - Environmental and Siting

- Division 5 - Materials and Plant Protection

- Division 8 - Occupational health

NRC reviews the application for construction permit and operation license, according to procedures and criteria documented in a Standard Review Plan (SRP). If the application is approved a license is issued. The license contains the amount and type of material that the facility is allowed to posses and any special conditions imposed by the NRC. The fuel cycle materials facilities licenses are typically issued for 10 years. The SRP applicable to the FFC is expected to be:

- Standard Review Plan for the review of a License Application for a Fuel Cycle Facility (NUREG-1520)

In order to obtain a license to build and operate a Fuel Fabrication facility, an applicant must submit an application for approval to the NRC. The contents of the application are specified in the Code of Federal Regulations (CFR) and reflect the requirements set down by Congress in the Atomic Energy Act.

The main regulation that apply to the FFC is Title 10 of the Code of Federal regulations, part 70 (i.e., 10 CFR 70), "Domestic Licensing of Special Nuclear Material". Under the regulation, NRC authorization must be received before construction via a Construction Permit (CP) and an Operating License (OL) before operation of the FFC. In addition the impacts of the construction and operation of the FFC on the quality of the human environment will have to be assessed in either an Environmental Assessment and or an Environmental Impact Statement.

\section{$\underline{\text { Construction Permit }}$}

Under NRC regulations an application for the construction of the FFC must be submitted to the NRC before construction of the facility. To request a Construction Permit (CP), the applicant must provide:

- an Environmental Report (ER)

- a description of the plant site

- a description of the safety assessment of the design of the principal structures, systems and components (ISA), and

- a description of the quality assurance program.

Utilizing the information provided in the Construction Permit (CP) application, the NRC performs the following:

- prepares the Environmental Impact Statement (EIS)

- performs a review of the ISA Report (Integrated Safety Analysis) 
- prepares a Safety Evaluation Report (SER)

- conducts public hearings

- issues Construction Permit (CP)

Operations License

To request and Operations License (OL) an applicant has to submit the following documents:

- Environmental Report (ER) supplement

- a final ISA

- technical specifications

Utilizing the information provided in the Operations License (OL) application, the NRC performs the following:

- Prepares an EIS supplement

- Performs review of the final ISA

- Prepares a SER

- Issues Operations License.

A proposed timeline for the NRC licensing process is included in Section 11 - Schedule estimate. The timeline is aggressive, and is based on a well characterized uncontaminated site, and early joint NRC/NNSA agreement of the regulatory frame work of the project for the design, construction and commissioning of the FFC. 


\subsection{RISK MANAGEMENT}

This section provides the high level programmatic and technical risks, and risk management strategies for the Greenfield FFC. This risk assessment is based on the initial scoping evaluation of the process and facility design as well as process hazards evaluations conducted as part of this Greenfield Alternative Study.

The primary objectives of the FFC mission are:

- Provide proliferation resistance fuel for use in US HPRRs - In accordance with NNSA's GTRI program directive to meet the US HEU minimization policy

- Convert the 5 US HPRR's that currently use HEU- based dispersion fuel to a new monolithic LEU-Mo fuel by 2014. The ultra high density LEU-Mo fuel provides the proliferation resistance and is appropriate for research reactor use.

- The Fuel Fabrication Capability (FFC) facility to be designed, built and commissioned in time to support fabrication of LUA's and HPRR cores in order to complete conversion of the HPRR's per plan.

- The FFC will be licensed by the NRC; likewise the LEU-Mo fuel will require NRC approval via submittal initially of the generic Fuel Qualification Report followed by the specific data of the performance of the LTA's irradiated in each of the HPRRs.

The above FFC mission goals in conjunction with the initial hazards evaluation (See section 6.0) provide the basis for identifying the high level programmatic and technical risks of the FFC. These risks impact the ability of the FFC to be completed on schedule (1/1/2013) to support the conversion of the HPRRs by 2014, as well as contribute to cost growth. Hence these risks must be managed/mitigated so as to deploy an on-time, cost-effective FFC that meets the mission objectives. The risks identified in this section are specific to the Greenfield Alternative for the LEU-Mo FFC project, and given the quick initial assessment, the risks are preliminary.

\subsection{Programmatic Risks}

The programmatic risks comprise of those high level risks which require management by the NNSA. These risks involve (1) Performing the FFC alternatives evaluation for the selection of the acquisition strategy, (2) Providing adequate project funding, (3) Establishing high-level project interfaces, (4) Facilitating early involvement of the NRC to establish the licensing strategy for the project, and (5) Timely site selection.

Evaluation of the project schedule indicates that even with the very short performance period for completion of the FFC project, it is still possible to meet the GTRI milestone for reactor conversion. The successful early mitigation of the programmatic risks is essential to establish the framework for the successful deployment of the FFC project and meet the FFC mission goals.

The key programmatic risks are summarized below:

- Acquisition Strategy -The NNSA has a process in place for the selection of either the Commercial alternative or the National Laboratory alternative for the deployment of the FFC. An expeditious selection is key and crucial for the downstream project initiation activities, in- particular the start of the conceptual design in October 2008 leading to the DOE approval of CD-1 by July 2009. This is required in-order to complete the timecritical licensing, design, construction and commissioning of the FFC to support completion of hot operations by $1 / 1 / 2013$. 
To mitigate this risk, a dual path conceptual design of the National Laboratory Alternative and the Commercial Alternative should commence in October 2008, in the event the acquisition strategy decision is not made on time. The project planning level schedule included in Section 8.0, provides an aggressive schedule that is compliant with DOE O 413.3A for the acquisition of the FFC assets. However there is little float in the schedule, and any delay in the critical path design and equipment procurement activities will delay the start of the FFC operations.

- Adequate Project Funding - Given the important proliferation resistance mission of the FFC in support of the NNSA, it is necessary that the project be funded fully to allow uninterrupted execution of the planned $\mathrm{R} \& \mathrm{D}$, design, licensing, construction and commissioning activities. Any funding cuts will cause delays to project completion, and contribute to adding to the cost of the project. The project cost estimate is presented in Section 10.0 of this report.

- Establish High-Level Project Interfaces - Early definition of the high-level project interfaces is necessary to establish the firm project scope and requirements from the outset. The interface definition will require either trade studies or programmatic decisions. Examples of interface include (1) casting operations (2) waste treatment and disposal plan (3) Make-buy evaluations for the supply of fabricated materials for the project.

- Early Involvement of the NRC - The FFC will be licensed by the NRC. This includes obtaining a Construction Permit in order to commence with the construction and subsequently the Operations License to commence with the operations. Early engagement of the NRC to establish the FFC licensing strategy including establishing the Standard Review Plan Requirements for Licensing documents approval, as well as requirements for plant commissioning would be very beneficial. A stable regulatory process is necessary to avoid rework and delays. It is recommended the NRC involvement and familiarity with the process operations, commence in the conceptual design phase.

- Timely site Selection - If the NNSA selects the National Laboratory site for housing the $\mathrm{FFC}$, it is preferred the selected site meet the following requirements to minimize impacts to project cost and schedule : (1) Fuel fabrication activities are currently performed at the site, (2) There is a programmatic EIS at the site that allows new fuel fabrication activities (3) About 15 acres of well characterized uncontaminated land adjoining the national laboratory site is available for siting the FFC (4) utilities, emergency management facilities and facilities for treatment, packaging and disposal of waste are available at the site, and (5) Allows cost effective construction and operations of the facility. Site selection is required to be completed by $4 / 2009$, in order to allow for the start of the preparation of the construction permit application as planned.

Note: if the NEPA process is involved for site selection, it could result in cost and schedule impacts.

It must be mentioned, that the above high level programmatic risks be managed by the NNSA, so as to allow the project to allow the critical path design and licensing activities to commence in October 08 in order to not jeopardize the completion of the hot ops of the FFC by 1/2013 


\subsection{Technical Risks}

The project technical risks involve challenges related primarily to technology development and demonstration as well as the NRC acceptance of the Fuel Qualification report and to a lesser degree technical issues resolution.

A number of technology development topics and demonstration of the fabrication processes have been noted in Section 1.4.2 of this report. The National Labs should in FY-09 and FY-10 complete the on-going development and demonstration of these processes to allow selection of the equipment and processes for the FFC. Key processes to be down-selected based on performance during technology demonstration includes the following:

- Diffusion Barrier material (zirconium or silicon layer)

- Fuel plate bonding equipment/process (Friction Bonding or Hot Isostatic Pressing)

- Non-destructive fuel plate examination process for monolithic fuels

Another key work item for the national labs is the preparation of the Generic Fuel Qualification report to be submitted to the NRC. This includes irradiation testing data of mini plates, full-size plates and a full element required for the qualification of the generic LEU-MO monolithic alloy fuel.

The National Labs engaged in supporting the development and maturity of the FFC processes and equipment designs have to be adequately funded to allow completion of these key development activities of the FFC processes in the near term. The Technology Readiness Levels (TRL) for all processes to be deployed in the FFC at CD-2 should be at 8 or higher.

Key technical risks for the FFC much like other Fuel Fabrication Facilities include the following topical areas:

- Safety

- Safeguards and Security

- Waste Management

- Cost

- Technology

The risks involved with these areas must be addressed from the outset, so as to establish the project requirements and features necessary to handle these risks and gain DOE and NRC authorization for the construction and operations of the FFC. Based on the initial scoping hazards evaluation included in section 6.0 of this report, safety concerns include chemical, fire and criticality hazards along with normal industrial hazards. Our evaluations indicate these risks pose a low risk to the public, most at risks from these hazards are the plant workers, and most of the worker safety risks are non-radiological in nature.

The risks are quantified as High, Medium or low based on a qualitative assessment of the level of likelihood that an event will occur and the level of consequence if that event does occur. The risk level matrix is shown in Figure 9-1 


\section{Risk Consequences}

\begin{tabular}{|c|c|c|c|c|c|c|}
\hline & Negligible & Marginal & Significant & Critical & Crisis \\
\hline \multirow{4}{*}{ 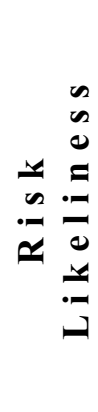 } & $\begin{array}{l}\text { Very } \\
\text { Likely }\end{array}$ & Low & Medium & Medium & High & High \\
\hline & Likely & Low & Low & Medium & High & High \\
\hline & Unlikely & Low & Low & Medium & Medium & High \\
\hline & $\begin{array}{c}\text { Very } \\
\text { Unlikely }\end{array}$ & Low & Low & Low & Medium & Medium \\
\hline
\end{tabular}

FIG. 9-1 RISK LEVEL MATRIX

A scoping level assessment of the risks involved in each of the technical areas identified above, and near term actions required to address these risks is identified in Table 9-1. 


\begin{tabular}{|c|c|c|c|}
\hline \multicolumn{4}{|c|}{$\begin{array}{l}\text { TABLE 9-1 } \\
\text { FFC PRELIMINARY PROJECT TECHNICAL RISKS AND MITIGATION }\end{array}$} \\
\hline $\begin{array}{l}\text { Item } \\
\#\end{array}$ & $\begin{array}{c}\text { Technical } \\
\text { Risk Area \& } \\
\text { Risk level }\end{array}$ & Risk & Risk Mitigation Strategies \\
\hline 1 & Safety - Low & $\begin{array}{l}\text { Key hazards which } \\
\text { provide safety risks, } \\
\text { include: } \\
\text { - Criticality, } \\
\text { - Fire, } \\
\text { - Natural } \\
\text { Phenomena, and } \\
\text { - Industrial hazards } \\
\text { (burns, electrical } \\
\text { shocks etc.) } \\
\text { NRC licensing } \\
\text { requirements for } \\
\text { project not established. } \\
\text { NRC staffing for } \\
\text { review of Project } \\
\text { documents is critical to } \\
\text { avoid delays }\end{array}$ & $\begin{array}{l}\text { Perform a preliminary Hazards analysis } \\
\text { during conceptual design. Provide engineered } \\
\text { safety features to mitigate the risks. } \\
\text { - Perform fire Hazards and Life Safety } \\
\text { evaluations. Provide engineered features to } \\
\text { address all fire and life safety code } \\
\text { requirements. } \\
\text { - Prepare criticality hazards calculations to } \\
\text { establish criticality control features for the } \\
\text { project } \\
\text { - Integrate safety into design early, follow } \\
\text { requirements of DOE STD } 1189 \text {. } \\
\text { - NRC/NNSA establish the regulatory } \\
\text { framenwork for the project early in the } \\
\text { conceptual design } \\
\text { - Early involvement of the NRC during the } \\
\text { developemnt of the design } \\
\text { - NRC to be adequaltely staffed to support } \\
\text { timely review of project licensing dcouemnts }\end{array}$ \\
\hline 2 & $\begin{array}{l}\text { Safeguards } \\
\text { and security - } \\
\text { Low }\end{array}$ & $\begin{array}{l}\text { Concerns over safe and } \\
\text { secure transport of } \\
\text { nuclear materials and } \\
\text { security of the FFC } \\
\text { from terrorist attacks. }\end{array}$ & $\begin{array}{l}\text { - Use only low enriched material which has a } \\
\text { much lower attractiveness level. } \\
\text { - Perform vulnerability assessment to provide } \\
\text { the appropriate protection of the nuclear } \\
\text { materials in the FFC } \\
\text { - FFC located at a national Laboratory site, } \\
\text { which has established security. } \\
\text { - The LEU-Mo alloy material will be packaged } \\
\text { in accordance with } 10 \text { CFR 71, security for } \\
\text { the material in transit will be in accordance } \\
\text { with } 10 \text { CFR } 73 \text {. }\end{array}$ \\
\hline 3 & $\begin{array}{l}\text { Waste } \\
\text { management - } \\
\text { - Low }\end{array}$ & $\begin{array}{l}\text { Concerns over } \\
\text { management (treatment } \\
\text { and disposal) of } \\
\text { hazardous waste }\end{array}$ & $\begin{array}{l}\text { Perform trade studies to establish path } \\
\text { forward relative to utilizing the host site } \\
\text { capabilities or including these capabilities in } \\
\text { the FFC: } \\
\text { Treatment of hazardous liquid waste } \\
\text { Characterization, Packaging and shipping } \\
\text { the waste to disposal facilities } \\
\text { - Perform waste minimization studies, with }\end{array}$ \\
\hline
\end{tabular}




\begin{tabular}{|c|c|c|c|}
\hline & & & $\begin{array}{l}\text { emphasis on internal recycling to the } \\
\text { maximum extent feasible. Include the } \\
\text { selected approach in the facility design basis. }\end{array}$ \\
\hline 4 & $\begin{array}{l}\text { Cost - } \\
\text { Medium }\end{array}$ & $\begin{array}{l}\text { Concerns over cost } \\
\text { growth }\end{array}$ & $\begin{array}{l}\text { - Perform trade studies as identified in Section } \\
\text { 1, to optimize the facility layout } \\
\text { - Avoid any project implementation and site } \\
\text { activities as scheduled. Project escalation } \\
\text { costs amount to approx. } \$ 1.8 \text { million a year } \\
\text { - Freeze project functions and operations } \\
\text { requirements during conceptual design, } \\
\text { likewise establish the project licensing and } \\
\text { permitting strategies approved by the NRC } \\
\text { early in the design process, to establish a firm } \\
\text { regulatory basis from the outset. This is } \\
\text { necessary to avoid rework. } \\
\text { - Prepare a rigorous Project risk management } \\
\text { plan and risk management strategies. } \\
\text { - Adopt a design-build strategy to realize } \\
\text { efficiencies and cost savings. } \\
\text { - Implement a design-to-cost to manage } \\
\text { changes. } \\
\text { - Utilize the draft NNSA PDRI process to } \\
\text { ensure design deliverables provide the } \\
\text { required maturity before authorization is } \\
\text { provided to advance to the next phase. } \\
\text { - Perfrom source surveys to selct qualified } \\
\text { vendors of process equipment. Seek } \\
\text { authorization to procure long lead equipment } \\
\text { upon completion of CD-2 doucmentation. }\end{array}$ \\
\hline 5 & $\begin{array}{l}\text { Technology - } \\
\text { Medium }\end{array}$ & $\begin{array}{l}\text { Uncertainties exist in: } \\
\text { - Selection of } \\
\text { diffusion barrier } \\
\text { - Selection of the } \\
\text { plate bonding } \\
\text { process } \\
\text { - Selection of the } \\
\text { non-destructive } \\
\text { examination } \\
\text { process } \\
\text { NRC acceptance of the } \\
\text { Generic Fuel } \\
\text { Qualification report }\end{array}$ & $\begin{array}{l}\text { - Perform the required demonstration/testing in } \\
\text { FY-09 in-order to down-select the processes } \\
\text { to be incorporated in the FFC } \\
\text { - Prepare a technology maturation plan/road } \\
\text { map to define the pathway to be used to } \\
\text { mature the technologies selected for } \\
\text { deployment in the FFC. (Require TRL-5 for } \\
\text { CD-1 approval and TRL-7 or better for CD-2 } \\
\text { approval) } \\
\text { - Early involvement of the NRC realtive to the } \\
\text { developemnt of the Fuel Qualifcation test } \\
\text { data and perfromance evaluation } \\
\text { - Complete Fuel Qualification documentation } \\
\text { for submittal to the NRC by } 12 / 2010\end{array}$ \\
\hline
\end{tabular}




\subsection{Risk Management Plan}

A risk management plan will be prepared during the conceptual design phases, so that all FFC project risks are identified and that all events that might adversely impact the performance of the project are addressed by incorporating appropriate efficient and cost effective measures to manage the unacceptable project related risks. The risk management plan will describe the roles and responsibilities of the project personnel in performing risk management functions, and will define reporting and tracking requirements for risk related information. A product of the risk management process is a report on project risks. The report will list the high and moderate level risks and includes the risk handling strategies and project impacts.

The risk management process will include the following:

- Preparing a risk management plan

- Risk identification; risk analysis and reporting

- Project impacts determination - Cost and schedule

- Risk tracking, reporting and closure

The risk management process will:

- Assess individual risks and their impact on project and facility performance, cost and schedule.

- Evaluate alternate approaches to manage high risks.

- Develop action plans to handle (i.e., avoid, reduce, transfer, or accept individual risks).

- Interface risks with other projects/ programs.

Secondarily, the project risk management process will:

- Provide risk-informed feedback regarding the setting of project milestones and goals.

- Identify actions that can be taken to help achieve cost, schedule and performance goals.

- Assist in making decisions on funding priorities.

- Assist in monitoring the status of the project as it proceeds.

- Provide evidence of compliance with NNSA project risk management requirements.

- Evaluates TRL's for key process/equipment items, to mange technology related risks. 


\subsection{COST ESTIMATE}

This section provides the estimate basis comprising of assumptions, inclusion/exclusions as well as the basis used for estimating the quantities and prices used in this ROM scoping level cost estimate. The estimate is based on the site, facility and process design and layout drawings prepared for this Greenfield Alternative Study. A detail process and mechanical equipment list was prepared and used to estimate the pricing of the equipment items. Also included are the estimate summaries and details along with a project contingency analysis based on the risk evaluations for the various cost accounts included in the estimate. ROM estimate ranges were prepared for the following:

- Base Case Greenfield Facility using the Friction Bonding process

- Greenfield Facility using the Alternate HIP Process.

A summary level operations cost estimate was also prepared.

\subsection{Assumptions.}

The following are the assumptions used for the preparation of the ROM type cost estimates.

- All costs are in constant un-escalated CY 2008 dollars.

- FFC is sited at an uncontaminated well characterized site at a US National Laboratory with an on-going fuel fabrication program.

- Cost of land, access roads, utilities (Power, water, sewer) to the site boundary is not included in the estimate

- Adequate annual funding will be provided for un-interrupted progress to completion.

- Owner's costs are not included .This includes costs for project management and control, site security, environmental permits and safety documentation, start-up, testing and acceptance cost.

- Cost for fuel qualification and technology development and demonstration is not included in the estimate.

- Cost of LEU-Mo feed material including transportation from Y-12 is not included.

- Costs of shipping casks and containers are not included in this estimate.

- Costs for the treatment, packaging and disposal of waste are not included.

\subsection{Cost Estimate Basis}

The construction approach envisions one General Construction Contractor with fully subcontracted union shop labor. A standard 40 hour work week is assumed @ 5 days 8 hours/day.

\subsubsection{Quantity Basis}

Quantities for the civil/structural/architectural works were largely based on the site and facility layout drawings included in Appendix A. The equipment quantities and design parameters were based on a detail mechanical and process equipment list included in section 3 of the estimate. All other accounts utilized factored estimates considering building size and historical data for industrial facilities. The quantity basis for each of the construction code of accounts is shown below: 


\begin{tabular}{|c|c|c|}
\hline $\begin{array}{l}\text { Code of } \\
\text { Account }\end{array}$ & Description & Quantity basis \\
\hline 02 & $\begin{array}{l}\text { Improvement to } \\
\text { site }\end{array}$ & $\begin{array}{l}\text { This account includes site preparation activities comprising of } \\
\text { clearing and grubbing, site drainage, installing yard fencing, } \\
\text { building parking lot and site roads including asphalt wearing } \\
\text { coat. Quantities were estimated using the site layout drawings. }\end{array}$ \\
\hline 03 & Earthwork & $\begin{array}{l}\text { This account includes excavation and backfill for the building } \\
\text { foundation and slabs on grade, and all earthwork required for the } \\
\text { roads and parking lots. Quantities were estimated using the site } \\
\text { layout drawings. }\end{array}$ \\
\hline 04 & Concrete & 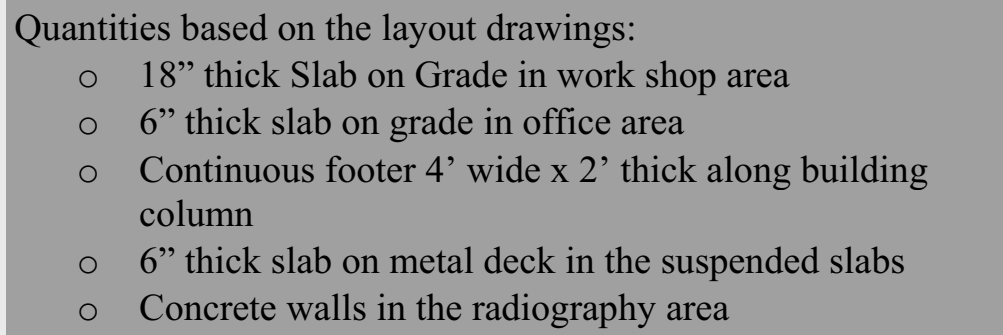 \\
\hline 05 & Structural Steel & $\begin{array}{l}\text { Quantities include structural framework to support the cranes } \\
\text { and hoists. Quantities were estimated using the site layout } \\
\text { drawings. }\end{array}$ \\
\hline 06 & $\begin{array}{l}\text { Permanent } \\
\text { equipment }\end{array}$ & $\begin{array}{l}\text { This section includes the mechanical and process equipment per } \\
\text { the equipment list included in section } 3 \text { of this report. All the } \\
\text { equipment is identified in the facility layout drawings included } \\
\text { in the Appendices of this report. }\end{array}$ \\
\hline 11 & Piping Bulks & $\begin{array}{l}\text { The quantities were estimated using a factored approach } \\
\text { considering the facility size. }\end{array}$ \\
\hline 12 & Electrical Bulks & $\begin{array}{l}\text { The quantities were estimated using a factored approach } \\
\text { considering the facility size }\end{array}$ \\
\hline 13 & $\begin{array}{l}\text { Instrumentation } \\
\text { Bulks }\end{array}$ & $\begin{array}{l}\text { The quantities were estimated using a factored approach } \\
\text { considering the facility size. }\end{array}$ \\
\hline 16 & Buildings & $\begin{array}{l}\text { This account was based on quantity take off from the facility } \\
\text { layout drawings including elevations and sections. The account } \\
\text { includes building siding \& roofing (insulated metal panels), } \\
\text { interior walls and structural steel building framing. Also } \\
\text { included is the personnel amenities, as well as standard building } \\
\text { HVAC. }\end{array}$ \\
\hline
\end{tabular}


10.2.2 Pricing Basis.

The prices for the construction accounts was based upon current project costs such as from the Integrated Waste Treatment Unit Project and other on going URS WD projects. The pricing for the specialty equipment items such as HIP, Electron Beam Welder, milling and rolling machines, friction bonding machine, X-ray and fluoroscopy equipment were based on preliminary vendor data. All other standard off-the shelf items were based on URS WD project data base pricing and or the Means hand book.

- The construction labor rates are "all in" labor rates for union craft labor and includes:

- Direct Labor wages: W2's, fringes, legalities, small tools, consumables, safety items/training, construction equipment and maintenance.

- Site in directs : Site office, mobilization and de-mobilization; indirect craft support such as scaffolding, clean-up, material receiving and storage.

- General Construction contractor's overhead and profit.

- A labor factor of 1.2 is applied to account for Quality assurance (NQA-1 for Quality level 2 items) and protocols for work at a federal site

- Construction Management Services includes costs for site construction manager, and personnel for safety, project controls and superintendents for civil/structural, piping/mechanical and electrical/I\&C.

- Design/Engineering (Home Office Services) costs are based on a factored percent of the direct field cost for preparing the Preliminary and final design of the project.

- Insurance is calculated at $0.42 \%$ of the Total Field Cost and Home Office Services Cost

- Taxes for materials and services have not been included as the FFC is a federal project.

- Freight is applied at 5\% on all materials cost.

\subsection{Contingency}

\section{Contingency Development Approach}

Contingency is a specific provision of resources to be included in an estimate for undefined items, which statistical studies of historical data have shown, will likely be required within a defined scope of work. Contingency covers inadequacies in the estimate basis definition (level of completeness of the design) and inadequacies in the estimating methods and data (Quantity and pricing data). The methodology used for estimating the contingency is the Risk Analysis method.

\section{$\underline{\text { Risk Analysis }}$}

The Risk Analysis method for estimating project contingency utilizes the Monte Carlo Simulation Model. The contingency development process follows the following steps:

- Develop the cost of the project. Group the cost into discrete work items (See Risk Analysis work sheet) 
- Next identify uncertainties in the various estimate accounts for labor, materials, subcontract etc. as shown in the risk analysis work sheet, by specifying the possible $+/$ - values of the variables in the estimate with probability ranges.

- Discrete values of the uncertainties (Low and high values) were established based on the consensus of the estimator, the INL project team and the project design team. The uncertainty ranges were established by examining for each estimate account the type of work activity, the completeness of the design and the source of the pricing/cost data.

- Analyze the estimate with simulation. The simulation model is run (2000 to 3000) iterations to determine the range and probabilities of all possible outcomes of the model. During each iteration, a value for each variable is selected randomly based on the specified probability distribution.

- The results are presented as overall probability distribution for the simulation. The estimate range is established based on the desired confidence level, which represents the confidence that the estimate will not be exceeded

The estimate range for the FFC is established as follows:

- Confidence Level of 99\% for the high end

- Confidence Level of $70 \%$ for the low end

- Confidence Level of $80 \%$ for the median end 


\subsection{Estimate Summary}

Estimate sheets for the summary and details of the estimate are provided in the Attachment to this section for (a) The base case using the Friction bonding process and (2) The HIP alternate. Capsule summaries are presented below:

10.4.1 Base Case Estimate summary.

The base case (friction bonding) estimate summary is shown in Table 10-2

TABLE 10-2 FFC BASE CASE COST ESTIMATE SUMMARY (ALL COSTS ARE IN MILLIONS OF DOLLARS)

\begin{tabular}{|c|c|c|}
\hline Cost Elements & & \\
\hline Yard / Civil Works & $\$ 2.3$ & \\
\hline 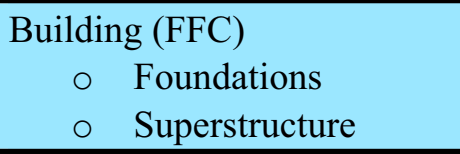 & $\begin{array}{l}\$ 4.6 \\
\$ 6.5 \\
\end{array}$ & \\
\hline Equipment & $\$ 12.0$ & \\
\hline \begin{aligned} & \multicolumn{2}{l}{ Utilities } \\
&$\quad$ HVAC \\
&$\circ$ Others \end{aligned} & $\begin{array}{l}\$ 3.9 \\
\$ 9.6\end{array}$ & \\
\hline $\begin{aligned} & \text { Other Buildings } \\
& \circ \text { Warehouse } \\
& \circ \text { Substation } \\
&\end{aligned}$ & $\begin{array}{l}\$ 0.8 \\
\$ 0.5\end{array}$ & \\
\hline Total Direct Field Cost & & $\$ 40.2$ \\
\hline $\begin{array}{l}\text { Professional services } \\
\begin{array}{l}\text { Design/engineering } \\
\text { services } \\
\circ \\
\text { Construction } \\
\text { management }\end{array}\end{array}$ & $\begin{array}{l}\$ 7.2 \\
\$ 1.7\end{array}$ & \\
\hline $\begin{array}{l}\text { Field \& Other Professional } \\
\text { Service Cost }\end{array}$ & & $\$ 49.1$ \\
\hline $\begin{array}{l}\text { Other cost } \\
\quad \circ \text { Insurance/Freight }\end{array}$ & $\$ 1.4$ & \\
\hline $\begin{array}{l}\text { Contingency } \\
\qquad \begin{array}{l}\text { Median } \\
\circ \text { High } \\
\circ \text { Low }\end{array}\end{array}$ & $\begin{array}{l}\$ 13.9 \\
\$ 22.3 \\
\$ 11.6\end{array}$ & \\
\hline $\begin{array}{cl}\text { Total Project Cost } \\
\circ & \text { Median } \\
\circ & \text { High } \\
\circ & \text { Low }\end{array}$ & & $\begin{array}{l}\$ 64.4 \\
\$ 72.8 \\
\$ 62.1\end{array}$ \\
\hline
\end{tabular}

The Base case (friction bonding) total estimate cost range is $\$ 62 \mathrm{M}-\$ 73 \mathrm{M}$

The segregated costs for the casting area are approximately $\$ 5 \mathrm{M}$. 
10.4.2 HIP case (HIP bonding) estimate summary

The HIP case estimate summary is shown in table 10-2

TABLE 10-2 FFC HIP CASE ESTIMATE SUMMARY (ALL COSTS ARE IN MILLION DOLLARS)

\begin{tabular}{|c|c|c|}
\hline Cost Elements & & \\
\hline Yard / Civil Works & $\$ 2.4$ & \\
\hline $\begin{array}{cl}\text { Building }(\mathrm{FFC}) \\
\circ & \text { Foundations } \\
\circ & \text { Superstructure }\end{array}$ & $\begin{array}{l}\$ 4.8 \\
\$ 6.8\end{array}$ & \\
\hline Equipment & $\$ 23.6$ & \\
\hline \begin{aligned} & \multicolumn{2}{l}{ Utilities } \\
&$\quad$ HVAC \\
&$\circ$ Others \end{aligned} & $\begin{array}{l}\$ 4.4 \\
\$ 9.7\end{array}$ & \\
\hline $\begin{aligned} & \text { Other Buildings } \\
& \circ \text { Warehouse / ECF } \\
& \circ \text { Substation } \\
&\end{aligned}$ & $\begin{array}{l}\$ 0.8 \\
\$ 0.5\end{array}$ & \\
\hline Total Direct Field Cost & & $\$ 53.0$ \\
\hline $\begin{array}{cl}\text { Professional services } \\
\quad \text { Design/engineering } \\
\text { services } \\
\circ \begin{array}{l}\text { Construction } \\
\text { management }\end{array} \\
\end{array}$ & $\begin{array}{l}\$ 9.0 \\
\$ 1.7\end{array}$ & \\
\hline $\begin{array}{l}\text { Field \& Other Professional } \\
\text { Service Cost }\end{array}$ & & $\$ 63.9$ \\
\hline $\begin{array}{l}\text { Other cost } \\
\quad \circ \quad \text { Insurance/Freight }\end{array}$ & $\$ 1.8$ & \\
\hline 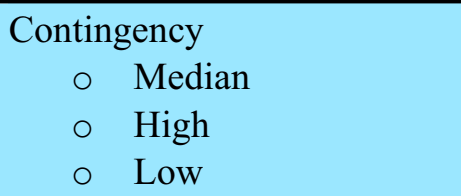 & $\begin{array}{l}\$ 17.7 \\
\$ 27.3 \\
\$ 15.2\end{array}$ & $\begin{array}{l}\$ 83.4 \\
\$ 93.0 \\
\$ 80.9\end{array}$ \\
\hline $\begin{array}{cl}\text { Total Project Cost } \\
\circ & \text { Median } \\
\circ & \text { High } \\
\circ & \text { Low }\end{array}$ & & $\begin{array}{l}\$ 83.4 \\
\$ 93.0 \\
\$ 80.9\end{array}$ \\
\hline
\end{tabular}

The HIP case (HIP bonding process) total estimate cost range is $\$ 81 \mathrm{M}$ - $\$ 93 \mathrm{M}$.

The additional cost range for the HIP option is $\$ 13 \mathrm{M}-\$ 20 \mathrm{M}$. 


\subsection{Operations Cost}

The annual operations cost is comprised of the annual labor cots and annual non-labor costs.

The staffing estimate for the FFC is provided in table 5-3 - FFC Shop personnel tally and Table 5-4 FFC Office personnel tally. The staffing estimate includes 55 shop floor personnel and 17 management and administrative personnel for a total FFC head count of 72 personnel.

Based on historical data the annual labor cost estimate for the 72 personnel is: $\$ 8 \mathrm{M}--\$ 10 \mathrm{M}$

The annual non-labor costs include costs for:

- Utilities

- Materials and consumables

- Equipment replacement.

Section 5.19 of this report provides a detail listing of materials and consumables.

Using historical data for industrial facilities, the annual non-labor costs are estimated to be as follows:

- Utilities -- \$1M - \$2M

- Materials \& Consumables -- $\$ 5 \mathrm{M}--\$ 6 \mathrm{M}$

- Equipment Replacement --\$1M -- \$2M

Hence the annual operations cost is estimated to be $\$ 15 \mathrm{M}-\mathrm{\$} 20 \mathrm{M}$

\subsection{Startup \& Testing}

Startup and Testing costs are included in the owner's cost and based on historical data are anticipated to be $\$ 10 \mathrm{M}-\$ 15 \mathrm{M}$. 


\section{Estimate Summary}

Base Case (Friction Bonding) 


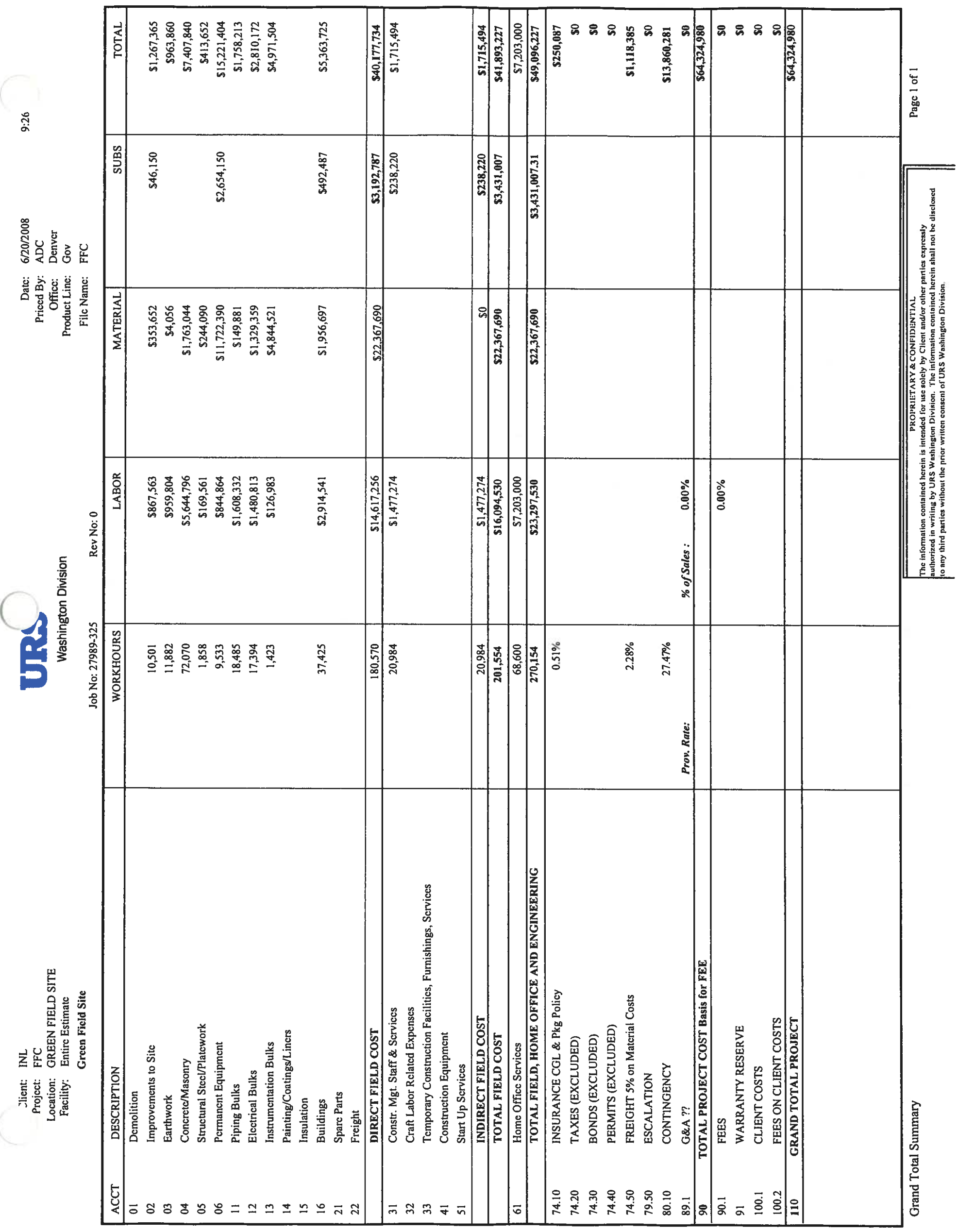




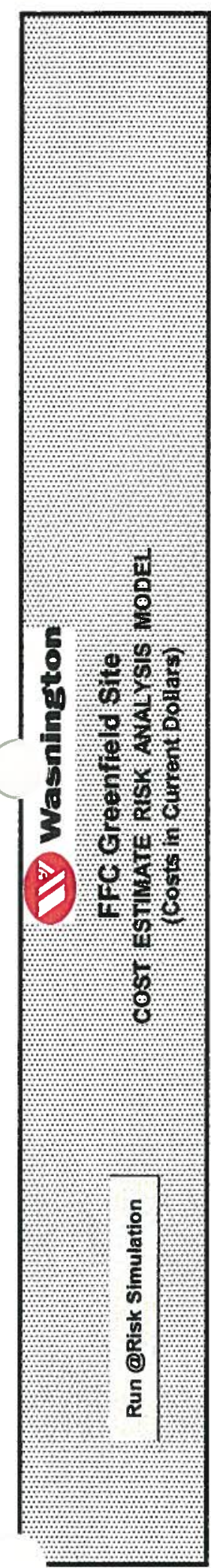

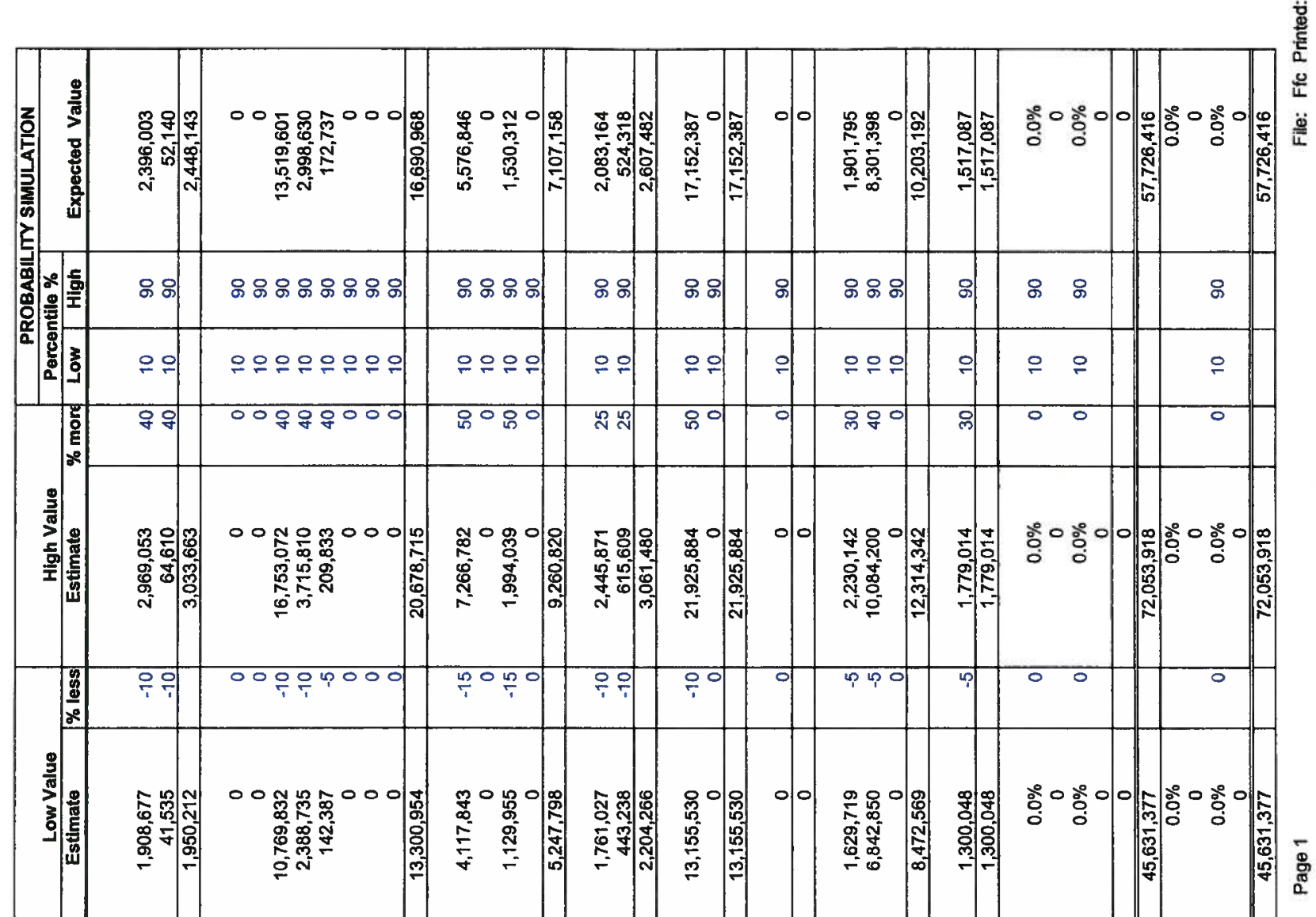




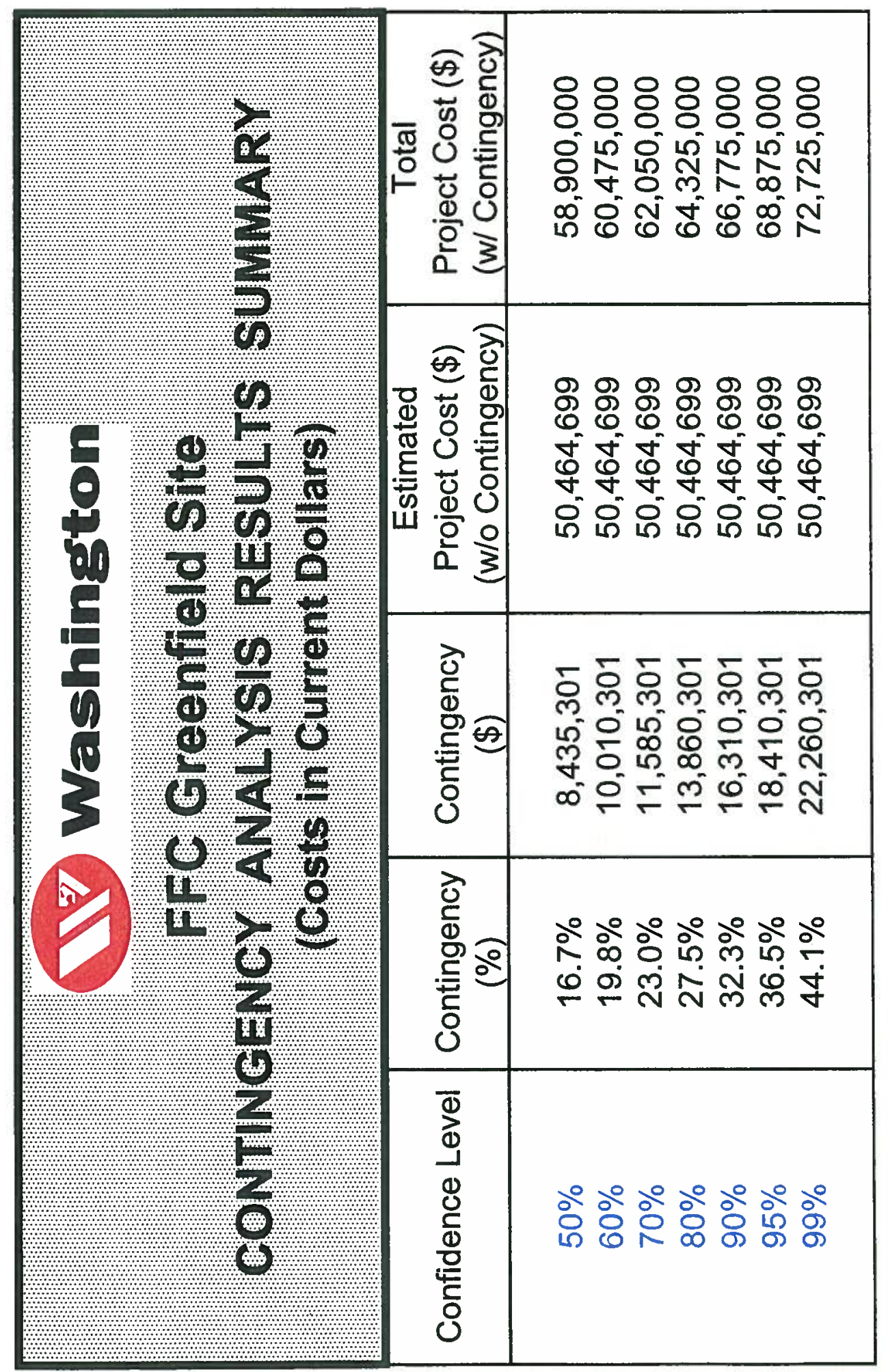

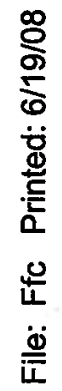

훔 


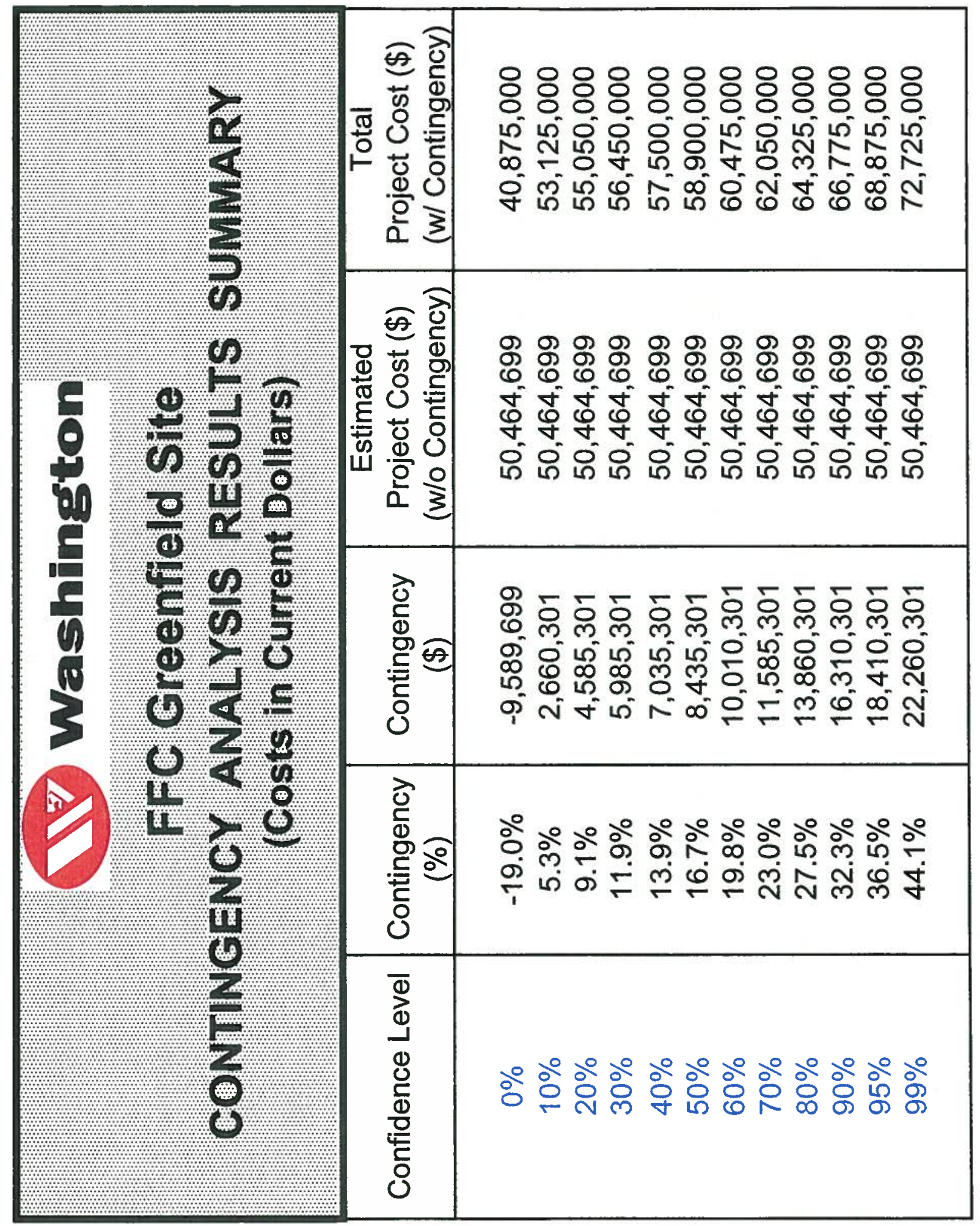

N
$\stackrel{0}{0}$
0 


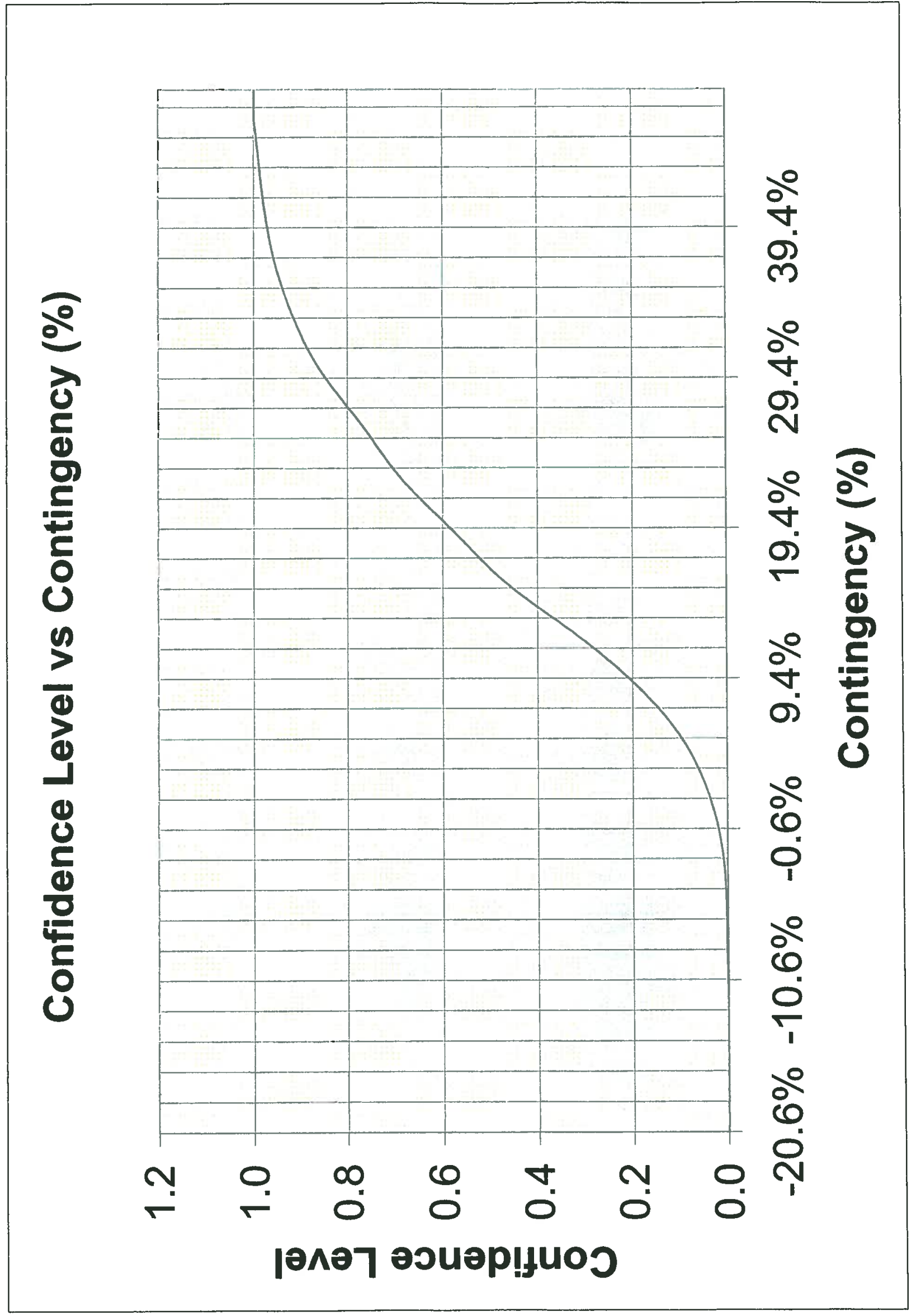




\title{
Estimate Summary
}

\author{
HIP Alternate
}




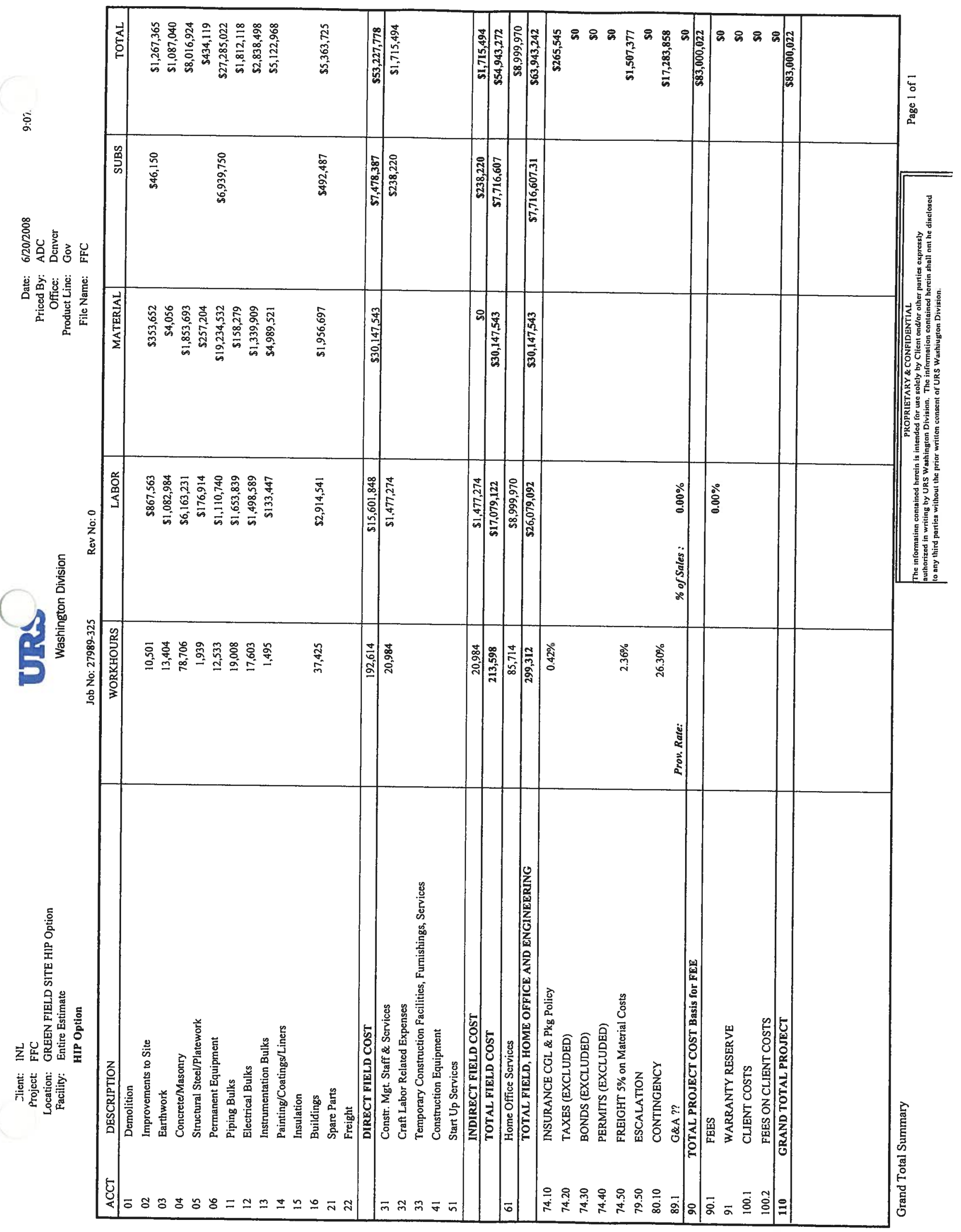




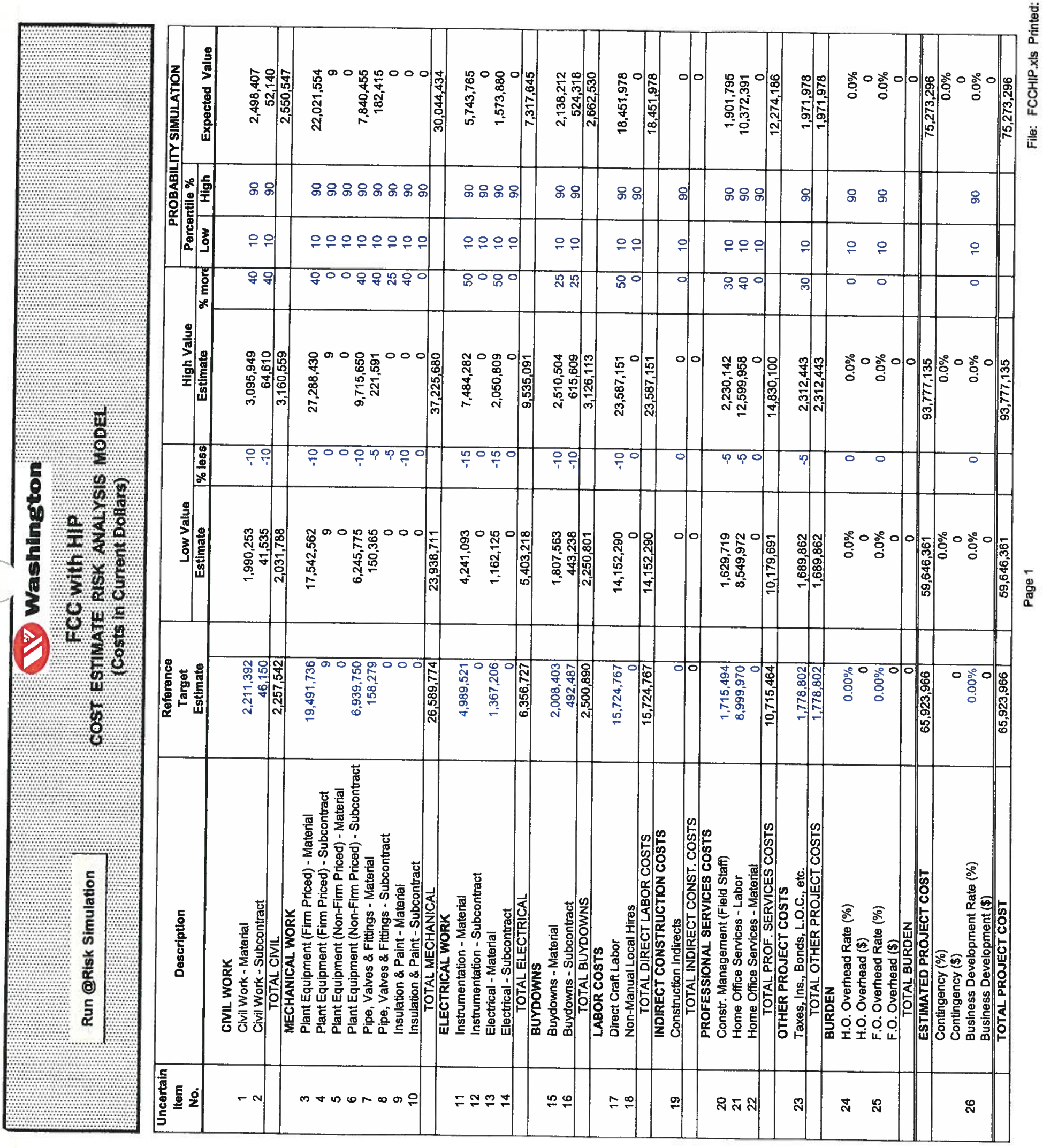




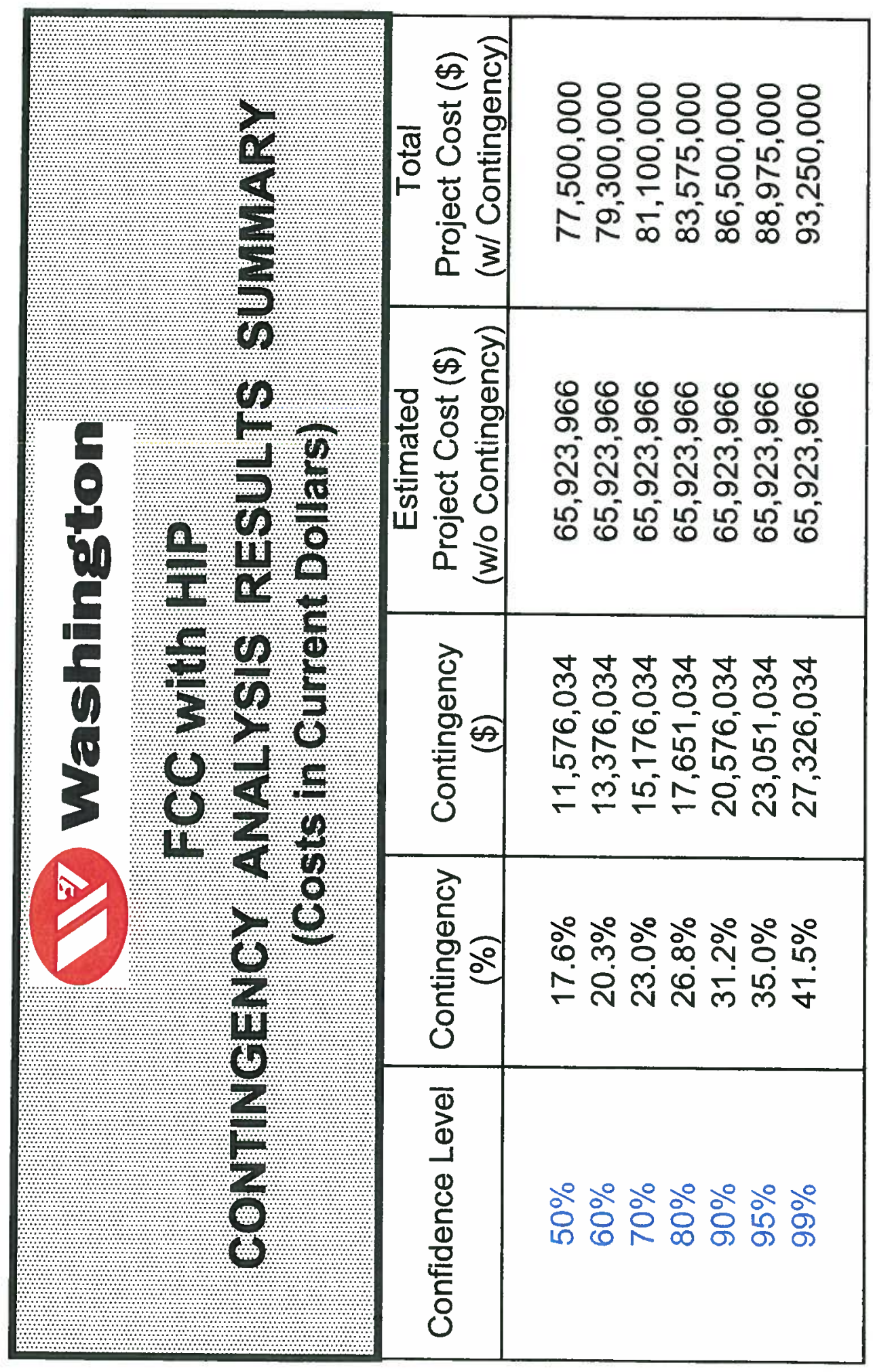




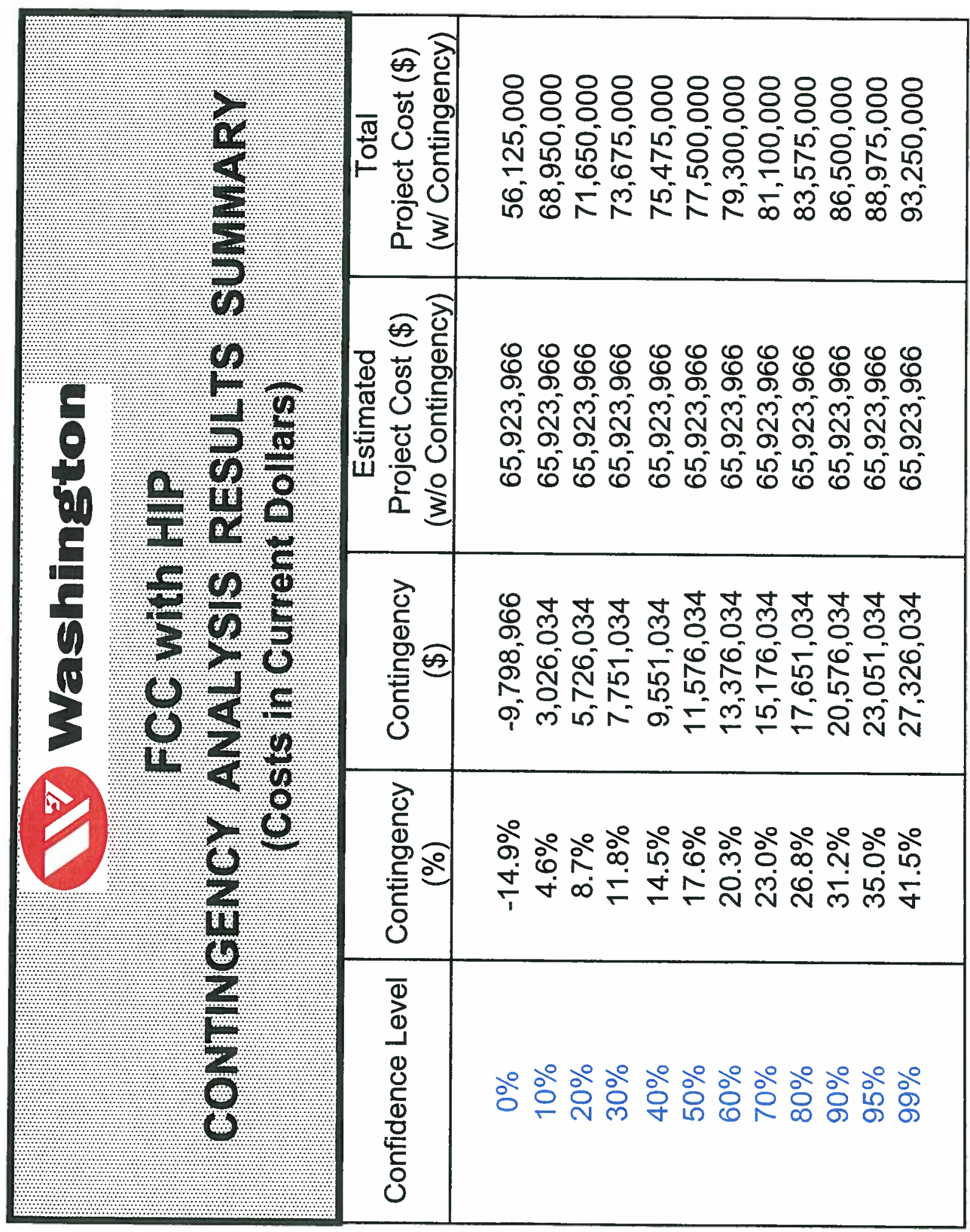




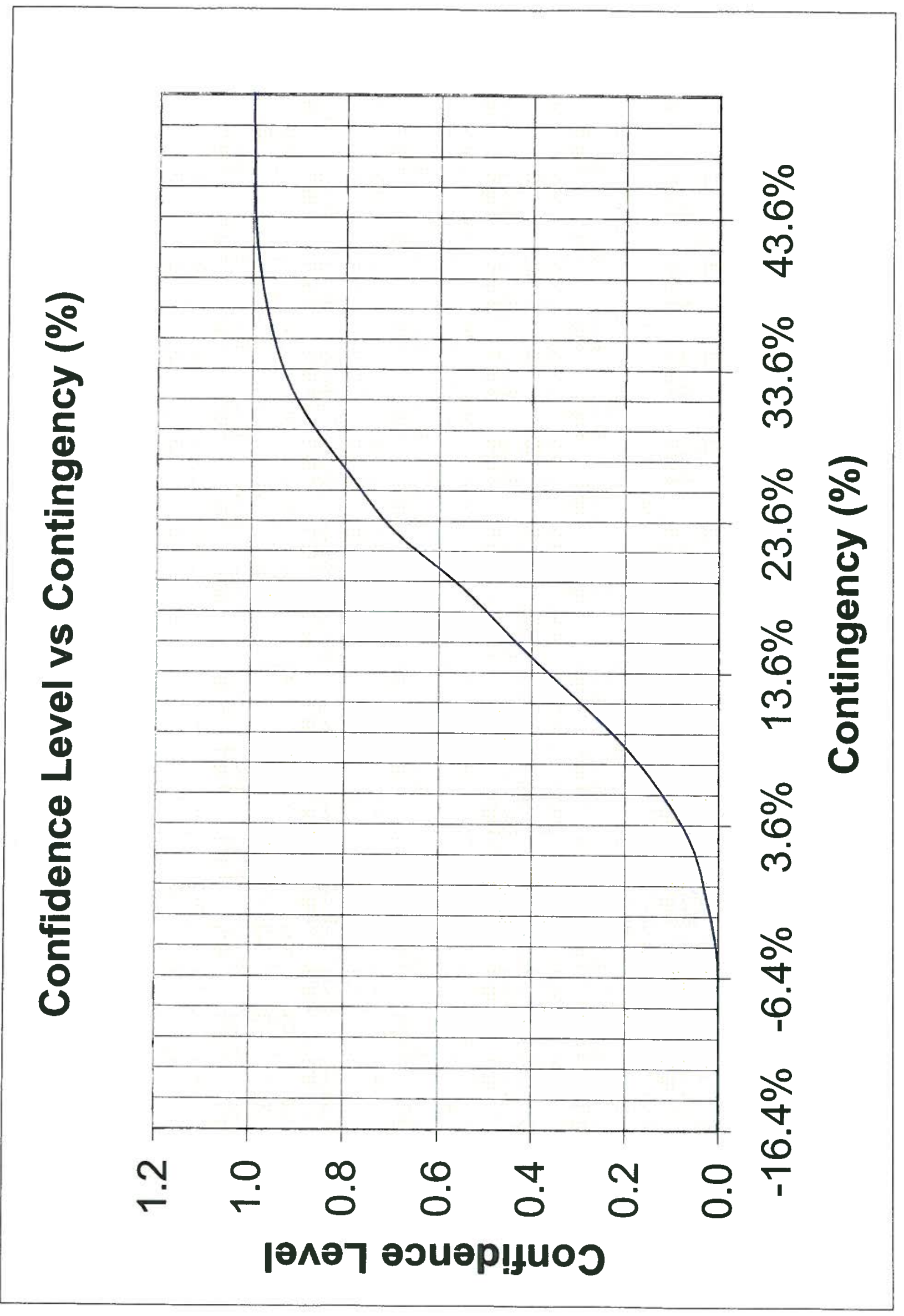




\subsection{SCHEDULE ESTIMATE}

This section provides a scoping level integrated FFC project schedule. The schedule defines key activities and their durations, along with required project approvals necessary to meet the desired FFC hot ops by 12/31/2012 in-order to complete the conversion of the fuel of the 5 HPRR's from HEU to LEU by $10 / 1 / 2014$.

\subsection{Introduction}

An integrated summary level project scoping type schedule has been developed for the FFC. The schedule is presented in Fig. 11-1. The schedule covers key activities relative to securing project funding/approvals, NRC licensing, environmental permitting, construction including start-up and commissioning as well as fuel qualification. The schedule conforms to the requirements of DOE O 413.3A - Project Management for the Acquisition of Capital Assets, which mandates compliance with the critical decisions and baseline change control as the fundamental processes for DOE project authorization and change management. The schedule defines the major activities on an annual basis, commencing with the completion of the Greenfield Alternative Study leading to the start of the conceptual design in October of 2008, and ends with the conversion reactor hot ops by $10 / 1 / 2014$.

The DOE's critical decision process for project authorization/funding requires careful planning and scheduling of work activities to facilitate DOE's review of project performance and approval of key review documents. The critical decision approval process provides the DOE with a structured approach to ascertain that the project is on schedule, within budget and fully capable of meeting the project mission, functions and operations requirements and safety and ES\&H standards before approving funds to proceed to the next phase of the project

In this regard it is noteworthy to mention that the NNSA (NA-50 and NA-54) is proposing to utilize the Project Definition Rating Index (PDRI) process as a management tool. The PDRI has been designed by industry and used by the DOE to increase the likelihood of project success by improving project scope definition, and achieving project objectives within established budget and schedule. The NNSA PDRI has five rating areas: Scope/technical, Safety/quality assurance; Cost; Schedule, and Management Planning and Control. Each rating area has a number of subelements, which are evaluated/ rated by a team of subject matter experts to gauge the maturity of the design products to meet established thresholds. This data is utilized by the acquisition executive to authorize appropriate critical decision approval, thus enabling the project to advance to the next phase.

The goal of the NNSA PDRI process is to significantly improve up-front planning in the areas of alternatives evaluation, safety evaluation, and technology maturity as well as nailing down the project requirements and design criteria so as to define the project scope during the conceptual design phase of the project which concludes at CD-1. By CD-2 (completion of preliminary design), the project scope definition must be complete, to allow establishment of a firm technical, cost and schedule performance baseline for the project. This baseline will be independently validated by an external review team per DOE 413.3A. The importance of a well defined project scope at CD-2 is highlighted by the NNSA's firm expectations that the Performance Baseline cost and schedule will not be exceeded. 


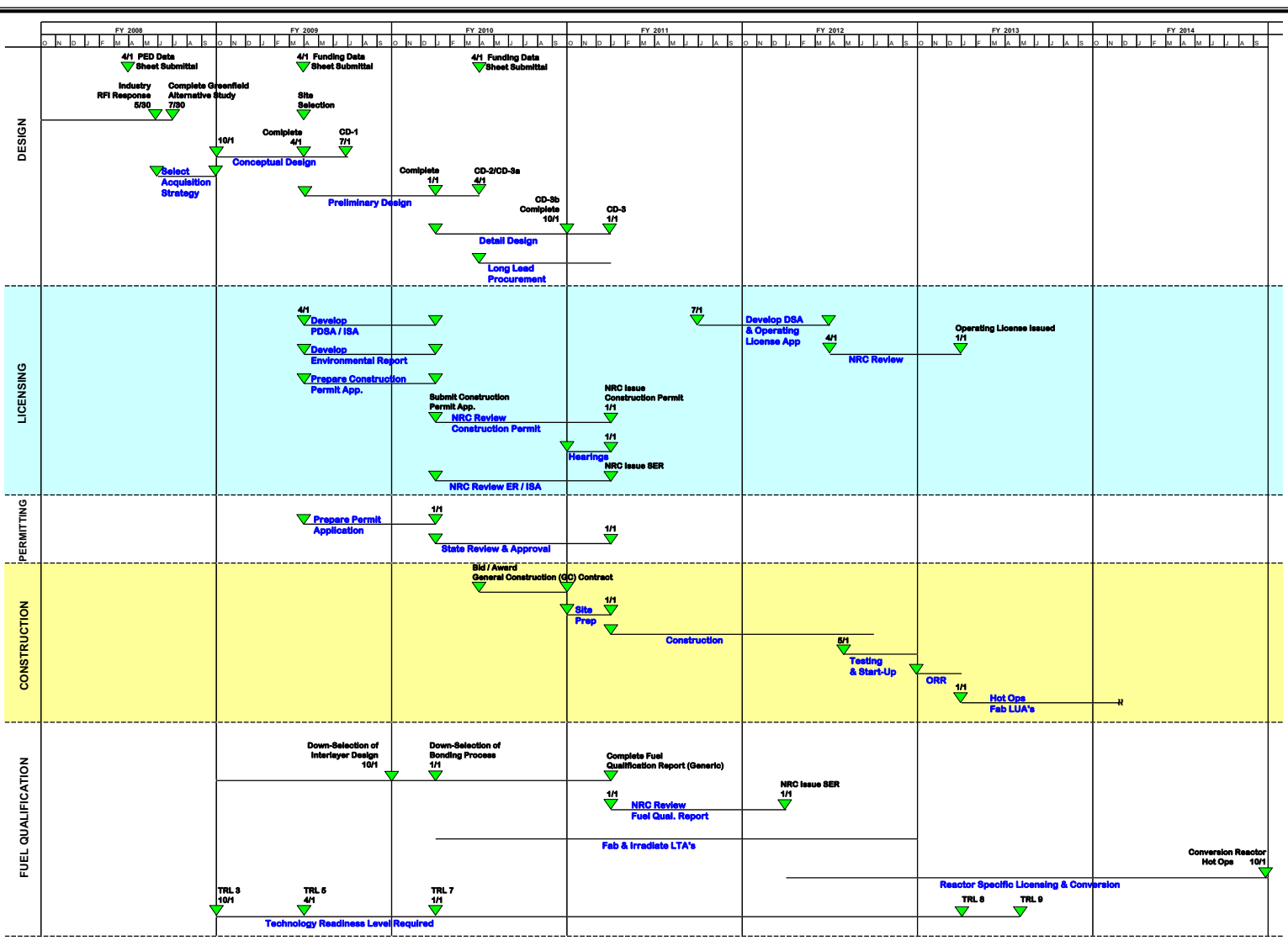

FIGURE 11-1 INTEGRATED FFC SCHEDULE 
DOE O 413.3A permits projects to propose partial or phased critical decision approval as well as to propose combined CD reviews. Accordingly in order to facilitate early procurement of Long Lead Equipment (LLE), a combined CD-3a (Authorization to procure Long Lead Equipment) and CD-2 (Approve Performance Baseline) will be secured at the completion of the Preliminary Design (Title I). This will enable the LLE to be procured early in time to allow for the timely installation and integration into the construction process. Likewise early approval for site work (grading and drainage, fencing, parking, temporary utilities, excavation for foundations) will be sought (CD-3b) prior to the completion of detail design and receipt of CD-3 - Approval to begin Construction. Early approval to commence site preparation work is required in order to facilitate start of the permanent plant construction upon approval of the CD-3 authorization.

Actions authorized by the DOE's Critical decision approval process are summarized in Table 11-1.

TABLE 11-1 ACTIONS AUTHORIZED BY DOE CRITICAL DECISION APPROVAL

\begin{tabular}{|c|c|c|c|c|c|c|}
\hline \multirow[t]{2}{*}{$C D-0$} & \multirow[t]{2}{*}{$C D-1$} & \multirow[t]{2}{*}{$C D-2$} & \multicolumn{3}{|c|}{$C D-3$} & \multirow[t]{2}{*}{$C D-4$} \\
\hline & & & $C D-3 a$ & $C D-3 b$ & $C D-3 c$ & \\
\hline $\begin{array}{l}\text { Proceed with } \\
\text { conceptual } \\
\text { design using } \\
\text { program funds } \\
\text { Request PED } \\
\text { funding }\end{array}$ & $\begin{array}{l}\text { Allow } \\
\text { expenditure of } \\
\text { PED funds for } \\
\text { design }\end{array}$ & $\begin{array}{l}\text { Establish } \\
\text { Baseline } \\
\text { budget for } \\
\text { construction } \\
\text { Continue } \\
\text { design } \\
\text { Request } \\
\text { construction } \\
\text { funding }\end{array}$ & $\begin{array}{l}\text { Approve } \\
\text { expenditure } \\
\text { of funds } \\
\text { to procure } \\
\text { LLE items }\end{array}$ & $\begin{array}{l}\text { Approve } \\
\text { expenditure } \\
\text { of funds } \\
\text { for early site } \\
\text { prepration } \\
\text { construction }\end{array}$ & $\begin{array}{l}\text { Approve } \\
\text { expenditure } \\
\text { of funds } \\
\text { for } \\
\text { construction }\end{array}$ & $\begin{array}{l}\text { Allow start } \\
\text { of } \\
\text { operations }\end{array}$ \\
\hline
\end{tabular}

\subsection{Assumptions and Design Basis}

The following provides key assumptions used to develop the integrated project schedule:

- Conceptual design will commence on 10/1/08 and complete 4/1/09 to allow for timely approval of CD-1 by 7/1/09. This is required to complete the Critical Path design activities to allow start of construction by $1 / 11$.

- Site selection will be completed by 4/2009 to allow for the preparation of the required Environmental Report to support the Construction Permit application to the NRC. The site selected will be a well characterized site with no contamination.

- Early interaction (during conceptual design) with the NRC to establish the project licensing basis and requirements.

- On going involvement of the NRC during the development of the preliminary and detail design to allow NRC staff to gain familiarity with the design.

- NRC will provide a NUREG 1313 type SER signifying the approval of the Generic Fuel Qualification Report.

- NRC has sufficient staff, to allow for timely review and approval of Construction Permit, Operations license, Fuel Qualification report with 12 months of the submission of these licensing documents 
- LTA's fabricated through the INL's lead test assembly plan, will be used for generic fuel qualification. LUA's fabricated at the FFC will be used for the qualification of the fuel fabrication processes.

- Adequate funding required for each of the phases will be provided to allow for uninterrupted execution of the design, permitting, construction and start-up/ commissioning activities. A period of 12 weeks has been allocated at the end of each of the design phases for the approval of the DOE's critical decisions. The approval process will utilize Independent Review Teams and the PDRI. During this 12-week period the design effort will continue un-interrupted.

\subsection{Key Activity Durations}

Durations of Key Activities is presented in Table 11-2 below:

TABLE 11-2 FFC PROJECT DURATIONS - KEY ACTIVITIES

\begin{tabular}{|l|c|}
\hline \multicolumn{1}{|c|}{ Activity } & Duration \\
\hline Conceptual Design & 6 months \\
\hline Preliminary Design & $\mathbf{9}$ months \\
\hline Detail Design & 9 months \\
\hline NRC Construction Permit (Prep, Review) & $\mathbf{2 1 ~ m o n t h s ~}$ \\
\hline NRC Operations License (Prep, Review) & 18 months \\
\hline Construction $\quad$ & $\mathbf{1 8}$ months \\
\hline Start-up \& Commissioning & 8 months \\
\hline
\end{tabular}

The proposed activity durations are workable and represent the minimum durations consistent with the scope of the project. Although this scoping-level schedule has not been developed using resource loadings, it is believed that the schedule as presented approximately represents the shortest reasonable overall duration possible for the project. Any delays encountered are expected to result in an extension of the completion date. 


\subsection{REFERENCES}

The following INL documents were key reference documents used for the Greenfield Alternative Study:

- Statement of Work for an Engineering Alternative Study for a Greenfield LEU Molybdenum Fuel Fabrication Capability - April 208

- Conceptual process description for the Manufacture of Low Enriched Uranium Molybdenum Fuel; Wachs et al; INL/EXT-08-13840; February 2008

Guidance and specific project design basis and requirements was provided by cognizant INL and Y-12 personnel involved with the development of the monolithic LEU-Mo fuel. Notes of meetings and motes of weekly teleconference document the programmatic and technical direction relative to the process and equipment requirements. In addition a 35\% complete design review was conducted by the cognizant INL and Y-12 personnel. Comments and directions resulting from the design review were recorded in the Notes of the $35 \%$ complete design review meeting.

The directions, comments received from the cognizant INL and Y-12 personnel during the weekly teleconference, as well as at the design review and Kick-off meetings were considered as design basis requirements and incorporated into the facility and process design. 


\section{APPENDIX A - DRAWINGS AND DATA}

\section{SITE LAYOUT}

SK-ME-06, Green Field Alternative, FFC Site Plan

Architectural Prospective, 4 Drawings

FACILITY LAYOUT

SK-ME-01, Green Field Alternative, General Arrangement, Plan

SK-ME-02, Green Field Alternative, Storage Arrangements, Plan Views

SK-ME-03, Green Field Alternative, General Arrangement, HIP Layout, Plan

SK-ME-04, Green Field Alternative, Building Sections

SK-ME-05, Green Field Alternative, Building Sections and Details

SK-ME-07, Green Field Alternative, Miscellaneous Building Sections and Details

SK-ME-08, Green Field Alternative, Miscellaneous Building Sections and Details

SK-AR-01, Green Field Alternative, FFC Building Elevations with HIP Option

\section{BLOCK FLOW DIAGRAMS}

BF-1, Overall Block Flow Diagram

\section{PROCESS FLOW DIAGRAMS}

SK-1, Flow sheet. Legend and Symbols

SK-2, Front-End Processing, Process Flow Diagram

SK-3, Fuel Zr Cladding, Process Flow Diagram

SK-4, Fuel AL Cladding, Process Flow Diagram

SK-5, Final Assembly, Process Flow Diagram

SK-6, Inspection, Process Flow Diagram

SK-8, Waste Characterization and Packaging, Process Flow Diagram

SK-9, Shipping, Receiving, and Storage, Process Flow Diagram

SK-10, Alternate HIP Process, Process Flow Diagram

SK-11, Argon Purification and DI Waster Supply, Process Flow Diagram

SK-12, Final Assembly Machining, Process Flow Diagram 
4. EQUIPMENT CUT SHEETS

CS-800 Carbon/Sulfur Determinator

PAXcam Digital USB 2.0 Camera System

Pegasus Floor Scale System

Raymond Forklifts

Hass Automation, CNC Turning Centers

Hass VR Series, 5-Axis Contouring VMCs

Elan 9000 ICP-Mass Spectrometer

Olympus GX Series, Metallurgical Microscopes

Raymond Walkie Pallet Truck, Model 102XM

Spectrum System 1000, Grinder/Polisher

Pre-Engineered Tarca Crane System

Hot Isostatic Pressing System 
SITE LAYOUT 


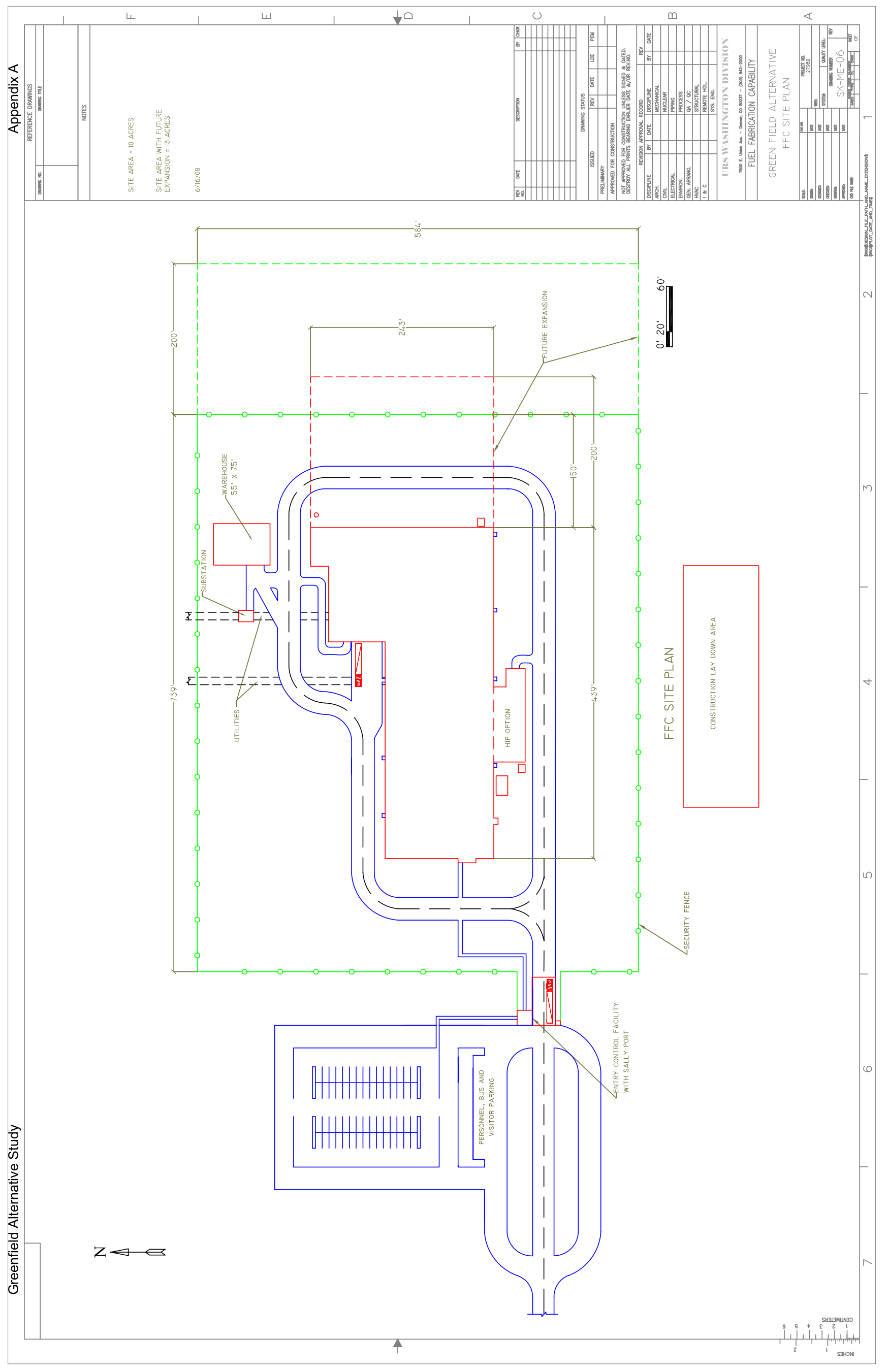


$\frac{x}{x}$
$\frac{x}{0}$
$\frac{0}{0}$
$\frac{0}{2}$
$\frac{1}{2}$

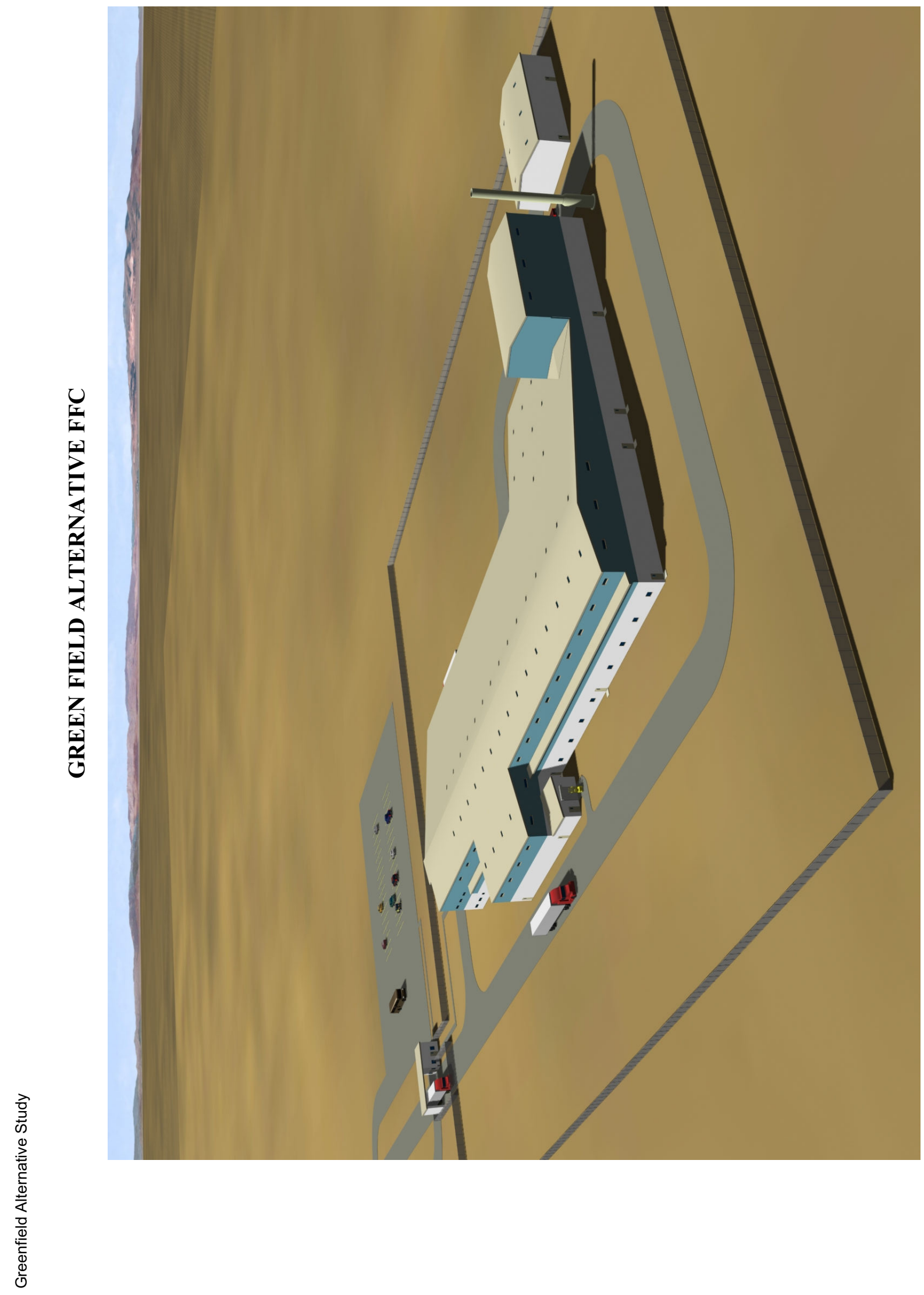


$\varangle$
$\frac{x}{0}$
$\frac{0}{0}$
$\frac{0}{0}$
$\frac{0}{2}$

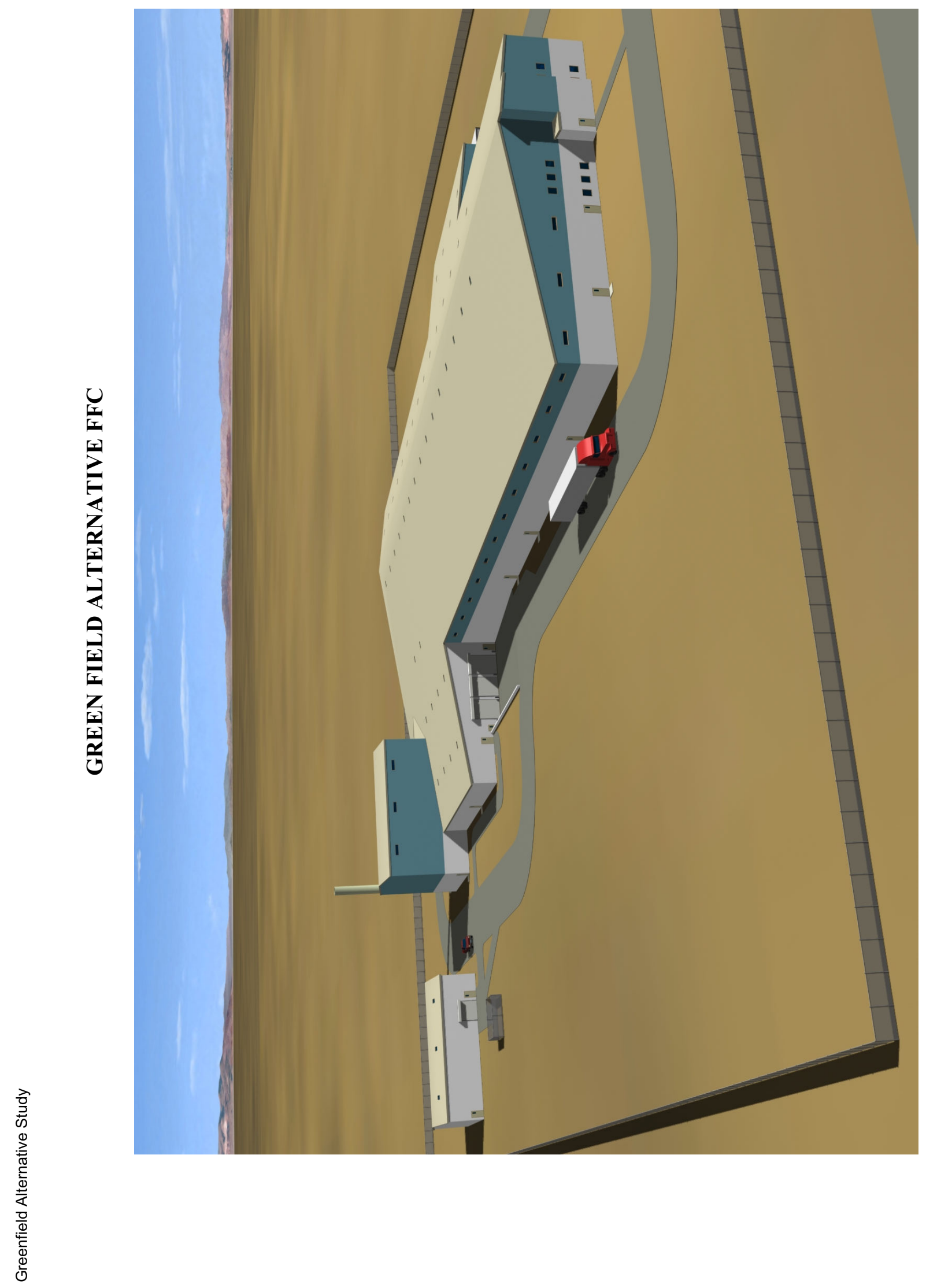


$\varangle$
$\frac{x}{0}$
$\frac{0}{0}$
$\frac{0}{0}$
$\frac{0}{2}$

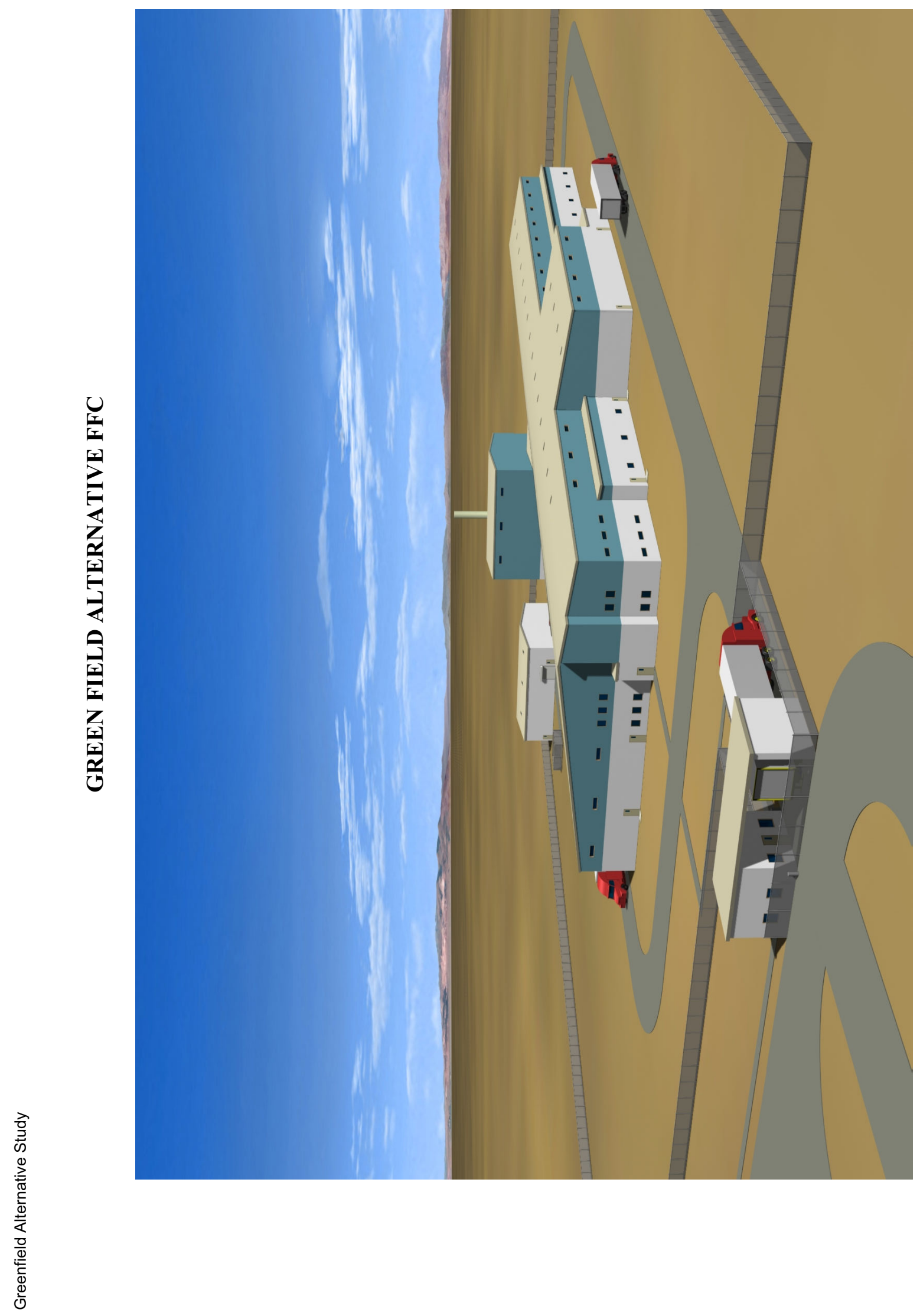


$\varangle$
$\frac{x}{0}$
$\frac{0}{0}$
$\frac{0}{0}$
$\frac{0}{2}$

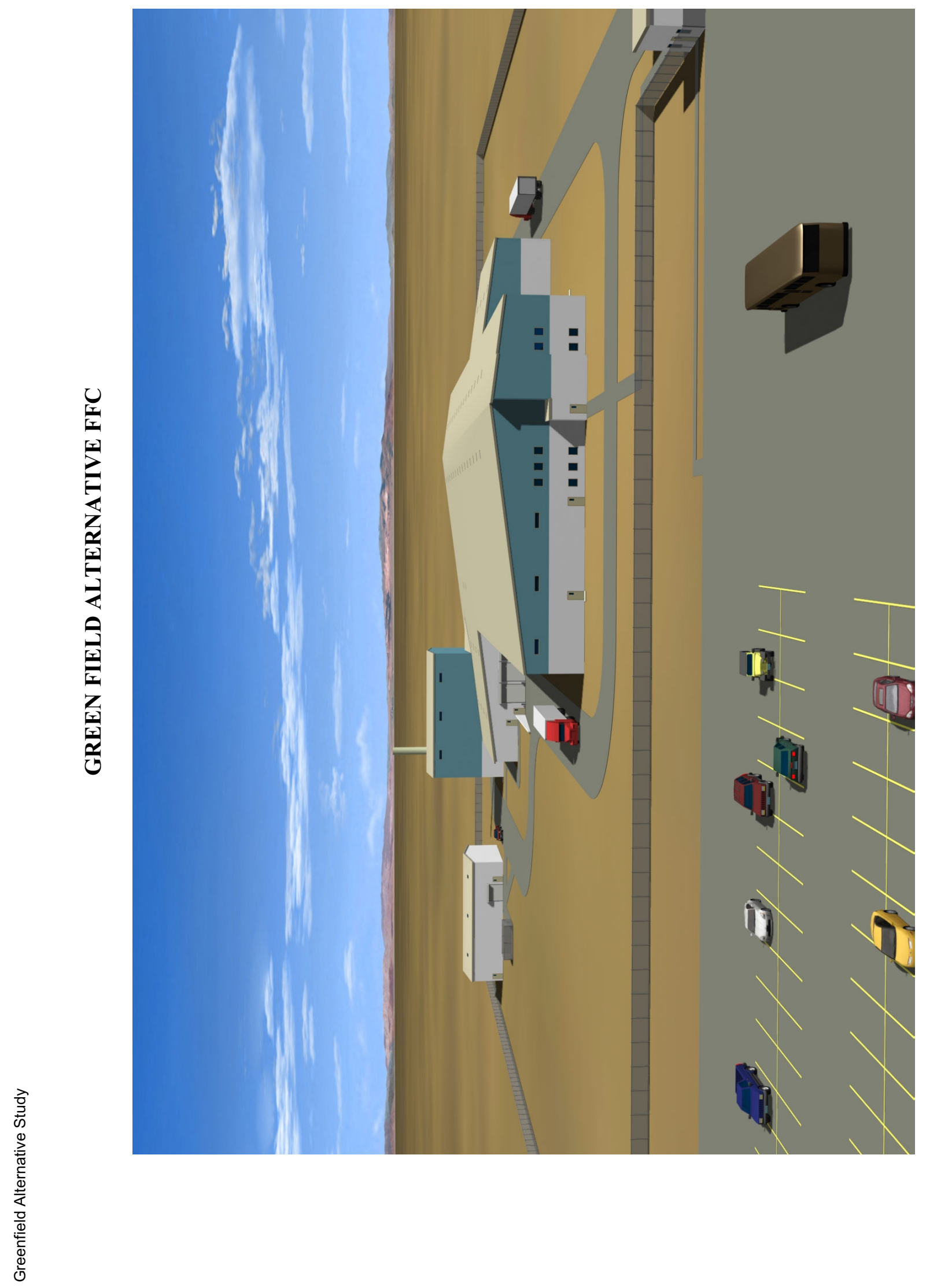


FACILITY LAYOUT 

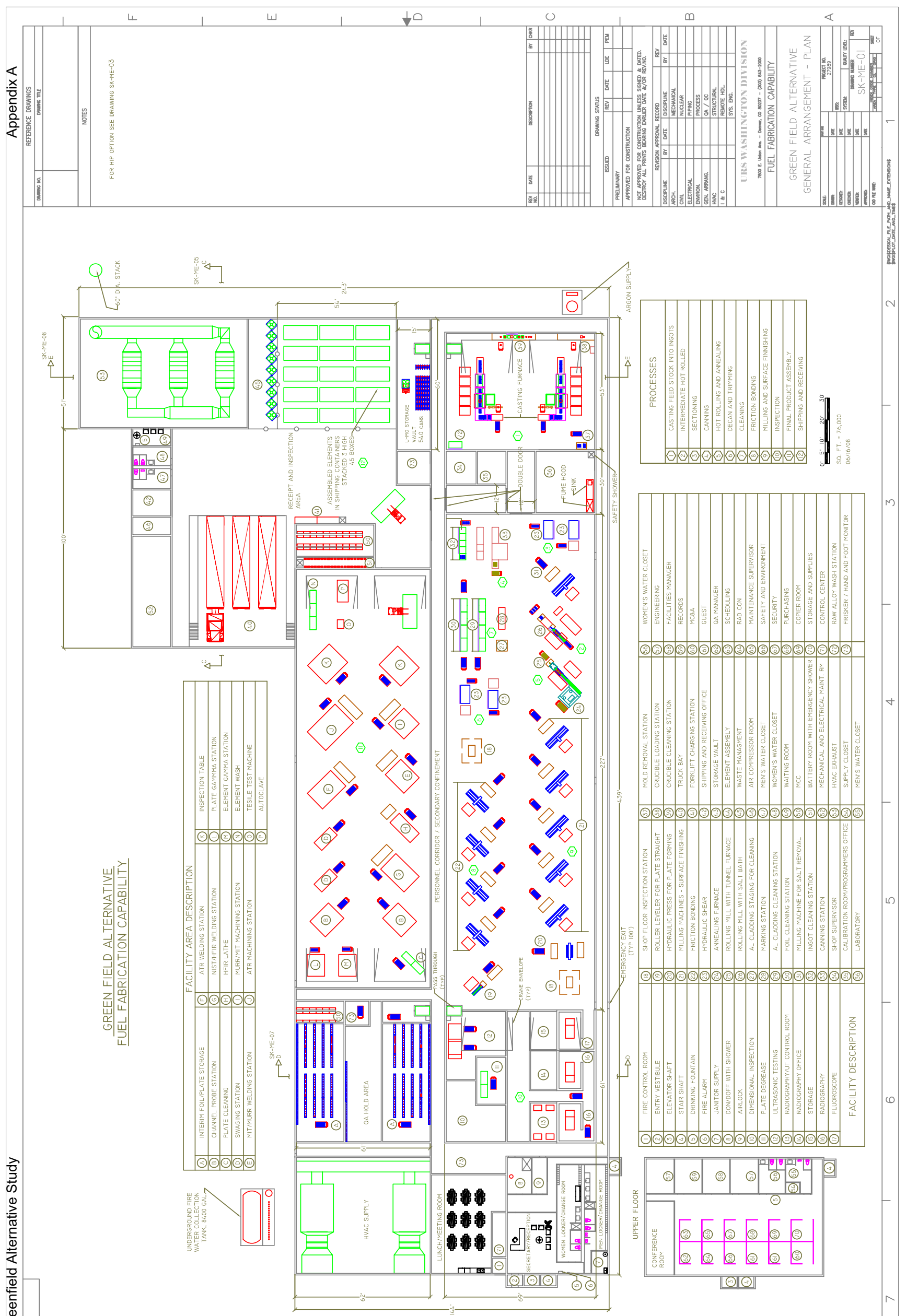

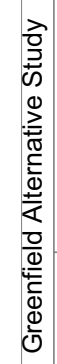



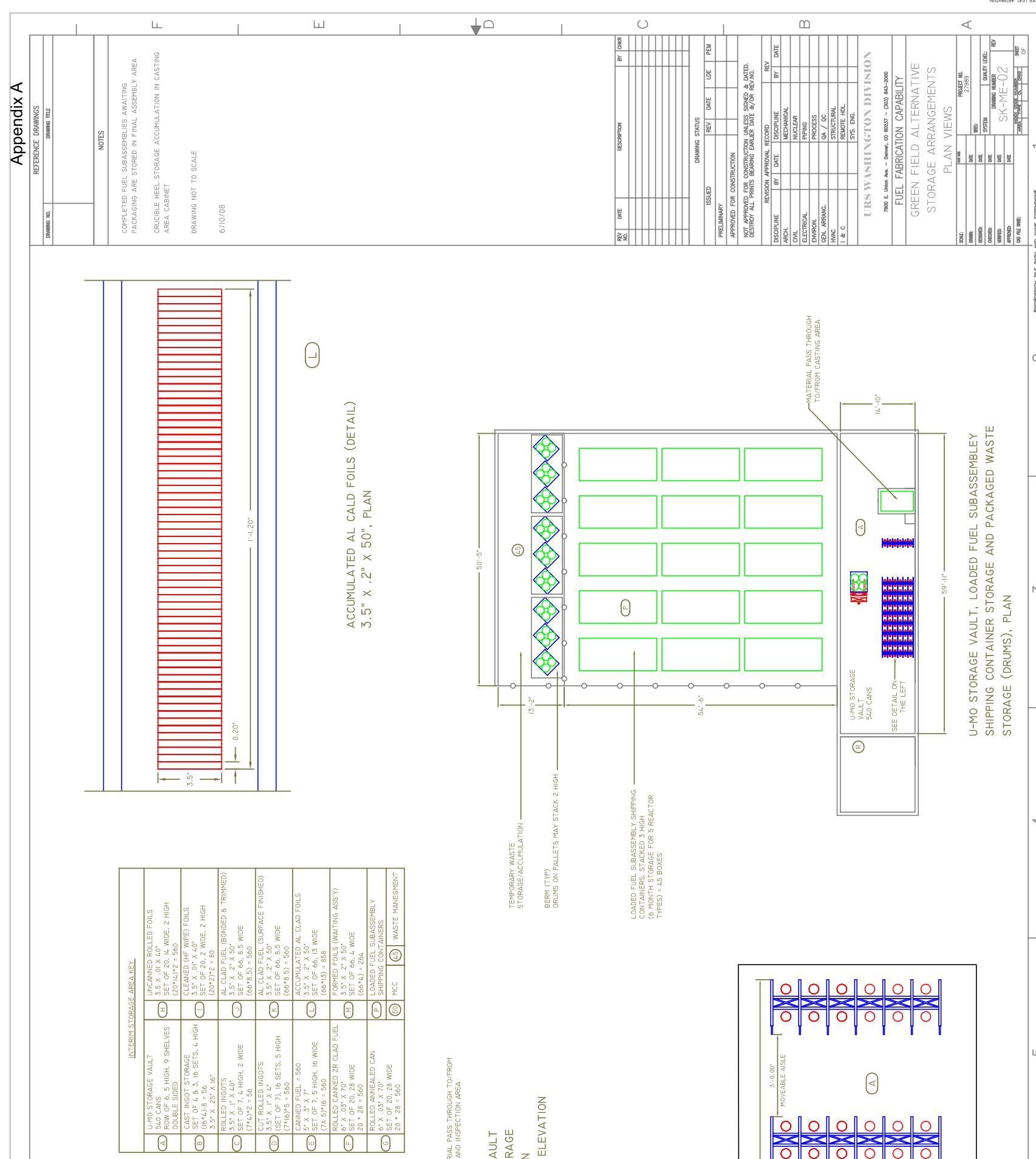

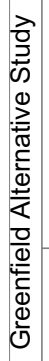
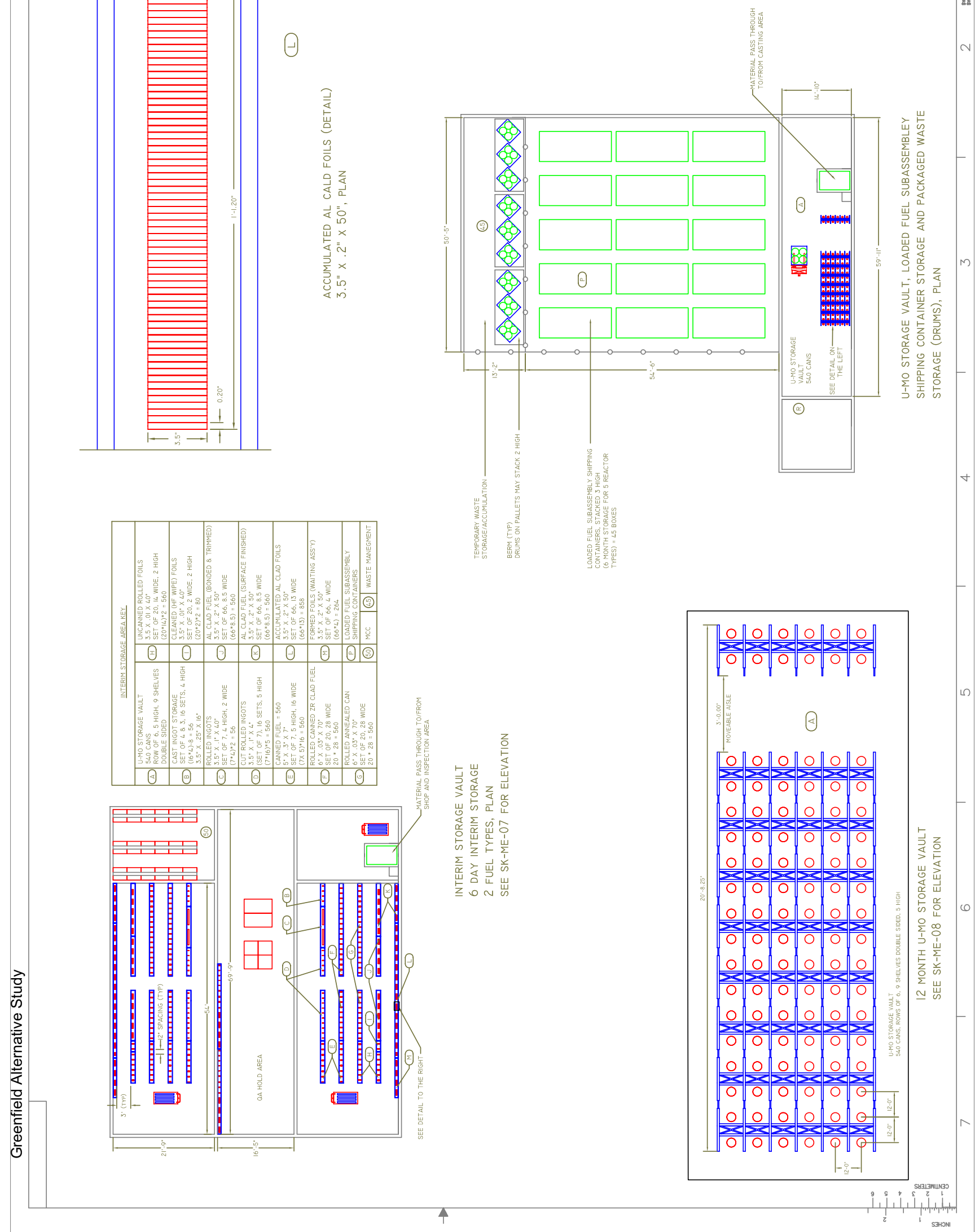

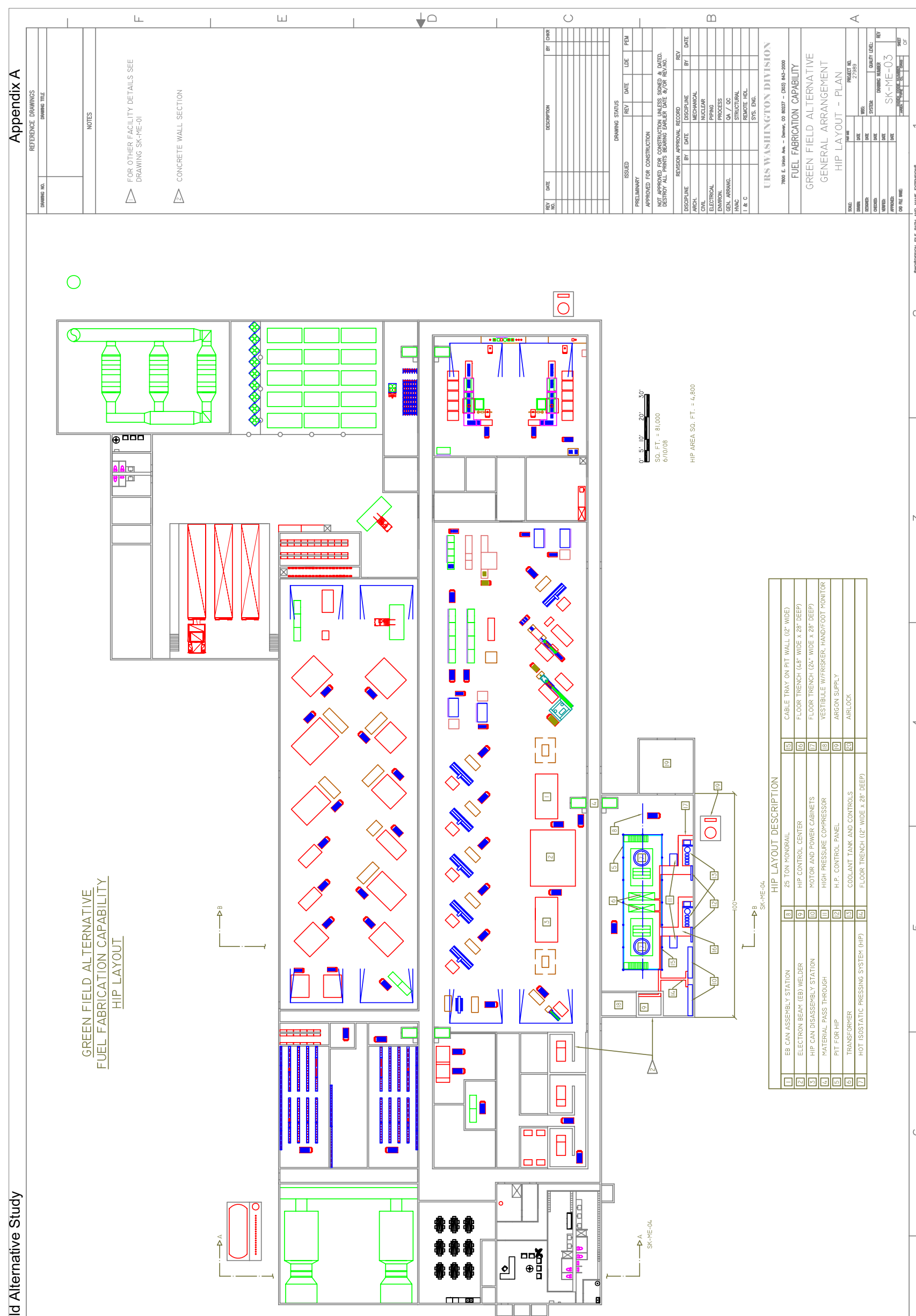


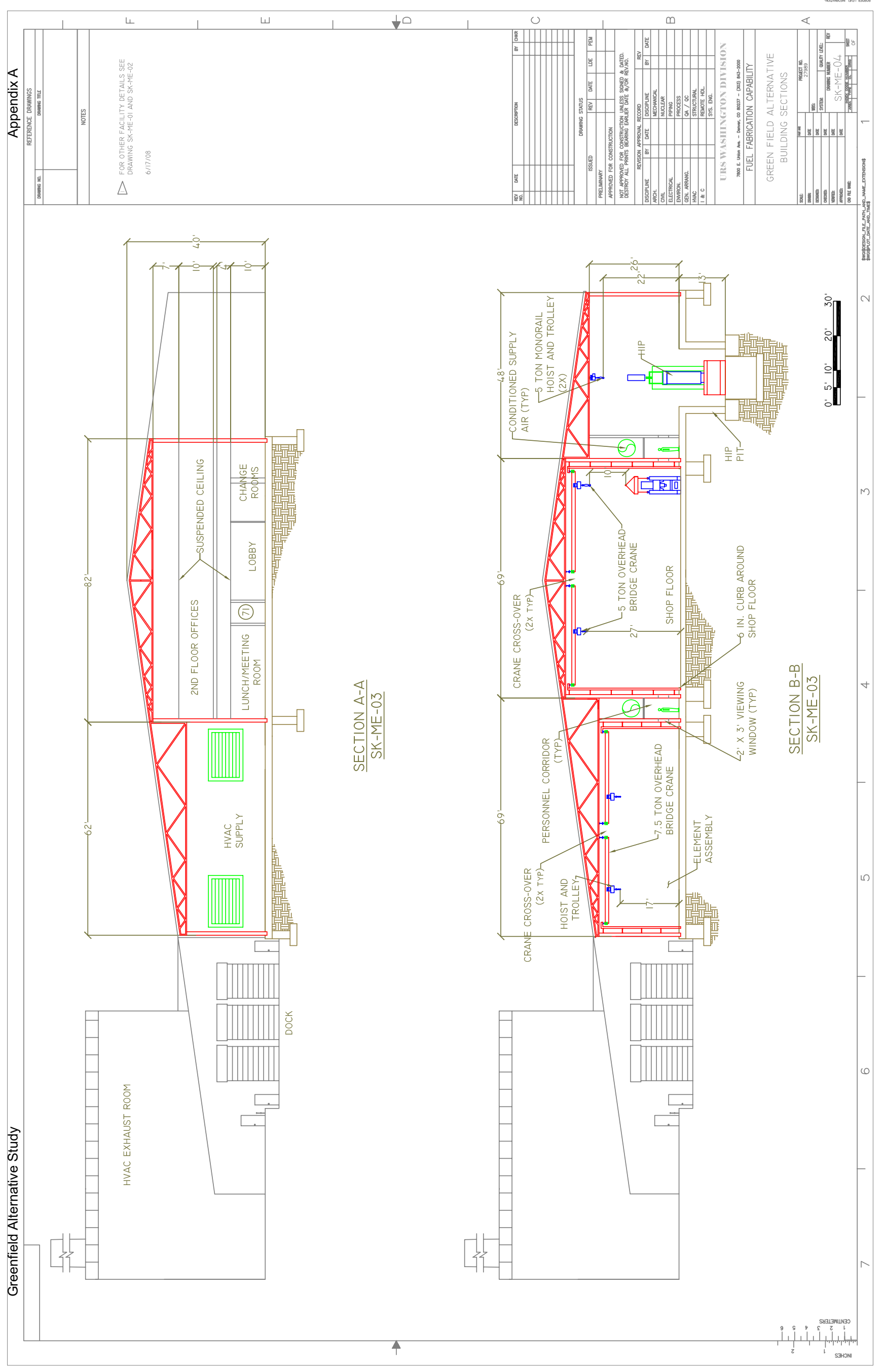




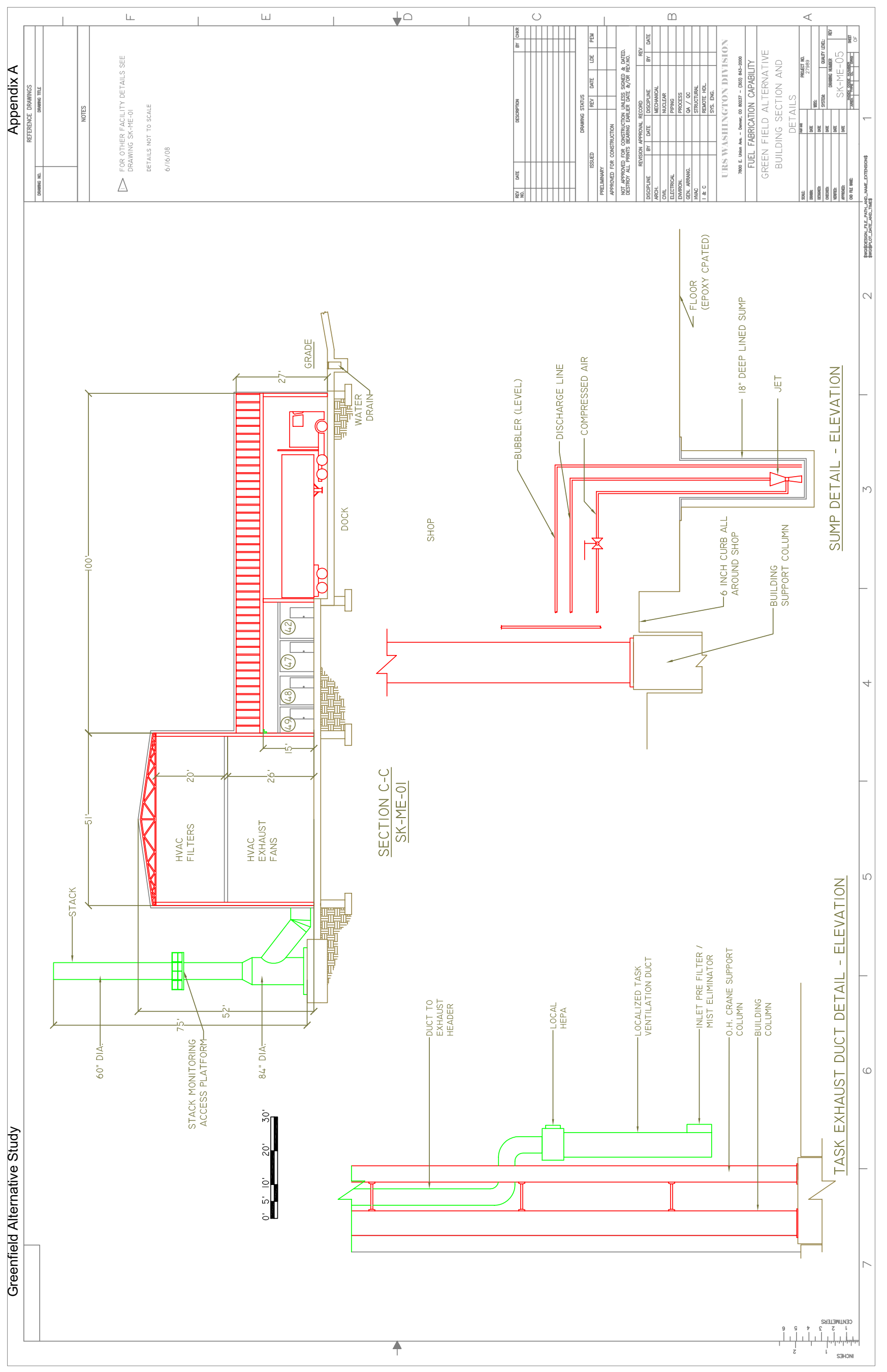



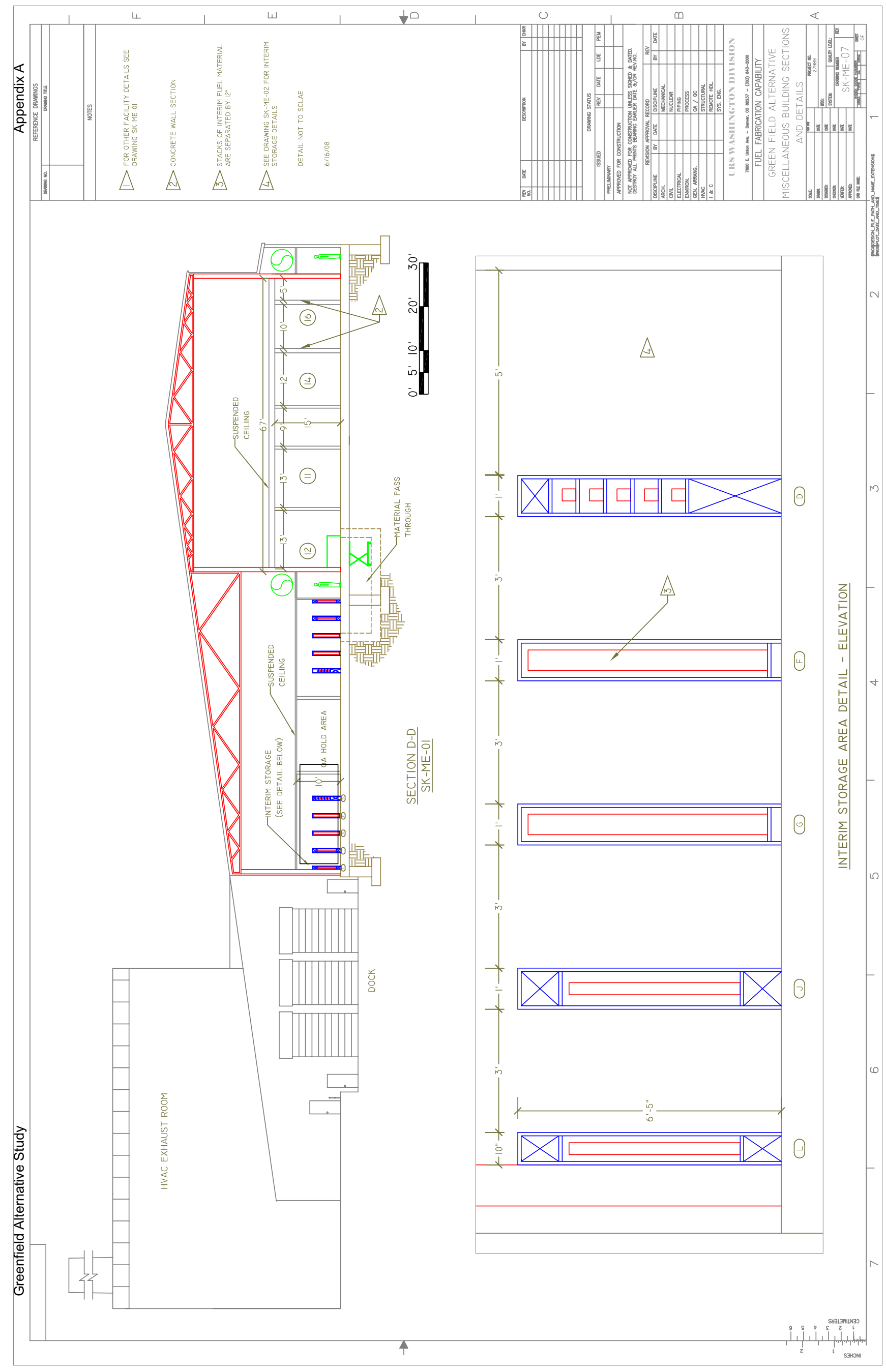

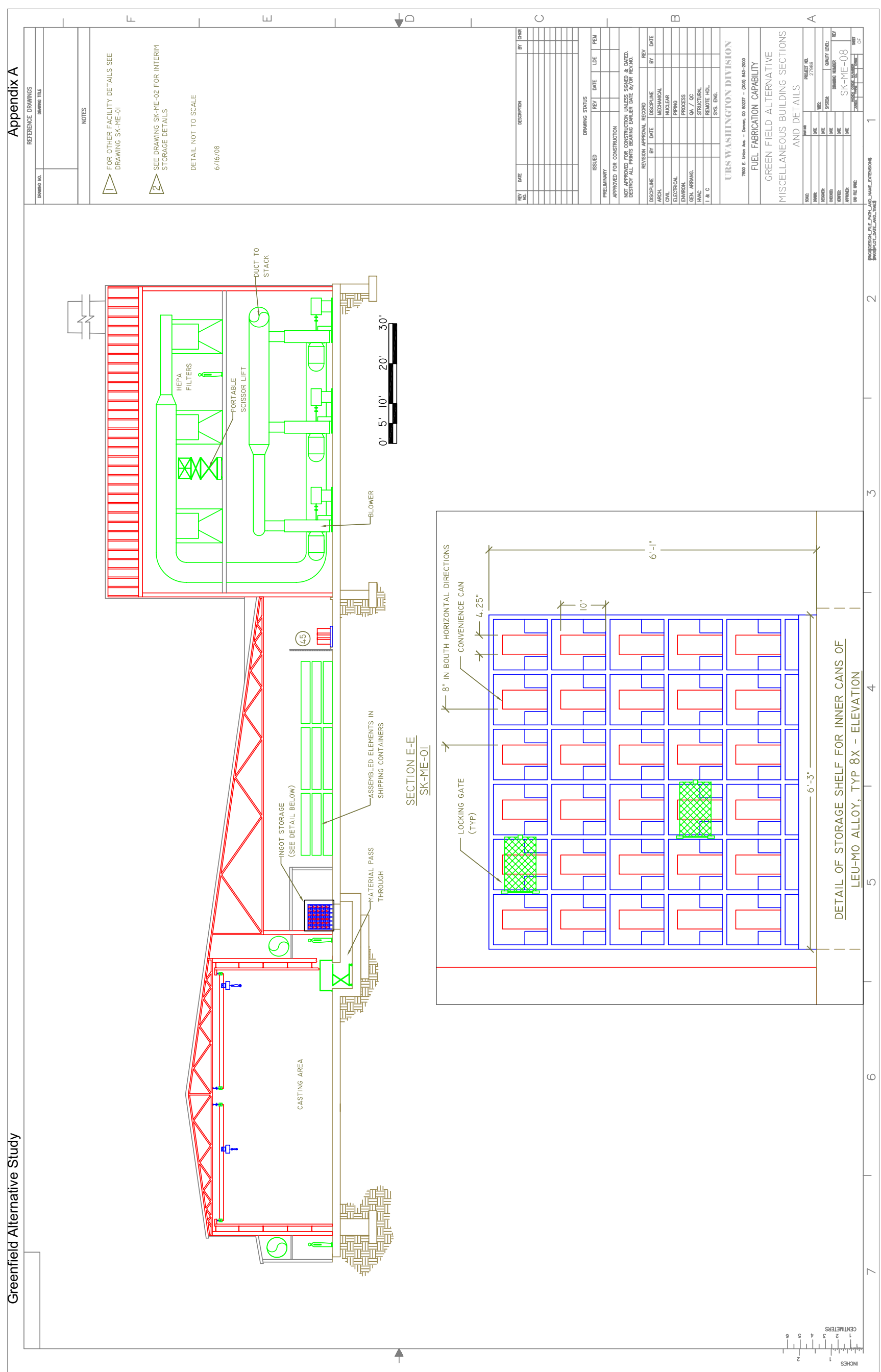


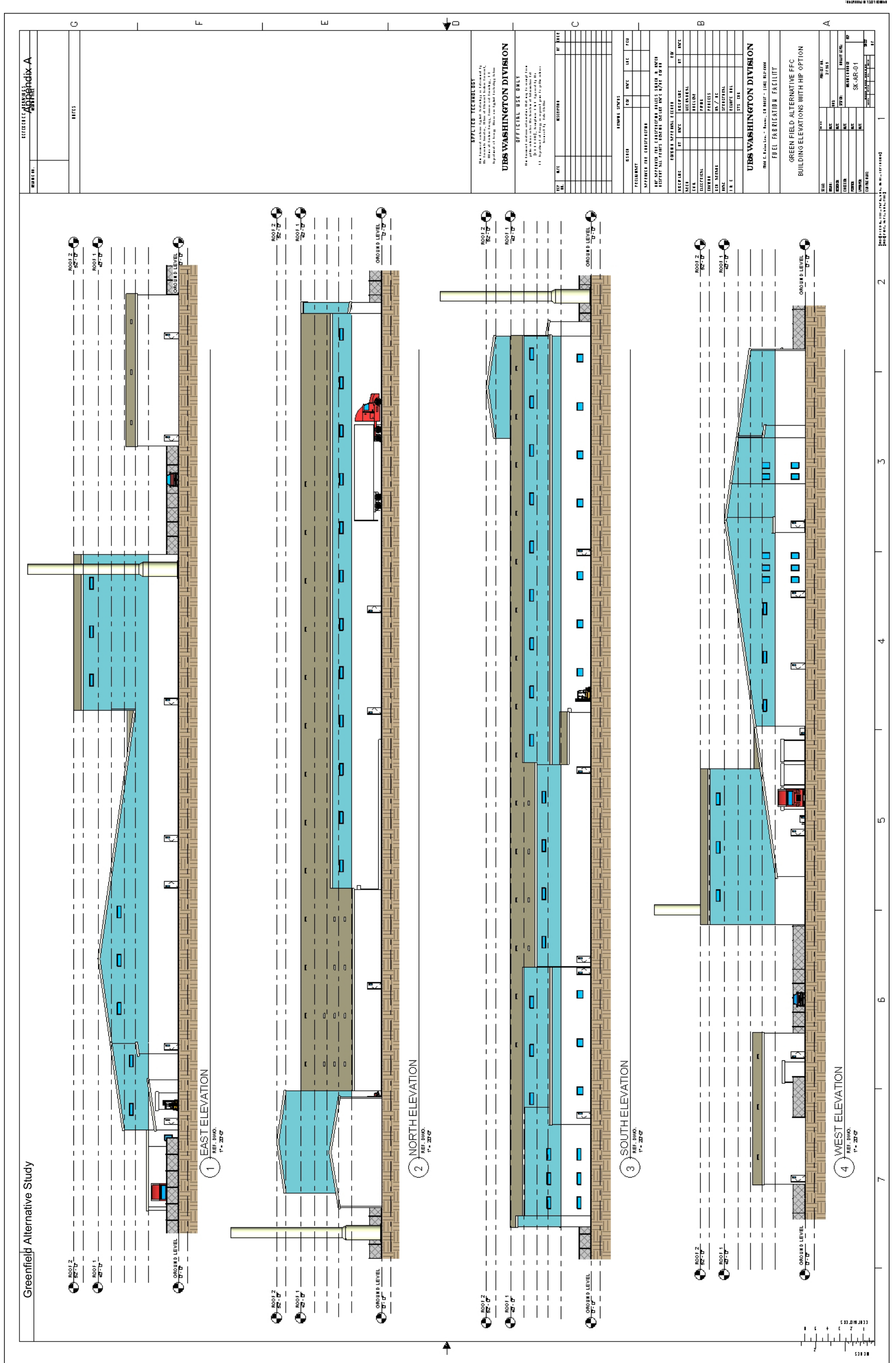




\section{BLOCK FLOW DIAGRAMS}




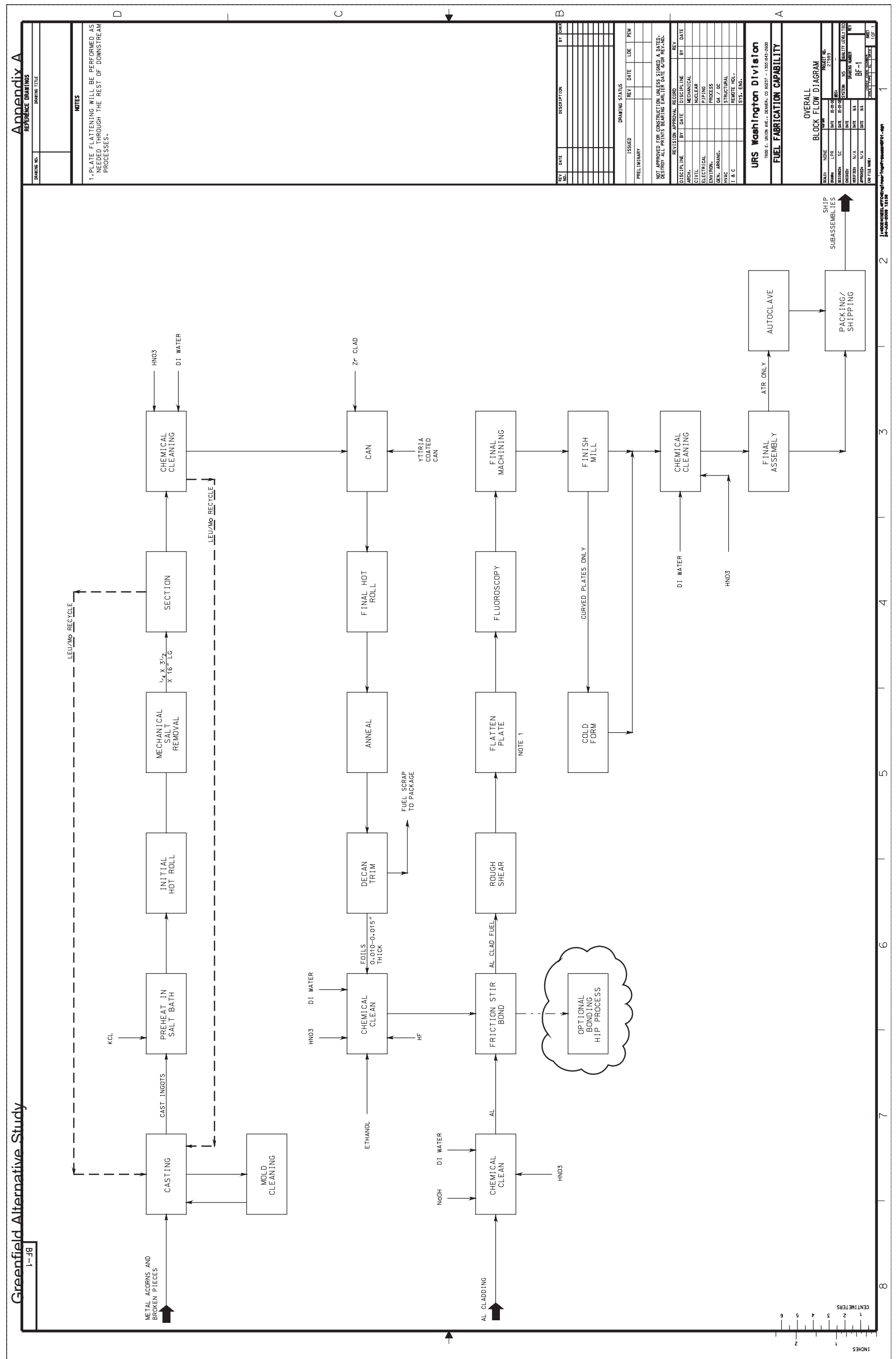


PROCESS FLOW DIAGRAMS 


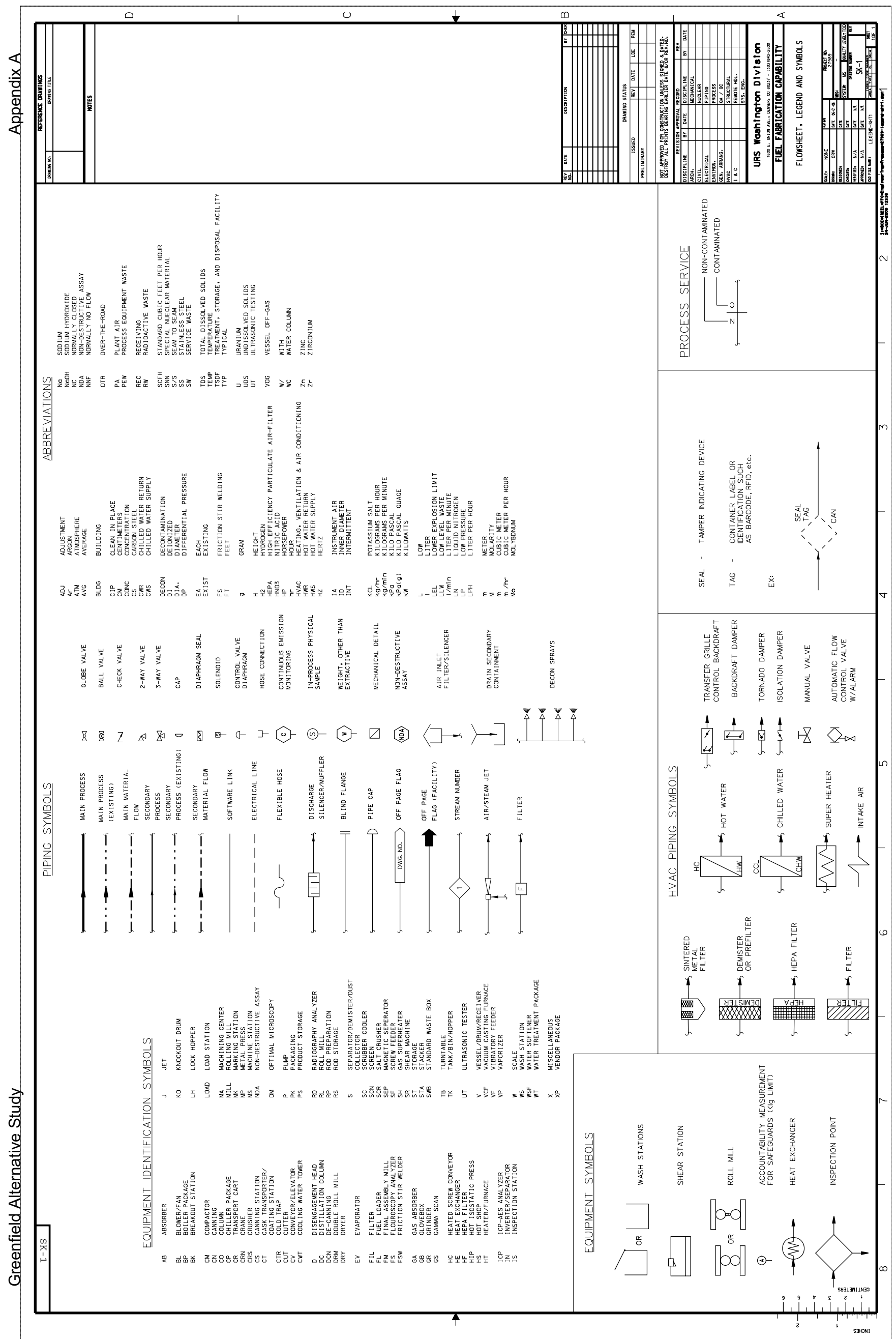




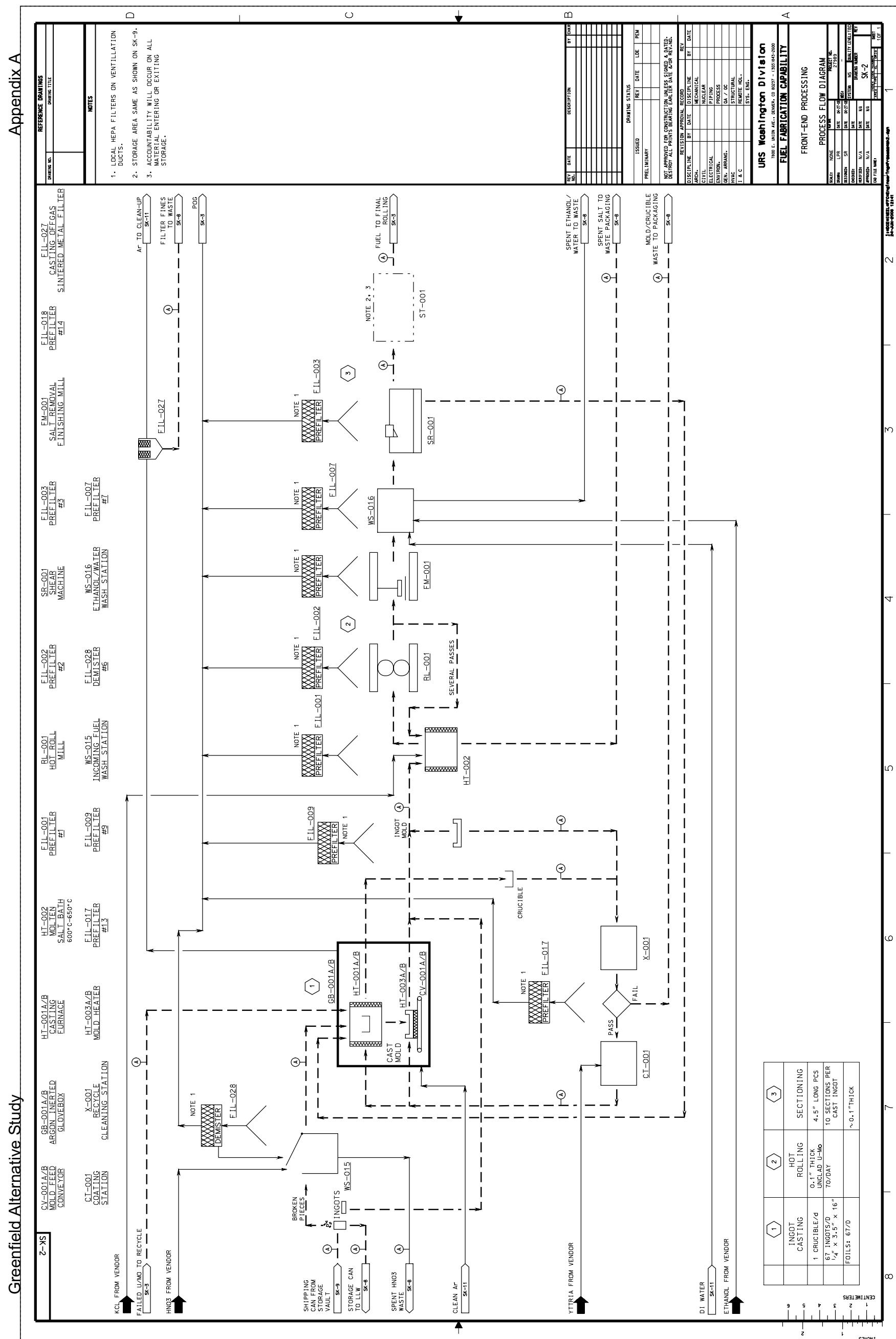




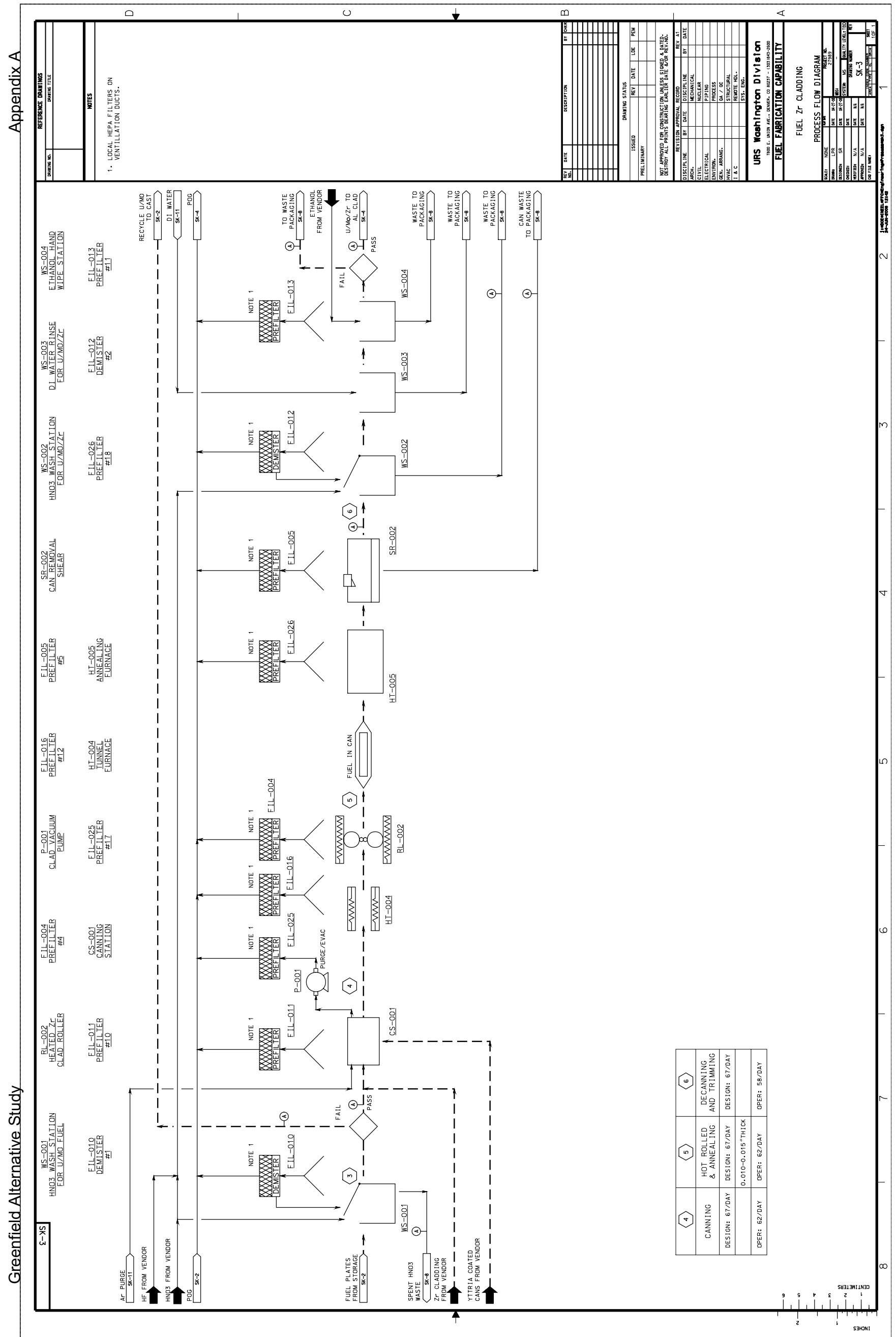




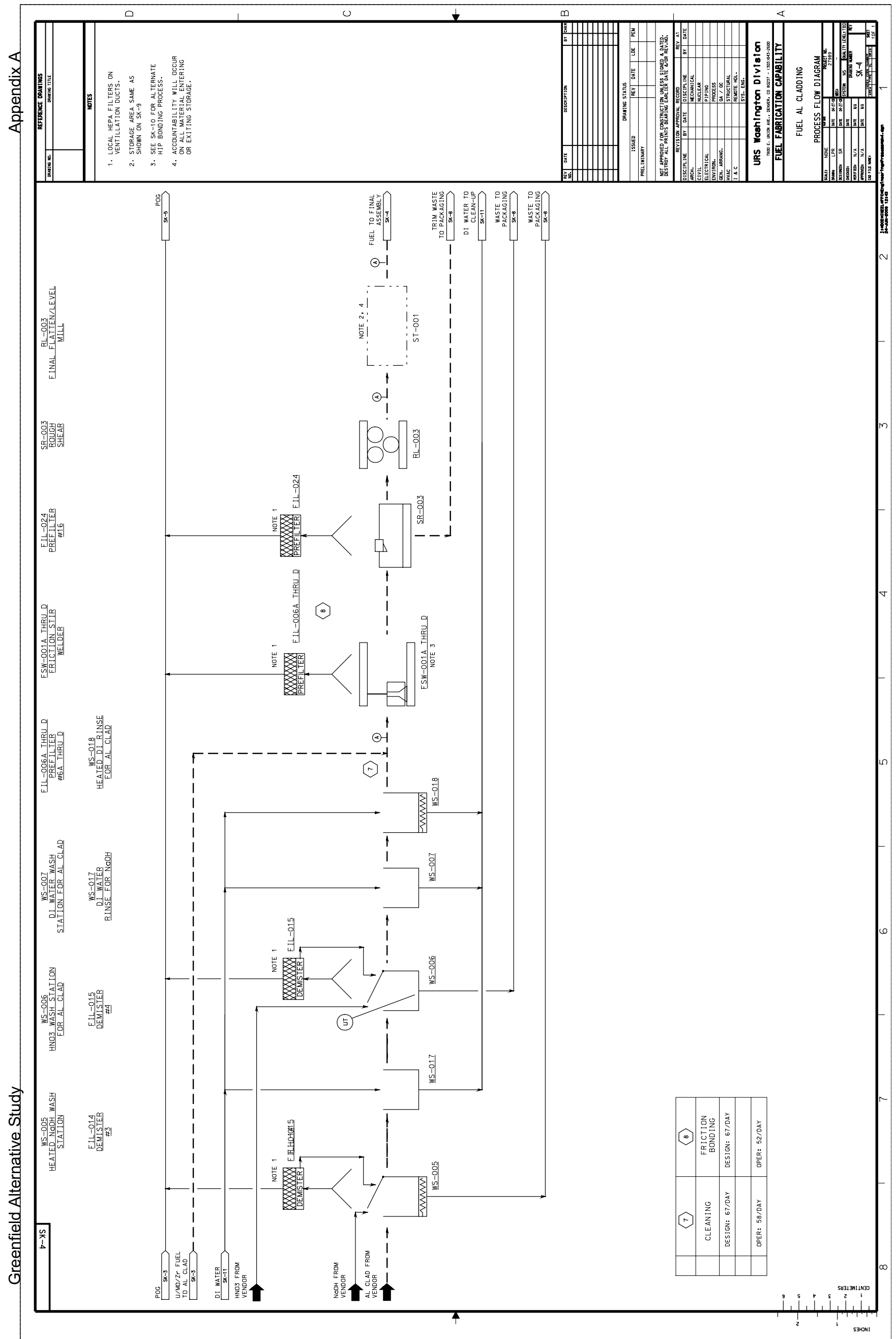




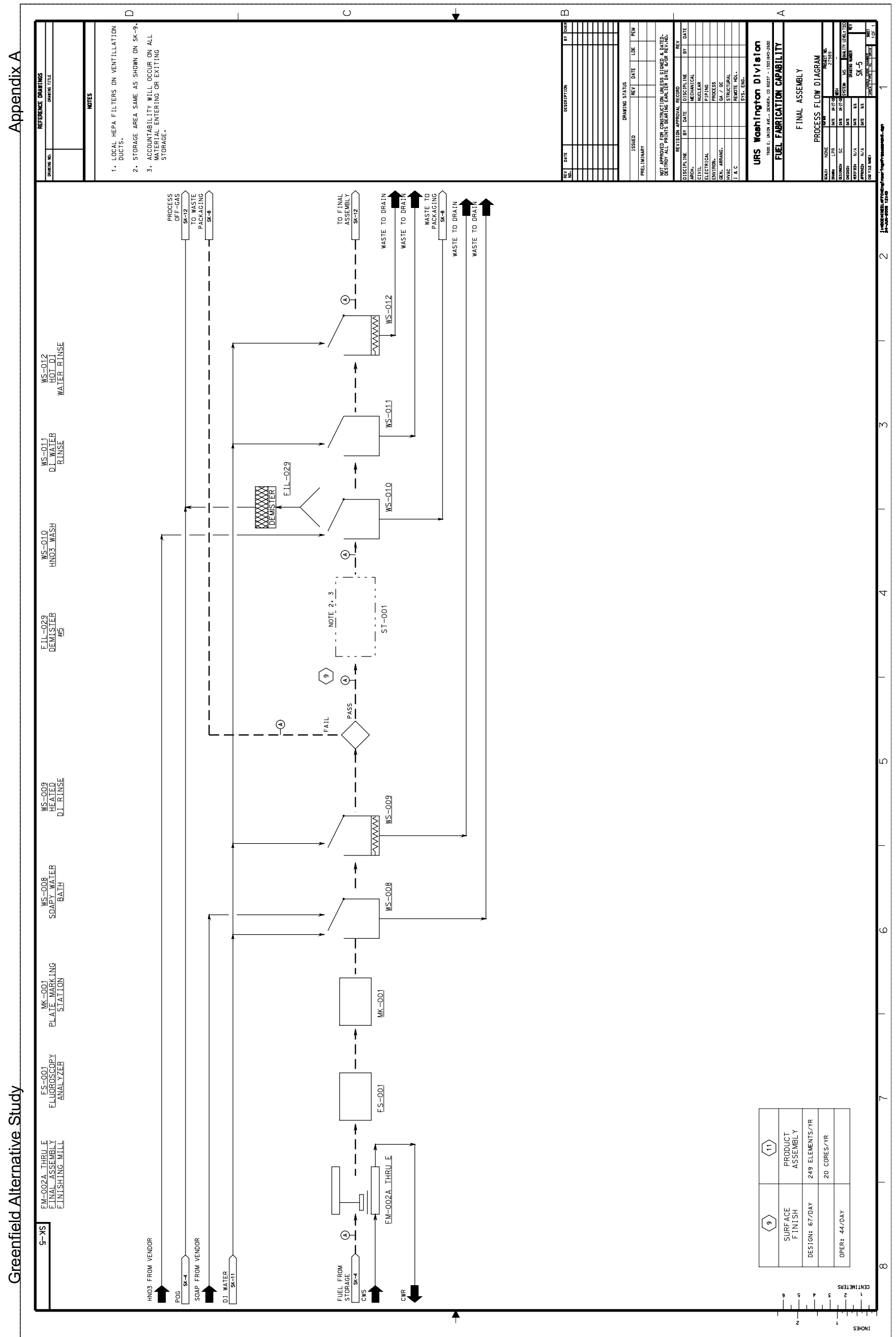




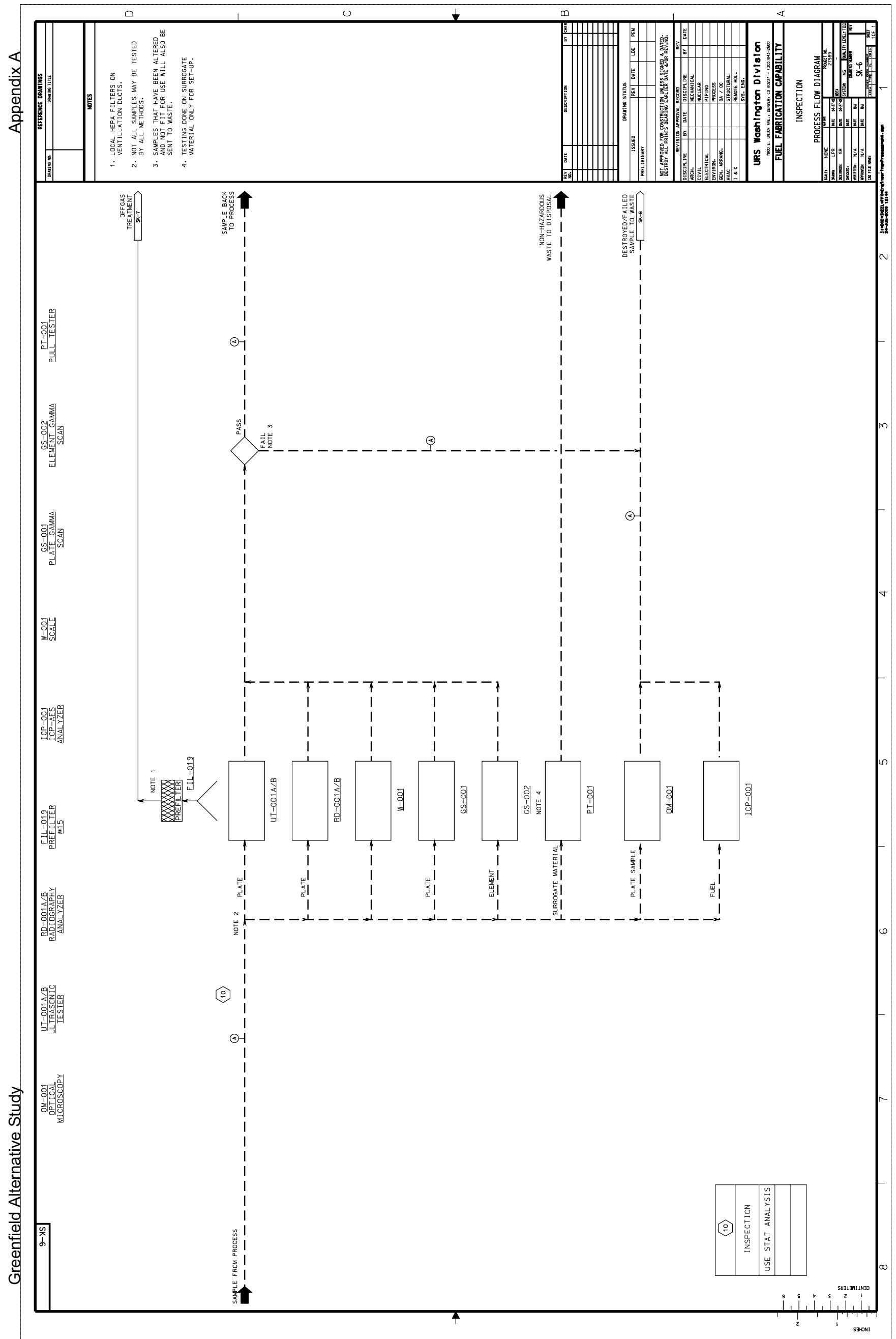




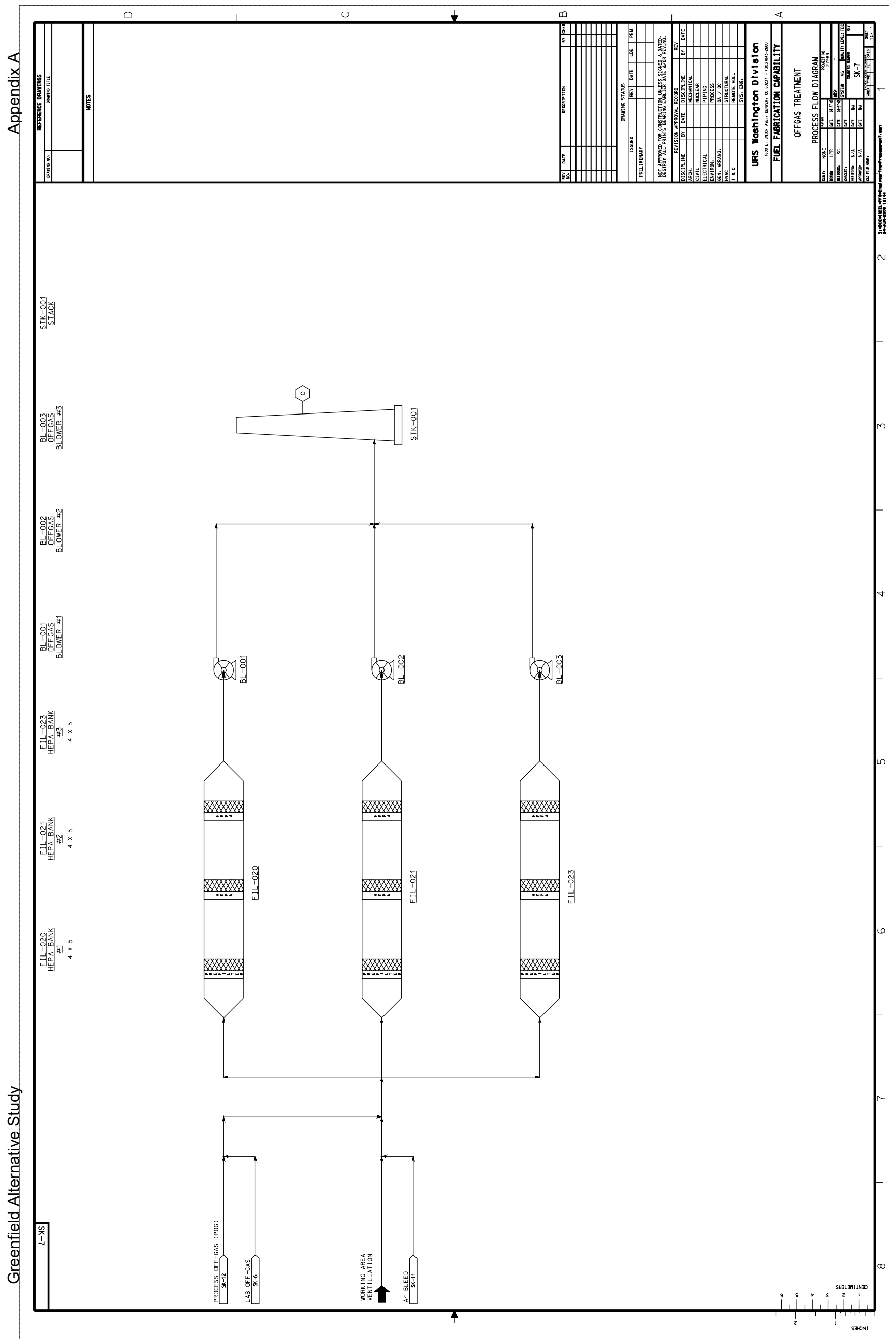




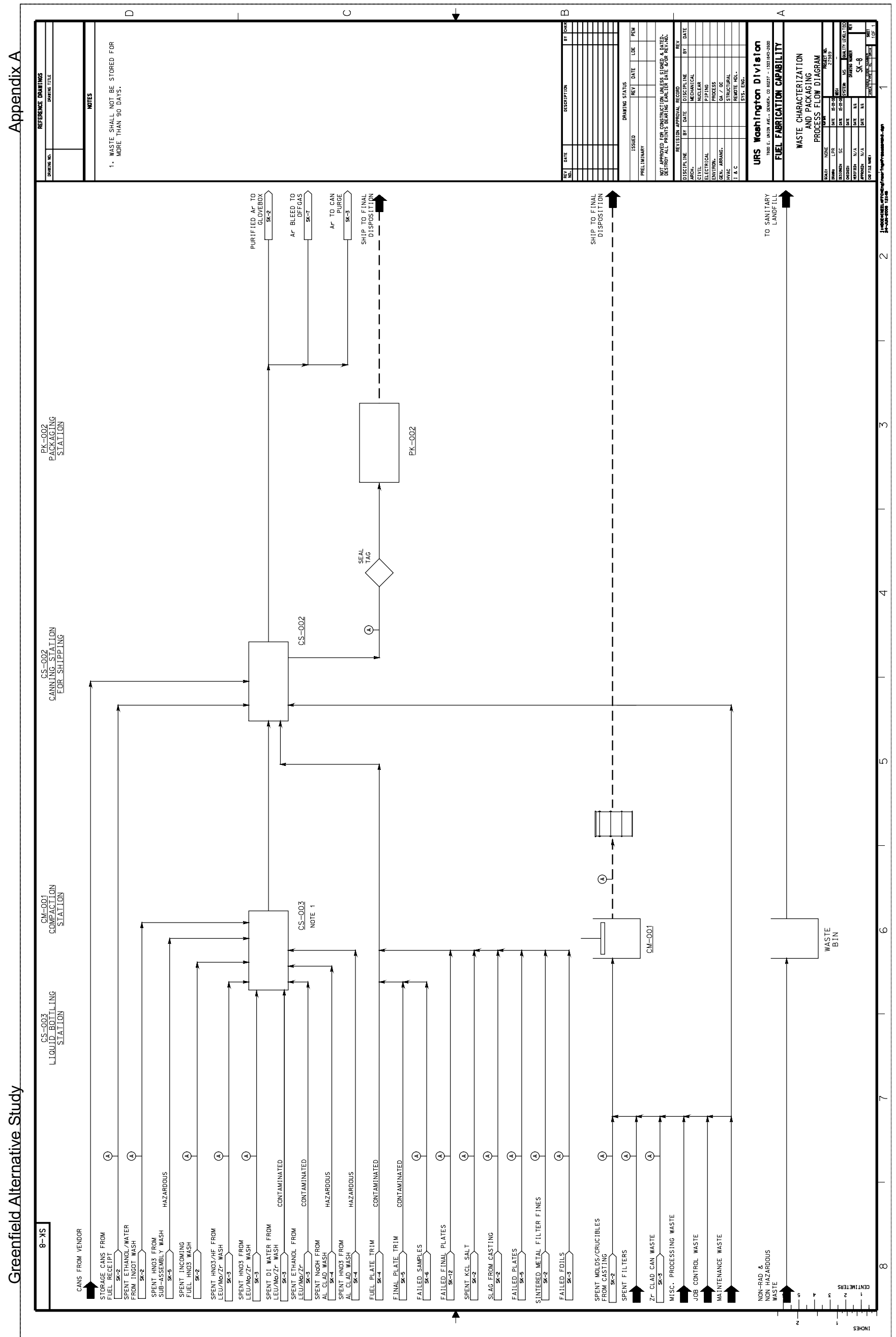




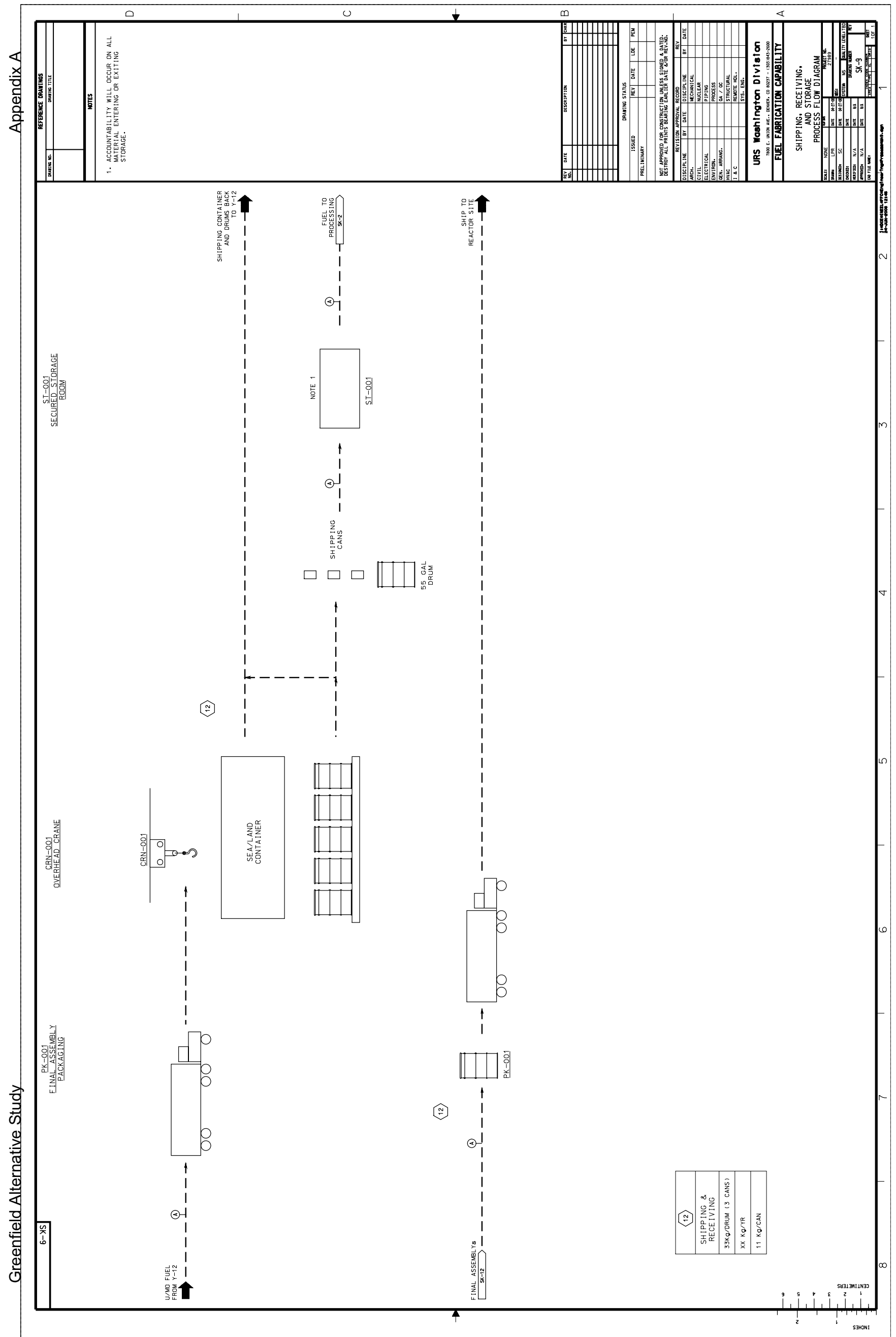




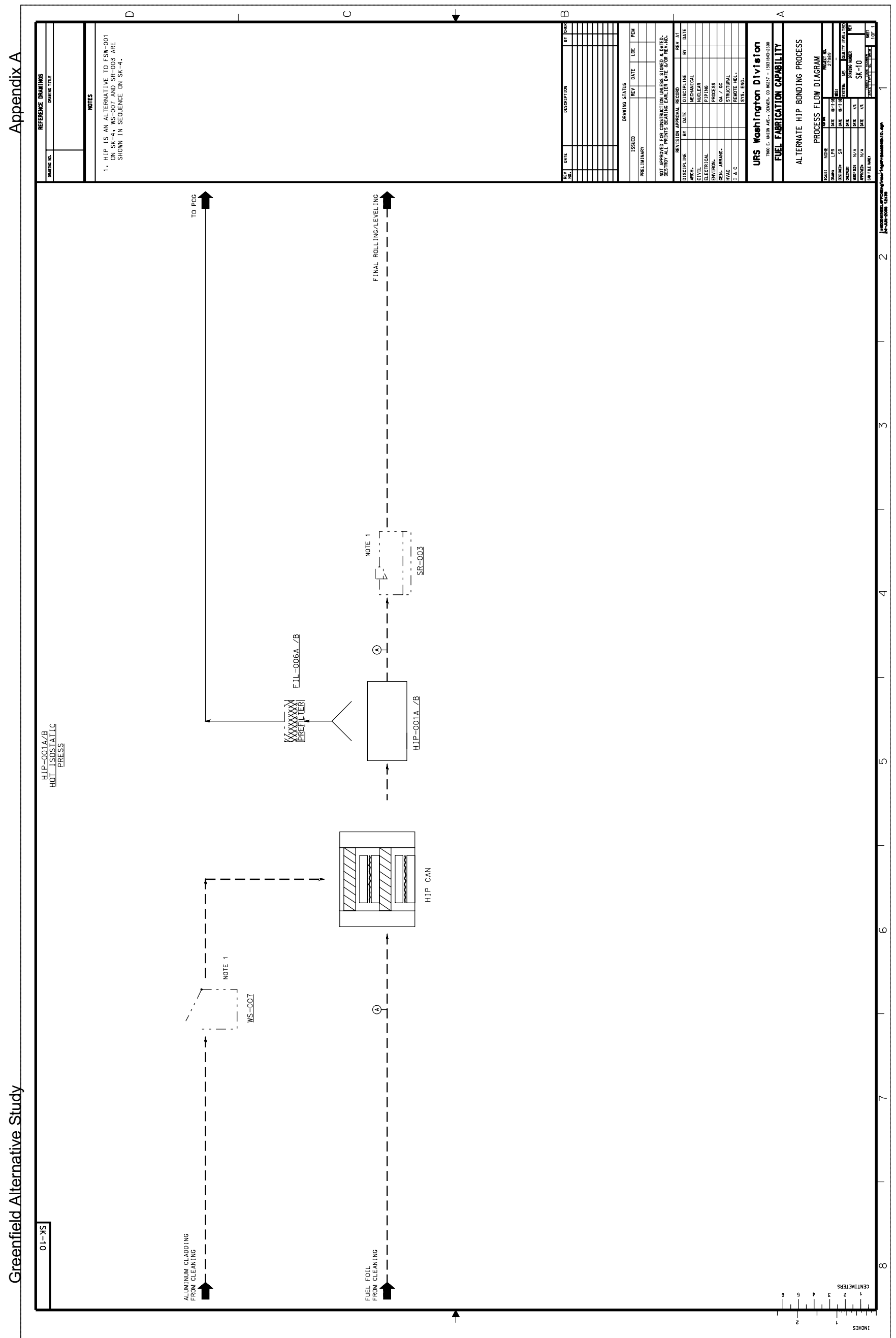




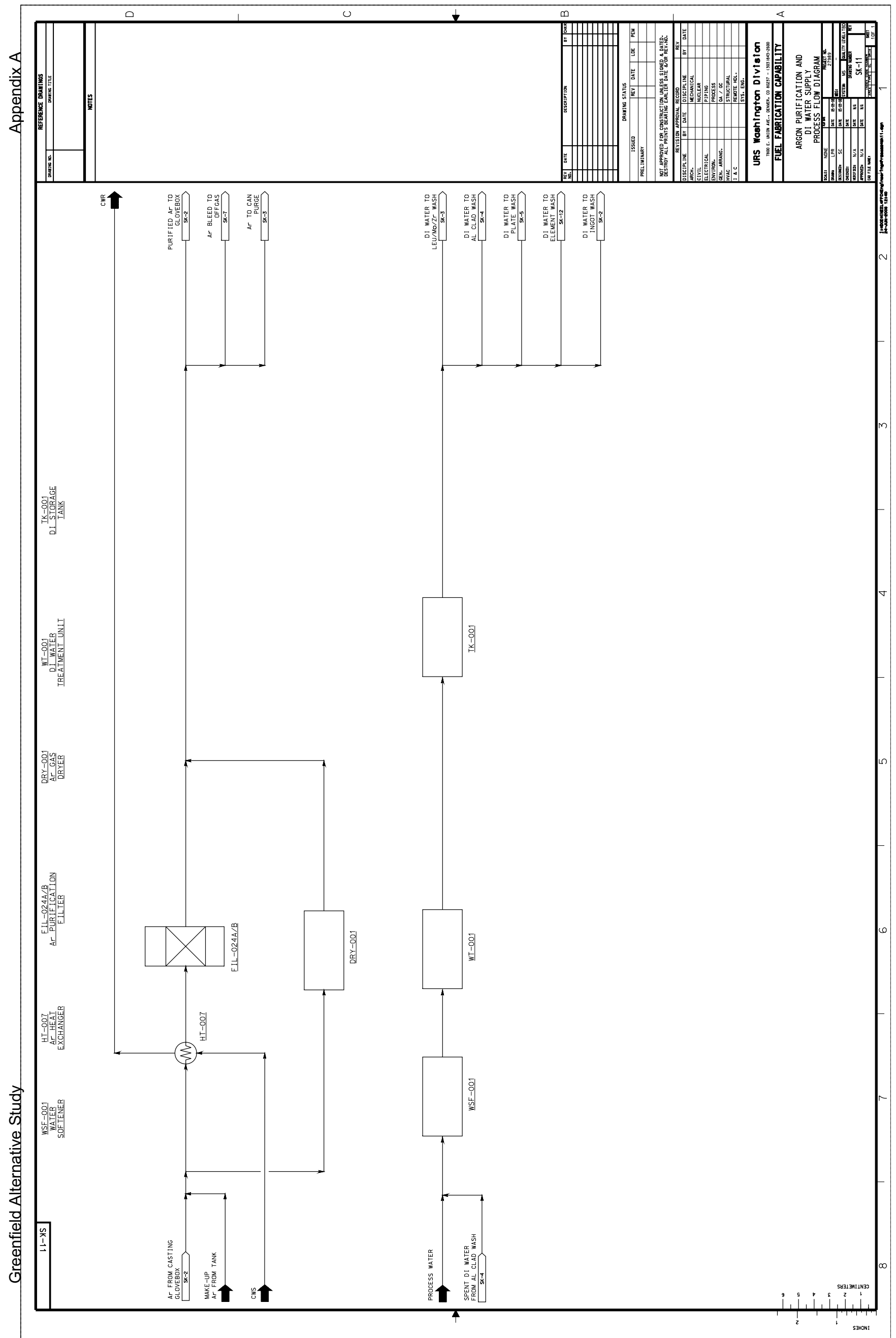




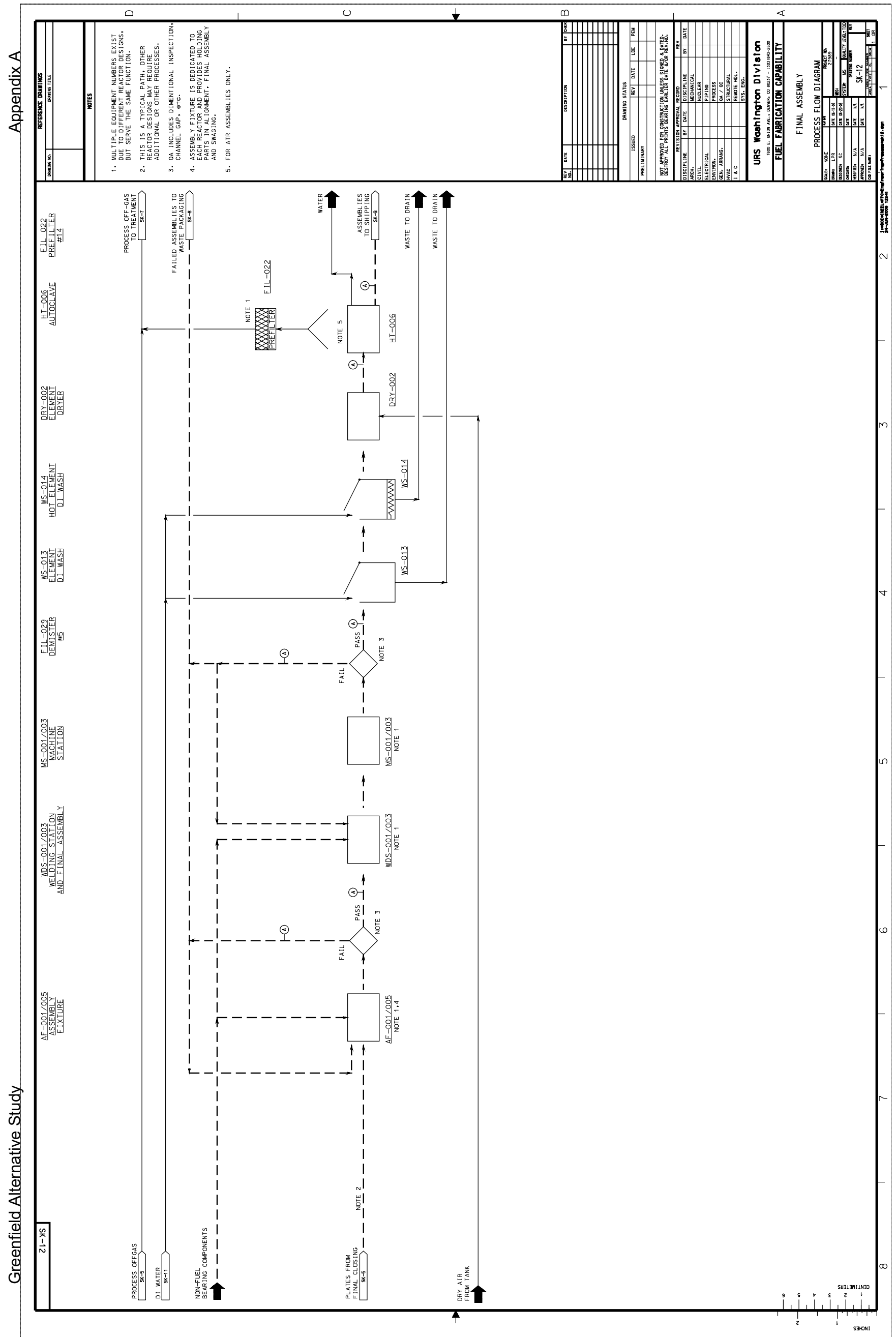




\section{EQUIPMENT CUT SHEETS}




\section{CS - 800 Double Dual Range}

Carbon / Sulfur Determinator

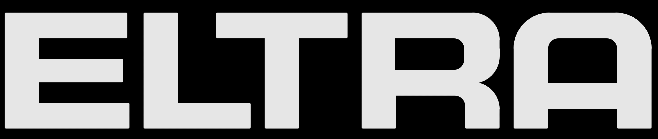

Analysers made in Germany

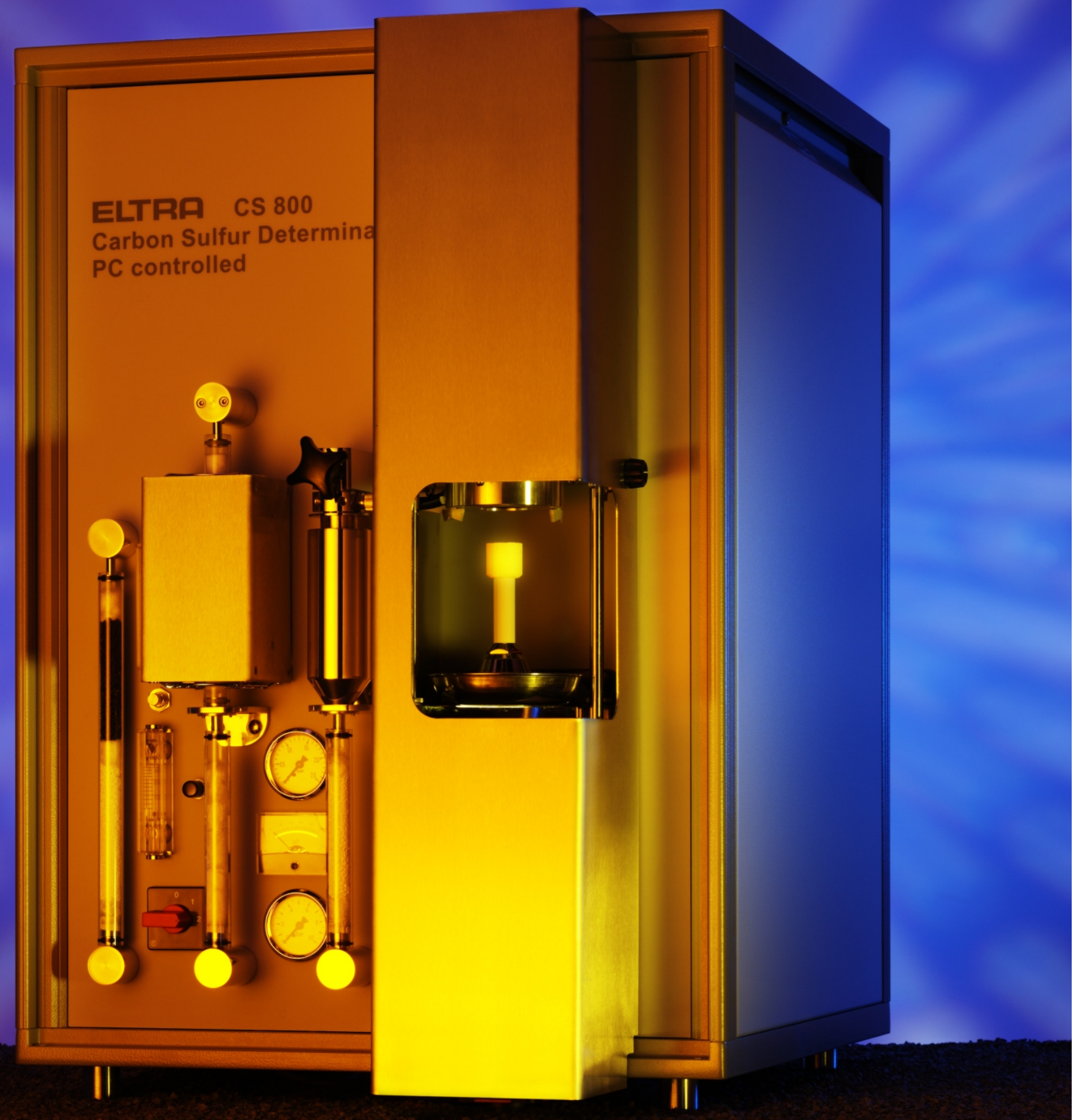

- Four solid state infrared cells

No halogen trap required

- Automatic furnace cleaning

Power controlled induction furnace

- PC controlled

Up to 20 grams of copper samples without accelerators

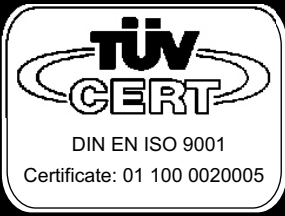




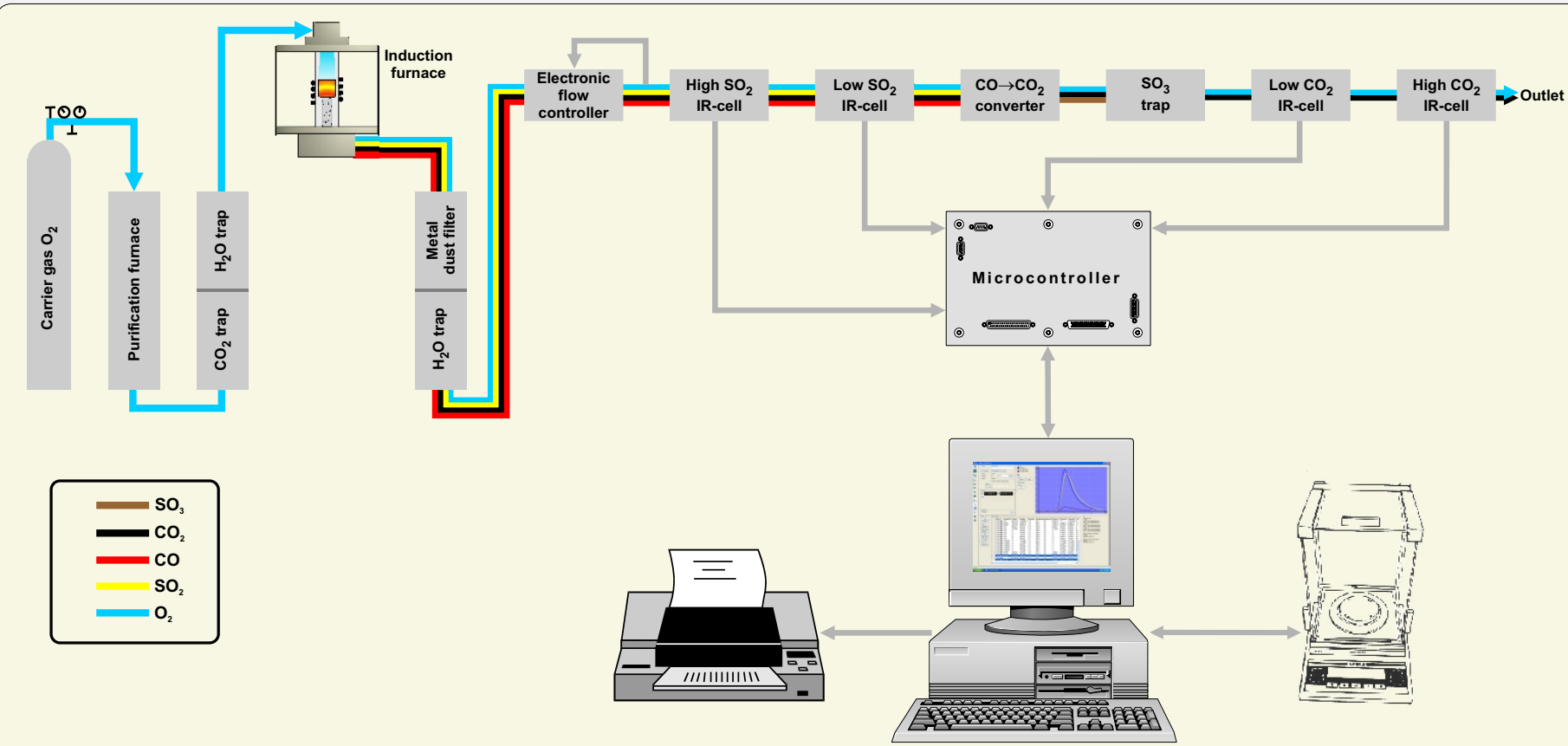

\section{System overview}

The CS-800 incorporates the latest in combustion technology. It is designed for the rapid, simultaneous determination of carbon and sulfur in steel, cast iron, copper, alloys, ores, cement, ceramics, carbides, minerals, sand, glass, etc.

The CS-800 can be supplied with up to four independent infrared cells. The respective cells with the appropriate path length and sensitivity can be individually selected to offer optimum precision for the analysis of high and low levels of both sulfur and carbon. The CS-800 features a microcontroller, induction furnace and solid state infrared detectors with auto zero and auto range control.

\section{Operating technique}

Samples are accurately weighed in the ceramic crucible on the interfaced electronic balance. By pressing a key, the sample weight is automatically transferred to the PC. If required, sample weights can also be entered manually. By pressing the start key the analysis cycle begins and the sample enters the furnace. Depending on sample weight and material analysed, the typical analysis time is 50 seconds. During the analysis cycle instructions are displayed to ensure easy and reliable performance. The CS-800 requires minimal maintenance for which access is simple.

\section{PC control with Windows 2000/XP software}

Comprehensive analyser control and easy operation are provided by the PC and software connected to the computer.

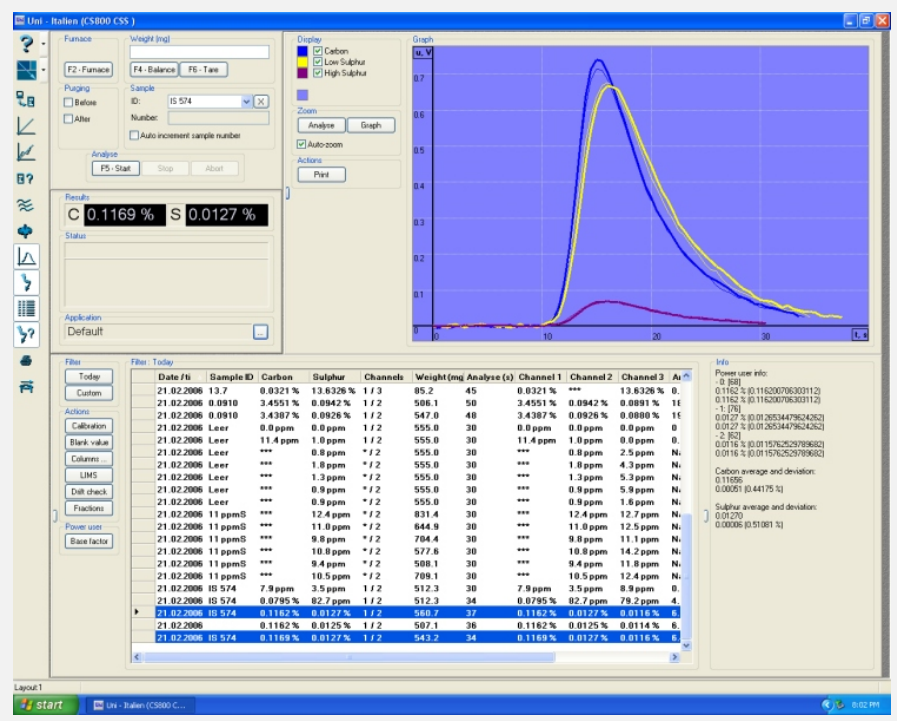

The multilingual software provides the user with the following features:

- Optional display layout - adjustable screen appearance of the program windows.

- User profiles with multi-level access - parameter changes protected by unauthorized acces.
- Sample ID memory - supplemented with running analysis number.

- Data base (analysis results storage) - all data for each analysis is stored and can be recalled later for review, report creation, statistical calculations or results recalculation with modified parameters.

- Optional data base configuration - displays only results meeting specified conditions, for example, certain date/time period, specific sample I.D. etc.

- Visualisation of the results consistency.

- Peak separation calculation procedure for fractional analysis.

- LIMS communication and data export (Notepad, Excel etc.).

- Basic one-point and advanced multi-point calibration.

- Barometric pressure compensation.

- Simultaneous calibration of more than one measuring range.

- Procedure for automatic linearity correction calculation.

- Applications memory and deficiency checks - adjustable analysis counters to prompt the changing of reagents, cleaning of filters and other maintenance procedures.

- Hardware diagnostics display and technical report printouts.

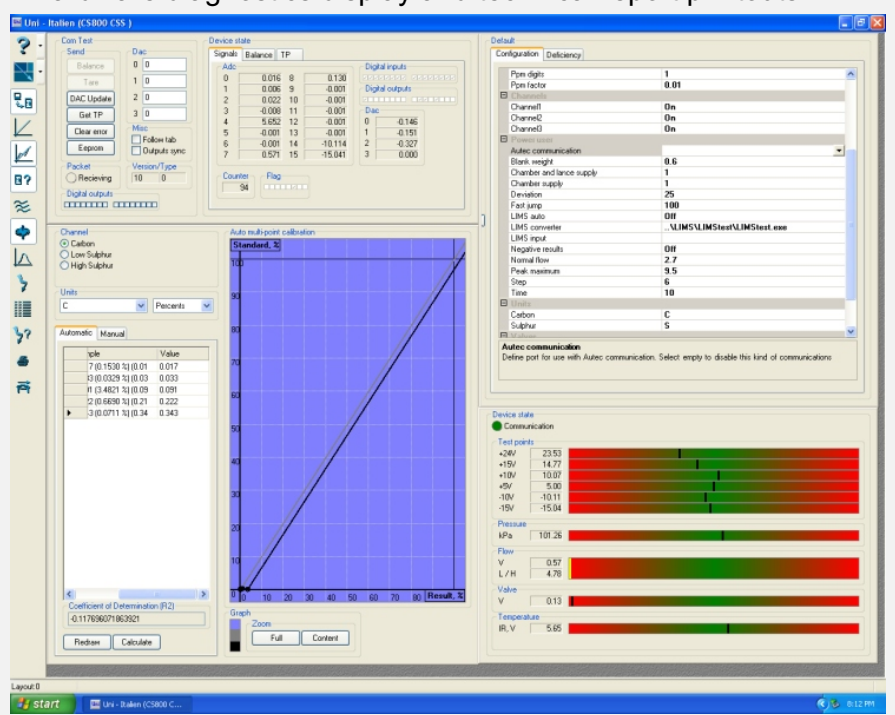




\section{Infrared cells}

The infrared cells of the CS-800 do not require any manual zero adjustments. The zero and sensitivity adjustments of the infrared cells are permanently and automatically controlled by the electronics. The detectors utilize solid state sensors combined with infrared filters. The sensors are not gas filled, thus eliminating long term problems due to gas leakage. The CS-800 can be equipped with up to four independent infrared cells.

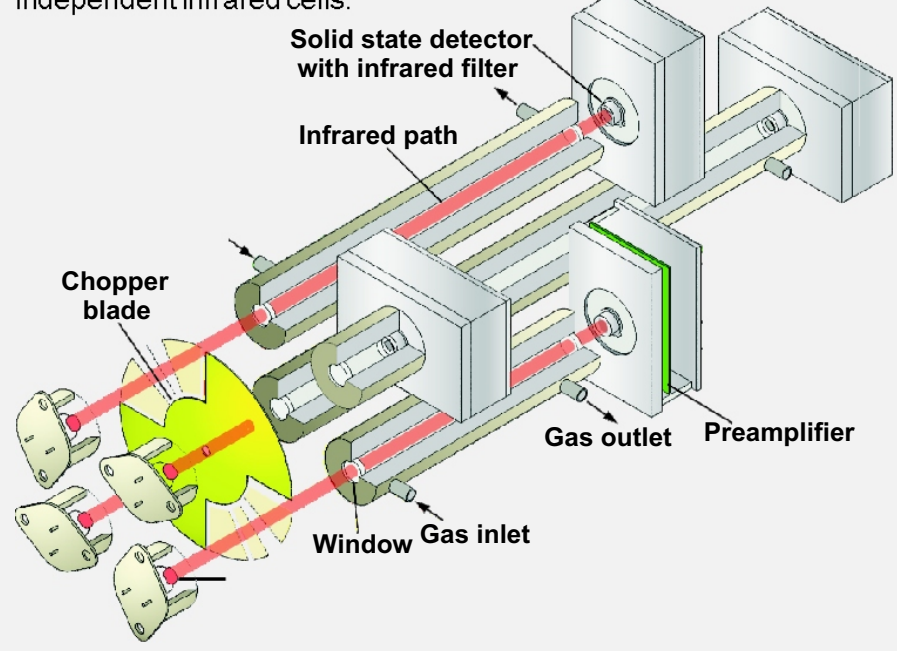

The lengths of all four cells can be individually optimized, to obtain maximum precision for the target analysis levels of each customer. Each of the cells can be installed with infrared absorption lengths ranging between $1 \mathrm{~mm}$ and $320 \mathrm{~mm}$.

\section{Preheating crucibles}

The ceramic crucibles for the induction furnace can be preheated in the high temperature furnace HTF540. The preheating reduces the blank value of the crucibles. This is important for analysis in the very low ppm range.

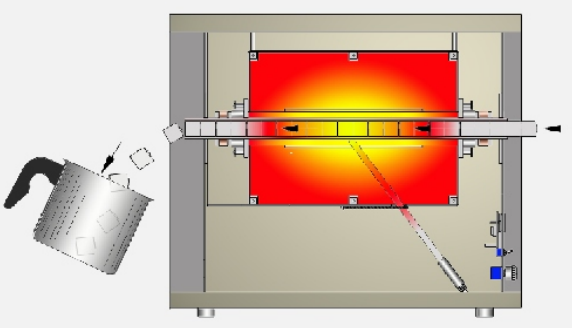

The crucibles are inserted into the furnace tube and they remain preheated in the tube until needed. Each time a crucible is needed, a new one is inserted into the tube, and a preheated crucible falls out the other end of the furnace tube. The recommended preheating temperature is between $1250^{\circ} \mathrm{C}$ and $1350^{\circ} \mathrm{C}$.

\section{Auto loader}

The CS-800 can be supplied with an automatic sample loading system. This loading system may also be retrofitted at a later date. Unlike many other auto loaders the ELTRA system can accommodate 130 samples giving hours of unattended operation. On request, the loader can be delivered for more crucibles. The auto loader, which does not occupy any additional bench space, is mounted above the area where the balance, PC, monitor and consumables are normally situated. The crucibles positions in the loader are easily accessible to the operator even from a sitting position. Three available models for 130, 104 and 36 crucibles. Dispensers for accelerators are available.

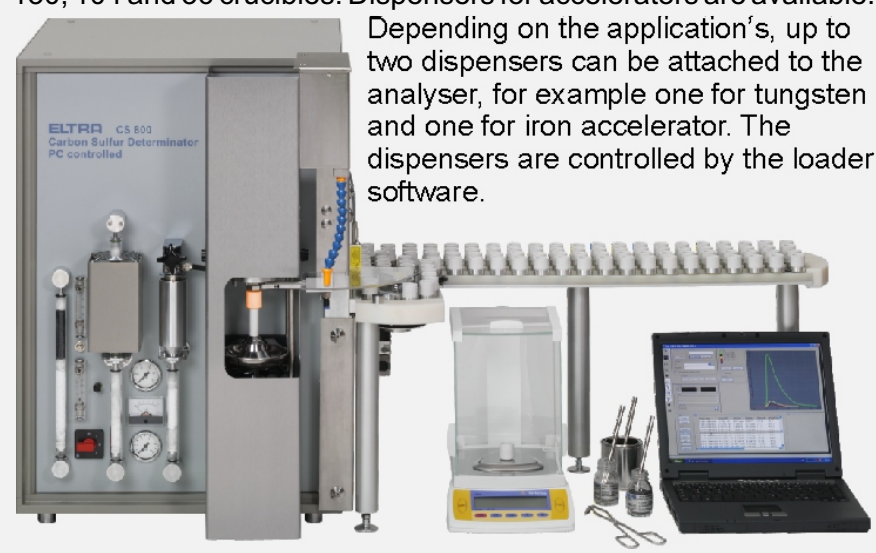

\section{Automatic induction furnace cleaning}

Users of carbon and sulfur analysers with induction furnaces know that dust accumulates during combustion and forms deposits (mainly of iron and tungsten oxides) in the combustion chamber.

The CS-800 furnace is cleaned automatically after each analysis, thus ensuring repeatable and accurate results without the time consuming and unpleasant task of manual furnace cleaning.

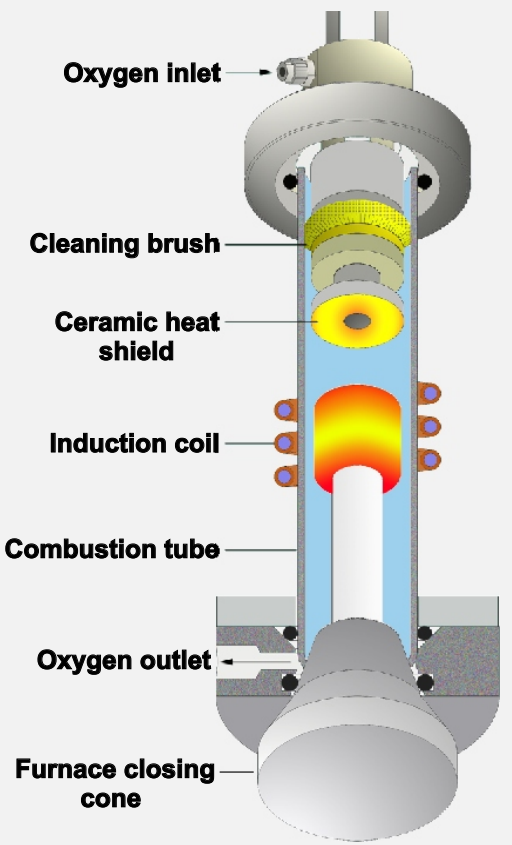

The standard cleaning apparatus is mechanically attached to the furnace open / close system, thus ensuring that it is not possible for the cleaning brush to collide with a hot crucible.

\section{The cleaning brushes won't burn !}

The efficient design of the cleaning mechanism rules out any possibility of the cleaning brush catching fire. To confirm this fact, ELTRA offers free replacement of each burned cleaning brush, during the entire working life of the analyser.

\section{Electronic flow controller}

An essential part of the gas flow system is the electronic flow controller. This provides a stable gas flow by eliminating the known disadvantages of mechanical controllers.

\section{Up to $\mathbf{2 0}$ grams of copper sample without accelerators}

The unique design of the induction furnace combined with intelligent power control electronics enables the analysis of copper samples up to a weight of 20 grams without the need of any accelerators. This is very important in case of samples with extremely low $C$ and $S$ contents, like copper and copper alloys. The higher the weight, the higher the amount of $\mathrm{C}$ and $\mathrm{S}$ present. The elimination of the need for an accelerator is a breakthrough in the analysis of very low $C$ and $S$. The use of accelerators can badly affect the results when the very low $C$ and $S$ in the sample is similar to the $C$ and $S$ content of the accelerators (blanks). The CS-800 eliminates this problem.

\section{Carrier gas conservation}

If an analysis has not been carried out for a period of time, the analyser will automatically switch to "gas conservation mode." This effectively means that the carrier gas flow rate is reduced to a minimum, only allowing a small amount of oxygen to circulate through the IR cells etc. The period of time before the gas conservation is activated, can be modified via the PC software. It is also possible to have carrier gas flow only during combustion. As soon as the next sample is analysed, the carrier gas returns to normal flow rate.

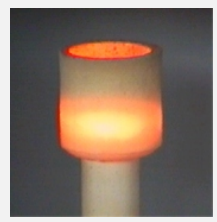

The induction furnace and the auto loader use standard ceramic crucibles, which are 1 " or $25 \mathrm{~mm}$ in diameter. 


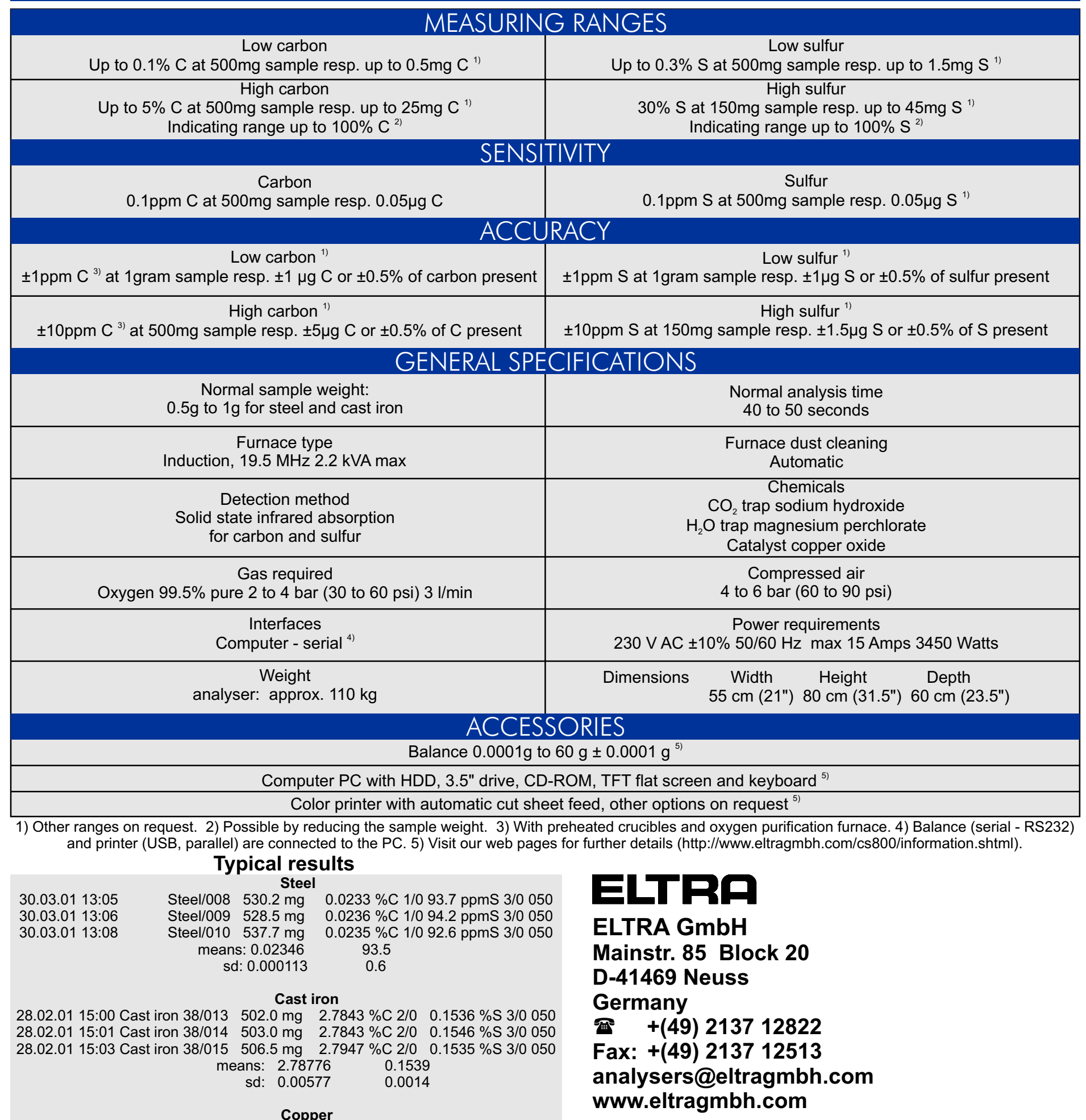

28.02.01 14:44

28.02.01 14:49

28.02.01 14:53

Copper/031 $5012.7 \mathrm{mg}$

Copper/032 $5132.5 \mathrm{mg} \quad 54.7 \mathrm{ppm} \mathrm{1/0} \quad 4.2 \mathrm{ppm} \mathrm{S} 3 / 1047$

Copper/033 $4983.1 \mathrm{mg} \quad 56.3 \mathrm{ppm} \mathrm{1/0} \quad 4.3 \mathrm{ppm} \mathrm{S} 3 / 1046$

means: $54.4 \quad 4.3$

sd: $\quad 1.7 \quad 0.0$

Ore $25 \mathrm{C} / 026 \quad 87.6 \mathrm{mg}$

31.03.01 09:57

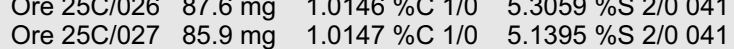

Ore $25 \mathrm{C} / 028 \quad 81.5 \mathrm{mg} \quad 1.0264 \% \mathrm{C} \quad 1 / 0 \quad 5.3230 \% \mathrm{~S} 2 / 0039$
31.03.01 09:52

31.03.01 09:55

$$
\begin{array}{rcr}
\text { means: } & 1.01856 & 5.25613 \\
\text { sd: } & 0.00522 & 0.07775
\end{array}
$$

Cement

31.03.01 09:43 Cement B8/023 $183.7 \mathrm{mg} \quad 1.0629 \% \mathrm{C} \quad 1 / 0 \quad 0.5446 \% \mathrm{~S} 2 / 0050$

31.03.01 09:46 Cement B8/024 $181.6 \mathrm{mg} \quad 1.0366 \%$ C $1 / 0 \quad 0.5463 \%$ S 2/0 050

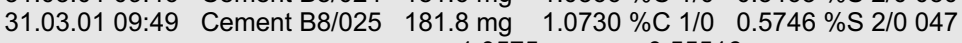
means: $1.0575 \quad 0.55516$

sd: $0.0139 \quad 0.01295$

The contents of the catalogue are subject to change without prior notice for further improvement. 


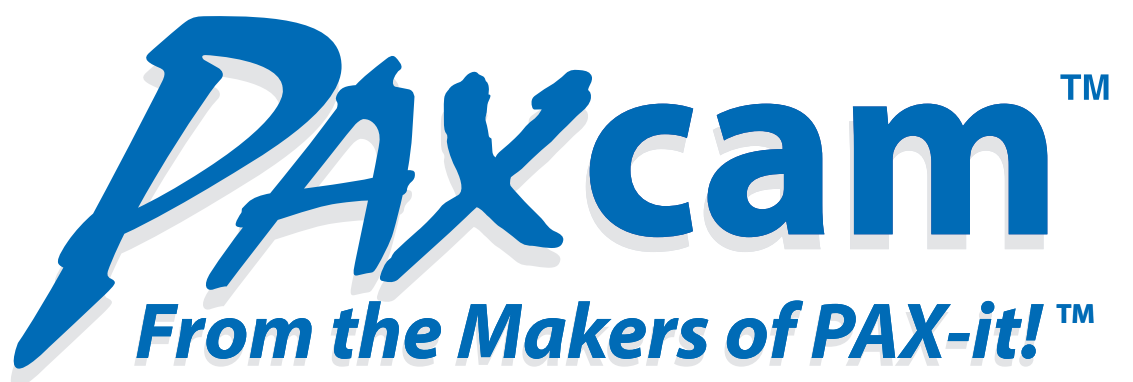

\section{Digital Imaging Workflow for Industrial Applications}

\section{The PAXcam Digital USB 2.0 Camera System}

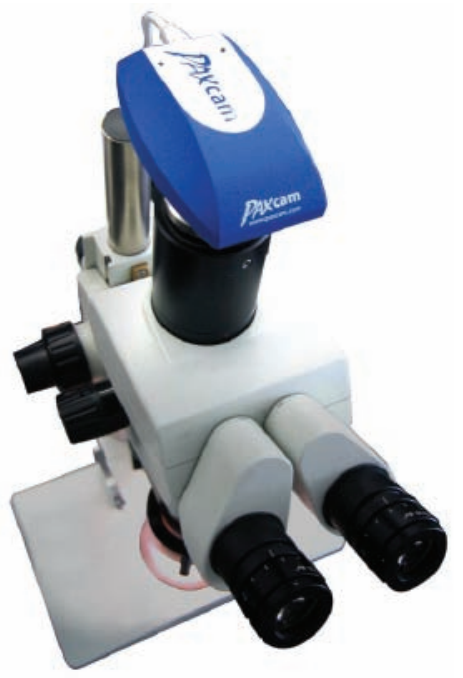

- Affordable camera for microscopy, with an easy-to-use interface

- Beautiful, high-resolution images; true color rendition

- Fully integrated package with camera and software

- USB 2.0 interface for the fastest live digital color preview on the market

- Easy-to-use interface for color balance, exposure \& contrast control, including focus indicator tool

- Adjustable capture resolution settings (true optical resolution -- no interpolation)

- Auto exposure, auto white balance and manual color adjustment are supported

- Create and apply templates and transparencies over the live image

- Acquire images directly into the PAX-it archive for easy workflow

- Easy one-cable connection to computer; can also be used on a laptop

- Adjustable region of interest means smaller file sizes when capturing images

- PAXcam interface can control multiple cameras from the same computer

- Stored presets may be used to save all camera settings for repeat conditions

\section{Capture Images Directly to PAX-it Image Database Software}

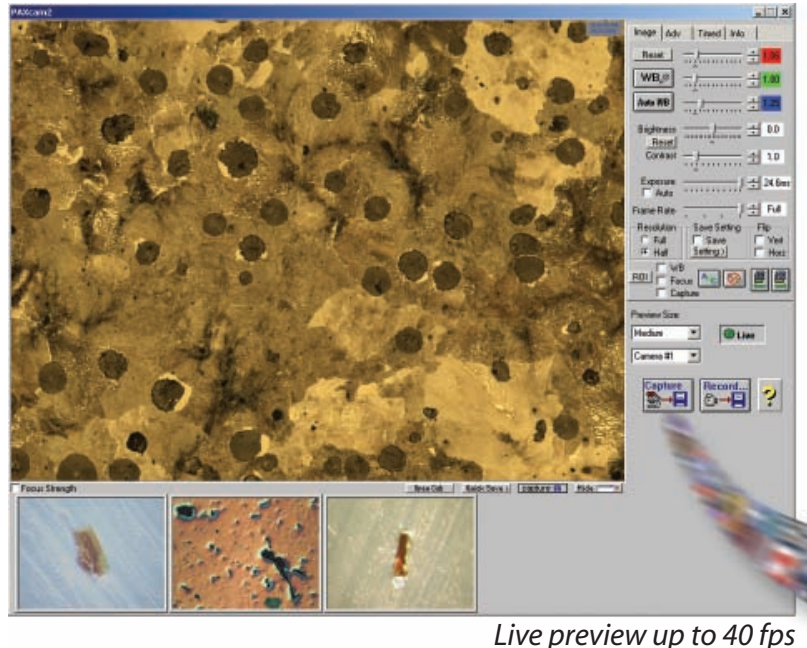

Live preview up to $40 \mathrm{fps}$
Includes PAXcam Video Agent for motion video capture

- Time lapse image capture

- Combine still images to create movie files

- Extract individual frames of video clips as bitmap images

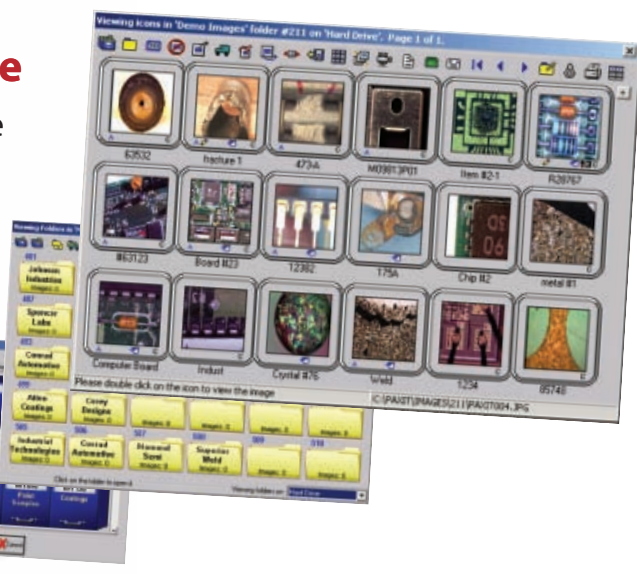

\section{PAX-it!}

- File \& retrieve images in easy-to-use cabinet/folder structure

- Store images, video clips, documents, and other standard digital file types

- Images and other files are in a searchable database that you design!

- Annotate images with circles, arrows, text; add a calibrated scale bar

- Automatically stitch overlapping adjacent images together (mosaic)

- Add written notes \& dictated messages to images

- Link to MS Wordтм and Excelтм for easy report generation

- Link to MS PowerPoint ${ }^{\mathrm{TM}}$ for easy construction of presentations

- Link to email service for sending images, notes, \& data as attachments

- Additional modules for measurement, image analysis, network sharing, web-sharing, tutorial production, CD/DVD Archiving, Extended Security

Special Package Pricing PAXcam USB 2.0 Digital Camera System includes:

- PAX-it Image Database Software / interface

- USB 2.0 digital camera, card and cable

- Free tech support \& software updates for one year

PAXcam2 (1600 x 1200)

PAXcam3 (2048 x 1536)

PAXcamARC

(Adjustable Resolution to 5120 x 4096) 


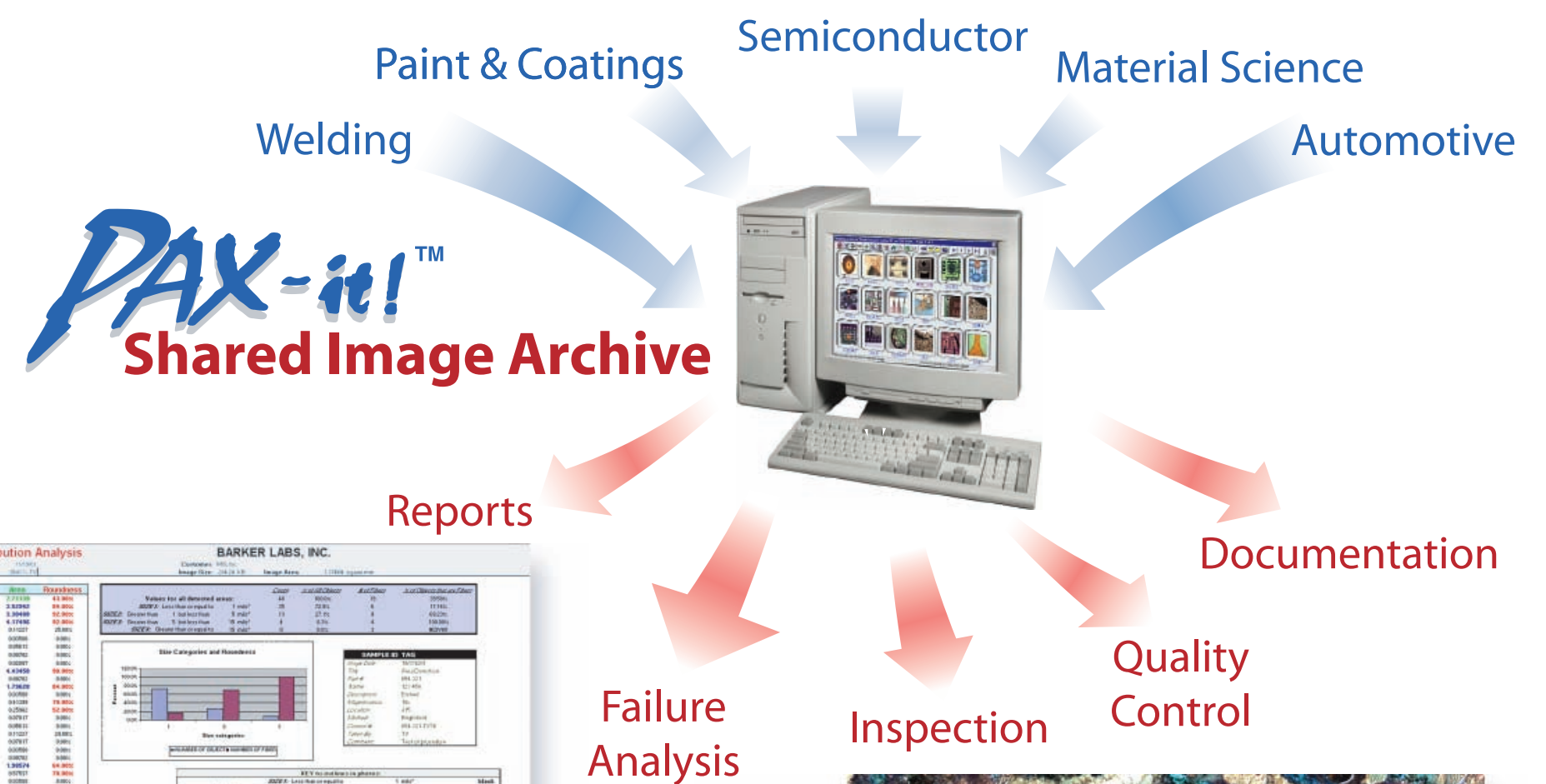

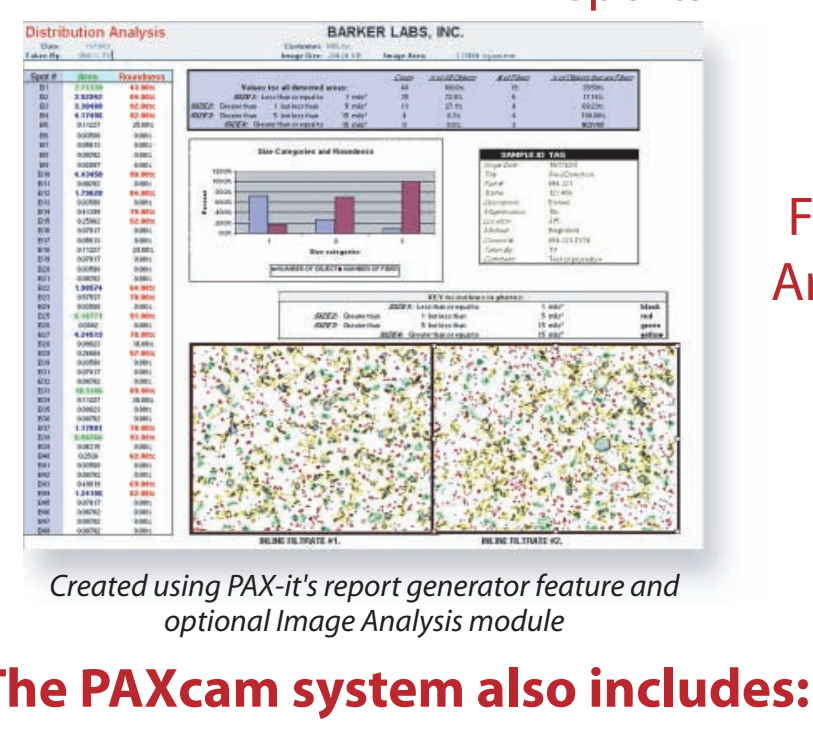

Email: link to your email service to send images \& data as attachments

Videotext Overlays: annotate and edit images with text, shapes, arrows and calibrated scale bar; overlays can be placed on the live preview image for alignment and comparison

Reporting: create powerful, customizable presentations and written reports via link to MS Office ${ }^{\mathrm{TM}}$ programs

Image Stitching: automatically stitch overlapping adjacent images into one mosaic image

Image Fusion: fuse multiple images at different focal planes into one focused image, solving problematic depth-of-field issues

PAXcam Minimum System Requirements:

P2 - 400Mhz, Win 2000, Win XP. Must have PCl slot or USB 2.0 port available. For best performance, a $0.5 \times$ c-mount coupler is recommended.

\section{LECO Corporation 800-292-6141 www.leco.com}

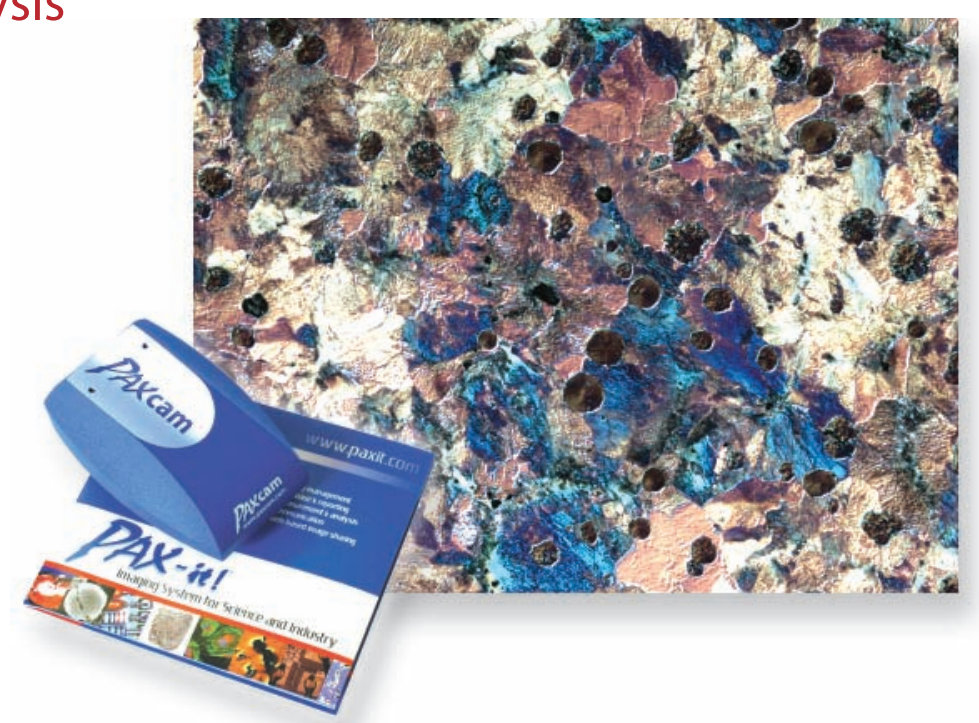

\section{PAXcam seamlessly integrates with PAX-it's optional modules:}

Networking: share images, reports, presentations and more with a PAX-it site license

Web: PAXnet web-based asset sharing over intranet or internet; no additional software required at remote sites

Basic Measurement: manual point-to-point, fixed area, parallel line, ellipse arc, and angle measurements. Easy to calibrate.

Image Analysis: user-defined area fractioning, porosity, nodularity, grainsizing, plating thickness, flake size classification, ferrite/pearlite, color and grayscale thresholding

CD/DVD Creator: back up, burn partial or entire PAX-it collection to $C D$ or DVD, store images off-line while leaving database information and thumbnails on-line.

Extended Security: increased user/group permission controls, electronic image signature and validation, and logging of audit trails in a network environment 


\section{Pegasus \\ Floor Scale Systems}

Calibrated Platform Scale with Indicator
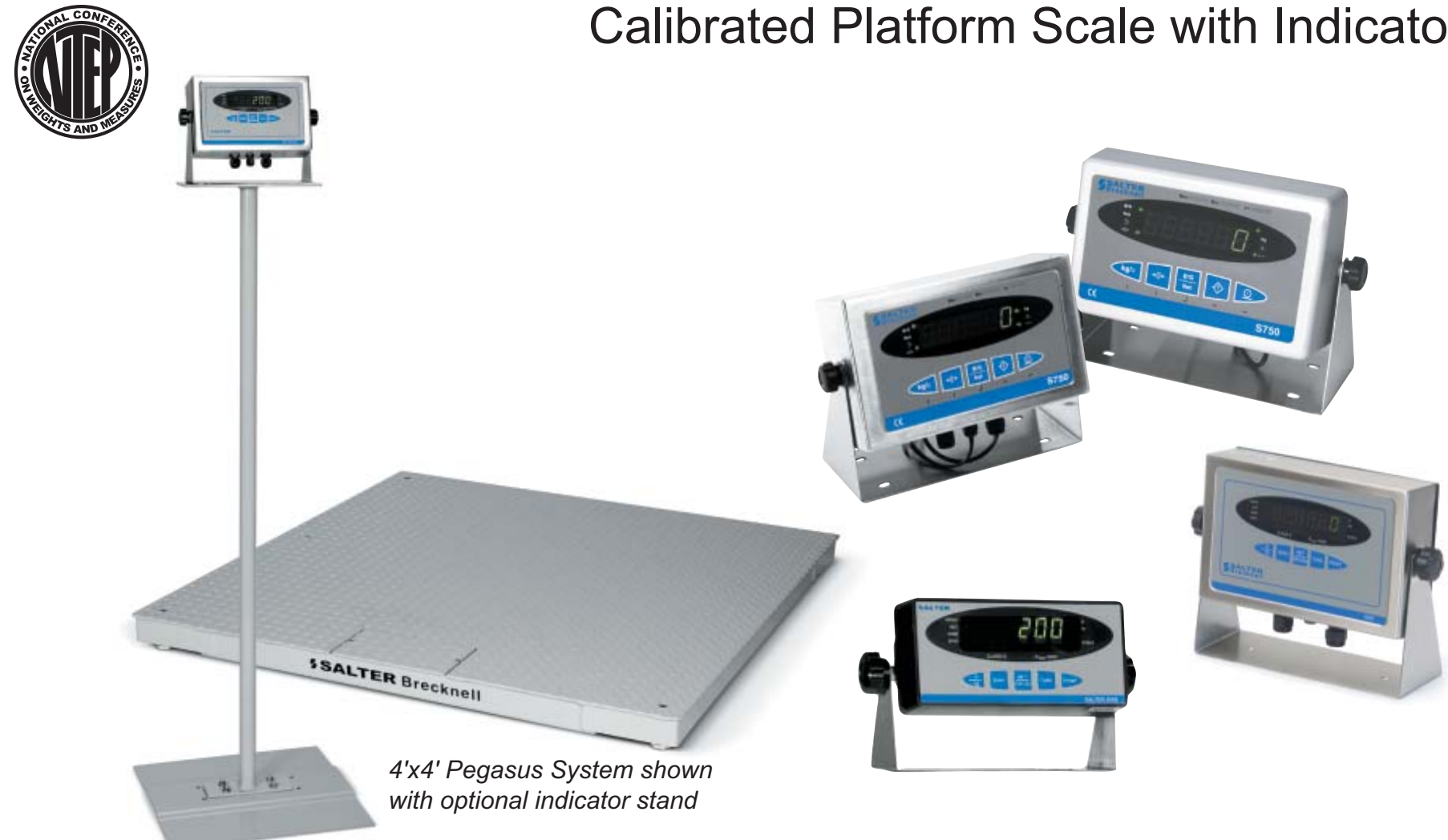

- Pre-calibrated-Indicator and floor scale calibrated at the factory prior to shipment

- Capacity, Size- Match a capacity and size with one of four Salter Brecknell indicators.

$\checkmark$ Easy Installation- Units can be placed into service with no on-site installation required.

$\checkmark$ A/D Conversion Rate- Up to 25 measurements per second. 


\section{Capacities \& Resolution}

\begin{tabular}{|l|c|c|}
\hline \multicolumn{1}{l}{ Capacity } & \multicolumn{1}{c}{ Dimension-in. } & \multicolumn{1}{c|}{ Dimension-cm } \\
\hline $5,000 \times 1 \mathrm{lb}$ & $48 \times 48 \times 3.1$ & $122 \times 122 \times 7.9$ \\
$(2500 \times .5 \mathrm{~kg})$ & $60 \times 60 \times 3.1$ & $152 \times 152 \times 7.9$ \\
\hline $10,000 \times 2 \mathrm{lb}$ & $48 \times 48 \times 3.1$ & $122 \times 122 \times 7.9$ \\
$(5000 \times 1 \mathrm{~kg})$ & $60 \times 60 \times 3.1$ & $152 \times 152 \times 7.9$ \\
\hline
\end{tabular}

ALL capacities and resolutions shown above are based on 5000d.

\section{Indicator Interface Cable}

Models 750S/200ES- Includes quick disconnect, $25 \mathrm{ft}$ Models 200E/750 - 14 pin Centronics connector, $15 \mathrm{ft}$
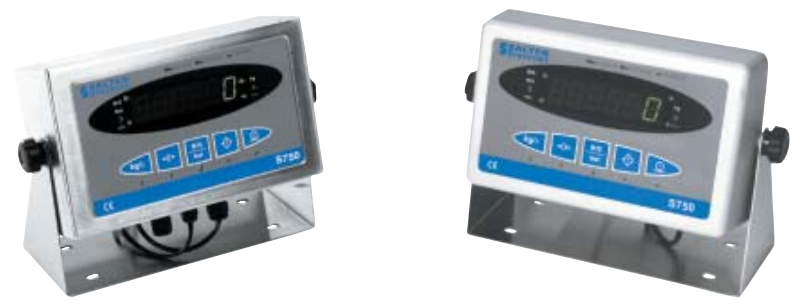

\section{Model 750 / 750 S Indicator}

Easy to Read- Large 1" (25 mm) LED display.

\section{Construction-}

Model 750: ABS NEMA 12 enclosure

Model 750S: stainless steel NEMA 4X enclosure

Power-

Model 750: 115 VAC $\pm 10 \%$

Model 750S: $115 / 230$ VAC $\pm 10 \%$

Excitation Voltage- \pm 10 VDC, $8 \times 350$ ohm loadcells

Serial Port- One full duplex RS-223; One simplex RS-232C; One simplex passive $20 \mathrm{~mA}$ current loop

Digital Calibration- Front panel calibration and configuration

Keyboard- Units, Zero, Net/Gross, Tare, Print

Functions- Gross/Net/Tare; Print ticket mode with 6-digit ID number, Simple piece counting

Units- Custom unit configuration

Stand-Stainless steel swivel stand included

Display- Up to 50,000 graduations

Certifications- NTEP approved at 10,000 divisions, Class III / IIIL, COC \#00-064A1

\section{Material Specifications}

Platform- Mild steel

J-Box- ABS Plastic

Finish-Powder coat paint

\section{Loadcell Specifications}

Material- Alloy tool steel potted

Rated Output- $3 \mathrm{mv} / \mathrm{V}$

Output Resistance- 350 ohm
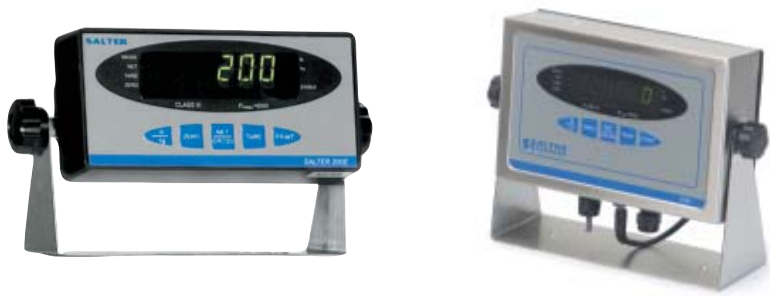

\section{Model 200E / 200ES Indicator}

Easy to Read- 0.6" (15 mm) LED display

\section{Construction-}

200E: ABS NEMA 12 enclosure

200ES: NEMA 4X stainless steel enclosure

Power-

200E: 115 VAC $\pm 10 \%$

200ES: $115 / 230$ VAC $\pm 10 \%$

Excitation Voltage- \pm 10 VDC, $4 \times 350$ ohm loadcells

Serial Port- One full duplex RS-232.

Digital Calibration- Front panel calibration and configuration

Keyboard- Units, Zero, Net/Gross, Tare, Print

Functions- Gross/Net/Tare; Print ticket mode; Simple piece counting

Units-Switch between $\mathrm{lb}$ and $\mathrm{kg}$

Stand-Stainless steel swivel stand included

Display- Up to 50,000 graduations

Certifications- NTEP at 5,000 divisions, Class III, COC \#00-065A1

Salter Brecknell Weighing Products a division of Avery Weigh-Tronix

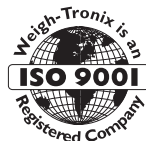
1000 Armstrong Dr.

Fairmont, MN 56031

Toll free: $800-637-0529$

Tel: 507-238-8702 Fax: 507-238-8271

e-mail: sales@salterbrecknell.com

www.salterbrecknell.com 


\section{- HAAS AUTOMATION, INC. WMAS CNC TURNING CENTERS}

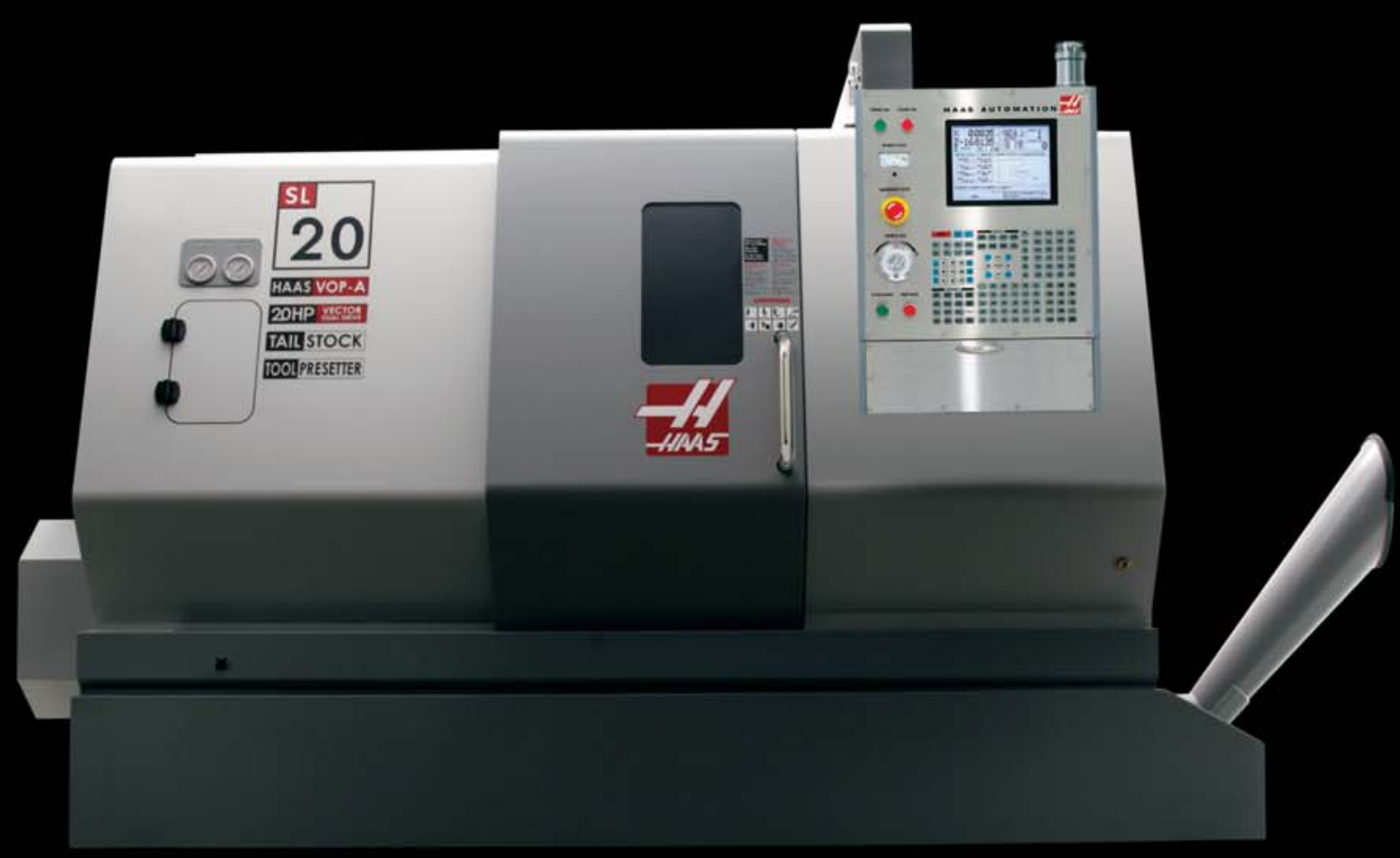




\section{HIGH-PERFORMANCE TURNING CENTERS}

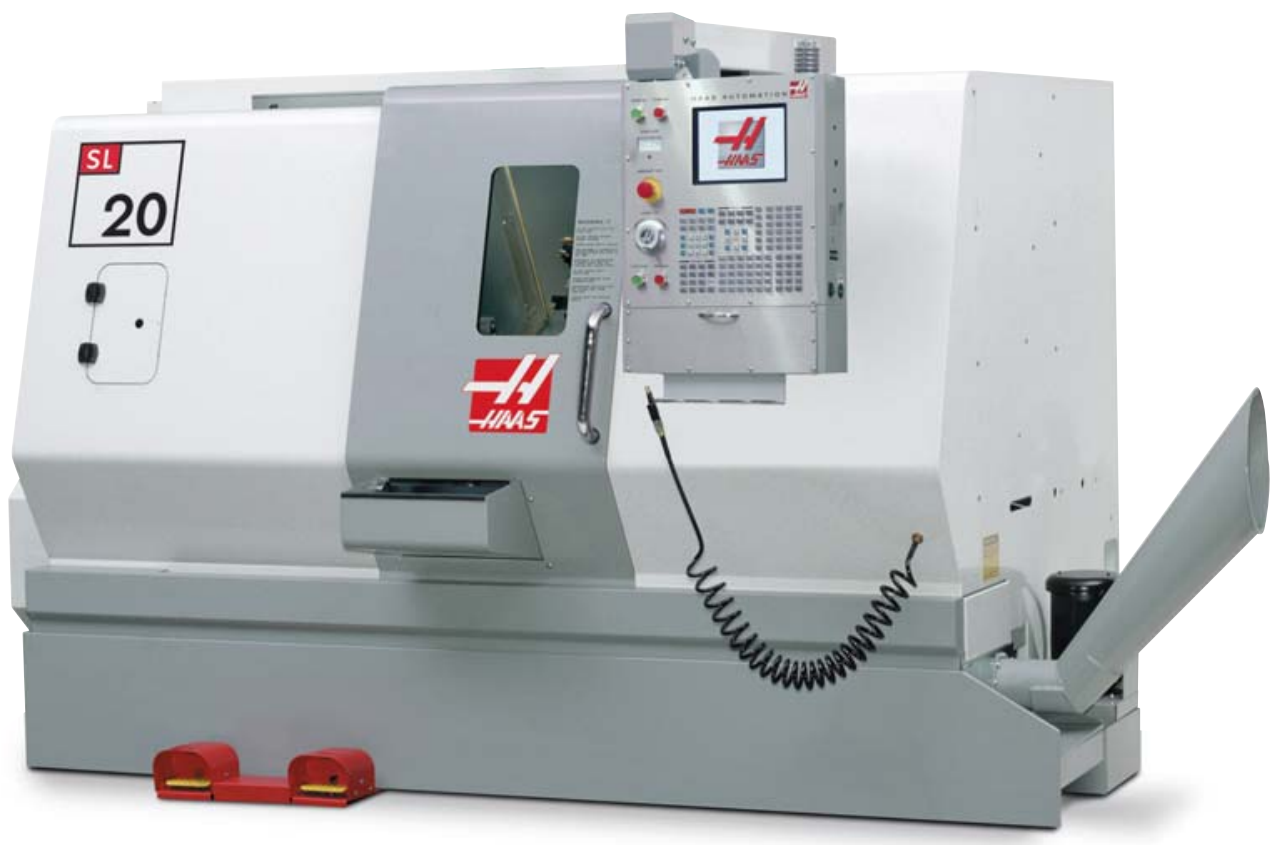

\begin{tabular}{lc}
\hline Special turret design & $5-7$ \\
\hline Live/driven tools \& C axis & $8-9$ \\
\hline Rigid foundation & $10-11$ \\
\hline Main spindle \& spindle drive & $12-13$ \\
\hline Motion control & $14-15$ \\
\hline Part setup \& support & $16-17$ \\
\hline Part-holding variations & $18-19$ \\
\hline Chip removal \& automated operation & $20-23$ \\
\hline Operator convenience & $24-25$ \\
\hline Model variations & $26-27$ \\
\hline Toolroom lathes & $28-29$ \\
\hline The Haas control & $30-33$ \\
\hline Specifications & $34-39$ \\
\hline
\end{tabular}

$\mathrm{H}$ aas Automation manufactures a complete line of CNC lathes designed to meet the ever-increasing needs of modern machine shops - both now and long into the future. The SL Series of CNC lathes is the culmination of extensive design and real-world testing aimed at making Haas lathes the finest available. At present, more than 12,000 high-performance Haas turning centers are installed worldwide, proving their capabilities every day in machine shops around the globe. The addition of the Toolroom line opens up new possibilities and provides additional capabilities. With these machines, we have raised CNC turning to new levels of reliability, ease and productivity.

Haas CNC turning centers include such innovations as massive headstock castings with symmetric ribs for rigidity and stability; large tailstock castings with a two-piece design for easy realignment; embedded chip trays for improved sealing and efficient chip removal; increased-volume, roll-out coolant tanks; and double-wall doors riding on precision bearings for smooth, reliable operation. 


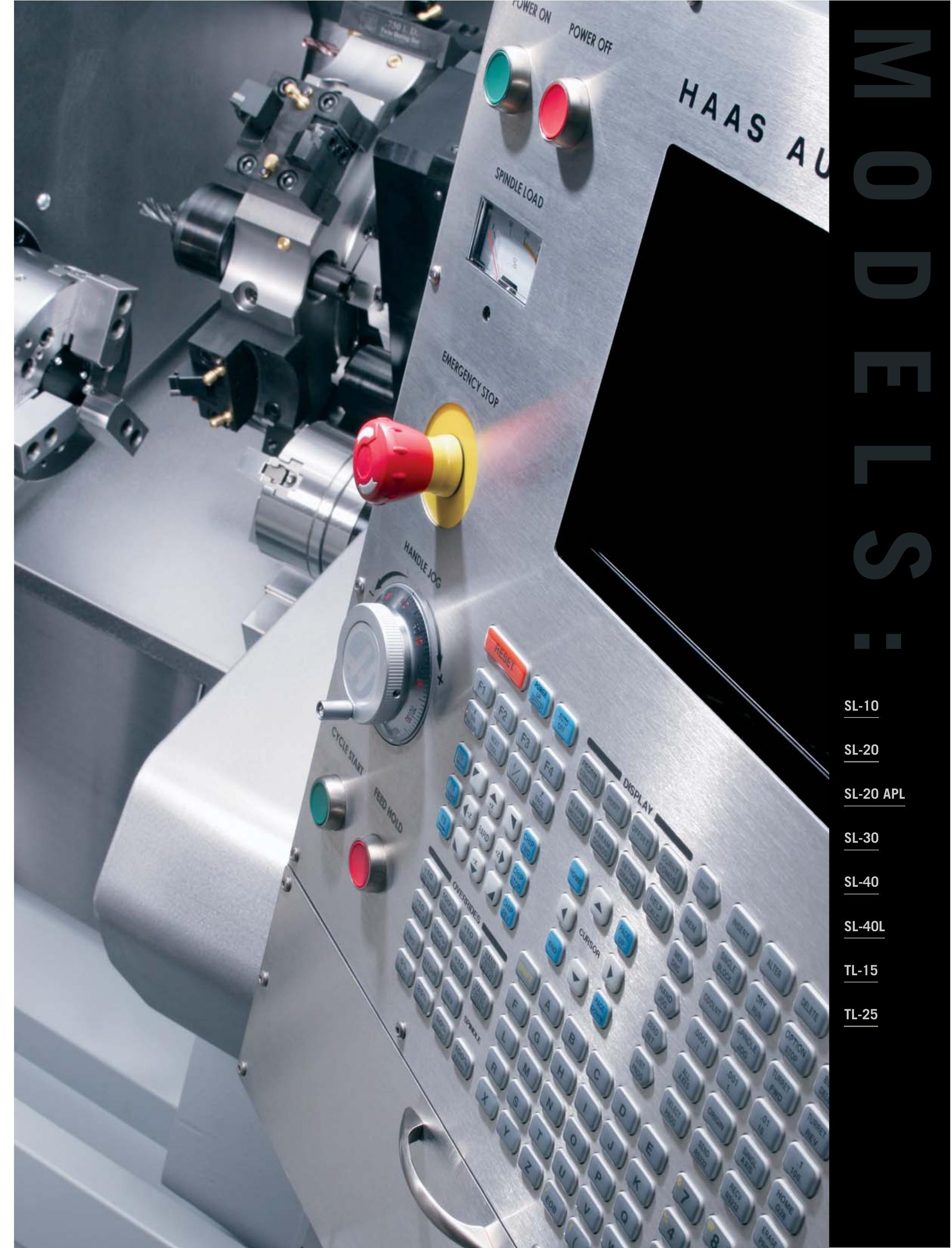




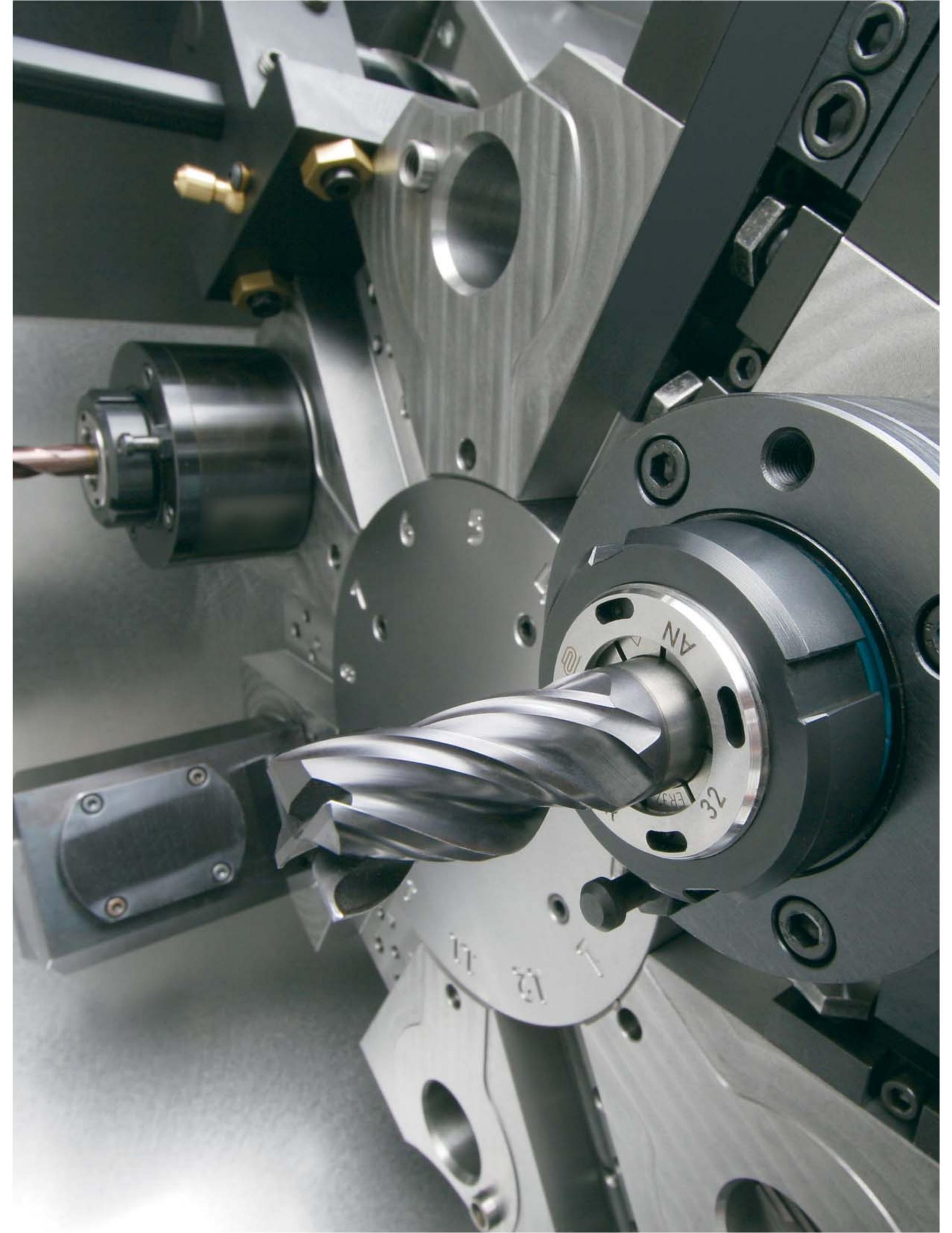




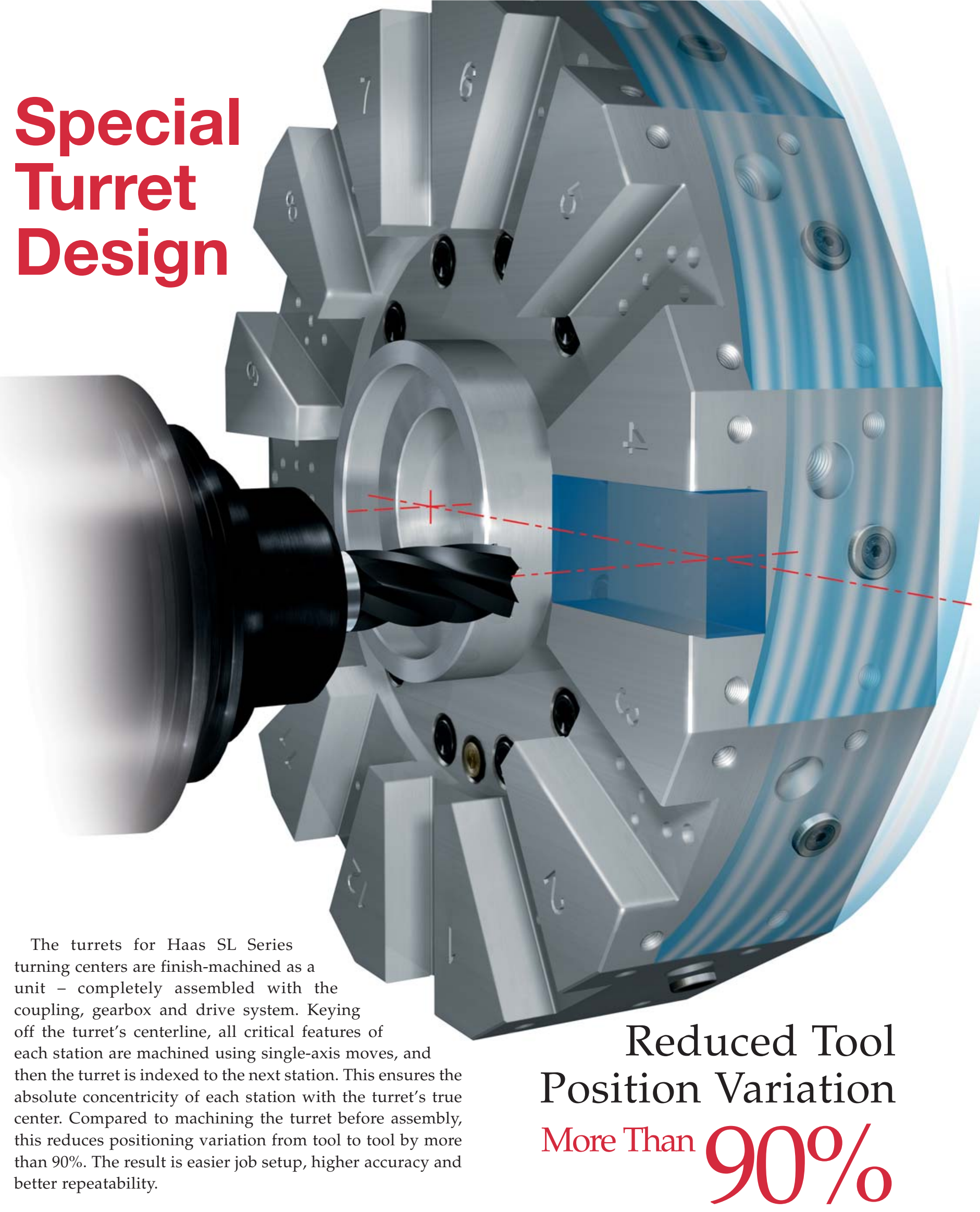

\section{New, faster hydraulic turret for the SL-40 reduces tool change times by $60 \%$.}




\section{Special Turret Design}

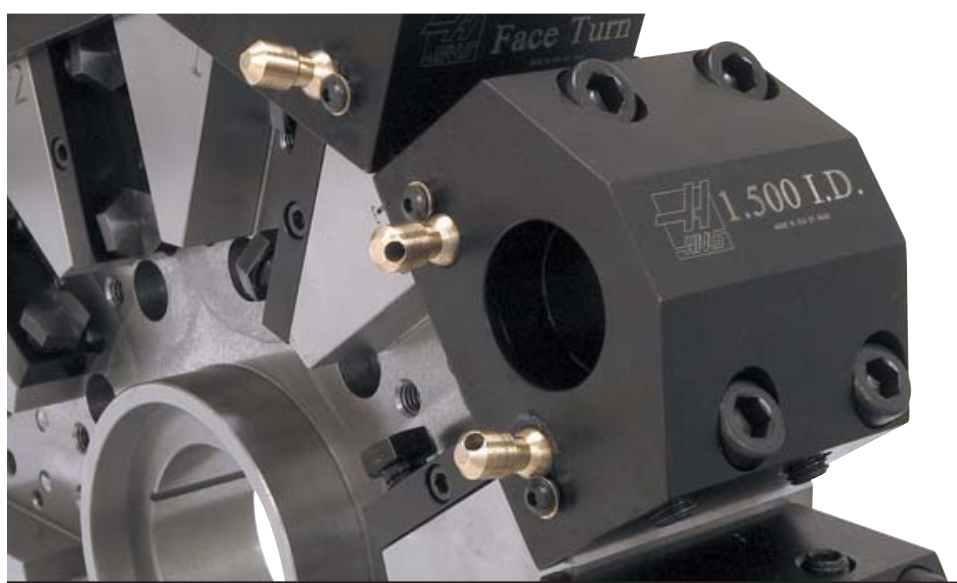

\section{On-The-Fly Indexing}

This standard feature allows tool changes on the fly, while the axes are in motion retracting from the part. This feature, combined with high-speed rapid movements, results in faster tool changes and shorter cycle times by reducing non-cutting time to an absolute minimum.

\section{Improve your cycle times by keeping the machine in constant motion.}

\section{THREE TYPES OF TURRET ${ }^{1}$}

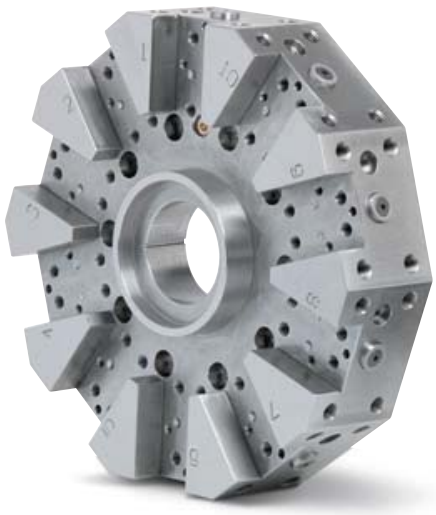

Haas SL Series turning centers come standard with a BOLT-ON TOOL TURRET that provides super-rigid mounting of turning tools and boring bars. This turret accepts tools around the perimeter, and has an equal number of standard radial slots on the face for mounting turning tools in either the right- or left-hand direction.

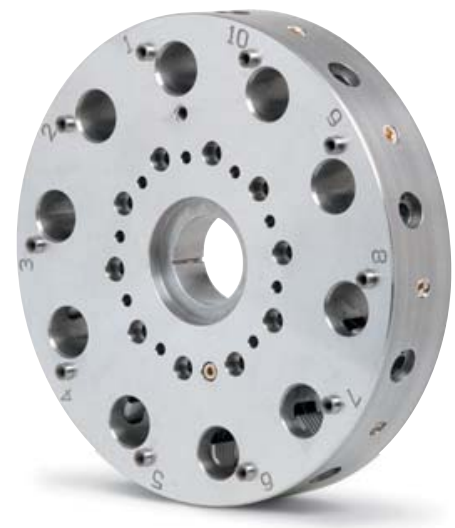

The optional HAAS VDI TURRET accepts standard VDI40 tools, including axial and radial live tools when combined with the versatile live tooling option.

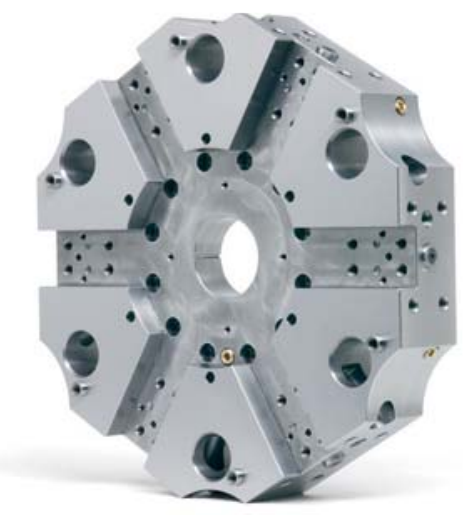

Dual-spindle TL-15 and TL-25 models feature the VB HYBRID TURRET, a 12-station turret that allows the use of VDI40 tools (including live tools), as well as standard bolt-on tools. The turret has 6 VDI stations and 6 bolt-on stations (both radial slots for turning tools and perimeter stations for ID tools). The VB turret is optional on the SL-20, SL-30 and SL-40.

${ }^{1}$ Turret type may affect maximum turning diameter. 


\section{Toolholders}
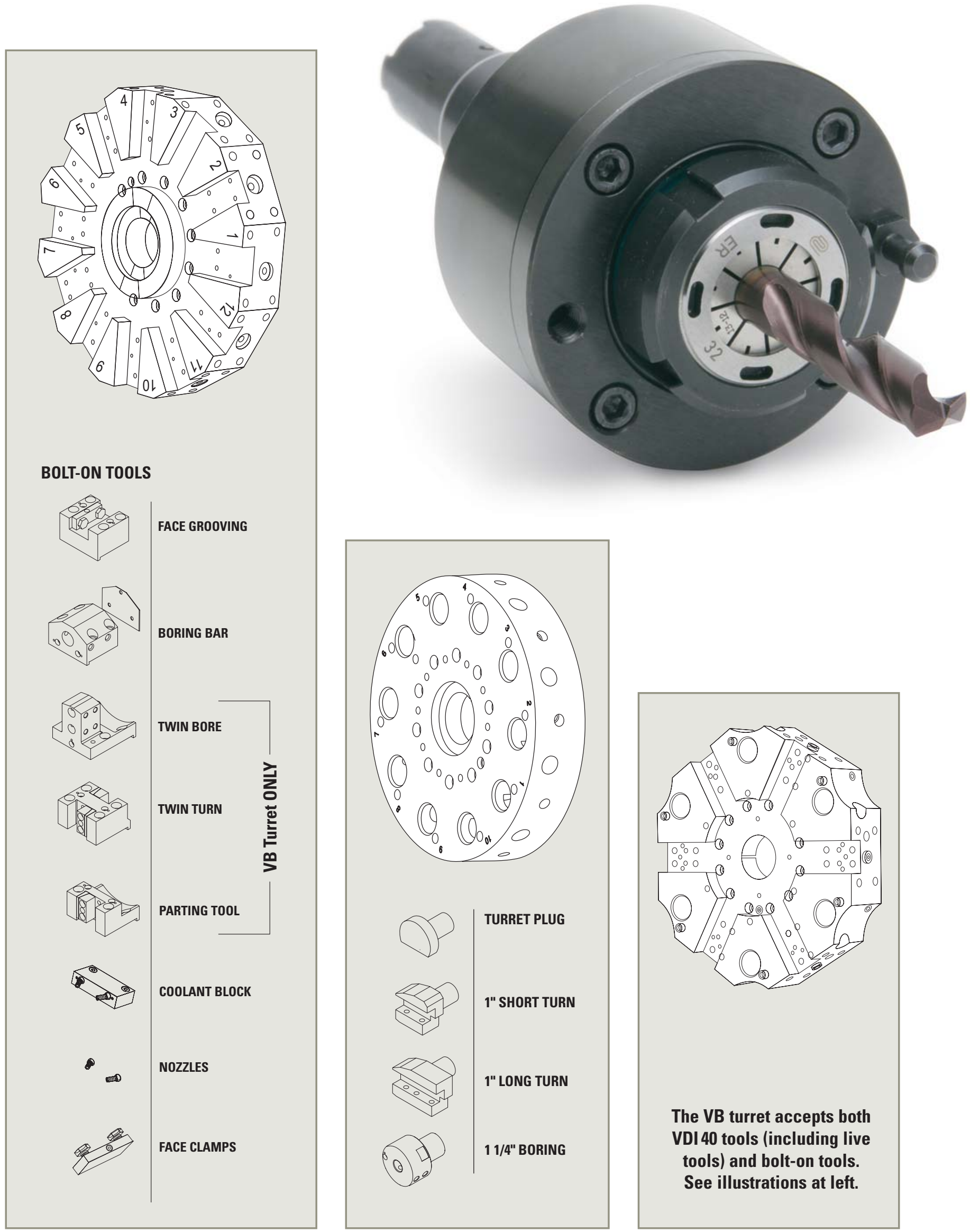


\section{Live Tooling}

Live Tooling with C Axis

The live tooling option for Haas lathes allows you to use standard VDI40 axial or radial driven tools to perform secondary operations such as milling, drilling, flatting and tapping - both on the face of the part and around the diameter. This option includes a full $\mathrm{C}$ axis, which provides interpolated bidirectional motion at precise speeds and feeds for part positioning and repeatability. A powerful hydraulic brake locks the main spindle during secondary operations, and disengages for turning and indexing.

Live tooling is not available with a standard bolt-on style tool turret. It is only available with the VDI or VB Hybrid turret.

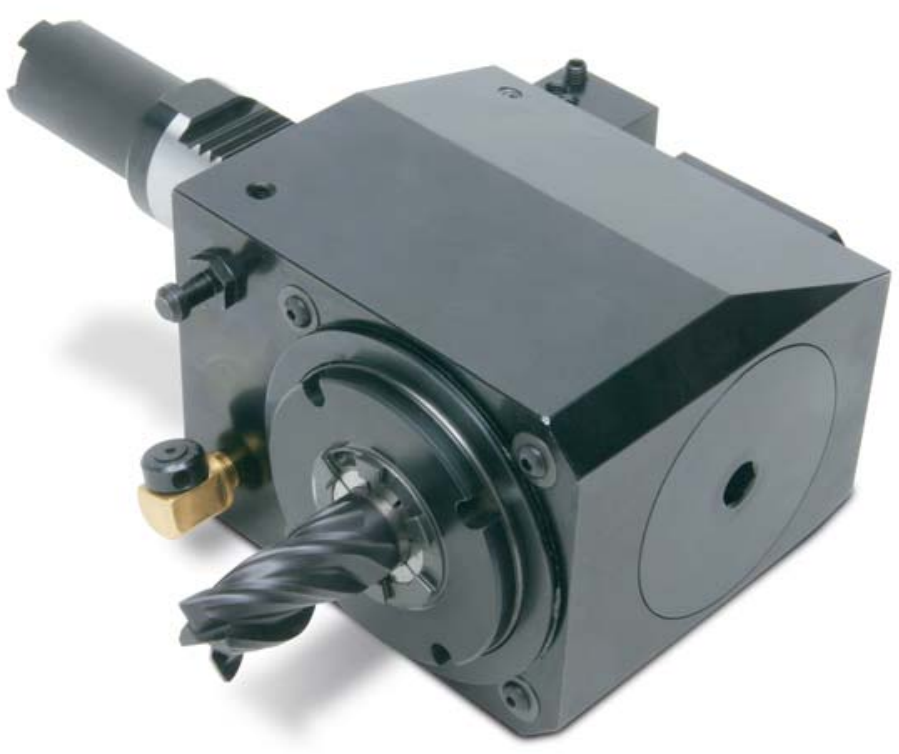

\section{Simple, Modular Live Tool Drive System}

The Haas live tooling drive system features a simple, modular design that places the drive motor outside of the tool changer mechanism. This setup simplifies maintenance and service while providing a robust, reliable drive for secondary operations.

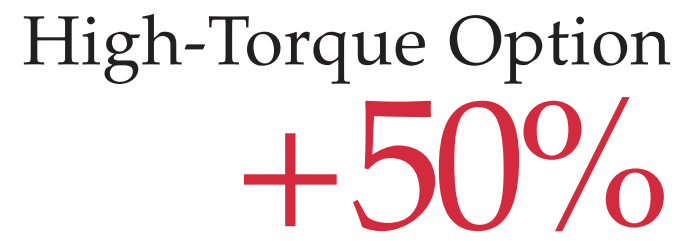

torque increase over standard live tools.

\section{High-Torque Live Tool Option}

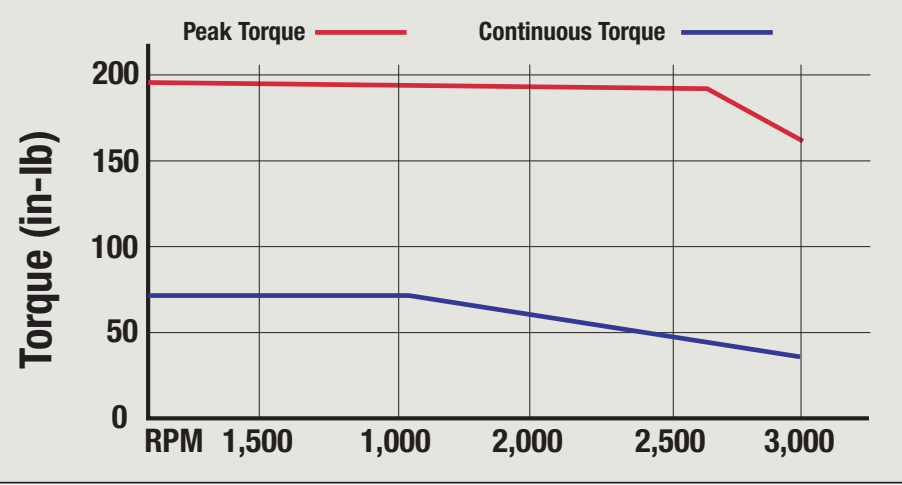



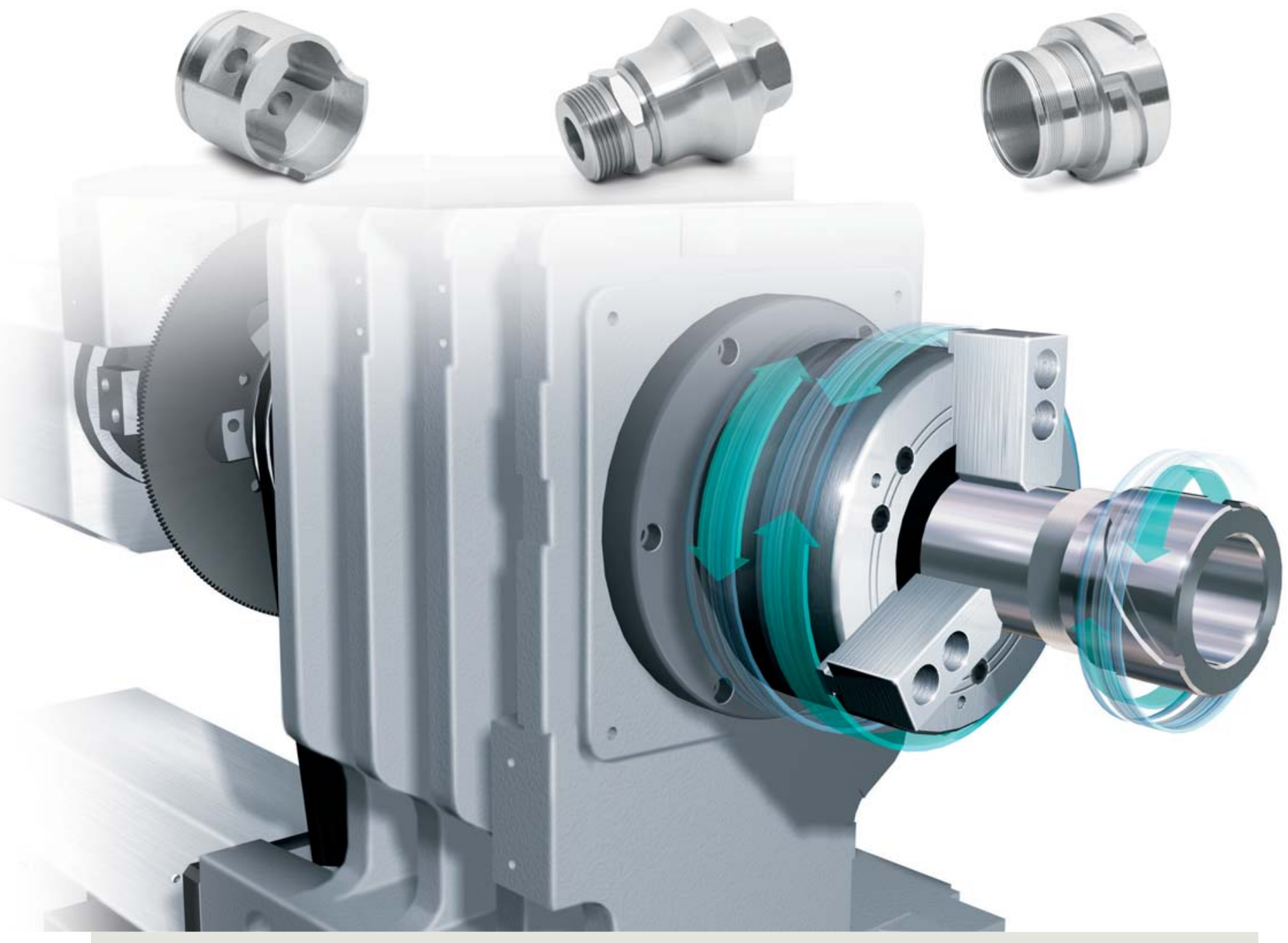

\section{C-AXIS MOTION}

Eliminate secondary operations and increase your productivity by adding the ability to mill parts on your turning center. The $\mathrm{C}$ axis provides high-precision $( \pm 0.01$ degree) bidirectional spindle motion that is fully interpolated with $\mathrm{X}$ and/or $\mathrm{Z}$ motion. It is servo driven through a back-gear reduction to provide outstanding torque for precision milling. Speeds are programmable from 0.1 to $60 \mathrm{rpm}$, and Cartesian-to-polar interpolation allows programming of face machining operations using traditional $\mathrm{X}$ and $\mathrm{Y}$ coordinates.

Cartesian-to-polar coordinate programming converts $\mathrm{X}, \mathrm{Y}$ position commands into rotary $\mathrm{C}$-axis and linear $\mathrm{X}$ axis moves, which greatly reduces the amount of code required to command complex moves. In the polar coordinate system, milling a straight line on the face of the part would require many points to define the path, but in Cartesian, only the end points are necessary.

\section{Spindle Orientation}

This option enables orientation of the main spindle for automatically loading nonround bar stock (hex, square, etc.) via a bar feeder. An M19 specified in the part program orients the spindle to within \pm 0.1 degree, with a repeatability of \pm 0.045 degree.

Spindle orientation is included in the Live Tooling option, and is standard on APL, dual-spindle models and CE machines.

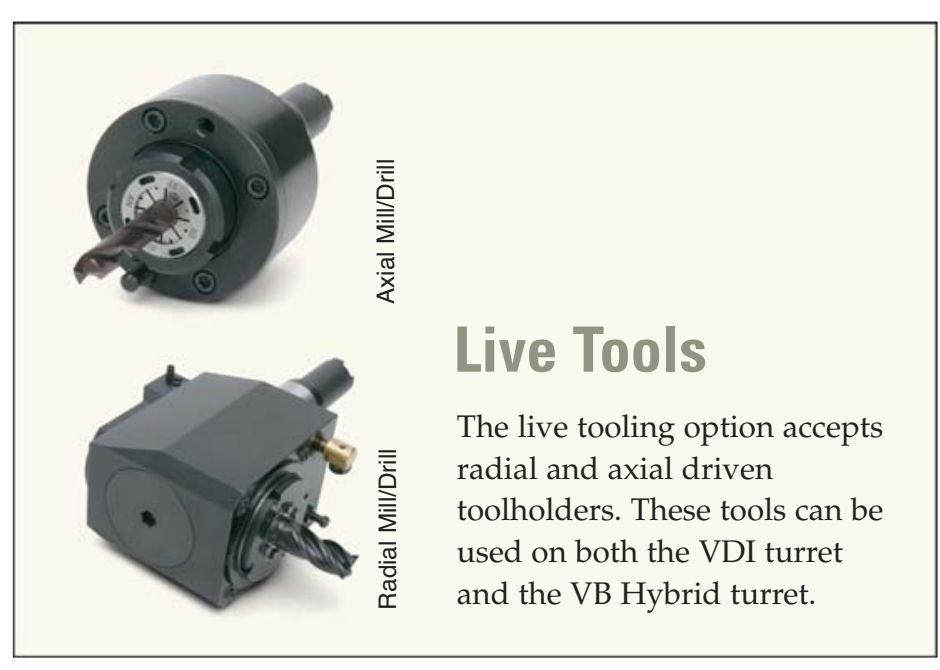




\section{Rigid Foundation}

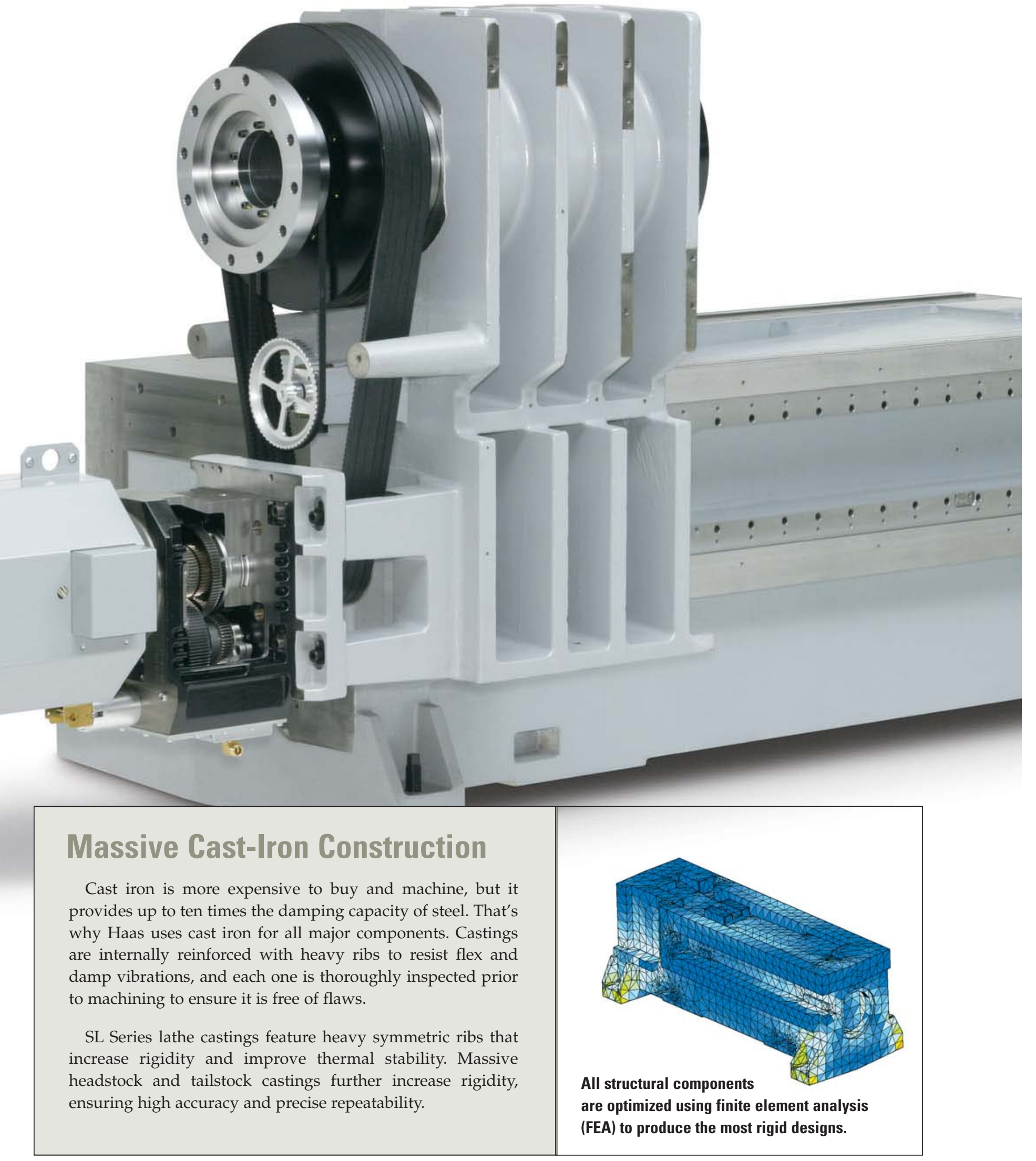




\section{Setup, 5 Sides - surfaced, drilled and tapped}

All Haas base castings are machined on state-of-the-art, 5-side CNC machines. Each casting is completely machined, drilled, tapped and inspected in a single setup, which provides higher accuracies and smoother production. Our ability to precisely machine all critical features of each casting eliminates alignment errors in final assembly. High-accuracy in-process inspection guarantees that every casting meets our stringent quality standards.

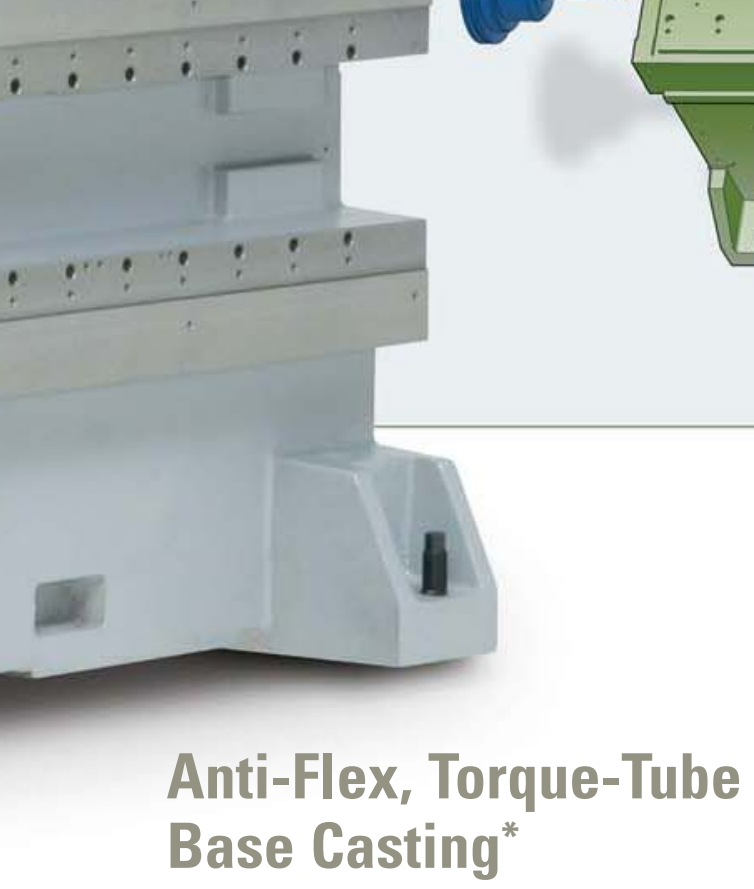

Our unique, specially designed, Haas torque-tube base casting yields superior rigidity and gives the most anti-flex properties of any base design. It's so stable, in fact, that the machine will cut accurately with one leveling screw removed, and no special foundation is required for installation.

*Except the SL-10, which is built using a heavily-ribbed and reinforced structure that incorporates the coolant tank into the base, and the Toolroom Lathes.

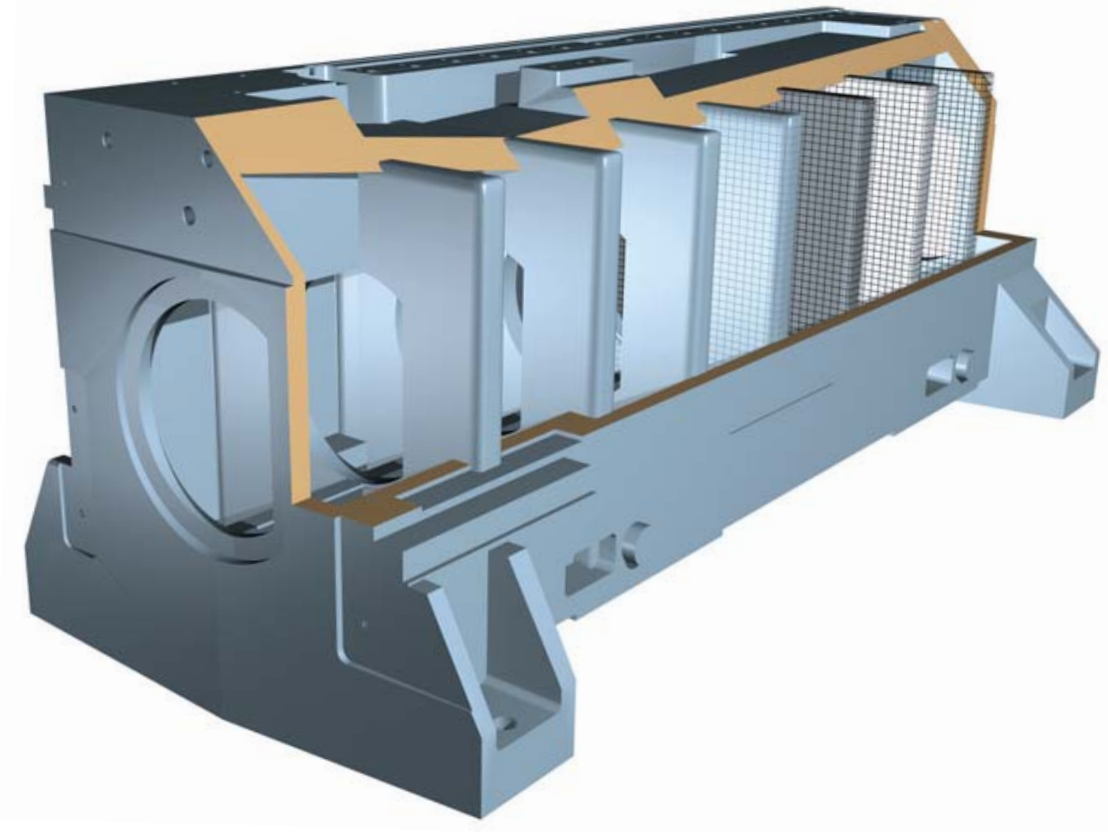

\section{Rigid, Cost-Effective Design Solutions.}




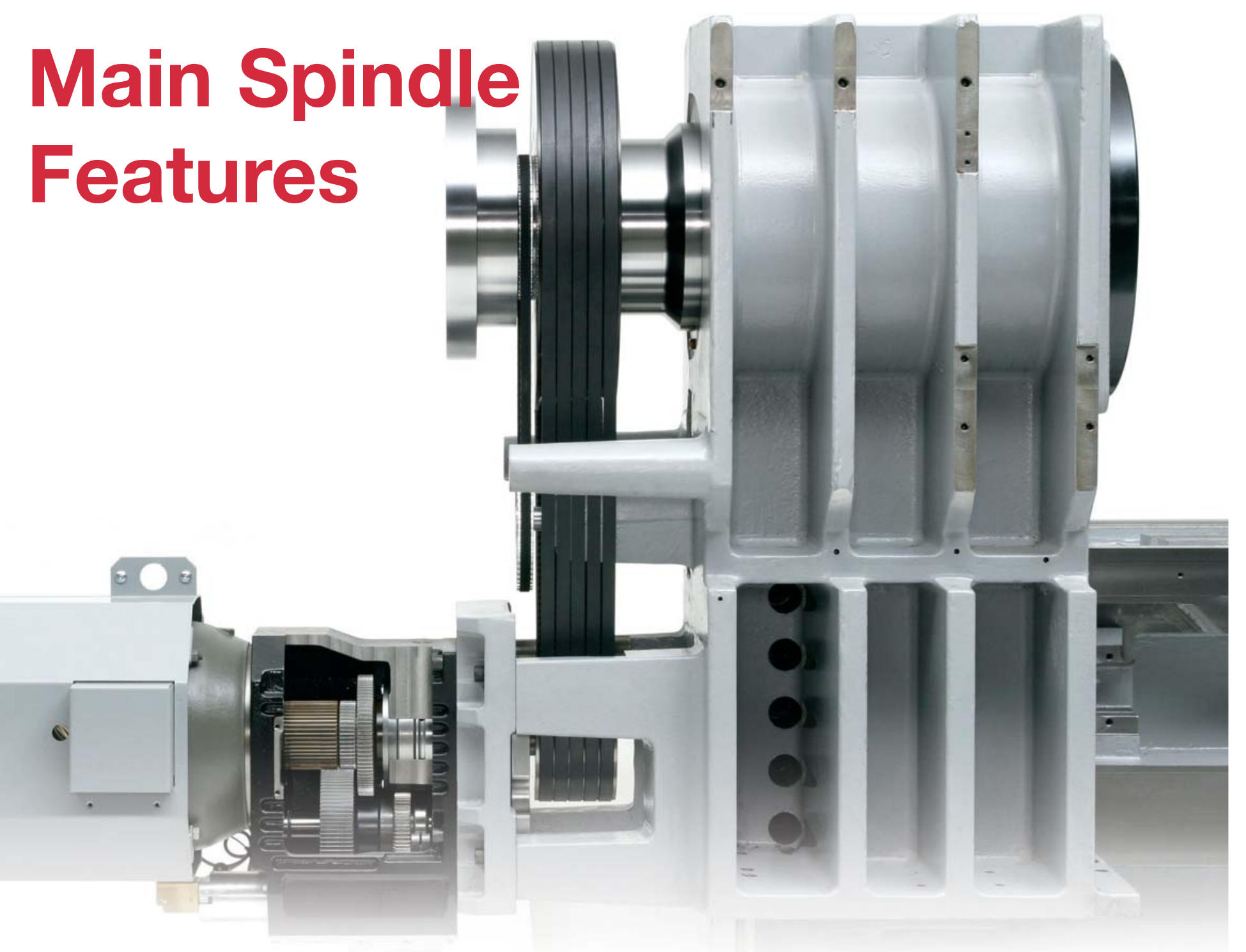

\section{Powerful Vector Spindle Drives}

The Haas-designed vector spindle drive uses the same technology as our brushless servo motors to provide peak performance and speed control under heavy cutting loads. This true closed-loop system optimizes the slip angle between the rotor and stator of the spindle motor to double low-speed torque and acceleration, resulting in the most powerful spindle output ever. These drives allow you to push the spindle to 150 percent of the motor's continuous horsepower for 15 minutes, and to 200 percent for 5 minutes.

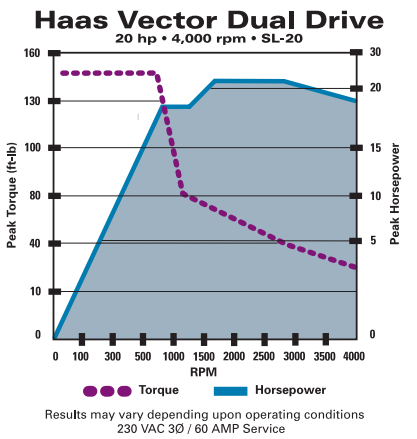

Haas Vector Dual Drive

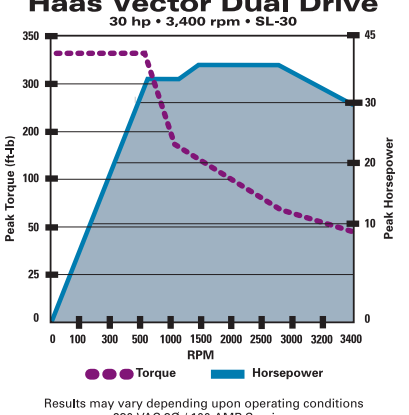

vary depending upon operating
230 VAC $3 \varnothing / 60$ AMP Service

That's more performance headroom than any other drive on the market.

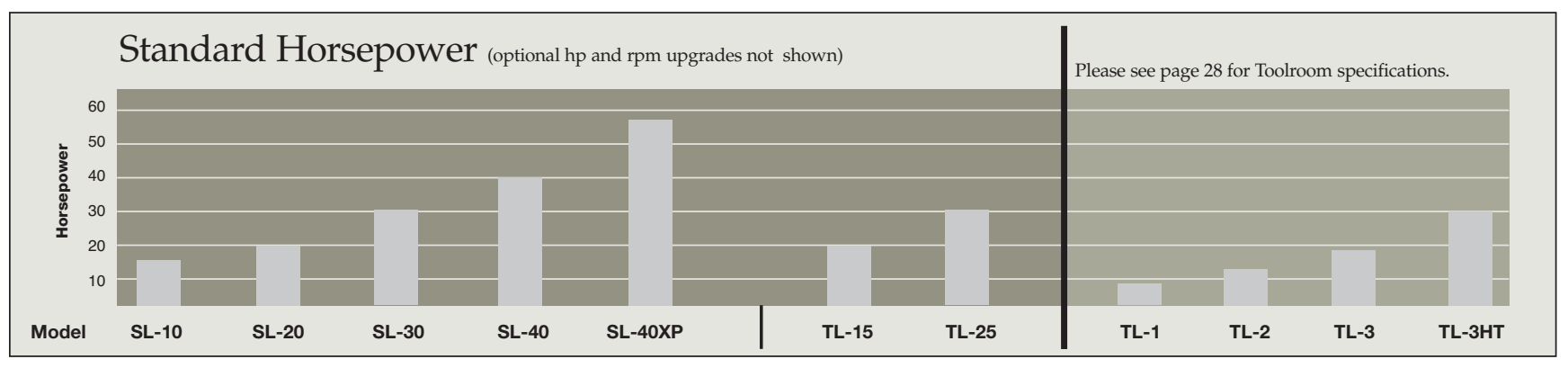




\section{Built for Extra Torque}

This Haas-made gearbox is standard on the SL-40, SL-40L Long Bed, SL-30 Big Bore and TL-25 Big Bore. Designed and built entirely in-house to ensure superior quality and precision, Haas gears are CNC machined and hobbed out of 8620 steel, then heattreated to $60 \mathrm{Rc}$ and precision ground to AGMA Class 13 quality.

The gearbox yields $1,400 \mathrm{ft}-\mathrm{lb}$ of spindle torque at $150 \mathrm{rpm}$ for low-speed, heavy cutting - and it also allows speeds to 2,400 rpm for finish cuts and turning aluminum.

Gearbox shown with cutaway for illustration purposes.

\section{On-The-Fly WYE-DELTA Switching}

Most Haas lathes feature the Haas Dual-Drive system, which consists of a dual-winding motor Wye and Delta - and an electronic switch to change between the two windings. Selecting the best winding for low-rpm cutting and the best winding for high-rpm cutting yields higher torque values over a wider rpm range. In addition, the Haas Dual-Drive system provides constant horsepower over a much wider rpm range. On-the-fly winding change provides the wide power range needed during constant surface speed cuts.

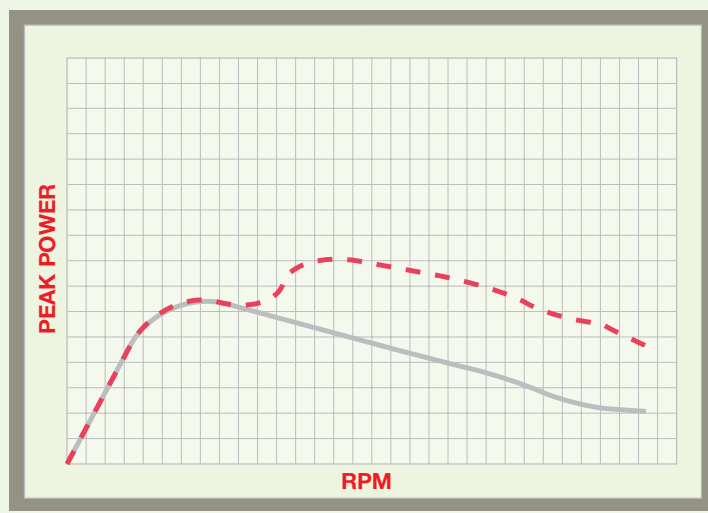

Typical power curve Enhanced power range with on-the-fly Wye-Delta

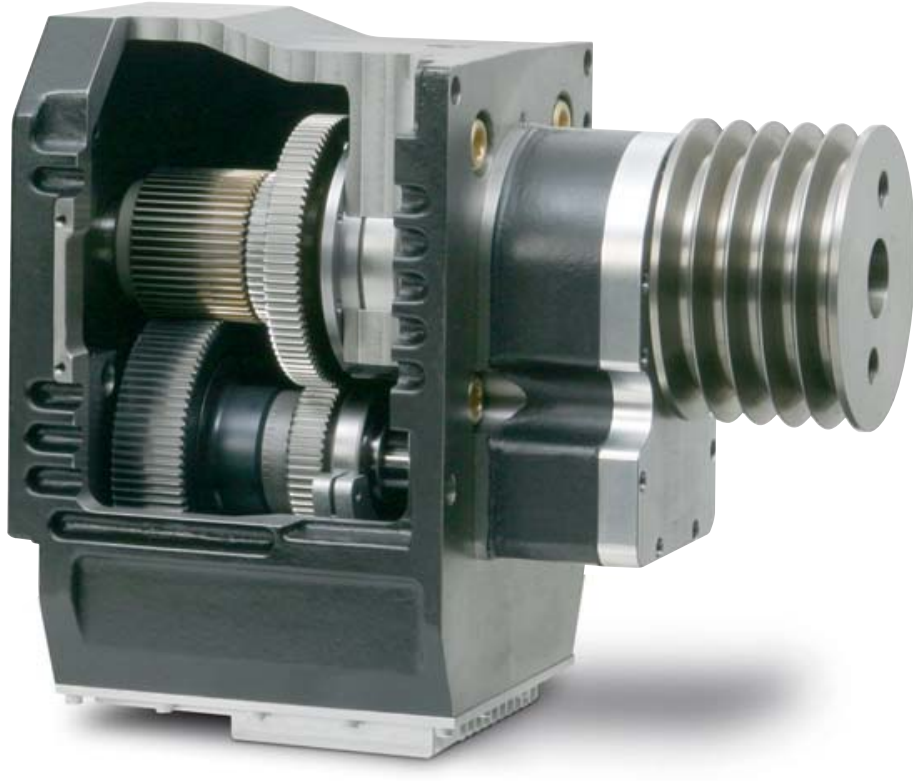

Spindle Options

\section{5,000-rpm / 30-hp Spindle}

For high-speed turning of smaller parts, the 5,000rpm/30-hp option for the SL-20 and TL-15 reduces cycle times and provides high surface feedrates. The 30-hp vector drive spindle provides faster acceleration to full speed, and yields more torque at higher rpm.

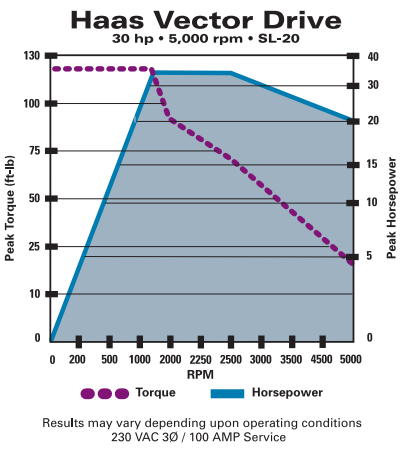

\section{7,000-rpm / 20-hp Spindle}

When even higher spindle speeds are needed, a smooth-running 7,000-rpm/20-hp option is available for the SL-20 and TL-15. This 20-hp vector drive spindle has an A2-5 spindle nose, and comes with a 5" chuck with 1.3" bar capacity. It accelerates quickly to top speed, and yields the high surface feedrates needed for excellent finishes on small parts.

\section{5-hp Extra-Performance Option}

Available for the SL-40 and SL-40L Long Bed, this option boosts spindle power to $55 \mathrm{hp}$, with $2,100 \mathrm{ft}-\mathrm{lb}$ of torque. It includes a larger servomotor on the $\mathrm{Z}$ axis for increased $\mathrm{Z}$-axis thrust of $7,847 \mathrm{lb}$. 


\section{Motion Control}

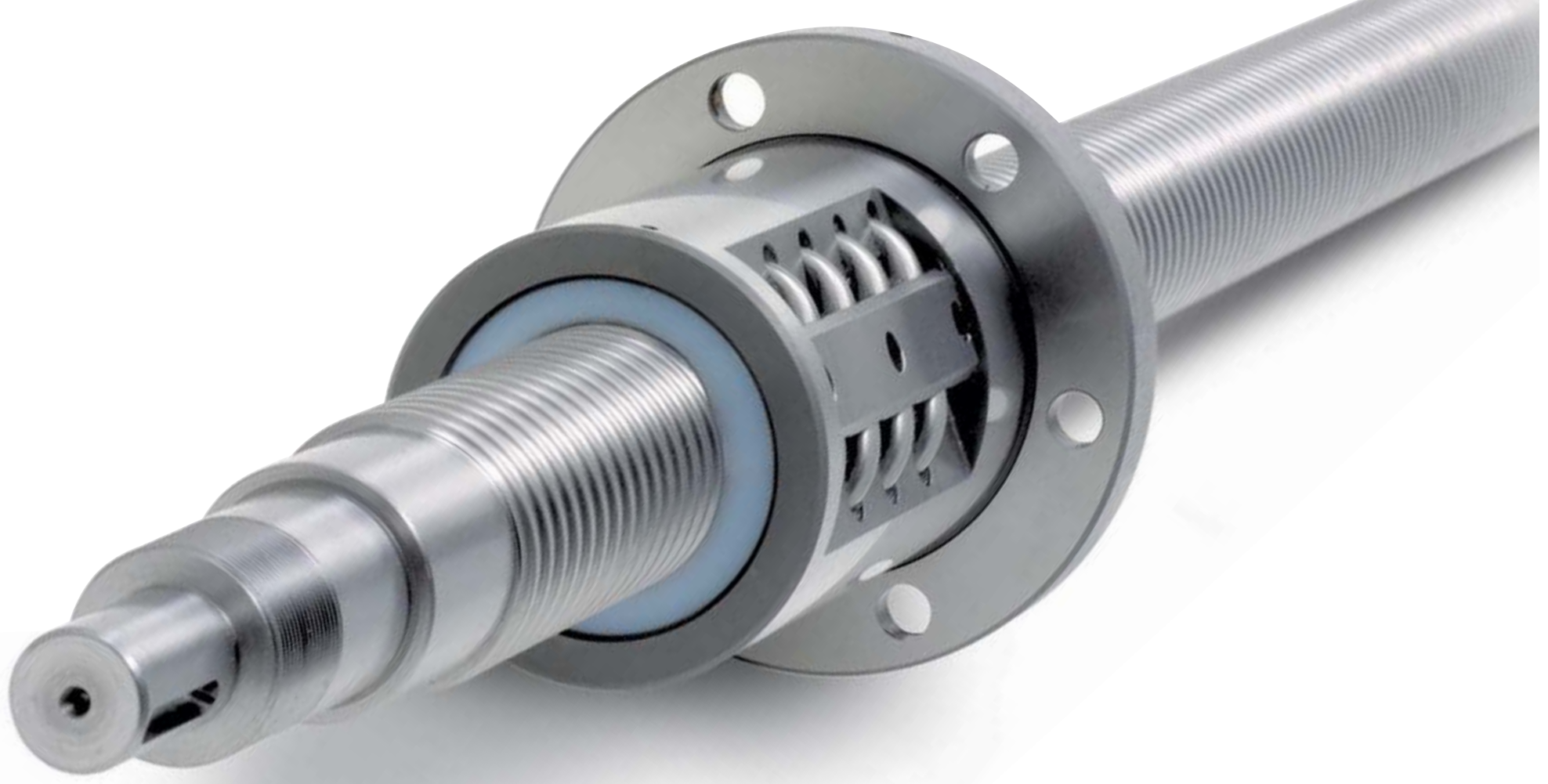

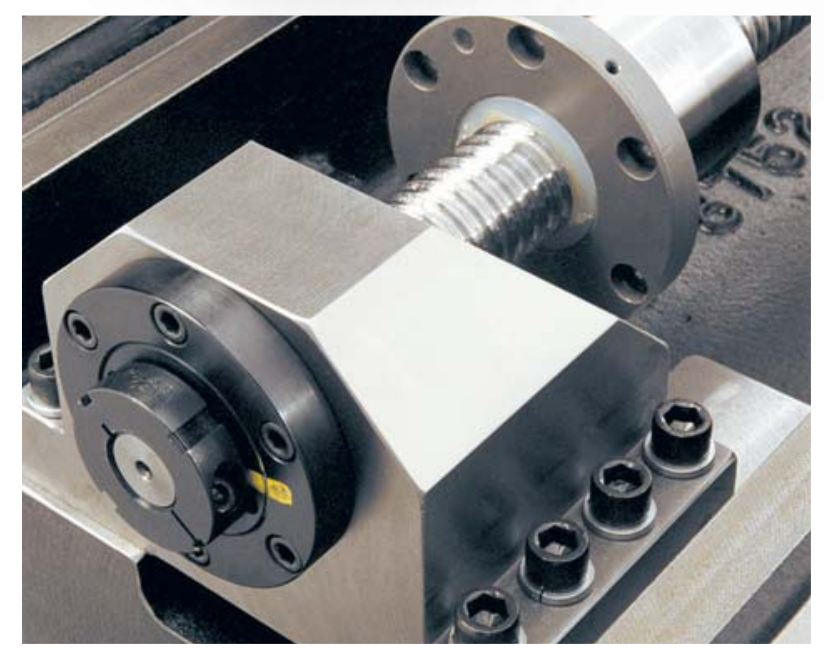

\section{Double-Anchored Ballscrews}

Haas uses only premium-quality ballscrews and guides from the world's top manufacturers. Although these suppliers are more expensive than our competitors' sources, their premium quality is the only way to achieve the high accuracy and long life that our customers demand. Our ballscrews are anchored at both ends and inspected for $100 \%$ parallelism to the axis guides. Preloaded ball nuts are used to eliminate backlash.

\section{Thermal Stability/ Temp Track}

Our temperature-tracking system uses a temperature probe attached to the ballscrew nut combined with electronic modeling to compensate for thermal growth. Temp-Track monitors temperature variations of the ballscrew and uses a sophisticated control algorithm to electronically adjust for thermal expansion, ensuring that the machine maintains accuracy and repeatability throughout the day.

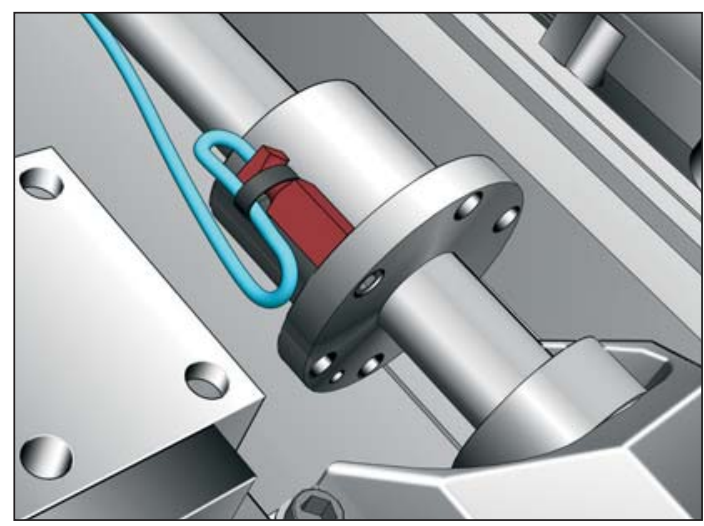




\section{Recirculating Ball Guideways}

Haas uses recirculating ball guideways for each axis of every CNC turning center. These guideways are preloaded for zero clearance, and provide full loadcarrying capacity in all directions. They consume less power, do not require adjustment and are proven to outperform sliding box ways for accuracy and speed. Each guideway is automatically lubricated to guarantee long life.
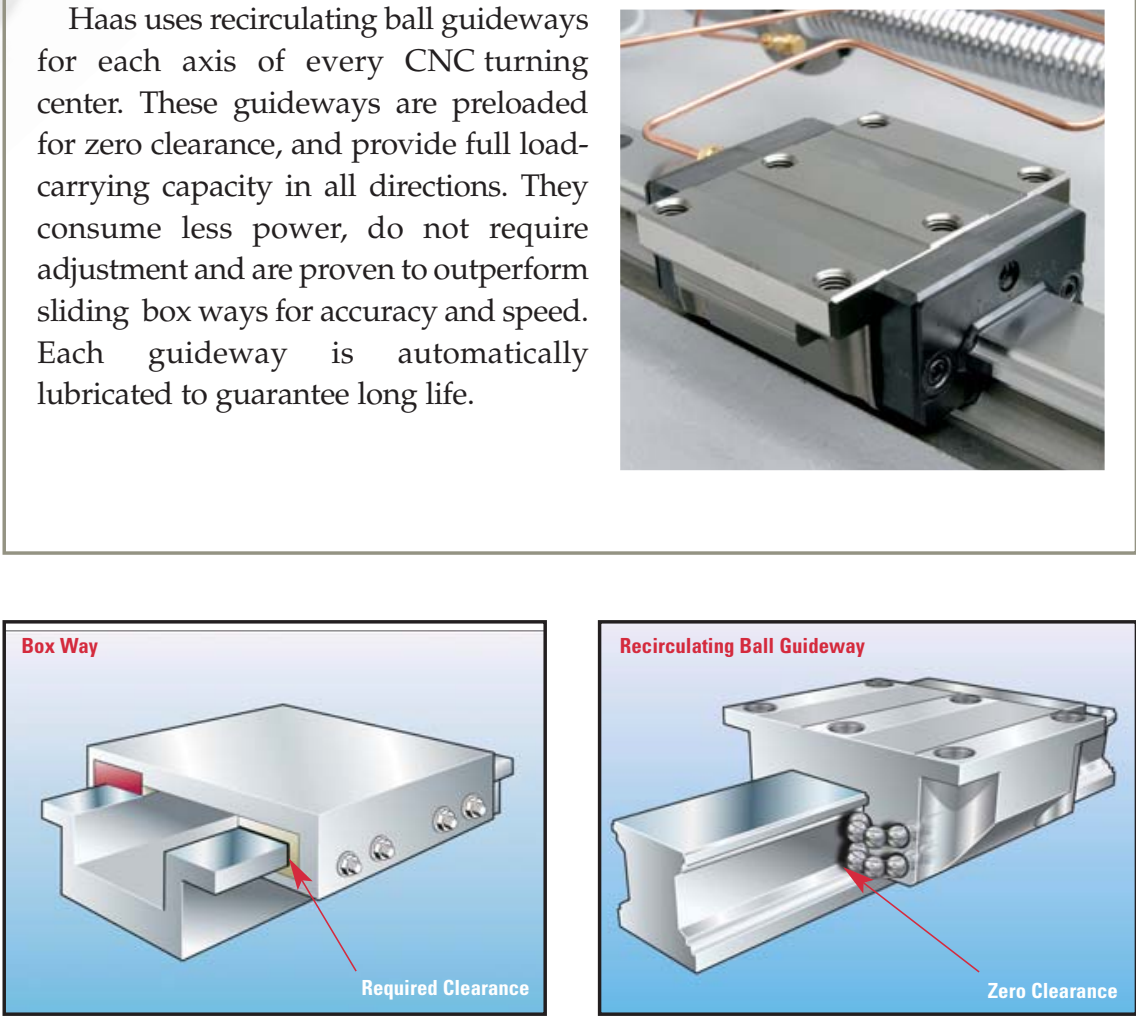

\section{Recirculating Ball Guideways Outperform Box Ways}

Recirculating ball guideways are preloaded to provide zero clearance between the moving surfaces. This increases rigidity and stiffness, while providing higher accuracy and reliability. Box ways require clearance between the moving surfaces in order to operate. Recirculating ball guides have a very low coefficient of friction, which allows faster movements without sacrificing repeatability or positioning accuracy. Box ways have a high coefficient of friction, which results in stick-slip effects that can lead to machine errors.

\section{Automatic Lubrication}

The Haas automatic lubrication system meters and distributes oil to guideways and ballscrews as the machine operates. This ensures that all critical components are lubricated with the correct amount of oil at all times, saving time and reducing maintenance costs.

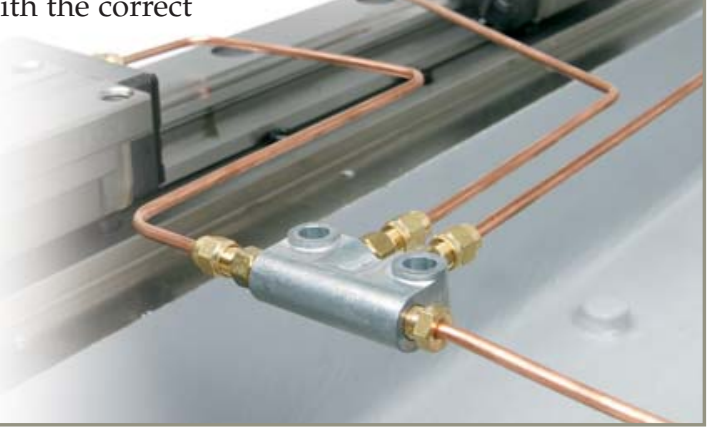

\section{Brushless Servomotors}

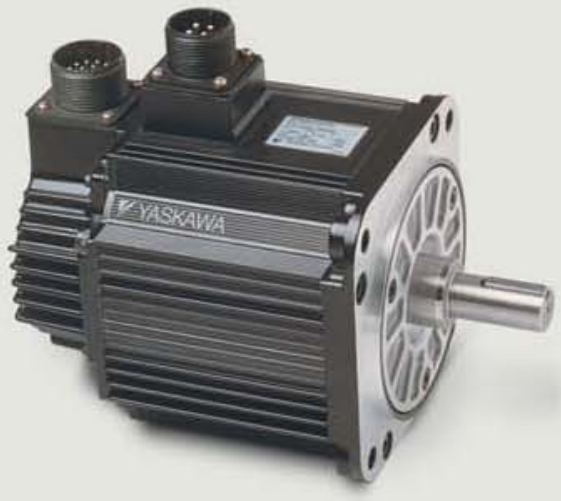

Haas uses state-of-the-art brushless servomotors that offer more torque in a smaller frame than almost any other motor available. These high-performance servos provide more power for heavy cuts, and higher acceleration/deceleration rates to reduce cycle times. With no brushes to wear and generate dust, maintenance is reduced and reliability is substantially increased. These compact motors also run cooler, have a higher duty rating and perform longer than brushtype motors.

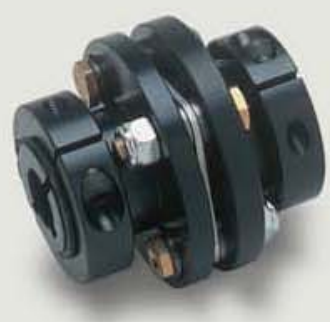

\section{Haas Direct-Coupled Servomotors}

Our servomotors are directly coupled to the ballscrews with steel disc couplings to eliminate windup - even under severe loading. This results in greatly improved positioning accuracy, and more accurate threading and contouring. Unlike belt- or rubber-coupled joints, our couplings don't wear out or lose accuracy over time. 


\section{Quick Setup \& Part Support}

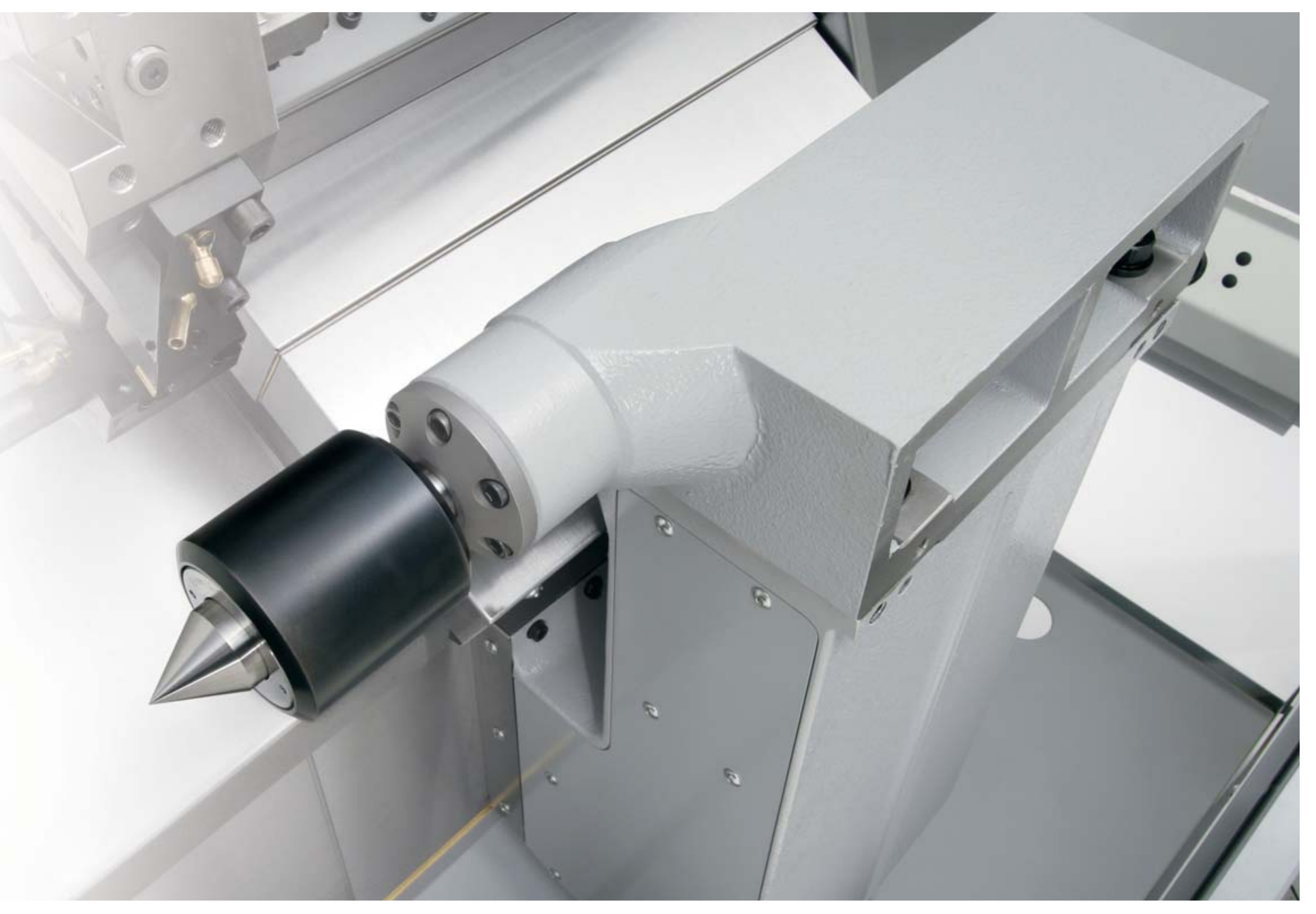

\section{Programmable Hydraulic Taillstock}

The Haas fully programmable hydraulic tailstock can be activated via the part program or controlled directly by the operator with the standard foot switch. Closedloop positioning allows you to stop anywhere along the tailstock's travel. Massive ribbed castings provide superior rigidity, and a two-piece design simplifies realignment if required. (The optional tailstock for the SL-10 features manual coarse adjustment and a hydraulic quill.)

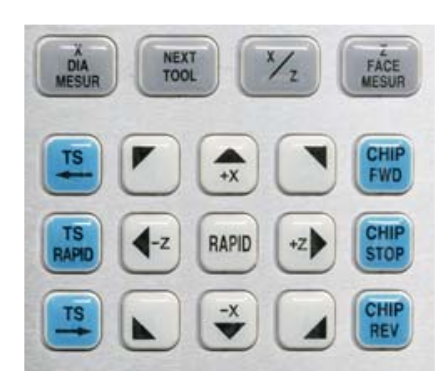

Simple three-step setup procedure saves time and lets you fine tune the working position for the tailstock. Retract, advance and hold point are easily specified, and the system also has an automatic missing part detection feature.

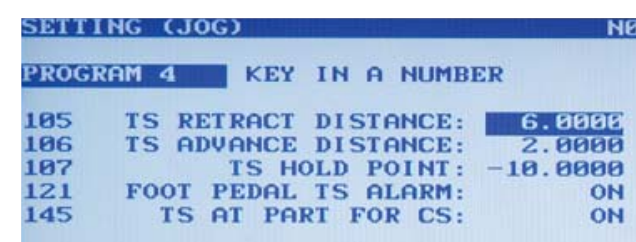




\section{Big-Bore Capacity}

The Haas Big Bore option provides larger bar capacity and more power without moving up to a larger machine. For the SL-10, SL-20 and SL-30, this is done by taking the spindle and drive system off the next larger machine and putting it on the smaller platform. The SL-20 Big Bore, for example, gets the SL-30's drive system, and the SL-30 Big Bore gets the SL-40's drive system. The SL-40 Big Bore maintains the same drive system, but with higher ratio gearing, and upgrades to an A2-11 spindle with up to 7.0" bar capacity (6.5" with a draw tube).

\begin{tabular}{|c|c|c|c|c|c|c|c|}
\hline Model & SL-10 & SL-20 & TL-15 & SL-30 & TL-25 & SL-40 & SL-40L \\
\hline Std Bore & $\begin{array}{l}1.75^{\prime \prime} \\
44 \mathrm{~mm}\end{array}$ & $\begin{array}{l}2.0 " \\
51 \mathrm{~mm}\end{array}$ & $\begin{array}{l}2.0 " \\
51 \mathrm{~mm}\end{array}$ & $\begin{array}{l}3.0 " \\
76 \mathrm{~mm}\end{array}$ & $\begin{array}{l}3.0^{\prime \prime} \\
76 \mathrm{~mm}\end{array}$ & $\begin{array}{l}4.0 " \\
102 \mathrm{~mm}\end{array}$ & $\begin{array}{l}4.0 " \\
102 \mathrm{~mm}\end{array}$ \\
\hline Big Bore & $\begin{array}{l}2.0 " \\
51 \mathrm{~mm}\end{array}$ & $\begin{array}{l}2.5 " \\
64 \mathrm{~mm}\end{array}$ & $\begin{array}{l}2.5^{\prime \prime} \\
64 \mathrm{~mm}\end{array}$ & $\begin{array}{l}4.0 " \\
102 \mathrm{~mm}\end{array}$ & $\begin{array}{l}4.0 " \\
102 \mathrm{~mm}\end{array}$ & $\begin{array}{l}7.0^{\prime \prime \dagger} \\
178 \mathrm{~mm}\end{array}$ & $\begin{array}{l}7.0 " \dagger \\
178 \mathrm{~mm}\end{array}$ \\
\hline
\end{tabular}

\section{SL-40 Extra- Performance Option}

Available for the SL-40 and SL-40L Long Bed, this option boosts spindle power to $55 \mathrm{hp}$, with $2,100 \mathrm{ft}-\mathrm{lb}$ of torque. It includes a larger servomotor on the $\mathrm{Z}$ axis for increased $\mathrm{Z}$-axis thrust of $7,847 \mathrm{lb}$.

\section{Steady Rest Provision}

The Haas steady rest platform provides increased support for turning and boring long shafts on SL-30 and SL-40 lathes. It features industry-standard mounting holes to accept a variety of aftermarket automatic hydraulic steady rests. Hydraulic power is provided by the lathe, and the gripper is activated by $\mathrm{M}$ code.
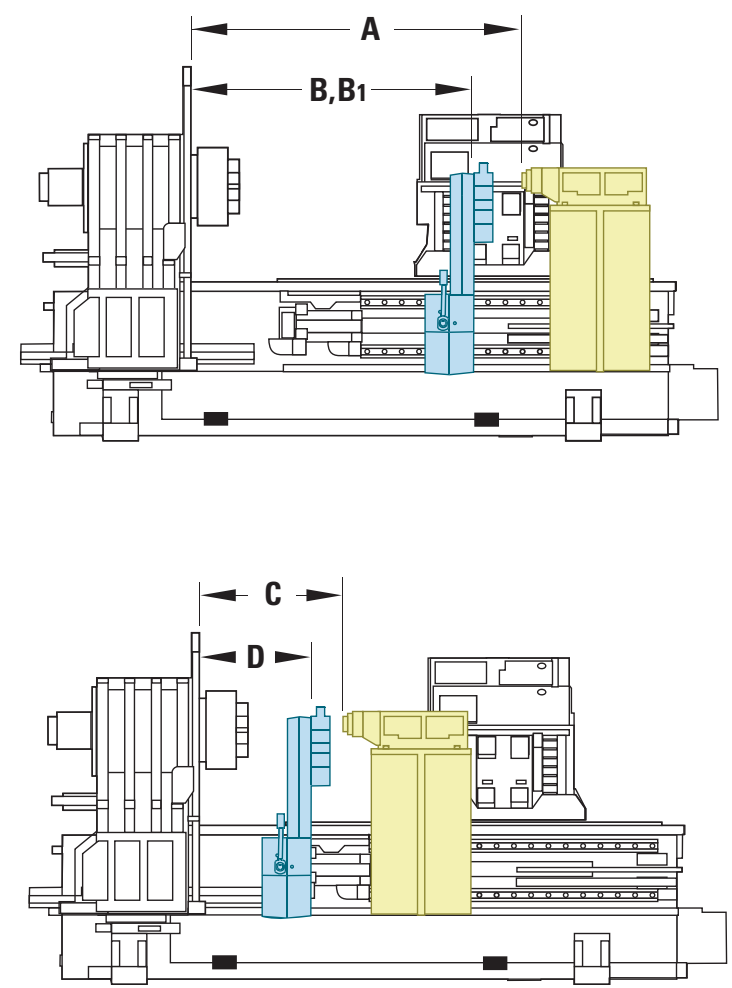

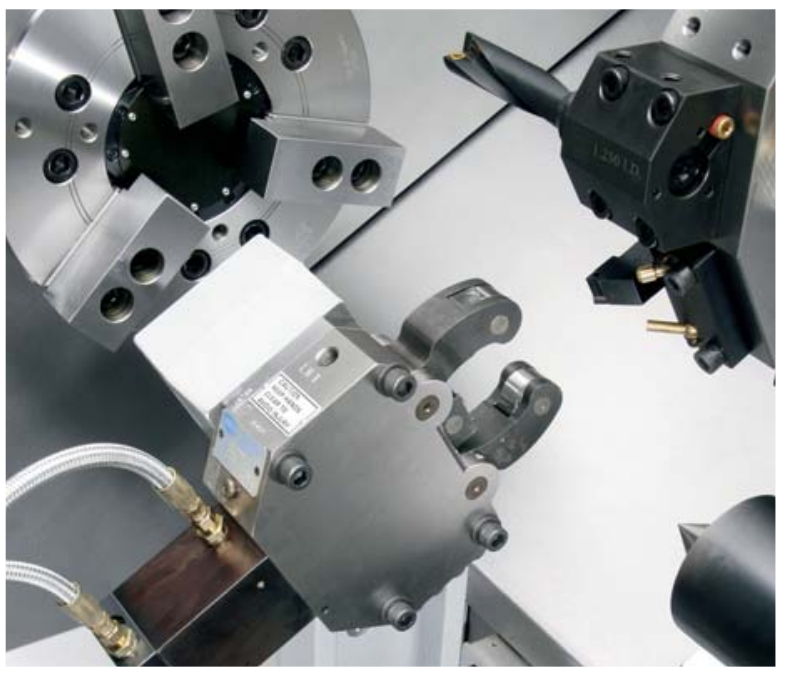

\begin{tabular}{llll}
\multicolumn{4}{c}{ Steady Rest and Tailstock Travels } \\
& SL-30 & SL-40 & SL-40L \\
\hline A & $51.8^{\prime \prime}$ & $59.4^{\prime \prime}$ & $94.9^{\prime \prime}$ \\
& $1316 \mathrm{~mm}$ & $1509 \mathrm{~mm}$ & $2410 \mathrm{~mm}$ \\
\hline B (w/tailstock) & $41.5^{\prime \prime}$ & $52.6^{\prime \prime}$ & $67.8^{\prime \prime}$ \\
& $1054 \mathrm{~mm}$ & $1336 \mathrm{~mm}$ & $1722 \mathrm{~mm}$ \\
\hline B (w/o tailstock) & $47.1^{\prime \prime}$ & $67.8^{\prime \prime}$ & $67.8^{\prime \prime}$ \\
& $1196 \mathrm{~mm}$ & $1722 \mathrm{~mm}$ & $1722 \mathrm{~mm}$ \\
\hline C (w/steady rest) & $26.6^{\prime \prime}$ & $33.3^{\prime \prime}$ & $38.0^{\prime \prime}$ \\
& $676 \mathrm{~mm}$ & $846 \mathrm{~mm}$ & $965 \mathrm{~mm}$ \\
\hline $\mathrm{D}$ & $20.9^{\prime \prime}$ & $26.5^{\prime \prime}$ & $26.5^{\prime \prime}$ \\
& $531 \mathrm{~mm}$ & $673 \mathrm{~mm}$ & $673 \mathrm{~mm}$
\end{tabular}

The steady rest provision does not include the gripper. Minimum and maximum part diameters are dependent on the size and style of gripper unit mounted on the platform. Minimum and maximum travel limits are measured from the spindle bulkhead, and do not include chuck dimensions or other workholding. 


\section{Part-Holding Variations}

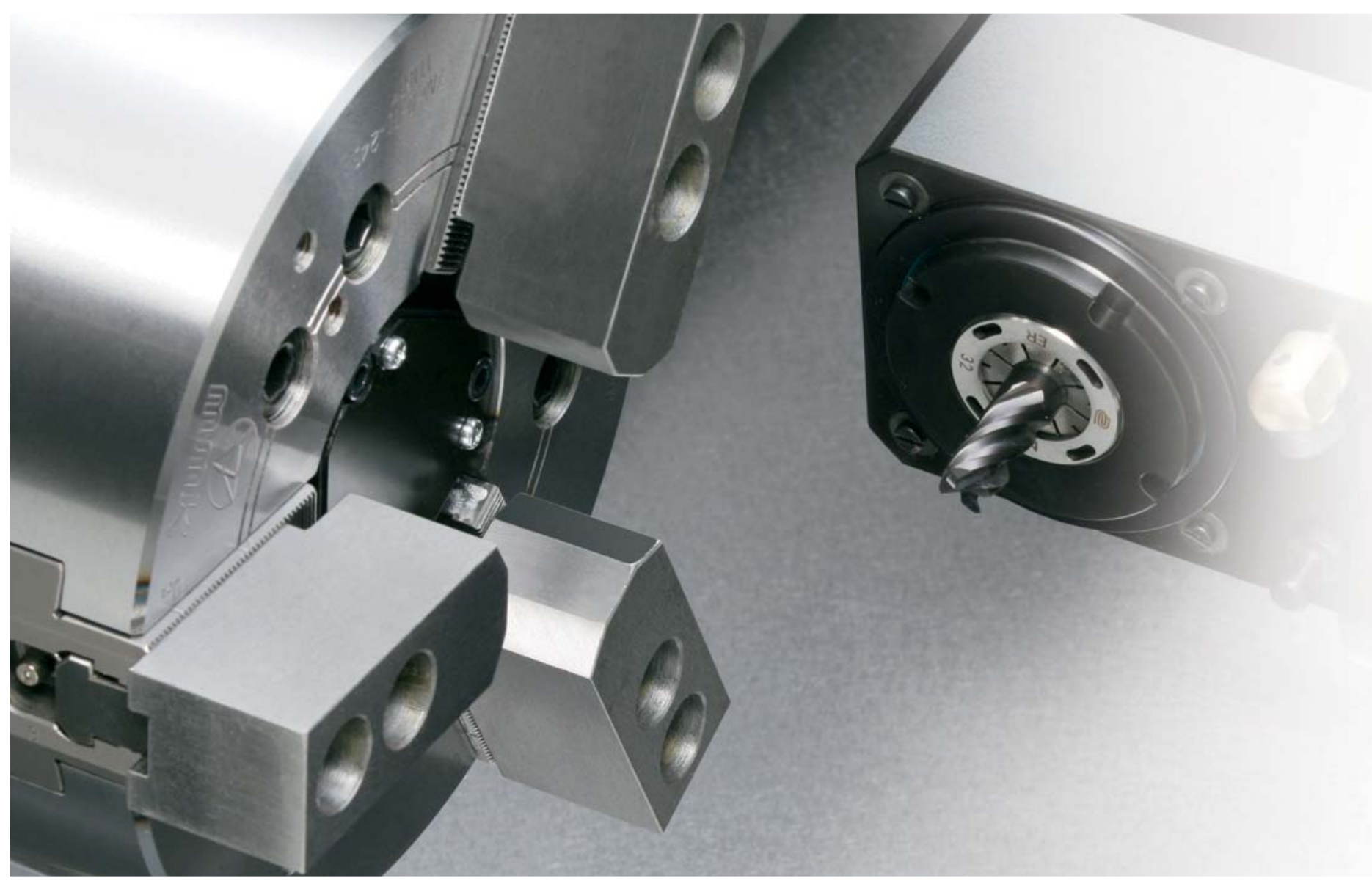

\section{Hydraulic Chuck}

Every standard Haas turning center comes with a hydraulic, through-hole chuck. The SL-10 comes equipped with a $6 "$ chuck, the SL-20 comes with an 8 " chuck, the SL-30 comes with a 10" chuck and the SL-40 comes with a 15" chuck. Bar capacities range from 1.75" for the standard SL-10 up to a massive 7.0" for the SL-40 with Big Bore.

\begin{tabular}{|c|c|c|c|c|c|c|c|}
\hline Model & SL-10 & SL-20 & TL-15 & SL-30 & TL-25 & SL-40 & SL-40L \\
\hline Standard Chuck & $\begin{array}{l}6.5^{\prime \prime} \\
165 \mathrm{~mm}\end{array}$ & $\begin{array}{l}8.3^{\prime \prime} \\
210 \mathrm{~mm}\end{array}$ & $\begin{array}{l}8.3^{\prime \prime} \\
210 \mathrm{~mm}\end{array}$ & $\begin{array}{l}10.0 " \\
254 \mathrm{~mm}\end{array}$ & $\begin{array}{l}10.0 " \\
254 \mathrm{~mm}\end{array}$ & $\begin{array}{l}15.0 " \\
381 \mathrm{~mm}\end{array}$ & $\begin{array}{l}15.0 " \\
381 \mathrm{~mm}\end{array}$ \\
\hline Big Bore Upgrade & $\begin{array}{l}8.3^{\prime \prime} \\
210 \mathrm{~mm}\end{array}$ & $\begin{array}{l}10.0 " \\
254 \mathrm{~mm}\end{array}$ & $\begin{array}{l}10.0 " \\
254 \mathrm{~mm}\end{array}$ & $\begin{array}{l}15.0 " \\
381 \mathrm{~mm}\end{array}$ & $\begin{array}{l}15.0 " \\
381 \mathrm{~mm}\end{array}$ & Optional † & Optional $\dagger$ \\
\hline
\end{tabular}

\section{Rear Chuck Options}

The SL-40BB and SL-40LBB models are available with optional rear chuck capability for gripping long, tubular parts at both ends of the spindle. Chuck not included. 


\section{Chip Removal}
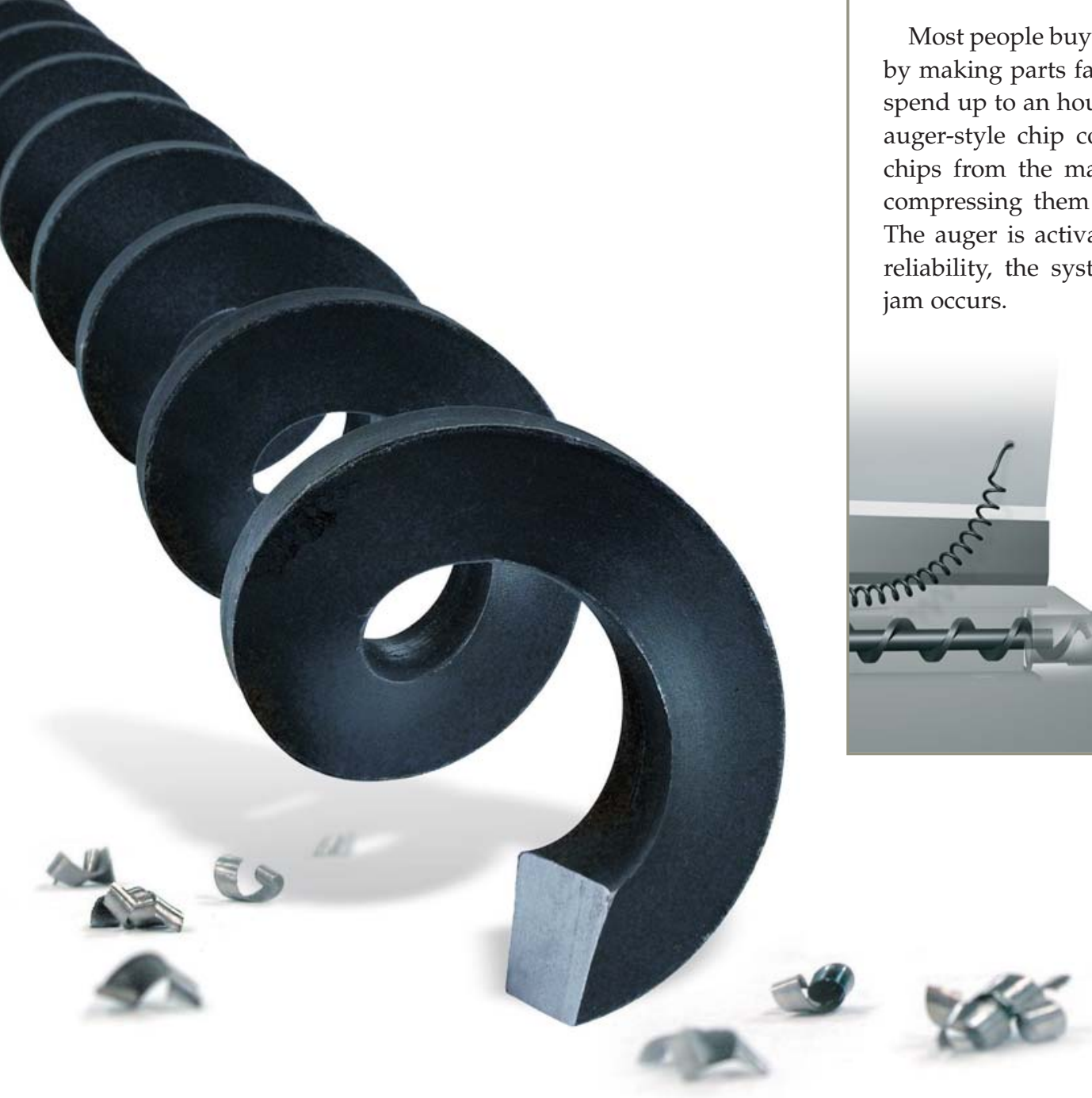

Most people buy a CNC machine to make money by making parts faster. Yet those same people will spend up to an hour per shift removing chips. Our auger-style chip conveyor automatically removes chips from the machine, while at the same time compressing them and wringing out the coolant. The auger is activated by $\mathrm{M}$ code, and for added reliability, the system reverses automatically if a jam occurs.

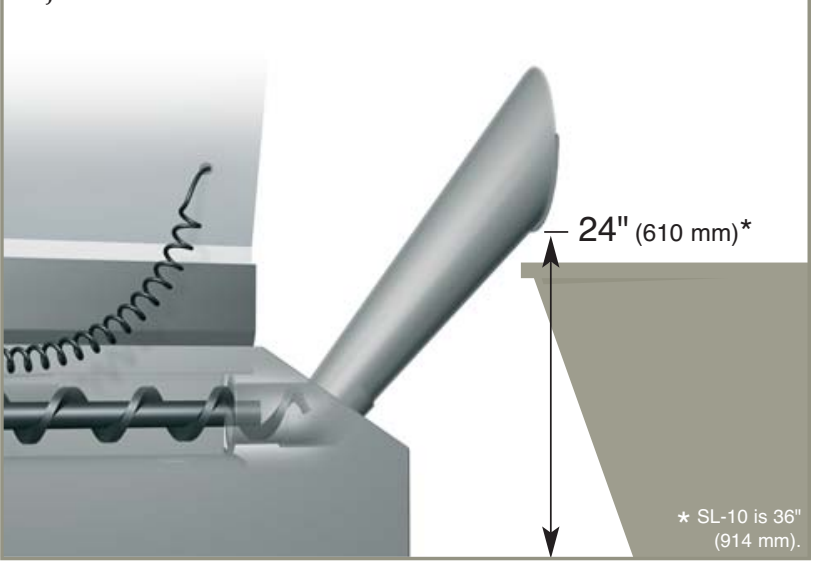

\section{Optional Chip Conveyor}

For high-production work using harder materials (such as tool steel or cast iron), a belt-type conveyor is available to clear chips from your machine's interior. Chips may be discharged into a standard 55-gallon (208 liter) drum.

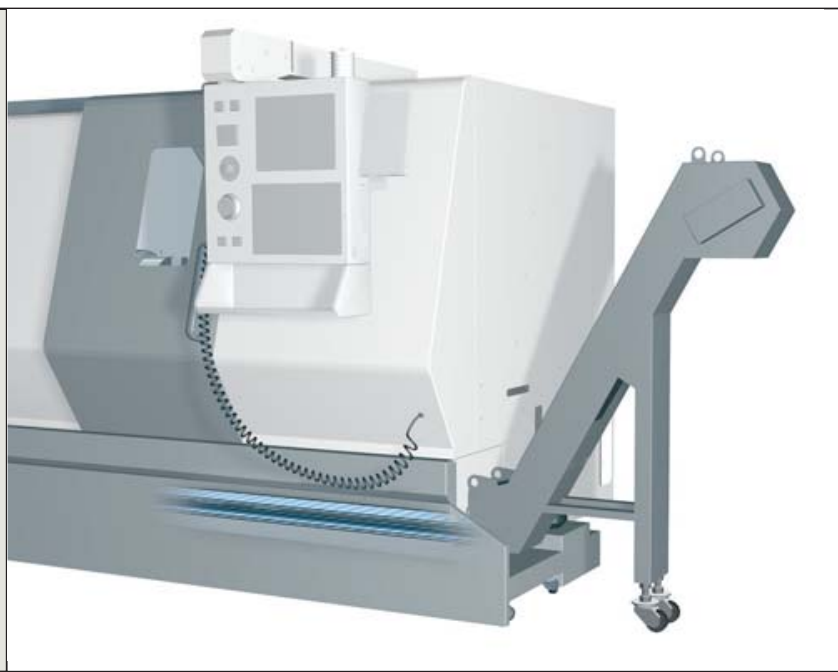




\section{Coolant System Features}

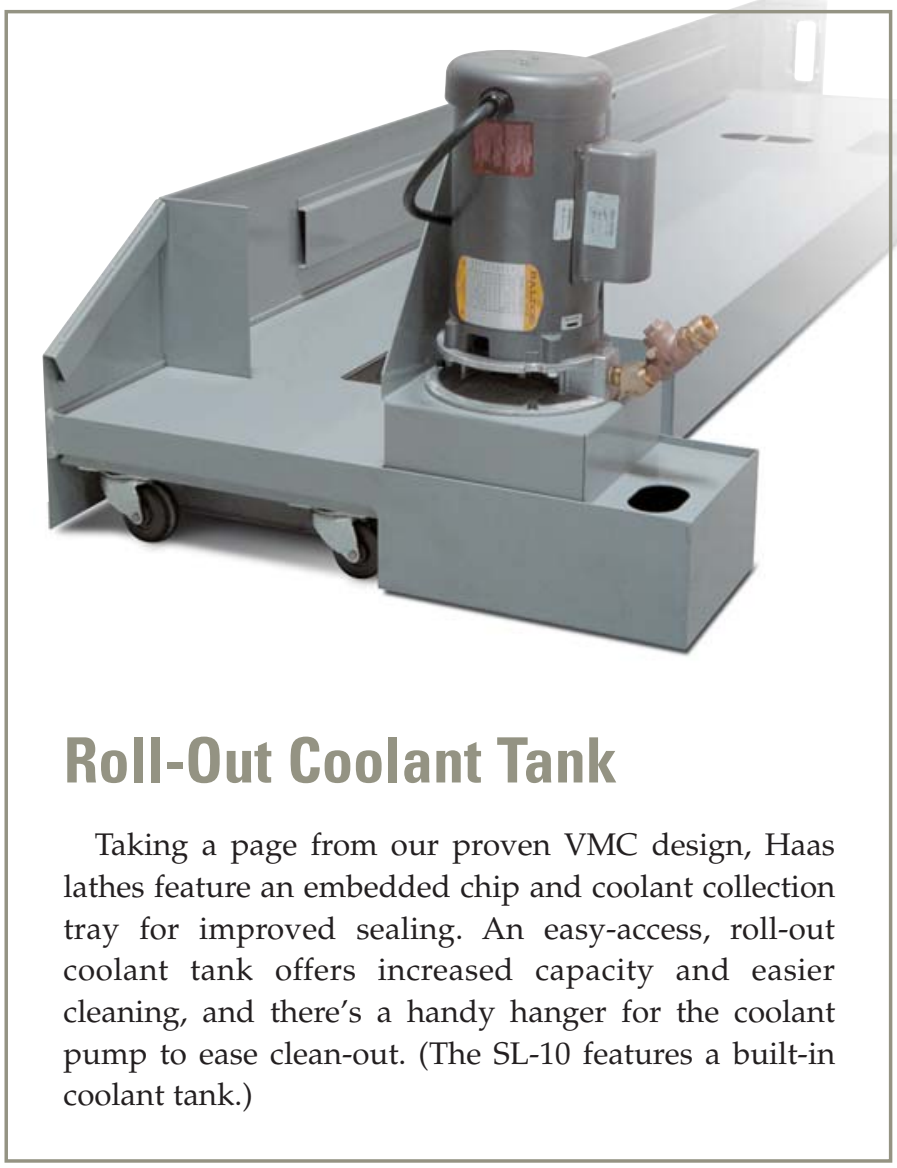

\section{Coolant Level Sensor}

A mechanical level sensor in the coolant tank allows you to monitor your coolant level directly on the control screen, preventing damaged tools

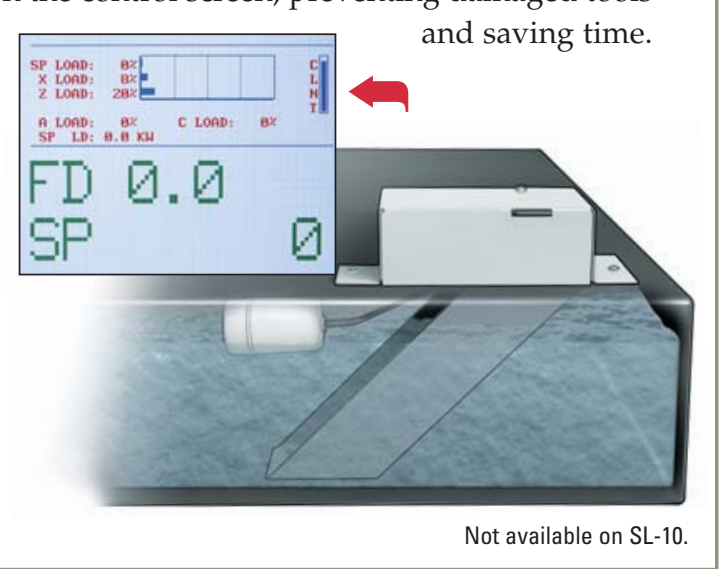

\section{Auto Jet Blast}

An M-code activated air

blast clears chips and

coolant from the chuck and workpiece while the doors are closed.

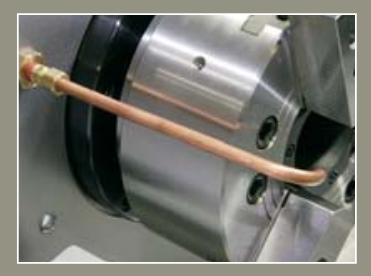

\section{High-Pressure Coolant}

Our high-pressure coolant systems provide up to 300 psi or 1,000 psi of coolant to the cutting edge for deep-hole drilling, heavy cuts, faster feedrates and better surface finishes. The standard HPC

option provides up to 300 psi at a flow rate of 1.5 gallons per minute, and 250 psi at $4 \mathrm{gpm}$. The 1,000-psi option (shown at left) has a max flow rate of $6 \mathrm{gpm}$, and requires separate (customer-supplied) 3-phase power. In both systems, actual pressure will vary with the diameter of the coolant passages in the tool.

\section{Auxiliary Coolant Filter}

This 25-micron, \#2 bag-type filter system removes contamination and minute particles from the coolant before they can be recirculated through the coolant pump. The filter is mandatory for HPC-equipped machines, but may be used on non-HPC machines as well. It is included with the 1,000 psi coolant option. 


\section{Autornated Operation Increase throughput by $200 \%$ or more.}

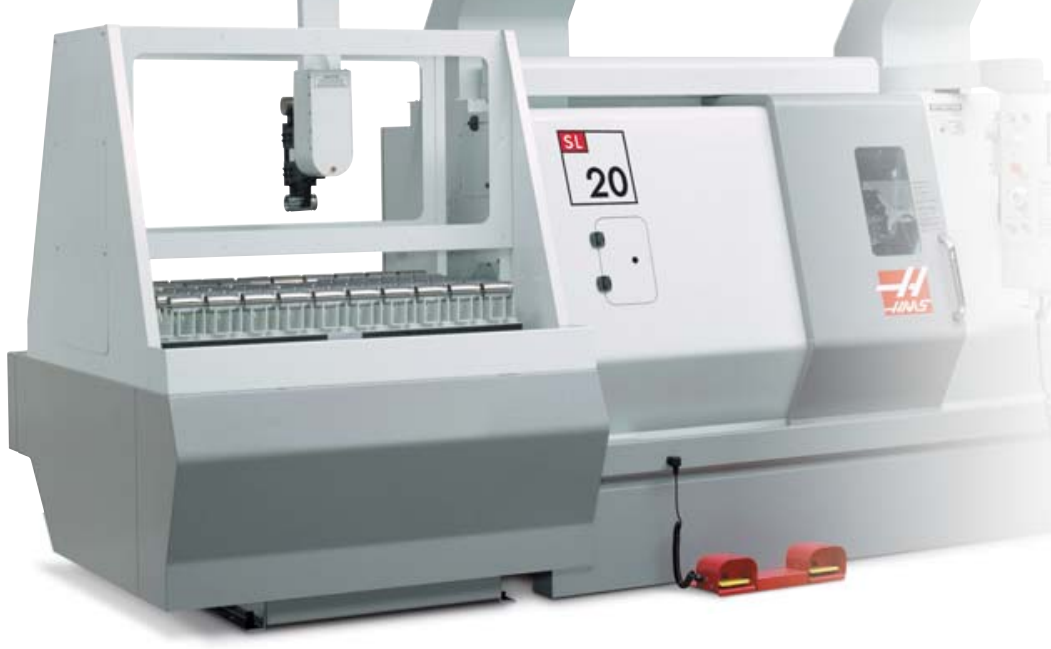

\section{SL-20APL Automatic Parts Loader}

The Haas APL is a fully automatic lathe parts loader with opposing two-jaw grippers. Two gripper configurations come standard: one for shaft work and one stacker system. The APL is programmed directly through the lathe's control, and operates in the background of normal turning operations to deliver nearcontinuous unattended machining. The APL's double-sided rotating gripper loads and unloads parts with a single trip to the spindle, or flips parts for double-ended turning. The APL is available only with a new SL-20 lathe.

\section{Features}

- Allows unattended operation

- Flexible gripper configurations

- Up to 5.0 " OD shafts

- Up to 7.0" OD disks

- Easy setup via the Haas control

- Loads/unloads parts in one trip

- Rotates parts for double-ended turning capability*

*Shaft loader

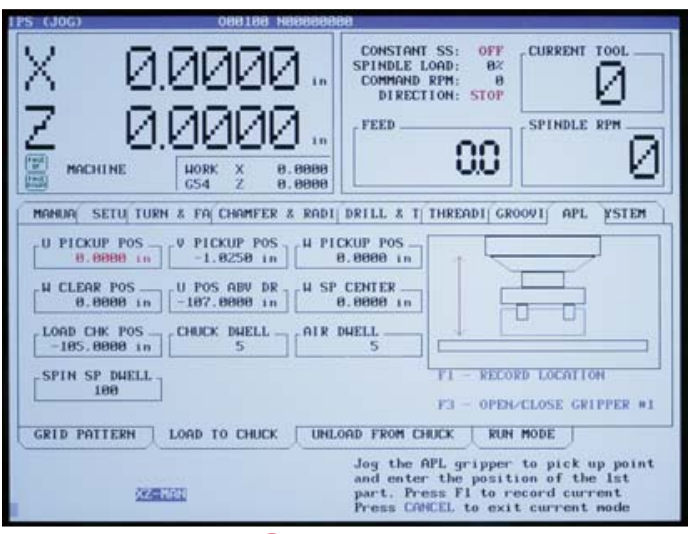

Simplified Setup

APL setup has been vastly simplified through Haas' Intuitive Programming System, which guides the operator through the steps necessary to quickly program the APL. Answering simple questions, the operator enters basic information into the system by either positioning the part grippers and pushing a single button or entering basic numeric dimensions. All values are calculated automatically by the control, and the APL is ready to go.
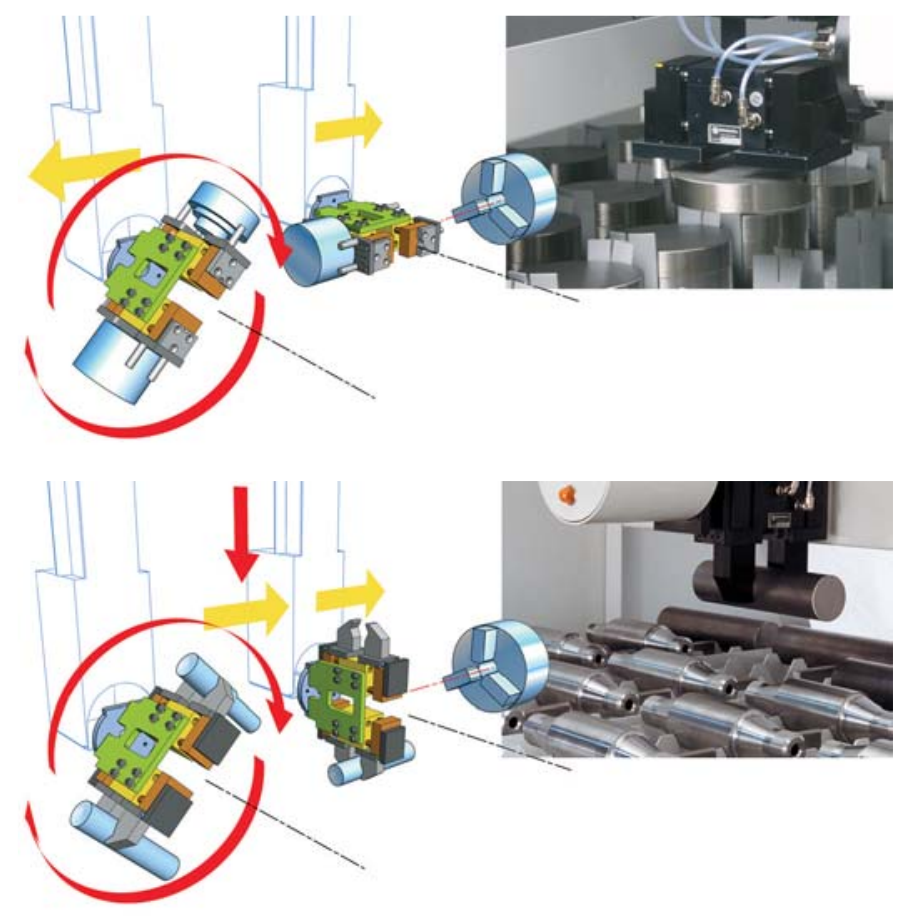


\section{Haas Servo Bar 300 Automatic Bar Feeder}

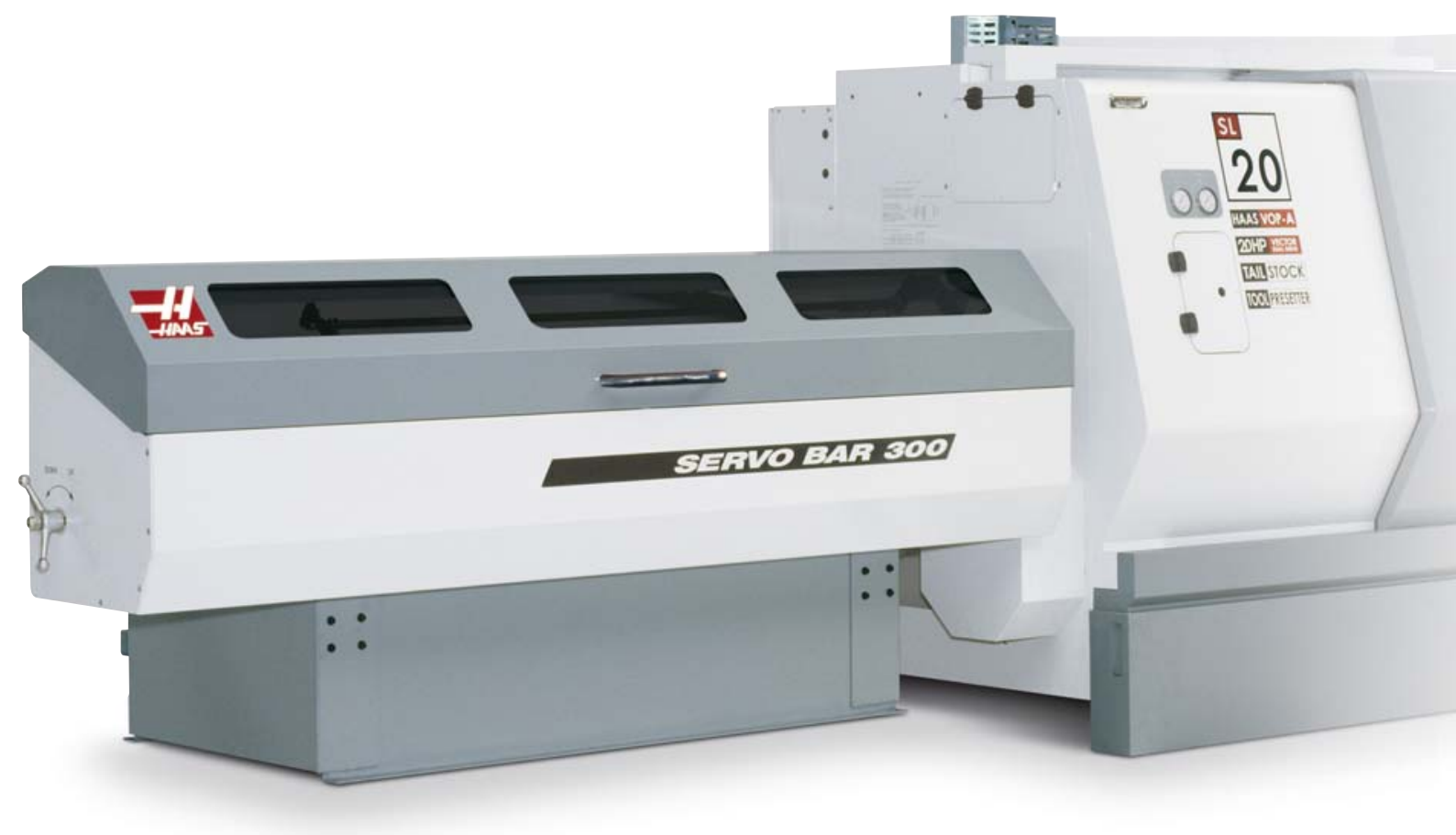

Designed to boost productivity and streamline turning operations, this servo-driven bar feeder is built by Haas exclusively for Haas $\mathrm{CNC}$ lathes. It runs directly from the Haas control, making it the only "smart" bar feeder on the market. Unique features make setup and operation simple, like a large access door for spindle liner change-out and a single adjustment for setting bar diameter. All bar feed parameters are set at the lathe control.

See page 37 for bar feeder specifications.

\section{Parts Catcher}

For bar feed applications, or when using a bar puller, the parts catcher rotates into position to catch the finished part and direct it into a bin located on the front door. There's no need to stop the machine and open the door to retrieve parts.

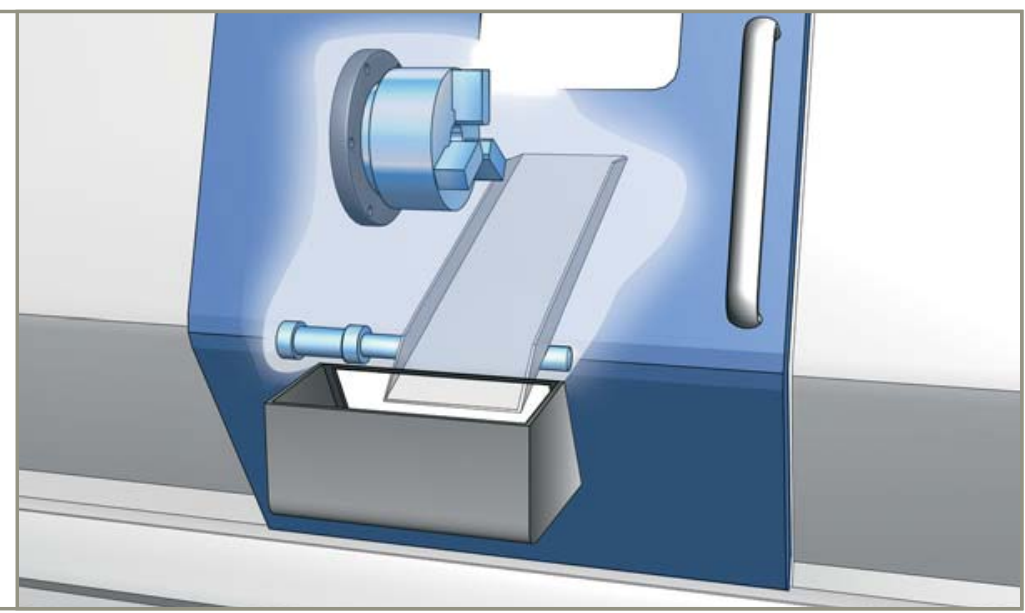




\section{Operator Convenience}

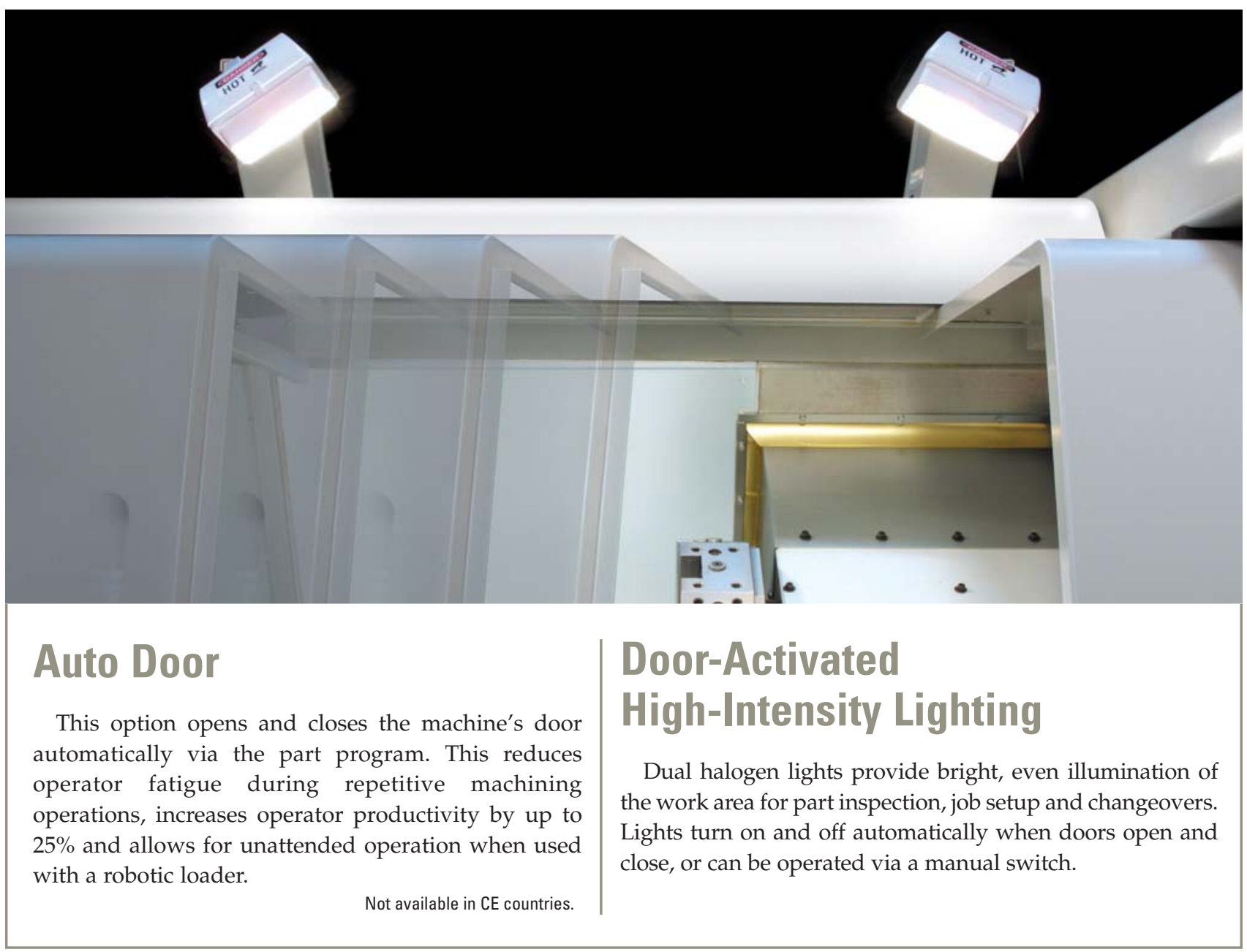

\section{Convenient Storage Box}

This covered box provides convenient storage for toolholders, cutting tools and accessories right at the machine, eliminating the need for a separate tool cart.

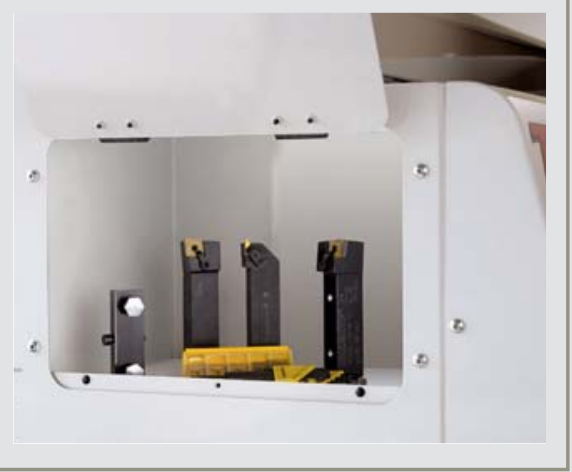

\section{Note Pad \& Disk Storage}

This handy bracket on the side of the control pendant holds a pad of paper for jotting notes, and has slots for holding pencils and storing up to 6 floppy disks.

\section{Storage Area}

Another operator-focused feature is this convenient "glove box" below the pendant. The hinged door folds down to form a handy shelf, and closes securely to keep items out of sight.
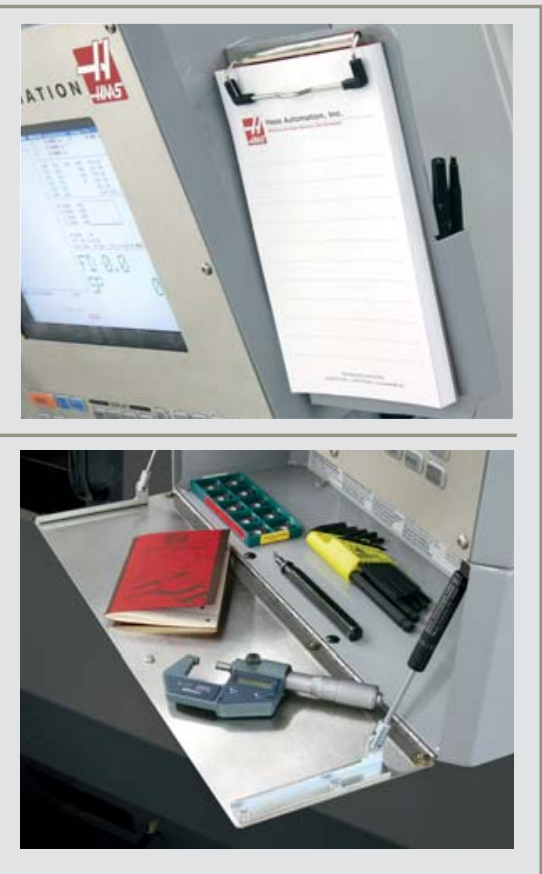


\section{Remote Jog Handle}

Extend your reach into the work envelope to simplify setup when working on our larger turning centers. Can be used to move all axes, as well as override spindle speeds and feedrates, single-block scroll through programs and more.

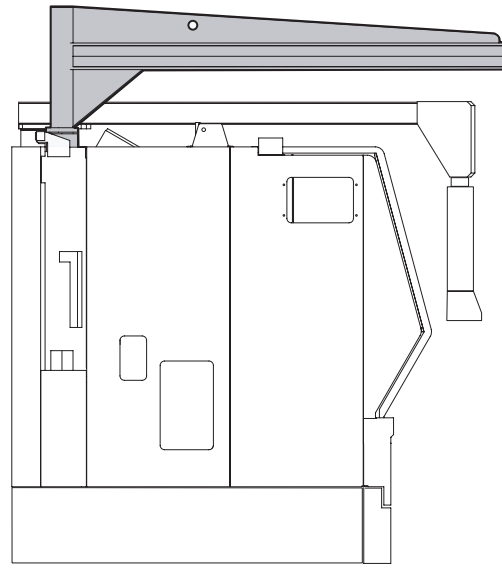

\section{Part Hoist Boom}

This convenient crane arm allows the operator to easily load raw materials and unload finished parts. It has a $300 \mathrm{lb}(136 \mathrm{~kg})$ capacity.

For TL-25, SL-30, SL-40 and SL-40L only. Hoist and trolley not included.

\section{Tool Presetter}

The manual tool-probe arm swings down for fast tool setting. Touch the tool tip to the probe, and offsets are automatically entered.

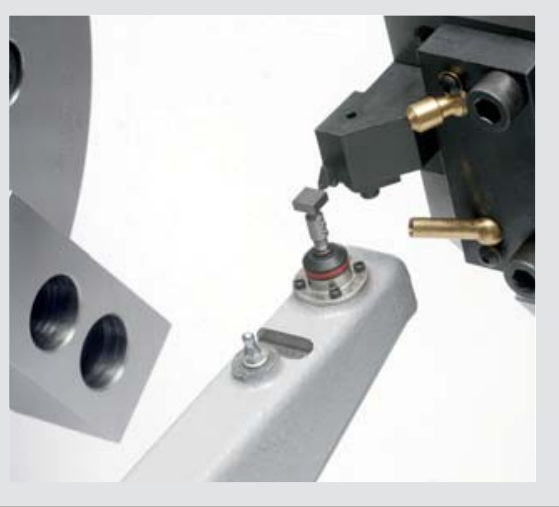

\section{Work Probing}

The Haas work probing option incorporates a turret probe, receiver and macros. This option can be used to perform rapid first-off part inspection, inprocess reporting and unattended machining operations. It can also be used to update work offsets to ensure ultimate part accuracy and keep tool wear in check.

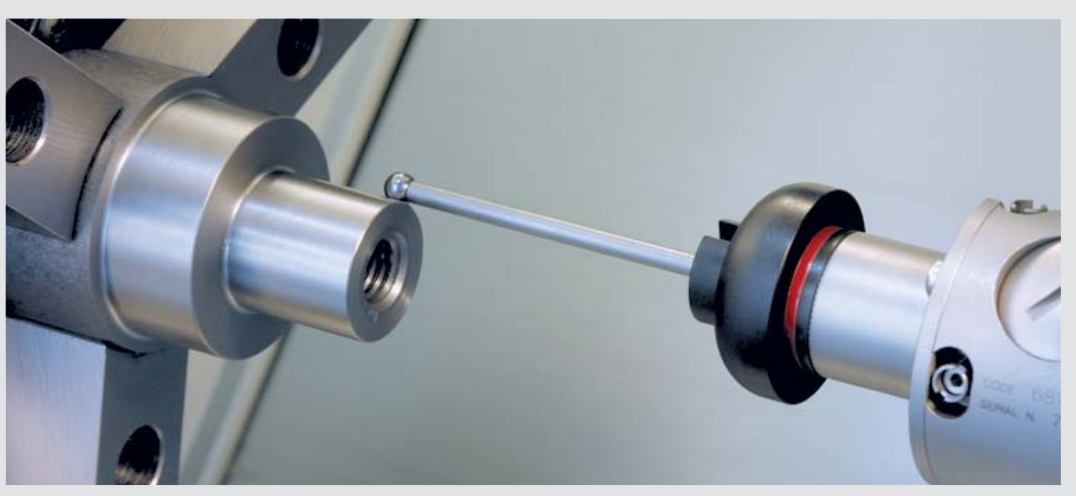



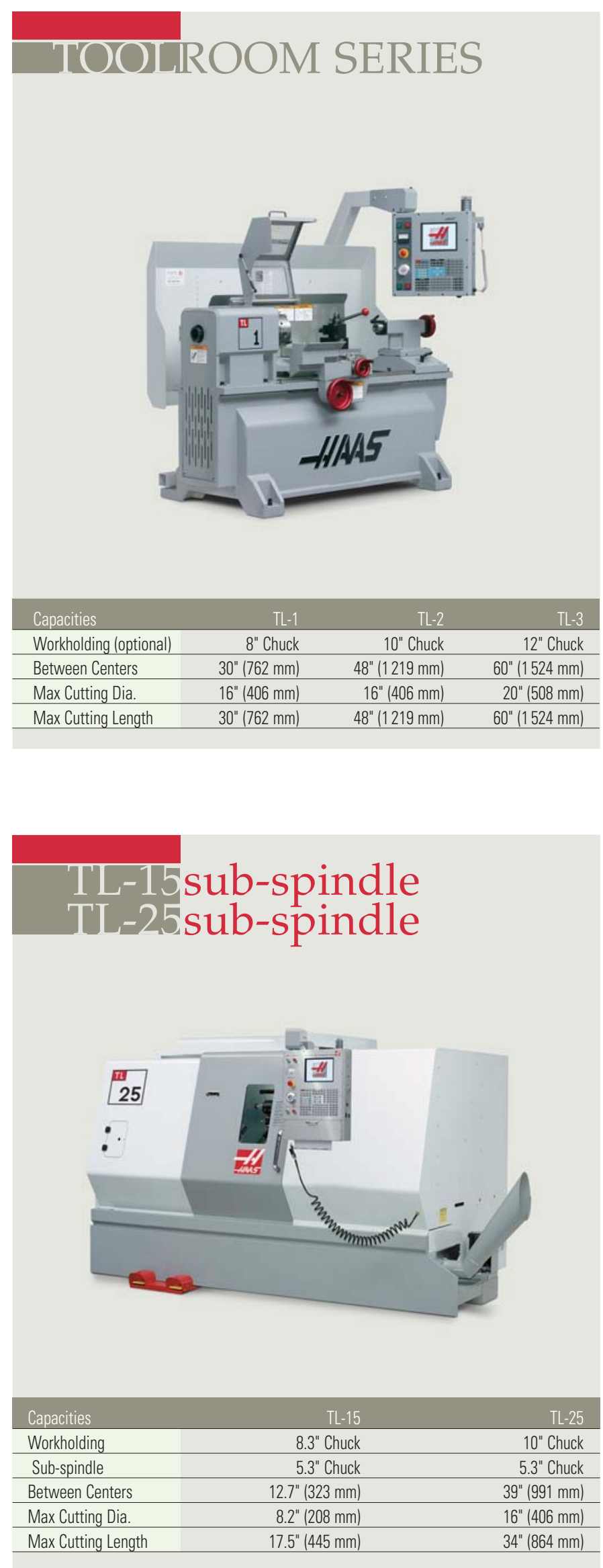

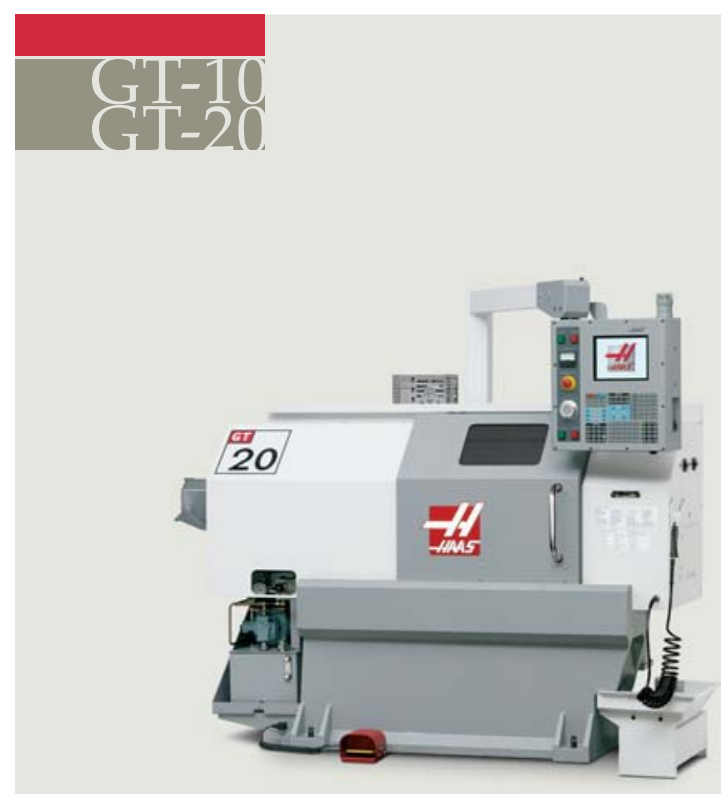

\begin{tabular}{lrr} 
Capacities & GT-10 & GT-20 \\
\hline Workholding (optional) & $5 C$ & $8.3^{\prime \prime}$ Chuck \\
\hline Max Cutting Dia. & $10 "(254 \mathrm{~mm})$ & $11^{\prime \prime}(279 \mathrm{~mm})$ \\
\hline Max Cutting Length & $8^{\prime \prime}(203 \mathrm{~mm})$ & $12 "(305 \mathrm{~mm})$ \\
\hline
\end{tabular}

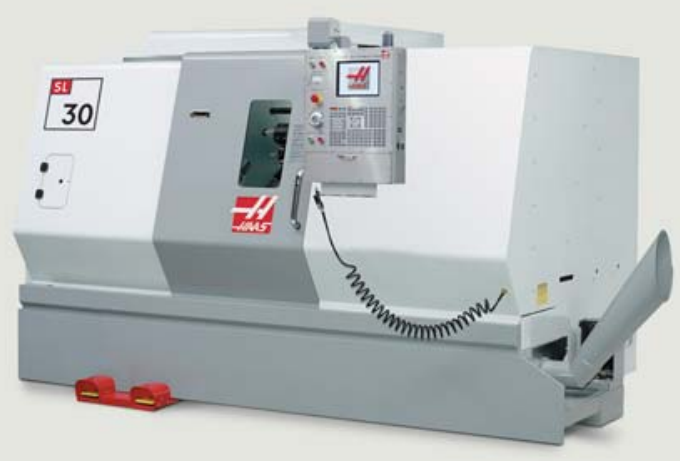

\begin{tabular}{lr}
\hline Capacities & SL-30 \\
\hline Workholding & $10 "$ Chuck \\
\hline Between Centers & $39 "(991 \mathrm{~mm})$ \\
\hline Max Cutting Dia. & $17^{\prime \prime}(432 \mathrm{~mm})$ \\
\hline Max Cutting Length & $34^{\prime \prime}(864 \mathrm{~mm})$ \\
\hline
\end{tabular}




\section{Model Variations}
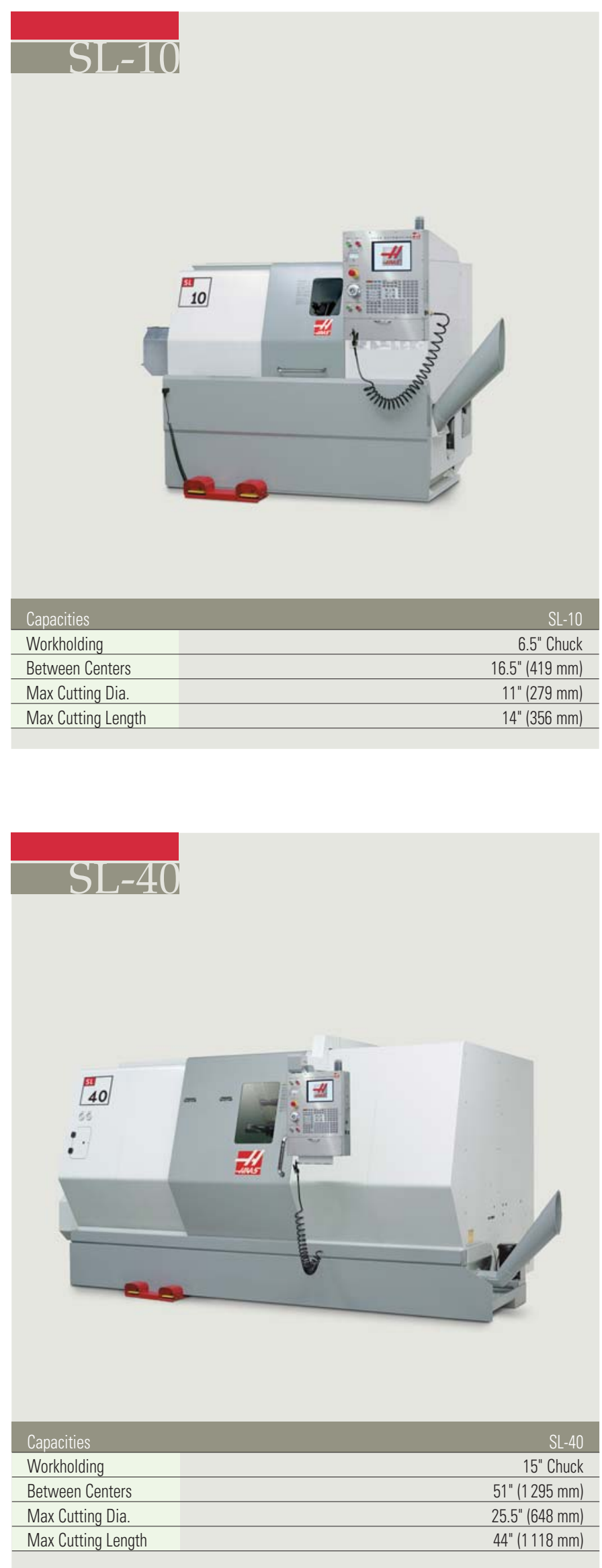
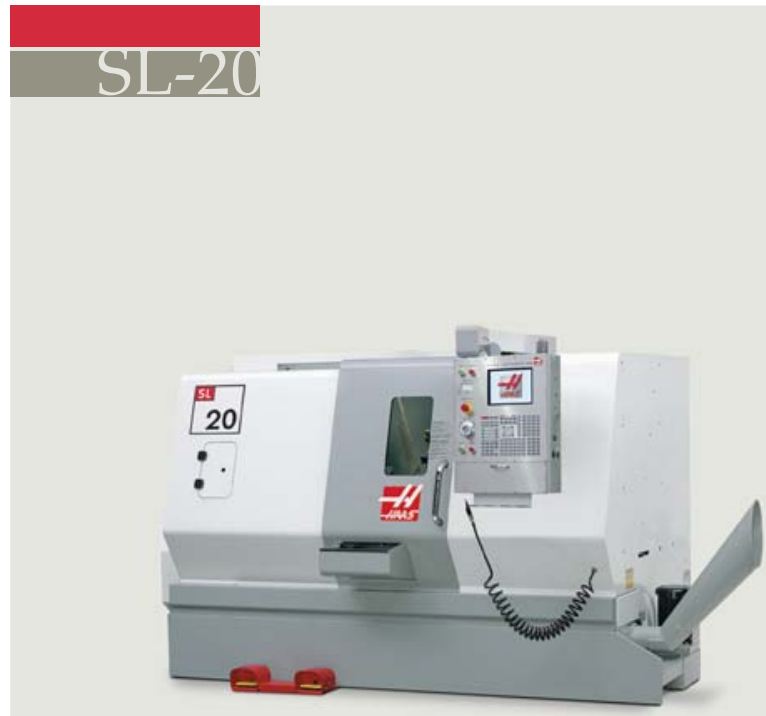

Capacities

SL-20

\begin{tabular}{lr}
\hline Workholding & $8.3^{\prime \prime}$ Chuck \\
\hline Between Centers & $24^{\prime \prime}(610 \mathrm{~mm})$ \\
\hline Max Cutting Dia. & $10.3^{\prime \prime}(262 \mathrm{~mm})$ \\
\hline Max Cutting Length & $20 "(508 \mathrm{~mm})$ \\
\hline
\end{tabular}

SL-40L

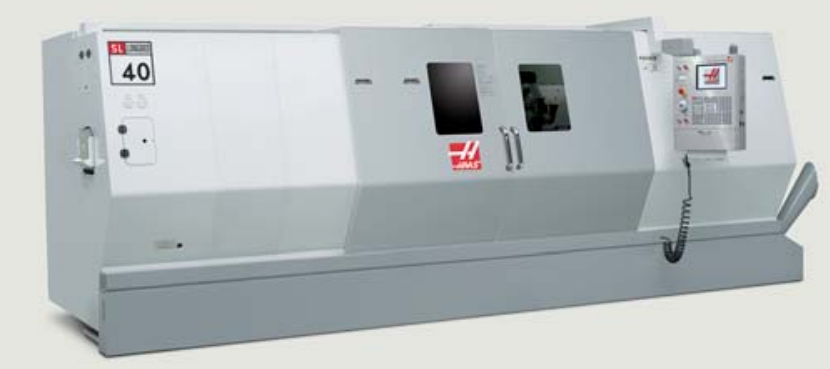

\begin{tabular}{lr} 
Capacities & SL-40L \\
\hline Workholding & $15 "$ Chuck \\
\hline Between Centers & $89 "(2261 \mathrm{~mm})$ \\
\hline Max Cutting Dia. & $25.5^{\prime \prime}(648 \mathrm{~mm})$ \\
\hline Max Cutting Length & $80 "(2032 \mathrm{~mm})$ \\
\hline
\end{tabular}




\section{Toolroom Solutions}

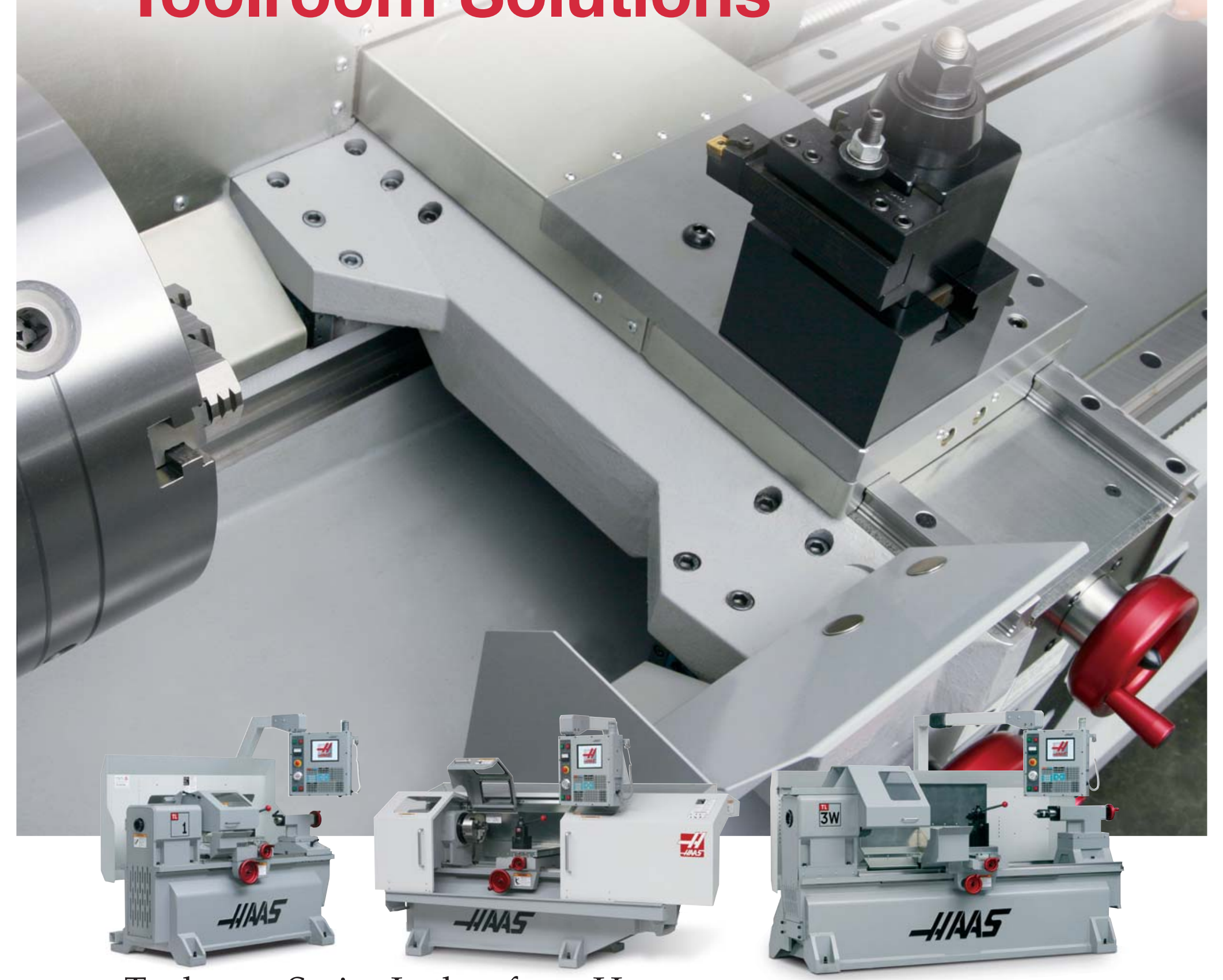

\section{Toolroom Series Lathes from Haas}

The Haas Toolroom Lathes combine the full functionality and simplicity of a manual lathe with the power and flexibility of the easyto-use Haas CNC system. With standard handwheels and highaccuracy DRO, manual machinists will be cutting parts in minutes. And with the new Haas Intuitive Programming System, they'll transition quickly and easily into automatic modes and full CNC. Even novice machinists will find the TL Series lathes very easy to learn and operate.
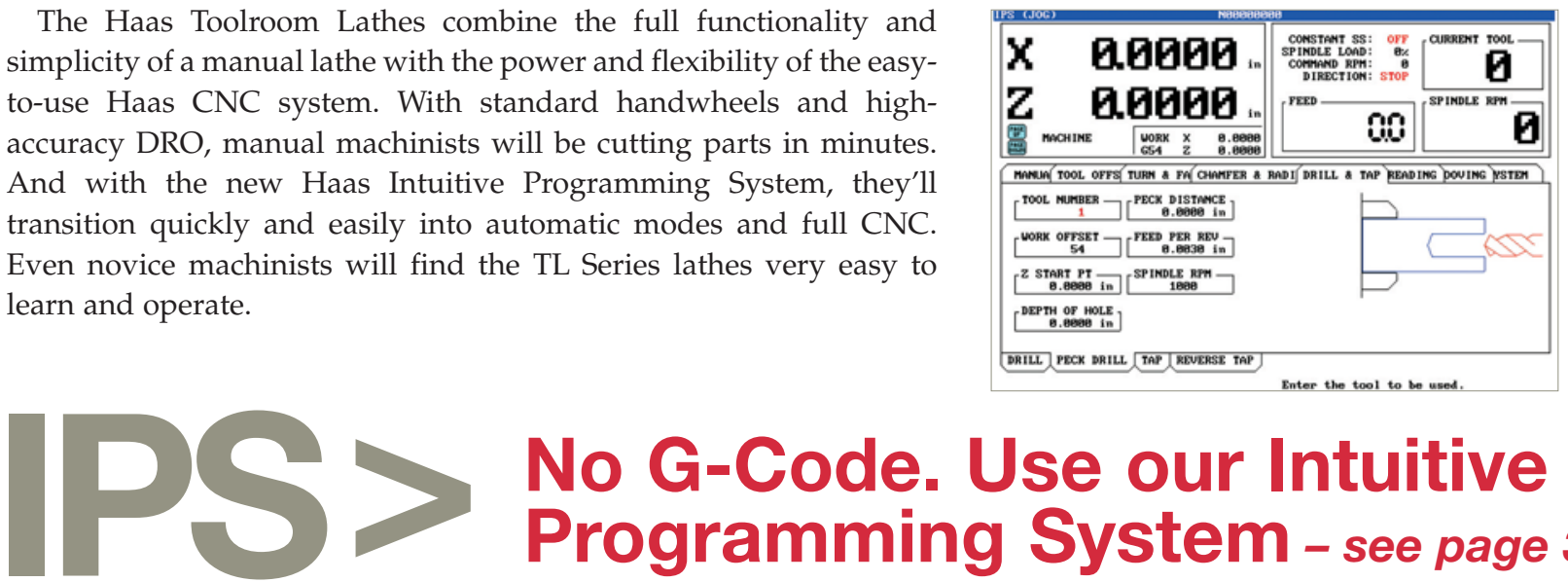

\section{No G-Code. Use our Intuitive Programming System - see page 32.}




\section{Operate Haas Toolroom Lathes in any one of four modes:}

In manual mode, standard handwheels move both axes, with accurate digital readout (DRO) of position. In automatic mode, the Toolroom Lathes have built-in cycles for profiling, chamfering, corner radius, grooving, threading, drilling and tapping. In recorder or "Teach" mode, a series of operations can be easily recorded, and then saved and/or edited for running the same part program to duplicate parts in the future. In full CNC mode, Haas Toolroom Lathes are programmed using standard G-code via the Haas control.

Toolrooms and small machine shops have long been bastions of manual machine tools. But to survive in today's competitive market, it is increasingly necessary for these shops to make the move to CNC. The Haas Toolroom Lathes ease the transition from manual machines to $\mathrm{CNC}$ - without breaking the bank.

\section{Toolroom Series Specifications}

\begin{tabular}{llll} 
Capacities & TL-1 & TL-2 & TL-3* \\
Workholding (optional) & 8" Chuck & $10^{\prime \prime}$ Chuck & $12^{\prime \prime}$ Chuck \\
\hline Between Centers & $30 "(762 \mathrm{~mm})$ & $48^{\prime \prime}(1219 \mathrm{~mm})$ & $60^{\prime \prime}(1524 \mathrm{~mm})$ \\
\hline Max Cutting Dia. & $16^{\prime \prime}(406 \mathrm{~mm})$ & $16^{\prime \prime}(406 \mathrm{~mm})$ & $20^{\prime \prime}(508 \mathrm{~mm})$ \\
\hline Max Cutting Length & $30 "(762 \mathrm{~mm})$ & $48^{\prime \prime}(1219 \mathrm{~mm})$ & $60 "(1524 \mathrm{~mm})$ \\
\hline
\end{tabular}

\section{Spindle}

\begin{tabular}{|c|c|c|c|}
\hline Max Speed & 1,800 rpm & 1,800 rpm & $1,800 \mathrm{rpm}$ \\
\hline Max Motor Rating & 7.5 hp (5.6 kW) & $12 \mathrm{hp}(8.9 \mathrm{~kW})$ & 18 hp (13.4 kW) \\
\hline Max Torque & $\begin{array}{l}100 \text { ft-lb @ } 375 \text { rpm } \\
\text { (136 Nm @ } 375 \text { rpm) }\end{array}$ & $\begin{array}{l}150 \text { ft-lb @ } 150 \text { rpm } \\
\text { (203 Nm @ } 150 \text { rpm) }\end{array}$ & $\begin{array}{l}204 \mathrm{ft}-\mathrm{lb} @ 417 \mathrm{rpm} \\
\text { (227 Nm @ } 417 \mathrm{rpm})\end{array}$ \\
\hline Spindle Nose & A2-5 & A2-6 & A2-6 \\
\hline Spindle Bore & $\emptyset 2.31 "(59 \mathrm{~mm})$ & Ø3.00" (76 mm) & Ø3.50" (89 mm) \\
\hline
\end{tabular}

Swing Diameter

\begin{tabular}{llll}
\hline Over Front Apron & $16.00 "(406 \mathrm{~mm})$ & $16.00 "(406 \mathrm{~mm})$ & $20.00 "(508 \mathrm{~mm})$ \\
\hline Over Cross Slide & $9.50 "(241 \mathrm{~mm})$ & $11.10^{\prime \prime}(282 \mathrm{~mm})$ & $13.00 "(330 \mathrm{~mm})$ \\
\hline
\end{tabular}

Travels \& Feedrates

\begin{tabular}{llll}
\hline X Axis Travels & $8 "(203 \mathrm{~mm})$ & $8^{\prime \prime}(203 \mathrm{~mm})$ & $12 "(305 \mathrm{~mm})$ \\
\hline Z Axis Travels & $30 "(762 \mathrm{~mm})$ & $48 "(1219 \mathrm{~mm})$ & $60 "(1524 \mathrm{~mm})$ \\
\hline X Axis Max Thrust & $3,096 \mathrm{lb}(13772 \mathrm{~N})$ & $3,096 \mathrm{lb}(13772 \mathrm{~N})$ & $3,096 \mathrm{lb}(13772 \mathrm{~N})$ \\
\hline Z Axis Max Thrust & $1,548 \mathrm{lb}(6886 \mathrm{~N})$ & $2,340 \mathrm{lb}(10409 \mathrm{~N})$ & $3,096 \mathrm{lb}(13772 \mathrm{~N})$ \\
\hline X Axis Rapids & $75 \mathrm{ipm}(1.9 \mathrm{~m} / \mathrm{min})$ & $75 \mathrm{ipm}(1.9 \mathrm{~m} / \mathrm{min})$ & $75 \mathrm{ipm}(1.9 \mathrm{~m} / \mathrm{min})$ \\
\hline Z Axis Rapids & $150 \mathrm{ipm}(3.8 \mathrm{~m} / \mathrm{min})$ & $150 \mathrm{ipm}(3.8 \mathrm{~m} / \mathrm{min})$ & $150 \mathrm{ipm}(3.8 \mathrm{~m} / \mathrm{min})$ \\
\hline
\end{tabular}

\begin{tabular}{llll} 
General & & & \\
\hline Power & 1-Phase - 240V @ 40A & 1-Phase - 240V @ 40A & 200 - 250VAC \\
& 3-Phase - 208V @ 25A & 3-Phase - 208V @ 25A & 3-Phase, 40A \\
\hline Machine Weight & $4,100 \mathrm{lb}(1860 \mathrm{~kg})$ & $4,600 \mathrm{lb}(2087 \mathrm{~kg})$ & $6,500 \mathrm{lb}(2948 \mathrm{~kg})$ \\
\hline Footprint & $83^{\prime \prime} \times 55^{\prime \prime}$ & $101 " \times 55 "$ & $118^{\prime \prime} \times 62^{\prime \prime}$ \\
& $(2108 \times 1397 \mathrm{~mm})$ & $(2565 \times 1397 \mathrm{~mm})$ & $(2997 \times 1575 \mathrm{~mm})$ \\
\hline
\end{tabular}

Note: Specifications subject to change without notice. No bar feed provision.

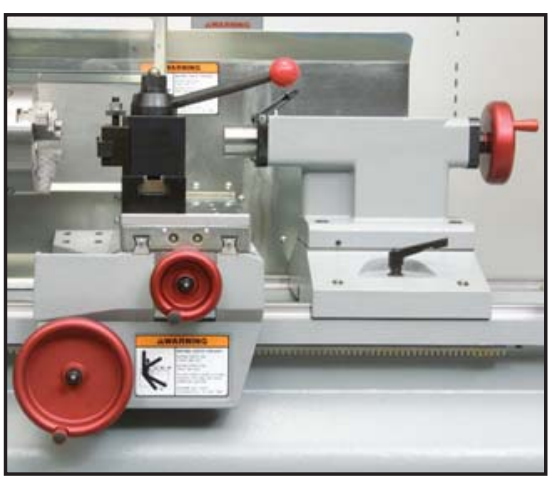

Handwheels and precise DRO make manual operation simple, and the heavy-duty cross-slide accepts a wide selection of tool post options.

The optional tailstock, with a manually adjustable quill, provides additional support for long workpieces.

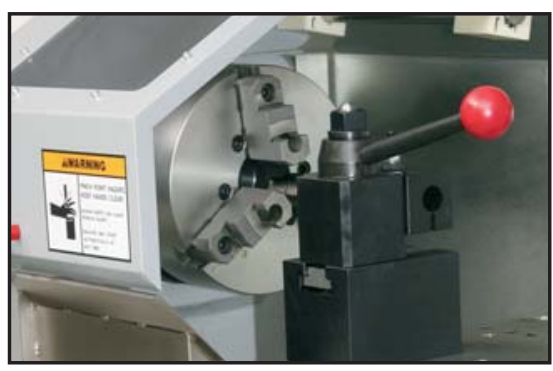

The spindle nose (A2-5 or A2-6) accepts a variety of optional chucks and collets. An integral chip-flow channel prevents chips from building up in the work area and simplifies clean-up.

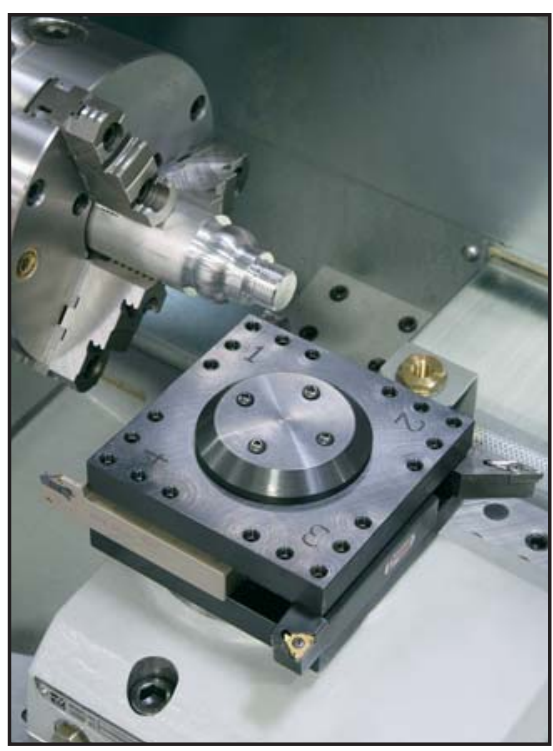

An optional four-station automatic tool turret (TL-1 and TL-2) eliminates manual tool changes, allowing fully automatic operation to increase productivity and decrease cycle times for longer part runs.

*High-torque and wide-swing versions of the TL-3 are available. 


\section{The Haas Control}

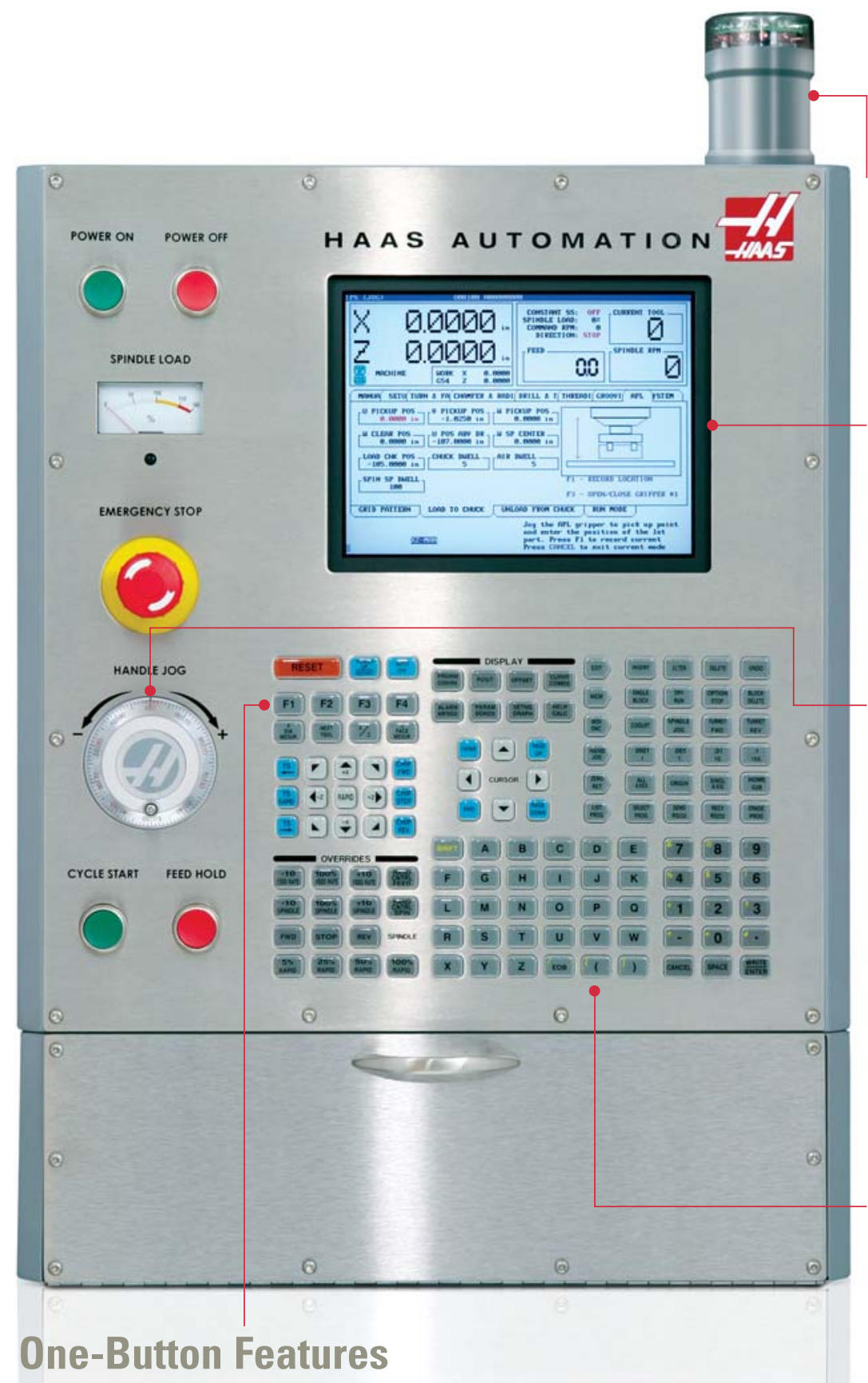

Common multi-step functions, such as powering up in the morning or setting tool offsets, have been reduced to the push of a single button. Other often used functions, such as setting part zeros, homing the machine, setting the offset to centerline and resetting the tool turret, are also one-button commands.

\section{Dedicated Closed System}

Our focus is to provide Haas users with a robust, dependable control that is seamlessly integrated with the machine. Our closed system is not reliant on PC-based controls or third-party NC suppliers. When you call Haas, you get a company that takes full responsibility for the entire machine.

\section{LED Beacon Light}

The Haas-designed beacon light features ultra-bright LEDS that can easily be seen from anywhere in the shop. The LEDs generate very little heat and have a life expectancy of more than 10 years.

\section{Color LCD Screen}

Our full-color TFT LCD display is designed to work in the machine shop environment. The highintensity, high-contrast LCD features a very wide viewing angle, and will not fade out in bright light. The panel is mounted behind anti-glare tempered glass for protection and easy viewing.

\section{Multi-Function Jog Handlle}

Most machines use the jog handle only to move the axes around. On Haas machines, the jog handle can also be used in other modes to cursor through the program for faster editing, override spindle speeds and feedrates, or scan through offsets, parameters, etc.

\section{Advanced Program Editing}

A unique feature of the Haas control is an advanced program editor that allows the operator to edit programs in a dual-window format.

\section{Dedicated Keypad}

The Haas keypad has 131 keys. All common functions, including a full alphanumeric keypad, are clearly labeled for operator ease. If you have ever fumbled with hot keys, or had to page through screens looking for a specific function, then you will appreciate the simplicity of the Haas control. There are no encrypted codes to memorize, and many functions can be performed simply with the push of a single button.

\section{Stainless Steel Bezel*}

Constructed of heavy-gauge T304 stainless steel with a fine-grained finish, the bezel of the Haas control not only looks great, it's also easy to clean, resists wear and will not corrode. 


\section{Control Features}

- User friendly

- Advanced program editor

- Brushless AC servo drives

- Triple 32-bit processors

- Executes up to 1,000 blocks/second

- ISO standard G-code compatibility

- $1 \mathrm{MB}$ standard program memory

- Haas one-button power-up

- Haas one-button tool offset entry

- 50 tool-geometry offsets

- Tool load monitoring

- Advanced tool life management

- Background editing

- Split screen program review

- Trig calculator

- Speeds and feeds calculator

- Arc calculator

- RS-232 / DNC link / opt. Ethernet + USB

- Mid-program restart

- Inch or metric programming

- Message page

- Selectable languages

- Fully descriptive alarms

- Self-diagnostics

- Graphic dry run

- 5 spare M functions

- Electronic thermal compensation

- One-year parts and labor warranty

- Made in the USA

\section{Control Specifications}

\begin{tabular}{|c|c|c|}
\hline Microprocessor & & Triple high-speed 32-bit \\
\hline Program execution speed & & 1,000 blocks/sec standard \\
\hline Axis control & & 2 axes linear, $C$ axis optional \\
\hline Interpolation & & G01, G02, G03 \\
\hline Min. input increment & $\begin{array}{r}\text { - inch mode } \\
\text { - metric mode }\end{array}$ & $\begin{array}{l}0.0001 " \\
0.001 \mathrm{~mm}\end{array}$ \\
\hline Min. output resolution & & $0.0000072 "(0.00018 \mathrm{~mm})$ \\
\hline
\end{tabular}

Feed Functions

\begin{tabular}{|c|c|c|}
\hline Rapid Traverse Override & & $5 \%, 25 \%, 50 \%, 100 \%$ \\
\hline Feedrate Override & & $0 \%$ to $200 \%$ in $1 \%$ increments \\
\hline Jog Handle Resolution & $\begin{array}{r}\text { - inch mode } \\
\text { - metric mode }\end{array}$ & $\begin{array}{l}0.0001 " / 0.001 " / 0.01 " / 0.1 " \text { per handle division } \\
0.001 / 0.01 / 0.1 / 1.0 \mathrm{~mm} \text { per handle division }\end{array}$ \\
\hline Jog Feeds & $\begin{array}{r}\text { - inch mode } \\
\text { - metric mode }\end{array}$ & $\begin{array}{l}0.1 / 1.0 / 10.0 / 100.0 \mathrm{ipm} \\
1.0 / 10 / 100 / 1000 \mathrm{~mm} / \mathrm{min}\end{array}$ \\
\hline Zero Return & & One key (G28) \\
\hline \multicolumn{3}{|l|}{ Spindle Functions } \\
\hline Speed Command & & $S=1$ to 7,000 (max determined by model) \\
\hline Override & & $0 \%$ to $200 \%$ in $1 \%$ increments \\
\hline
\end{tabular}

\section{Tool Functions}

\begin{tabular}{ll}
\hline Length Compensation & 50 sets, geometry \& wear \\
\hline Diameter/Radius Compensation & 50 sets, geometry \& wear \\
\hline Length Measurement & Automatic length storage \\
\hline Life Management & 10 or 12 tools \\
\hline Selection & Txx command \\
\hline
\end{tabular}

Programming

\begin{tabular}{ll} 
Compatibility & ISO standard G code \\
\hline Positioning & X, Z absolute and U, W incremental \\
\hline Canned Cycles & 17 functions, standard \\
\hline Inch/Metric & Switchable \\
\hline Work Coordinates & 105 sets \\
\hline Part Zero Set & Automatic input and storage \\
\hline M Functions & 5 spare ${ }^{\dagger}$ \\
\hline Cutter Compensation & G40, G41, G42 \\
\hline
\end{tabular}

\section{Data Input/Output}

\begin{tabular}{ll}
\hline Communications Port & RS-232 \\
\hline Data Rate & To 115,200 bits/second $\ddagger$ \\
\hline
\end{tabular}

\section{Memory Capacity}

\begin{tabular}{ll} 
Standard & $1 \mathrm{MB}$; up to $16 \mathrm{MB}$ optional \\
\hline Number of Programs & 500
\end{tabular}

$\dagger$ May be used by options. $\ddagger$ With 6 ' or shorter cable. 


\section{Intuitive Programming System}

\section{Simple, intuitive and more powerful than ever ...}

It's getting more and more difficult to find machine operators who are experts in G-code programming. In fact, it's often impossible. Now, thanks to Haas' Intuitive Programming System (IPS), it's no longer necessary for a machine operator to know G-code for basic turning operations. The IPS software is available for our complete line of turning centers. It is standard on our Toolroom machines, and optional on our SL Series machines.

The Haas Intuitive Programming System is a proprietary conversational operating system that makes cutting parts and creating part programs nearly effortless. Using an easy-to-read format with full-color graphics, the IPS guides the operator through the steps necessary to machine a part. The operator simply chooses an operation, touches off the $\mathrm{X}$ and $\mathrm{Z}$ surfaces, and then enters basic dimension information. Default values for feed per revolution, spindle speed and depth of cut are entered automatically by the control, but can be modified by the operator. Once all necessary information is entered, pushing Cycle Start performs the operation. A Recorder function allows multiple operations to be recorded and saved, so that the information can be played back to duplicate the part. Operations that would be difficult or impossible on a manual machine - radii, tapers, profiles, grooving, threading, rigid tapping - are all possible on Haas lathes without knowledge of G-code programming. Help menus are available directly on-screen, and a dry-run graphics feature allows operators to check their work before running a part.

\section{Powerful new features:}

Programmed from the centerline using tool offsets.

- Allows programs created in the Intuitive Programming System to be saved and rerun at a later date, or transferred to a Haas SL series production turning center as standard G-code.

- IPS upgraded threading features:

- Machine tapered threads based on the amount of taper per foot.

- Choose right- or left-hand threads.

- Chamfer out or straight pull-out at thread's end.

- Thread repair operations for straight, tapered, right-hand or left-hand, inside or outside diameter threads, without the need to orient the spindle to a known position.

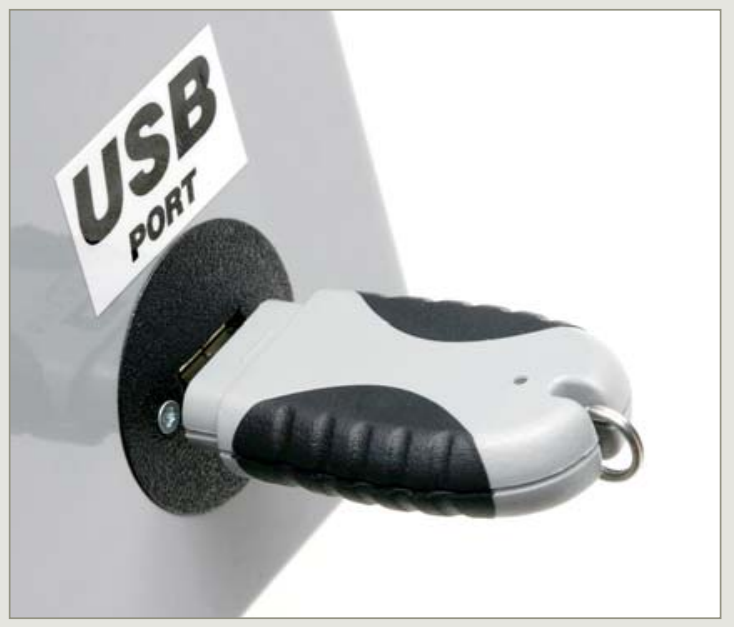

\section{Hard Drive \& Ethernet Interface with USB Support}

Store and transfer data between your Haas and a network or PC with the Ethernet connection. Program files are easily transferred to and from memory or the hard drive, and large files can be accessed by multiple machines. High-speed data transfers allow DNC of large files at up to 1,000 blocks per second. USB support allows the use of customer-supplied Zip ${ }^{\mathrm{TM}}$ drives or flash memory devices. Compatible with Windows 95, 98, ME, 2000 and XP. Also works with server versions NT 4.0, 2000 and 2003. IPX/SPX or TCP/IP protocol. Easy setup from the control screen. 


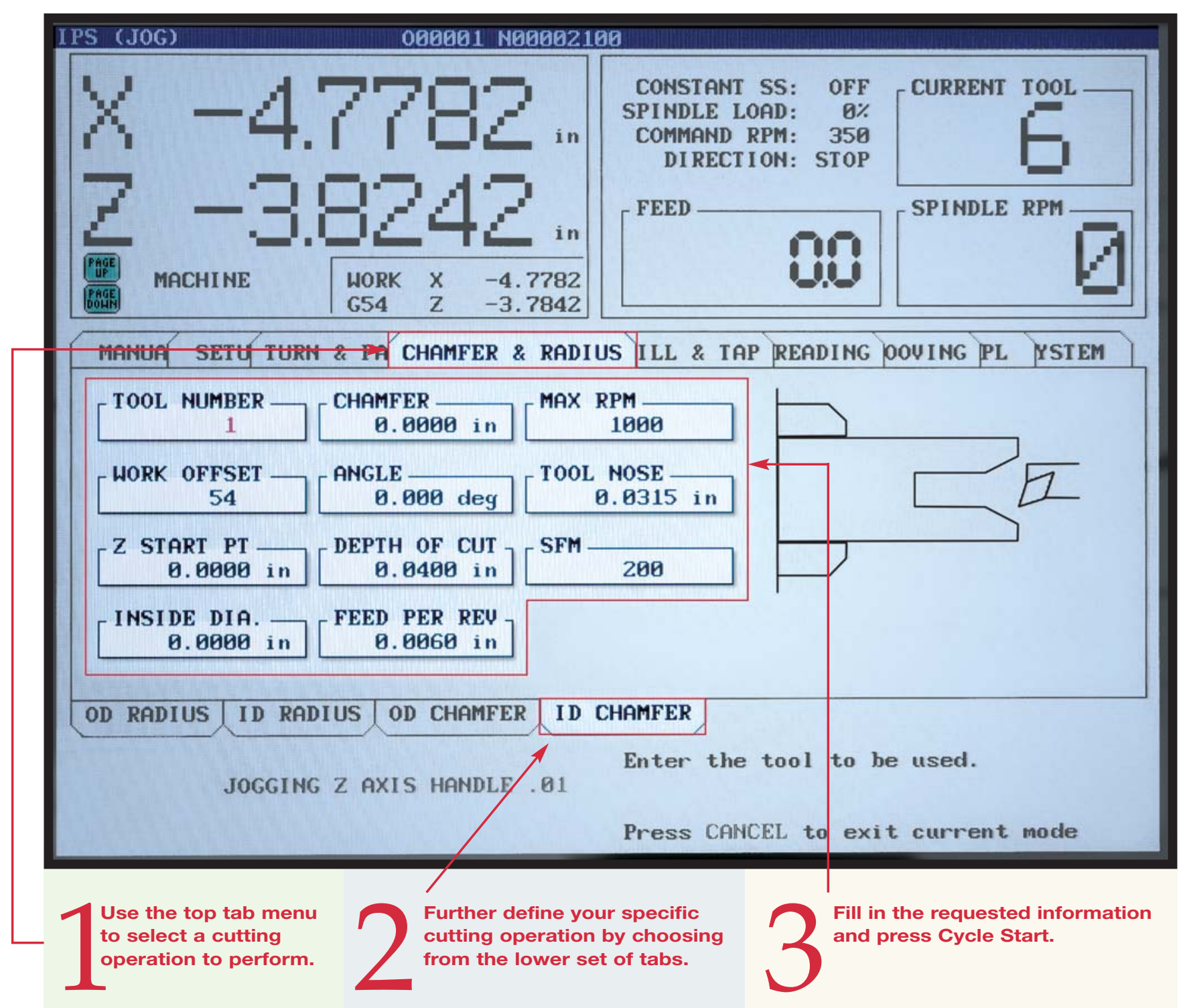

Using plain language, simple graphics and easy-to-follow steps, the Haas Intuitive Programming System guides the operator through the steps necessary to machine a part.

\section{PC-Compatible $1.44 \mathrm{MB} / 3.5 "$ Floppy Drive}

Conveniently located on the side of the control pendant, this drive uses standard PC floppies to easily upload programs for machining, download for storage off the machine or DNC directly off the disk.

\section{M Functions}

Adds 8 additional $\mathrm{M}$ functions for a total of up to 13 user interfaces. Use these to activate probes, auxiliary pumps, clamping devices, part loaders, etc.

\section{Memory Upgrade - 16 MB}

Allows loading of large programs directly into the machine, rather than from DNC, for faster feedrates. Batterypowered, high-speed static RAM is faster than dynamic RAM and retains data even when the power is turned off.

\section{Memory Lock Keyswitch}

Locks memory to prevent program editing by unauthorized personnel. It can also be used to lock settings, parameters, offsets and macro variables. 


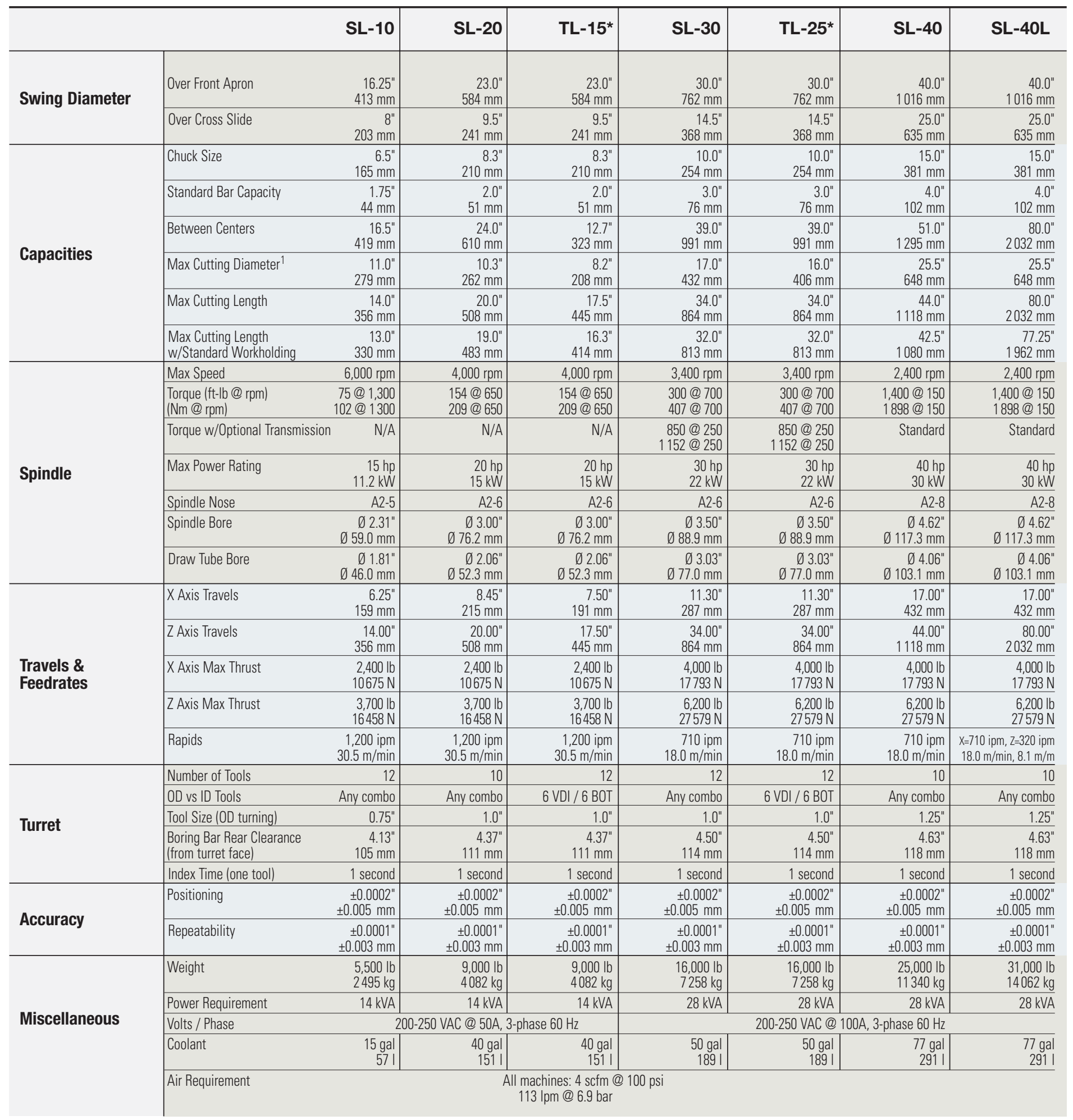

${ }^{1}$ Maximum turning diameter may be affected by machine options. 


\begin{tabular}{|c|c|c|c|c|c|c|c|c|}
\hline & & SL-10 & SL-20 & TL-15* & SL-30 & TL-25* & SL-40 & SL-40L \\
\hline \multirow{6}{*}{$\begin{array}{l}\text { Big Bore Option } \\
\text { Capacities }\end{array}$} & Chuck Size & $\begin{array}{r}8.3^{\prime \prime} \\
210 \mathrm{~mm}\end{array}$ & $\begin{array}{r}10.0 " \\
254 \mathrm{~mm}\end{array}$ & $\begin{array}{r}10.0 " \\
254 \mathrm{~mm}\end{array}$ & $\begin{array}{r}15.0 " \\
381 \mathrm{~mm}\end{array}$ & $\begin{array}{r}15.0 " \\
381 \mathrm{~mm}\end{array}$ & † optional & † optional \\
\hline & Maximum Bar Capacity & $\begin{array}{r}2.0^{\prime \prime} \\
51 \mathrm{~mm}\end{array}$ & $\begin{array}{r}2.5 " \\
64 \mathrm{~mm}\end{array}$ & $\begin{array}{r}2.5^{\prime \prime} \\
64 \mathrm{~mm} \\
\end{array}$ & $\begin{array}{r}4.0 " \\
102 \mathrm{~mm}\end{array}$ & $\begin{array}{r}4.0^{\prime \prime} \\
102 \mathrm{~mm} \\
\end{array}$ & $\begin{array}{r}7.0 " \dagger \\
178 \mathrm{~mm} \\
\end{array}$ & $\begin{array}{r}7.0 " \dagger \\
178 \mathrm{~mm} \\
\end{array}$ \\
\hline & Between Centers & $\begin{array}{r}16.5^{\prime \prime} \\
419 \mathrm{~mm}\end{array}$ & $\begin{array}{r}24.0^{\prime \prime} \\
610 \mathrm{~mm}\end{array}$ & $\begin{array}{r}12.7 " \\
323 \mathrm{~mm}\end{array}$ & $\begin{array}{r}39.0 " \\
991 \mathrm{~mm}\end{array}$ & $\begin{array}{r}39.0 " \\
991 \mathrm{~mm}\end{array}$ & $\begin{array}{r}51.0 " \\
1295 \mathrm{~mm}\end{array}$ & $\begin{array}{r}89.0 " \\
2261 \mathrm{~mm}\end{array}$ \\
\hline & Maximum Cutting Diameter & $\begin{array}{r}11.0^{\prime \prime} \\
279 \mathrm{~mm} \\
\end{array}$ & $\begin{array}{r}10.3^{\prime \prime} \\
262 \mathrm{~mm} \\
\end{array}$ & $\begin{array}{r}8.2^{\prime \prime} \\
208 \mathrm{~mm} \\
\end{array}$ & $\begin{array}{r}16.0 " \\
406 \mathrm{~mm}\end{array}$ & $\begin{array}{r}16.0 " \\
406 \mathrm{~mm} \\
\end{array}$ & $\begin{array}{r}25.5^{\prime \prime} \\
648 \mathrm{~mm} \\
\end{array}$ & $\begin{array}{r}25.5^{\prime \prime} \\
648 \mathrm{~mm} \\
\end{array}$ \\
\hline & Maximum Cutting Length & $\begin{array}{r}14.0^{\prime \prime} \\
356 \mathrm{~mm} \\
\end{array}$ & $\begin{array}{r}20.0 " \\
508 \mathrm{~mm}\end{array}$ & $\begin{array}{r}17.5^{\prime \prime} \\
445 \mathrm{~mm} \\
\end{array}$ & $\begin{array}{r}34.0 " \\
864 \mathrm{~mm}\end{array}$ & $\begin{array}{r}34.0 " \\
864 \mathrm{~mm} \\
\end{array}$ & $\begin{array}{r}44.0^{\prime \prime} \\
1118 \mathrm{~mm} \\
\end{array}$ & $\begin{array}{r}80.0 " \\
2032 \mathrm{~mm} \\
\end{array}$ \\
\hline & \begin{tabular}{|l|} 
Max Cutting Length \\
w/Standard Workholding \\
\end{tabular} & $\begin{array}{r}12.0 " \\
305 \mathrm{~mm} \\
\end{array}$ & $\begin{array}{r}17.5 " \\
444 \mathrm{~mm} \\
\end{array}$ & $\begin{array}{r}15.0^{\prime \prime} \\
381 \mathrm{~mm} \\
\end{array}$ & $\begin{array}{r}31.0 " \\
787 \mathrm{~mm} \\
\end{array}$ & $\begin{array}{r}29.0 " \\
737 \mathrm{~mm} \\
\end{array}$ & $\begin{array}{r}42.5^{\prime \prime} \\
1080 \mathrm{~mm} \\
\end{array}$ & $\begin{array}{r}77.25 " \\
1962 \mathrm{~mm} \\
\end{array}$ \\
\hline \multirow{8}{*}{$\begin{array}{l}\text { Big Bore Option } \\
\text { Spindle }\end{array}$} & Maximum Speed & 4,000 rpm & $3,400 \mathrm{rpm}$ & 3,400 rpm & $2,400 \mathrm{rpm}$ & $2,400 \mathrm{rpm}$ & $1,400 \mathrm{rpm}$ & $1,400 \mathrm{rpm}$ \\
\hline & $\begin{array}{l}154 \mathrm{ft}-\mathrm{lb} \\
209 \mathrm{Nm}\end{array}$ & lb @ 650 rpm & \multicolumn{2}{|c|}{$\begin{array}{l}300 \mathrm{ft}-\mathrm{lb} @ 700 \mathrm{rpm} \\
407 \mathrm{Nm} @ 700 \mathrm{rpm}\end{array}$} & \multicolumn{4}{|c|}{$\begin{array}{r}1,400 \mathrm{ft}-\mathrm{lb} @ 150 \mathrm{rpm} \\
1898 \mathrm{Nm} @ 150 \mathrm{rpm} \\
\end{array}$} \\
\hline & Transmission & $\mathrm{N} / \mathrm{A}$ & N/A & $\mathrm{N} / \mathrm{A}$ & Standard & Standard & Standard & Standard \\
\hline & Max Power Rating & $\begin{array}{r}20 \mathrm{hp} \\
15 \mathrm{~kW}\end{array}$ & $\begin{array}{r}30 \mathrm{hp} \\
22 \mathrm{~kW}\end{array}$ & $\begin{array}{r}30 \mathrm{hp} \\
22 \mathrm{~kW}\end{array}$ & $\begin{array}{r}40 \mathrm{hp} \\
30 \mathrm{~kW} \\
\end{array}$ & $\begin{array}{r}40 \mathrm{hp} \\
30 \mathrm{~kW} \\
\end{array}$ & $\begin{array}{r}40 \mathrm{hp} \\
30 \mathrm{~kW} \\
\end{array}$ & $\begin{array}{r}40 \mathrm{hp} \\
30 \mathrm{~kW} \\
\end{array}$ \\
\hline & Spindle Nose & A2-6 & A2-6 & A2-6 & A2-8 & A2-8 & A2-11 & A2-11 \\
\hline & Spindle Bore & $\begin{array}{r}\emptyset 3.00 " \\
76.2 \mathrm{~mm}\end{array}$ & $\begin{array}{r}\emptyset 3.00 " \\
76.2 \mathrm{~mm}\end{array}$ & $\begin{array}{r}\emptyset 3.00 " \\
76.2 \mathrm{~mm}\end{array}$ & $\begin{array}{r}\emptyset 4.62 " \\
117.3 \mathrm{~mm} \\
\end{array}$ & $\begin{array}{r}\emptyset 4.62 " \\
117.3 \mathrm{~mm} \\
\end{array}$ & $\begin{array}{c}\emptyset 7.08 " \\
180 \mathrm{~mm}\end{array}$ & $\begin{array}{r}\emptyset 7.08 " \\
180 \mathrm{~mm} \\
\end{array}$ \\
\hline & Draw Tube Bore & $\begin{array}{r}\emptyset 2.06 " \\
\emptyset 52.3 \mathrm{~mm} \\
\end{array}$ & $\begin{array}{r}\emptyset 2.56 " \\
\emptyset 65.0 \mathrm{~mm} \\
\end{array}$ & $\begin{array}{r}\emptyset 2.56 " \\
\emptyset 65.0 \mathrm{~mm} \\
\end{array}$ & $\begin{array}{r}\emptyset 4.06 " \\
\emptyset 103.1 \mathrm{~mm} \\
\end{array}$ & $\begin{array}{r}\emptyset 4.06 " \\
\emptyset 103.1 \mathrm{~mm} \\
\end{array}$ & $\begin{array}{r}\emptyset 6.5^{\prime \prime} \\
\emptyset 165 \mathrm{~mm} \\
\end{array}$ & $\begin{array}{r}\emptyset 6.5^{\prime \prime} \\
\emptyset 165 \mathrm{~mm} \\
\end{array}$ \\
\hline & Power Requirement & $14 \mathrm{kVA}$ & $28 \mathrm{kVA}$ & $28 \mathrm{kVA}$ & $28 \mathrm{kVA}$ & $28 \mathrm{kVA}$ & $28 \mathrm{kVA}$ & $28 \mathrm{kVA}$ \\
\hline \multirow{3}{*}{ Tailstock Option } & Taper & MT3 & MT4 & - & MT4 (MT5 opt) & - & MT5 & MT5 \\
\hline & $\begin{array}{cc}\text { Travels } & 9.75 " \text { manual } \\
& 248 \mathrm{~mm}\end{array}$ & $\begin{array}{r}4.0 " \text { hydraulic } \\
102 \mathrm{~mm}\end{array}$ & $\begin{array}{r}20.0 " \\
508 \mathrm{~mm}\end{array}$ & - & $\begin{array}{r}33.5^{\prime \prime} \\
851 \mathrm{~mm}\end{array}$ & - & $\begin{array}{r}44.0^{\prime \prime} \\
1118 \mathrm{~mm}\end{array}$ & $\begin{array}{r}80.0^{\prime \prime} \\
2032 \mathrm{~mm}\end{array}$ \\
\hline & \begin{tabular}{|l|} 
Thrust \\
\end{tabular} & $\begin{array}{r}225-900 \mathrm{lb} \\
001-4003 \mathrm{~N}\end{array}$ & $\begin{array}{r}300-1,500 \mathrm{lb} \\
1334-6672 \mathrm{~N} \\
\end{array}$ & - & $\begin{array}{r}300-1,500 \mathrm{lb} \\
1334-6672 \mathrm{~N} \\
\end{array}$ & - & $\begin{array}{r}300-1,500 \mathrm{lb} \\
1334-6672 \mathrm{~N} \\
\end{array}$ & $\begin{array}{r}300-4,500 \mathrm{lb} \\
1334-20017 \mathrm{~N} \\
\end{array}$ \\
\hline \multirow{4}{*}{ Spindle Options } & w/ 5,000-rpm / 30-hp & & & & & & & \\
\hline & Torque & - & \multicolumn{2}{|c|}{$\begin{array}{l}120 \mathrm{ft}-\mathrm{lb} @ 1,400 \text { rpm } \\
163 \mathrm{Nm} @ 1400 \text { rpm }\end{array}$} & - & - & - & - \\
\hline & \multicolumn{2}{|l|}{ w/ 7,000-rpm / 20-hp } & \multirow{2}{*}{\multicolumn{2}{|c|}{$\begin{array}{l}80 \mathrm{ft}-\mathrm{lb} @ 1,400 \mathrm{rpm} \\
108 \mathrm{Nm} @ 1400 \mathrm{rpm} \\
\end{array}$}} & & & & \\
\hline & Torque & - & & & - & - & - & - \\
\hline \multirow{7}{*}{ Live Tooling } & Speed & - & $0-3,000 \mathrm{rpm}$ & $0-3,000 \mathrm{rpm}$ & $0-3,000 \mathrm{rpm}$ & $0-3,000 \mathrm{rpm}$ & $0-3,000 \mathrm{rpm}$ & $0-3,000 \mathrm{rpm}$ \\
\hline & Peak Torque Standard & - & $\begin{array}{l}132 \mathrm{in}-\mathrm{Ib} \\
14.9 \mathrm{Nm}\end{array}$ & $\begin{array}{l}132 \mathrm{in}-\mathrm{lb} \\
14.9 \mathrm{Nm}\end{array}$ & $\begin{array}{l}132 \mathrm{in}-\mathrm{lb} \\
14.9 \mathrm{Nm}\end{array}$ & $\begin{array}{l}132 \mathrm{in}-\mathrm{lb} \\
14.9 \mathrm{Nm}\end{array}$ & $\begin{array}{l}132 \mathrm{in}-\mathrm{lb} \\
14.9 \mathrm{Nm}\end{array}$ & $\begin{array}{l}132 \mathrm{in}-\mathrm{lb} \\
14.9 \mathrm{Nm}\end{array}$ \\
\hline & Peak Torque w/HT option & - & $\begin{array}{l}195 \mathrm{in}-\mathrm{lb} \\
22.0 \mathrm{Nm}\end{array}$ & $\begin{array}{l}195 \mathrm{in}-\mathrm{lb} \\
22.0 \mathrm{Nm}\end{array}$ & $\begin{array}{l}195 \mathrm{in}-\mathrm{lb} \\
22.0 \mathrm{Nm}\end{array}$ & $\begin{array}{l}195 \mathrm{in}-\mathrm{lb} \\
22.0 \mathrm{Nm}\end{array}$ & $\begin{array}{l}195 \mathrm{in}-\mathrm{lb} \\
22.0 \mathrm{Nm}\end{array}$ & $\begin{array}{l}195 \mathrm{in}-\mathrm{Ib} \\
22.0 \mathrm{Nm}\end{array}$ \\
\hline & Drive Ratio & - & $1: 1$ & $1: 1$ & $1: 1$ & $1: 1$ & $1: 1$ & $1: 1$ \\
\hline & Brake Diameter & - & $\begin{array}{r}13.25 " \\
337 \mathrm{~mm}\end{array}$ & $\begin{array}{r}13.25 " \\
337 \mathrm{~mm}\end{array}$ & $\begin{array}{l}\ddagger 13.25 " \\
337 \mathrm{~mm}\end{array}$ & $\begin{array}{l}\ddagger 13.25 " \\
337 \mathrm{~mm}\end{array}$ & $\begin{array}{r}21.5^{\prime \prime} \\
546 \mathrm{~mm} \\
\end{array}$ & $\begin{array}{r}21.5^{\prime \prime} \\
546 \mathrm{~mm} \\
\end{array}$ \\
\hline & Brake Clamp Force & - & $\begin{array}{l}1,000 \mathrm{lb} \\
4448 \mathrm{~N}\end{array}$ & $\begin{array}{l}1,000 \mathrm{lb} \\
4448 \mathrm{~N}\end{array}$ & $\begin{array}{r}1,000 \mathrm{lb} \\
4448 \mathrm{~N} \\
\end{array}$ & $\begin{array}{l}1,000 \mathrm{lb} \\
4448 \mathrm{~N} \\
\end{array}$ & $\begin{array}{l}1,000 \mathrm{lb} \\
4448 \mathrm{~N}\end{array}$ & $\begin{array}{l}1,000 \mathrm{lb} \\
4448 \mathrm{~N}\end{array}$ \\
\hline & Tooling & - & \multicolumn{6}{|c|}{ All machines: Standard VDI 40 driven tools } \\
\hline
\end{tabular}

* See page 36 for specifications on the TL-15 \& TL-25 sub-spindle.

† Chuck, rotating union and draw tube not included; accepts chucks up to 24". Bar capacity 6.5" w/ draw tube.

$\ddagger$ SL-30 and TL-25 Big Bore have 21.5" brake disc. 


\section{Sub-Spindle Machines}

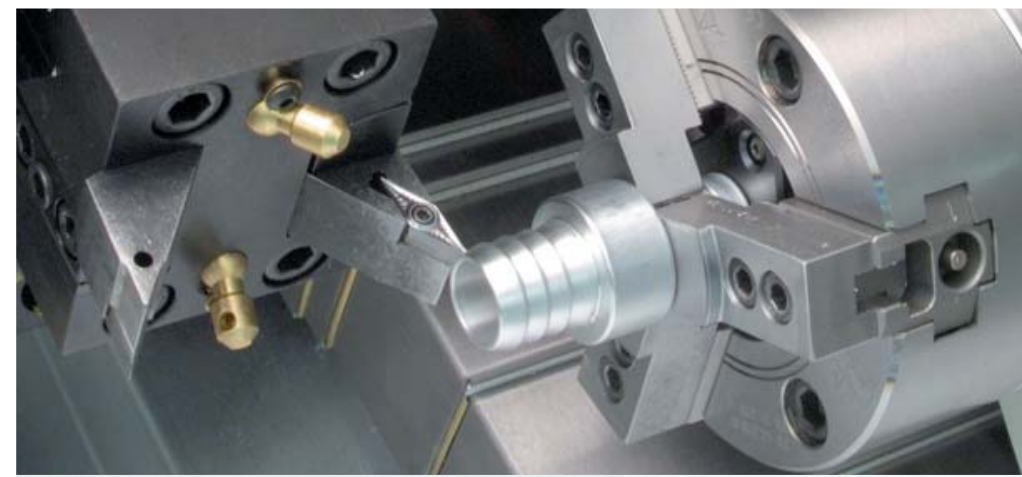

\section{Working Specifications}

\begin{tabular}{|c|c|c|}
\hline Specs & TL-15 & TL-25 \\
\hline Chuck Size & $\begin{array}{l}8.3^{\prime \prime} \\
210 \mathrm{~mm} \\
\end{array}$ & $\begin{array}{l}10 " \\
254 \mathrm{~mm}\end{array}$ \\
\hline Spindle Rating (peak) & $\begin{array}{l}20 \mathrm{hp} \\
14.9 \mathrm{~kW}\end{array}$ & $\begin{array}{l}30 \mathrm{hp} \\
22.4 \mathrm{~kW}\end{array}$ \\
\hline Spindle Speeds & $50-4,000$ rpm & 50-3,400 rpm \\
\hline Standard Bar Capacity & $\begin{array}{l}2 " \\
51 \mathrm{~mm}\end{array}$ & $\begin{array}{l}3^{\prime \prime} \\
76 \mathrm{~mm}\end{array}$ \\
\hline Between Centers & $\begin{array}{l}12.7^{\prime \prime} \\
323 \mathrm{~mm}\end{array}$ & $\begin{array}{l}39 " \\
991 \mathrm{~mm}\end{array}$ \\
\hline Max Cutting Diameter & $\begin{array}{l}8.2^{\prime \prime} \\
208 \mathrm{~mm} \\
\end{array}$ & $\begin{array}{l}16 " \\
406 \mathrm{~mm}\end{array}$ \\
\hline Max Cutting Length & $\begin{array}{l}17.5^{\prime \prime} \\
445 \mathrm{~mm}\end{array}$ & $\begin{array}{l}34 " \\
864 \mathrm{~mm}\end{array}$ \\
\hline Tool Turret & \multicolumn{2}{|c|}{$\begin{array}{c}\text { Hybrid } \\
6 \text { VDI-40, } 6 \text { Bolt-On }\end{array}$} \\
\hline \multicolumn{3}{|l|}{ Sub-Spindle } \\
\hline Chuck Size & $\begin{array}{l}5.3^{\prime \prime} \\
135 \mathrm{~mm}\end{array}$ & $\begin{array}{l}5.3^{\prime \prime} \\
135 \mathrm{~mm}\end{array}$ \\
\hline Bar Capacity & $\begin{array}{l}1.18 " \\
30 \mathrm{~mm} \\
\end{array}$ & $\begin{array}{l}1.18 " \\
30 \mathrm{~mm}\end{array}$ \\
\hline Spindle Rating (peak) & $\begin{array}{l}8 \mathrm{hp} \\
6 \mathrm{~kW}\end{array}$ & $\begin{array}{l}8 \mathrm{hp} \\
6 \mathrm{~kW}\end{array}$ \\
\hline Spindle Speeds & \multicolumn{2}{|c|}{$0-4,000$ rpm } \\
\hline
\end{tabular}

\section{Working Dimensions}

\begin{tabular}{|c|c|c|}
\hline Dim. & TL-15 & TL-25 \\
\hline A & $\begin{array}{l}ø 8.27 " \\
\emptyset 210 \mathrm{~mm}\end{array}$ & $\begin{array}{l}\emptyset 10.00 " \\
\emptyset 254 \mathrm{~mm}\end{array}$ \\
\hline B & $\begin{array}{l}2.11 " \\
54 \mathrm{~mm}\end{array}$ & $\begin{array}{l}2.57 " \\
65 \mathrm{~mm}\end{array}$ \\
\hline C & $\begin{array}{l}5.48^{\prime \prime} \\
139 \mathrm{~mm}\end{array}$ & $\begin{array}{l}6.26 " \\
159 \mathrm{~mm}\end{array}$ \\
\hline D & $\begin{array}{l}20.00 " \\
508 \mathrm{~mm}\end{array}$ & $\begin{array}{l}33.50 " \\
851 \mathrm{~mm}\end{array}$ \\
\hline$E$ & $\begin{array}{l}27.59 " \\
701 \mathrm{~mm}\end{array}$ & $\begin{array}{l}42.33^{\prime \prime} \\
1075 \mathrm{~mm}\end{array}$ \\
\hline$F$ & $\begin{array}{l}ø 5.31^{\prime \prime} \\
ø 135 \mathrm{~mm}\end{array}$ & $\begin{array}{l}ø 5.31 " \\
\emptyset 135 \mathrm{~mm}\end{array}$ \\
\hline G & $\begin{array}{l}1.38 " \\
35 \mathrm{~mm}\end{array}$ & $\begin{array}{l}1.42^{\prime \prime} \\
36 \mathrm{~mm}\end{array}$ \\
\hline $\mathrm{H}$ & $\begin{array}{l}ø 23.00 " \\
ø 584 \mathrm{~mm}\end{array}$ & $\begin{array}{l}\emptyset 29.50 " \\
\varnothing 749 \mathrm{~mm}\end{array}$ \\
\hline J & $\begin{array}{l}ø 10.50 " \\
ø 267 \text { mm }\end{array}$ & $\begin{array}{l}ø 14.50 " \\
ø 368 \mathrm{~mm}\end{array}$ \\
\hline K & $\begin{array}{l}8.25 " \\
210 \mathrm{~mm}\end{array}$ & $\begin{array}{l}5.82 " \\
148 \mathrm{~mm}\end{array}$ \\
\hline
\end{tabular}
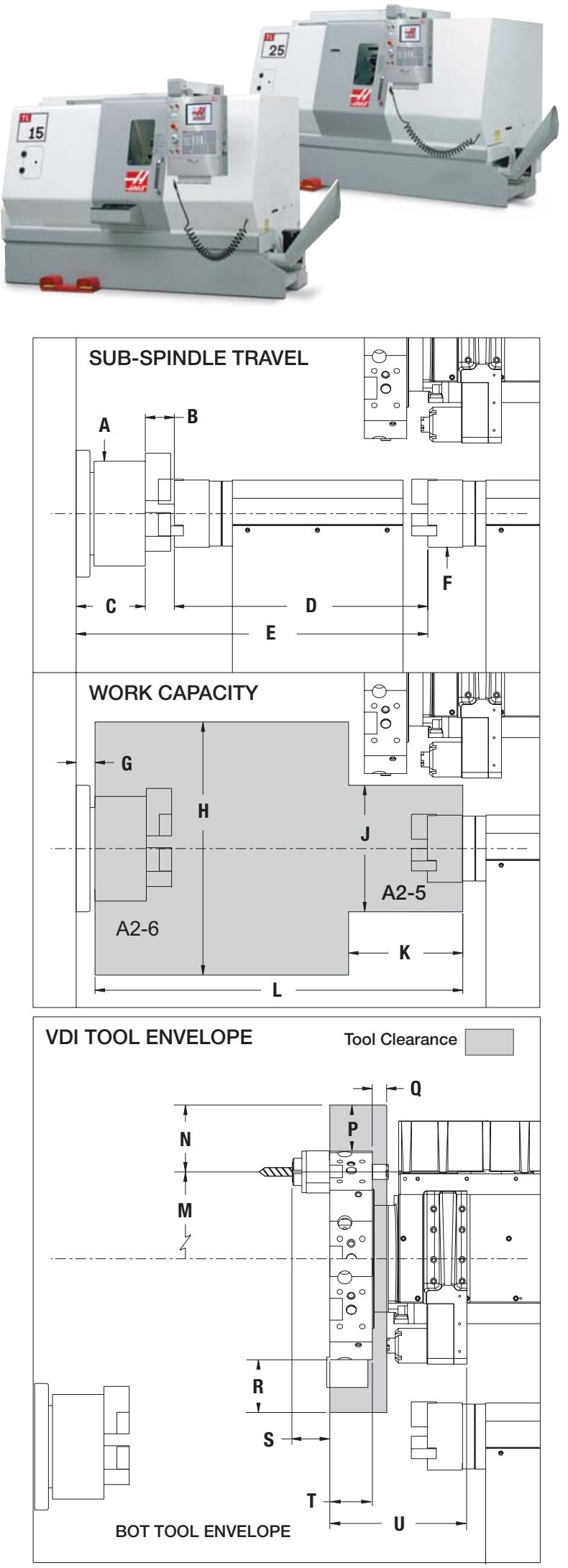

Warning: There are numerous work envelope restrictions on the TL-15 \& TL-25, depending upon the tooling being used. A complete set of work envelope drawings is available from Haas or your local Haas Factory Outlet 


\section{Automated Operation}

\section{SL-20 with APL}

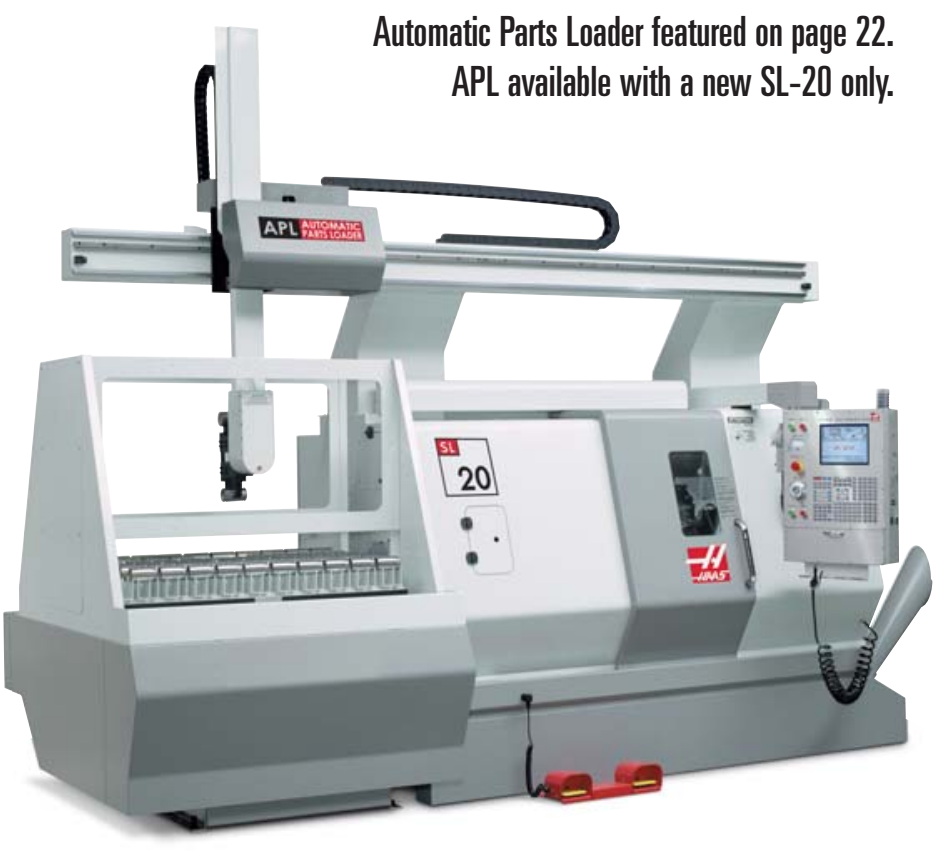

\begin{tabular}{|c|c|}
\hline \multicolumn{2}{|l|}{ Feedrates (Part Loader)* } \\
\hline $\begin{array}{l}\text { U-Axis Rapid } \\
\text { V-Axis Rapid } \\
\text { W-Axis Rapid } \\
\text { Change Time }\end{array}$ & $\begin{array}{l}\text { 1,300 ipm (33.0 m/min) } \\
750 \mathrm{ipm}(19.1 \mathrm{~m} / \mathrm{min}) \\
1,880 \mathrm{ipm}(47.8 \mathrm{~m} / \mathrm{min}) \\
30 \mathrm{sec} \text { (chip-to-chipt) }\end{array}$ \\
\hline \multicolumn{2}{|l|}{ APL } \\
\hline $\begin{array}{l}\text { Table Size } \\
\text { Table Load Capacity }\end{array}$ & $\begin{array}{l}48^{\prime \prime} \times 24^{\prime \prime}(1219 \times 610 \mathrm{~mm}) \\
1,000 \mathrm{lb}(453.6 \mathrm{~kg})\end{array}$ \\
\hline \multicolumn{2}{|c|}{ Double Gripper / Double Part } \\
\hline $\begin{array}{l}\text { Part Weight } \\
\text { Combined Weight } \\
\text { Max Part Diameter } \\
\text { Max Part Length }\end{array}$ & $\begin{array}{l}15 \mathrm{lb} \max (6.8 \mathrm{~kg}) \\
30 \mathrm{lb} \max (13.6 \mathrm{~kg}) \\
5 "(127 \mathrm{~mm}) \\
16 "(406 \mathrm{~mm})\end{array}$ \\
\hline \multicolumn{2}{|l|}{ Disc Gripper } \\
\hline $\begin{array}{l}\text { Part Weight } \\
\text { Max Part Diameter }\end{array}$ & $\begin{array}{l}15 \mathrm{lb} \max (6.8 \mathrm{~kg}) \\
7 "(178 \mathrm{~mm})\end{array}$ \\
\hline \multicolumn{2}{|c|}{$\begin{array}{l}\text { Grippers convert to OD or ID. } \\
\text { Part configuration determines stacking height. }\end{array}$} \\
\hline \multicolumn{2}{|l|}{ Dimensions } \\
\hline $\begin{array}{l}\text { Width } \\
\text { Depth } \\
\text { Height }\end{array}$ & $\begin{array}{l}179 " \text { " (4547 mm) } \\
84 " \text { (2 } 134 \mathrm{~mm}) \\
146 " \text { (3708 mm) }\end{array}$ \\
\hline
\end{tabular}

\section{Automatic Bar Feeder}

Servo Bar featured on page 23.

Servo Bar available with SL-10, -20, -30 and -40.

\section{Servo Bar Specifications}

\begin{tabular}{ll} 
Capacity & $\begin{array}{l}3 / 8 \text { " to } 31 / 8 \text { " dia., up to } 60 " \text { length } \\
9.53 \mathrm{~mm} \text { to } 79.38 \mathrm{~mm} \text {, up to } 1524 \mathrm{~mm}\end{array}$ \\
\hline Control & Via the Haas CNC control \\
\hline Pushers & Standard $3 / 8 "$ and $3 / 4^{\prime \prime}$ \\
\hline Tray capacity & $1 "(25 \mathrm{~mm})$ dia. bars $\times 30 \mathrm{pcs}$. \\
\hline Spindle liner & Most sizes available; \\
\hline Weight & depends on lathe model. \\
\hline Note: Specifications subject to change. & $900 \mathrm{lb}(408 \mathrm{~kg})$ \\
Maximum bar diameter and length determined by lathe model.
\end{tabular}

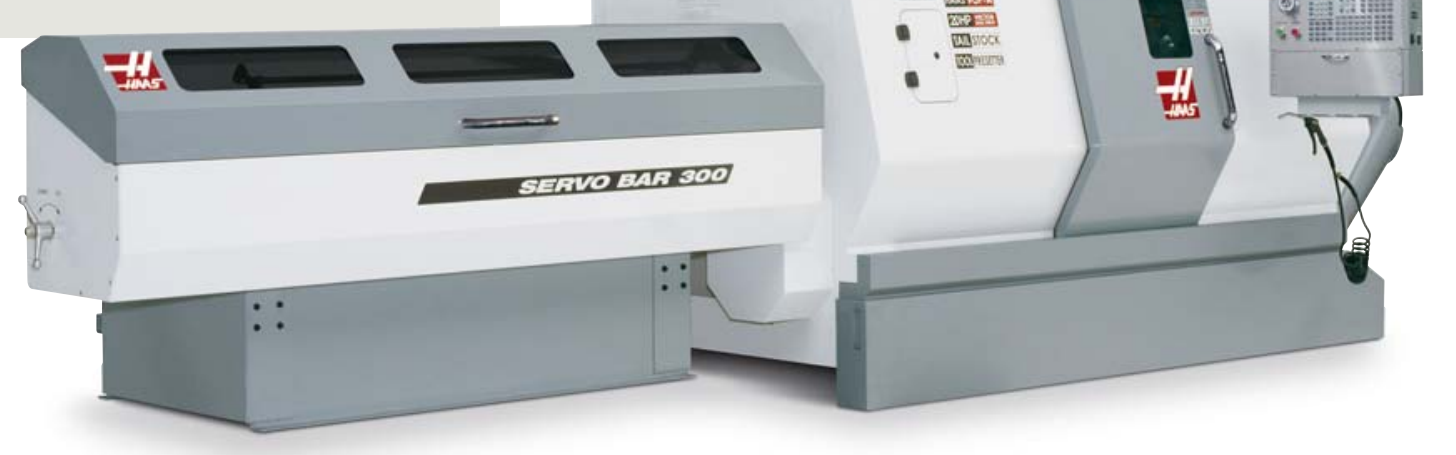




\section{Installation Dimensions}

\begin{tabular}{|c|c|c|c|c|c|}
\hline Dim. & SL-10 & SL-20/TL-15 & SL-30/TL-25 & SL-40 & SL-40L \\
\hline A & $\begin{array}{l}70 " \\
1778 \mathrm{~mm}\end{array}$ & $\begin{array}{l}96 " \\
2438 \mathrm{~mm}\end{array}$ & $\begin{array}{l}119 " \\
3023 \mathrm{~mm}\end{array}$ & $\begin{array}{l}150 " \\
3810 \mathrm{~mm}\end{array}$ & $\begin{array}{l}209 " \\
5309 \mathrm{~mm}\end{array}$ \\
\hline B & $\begin{array}{l}102 " \\
2591 \mathrm{~mm}\end{array}$ & $\begin{array}{l}126.5 " \\
3505 \mathrm{~mm}\end{array}$ & $\begin{array}{l}* 149.5 " \\
3797 \mathrm{~mm}\end{array}$ & $\begin{array}{l}189.5 " \\
4813 \mathrm{~mm}\end{array}$ & $\begin{array}{l}234 " \\
5944 \mathrm{~mm}\end{array}$ \\
\hline B1 & $\begin{array}{l}121 " \\
3073 \mathrm{~mm}\end{array}$ & $\begin{array}{l}138 " \\
3505 \mathrm{~mm}\end{array}$ & $\begin{array}{l}161.5^{\prime \prime} \\
4102 \mathrm{~mm}\end{array}$ & $\begin{array}{l}192.5^{\prime \prime} \\
4889 \mathrm{~mm}\end{array}$ & $\begin{array}{l}252 " \\
6401 \mathrm{~mm}\end{array}$ \\
\hline C & $\begin{array}{l}69^{\prime \prime} \\
1753 \mathrm{~mm}\end{array}$ & $\begin{array}{l}72 " \\
1829 \mathrm{~mm}\end{array}$ & $\begin{array}{l}74 " \\
1880 \mathrm{~mm}\end{array}$ & $\begin{array}{l}86 " \\
2184 \mathrm{~mm}\end{array}$ & $\begin{array}{l}86 " \\
2184 \mathrm{~mm}\end{array}$ \\
\hline D & $\begin{array}{l}36.34 " \\
923 \mathrm{~mm}\end{array}$ & $\begin{array}{l}24 " \\
610 \mathrm{~mm}\end{array}$ & $\begin{array}{l}24 " \\
610 \mathrm{~mm}\end{array}$ & $\begin{array}{l}24 " \\
610 \mathrm{~mm}\end{array}$ & $\begin{array}{l}24 " \\
610 \mathrm{~mm}\end{array}$ \\
\hline D1 & $\begin{array}{l}43 " \\
1092 \mathrm{~mm}\end{array}$ & $\begin{array}{l}48 " \\
1219 \mathrm{~mm}\end{array}$ & $\begin{array}{l}50 " \\
1270 \mathrm{~mm}\end{array}$ & $\begin{array}{l}50 " \\
1270 \mathrm{~mm}\end{array}$ & $\begin{array}{l}50 " \\
1270 \mathrm{~mm}\end{array}$ \\
\hline$E$ & $\begin{array}{l}55 " \\
1397 \mathrm{~mm}\end{array}$ & $\begin{array}{l}62 " \\
1575 \mathrm{~mm}\end{array}$ & $\begin{array}{l}73 " \\
1854 \mathrm{~mm}\end{array}$ & $\begin{array}{l}92 " \\
2337 \mathrm{~mm}\end{array}$ & $\begin{array}{l}95 " \\
2413 \mathrm{~mm}\end{array}$ \\
\hline$F$ & $\begin{array}{l}14.5^{\prime \prime} \\
368 \mathrm{~mm}\end{array}$ & $\begin{array}{l}8.0 " \\
203 \mathrm{~mm}\end{array}$ & $\begin{array}{l}{ }^{*} 8.0 " \\
203 \mathrm{~mm}\end{array}$ & $\begin{array}{l}14.5^{\prime \prime} \\
368 \mathrm{~mm}\end{array}$ & - \\
\hline G & $\begin{array}{l}76 " \\
1930 \mathrm{~mm}\end{array}$ & $\begin{array}{l}106 " 1 \\
2692 \mathrm{~mm}\end{array}$ & $\begin{array}{l}128 " \\
3251 \mathrm{~mm}\end{array}$ & $\begin{array}{l}160 " \\
4064 \mathrm{~mm}\end{array}$ & $\begin{array}{l}219 " \\
5563 \mathrm{~mm}\end{array}$ \\
\hline H & $\begin{array}{l}55 " \\
1397 \mathrm{~mm}\end{array}$ & $\begin{array}{l}74 " \\
1880 \mathrm{~mm}\end{array}$ & $\begin{array}{l}77 " \\
1956 \mathrm{~mm}\end{array}$ & $\begin{array}{l}98 " \\
2489 \mathrm{~mm}\end{array}$ & $\begin{array}{l}107^{\prime \prime} \\
2718 \mathrm{~mm}\end{array}$ \\
\hline
\end{tabular}

*With optional two-speed gearbox add 8". With Big Bore option add 14" B1, D1 = With optional chip conveyor
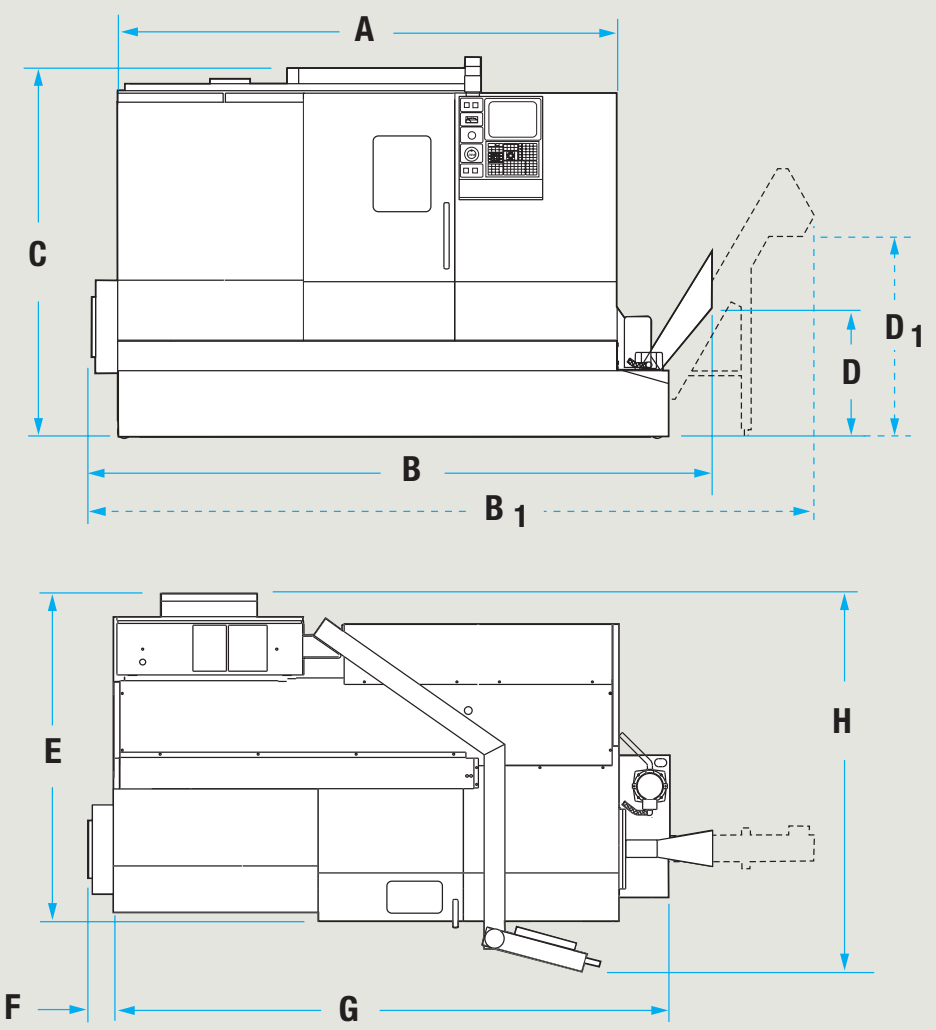

\begin{tabular}{|c|c|c|c|c|}
\hline Dim. & SL-10 & SL-20 & SL-30 & SL-40† \\
\hline $\begin{array}{l}\text { A - Turret Dia } \\
\text { VDI }\end{array}$ & $\begin{array}{l}7.937 " \\
201.6 \mathrm{~mm}\end{array}$ & $\begin{array}{l}15.374 " \\
390.5 \mathrm{~mm}\end{array}$ & $\begin{array}{l}19.000 " \\
482.6 \mathrm{~mm}\end{array}$ & $\begin{array}{l}23.750 " \\
603.3 \mathrm{~mm}\end{array}$ \\
\hline $\begin{array}{l}\text { B- Pocket Dia } \\
\text { VDI }\end{array}$ & $\begin{array}{l}{ }^{* * 1} 12.000 " \\
304.8 \mathrm{~mm}\end{array}$ & $\begin{array}{l}12.000 " \\
304.8 \mathrm{~mm}\end{array}$ & $\begin{array}{l}15.280 " \\
388.1 \mathrm{~mm}\end{array}$ & $\begin{array}{l}20.000 " \\
508.0 \mathrm{~mm}\end{array}$ \\
\hline VB & - & $\begin{array}{l}13.720 " \\
348.5 \mathrm{~mm} \\
\end{array}$ & $\begin{array}{l}13.720 " \\
348.5 \mathrm{~mm} \\
\end{array}$ & $\begin{array}{l}20.000 " \\
508 \mathrm{~mm}\end{array}$ \\
\hline $\begin{array}{l}\text { C- Pocket Spacing } \\
\text { VDI }\end{array}$ & $\begin{array}{l}* * 6.000 " \\
152.4 \mathrm{~mm}\end{array}$ & $\begin{array}{l}3.669^{\prime \prime} \\
93.2 \mathrm{~mm}\end{array}$ & $\begin{array}{l}3.955 " \\
100.5 \mathrm{~mm}\end{array}$ & $\begin{array}{l}6.180 " \\
156.9 \mathrm{~mm}\end{array}$ \\
\hline VB & - & $\begin{array}{l}6.860 " \\
174.2 \mathrm{~mm}\end{array}$ & $\begin{array}{l}6.860 " \\
174.2 \mathrm{~mm}\end{array}$ & $\begin{array}{l}10.000 " \\
254 \mathrm{~mm}\end{array}$ \\
\hline $\begin{array}{l}\text { D - TC Clearance } \\
\text { VDI }\end{array}$ & 0.000 & $\begin{array}{l}3.79^{\prime \prime} \\
96 \mathrm{~mm}\end{array}$ & $\begin{array}{l}4.90 " \\
124 \mathrm{~mm}\end{array}$ & $\begin{array}{l}3.66 " 1 \\
93 \mathrm{~mm}\end{array}$ \\
\hline E - TC Clearance (LT) & - & - & - & $\begin{array}{l}5.70 " \dagger \\
145 \mathrm{~mm}\end{array}$ \\
\hline $\begin{array}{l}\text { F- Max Tool Stickout, } \\
\text { Rear }\end{array}$ & $\begin{array}{l}4.13^{\prime \prime} \\
105 \mathrm{~mm}\end{array}$ & $\begin{array}{l}4.37 " \\
111 \mathrm{~mm}\end{array}$ & $\begin{array}{l}4.50 " \\
114 \mathrm{~mm}\end{array}$ & $\begin{array}{l}4.63 " \\
118 \mathrm{~mm}\end{array}$ \\
\hline $\begin{array}{l}\text { G - Max Tool Stickout, } \\
\text { Front (full Z travel) }\end{array}$ & $\begin{array}{l}4.96 " \\
126 \mathrm{~mm}\end{array}$ & $\begin{array}{l}5.73^{\prime \prime} \\
146 \mathrm{~mm}\end{array}$ & $\begin{array}{l}5.90 " \\
150 \mathrm{~mm}\end{array}$ & $\begin{array}{l}10.91 " \dagger \\
277 \mathrm{~mm}\end{array}$ \\
\hline H - Live Tool Stickout & - & - & - & $\begin{array}{l}5.50 " \\
140 \mathrm{~mm}\end{array}$ \\
\hline J - Live Tool Pocket Width & - & - & - & $\begin{array}{l}8.00 " \\
203 \mathrm{~mm}\end{array}$ \\
\hline K - BOT ID Tool Pocket Dia & $\begin{array}{l}15.28 " \\
388 \mathrm{~mm}\end{array}$ & $\begin{array}{l}15.28 " \\
388 \mathrm{~mm}\end{array}$ & $\begin{array}{l}18.56 " \\
471 \mathrm{~mm}\end{array}$ & $\begin{array}{l}21.42 " \\
544 \mathrm{~mm}\end{array}$ \\
\hline VB & - & $\begin{array}{l}18.42^{\prime \prime} \\
467.9 \mathrm{~mm}\end{array}$ & $\begin{array}{l}18.42 " \\
467.9 \mathrm{~mm}\end{array}$ & $\begin{array}{l}25.52 " \\
648.2 \mathrm{~mm}\end{array}$ \\
\hline L - BOT Pocket Spacing & $\begin{array}{l}3.95 " \\
100 \mathrm{~mm}\end{array}$ & $\begin{array}{l}4.72 " \\
120 \mathrm{~mm}\end{array}$ & $\begin{array}{l}4.80 " \\
122 \mathrm{~mm}\end{array}$ & $\begin{array}{l}6.62 " \\
168 \mathrm{~mm}\end{array}$ \\
\hline VB & - & $\begin{array}{l}9.21 " \\
234 \mathrm{~mm}\end{array}$ & $\begin{array}{l}9.21 " \\
234 \mathrm{~mm}\end{array}$ & $\begin{array}{l}12.76 " 1 \\
324 \mathrm{~mm}\end{array}$ \\
\hline M - BOT TC Clearance & $\begin{array}{l}2.47 " \\
63 \mathrm{~mm}\end{array}$ & $\begin{array}{l}5.00 " \\
127 \mathrm{~mm}\end{array}$ & $\begin{array}{l}6.40 " \\
163 \mathrm{~mm}\end{array}$ & $\begin{array}{l}6.40 " \\
163 \mathrm{~mm}\end{array}$ \\
\hline
\end{tabular}

* Exceeding this stickout may result in collision with the front bulkhead.

** SL-10 VDI pockets are staggered. B1 = 15.280" (388.1 mm); corresponding C dimension = 7.640" (194.1 mm). † All dimensions for the SL-40L are the same as the SL-40, except E, which is 2.80 " $(71 \mathrm{~mm})$, and G, which is $35.00^{\prime \prime}(889 \mathrm{~mm})$. A pocket in the bulkhead of the SL-40L allows clearance for boring bars and long tools.

Specifications subject to change without notice. Not responsible for typographical errors. Note: Overall shipping dimensions vary; contact Haas for details. 


\section{Work Envelope}

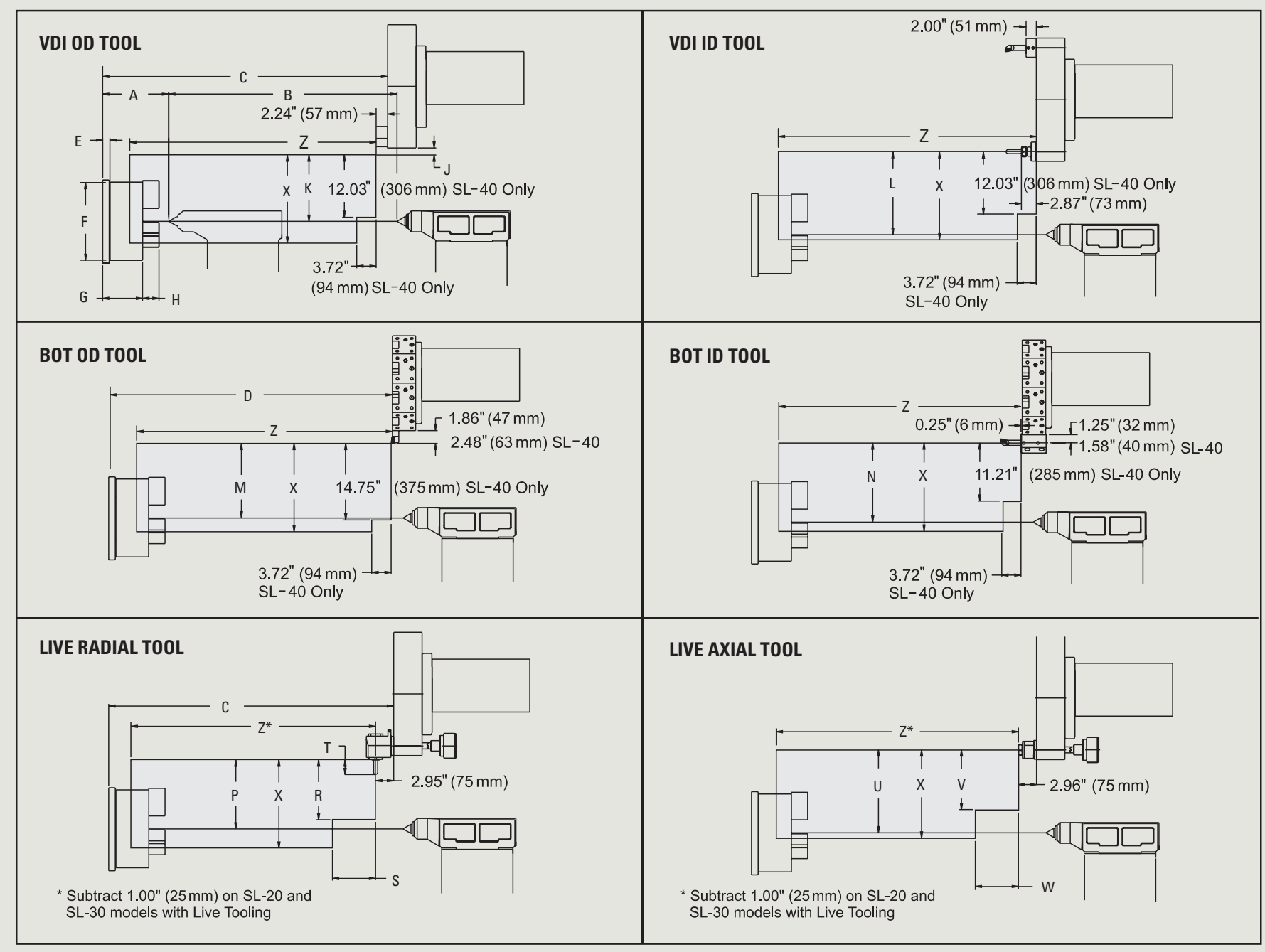

\begin{tabular}{|c|c|c|c|c|c|}
\hline Dimensions & SL-10 & SL-20 & SL-30 & SL-40 & SL-40L \\
\hline A - Tailstock at Full Travel & $\begin{array}{l}9.35^{\prime \prime} \\
237 \mathrm{~mm}\end{array}$ & $\begin{array}{l}10.96 " \\
278 \mathrm{~mm}\end{array}$ & $\begin{array}{l}12.03^{\prime \prime} \\
306 \text { mm }\end{array}$ & $\begin{array}{l}12.72 " \\
323 \text { mm }\end{array}$ & $\begin{array}{l}31.36 " \\
797 \mathrm{~mm}\end{array}$ \\
\hline B - Tailstock Travel & $\begin{array}{l}13.55 " \\
344 \text { mm }\end{array}$ & $\begin{array}{l}20.00 " \\
508 \mathrm{~mm}\end{array}$ & $\begin{array}{l}34.00 " \\
867 \mathrm{~mm}\end{array}$ & $\begin{array}{l}44.00 " \\
1118 \mathrm{~mm}\end{array}$ & $\begin{array}{l}62.00^{\prime \prime} \\
1575 \mathrm{~mm}\end{array}$ \\
\hline C - Turret Home VDI & $\begin{array}{l}18.96 " \\
482 \mathrm{~mm}\end{array}$ & $\begin{array}{l}25.73^{\prime \prime} \\
654 \mathrm{~mm}\end{array}$ & $\begin{array}{l}39.90 " \\
1013 \mathrm{~mm}\end{array}$ & $\begin{array}{l}54.91^{\prime \prime} \\
1395 \mathrm{~mm}\end{array}$ & $\begin{array}{l}90.21 " \\
2291 \mathrm{~mm}\end{array}$ \\
\hline D - Turret Home BOT & $\begin{array}{l}18.96 " \\
482 \mathrm{~mm}\end{array}$ & $\begin{array}{l}25.73^{\prime \prime} \\
654 \mathrm{~mm}\end{array}$ & $\begin{array}{l}39.90 " \\
1013 \mathrm{~mm}\end{array}$ & $\begin{array}{l}54.64 " \\
1388 \mathrm{~mm}\end{array}$ & $\begin{array}{l}89.94 " \\
2284 \mathrm{~mm}\end{array}$ \\
\hline E - Spindle Face & $\begin{array}{l}2.12^{\prime \prime} \\
54 \mathrm{~mm}\end{array}$ & $\begin{array}{l}1.39^{\prime \prime} \\
35 \mathrm{~mm}\end{array}$ & $\begin{array}{l}1.39^{\prime \prime} \\
35 \mathrm{~mm}\end{array}$ & $\begin{array}{l}1.40 " \\
36 \mathrm{~mm}\end{array}$ & $\begin{array}{l}1.40 " \\
36 \mathrm{~mm}\end{array}$ \\
\hline F-Chuck Diameter & $\begin{array}{l}6.50 " \\
165 \mathrm{~mm}\end{array}$ & $\begin{array}{l}8.27^{\prime \prime} \\
210 \mathrm{~mm}\end{array}$ & $\begin{array}{l}10.00 " \\
254 \mathrm{~mm}\end{array}$ & $\begin{array}{l}15.00 " \\
381 \text { mm }\end{array}$ & $\begin{array}{l}15.00 " \\
381 \mathrm{~mm}\end{array}$ \\
\hline G - Chuck Face & $\begin{array}{l}5.66 " \\
144 \mathrm{~mm}\end{array}$ & $\begin{array}{l}5.45^{\prime \prime} \\
139 \mathrm{~mm}\end{array}$ & $\begin{array}{l}6.12^{\prime \prime} \\
155 \mathrm{~mm}\end{array}$ & $\begin{array}{l}7.70 " \\
196 \mathrm{~mm}\end{array}$ & $\begin{array}{l}7.70 " \\
196 \mathrm{~mm}\end{array}$ \\
\hline H - Chuck Jaws & $\begin{array}{l}1.26 " 1 \\
32 \mathrm{~mm}\end{array}$ & $\begin{array}{l}2.00 " \\
51 \mathrm{~mm}\end{array}$ & $\begin{array}{l}1.81 " \\
46 \mathrm{~mm}\end{array}$ & $\begin{array}{l}3.14 " \\
80 \mathrm{~mm}\end{array}$ & $\begin{array}{l}3.14 " \\
80 \mathrm{~mm}\end{array}$ \\
\hline J - VDI OD Tool Stickout & $\begin{array}{l}1.00 " \\
25 \mathrm{~mm}\end{array}$ & $\begin{array}{l}1.26 " 1 \\
32 \mathrm{~mm}\end{array}$ & $\begin{array}{l}1.15^{\prime \prime} \\
29 \mathrm{~mm}\end{array}$ & $\begin{array}{l}1.33 " \\
34 \text { mm }\end{array}$ & $\begin{array}{l}1.33 " \\
34 \text { mm }\end{array}$ \\
\hline K - VDI OD Tool Home & $\begin{array}{l}9.93^{\prime \prime} \\
252 \mathrm{~mm}\end{array}$ & $\begin{array}{l}4.44^{\prime \prime} \\
113 \mathrm{~mm}\end{array}$ & $\begin{array}{l}7.29 " \\
185 \mathrm{~mm}\end{array}$ & $\begin{array}{l}12.79 " \\
325 \mathrm{~mm}\end{array}$ & $\begin{array}{l}12.79^{\prime \prime} \\
325 \mathrm{~mm}\end{array}$ \\
\hline L - VDI ID Tool Home & $\begin{array}{l}7.44^{\prime \prime} \\
189 \mathrm{~mm}\end{array}$ & $\begin{array}{l}7.45 " \\
190 \mathrm{~mm}\end{array}$ & $\begin{array}{l}10.30 " \\
262 \mathrm{~mm}\end{array}$ & $\begin{array}{l}16.00 " \\
406 \mathrm{~mm}\end{array}$ & $\begin{array}{l}16.00 " \\
406 \mathrm{~mm}\end{array}$ \\
\hline
\end{tabular}

\begin{tabular}{|c|c|c|c|c|c|}
\hline \multicolumn{2}{|c|}{ SL-10 } & & & SL-40 & \\
\hline M - BOT OD Tool Home & $\begin{array}{l}5.55^{\prime \prime} \\
141 \mathrm{~mm}\end{array}$ & $\begin{array}{l}5.14^{\prime \prime} \\
131 \mathrm{~mm}\end{array}$ & $\begin{array}{l}8.50 " \\
216 \mathrm{~mm}\end{array}$ & $\begin{array}{l}14.37^{\prime \prime} \\
365 \mathrm{~mm}\end{array}$ & $\begin{array}{l}14.37^{\prime \prime} \\
365 \mathrm{~mm}\end{array}$ \\
\hline N - BOT ID Tool Home & $\begin{array}{l}5.80 " \\
147 \mathrm{~mm}\end{array}$ & $\begin{array}{l}5.75^{\prime \prime} \\
146 \mathrm{~mm}\end{array}$ & $\begin{array}{l}8.66^{\prime \prime} \\
220 \mathrm{~mm}\end{array}$ & $\begin{array}{l}15.28 " 1 \\
388 \mathrm{~mm}\end{array}$ & $\begin{array}{l}15.28 " 1 \\
388 \mathrm{~mm}\end{array}$ \\
\hline P - Radial Live Tool Home & - & $\begin{array}{l}4.74 " \\
120 \mathrm{~mm}\end{array}$ & $\begin{array}{l}7.59^{\prime \prime} \\
193 \mathrm{~mm}\end{array}$ & $\begin{array}{l}13.29 " \\
338 \mathrm{~mm}\end{array}$ & $\begin{array}{l}13.29 " \\
338 \mathrm{~mm}\end{array}$ \\
\hline R - Radial Live Tool X T/S Zone & - & $\begin{array}{l}3.52 " \\
89 \mathrm{~mm}\end{array}$ & $\begin{array}{l}5.83^{\prime \prime} \\
148 \mathrm{~mm}\end{array}$ & $\begin{array}{l}11.53^{\prime \prime} \\
293 \mathrm{~mm}\end{array}$ & $\begin{array}{l}11.53^{\prime \prime} \\
293 \mathrm{~mm}\end{array}$ \\
\hline S - Radial Live Tool Z T/S Zone & - & $\begin{array}{l}4.40 " \\
112 \mathrm{~mm}\end{array}$ & $\begin{array}{l}4.01 " \\
102 \mathrm{~mm}\end{array}$ & $\begin{array}{l}8.32 " \\
211 \mathrm{~mm}\end{array}$ & $\begin{array}{l}8.32 " \\
211 \mathrm{~mm}\end{array}$ \\
\hline T - Radial Live Tool Stickout & - & $\begin{array}{l}2.83^{\prime \prime} \\
72 \mathrm{~mm}\end{array}$ & $\begin{array}{l}4.12^{\prime \prime} \\
105 \mathrm{~mm}\end{array}$ & $\begin{array}{l}4.86 " \\
123 \mathrm{~mm}\end{array}$ & $\begin{array}{l}4.86 " \\
123 \mathrm{~mm}\end{array}$ \\
\hline U - Axial Live Tool Home & - & $\begin{array}{l}7.45^{\prime \prime} \\
189 \mathrm{~mm}\end{array}$ & $\begin{array}{l}10.30 " \\
262 \mathrm{~mm}\end{array}$ & $\begin{array}{l}16.00 " \\
406 \mathrm{~mm}\end{array}$ & $\begin{array}{l}16.00 " \\
406 \mathrm{~mm}\end{array}$ \\
\hline V - Axial Live Tool X T/S Zone & - & $\begin{array}{l}3.52 " \\
89 \mathrm{~mm}\end{array}$ & $\begin{array}{l}5.83^{\prime \prime} \\
148 \mathrm{~mm}\end{array}$ & $\begin{array}{l}11.53^{\prime \prime} \\
293 \mathrm{~mm}\end{array}$ & $\begin{array}{l}11.53^{\prime \prime} \\
293 \mathrm{~mm}\end{array}$ \\
\hline W - Axial Live Tool Z T/S Zone & - & $\begin{array}{l}4.40^{\prime \prime} \\
112 \mathrm{~mm}\end{array}$ & $\begin{array}{l}4.01 " \\
102 \mathrm{~mm}\end{array}$ & $\begin{array}{l}8.32^{\prime \prime} \\
211 \mathrm{~mm}\end{array}$ & $\begin{array}{l}7.32 " \\
186 \mathrm{~mm}\end{array}$ \\
\hline$X$-X-Axis Travel & $\begin{array}{l}6.25 " \\
159 \mathrm{~mm}\end{array}$ & $\begin{array}{l}8.45^{\prime \prime} \\
215 \mathrm{~mm}\end{array}$ & $\begin{array}{l}11.30 " \\
287 \mathrm{~mm}\end{array}$ & $\begin{array}{l}17.00 " \\
432 \mathrm{~mm}\end{array}$ & $\begin{array}{l}17.00 " \\
432 \mathrm{~mm}\end{array}$ \\
\hline Z-Z-Axis Travel & $\begin{array}{l}14.00 " \\
356 \mathrm{~mm}\end{array}$ & $\begin{array}{l}20.00 " \\
508 \mathrm{~mm}\end{array}$ & $\begin{array}{l}34.00 " \\
864 \mathrm{~mm}\end{array}$ & $\begin{array}{l}44.00^{\prime \prime} \\
1118 \mathrm{~mm}\end{array}$ & $\begin{array}{l}80.00 " \\
2032 \mathrm{~mm}\end{array}$ \\
\hline
\end{tabular}




\title{
Worldwide Support
}

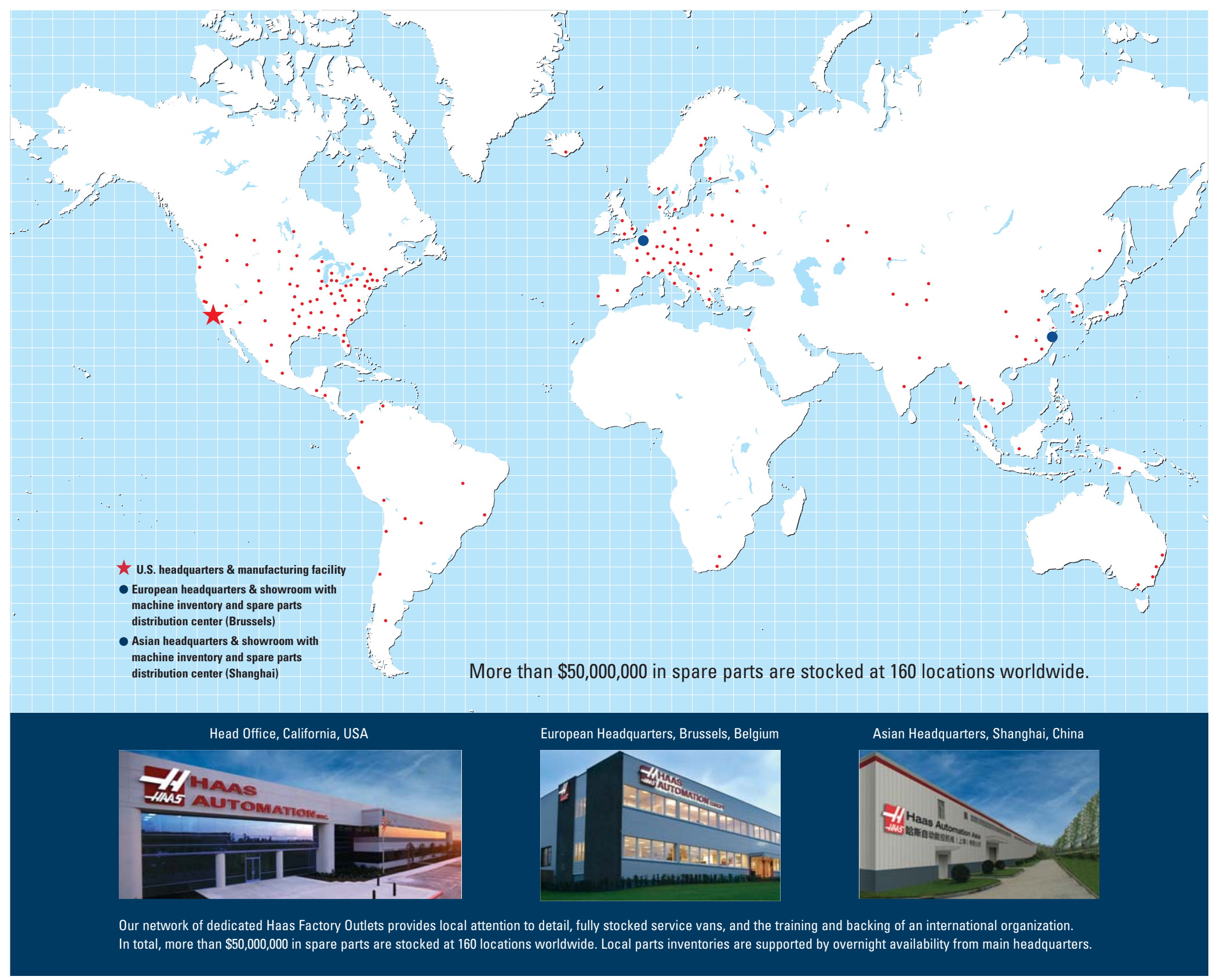

\section{Haas Automation, Inc.}

2800 Sturgis Road, Oxnard, California 93030 Toll Free: 800-331-6746 Fax: 805-278-8540

www.HaasCNC.com

\author{
Haas Automation, Europe
}

Mercuriusstraat 28, B-1930 Zaventem, Belgium• Tel: 011-32 25229905 Fax: 011-32 25230855

\section{Haas Automation, Asia}



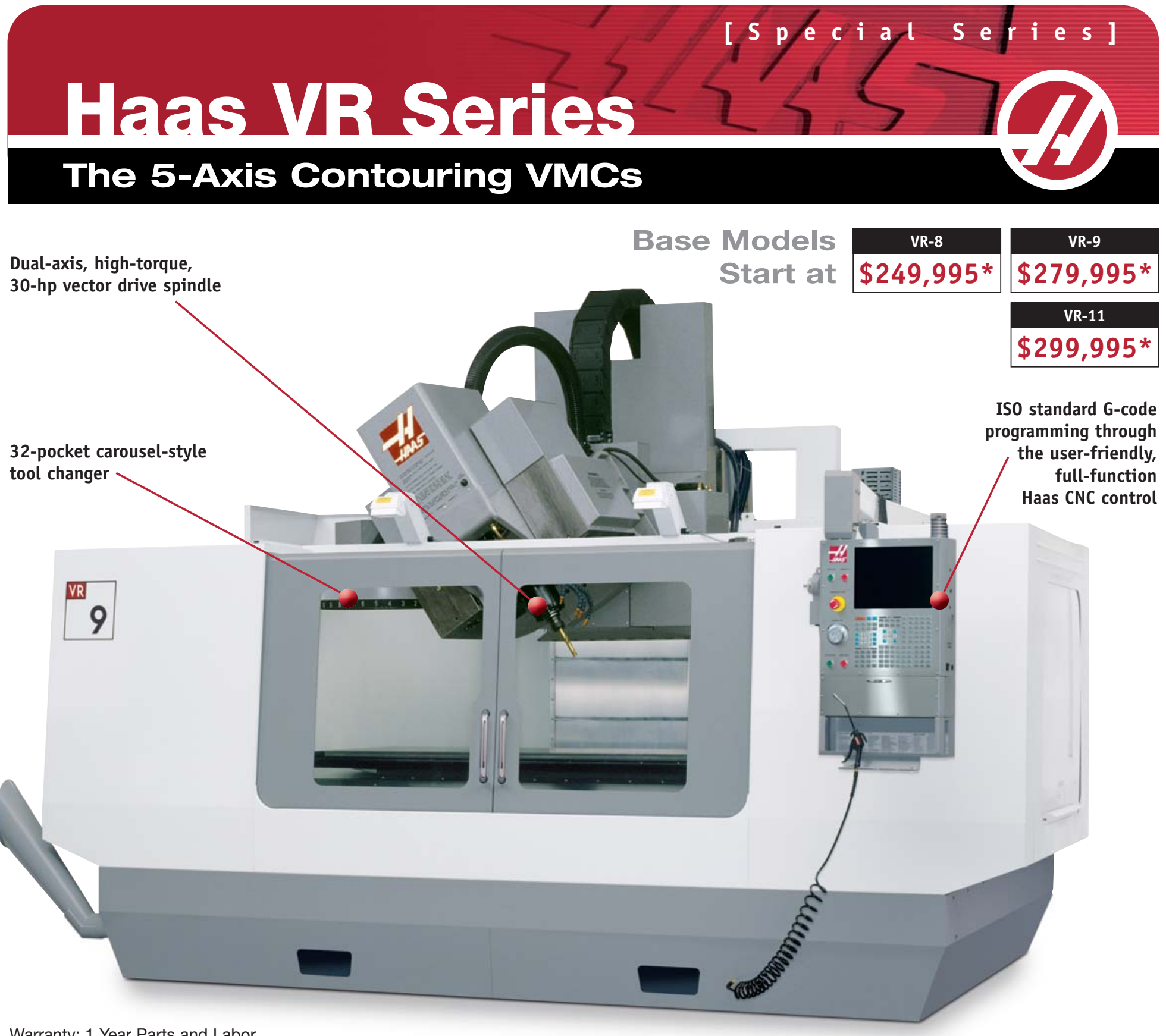

Warranty: 1 Year Parts and Labor

\section{[ Standard Features ]}

- High-Torque 30-hp Vector Drive Spindle

- 32-Pocket Automatic Tool Changer

- Programmable Coolant Nozzle

- Chip Auger System

- 16 MB Program Memory

- Remote Jog Handle

- Visual Quick Code Programming

- Rigid Tapping

- 15" Color LCD Monitor w/USB Port

- High-Speed Machining w/Look-Ahead

- User-Defined 2nd Home Button

- Anchor Kit
[ Options ] p a r t i a I l is t

- 15,000-rpm Spindle

- 10,000-rpm Spindle

- Through-Spindle Coolant

- Linear Scales (X,Y and Z axes)

- Hard Disk Drive with Ethernet Interface

- User-Definable Macros

- Spindle Orientation

- Coordinate Rotation and Scaling

- Memory Lock Keyswitch 


\section{Haas VR Series}

\section{The 5-Axis Contouring VMCs}

The Haas VR Series five-axis contouring VMCs provide a cost-effective solution for large 5 -axis machining operations. With travels ranging from $64 "$ " $40 "$ " $\times 30$ " for the VR-8 to $120 "$ " $40 \times 30$ " for the VR-11, these machines can handle the demands of producing large, complicated parts. Each VR is equipped with a unique dual-axis spindle that features a fully enclosed gimbal design to protect gears from chips and coolant. The VR head provides \pm 32 degrees of travel on the A and $B$ axes for machining complex geometries and undercuts.

Each VR features a 32-pocket automatic tool changer that swings out of the enclosure for unobstructed machining, and a 30-hp, high-torque spindle that features a vector drive for the absolute best performance throughout the entire rpm range.
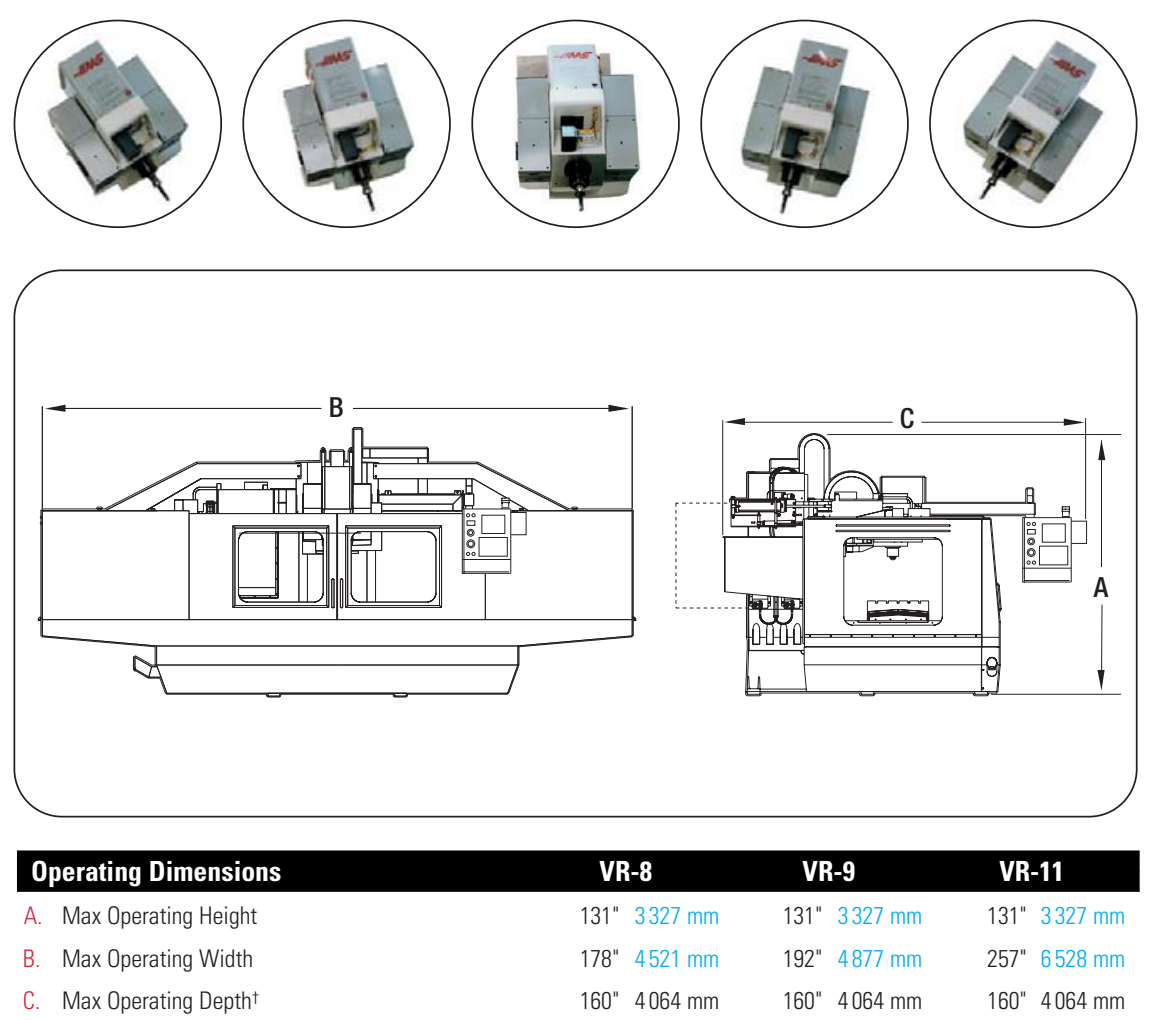

† Requires additional $36^{\prime \prime}(914 \mathrm{~mm})$ to open rear service panel.

\section{[ Specifications ]}

\begin{tabular}{|c|c|c|c|}
\hline Travels & VR-8 & VR-9 & VR-11 \\
\hline$x$ & $\begin{array}{r}64^{\prime \prime} \\
1626 \mathrm{~mm}\end{array}$ & $\begin{array}{r}84 " \\
2134 \mathrm{~mm}\end{array}$ & $\begin{array}{r}120 " \\
3048 \mathrm{~mm}\end{array}$ \\
\hline Y & $\begin{array}{r}40 " \\
1016 \mathrm{~mm}\end{array}$ & $\begin{array}{r}40 " \\
1016 \mathrm{~mm}\end{array}$ & $\begin{array}{r}40 " \\
1016 \mathrm{~mm}\end{array}$ \\
\hline Z & $\begin{array}{r}30 " \\
762 \mathrm{~mm}\end{array}$ & $\begin{array}{r}30 " \\
762 \mathrm{~mm}\end{array}$ & $\begin{array}{r}30 " \\
762 \mathrm{~mm}\end{array}$ \\
\hline$A, B$ & $\pm 32^{\circ}$ & $\pm 32^{\circ}$ & $\pm 32^{\circ}$ \\
\hline $\begin{array}{l}\text { Spindle Nose to Table } \\
\text { (approximate) }\end{array}$ & $\begin{array}{r}4 " \text { - } 34 " \\
102-864 \mathrm{~mm}\end{array}$ & $\begin{array}{c}4 " \text { - } 34 " \\
2-864 \mathrm{~mm} 1\end{array}$ & $\begin{array}{r}4 "-34 " \\
2-864 \mathrm{~mm}\end{array}$ \\
\hline
\end{tabular}

\section{Table}

\begin{tabular}{|c|c|c|c|}
\hline Length & $\begin{array}{r}64 " \\
1626 \text { mm }\end{array}$ & $\begin{array}{r}84 " \\
2134 \text { mm }\end{array}$ & $\begin{array}{r}120 " \\
3048 \text { mm }\end{array}$ \\
\hline Width & $\begin{array}{r}36 " \\
914 \mathrm{~mm}\end{array}$ & $\begin{array}{r}36 " \\
914 \text { mm }\end{array}$ & $\begin{array}{r}28 " \\
711 \mathrm{~mm}\end{array}$ \\
\hline Max Weight on Table & $\begin{array}{l}4,000 \mathrm{lb} \\
1814 \mathrm{~kg}\end{array}$ & $\begin{array}{l}4,000 \mathrm{lb} \\
1814 \mathrm{~kg}\end{array}$ & $\begin{array}{l}4,000 \mathrm{lb} \\
1814 \mathrm{~kg}\end{array}$ \\
\hline \multicolumn{4}{|l|}{ T-Slots } \\
\hline Width & \multicolumn{3}{|c|}{ 5/8" 16 mm } \\
\hline Center Distance & \multicolumn{3}{|c|}{ 4.92" $125.0 \mathrm{~mm}$} \\
\hline \multicolumn{4}{|l|}{ Spindle } \\
\hline Taper Size & \multicolumn{3}{|c|}{ \#40 Taper } \\
\hline Speed & \multicolumn{3}{|c|}{$7,500 \mathrm{rpm}$} \\
\hline
\end{tabular}

Max Torque

75 ft-lb @ 1,000 rpm 102 Nm @ 1000 rpm

Motors

$\begin{array}{lc}\text { Spindle Max Rating } & 30 \mathrm{hp} 22.4 \mathrm{~kW} \\ \text { Axis Max Thrust Rating } & 3,400 \mathrm{lb} 15124 \mathrm{~N}\end{array}$

Feedrates

$\begin{array}{crrr}\text { Rapids } & & & \\ \text { X } & 540 \mathrm{ipm} & 600 \mathrm{ipm} & 360 \mathrm{ipm} \\ & 13.7 \mathrm{~m} / \mathrm{min} & 15.2 \mathrm{~m} / \mathrm{min} & 9.1 \mathrm{~m} / \mathrm{min} \\ \text { Y, Z } & 600 \mathrm{ipm} & 600 \mathrm{ipm} & 600 \mathrm{ipm} \\ & 15.2 \mathrm{~m} / \mathrm{min} & 15.2 \mathrm{~m} / \mathrm{min} & 15.2 \mathrm{~m} / \mathrm{min} \\ \text { A, B } & & 1,900^{\circ} / \mathrm{min} & \\ \text { Cutting Feedrates } & & & \\ \text { X, Y, Z } & 500 \mathrm{ipm} & 500 \mathrm{ipm} & 360 \mathrm{ipm} \\ & 12.7 \mathrm{~m} / \mathrm{min} & 12.7 \mathrm{~m} / \mathrm{min} & 9.1 \mathrm{~m} / \mathrm{min} \\ \text { A, B } & & 600^{\circ} / \mathrm{min} & \end{array}$

Tool Changer

$\begin{array}{lc}\text { Capacity } & 32 \\ \text { Type } & \text { CT } 40 \text { (BT } 40 \text { optional) }\end{array}$

General

\begin{tabular}{lcrr} 
Door Open Width & $69 "$ & $85 "$ & $120 "$ \\
& $1753 \mathrm{~mm}$ & $2159 \mathrm{~mm}$ & $3048 \mathrm{~mm}$ \\
Machine Weight & $27,100 \mathrm{lb}$ & $28,100 \mathrm{lb}$ & $32,500 \mathrm{lb}$ \\
& $12293 \mathrm{~kg}$ & $12746 \mathrm{~kg}$ & $14742 \mathrm{~kg}$ \\
Air Required & $9 \mathrm{scfm} @ 100 \mathrm{psi} 255 \mathrm{Lpm} @ 6.9 \mathrm{bar}$ \\
Power Required (min) & $28 \mathrm{kVA}$ \\
& \multicolumn{3}{c}{$200-250 \mathrm{VAC}$ or } \\
& $380-480 \mathrm{VAC}$
\end{tabular}




\section{Preparing Your Laboratory for the}

PerkinElmer SCIEX ICP-MS instruments are complete systems with the exception of the following items which must be provided by the customer: electrical power, exhaust vents, argon gas supplies with approved regulator, and coolant system. The items shown in the following checklist need to be considered when preparing the laboratory for the instrument.

- Environmental conditions

- Electrical requirements

- Space requirements

- Exhaust ventilation

- Coolant requirements

- Gases

- Computer and printer table

\section{Environmental Conditions}

The environment in which the instrument is installed should meet the following conditions.

- The room temperature should be between 15 and $30^{\circ} \mathrm{C}\left(59-86^{\circ} \mathrm{F}\right)$ with a maximum rate of change of $2.8^{\circ} \mathrm{C}\left(5^{\circ} \mathrm{F}\right)$ per hour.

- The relative humidity should be between 20 and $80 \%$, noncondensing. For optimum performance, the room temperature should be controlled at $20 \pm 2^{\circ} \mathrm{C}\left(68 \pm 3.6^{\circ} \mathrm{F}\right)$ and the relative humidity should be between 35 and $50 \%$.

In order to minimize contamination problems, a relatively dust-free environment is necessary. This is especially important when working with ultra-trace techniques, such as
ICP-MS. Maximum dust levels can not exceed $1,000,000$ particles (0.5 micron or larger) per cubic foot of air (Class 1,000,000). A normal office environment would be 500,000 to $1,000,000$ particles per cubic foot.

If the ELAN 9000 is going to be used to carry out ultra-trace determinations, such as in the semiconductor industry, it is advised that the instrument be installed in at least a Class 1000 clean room.

In addition, the instrument should be located in an area that is:
- Free of smoke and corrosive fumes

- Not prone to excessive vibration

- Out of direct sunlight

- Away from heat radiators

PerkinElmer SCIEX ICP-MS instruments have been designed for indoor use. The ELAN 9000 can be installed into a mobile laboratory if the instrument is isolated from vibrations.

WARNING:

Do not use the instrument in an area where explosion hazards may exist.

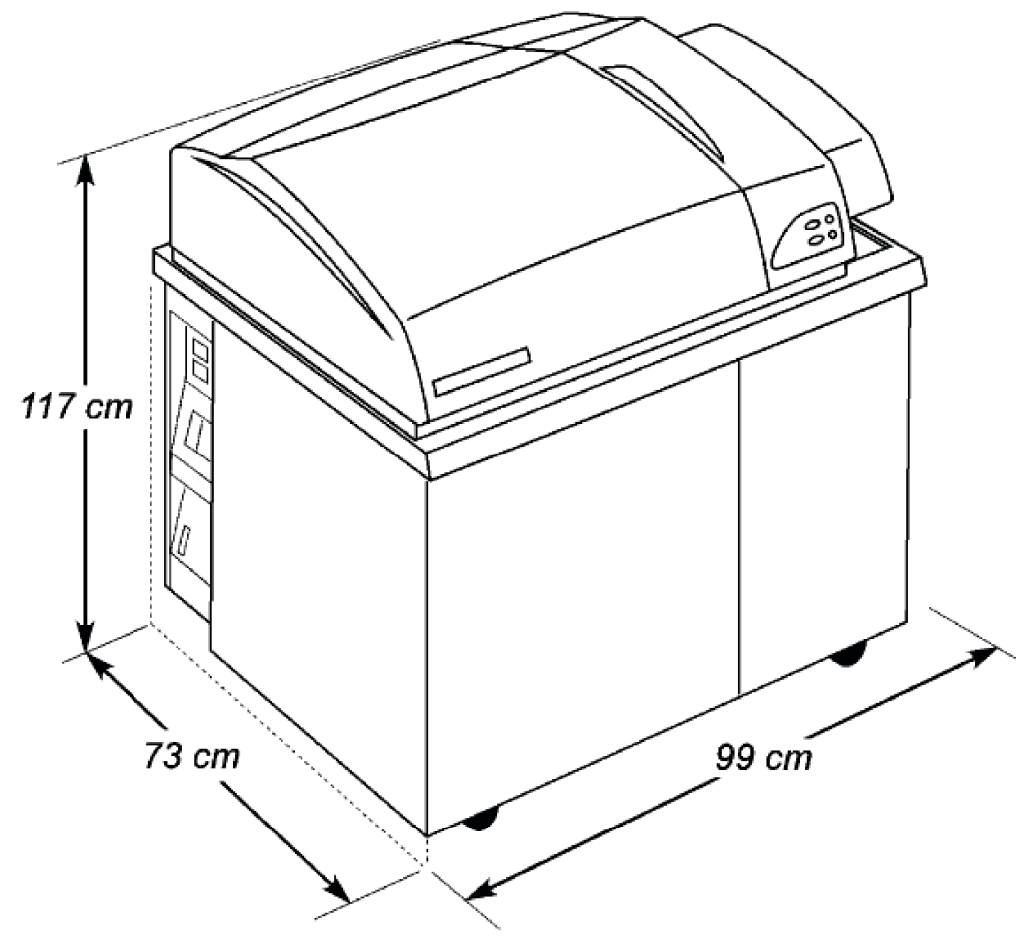

Figure 1. Dimensions of the ELAN 9000 ICP-MS. 


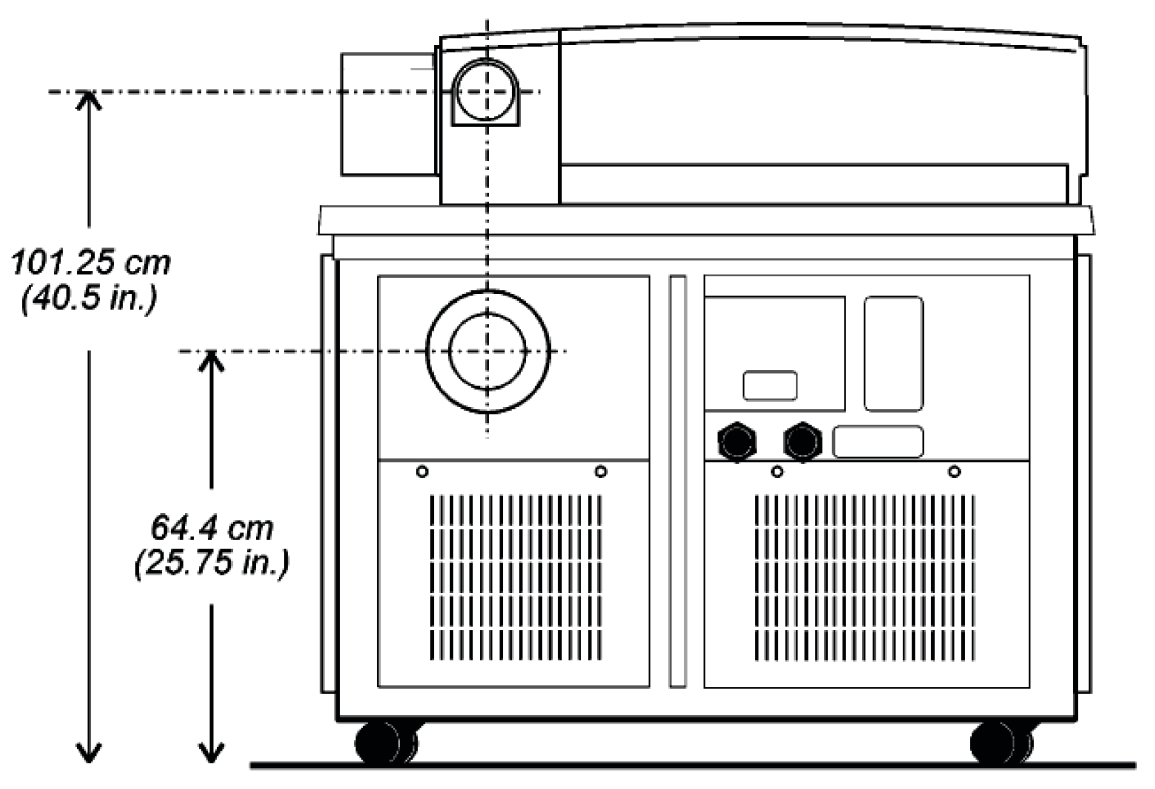

Figure 2. Vent locations, rear of the ELAN 9000 ICP-MS.

- Equip the outlet end of the system with a backdraft damper. Keep the exhaust outlet away from open windows or inlet vents and to extend it above the roof of the building for proper dispersal of the exhaust.

- Equip the exhaust end of the system with an exhaust stack to improve the overall efficiency of the system.

- For best efficiency, make sure the length of the duct that enters into the blower is a straight length at least ten times the duct diameter. An elbow entrance into the blower inlet causes a loss in efficiency.

- Provide make-up air in the same quantity as is exhausted by the system. An "airtight" lab will cause an efficiency loss in the exhaust system.

- Ensure that the system is drawing properly by placing a piece of cardboard over the mouth of the vent.

- Equip the blower with an indicator light located near the instrument to indicate to the operator when the blower is on.
If laboratory temperatures do not exceed $30^{\circ} \mathrm{C}\left(86^{\circ} \mathrm{F}\right)$, the heat exchanger can be used in place of a refrigerated chiller. For laboratories where the temperature can exceed $30^{\circ} \mathrm{C}\left(86^{\circ} \mathrm{F}\right)$. a refrigerated chiller is required. The heat exchanger must be located in a well ventilated area where the air temperature will not exceed $30^{\circ} \mathrm{C}\left(86^{\circ} \mathrm{F}\right)$.

\section{Argon Gas Requirements}

Argon is used as the ICP torch gas with the ELAN 9000 . The quality criteria for argon is listed below.

$\begin{array}{lr}\text { Purity } & \geq 99.996 \% \\ \text { Oxygen } & <5 \mathrm{ppm} \\ \text { Hydrogen } & <1 \mathrm{ppm} \\ \text { Nitrogen } & <20 \mathrm{ppm} \\ \text { Water } & <4 \mathrm{ppm}\end{array}$

Either liquid or gaseous argon can be used with an ICP-MS system. The choice of liquid argon or gaseous argon tanks is determined primarily by the availability of each and the usage rate. Liquid argon is usually less expensive per unit volume to purchase, but cannot be stored for extended periods. If liquid argon is used, the tank should be fitted with an over-pressure regulator which will vent the tank as necessary in order to prevent the tank from becoming a safety hazard.

\section{Coolant Requirements}

The ELAN 9000 system requires a regulated source of filtered coolant for the purpose of cooling. The coolant supply should be filtered (free of sediment) and have a $\mathrm{pH}$ between 6.5 and 8.5 . It should be hardness-free with $<1 \mathrm{ppm}$ of heavy minerals. The recirculator operating pressure should be $344 \pm 13 \mathrm{kPa}$ $(50 \pm 2 \mathrm{psi})$. A coolant flow of at least $3.8 \mathrm{~L} / \mathrm{min}(1.0 \mathrm{gpm})$ is required.

A recirculating system containing a corrosion inhibitor is specified to protect the aluminum components of the cooling system and the interface. Sufficient pre-mixed coolant (Part No. WE01-6558) is supplied for the Heat Exchanger or Refrigerated chiller.
Gaseous argon tanks do not require venting and consequently can be stored for extended periods without loss. A tank of liquid argon containing 4300 cubic feet will last for approximately 100 hours of continuous ICP running time. A tank of gaseous argon will last 5 to 6 hours of ICP running time. The normal argon gas usage is $14-20 \mathrm{~L} / \mathrm{min}$.

A cylinder regulator (Part No. 03030284) which can be used with argon is available from PerkinElmer. The regulator can be used with CGA 580 or CGA 590 fittings and includes a color-coded hose with $1 / 4$-inch Swagelok fittings to permit direct connection to the regulator and to the instrument gas controls. Liquid argon may be purchased from your gas supplier. 


\section{Exhaust Vents}

The ELAN 9000 ICP-MS requires two separate vents, one for the ICP Power Supply/Roughing Pump exhaust and another vent for the Torch Box exhaust. The main venting system is required to remove combustion fumes and vapors from the torch housing. Exhaust venting is important for the following reasons:

- It protects laboratory personnel from toxic vapors that may be produced by some samples.

- It will minimize the effects of room drafts and the laboratory atmosphere on ICP torch stability.

- It will help protect the instrument from corrosive vapors which may originate from the samples.

- It will remove dissipated heat which is produced by the ICP torch, RF power supply, and the pump motors.

\section{WARNING:}

The use of ICP-MS instruments without adequate ventilation to outside air may constitute a health hazard. For example, the combustion of halogenated hydrocarbons produces toxic vapors. Extreme care should be taken that exhaust gases are vented properly.
The main $100 \mathrm{~mm}$ (4 inch) venting system must provide a flow rate of approximately 70 liters/sec $\pm 10 \%$ (150 cubic feet/min). The $150 \mathrm{~mm}$ (6 inch) venting system must provide a flow rate of approximately 210 liters/sec $\pm 10 \%$ (450 cubic feet/min). Both of the exhaust ports should be connected directly to flexible exhaust hoses.

The main torch box must be installed, but there is an option for the coaxial $\mathrm{RF}$ generator and roughing pump exhaust. If a 150-mm (6-in.) duct is not available, a $100-\mathrm{mm}$ (4-in., $150 \mathrm{ft}^{3} / \mathrm{min}$ ) duct can be connected to the inner duct. Only the RF generator is exhausted in this case. The heat from the roughing pumps is released into the laboratory.

We recommend a $100 \mathrm{~mm}$ (4 inch) ID torch box exhaust hose and a 150 mm (6 inch) ID ICP Power Supply/ Roughing Pump air exhaust hose. The ELAN 9000 is supplied with $3 \mathrm{~m}$ (10 ft.) of $100 \mathrm{~mm}$ (4 inch) and $3 \mathrm{~m}$ (10 ft.) of $150 \mathrm{~mm}$ (6 inch) flexible tubing. This tubing permits the movement of the instrument without disconnecting the vents from the laboratory system. See Tables VI and VII for vent specifications.

Table VI. Flow Rates and Anemometer Readings

\begin{tabular}{lcc}
\hline Hose Diameter & Flow Rate & Anemometer Reading \\
\hline $100 \mathrm{~mm}(4 \mathrm{in})$. & $70 \mathrm{~L} / \mathrm{s}\left(150 \mathrm{ft}^{3} / \mathrm{min}\right)$ & $9 \mathrm{~m} / \mathrm{s}(1695 \mathrm{fpm})$ \\
\hline $150 \mathrm{~mm}(6 \mathrm{in})$. & $210 \mathrm{~L} / \mathrm{s}\left(450 \mathrm{ft}^{3} / \mathrm{min}\right)$ & $11.5 \mathrm{~m} / \mathrm{s}(2250 \mathrm{fpm})$ \\
\hline
\end{tabular}

Table VII. Hose Diameter and Venting Capabilities

\begin{tabular}{lcc}
\hline Hose & Hose Diameter & $\begin{array}{c}\text { Vented Outside Lab } \\
\text { Watts (BTU/hr) }\end{array}$ \\
\hline Torch Box Exhaust & $100 \mathrm{~mm}(4 \mathrm{in})$. & $200(680)$ \\
\hline ICP Power Supply/Roughing Pump & $150 \mathrm{~mm}(6 \mathrm{in} .)^{*}$ & $2800(9400)$ \\
\hline ICP Power Supply Only & $100 \mathrm{~mm}(4 \mathrm{in})$. & $1400(4700)$ \\
\hline *If only the 100-mm (4-in.) ID ICP power supply exhaust hose is used, approximately $1400 \mathrm{~W}$ \\
$\begin{array}{l}(4700 \mathrm{BTU} / \mathrm{hr}) \text { of heat is vented into the lab. An independent room air conditioner [3000 W } \\
(10000 \mathrm{BTU} / \mathrm{hr})] \text { is recommended to remove this additional heat. }\end{array}$
\end{tabular}

\section{Vent Positions}

Both of the ELAN 9000 vents are located on the back of the instrument. See Figure 2. The Torch Box exhaust vent is $23.75 \mathrm{~cm}(9.5 \mathrm{in})$ from the left side of the instrument when viewed from the rear and $101.25 \mathrm{~cm}$ (40.5 in) above the floor. The ICP Power Supply/Roughing pump exhaust vent is $23.75 \mathrm{~cm}$ (9.5 in) from the left side of the instrument (rear view) and $64.4 \mathrm{~cm}$ (25.75 in) above the floor.

\section{Venting System Recommendations}

The exhaust flow rate at the instrument (the ability to vent the system) is dependent on customer provided blower, the duct length, material and the number of elbows or bends used. If an excessively long duct system or a system with many bends is used, a stronger blower may be necessary to provide sufficient exhaust volume at the instrument. Smooth stainless steel tubing should be used instead of flexible stainless steel tubing where flexibility is not required to reduce system friction loss or "drag." A length of smooth stainless steel ducting has $20-30 \%$ less friction loss than a comparable length of flexible ducting. When smooth stainless steel tubing is used, elbows must be used to turn corners. These elbows should turn at no more than 45 degrees between straight sections to reduce friction losses, and the number of elbows should be minimized.

Additional recommendations on the venting system include:

- The duct casing and venting system should be made of materials suitable for temperatures as high as $70^{\circ} \mathrm{C}$ $\left(160^{\circ} \mathrm{F}\right)$ and be installed to meet local building code requirements.

- Locate the blower as close to the discharge outlet as possible. All joints on the discharge side should be airtight, especially if toxic vapors are being carried. 
- When storing cylinders external to a building, the cylinders should be stored so that they are protected against temperature extremes (including the direct rays of the sun) and should be stored above ground on a suitable floor.

- Mark gas cylinders clearly to identify the contents and status (full, empty, etc.).

- Do not attempt to refill gas cylinders yourself.

- Use only approved regulators and hose connectors. Left-hand thread fittings are used for fuel gas tank connections whereas right-hand fittings are used for oxidant and support gas connections.

- Arrange gas hoses where they will not be damaged or stepped on and where things will not be dropped on them.

- Perform periodic gas leak tests by applying a soap solution to all joints and seals.

\section{Facilities Requirements}

Table III provides information on the gas and liquid services required for the ELAN 9000. Tables IV and $\mathrm{V}$ show the electrical supply requirements and approximate power consumption of the ELAN 9000 and its major accessories.

\section{Electrical Requirements}

Power to the ELAN 9000 is to be delivered from two 30A single-phase 200-240V dedicated electrical branch circuits according to the power specifications in Table IV. Table V provides the electrical supply requirements and approximate power consumption of the major accessories and options. If the power line is unstable, fluctuates or is subject to surges, additional control of the incoming power may be required. A means of electrically grounding the instrument must be available.
60-Hertz-Operation Connections

The instrument is shipped with two $400 \mathrm{~cm}$ line cord cables. The installation kit includes two Hubbell No. 2621 plugs and two Hubbell No. 2620 receptacles for use with two $60 \mathrm{~Hz}$ single phase outlets. The instrument is wired for power at the time

\section{0-Hertz-Operation Connections}

The instrument is shipped with two $400 \mathrm{~cm}$ line cord cables. It is up to the service person installing the instrument to wire it according to the power available at the lab. The single phase connectors must be supplied by either the customer or the local PerkinElmer office. For $50 \mathrm{~Hz}$ operation, Hubbell Number 2351 plugs and of installation.
Hubbell No. 2350 receptacles are recommended. Note that the installation kit includes 2 Hubbell No. 2621 plugs and a No. 6 AWG ground wire. A means of electrically grounding the instrument must be available.

\section{Three-Phase-Operation Connections} If a three phase connection is required (by local electrical code), the instrument can be connected via a single line cord to two of the three sides of the three phase line. The three-phase line cord and connectors must be supplied by either the customer or the local PerkinElmer office. A means of electrically grounding the instrument must be available. Four meters of grounding wire are provided with the instrument.
Table III. Services Required for the ELAN 9000

\begin{tabular}{ll}
\hline Gases & Argon, $350 \pm 7 \mathrm{kPa}(51 \pm 1 \mathrm{psi})$ at $20 \mathrm{~L} / \mathrm{min}$ flow \\
\hline Cooling & $\begin{array}{l}\text { Coolant circulation of at least } 3.8 \mathrm{~L} / \mathrm{min}(1.0 \mathrm{gpm}) \text { at an operating } \\
\text { pressure of } 344 \pm 14 \mathrm{kPa}(50 \pm 2 \mathrm{psi})\end{array}$ \\
\hline
\end{tabular}

Table IV. ELAN 9000 Power Specifications

\begin{tabular}{lc}
\hline Power Consumption & \\
\hline Maximum Volt Amperes (total, both circuits) & $6000 \mathrm{VA}$ \\
\hline Maximum Continuous Current (per circuit) & $20 \mathrm{~A}$ \\
\hline Voltage Amplitude Specification & \\
\hline Operating Voltage & $200-240 \mathrm{~V}$ \\
\hline Maximum Allowable Percent Sag & $5 \%$ \\
\hline Maximum Allowable Percent Swell & $5 \%$ \\
\hline Phase (single or three) & \\
\hline Frequency Specification & 50 or $60 \mathrm{~Hz}$ \\
\hline Operating Frequency & $\pm 1 \mathrm{~Hz}$ \\
\hline Allowable Frequency Variance & \\
\hline Waveform Specification & $5 \%$ \\
\hline Maximum Supply Voltage Total Distortion & $3 \%$ \\
\hline Maximum Supply Voltage Distortion by Single Harmonic & \\
\hline
\end{tabular}

Table V. Electrical Requirements of ELAN Accessories

\begin{tabular}{lcc}
\hline Equipment & Voltage (AC) & Power \\
\hline Computer & Depends on Model & Depends on Model \\
\hline Printer: HP LaserJet 4100 & $100-127 \mathrm{~V} / 220-240 \mathrm{~V}, 50 / 60 \mathrm{~Hz}$ & $330 \mathrm{~W}$ \\
\hline $\begin{array}{l}\text { Cooling System: } \\
\begin{array}{l}\text { Heat Exchanger } \\
\text { (PolyScience 3370) }\end{array}\end{array}$ & $208-240 \mathrm{~V}, 50 / 60 \mathrm{~Hz}$ & $800 \mathrm{~W}$ \\
$\begin{array}{l}\text { Refrigerated Chiller } \\
\text { (PolyScience 6105PE) }\end{array}$ & $\begin{array}{c} \\
\end{array}$ & $208-230 \mathrm{~V}, 60 \mathrm{~Hz}, 8 \mathrm{~A}$ \\
\hline FIAS 400MS & $240 \mathrm{~V}, 50 \mathrm{~Hz}, 8.5 \mathrm{~A}$ & $2000 \mathrm{~W}$ \\
\hline
\end{tabular}




\section{Space Requirements}

The system should be located near the required electrical and gas supplies. The spectrometer also requires liquid cooling. A suitable cooling system can be purchased from PerkinElmer.

The ELAN 9000 is on wheels and can be moved for service and preventative maintenance. However, a space of at least $30 \mathrm{~cm}$ (12 in.) behind the instrument is recommended. This space behind the instrument provides clearance for the vent hoses. Access for most service procedures will be through the front of the instrument. Allow space on the right side of the instrument for an accessory cart or table.

\section{System Layout}

The ICP-MS consists of the main instrument, the computer controller assembly and a printer, the dimensions of which are given in Figure 1 and Table I.

The ELAN 9000 ICP-MS can be positioned in either a linear or an L-shaped configuration. In the L-shaped configuration, the computer and printer are positioned on one leg of the L. The instrument and an accessory table make up the other leg.

There should be sufficient space near the spectrometer for the various accessories (autosampler, FIAS, electrothermal vaporizer, laser sampler, ultrasonic nebulizer, etc). It is recommended that the accessories be placed on a movable cart or table to allow for easy servicing access. Table II lists the dimensions of the accessories.

The system computer may be placed on a bench or a separate computer table. A suitable computer worktable is available from PerkinElmer (Part No. N058-1451).

\section{Drain Vessels}

A 15-liter drain vessel is supplied with the ELAN 9000 ICP-MS. The vessel is made of HDPE (high density polyethylene) and is used to collect the effluent from the ICP sample introduction system.

The drain vessel should be placed to the right of the instrument. The drain vessel should NOT be stored in an enclosed storage area. The drain system should be checked regularly and replaced when necessary. Should it become necessary to replace the drain vessel, it should be made from a material not likely to be attacked by samples being analyzed. Glass or other brittle materials must not be used.

Liquid waste should always be segregated and clearly labeled. Never mix organic and inorganic liquids in the same drain vessel. Organic and inorganic drain vessels should never be stored in the same area.

\section{Safe Handling of Gas Cylinders}

Notice: The permanent installation of gas supplies is the responsibility of the user and should conform to local safety and building codes.

- Fasten all gas cylinders securely to an immovable bulkhead or a permanent wall.

- When gas cylinders are stored in confined areas, such as a room, ventilation should be adequate to prevent toxic or explosive accumulations. Move or store gas cylinders only in a vertical position with the valve cap in place.

- Locate gas cylinders away from heat or ignition sources, including heat lamps. Cylinders have a pressure-relief device that will release the contents of the cylinder if the temperature exceeds $52^{\circ} \mathrm{C}\left(125^{\circ} \mathrm{F}\right)$.
Table I. Dimensions of the Instrument and Computer

\begin{tabular}{lcccc}
\hline Instrument & $\begin{array}{c}\text { Width } \\
\text { cm (in.) }\end{array}$ & $\begin{array}{c}\text { Height } \\
\text { cm (in.) }\end{array}$ & $\begin{array}{c}\text { Depth } \\
\text { cm (in.) }\end{array}$ & $\begin{array}{c}\text { Weight } \\
\text { kg (lb.) }\end{array}$ \\
\hline ELAN 9000 & $99(39)$ & $\begin{array}{c}117(46) \\
87(34)\end{array}$ & $73(29)$ & $295(650)$ \\
Work Surface Height & \multicolumn{5}{c}{ Dimensions will vary by model } \\
\hline DELL Computer & \multicolumn{4}{c}{ Dimensions will vary by model } \\
\hline DELL Monitor & \multicolumn{5}{c}{$34.6(13.6)$} & $50.7(19.9)$ & $17(37.5)$ \\
\hline HP Laser Jet 4100 & $39(15.3)$ & \multicolumn{5}{c}{} \\
\hline
\end{tabular}

Table II. Dimensions of the Accessories

\begin{tabular}{lcccc}
\hline Accessory & $\begin{array}{c}\text { Width } \\
\text { cm (in.) }\end{array}$ & $\begin{array}{c}\text { Height } \\
\text { cm (in.) }\end{array}$ & $\begin{array}{c}\text { Depth } \\
\text { cm (in.) }\end{array}$ & $\begin{array}{l}\text { Weight } \\
\text { kg (Ib.) }\end{array}$ \\
\hline $\begin{array}{l}\text { Cooling System: } \\
\text { Heat Exchanger } \\
\text { (PolyScience 3370) }\end{array}$ & $38(15)$ & $63.5(25)$ & $38(15)$ & $31.3(69)$ \\
$\begin{array}{l}\text { Refrigerated Chiller } \\
\text { (PolyScience 6105PE) }\end{array}$ & $38(15)$ & $63.5(25)$ & $67.3(26.5)$ & $81(178)$ \\
\hline FIAS 400MS & $41.5(16)$ & $18.4(7)$ & $41(16)$ & $11(24)$ \\
\hline $\begin{array}{l}\text { AS 93plus } \\
\text { Autosampler }\end{array}$ & $44(17)$ & $37(15)$ & $34(14)$ & $4(9)$ \\
\hline
\end{tabular}


PerkinElmer SCIEX ICP-MS instruments include the hoses necessary for connecting the argon to the instrument.

\section{Suggested Safety Practices}

This advice is intended to supplement, not supersede, the normal safety codes in the user's country. The information provided here does not cover every safety procedure that should be practiced. Ultimately, maintenance of a safe laboratory environment is the responsibility of the analyst and the analyst's organization.

\section{General}

- Never view the ICP discharge directly without protective eye wear. Potentially hazardous ultraviolet radiation may be emitted. Ordinary safety glasses will in general provide sufficient protection, but additional side shields will insure a further margin of safety. Safety glasses will also provide mechanical protection for the eyes.

- ICP-MS instruments generate high amounts of radio frequency energy in their RF power supply and torch boxes, which is potentially hazardous if allowed to escape. Safety devices and screening interlocks should not be bypassed or disconnected.

- The power supply of an ICP-MS is capable of generating potentially lethal voltages. No maintenance should be performed by anyone other than a PerkinElmer Service Specialist or the customer's own PerkinElmer trained maintenance personnel.

- Coolant lines should be located away from electrical connections. Condensation and possible leaks may create an unsafe situation if in proximity to electrical connections. 


\section{OLYMPUS}

Your Vision, Our Future

INVERTED METALLURGICAL MICROSCOPES

GX SERIES
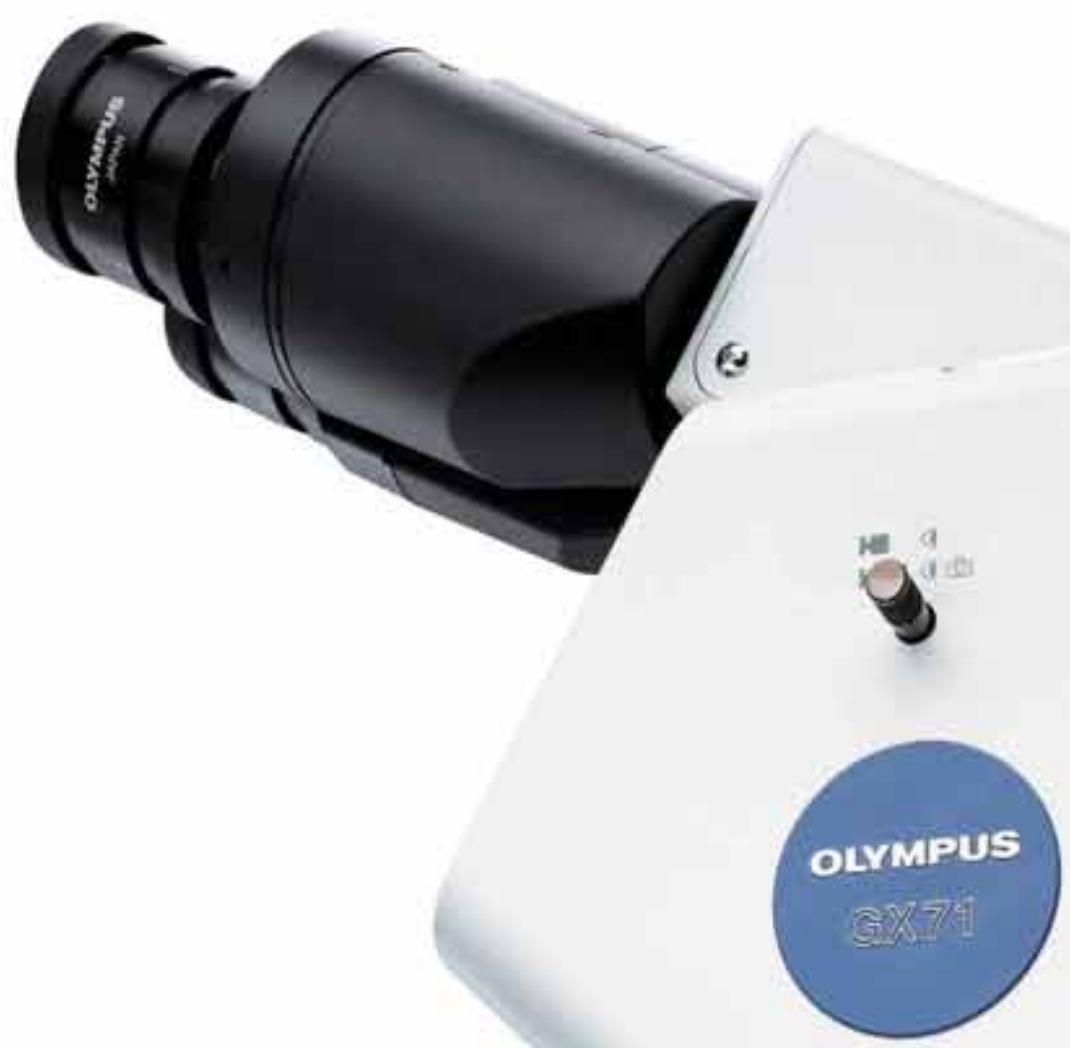

INVERTED METALLURGICAL MICROSCOPES

$\theta$

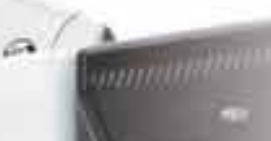

U1S2

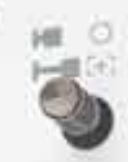

6

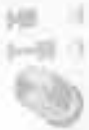


Images of the world's highest order created with UIS2 wavefront aberration control
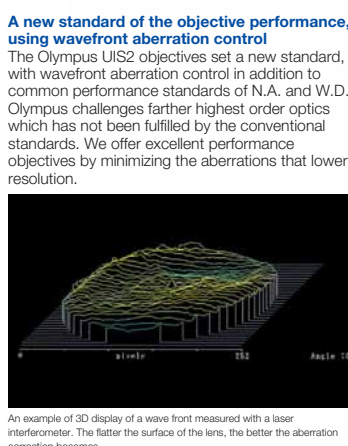

Natural color reproduction faithful to the Specimen

atural color reproduction selected high transmittance glass and advance coating technology that provides high transmittance In addition, since the total bacal system, ingluding color, clear images faithful to the specimen are obtained even with digital imaging.
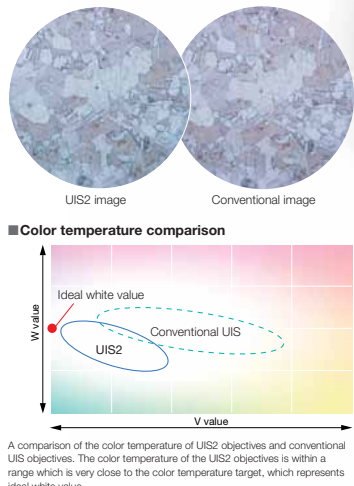

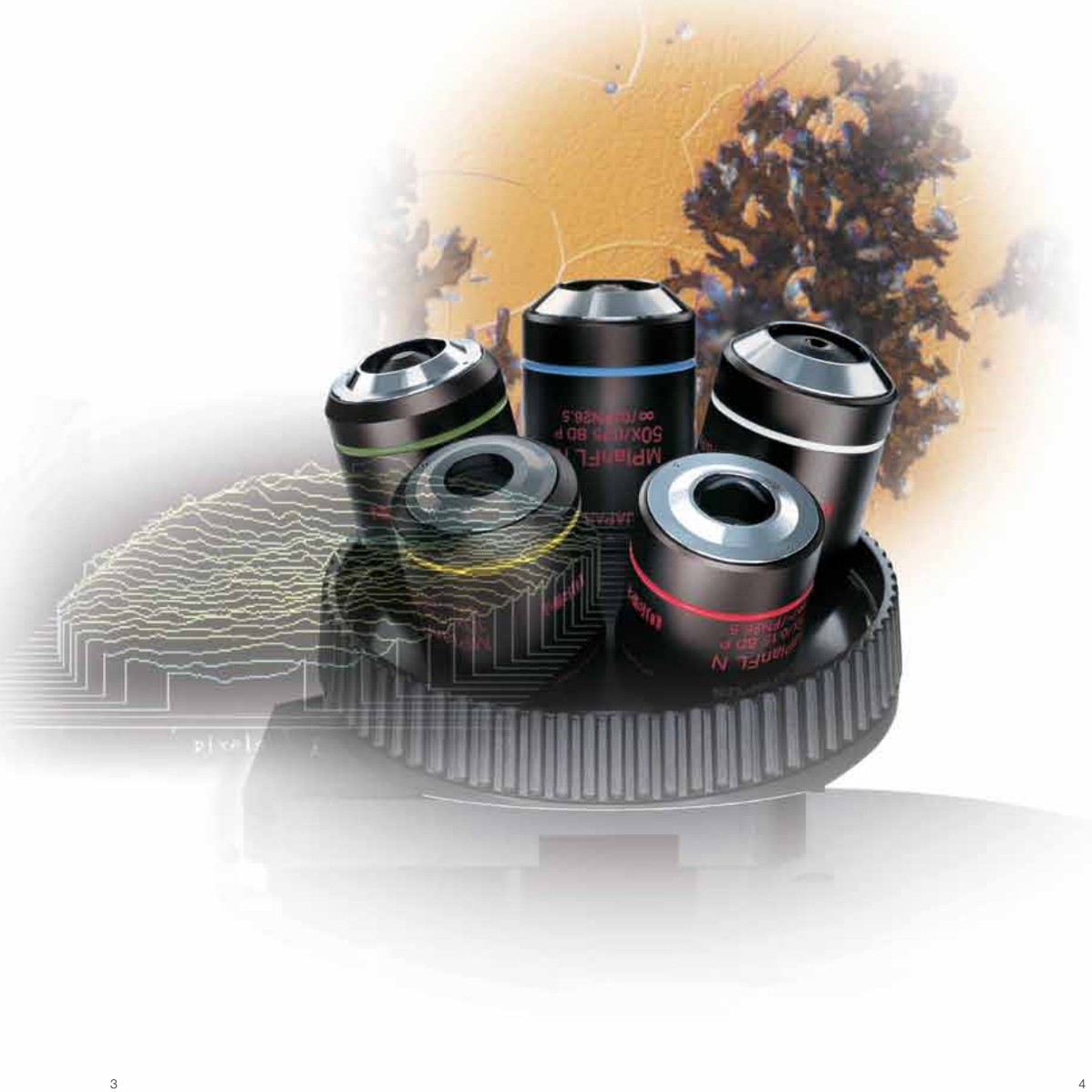

Removes spot flare during ultra low

When a low reflection specimen is observed in ultra low power magnification, spot flare may hinder precise observation. In UIS2 ultra low magnification observation, a depolanizer built into the objective image is obtained by combining a set of polarizer and analyzer plate.

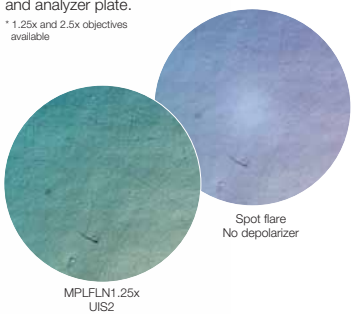

Spot flare removal principle conceptual diagram Since the light reflected from the
surfaces

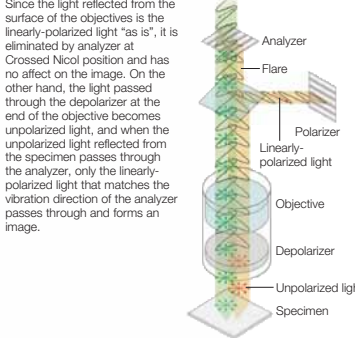
Promotes environmentally-friendly
ecologization and weight reduction olympus was the first to consider the environment of this on introduction of UIS 2 optical systen eco-friendly glass free of lead and arsenic is used in the objectives and the major Semi-apochromatic UIS2 objectives are lightened by approximately of objectives replacement, etc. 
High-performance research and quality control are enhanced by automated modules

Operations that you want to save - various powered modules fulfill your requirements magnification change, easy observation mode selection from brightifield to simple polanizing and
illumination filter switching are performed through hand control panel or PC. Automation allows the
operator to focus on the crisp UIS2 images. You only need to add the automation you need withou only need to add
adding any extras.

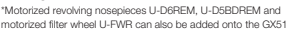

Getting the optimized image with any observation method

ical system was and the GX series is designed to maximize its (a) images with excellent contrast and consiste high clarity with any and all observation methods. Equipped with 100W halogen lamp and newly provide the intens, he Gx series microscopes

The brightest Darkfield images than ever The UIS2 contrast has improved brightness an delivers better sensitivities for holes or flaws on

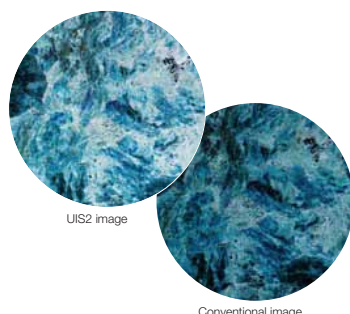

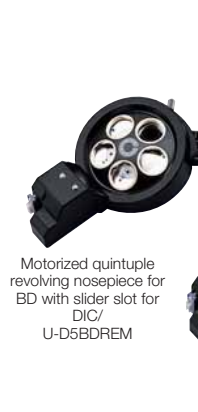

$$
\text { Motorized }
$$
objective switching

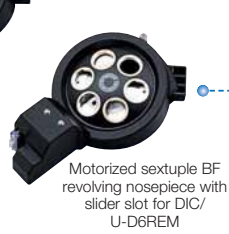

(2)

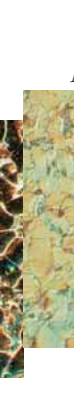

Nomarski DIC
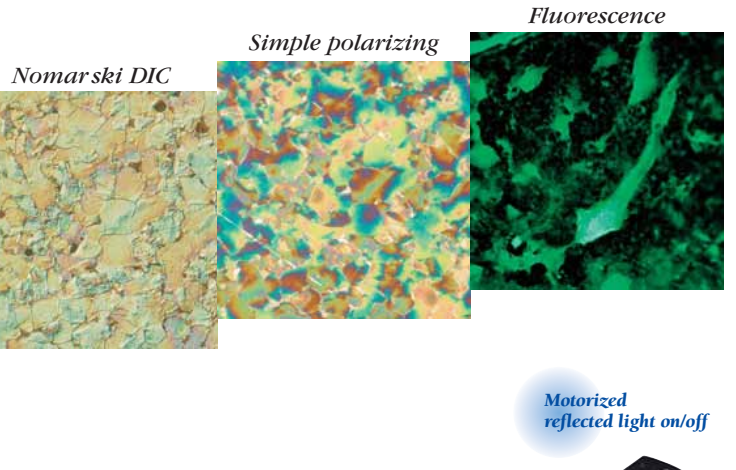$$
\text { Slider Slot fREM }
$$

Motorized
observatio observation method switching

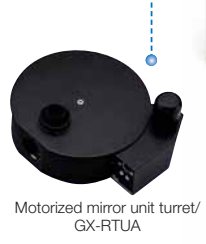

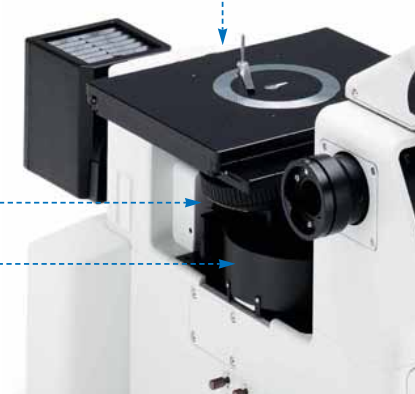

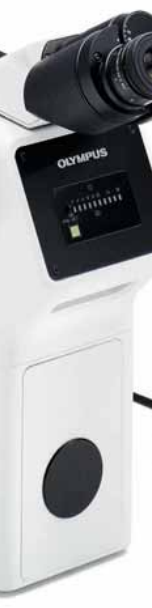

Motorized

Fluorescence
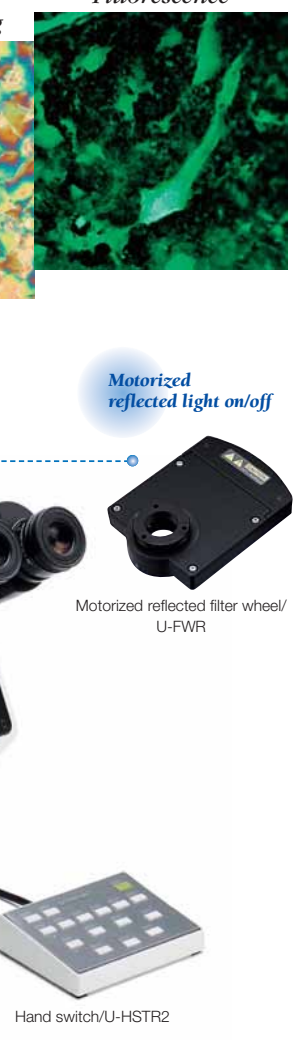

Nomarski DIC system provides an optimum Olympus Nomarski DIC observation switching slider type single prism system. Three different DIC prisms are provided: the U-Dich for all inaging applications, high U-DICRHC, so that the best resolution contrast matched to the state of the sample are obtained. Since the exit pupil position of the for when the magnification was changed by switching the objective.
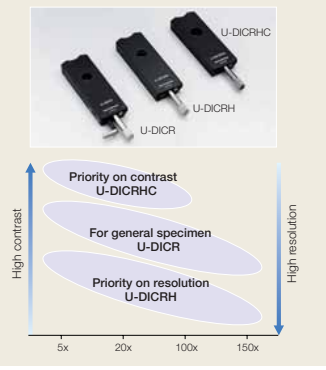

Polarized light: optimizing contrast in the The combin

ts enables with a sensitive tint: the rotating stage GX-SRG for plate, and an analyzer slider, GX-AN360 or GX-AN. In addition, use of the binocular tube U-BI9OCT (with GX51 only) makes it possible to observe an anisotropy on the specimen surface caused by reflection (also known as conoscopic image provides an unrestricted choice of framing angles when taking in photomicrography.

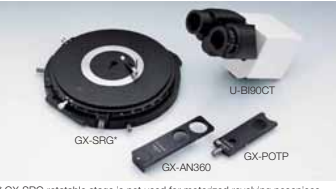


Digital micro imaging solutions for obtaining high quality microscopic images

Digital imaging ? No, it is digital micro imaging

High resolution objectives, high transmittance optical

extract maximum performance from the digital camera Our microscope digital cameras offer high contrast images with color reproduction faithful to the sample. The DP20 microscope digital camera, which can als
be used alone, and the DP71 microscope digital camera, which is completely controlled via PC for observation methods from brightfield to fluorescence, are available. Choose the camera matched to your imaging solutions for microscopes based on many years of optoelectronics technologies

Simultaneous attachment of digital camera and video camera

ital cameras and a video camera GX. The BX2M Series video system can be used with a trinocular observation tube combination. The GX71 cameras.

CX51 required an optional side port intermediate tube \{GX-SPU.

UIS2 objectives with excellent image

parcentricity

High power Semi-apochromatic UIS2 objectives make

microscope nosepiece keep the image within the

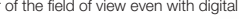

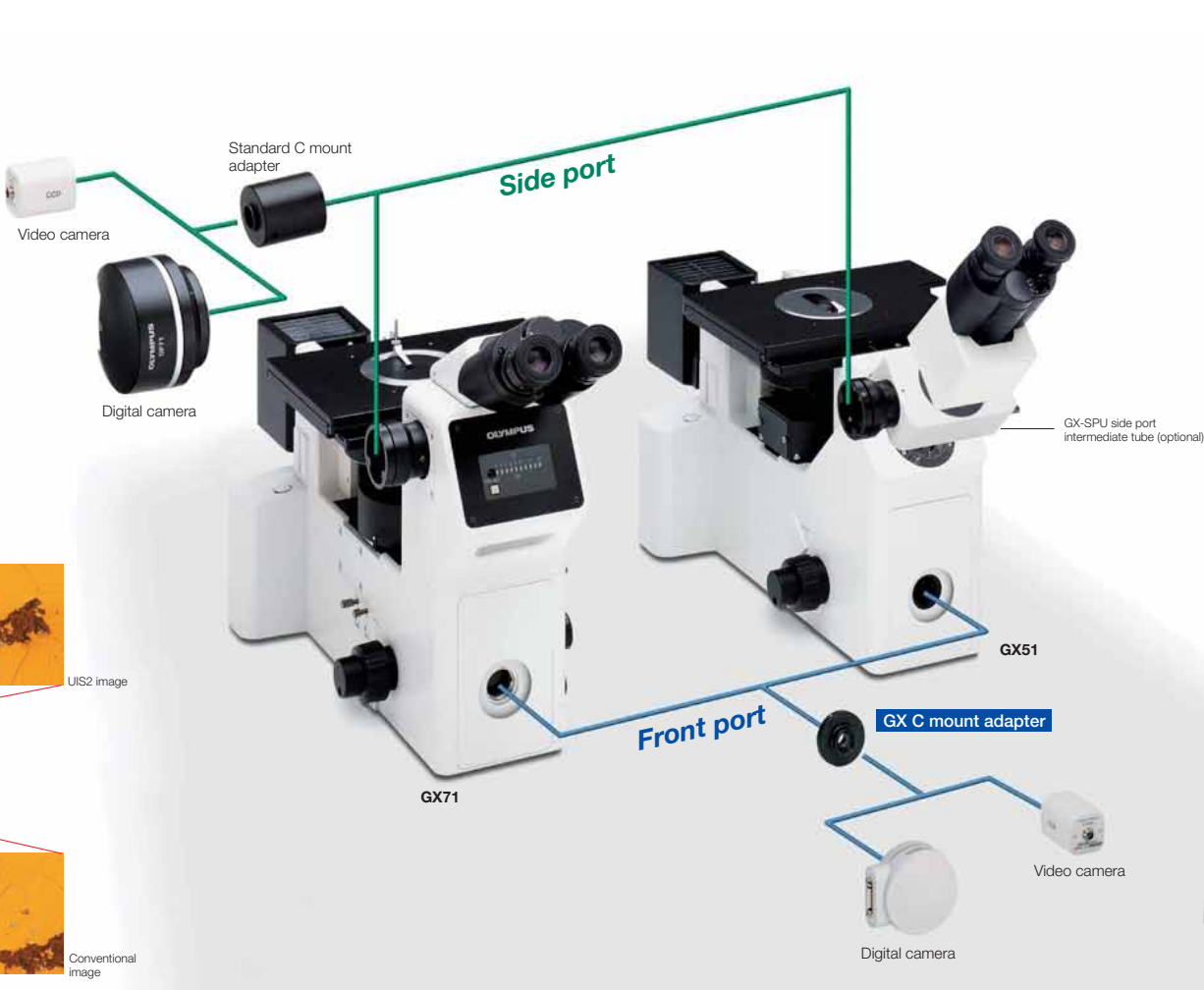

Microscope digital camera DP20 Smooth live image display. igh-speed image capturing which allows

igh definition television class resolution so that focusing on the monitor is performed easily without observation and faithful color is obtained a high tosolving power. Also, the DP20 can be connected analysis can be performed using our image

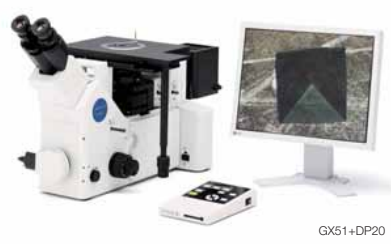

Microscope digital camera DP71 Captures high-resolution, high-sensitivity digital images fast - equivalent to 12.5
million pixels in approximately 3 seconds The digital camera DP7, thanks to its high speed mages equivalent to 12.5 million pixels in as little as (approx.) 3 seconds. The DP71's multiple functions make every phase of the operation simple, from image acquistion through to data microscopic detail, with unparalleled clarity and resolution accuracy.

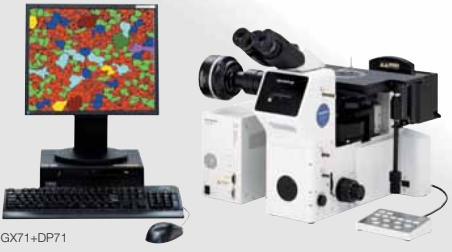


Making the best use of microscope digital imaging, the more freedom, the more comfort

Seamless operation for image acquisition, and analytical solutions

The image analysis software analySIS FIVE has made possible seamless operation from image processing, measurement, and analysis to FIVE comes in 3 types: " "imager" "docu", and "auto", according to the difference of the functions incorporated. The type can be
chosen according to the application. The chosen according to the application. The
"auto" type has all functions, including particle
analysis, etc. Customizing to more pleasant software is possible by freely adding the desired functions

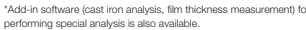

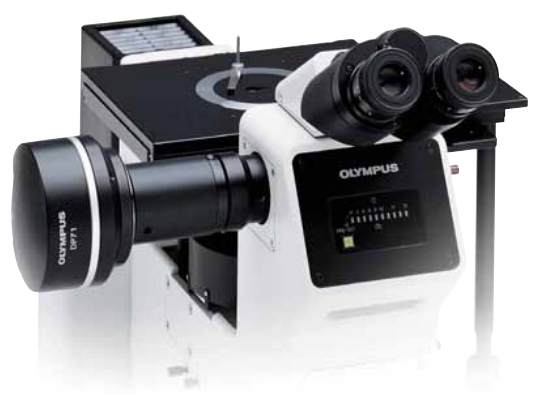

\section{Particle Analysis}

Automatic separation of particles within a given image is possible using the integrated Many other parameters can be used to measure all particles automatically, or carny out statistical data processing
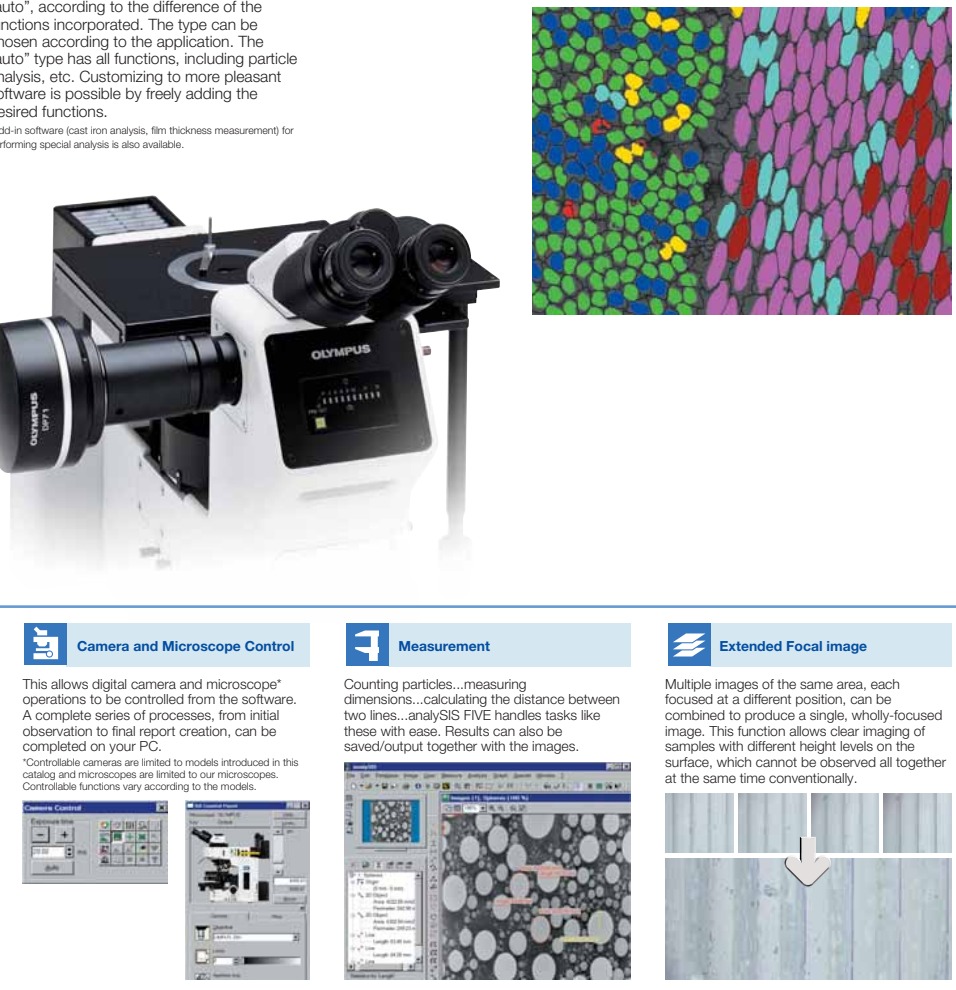

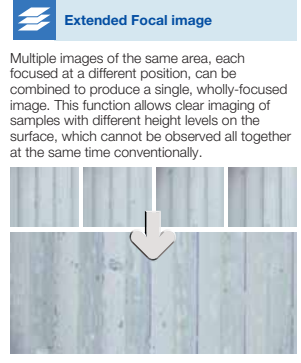

NOTE: Olympus IA software as shown is not available in the U.S.A. Contact LECO for more information on Image Analysis Systems.

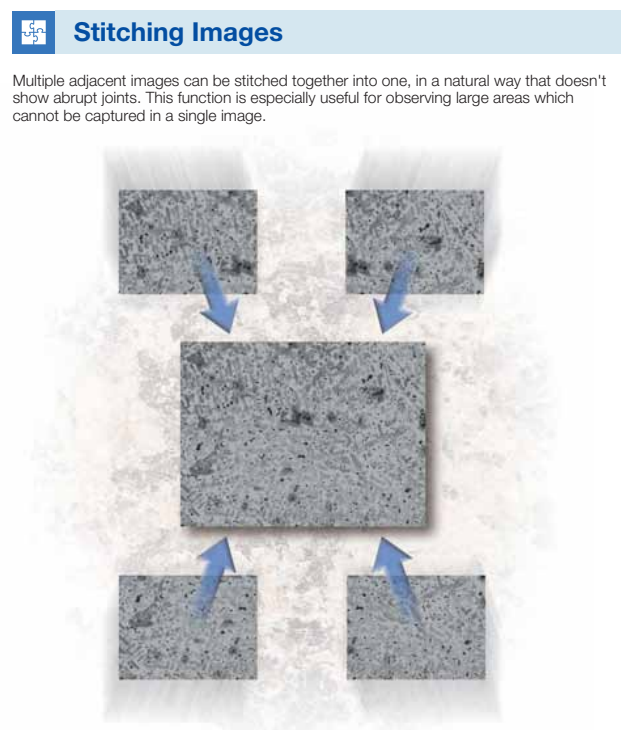

\begin{tabular}{|c|c|}
\hline \multicolumn{2}{|c|}{$\begin{array}{l}\text { WhalySIS FIVE function } \\
\text { The softwere package varies by area. }\end{array}$} \\
\hline \multicolumn{2}{|c|}{ imager } \\
\hline 5 & Camera and Microscope Control \\
\hline 9 & Measurement \\
\hline a & Database \\
\hline 0 & Report \\
\hline \multicolumn{2}{|c|}{ docu } \\
\hline 祀 & Camera and Microscope Control \\
\hline 7 & Measurement \\
\hline 뭅 & Stitching Images \\
\hline 3 & Extended Focal image \\
\hline i. & 3D Image \\
\hline a & Database \\
\hline $\mathbb{Q}$ & Report \\
\hline \multicolumn{2}{|c|}{ auto } \\
\hline 国 & Camera and Microscope Control \\
\hline 7 & Measurement \\
\hline 国 & Stitching Images \\
\hline 3 & Extended Focal image \\
\hline a. & 3D Image \\
\hline gi & Particle Analysis \\
\hline$\overline{0}$ & Database \\
\hline 0 & Report \\
\hline
\end{tabular}

3D Image

By adding height/texture information to multi-
focused images obtained with the "Extended

Focal Image" dimensions...calculating the distance betwe wo lines....analySIS FIVE handles tasks saved/output together with the images.

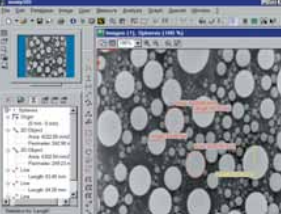

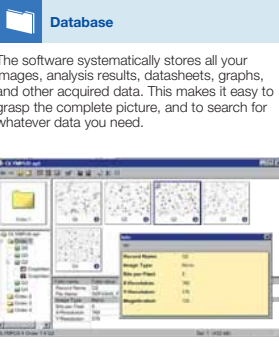

Report

ges can be freely laid out and edited. Some entation templates are producing professionally-finished reports and

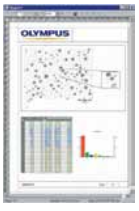

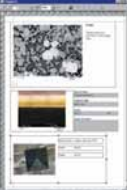




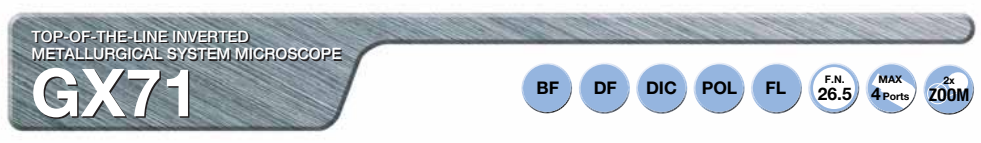

Top-notch performance for today's leading-edge research

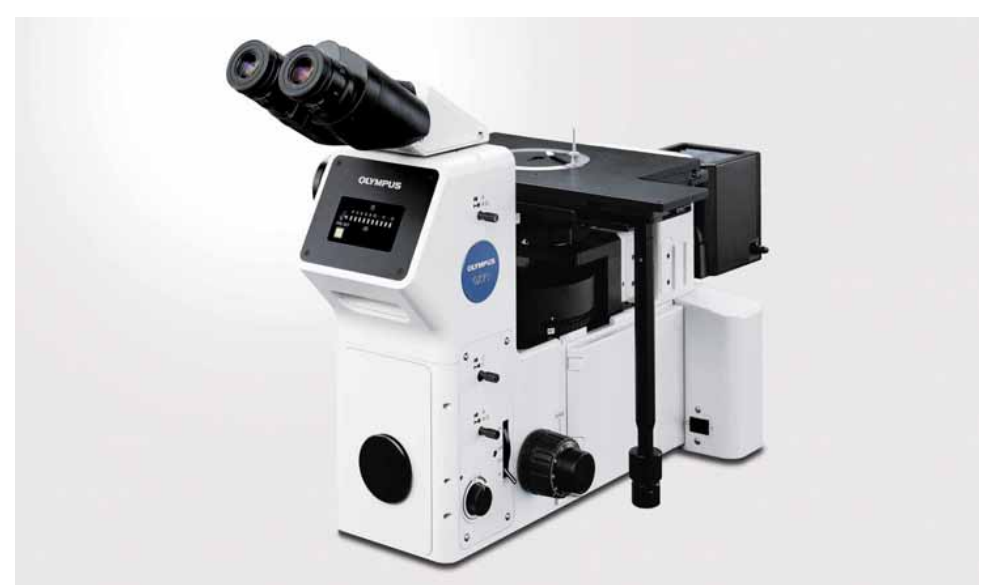

Zoom function for easy framing
The $1 x-2 \times$ zoom facility acts on all ports, shows critical specimen detail more clearly and makes accurate framing especially easy same magnification as the

visual observation.

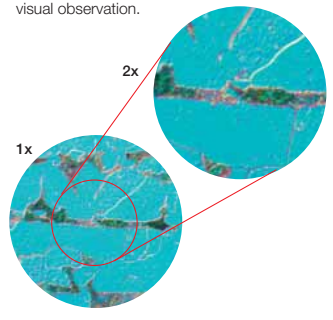

Truthful reproduction of specimen in Viewing images and acquisition rewing images are not reversal, the horizontal directions. The true reproduction makes it easier to compare the images with gital photos.

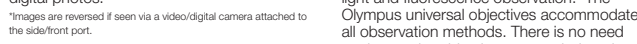
all observation methods. There is no need observation method is changed.

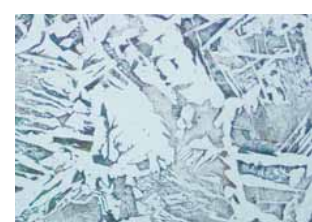
eyepieces (F.N.26.5), for an efficient

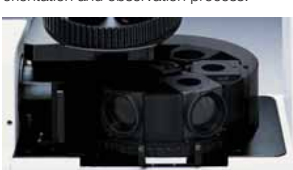

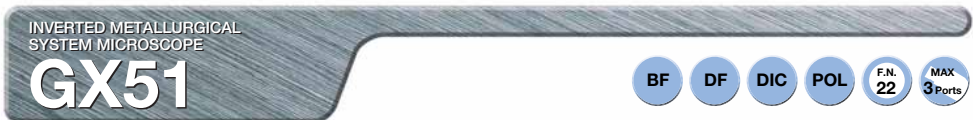

Superb performance and reliability for all kinds of routine observation and documentation

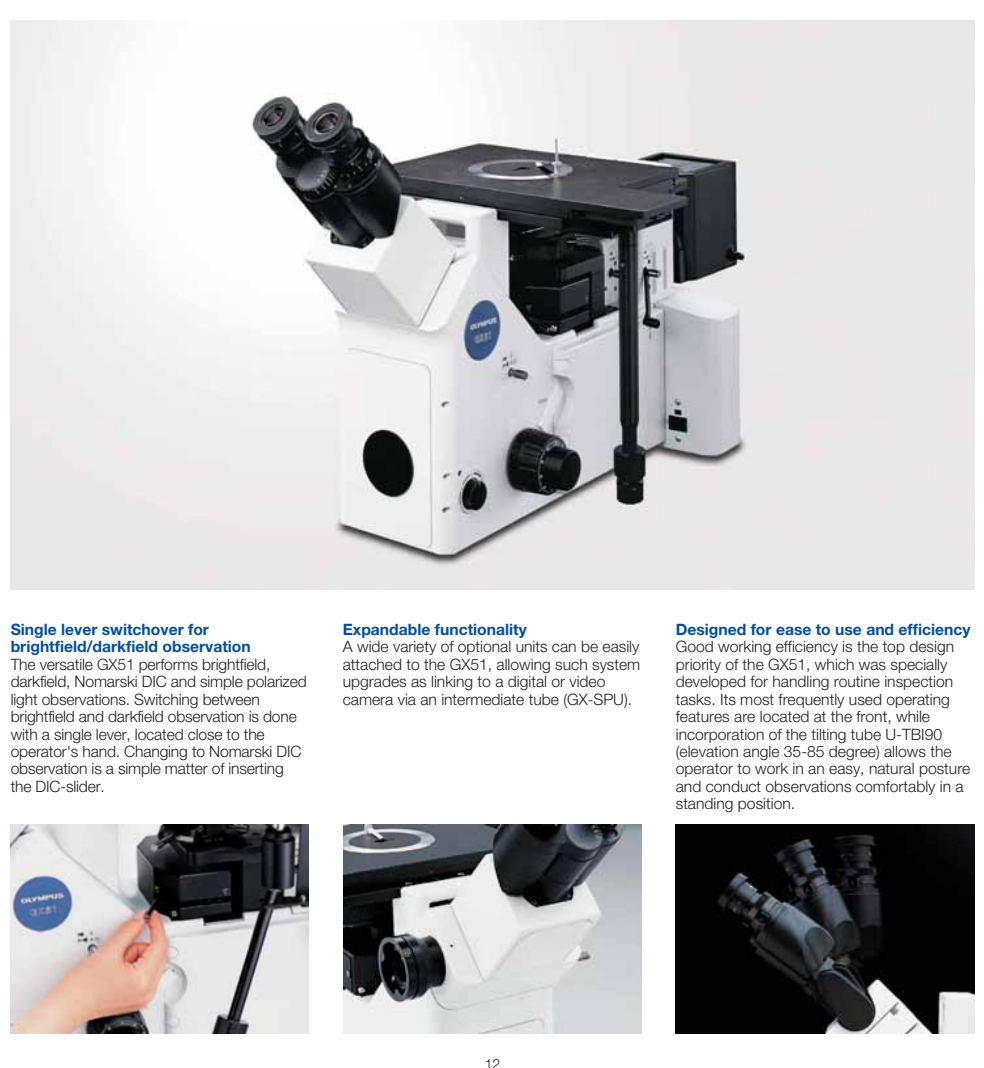




\section{Compatible with transmitted light polarized observation}

Transmitted light polarized observation combination

Transmitted light polarized observation, which is ideal for transparent specimens or fine powders, can be performed by combining illumination pillar IX2-ILL100.

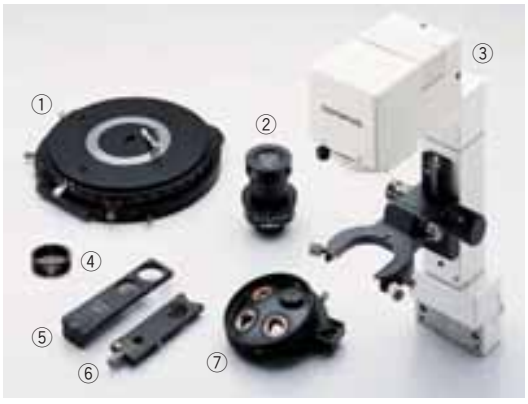

(1) GX-SRG (2)PMG3-LWCD (3)IX2-ILL100 (4)U-POT (5)GX-AN360 (6)GX-POTP (7)U-P4RE

\section{GX51}

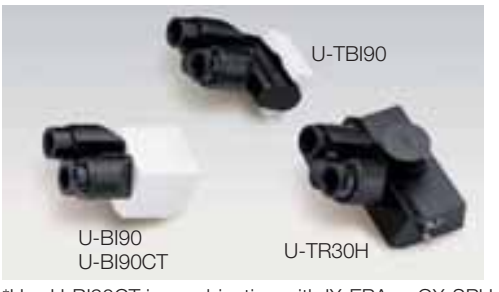

*Use U-BI90CT in combination with IX-EPA or GX-SPU.
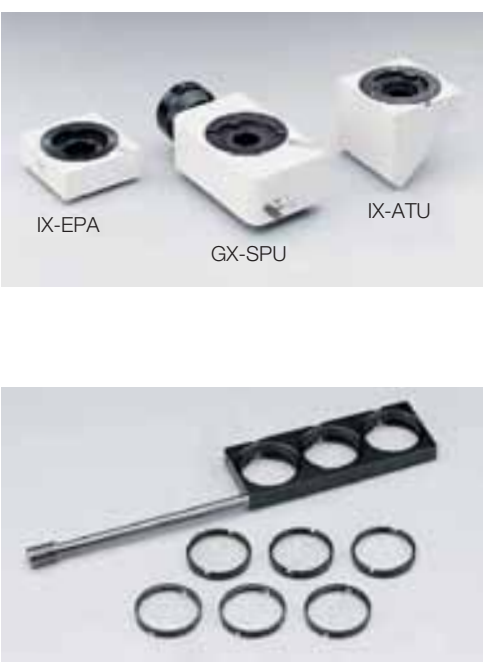

GX51 observation tube

Besides trinocular tube U-TR3OH, the lineup includes binocular tube $\mathrm{U}-\mathrm{Bl} 190$, for use in combination with to be made in whatever posture suits the individual user.

Intermediate tubes

Other high-performance

accessories are available to meet a variety of applications. Included which allows attachment of a trinocular observation tube, a side port intermediate tube (GX-SPU) and an eyepoint adjuster (IX-EPA).

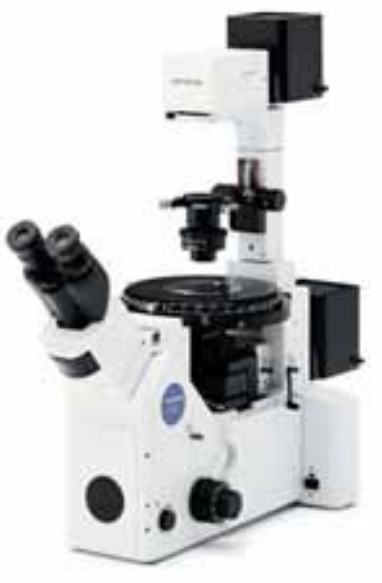

GX71

GX71 observation tubes The super widefield binocular observation tube (U-SWBI30) and super widefield trinocular observation tube (U-SWTR-3) are provided for the GX71.

\section{Compatible with macro observation and photographing}

Drawing attachment / U-DA

As well as its conventional use as drawing attachment, this accessory also provides a macro observation function. When combined with a trinocular observation tube, the macro images are stored as photomicrographs or retained in the digital camera.

*Use in combination with 10x lens for drawing attachment U-DAL10x.

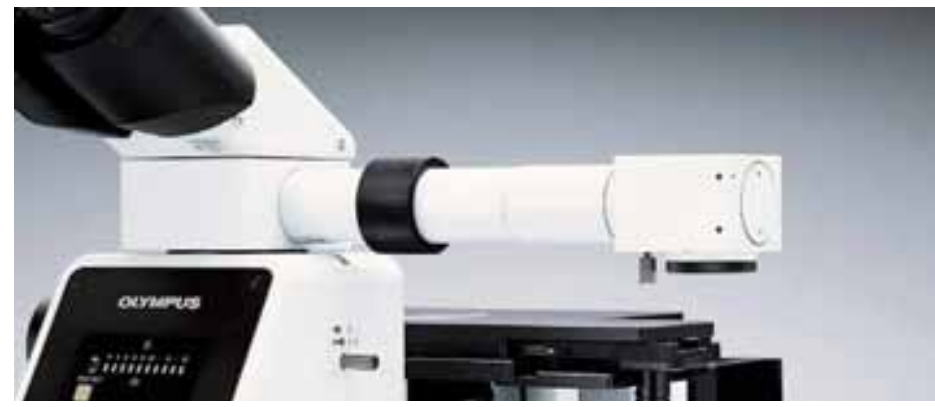
an eyepoint adjuster, and tilting tube U-TBI90, which allows observations are an intermediate tube (IX-ATU),

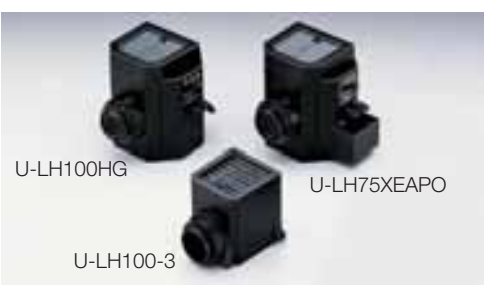

Lamp housing

A variety of light sources to accomplish bright and even illumination are provided, according to your purpose.

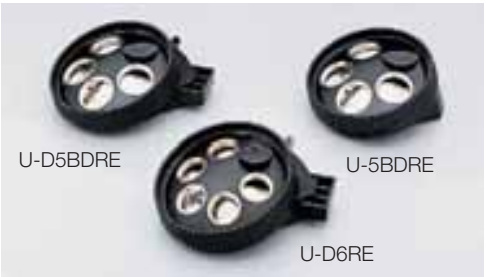

Revolving nosepieces Sextuple revolving nosepieces and quintuple revolving nosepieces with DIC slider compatibility are also provided.

GX71 GX51

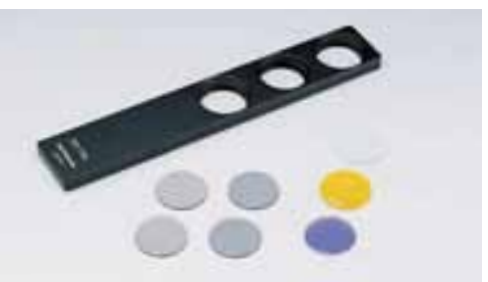

Filters

The GX series comes with a select range of filters, including neutral density, color temperature conversion and green filters. Two slider slots are provided, each allowing introduction of up to three filters. 
GX series specifications

\begin{tabular}{|c|c|c|c|}
\hline & & GX71 & GX51 \\
\hline \multicolumn{2}{|l|}{ Optics } & \multicolumn{2}{|c|}{ UIS2 optical system (infinity-corrected) } \\
\hline \multirow[t]{6}{*}{ Microscope body } & Intermediate magnification & $\begin{array}{c}\text { Zoom incorporated }(1 \mathrm{x}-2 \mathrm{x}) \\
\text { Clicks in the two intermediate positions (can be released) }\end{array}$ & - \\
\hline & Imprinting of scale & $\begin{array}{l}\text { All ports } \\
\text { Reversed positions (up/down/left/right) from observation } \\
\text { positions seen through the eyepiece }\end{array}$ & $\begin{array}{c}\text { All ports } \\
\text { Reversed positions (up/down) from observation } \\
\text { positions seen through the eyepiece }\end{array}$ \\
\hline & Power source & \multicolumn{2}{|c|}{ Power source for illuminator (12V100 halogen) incorporated } \\
\hline & Focusing & \multicolumn{2}{|c|}{ Manual, Coarse and Fine coaxial handle. Focus stroke $9 \mathrm{~mm}$ ( $2 \mathrm{~mm}$ above and $7 \mathrm{~mm}$ below the stage surface) } \\
\hline & \multirow[t]{2}{*}{ Output port } & \multicolumn{2}{|c|}{ Front port — Video and DP system (reversed image, special video adapter for GX) } \\
\hline & & Side port — Video, DP system (reversed image) & Side port (option) — Video, DP system (upright image) \\
\hline \multirow[t]{2}{*}{ Observation tube } & Super widefield (F.N. 26.5) & U-SWBI30, U-SWTR-3 & - \\
\hline & Widefield (F.N. 22) & - & U-BI90, U-TR3OH \\
\hline \multirow[t]{3}{*}{ Illuminator } & Observation method & Brightfield, darkfield, simple polarized light, DIC, fluorescence & Brightfield, darkfield, simple polarized light, DIC \\
\hline & Illuminator diaphragm & \multicolumn{2}{|c|}{ FS/AS manually controlled, with centering adjustment } \\
\hline & Light source & \multicolumn{2}{|c|}{ 100W halogen (standard), 100W mercury, $75 \mathrm{~W}$ xenon (option) } \\
\hline \multirow[t]{2}{*}{ Revolving nosepiece } & Manual operation & \multicolumn{2}{|c|}{ Sextuple for BF/DIC, quintuple for BF/DF, quintuple for BF/DF/DIC, Quadruple for BF with centering } \\
\hline & Motorized operation & \multicolumn{2}{|c|}{ Sextuple for BF/DIC, quintuple for BF/DF/DIC } \\
\hline \multirow[t]{3}{*}{ Stage } & Standard type & \multicolumn{2}{|c|}{ Right handle stage for GX (XY stroke: 50x50mm) } \\
\hline & Option & \multicolumn{2}{|c|}{$\begin{array}{l}\text { Flexible right handle stage, left short handle stage (each XY stroke: } 50 \times 50 \mathrm{~mm}) \\
\text { Gliding stage, rotatable stage for GX }\end{array}$} \\
\hline & Stage insert plate & \multicolumn{2}{|c|}{ A set of teardrop and long hole types } \\
\hline Image recording & Digital camera, video camera & \multicolumn{2}{|c|}{ OLYMPUS DP series etc, attachable using appropriate adapters } \\
\hline \multicolumn{2}{|l|}{ Combined weight } & Approx. 39kg (BF, DF and DIC observations, combined with DP71) & Approx. 28kg (BF, DF and DIC observations, combined with DP20) \\
\hline Power consumption & & \multicolumn{2}{|c|}{$170 \mathrm{VA}, 140 \mathrm{~W}$} \\
\hline
\end{tabular}

\section{UIS2 objective specifications}

\begin{tabular}{|c|c|c|c|c|c|}
\hline Objectives & Magnifications & N.A. & $\begin{array}{l}\text { W.D. } \\
(\mathrm{mm})\end{array}$ & $\begin{array}{c}\text { Cover Glass } \\
\text { Thickness } \\
\text { (mm) }\end{array}$ & $\begin{array}{l}\text { Resolution }^{\star 2} \\
\quad(\mu \mathrm{m})\end{array}$ \\
\hline MPLFLN & $\begin{array}{c}1.25 x^{\star 3 * 4 * 5} \\
2.5 x^{\star 4 * 5} \\
5 x \\
10 x \\
20 x \\
50 x \\
100 x \\
\end{array}$ & $\begin{array}{l}0.04 \\
0.08 \\
0.15 \\
0.30 \\
0.45 \\
0.80 \\
0.90 \\
\end{array}$ & $\begin{array}{c}3.5 \\
10.7 \\
20.0 \\
11.0 \\
3.1 \\
1.0 \\
1.0 \\
\end{array}$ & $\begin{array}{l}- \\
- \\
\overline{-} \\
0 \\
0 \\
0\end{array}$ & $\begin{array}{l}8.39 \\
4.19 \\
2.24 \\
1.12 \\
0.75 \\
0.42 \\
0.37 \\
\end{array}$ \\
\hline MPLFLN-BD & $\begin{array}{c}5 x \\
10 x \\
20 x \\
50 x \\
100 x \\
150 x \\
\end{array}$ & $\begin{array}{l}0.15 \\
0.30 \\
0.45 \\
0.80 \\
0.90 \\
0.90\end{array}$ & $\begin{array}{l}12.0 \\
6.5 \\
3.0 \\
1.0 \\
1.0 \\
1.0\end{array}$ & $\begin{array}{l}- \\
\overline{0} \\
0 \\
0\end{array}$ & $\begin{array}{l}2.24 \\
1.12 \\
0.75 \\
0.42 \\
0.37 \\
0.37\end{array}$ \\
\hline MPLFLN-BDP & $\begin{array}{c}5 x \\
10 x \\
20 x \\
50 x \\
100 x\end{array}$ & $\begin{array}{l}0.15 \\
0.25 \\
0.40 \\
0.75 \\
0.90\end{array}$ & $\begin{array}{c}12.0 \\
6.5 \\
3.0 \\
1.0 \\
1.0\end{array}$ & $\begin{array}{l}- \\
0 \\
0 \\
0\end{array}$ & $\begin{array}{l}2.24 \\
1.34 \\
0.84 \\
0.45 \\
0.37\end{array}$ \\
\hline LMPLFLN & $\begin{array}{c}5 x \\
10 x \\
20 x \\
50 x \\
100 x \\
\end{array}$ & $\begin{array}{l}0.13 \\
0.25 \\
0.40 \\
0.50 \\
0.80 \\
\end{array}$ & $\begin{array}{l}22.5 \\
21.0 \\
12.0 \\
10.6 \\
3.4 \\
\end{array}$ & $\begin{array}{l}- \\
\overline{0} \\
0 \\
0\end{array}$ & $\begin{array}{l}2.58 \\
1.34 \\
0.84 \\
0.67 \\
0.42 \\
\end{array}$ \\
\hline LMPLFLN-BD & $\begin{array}{c}5 x \\
10 x \\
20 x \\
50 x \\
100 x \\
\end{array}$ & $\begin{array}{l}0.13 \\
0.25 \\
0.40 \\
0.50 \\
0.80 \\
\end{array}$ & $\begin{array}{c}15.0 \\
10.0 \\
12.0 \\
10.6 \\
3.3 \\
\end{array}$ & $\begin{array}{l}- \\
0 \\
0 \\
0\end{array}$ & $\begin{array}{l}2.58 \\
1.34 \\
0.84 \\
0.67 \\
0.42 \\
\end{array}$ \\
\hline MPLN³ & $\begin{array}{c}5 x \\
10 x \\
20 x \\
50 x \\
100 x\end{array}$ & $\begin{array}{l}0.10 \\
0.25 \\
0.40 \\
0.75 \\
0.90\end{array}$ & $\begin{array}{c}20.0 \\
10.6 \\
1.3 \\
0.38 \\
0.21\end{array}$ & $\begin{array}{l}- \\
0 \\
0 \\
0\end{array}$ & $\begin{array}{l}3.36 \\
1.34 \\
0.84 \\
0.45 \\
0.37\end{array}$ \\
\hline
\end{tabular}

\begin{tabular}{|c|c|c|c|c|c|}
\hline Objectives & Magnifications & N.A. & $\begin{array}{l}\text { W.D. } \\
(\mathrm{mm})\end{array}$ & $\begin{array}{c}\text { Cover Glass } \\
\text { Thickness } \\
(\mathrm{mm})\end{array}$ & $\begin{array}{l}\text { Resolution }{ }^{\star 2} \\
(\mu \mathrm{m})\end{array}$ \\
\hline MPLN-BD ${ }^{* 1 * 3}$ & $\begin{array}{c}5 x \\
10 x \\
20 x \\
50 x \\
100 x\end{array}$ & $\begin{array}{l}0.10 \\
0.25 \\
0.40 \\
0.75 \\
0.90\end{array}$ & $\begin{array}{c}12.0 \\
6.5 \\
1.3 \\
0.38 \\
0.21\end{array}$ & $\begin{array}{l}- \\
0 \\
0 \\
0\end{array}$ & $\begin{array}{l}3.36 \\
1.34 \\
0.84 \\
0.45 \\
0.37\end{array}$ \\
\hline LCPLFLN-LCD ${ }^{\star 5}$ & $\begin{array}{c}20 x \\
50 x \\
100 x\end{array}$ & $\begin{array}{l}0.45 \\
0.70 \\
0.85\end{array}$ & $\begin{array}{l}8.3 \sim 7.4 \\
3.0 \sim 2.2 \\
1.2 \sim 0.9\end{array}$ & $\begin{array}{l}0 \sim 1.2 \\
0 \sim 1.2 \\
0 \sim 0.7\end{array}$ & $\begin{array}{l}0.75 \\
0.48 \\
0.39\end{array}$ \\
\hline
\end{tabular}

\section{U/S}

\begin{tabular}{c|c|c|c|c|c}
\hline Objectives & Magnifications & N.A. & $\begin{array}{c}\text { W.D. } \\
(\mathrm{mm})\end{array}$ & $\begin{array}{c}\text { Cover Glass } \\
\text { Thickness } \\
(\mathrm{mm})\end{array}$ & $\begin{array}{c}\text {Resolution }^{* 2} \\
(\mu \mathrm{m})\end{array}$ \\
\hline & $20 \mathrm{x}$ & 0.60 & 0.9 & 0 & 0.56 \\
MPlanApo & $50 \mathrm{x}$ & 0.95 & 0.3 & 0 & 0.35 \\
& $100 \mathrm{x}$ & 0.95 & 0.35 & 0 & 0.35 \\
& $100 x \mathrm{xil}$ & 1.40 & 0.1 & 0 & 0.24 \\
\hline MPlanApo-BD & $100 \mathrm{x}$ & 0.90 & 0.31 & 0 & 0.37 \\
\hline SLMPlan & $20 \mathrm{x}$ & 0.35 & 21.0 & 0 & 0.96 \\
& $50 \mathrm{x}$ & 0.45 & 15.0 & 0 & 0.75 \\
\hline & $5 \mathrm{x}$ & 0.10 & 20.0 & - & - \\
& $10 \mathrm{x}$ & 0.25 & 18.5 & - & - \\
LMPlan-IR & $20 \mathrm{x}$ & 0.40 & 8.1 & - & - \\
& $50 \mathrm{x}$ & 0.55 & 6.0 & - & - \\
\hline MPlan-IR $^{* 3}$ & $100 \mathrm{x}$ & 0.80 & 3.4 & - & - \\
\hline
\end{tabular}

"BD" refers to brightfield and darkfield objectives

*1 Slight vignetting may occur in the periphery of the field when MPLN-BD series objectives are used with high-intensity light sources such as mercury and xenon for darkfield observation.

*2 Resolution values are calculated with the aperture diaphragm fully opened.

*3 Field numbers are limited (up to F.N.22). Not compatible with F.N.26.5

*4 Analyzer and polarizer are recommended to the usage with MPLFLN1.25x or 2.5x.

$\star 5$ Available in the beginning of 2007.

GX71 dimensions

(unit:mm)

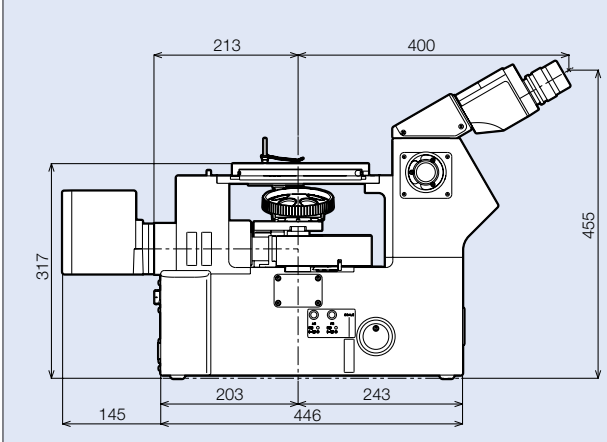

GX51 dimensions

(unit:mm)

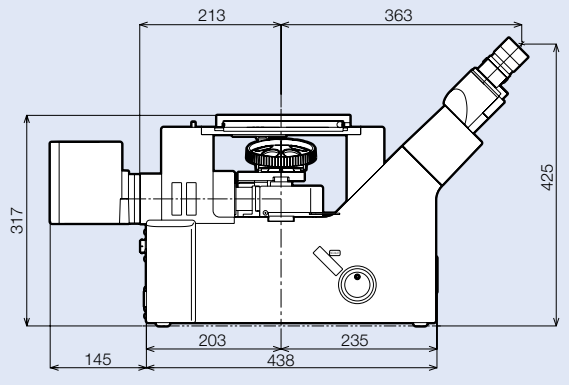




\section{SYSTEM DIAGRAM}

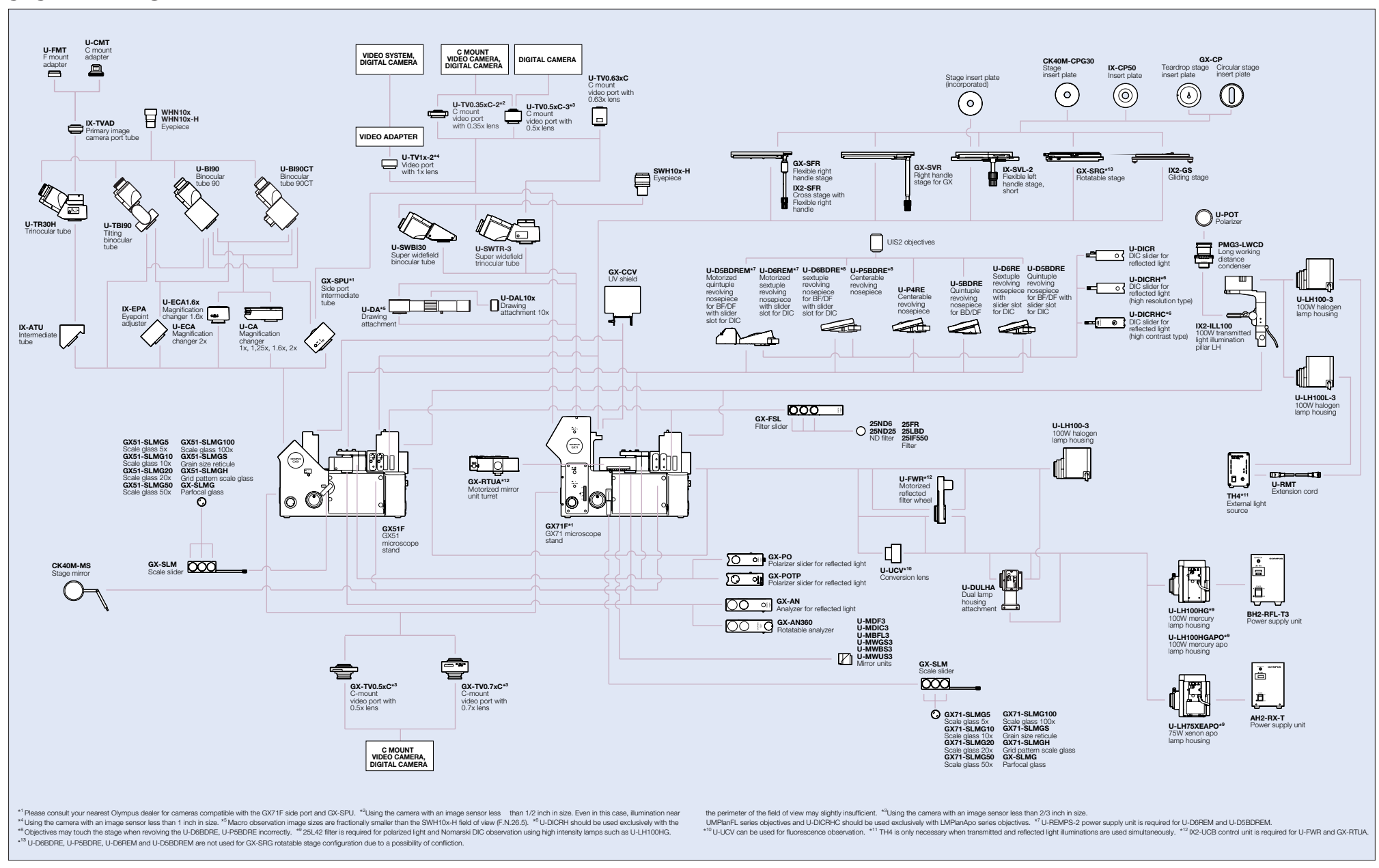




\section{LECO Corporation}

3000 Lakeview Avenue

St. Joseph, MI 49085-2396 U.S.A.

800-292-6141 • Fax: 269-982-8977

www.leco.com•info@leco.com

Form No. 209-127 5/07

OLYMPUS CORPORATION has obtained the ISO9001/ISO14001.

Specifications are subject to change without any obligation on the part of the manufacturer.

\section{OLYMPUS}

www.olympus.com
OLYMPUS CORPORATION

Shinjuku Monolith, 3-1, Nishi Shinjuku 2-chome, Shinjuku-ku, Tokyo, Japan

OLYMPUS LIFE AND MATERIAL SCIENCE EUROPA GMBH Postfach 1049 08, 20034, Hamburg, Germany OLYMPUS SURGICAL \& INDUSTRIAL AMERICA INC.

OLYMPUS UK ITD

2-8 Honduras Street, London EC1Y OTX, United Kingdom.
OLYMPUS AUSTRALIA PTY. LTD.

31 Gilby Road, Mt. Waverley, VIC 3149, Melbourne, Australia.

OLYMPUS LATIN AMERICA, INC.

(1) 


\section{Spectrum System $1000^{\circledR}$}

\section{Grinder/Polisher}

For your low- to medium-volume laboratory, choose the Spectrum System 1000 (SS-1000) Grinder/Polisher. This system features a dual 8-inch design that can be purchased as a manual stand-alone base unit or with a grinding/polishing head for semi-automatic sample preparation. A rugged 3/4 HP motor drives the simultaneous operation of both 8-inch wheels. Add the head assembly and an optional dispenser to maximize efficiency.

- Built-in timer

- Variable-speed base

- Adjustable water faucet for each wheel

- Compact, durable design

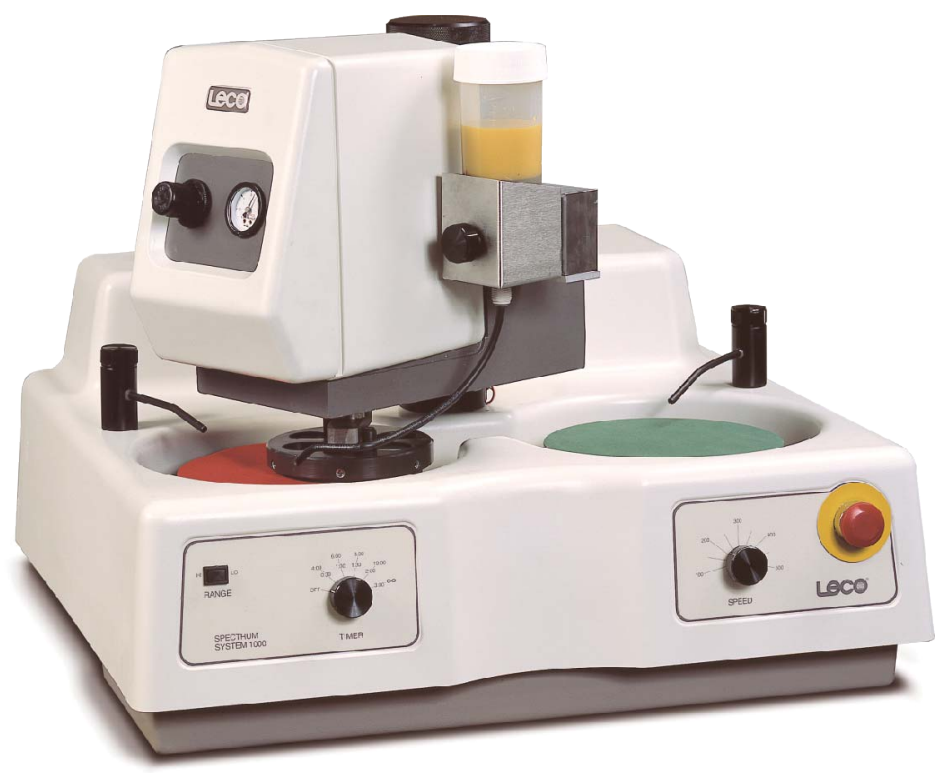

\section{Dispenser}

The optional automatic dispenser unit gives you even more freedom to walk away from the unit during sample preparation-allowing more time for other tasks. The dispenser bottle refills quickly and easily from the top, and dispenses colloidal silica without clogging. The gravity-drip design and flexible applicator provide even diamond suspension distribution anywhere on the wheel. Manual application of the suspension or solution to the wheel allows you to accurately control the duration and frequency.
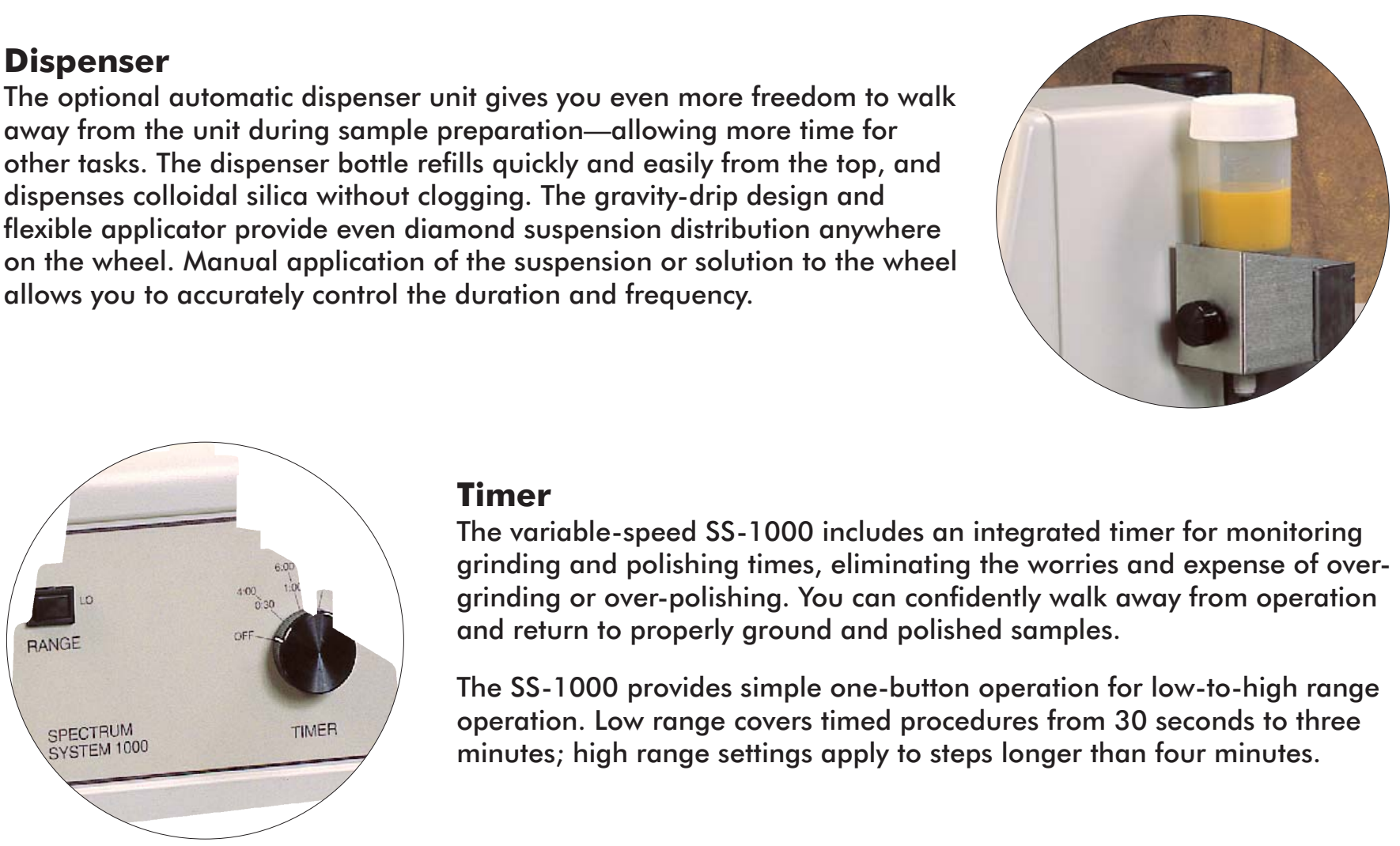

\section{Timer}

The variable-speed SS-1000 includes an integrated timer for monitoring grinding and polishing times, eliminating the worries and expense of overgrinding or over-polishing. You can confidently walk away from operation and return to properly ground and polished samples.

The SS-1000 provides simple one-button operation for low-to-high range operation. Low range covers timed procedures from 30 seconds to three minutes; high range settings apply to steps longer than four minutes.

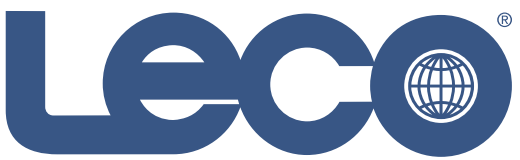




\section{Specifications}

\section{Base Unit}

\section{Weight}

Height

Width

Depth

Voltage Input

Operating Conditions

Motor Output

Wheel Size

Rotational Speed

Rotational Direction

Timer

Water Supply

Grinder/Polisher Discs

Semi-Automatic Grinder/Polisher Head Unit

Weight

Height

Width

Depth

Voltage Input

Operating Conditions

Motor Output

Rotational Speed

Rotational Direction

Pneumatic Supply

Sample Loading

Liquid Dispenser (optional)
90 lb. $(41 \mathrm{~kg})$

12.5 in. $(32 \mathrm{~cm})$

27 in. $(69 \mathrm{~cm})$

24 in. $(61 \mathrm{~cm})$

$120 / 220 \mathrm{~V} \sim, 50 / 60 \mathrm{~Hz}$, single phase

$50^{\circ}$ to $104^{\circ} \mathrm{F}\left(10^{\circ}\right.$ to $\left.40^{\circ} \mathrm{C}\right)$

$3 / 4 \mathrm{HP}$

Dual, 8-inch, belt driven

0 to 500 RPM

CCW

$00: 30,1: 00,1: 30,2: 00$, 3:00, 4:00, 6:00, 8:00,

10:00; continuous operation, microprocessor controlled

20 to 80 psi

8-inch single phase

$50^{\circ}$ to $104^{\circ} \mathrm{F}\left(10^{\circ}\right.$ to $\left.40^{\circ} \mathrm{C}\right)$

$1 / 6 \mathrm{HP}$

24 RPM constant

CW

40 to 100 psi, regulated free of oil and water

1 to $50 \mathrm{lb}$. (force)

Manual control

\section{Part Numbers}

SS-1000BD

SS-1000 Base Unit; 120V , $60 \mathrm{~Hz}$, single phase

SS-1000HD

SS-1000 8-inch Semi-Automatic Polishing Head; $120 \mathrm{~V} \sim, 60 \mathrm{~Hz}$, single phase

SS-1000BHD

SS-1000 Semi-Automatic

Grinder/Polisher System; 120V , $60 \mathrm{~Hz}$, single phase

\section{Options}

804-085

804-088

804-091

806-793

806-796

$801-424$

800-458

$803-795$

808-655

810-217-PRM

810-219-PRM

810-221-PRM

810-223-PRM

810-225-PRM

810-227-PRM

810-229-PRM
Specimen Holder Kit for eight 1 -inch diameter samples

Specimen Holder Kit for six 1.25-inch diameter samples

Specimen Holder Kit for four 1.5-inch diameter samples

Specimen Holder Kit for two 2-inch diameter samples

Specimen Leveler

8-inch Cloth Clamp

8-inch Paper Retainer

8 -inch Aluminum Wheel

Gravity Feed Dispenser (250 ml capacity) Abrasive Paper Disc, 60 grit (100/box) Abrasive Paper Disc, 120 grit (100/box) Abrasive Paper Disc, 180 grit (100/box) Abrasive Paper Disc, 240 grit (100/box) Abrasive Paper Disc, 320 grit (100/box) Abrasive Paper Disc, 400 grit (100/box) Abrasive Paper Disc, 600 grit (100/box)
To see a complete listing of all the high-quality consumables and supplies LECO offers, request our Met Supplies Catalog (form no. 203-826)

$V \sim$ denotes VAC.

Specifications and part numbers may change.

Consult LECO for latest information.

LECO Corporation

3000 Lakeview Avenue - St. Joseph, MI 49085-2396 Phone: 800-292-6141 • Fax: 269-982-8977 info@leco.com • www.leco.com・ISO-9001:2000 - No. FM 24045 - LECO is a registered trademark of LECO Corporation.

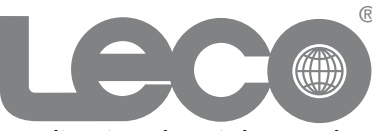

Delivering the Right Results

(C) 2006 LECO Corporation 


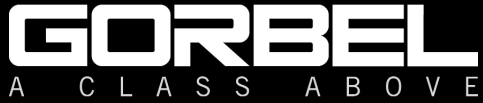
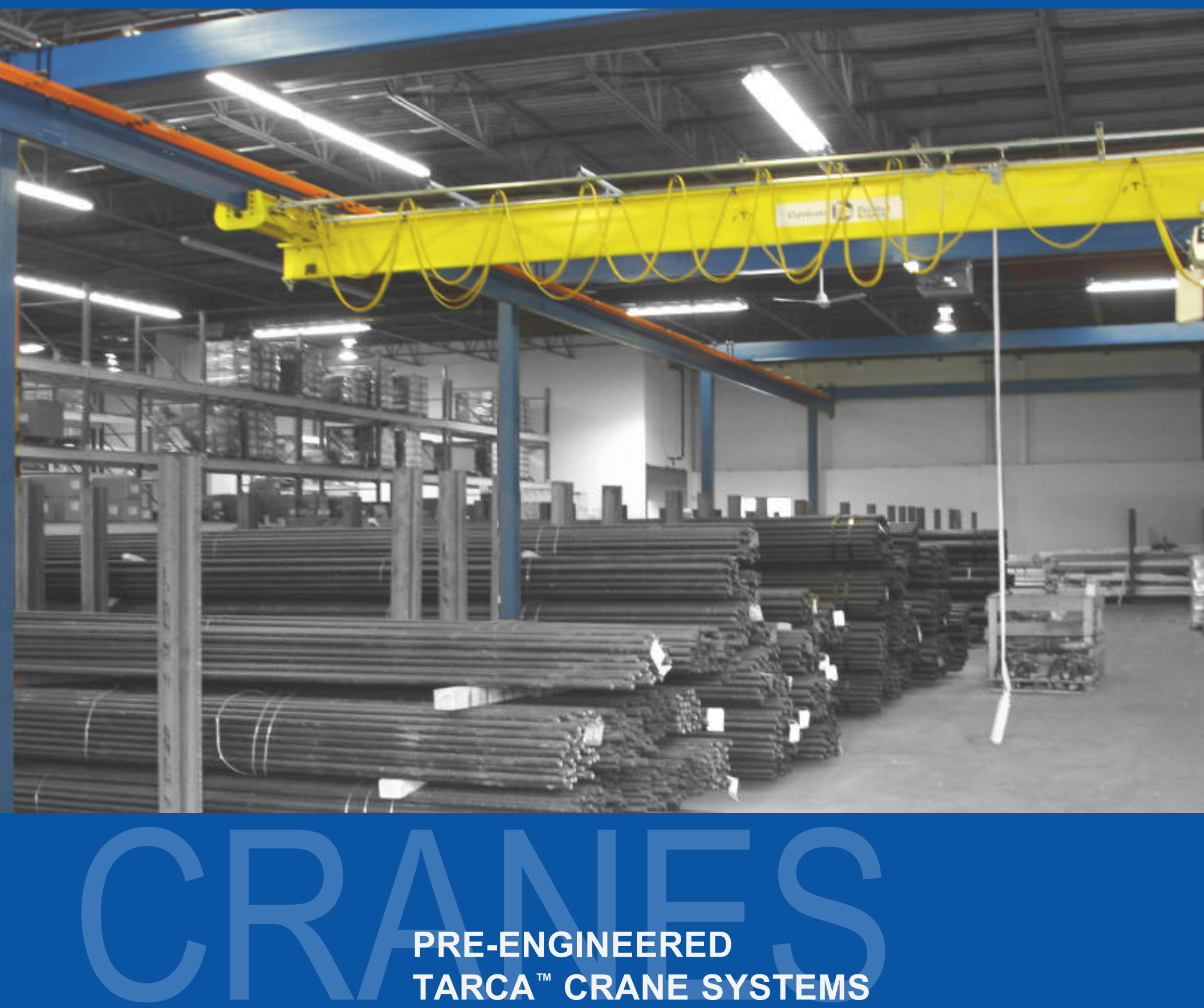

STYLE CAPACITIES SPANS TRACKS
FREE STANDING \& CEILING MOUNTED 2 TONS AND UP UP TO 60

TARCA $^{\text {TM }}$ PATENTED TRACK 


\section{Gorbel Pre-Engineered Tarca $^{\text {TM }}$ Crane Systems}

\section{A Name Built on Quality}

Since 1977, Gorbel Inc. has specialized in overhead material handling solutions, providing the highest quality and highest performance products on the market today. We are the leading supplier of Work Station Crane systems, offering near perfect on-time delivery, a focus on customer service and the industry's best warranty.

\section{Why Choose a Pre-Engineered Tarca" Crane System?}

\section{Industry's Best Warranty}

2 Years on all Crane systems.

\section{Design Responsibility}

Gorbel Crane Systems specify which beam to use for each application. We do not "Suggest" or "Recommend" any materials be used.

\section{Ease of Installation}

All components are built and assembled with jigs and fixtures which ensure easy installation.

\section{Improved Performance}

Tarca $^{\mathrm{TM}}$ Systems are designed to be superior to I-beam cranes in strength, durability, and consistency.

\section{Local Representation}

Gorbel cranes are sold and serviced by a nationwide network of material handling specialists that provide solutions suited to your needs and your budget.

\section{Versatility}

Gorbel makes a complete line of cranes to meet your individual needs

\section{Safety}

All cranes are pre-engineered for powered hoist operation with an impact factor of $15 \%$.

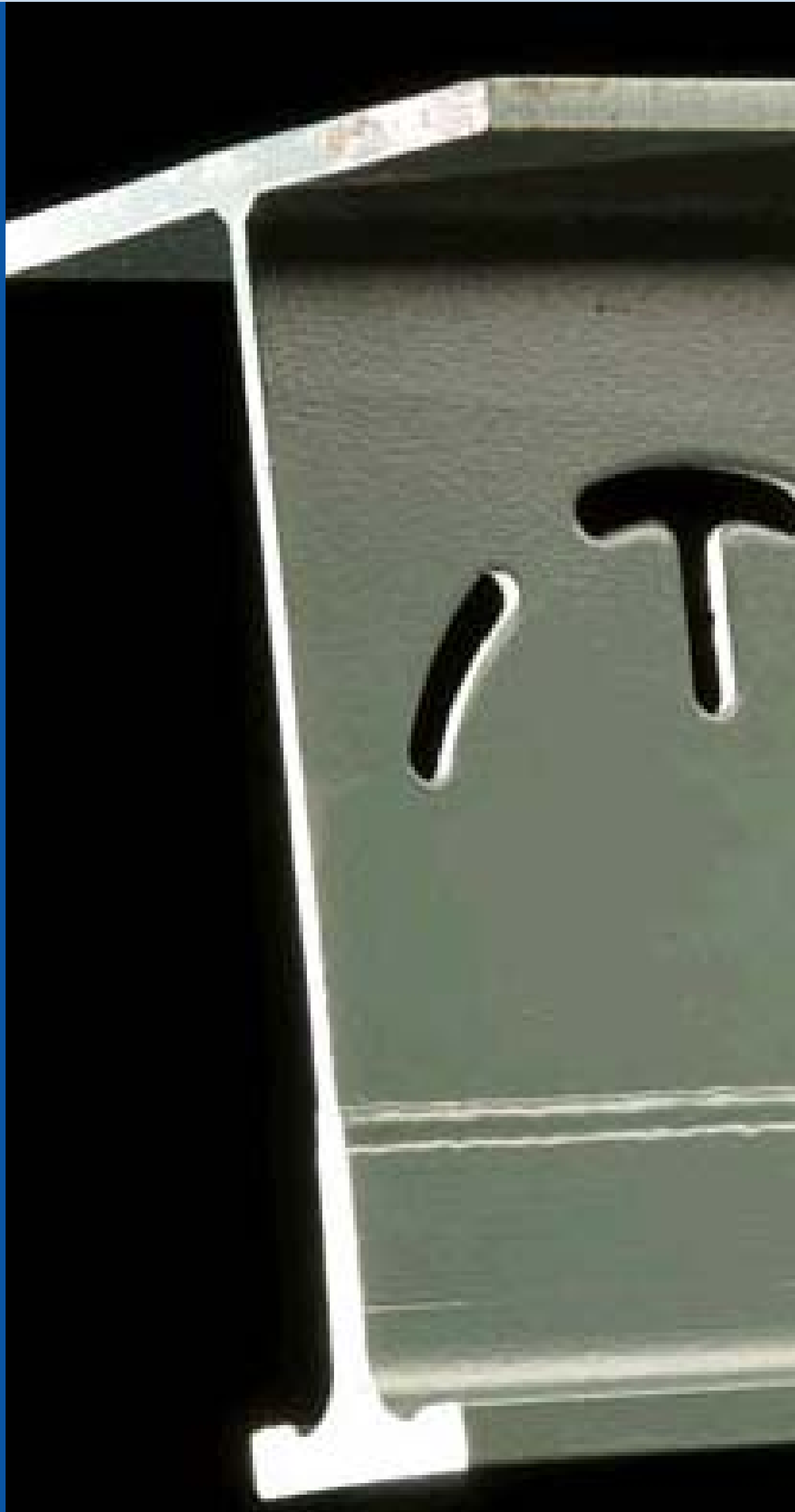




\section{Tarca $^{\mathrm{TM}}$ Track For Durability and Strength}

Gorbel's pre-engineered crane systems are built using our unique patented Tarca ${ }^{\mathrm{Tm}}$ Track, which continues to be the benchmark of the overhead material handling industry.

Its three piece welded construction is a compound section of a mild steel top flange and web and a specially rolled high-carbon steel lower rail. Tarca"' systems are characterized by consistently straight rail sections and durable, high quality Tarca ${ }^{\text {Tw }}$ components. Our Tarca"' systems offer unmatched versatility, durability and ease of installation.

\section{Why Tarca ${ }^{\text {Tm }}$ Track Over Structural Steel Track?}

A structural l-beam is rolled from soft, mild steel according to fairly loose steel mill tolerances. Its beveled flange prevents wheels from making balanced contact, causing uneven wear and a shortened track life.

Gorbel's unique Tarca"m Track, with its special raised tread and high carbon track, is superior to I-beams in strength, durability and consistency. Our exclusive rails permit the use of:

- Underhung carriers operating on a single straight, curved or inclined track

- Underhung cranes operating on two or more straight track runways.

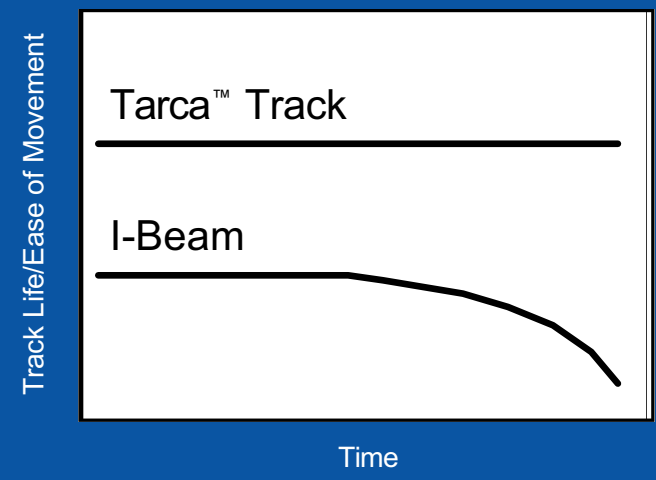

Why choose Gorbel Pre-Engineered Tarca"' Track Systems for extended capacities?

- Tarca ${ }^{\mathrm{m}}$ Track \& components stand the test of time

- Lower installation costs

- High quality designs mean reduced maintenance costs

Tarca $^{\text {TM }}$ Track has a mild steel top flange and web and a high carbon steel lower rail with raised tread to resist peening.

Continuous welding adds rigidity needed to cope with bending and twisting stresses of the most demanding applications.

The web and flange dimensions of each size are proportioned for maximum strength and spanning efficiency. 


\section{Tarca $^{\text {tm }}$ Track: Engineered for Crane Applications}

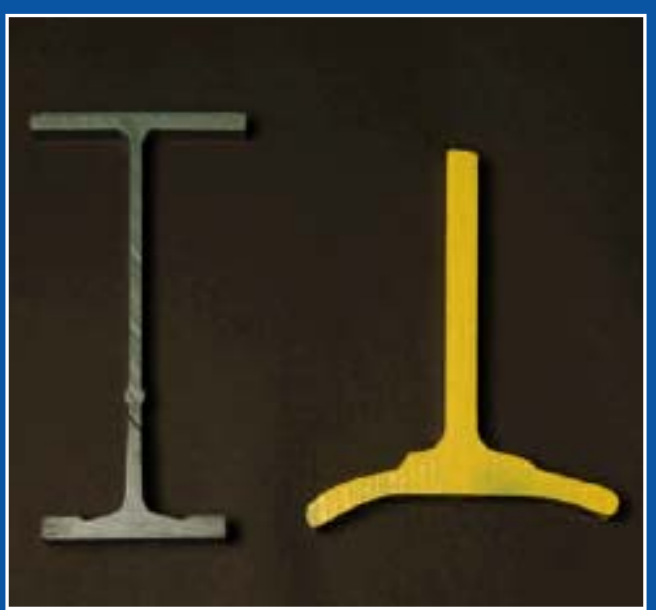

Peening caused the mild steel flange on this I-beam (on right) to bend, making it completely unsafe for crane travel.

\section{Strong, Durable Rail}

Tarca $^{\text {TM }}$ vs. I-beam

High carbon flange means longer track life

A common source of track wear is a process known as peening. Peening is the gradual movement of metal over time, caused by the rolling action of wheels. Because of I-beam's mild steel construction, peening tends to occur unevenly, weakening the beam flange and restricting smooth easy travel.

Tarca $^{\mathrm{m}}$ Track's raised tread design and extra hard alloy steel construction slow down the peening process. The full width of the raised tread wears evenly, extending track life well beyond that of I-beams.

\section{Our raised tread provides durability and superior safety} The raised tread on Tarca ${ }^{\mathrm{T}}$ Track is $20-25 \%$ of the Tarca ${ }^{\mathrm{T}}$ rail thickness. And though this does add some strength, our stress calculations do not include the strength added by the raised wearing tread. That means you get even more strength and dependability because the rated capacity of the track is not affected by wear.

Since l-beams have virtually no raised tread, their initial load carrying capacity is in the total cross section. As a result, capacity and safety are greatly reduced as soon as the tread begins to wear.

\section{$100 \%$ weld penetration ensures quality}

Tarca $^{\mathrm{TM}}$ Track is manufactured using stringent quality assurance procedures. This is the basis for certifying $100 \%$ weld penetration for maximum strength, safety and rigidity. Extreme care is taken during track fabrication to maintain dimensional tolerances. Our tracks are straight and true with minimum distortion, so:

- Installs are quick and easy

- Load distribution is uniform

- Tracks and components last longer

\section{Stop Throwing Away Your Installation Dollars!}

Our stringent quality standards in manufacturing guarantee consistently straight, high quality beams. Plate is cut to size and leveled by precision machinery. Special equipment uses continuous welds to insure absolutely straight, uniform track. Standard mill practice dimensions for I-beams have tolerances more than twice those for Tarca $^{\text {TM }}$ rail. For example, allowable sweep per 10' of structural beam may be as high as $1 / 4 "$ while $\operatorname{Tarca}^{\mathrm{Tm}}$ rail is less than half that at $3 / 32$ ".

\section{Is It Really Cheaper to Use an I-Beam?}

Consider the time and money you're throwing away during installation: redrilling holes and cutting, fitting, and shimming rails in order to align I-beams that aren't straight. Our consistently straight rails result in easy, predictable, cost effective installations.

\section{Straight Rails Make System Expansions Easy}

These tight quality standards even make system expansion and reorganization easier and more cost effective. Consistently straight beams will reduce labor costs when you want to expand a crane system or rearrange a work area. 


\section{Our Weight Is In All the Right Places}

Efficient Design for Spanning

The thickness and width of Tarca ${ }^{\text {Tw' }} \mathrm{s}$ web and flange for each size have been carefully engineered to maximize strength and loading capability while minimizing weight. Tarca "'s's three piece welded construction:

- Delivers maximum load carrying capacity to dead weight ratios

- Provides ability to cost effectively span longer distances

- Eliminates costly additional supporting structures

- Reduces drag in a manual system

I-beam simply can not match Tarca ${ }^{\text {Tm' }} \mathrm{s}$ carrying capacity to dead weight ratio. Structural I-Beams are manufactured for a purpose other than overhead material handling. Because of this, they are manufactured to much looser mill tolerances than many crane and monorail applications require.

\section{Standardized Lower Flange}

Standardized lower flange provides compatibility

Tarca $^{\text {Tm }}$ track features the same 3-1/4" wide lower flange regardless of rail height or load carrying capacity.

- Allows systems to be easily expanded or rearranged

- Ensures compatibility of components

- Lower costs when suspension points vary

The lower flange of an I-beam increases in width and thickness as its depth increases. Beams of different sizes are therefore not compatible. The result? I-beam crane systems are costly and difficult to expand or relocate.

\section{Rugged, Long Lasting Components}

Hardened wheels to ride on high carbon track

Our forged, heat-treated wheels are built to last. They provide years of smooth, easy movement and reliable service. They have been:

- Designed to roll with minimal resistance (2 times easier than I-beam)

- Machined to meet the surface of the track for consistent, full contact and longer life

- Engineered to avoid flat spots for smooth, uniform rolling

\section{Rugged end trucks and carriers} Gorbel end trucks have been carefully engineered to provide the finest performance with little or no maintenance.

\section{Extended drive life}

All gears and shafts in Gorbel drives are made with a remarkable alloy-steel that was chosen after long, grueling tests. This special alloy allows the drives to hold up to the wear and tear of constant service. This adds many years of reliable service to the motor head.

\section{Flexible Suspensions}

Flexible suspensions provide longer system performance and lower maintenance costs

Tarca" Track is suspended using ball and socket connections, permitting the track to float in all directions. This means it can compensate for structural movement by allowing tracks to move and adjust to crane wheel centers. This:

- Prevents damaging stress

- Dampens shock loads

- Allows for smoother operation

In other words, Tarca ${ }^{\text {tw }}$ rails can adjust to loads as they move, enabling carrier wheels to maintain consistent contact. This "load balance" of the wheels and components allows for longer life with less maintenance.

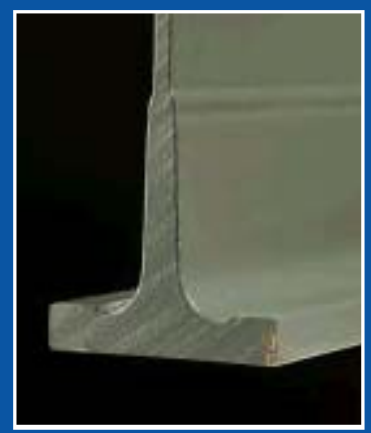

Our Tarca"w Track's standardized lower flange provides compatibility. 


\section{Case Study: Tarca ${ }^{\mathrm{m}}:$ A Better Solution than I-Beam}

\section{Gorbel Keeps Paper Industry Moving}

Seven Gorbel crane systems are the key to moving product around a Pittsburgh manufacturing plant. This plant manufactures fabric that is used in the paper industry to drain water out of the pulp mixture. On one end of the facility, the fabric is woven on looms using fishing wire. These pieces of woven wire are then seamed together in widths up to 40', taken off the loom and draped onto aluminum rolls.

These rolls weigh hundreds of pounds and their width makes them quite awkward to manipulate. It is vital to the quality of the paper that this fabric is free from impurities, so extreme care must be taken when handling these rolls.

Why was Gorbel the best choice for this application?

\section{Capacity 3-ton double girder cranes \\ Durability I-beam wears too quickly for a high duty cycle application like this where cranes are lifting and positioning rolls at a rate of 15 to 20 lifts per shift.}

\section{Building Design}

Headroom Constraints

\section{Interlocks were needed} to move the fabric rolls around building columns between the two systems. Gorbel interlocks are so safe and simple to operate that transferring loads from one system to another is quick and easy.

The design of the building created headroom constraints. The size of I-beam needed for these loads would not have fit within the constraints of the building.

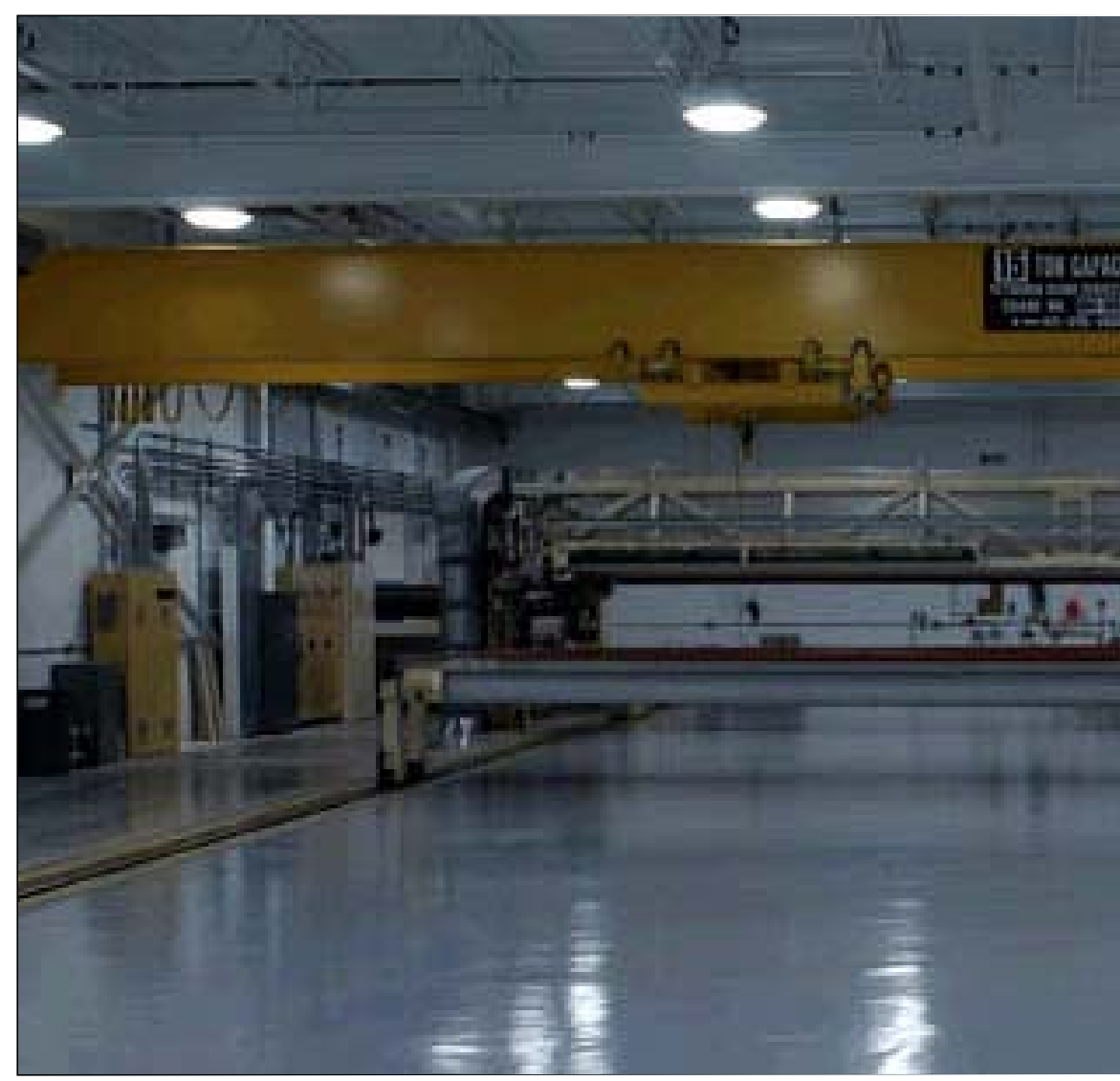

Our interlocks let you move your loads around anything - even building columns.

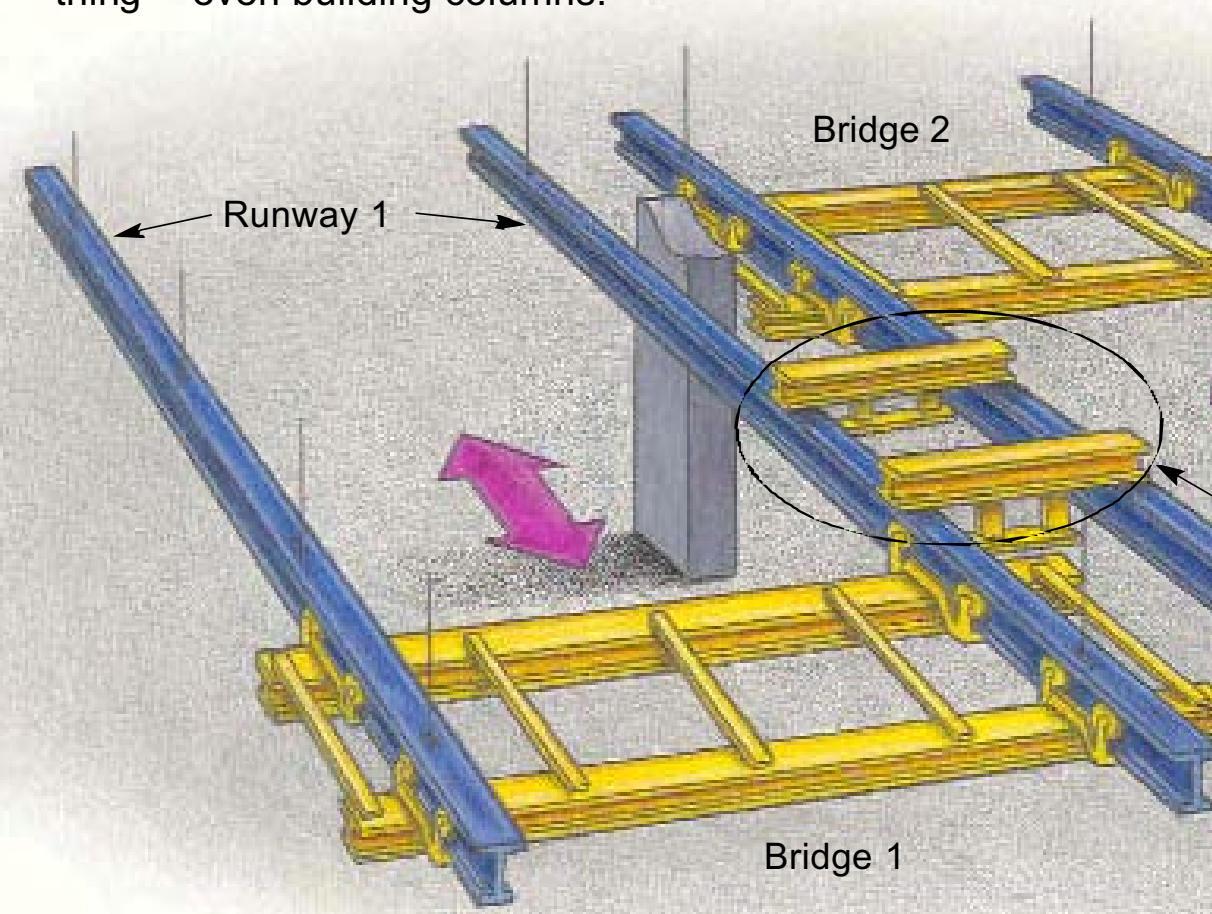




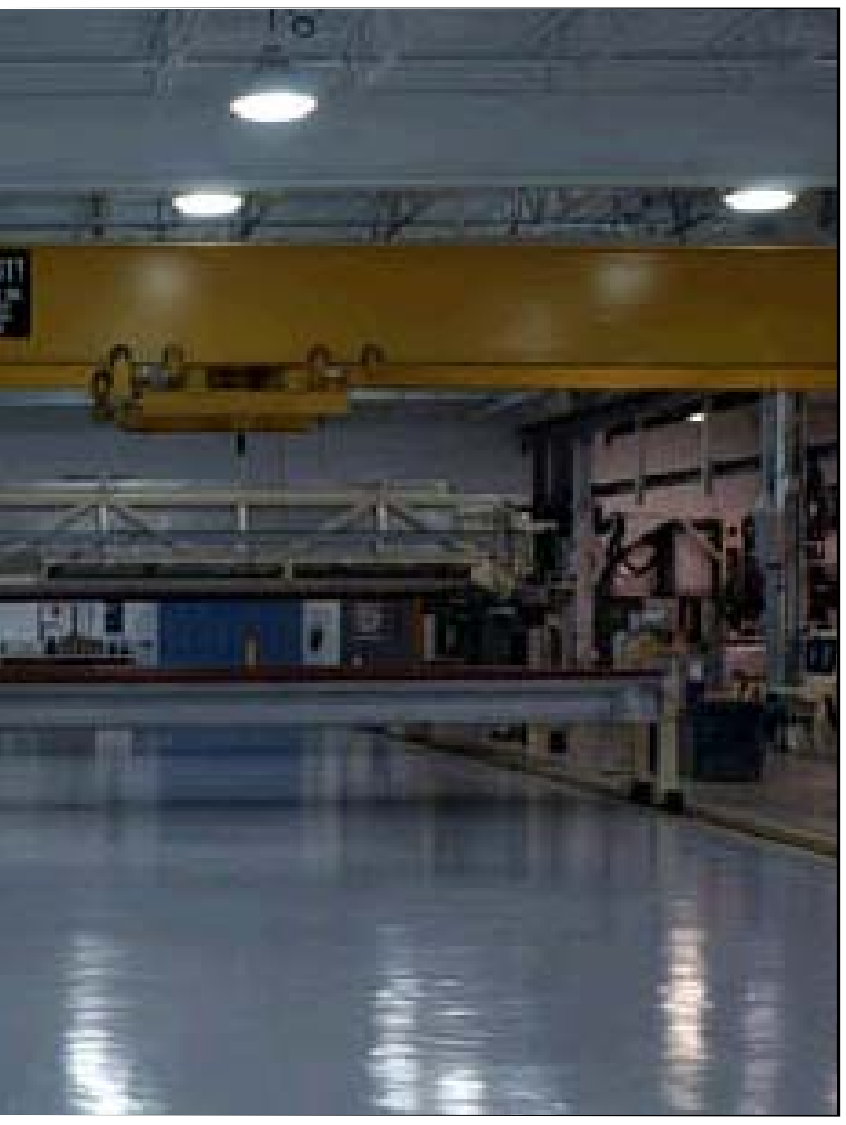

\section{How do our interlocks operate?}
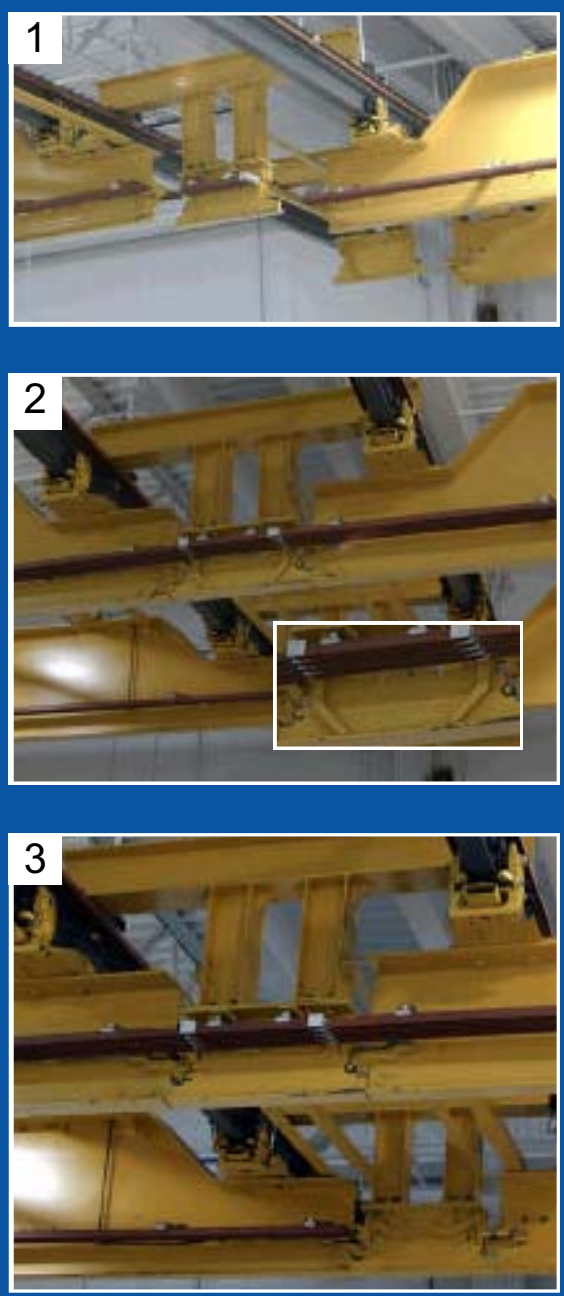

1. Both bridge girders approach the crossover track. The operator pushes a button powering a gear motor that activates a pair of rollers.

2. The bridges line up with the crossover track. The rollers move from the interlocking beam toward a wedge type engaging mechanism on the crossover track (see Photo 2).

3. The rollers make contact with the wedge and selfalign within 1-1/4" horizontal range.

As both beams are interlocked, forks on powered and nonpowered beams are raised for free passage of carrier (see Photo 3).

4. To disengage the interlock, the operator pushes a second button. Motor driven rollers move back and away from wedge, allowing forks to drop and make contact with rail tread. This prevents the carrier from travelling off the open end of the beam. 


\section{Gorbel Bridge Components}

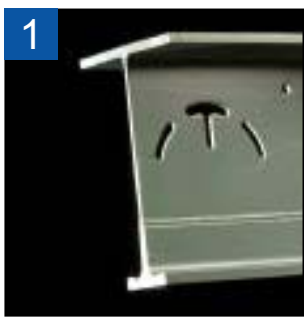

\section{Tarca $^{\text {TM }}$ Rail}

Tarca $^{m}$ Track features specially rolled high-carbon alloy steel rail with raised treads welded to a steel flange and web.

Tarca $^{\text {tw }}$ is designed to provide maximum spanning capability for heavy loads while minimizing the weight of required material. Its material properties resist peening and assure a longer operating life than ordinary track designs.

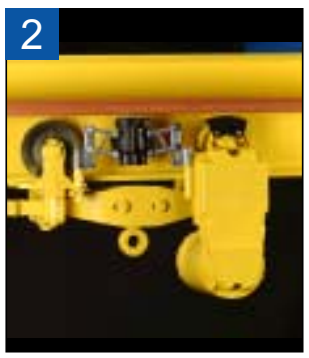

Photo shows carrier with drive and SAFPOWRBAR ${ }^{\mathrm{TM}}$

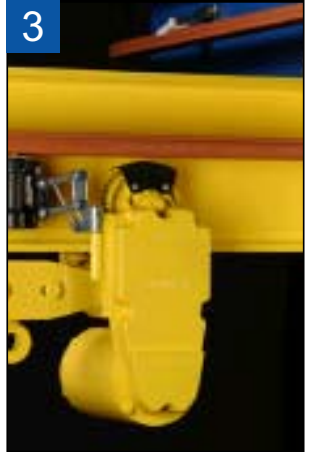

Belt-driven model show here.
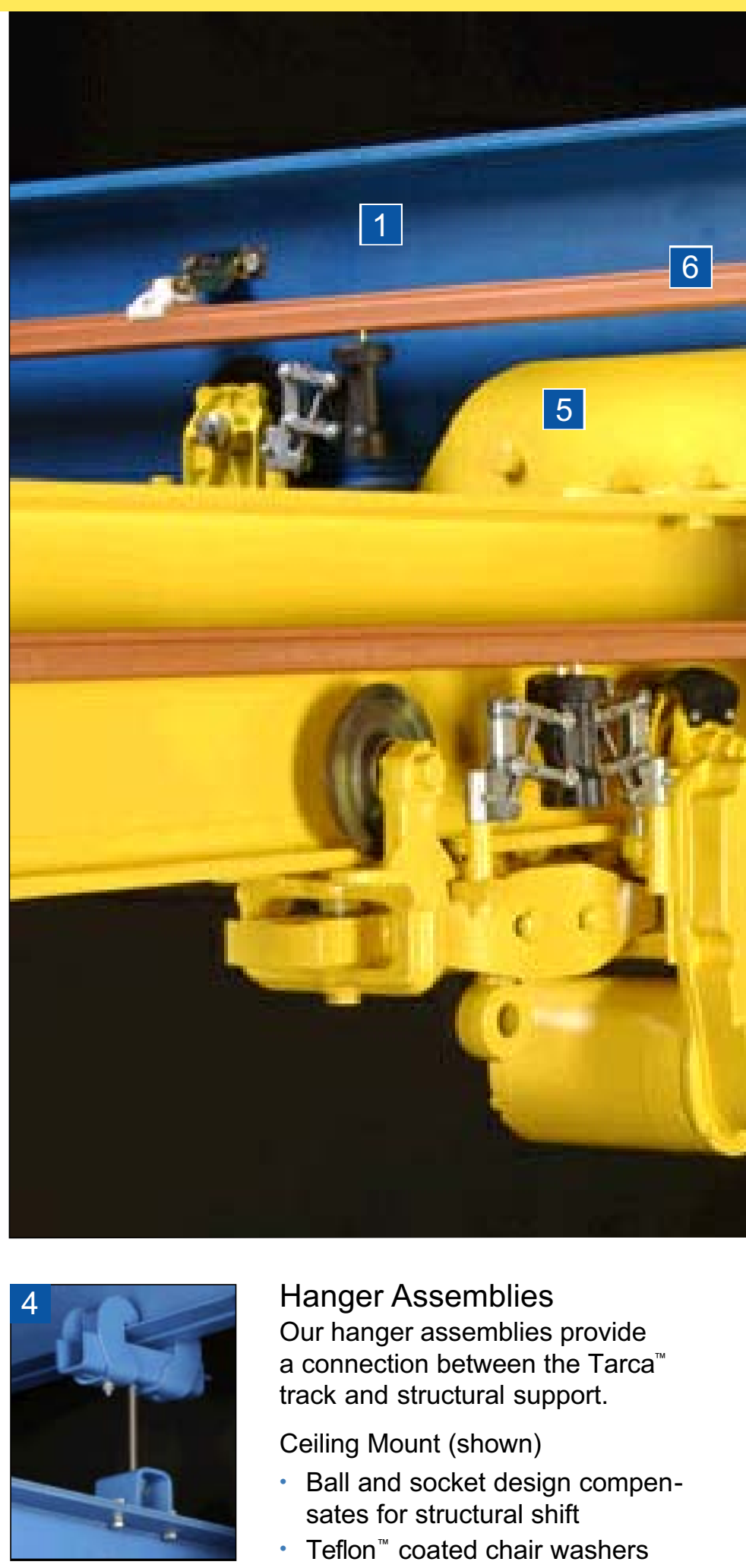

Hanger Assemblies

Our hanger assemblies provide a connection between the Tarca ${ }^{m}$ track and structural support.

Ceiling Mount (shown)

- Ball and socket design compensates for structural shift

- Teflon ${ }^{m}$ coated chair washers provide durable, long lasting performance

Free Standing Mount (not shown)

- Rigid mounted, bolted connection between the header and Tarca ${ }^{\mathrm{TM}}$ rail

- Easy to install

- Maximizes headroom 

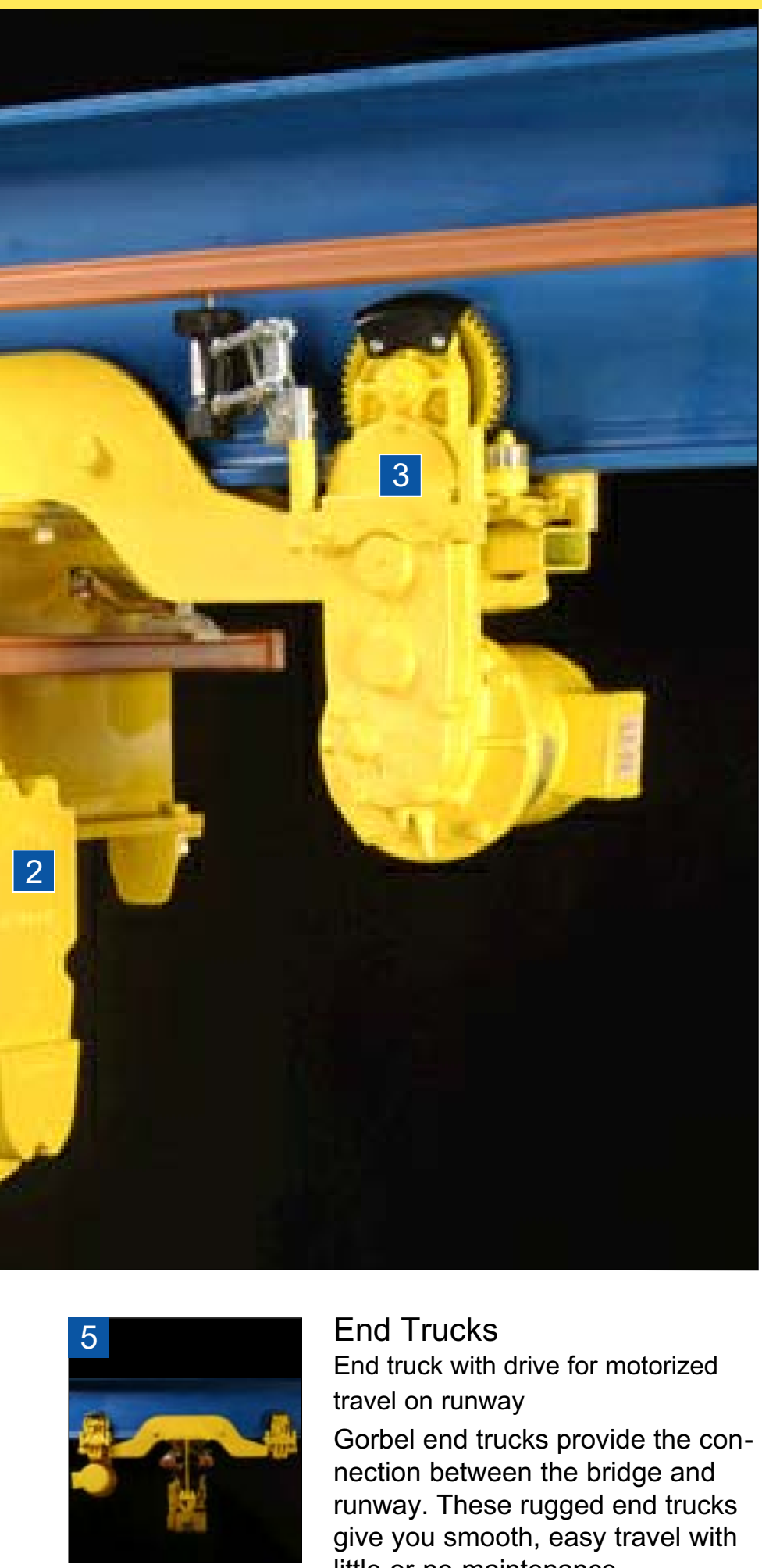

Motorized end truck shown here.

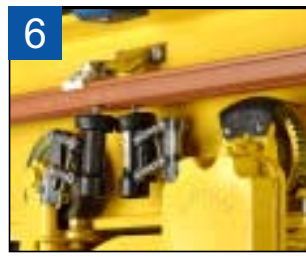

The photo at left shows a system with SAFPOWRBAR ${ }^{\mathrm{TM}}$ electrification.

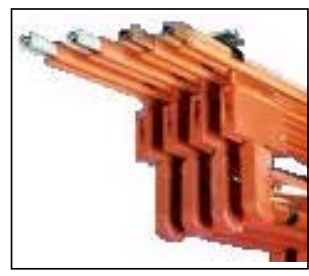

You can also choose Insul-8 Safe-Lec $2^{\text {TM }}$ conductor bar for your runway electrification.

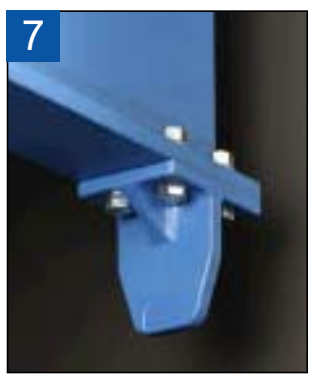

\section{Electrification Options}

(Brackets, Collector Shoe and Bar) Gorbel SAFPOWRBAR ${ }^{\text {tw }}$ Electrification is used to deliver electric power to drives and hoists. This rugged, durable system is made to perform in the most demanding applications and environments.

- Inverted U-shaped conductor bars enclosed by flame resistant insulated covers

- Fiberglass molded insulators for double insulation, making them safer and more efficient

- Sliding current collector shoes inside the bars make positive, continuous contact with three surfaces of the bar for longer life and easier maintenance.

\section{End Stop}

End stops are required and must be provided at the ends of the carrier or trolley travel and at the end of crane travel on runways.

Our end stops are unique in that they strike the end of the load bar, rather than the wheels. This prevents the wheels from absorbing the force of the load and reduces the areas of wear.

We offer standard wheel end stops for lighter applications and rubber, spring or hydraulic bumpers for higher speed conditions.
End truck with drive for motorized travel on runway

Gorbel end trucks provide the connection between the bridge and runway. These rugged end trucks give you smooth, easy travel with little or no maintenance.

One advantage of our end trucks are their wheels. While other manufacturers have fixed wheels on their end trucks, our articulating wheels allow for irregularities in track and runways. End trucks can also be manually driven. 
Designed to be

Low Maintenance!

“We've experienced less maintenance with the

Gorbel installation in

one year's operation

than had been required

in a single week of

operation with the

I-beam runway just

prior to its replacement."

Plant Manager

Babcock \& Wilcox unit of

J. Ray McDermott \& Co.

\section{Free Standing Pre-Engineered Systems Tarca ${ }^{\mathrm{T}}$ Track Systems}

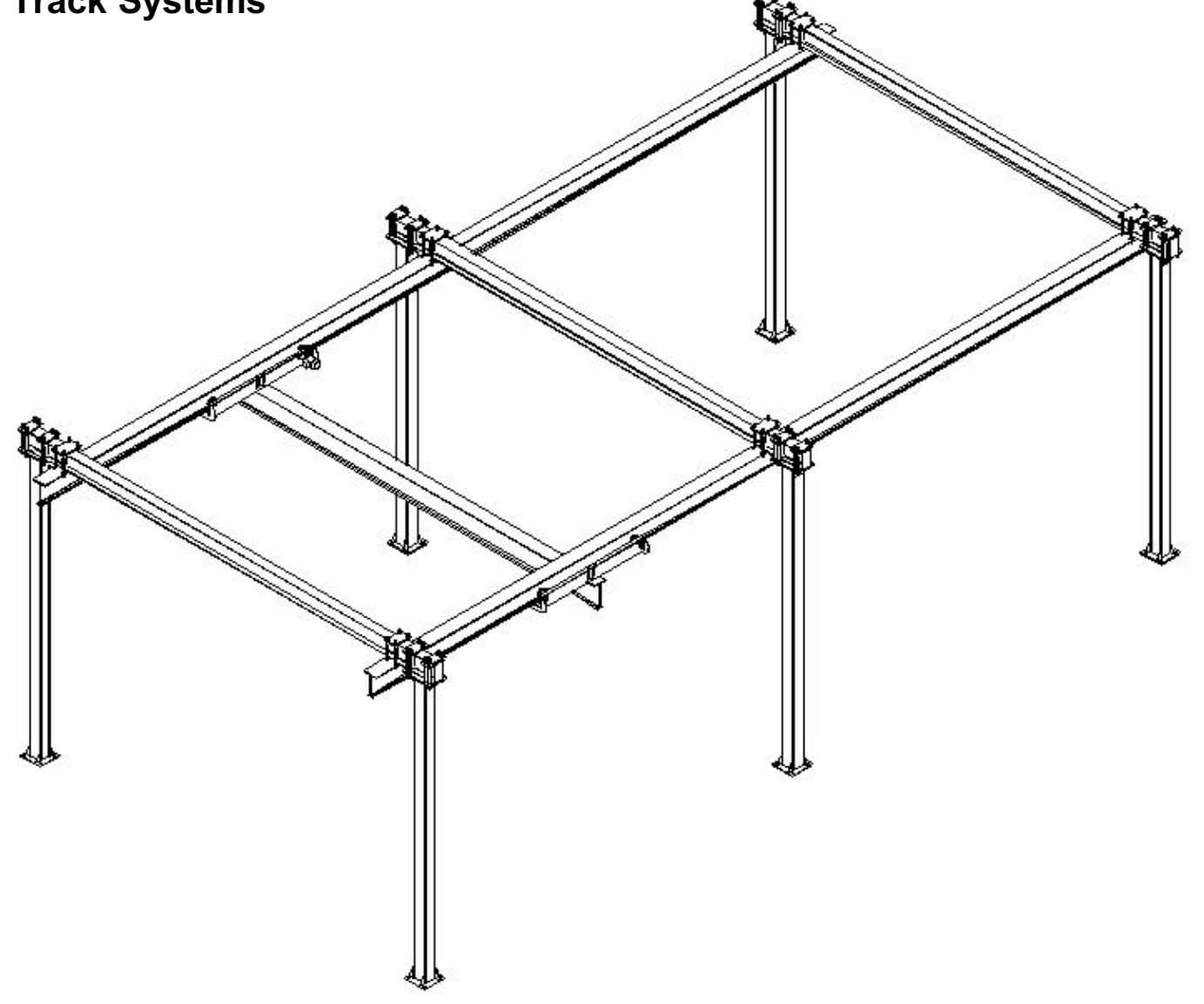

\section{SYSTEMS SPECIFICATIONS}

Capacities

Spans

Height Under Bridge

Runway Support Centers

* Consult factory for higher capacities

** Custom support centers available at no extra charge
Up to 5 ton

Up to $60^{\prime}$

$10^{\prime}-20$ '

$20^{\prime}-60^{\prime}$ in increments"
Free Standing System available in Braced designs or completely Free Standing designs:

Free Standing Braced Systems

- Require the crane support steel to be tied back to another structure (ie building columns) capable of resisting lateral and longitudinal forces.

- Braced systems tend to be less expensive than completely Free Standing systems.

Completely Free Standing Systems

- Completely independent of other systems

- Ideal for applications where there is no adequate support steel 


\section{Ceiling Mounted Pre-Engineered Systems for Extended Capacities}

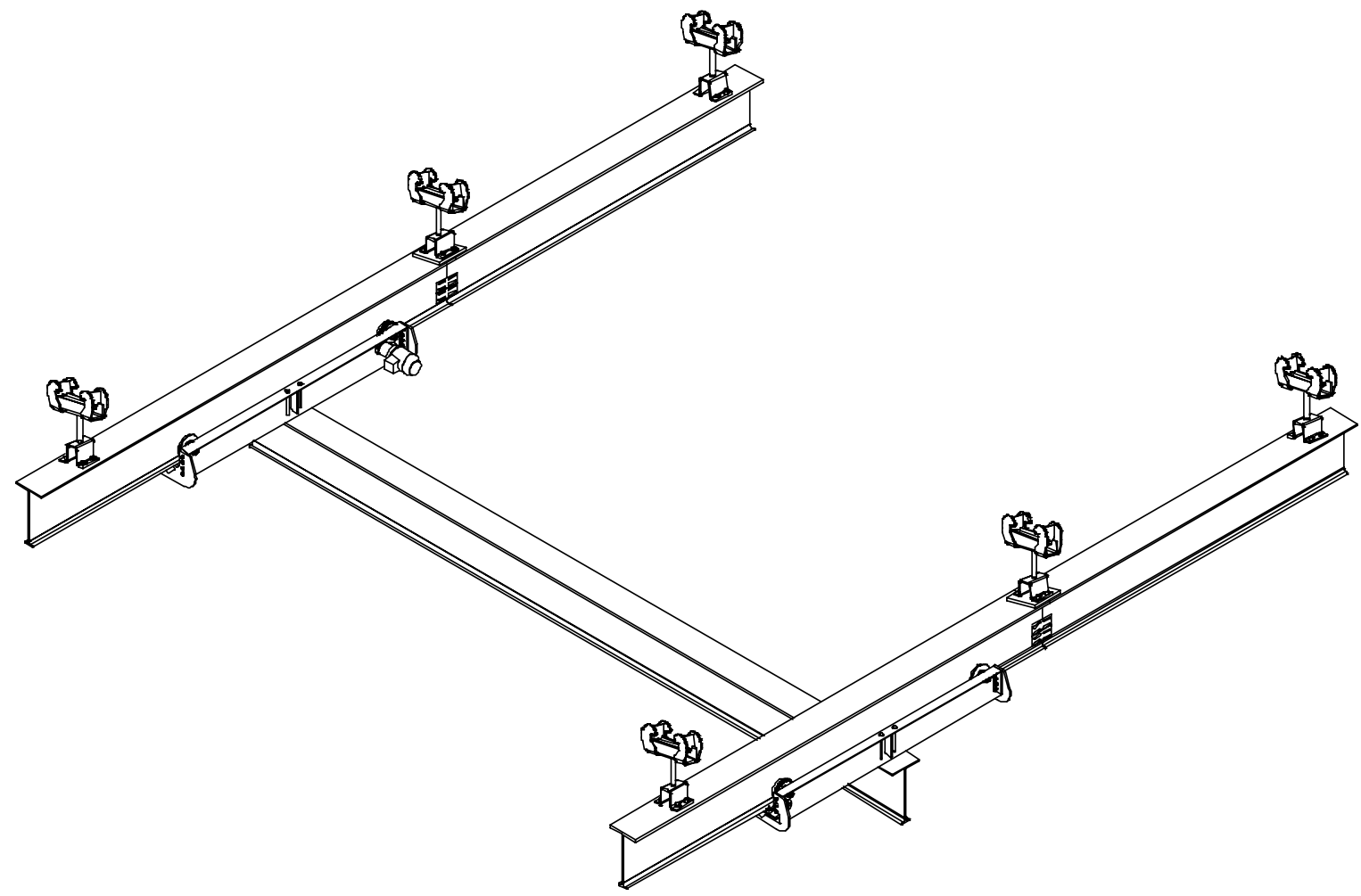

\section{Systems SpeCificATIONS}

\section{Capacities}

Spans

Height Under Bridge

Runway Support Centers
Up to 5 ton

Up to $60^{\prime}$

$10^{\prime}-20^{\prime}$

$20^{\prime}-60^{\prime}$ in increments"

* Consult factory for higher capacities

** Custom support centers available at no extra charge

Both Free Standing and Ceiling Mounted Systems Include:

- Runways, Bridge(s), End Trucks, Hanger Assemblies, runway electrification, bridge festooning, crane drives, and controls.

- Free Standing systems also include free standing structure

- Bracing and hoists by others. 
Your authorized Gorbel dealer can give you more information on what makes Gorbel's Ergonomic Work Station Cranes and other material handling products "A Class Above."

Bridge Cranes (Enclosed Track)

Jib and Gantry Cranes

Specialty Products (Enclosed Track)

Intelligent Assist Devices

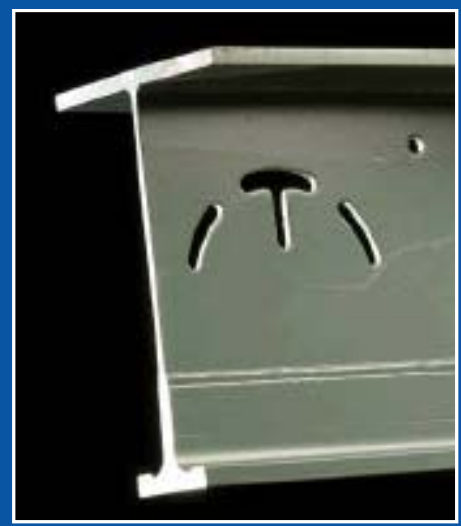

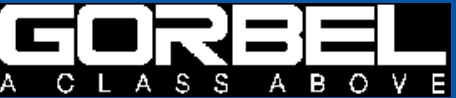

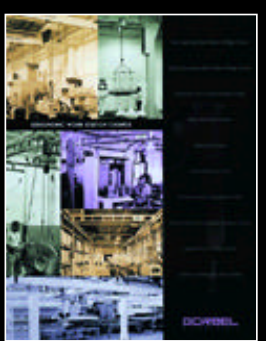

Capabilities

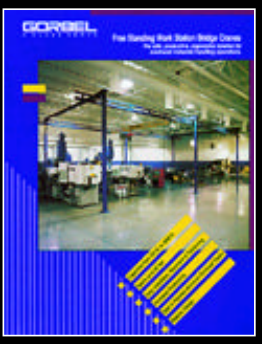

Free Standing

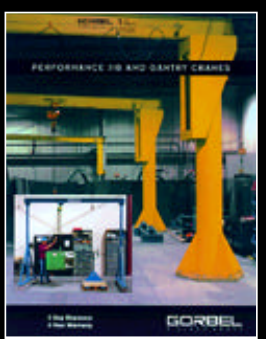

Jib and Gantry Cranes

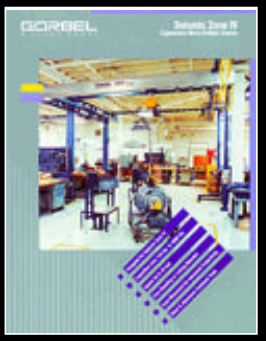

Seismic Zone IV

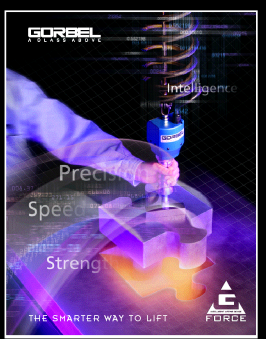

G-Force

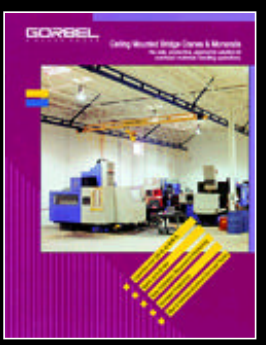

Ceiling Mounted

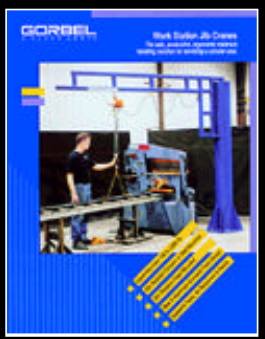

Work Station

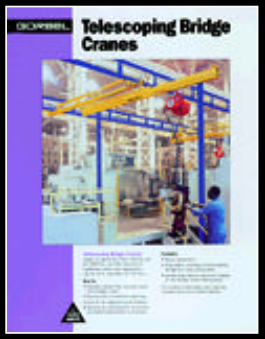

Telescoping

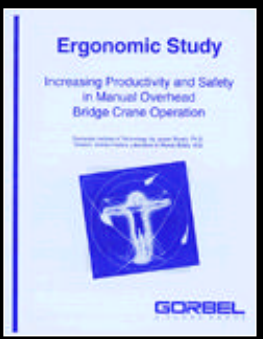

Productivity \& Safety

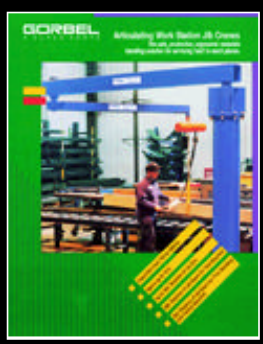

Articulating

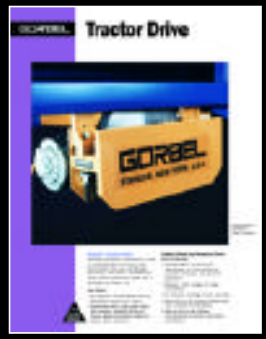

Tractor Drives

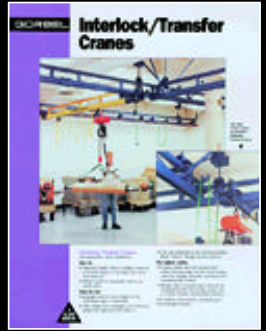

Interlock/Transfer

600 Fishers Run

P.O. Box 593

Fishers, NY 14453-0593 USA

Phone: 585-924-6262

Toll Free: (800) 821-0086

Toll Free Fax (800) 828-1808

www.gorbel.com 


\section{EPS \\ Engineered \\ Pressure Systems, Inc.}

PROPOSAL FOR

A

\section{Hot Isostatic Pressing System}

\section{PREPARED FOR \\ Idaho National Laboratory}

2008-53-13

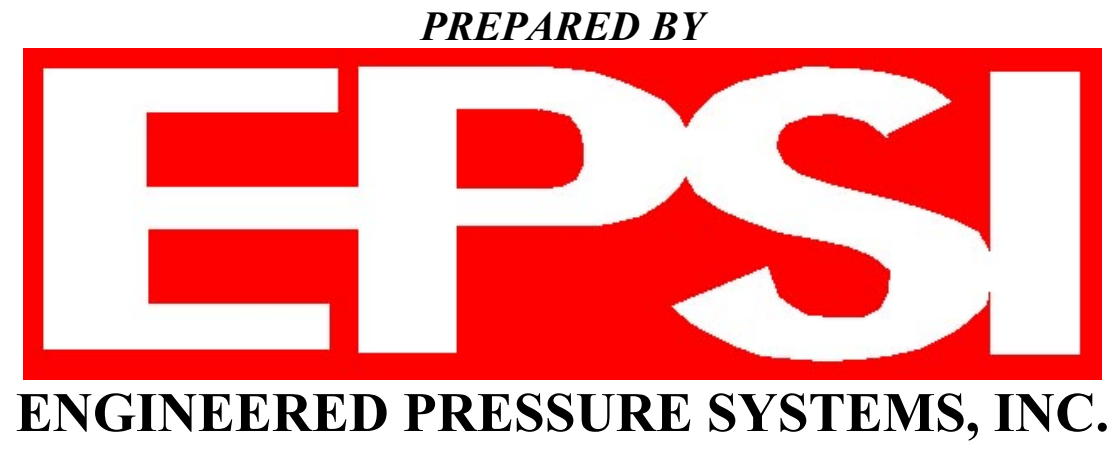

165 Ferry Road

Haverhill, MA 01835

Tel: 978-469-8280

Fax: 978-373-5628

March 27, 2008 


\section{뭉 \\ Engineered \\ Pressure Systems, Inc. \\ HIP Summary of Quotation 2008-53-13}

\section{Basic Hip System Configuration}

\begin{tabular}{|c|c|}
\hline Pressure Vessel & _Plate Yoke Vessel Assembly \\
\hline Design Basis & ASME Section VIII, Div. 3; U3 stamped \\
\hline Material & SA 723, \\
\hline Maximum Operating Pressure & 15,000 psi \\
\hline Design Pressure & 16,500 psi \\
\hline Hydrotest Pressure & 20,625 psi \\
\hline Vessel Design Temperature & $600^{\circ} \mathrm{F}$ \\
\hline Calculated Cycle Fatigue Life & 15,000 per \\
\hline Furnace/Heatsheild & Moly $1,260^{\circ} \mathrm{C} \pm 10^{\circ} \mathrm{C}\left(2,300^{\circ} \mathrm{F}\right)$ \\
\hline Workload diameter & 10" workload OD Clear \& Obstructed \\
\hline Workload length & 60" Clear \& Obstructed \\
\hline Workload weight_ & _1,000 lbs for BZH $@ 2,300^{\circ} \mathrm{F}$ \\
\hline Parts per Cycle & See Chart on Page 16 \\
\hline Process Gas & Argon \\
\hline Cooling system & Closed-loop \\
\hline Control System & Fully Automatic PLC Controls \\
\hline Vacuum System & 100 micron within 30 minutes \\
\hline \multicolumn{2}{|l|}{ Commercial } \\
\hline Price: & 10"X60"@15,000 psi HIP System \$1,300,000 \\
\hline Payment Terms: & See Page 48 \\
\hline Delivery: & $\begin{array}{l}12 \text { To } 14 \text { Months Ready for Shipment. Deliveries } \\
\text { are subject to allocation of melting capacity. } \\
\text { Deliveries to be adjusted after meeting melting } \\
\text { commitment are secured. }\end{array}$ \\
\hline Fob: & EPSI, Haverhill, MA \\
\hline Installation Included: & Supervision, of Start-up and Training \\
\hline $\begin{array}{l}\text { Warranty: } \\
\text { Validitv: }\end{array}$ & One Year \\
\hline $\begin{array}{l}\text { Validity: } \\
\text { Utilities: }\end{array}$ & 30 Days after Firm Pricing \\
\hline $\begin{array}{l}\text { Utilities: } \\
\text { System Accentance: }\end{array}$ & See page 43 \\
\hline System Acceptance: & System Functional Testing Only \\
\hline
\end{tabular}

Vessel Inspections should be conducted periodically. 


\section{EPST \\ Engineered \\ Pressure Systems, Inc.}

Engineered Pressure Systems, Inc. is pleased to provide the following proposal for your Hot Isostatic Pressing (HIP) System. Specifically, we propose the following:

1 Introduction 5

1.1 EPSI's HIP System Safety Items __ 6

1.2 HIP System Operation___ 8

2 Plate Yoke HIP Pressure Vessel Assembly

$2.1 \quad$ Pressure vessel Assembly __ 9

2.2 Pressure Vessel_ 10

2.3 Top \& Bottom Closures 11

2.4 Top \& Bottom Seal plates__ 13

2.5 Pressure Vessel Support Stand___ 13

2.6 User Design Specification 13

2.7 Safety Rupture Disc Assembly___ 14

3 Top Closure Hoist Assembly

3.1 Top Closure Hoist__ 15

3.2 Load Insertion Tooling__ 16

$4 \quad$ Furnace \& Heat shield Assembly

4.1 Moly Furnace Assembly__ 21

4.2 Furnace Plug-In Assembly___ 23

4.2.1. Furness Shells__ 23

4.2.2 Heating Elements _ 23

4.2.3 Furnace Ceramics_ 23

4.2.4 Furnace Thermocouples_ 23

4.2.5 Furnace Side Shield Insulation Package___ 23

4.2.6 Furnace Plug-In Components__ 23

4.3 High Performance Clopen Top Mantel__ 24

4.3.1 Mantle High Performance Clopen Top Design__ 24

4.3.2 Mantle Insulation Package__ 25

4.3.3 Furnace/Mantle Lifting Fixture__ 25

4.3.4 Gas Purity_ 25

4.4 Plug-In Bottom Zone Heater 25

4.4.1 Bottom Zone Heater (BZH)__ 25

4.4.2 Load Thermocouples_ 26

4.4.3 BZH (Bottom Zone Heaters) Ceramics___ 26

4.5 BZH/Tooling Lifting Fixture___ 26

4.5.1 BZH/Tooling Lift Fixture_ 26

4.5.2 BZH Tooling Support Plate__ 26

4.5.3 Optional BZH Support Plate__ 27

4.6 Seal Plate_ 27

4.6.1 EPSI Supplied Bottom Seal Plate___ 27

4.6.2 Plug-In Design Seal Plate with Components___ 27

4.7 Electrical Power Distribution __ 28 
Engineered

Pressure Systems, Inc.

$5 \quad$ Cooling System \& Vacuum System

5.1 Cooling__ 29

5.2 Vacuum System__ 29

$6 \quad$ Argon Delivery System

6.1 Description of Delivery System__ 30

6.2 High Pressure Piping, valves and Low Pressure Piping__ 31

$7 \quad$ Process Controls

7.1 PLC Controls___ 31

7.2 Control Process__ 31

7.3 The process steps will include_ 38

8 Installation

8.1 Supervision of Installation 38

8.1.1 Optional Turn Key Installation___ 39

8.2 Acceptance Cycles__ 39

8.3 Training__ 41

$9 \quad$ Miscellaneous

9.1 Documentation _ 43

9.2 Utilities__ 43

9.3 References 43

9.4 Vessel Inspection__ 46

10 General Conditions of Sale

10.1 Price__ 47

10.2 Delivery _ 47

10.3 Validity of the offer___ 47

10.4 Payment Terms _ 48

10.5 Warranty 48

10.6 Conditions of Sale 48 


\section{INTRODUCTION}

\section{EPS \\ Engineered \\ Pressure Systems, Inc.}

EPSI is pleased to present Idaho National Laboratory with this proposal for a Hot Isostatic Pressing System .

This system is configured in accordance with the Idaho National Laboratory Performance Specification for Hot Isostatic Press. Please note EPSI is offering a 10 inch diameter by 60 inch void length work zone to utilize the productivity of a HIP system. We are also offering 14 " and 12 " HIP systems as options.

Our proposal is divided into several sections relative to describing scope of supply, configuration, operating considerations, and technical features of the major sub assemblies and subsystems. Other sections of interest are a listing of our references for similar presses.

Please note that our review of your technical specifications indicates that the design, safety and performance requirements for the proposed system are similar to the ones we have manufactured for GE groups and Los Alamos National Laboratory. Based on the fact that these presses have proven to be operationally reliable, it is our intentions to supply Idaho National Laboratory essentially with the same equipment to meet your production and safety requirements.

We appreciate this opportunity to submit our proposal for your consideration. Please note that we have prepared it in a comprehensive manner to provide you with the information necessary to conduct your evaluation of the system we intend to supply. However, if you need additional data, or if you have any questions, please do not hesitate to contact EPSI.

Engineered Pressure Systems, Inc. will design, assemble, install and test a complete Hot Isostatic Pressing (HIP) system. The HIP system will consist of the following major components.

- Pressure Vessel Assembly

- Furnace \& Heatshield Assembly

- Part Support Tooling

- Control System

- Process Gas Handling \& Compression System

- Vacuum System

- Electrical Power Distribution System 


\section{EPS \\ Engineered \\ Pressure Systems, Inc.}

The proposed HIP System comprises several modules, which are interconnected with electrical wiring and piping to form the Hot Isostatic Pressing System. The modular design simplifies system installation in your facility. EPSI will supervise the installation the HIP equipment components.

EPSI will furnish facility layout drawings to be mutually agreed upon.

\subsection{HIP System Safety Items}

\section{HIP Vessel Equipment Safety}

EPSI believes we provided the safest highpressure equipment in the world, but no matter how safe our equipment is the ultimate responsibility is of the end user.

Our vessel assembly and yoke plates are designed to be properly NDT inspectable. What this means is that our vessel, yoke plates and pressure components can be inspected by means of ultrasonic testing, MAG practical testing, die penetrant and acoustic emission testing.

We recommend the pressure vessel assembly have a valid inspection every year. The vessel inspection is the responsibility of the end user. We have included a budget price and description to do a vessel assembly inspection one year after the system has been in service.

EPSI has some unique features in our vessel design, which include $99 \%$ inspect ability of all cooling water passages and $100 \%$ of all high stress areas.

There are some drill throughs that can only be visually inspected for corrosion. Corrosion is a concern that all HIP operators should be aware of and should be eliminated. The vessel cooling water never comes in contact with the vessel. We do this by using stainless steel jackets around the OD of the vessel.

We have yoke locating guide cylinders with limit switches to confirm the yoke is properly centered over the vessel before pressurization occurs. This is a feature we have included in every one of our yoke vessel assembly's.

In our controls we have incorporated safety interlock and warning notifications and $\operatorname{logs}$ to the operators. Proper training is crucial to the safety of operating HIP equipment.

Over the past few years EPSI has been awarded contracts over other suppliers because of our vessel design and long-standing safety record with them. Los Alamos National Laboratory placed in order with EPSI for a warm isostatic press to press high explosives. DuPont recently ordered a cold isostatic press from EPSI strictly based on our vessel design versus our competitors. 


\section{Facility}

\section{를 \\ Engineered \\ Pressure Systems, Inc.}

There are several safety areas to be considered when operating a HIP facility. As a minimum we bring to your attention the following:

- We recommend that the Pressure Vessel be placed in a pit or a barricaded area that is wel vented to confine high-pressure gas in the event of a leak. Placing the majority of the Vessel below floor level also facilitates loading and unloading of the load chamber.

- Argon is heavier than air, and therefore a suffocation hazard is more prevalent in a pit versus a barricaded well ventilated area. All enclosed areas, and the vessel pit, must contain oxygen monitors that give both an audible and visual alarm when oxygen is below safe levels. Additionally, all operators and maintenance personnel will need comprehensive training by our experts concerning this potential hazard. Should you wish EPSI to supply oxygen monitors or additional provisions within the controls to monitor these alarms we will offer this equipment at additional cost.

- All enclosed areas and the vessel pit will need exhaust fans that exhaust the pits of potentially suffocating gasses.

- Recommendations will be made concerning the location of system components relative to one another. This will permit safe access only to certain components while the unit is operational.

- The control panel should be located in an area away from high-pressure equipment.

- Appropriate electrical interlocks will be utilized to prevent operators and maintenance personnel from working on the unit while in operation. 


\section{EPS \\ Engineered \\ Pressure Systems, Inc.}

\subsection{HIP System Operation}

The following paragraphs provide a fundamental description of an operating cycle for the proposed HIP System. This description is presented as a sequence of events listed in chronological order. The HIP System we are proposing is a top loaded unit. This means that the materials to be processed are placed in the vessel from the top using a customer supplied overhead crane. Assuming the pressure vessel is open and ready for loading, the steps for a typical processing cycle are:

1. The product to be HIP'ed is placed in a EPSI supplied load tray set and it is in turn placed into the vessel while attached to the bottom zone, plug in heater (EPSI will supply one load can and IDAHO NATIONAL LABORATORY will provide 1,000 pound load or parts for test purposes).

2. The heat shield and furnace assembly is installed over the load and the vessel is closed in preparation for evacuation and pressurization.

3. The vacuum system is activated to evacuate the pressure vessel to a predetermined level. Vacuum level is indicated at the control console screen by a vacuum transducer gauge. Better cleanliness can be achieved by argon purging and re-evacuating.

4. Once the required vacuum level is reached, the vacuum system is turned off. Next, argon flows from Idaho National Laboratory storage to the pressure vessel through appropriate valves and piping. This flow will continue until storage and vessel pressures are nearly equal or liquid pump in turned on depending on pressurization system.

5. After equalization, the high-pressure gas compressors or liquid pump and furnace are turned on. Argon flows from storage to the argon compressor, which in turn fills the vessel. When the desired vessel pressure is reached, the pump is switched off automatically. Pressure \& temperature is reached at about the same time.

6. As soon as a positive pressure is sensed in the vessel, the furnace may be turned on. Power is applied to the furnace heating zones in a controlled manner to elevate the work zone temperature to a specified value. As the work zone temperatures increase, the argon within the vessel expands and consequently increases vessel pressure. Our controls are designed to work with step 5 to save time and energy.

7. The system is held at the required temperature and pressure for a specific dwell time (IDAHO NATIONAL LABORATORY specified 4 hours). The work zone temperature and pressure is automatically maintained at set point by the control system, which regulates power to the heating zones.

8. After the dwell period is over, furnace power is switched off, and the workload is cooled normally or thru EPSI's clopen top enhanced cooling mantle and valves. 


\section{EPS \\ Engineered \\ Pressure Systems, Inc.}

9. When the furnace and work zone have reached a specified temperature, argon is removed from the vessel by venting to atmosphere.

NOTE: Gas recovery can be offered as an option, but would add about $\$ 100,000$.

10. Subsequently, the vessel reaches atmospheric pressure, and it is opened.

11. The mantle and furnace assembly are removed, and the load can is lifted from the vessel.

2 Plate Yoke HIP Pressure Vessel Assembly

2.1 Plate Yoke Pressure Vessel Assembly ASME Section VIII, Division 3

- Pressure Vessel

The pressure vessel will be designed as a double ended, cylindrical monobloc vessel. The internal dimensions and basic design considerations for the pressure vessel load are:

\section{0 inch ID by 60 inch IL load Clear and Unobstructed Vessel and Yoke Assembly Weight is approximately 25,000 lbs}

The vessel and closures will be manufactured from SA-723, Class 2 material. This material will be melted, forged, machined, and tested to EPSI's specifications to ensure the highest level of quality control.

- Yoke Assembly

The Yoke Assembly with out any welding is a rigid structure that withstands the full thrust of both end Closures during pressure cycling with minimal movement.

- Yoke Pressure Vessel Assembly

Basic design guidelines, which assume $2,100^{\circ} \mathrm{F}$ furnace operation temperature, are:

ASME Code Stamped, Section VIII, Division 3

$\begin{array}{ll}\text { Operating Pressure: } & 15,000 \text { psi } \\ \text { Design Pressure: } & 16,500 \text { psi } \\ \text { Hydrotest Pressure: } & 20,625 \text { psi } \\ \text { Design Temperature: } & 40^{\circ} \mathrm{F} \text { to } 600^{\circ} \mathrm{F}\end{array}$

Where feasible, the analytical methods and safety factors incorporated in Section VIII, Division 3 of the ASME Boiler and Pressure Vessel Code are used. 


\section{EPS \\ Engineered \\ Pressure Systems, Inc.}

EPSI is offering an ASME, Div. 3, Vessel and Yoke. The following materials will be employed:

- Vessel and Closures: SA 723

- Vessel Yoke Plates and closures: SA543, or SA 516-70

Upon completion of all manufacturing steps, the vessel and closures will be assembled and hydrostatically tested to the value noted above. Of course, you are also invited to witness this test.

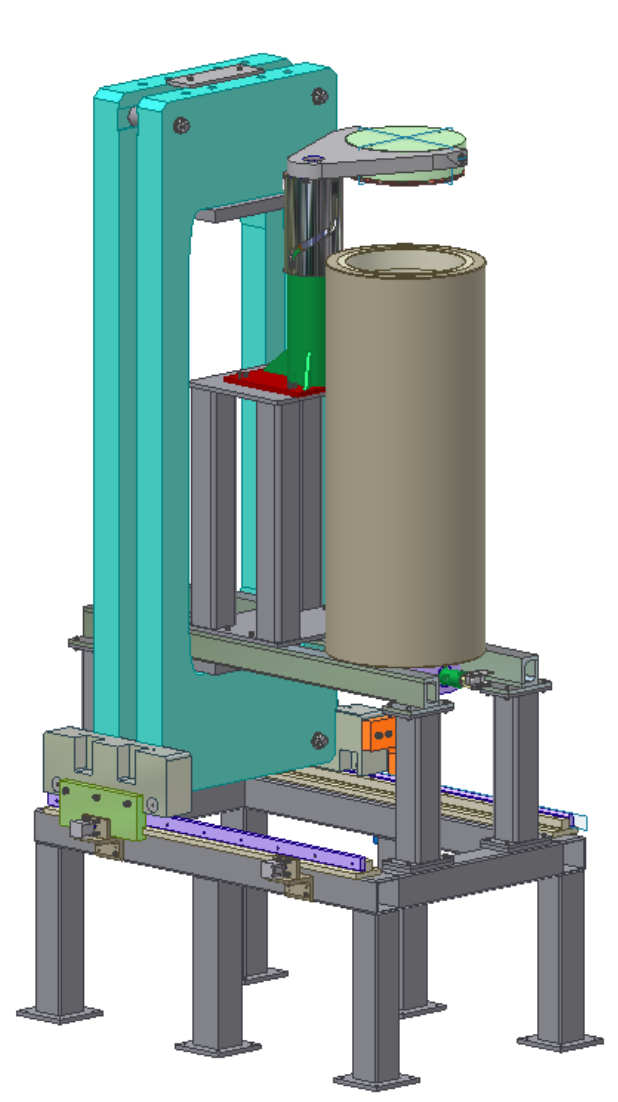

\subsection{Pressure Vessel}

The pressure vessel will be a double ended, monobloc cylinder.

Dimensions:

$$
\begin{aligned}
& \text { I.D. }-20 " \\
& \text { V.L. }-90 "
\end{aligned}
$$

The pressure vessel will be designed, manufactured, tested and inspected. The material of construction for the vessel body, and end closures will be SA-723 steel.

The pressure bearing seal plate assemblies at the top and bottom closures are easily split and will be $99 \%$ inspectable to prevent any corrosion of these water- 


\section{Engineered}

\section{Pressure Systems, Inc.}

cooled components in the future and $100 \%$ of the highly stresses areas will be inspectable. Chemical and mechanical property evaluations, hydrostatic testing, and non-destructive examinations will be performed. Naturally, all data will be supplied to Idaho National Laboratory.

\section{Vessel Inspections should be conducted periodically}

\subsection{Top \& Bottom End Closures}

The bottom closure of the vessel is an end plug that bolts into the vessel body from the lower side.

The bottom closure to vessel body seal is accomplished using our standard " $\mathrm{L}$ " shaped seal ring. Each leg of the "L" contains an "O" ring in a properly sized groove. One "O" ring seals against the top surface of the bottom seal plate, and the other against the vessel bore. The seal is clamped to the bottom seal plate using retaining washers.

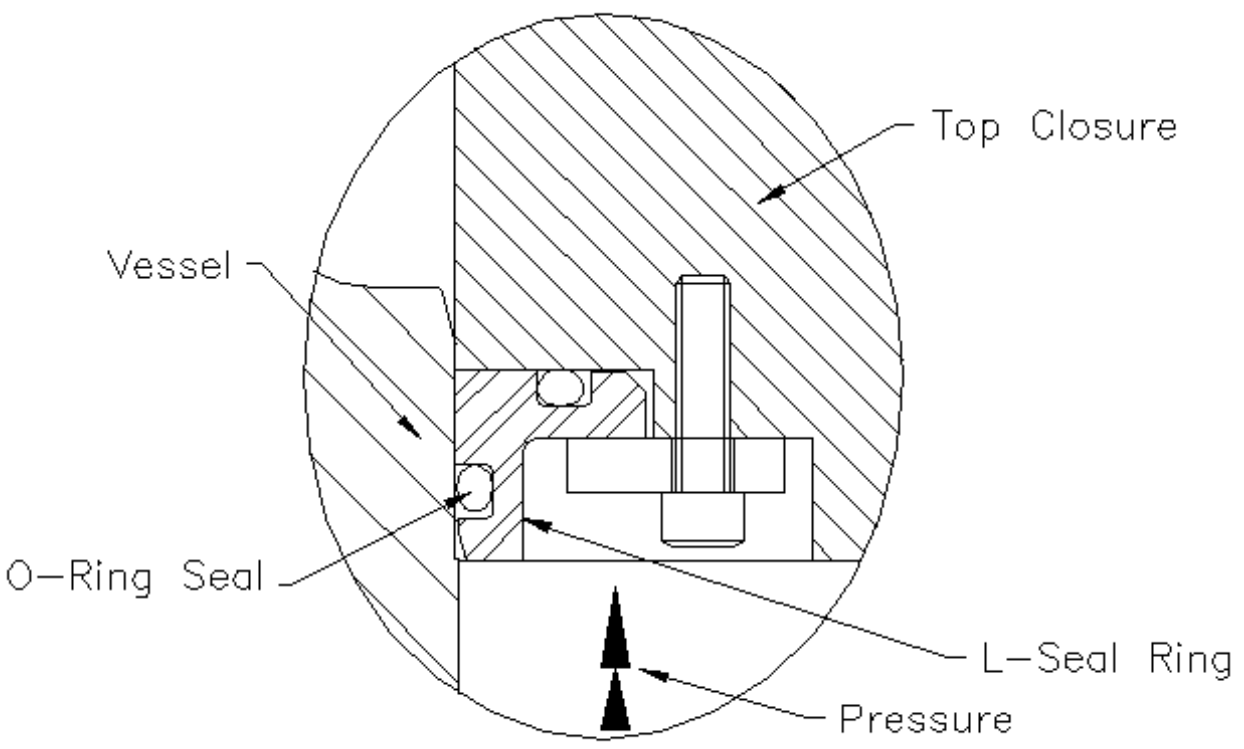

The bottom closure seal plate assembly contains the electrical power and thermocouple circuit feed throughs necessary for the HIP furnace assembly. Automatic plug-in components connect the furnace with the feed throughs when the furnace is installed in the vessel.

On the external side of the bottom seal plate, power cables and thermocouple wiring are permanently connected to the individual feed throughs as required. The bottom seal plate is cooled in the region of the " $\mathrm{O}$ " ring seals. 


\section{EPS \\ Engineered \\ Pressure Systems, Inc.}

The bottom closure also has the penetrations required to evacuate and route argon into and out of the vessel. Gas flow penetrations are simply drill throughs with proper machining on the external face of the closure for attachment of highpressure piping.

Vessel evacuation is accomplished through a large orifice valve that is fastened to the lower face of the closure.

\section{Vacuum Valve Assembly}

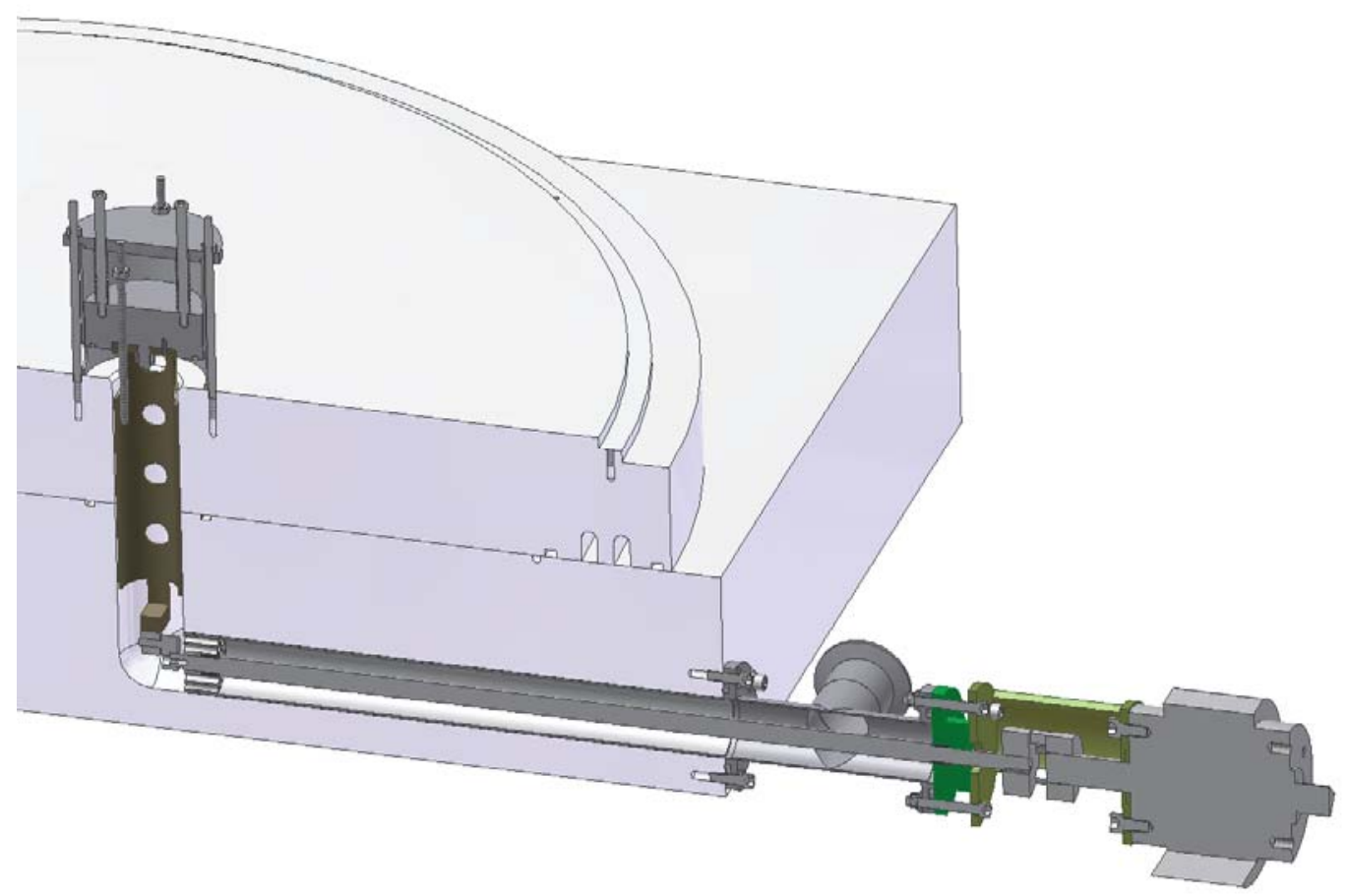

The top closure will be similar to the bottom closure without feed throughs. The sealing arrangement for the top closure is identical to the bottom closure. For heat transfer considerations, the top seal is cooled by a grooved plate, which is bolted to the seal plate. This assembly is suspended from a lifting device allowing the seal plate assembly to be put in \& out of the vessel thus minimizing the potential of damaging the sealing surface. The cooling water lines for the top closure are connected through the top closure hoist. 


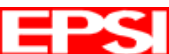 \\ Engineered \\ Pressure Systems, Inc. \\ 2.4 Top and Bottom Seal Plate Cooling and Vessel Body Cooling Components}

The top seal plate assembly will consist of two steel plates, which are bolted together. One half of the seal plate assembly will be supplied with concentric machined grooves, which are interconnected. This labyrinth of passages will be used to pass cooling water to the seal area where the top closure mates to the vessel body.

The bottom seal plate assembly will be supplied with cooling rings with similar concentric passages. These cooling rings will be spaced such that the seal area where the bottom seal ring mates with the vessel body will be water-cooled. Material of fabrication for the bottom seal plate and rings will be low alloy steel.

The vessel body will be cooled dry jackets. The dry jackets will be water cooled on the OD by means of multiple concentric stainless steel cooling panels, strapped one above the other along the length of the vessel.

In order to obtain proper heat transfer properties between the inside vessel wall and the vessel OD, we will use a special mastic between the vessel steel and the cooling coils.

These components are fabricated from stainless steel to eliminate the potential of corrosion of the vessel components. The cooling ring manufacturing process is held to very close tolerances so that proper heat transfer is accomplished.

\subsection{Pressure Vessel Support Stand}

The vessel assembly will be supported on a steel stand. This stand will be designed from steel beams and will support the weight of the vessel \& yoke assembly, furnace and heat shield assembly, as well as the workload and argon.

The height of the pressure vessel assembly stand can vary depending on the type of installation and location of the system.

\subsection{User Design Specification}

ASME Section VIII Div 3 vessels require a certified Users Design Specification. EPSI has included the cost to certify a mutually agreed Users Design Specification. 


\section{Engineered}

\section{Pressure Systems, Inc.}

\subsection{Dual Safety Rupture Disc Assembly}

To safeguard the HP Pumping System and the Pressure Vessel Assembly against overpressure at all times, we will provide Rupture Discs at appropriate locations in the associated piping. This will include Rupture Discs in the high-pressure inlet lines immediately adjacent to the Pressure Vessel.

Rupture Discs will be located in the system piping so as to facilitate inspection and maintenance. From a rating standpoint, the Rupture Disc on the inlet line of the Vessel will be $1 / 4$ Range Discs stamped at between 16,000 and 16,500 Psi since it is relatively isolated from pressure fluctuations caused by the High Pressure.

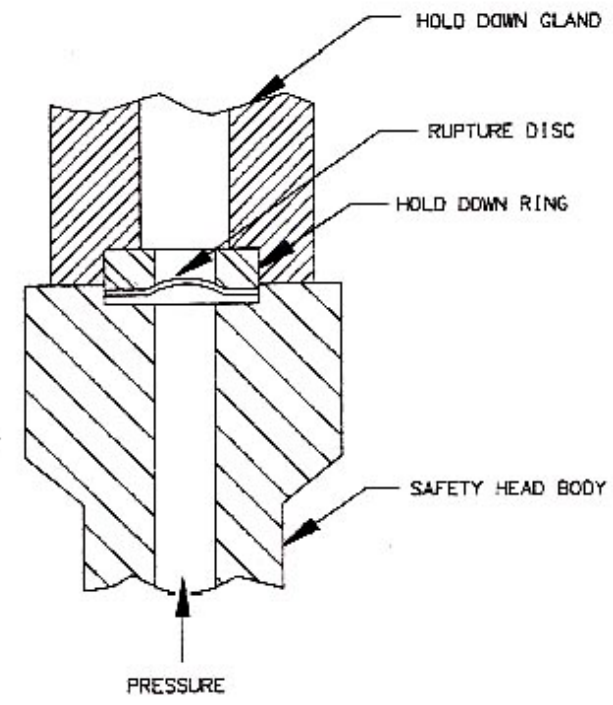

In addition as a result of the ASME rules related to rupture discs (relief device).

- No isolation valve is allowed in the relief valve line.

- Rupture discs will eventually fatigue and burst after repeated use at pressures below the $\pm 5 \%$ from published burst ranges.

EPSI has developed a special dual rupture disc assembly. The assembly includes two rupture discs arranged in one relief line. The first disc is always subjected to internal vessel pressure. The second is never subjected to internal vessel pressure. If the first disc breaks as a result of fatigue a small leak between the first and second disc indicates the occurrence. The leak can be isolated with a shut off valve. If the second disc burst it would only burst from an actual over pressure condition.

If there is no actual over pressure condition the process cycle can be completed. Both the discs (isolated and unisolated) can be replaced allowing continuous operation without loss of a process cycle. 


\section{EPS \\ Engineered \\ Pressure Systems, Inc. \\ 3 Top Closure Hoist Assembly}

\subsection{Top Closure Hoist}

EPSI will provide assembly drawings and installation specification for mounting and locating vessel and yoke supports.

The Top Closure is removed from the vessel body by employing hydraulically operated cylinder. A hydraulic cylinder is utilized for vertical lifting and lowering the top closure. The top closure can only be removed from the vessel when a limit switch is activated by an alignment pin confirmation the yoke assembly is in the open position.

The opening and closing of the vessel can be done at the local control console in an automatic mode by pressing and holding a single pushbutton. In the maintenance mode, the closure movements can be operated manually. The top closure and yoke movements can be stepped individually by appropriately trained personnel.

Limit switches are located on the yoke and top closure removal device to confirm location of top closure and position of yoke plates. These limit switches need to sense when the vessel and yoke are in the correct position before pressurization of the system can occur.

EPSI will provide limit switches on top and bottom of the vessel assembly. Each proximity switch will need to sense the proper positioning of the vessel relative to the yoke at all times. The bottom of the vessel will have the same proximity switch arrangement as the top.

A packaged hydraulic module provides hydraulic power for the closure and yoke movements. This module, connects to the yoke and closure hydraulic cylinders using fixed tubing, consists of the following:

- 1200 to 1500 psi gear pump with $480 \mathrm{~V}, 3$ phase, $60 \mathrm{~Hz}$ motor

- Reservoir with filters, sight glass, suction strainer, air vent and other standard components

- Solenoid directional valves plus adjustable flow control valves 


\subsection{Load Insertion Tooling}

\section{EPS \\ Engineered \\ Pressure Systems, Inc.}

The load tray shelves attach to the bottom support plate and will be secured in a load insertion tooling system. This system will support these components from the bottom base plate and will index from the vessel wall to assure axial alignment.

Rotational orientation will be obtained from a register bar on the insertion system. In addition, this system will ensure sufficient clearance of the load to the furnace for subsequent furnace/mantle installation.

A similar arrangement will be utilized for the furnace and mantle insertion, assuring axial rotational alignment with to the vessel.

\section{Furnace \& Heat Shield Assembly}

\section{$4 \quad$ EPSI Furnace and Heat Shield Assembly}

\subsection{Furnace and Heat Shield Assembly}

We will supply a moly, 3 zone Furnace Assembly for your HIP system. The Furnace Assembly will consist of a heatshield, main heating module and base module. Furnace specifications are listed in Table 1.

The furnace heat shield which will be a closed top design that controls the convective, radiative, and conductive heat transfer from the furnace hot-zone to the top closure and vessel bore. Consequently, the heatshield contributes to the control of the temperature uniformity within the hot-zone. The heat shield is designed to maintain the furnace at maximum temperature within a specified range of total power input to the heating zones.

This power input is typically referred to as the steady-state power, which varies as a function of furnace temperature and vessel pressure. The steady-state power at maximum rated operating conditions is always less than the total installed power available for furnace heating. This allows the correct furnace heating rates during the HIP cycle regardless of furnace temperature or vessel pressure.

Each heating element is rated for a maximum power output of $75 \mathrm{~kW} .3$ elements in the furnace control work-zone control temperature uniformity, and if one heating element becomes inoperative under certain circumstances the other heating element can make up the power loss and avoid aborting the cycle. The furnace design affords this redundancy of operation. The heating elements are fabricated from moly. 


\section{Engineered}

\section{Pressure Systems, Inc.}

The furnace elements are protected by individual ground fault detection units. These disconnect the power from the faulty element before significant otherwise undetectable damage to the furnace internals can occur.

The temperature of each heating zone is monitored by two Type "S" thermocouples. Heating zone power input can be controlled using either of the two thermocouples since both are contained in relatively the same location within the zone. This design provides the redundancy required to continue a cycle even if one thermocouple fails. A furnace stand is included which supports the furnace when it is removed from the vessel. The stand can be used for furnace storage or furnace maintenance.

\section{Table 1}

\section{Moly Furnace Assembly Specifications}

Furnace Inside Diameter $11 "$

Nominal Workload Weight 1,000

Maximum Workload OD $10 "$

Maximum Workload Inside Height $60 "$

Work Tooling approximate weight $600 \mathrm{lbs}$ (customer supplied)

Rated Operating Temperature $2300^{\circ} \mathrm{F}$

Dwell temperature tolerance: $\pm 20^{\circ} \mathrm{F}$

Number Heating Elements 6

Number Thermal Zones 3

Power Available Each Element $75 \mathrm{~kW}$

Steady-State Power $100 \mathrm{~kW} @ 2300^{\circ} \mathrm{F}$ and 15,000 psi argon

Normal Heating Time $100^{\circ} \mathrm{F}$ per hour $\left(15^{\circ} \mathrm{F} / \mathrm{min}\right)$

Normal Cooling Time 4 to 5 hours

Rapid Cool Estimated less than $3 \mathrm{hrs}$ cooling time from $2300^{\circ}$ to $700^{\circ}$ with $1,000 \mathrm{lbs}$ load with tooling

Furnace Control Thermocouples Type "S"; 2 per zone

Furnace T/C Temperature Uniformity $\pm 20^{\circ} \mathrm{F}$ 


\section{Engineered}

\section{Pressure Systems, Inc.}

4.2 Furnace Plug-In Assembly

\subsubsection{Furnace Shells}

- Moly furnace shell, Note: easily removed from the vessel.

\subsubsection{Heating Elements}

- Estimated one moly furnace elements for each zone will be provided complete with buss bars.

\subsubsection{Furnace Ceramics}

- EPSI will supply furnace ceramics.

- Furnace ceramics are not covered under any warranty.

\subsubsection{Furnace Thermocouples}

- EPSI will supply furnace T/C type "S" wire

- EPSI will not provide any thermocouple wire for loads. Idaho National Laboratory can purchase the $\mathrm{T} / \mathrm{C}$ directly from the suppliers.

- EPSI will provide one set of ceramics and moly T/C support and T/C brackets for " $\mathrm{K}$ " type load $\mathrm{T} / \mathrm{C}$.

4.2.5 Furnace Side Shield Insulation Package

- EPSI will provide a moly side shield with an insulation package.

\subsubsection{Furnace Plug-In Components}

- EPSI will provide knife blade plug-in connections.

- EPSI will provide thermocouples plug-in connection blocks for furnace and load $\mathrm{T} / \mathrm{C}$. 


\section{Engineered}

Pressure Systems, Inc.

\subsection{High Performance Clopen Top Mantle for Furnace Plug-In Design with Enhanced Cooling}

\subsubsection{Mantle High Performance Clopen Top Design}

EPSI will provide a moly-closed top mantle with a High Performance open top design valve in the top center of the heatshield assembly.

This valve disperses the hot gases directly onto the top center closure enhanced cooling plate. This is a maintenance free valve with no moving components. We also incorporate a valve in the bottom seal plate. See figure 5

With this design we eliminate the removable mantle top, and we add a second moly shell. EPSI will plasma spray the outside of moly mantle top. EPSI will provide outer inconnel and stainless steel shells for optimum performance.

Figure 5

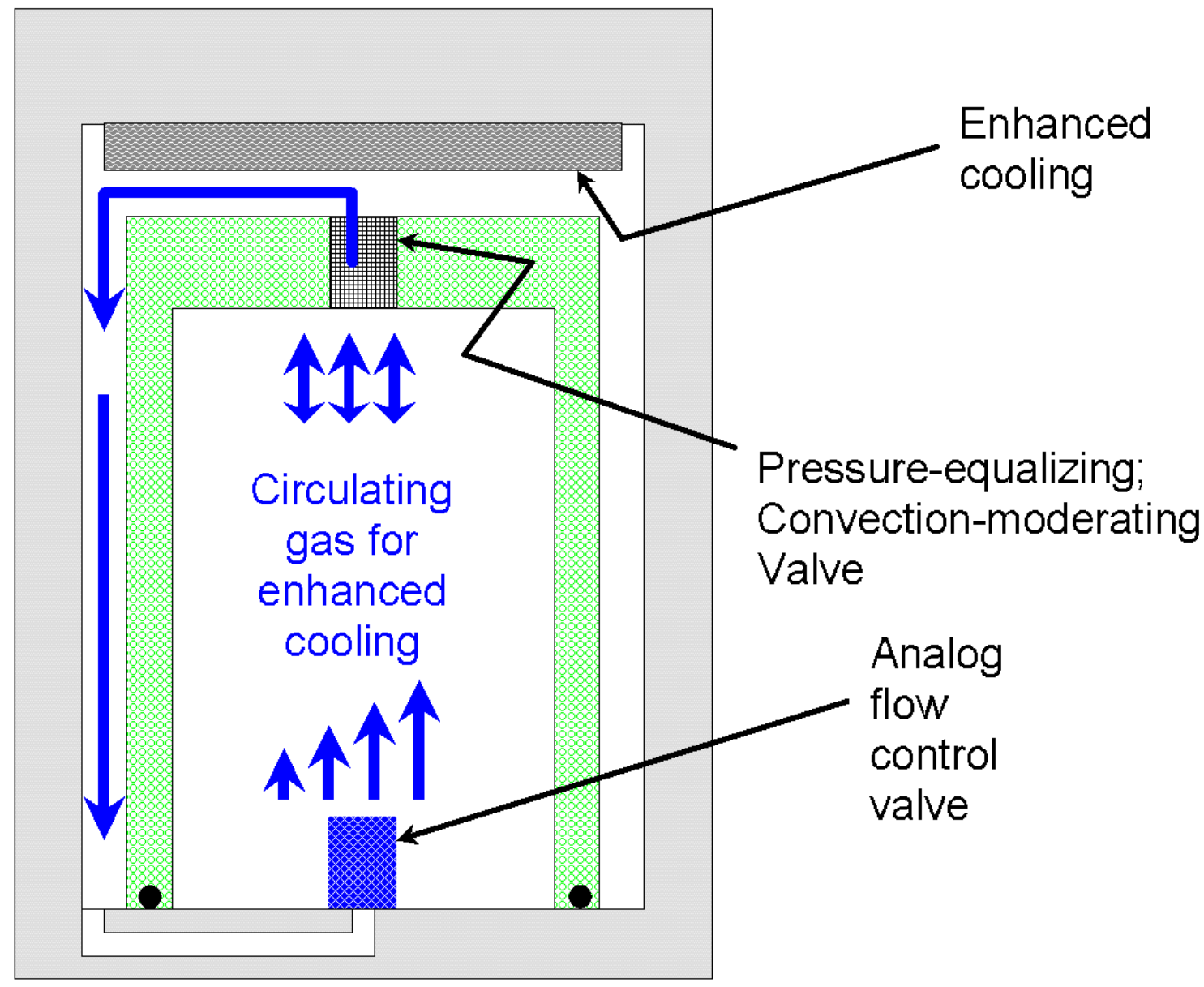




\subsubsection{Mantle Insulation Package}

\section{Pressure Systems, Inc.}

- EPSI will wrap Mantle shells with $2,600^{\circ} \mathrm{F}$ rated ceramic paper insulation.

\subsubsection{Furnace/Mantle Lifting Fixture}

- EPSI will provide a guided self-aligning furnace and mantle-lifting fixture.

- The furnace and mantle lifting fixture will be attached by the operator to the top of the mantle and lowered into the vessel repeated for the furnace. We will provide a guide pin for proper alignment on the top of the vessel.

\subsubsection{Gas Purity}

Pressurization medium: argon, purity requested by EPSI: Total impurities $<350$ ppm.

Maximum impurities for the furnace:

$\begin{array}{ll}\mathrm{O}_{2} & <=25 \mathrm{ppm} \\ \mathrm{H}_{2} \mathrm{O} & <=30 \mathrm{ppm} \\ \mathrm{N}_{2} & <=150 \mathrm{ppm} \\ \mathrm{H}_{2} & <=200 \mathrm{ppm} \\ \mathrm{C}_{\mathrm{n}} \mathrm{H}_{\mathrm{m}} & <=50 \mathrm{ppm} \\ \mathrm{CO} & <=100 \mathrm{ppm} \\ \mathrm{CO}_{2} & <=25 \mathrm{ppm}\end{array}$

\subsection{Plug-In Bottom Zone Heater}

\subsubsection{Bottom Zone Heater (BZH)}

- EPSI will provide a BZH that will plug into the seal plate connecting the load thermocouples, BZH thermocouples and knife blade power connections.

- The BZH will be designed to hold up to a 2,000 pound load.

- Design temperature: $2300^{\circ}$ Fahrenheit

- Estimated work zone void length 60 in. 


\subsubsection{Load Thermocouples}

\section{를 \\ Engineered \\ Pressure Systems, Inc.}

- EPSI will not provide load " $K$ " type thermocouple wire. We will supply the feedthroughs and plug-ins.

- EPSI will supply one " $K$ " type thermocouple feedthru (5 T/Cs)

- EPSI will provide one thermocouple plug-in racks to support 5 " $\mathrm{K}$ " type load thermocouples.

EPSI will provide 5 sets of " $K$ " type thermocouple wire for trials. EPSI will put supplied thermocouple wire into $\mathrm{T} / \mathrm{C}$ assemblies corresponding with each zone for acceptance cycles. Load thermocouple location will be proposed by EPSI for Idaho National Laboratory's approval.

\subsubsection{BZH (Bottom Zone Heaters) Ceramics}

- EPSI will supply standard ceramics. These ceramics performed excellent under normal hip cycles.

- BZH ceramics are not covered under any warranty

\subsection{BZH/Tooling Lifting Fixture}

\subsubsection{BZH/Tooling Lifting Fixture}

- EPSI will supply tooling that aligns on the vessel bore that will lift the BZH and the tooling. This fixture will center the tooling in the vessel and plug the BZH into the seal plate.

- The BZH/Tooling lifting fixture is designed to lift a maximum load of 1,000 pound load.

- The BZH load-lifting fixture is designed to be used when the load has cooled below $700^{\circ}$

\subsubsection{BZH Tooling Support Plate}

- EPSI will provide a BZH tooling support plate. This plate will be used to place the BZH on the floor to emulate the seal plate. The BZH will be placed on this plate with a specific orientation. The BZH/Tooling lifting fixture will be lowered over the load and tooling. The operator will have to lineup arrows, orientate the $\mathrm{BZH} /$ Tooling with the $\mathrm{BZH} /$ Tooling lifting fixture in the correct orientation. The BZH/load lifting fixture will then be turned into a locked position allowing load-lifting fixture to lift to the $\mathrm{BZH} /$ Tooling.

- This will allow for proper alignment when the BZH/Tooling is plug into the seal plate. 


\section{EPS \\ Engineered \\ Pressure Systems, Inc.}

4.5.3 Optional BZH Support Plate T/C Testing Junction Box

- EPSI will provide thermocouple connection plug-in blocks located on BZH/Tooling support plate, with thermocouple extension wire connecting to a display box mounted on a nearby wall or post. The display will provide a continuity check.

- The main purpose for this junction box and additional T/C plug-ins are used for when the operators are making up the loads. They will be able to bring their T/C testing meter over to the Junction box and check load T/C's before the BZH/Tooling is lowered into the vessel. See Concept Figure 6

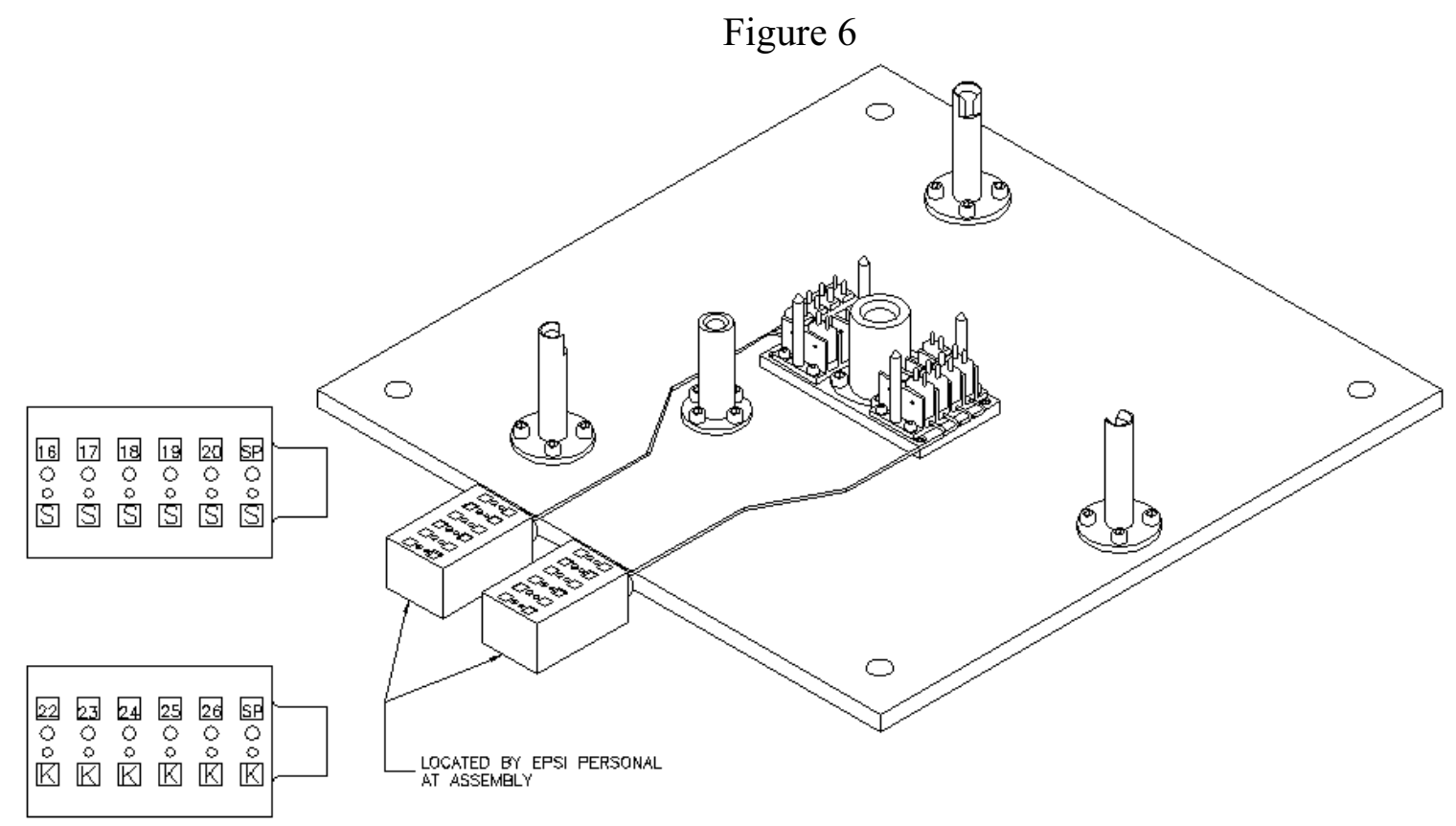

\subsection{Seal Plate}

\subsubsection{EPSI Supplied Bottom Seal Plate}

EPSI will supply a seal plate so that there is minimal drill through cooling passages.

\subsubsection{Plug-In Design Seal Plate with Components}

EPSI will supply a complete seal plate with the following components.

- T/C feedthroughs (estimated 3 five channel furnace "S" Type, 1 five channel Loads, "K" Type) 
Engineered

Pressure Systems, Inc.

- Power feedthroughs

- Furnace and BZH power plug-ins

- All necessary guide pins

- Thermocouple connection blocks for BZH and furnace

- Cooling line connection pipes

- Seal plate-lifting fixture will be provided.

- EPSI will provide 2 separate BZH plug-in racks "S" type for furnace control and " $\mathrm{K}$ " type for loads.

\subsection{Electrical Power Distribution System}

Idaho National Laboratory will be required to provide the electrical power required to operate the overall HIP system to an EPSI supplied disconnect. From there, we will supply the equipment necessary to convert this electrical power to the various levels and phases required for all of the HIP system components that we are supplying.

This will encompass the use of standard switches, transformers, relays, SCR power controllers with GFI protection and motor starters. The furnace power will be wired for 480 volt, 3 phase, $60 \mathrm{~Hz}$. All wiring will be in accordance with the National Electrical Code and state and local ordinances. Note: the motor control center will use IDAHO NATIONAL LABORATORY Electrical.

\section{Isolation Transformer}

EPSI believes the best way to protect the furnace from unforeseen short circuits is to provide ground fault protection. The ground fault protection is not the same as used in residential wiring for personnel protection, but is intended to protect the furnace. High resistance shorts can damage expensive internal furnace or vessel components. Providing ground fault protection provides an additional safety margin against otherwise undetectable short circuits.

It is necessary to have an isolated neutral to complete the ground fault circuit. Therefore, an isolation transformer is required to take conventional 3 phase power and provide a grounded neutral. It is necessary for EPSI to include the transformer in the scope of supply so that we can test the system with ground fault protection in our facility. The transformer will use 480 volt 3 phase inputs and will be procured that has the correct voltage to match the design voltage requested by Idaho National Laboratory. 


\section{EPS \\ Engineered \\ Pressure Systems, Inc.}

If Idaho National Laboratory wished to purchase the unit we would match the transformer to the requested 3 phase power and have 480 volt 3 phase 4 wire output to the EPSI furnace. The cost of the transformer is approximately $\$ 15,000$

\section{$5 \quad$ Cooling System \& Vacuum System}

\section{$5.1 \quad$ Cooling}

The water-cooling system is designed to cool the vessel assembly, compressor and power supply, if required. The system will consist of a circulation pump, heat exchanger, water reservoir and appropriate flow switches for monitoring and alarming. EPSI will be supplying a cooling tower.

The HIP system components requiring cooling water are:

- Pressure Vessel Body OD (by means of stainless steel cooling coils banded on the vessel OD)

- Pressure Vessel Top Seal Plate

- Pressure Vessel Bottom Seal Plate

- High Pressure Compressors TBD

- Vacuum Pump

Coolant on the secondary side is pumped from the reservoir tank to the components listed above. Sensors on the outlet side of each component and in the reservoir ensure that the coolant temperatures do not exceed safe system operating limits. Flow meters and switches also monitor the system to make sure coolant flow is continuous.

Coolant returning from the HIP system components to the reservoir is first circulated through a heat exchanger to decrease its temperature.

\subsection{Vacuum System}

Before each HIP cycle, most of the time it is necessary to evacuate the vessel. To meet this requirement, we will provide a complete vacuum system. The vacuum system will consist of a mechanical pump, a bottom closure vacuum valve, vacuum pressure gauge, interconnecting valves and piping, and a pressure relief device vacuum pump isolation valve. All these items shall be located in the pit or in proximity to the vessel. The discharge of the vacuum system will be to atmosphere. 


\section{Engineered}

Pressure Systems, Inc.

Vacuum system capabilities are 100 Microns in 30 minutes

Note: Vacuum performance is affected by moisture and humidity. The performance estimated is based on successive production cycles where the system has not be idle or the furnace / heat shield has not been left out of the vessel for more than 8 hours. When starting the system after periods of down time the system will reach 100 microns but it will take a longer time period to reach.

\subsection{Included Liquid Pump System}

As a point of interest, the argon system we are proposing here is similar to the systems used by other HIP customers. This type of argon delivery system saves the cost of bottles and provides no means of reclaim and is the cleanest gas source.

1. $1.5 \mathrm{gpm}$ at $15,000 \mathrm{PSI}$ (103.4 MPa) Liquid Argon Pump.

2. Pump is model 3-GMPD, 1.0" bore x 0.905 " stroke, high pressure reciprocating pump with purgeable intermediate and standard suction manifold.

3. $25 \mathrm{HP}(37 \mathrm{~kW}), 480 \mathrm{~V}, 3 \mathrm{ph}, 60 \mathrm{~Hz}, \mathrm{TEFC}$, inverter duty motor complete with belt drive and steel baseplate. Skid will be complete with forced oil system including oil pump and reservoir, lube piping, \& automatic low pressure cut off switch, along with surge chamber, high pressure relief valve and combination pressure switch gauge. A boost pump to insure positive NPSH to inlet of 3-Head pump will also be included on skid, along with control panel.

4. Natural draft ambient vaporizer rated at $1,000 \mathrm{scfh}\left(606 \mathrm{Nm}^{3}\right)(1.5 \mathrm{GPM})$ for 8 hours in LA2 service, Aluminum finned construction with stainless steel wetted liner for high pressure cryogenic service. Vaporizer will be mounted out side near pump skid which will also be mounted out side.

5. Nomenclature

$$
\begin{aligned}
& \text { TEFC - Totally Enclosed Fan Cooled } \\
& \text { NPSH - Net Positive Suction Head } \\
& \text { SCFH - Standard Cubic Feet per Hour } \\
& \text { NM }^{3} \text { - Normal Cubic Meters } \\
& \text { SCF - Standard Cubic Feet } \\
& \text { CFM - Cubic Feet per Minute }
\end{aligned}
$$

The gas systems are used in operations when gas reclamation is desired. In a small HIP unit, this is not the case, which allows use of the simpler lower cost liquid system. 


\section{EPS \\ Engineered \\ Pressure Systems, Inc.}

The primary function of the process gas handling System is to route argon gas to the HIP Vessel. The system consists of a high pressure liquid argon pump with integral vaporizer, valves, fittings, piping relief devices and pressure transducers. Idaho National Laboratory will need to supply liquid argon storage. A minimum of $200 \mathrm{Nm}^{3}$ (7,000 SCF) of argon will be needed for each cycle. For HIP operation it is typical to vent or throw away all gas after each cycle.

Therefore, a reasonable estimate of consumption per cycle is 100 gallon of liquid argon. For HIP operation it is typical to vent or throw away all gas after each cycle.

The bulk liquid storage volume should be larger than 1500 gallons

\subsection{High Pressure Piping, Valves and Low Pressure Piping}

EPSI will provide all high-pressure piping and control valves. EPSI will supply all high-pressure connections and installation.

Gas flow control valves for both high and low pressure levels are of standard airto-open or air-to-close design. You can use a standard compressor to supply air (100 psi@100 CFM) to these valves. All high-pressure valves, piping, and fittings will be rated for 20,000 psi.

\section{$7 \quad$ Controls}

\subsection{PLC Controls}

- $\quad$ PLC for control and monitoring of all discrete and analog signals.

- An Ethernet network for communicating all discrete and analog signals with the PLC and PC.

\subsection{HMI (Operator Interface)}

- Windows based software on a personal computer for operator interface, report generation, alarm logging, data archiving

- The control system will contain the necessary hardware and software to connect to the internet. The customer has to agree to provide an internet connection and to assign a static IP address to the HIP PC.

- System can be setup for password protection. This would prohibit unauthorized personnel from operating the equipment.

- Typical screens would be as described below. Screens may vary per customer's application and software package selected. 


\section{Engineered}

Pressure Systems, Inc.

Overview - This screen is intended to give a quick overview of the process. Typical items displayed are analog and TC signals. The load thermocouples can be viewed from this screen. This is the default screen on power up. This screen will allow the operator to view the current segment status, process values, place on HOLD, Restart or Stop the HIP Unit. 
EPS

Engineered

Pressure Systems, Inc.

Over View

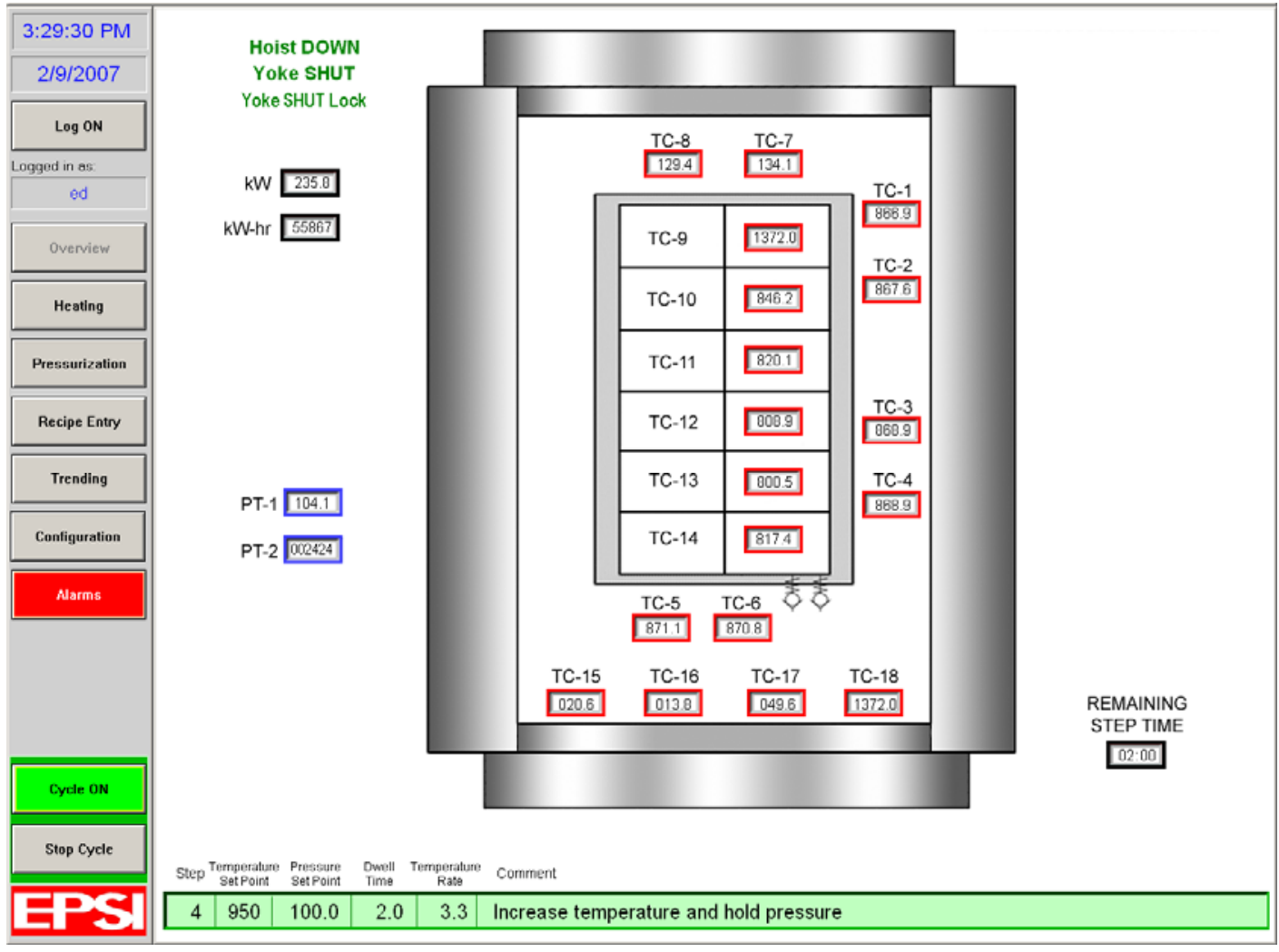

- Alarm - The screen will show all current and recent alarms from the HIP system. The operator will be able to select either current alarms or historical alarms for the selected source(s). 
EPS

Engineered

Pressure Systems, Inc.

Alarms

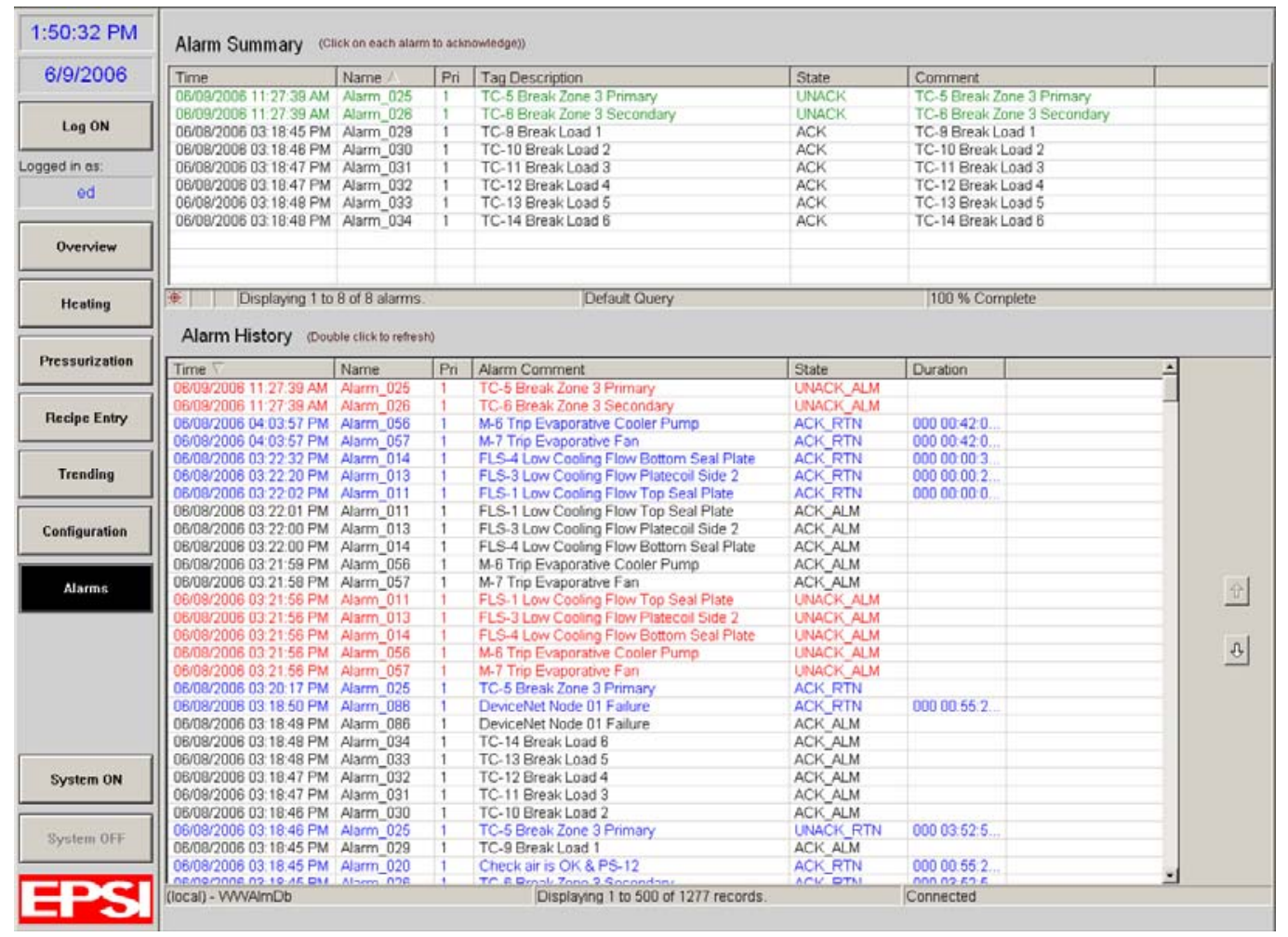

- Heating - This screen allows the operator to adjust tuning parameters and review the furnace operation.

- Active Ground Fault Status

- The screen indicates the current temperature and whether the thermocouple is enabled or disabled. 


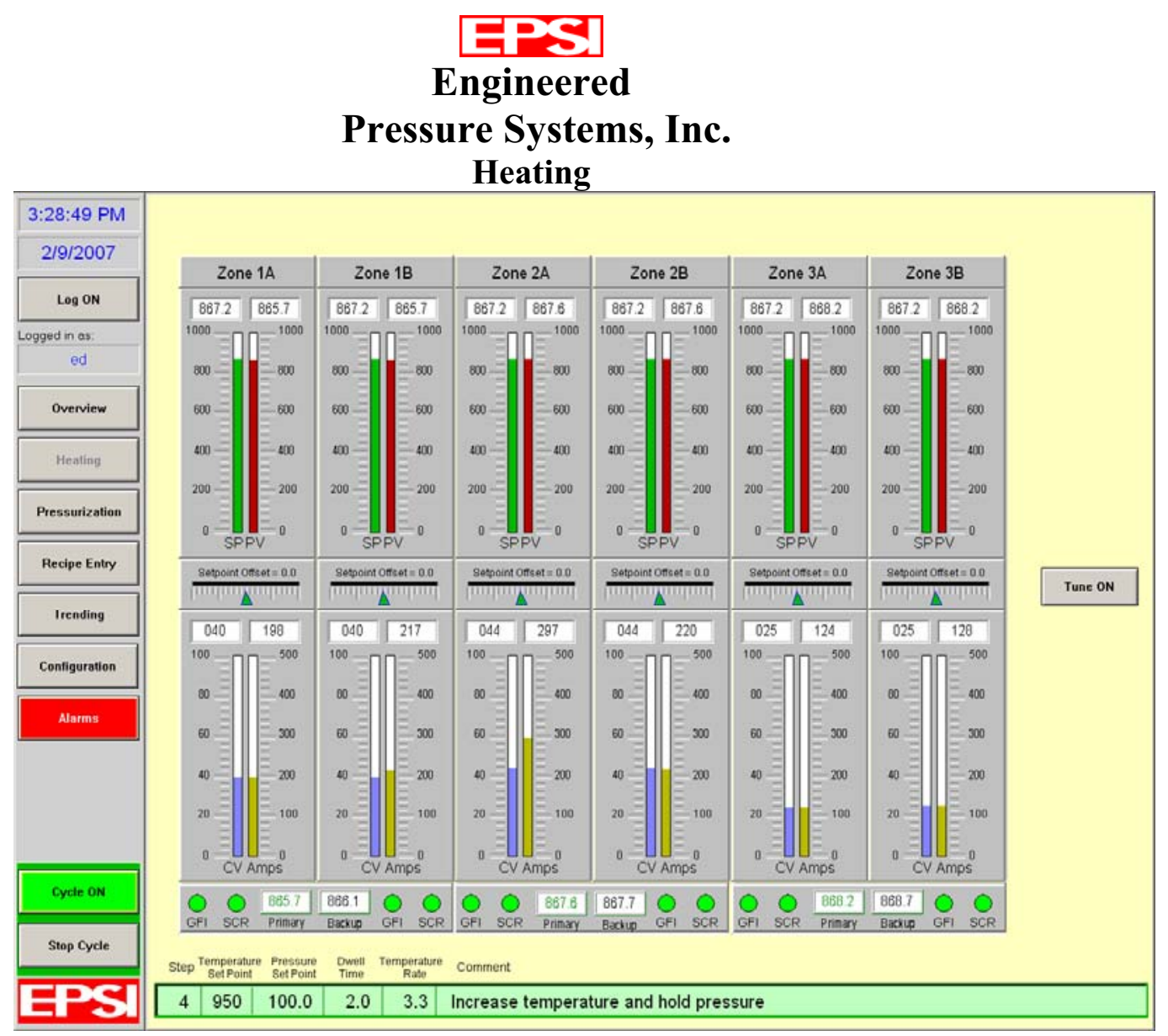

- Pressurization - This will be a P\&ID representation of the vessel's current status. This screen will be modified to reflect this unit P\&ID (screen shown is of a cryogenic liquid pressurization system). Some other key functions or displays are:

- Water Temperature

- Water Flow Indication

- Vessel Pressure

- Closure Status

- Start and stop Semi-Automatic cycles

- Manual Control of valves and pumps with safety interlocks

- Provide ability for the operator to restart (actually continue) the cycle at the beginning of a specified segment 
EPS

\section{Engineered}

Pressure Systems, Inc.

Pressurization

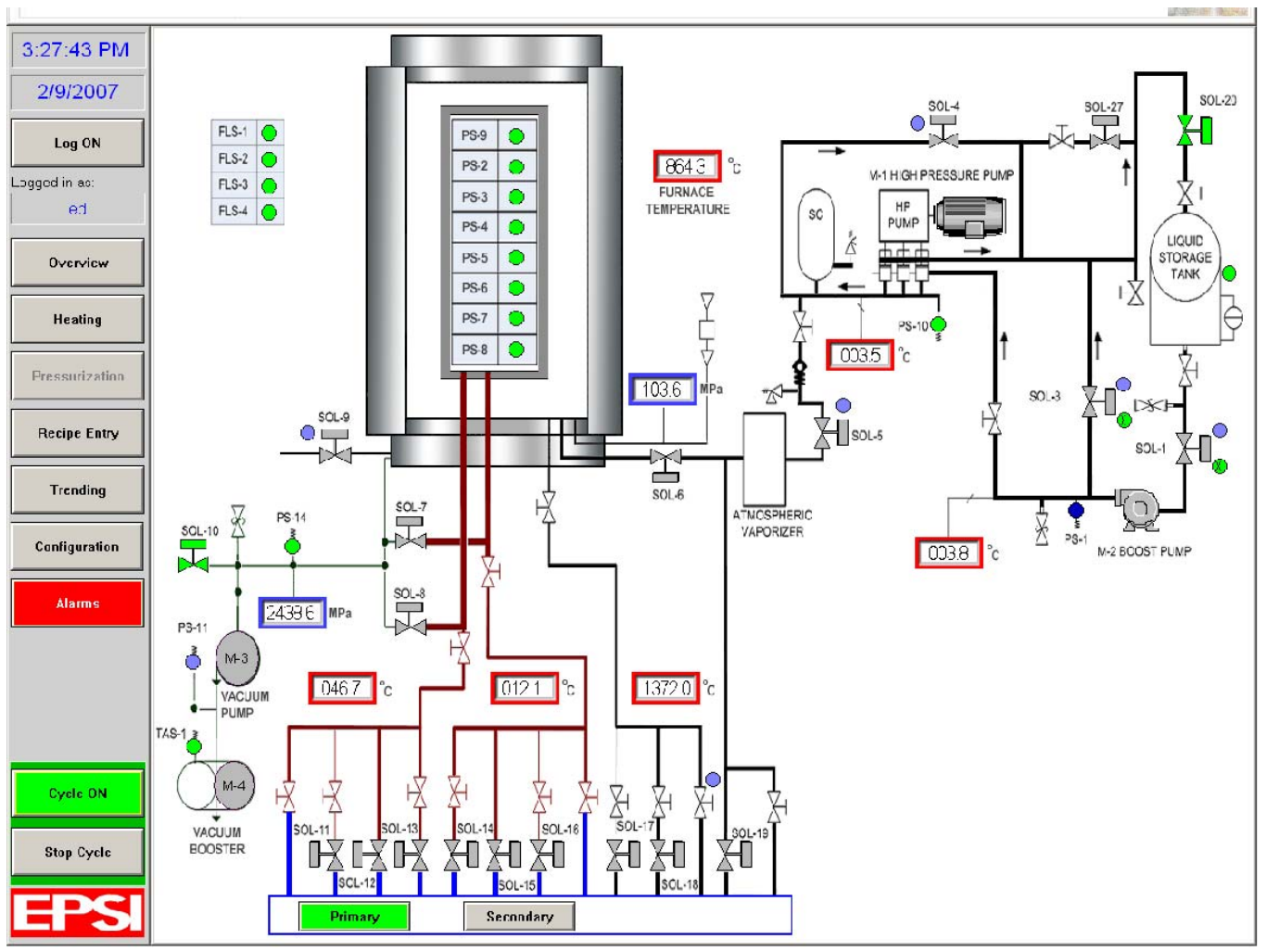

- Recipe Entry - This screen allows the operator to input the cycle parameters. The system has the capability that will allow the operator to change parameters even mid step of a cycle. 


\section{Engineered}

Pressure Systems, Inc.

Recipe Entry

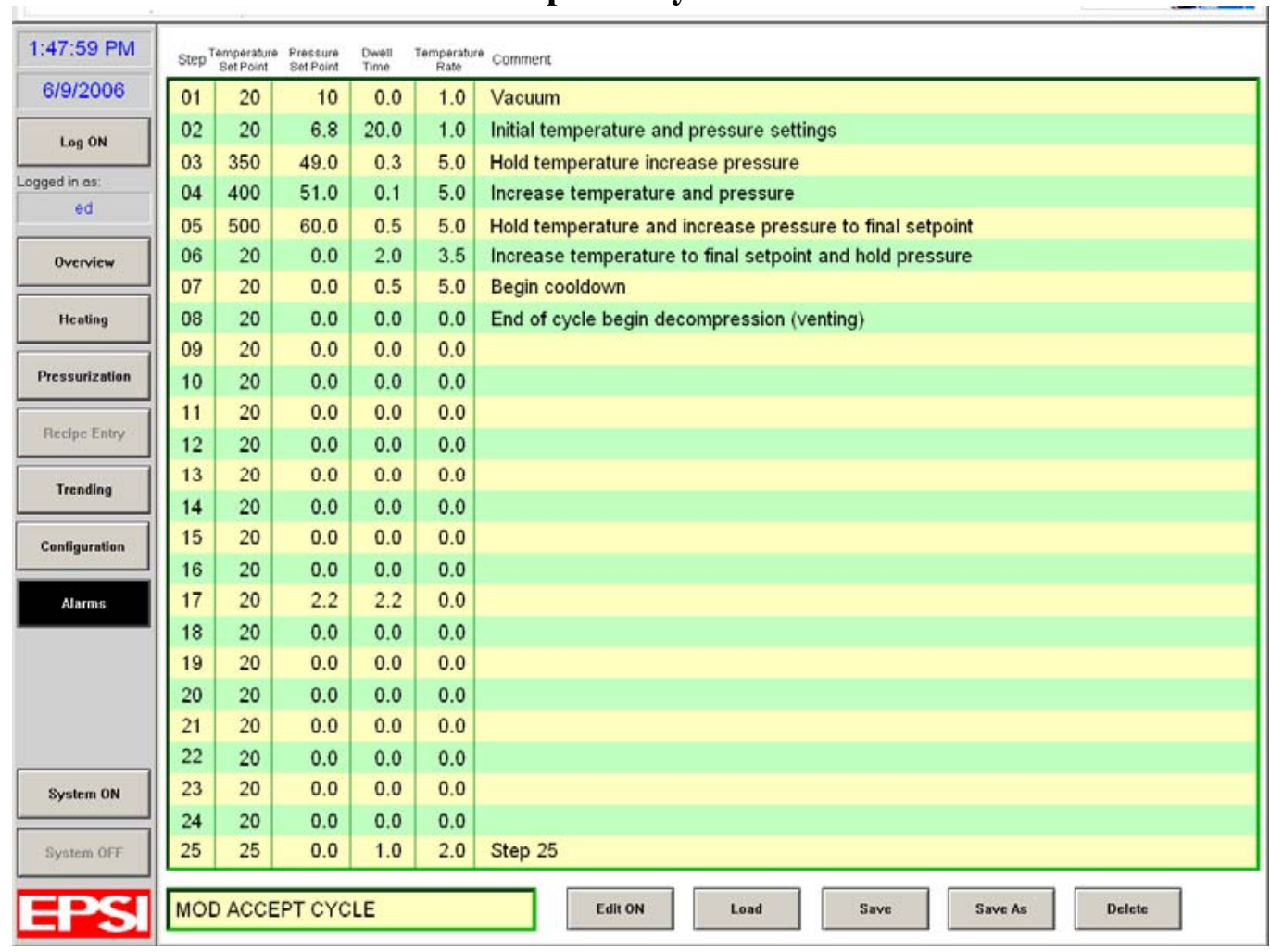

- Historical Trend - This screen allows viewing of locally logged process data in a trend screen format. The operator can choose up to 8 analog devices to be shown on the trend screen at one time. The system also has the ability to save a .csv file based on the current date. 


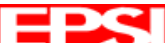

Engineered

Pressure Systems, Inc.

Trending

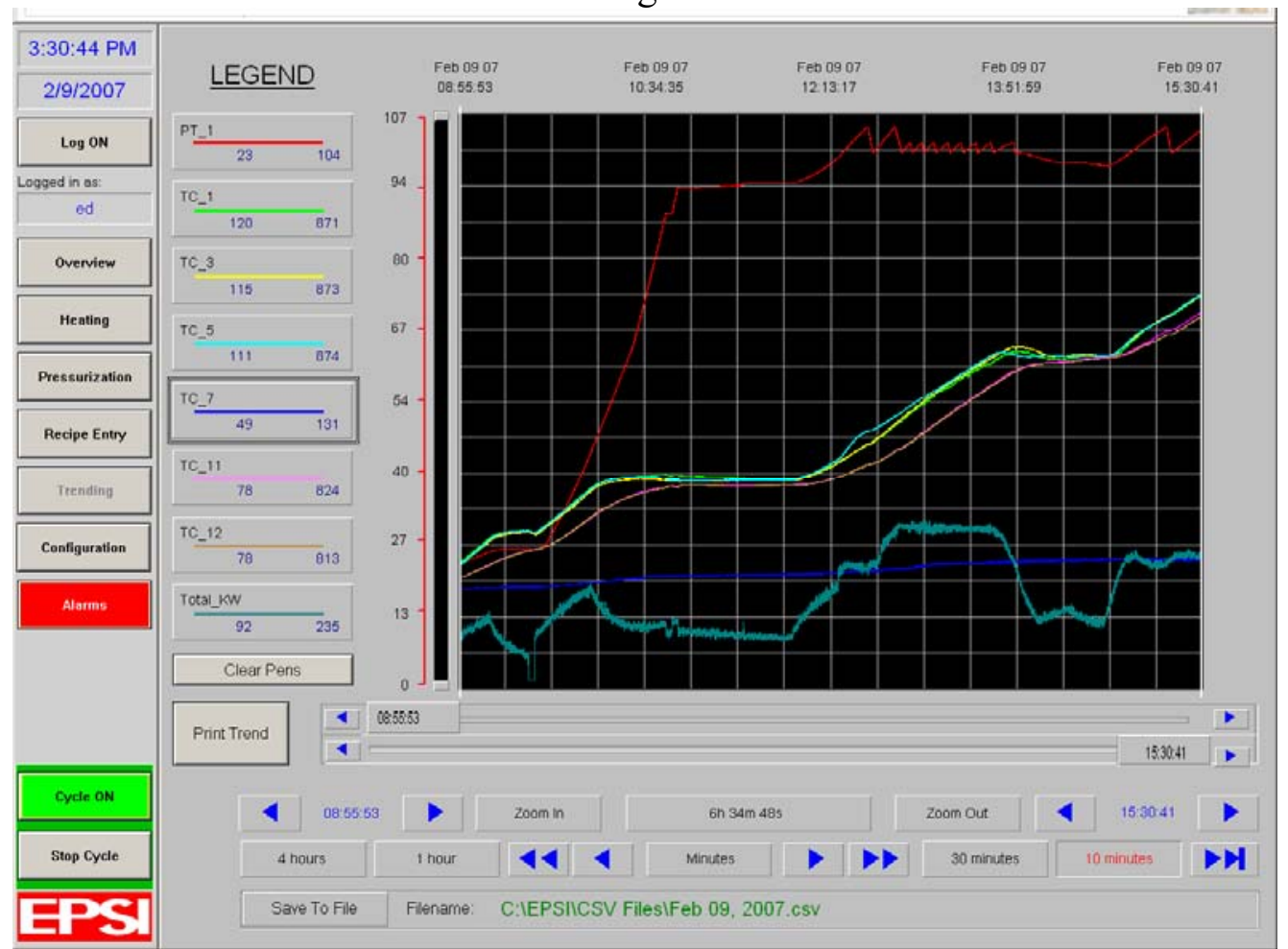

- Configuration Screen - This screen allows appropriate personnel to adjust process parameters that are not included in the recipe functions. These parameters are not expected to have to be changed each cycle. 


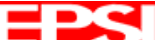

Engineered

Pressure Systems, Inc.

Configuration

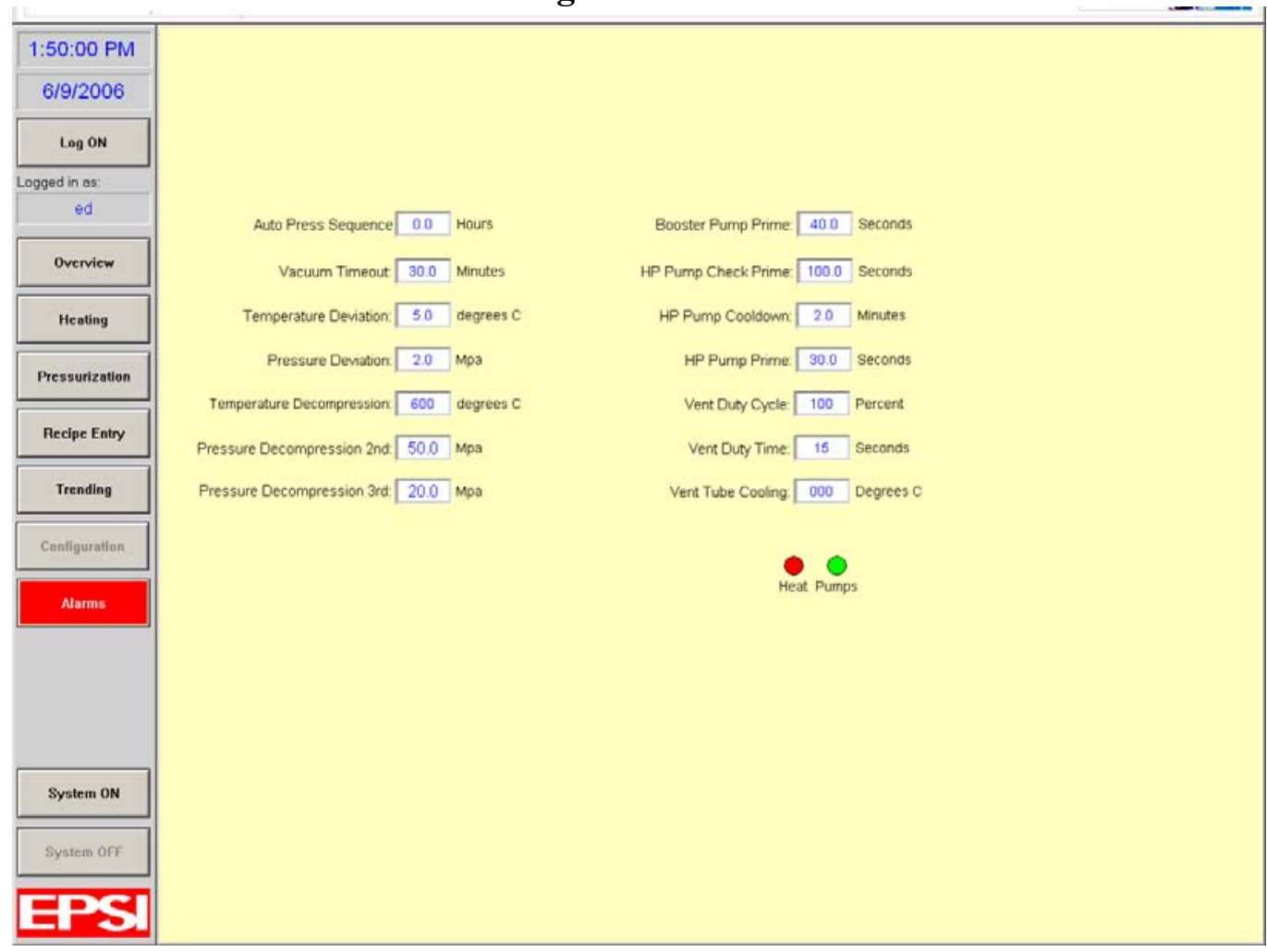

\subsection{The process steps will include:}

- Load and close vessel - Manual function

- Evacuate pressure vessel

- Pressurize with gas compressors

- Start furnace (Ramp/Soak)

- Stop furnace

- Vent

- Cycle complete

8 Installation

\subsection{Supervision of Installation}

EPSI will provide supervision of installation for vessel system, hoist alignment, controls and training. EPSI will be responsible for all high pressure piping.

Idaho National Laboratory will be responsible for all electrical interconnections, low pressure piping, installing vessel, controls and miscellaneous support equipment. 


\section{EPS \\ Engineered \\ Pressure Systems, Inc. \\ 8.1.1 Optional Turnkey Installation}

EPSI has provided budget turnkey installation price as an option. We are assuming the location will be in the Shanghai, China area. When the location has been disclosed and building details have been released we will need to reevaluate our proposed price.

Shipping: includes shipping all equipment to your facility in Shanghai, China

Rigging: EPSI to include offloading and transportation to your facility and for installation of all equipment and to be located and anchored.

Interconnections: EPSI to provide power and signal wiring between main power panels and the subassemblies including: furnace, compressor, vacuum pump, hoist hydraulic system. High pressure piping and low pressure piping is included.

\section{Cost of power wiring from the customer's substation to EPSI main power panels is excluded.}

Our intentions are to set up this HIP System in our facility for trials and the hydrotest per ASME specifications will be performed.

Idaho National Laboratory provides:

- Adequate access to the plant site.

- Reinforcement on floors, overhead protection from the elements and otherwise, and such modifications in user's building or premises as are necessary for proper installation of the apparatus covered by this proposal.

- Air, and water piping not furnished as an integral part of the apparatus.

- Electrical power, compressed air, compressor, cooling water and other utilities.

- Lifting equipment (Crane with Cane operator) with appropriate capacity to lift the vessel and yokes from a flat bed truck into position in Idaho National Laboratory's building

\subsection{Acceptance Cycles}

- EPSI will confirm set up of HIP system and perform operational test cycles with test load or if supplied customers product.

- These tests will be functional tests based on this proposal and Idaho National Laboratory specification not product quality tests. 


\section{EPS \\ Engineered \\ Pressure Systems, Inc.}

\subsection{Training}

Five days of hands on operational and maintenance training will be supplied by EPSI at EPSI Haverhill, MA. We suggest that Idaho National Laboratory personnel be available during the installation to receive maintenance training during that time.

- Our technicians will provide training at Idaho National Laboratory.

- We will provide 2 days of training after functional tests are completed.

- Training will contain controls, operation of hoist and pressurization system.

- Coordination of training will be Idaho National Laboratory responsibility and time will be mutually agreed with EPSI. 


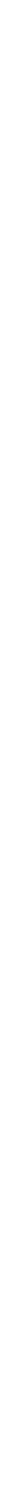




\section{$9 \quad$ Miscellaneous}

\section{EPS \\ Engineered \\ Pressure Systems, Inc.}

\subsection{Documentation}

EPSI will supply the following updated and current documentation:

- Overall dimensional drawings showing equipment location and interconnecting piping

- All component assembly drawings load support trays, furnace, load support, vessel, yoke, top and bottom closures, $\mathrm{t} / \mathrm{c}$ and power feed throughs, hoist, load assembly tooling, furnace assembly tooling. Schematics: argon gas system, source code: PLC and PC on CD.

- Hoist control schematic

- Vacuum piping schematic

- Electrical schematics

- Control ladder logic

- Control layout drawings

- Complete manuals for operation and maintenance.

Excluded are:

- Detail drawings

- Foundation engineering

- Building modifications

\section{$9.2 \quad$ Utilities}

The utility requirements for your HIP are:

Electrical: $\quad$ 480VAC, 3 phase, 400 -amp service.

Air: $\quad 100$ psi minimum, nominal volume

Water: To fill the reservoir and cooling exchangers

\subsection{References}

\section{DuPont Engineering Polymers}

Equipment

20" ID x 60" Void Length, 20,000 PSI HIP (hot isostatic press)

20 " ID x 60" Void Length, 20,000 PSI HIP

Yr Supplied

20" ID x 60" Void Length, 20,000 PSI HIP

1967

24" ID x 60" Void Length, 20,000 PSI HIP

24" ID x 60" Void Length, 20,000 PSI HIP

24" ID x 96" Void Length, 15,000 PSI CIP

24" ID x 60" Void Length, 20,000 PSI HIP 


\section{Engineered}

Pressure Systems, Inc.

\section{Los Alamos National Laboratory}

Equipment

Yr Supplied

2003

1985

12 ” ID x 36 Void Length $70^{\circ} \mathrm{F} 30,000$ psi CIP/Drybag

BWXT Pantex (Mason \& Hanger-Silas Mason Company, Inc.)

Equipment

20" ID x 30" VL, 20,000 PSI WIP

19.5 ID x 30" VL, 20,000 PSI WIP

25 " ID x 35" VL, 30,000 PSI WIP

\section{Schlumberger Well Services}

Equipment

Yr Supplied

5" ID x 36" Void Length, 650F 30,000 PSI Deep Well Simulator 1973

8" ID x 360" Void Length, 650 F 35,000 PSI Deep Well Simulator 1974

7.5" ID x 30" Void Length, 650F 35,000 PSI Deep Well Simulator 1977

8" ID x 16" Void Length, $650^{\circ} \mathrm{F} 35,000$ PSI Deep Well Simulator 1981

14" ID x 388" Void Length, 650F 35,000 PSI Deep Well Simulator 2003

14" ID x 388" Void Length, 650 F 35,000 PSI Deep Well Simulator 2005

14” ID x 342” Void Length, 450F 20,000 PSI Deep Well Simulator 2006

\section{Bodycote IMT Andover}

Equipment (Please Note all HIP Systems have Updated Plug-in Furnaces since 1998)

16" ID x 48" Void Length, 15,000 PSI HIP Yr Supplied

16 " ID x 88" Void Length, 15,000 PSI HIP 1968

16 " ID x 48" Void Length, 15,000 PSI HIP

1972

1974

48” ID x 132" Void Length, 15,000 PSI HIP/650C. HIPIC 1979

25" ID x 100" Void Length, 45,000 PSI HIP

1982

48" ID x 132" Void Length, 15,000 PSI HIP

1985

10.5 " ID x 60" Void Length, 60,000 PSI CIP

1998

\section{Alliant Tech Systems (ATK)}

Equipment

12" ID x 36"Void Length, 30,000 PSI CIP

$38^{\prime \prime}$ ID x 140" Void Length, 15,000 PSI, $650^{\circ} \mathrm{C}$. HIPIC

Yr Supplied

1982

1988

(28" ID x 102" Void Length, Isolation Chamber)

Fiber Materials, Inc. (FMI)

Equipment

38.5" ID x 77" Void Length, 15,000 PSI, $1000^{\circ} \mathrm{C}$, HIPIC

Yr Supplied

36" ID x 110" Void Length, 15,000 PSI HIPIC

1976

1983

24" ID x 82" Void Length, 15,000 PSI HIPIC

1983

36 " ID x 110" Void Length, 15,000 PSI HIPIC 


\subsection{Vessel Inspection}

\section{EPS \\ Engineered \\ Pressure Systems, Inc.}

EPSI suggests a vessel inspection periodically. EPSI will review and approve the inspection procedure and report for a minimal charge.

Inspection should consist of the following:

Mag Particle and UT inspection. If unable to perform a mag particle inspection of the entire vessel bore. Idaho National Laboratory should substitute a liquid penetrant exam for the areas not accessible with magnetic particle.

The vessel will have to be completely disassembled, cleaned and dried for the purposes of the inspection.

A complete disassembly of the vessel means that all vessel components, such as closures, seal plates, vacuum valves, etc. have to be taken apart, free of any oil or grease and be dried. The top and bottom closure needs to be disassembled. Depending on the inspectors the yoke plates may not need to be disassembled.

Should you wish to hire EPSI to perform any of the functions of dismantling the vessel, we will charge our standard labor rate of $\$ 1,000$ per 8-hour day, travel time at $\$ 90$ per hour, overtime at $\$ 187.50$ per hour and all travel related expenses at cost.

Estimated cost for qualified inspectors to perform the above inspection: $\$ 15,000$

Idaho National Laboratory will need to be able to supply a source of power for our inspector and a clean surface to work from. 
Pressure Systems, Inc.

10.1 Price:

Complete 10" HIP system with out turnkey installation:

$\$ 1,300,000$

Spare Furnace/Mantle and BZH Assembly

$\$ 250,000$

Budget Turnkey Installation without Crane

$\$ 75,000$ to $\$ 150,000$

Optional Base Plate T/C Plug-In Test Plate $\$ 10,000$

Future Vessel Assembly NDT Inspection

$\$ 15,000$

Reclaimed Argon gas System

$\$ 120,000$

Budget 14" HIP system with out turnkey installation:

$\$ 1,750,000$

Budget 12" HIP system with out turnkey installation: $\$ 1,500,000$

Budget Spare Parts:

$\$ 15,000$

Suggested spare parts to be, $\$ 5,000$ of consumable spare parts not counting load thermocouples and $\$ 10,000$ of strategic spare parts on hand. EPSI will provide a complete list of spare parts before shipment of HIP system

Not included in this proposal are:

- Pit Design

- Civil Engineering

- Grating, stairways or other access means to or around the vessel.

- Workload tooling

\subsection{Delivery}

- Delivery: 12 to 14 months after export license, Deliveries are subject to allocation of melting capacity. Deliveries to be adjusted after meeting melting commitment are secured.

- Delivery is Ex works EPSI Haverhill, MA

- Transportation, rigging and installation of vessel and components to be responsibility of EPSI - if turnkey is purchased. Not Duties

- Commissioning by end of TBD 


\subsection{Validity:}

\section{EPS \\ Engineered \\ Pressure Systems, Inc.}

- This proposal is valid for $\underline{30}$ days after the firm pricing has been provided. Orders placed after the validity periods are subject to our confirmation of both the quoted price and delivery time given above.

\subsection{Payment terms:}

- Our Standard Terms and Conditions are attached for your review.

Payment terms are suggested as follows:

$30 \%$ - At time of order placement, Net 30

$20 \%$ - At time of mechanical test of steel, test certificates to prove milestone achievement, Net 30

$30 \%$ - At time of hydrostatic test, test certificates to prove milestone Achievement, Net 30

$10 \%$ - At time of acceptance at EPSI, Net 30

$10 \%$ - At time of acceptance, no later than 30 days after completion of installation or 60 days after delivery, Net 30

\subsection{Warranty}

EPSI warrants all components, which will be supplied new, as outlined in this detailed proposal will be warrantied for one year after start up or 14 months after delivery.

\section{Exceptions to warranty components}

Wear items that fail under normal operation are not covered under the one year warranty. Example: furnace and load thermocouples, O-rings, packing for compressor or liquid pump and ceramics.

\subsection{Conditions of Sale}

\section{PRICES}

Unless otherwise specified, the prices quoted are firm and are not subject to adjustment unless the

Buyer causes shipment to be delayed.

2. TAXES

The Buyer agrees to pay the amount of any excise, sales gross receipts, use or occupation tax or other tax levied upon this transaction or upon any sale, contract, shipment or delivery incident thereto, or to furnish Seller with necessary funds for such payment if payable by Seller, in addition to the price or prices provided herein.

3. DELAY

Delivery is subject to delays due to war, Acts of God or of the Public Enemy, Acts of Government, fire, floods, strikes, labor trouble, sabotage, freight and transportation delays, inability to obtain materials, procurement elements for fuel at the planned time during order execution, or any cause of the same or any other kind beyond the control of the Seller.

4. IDAHO NATIONAL LABORATORYNERAL PROVISIONS

a. The Seller shall be liable only to replace such products as may be found to be defective or to allow credit for such products at its option, and shall not be liable for transportation or installation charges, expenses for repairs or replacements, including, but not limited to Buyer's machining expense, for any loss or reduction of profits or for loss of use or for indirect or consequential damages of any kind, whether arising from delay in delivery, breach of warranty or from any other cause whatsoever. 


\section{EPS \\ Engineered \\ Pressure Systems, Inc.}

b. In the event of a conflict between the terms and conditions of the Buyer's purchase order and these Conditions of Sale, the latter shall govern the contract between the Buyer and the Seller.

c. This quotation is submitted solely for the information of the Buyer and shall become a contract only after approval by the Buyer and acceptance in writing by the Seller at its home office in Haverhill,

Massachusetts. Upon such acceptance by the Seller, the above terms and conditions shall constitute the entire agreement between the parties and there are no terms obligations, covenants, representations, statements or other conditions other than those contained herein or in any specifications attached thereto. This contract shall be construed and governed by the laws of the Commonwealth of Massachusetts.

d. When material or equipment is supplied by the Buyer in connection with performance of labor or services by EPSI on such material or equipment or for any other reason, whether or not such material or equipment is listed as a part of this order, the following additional and supplementary terms and conditions shall apply and shall supersede for foregoing terms and conditions to the degree they conflict with such terms and conditions, if any:

EPSI shall not, under any circumstances, be liable either for any direct or consequential damages which may result from acts which it performs or from its failure to perform any act with respect to such material or equipment or for any loss of or any damage PSI shall not be entitled to compensation for processing material furnished by the buyer when such material has been rendered defective by such processing. If a defect in the processed product is caused by a defect in the material supplied or by error in the drawings or instructions furnished by the Buyer, EPSI shall, nevertheless, be entitled to full payment of the costs of processing. Defective parts and material shall be returned to the Buyer.

5. $\quad$ CREDIT APPROVAL

Shipments, deliveries and performance of work shall at all times be subject of the Seller's Credit Department and the Seller may at any time decline to make any shipment or delivery or perform any work except upon receipt of payment or upon terms and conditions or security satisfactory to such Department.

6. $\quad$ COMPLIANCE WITH LAWS

The seller intends to comply with all laws applicable to its performance of any accepted order resulting form the accompanying quotation.

\section{CANCELLATION}

If any order placed against this quotation shall be canceled or suspended by Buyer and agreed to by Seller, Buyer will pay to Seller a cancellation or suspension charge amount to: a. A minimum of $20 \%$ of the base selling price, plus increased by: b. If prior to cancellation, the order has been placed in engineering and/or procurement stage, additional charges based on escalating that portion of work completed or committed through purchase ordering by EPSI from sub-suppliers this portion to be calculated a percentage of the total selling price.

8. GUARANTEE

Engineered Pressure Systems, Inc., guarantees the quoted equipment except expendable items such as o-rings, packing, seals, heating elements, thermocouples, etc., to be free from defects in materials and workmanship for a period of one (1) year from date of delivery except for the vessel, provided the equipment is used in accordance with EPSI recommendations. Defects discovered while the system is under guarantee shall be remedied by the Seller at his expense. The Seller shall have the option of repairing or replacing the defective part or component at the Buyer's plant or when returned PREPAID to the Seller's plant.

The guarantee is restricted to the replacement of defective part and shall in no case give the buyer the right to claim reimbursement of consequential damages caused by the defect or losses caused by the non-operative time of the system.

For the parts not manufactured by EPSI, EPSI attempts to obtain from its suppliers, guarantee provisions equivalent to its own. Therefore, this guarantee is limited to those guarantees which are given to us by each supplier.

Except as expressly set forth herein, Seller makes no warranty, express or implied, as to the fitness of the goods for a particular purpose, there are no warranties which extend beyond the description set forth in any specification mutually agreed on by the parties hereto. By acceptance hereof, Buyer waives all rights it may have under any other express or implied warranty provided by law.

\section{If you have any questions or concerns, please do not hesitate in contacting EPSI.}

\section{Sincerely,}

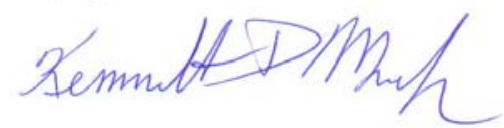

Kenneth D. Morse Jr. Sales Manger 
APPENDIX B- ESTIMATE DETAILS

REVISION 1

$\begin{array}{lll}\text { 1. ESTIMATE DETAILS } & \text { BASE CASE (FRICTION BONDING) } \\ \text { 2. ESTIMATE DETAILS } & \text { HIP ALTERNATE }\end{array}$




\title{
Estimate Summary
}

\section{Base Case (Friction Bonding)}

\author{
Revision 1
}


Client: INL

Project: FFC

Location: GREEN FIELD SITE

Facility: Entire Estimate

Green Field Site

\begin{tabular}{|c|c|c|c|c|c|c|}
\hline ACCT & DESCRIPTION & WORKHOURS & LABOR & MATERIAL & SUBS & TOTAL \\
\hline 01 & Demolition & & & & & \\
\hline 02 & Improvements to Site & 10,501 & $\$ 867,563$ & $\$ 353,652$ & $\$ 46,150$ & $\$ 1,267,365$ \\
\hline 03 & Earthwork & 11,882 & $\$ 959,804$ & $\$ 4,056$ & & $\$ 963,860$ \\
\hline 04 & Concrete/Masonry & 72,070 & $\$ 5,644,796$ & $\$ 1,763,044$ & & $\$ 7,407,840$ \\
\hline 05 & Structural Steel/Platework & 1,858 & $\$ 169,561$ & $\$ 244,090$ & & $\$ 413,652$ \\
\hline 06 & Permanent Equipment & 9,533 & $\$ 844,864$ & $\$ 11,722,390$ & $\$ 2,654,150$ & $\$ 15,221,404$ \\
\hline 11 & Piping Bulks & 18,485 & $\$ 1,608,332$ & $\$ 149,881$ & & $\$ 1,758,213$ \\
\hline 12 & Electrical Bulks & 17,394 & $\$ 1,480,813$ & $\$ 1,329,359$ & & $\$ 2,810,172$ \\
\hline 13 & Instrumentation Bulks & 1,423 & $\$ 126,983$ & $\$ 4,844,521$ & & $\$ 4,971,504$ \\
\hline 14 & Painting/Coatings/Liners & & & & & \\
\hline 15 & Insulation & & & & & \\
\hline 16 & Buildings & 37,425 & $\$ 2,914,541$ & $\$ 1,956,697$ & $\$ 492,487$ & $\$ 5,363,725$ \\
\hline 21 & Spare Parts & & & & & \\
\hline \multirow[t]{2}{*}{22} & Freight & & & & & \\
\hline & DIRECT FIELD COST & 180,570 & $\$ 14,617,256$ & $\$ 22,367,690$ & $\$ 3,192,787$ & $\$ 40,177,734$ \\
\hline 31 & Constr. Mgt. Staff \& Services & 20,984 & $\$ 1,477,274$ & & $\$ 238,220$ & $\$ 1,715,494$ \\
\hline 32 & Craft Labor Related Expenses & & & & & \\
\hline 33 & Temporary Construction Facilities, Furnishings, Services & & & & & \\
\hline 41 & Construction Equipment & & & & & \\
\hline \multirow[t]{3}{*}{51} & Start Up Services & & & & & \\
\hline & INDIRECT FIELD COST & 20,984 & $\$ 1,477,274$ & $\$ 0$ & $\$ 238,220$ & $\$ 1,715,494$ \\
\hline & TOTAL FIELD COST & 201,554 & $\$ 16,094,530$ & $\$ 22,367,690$ & $\$ 3,431,007$ & $\$ 41,893,227$ \\
\hline \multirow[t]{2}{*}{61} & Home Office Services & 68,600 & $\$ 7,203,000$ & & & $\$ 7,203,000$ \\
\hline & TOTAL FIELD, HOME OFFICE AND ENGINEERING & 270,154 & $\$ 23,297,530$ & $\$ 22,367,690$ & $\$ 3,431,007.31$ & $\$ 49,096,227$ \\
\hline 74.10 & INSURANCE CGL \& Pkg Policy & $0.51 \%$ & & & & $\$ 250,087$ \\
\hline 74.20 & TAXES (EXCLUDED) & & & & & $\$ 0$ \\
\hline 74.30 & BONDS (EXCLUDED) & & & & & \$0 \\
\hline 74.40 & PERMITS (EXCLUDED) & & & & & \$0 \\
\hline 74.50 & FREIGHT $5 \%$ on Material Costs & $2.28 \%$ & & & & $\$ 1,118,385$ \\
\hline 79.50 & ESCALATION & & & & & $\$ 0$ \\
\hline 80.10 & CONTINGENCY & $27.47 \%$ & & & & $\$ 13,860,281$ \\
\hline 89.1 & G\&A ?? & Prov. Rate: & $\%$ of Sales: & & & \$0 \\
\hline 90 & TOTAL PROJECT COST Basis for FEE & & & & & $\$ 64,324,980$ \\
\hline 90.1 & FEES & & $0.00 \%$ & & & $\$ 0$ \\
\hline 91 & WARRANTY RESERVE & & & & & \$0 \\
\hline 100.1 & CLIENT COSTS & & & & & \$o \\
\hline 100.2 & FEES ON CLIENT COSTS & & & & & \$o \\
\hline 110 & GRAND TOTAL PROJECT & & & & & $\$ 64,324,980$ \\
\hline & & & & & & \\
\hline & & & & & & \\
\hline & & & & & & \\
\hline & & & & & & \\
\hline
\end{tabular}

Grand Total Summary
URS

Washington Division

Date: 7/17/2008

Priced By: ADC

Office: Denver 


\title{
Estimate Details
}

\section{Base Case (Friction Bonding)}

\author{
Revision 1
}




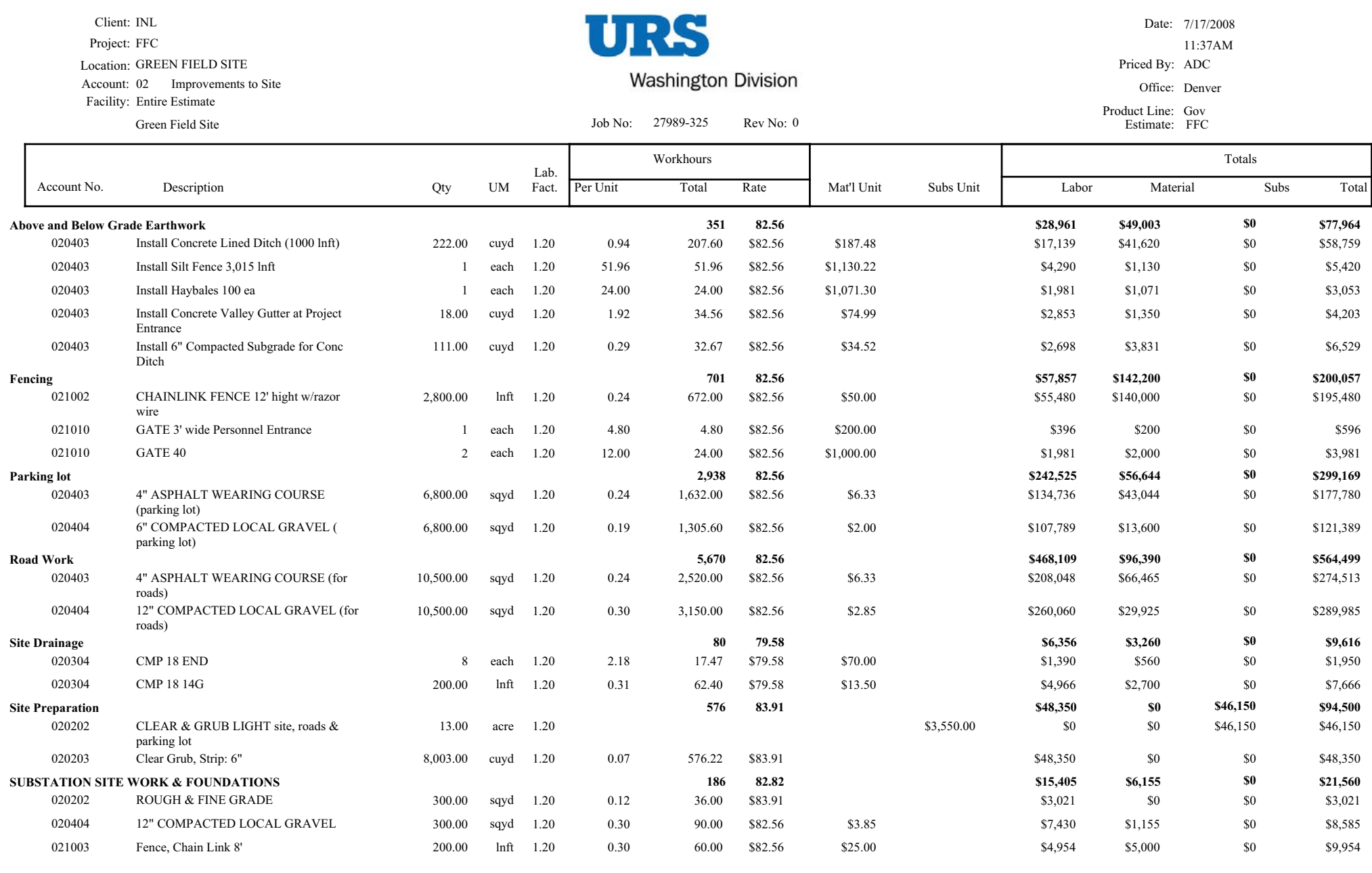

Totals 02-Improvements to Site Entire Estimate

\begin{tabular}{|l|l|}
\hline & \\
\hline
\end{tabular}

$\$ 867,563$

$\$ 353,652$

$\$ 46,150$

$\$ 1,267,365$ 
Client: INL

Project: FFC

Location: GREEN FIELD SITE

Account: 03 Earthwork

Facility: Entire Estimate

URS

Washington Division
Date: 7/17/2008

11:37AM

Priced By: ADC

Office: Denver

Product Line: Gov
Estimate: FFC

\begin{tabular}{|c|c|c|c|c|c|c|c|c|c|c|c|c|c|}
\hline \multirow[b]{2}{*}{ Account No. } & \multirow[b]{2}{*}{ Description } & \multirow[b]{2}{*}{ Qty } & \multirow[b]{2}{*}{ UM } & \multirow{2}{*}{$\begin{array}{l}\text { Lab. } \\
\text { Fact. }\end{array}$} & \multicolumn{3}{|c|}{ Workhours } & \multirow[b]{2}{*}{ Mat'l Unit } & \multirow[b]{2}{*}{ Subs Unit } & \multicolumn{4}{|c|}{ Totals } \\
\hline & & & & & Per Unit & Total & Rate & & & Labor & Material & Subs & Total \\
\hline \multicolumn{2}{|c|}{ Sanitary Sewer Line 475 Inft by $6^{\prime}$ deep } & & & & & 545 & 79.65 & & & $\$ 43,400$ & \$o & \$0 & $\$ 43,400$ \\
\hline 030102 & REMOVE SURPLUS EXCAVATION & 316.67 & cuyd & 1.20 & 0.04 & 11.40 & $\$ 83.91$ & & & $\$ 957$ & $\$ 0$ & $\$ 0$ & $\$ 957$ \\
\hline 030104 & HAND EXCAVATION & 95.00 & cuyd & 1.20 & 1.62 & 153.90 & $\$ 74.33$ & & & $\$ 11,439$ & $\$ 0$ & $\$ 0$ & $\$ 11,439$ \\
\hline 030105 & TRENCH EXCAVATION & 855.00 & cuyd & 1.20 & 0.13 & 112.86 & $\$ 79.58$ & & & $\$ 8,981$ & $\$ 0$ & $\$ 0$ & $\$ 8,981$ \\
\hline 030204 & HAND BACKFILL & 63.33 & cuyd & 1.20 & 2.16 & 136.80 & $\$ 82.56$ & & & $\$ 11,294$ & $\$ 0$ & $\$ 0$ & $\$ 11,294$ \\
\hline 030205 & TRENCH BACKFILL & 570.00 & cuyd & 1.20 & 0.23 & 129.96 & $\$ 82.56$ & & & $\$ 10,729$ & $\$ 0$ & $\$ 0$ & $\$ 10,729$ \\
\hline \multicolumn{2}{|c|}{ Concrete Footing by Dimension } & & & & & 2,725 & 81.24 & & & $\$ 221,387$ & \$o & \$o & $\$ 221,387$ \\
\hline 030102 & REMOVE SURPLUS EXCAVATION & 750.00 & cuyd & 1.20 & 0.16 & 117.00 & $\$ 83.91$ & & & $\$ 9,817$ & $\$ 0$ & $\$ 0$ & $\$ 9,817$ \\
\hline 030103 & STRUCTURAL EXCAVATION & $3,037.50$ & cuyd & 1.20 & 0.18 & 546.75 & $\$ 83.91$ & & & $\$ 45,878$ & $\$ 0$ & $\$ 0$ & $\$ 45,878$ \\
\hline 030104 & HAND EXCAVATION & 337.50 & cuyd & 1.20 & 1.62 & 546.75 & $\$ 74.33$ & & & $\$ 40,640$ & $\$ 0$ & $\$ 0$ & $\$ 40,640$ \\
\hline 030203 & STRUCTURAL BACKFILL & $3,037.50$ & cuyd & 1.20 & 0.31 & 947.70 & $\$ 82.56$ & & & $\$ 78,241$ & $\$ 0$ & $\$ 0$ & $\$ 78,241$ \\
\hline 030204 & HAND BACKFILL & 262.50 & cuyd & 1.20 & 2.16 & 567.00 & $\$ 82.56$ & & & $\$ 46,811$ & $\$ 0$ & $\$ 0$ & $\$ 46,811$ \\
\hline \multicolumn{2}{|c|}{ Concrete Footing by Dimension Security Bldg } & & & & & 106 & 81.26 & & & $\$ 8,589$ & \$o & $\$ 0$ & $\$ 8,589$ \\
\hline 030102 & REMOVE SURPLUS EXCAVATION & 44.00 & cuyd & 1.20 & 0.16 & 6.86 & $\$ 83.91$ & & & $\$ 576$ & $\$ 0$ & $\$ 0$ & $\$ 576$ \\
\hline 030103 & STRUCTURAL EXCAVATION & 118.80 & cuyd & 1.20 & 0.18 & 21.38 & $\$ 83.91$ & & & $\$ 1,794$ & $\$ 0$ & $\$ 0$ & $\$ 1,794$ \\
\hline 030104 & HAND EXCAVATION & 13.20 & cuyd & 1.20 & 1.62 & 21.38 & $\$ 74.33$ & & & $\$ 1,589$ & $\$ 0$ & $\$ 0$ & $\$ 1,589$ \\
\hline 030203 & STRUCTURAL BACKFILL & 118.80 & cuyd & 1.20 & 0.31 & 37.07 & $\$ 82.56$ & & & $\$ 3,060$ & $\$ 0$ & $\$ 0$ & $\$ 3,060$ \\
\hline 030204 & HAND BACKFILL & 8.80 & cuyd & 1.20 & 2.16 & 19.01 & $\$ 82.56$ & & & $\$ 1,569$ & $\$ 0$ & $\$ 0$ & $\$ 1,569$ \\
\hline \multicolumn{2}{|c|}{ Concrete Footing by Dimension under Warehouse Slab } & & & & & 139 & 81.26 & & & $\$ 11,322$ & so & \$o & $\$ 11,322$ \\
\hline 030102 & REMOVE SURPLUS EXCAVATION & 58.00 & cuyd & 1.20 & 0.16 & 9.05 & $\$ 83.91$ & & & $\$ 759$ & $\$ 0$ & $\$ 0$ & $\$ 759$ \\
\hline 030103 & STRUCTURAL EXCAVATION & 156.60 & cuyd & 1.20 & 0.18 & 28.19 & $\$ 83.91$ & & & $\$ 2,365$ & $\$ 0$ & $\$ 0$ & $\$ 2,365$ \\
\hline 030104 & HAND EXCAVATION & 17.40 & cuyd & 1.20 & 1.62 & 28.19 & $\$ 74.33$ & & & $\$ 2,095$ & $\$ 0$ & $\$ 0$ & $\$ 2,095$ \\
\hline 030203 & STRUCTURAL BACKFILL & 156.60 & cuyd & 1.20 & 0.31 & 48.86 & $\$ 82.56$ & & & $\$ 4,034$ & $\$ 0$ & $\$ 0$ & $\$ 4,034$ \\
\hline 030204 & HAND BACKFILL & 11.60 & cuyd & 1.20 & 2.16 & 25.06 & $\$ 82.56$ & & & $\$ 2,069$ & $\$ 0$ & $\$ 0$ & $\$ 2,069$ \\
\hline \multicolumn{2}{|c|}{ CONCRETE SOG - BY VOLUME Security BIdg } & & & & & 15 & 80.91 & & & $\$ 1,192$ & so & \$o & $\$ 1,192$ \\
\hline 030102 & REMOVE SURPLUS EXCAVATION & 17.00 & cuyd & 1.20 & 0.16 & 2.65 & $\$ 83.91$ & & & $\$ 223$ & $\$ 0$ & $\$ 0$ & $\$ 223$ \\
\hline 030103 & STRUCTURAL EXCAVATION & 22.50 & cuyd & 1.20 & 0.18 & 4.05 & $\$ 83.91$ & & & $\$ 340$ & $\$ 0$ & $\$ 0$ & $\$ 340$ \\
\hline 030104 & HAND EXCAVATION & 2.50 & cuyd & 1.20 & 1.62 & 4.05 & $\$ 74.33$ & & & $\$ 301$ & $\$ 0$ & $\$ 0$ & $\$ 301$ \\
\hline 030203 & STRUCTURAL BACKFILL & 7.20 & cuyd & 1.20 & 0.31 & 2.25 & $\$ 82.56$ & & & $\$ 185$ & $\$ 0$ & $\$ 0$ & $\$ 185$ \\
\hline 030204 & HAND BACKFILL & 0.80 & cuyd & 1.20 & 2.16 & 1.73 & $\$ 82.56$ & & & $\$ 143$ & $\$ 0$ & $\$ 0$ & $\$ 143$ \\
\hline \multicolumn{2}{|c|}{ CONCRETE SOG - BY VOLUME Sidewalk to Bldg } & & & & & 37 & 80.92 & & & $\$ 3,026$ & so & $\$ 0$ & $\$ 3,026$ \\
\hline 030102 & REMOVE SURPLUS EXCAVATION & 42.00 & cuyd & 1.20 & 0.16 & 6.55 & $\$ 83.91$ & & & $\$ 550$ & $\$ 0$ & $\$ 0$ & $\$ 550$ \\
\hline 030103 & STRUCTURAL EXCAVATION & 56.70 & cuyd & 1.20 & 0.18 & 10.21 & $\$ 83.91$ & & & $\$ 856$ & $\$ 0$ & $\$ 0$ & $\$ 856$ \\
\hline 030104 & HAND EXCAVATION & 6.30 & cuyd & 1.20 & 1.62 & 10.21 & $\$ 74.33$ & & & $\$ 759$ & $\$ 0$ & $\$ 0$ & $\$ 759$ \\
\hline 030203 & STRUCTURAL BACKFILL & 18.90 & cuyd & 1.20 & 0.31 & 5.90 & $\$ 82.56$ & & & $\$ 487$ & $\$ 0$ & $\$ 0$ & $\$ 487$ \\
\hline
\end{tabular}




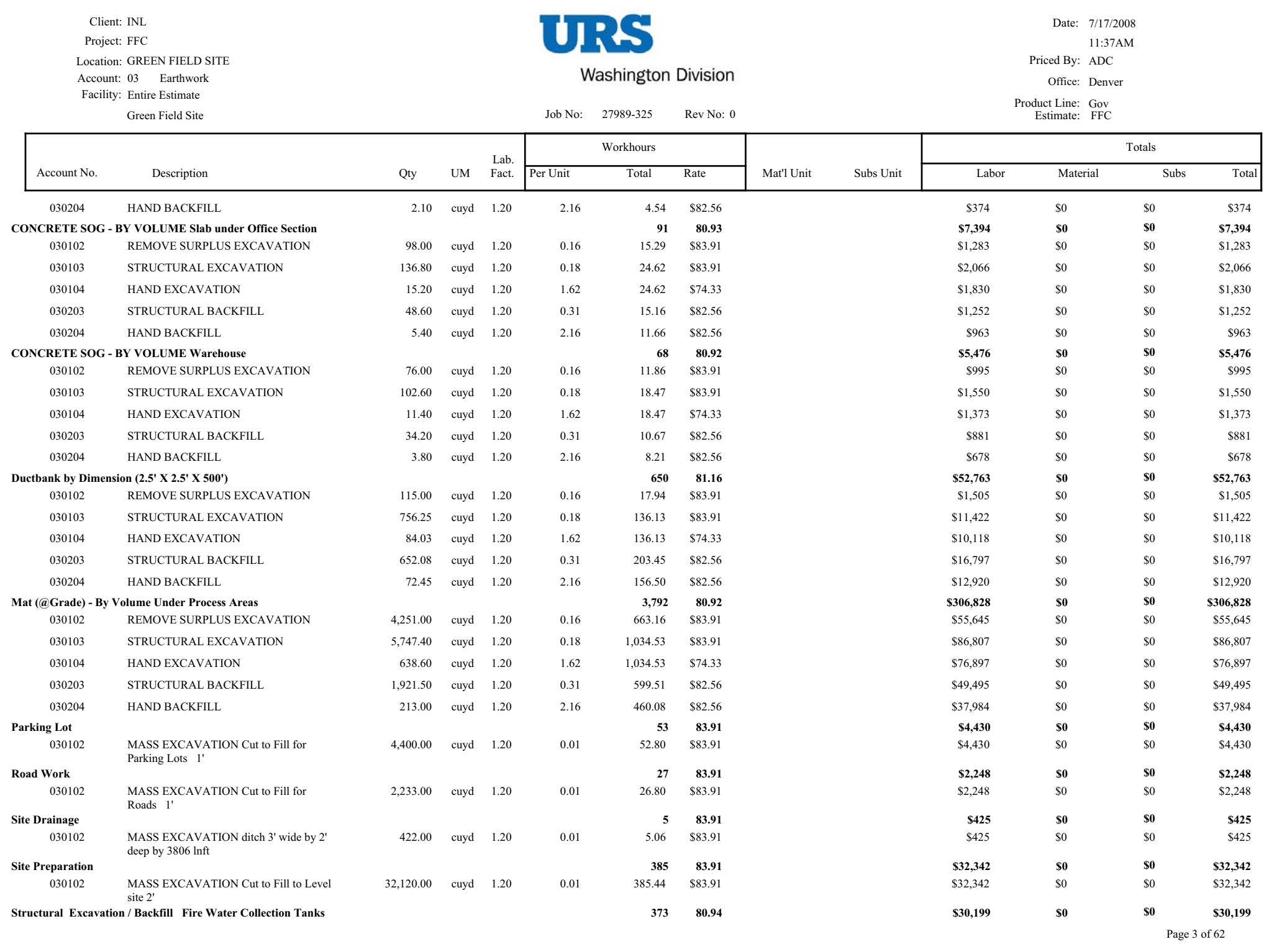




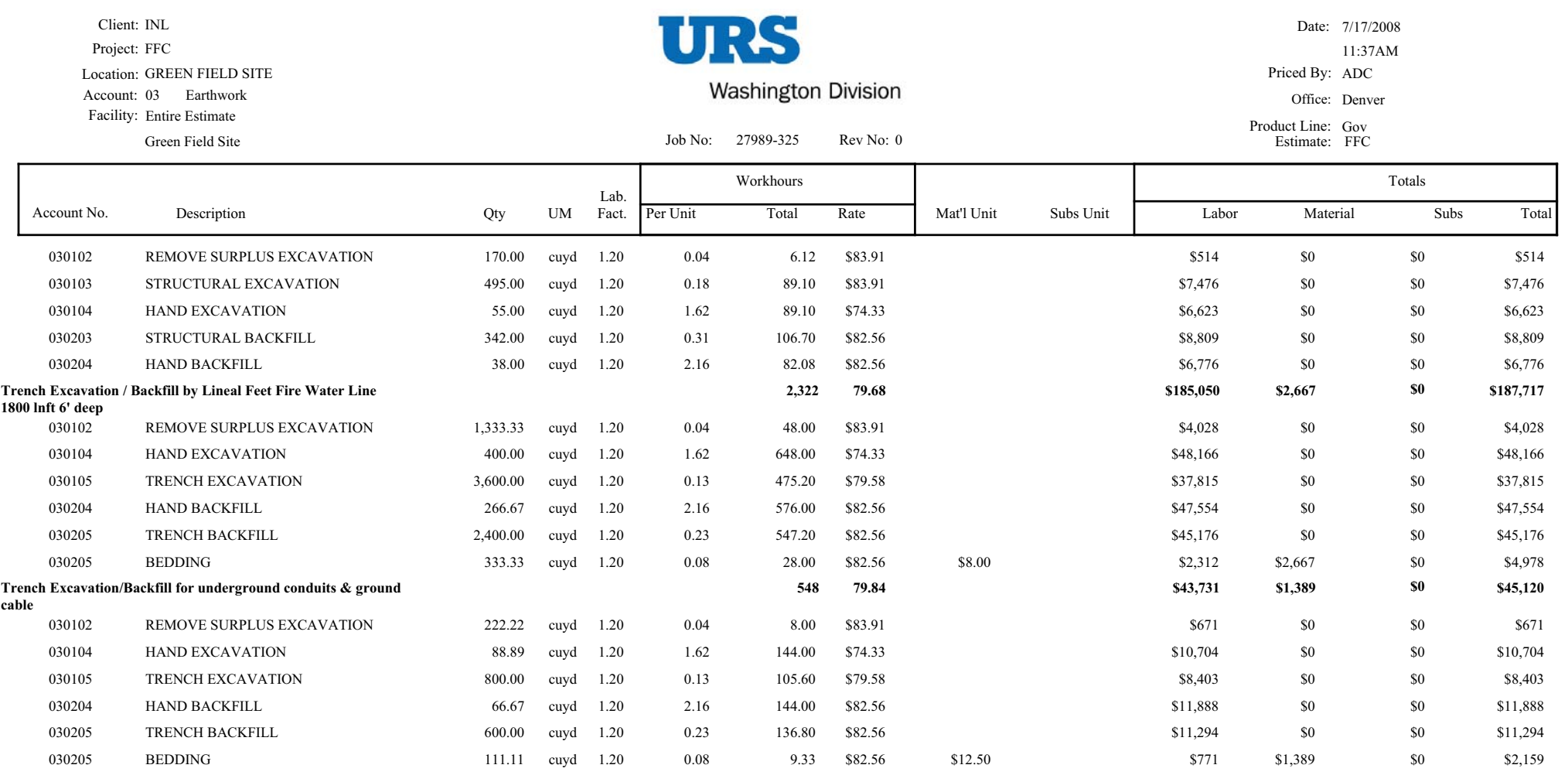

\begin{tabular}{|l|r|}
\hline$\$ 959,804$ \\
\hline
\end{tabular}




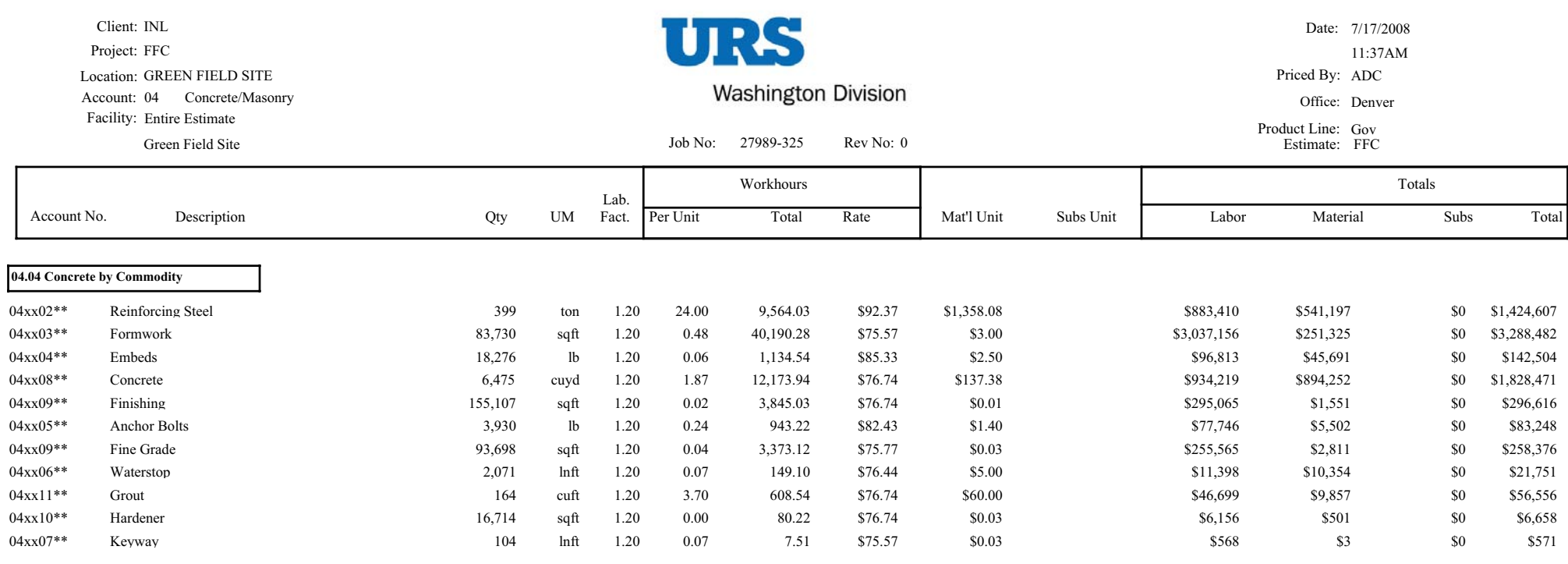

\begin{tabular}{|c|c|c|c|}
\hline \multicolumn{4}{|c|}{ Overall Ratios: } \\
\hline FormWork: & & 12.93 & SF/CUYD \\
\hline Rebar: & & 123.08 & LB/CUYD \\
\hline \multirow[t]{3}{*}{ Embedments: } & & 2.82 & LB/CUYD \\
\hline & Wkhr/CY & & 11.13 \\
\hline & Total/CUYD & & $\$ 1,144.01$ \\
\hline
\end{tabular}

\begin{tabular}{|c|c|c|c|c|c|c|c|c|c|c|c|}
\hline Grand Total: & Concrete / Masonry & 6,475 & CUYD & 1.20 & 11.13 & 72,070 & $\$ 78.32$ & $\begin{array}{r}\mathbf{\$ 5 , 6 4 4 , 7 9 6} \\
\$ 871.74 / \mathrm{CY}\end{array}$ & $\begin{array}{c}\$ 1,763,044 \\
\$ 272.27 / C Y\end{array}$ & $\begin{array}{c}\$ 0 \\
\$ 0.00 / \mathrm{CY}\end{array}$ & $\$ 7,407,840$ \\
\hline
\end{tabular}

Page 5 of 62 


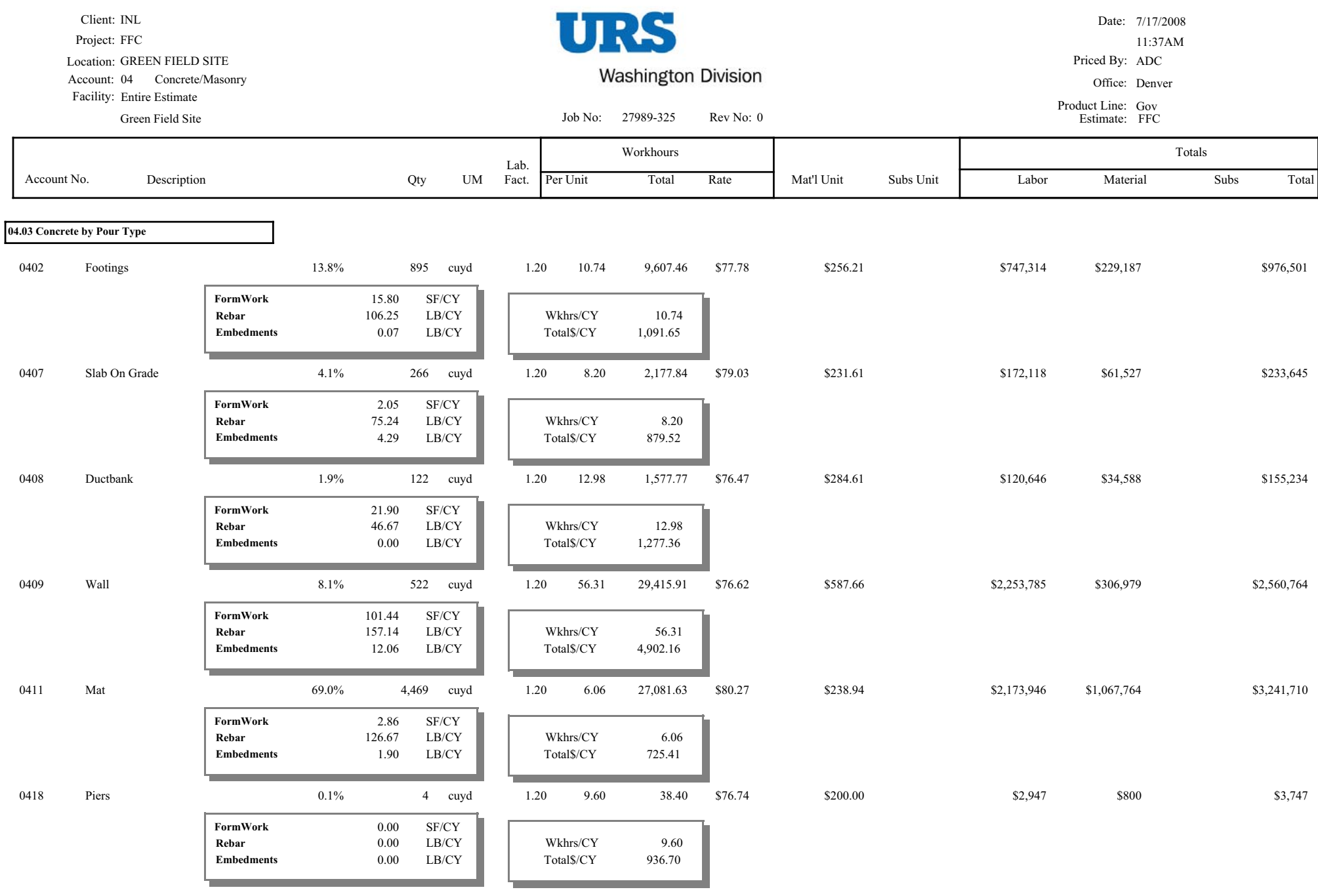




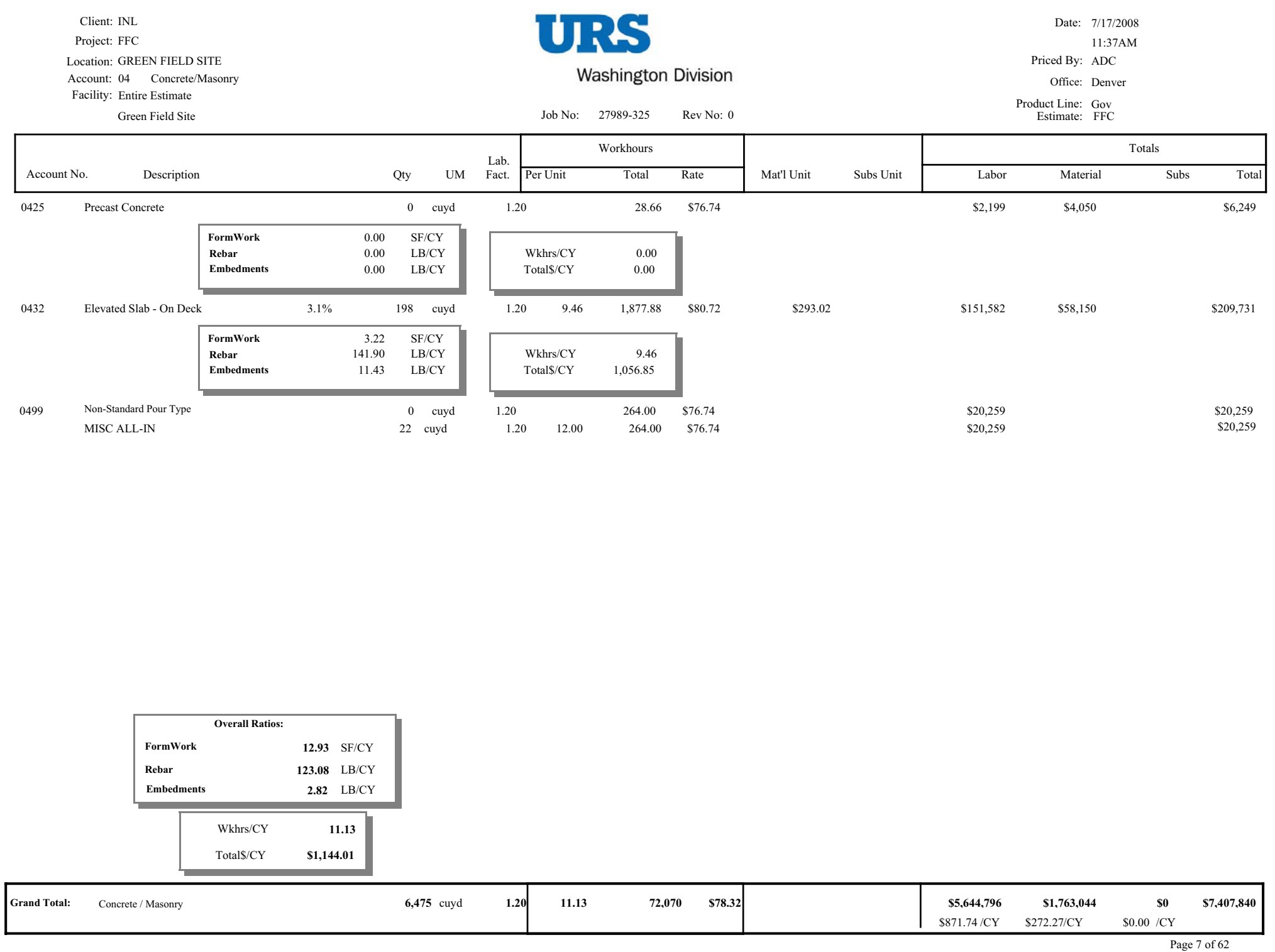


Client: INL

Project: FFC

Location: GREEN FIELD SITE

Account: 04 Concrete/Masonry

URS

Date: $7 / 17 / 2008$

11:37AM

Washington Division

Priced By: ADC

Office: Denver

Facility: Entire Estimate

Job No: $\quad 27989-325 \quad$ Rev No: 0

Product Line: Gov
Estimate: FFC

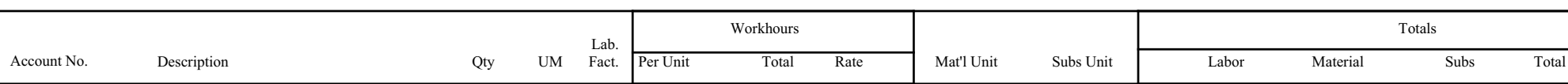

04.02 Concrete by Assembly - Summary

Concrete Footing by Dimension

Concrete Footing by Dimension Security Bldg

Concrete Footing by Dimension under Warehouse Slab

CONCRETE SOG - BY VOLUME Fire water Collection

Tank

CONCRETE SOG - BY VOLUME Security BIdg

CONCRETE SOG - BY VOLUME Sidewalk to Bldg

CONCRETE SOG - BY VOLUME Slab under Office

Section

CONCRETE SOG - BY VOLUME Warehouse

Ductbank by Dimension $\left(2.5^{\prime}\right.$ X 2.5' X 500')

Elevated Slab on Metal Deck- by Volume

Elevated Slab on Metal Deck- by Volume HVAC Filter

Floor

Elevated Slab on Metal Deck- by Volume Security Bldg

Roof

Fence/Fence lighting Pole

Mat (@Grade) - By Volume Under Process Areas

Roofing Fire water Collection Tank Sump

SUBSTATION SITE WORK \& FOUNDATIONS

Wall by Volume Greater than 8' high Fire Water Collection.

Tank

Wall by Volume Greater than 8' high Radiography Area

\begin{tabular}{|c|c|c|c|c|c|c|}
\hline 787 & cuyd & 1.20 & 9.27 & 7,298 & $\$ 78.10$ & $\$ 246.45$ \\
\hline 47 & cuyd & 1.20 & 28.88 & 1,350 & $\$ 76.58$ & $\$ 382.26$ \\
\hline 61 & cuyd & 1.20 & 15.80 & 959 & $\$ 77.06$ & $\$ 285.73$ \\
\hline 20 & cuyd & 1.20 & 6.87 & 137 & $\$ 79.47$ & $\$ 230.18$ \\
\hline 18 & cuyd & 1.20 & 7.71 & 138 & $\$ 79.18$ & $\$ 231.08$ \\
\hline 44 & cuyd & 1.20 & 11.04 & 487 & $\$ 78.44$ & $\$ 234.68$ \\
\hline 104 & cuyd & 1.20 & 7.71 & 801 & $\$ 79.18$ & $\$ 231.08$ \\
\hline 80 & cuyd & 1.20 & 7.71 & 615 & $\$ 79.18$ & $\$ 231.08$ \\
\hline 122 & cuyd & 1.20 & 12.98 & 1,578 & $\$ 76.47$ & $\$ 284.61$ \\
\hline 95 & cuyd & 1.20 & 9.46 & 894 & $\$ 80.72$ & $\$ 293.02$ \\
\hline 86 & cuyd & 1.20 & 9.46 & 815 & $\$ 80.72$ & $\$ 293.02$ \\
\hline 18 & cuyd & 1.20 & 9.46 & 169 & $\$ 80.72$ & $\$ 293.02$ \\
\hline 0 & cuyd & 1.20 & & 264 & $\$ 76.74$ & \\
\hline 4,469 & cuyd & 1.20 & 6.06 & 27,082 & $\$ 80.27$ & $\$ 238.94$ \\
\hline 0 & cuyd & 1.20 & & 29 & $\$ 76.74$ & \\
\hline 4 & cuyd & 1.20 & 9.60 & 38 & $\$ 76.74$ & $\$ 200.00$ \\
\hline 26 & cuyd & 1.20 & 42.50 & 1,116 & $\$ 76.93$ & $\$ 506.75$ \\
\hline 496 & cuyd & 1.20 & 57.04 & 28,300 & $\$ 76.61$ & $\$ 591.94$ \\
\hline
\end{tabular}

\begin{tabular}{|lrl|}
\hline & Entire Estimate & \\
Formwork & $\mathbf{1 2 . 9 3}$ & $\mathrm{SF} / \mathrm{CY}$ \\
Rebar & $\mathbf{1 2 3 . 0 8}$ & $\mathrm{LB} / \mathrm{CY}$ \\
Embeds & $\mathbf{2 . 8 2}$ & $\mathrm{LB} / \mathrm{CY}$ \\
\hline & & \\
& WKHR/CUYD & $\mathbf{1 1 . 1 3}$ \\
& Total/CY & $\mathbf{\$ 1 , 1 4 4 . 0 1}$ \\
\hline
\end{tabular}

Grand Total: Entire Estimate

6,475 cuyd

\begin{tabular}{l|l}
1.20 & 11.13
\end{tabular}

72,070

$\$ 78.32$

$55,644,796$

$\$ 1,763,044$

\$0 $\$ \mathbf{\$}, 407,840$

$\$ \mathbf{\$ 8 7 1 . 7 4} / \mathrm{CY} \quad \$ 272.27 / \mathrm{CY} \quad \mathbf{\$ 0 . 0 0} / \mathrm{CY}$




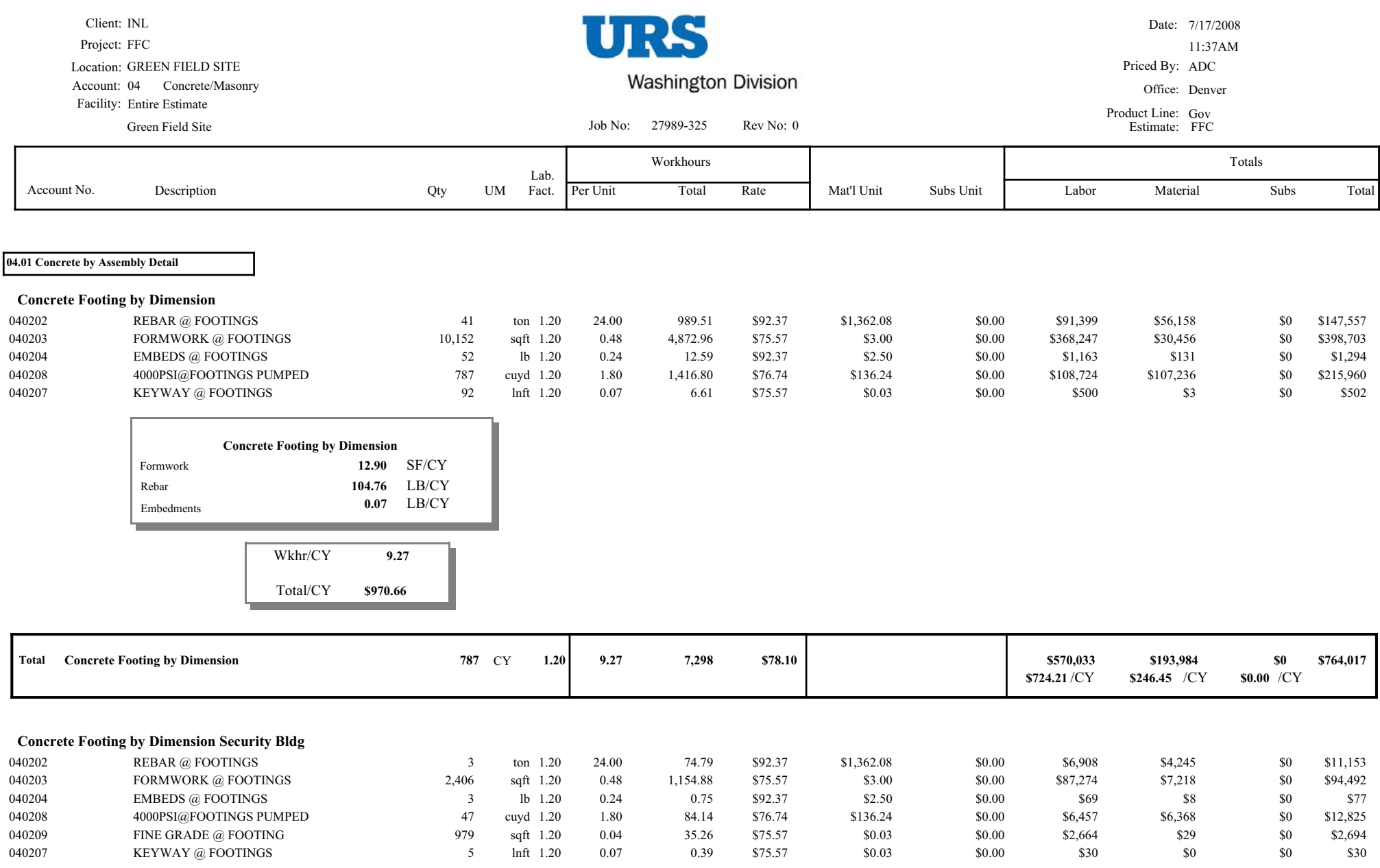




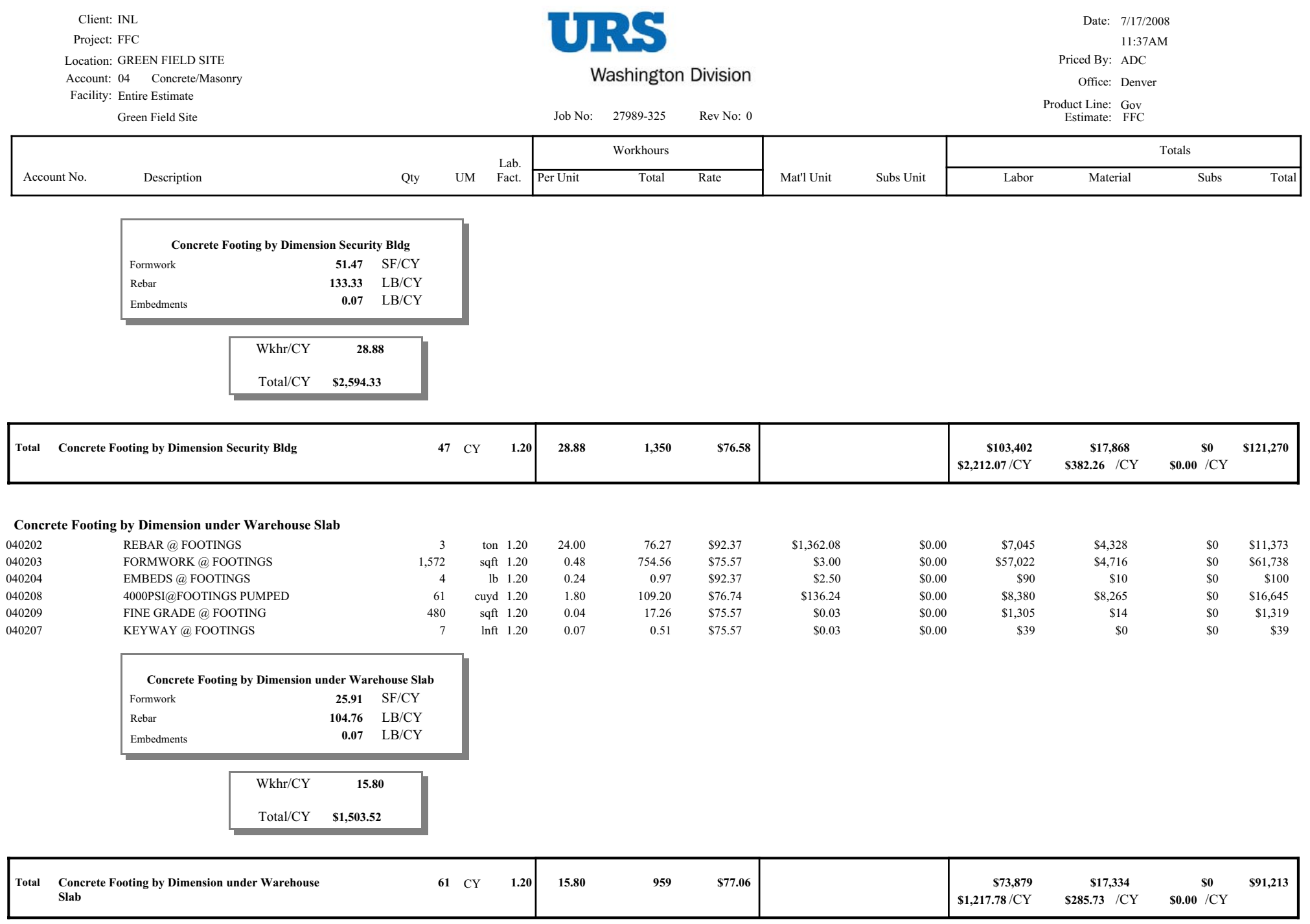


Client: INL

Project: FFC

Location: GREEN FIELD SITE

Account: 04 Concrete/Masonry

URS

Washington Division
Date: $7 / 17 / 2008$

$11: 37 \mathrm{AM}$

Priced By: ADC

Office: Denver

Product Line: Gov
Estimate: FFC

\begin{tabular}{|c|c|c|c|c|c|c|c|c|c|c|}
\hline \multirow[b]{2}{*}{ Account No. } & \multirow[b]{2}{*}{ Description } & \multirow[b]{2}{*}{ Qty } & \multirow[b]{2}{*}{ UM } & \multirow{2}{*}{$\begin{array}{l}\text { Lab. } \\
\text { Fact. }\end{array}$} & & rkhours & \multirow[b]{2}{*}{ Mat'l Unit } & \multirow[b]{2}{*}{ Subs Unit } & \multicolumn{2}{|c|}{ Totals } \\
\hline & & & & & Per Unit & Total & & & Labor & Material \\
\hline
\end{tabular}

CONCRETE SOG - BY VOLUME Fire water Collection Tank

\begin{tabular}{|c|c|c|c|c|c|c|c|}
\hline 040702 & REBAR@SOG & 1 & ton & 1.20 & 24.00 & 18.01 & $\$ 92.37$ \\
\hline 040703 & FORMWORK@SOG & 41 & sqft & 1.20 & 0.48 & 19.61 & $\$ 75.57$ \\
\hline 040704 & EMBEDS@SOG & 86 & $\mathrm{lb}$ & 1.20 & 0.06 & 5.13 & $\$ 92.37$ \\
\hline 040708 & 4000PSI@SOG PUMPED & 20 & cuyd & 1.20 & 1.80 & 35.91 & $\$ 76.74$ \\
\hline 040709 & FINISHING@SOG & 770 & sqft & 1.20 & 0.02 & 18.47 & $\$ 76.74$ \\
\hline 040705 & ANCHOR BOLTS@SOG & 10 & $\mathrm{lb}$ & 1.20 & 0.24 & 2.31 & $\$ 92.37$ \\
\hline 040709 & FINE GRADE @ SOG & 770 & sqft & 1.20 & 0.04 & 27.70 & $\$ 76.74$ \\
\hline 040706 & WATERSTOP@SOG & 88 & $\operatorname{lnft}$ & 1.20 & 0.07 & 6.31 & $\$ 76.74$ \\
\hline 040710 & HARDENER@SOG & 770 & sqft & 1.20 & 0.00 & 3.69 & $\$ 76.74$ \\
\hline
\end{tabular}

HARDENER@SOG

sqft 1.20

\begin{tabular}{|lrll|}
\hline \multicolumn{4}{|c|}{} \\
\multicolumn{4}{|c|}{ CONCRETE SOG - BY VOLUME } \\
Formwork & Tank & 2.05 & SF/CY \\
Rebar & & 75.24 & $\mathrm{LB} / \mathrm{CY}$ \\
Embedments & 4.29 & $\mathrm{LB} / \mathrm{CY}$ \\
\hline
\end{tabular}

\begin{tabular}{|rr|}
\hline Wkhr/CY & $\mathbf{6 . 8 7}$ \\
Total/CY & $\$ \mathbf{\$ 7 6 . 5 0}$
\end{tabular}

Total CONCRETE SOG - BY VOLUME Fire water Collection Tank
$20 \mathrm{CY}$

1.20

6.87

6.87

137

$\$ 1,362.08$
$\$ 3.25$
$\$ 2.50$
$\$ 136.24$
$\$ 0.01$
$\$ 1.40$
$\$ 0.03$
$\$ 5.00$
$\$ 0.03$

$\begin{array}{lrr}\$ 0.00 & \$ 1,664 & \$ 1,022 \\ \$ 0.00 & \$ 1,482 & \$ 133 \\ \$ 0.00 & \$ 474 & \$ 214 \\ \$ 0.00 & \$ 2,756 & \$ 2,718 \\ \$ 0.00 & \$ 1,417 & \$ 8 \\ \$ 0.00 & \$ 214 & \$ 13 \\ \$ 0.00 & \$ 2,126 & \$ 23 \\ \$ 0.00 & \$ 484 & \$ 438 \\ \$ 0.00 & \$ 283 & \$ 23\end{array}$

$\begin{array}{lr}\$ 0 & \$ 2,686 \\ \$ 0 & \$ 1,615 \\ \$ 0 & \$ 688 \\ \$ 0 & \$ 5,474 \\ \$ 0 & \$ 1,425 \\ \$ 0 & \$ 227 \\ \$ 0 & \$ 2,149 \\ \$ 0 & \$ 922 \\ \$ 0 & \$ 307\end{array}$

\section{CONCRETE SOG - BY VOLUME Security BIdg}

$\begin{array}{ll}040702 & \text { REBAR @ SOG } \\ 040703 & \text { FORMWORK @ SOG } \\ 040704 & \text { EMBEDS @ SOG } \\ 040708 & \text { 4000PSI@SOG PUMPED } \\ 040709 & \text { FINISHING @ SOG } \\ 040705 & \text { ANCHOR BOLTS @ SOG } \\ 040709 & \text { FINE GRADE @ SOG } \\ 040706 & \text { WATERSTOP @ SOG } \\ 040710 & \text { HARDENER @ SOG }\end{array}$

$\begin{array}{rrrrrr}1 & \text { ton } 1.20 & 24.00 & 16.12 & \$ 92.37 \\ 37 & \text { sqft } 1.20 & 0.48 & 17.54 & \$ 75.57 \\ 77 & \text { Ib } & 1.20 & 0.06 & 4.59 & \$ 92.37 \\ 18 & \text { cuyd } 1.20 & 1.80 & 32.13 & \$ 76.74 \\ 918 & \text { sqft } 1.20 & 0.02 & 22.03 & \$ 76.74 \\ 9 & \text { lb } & 1.20 & 0.24 & 2.07 & \$ 92.37 \\ 918 & \text { sqft } 1.20 & 0.04 & 33.05 & \$ 76.74 \\ 78 & \text { Inft } 1.20 & 0.07 & 5.64 & \$ 76.74 \\ 918 & \text { sqft } 1.20 & 0.00 & 4.41 & \$ 76.74\end{array}$

$\$ 1,362.08$
$\$ 3.25$
$\$ 2.50$
$\$ 136.24$
$\$ 0.01$
$\$ 1.40$
$\$ 0.03$
$\$ 5.00$
$\$ 0.03$

$\begin{array}{lr}\$ 0.00 & \$ 1,489 \\ \$ 0.00 & \$ 1,326 \\ \$ 0.00 & \$ 424 \\ \$ 0.00 & \$ 2,466 \\ \$ 0.00 & \$ 1,691 \\ \$ 0.00 & \$ 191 \\ \$ 0.00 & \$ 2,536 \\ \$ 0.00 & \$ 433 \\ \$ 0.00 & \$ 338\end{array}$

$\$ 4,592$

$\$ 230.18 / C Y$

\$o

$\$ 0.00 / \mathrm{CY}$

$\$ 15,491$

$\$ 546.32 / \mathrm{CY}$

$\$ 915$
$\$ 119$
$\$ 191$
$\$ 2,432$
$\$ 9$
$\$ 12$
$\$ 28$
$\$ 392$
$\$ 28$

$\begin{array}{lr}\$ 0 & \$ 2,403 \\ \$ 0 & \$ 1,445 \\ \$ 0 & \$ 615 \\ \$ 0 & \$ 4,898 \\ \$ 0 & \$ 1,700 \\ \$ 0 & \$ 203 \\ \$ 0 & \$ 2,564 \\ \$ 0 & \$ 825 \\ \$ 0 & \$ 366\end{array}$


Client: INL

Project: FFC

URS

Washington Division
Date: $7 / 17 / 2008$

11:37AM Priced By: ADC

Office: Denver

Product Line: Gov
Estimate: FFC

Account: 04 Concrete/Masonry

Job No: $\quad 27989-325 \quad$ Rev No: 0

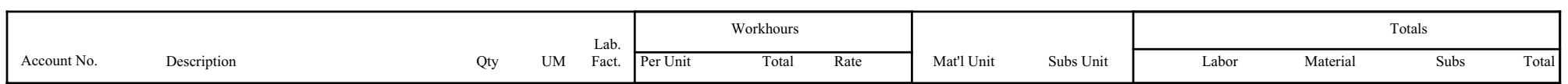

CONCRETE SOG - BY VOLUME Security BIdg

$\begin{array}{lrl}\text { Formwork } & \mathbf{2 . 0 5} & \mathrm{SF} / \mathrm{CY} \\ \text { Rebar } & \mathbf{7 5 . 2 4} & \mathrm{LB} / \mathrm{CY} \\ \text { Embedments } & \mathbf{4 . 2 9} & \mathrm{LB} / \mathrm{CY}\end{array}$

\begin{tabular}{|cr|}
\hline $\mathrm{Wkhr} / \mathrm{CY}$ & $\mathbf{7 . 7 1}$ \\
$\mathrm{Total} / \mathrm{CY}$ & $\mathbf{\$ 8 4 1 . 3 4}$ \\
\hline
\end{tabular}

Total CONCRETE SOG - BY VOLUME Security BIdg

$18 \mathrm{CY}$

1.20

7.71

138

$\$ 79.18$

\begin{tabular}{l|l}
\hline 18 & \\
&
\end{tabular}

$\$ 10,893$

$\$ 610.26 / \mathrm{CY}$

$\$ 4,125$
$\$ 231.08 / C Y$

$\$ 0$
$\$ 0.00 / \mathrm{CY}$

CONCRETE SOG - BY VOLUME Sidewalk to BIdg

$\begin{array}{ll}040702 & \text { REBAR @ SOG } \\ 040703 & \text { FORMWORK @ SOG } \\ 040704 & \text { EMBEDS @ SOG } \\ 040708 & \text { 4000PSI@SOG PUMPED } \\ 040709 & \text { FINISHING @ SOG } \\ 040705 & \text { ANCHOR BOLTS @ SOG } \\ 040709 & \text { FINE GRADE @ SOG } \\ 040706 & \text { WATERSTOP @ SOG } \\ 040710 & \text { HARDENER @ SOG }\end{array}$

$\begin{array}{rrrrrr}2 & \text { ton } & 1.20 & 24.00 & 39.82 & \$ 92.37 \\ 90 & \text { sqft } & 1.20 & 0.48 & 43.34 & \$ 75.57 \\ 189 & \text { lb } & 1.20 & 0.06 & 11.34 & \$ 92.37 \\ 44 & \text { cuyd } & 1.20 & 1.80 & 79.38 & \$ 76.74 \\ 4,536 & \text { sqft } & 1.20 & 0.02 & 108.86 & \$ 76.74 \\ 21 & \text { lb } & 1.20 & 0.24 & 5.11 & \$ 92.37 \\ 4,536 & \text { sqft } & 1.20 & 0.04 & 163.30 & \$ 76.74 \\ 194 & \text { Inft } & 1.20 & 0.07 & 13.94 & \$ 76.74 \\ 4,536 & \text { sqft } & 1.20 & 0.00 & 21.77 & \$ 76.74\end{array}$

$\$ 1,362.08$
$\$ 3.25$
$\$ 2.50$
$\$ 136.24$
$\$ 0.01$
$\$ 1.40$
$\$ 0.03$
$\$ 5.00$
$\$ 0.03$

$\$ 0.00$
$\$ 0.00$
$\$ 0.00$
$\$ 0.00$
$\$ 0.00$
$\$ 0.00$
$\$ 0.00$
$\$ 0.00$
$\$ 0.00$

$\$ 3,678$
$\$ 3,275$
$\$ 1,047$
$\$ 6,092$
$\$ 8,354$
$\$ 472$
$\$ 12,531$
$\$ 1,070$
$\$ 1,671$

$\$ 2,260$
$\$ 293$
$\$ 473$
$\$ 6,008$
$\$ 45$
$\$ 30$
$\$ 136$
$\$ 968$
$\$ 136$

$\begin{array}{lr}\$ 0 & \$ 5,937 \\ \$ 0 & \$ 3,569 \\ \$ 0 & \$ 1,520 \\ \$ 0 & \$ 12,100 \\ \$ 0 & \$ 8,400 \\ \$ 0 & \$ 502 \\ \$ 0 & \$ 12,667 \\ \$ 0 & \$ 2,038 \\ \$ 0 & \$ 1,807\end{array}$


Client: INL

Project: FFC

URS

Washington Division
Date: $7 / 17 / 2008$

11:37AM Priced By: ADC

Office: Denver

Product Line: Gov
Estimate: FFC

Account: 04 Concrete/Masonry

Job No: $\quad 27989-325 \quad$ Rev No: 0

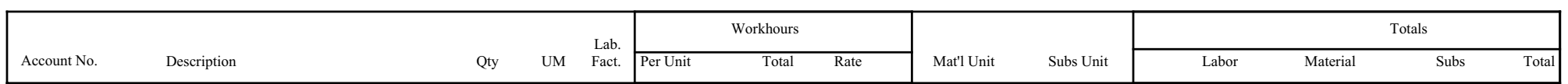

\begin{tabular}{|l|rl|}
\hline \multicolumn{4}{|c|}{ CONCRETE SOG - BY VOLUME Sidewalk to BIdg } \\
Formwork & 2.05 & SF/CY \\
Rebar & 75.24 & $\mathrm{LB} / \mathrm{CY}$ \\
Embedments & 4.29 & $\mathrm{LB} / \mathrm{CY}$ \\
\hline
\end{tabular}

\begin{tabular}{rr|}
\hline Wkhr/CY & $\mathbf{1 1 . 0 4}$ \\
Total/CY & $\mathbf{\$ 1 , 1 0 0 . 6 8}$ \\
\hline
\end{tabular}

\begin{tabular}{|c|c|c|c|c|c|c|c|c|c|}
\hline Total & $\begin{array}{l}\text { CONCRETE SOG - BY VOLUME Sidewalk to } \\
\text { BIdg }\end{array}$ & $44 \mathrm{CY}$ & 1.20 & 11.04 & $\$ 78.44$ & $\begin{array}{r}\$ 38,191 \\
\$ 866.00 / C Y\end{array}$ & $\begin{array}{c}\$ 10,349 \\
\$ 234.68 / C Y\end{array}$ & $\begin{array}{c}\text { so } \\
\$ \mathbf{s 0 . 0 0} / \mathrm{CY}\end{array}$ & $\$ 48,540$ \\
\hline
\end{tabular}

CONCRETE SOG - BY VOLUME Slab under Office Section

$\begin{array}{ll}040702 & \text { REBAR @ SOG } \\ 040703 & \text { FORMWORK @ SOG } \\ 040704 & \text { EMBEDS @ SOG } \\ 040708 & \text { 4000PSI@SOG PUMPED } \\ 040709 & \text { FINISHING @ SOG } \\ 040705 & \text { ANCHOR BOLTS @ SOG } \\ 040709 & \text { FINE GRADE @ SOG } \\ 040706 & \text { WATERSTOP @ SOG } \\ 040710 & \text { HARDENER @ SOG }\end{array}$

$\begin{array}{rrrrrr}4 & \text { ton } & 1.20 & 24.00 & 93.85 & \$ 92.37 \\ 213 & \text { sqft } & 1.20 & 0.48 & 102.17 & \$ 75.57 \\ 446 & \text { lb } & 1.20 & 0.06 & 26.73 & \$ 92.37 \\ 104 & \text { cuyd } & 1.20 & 1.80 & 187.11 & \$ 76.74 \\ 5,346 & \text { sqft } & 1.20 & 0.02 & 128.30 & \$ 76.74 \\ 50 & \text { lb } & 1.20 & 0.24 & 12.05 & \$ 92.37 \\ 5,346 & \text { sqft } & 1.20 & 0.04 & 192.46 & \$ 76.74 \\ 456 & \text { Inft } & 1.20 & 0.07 & 32.86 & \$ 76.74 \\ 5,346 & \text { sqft } & 1.20 & 0.00 & 25.66 & \$ 76.74\end{array}$

$\$ 1,362.08$
$\$ 3.25$
$\$ 2.50$
$\$ 136.24$
$\$ 0.01$
$\$ 1.40$
$\$ 0.03$
$\$ 5.00$
$\$ 0.03$

$\begin{array}{lr}\$ 0.00 & \$ 8,669 \\ \$ 0.00 & \$ 7,721 \\ \$ 0.00 & \$ 2,469 \\ \$ 0.00 & \$ 14,359 \\ \$ 0.00 & \$ 9,846 \\ \$ 0.00 & \$ 1,113 \\ \$ 0.00 & \$ 14,769 \\ \$ 0.00 & \$ 2,522 \\ \$ 0.00 & \$ 1,969\end{array}$

$\$ 5,326$
$\$ 692$
$\$ 1,114$
$\$ 14,162$
$\$ 53$
$\$ 70$
$\$ 160$
$\$ 2,282$
$\$ 160$

$\begin{array}{lr}\$ 0 & \$ 13,995 \\ \$ 0 & \$ 8,413 \\ \$ 0 & \$ 3,583 \\ \$ 0 & \$ 28,521 \\ \$ 0 & \$ 9,899 \\ \$ 0 & \$ 1,184 \\ \$ 0 & \$ 14,929 \\ \$ 0 & \$ 4,804 \\ \$ 0 & \$ 2,130\end{array}$


Client: INL

Project: FFC

URS

Washington Division
Date: $7 / 17 / 2008$

11:37AM Priced By: ADC

Office: Denver

Product Line: Gov
Estimate: FFC

Account: 04 Concrete/Masonry

Facility: Entire Estimate

Job No: $\quad 27989-325 \quad$ Rev No: 0

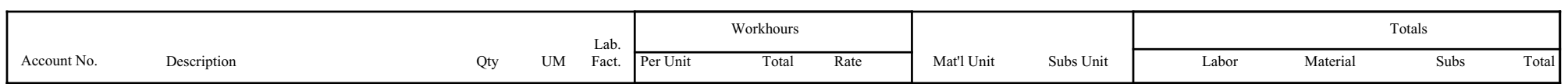

CONCRETE SOG - BY VOLUME Slab under Office Section

$\begin{array}{lrl}\text { Formwork } & \mathbf{2 . 0 5} & \mathrm{SF} / \mathrm{CY} \\ \text { Rebar } & \mathbf{7 5 . 2 4} & \mathrm{LB} / \mathrm{CY} \\ \text { Embedments } & \mathbf{4 . 2 9} & \mathrm{LB} / \mathrm{CY}\end{array}$

\begin{tabular}{|cr|}
\hline $\mathrm{Wkhr} / \mathrm{CY}$ & $\mathbf{7 . 7 1}$ \\
$\mathrm{Total} / \mathrm{CY}$ & $\mathbf{\$ 8 4 1 . 3 4}$ \\
\hline
\end{tabular}

Total CONCRETE SOG - BY VOLUME Slab under

$104 \mathrm{CY}$ Office Section

\begin{tabular}{lll|l}
104 & $\mathrm{CY}$ & $\mathbf{1 . 2 0}$ & $\mathbf{7 . 7 1}$
\end{tabular}

CONCRETE SOG - BY VOLUME Warehouse

$\begin{array}{ll}040702 & \text { REBAR @ SOG } \\ 040703 & \text { FORMWORK @ SOG } \\ 040704 & \text { EMBEDS @ SOG } \\ 040708 & \text { 4000PSI@SOG PUMPED } \\ 040709 & \text { FINISHING @ SOG } \\ 040705 & \text { ANCHOR BOLTS @ SOG } \\ 040709 & \text { FINE GRADE @ SOG } \\ 040706 & \text { WATERSTOP @ SOG } \\ 040710 & \text { HARDENER @ SOG }\end{array}$

$\begin{array}{rrrrrr}3 & \text { ton } & 1.20 & 24.00 & 72.05 & \$ 92.37 \\ 163 & \text { sqft } & 1.20 & 0.48 & 78.43 & \$ 75.57 \\ 342 & \text { lb } & 1.20 & 0.06 & 20.52 & \$ 92.37 \\ 80 & \text { cuyd } & 1.20 & 1.80 & 143.64 & \$ 76.74 \\ 4,104 & \text { sqft } & 1.20 & 0.02 & 98.50 & \$ 76.74 \\ 39 & \text { lb } & 1.20 & 0.24 & 9.25 & \$ 92.37 \\ 4,104 & \text { sqft } & 1.20 & 0.04 & 147.74 & \$ 76.74 \\ 350 & \text { Inft } & 1.20 & 0.07 & 25.23 & \$ 76.74 \\ 4,104 & \text { sqft } & 1.20 & 0.00 & 19.70 & \$ 76.74\end{array}$

$\$ 1,362.08$
$\$ 3.25$
$\$ 2.50$
$\$ 136.24$
$\$ 0.01$
$\$ 1.40$
$\$ 0.03$
$\$ 5.00$
$\$ 0.03$

$\$ 0.00$
$\$ 0.00$
$\$ 0.00$
$\$ 0.00$
$\$ 0.00$
$\$ 0.00$
$\$ 0.00$
$\$ 0.00$
$\$ 0.00$

$\$ 6,655$
$\$ 5,927$
$\$ 1,895$
$\$ 11,023$
$\$ 7,559$
$\$ 855$
$\$ 11,338$
$\$ 1,936$
$\$ 1,512$

$\$ 4,089$
$\$ 531$
$\$ 855$
$\$ 10,872$
$\$ 41$
$\$ 54$
$\$ 123$
$\$ 1,752$
$\$ 123$

$\begin{array}{rr}\$ 0 & \$ 10,744 \\ \$ 0 & \$ 6,458 \\ \$ 0 & \$ 2,750 \\ \$ 0 & \$ 21,895 \\ \$ 0 & \$ 7,600 \\ \$ 0 & \$ 909 \\ \$ 0 & \$ 11,461 \\ \$ 0 & \$ 3,688 \\ \$ 0 & \$ 1,635\end{array}$




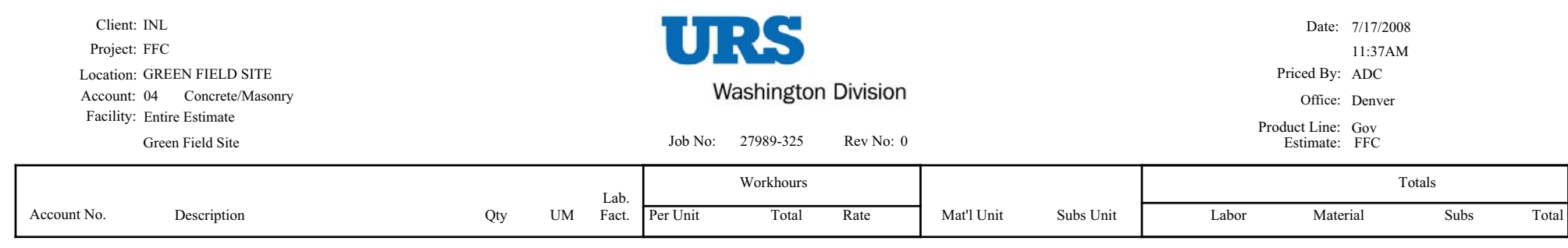

\begin{tabular}{|llrl}
\multicolumn{4}{c}{ CONCRETE SOG - BY VOLUME Warehouse } \\
Formwork & 2.05 & SF/CY \\
Rebar & 75.24 & $\mathrm{LB} / \mathrm{CY}$ \\
Embedments & 4.29 & $\mathrm{LB} / \mathrm{CY}$ \\
\hline
\end{tabular}

\begin{tabular}{rr}
\hline Wkhr/CY & 7.71 \\
Total/CY & $\$ 841.34$
\end{tabular}

\begin{tabular}{|c|c|c|c|c|c|c|c|c|c|c|}
\hline Total & CONCRETE SOG - BY VOLUME Warehouse & $80 \mathrm{CY}$ & 1.20 & 7.71 & 615 & $\$ 79.18$ & $\begin{array}{r}\$ 48,699 \\
\$ 610.26 / C Y\end{array}$ & $\begin{array}{c}\$ 18,440 \\
\$ 231.08 / C Y\end{array}$ & $\begin{array}{c}\text { so } \\
\$ 0.00 / \mathrm{CY}\end{array}$ & $\$ 67,139$ \\
\hline
\end{tabular}

\begin{tabular}{|c|c|c|c|c|}
\hline \multicolumn{5}{|c|}{ Ductbank by Dimension (2.5' X 2.5' X 500') } \\
\hline 040802 & \multicolumn{4}{|c|}{ REBAR@DUCTBANK } \\
\hline 040803 & \multicolumn{4}{|c|}{ FORMWORK@DUCTBANK } \\
\hline 040808 & \multicolumn{4}{|c|}{ 4000PSI@DUCTBANK PUMPED } \\
\hline \multirow[t]{7}{*}{040810} & \multicolumn{4}{|c|}{ ADDED COLOR@DUCTBANK } \\
\hline & \multicolumn{4}{|c|}{ Ductbank by Dimension (2.5' X 2.5' X 500') } \\
\hline & \multirow{3}{*}{$\begin{array}{l}\text { Formwork } \\
\text { Rebar } \\
\text { Embedments }\end{array}$} & & 21.90 & $\mathrm{SF} / \mathrm{CY}$ \\
\hline & & & 46.67 & $\mathrm{LB} / \mathrm{CY}$ \\
\hline & & & 0.00 & $\mathrm{LB} / \mathrm{CY}$ \\
\hline & & $\mathrm{Wkhr} / \mathrm{CY}$ & 12. & \\
\hline & & Total/CY & $\$ 1,277$. & \\
\hline
\end{tabular}

\begin{tabular}{|c|c|c|c|c|c|c|c|c|c|c|}
\hline Total & Ductbank by Dimension (2.5' X 2.5' X 500') & $122 \mathrm{CY}$ & 1.20 & 12.98 & 1,578 & $\$ 76.47$ & $\begin{array}{r}\$ 120,646 \\
\$ 992.75 / \mathrm{CY}\end{array}$ & $\begin{array}{c}\$ 34,588 \\
\$ 284.61 / C Y\end{array}$ & $\begin{array}{c}\$ 0 \\
\$ 0.00 / C Y\end{array}$ & $\$ 155,234$ \\
\hline
\end{tabular}

\begin{tabular}{|c|c|c|c|c|c|c|c|c|c|c|c|c|}
\hline \multicolumn{13}{|c|}{ Elevated Slab on Metal Deck- by Volume } \\
\hline 043202 & REBAR@ ELEV SLAB ON DECK & 7 & ton 1.20 & 24.00 & 160.92 & $\$ 92.37$ & $\$ 1,362.08$ & $\$ 0.00$ & $\$ 14,864$ & $\$ 9,133$ & $\$ 0$ & $\$ 23,997$ \\
\hline
\end{tabular}


Client: INL

Project: FFC

Location: GREEN FIELD SITE

Account: 04 Concrete/Masonry

URS

Date: 7/17/2008

11:37AM

Facility: Entire Estimate

Washington Division

Priced By: ADC

Office: Denver

Green Field Site

Job No: $\quad 27989-325 \quad$ Rev No: 0

Product Line: Gov
Estimate: FFC

\begin{tabular}{|c|c|c|c|c|c|c|c|c|c|c|c|c|c|c|}
\hline \multirow[b]{2}{*}{ Account No. } & \multirow[b]{2}{*}{ Description } & \multirow[b]{2}{*}{ Qty } & \multirow[b]{2}{*}{ UM } & \multirow{2}{*}{\multicolumn{2}{|c|}{$\begin{array}{l}\text { Lab. } \\
\text { Fact. }\end{array}$}} & \multicolumn{3}{|c|}{ Workhours } & \multirow[b]{2}{*}{ Mat'l Unit } & \multirow[b]{2}{*}{ Subs Unit } & \multicolumn{4}{|c|}{ Totals } \\
\hline & & & & & & Per Unit & Total & Rate & & & Labor & Material & Subs & Total \\
\hline 43203 & FORMWORK@ELEV SLAB ON DECK & 304 & & sqft & 1.20 & 0.48 & 146.02 & $\$ 75.57$ & $\$ 3.00$ & $\$ 0.00$ & $\$ 11,034$ & $\$ 913$ & $\$ 0$ & $\$ 11,947$ \\
\hline 043204 & EMBEDS @ ELEV SLAB ON DECK & 1,080 & & $\mathrm{lb}$ & 1.20 & 0.07 & 77.76 & $\$ 92.37$ & $\$ 2.50$ & $\$ 0.00$ & $\$ 7,183$ & $\$ 2,700$ & $\$ 0$ & $\$ 9,883$ \\
\hline 043208 & 4000PSI@ESOMD PUMPED & 95 & & cuyd & 1.20 & 2.74 & 258.55 & $\$ 76.74$ & $\$ 136.24$ & $\$ 0.00$ & $\$ 19,841$ & $\$ 12,875$ & $\$ 0$ & $\$ 32,716$ \\
\hline 43209 & FINISHING,CURE,HRDNR@ESOMD & 4,860 & & sqft & 1.20 & 0.04 & 174.96 & $\$ 76.74$ & $\$ 0.01$ & $\$ 0.00$ & $\$ 13,426$ & $\$ 49$ & $\$ 0$ & $\$ 13,475$ \\
\hline 043211 & GROUT @ ESOMD & 34 & & cuft & 1.20 & 2.26 & 76.02 & $\$ 76.74$ & $\$ 60.00$ & $\$ 0.00$ & $\$ 5,834$ & $\$ 2,022$ & $\$ 0$ & $\$ 7,855$ \\
\hline
\end{tabular}

\begin{tabular}{|lrl|}
\hline \multicolumn{4}{|c}{ Elevated Slab on Metal Deck- by Volume } \\
Formwork & $\mathbf{3 . 2 2}$ & $\mathrm{SF} / \mathrm{CY}$ \\
Rebar & $\mathbf{1 4 1 . 9 0}$ & $\mathrm{LB} / \mathrm{CY}$ \\
Embedments & $\mathbf{1 1 . 4 3}$ & $\mathrm{LB} / \mathrm{CY}$ \\
\hline
\end{tabular}

\begin{tabular}{rr|}
\hline Wkhr/CY & $\mathbf{9 . 4 6}$ \\
Total/CY & $\mathbf{\$ 1 , 0 5 6 . 8 5}$ \\
\hline
\end{tabular}

\begin{tabular}{|c|c|c|c|c|c|c|c|c|c|c|}
\hline Total & Elevated Slab on Metal Deck- by Volume & $95 \mathrm{CY}$ & 1.20 & 9.46 & 894 & $\$ 80.72$ & $\begin{array}{r}\$ 72,182 \\
\$ 763.83 / C Y\end{array}$ & $\begin{array}{c}\$ 27,690 \\
\$ 293.02 / C Y\end{array}$ & $\begin{array}{c}\text { \$0 } \\
\text { \$0.00 } / \mathrm{CY}\end{array}$ & $\$ 99,872$ \\
\hline
\end{tabular}

\begin{tabular}{|c|c|}
\hline \multicolumn{2}{|c|}{ Elevated Slab on Metal Deck- by Volume HVAC Filter } \\
\hline 043202 & REBAR@ELEV SLAB ON DECK \\
\hline 043203 & FORMWORK@ELEV SLAB ON DECK \\
\hline 043204 & EMBEDS@ ELEV SLAB ON DECK \\
\hline 043208 & 4000PSI@ESOMD PUMPED \\
\hline 043209 & FINISHING,CURE,HRDNR@ESOMD \\
\hline 043211 & GROUT@ ESOMD \\
\hline
\end{tabular}

$\begin{array}{rrrrrr}6 & \text { ton } 1.20 & 24.00 & 146.62 & \$ 92.37 \\ 277 & \text { sqft } & 1.20 & 0.48 & 133.04 & \$ 75.57 \\ 984 & \text { lb } & 1.20 & 0.07 & 70.85 & \$ 92.37 \\ 86 & \text { cuyd } 1.20 & 2.74 & 235.57 & \$ 76.74 \\ 4,428 & \text { sqft } & 1.20 & 0.04 & 159.41 & \$ 76.74 \\ 31 & \text { cuft } 1.20 & 2.26 & 69.26 & \$ 76.74\end{array}$

$\$ 1,362.08$
$\$ 3.00$
$\$ 2.50$
$\$ 136.24$
$\$ 0.01$
$\$ 60.00$

$\begin{array}{lr}\$ 0.00 & \$ 13,543 \\ \$ 0.00 & \$ 10,054 \\ \$ 0.00 & \$ 6,544 \\ \$ 0.00 & \$ 18,077 \\ \$ 0.00 & \$ 12,233 \\ \$ 0.00 & \$ 5,315\end{array}$

$\$ 8,321$
$\$ 831$
$\$ 2,460$
$\$ 11,730$
$\$ 44$
$\$ 1,842$

$\begin{array}{lr}\$ 0 & \$ 21,864 \\ \$ 0 & \$ 10,885 \\ \$ 0 & \$ 9,004 \\ \$ 0 & \$ 29,808 \\ \$ 0 & \$ 12,277\end{array}$

FINISHING,CURE,H
GROUT @ ESOMD

31 cuft 1.20 


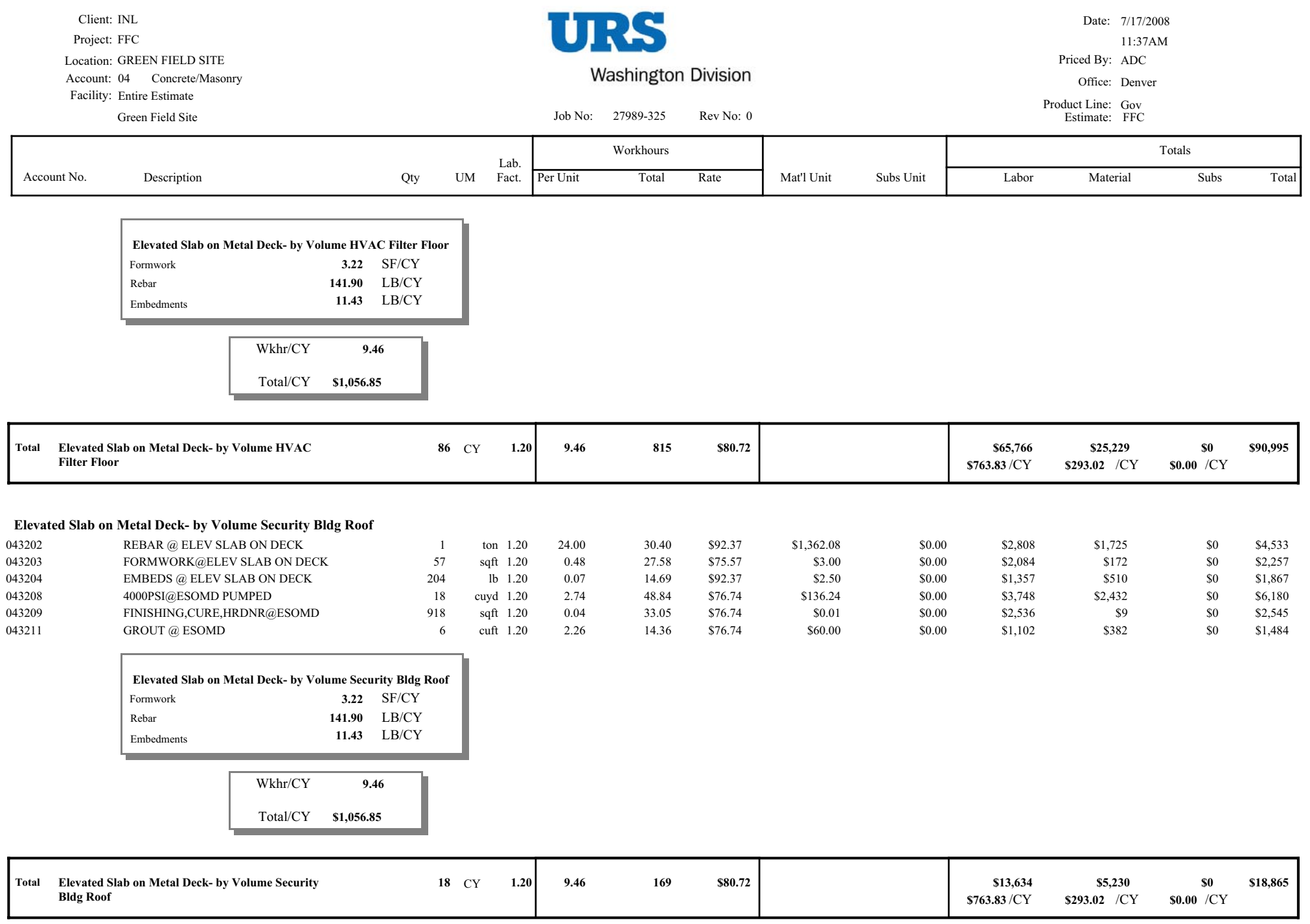


Client: INL

Project: FFC

URS

Washington Division
Date: $7 / 17 / 2008$

11:37AM Priced By: ADC

Office: Denver

Product Line: Gov

Account: 04 Concrete/Masonry

Job No: $\quad 27989-325 \quad$ Rev No: 0

\begin{tabular}{|c|c|c|c|c|c|c|c|c|c|c|c|c|}
\hline \multirow[b]{2}{*}{ Account No. } & \multirow[b]{2}{*}{ Description } & \multirow[b]{2}{*}{ Qty } & \multirow[b]{2}{*}{ UM } & \multirow{2}{*}{$\begin{array}{l}\text { Lab. } \\
\text { Fact. }\end{array}$} & \multicolumn{2}{|c|}{ Workhours } & \multirow[b]{2}{*}{ Mat'l Unit } & \multirow[b]{2}{*}{ Subs Unit } & \multicolumn{4}{|c|}{ Totals } \\
\hline & & & & & Per Unit & Total & & & Labor & Material & Subs & Total \\
\hline
\end{tabular}

Fence/Fence lighting Pole

$22 \quad$ cuyd 1.20

12.00

$264.00 \quad \$ 76.74$

$\$ 0.00$

$\$ 0.00$

$\$ 20,259$

$\$ 0$

$\$ 0 \$ 20,259$

\begin{tabular}{|lrl|}
\hline \multicolumn{3}{|c|}{ Fence/Fence lighting Pole } \\
Formwork & $\mathbf{0 . 0 0}$ & $\mathrm{SF} / \mathrm{CY}$ \\
Rebar & $\mathbf{0 . 0 0}$ & $\mathrm{LB} / \mathrm{CY}$ \\
Embedments & $\mathbf{0 . 0 0}$ & $\mathrm{LB} / \mathrm{CY}$ \\
\hline
\end{tabular}

\begin{tabular}{rr|} 
Wkhr/CY & $\mathbf{0 . 0 0}$ \\
Total/CY & $\mathbf{\$ 0 . 0 0}$ \\
\hline
\end{tabular}

Total Fence/Fence lighting Pole

o $\quad \mathrm{CY}$

\begin{tabular}{l|l}
1.20 & 0.00 \\
\hline
\end{tabular}

264

$\$ 76.74$

(2)

$\$ 20,259$ $\$ 0.00 / \mathrm{CY}$

/CY $\$ \mathbf{\$ 0 . 0 0 / C Y}$

$\begin{array}{rrrrrr}283 & \text { ton } 1.20 & 24.00 & 6,792.58 & \$ 92.37 \\ 12,768 & \text { sqft } 1.20 & 0.48 & 6,128.64 & \$ 75.57 \\ 8,512 & \text { Ib } & 1.20 & 0.06 & 510.72 & \$ 76.74 \\ 4,469 & \text { cuyd } 1.20 & 1.80 & 8,043.84 & \$ 76.74 \\ 76,565 & \text { sqft } 1.20 & 0.02 & 1,837.57 & \$ 76.74 \\ 2,500 & \text { Ib } & 1.20 & 0.24 & 599.99 & \$ 76.74 \\ 76,565 & \text { sqft } 1.20 & 0.04 & 2,756.36 & \$ 75.57 \\ 521 & \text { Inft } 1.20 & 0.07 & 37.54 & \$ 75.57 \\ 78 & \text { cuft } 1.20 & 4.80 & 374.40 & \$ 76.74\end{array}$

$\$ 1,362.08$
$\$ 3.00$
$\$ 2.50$
$\$ 136.24$
$\$ 0.01$
$\$ 1.40$
$\$ 0.03$
$\$ 5.00$
$\$ 60.00$

$\$ 0.00$
$\$ 0.00$
$\$ 0.00$
$\$ 0.00$
$\$ 0.00$
$\$ 0.00$
$\$ 0.00$
$\$ 0.00$
$\$ 0.00$
$\$ 627,417$
$\$ 463,138$
$\$ 39,192$
$\$ 617,278$
$\$ 141,014$
$\$ 46,043$
$\$ 208,296$
$\$ 2,837$

$\$ 28,731$

$\$ 385,501$
$\$ 38,304$
$\$ 21,280$
$\$ 608,829$
$\$ 766$
$\$ 3,500$
$\$ 2,297$
$\$ 2,607$
$\$ 4,680$

$\begin{array}{lr}\$ 0 & \$ 1,012,918 \\ \$ 0 & \$ 501,442 \\ \$ 0 & \$ 60,472 \\ \$ 0 & \$ 1,226,108 \\ \$ 0 & \$ 141,779 \\ \$ 0 & \$ 49,543 \\ \$ 0 & \$ 210,593 \\ \$ 0 & \$ 5,444 \\ \$ 0 & \$ 33,411\end{array}$ 


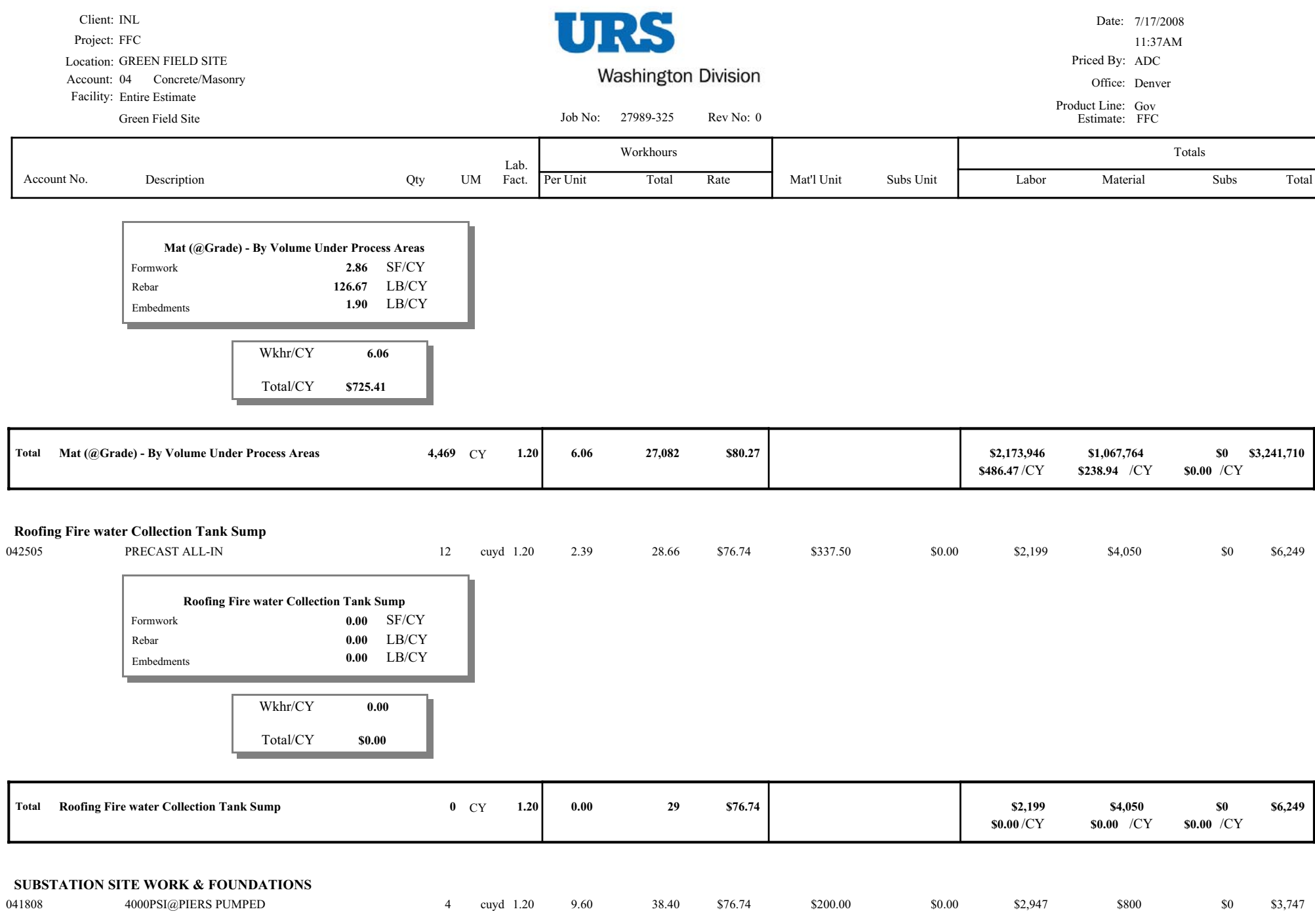


Client: INL

Project: FFC

Location: GREEN FIELD SITE

Account: 04 Concrete/Masonry

Facility: Entire Estimate

URS

Washington Division
Date: $7 / 17 / 2008$

$11: 37 \mathrm{AM}$ Priced By: ADC

Office: Denver

Product Line: Gov

Job No: $\quad 27989-325 \quad$ Rev No: 0

\begin{tabular}{|c|c|c|c|c|c|c|c|c|c|c|}
\hline \multirow[b]{2}{*}{ Description } & \multirow[b]{2}{*}{ Qty } & \multirow[b]{2}{*}{ UM } & \multirow{2}{*}{$\begin{array}{l}\text { Lab. } \\
\text { Fact. }\end{array}$} & & rkhours & \multirow[b]{2}{*}{ Mat'l Unit } & \multirow[b]{2}{*}{ Subs Unit } & \multicolumn{3}{|c|}{ Totals } \\
\hline & & & & Per Unit & Total & & & Labor & Material & Subs \\
\hline
\end{tabular}

SUBSTATION SITE WORK \& FOUNDATIONS

Formwork $\quad \mathbf{0 . 0 0} \quad \mathrm{SF} / \mathrm{CY}$

$\begin{array}{lll}\text { Rebar } & \mathbf{0 . 0 0} & \mathrm{LB} / \mathrm{CY}\end{array}$

$\begin{array}{lll}\text { Embedments } & \mathbf{0 . 0 0} & \text { LB/CY }\end{array}$

\begin{tabular}{|rr|}
\hline Wkhr/CY & $\mathbf{9 . 6 0}$ \\
& \\
Total/CY & $\$ \mathbf{9 3 6 . 7 0}$ \\
\hline
\end{tabular}

Total SUBST ATION SITE WORK \& FOUNDATIONS

\begin{tabular}{lll|ll}
4 & $\mathrm{CY}$ & 1.20 & 9.60 & 38
\end{tabular}

$\$ 76.74$

$\$ 2,947$

$\$ 736.70 / \mathrm{CY}$

$\$ \mathbf{\$ 2 0 0 . 0 0} / \mathrm{CY}$

$\$ 3,747$

Wall by Volume Greater than $8^{\prime}$ high Fire Water Collection Tank

$\begin{array}{ll}040902 & \text { REBAR @ WALLS } \\ 040903 & \text { FORMWORK @ WALLS OVER 8' HIGH } \\ 040904 & \text { EMBEDS @ WALLS } \\ 040908 & \text { 400OPSI@WALLS PUMPED } \\ 040909 & \text { FINISHING @ WALLS } \\ 040905 & \text { ANCHOR BOLTS @ WALLS } \\ 040906 & \text { WATERSTOP @ WALL } \\ 040911 & \text { GROUT @ WALLS }\end{array}$

$\begin{array}{rrrrrr}2 & \text { ton } & 1.20 & 24.00 & 49.50 & \$ 92.37 \\ 1,958 & \text { sqft } & 1.20 & 0.48 & 939.89 & \$ 75.57 \\ 317 & \text { lb } & 1.20 & 0.06 & 18.99 & \$ 92.37 \\ 26 & \text { cuyd } & 1.20 & 1.80 & 47.25 & \$ 76.74 \\ 1,632 & \text { sqft } & 1.20 & 0.02 & 39.16 & \$ 76.74 \\ 65 & \text { lb } & 1.20 & 0.24 & 15.70 & \$ 92.37 \\ 19 & \text { Inft } & 1.20 & 0.07 & 1.39 & \$ 76.74 \\ 1 & \text { cuft } & 1.20 & 4.80 & 3.74 & \$ 76.74\end{array}$

$\$ 1,362.08$
$\$ 3.00$
$\$ 2.50$
$\$ 136.24$
$\$ 0.01$
$\$ 1.40$
$\$ 5.00$
$\$ 60.00$

$\begin{array}{lr}\$ 0.00 & \$ 4,572 \\ \$ 0.00 & \$ 71,027 \\ \$ 0.00 & \$ 1,754 \\ \$ 0.00 & \$ 3,626 \\ \$ 0.00 & \$ 3,005 \\ \$ 0.00 & \$ 1,450 \\ \$ 0.00 & \$ 106 \\ \$ 0.00 & \$ 287\end{array}$

$\$ 2,809$
$\$ 5,874$
$\$ 791$
$\$ 3,576$
$\$ 16$
$\$ 92$
$\$ 96$
$\$ 47$

$\begin{array}{lr}\$ 0 & \$ 7,382 \\ \$ 0 & \$ 76,901 \\ \$ 0 & \$ 2,545 \\ \$ 0 & \$ 7,202 \\ \$ 0 & \$ 3,022 \\ \$ 0 & \$ 1,542 \\ \$ 0 & \$ 203 \\ \$ 0 & \$ 334\end{array}$


Client: INL

Project: FFC

URS

Washington Division
Date: $7 / 17 / 2008$

11:37AM Priced By: ADC

Office: Denver

Product Line: Gov
Estimate: FFC

Account: 04 Concrete/Masonry

Facility: Entire Estimate

Job No: $\quad 27989-325 \quad$ Rev No: 0

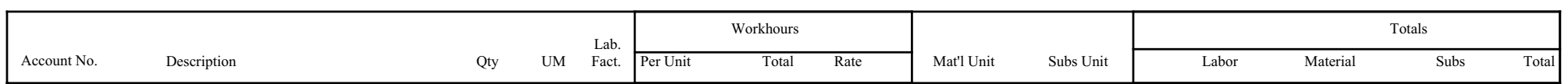

Wall by Volume Greater than $8^{\prime}$ high Fire Water Collection

$\begin{array}{lrrl}\text { Formwork } & \text { Tank } & \mathbf{7 4 . 5 9} & \mathrm{SF} / \mathrm{CY} \\ \text { Rebar } & & \mathbf{1 5 7 . 1 4} & \mathrm{LB} / \mathrm{CY}\end{array}$

Embedments $\quad \mathbf{1 2 . 0 6} \quad \mathrm{LB} / \mathrm{CY}$

$\mathrm{Wkhr} / \mathrm{CY} \quad \mathbf{4 2 . 5 0}$

Total/CY $\$ \mathbf{\$ 3 , 7 7 6 . 3 9}$

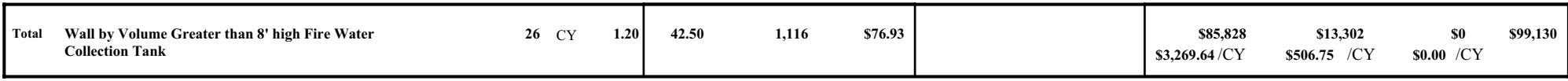

Wall by Volume Greater than 8' high Radiography Area

$\begin{array}{ll}040902 & \text { REBAR @ WALLS } \\ 040903 & \text { FORMWORK @ WALLS OVER 8' HIGH } \\ 040904 & \text { EMBEDS @ WALLS } \\ 040908 & \text { 400OPSI@WALLS PUMPED } \\ 040909 & \text { FINISHING @ WALLS } \\ 040905 & \text { ANCHOR BOLTS @ WALLS } \\ 040906 & \text { WATERSTOP @ WALL } \\ 040911 & \text { GROUT @ WALLS }\end{array}$

$\begin{array}{rrrrrr}39 & \text { ton } & 1.20 & 24.00 & 935.55 & \$ 92.37 \\ 51,030 & \text { sqft } & 1.20 & 0.48 & 24,494.40 & \$ 75.57 \\ 5,982 & \text { lb } & 1.20 & 0.06 & 358.91 & \$ 92.37 \\ 496 & \text { cuyd } & 1.20 & 1.80 & 893.03 & \$ 76.74 \\ 51,030 & \text { sqft } & 1.20 & 0.02 & 1,224.72 & \$ 76.74 \\ 1,236 & \text { lb } & 1.20 & 0.24 & 296.72 & \$ 92.37 \\ 364 & \text { Inft } & 1.20 & 0.07 & 26.20 & \$ 76.74 \\ 15 & \text { cuft } & 1.20 & 4.80 & 70.76 & \$ 76.74\end{array}$

$\$ 1,362.08$
$\$ 3.00$
$\$ 2.50$
$\$ 136.24$
$\$ 0.01$
$\$ 1.40$
$\$ 5.00$
$\$ 60.00$

$\begin{array}{lr}\$ 0.00 & \$ 86,415 \\ \$ 0.00 & \$ 1,851,028 \\ \$ 0.00 & \$ 33,152 \\ \$ 0.00 & \$ 68,530 \\ \$ 0.00 & \$ 93,984 \\ \$ 0.00 & \$ 27,408 \\ \$ 0.00 & \$ 2,010 \\ \$ 0.00 & \$ 5,430\end{array}$

$\$ 53,096$

$\$ 153,090$

$\$ 14,955$

$\$ 67,592$

$\$ 93,984 \quad \$ 510$

$\$ 27,408 \quad \$ 1,731$

$\$ 2,010 \quad \$ 1,819$

$\$ 1,819$

$\$ 48,107$
$\$ 0$

$\$ 0 \quad \$ 94,494$

$\$ 0 \quad \$ 29,139$

$\$ 0 \quad \$ 3,829$

$\$ 0 \quad \$ 6,315$ 
Client: INL

Project: FFC

URS

Date: 7/17/2008

11:37AM

Location: GREEN FIELD SITE

Washington Division

Priced By: ADC

Office: Denver

Facility: Entire Estimate

Job No: $27989-325 \quad$ Rev No: 0

Product Line: Gov
Estimate:

Green Field Site

\begin{tabular}{|c|c|c|c|c|c|c|c|c|c|c|c|c|}
\hline \multirow[b]{2}{*}{ Account No. } & \multirow[b]{2}{*}{ Description } & \multirow[b]{2}{*}{ Qty } & \multirow[b]{2}{*}{ UM } & \multirow{2}{*}{$\begin{array}{l}\text { Lab. } \\
\text { Fact. }\end{array}$} & \multicolumn{2}{|c|}{ Workhours } & \multirow[b]{2}{*}{ Mat'l Unit } & \multirow[b]{2}{*}{ Subs Unit } & \multicolumn{4}{|c|}{ Totals } \\
\hline & & & & & \begin{tabular}{|l} 
Per Unit \\
\end{tabular} & Total & & & Labor & Material & Subs & Total \\
\hline
\end{tabular}

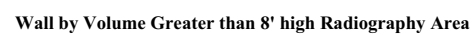

$\begin{array}{lll}\text { Formwork } & \mathbf{1 0 2 . 8 6} & \mathrm{SF} / \mathrm{CY}\end{array}$

Rebar $\quad \mathbf{1 5 7 . 1 4}$ LB/CY

Embedments $\quad \mathbf{1 2 . 0 6} \quad \mathrm{LB} / \mathrm{CY}$

Exh

Wkhr/CY $\quad \mathbf{5 7 . 0 4}$

Total/CY $\$ 4,961.72$ 
Client: INL

Project: FFC

Location: GREEN FIELD SITE

URS

Date: 7/17/2008

11:37AM

Account: 05 Structual Steel/Platework

Washington Division

Priced By: ADC

Office: Denver

Facility: Entire Estimate

Job No: $\quad 27989-325 \quad$ Rev No: 0

Product Line: Gov
Estimate: FFC

\begin{tabular}{|c|c|c|c|c|c|c|c|c|c|c|c|c|c|c|}
\hline \multirow[b]{2}{*}{ Account No. } & \multirow{2}{*}{\multicolumn{2}{|c|}{ Description }} & \multirow[b]{2}{*}{ Qty } & \multirow[b]{2}{*}{ UM } & \multirow{2}{*}{$\begin{array}{l}\text { Lab. } \\
\text { Fact. }\end{array}$} & \multicolumn{3}{|c|}{ Workhours } & \multirow[b]{2}{*}{ Mat'l Unit } & \multirow[b]{2}{*}{ Subs Unit } & \multicolumn{4}{|c|}{ Totals } \\
\hline & & & & & & Per Unit & Total & Rate & & & Labor & Material & Subs & Total \\
\hline \multicolumn{15}{|c|}{05 Structural Steel by Class } \\
\hline Structural Steel: Su & ub Total (incl xLight - $x$ & Hvy) & 43 & tons & 1.20 & 27.15 & 1,167 & $\$ 91.25$ & $\$ 4,462$ & & $\$ 106,512$ & $\$ 191,871$ & $\$ 0$ & $\$ 298,383$ \\
\hline \multicolumn{3}{|c|}{$05 \times 03^{* *}$ Light Steel $-5-20 \# / L F$} & 15 & tons & 1.20 & 33.30 & 500 & $\$ 91.25$ & $\$ 5,564$ & & $\$ 45,578$ & $\$ 83,465$ & $\$ 0$ & $\$ 129,043$ \\
\hline \multicolumn{15}{|c|}{ Light Steel - 5-20\#/LF } \\
\hline & $\$ 8,603$ & Total Cost/Ton & & & & & & & & & & & & \\
\hline \multirow[t]{3}{*}{$05 \times x 04 * *$} & Medium Steel - 20-40\#/I & & \multirow[t]{3}{*}{28} & \multirow[t]{3}{*}{ tons } & \multirow[t]{3}{*}{1.20} & \multirow[t]{3}{*}{23.85} & \multirow[t]{3}{*}{668} & \multirow[t]{3}{*}{$\$ 91.25$} & \multirow[t]{3}{*}{$\$ 3,872$} & & \multirow[t]{3}{*}{$\$ 60,934$} & \multirow[t]{3}{*}{$\$ 108,406$} & \multirow[t]{3}{*}{$\$ 0$} & \multirow[t]{3}{*}{$\$ 169,340$} \\
\hline & Mediun & n Steel - 20-40\#/LF & & & & & & & & & & & & \\
\hline & $\$ 6,048$ & Total Cost/Ton & & & & & & & & & & & & \\
\hline \multicolumn{3}{|c|}{ Minor Struct Steel : Sub Total (incl xLight - xHvy) } & 5 & tons & 1.20 & 48.00 & 242 & $\$ 91.25$ & $\$ 6,454$ & & $\$ 22,118$ & $\$ 32,594$ & $\$ 0$ & $\$ 54,712$ \\
\hline $05 \times x 03 * *$ & Light Steel - 5-20\#/LF & & 2 & tons & 1.20 & 48.00 & 108 & $\$ 91.25$ & $\$ 6,454$ & & $\$ 9,855$ & $\$ 14,522$ & $\$ 0$ & $\$ 24,377$ \\
\hline \multirow[t]{4}{*}{$05 \times x 04 * *$} & Medium Steel - 20-40\#/L & & 3 & tons & 1.20 & 48.00 & 134 & $\$ 91.25$ & $\$ 6,454$ & & $\$ 12,263$ & $\$ 18,072$ & $\$ 0$ & $\$ 30,335$ \\
\hline & \multicolumn{5}{|c|}{ Minor Struct Steel : Sub Total (incl xLight - xHvy) } & & & & & & & & & \\
\hline & 48.00 & Workhours/Ton & & & & & & & & & & & & \\
\hline & $\$ 10,834$ & Total Cost/Ton & & & & & & & & & & & & \\
\hline \multicolumn{3}{|l|}{ Decking and Flooring } & 9,345 & sqft & 1.20 & & 449 & $\$ 91.25$ & & & $\$ 40,931$ & $\$ 19,625$ & $\$ 0$ & $\$ 60,557$ \\
\hline
\end{tabular}

\begin{tabular}{|c|c|c|c|c|c|c|c|c|c|c|c|c|c|c|}
\hline \multirow[b]{2}{*}{ Account No. } & \multirow{2}{*}{\multicolumn{2}{|c|}{ Description }} & \multirow[b]{2}{*}{ Qty } & \multirow[b]{2}{*}{ UM } & \multirow{2}{*}{$\begin{array}{l}\text { Lab. } \\
\text { Fact. }\end{array}$} & \multicolumn{3}{|c|}{ Workhours } & \multirow[b]{2}{*}{ Mat'l Unit } & \multirow[b]{2}{*}{ Subs Unit } & \multicolumn{4}{|c|}{ Totals } \\
\hline & & & & & & Per Unit & Total & Rate & & & Labor & Material & Subs & Total \\
\hline \multicolumn{15}{|c|}{05 Structural Steel by Class } \\
\hline Structural Steel: Su & ub Total (incl xLight - $x$ & Hvy) & 43 & tons & 1.20 & 27.15 & 1,167 & $\$ 91.25$ & $\$ 4,462$ & & $\$ 106,512$ & $\$ 191,871$ & $\$ 0$ & $\$ 298,383$ \\
\hline \multicolumn{3}{|c|}{$05 \times 03^{* *}$ Light Steel $-5-20 \# / L F$} & 15 & tons & 1.20 & 33.30 & 500 & $\$ 91.25$ & $\$ 5,564$ & & $\$ 45,578$ & $\$ 83,465$ & $\$ 0$ & $\$ 129,043$ \\
\hline \multicolumn{15}{|c|}{ Light Steel - 5-20\#/LF } \\
\hline & $\$ 8,603$ & Total Cost/Ton & & & & & & & & & & & & \\
\hline \multirow[t]{3}{*}{$05 \times x 04 * *$} & Medium Steel - 20-40\#/I & & \multirow[t]{3}{*}{28} & \multirow[t]{3}{*}{ tons } & \multirow[t]{3}{*}{1.20} & \multirow[t]{3}{*}{23.85} & \multirow[t]{3}{*}{668} & \multirow[t]{3}{*}{$\$ 91.25$} & \multirow[t]{3}{*}{$\$ 3,872$} & & \multirow[t]{3}{*}{$\$ 60,934$} & \multirow[t]{3}{*}{$\$ 108,406$} & \multirow[t]{3}{*}{$\$ 0$} & \multirow[t]{3}{*}{$\$ 169,340$} \\
\hline & Mediun & n Steel - 20-40\#/LF & & & & & & & & & & & & \\
\hline & $\$ 6,048$ & Total Cost/Ton & & & & & & & & & & & & \\
\hline \multicolumn{3}{|c|}{ Minor Struct Steel : Sub Total (incl xLight - xHvy) } & 5 & tons & 1.20 & 48.00 & 242 & $\$ 91.25$ & $\$ 6,454$ & & $\$ 22,118$ & $\$ 32,594$ & $\$ 0$ & $\$ 54,712$ \\
\hline $05 \times x 03 * *$ & Light Steel - 5-20\#/LF & & 2 & tons & 1.20 & 48.00 & 108 & $\$ 91.25$ & $\$ 6,454$ & & $\$ 9,855$ & $\$ 14,522$ & $\$ 0$ & $\$ 24,377$ \\
\hline \multirow[t]{4}{*}{$05 \times x 04 * *$} & Medium Steel - 20-40\#/L & & 3 & tons & 1.20 & 48.00 & 134 & $\$ 91.25$ & $\$ 6,454$ & & $\$ 12,263$ & $\$ 18,072$ & $\$ 0$ & $\$ 30,335$ \\
\hline & \multicolumn{5}{|c|}{ Minor Struct Steel : Sub Total (incl xLight - xHvy) } & & & & & & & & & \\
\hline & 48.00 & Workhours/Ton & & & & & & & & & & & & \\
\hline & $\$ 10,834$ & Total Cost/Ton & & & & & & & & & & & & \\
\hline \multicolumn{3}{|l|}{ Decking and Flooring } & 9,345 & sqft & 1.20 & & 449 & $\$ 91.25$ & & & $\$ 40,931$ & $\$ 19,625$ & $\$ 0$ & $\$ 60,557$ \\
\hline
\end{tabular}

Decking and Flooring

9,345

449

$\$ 40,931$

$\$ 19,625$

$\$ 0 \$ 60,557$

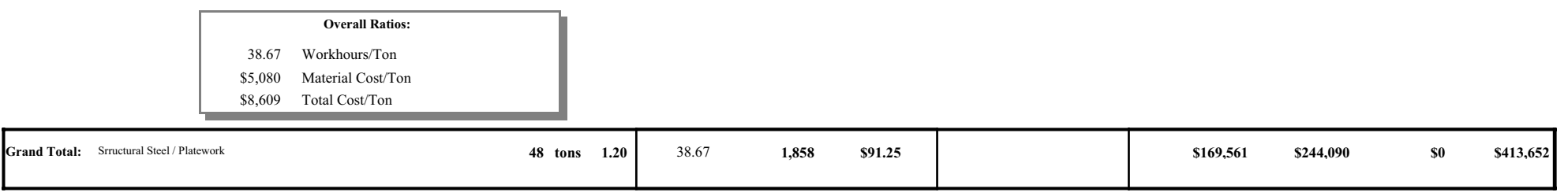


Client: INL

Project: FFC

URS

Washington Division

Account: 05 Structual Steel/Platework

Facility: Entire Estimate

Green Field Site

Job No: $27989-325 \quad$ Rev No: 0

Workhours

$\begin{array}{ll} & \text { Lab. } \\ \text { Qty UM Fact. }\end{array}$

Description

Per Unit

Per Unit Total Rate

\section{Structural Steel Summary \\ $\%$ of total $\%$ of commodity}

0501 Building Steel

$100.00 \%$

$$
\begin{aligned}
& \text { Light Steel - 5-20\#/LF } \\
& \text { Medium Steel - 20-40\#/LF }
\end{aligned}
$$

$35.90 \%$
$64.10 \%$

$48 \quad$ tons $\quad 1.20 \quad 29.34$

$\begin{array}{llll}48 & \text { tons } & 1.20 & 29.34 \\ 17 & \text { tons } & 1.20 & 35.22\end{array}$

31 tons $1.20 \quad 26.05$

$\$ 7,348$ Total Cost/Ton

0505 Decking and Flooring

$\begin{array}{rr}1,410 & \$ 91.25 \\ 608 & \$ 91.25\end{array}$

$\$ 91.25$

$\$ 91.25$

449
$\$ 4,671$
$\$ 5,680$

$\$ 4,106$

0.05

.05

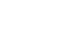

Date: 7/17/2008

11:37AM

Priced By: ADC

Office: Denver

\begin{tabular}{|c|c|c|c|c|c|}
\hline \multirow[b]{2}{*}{ at'l Unit } & \multirow[b]{2}{*}{ Subs Unit } & \multicolumn{4}{|c|}{ Totals } \\
\hline & & Labor & Material & Subs & Total \\
\hline
\end{tabular}

Product Line: Gov
Estimate: FFC

$\$ 4,671$
$\$ 5,680$
$\$ 4,106$

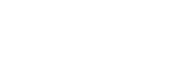




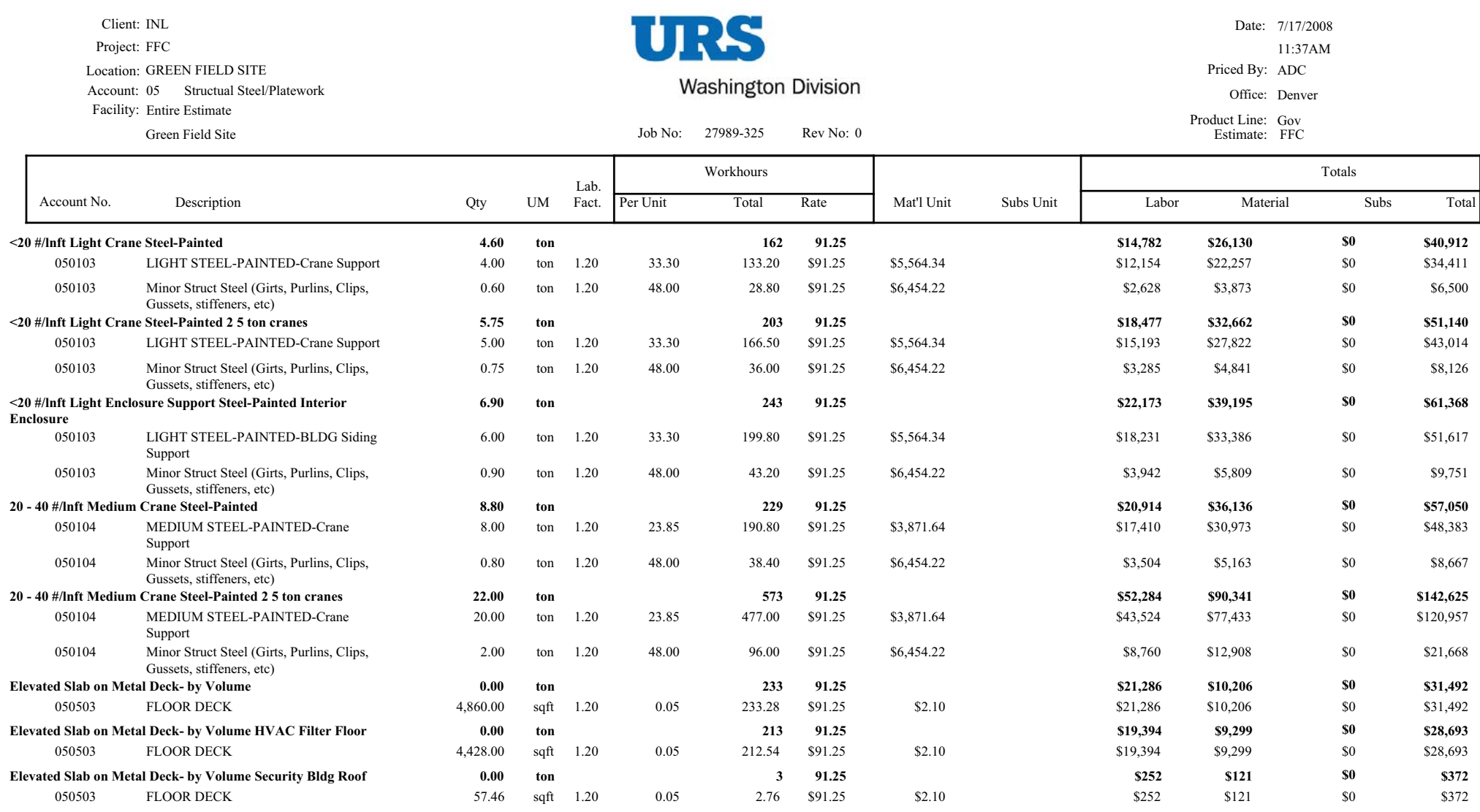

\begin{tabular}{|c|c|c|c|c|c|c|c|c|c|c|c|}
\hline & Steel Ratios: & $\begin{array}{c}\text { Entir } \\
38.67 \\
\$ 5,080 \\
\$ 8,609\end{array}$ & $\begin{array}{l}\text { Estimate } \\
\text { Workhours/Ton } \\
\text { Material Cost/Ton } \\
\text { Total Cost/Ton }\end{array}$ & & & & & & & & \\
\hline Totals & $\begin{array}{l}\text { Structual SteelPlatework } \\
\text { Entrire Estimate }\end{array}$ & & 48 Tons & 38.67 & 1,858 & $\$ 91.25$ & $5,079.92$ & $\$ 169,561$ & $\$ 244,090$ & so & $\$ 413,652$ \\
\hline
\end{tabular}




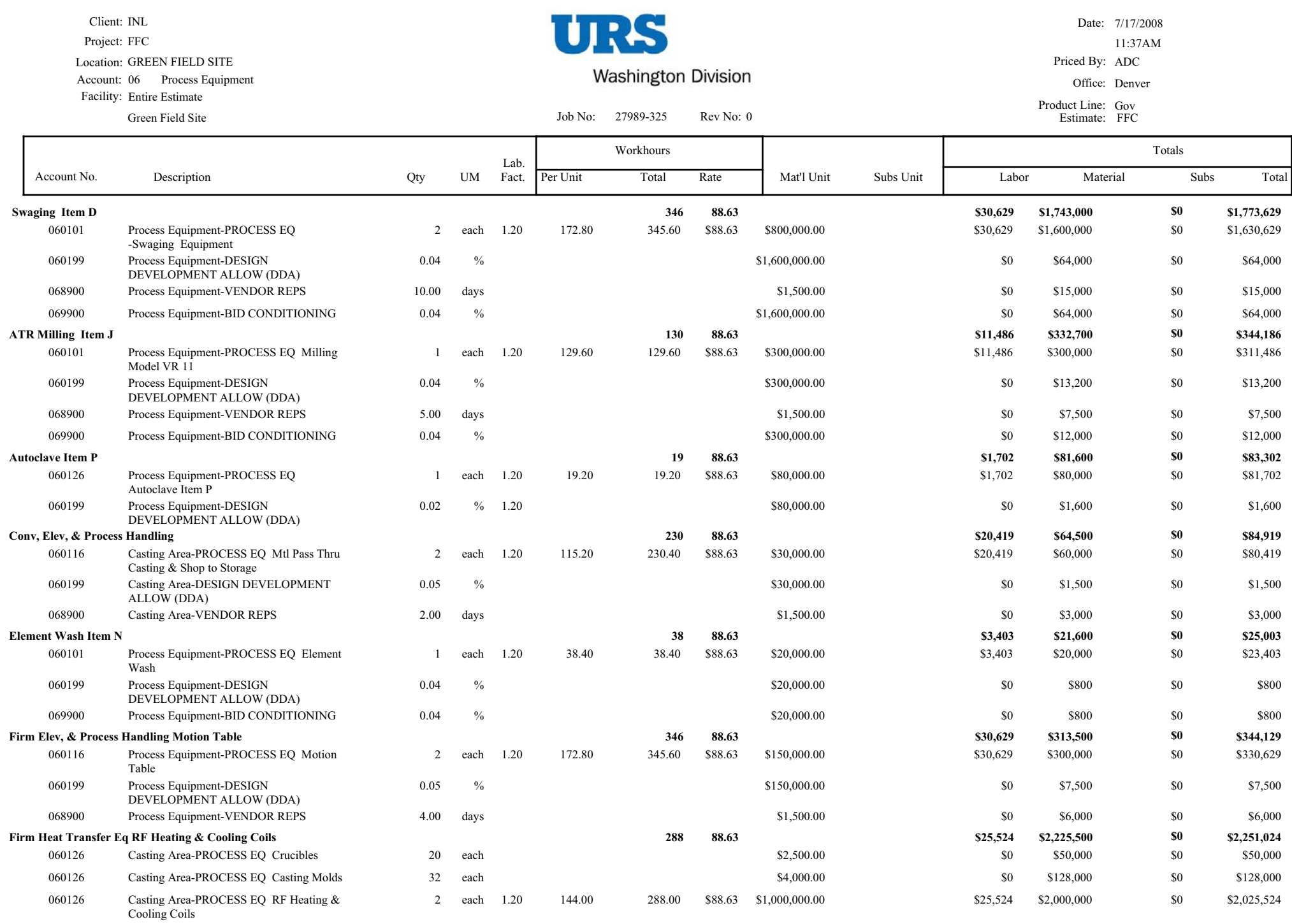


Client: INL

Project: FFC

Location: GREEN FIELD SITE

Account: 06 Process Equipment

URS

Date: $7 / 17 / 2008$

11:37AM

Washington Division

Priced By: ADC

Office: Denver

Facility: Entire Estimate

Job No: $\quad 27989-325 \quad$ Rev No: 0

Product Line: Gov
Estimate: FFC

\begin{tabular}{|c|c|c|c|c|c|c|c|c|c|c|c|c|c|}
\hline \multirow[b]{2}{*}{ Account No. } & \multirow[b]{2}{*}{ Description } & \multirow[b]{2}{*}{ Qty } & \multirow[b]{2}{*}{ UM } & \multirow{2}{*}{$\begin{array}{l}\text { Lab. } \\
\text { Fact. }\end{array}$} & \multicolumn{3}{|c|}{ Workhours } & \multirow[b]{2}{*}{ Mat'l Unit } & \multirow[b]{2}{*}{ Subs Unit } & \multicolumn{4}{|c|}{ Totals } \\
\hline & & & & & \begin{tabular}{|l|l} 
Per Unit \\
\end{tabular} & Total & Rate & & & Labor & Material & Subs & Total \\
\hline 060199 & $\begin{array}{l}\text { Casting Area-DESIGN DEVELOPMENT } \\
\text { ALLOW (DDA) }\end{array}$ & 0.02 & $\%$ & & & & & $\$ 2,000,000.00$ & & $\$ 0$ & $\$ 40,000$ & $\$ 0$ & $\$ 40,000$ \\
\hline 068900 & Casting Area-VENDOR REPS & 5.00 & days & & & & & $\$ 1,500.00$ & & $\$ 0$ & $\$ 7,500$ & $\$ 0$ & $\$ 7,500$ \\
\hline \multicolumn{2}{|c|}{ irm Price-Al Cladding Cleaning Station Item 29} & & & & & 38 & 88.63 & & & $\$ 3,403$ & $\$ 108,000$ & \$o & $\$ 111,403$ \\
\hline 060101 & $\begin{array}{l}\text { Process Equipment-PROCESS EQ Al } \\
\text { Cladding Cleaning Station }\end{array}$ & 1 & each & 1.20 & 38.40 & 38.40 & $\$ 88.63$ & $\$ 100,000.00$ & & $\$ 3,403$ & $\$ 100,000$ & $\$ 0$ & $\$ 103,403$ \\
\hline 060199 & $\begin{array}{l}\text { Process Equipment-DESIGN } \\
\text { DEVELOPMENT ALLOW (DDA) }\end{array}$ & 0.04 & $\%$ & & & & & $\$ 100,000.00$ & & $\$ 0$ & $\$ 4,000$ & $\$ 0$ & $\$ 4,000$ \\
\hline 069900 & Process Equipment-BID CONDITIONING & 0.04 & $\%$ & & & & & $\$ 100,000.00$ & & $\$ 0$ & $\$ 4,000$ & $\$ 0$ & $\$ 4,000$ \\
\hline \multicolumn{2}{|c|}{ irm Price-AL Cladding Washing Item 27} & & & & & 19 & 88.63 & & & $\$ 1,702$ & $\$ 10,800$ & $\$ 0$ & $\$ 12,502$ \\
\hline 060101 & $\begin{array}{l}\text { Process Equipment-PROCESS EQ } \\
\text { Washing Station }\end{array}$ & 1 & each & 1.20 & 19.20 & 19.20 & $\$ 88.63$ & $\$ 10,000.00$ & & $\$ 1,702$ & $\$ 10,000$ & $\$ 0$ & $\$ 11,702$ \\
\hline 060199 & $\begin{array}{l}\text { Process Equipment-DESIGN } \\
\text { DEVELOPMENT ALLOW (DDA) }\end{array}$ & 0.04 & $\%$ & & & & & $\$ 10,000.00$ & & $\$ 0$ & $\$ 400$ & $\$ 0$ & $\$ 400$ \\
\hline 069900 & Process Equipment-BID CONDITIONING & 0.04 & $\%$ & & & & & $\$ 10,000.00$ & & $\$ 0$ & $\$ 400$ & $\$ 0$ & $\$ 400$ \\
\hline \multicolumn{2}{|c|}{ irm Price-Annealing Furnace 24} & & & & & 43 & 88.63 & & & $\$ 3,829$ & $\$ 40,800$ & \$o & $\$ 44,629$ \\
\hline 060126 & $\begin{array}{l}\text { Process Equipment-PROCESS EQ } \\
\text { Annealing Furnace }\end{array}$ & 1 & each & 1.20 & 43.20 & 43.20 & $\$ 88.63$ & $\$ 40,000.00$ & & $\$ 3,829$ & $\$ 40,000$ & $\$ 0$ & $\$ 43,829$ \\
\hline 060199 & $\begin{array}{l}\text { Process Equipment-DESIGN } \\
\text { DEVELOPMENT ALLOW (DDA) }\end{array}$ & 0.02 & $\%$ & & & & & $\$ 40,000.00$ & & $\$ 0$ & $\$ 800$ & $\$ 0$ & $\$ 800$ \\
\hline \multicolumn{2}{|c|}{ irm Price-Argon Supply \& Distrib Sys } & & & & & 38 & 88.63 & & & $\$ 3,403$ & $\$ 42,500$ & \$o & $\$ 45,903$ \\
\hline 060150 & $\begin{array}{l}\text { Casting Area-PROCESS EQ Argon } \\
\text { Recycle System }\end{array}$ & 1 & each & 1.20 & 38.40 & 38.40 & $\$ 88.63$ & $\$ 40,000.00$ & & $\$ 3,403$ & $\$ 40,000$ & $\$ 0$ & $\$ 43,403$ \\
\hline 060199 & $\begin{array}{l}\text { Casting Area-DESIGN DEVELOPMENT } \\
\text { ALLOW (DDA) }\end{array}$ & 0.03 & $\%$ & 1.20 & & & & $\$ 40,000.00$ & & $\$ 0$ & $\$ 1,000$ & $\$ 0$ & $\$ 1,000$ \\
\hline 068900 & Casting Area-VENDOR REPS & 1.00 & days & 1.20 & & & & $\$ 1,500.00$ & & $\$ 0$ & $\$ 1,500$ & $\$ 0$ & $\$ 1,500$ \\
\hline \multicolumn{2}{|c|}{ irm Price-Canning Machines Item 33} & & & & & 77 & 88.63 & & & $\$ 6,806$ & $\$ 165,000$ & \$o & $\$ 171,806$ \\
\hline 060101 & $\begin{array}{l}\text { Process Equipment-PROCESS EQ Canning } \\
\text { Machine } 33\end{array}$ & 1 & each & 1.20 & 76.80 & 76.80 & $\$ 88.63$ & $\$ 150,000.00$ & & $\$ 6,806$ & $\$ 150,000$ & $\$ 0$ & $\$ 156,806$ \\
\hline 060199 & $\begin{array}{l}\text { Process Equipment-DESIGN } \\
\text { DEVELOPMENT ALLOW (DDA) }\end{array}$ & 0.04 & $\%$ & & & & & $\$ 150,000.00$ & & $\$ 0$ & $\$ 6,000$ & $\$ 0$ & $\$ 6,000$ \\
\hline 068900 & Process Equipment-VENDOR REPS & 2.00 & days & & & & & $\$ 1,500.00$ & & $\$ 0$ & $\$ 3,000$ & $\$ 0$ & $\$ 3,000$ \\
\hline 069900 & Process Equipment-BID CONDITIONING & 0.04 & $\%$ & & & & & $\$ 150,000.00$ & & $\$ 0$ & $\$ 6,000$ & $\$ 0$ & $\$ 6,000$ \\
\hline \multicolumn{2}{|c|}{ irm Price-Conv, Cranes 25 ton Cap Units } & & & & & 192 & 88.63 & & & $\$ 17,016$ & $\$ 136,620$ & \$o & $\$ 153,636$ \\
\hline 060116 & $\begin{array}{l}\text { Mechanical Equipment-PROCESS EQ } \\
\text { Cranes } 5 \text { ton cal } 30^{\prime} \text { span }\end{array}$ & 2 & each & 1.20 & 96.00 & 192.00 & $\$ 88.63$ & $\$ 62,200.00$ & & $\$ 17,016$ & $\$ 124,400$ & $\$ 0$ & $\$ 141,416$ \\
\hline 060199 & $\begin{array}{l}\text { Mechanical Equipment-DESIGN } \\
\text { DEVELOPMENT ALLOW (DDA) }\end{array}$ & 0.05 & $\%$ & & & & & $\$ 124,400.00$ & & $\$ 0$ & $\$ 6,220$ & $\$ 0$ & $\$ 6,220$ \\
\hline 068900 & Mechanical Equipment-VENDOR REPS & 4.00 & days & & & & & $\$ 1,500.00$ & & $\$ 0$ & $\$ 6,000$ & $\$ 0$ & $\$ 6,000$ \\
\hline \multicolumn{2}{|c|}{ irm Price-Conv, Cranes 5.0 ton Cap Monorail 2Units } & & & & & 77 & 88.63 & & & $\$ 6,806$ & $\$ 39,900$ & so & $\$ 46,706$ \\
\hline 060116 & $\begin{array}{l}\text { Mechanical Equipment-PROCESS EQ } \\
\text { Cranes } 5.0 \text { ton cal Monorail Type }\end{array}$ & 2 & each & 1.20 & 38.40 & 76.80 & $\$ 88.63$ & $\$ 18,000.00$ & & $\$ 6,806$ & $\$ 36,000$ & $\$ 0$ & $\$ 42,806$ \\
\hline 060199 & Mechanical Equipment-DESIGN & 0.05 & $\%$ & 1.20 & & & & $\$ 18,000.00$ & & $\$ 0$ & $\$ 900$ & $\$ 0$ & $\$ 900$ \\
\hline
\end{tabular}




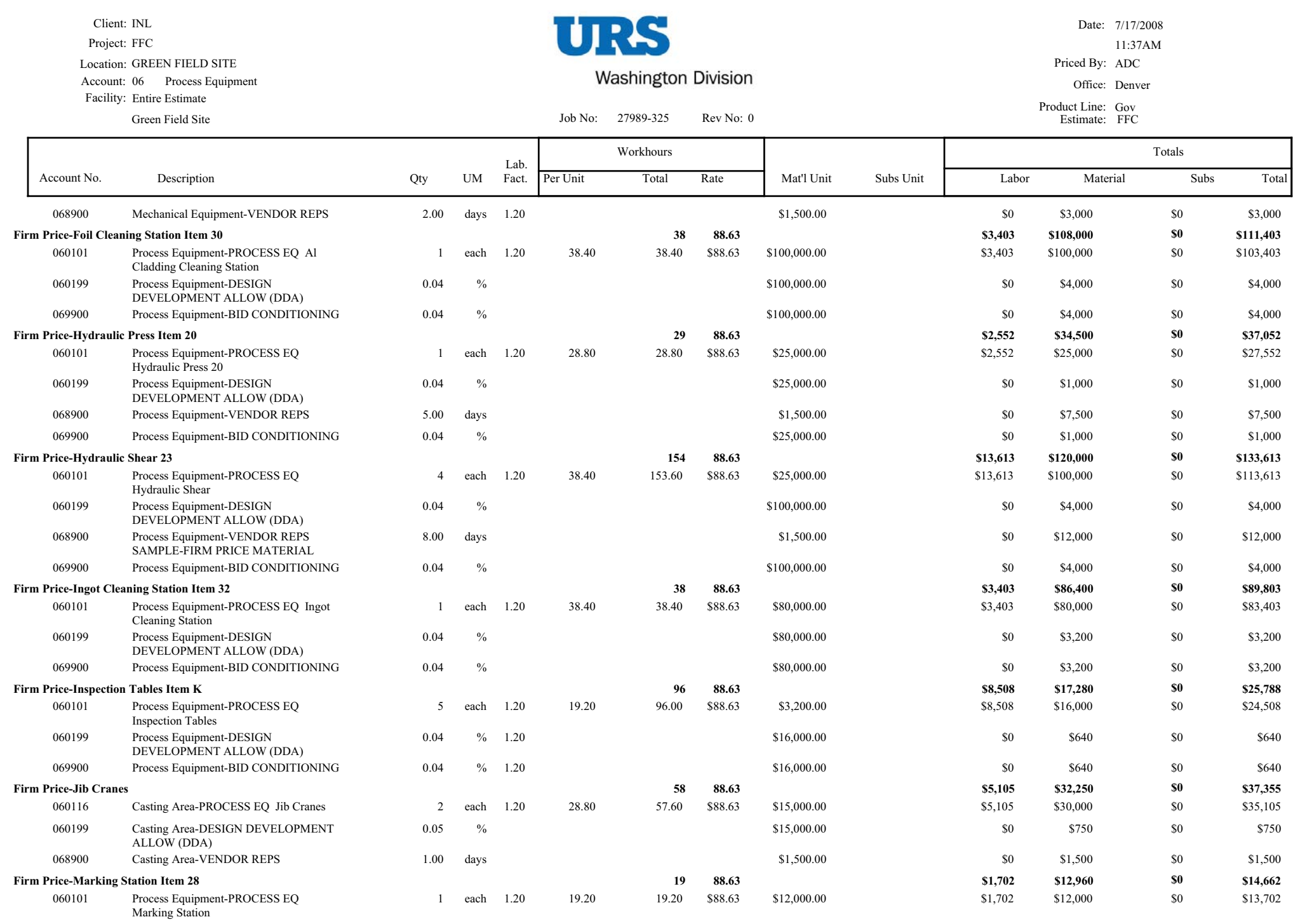


Client: INL

Project: FFC

Location: GREEN FIELD SITE

Account: 06 Process Equipment

URS

Date: $7 / 17 / 2008$

11:37AM

Priced By: ADC

Washington Division

Office: Denver

Facility: Entire Estimate

Job No: $\quad 27989-325 \quad$ Rev No: 0

Product Line: Gov

\begin{tabular}{|c|c|c|c|c|c|c|c|c|c|c|c|c|c|}
\hline \multirow[b]{2}{*}{ Account No. } & \multirow[b]{2}{*}{ Description } & \multirow[b]{2}{*}{ Qty } & \multirow[b]{2}{*}{ UM } & \multirow{2}{*}{$\begin{array}{l}\text { Lab. } \\
\text { Fact. }\end{array}$} & \multicolumn{3}{|c|}{ Workhours } & \multirow[b]{2}{*}{ Mat'l Unit } & \multirow[b]{2}{*}{ Subs Unit } & \multicolumn{4}{|c|}{ Totals } \\
\hline & & & & & \begin{tabular}{|l|l} 
Per Unit \\
\end{tabular} & Total & Rate & & & Labor & Material & Subs & Total \\
\hline 060199 & $\begin{array}{l}\text { Process Equipment-DESIGN } \\
\text { DEVELOPMENT ALLOW (DDA) }\end{array}$ & 0.04 & $\%$ & & & & & $\$ 12,000.00$ & & $\$ 0$ & $\$ 480$ & $\$ 0$ & $\$ 480$ \\
\hline 069900 & Process Equipment-BID CONDITIONING & 0.04 & $\%$ & & & & & $\$ 12,000.00$ & & $\$ 0$ & $\$ 480$ & $\$ 0$ & $\$ 480$ \\
\hline \multicolumn{2}{|c|}{ irm Price-Milling Machines Salt Removal Item 31} & & & & & 43 & 88.63 & & & $\$ 3,829$ & $\$ 54,000$ & \$o & $\$ 57,829$ \\
\hline 060101 & $\begin{array}{l}\text { Process Equipment-PROCESS EQ Milling } \\
\text { Machines } 31\end{array}$ & 1 & each & 1.20 & 43.20 & 43.20 & $\$ 88.63$ & $\$ 50,000.00$ & & $\$ 3,829$ & $\$ 50,000$ & $\$ 0$ & $\$ 53,829$ \\
\hline 060199 & $\begin{array}{l}\text { Process Equipment-DESIGN } \\
\text { DEVELOPMENT ALLOW (DDA) }\end{array}$ & 0.04 & $\%$ & & & & & $\$ 50,000.00$ & & $\$ 0$ & $\$ 2,000$ & $\$ 0$ & $\$ 2,000$ \\
\hline 069900 & Process Equipment-BID CONDITIONING & 0.04 & $\%$ & & & & & $\$ 50,000.00$ & & $\$ 0$ & $\$ 2,000$ & $\$ 0$ & $\$ 2,000$ \\
\hline \multicolumn{2}{|c|}{ irm Price-Milling Machines Surface Finish 21} & & & & & 480 & 88.63 & & & $\$ 42,540$ & $\$ 979,500$ & $\$ 0$ & $\$ 1,022,040$ \\
\hline 060101 & $\begin{array}{l}\text { Process Equipment-PROCESS EQ Milling } \\
\text { Machines } 21\end{array}$ & 5 & each & 1.20 & 96.00 & 480.00 & $\$ 88.63$ & $\$ 180,000.00$ & & $\$ 42,540$ & $\$ 900,000$ & $\$ 0$ & $\$ 942,540$ \\
\hline 060199 & $\begin{array}{l}\text { Process Equipment-DESIGN } \\
\text { DEVELOPMENT ALLOW (DDA) }\end{array}$ & 0.04 & $\%$ & & & & & $\$ 900,000.00$ & & $\$ 0$ & $\$ 36,000$ & $\$ 0$ & $\$ 36,000$ \\
\hline 068900 & Process Equipment-VENDOR REPS & 5.00 & days & & & & & $\$ 1,500.00$ & & $\$ 0$ & $\$ 7,500$ & $\$ 0$ & $\$ 7,500$ \\
\hline 069900 & Process Equipment-BID CONDITIONING & 0.04 & $\%$ & & & & & $\$ 900,000.00$ & & $\$ 0$ & $\$ 36,000$ & $\$ 0$ & $\$ 36,000$ \\
\hline \multicolumn{2}{|c|}{ irm Price-MIT/MURR Welding Station Item 32} & & & & & 10 & 88.63 & & & $\$ 851$ & $\$ 10,800$ & \$0 & $\$ 11,651$ \\
\hline 060101 & $\begin{array}{l}\text { Casting Area-PROCESS EQ Ingot } \\
\text { Cleaning Station }\end{array}$ & 1 & each & 1.20 & 9.60 & 9.60 & $\$ 88.63$ & $\$ 10,000.00$ & & $\$ 851$ & $\$ 10,000$ & $\$ 0$ & $\$ 10,851$ \\
\hline 060199 & $\begin{array}{l}\text { Casting Area-DESIGN DEVELOPMENT } \\
\text { ALLOW (DDA) }\end{array}$ & 0.04 & $\%$ & & & & & $\$ 10,000.00$ & & $\$ 0$ & $\$ 400$ & $\$ 0$ & $\$ 400$ \\
\hline 069900 & Casting Area-BID CONDITIONING & 0.04 & $\%$ & & & & & $\$ 10,000.00$ & & $\$ 0$ & $\$ 400$ & $\$ 0$ & $\$ 400$ \\
\hline \multicolumn{2}{|c|}{ irm Price-Plate Cleaning System Item C } & & & & & 38 & 88.63 & & & $\$ 3,403$ & $\$ 12,300$ & \$o & $\$ 15,703$ \\
\hline 060101 & $\begin{array}{l}\text { Process Equipment-PROCESS EQ Plate } \\
\text { Degreaser }\end{array}$ & 1 & each & 1.20 & 38.40 & 38.40 & $\$ 88.63$ & $\$ 10,000.00$ & & $\$ 3,403$ & $\$ 10,000$ & $\$ 0$ & $\$ 13,403$ \\
\hline 060199 & $\begin{array}{l}\text { Process Equipment-DESIGN } \\
\text { DEVELOPMENT ALLOW (DDA) }\end{array}$ & 0.04 & $\%$ & & & & & $\$ 10,000.00$ & & $\$ 0$ & $\$ 400$ & $\$ 0$ & $\$ 400$ \\
\hline 068900 & Process Equipment-VENDOR REPS & 1.00 & days & & & & & $\$ 1,500.00$ & & $\$ 0$ & $\$ 1,500$ & $\$ 0$ & $\$ 1,500$ \\
\hline 069900 & Process Equipment-BID CONDITIONING & 0.04 & $\%$ & & & & & $\$ 10,000.00$ & & $\$ 0$ & $\$ 400$ & $\$ 0$ & $\$ 400$ \\
\hline \multicolumn{2}{|c|}{ irm Price-Plate Degreaser System Item 11} & & & & & 38 & 88.63 & & & $\$ 3,403$ & $\$ 12,300$ & $\$ 0$ & $\$ 15,703$ \\
\hline 060101 & $\begin{array}{l}\text { Process Equipment-PROCESS EQ Plate } \\
\text { Degreaser }\end{array}$ & 1 & each & 1.20 & 38.40 & 38.40 & $\$ 88.63$ & $\$ 10,000.00$ & & $\$ 3,403$ & $\$ 10,000$ & $\$ 0$ & $\$ 13,403$ \\
\hline 060199 & $\begin{array}{l}\text { Process Equipment-DESIGN } \\
\text { DEVELOPMENT ALLOW (DDA) }\end{array}$ & 0.04 & $\%$ & & & & & $\$ 10,000.00$ & & $\$ 0$ & $\$ 400$ & $\$ 0$ & $\$ 400$ \\
\hline 068900 & Process Equipment-VENDOR REPS & 1.00 & days & & & & & $\$ 1,500.00$ & & $\$ 0$ & $\$ 1,500$ & $\$ 0$ & $\$ 1,500$ \\
\hline 069900 & Process Equipment-BID CONDITIONING & 0.04 & $\%$ & & & & & $\$ 10,000.00$ & & $\$ 0$ & $\$ 400$ & $\$ 0$ & $\$ 400$ \\
\hline \multicolumn{2}{|c|}{ irm Price-Polishing Wheel } & & & & & 5 & 88.63 & & & $\$ 425$ & $\$ 5,250$ & \$0 & $\$ 5,675$ \\
\hline 060199 & $\begin{array}{l}\text { Process Equipment-DESIGN } \\
\text { DEVELOPMENT ALLOW (DDA) }\end{array}$ & 0.05 & $\%$ & 1.20 & & & & $\$ 5,000.00$ & & $\$ 0$ & $\$ 250$ & $\$ 0$ & $\$ 250$ \\
\hline 060201 & $\begin{array}{l}\text { Process Equipment-PROCESS EQ } \\
\text { Polishing Wheel }\end{array}$ & 1 & each & 1.20 & 4.80 & 4.80 & $\$ 88.63$ & $\$ 5,000.00$ & & $\$ 425$ & $\$ 5,000$ & $\$ 0$ & $\$ 5,425$ \\
\hline \multicolumn{2}{|c|}{ irm Price-Raw Alloy Wash Station tem 72} & & & & & 38 & 88.63 & & & $\$ 3,403$ & $\$ 12,300$ & \$o & $\$ 15,703$ \\
\hline 060101 & $\begin{array}{l}\text { Casting Area-PROCESS EQ Wash Station } \\
\text { Item } 72\end{array}$ & 1 & each & 1.20 & 38.40 & 38.40 & $\$ 88.63$ & $\$ 10,000.00$ & & $\$ 3,403$ & $\$ 10,000$ & $\$ 0$ & $\$ 13,403$ \\
\hline
\end{tabular}




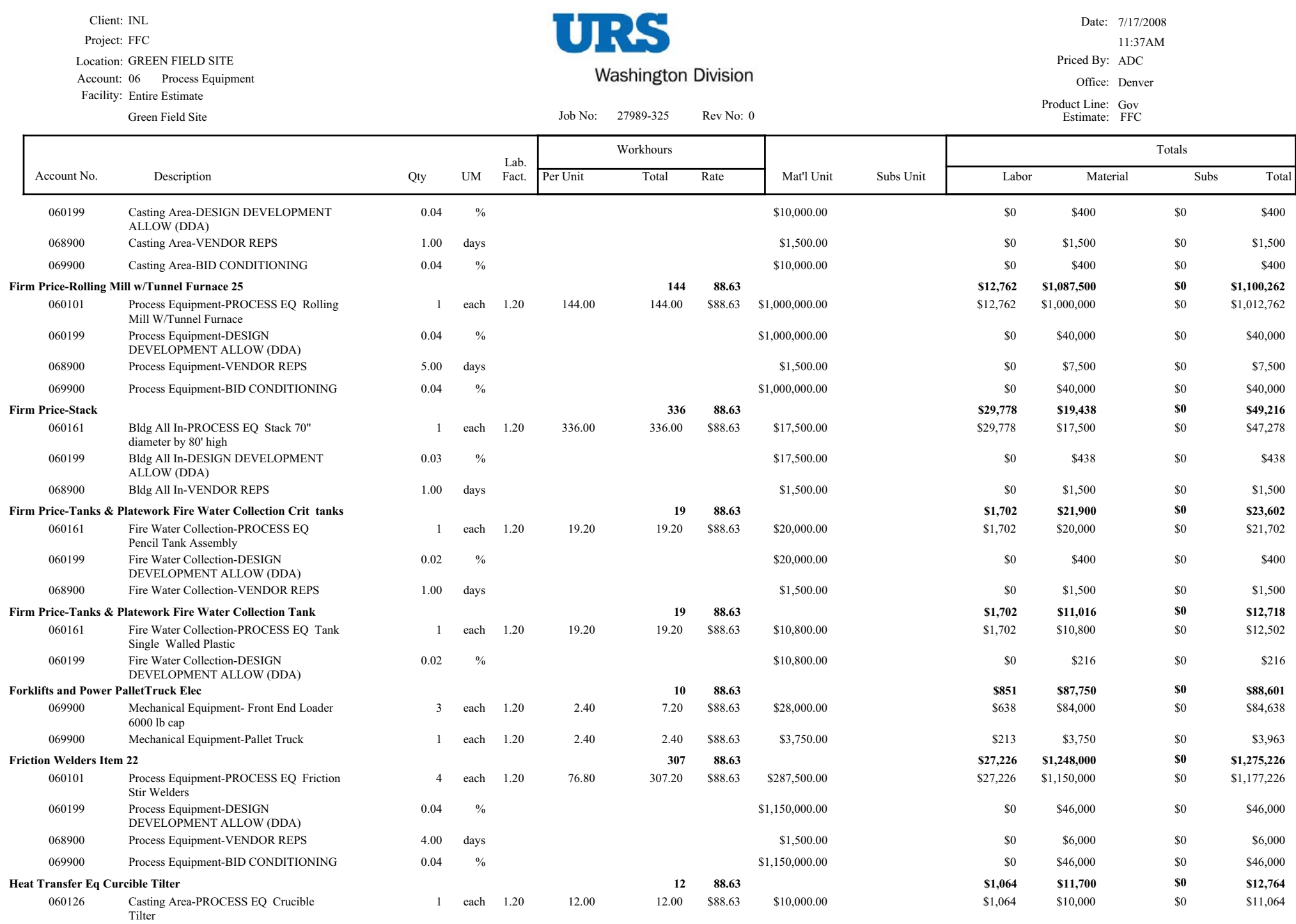


Client: INL

Project: FFC

Location: GREEN FIELD SITE

Account: 06 Process Equipment

URS

Date: 7/17/2008

11:37AM

Washington Division

Priced By: ADC

Office: Denver

Facility: Entire Estimate

Job No: $\quad 27989-325 \quad$ Rev No: 0

Product Line: Gov
Estimate: FFC

\begin{tabular}{|c|c|c|c|c|c|c|c|c|c|c|c|c|c|}
\hline \multirow[b]{2}{*}{ Account No. } & \multirow[b]{2}{*}{ Description } & \multirow[b]{2}{*}{ Qty } & \multirow[b]{2}{*}{ UM } & \multirow{2}{*}{$\begin{array}{l}\text { Lab. } \\
\text { Fact. }\end{array}$} & \multicolumn{3}{|c|}{ Workhours } & \multirow[b]{2}{*}{ Mat'l Unit } & \multirow[b]{2}{*}{ Subs Unit } & \multicolumn{4}{|c|}{ Totals } \\
\hline & & & & & Per Unit & Total & Rate & & & Labor & Material & Subs & Total \\
\hline 060199 & $\begin{array}{l}\text { Casting Area-DESIGN DEVELOPMENT } \\
\text { ALLOW (DDA) }\end{array}$ & 0.02 & $\%$ & & & & & $\$ 10,000.00$ & & $\$ 0$ & $\$ 200$ & $\$ 0$ & $\$ 200$ \\
\hline 068900 & Casting Area-VENDOR REPS & 1.00 & days & & & & & $\$ 1,500.00$ & & $\$ 0$ & $\$ 1,500$ & $\$ 0$ & $\$ 1,500$ \\
\hline EPA Filters and & I Handling Sys for Fuel Fabrication Area & & & & & 4,920 & 88.63 & & & $\$ 436,037$ & $\$ 154,017$ & $\$ 2,654,150$ & $\$ 3,244,204$ \\
\hline 060129 & $\begin{array}{l}\text { Mechanical Equipment-HVAC } \\
\text { EQUIPMENT Process Area craft labor } \\
\text { support ( } 38900 \text { sqft) }\end{array}$ & 1 & each & 1.20 & $4,548.00$ & $4,548.00$ & $\$ 88.63$ & $\$ 154,017.00$ & & $\$ 403,068$ & $\$ 154,017$ & $\$ 0$ & $\$ 557,085$ \\
\hline 060129 & $\begin{array}{l}\text { Mechanical Equipment-HVAC } \\
\text { EQUIPMENT Process Area Subcontract } \\
\text { furnish \& install ( } 38900 \mathrm{sqft})\end{array}$ & 1 & each & & & & & & $\$ 2,654,150.00$ & $\$ 0$ & $\$ 0$ & $\$ 2,654,150$ & $\$ 2,654,150$ \\
\hline 060129 & $\begin{array}{l}\text { Mechanical Equipment-HVAC } \\
\text { EQUIPMENT Process Area HEPA Filter } \\
\text { Install Craft Support }\end{array}$ & 1 & each & 1.20 & 372.00 & 372.00 & $\$ 88.63$ & & & $\$ 32,969$ & $\$ 0$ & $\$ 0$ & $\$ 32,969$ \\
\hline stron Item $\mathrm{O}$ & & & & & & 48 & 88.63 & & & $\$ 4,254$ & $\$ 64,800$ & \$0 & $\$ 69,054$ \\
\hline 060101 & Casting Area-PROCESS EQ Pull Testor & 1 & each & 1.20 & 48.00 & 48.00 & $\$ 88.63$ & $\$ 60,000.00$ & & $\$ 4,254$ & $\$ 60,000$ & $\$ 0$ & $\$ 64,254$ \\
\hline 060199 & $\begin{array}{l}\text { Casting Area-DESIGN DEVELOPMENT } \\
\text { ALLOW (DDA) }\end{array}$ & 0.04 & $\%$ & & & & & $\$ 60,000.00$ & & $\$ 0$ & $\$ 2,400$ & $\$ 0$ & $\$ 2,400$ \\
\hline 069900 & Casting Area-BID CONDITIONING & 0.04 & $\%$ & & & & & $\$ 60,000.00$ & & $\$ 0$ & $\$ 2,400$ & $\$ 0$ & $\$ 2,400$ \\
\hline \multicolumn{2}{|c|}{ IST/HIFR Welding Item G } & & & & & 48 & 88.63 & & & $\$ 4,254$ & $\$ 105,900$ & $\$ 0$ & $\$ 110,154$ \\
\hline 060101 & $\begin{array}{l}\text { Process Equipment-PROCESS EQ } \\
\text { Welding Station }\end{array}$ & 1 & each & 1.20 & 48.00 & 48.00 & $\$ 88.63$ & $\$ 100,000.00$ & & $\$ 4,254$ & $\$ 100,000$ & $\$ 0$ & $\$ 104,254$ \\
\hline 060199 & $\begin{array}{l}\text { Process Equipment-DESIGN } \\
\text { DEVELOPMENT ALLOW (DDA) }\end{array}$ & 0.00 & $\%$ & & & & & $\$ 100,000.00$ & & $\$ 0$ & $\$ 400$ & $\$ 0$ & $\$ 400$ \\
\hline 068900 & Process Equipment-VENDOR REPS & 1.00 & days & & & & & $\$ 1,500.00$ & & $\$ 0$ & $\$ 1,500$ & $\$ 0$ & $\$ 1,500$ \\
\hline 069900 & Process Equipment-BID CONDITIONING & 0.04 & $\%$ & & & & & $\$ 100,000.00$ & & $\$ 0$ & $\$ 4,000$ & $\$ 0$ & $\$ 4,000$ \\
\hline \multicolumn{2}{|c|}{ itric Acid Wash System (incl Piping) } & & & & & 94 & 88.63 & & & $\$ 8,295$ & $\$ 469$ & \$0 & $\$ 8,764$ \\
\hline 060101 & $\begin{array}{l}\text { Mechanical Equipment-Nitric Acid Wash } \\
\text { System }\end{array}$ & 1 & each & 1.20 & 93.60 & 93.60 & $\$ 88.63$ & $\$ 469.00$ & & $\$ 8,295$ & $\$ 469$ & $\$ 0$ & $\$ 8,764$ \\
\hline \multicolumn{2}{|c|}{ latework Crucible Cleaning Station Glovebox Item 39} & & & & & 19 & 88.63 & & & $\$ 1,702$ & $\$ 55,000$ & \$o & $\$ 56,702$ \\
\hline 060161 & $\begin{array}{l}\text { Casting Area-PROCESS EQ Crucible } \\
\text { Cleaning Station Glovebox Item } 39\end{array}$ & 1 & each & 1.20 & 19.20 & 19.20 & $\$ 88.63$ & $\$ 50,000.00$ & & $\$ 1,702$ & $\$ 50,000$ & $\$ 0$ & $\$ 51,702$ \\
\hline 060199 & $\begin{array}{l}\text { Casting Area-DESIGN DEVELOPMENT } \\
\text { ALLOW (DDA) }\end{array}$ & 0.04 & $\%$ & & & & & $\$ 50,000.00$ & & $\$ 0$ & $\$ 2,000$ & $\$ 0$ & $\$ 2,000$ \\
\hline 068900 & Casting Area-VENDOR REPS & 2.00 & days & & & & & $\$ 1,500.00$ & & $\$ 0$ & $\$ 3,000$ & $\$ 0$ & $\$ 3,000$ \\
\hline \multicolumn{2}{|c|}{ latework Crucible Loading Station Item 38} & & & & & 19 & 88.63 & & & $\$ 1,702$ & $\$ 34,200$ & $\$ 0$ & $\$ 35,902$ \\
\hline 060161 & $\begin{array}{l}\text { Casting Area-PROCESS EQ Crucible } \\
\text { Loading Station Item } 38\end{array}$ & 1 & each & 1.20 & 19.20 & 19.20 & $\$ 88.63$ & $\$ 30,000.00$ & & $\$ 1,702$ & $\$ 30,000$ & $\$ 0$ & $\$ 31,702$ \\
\hline 060199 & $\begin{array}{l}\text { Casting Area-DESIGN DEVELOPMENT } \\
\text { ALLOW (DDA) }\end{array}$ & 0.04 & $\%$ & & & & & $\$ 30,000.00$ & & $\$ 0$ & $\$ 1,200$ & $\$ 0$ & $\$ 1,200$ \\
\hline 068900 & Casting Area-VENDOR REPS & 2.00 & days & & & & & $\$ 1,500.00$ & & $\$ 0$ & $\$ 3,000$ & $\$ 0$ & $\$ 3,000$ \\
\hline \multicolumn{2}{|c|}{ latework Entry Glovebox } & & & & & 58 & 88.63 & & & $\$ 5,105$ & $\$ 55,000$ & \$o & $\$ 60,105$ \\
\hline 060161 & $\begin{array}{l}\text { Casting Area-PROCESS EQ Casting Mold } \\
\text { Entry Glovebox }\end{array}$ & 1 & each & 1.20 & 57.60 & 57.60 & $\$ 88.63$ & $\$ 50,000.00$ & & $\$ 5,105$ & $\$ 50,000$ & $\$ 0$ & $\$ 55,105$ \\
\hline 060199 & $\begin{array}{l}\text { Casting Area-DESIGN DEVELOPMENT } \\
\text { ALLOW (DDA) }\end{array}$ & 0.04 & $\%$ & & & & & $\$ 50,000.00$ & & $\$ 0$ & $\$ 2,000$ & $\$ 0$ & $\$ 2,000$ \\
\hline
\end{tabular}




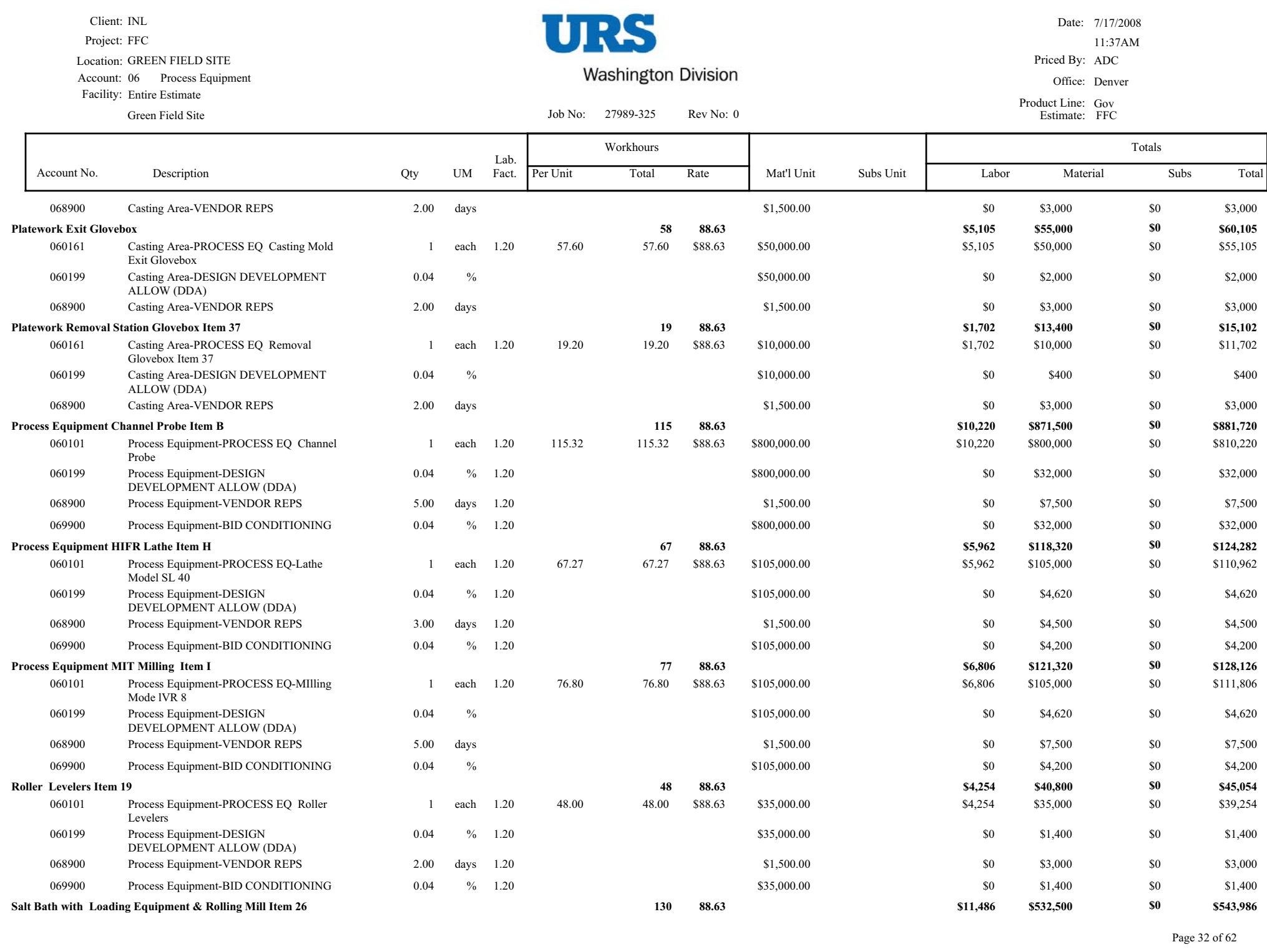


Client: INL

Project: FFC

URS

Date: 7/17/2008

11:37AM

Location: GREEN FIELD SITE

Washington Division

Priced By: ADC

Office: Denver

Facility: Entire Estimate

Job No: $\quad 27989-325 \quad$ Rev No: 0

Product Line: Gov
Estimate: FFC

\begin{tabular}{|c|c|c|c|c|c|c|c|c|c|c|c|c|c|}
\hline \multirow[b]{2}{*}{ Account No. } & \multirow[b]{2}{*}{ Description } & \multirow[b]{2}{*}{ Qty } & \multirow[b]{2}{*}{ UM } & \multirow{2}{*}{$\begin{array}{l}\text { Lab. } \\
\text { Fact. }\end{array}$} & \multicolumn{3}{|c|}{ Workhours } & \multirow[b]{2}{*}{ Mat'l Unit } & \multirow[b]{2}{*}{ Subs Unit } & \multicolumn{4}{|c|}{ Totals } \\
\hline & & & & & Per Unit & Total & Rate & & & Labor & Material & Subs & Total \\
\hline 060116 & $\begin{array}{l}\text { Process Equipment-PROCESS EQ Loading } \\
\text { Equipment }\end{array}$ & 1 & each & 1.20 & 129.60 & 129.60 & $\$ 88.63$ & $\$ 500,000.00$ & & $\$ 11,486$ & $\$ 500,000$ & $\$ 0$ & $\$ 511,486$ \\
\hline 060199 & $\begin{array}{l}\text { Process Equipment-DESIGN } \\
\text { DEVELOPMENT ALLOW (DDA) }\end{array}$ & 0.05 & $\%$ & & & & & $\$ 500,000.00$ & & $\$ 0$ & $\$ 25,000$ & $\$ 0$ & $\$ 25,000$ \\
\hline 068900 & Process Equipment-VENDOR REPS & 5.00 & days & & & & & $\$ 1,500.00$ & & $\$ 0$ & $\$ 7,500$ & $\$ 0$ & $\$ 7,500$ \\
\hline
\end{tabular}

Totals 06-Process Equipment

Entire Estimate

$\$ 844,864$

$\$ 11,533,390$

$\$ 2,654,150$

$\$ 15,032,404$ 


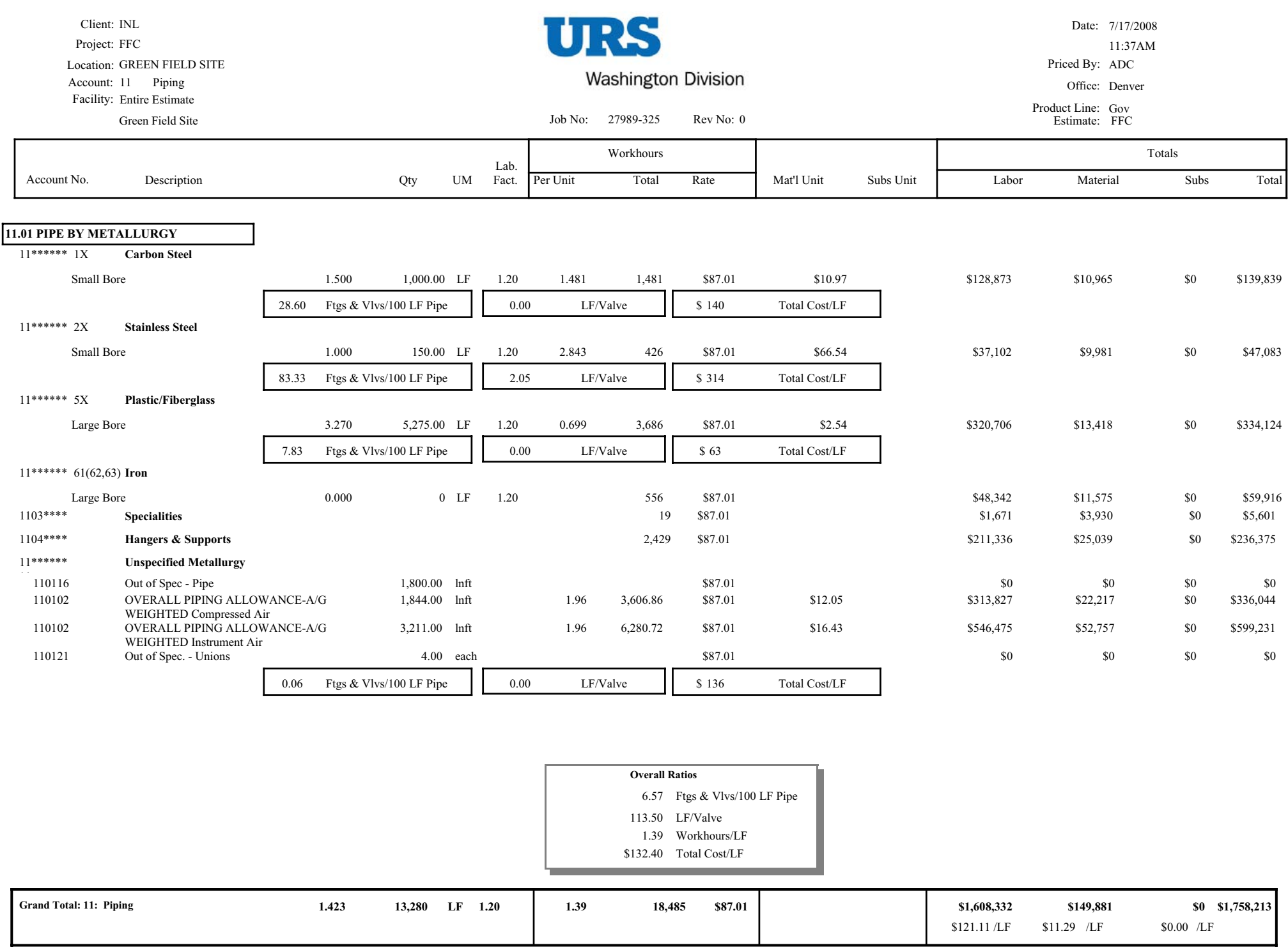


Client: INL

Project: FFC

URS

Location: GREEN FIELD SITE

Account: 11 Piping

Washington Division

Date: 7/17/2008

11:37AM

Facility: Entire Estimate

Job No: $\quad 27989-325 \quad$ Rev No: 0 Priced By: ADC

Office: Denver

Product Line: Gov
Estimate:

Green Field Site

No. 27989-325 Rov No. 0

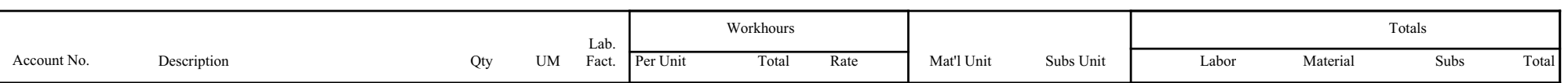

11.02 PIPE BY SERVICE SYSTEM

\begin{tabular}{|lrr}
1.423 & $13,280.00 \quad$ LF $\quad 1.20$ \\
\hline 6.57 & Ftgs \& Vlvs/100 LF 1 \\
\hline
\end{tabular}

\begin{tabular}{|lr|rr|}
\multicolumn{1}{rr}{1.392} & $18,484.80$ & $\$ 87.01$ & $\$ 11.29$ \\
\hline 113.50 & LF/Valve & $\$ 132.40$ & Total Cost/LF \\
\cline { 3 - 3 } & &
\end{tabular}

$\$ 0.00$

$\$ 1,608,332$

$\$ 149,881$

$\$ 0 \$ 1,758,213$

\begin{tabular}{|rl|}
\hline \multicolumn{2}{|c|}{ Overall Ratios } \\
6.57 & Ftgs \& Vlvs/100 LF P \\
113.50 & LF/Valve \\
1.39 & Workhours/LF \\
$\$ 132.40$ & Total Cost/LF \\
\hline
\end{tabular}

\begin{tabular}{|c|c|c|c|c|c|c|c|c|c|c|}
\hline Grand Total: & 1.423 & $13,280 \quad$ LF & 1.20 & 1.39 & 18,485 & $\$ 87.01$ & $\begin{array}{r}\mathbf{\$ 1 , 6 0 8 , 3 3 2} \\
\$ 121.11 / L F\end{array}$ & $\begin{aligned} & \$ 149,881 \\
\$ 11.29 & / L F\end{aligned}$ & $\begin{array}{r}\mathbf{\$ 0} \\
\$ 0.00 / \mathrm{LF}\end{array}$ & $\$ 1,758,213$ \\
\hline
\end{tabular}

Page 35 of 62 
Client: INL

Project: FFC
URS

Washington Division
Date: $7 / 17 / 2008$

$11: 37 \mathrm{AM}$ Priced By: ADC

Office: Denver

Product Line: Gov
Estimate: FFC

Job No: $\quad 27989-325 \quad$ Rev No: 0

(Workous

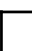

Mat'l Unit

Subs Unit

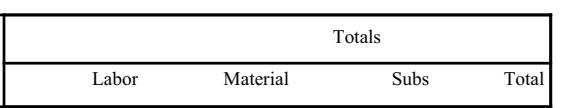

11.04 PIPE BY ASSEMBLY - SUMMARY

Bldg Plumbing

Carbon Steel (400s Specs) Plumbing

Water Pipe (50 lnft per fixture)L

Plastic (800s) Fire Protection Loop

Plastic (800s) Floor Drain Pipe (100 lnft

per floor drain)

Plastic (800s) Outside Sanitary Sewer

Line

Plastic (800s) Roof drain pipe( $70 \mathrm{lnft}$ per

roof drain)

Plastic (800s) Sanitary Sewer piping (50

Inft per fixture)

Stainless Steel (700s) Argon Supply

1.500
3.000
6.000
3.000
3.000
1.000

Qty UM Fact

Per Unit Total Rate

$\begin{array}{rlll}0.00 & \text { LF } & 1.20 & 0.00 \\ 1,000.00 & \text { LF } & 1.20 & 1.91 \\ & & & \\ 1,800.00 & \text { LF } & 1.20 & 0.89 \\ 2,400.00 & \text { LF } & 1.20 & 0.72 \\ 475.00 & \text { LF } & 1.20 & 1.14 \\ 1,400.00 & \text { LF } & 1.20 & 0.70 \\ 1,000.00 & \text { LF } & 1.20 & 1.54 \\ 150.00 & \text { LF } & 1.20 & 1.83 \\ & & & 1.96\end{array}$

$\begin{array}{rr}19 & \$ 87.01 \\ 1,908 & \$ 87.01 \\ 1,608 & \$ 87.01 \\ 1,723 & \$ 87.01 \\ 540 & \$ 87.01 \\ 986 & \$ 87.01 \\ 1,538 & \$ 87.01 \\ 275 & \$ 87.01 \\ 9,888 & \$ 87.01\end{array}$

$\begin{array}{rr}\$ 0.00 & \$ 0.00 \\ \$ 17.05 & \$ 0.00 \\ \$ 10.41 & \$ 0.00 \\ \$ 3.83 & \$ 0.00 \\ \$ 9.30 & \$ 0.00 \\ \$ 3.79 & \$ 0.00 \\ \$ 9.20 & \$ 0.00 \\ \$ 47.21 & \$ 0.00 \\ \$ 14.83 & \$ 0.00\end{array}$

$\$ 1,671$
$\$ 166,043$
$\$ 139,889$
$\$ 149,933$
$\$ 46,985$
$\$ 85,762$
$\$ 133,801$
$\$ 23,946$
$\$ 860,302$

$\$ 3,930$

$\$ 17,047$

$\$ 18,746$

$\$ 9,180$

$\$ 4,416$

$\$ 5,304$

$\$ 9,204$

$\$ 7,081$

$\$ 74,973$
$\$ 0 \quad \$ 5,601$

$\$ 0 \quad \$ 158,635$

$\$ 0 \quad \$ 159,113$

$\$ 0 \quad \$ 51,400$

$\$ 0 \quad \$ 91,066$

$\$ 0 \quad \$ 143,005$

$\$ 0 \quad \$ 31,028$

$\$ 0 \quad \$ 935,275$

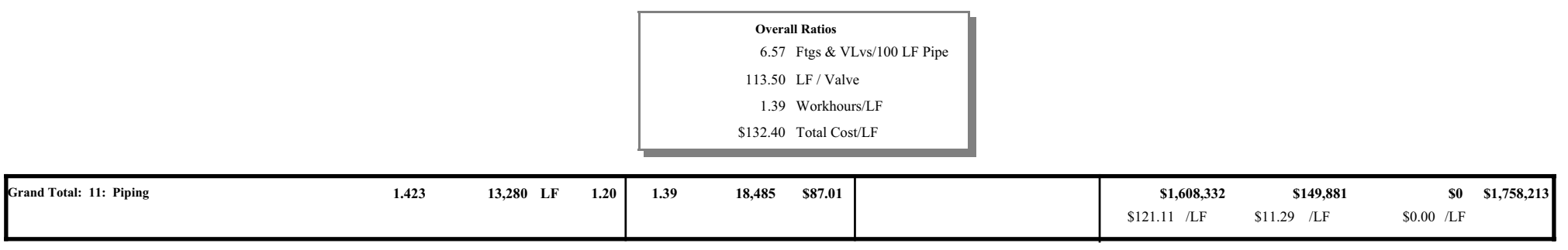


Client: INL

Project: FFC

URS

Date: 7/17/2008

Location: GREEN FIELD SITE

Account: 11 Piping

Washington Division

11:37AM

Facility: Entire Estimate

Job No: $\quad 27989-325 \quad$ Rev No: 0

Office: Denver

$\begin{array}{rr}\text { Product Line: } & \text { Gov } \\ \text { Estimate: } & \text { FFC }\end{array}$

\begin{tabular}{lllll}
\hline Account No. & & & & Lab. \\
& Description & UM & Fact.
\end{tabular}

Job No: 27989-325 Rev No: 0

\subsection{PIPE BY COMMODITY}

1101 Pipe, Fittings, Welds, and Shopcosts

1102 Valves

$1103 \quad$ Specialties

$1104 \quad$ Hangers \& Supports

$\begin{array}{rlrll}13,280.00 & \text { lnft } & 1.42 & 1.20 & 1.149 \\ 117.00 & \text { ea } & & 1.20 & 6.655 \\ & & & 1.20 & 9.600 \\ 640.00 & \text { ea } & & 1.20 & 3.795\end{array}$

$\begin{array}{rr}15,258 & \$ 87.01 \\ 779 & \$ 87.01 \\ 19 & \$ 87.01 \\ 2,429 & \$ 87.01\end{array}$

$\$ 7.70$
$\$ 159.83$
$\$ 1,965.00$
$\$ 39.12$

$\$ 1,327,573$

$\$ 102,211$

$\$ 18,701$

$\$ 3,930$

$\$ 25,039$

$\$ 211,336$

$\$ 25,039$

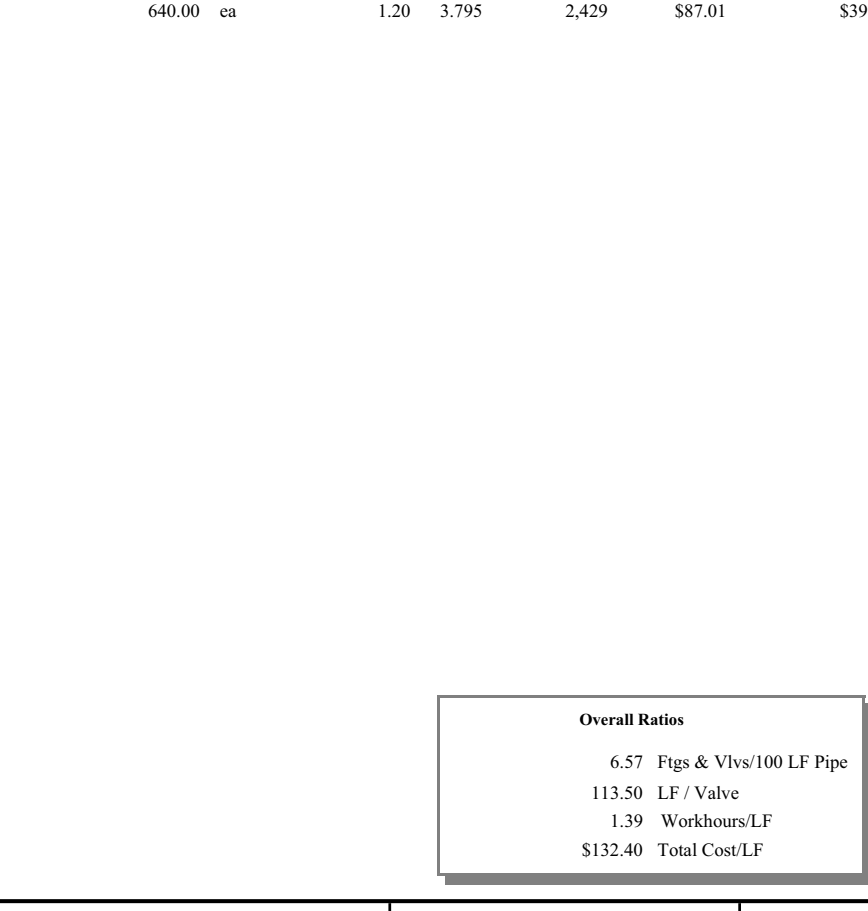

Grand Total: 11: Piping

$13,280 \quad$ LF $\quad 1.423$

1.20

$18,485 \quad \$ 87.01$

$\$ 1,608,332$

$\$ 121.11 / \mathrm{LF}$

$\$ 149,881$

$\$ 11.29 / \mathrm{LF}$

\$o $\$ 0.00 / \mathrm{LF}$

Page 37 of 62 


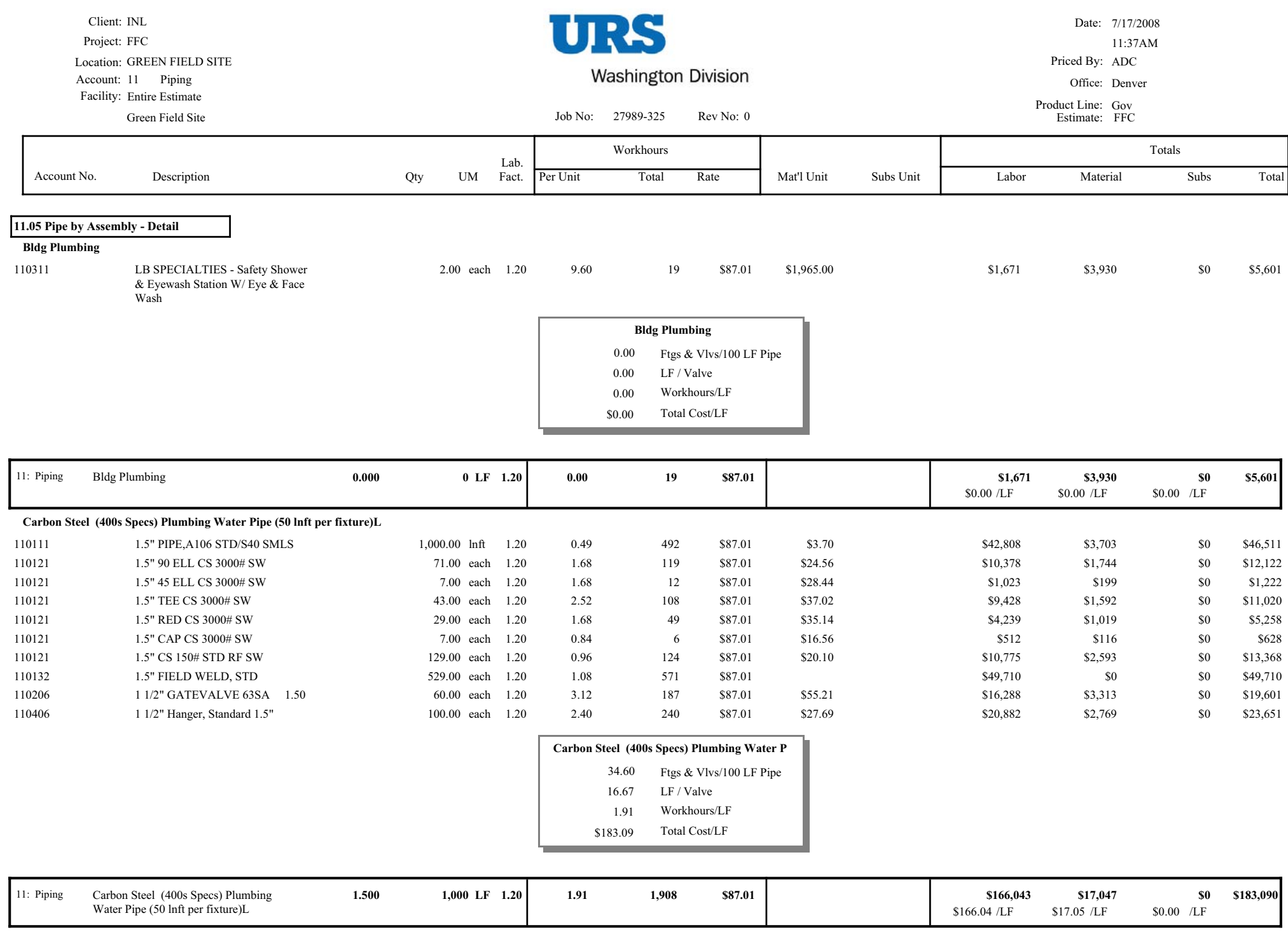


Client: INL

Project: FFC

Location: GREEN FIELD SITE

Account: 11 Piping

Facility: Entire Estimate

URS

Washington Division
Date: 7/17/2008

11:37AM

Priced By: ADC

Office: Denver

Product Line: Gov
Estimate: FFC

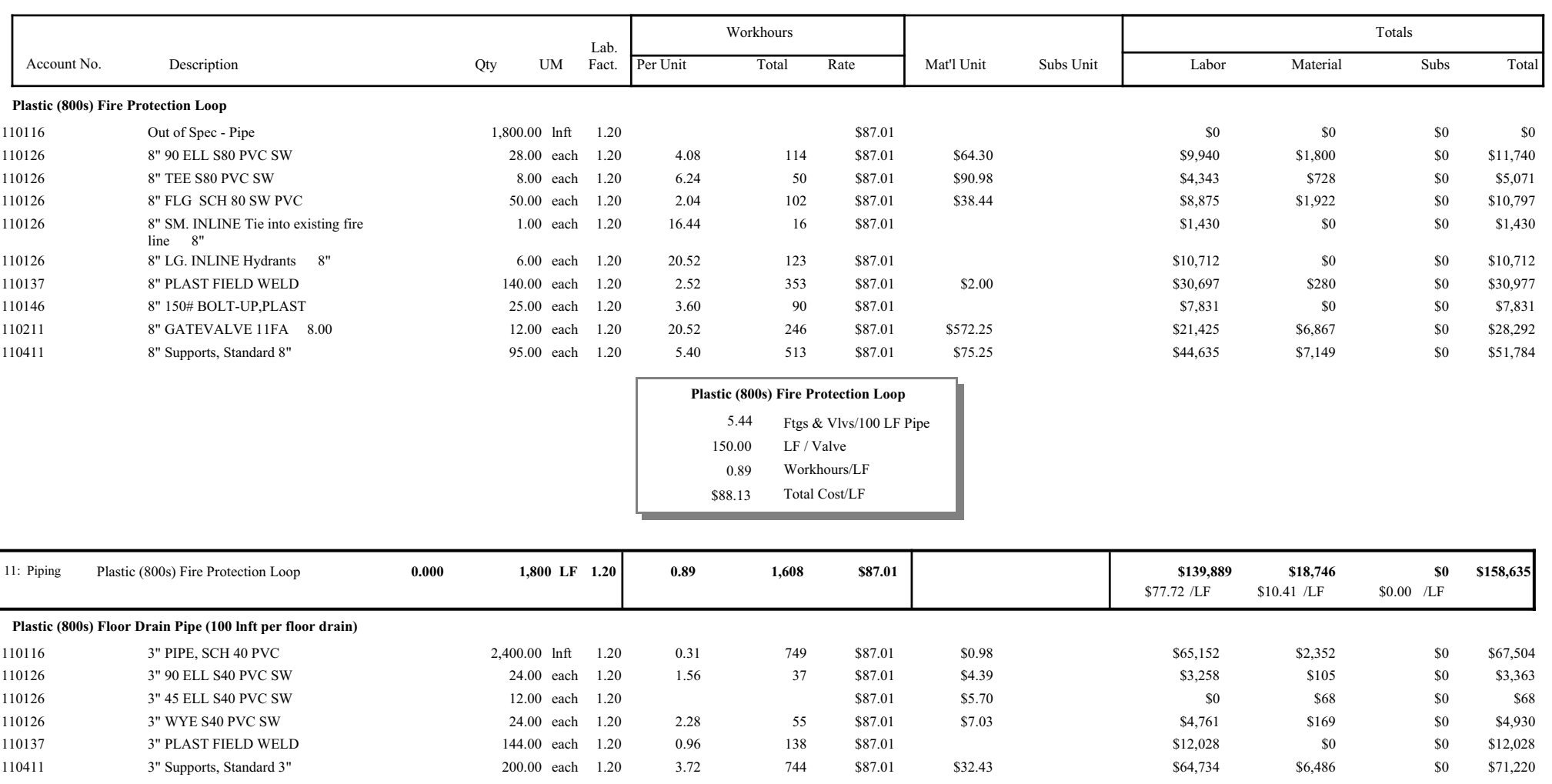




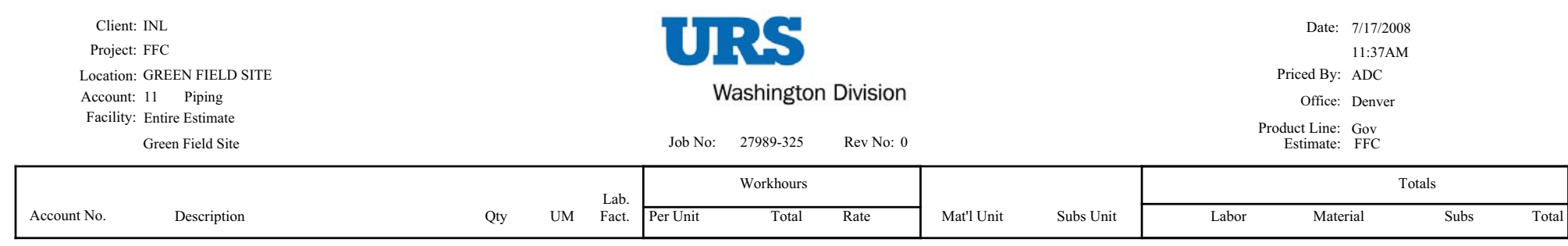

Plastic (800s) Floor Drain Pipe (100 lnft per fl

$\begin{aligned} 2.50 & \text { Ftgs \& Vlvs/100 LF Pipe } \\ 0.00 & \text { LF/Valve } \\ 0.72 & \text { Workhours/LF } \\ \$ 66.30 & \text { Total Cost/LF }\end{aligned}$

$\$ 66.30 \quad$ Total Cost/LF

\begin{tabular}{|c|c|c|c|c|c|c|c|c|c|c|c|c|c|c|}
\hline 11: Piping & $\begin{array}{l}\text { Plastic (800s) Floor Drain Pipe (100 lnft } \\
\text { per floor drain) }\end{array}$ & 3.000 & 2,400 & $0 \mathrm{LF}$ & 1.20 & 0.72 & 1,723 & $\$ 87.01$ & & $\begin{array}{l}\$ 149,933 \\
\$ 62.47 / \mathrm{LF}\end{array}$ & $\begin{array}{l}\$ 9,180 \\
\$ 3.83 / L F\end{array}$ & $\$ 0.00$ & $\begin{array}{l}\mathbf{\$ 0} \\
/ \mathrm{LF}\end{array}$ & $\$ 159,113$ \\
\hline \multicolumn{15}{|c|}{ Plastic (800s) Outside Sanitary Sewer Line } \\
\hline 110116 & 6" PIPE, SCH 40 PVC & & 475.00 & $\operatorname{lnft}$ & 1.20 & 0.46 & 217 & $\$ 87.01$ & $\$ 2.56$ & $\$ 18,846$ & $\$ 1,216$ & & $\$ 0$ & $\$ 20,062$ \\
\hline 110126 & 6" 90 ELL S40 PVC SW & & 6.00 & each & 1.20 & 3.12 & 19 & $\$ 87.01$ & $\$ 24.97$ & $\$ 1,629$ & $\$ 150$ & & $\$ 0$ & $\$ 1,779$ \\
\hline 110126 & 6" 45 ELL S40 PVC SW & & 2.00 & each & 1.20 & & & $\$ 87.01$ & $\$ 25.26$ & $\$ 0$ & $\$ 51$ & & $\$ 0$ & $\$ 51$ \\
\hline 110126 & 6" TEE S40 PVC SW & & 1.00 & each & 1.20 & 4.68 & 5 & $\$ 87.01$ & $\$ 39.23$ & $\$ 407$ & $\$ 39$ & & $\$ 0$ & $\$ 446$ \\
\hline 110126 & 6" RED S40 PVC SW & & 1.00 & each & 1.20 & 3.12 & 3 & $\$ 87.01$ & $\$ 11.80$ & $\$ 271$ & $\$ 12$ & & $\$ 0$ & $\$ 283$ \\
\hline 110121 & Out of Spec. - Unions & & 4.00 & each & 1.20 & & & $\$ 87.01$ & & \$0 & $\$ 0$ & & $\$ 0$ & $\$ 0$ \\
\hline 110126 & 6" FLG SCH 40 SW PVC & & 31.00 & each & 1.20 & 1.56 & 48 & $\$ 87.01$ & $\$ 19.79$ & $\$ 4,208$ & $\$ 613$ & & $\$ 0$ & $\$ 4,821$ \\
\hline 110126 & $\begin{array}{l}\text { 6" LG. INLINE Tie In to Existing } \\
\text { Sewer Line } 6 "\end{array}$ & & 1.00 & each & 1.20 & 14.28 & 14 & $\$ 87.01$ & & $\$ 1,242$ & $\$ 0$ & & $\$ 0$ & $\$ 1,242$ \\
\hline 110137 & 6" PLAST FIELD WELD & & 30.00 & each & 1.20 & 2.04 & 61 & $\$ 87.01$ & $\$ 1.00$ & $\$ 5,325$ & $\$ 30$ & & $\$ 0$ & $\$ 5,355$ \\
\hline 110211 & 6" GATEVALVE 11FA $\quad 6.00$ & & 2.00 & each & 1.20 & 14.28 & 29 & $\$ 87.01$ & $\$ 316.75$ & $\$ 2,485$ & $\$ 634$ & & $\$ 0$ & $\$ 3,118$ \\
\hline 110411 & 6" Supports, Standard 6" & & 28.00 & each & 1.20 & 5.16 & 144 & $\$ 87.01$ & $\$ 59.70$ & $\$ 12,571$ & $\$ 1,672$ & & $\$ 0$ & $\$ 14,243$ \\
\hline
\end{tabular}

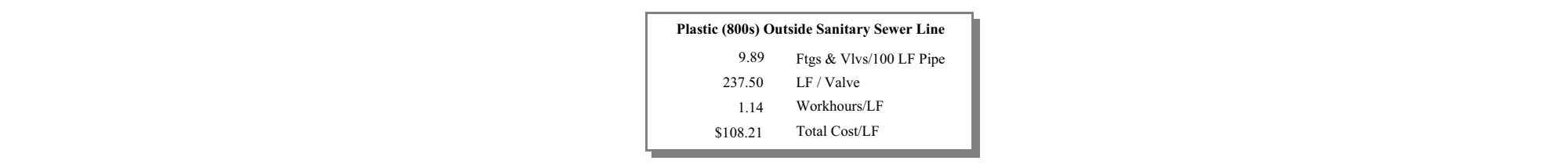

\begin{tabular}{|c|c|c|c|c|c|c|c|c|c|c|c|c|c|c|}
\hline 11: Piping & $\begin{array}{l}\text { Plastic (800s) Outside Sanitary Sewer } \\
\text { Line }\end{array}$ & 6.000 & & $175 \mathbf{L F}$ & 1.20 & 1.14 & 540 & $\$ 87.01$ & & $\begin{array}{l}\$ 46,985 \\
\$ 98.91 / L F\end{array}$ & $\begin{array}{l}\mathbf{\$ 4 , 4 1 6} \\
\$ 9.30 / \mathrm{LF}\end{array}$ & $\$ 0.00$ & $\begin{array}{l}\text { s0 } \\
/ \mathrm{LF}\end{array}$ & $\$ 51,400$ \\
\hline \multicolumn{15}{|c|}{ Plastic (800s) Roof drain pipe( $70 \mathrm{lnft}$ per roof drain) } \\
\hline 110116 & 3" PIPE, SCH 40 PVC & & $1,400.00$ & $0 \operatorname{lnft}$ & 1.20 & 0.31 & 437 & $\$ 87.01$ & $\$ 0.98$ & $\$ 38,005$ & $\$ 1,372$ & & $\$ 0$ & $\$ 39,377$ \\
\hline 110126 & 3" 90 ELL S40 PVC SW & & 20.00 & 0 each & 1.20 & 1.56 & 31 & $\$ 87.01$ & $\$ 4.39$ & $\$ 2,715$ & $\$ 88$ & & $\$ 0$ & $\$ 2,802$ \\
\hline 110126 & 3" 45 ELL S40 PVC SW & & 3.00 & 0 each & 1.20 & & & $\$ 87.01$ & $\$ 5.70$ & so & $\$ 17$ & & $\$ 0$ & $\$ 17$ \\
\hline
\end{tabular}




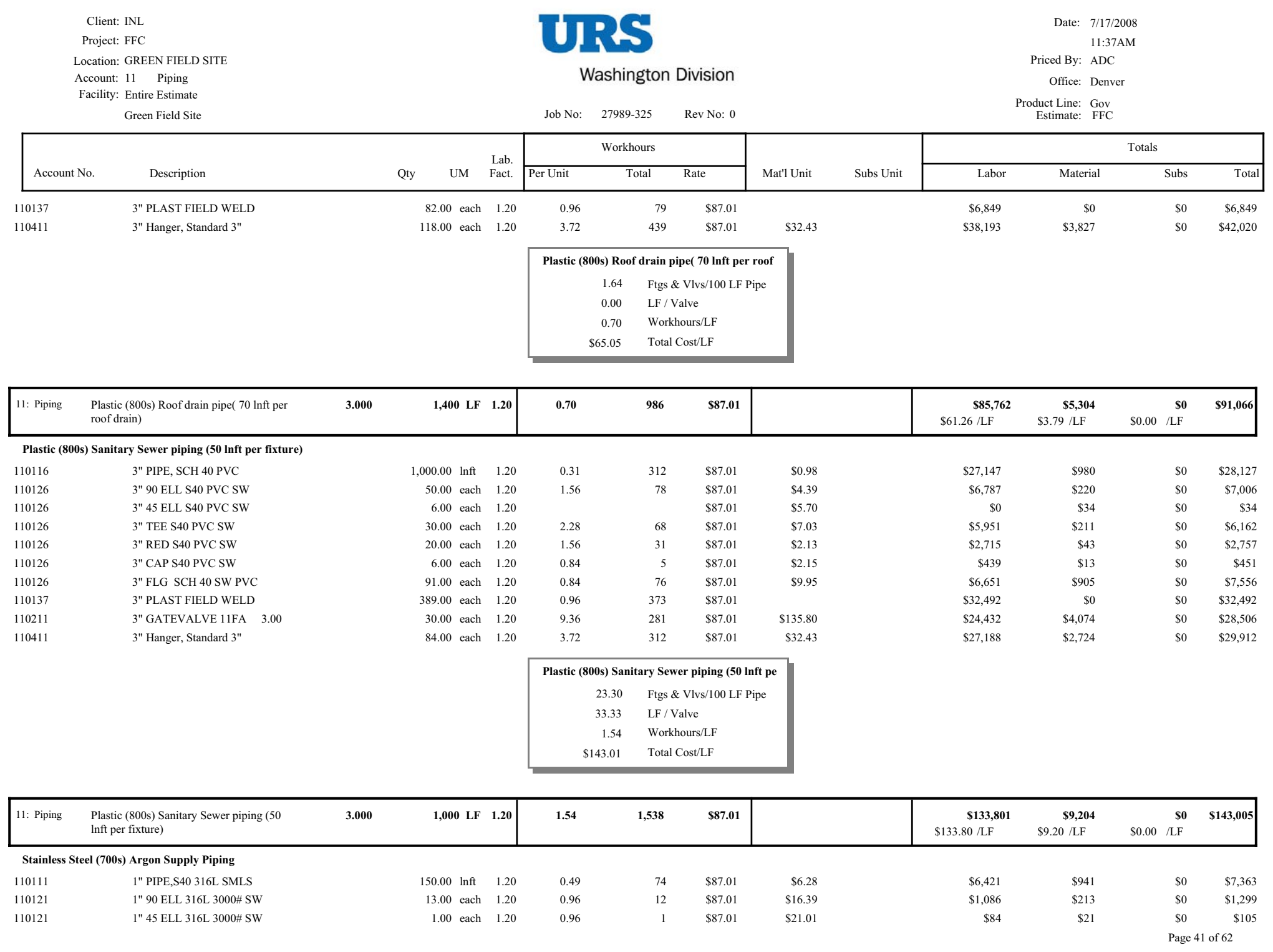




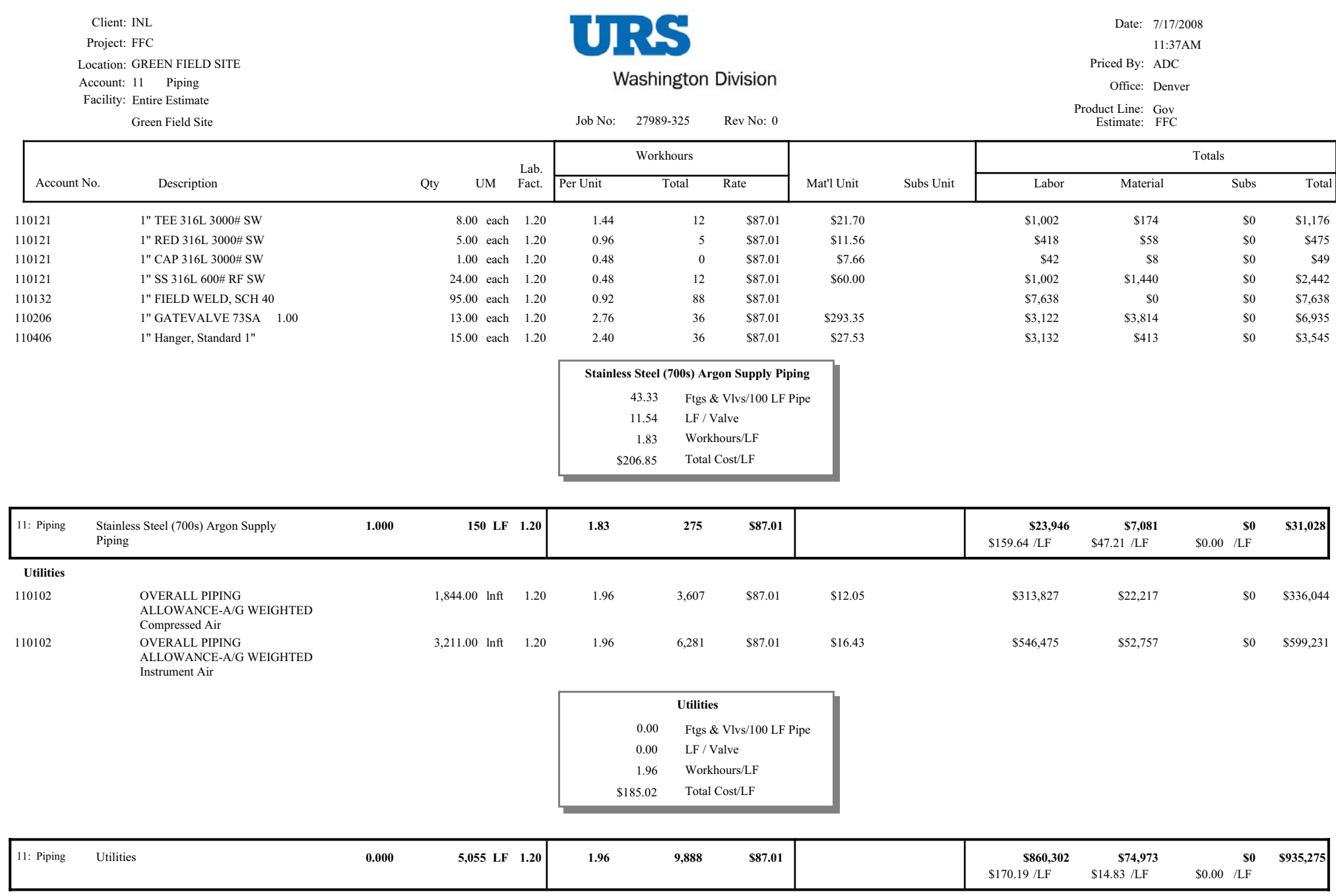


Client: INL

Project: FFC

URS

Washington Division

Account: 11 Piping

Facility: Entire Estimate

Green Field Site

Job No:

27989-325

Rev No: 0
Date: 7/17/2008

11:37AM

Priced By: ADC

Office: Denver

Product Line: Gov
Estimate: FFC

\begin{tabular}{|c|c|c|c|c|c|c|c|c|c|c|c|c|c|}
\hline \multirow[b]{2}{*}{ Account No. } & \multirow[b]{2}{*}{ Description } & \multirow[b]{2}{*}{ Qty } & \multirow[b]{2}{*}{ UM } & \multirow{2}{*}{$\begin{array}{l}\text { Lab. } \\
\text { Fact. }\end{array}$} & \multicolumn{3}{|c|}{ Workhours } & \multirow[b]{2}{*}{ Mat'l Unit } & \multirow[b]{2}{*}{ Subs Unit } & \multicolumn{4}{|c|}{ Totals } \\
\hline & & & & & \begin{tabular}{|l} 
Per Unit \\
\end{tabular} & Total & Rate & & & Labor & Material & Subs & Total \\
\hline
\end{tabular}

\begin{tabular}{|c|c|c|c|c|c|c|c|c|c|c|c|}
\hline Grand Total: & 11: Piping & 1.423 & $13,280 \mathrm{LF}$ & 1.20 & 1.96 & $18,485 \quad \$ 87.01$ & $\begin{array}{c}\mathbf{\$ 1 , 6 0 8 , 3 3 2} \\
\$ 121.11 / \mathrm{LF}\end{array}$ & $\begin{array}{r}\mathbf{\$ 1 4 9 , 8 8 1} \\
\$ 11.29 / \mathrm{LF}\end{array}$ & $\$ 0.0$ & $\begin{array}{l}\mathbf{\$ 0} \\
\text { /LF }\end{array}$ & $\$ 1,758,213$ \\
\hline
\end{tabular}




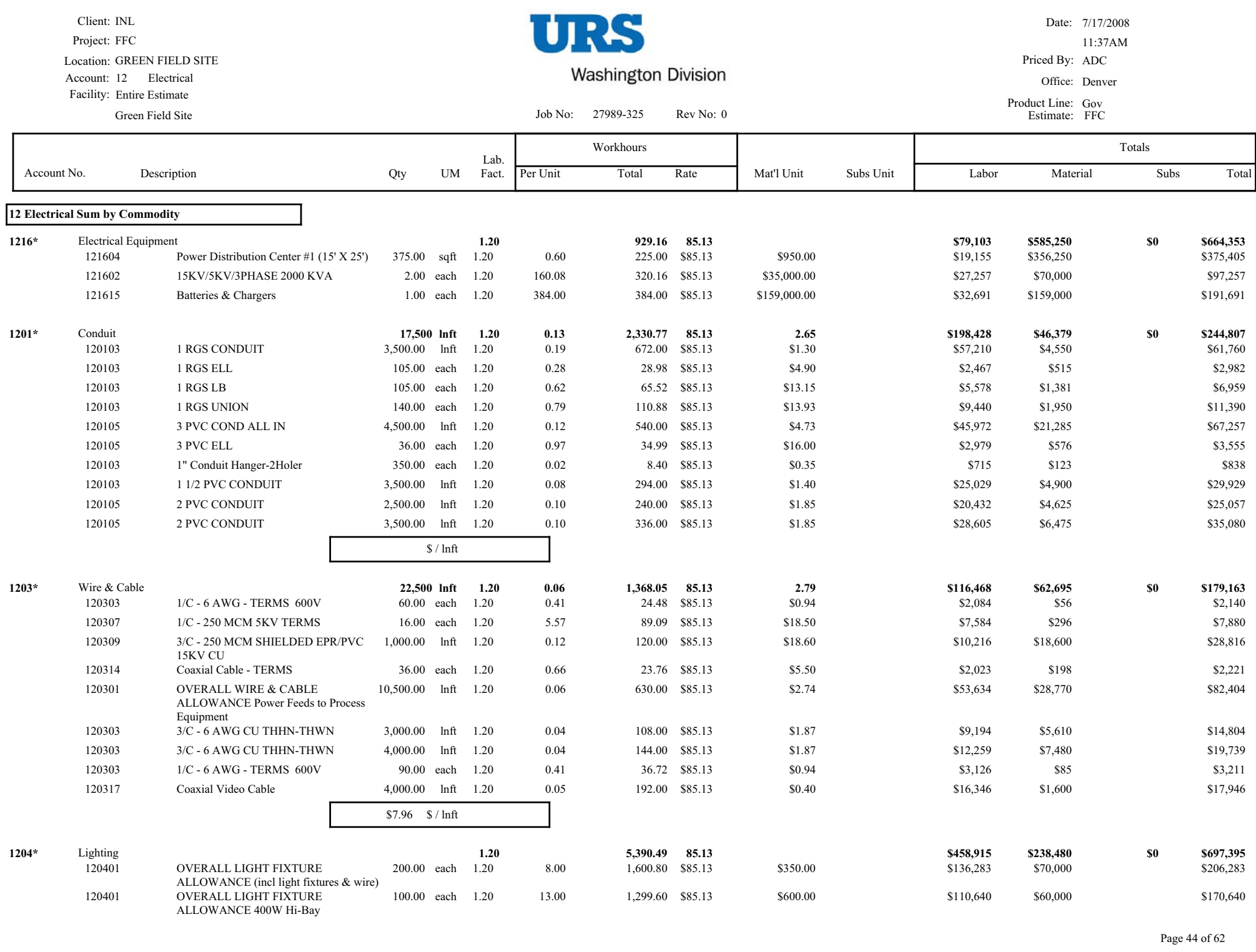




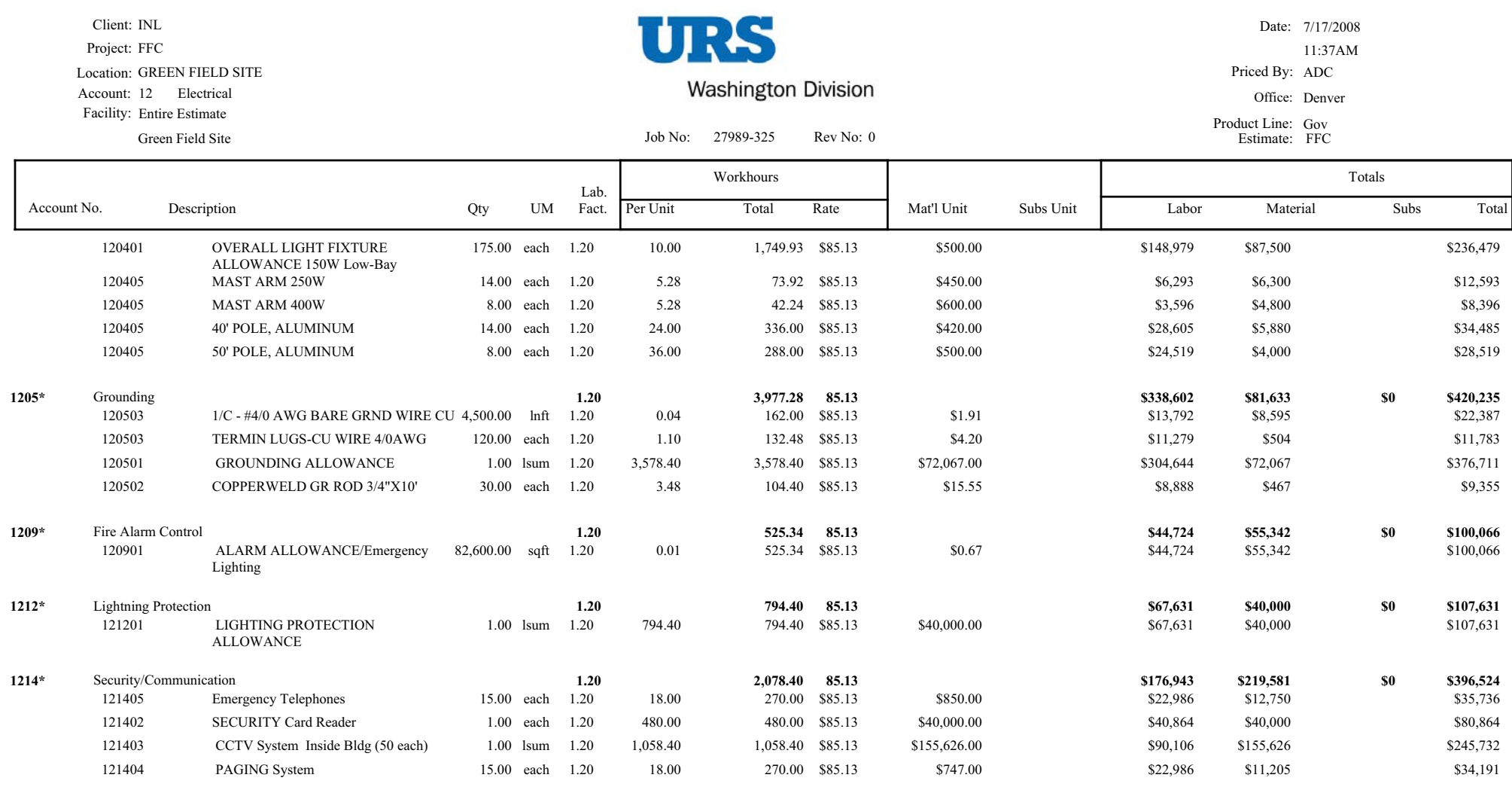

\begin{tabular}{|c|c|c|c|c|c|c|c|c|c|}
\hline Grand Total: 12: Electrical & 1.20 & 17,394 & $\$ 85.13$ & $\$ 33.23$ & $\$ 0.00$ & $\$ 1,480,813$ & $\$ 1,329,359$ & \$o & $\$ 2,810,172$ \\
\hline
\end{tabular}


Client: INL

Project: FFC

URS

Washington Division

ion: GREEN FIELD SITE

Account: 12 Electrical

Facility: Entire Estimate

Green Field Site

Job No: $\quad 27989-325 \quad$ Rev No: 0

Workons

Qty $\quad$ Lab.

Description

Qty UM Fact

12.01 Electrical Equipment

\begin{tabular}{lc}
\hline & Elec. Equip.-Transformers \\
121602 & $15 \mathrm{KV} / 5 \mathrm{KV} / 3 \mathrm{PHASE} 2000 \mathrm{KVA}$ \\
& Electrical Equipment \\
121615 & Batteries \& Chargers \\
& ELECTRICAL SUBSTATION \\
121604 & Power Distribution Center \#1 (15' X 25')
\end{tabular}

$\begin{array}{rrrrrrr}2 & \text { each } & 1.20 & 160.08 & 320.16 & \mathbf{3 8 5 . 1 3} & \\ & & & & \mathbf{3 8 4} & \mathbf{\$ 8 5 . 1 3} & 35,000.00 \\ 1 & \text { each } & 1.20 & 384.00 & 384.00 & 85.13 & 159,000.00 \\ & & & & \mathbf{2 2 5} & \mathbf{\$ 8 5 . 1 3} & \\ 375 & \text { sqft } & 1.20 & 0.60 & 225.00 & 85.13 & 950.00\end{array}$

\begin{tabular}{|c|c|c|c|c|c|c|}
\hline \multirow{3}{*}{2} & & & & 320 & $\$ 85.13$ & \multirow{3}{*}{$35,000.00$} \\
\hline & each & 1.20 & 160.08 & 320.16 & 85.13 & \\
\hline & & & & 384 & $\$ 85.13$ & \\
\hline \multirow[t]{2}{*}{1} & each & 1.20 & 384.00 & 384.00 & 85.13 & $159,000.00$ \\
\hline & & & & 225 & $\$ 85.13$ & \\
\hline 375 & sqft & 1.20 & 0.60 & 225.00 & 85.13 & 950.00 \\
\hline
\end{tabular}

$\begin{array}{lllllll}375 & \text { sqft } & 1.20 & 0.60 & 225.00 & 85.13 & 950.00\end{array}$

Date: 7/17/2008

11:37AM

Priced By: ADC

Office: Denver

Product Line: Gov
Estimate: FFC

\begin{tabular}{lll}
\multicolumn{3}{c}{ Totals } \\
\hline Material & Subs & Total
\end{tabular}

3

$\$ 79,103$

$\$ 585,250$ 
Client: INL

Project: FFC

URS

Date: 7/17/2008

11:37AM

Location: GREEN FIELD SITE

Washington Division

Priced By: ADC

Office: Denver

Facility: Entire Estimate

Job No: $27989-325 \quad$ Rev No: 0

Product Line: Gov
Estimate: FFC

Workous

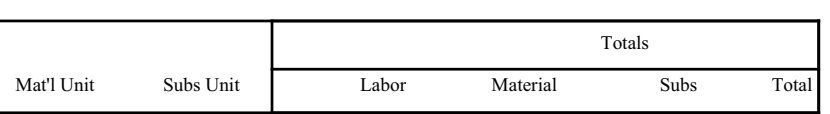

Account No.

Description

Qty UM Fact.

Per Unit Total Rate

$3,500.00 \quad \mathbf{L F}$

$\begin{array}{ll}3,500.00 & \text { Inft } \\ 3,500.00 & \text { LF }\end{array}$

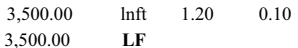

$3,500.00$

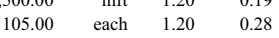

105.00 each $1.20 \quad 0.62$

$\begin{array}{llll}140.00 & \text { each } & 1.20 & 0.79 \\ 350.00 & \text { each } & 1.20 & 0.02\end{array}$

$4,500.00 \quad$ LF

$\begin{array}{llll}4,500.00 & \text { Inft } & 1.20 & 0.12\end{array}$

$\begin{array}{rrrr}36.00 & \text { each } & 1.20 & 0.12 \\ 3.20 & 0.97\end{array}$

36.00
$2,500.00$

$\begin{array}{llll}2,500.00 & \text { Inft } & 1.20 & 0.10\end{array}$

$3,500.00$

$\begin{array}{llll}3,500.00 & \text { lnft } & 1.20 & 0.08\end{array}$

$\begin{aligned} \mathbf{3 3 6} & \\ 336.00 & \$ 85.13 \\ \mathbf{8 8 6} & \\ 672.00 & \$ 85.13 \\ 28.98 & \$ 85.13 \\ 65.52 & \$ 85.13 \\ 110.88 & \$ 85.13 \\ 8.40 & \$ 85.13 \\ \mathbf{5 7 5} & \\ 540.00 & \$ 85.13 \\ 34.99 & \$ 85.13 \\ \mathbf{2 4 0} & \\ 240.00 & \$ 85.13 \\ \mathbf{2 9 4} & \\ 294.00 & \$ 85.13\end{aligned}$

$\$ 1.85$

$\$ 1.30$
$\$ 4.90$
$\$ 13.15$
$\$ 13.93$
$\$ 0.35$

$\$ 4.73$
$\$ 16.00$

$\$ 1.85$
$\$ 1.40$

28,605

$\$ 28,605$

$\mathbf{6 , 4 7 5}$

$\$ 6,475$

75,410

$\$ 57,210$

$\$ 2,467$

$\$ 5,578$

$\$ 9,440$
$\$ 715$

48,951

$\$ 45,972$

$\$ 45,972$
$\$ 2,979$

20,432

$\$ 20,432$
$\mathbf{2 5 , 0 2 9}$

1 1/2 PVC CONDUIT

$\$ 85.13$

$\$ 1.40$

$\$ 25,029$

$\$ 4,550$

$\$ 515$

$\$ 1,381$

$\$ 1,950$
$\$ 123$

21,861

$\$ 21,285$

$\$ 576$

4,625

$\$ 4,625$

$\$ 4,900$ $\mathbf{3 5 , 0 8 0}$

$\$ 35,080$

83,928

$\$ 61,760$

$\$ 2,982$

$\$ 6,959$

$\$ 11,390$

$\$ 838$
$\mathbf{7 0 , 8 1 2}$

$\$ 67,257$

$\$ 3,555$

$\$ 3,555$

$\mathbf{2 5 , 0 5 7}$
$\$ 25,057$

29,929

$\$ 29,929$

Grand Total:

$17,500 \quad$ LF

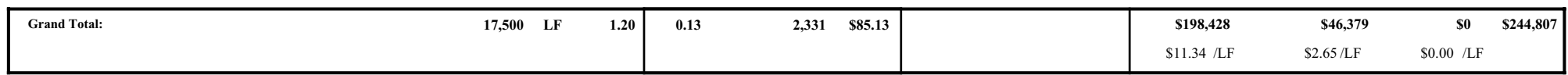

$2,331 \quad \$ 85.13$

Overall Ratios:

0.13 Workhours/LF

$\$ 2.65$ Material Cost/LF

$\$ 13.99$ Total Cost/LF

sis.ge Total $/ \mathrm{L}$ 
Client: INL

Project: FFC

Location: GREEN FIELD SITE

Account: 12 Electrical

URS

Date: 7/17/2008

11:37AM

Washington Division

Priced By: ADC

Office: Denver

Facility: Entire Estimate

Job No: 27989-325 Rev No: 0

Product Line: Gov
Estimate: FFC

\begin{tabular}{|c|c|c|c|c|c|c|c|c|c|c|c|c|c|}
\hline \multirow[b]{2}{*}{ Account No. } & \multirow[b]{2}{*}{ Description } & \multirow[b]{2}{*}{ Qty } & \multirow[b]{2}{*}{ UM } & \multirow{2}{*}{$\begin{array}{l}\text { Lab. } \\
\text { Fact. }\end{array}$} & \multicolumn{3}{|c|}{ Workhours } & \multirow[b]{2}{*}{ Mat'l Unit } & \multirow[b]{2}{*}{ Subs Unit } & \multicolumn{4}{|c|}{ Totals } \\
\hline & & & & & \begin{tabular}{|l|l} 
Per Unit \\
\end{tabular} & Total & Rate & & & Labor & Material & Subs & Total \\
\hline \multicolumn{14}{|c|}{ 12.03 Electrical Wire/Cable } \\
\hline & AREA FENCE LIGHTING CABLE & 4,000 & Inft & & & 168 & 85.13 & & & $\$ 14,343$ & $\$ 7,536$ & $\$ 0$ & $\$ 21,880$ \\
\hline \multirow{3}{*}{$\begin{array}{l}20303 \\
20303\end{array}$} & 3/C - 6 AWG CU THHN-THWN & 4,000 & $\operatorname{lnft}$ & 1.20 & 0.04 & 144.00 & 85.13 & 1.87 & & $\$ 12,259$ & $\$ 7,480$ & $\$ 0$ & $\$ 19,739$ \\
\hline & 1/C - 6 AWG - TERMS $600 \mathrm{~V}$ & 60 & ach & 1.20 & 0.41 & 24.48 & 85.13 & 0.94 & & $\$ 2,084$ & $\$ 56$ & $\$ 0$ & $\$ 2,140$ \\
\hline & Power Feeds Process Area & 10,500 & Inft & & & 630 & 85.13 & & & $\$ 53,634$ & $\$ 28,770$ & $\$ 0$ & $\$ 82,404$ \\
\hline \multirow[t]{2}{*}{20301} & $\begin{array}{l}\text { OVERALL WIRE \& CABLE ALLOWANCE } \\
\text { Power Feeds to Process Equipment }\end{array}$ & 10,500 & lnft & 1.20 & 0.06 & 630.00 & 85.13 & 2.74 & & $\$ 53,634$ & $\$ 28,770$ & $\$ 0$ & $\$ 82,404$ \\
\hline & ROADWAY LIGHTING CABLE & 3,000 & Inft & & & 145 & 85.13 & & & $\$ 12,321$ & $\$ 5,695$ & \$o & $\$ 18,015$ \\
\hline \multirow{3}{*}{$\begin{array}{l}20303 \\
20303\end{array}$} & 3/C - 6 AWG CU THHN-THWN & 3,000 & $\operatorname{lnft}$ & 1.20 & 0.04 & 108.00 & 85.13 & 1.87 & & $\$ 9,194$ & $\$ 5,610$ & $\$ 0$ & $\$ 14,804$ \\
\hline & 1/C - 6 AWG - TERMS $600 \mathrm{~V}$ & 90 & ach & 1.20 & 0.41 & 36.72 & 85.13 & 0.94 & & $\$ 3,126$ & $\$ 85$ & $\$ 0$ & $\$ 3,211$ \\
\hline & SECURITY CAMERA CABLE & 4,000 & Inft & & & 216 & 85.13 & & & $\$ 18,369$ & $\$ 1,798$ & \$o & $\$ 20,167$ \\
\hline \multirow{3}{*}{$\begin{array}{l}20314 \\
20317\end{array}$} & Coaxial Cable - TERMS & 36 & ach & 1.20 & 0.66 & 23.76 & 85.13 & 5.50 & & $\$ 2,023$ & $\$ 198$ & $\$ 0$ & $\$ 2,221$ \\
\hline & Coaxial Video Cable & 4,000 & $\operatorname{lnft}$ & 1.20 & 0.05 & 192.00 & 85.13 & 0.40 & & $\$ 16,346$ & $\$ 1,600$ & $\$ 0$ & $\$ 17,946$ \\
\hline & Wire \& Cable-Medium Voltage ( 5 \& $15 \mathrm{kV}$ ) & 1,000 & Inft & & & 209 & 85.13 & & & $\$ 17,801$ & $\$ 18,896$ & $\$ 0$ & $\$ 36,697$ \\
\hline 20307 & 1/C - 250 MCM 5KV TERMS & 16 & ach & 1.20 & 5.57 & 89.09 & 85.13 & 18.50 & & $\$ 7,584$ & $\$ 296$ & $\$ 0$ & $\$ 7,880$ \\
\hline 20309 & $\begin{array}{l}\text { 3/C - } 250 \text { MCM SHIELDED EPR/PVC } 15 \mathrm{KV} \\
\mathrm{CU}\end{array}$ & 1,000 & $\operatorname{lnft}$ & 1.20 & 0.12 & 120.00 & 85.13 & 18.60 & & $\$ 10,216$ & $\$ 18,600$ & $\$ 0$ & $\$ 28,816$ \\
\hline
\end{tabular}

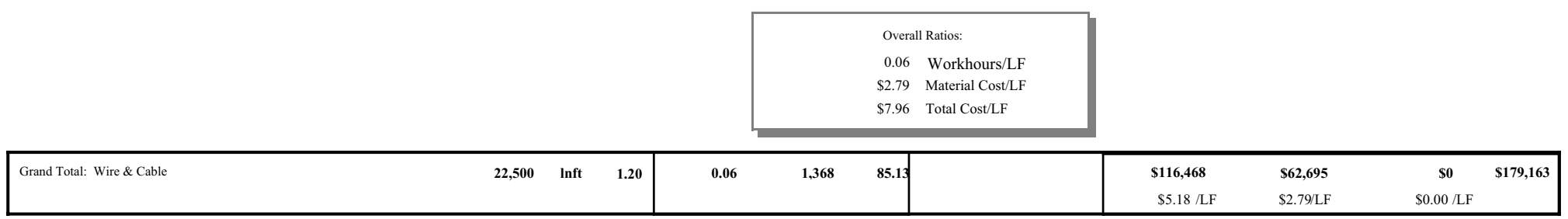

Page 48 of 62 


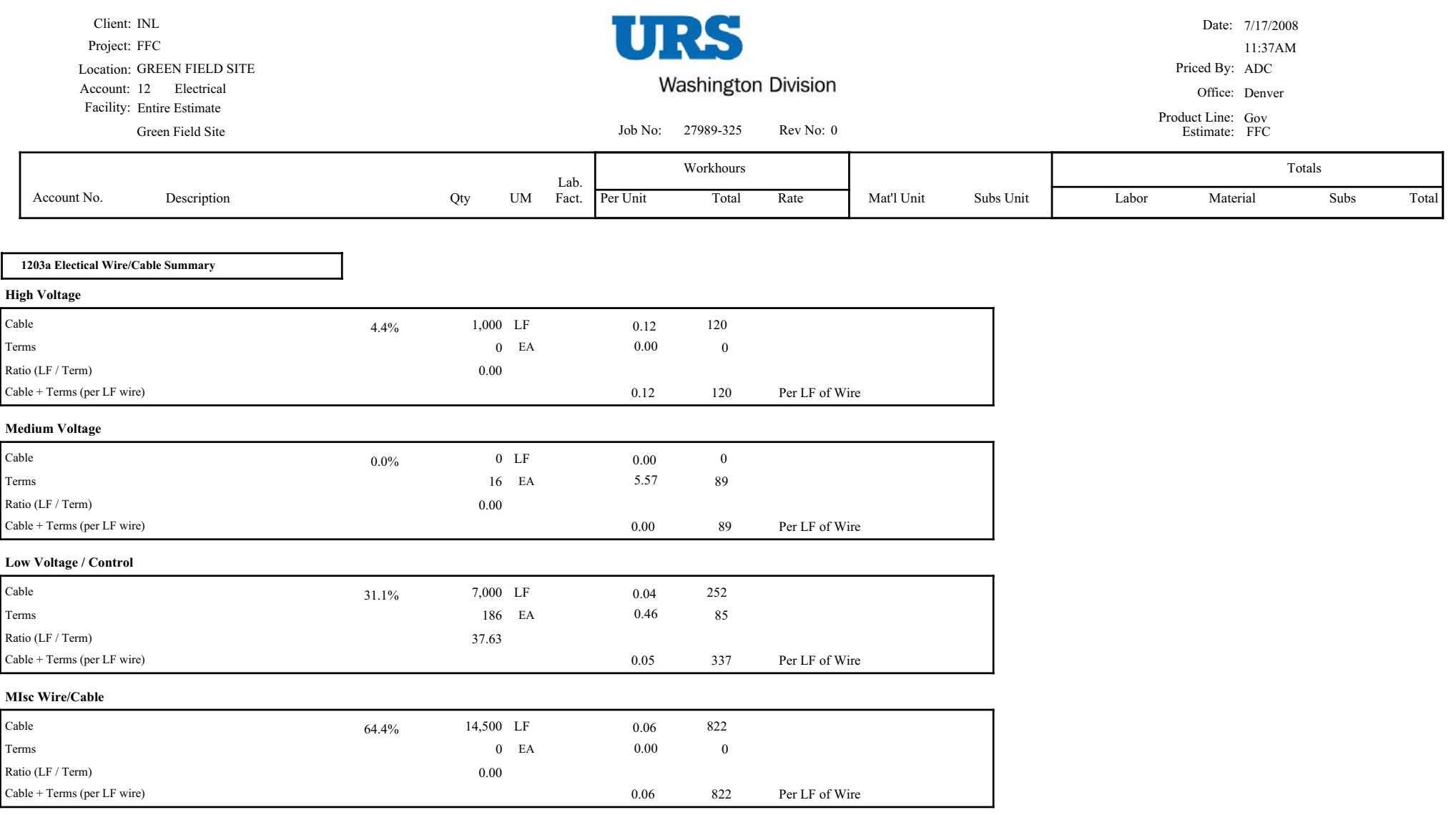

Overall
\begin{tabular}{|lrrrrr|r|}
\hline Cable & $100.0 \%$ & 22,500 & LF & 0.05 & 1,194 & \\
Terms & & 202 EA & 0.86 & 174 & \\
Ratio (LF / Term) & & 111.39 & & & & \\
Cable + Terms & & & 0.06 & 1,368 & Per LF of Wire \\
\hline
\end{tabular}


Client: INL

Project: FFC

Location: GREEN FIELD SITE

Account: 12 Electrical

URS

Washington Division

Job No: $\quad 27989-325 \quad$ Rev No: 0

Green Field Site

Description

Qty UM Fact.

Workhours

Per Unit

Total Rate

n.

12.04 Electrical Lighting

$\begin{array}{ll} & \text { AREA FENCE LIGHTING } \\ 120405 & \text { MAST ARM 400W } \\ 120405 & 50^{\prime} \text { POLE, ALUMINUM } \\ & \text { Finishes Office Area } \\ & \text { OVERALL LIGHT FIXTURE ALLOWANCE } \\ & \text { (incl light fixtures \& wire) } \\ & \text { Finishes Process Area } \\ & \text { OVERALL LIGHT FIXTURE ALLOWANCE } \\ 120401 & \text { 400W Hi-Bay } \\ & \text { OVERALL LIGHT FIXTURE ALLOWANCE } \\ 120401 & \text { 150W Low-Bay } \\ & \text { ROADWAY LIGHTING } \\ 120405 & \text { MAST ARM 250W } \\ 120405 & \text { 40' POLE, ALUMINUM }\end{array}$

$\begin{array}{rrrrrrr}8.00 & \text { each } & 1.20 & 5.28 & 43.24 & \$ 85.13 & \\ 8.00 & \text { each } & 1.20 & 36.00 & 288.00 & \$ 85.13 & \$ 600.13 \\ & & & & 1,601 & \$ 85.13 & \$ 500.00 \\ 200.00 & \text { each } & 1.20 & 8.00 & 1,600.80 & \$ 85.13 & \$ 350.00 \\ & & & & 3,050 & \$ 85.13 & \\ 100.00 & \text { each } & 1.20 & 13.00 & 1,299.60 & \$ 85.13 & \$ 600.00 \\ 175.00 & \text { each } & 1.20 & 10.00 & 1,749.93 & \$ 85.13 & \$ 500.00 \\ & & & & 410 & \$ 85.13 & \\ 14.00 & \text { each } & 1.20 & 5.28 & 73.92 & \$ 85.13 & \$ 450.00 \\ 14.00 & \text { each } & 1.20 & 24.00 & 336.00 & \$ 85.13 & \$ 420.00\end{array}$

\begin{tabular}{|c|c|c|c|c|}
\hline \multirow[b]{2}{*}{ Mat'l Unit } & \multirow[b]{2}{*}{ Subs Unit } & \multicolumn{3}{|c|}{ Totals } \\
\hline & & Labor & Material & Subs \\
\hline
\end{tabular}

$\begin{array}{lrr} & & \\ \$ 600.00 & \$ 3,115 & \$ 8,800 \\ \$ 500.00 & \$ 24,519 & \$ 4,800 \\ & \$ 136,283 & \$ 70,000 \\ \$ 350.00 & \$ 136,283 & \$ 70,000 \\ & \$ 259,619 & \$ 147,500 \\ \$ 600.00 & \$ 110,640 & \$ 60,000 \\ \$ 500.00 & \$ 148,979 & \$ 87,500 \\ & \$ 34,898 & \$ 12,180 \\ \$ 450.00 & \$ 6,293 & \$ 6,300 \\ \$ 420.00 & \$ 28,605 & \$ 5,880\end{array}$

Date: 7/17/2008

11:37AM

Priced By: ADC

Office: Denver

Estimate: Gov

$\begin{array}{lr}\$ 0 & \$ 36,915 \\ \$ 0 & \$ 8,396 \\ \$ 0 & \$ 28,519 \\ \$ 0 & \$ 206,283 \\ \$ 0 & \$ 206,283 \\ \$ 0 & \$ 407,119 \\ \$ 0 & \$ 170,640 \\ \$ 0 & \$ 236,479 \\ \$ 0 & \$ 47,078 \\ \$ 0 & \$ 12,593 \\ \$ 0 & \$ 34,485\end{array}$


Client: INL

Project: FFC

Location: GREEN FIELD SITE

Account: 12 Electrical

URS

Date: 7/17/2008

11:37AM

Facility: Entire Estimate

Washington Division

Priced By: ADC

Office: Denver

Green Field Site

Job No: $\quad 27989-325 \quad$ Rev No: 0

oduct Line: Gov
Estimate: FFC

\begin{tabular}{|c|c|c|c|c|c|c|c|c|c|c|}
\hline \multirow[b]{2}{*}{ Account No. } & \multirow[b]{2}{*}{ Description } & \multirow[b]{2}{*}{ Qty } & \multirow[b]{2}{*}{ UM } & \multirow{2}{*}{$\begin{array}{l}\text { Lab. } \\
\text { Fact. }\end{array}$} & & rkhours & \multirow[b]{2}{*}{ Mat'l Unit } & \multirow[b]{2}{*}{ Subs Unit } & \multicolumn{2}{|c|}{ Totals } \\
\hline & & & & & Per Unit & Total & & & Labor & Material \\
\hline
\end{tabular}

\section{Electrical Other}

\begin{tabular}{|c|c|}
\hline & Electrical Systems \\
\hline 120501 & GROUNDING ALLOWANCE \\
\hline & FENCE GROUND CABLE \\
\hline 120503 & $1 / \mathrm{C}$ - \#4/0 AWG BARE GRND WIRE CU \\
\hline 120503 & TERMIN LUGS-CU WIRE 4/0AWG \\
\hline & FENCE GROUND RODS \\
\hline 120502 & COPPERWELD GR ROD 3/4"X10' \\
\hline $1205^{*}$ & Grounding \\
\hline & Alarm Systems \\
\hline 120901 & ALARM ALLOWANCE/Emergency Lighting \\
\hline $1209^{*}$ & Fire Alarm Control \\
\hline & Electrical Systems \\
\hline 121201 & LIGHTING PROTECTION ALLOWANCE \\
\hline 1212 * & Lightning Protection \\
\hline & Access System \\
\hline 121402 & SECURITY Card Reader \\
\hline & Electrical Systems \\
\hline 121403 & CCTV System Inside Bldg (50 each) \\
\hline 121404 & PAGING System \\
\hline 121405 & Emergency Telephones \\
\hline
\end{tabular}

$\begin{array}{rrrrrrr} & & & & \mathbf{3 , 5 7 8} & \$ 85.13 & \\ 1.00 & \text { Isum } & 1.20 & 3,578.40 & 3,578.40 & \$ 85.13 & \$ 72,067.00 \\ & & & & \mathbf{2 9 4} & \$ 85.13 & \\ 4,500.00 & \text { Inft } & 1.20 & 0.04 & 162.00 & \$ 85.13 & \$ 1.91 \\ 120.00 & \text { each } & 1.20 & 1.10 & 132.48 & \$ 85.13 & \$ 4.20 \\ & & & & \mathbf{1 0 4} & \$ 85.13 & \\ 30.00 & \text { each } & 1.20 & 3.48 & 104.40 & \$ 85.13 & \$ 15.55 \\ \mathbf{4 , 5 0 0 . 0 0} & \text { Inft } & \mathbf{1 . 2 0} & & \mathbf{3 , 9 7 7} & \mathbf{\$ 8 5 . 1 3} & \\ & & & & \mathbf{5 2 5} & \$ 85.13 & \\ 82,600.00 & \text { sqft } & 1.20 & 0.01 & 525.34 & \$ 85.13 & \$ 0.67 \\ \mathbf{0 . 0 0} & \text { Inft } & \mathbf{1 . 2 0} & & \mathbf{5 2 5} & \mathbf{\$ 8 5 . 1 3} & \\ & & & & \mathbf{7 9 4} & \$ 85.13 & \\ 1.00 & \text { lsum } & 1.20 & 794.40 & 794.40 & \$ 85.13 & \$ 40,000.00 \\ \mathbf{0 . 0 0} & \text { Inft } & \mathbf{1 . 2 0} & & \mathbf{7 9 4} & \mathbf{\$ 8 5 . 1 3} & \\ & & & & \mathbf{4 8 0} & \$ 85.13 & \\ 1.00 & \text { each } & 1.20 & 480.00 & 480.00 & \$ 85.13 & \$ 40,000.00 \\ & & & & \mathbf{1 , 5 9 8} & \$ 85.13 & \\ 1.00 & \text { lsum } & 1.20 & 1,058.40 & 1,058.40 & \$ 85.13 & \$ 155,626.00 \\ 15.00 & \text { each } & 1.20 & 18.00 & 270.00 & \$ 85.13 & \$ 747.00 \\ 15.00 & \text { each } & 1.20 & 18.00 & 270.00 & \$ 85.13 & \$ 850.00 \\ \mathbf{0 . 0 0} & \text { Inft } & \mathbf{1 . 2 0} & & \mathbf{2 , 0 7 8} & \mathbf{\$ 8 5 . 1 3} & \end{array}$

\begin{tabular}{|c|c|}
\hline 304,644 & $\$ 72,067$ \\
\hline 304,644 & $\$ 72,067$ \\
\hline$\$ 25,070$ & $\$ 9,099$ \\
\hline$\$ 13,792$ & $\$ 8,595$ \\
\hline$\$ 11,279$ & $\$ 504$ \\
\hline$\$ 8,888$ & $\$ 467$ \\
\hline$\$ 8,888$ & $\$ 467$ \\
\hline 338,602 & $\$ 81,633$ \\
\hline$\$ 44,724$ & $\$ 55,342$ \\
\hline$\$ 44,724$ & $\$ 55,342$ \\
\hline$\$ 44,724$ & $\$ 55,342$ \\
\hline$\$ 67,631$ & $\$ 40,000$ \\
\hline$\$ 67,631$ & $\$ 40,000$ \\
\hline$\$ 67,631$ & $\$ 40,000$ \\
\hline$\$ 40,864$ & $\$ 40,000$ \\
\hline$\$ 40,864$ & $\$ 40,000$ \\
\hline 136,078 & $\$ 179,581$ \\
\hline$\$ 90,106$ & $\$ 155,626$ \\
\hline$\$ 22,986$ & $\$ 11,205$ \\
\hline$\$ 22,986$ & $\$ 12,750$ \\
\hline 176,943 & $\$ 219,581$ \\
\hline
\end{tabular}

$\$ 376,711$

$\$ 376,711$

$\$ 34,169$

$\$ 22,387$

$\$ 11,783$

$\$ \mathbf{9 , 3 5 5}$

$\$ 9,355$

$\$ 420,235$

$\$ 100,066$

$\$ 100,066$

$\$ 100,066$

$\$ 107,631$

$\$ 107,631$

$\$ 107,631$

$\$ 80,864$

$\$ 80,864$

$\$ 315,659$

$\$ 245,732$

$\$ 34,191$

$\$ 35,736$

$\mathbf{\$ 3 9 6 , 5 2 4}$ 


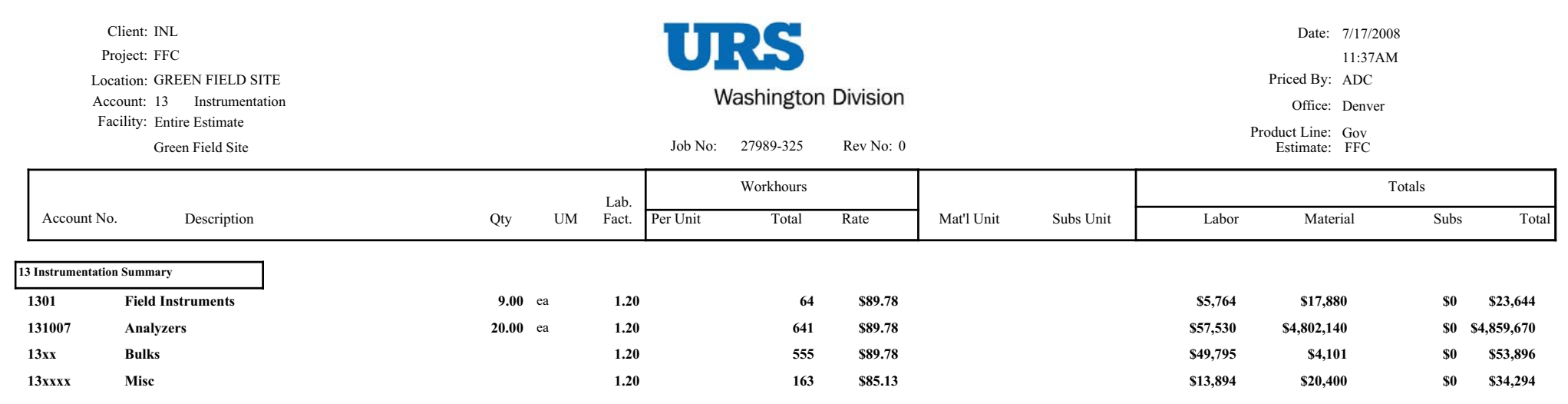

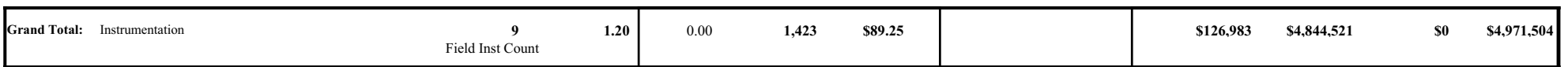




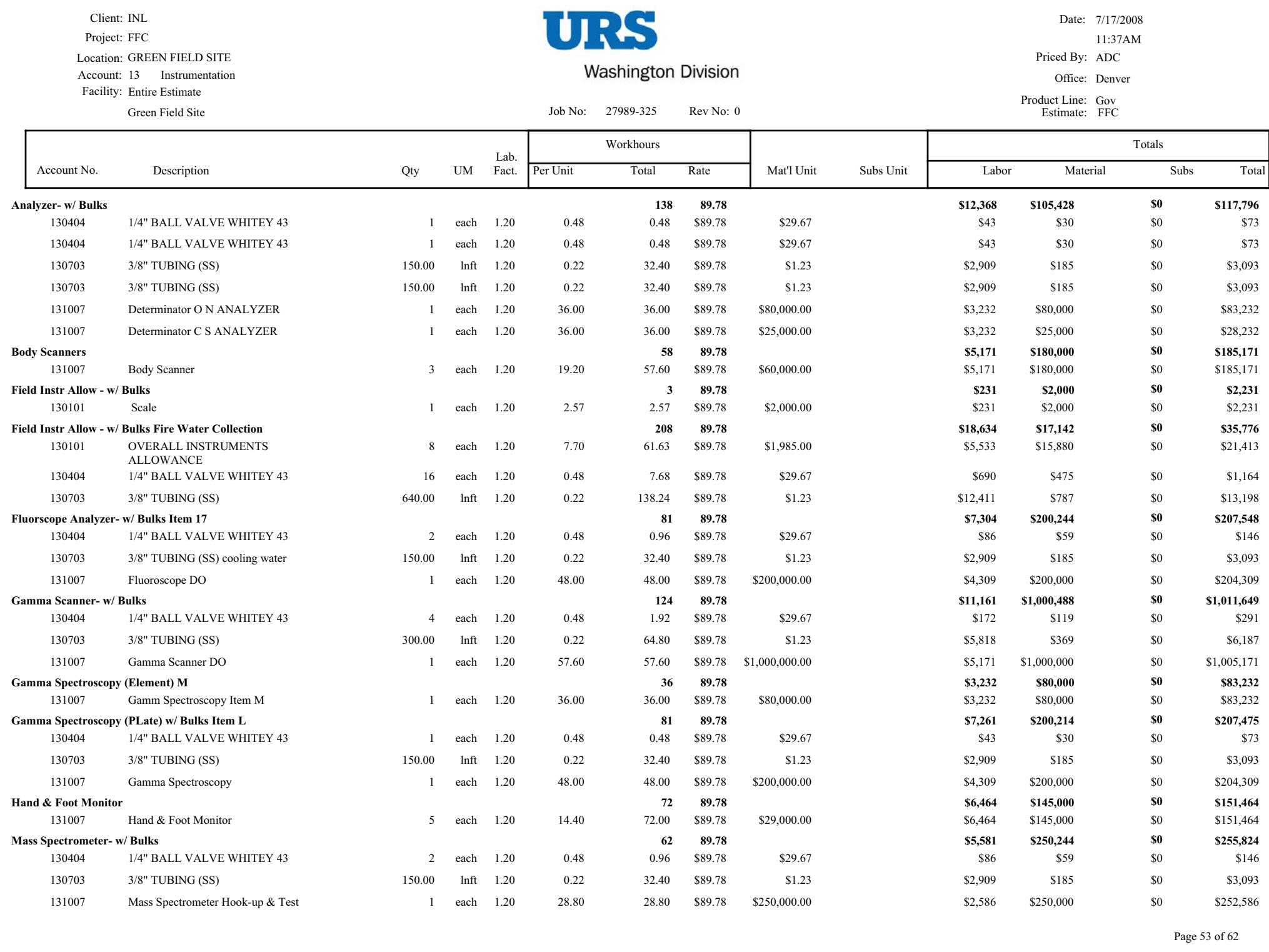




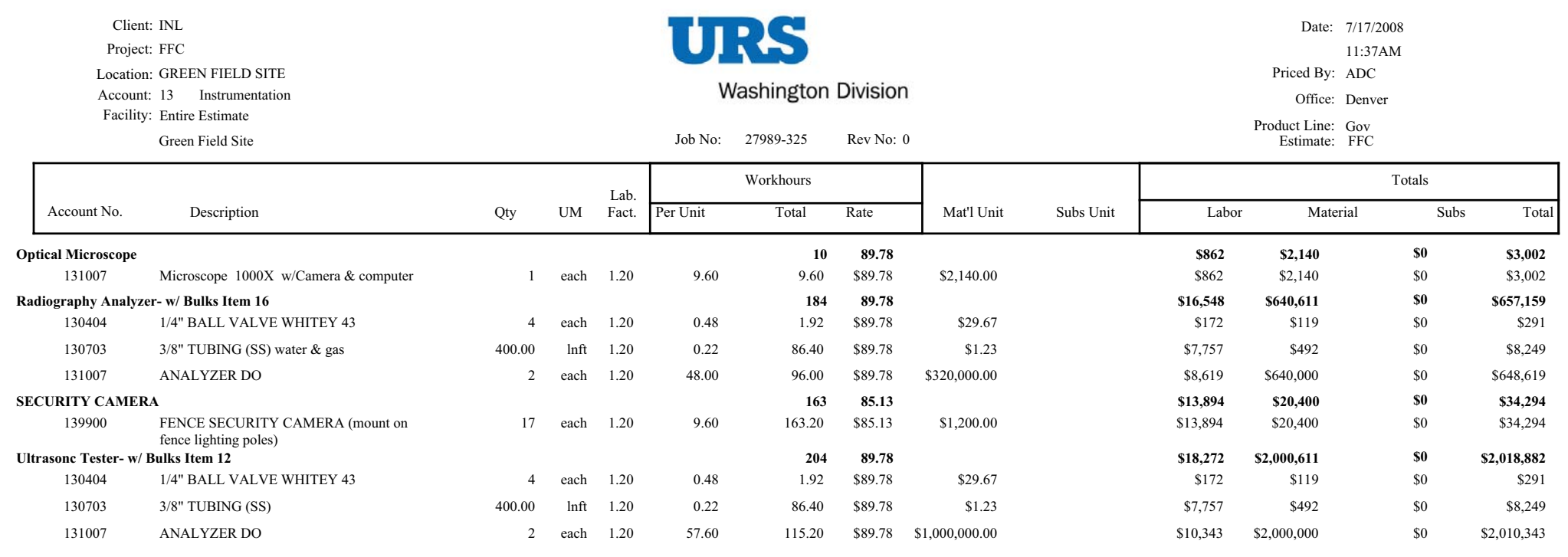

\begin{tabular}{|c|c|c|c|c|c|c|c|}
\hline Totals & $\begin{array}{l}\text { 13-Instrumentation } \\
\text { Entire Estimate }\end{array}$ & $\begin{array}{l}9 \\
\text { Field Inst Count }\end{array}$ & $1,423 \quad \$ 89.25$ & $\$ 126,983$ & $\$ 4,844,521$ & $\$ 0$ & $\$ 4,971,504$ \\
\hline
\end{tabular}




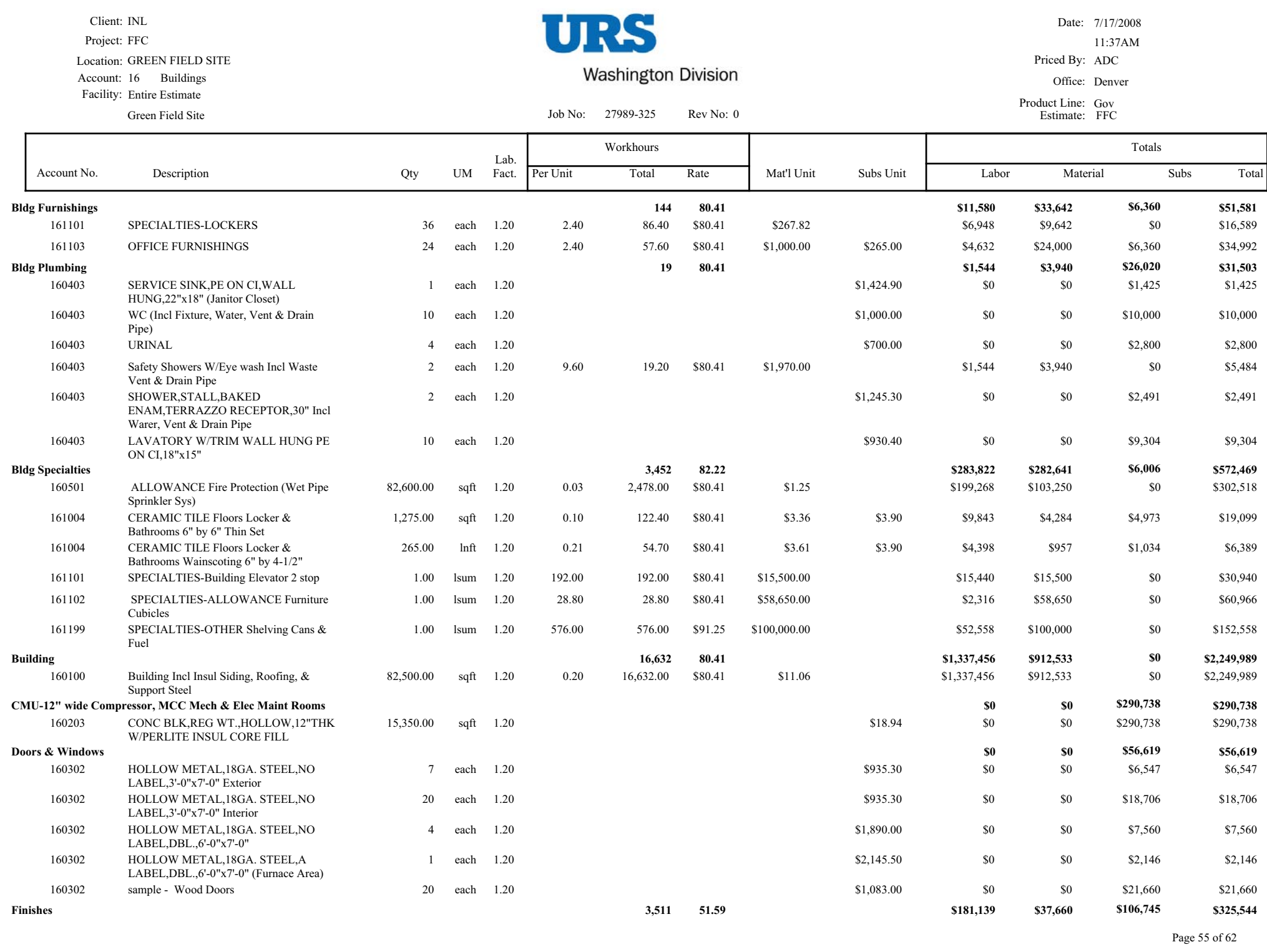




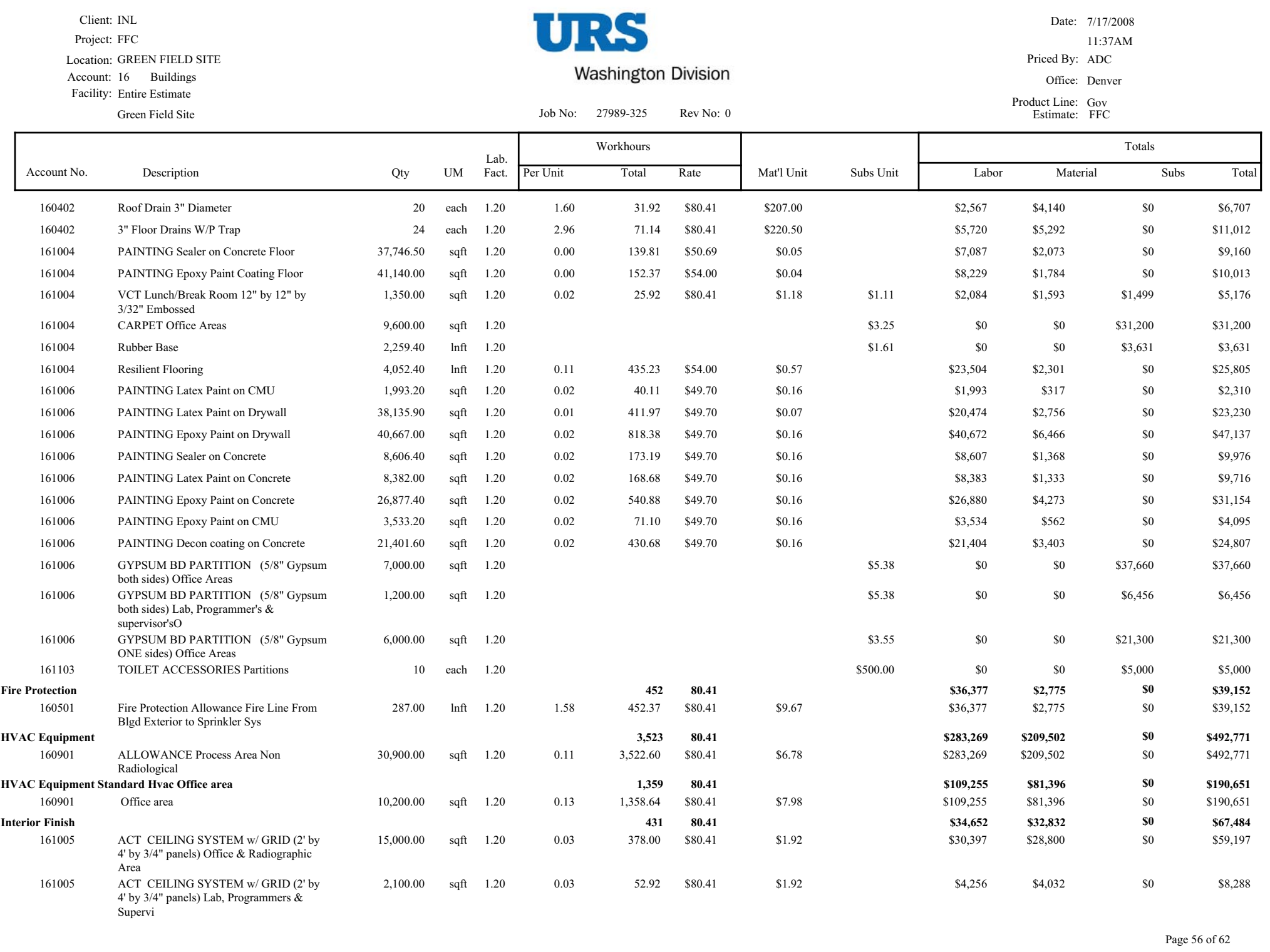




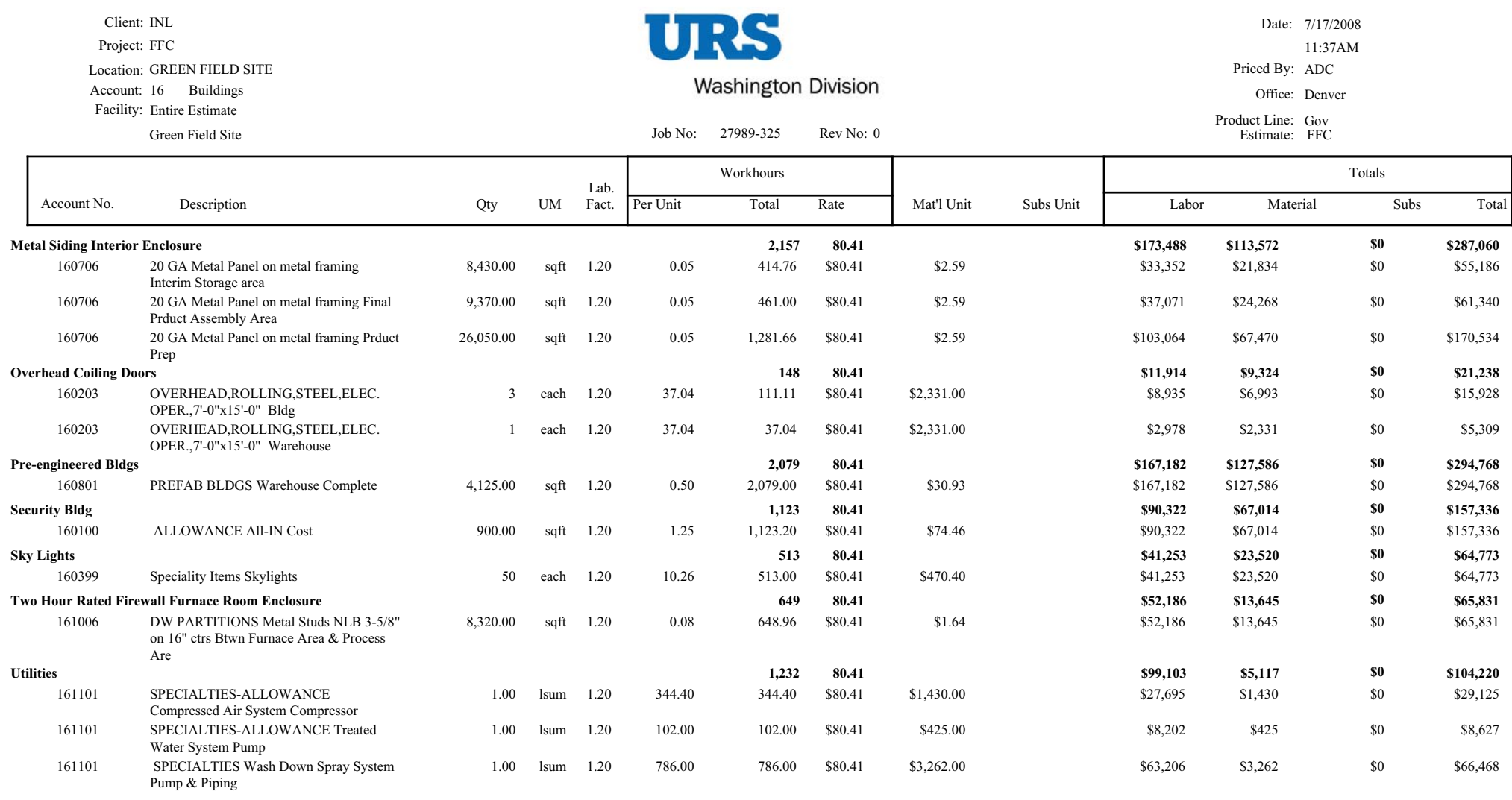

\begin{tabular}{|c|c|c|c|c|c|c|c|}
\hline Totals & $\begin{array}{l}\text { 16-Buildings } \\
\text { Entire Estimate }\end{array}$ & 37,425 & $\$ 77.88$ & $\$ 2,914,541$ & $\$ 1,956,697$ & $\$ 492,487$ & $\$ 5,363,725$ \\
\hline
\end{tabular}


Project: FFC

URS

Date: 7/17/2008

11:37AM

Location: GREEN FIELD SITE

Washington Division

Priced By: ADC

ccount: 21 Spare Part

Washington Division

Office: Denver

Facility: Entire Estimate

Job No: $\quad 27989-325 \quad$ Rev No: 0

Product Line:
Estimate: FFC

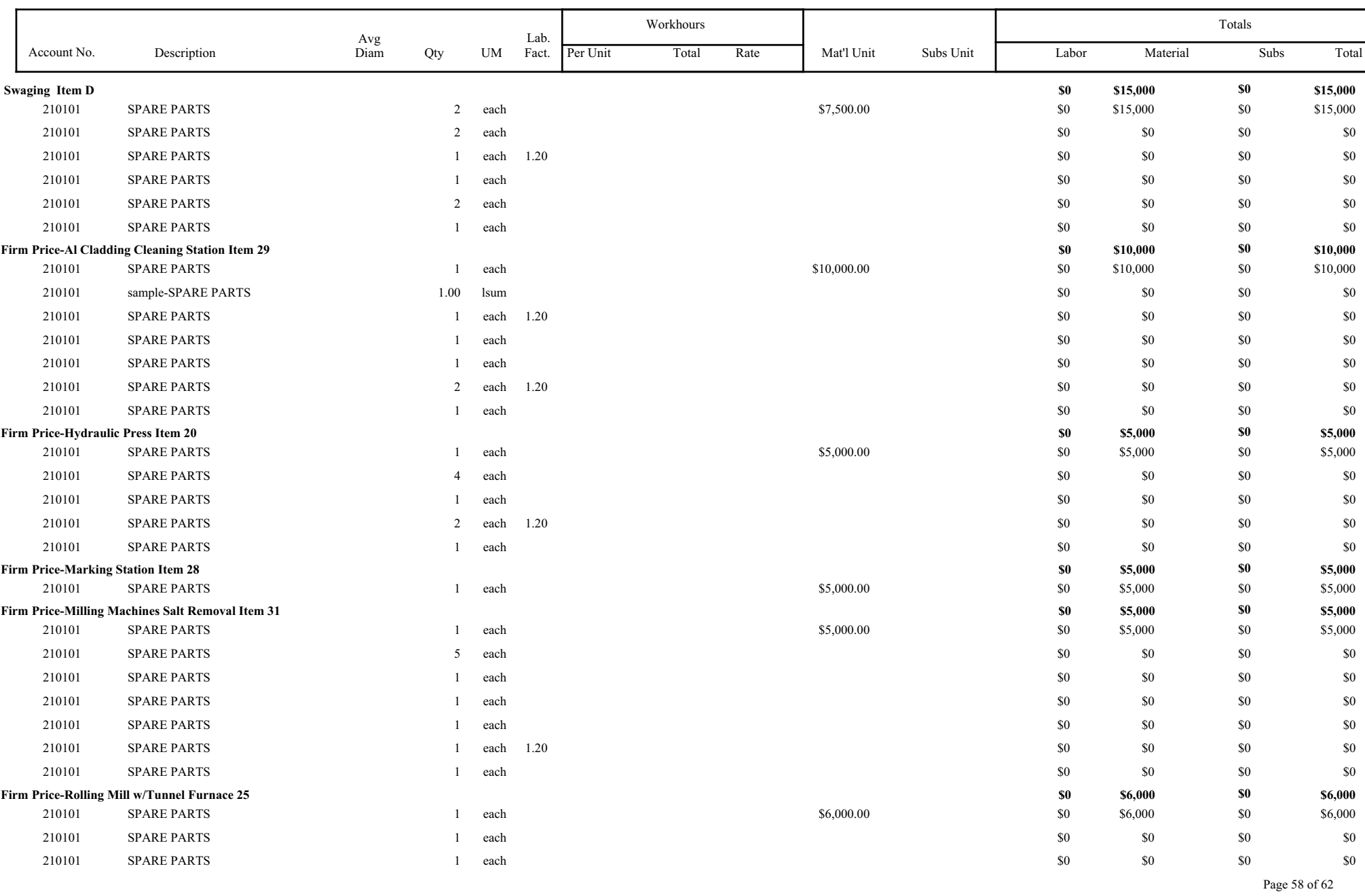


Client: INL

Project: FFC

URS

Date: 7/17/2008

11:37AM

Location: GREEN FIELD SITE

Washington Division

Priced By: ADC

Account: 21 Spare Part

Job No: $\quad 27989-325 \quad$ Rev No: 0

Office: Denver

Facility: Entire Estimate

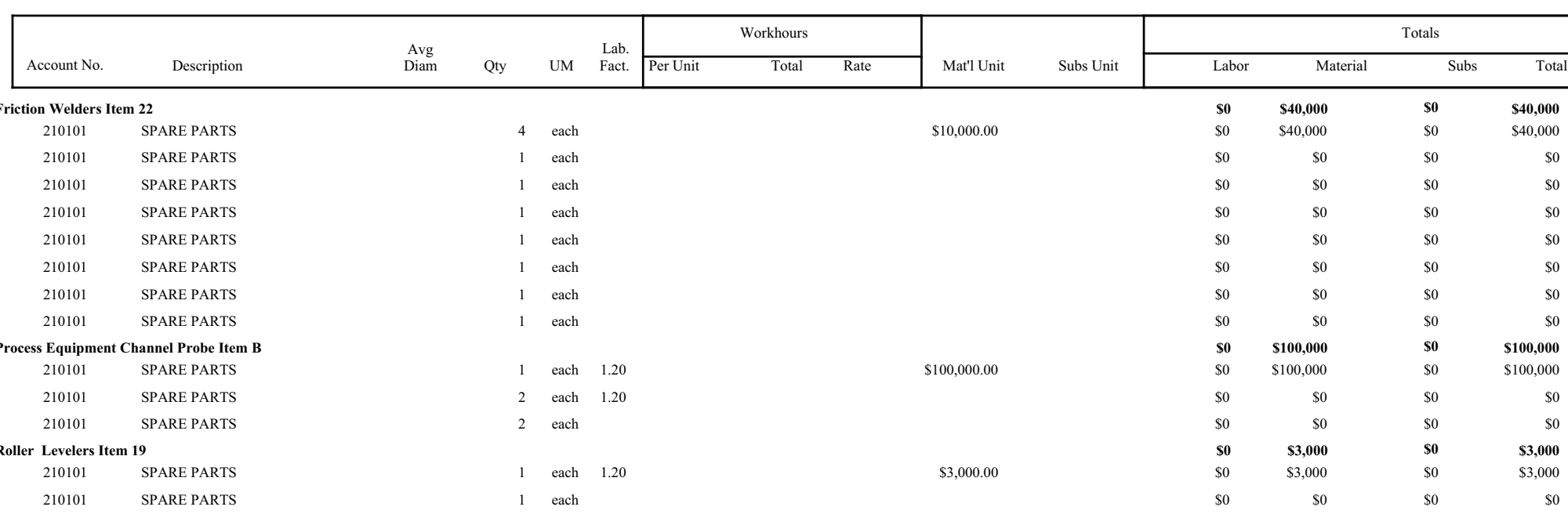

Product Line: Gov
Estimate: FFC

\begin{tabular}{|l|l}
\hline so & so \\
\hline
\end{tabular}


Client: INL

Project: FFC

URS

Washington Division

Job No: $\quad 27989-325 \quad$ Rev No: 0

Account: 31 Construction Management Staff \& Services

Facility: Entire Estimate

Green Field Site

Workhours

$\begin{array}{ll} & \\ \text { Qty Uab. }\end{array}$

Description

Construction Management - URS

$310101 \quad$ Site Manage

$310603 \quad$ Project Secretary (Local)

$310901 \quad$ Safety Professional

310901

310901

310901

310901

310901

Superientendant Civil/Structural (local)

Superientendant Piping/Mech (local)

Superientendant Elec/I\& C (local)

Project Controls

Superientendant Elec/I\& C (local)

24.00 mnth

24.00 mnth

24.00 mnth

$8.00 \mathrm{mnth}$

4.00 mnth

6.00 mnth

18.00 mnth

14.00 mnth

Per Unit

172.00

Total $\quad$ R

$\begin{array}{rr}\mathbf{2 0 , 9 8 4} & \mathbf{7 0 . 4 0} \\ 4,128.00 & \$ 95.00\end{array}$

172.00

$4,128.00$

$4,128.00 \quad \$ 38.20$

172.00

172.00

$4,128.00$

$1,376.00$

688.00

$1,032.00$

$3,096.00$

$2,408.00$

172.00
Date: 7/17/2008

11:37AM Priced By: ADC

Office: Denver

Product Line: Gov
Estimate: FFC

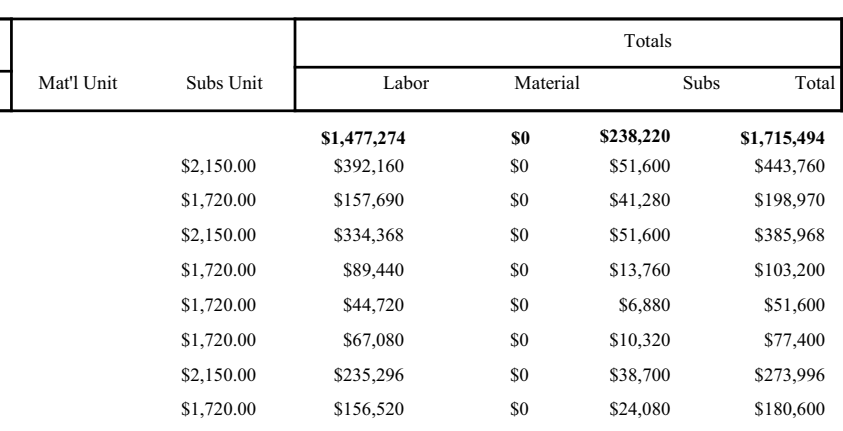

Totals 31-Construction Management Staff \& Services Entire Estimate

0

1

$\$ 1,477,274$

so

$\$ 238,220$

$\$ 1,715,494$ 
Client: INL

Project: FFC

URS

Washington Division

Account: 61 Home Office Services

Facility: Entire Estimate

Green Field Site

\begin{tabular}{|llll}
\hline & & & \\
Account No. Description & Qty $\quad$ UM & Fact. \\
\hline
\end{tabular}

All-In Home Office at 105s/hr

610000 ALL IN HOME OFFICE w/ Overhead \& other costs
Job No: $\quad 27989-325 \quad$ Rev No: 0

Per Unit
Workhours

$68,600.00 \quad \mathrm{mnhr}$

1.00

Total Rate

Date: $7 / 17 / 2008$

11:37AM

Priced By: ADC

Office: Denver

Product Line:
Estimate: FFC

\begin{tabular}{|c|c|c|c|c|}
\hline \multirow[b]{2}{*}{ Subs Unit } & \multicolumn{4}{|c|}{ Totals } \\
\hline & Labor & Material & Subs & Total \\
\hline & 000 & so & \$o & $\$ 7,203,000$ \\
\hline & 000 & $\$ 0$ & $\$ 0$ & $\$ 7,203,000$ \\
\hline
\end{tabular}

(1)

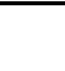




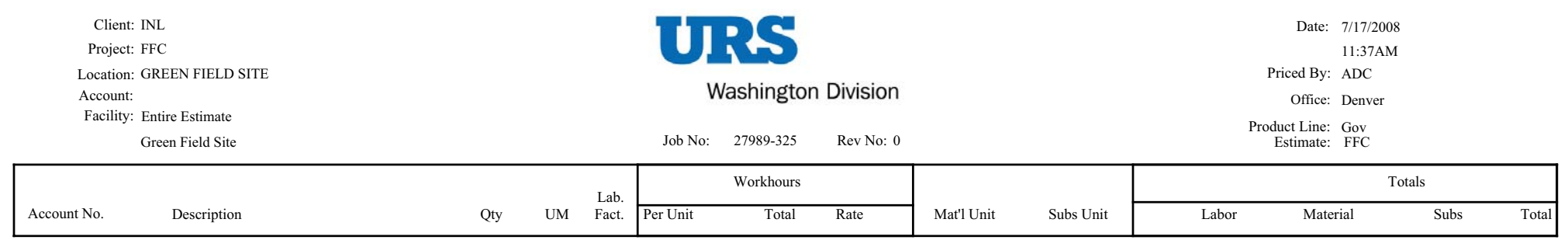

\begin{tabular}{|c|c|c|c|c|c|c|}
\hline Grand Total: Entire Estimate & 270,154 & 86.24 & $\$ 23,297,530$ & $\$ 22,367,690$ & $\$ 3,431,007$ & $\$ 49,096,227$ \\
\hline
\end{tabular}




\section{Estimate Summary}

\section{HIP Alternate}

\section{Revision 1}




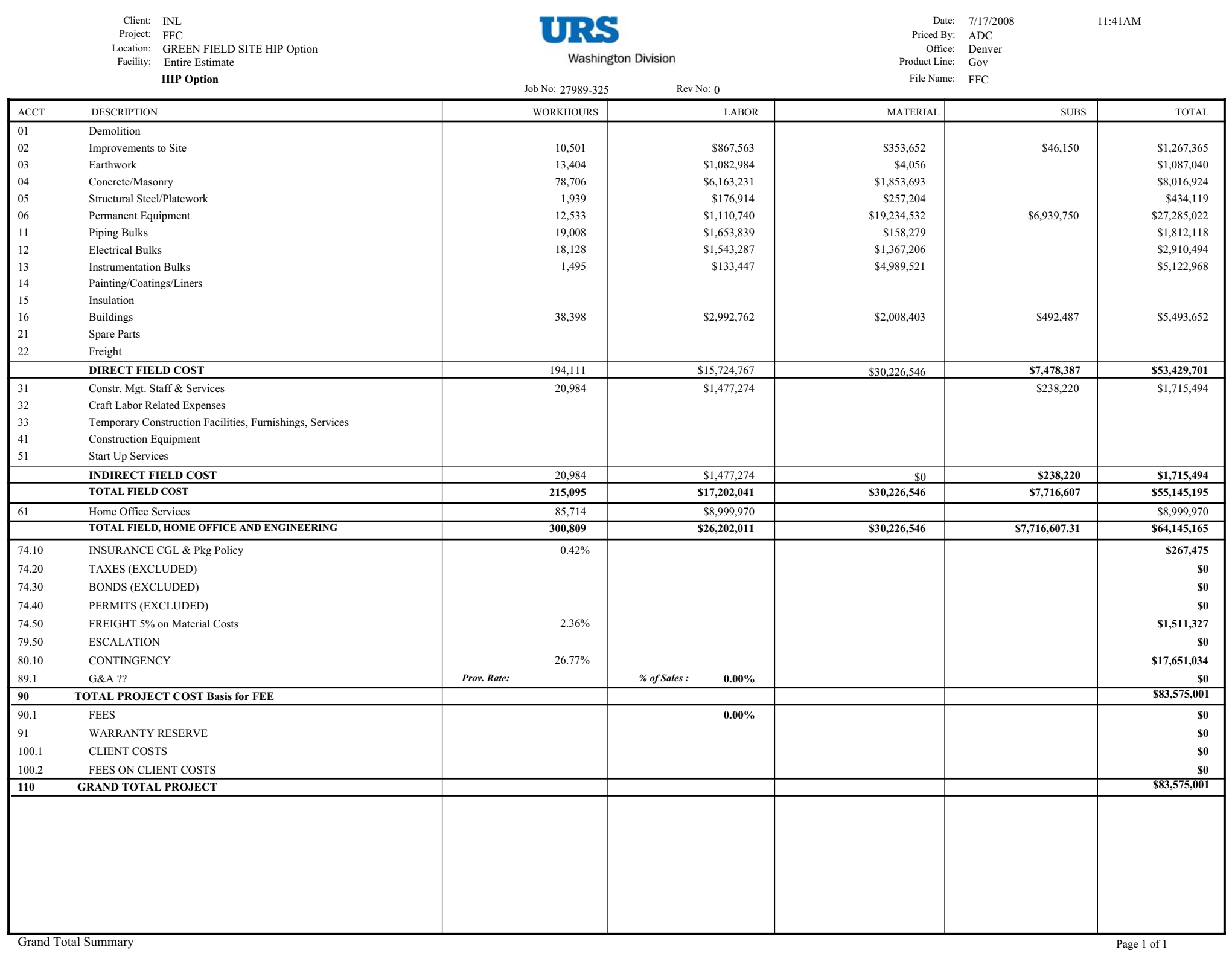




\section{Estimate Details}

\section{HIP Alternate}

\section{Revision 1}




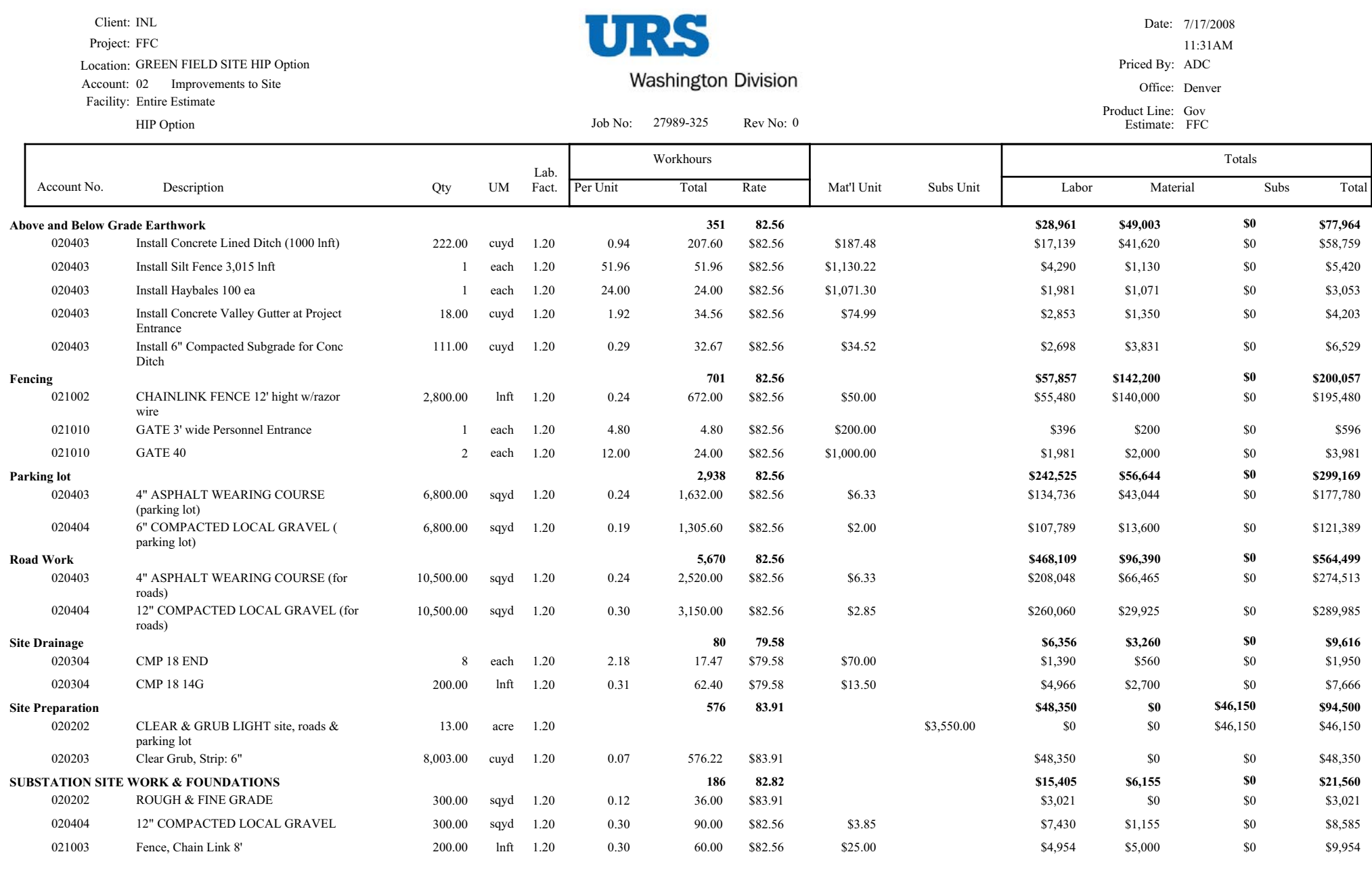

Totals 02-Improvements to Site Entire Estimate

\begin{tabular}{|l|l|}
\hline & \\
\hline
\end{tabular}

$\$ 867,563$

$\$ 353,652$

$\$ 46,150$

$\$ 1,267,365$ 
Client: INL

Project: FFC

Location: GREEN FIELD SITE HIP Option

Account: 03 Earthwork

Facility: Entire Estimate

URS

Washington Division
Date: 7/17/2008

11:31AM

Priced By: ADC

Office: Denver

$\begin{aligned} \text { Product Line: } & \text { Gov } \\ \text { Estimate: } & \text { FFC }\end{aligned}$

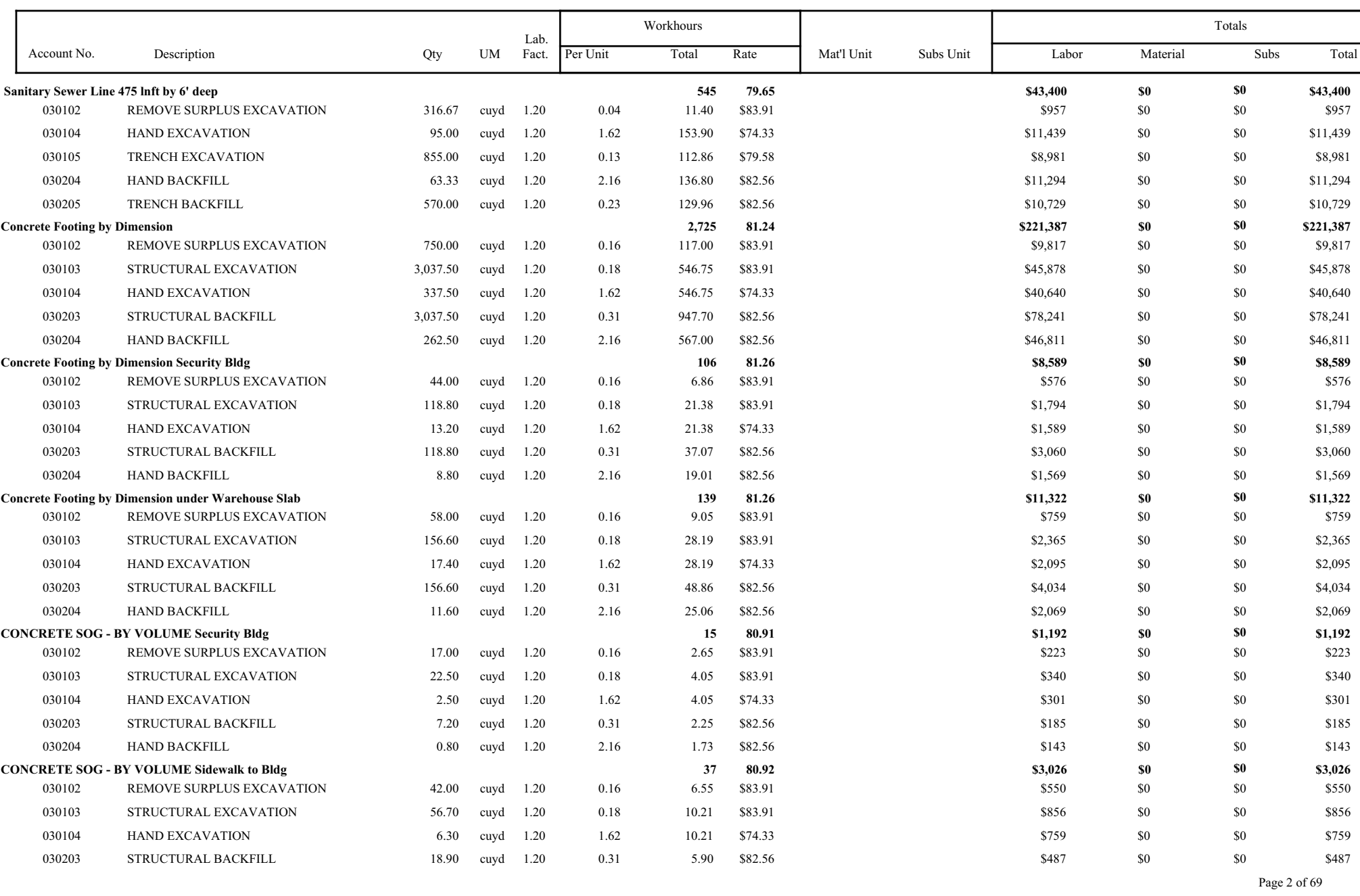




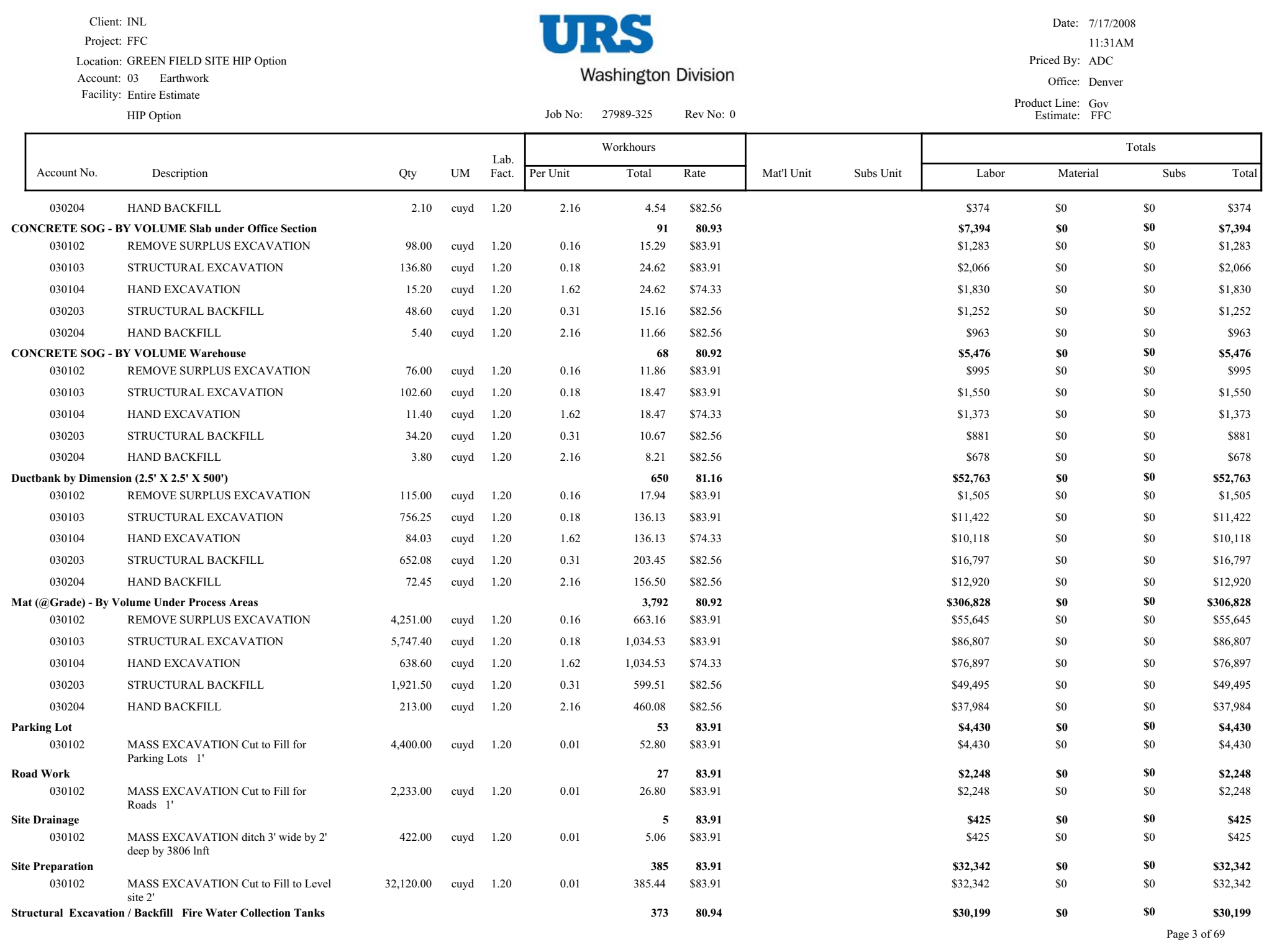


Client: INL

Project: FFC

Location: GREEN FIELD SITE HIP Option

Account: 03 Earthwork

Facility: Entire Estimate

URS

Washington Division
Date: 7/17/2008

11:31AM

Priced By: ADC

Office: Denver

Product Line: Gov
Estimate: FFC

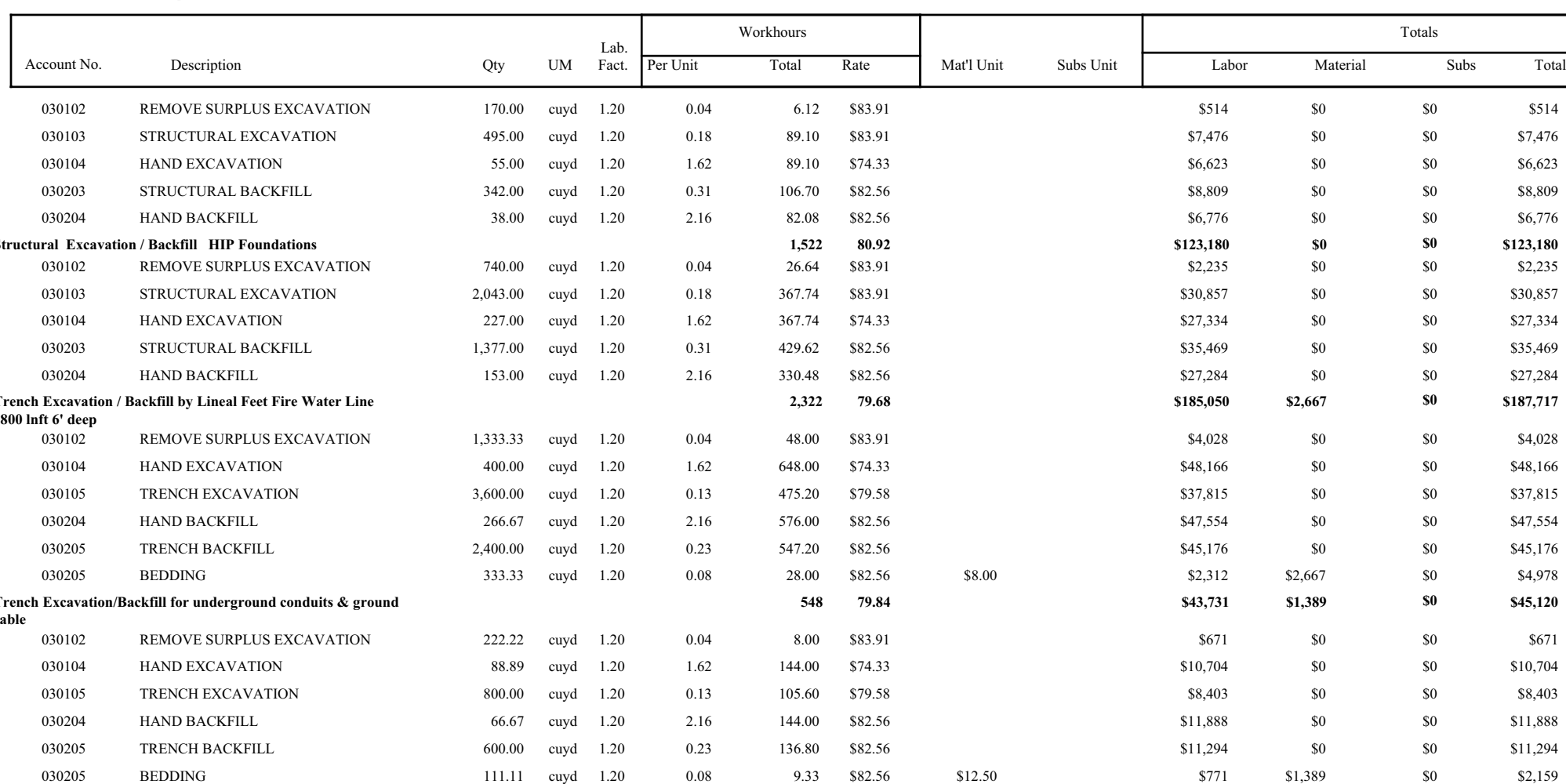

Totals 03-Earthwork

Entire Estimate

$13,404 \quad \$ 80.80$

$\$ 1,082,984$

$\$ 4,056$

\$0

$\$ 1,087,040$ 
Client: INL

Project: FFC

URS

Date: 7/17/2008

11:31AM

Location: GREEN FIELD SITE HIP Option

Washington Division

Priced By: ADC

Office: Denver

Facility: Entire Estimate

Job No: $\quad 27989-325 \quad$ Rev No: 0

Product Line: Gov
Estimate: FFC

HIP Option

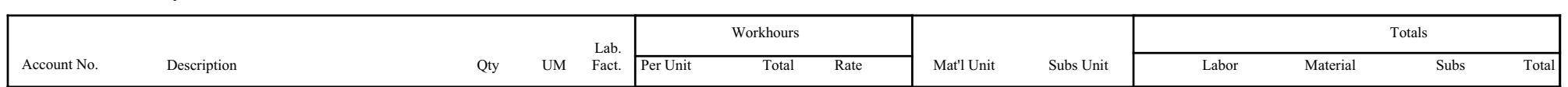

04.04 Concrete by Commodity

04xx02** Reinforcing Steel

04xx03** Formwork

04xx04** Embeds

04x $\times 08 * * \quad$ Concrete

04xx09** Finishing

04xx05** Anchor Bolts

04xx09** Fine Grade

$04 \times 06 * * \quad$ Waterstop

04xx11** Grout

04x $\times 10 * * \quad$ Hardener

$04 \times 07 * * \quad$ Keyway

$\begin{array}{rrrr}430 & \text { ton } & 1.20 & 24.00 \\ 92,570 & \text { sqft } & 1.20 & 0.48 \\ 20,497 & \text { lb } & 1.20 & 0.06 \\ 6,958 & \text { cuyd } & 1.20 & 1.87 \\ 66,707 & \text { sqft } & 1.20 & 0.02 \\ 4,448 & \text { lb } & 1.20 & 0.24 \\ 98,776 & \text { sqft } & 1.20 & 0.04 \\ 2,206 & \text { lnft } & 1.20 & 0.07 \\ 174 & \text { cuft } & 1.20 & 3.77 \\ 16,714 & \text { sqft } & 1.20 & 0.00 \\ 104 & \text { lnft } & 1.20 & 0.07\end{array}$

$\begin{array}{rrr}10,311.36 & \$ 92.37 & \$ 1,317.63 \\ 44,433.74 & \$ 75.57 & \$ 3.00 \\ 1,267.77 & \$ 85.57 & \$ 2.50 \\ 13,043.34 & \$ 76.74 & \$ 132.38 \\ 4,123.43 & \$ 76.74 & \$ 0.01 \\ 1,067.48 & \$ 82.89 & \$ 1.40 \\ 3,555.93 & \$ 75.76 & \$ 0.03 \\ 158.84 & \$ 76.44 & \$ 5.00 \\ 656.54 & \$ 76.74 & \$ 60.00 \\ 80.22 & \$ 76.74 & \$ 0.03 \\ 7.51 & \$ 75.57 & \$ 0.03\end{array}$

$\$ 952,440$
$\$ 3,357,832$
$\$ 108,486$
$\$ 1,000,936$
$\$ 316,429$
$\$ 88,479$
$\$ 269,379$
$\$ 12,142$
$\$ 50,383$
$\$ 6,156$
$\$ 568$

$\$ 566,108$

$\$ 277,847$

$\$ 51,243$

$\$ 925,647$

$\$ 1,667$

$\$ 6,227$

$\$ 2,963$

$\$ 11,031$

$\$ 10,457$

$\$ 501$

$\$ 0.03$

$\$ 3$

$\$ 1,518,548$

$\$ 3,635,679$

$\$ 159,728$

$\$ 1,926,583$

$\$ 318,096$

$\$ 94,706$

$\$ 272,343$

$\$ 23,172$

$\$ 60,840$

$\$ 6,658$

$\$ 571$

\begin{tabular}{|c|c|c|c|}
\hline \multicolumn{4}{|c|}{ Overall Ratios: } \\
\hline FormWork: & & 13.30 & SF/CUYD \\
\hline Rebar: & & 123.49 & LB/CUYD \\
\hline \multirow[t]{3}{*}{ Embedments: } & & 2.95 & LB/CUYD \\
\hline & Wkhr/CY & & 11.31 \\
\hline & Total/CUYD & & $\$ 1,152.13$ \\
\hline
\end{tabular}

Grand Total: Concrete / Masonry

6,958 CUYD $\quad 1.20$

11.31

78,706

$\$ 78.31$

$\$ 6,163,231$

$\$ 1,853,693$

$266.40 / \mathrm{CY}$

\$0

$\$ 8,016,924$

$\$ 885.73 / \mathrm{CY}$

$\$ 266.40 / \mathrm{CY} \quad \$ 0.00 / \mathrm{CY}$

Page 5 of 69 


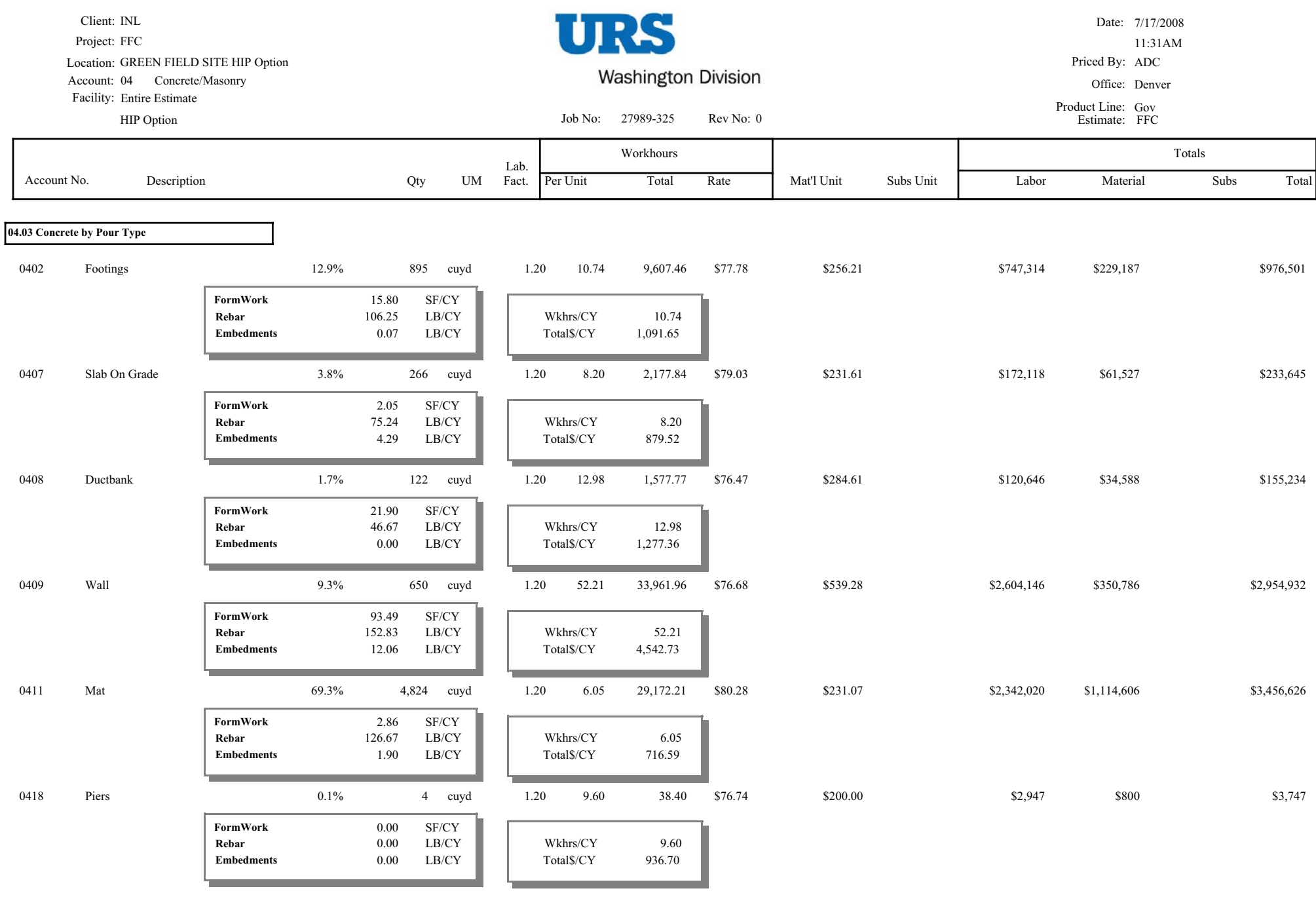


Client: INL

Project: FFC

URS

Date: 7/17/2008

11:31AM

Location: GREEN FIELD SITE HIP Option

Washington Division

Priced By: ADC

Office: Denver

Facility: Entire Estimate

Job No: $\quad 27989-325 \quad$ Rev No: 0

Product Line: Gov
Estimate: FFC

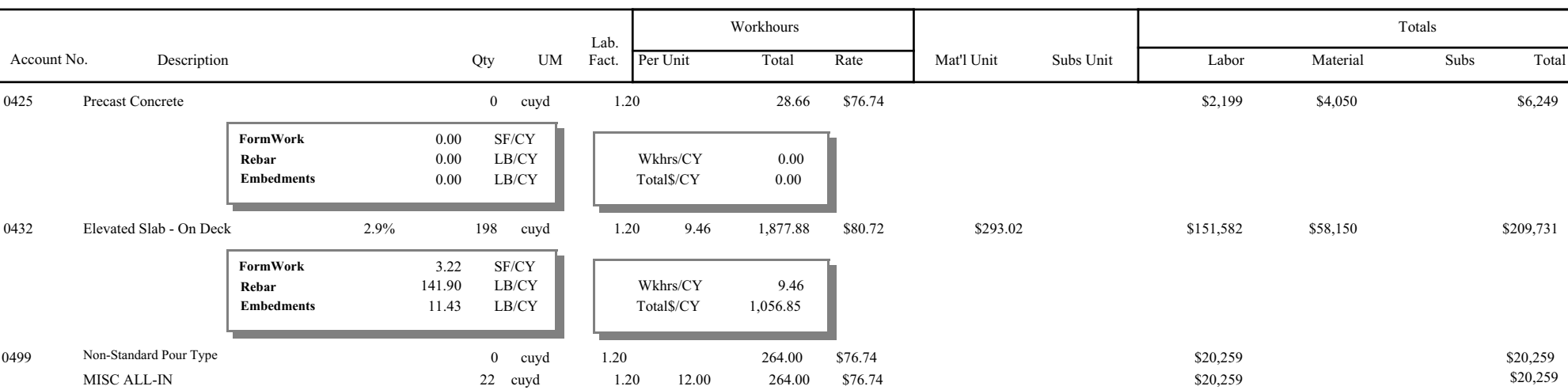

\begin{tabular}{|c|c|c|c|}
\hline \multirow{3}{*}{$\begin{array}{l}\text { FormWork } \\
\text { Rebar }\end{array}$} & \multicolumn{3}{|c|}{ Overall Ratios: } \\
\hline & & 13.30 & $\mathrm{SF} / \mathrm{CY}$ \\
\hline & & 123.49 & $\mathrm{LB} / \mathrm{CY}$ \\
\hline \multicolumn{2}{|l|}{ Embedments } & 2.95 & $\mathrm{LB} / \mathrm{CY}$ \\
\hline & Wkhrs/CY & & 1.31 \\
\hline & Total\$/CY & $\$ 1,15$ & 2.13 \\
\hline
\end{tabular}

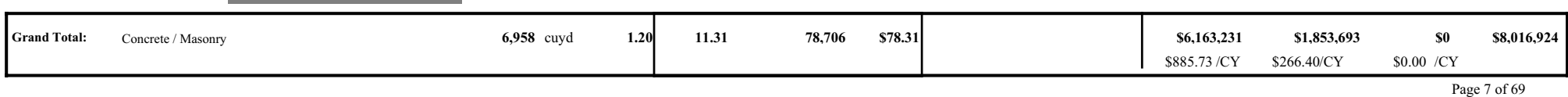


Client: INL

Project: FFC

Location: GREEN FIELD SITE HIP Option

Account: 04 Concrete/Masonry

Facility: Entire Estimate

HIP Option
URS

Washington Division
Date: $7 / 17 / 2008$

11:31AM

Priced By: ADC

Office: Denver

Product Line: Gov
Estimate: FFC

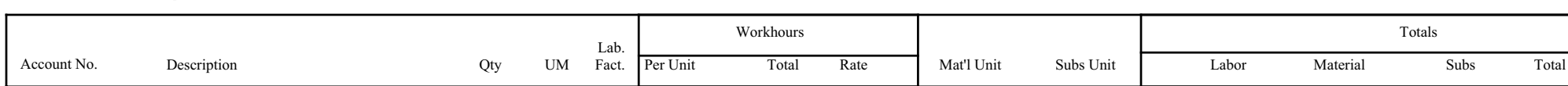

04.02 Concrete by Assembly - Summary

Concrete Footing by Dimension

Concrete Footing by Dimension Security Bldg

Concrete Footing by Dimension under Warehouse Sla

CONCRETE SOG - BY VOLUME Fire water Collection

Tank

CONCRETE SOG - BY VOLUME Security BIdg

CONCRETE SOG - BY VOLUME Sidewalk to Bldg

CONCRETE SOG - BY VOLUME Slab under Office

Section

CONCRETE SOG - BY VOLUME Warehouse

Ductbank by Dimension $\left(2.5^{\prime}\right.$ X $2.5^{\prime}$ X $\left.500^{\prime}\right)$

Elevated Slab on Metal Deck- by Volume

Elevated Slab on Metal Deck- by Volume HVAC Filter

Floor

Elevated Slab on Metal Deck- by Volume Security BIdg

Fence/Fence lighting Pole

Mat (@Grade) - By Volume Hip Fdn

Mat (@Grade) - By Volume HIP Fdn 18" Thk

Mat (@Grade) - By Volume Under Process Areas

Roofing Fire water Collection Tank Sump

SUBSTATION SITE WORK \& FOUNDATIONS

Wall by Volume Greater than $8^{\prime}$ high

Wall by Volume Greater than 8 ' high Fire Water Collection

Wall by Volume Greater than 8' high Radiography Area
Job No: $\quad 27989-325 \quad$ Rev No: 0

$\begin{array}{rlrrrrl}787 & \text { cuyd } & 1.20 & 9.27 & 7,298 & \$ 78.10 & \$ 246.45 \\ 47 & \text { cuyd } & 1.20 & 28.88 & 1,350 & \$ 76.58 & \$ 382.26 \\ 61 & \text { cuyd } & 1.20 & 15.80 & 959 & \$ 77.06 & \$ 285.73 \\ 20 & \text { cuyd } & 1.20 & 6.87 & 137 & \$ 79.47 & \$ 230.18 \\ & & & & & & \\ 18 & \text { cuyd } & 1.20 & 7.71 & 138 & \$ 79.18 & \$ 231.08 \\ 44 & \text { cuyd } & 1.20 & 11.04 & 487 & \$ 78.44 & \$ 234.68 \\ 104 & \text { cuyd } & 1.20 & 7.71 & 801 & \$ 79.18 & \$ 231.08 \\ & & & & & & \\ 80 & \text { cuyd } & 1.20 & 7.71 & 615 & \$ 79.18 & \$ 231.08 \\ 122 & \text { cuyd } & 1.20 & 12.98 & 1,578 & \$ 76.47 & \$ 284.61 \\ 95 & \text { cuyd } & 1.20 & 9.46 & 894 & \$ 80.72 & \$ 293.02 \\ 86 & \text { cuyd } & 1.20 & 9.46 & 815 & \$ 80.72 & \$ 293.02 \\ & & & & & & \\ 18 & \text { cuyd } & 1.20 & 9.46 & 169 & \$ 80.72 & \$ 293.02 \\ & & & & & & \\ 0 & \text { cuyd } & 1.20 & & 264 & \$ 76.74 & \\ 156 & \text { cuyd } & 1.20 & 5.68 & 888 & \$ 80.56 & \$ 131.84 \\ 198 & \text { cuyd } & 1.20 & 6.06 & 1,203 & \$ 80.27 & \$ 132.10 \\ 4,469 & \text { cuyd } & 1.20 & 6.06 & 27,082 & \$ 80.27 & \$ 238.94 \\ 0 & \text { cuyd } & 1.20 & & 29 & \$ 76.74 & \\ 4 & \text { cuyd } & 1.20 & 9.60 & 38 & \$ 76.74 & \$ 200.00 \\ 128 & \text { cuyd } & 1.20 & 35.49 & 4,546 & \$ 77.07 & \$ 341.98 \\ 26 & \text { cuyd } & 1.20 & 42.50 & 1,116 & \$ 76.93 & \$ 506.75 \\ & & & & & & \\ 496 & \text { cuyd } & 1.20 & 57.04 & 28,300 & \$ 76.61 & \$ 591.94\end{array}$

Entire Estimate

\begin{tabular}{|lrl|}
\hline & Entire Estimate & \\
Formwork & $\mathbf{1 3 . 3 0}$ & $\mathrm{SF} / \mathrm{CY}$ \\
Rebar & $\mathbf{1 2 3 . 4 9}$ & $\mathrm{LB} / \mathrm{CY}$ \\
Embeds & $\mathbf{2 . 9 5}$ & $\mathrm{LB} / \mathrm{CY}$ \\
\hline
\end{tabular}

WKHR/CUYD $\quad \mathbf{1 1 . 3 1}$

$\begin{array}{ll}\text { Grand Total: Entire Estimate } & 6,958 \text { cuyd }\end{array}$

1.20

11.3

78,706

$\$ 78.31$
$\$ 6,163,231$

$\$ 885.73 / \mathrm{CY}$
$\$ 1,853,693$

\$0 $\$ 8,016,924$ $\$ 266.40 / C Y$ 
Client: INL

Project: FFC

Location: GREEN FIELD SITE HIP Option

Account: 04 Concrete/Masonry

Facility: Entire Estimate

URS

Washington Division
Date: 7/17/2008

11:31AM

Priced By: ADC

Office: Denver

Product Line: Gov
Estimate: FFC

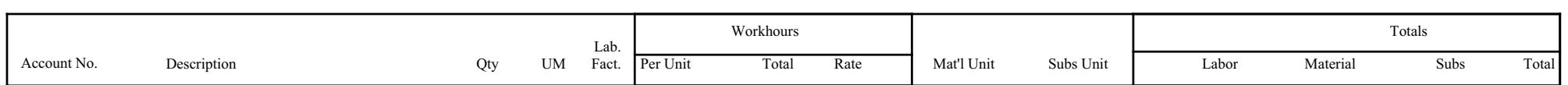

$$
\text { 04.01 Concrete by Assembly Detail }
$$

Concrete Footing by Dimension

\begin{tabular}{|c|c|c|c|c|}
\hline 040202 & \multicolumn{4}{|c|}{ REBAR@ FOOTINGS } \\
\hline 040203 & \multicolumn{4}{|c|}{ FORMWORK@FOOTINGS } \\
\hline 040204 & \multicolumn{4}{|c|}{ EMBEDS@ FOOTINGS } \\
\hline 040208 & \multicolumn{4}{|c|}{ 4000PSI@FOOTINGS PUMPED } \\
\hline \multirow[t]{7}{*}{040207} & \multicolumn{4}{|c|}{ KEYWAY@ FOOTINGS } \\
\hline & \multicolumn{4}{|c|}{ Concrete Footing by Dimension } \\
\hline & Formwork & & 12.90 & $\mathrm{SF} / \mathrm{CY}$ \\
\hline & Rebar & & 104.76 & $\mathrm{LB} / \mathrm{CY}$ \\
\hline & Embedments & & 0.07 & $\mathrm{LB} / \mathrm{CY}$ \\
\hline & & Wkhr/CY & 9. & \\
\hline & & Total/CY & $\$ 970$. & \\
\hline
\end{tabular}

Total Concrete Footing by Dimension

$787 \quad \mathrm{CY}$

ton $1.20 \quad 24.00$

sqft $1.20 \quad 0.48$

$\begin{array}{rrr}\text { lb } & 1.20 & 0.24\end{array}$

cuyd 1.20

lnft 1.20

1.80
0.07

$\begin{array}{rr}989.51 & \$ 92.37 \\ 4,872.96 & \$ 75.57 \\ 12.59 & \$ 92.37 \\ 1,416.80 & \$ 76.74 \\ 6.61 & \$ 75.57\end{array}$
$\$ 1,362.08$
$\$ 3.00$
$\$ 2.50$
$\$ 136.24$
$\$ 0.03$

$\begin{array}{lr}\$ 0.00 & \$ 91,399 \\ \$ 0.00 & \$ 368,247 \\ \$ 0.00 & \$ 1,163 \\ \$ 0.00 & \$ 108,724 \\ \$ 0.00 & \$ 500\end{array}$

$\$ 56,158$

$\$ 30,456$

$\$ 131$

$\$ 107,236$
$\$ 0$

$\$ 0 \quad \$ 215,960$

1.20

9.27

$\begin{array}{rrrr}3 & \text { ton } 1.20 & 24.00 \\ 2,406 & \text { sqft } & 1.20 & 0.48 \\ 3 & \text { lb } & 1.20 & 0.24 \\ 47 & \text { cuyd } 1.20 & 1.80 \\ 979 & \text { sqft } 1.20 & 0.04 \\ 5 & \text { Inft } 1.20 & 0.07\end{array}$

$\begin{array}{rr}74.79 & \$ 92.37 \\ 1,154.88 & \$ 75.57 \\ 0.75 & \$ 92.37 \\ 84.14 & \$ 76.74 \\ 35.26 & \$ 75.57 \\ 0.39 & \$ 75.57\end{array}$
$\$ 1,362.08$
$\$ 3.00$
$\$ 2.50$
$\$ 136.24$
$\$ 0.03$
$\$ 0.03$

REBAR@FOOTINGS

FORMWORK @ FOOTIN

4000PSI@FOOTINGS PUMPED

FINE GRADE @ FOOTING

KEYWAY@FOOTINGS

$\begin{array}{ll}\text { Concrete Footing by Dimension Security BIdg } \\ 040202 & \text { REBAR @ FOOTINGS } \\ 040203 & \text { FORMWORK @ FOOTINGS } \\ 040204 & \text { EMBEDS @ FOOTINGS } \\ 040208 & \text { 4000PSI@FOOTINGS PUMPED } \\ 040209 & \text { FINE GRADE @ FOOTING } \\ 040207 & \text { KEYWAY @ FOOTINGS }\end{array}$

$\$ 75.57$

$\$ 0.00$
$\$ 0.00$
$\$ 0.00$
$\$ 0.00$
$\$ 0.00$
$\$ 0.00$

$\$ 570,033$
$\$ 724.21 / C Y$

$\$ 193,984$

$\$ 246.45 / C Y$

$\$ \mathbf{\$ 0 0} / \mathrm{CY}$ 
Client: INL

Project: FFC

Location: GREEN FIELD SITE HIP Option

Account: 04 Concrete/Masonry

Facility: Entire Estimate

URS

Washington Division
Date: 7/17/2008

11:31AM

Priced By: ADC

Office: Denver

Product Line: Gov
Estimate: FFC

\begin{tabular}{|c|c|c|c|c|c|c|c|c|c|c|c|c|c|}
\hline \multirow[b]{2}{*}{ Account No. } & \multirow[b]{2}{*}{ Description } & \multirow[b]{2}{*}{ Qty } & \multirow[b]{2}{*}{ UM } & \multirow{2}{*}{$\begin{array}{l}\text { Lab. } \\
\text { Fact. }\end{array}$} & \multicolumn{3}{|c|}{ Workhours } & \multirow[b]{2}{*}{ Mat'l Unit } & \multirow[b]{2}{*}{ Subs Unit } & \multicolumn{4}{|c|}{ Totals } \\
\hline & & & & & Per Unit & Total & Rate & & & Labor & Material & Subs & Total \\
\hline
\end{tabular}

Concrete Footing by Dimension Security BIdg

$\begin{array}{lrl}\text { Formwork } & \mathbf{5 1 . 4 7} & \mathrm{SF} / \mathrm{CY} \\ \text { Rebar } & \mathbf{1 3 3 . 3 3} & \mathrm{LB} / \mathrm{CY} \\ \text { Embedments } & \mathbf{0 . 0 7} & \mathrm{LB} / \mathrm{CY}\end{array}$

Embedments

$\mathbf{0 . 0 7} \mathrm{LB} / \mathrm{CY}$

$\begin{array}{rr}\text { Wkhr/CY } & \mathbf{2 8 . 8 8} \\ \text { Total/CY } & \mathbf{\$ 2 , 5 9 4 . 3 3}\end{array}$

\begin{tabular}{|c|c|c|c|c|c|c|c|c|c|c|c|c|c|c|c|c|}
\hline Total & Concrete & ooting by Dimension Security Bldg & & 47 & $\mathrm{CY}$ & 1.20 & 28.88 & 1,350 & $\$ 76.58$ & & & $\begin{array}{r}\$ 103,402 \\
\$ 2,212.07 / C Y\end{array}$ & $\begin{array}{c}\$ 17,868 \\
\$ 382.26 / C Y\end{array}$ & $\$ 0.00$ & $\begin{array}{l}\$ 0 \\
/ \mathrm{CY}\end{array}$ & $\$ 121,270$ \\
\hline \multicolumn{17}{|c|}{ Concrete Footing by Dimension under Warehouse Slab } \\
\hline 040202 & & REBAR@ FOOTINGS & & 3 & ton & 1.20 & 24.00 & 76.27 & $\$ 92.37$ & $\$ 1,362.08$ & $\$ 0.00$ & $\$ 7,045$ & $\$ 4,328$ & & $\$ 0$ & $\$ 11,373$ \\
\hline 040203 & & FORMWORK@ FOOTINGS & & 1,572 & sqft & 1.20 & 0.48 & 754.56 & $\$ 75.57$ & $\$ 3.00$ & $\$ 0.00$ & $\$ 57,022$ & $\$ 4,716$ & & $\$ 0$ & $\$ 61,738$ \\
\hline 040204 & & EMBEDS@ FOOTINGS & & 4 & $\mathrm{lb}$ & 1.20 & 0.24 & 0.97 & $\$ 92.37$ & $\$ 2.50$ & $\$ 0.00$ & $\$ 90$ & $\$ 10$ & & $\$ 0$ & $\$ 100$ \\
\hline 040208 & & 4000PSI@FOOTINGS PUMPED & & 61 & cuyd & 1.20 & 1.80 & 109.20 & $\$ 76.74$ & $\$ 136.24$ & $\$ 0.00$ & $\$ 8,380$ & $\$ 8,265$ & & $\$ 0$ & $\$ 16,645$ \\
\hline 040209 & & FINE GRADE @ FOOTING & & 480 & sqft & 1.20 & 0.04 & 17.26 & $\$ 75.57$ & $\$ 0.03$ & $\$ 0.00$ & $\$ 1,305$ & $\$ 14$ & & $\$ 0$ & $\$ 1,319$ \\
\hline \multirow[t]{7}{*}{040207} & & KEYWAY@ FOOTINGS & & 7 & $\operatorname{lnft}$ & 1.20 & 0.07 & 0.51 & $\$ 75.57$ & $\$ 0.03$ & $\$ 0.00$ & $\$ 39$ & $\$ 0$ & & $\$ 0$ & $\$ 39$ \\
\hline & & Concrete Footing by Dimension un & ader Ware & house Slab & & & & & & & & & & & & \\
\hline & & Formwork & 25.91 & $\mathrm{SF} / \mathrm{CY}$ & & & & & & & & & & & & \\
\hline & & Rebar & 104.76 & $\mathrm{LB} / \mathrm{CY}$ & & & & & & & & & & & & \\
\hline & & Embedments & 0.07 & $\mathrm{LB} / \mathrm{CY}$ & & & & & & & & & & & & \\
\hline & & $\mathrm{Wkhr} / \mathrm{CY}$ & 15.8 & & & & & & & & & & & & & \\
\hline & & Total/CY & $\$ 1,503.5$ & & & & & & & & & & & & & \\
\hline Total & $\begin{array}{l}\text { Concrete } \\
\text { Slab }\end{array}$ & ooting by Dimension under Warehouse & & 61 & $\mathrm{CY}$ & 1.20 & 15.80 & 959 & $\$ 77.06$ & & & $\begin{array}{r}\$ 73,879 \\
\$ 1,217.78 / C Y\end{array}$ & $\begin{array}{c}\$ 17,334 \\
\$ 285.73 / C Y\end{array}$ & $\$ 0.00$ & $\begin{array}{l}\$ \mathbf{s o} \\
/ \mathrm{CY}\end{array}$ & $\$ 91,213$ \\
\hline
\end{tabular}


Client: INL

Project: FFC

Location: GREEN FIELD SITE HIP Option

Account: 04 Concrete/Masonry

URS

Washington Division
Date: $7 / 17 / 2008$

11:31AM

Priced By: ADC

Office: Denver

Product Line:
Estimate:

$$
\text { HIP Option }
$$

Job No: $\quad 27989-325 \quad$ Rev No: 0

\begin{tabular}{|c|c|c|c|c|c|c|c|c|c|c|c|c|c|c|c|}
\hline \multirow[b]{2}{*}{ Account No. } & \multirow[b]{2}{*}{ Description } & & & \multirow[b]{2}{*}{ Qty } & \multirow[b]{2}{*}{ UM } & \multirow{2}{*}{$\begin{array}{l}\text { Lab. } \\
\text { Fact. }\end{array}$} & \multicolumn{3}{|c|}{ Workhours } & \multirow[b]{2}{*}{ Mat'l Unit } & \multirow[b]{2}{*}{ Subs Unit } & \multicolumn{4}{|c|}{ Totals } \\
\hline & & & & & & & Per Unit & Total & Rate & & & Labor & Material & Subs & Total \\
\hline \multicolumn{16}{|c|}{ CONCRETE SOG - BY VOLUME Fire water Collection Tank } \\
\hline 040702 & \multicolumn{4}{|c|}{ REBAR@SOG } & ton & 1.20 & 24.00 & 18.01 & $\$ 92.37$ & $\$ 1,362.08$ & $\$ 0.00$ & $\$ 1,664$ & $\$ 1,022$ & $\$ 0$ & $\$ 2,686$ \\
\hline 040703 & \multicolumn{4}{|c|}{ FORMWORK@SOG } & sqft & 1.20 & 0.48 & 19.61 & $\$ 75.57$ & $\$ 3.25$ & $\$ 0.00$ & $\$ 1,482$ & $\$ 133$ & $\$ 0$ & $\$ 1,615$ \\
\hline 040704 & \multicolumn{4}{|c|}{ EMBEDS@ SOG } & $\mathrm{lb}$ & 1.20 & 0.06 & 5.13 & $\$ 92.37$ & $\$ 2.50$ & $\$ 0.00$ & $\$ 474$ & $\$ 214$ & $\$ 0$ & $\$ 688$ \\
\hline 040708 & \multicolumn{3}{|c|}{ 4000PSI@SOG PUMPED } & 20 & cuyd & 1.20 & 1.80 & 35.91 & $\$ 76.74$ & $\$ 136.24$ & $\$ 0.00$ & $\$ 2,756$ & $\$ 2,718$ & $\$ 0$ & $\$ 5,474$ \\
\hline 040709 & \multicolumn{3}{|c|}{ FINISHING@ SOG } & 770 & sqft & 1.20 & 0.02 & 18.47 & $\$ 76.74$ & $\$ 0.01$ & $\$ 0.00$ & $\$ 1,417$ & $\$ 8$ & $\$ 0$ & $\$ 1,425$ \\
\hline 040705 & \multicolumn{3}{|c|}{ ANCHOR BOLTS@SOG } & 10 & $\mathrm{lb}$ & 1.20 & 0.24 & 2.31 & $\$ 92.37$ & $\$ 1.40$ & $\$ 0.00$ & $\$ 214$ & $\$ 13$ & $\$ 0$ & $\$ 227$ \\
\hline 040709 & \multicolumn{3}{|c|}{ FINE GRADE@SOG } & 770 & sqft & 1.20 & 0.04 & 27.70 & $\$ 76.74$ & $\$ 0.03$ & $\$ 0.00$ & $\$ 2,126$ & $\$ 23$ & $\$ 0$ & $\$ 2,149$ \\
\hline 040706 & \multicolumn{3}{|c|}{ WATERSTOP@SOG } & 88 & $\operatorname{lnft}$ & 1.20 & 0.07 & 6.31 & $\$ 76.74$ & $\$ 5.00$ & $\$ 0.00$ & $\$ 484$ & $\$ 438$ & $\$ 0$ & $\$ 922$ \\
\hline \multirow[t]{7}{*}{040710} & \multicolumn{3}{|c|}{ HARDENER@SOG } & 770 & sqft & 1.20 & 0.00 & 3.69 & $\$ 76.74$ & $\$ 0.03$ & $\$ 0.00$ & $\$ 283$ & $\$ 23$ & $\$ 0$ & $\$ 307$ \\
\hline & \multicolumn{4}{|c|}{ CONCRETE SOG - BY VOLUME Fire water Collection } & & & & & & & & & & & \\
\hline & \multirow{3}{*}{\multicolumn{4}{|c|}{$\begin{array}{lrrl}\text { Formwork } & \text { Tank } & \mathbf{2 . 0 5} & \text { SF/CY } \\
\text { Rebar } & & \mathbf{7 5 . 2 4} & \text { LB/CY } \\
\text { Embedments } & & 4.29 & \text { LB/CY }\end{array}$}} & & & & & & & & & & & \\
\hline & & & & & & & & & & & & & & & \\
\hline & & & & & & & & & & & & & & & \\
\hline & & Wkhr/CY & 6 & & & & & & & & & & & & \\
\hline & & Total/CY & $\$ 776$. & & & & & & & & & & & & \\
\hline
\end{tabular}

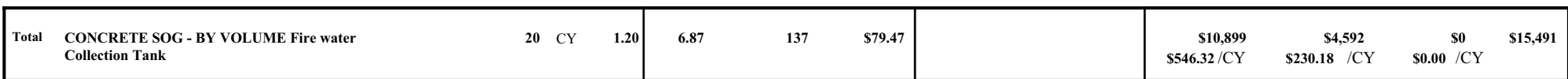

CONCRETE SOG - BY VOLUME Security BIdg

$\begin{array}{ll}040702 & \text { REBAR @ SOG } \\ 040703 & \text { FORMWORK @ SOG } \\ 040704 & \text { EMBEDS @ SOG } \\ 040708 & \text { 4000PSI@SOG PUMPED } \\ 040709 & \text { FINISHING @ SOG } \\ 040705 & \text { ANCHOR BOLTS @ SOG } \\ 040709 & \text { FINE GRADE @ SOG } \\ 040706 & \text { WATERSTOP @ SOG } \\ 040710 & \text { HARDENER @ SOG }\end{array}$

$\begin{array}{rrrr}1 & \text { ton } 1.20 & 24.00 \\ 37 & \text { sqft } 1.20 & 0.48 \\ 77 & \text { Ib } & 1.20 & 0.06 \\ 18 & \text { cuyd } 1.20 & 1.80 \\ 918 & \text { sqft } & 1.20 & 0.02 \\ 9 & \text { lb } & 1.20 & 0.24 \\ 918 & \text { sqft } 1.20 & 0.04 \\ 78 & \text { Inft } 1.20 & 0.07 \\ 918 & \text { sqft } 1.20 & 0.00\end{array}$

$\begin{aligned} 16.12 & \$ 92.37 \\ 17.54 & \$ 75.57 \\ 4.59 & \$ 92.37 \\ 32.13 & \$ 76.74 \\ 22.03 & \$ 76.74 \\ 2.07 & \$ 92.37 \\ 33.05 & \$ 76.74 \\ 5.64 & \$ 76.74 \\ 4.41 & \$ 76.74\end{aligned}$

$\$ 1,362.08$
$\$ 3.25$
$\$ 2.50$
$\$ 136.24$
$\$ 0.01$
$\$ 1.40$
$\$ 0.03$
$\$ 5.00$
$\$ 0.03$

$\$ 0.00$
$\$ 0.00$
$\$ 0.00$
$\$ 0.00$
$\$ 0.00$
$\$ 0.00$
$\$ 0.00$
$\$ 0.00$
$\$ 0.00$

$\$ 1,489$
$\$ 1,326$
$\$ 424$
$\$ 2,466$
$\$ 1,691$
$\$ 191$
$\$ 2,536$
$\$ 433$
$\$ 338$

$\$ 915$
$\$ 119$
$\$ 191$
$\$ 2,432$
$\$ 9$
$\$ 12$
$\$ 28$
$\$ 392$
$\$ 28$

$\begin{array}{lr}\$ 0 & \$ 2,403 \\ \$ 0 & \$ 1,445 \\ \$ 0 & \$ 615 \\ \$ 0 & \$ 4,898 \\ \$ 0 & \$ 1,700 \\ \$ 0 & \$ 203 \\ \$ 0 & \$ 2,564 \\ \$ 0 & \$ 825 \\ \$ 0 & \$ 366\end{array}$


Client: INL

Project: FFC

URS

Washington Division
Date: $7 / 17 / 2008$

$11: 31 \mathrm{AM}$ Priced By: ADC

Office: Denver

Product Line: Gov
Estimate: FFC

Account: 04 Concrete/Masonry

Job No: $\quad 27989-325 \quad$ Rev No: 0

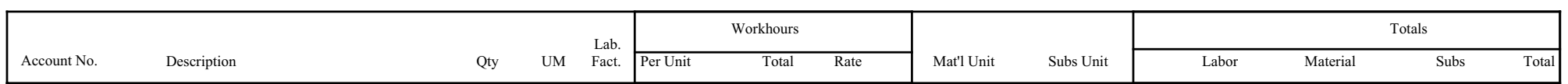

CONCRETE SOG - BY VOLUME Security BIdg $\begin{array}{lrl}\text { Formwork } & \mathbf{2 . 0 5} & \mathrm{SF} / \mathrm{CY} \\ \text { Rebar } & \mathbf{7 5 . 2 4} & \mathrm{LB} / \mathrm{CY}\end{array}$

$\begin{array}{lll}\text { Embedments } & 4.29 & \mathrm{LB} / \mathrm{CY}\end{array}$

\begin{tabular}{|cr|}
\hline Wkhr/CY & $\mathbf{7 . 7 1}$ \\
Total/CY & $\mathbf{\$ 8 4 1 . 3 4}$ \\
\hline
\end{tabular}

Total CONCRETE SOG - BY VOLUME Security BIdg

$18 \mathrm{CY}$

\begin{tabular}{l|l}
1.20 & 7.71
\end{tabular}

7.71

138

$\$ 79.18$

\begin{tabular}{|r|r}
\hline & $\$ 10,893$ \\
$\$ 610.26 / C Y$
\end{tabular}

$\$ 4,125$
$\$ 231.08 / C Y$

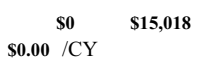

CONCRETE SOG - BY VOLUME Sidewalk to BIdg

$\begin{array}{ll}040702 & \text { REBAR @ SOG } \\ 040703 & \text { FORMWORK @ SOG } \\ 040704 & \text { EMBEDS @ SOG } \\ 040708 & \text { 4000PSI@SOG PUMPED } \\ 040709 & \text { FINISHING @ SOG } \\ 040705 & \text { ANCHOR BOLTS @ SOG } \\ 040709 & \text { FINE GRADE @ SOG } \\ 040706 & \text { WATERSTOP @ SOG } \\ 040710 & \text { HARDENER @ SOG }\end{array}$

$\begin{array}{rrrrrr}2 & \text { ton } & 1.20 & 24.00 & 39.82 & \$ 92.37 \\ 90 & \text { sqft } & 1.20 & 0.48 & 43.34 & \$ 75.57 \\ 189 & \text { lb } & 1.20 & 0.06 & 11.34 & \$ 92.37 \\ 44 & \text { cuyd } & 1.20 & 1.80 & 79.38 & \$ 76.74 \\ 4,536 & \text { sqft } & 1.20 & 0.02 & 108.86 & \$ 76.74 \\ 21 & \text { lb } & 1.20 & 0.24 & 5.11 & \$ 92.37 \\ 4,536 & \text { sqft } & 1.20 & 0.04 & 163.30 & \$ 76.74 \\ 194 & \text { Inft } & 1.20 & 0.07 & 13.94 & \$ 76.74 \\ 4,536 & \text { sqft } & 1.20 & 0.00 & 21.77 & \$ 76.74\end{array}$

$\$ 1,362.08$
$\$ 3.25$
$\$ 2.50$
$\$ 136.24$
$\$ 0.01$
$\$ 1.40$
$\$ 0.03$
$\$ 5.00$
$\$ 0.03$

$\$ 0.00$
$\$ 0.00$
$\$ 0.00$
$\$ 0.00$
$\$ 0.00$
$\$ 0.00$
$\$ 0.00$
$\$ 0.00$
$\$ 0.00$

$\$ 3,678$

$\$ 3,275$

$\$ 1,047$

$\$ 6,092$

$\$ 8,354$

$\$ 472$

$\$ 12,531$

$\$ 1,070$

$\$ 1,671$

$\$ 2,260$
$\$ 293$
$\$ 473$
$\$ 6,008$
$\$ 45$
$\$ 30$
$\$ 136$
$\$ 968$
$\$ 136$

$\begin{array}{lr}\$ 0 & \$ 5,937 \\ \$ 0 & \$ 3,569 \\ \$ 0 & \$ 1,520 \\ \$ 0 & \$ 12,100 \\ \$ 0 & \$ 8,400 \\ \$ 0 & \$ 502 \\ \$ 0 & \$ 12,667 \\ \$ 0 & \$ 2,038 \\ \$ 0 & \$ 1,807\end{array}$


Client: INL

Project: FFC

Location: GREEN FIELD SITE HIP Option

Account: 04 Concrete/Masonry

Facility: Entire Estimate

URS

Washington Division
Date: $7 / 17 / 2008$

11:31AM

Priced By: ADC

Office: Denver

Product Line: Gov
Estimate: FFC

$$
\text { HIP Option }
$$

Job No:

Rev No: 0

\begin{tabular}{|c|c|c|c|c|c|c|c|c|c|c|}
\hline \multirow[b]{2}{*}{ Account No. } & \multirow[b]{2}{*}{ Description } & \multirow[b]{2}{*}{ Qty } & \multirow[b]{2}{*}{ UM } & \multirow{2}{*}{$\begin{array}{l}\text { Lab. } \\
\text { Fact. }\end{array}$} & & rkhours & \multirow[b]{2}{*}{ Mat'l Unit } & \multirow[b]{2}{*}{ Subs Unit } & \multicolumn{2}{|c|}{ Totals } \\
\hline & & & & & Per Unit & Total & & & Labor & Material \\
\hline
\end{tabular}

\begin{tabular}{|lrll|}
\hline \multicolumn{4}{|c|}{ CONCRETE SOG - BY VOLUME Sidewalk to BIdg } \\
Formwork & 2.05 & SF/CY \\
Rebar & 75.24 & LB/CY \\
Embedments & 4.29 & LB/CY \\
\hline
\end{tabular}

\begin{tabular}{rr|} 
Wkhr/CY & $\mathbf{1 1 . 0 4}$ \\
Total/CY & $\mathbf{\$ 1 , 1 0 0 . 6 8}$ \\
\hline
\end{tabular}

\begin{tabular}{|c|c|c|c|c|c|c|c|c|c|}
\hline Total & $\begin{array}{l}\text { CONCRETE SOG - BY VOLUME Sidewalk to } \\
\text { Bldg }\end{array}$ & $44 \mathrm{CY}$ & 1.20 & 11.04 & $\$ 78.44$ & $\begin{array}{r}\$ 38,191 \\
\$ 866.00 / C Y\end{array}$ & $\begin{array}{c}\$ 10,349 \\
\$ 234.68 / C Y\end{array}$ & $\begin{array}{c}\$ 0 \\
\$ 0.00\end{array}$ & $\$ 48,540$ \\
\hline
\end{tabular}

CONCRETE SOG - BY VOLUME Slab under Office Section

$\begin{array}{ll}040702 & \text { REBAR @ SOG } \\ 040703 & \text { FORMWORK @ SOG } \\ 040704 & \text { EMBEDS @ SOG } \\ 040708 & \text { 4000PSI@SOG PUMPED } \\ 040709 & \text { FINISHING @ SOG } \\ 040705 & \text { ANCHOR BOLTS @ SOG } \\ 040709 & \text { FINE GRADE @ SOG } \\ 040706 & \text { WATERSTOP @ SOG } \\ 040710 & \text { HARDENER @ SOG }\end{array}$

$\begin{array}{rrrrrr}4 & \text { ton } 1.20 & 24.00 & 93.85 & \$ 92.37 \\ 213 & \text { sqft } & 1.20 & 0.48 & 102.17 & \$ 75.57 \\ 446 & \text { lb } & 1.20 & 0.06 & 26.73 & \$ 92.37 \\ 104 & \text { cuyd } 1.20 & 1.80 & 187.11 & \$ 76.74 \\ 5,346 & \text { sqft } & 1.20 & 0.02 & 128.30 & \$ 76.74 \\ 50 & \text { lb } & 1.20 & 0.24 & 12.05 & \$ 92.37 \\ 5,346 & \text { sqft } & 1.20 & 0.04 & 192.46 & \$ 76.74 \\ 456 & \text { Inft } 1.20 & 0.07 & 32.86 & \$ 76.74 \\ 5,346 & \text { sqft } & 1.20 & 0.00 & 25.66 & \$ 76.74\end{array}$

$\$ 1,362.08$
$\$ 3.25$
$\$ 2.50$
$\$ 136.24$
$\$ 0.01$
$\$ 1.40$
$\$ 0.03$
$\$ 5.00$
$\$ 0.03$

$\begin{array}{lr}\$ 0.00 & \$ 8,669 \\ \$ 0.00 & \$ 7,721 \\ \$ 0.00 & \$ 2,469 \\ \$ 0.00 & \$ 14,359 \\ \$ 0.00 & \$ 9,846 \\ \$ 0.00 & \$ 1,113 \\ \$ 0.00 & \$ 14,769 \\ \$ 0.00 & \$ 2,522 \\ \$ 0.00 & \$ 1,969\end{array}$

$\$ 5,326$
$\$ 692$
$\$ 1,114$
$\$ 14,162$
$\$ 53$
$\$ 70$
$\$ 160$
$\$ 2,282$
$\$ 160$

$\begin{array}{lr}\$ 0 & \$ 13,995 \\ \$ 0 & \$ 8,413 \\ \$ 0 & \$ 3,583 \\ \$ 0 & \$ 28,521 \\ \$ 0 & \$ 9,899 \\ \$ 0 & \$ 1,184 \\ \$ 0 & \$ 14,929 \\ \$ 0 & \$ 4,804 \\ \$ 0 & \$ 2,130\end{array}$


Client: INL

Project: FFC

URS

Washington Division
Date: 7/17/2008

11:31AM Priced By: ADC

Office: Denver

Product Line: Gov
Estimate: FFC

Account: 04 Concrete/Masonry

Job No: $\quad 27989-325 \quad$ Rev No: 0

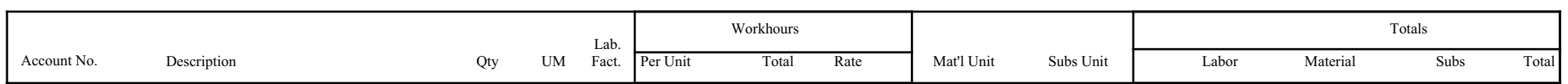

\begin{tabular}{|c|c|c|}
\hline Formwork & 2.05 & $\mathrm{SF} / \mathrm{CY}$ \\
\hline Rebar & 75.24 & $\mathrm{LB} / \mathrm{CY}$ \\
\hline Embedments & 4.29 & $\mathrm{LB} / \mathrm{CY}$ \\
\hline
\end{tabular}

Embedments

\begin{tabular}{|rr|} 
Wkhr/CY & 7.71 \\
Total/CY & $\$ 841.34$ \\
\hline
\end{tabular}

\begin{tabular}{|c|c|c|c|c|c|c|c|c|c|c|c|c|c|}
\hline Total & $\begin{array}{l}\text { CONCRETE SOG - BY VOLUME Slab under } \\
\text { Office Section }\end{array}$ & 104 & $\mathrm{CY}$ & 1.20 & 7.71 & 801 & $\$ 79.18$ & & & $\begin{array}{r}\$ 63,436 \\
\$ 610.26 / C Y\end{array}$ & $\begin{array}{c}\$ 24,021 \\
\$ 231.08 / C Y\end{array}$ & $\begin{array}{c}\$ 0 \\
\$ 0.00 / \mathrm{CY}\end{array}$ & $\$ 87,457$ \\
\hline \multicolumn{14}{|c|}{ CONCRETE SOG - BY VOLUME Warehouse } \\
\hline 040702 & REBAR@SOG & 3 & ton & 1.20 & 24.00 & 72.05 & $\$ 92.37$ & $\$ 1,362.08$ & $\$ 0.00$ & $\$ 6,655$ & $\$ 4,089$ & $\$ 0$ & $\$ 10,744$ \\
\hline 040703 & FORMWORK@SOG & 163 & sqft & 1.20 & 0.48 & 78.43 & $\$ 75.57$ & $\$ 3.25$ & $\$ 0.00$ & $\$ 5,927$ & $\$ 531$ & $\$ 0$ & $\$ 6,458$ \\
\hline 040704 & EMBEDS@SOG & 342 & $\mathrm{lb}$ & 1.20 & 0.06 & 20.52 & $\$ 92.37$ & $\$ 2.50$ & $\$ 0.00$ & $\$ 1,895$ & $\$ 855$ & $\$ 0$ & $\$ 2,750$ \\
\hline 040708 & 4000PSI@SOG PUMPED & 80 & cuyd & 1.20 & 1.80 & 143.64 & $\$ 76.74$ & $\$ 136.24$ & $\$ 0.00$ & $\$ 11,023$ & $\$ 10,872$ & $\$ 0$ & $\$ 21,895$ \\
\hline 040709 & FINISHING @ SOG & 4,104 & sqft & 1.20 & 0.02 & 98.50 & $\$ 76.74$ & $\$ 0.01$ & $\$ 0.00$ & $\$ 7,559$ & $\$ 41$ & $\$ 0$ & $\$ 7,600$ \\
\hline 040705 & ANCHORBOLTS@SOG & 39 & $\mathrm{lb}$ & 1.20 & 0.24 & 9.25 & $\$ 92.37$ & $\$ 1.40$ & $\$ 0.00$ & $\$ 855$ & $\$ 54$ & $\$ 0$ & $\$ 909$ \\
\hline 040709 & FINE GRADE@SOG & 4,104 & sqft & 1.20 & 0.04 & 147.74 & $\$ 76.74$ & $\$ 0.03$ & $\$ 0.00$ & $\$ 11,338$ & $\$ 123$ & $\$ 0$ & $\$ 11,461$ \\
\hline 040706 & WATERSTOP@SOG & 350 & $\operatorname{lnft}$ & 1.20 & 0.07 & 25.23 & $\$ 76.74$ & $\$ 5.00$ & $\$ 0.00$ & $\$ 1,936$ & $\$ 1,752$ & $\$ 0$ & $\$ 3,688$ \\
\hline 040710 & HARDENER@ SOG & 4,104 & sqft & 1.20 & 0.00 & 19.70 & $\$ 76.74$ & $\$ 0.03$ & $\$ 0.00$ & $\$ 1,512$ & $\$ 123$ & $\$ 0$ & $\$ 1,635$ \\
\hline
\end{tabular}


Client: INL

Project: FFC

Location: GREEN FIELD SITE HIP Option

Account: 04 Concrete/Masonry

Facility: Entire Estimate

URS

Washington Division
Date: 7/17/2008

11:31AM

Priced By: ADC

Office: Denver

Product Line: Gov
Estimate: FFC

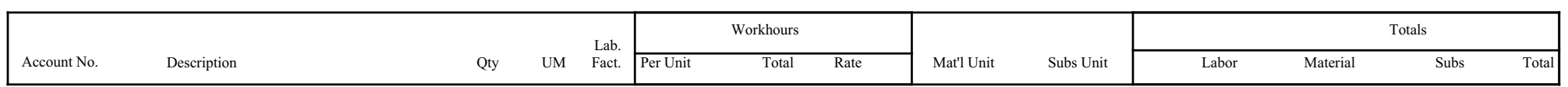

CONCRETE SOG - BY VOLUME Warehouse

$\begin{array}{lrl}\text { Formwork } & \mathbf{2 . 0 5} & \mathrm{SF} / \mathrm{CY} \\ \text { Rebar } & \mathbf{7 5 . 2 4} & \mathrm{LB} / \mathrm{CY} \\ \text { Embedments } & \mathbf{4 . 2 9} & \mathrm{LB} / \mathrm{CY}\end{array}$

Embedments

\begin{tabular}{|cr|}
\hline $\mathrm{Wkhr} / \mathrm{CY}$ & $\mathbf{7 . 7 1}$ \\
$\mathrm{Total} / \mathrm{CY}$ & $\mathbf{\$ 8 4 1 . 3 4}$ \\
\hline
\end{tabular}

\begin{tabular}{|c|c|c|c|c|c|c|c|c|c|c|}
\hline Total & CONCRETE SOG - BY VOLUME Warehouse & $80 \mathrm{CY}$ & 1.20 & 7.71 & 615 & $\$ 79.18$ & $\begin{array}{r}\$ 48,699 \\
\$ 610.26 / C Y\end{array}$ & $\begin{array}{c}\$ 18,440 \\
\$ 231.08 / C Y\end{array}$ & $\begin{array}{cc} & \$ 0 \\
\$ 0.00 & / C\end{array}$ & $\$ 67,139$ \\
\hline
\end{tabular}

Ductbank by Dimension (2.5' X 2.5' X 500')

\begin{tabular}{|c|c|c|c|}
\hline 040802 & REBAR@D & & \\
\hline 040803 & FORMWORF & & \\
\hline 040808 & 4000PSI@DL & & \\
\hline \multirow[t]{5}{*}{040810} & ADDED COI & & \\
\hline & \multicolumn{3}{|c|}{ Ductbank by Dimension (2.5' X 2.5' X 500') } \\
\hline & Formwork & 21.90 & $\mathrm{SF} / \mathrm{CY}$ \\
\hline & Rebar & 46.67 & $\mathrm{LB} / \mathrm{CY}$ \\
\hline & Embedments & 0.00 & $\mathrm{LB} / \mathrm{CY}$ \\
\hline
\end{tabular}

\begin{tabular}{rr|} 
Wkhr/CY & $\mathbf{1 2 . 9 8}$ \\
Total/CY & $\mathbf{\$ 1 , 2 7 7 . 3 6}$
\end{tabular}

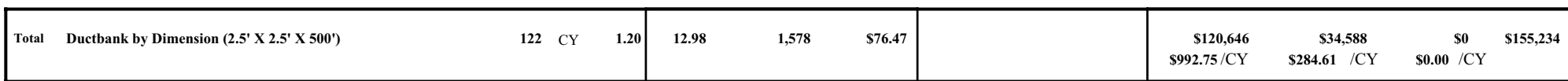

Elevated Slab on Metal Deck- by Volume

$043202 \quad$ REBAR@ELEV SLAB ON

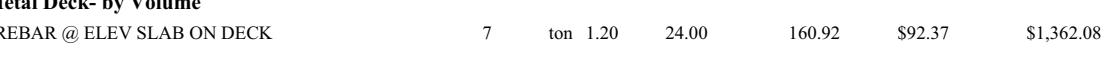

$\$ 0.00 \quad \$ 14,864 \quad \$ 9,133$

$\$ 0 \quad \$ 23,997$

Page 15 of 69 
Client: INL

Project: FFC

URS

Washington Division
Date: 7/17/2008

$11: 31 \mathrm{AM}$

Priced By: ADC

Office: Denver

Product Line: Gov
Estimate: FFC

Account: 04 Concrete

Job No: 27989-325 Rev No: 0

\begin{tabular}{|c|c|c|c|c|c|c|c|c|c|c|c|c|c|}
\hline \multirow[b]{2}{*}{ Account No. } & \multirow[b]{2}{*}{ Description } & \multirow[b]{2}{*}{ Qty } & \multirow[b]{2}{*}{ UM } & \multirow{2}{*}{$\begin{array}{l}\text { Lab. } \\
\text { Fact. }\end{array}$} & \multicolumn{3}{|c|}{ Workhours } & \multirow[b]{2}{*}{ Mat'l Unit } & \multirow[b]{2}{*}{ Subs Unit } & \multicolumn{4}{|c|}{ Totals } \\
\hline & & & & & Per Unit & Total & Rate & & & Labor & Material & Subs & Total \\
\hline 043203 & FORMWORK@ELEV SLAB ON DECK & 304 & sqft & 1.20 & 0.48 & 146.02 & $\$ 75.57$ & $\$ 3.00$ & $\$ 0.00$ & $\$ 11,034$ & $\$ 913$ & $\$ 0$ & $\$ 11,947$ \\
\hline 043204 & EMBEDS @ ELEV SLAB ON DECK & 1,080 & $\mathrm{lb}$ & 1.20 & 0.07 & 77.76 & $\$ 92.37$ & $\$ 2.50$ & $\$ 0.00$ & $\$ 7,183$ & $\$ 2,700$ & $\$ 0$ & $\$ 9,883$ \\
\hline 043208 & 4000PSI@ESOMD PUMPED & 95 & cuyd & d 1.20 & 2.74 & 258.55 & $\$ 76.74$ & $\$ 136.24$ & $\$ 0.00$ & $\$ 19,841$ & $\$ 12,875$ & $\$ 0$ & $\$ 32,716$ \\
\hline 043209 & FINISHING,CURE,HRDNR@ESOMD & 4,860 & sqft & 1.20 & 0.04 & 174.96 & $\$ 76.74$ & $\$ 0.01$ & $\$ 0.00$ & $\$ 13,426$ & $\$ 49$ & $\$ 0$ & $\$ 13,475$ \\
\hline 043211 & GROUT@ESOMD & 34 & cuft & 1.20 & 2.26 & 76.02 & $\$ 76.74$ & $\$ 60.00$ & $\$ 0.00$ & $\$ 5,834$ & $\$ 2,022$ & $\$ 0$ & $\$ 7,855$ \\
\hline
\end{tabular}

Elevated Slab on Metal Deck- by Volume

Formwork

$\begin{array}{lrl}\text { Rebar } & \mathbf{1 4 1 . 9 0} & \mathrm{LB} / \mathrm{CY} \\ \text { Embedments } & \mathbf{1 1 . 4 3} & \mathrm{LB} / \mathrm{CY}\end{array}$

\begin{tabular}{|rr}
\hline $\mathrm{Wkhr} / \mathrm{CY}$ & $\mathbf{9 . 4 6}$ \\
& \\
Total/CY & $\mathbf{\$ 1 , 0 5 6 . 8 5}$
\end{tabular}

\begin{tabular}{|c|c|c|c|c|c|c|c|c|c|c|c|c|c|}
\hline Total & Elevated Slab on Metal Deck- by Volume & 95 & $\mathrm{CY}$ & 1.20 & 9.46 & 894 & $\$ 80.72$ & & & $\begin{array}{r}\$ 72,182 \\
\$ 763.83 / C Y\end{array}$ & $\begin{array}{c}\$ 27,690 \\
\$ 293.02 / C Y\end{array}$ & $\begin{array}{c}\text { \$0 } \\
\$ 0.00 / \mathrm{CY}\end{array}$ & $\$ 99,872$ \\
\hline \multicolumn{14}{|c|}{ Elevated Slab on Metal Deck- by Volume HVAC Filter Floor } \\
\hline 043202 & REBAR@ ELEV SLAB ON DECK & 6 & ton & 1.20 & 24.00 & 146.62 & $\$ 92.37$ & $\$ 1,362.08$ & $\$ 0.00$ & $\$ 13,543$ & $\$ 8,321$ & $\$ 0$ & $\$ 21,864$ \\
\hline 043203 & FORMWORK@ELEV SLAB ON DECK & 277 & sqft & 1.20 & 0.48 & 133.04 & $\$ 75.57$ & $\$ 3.00$ & $\$ 0.00$ & $\$ 10,054$ & $\$ 831$ & $\$ 0$ & $\$ 10,885$ \\
\hline 043204 & EMBEDS@ ELEV SLAB ON DECK & 984 & $\mathrm{lb}$ & 1.20 & 0.07 & 70.85 & $\$ 92.37$ & $\$ 2.50$ & $\$ 0.00$ & $\$ 6,544$ & $\$ 2,460$ & $\$ 0$ & $\$ 9,004$ \\
\hline 043208 & 4000PSI@ESOMD PUMPED & 86 & cuyd & 1.20 & 2.74 & 235.57 & $\$ 76.74$ & $\$ 136.24$ & $\$ 0.00$ & $\$ 18,077$ & $\$ 11,730$ & $\$ 0$ & $\$ 29,808$ \\
\hline 043209 & FINISHING,CURE,HRDNR@ESOMD & 4,428 & sqft & 1.20 & 0.04 & 159.41 & $\$ 76.74$ & $\$ 0.01$ & $\$ 0.00$ & $\$ 12,233$ & $\$ 44$ & $\$ 0$ & $\$ 12,277$ \\
\hline 043211 & GROUT @ ESOMD & 31 & cuft & 1.20 & 2.26 & 69.26 & $\$ 76.74$ & $\$ 60.00$ & $\$ 0.00$ & $\$ 5,315$ & $\$ 1,842$ & $\$ 0$ & $\$ 7,157$ \\
\hline
\end{tabular}


Client: INL

Project: FFC

Location: GREEN FIELD SITE HIP Option

Account: 04 Concrete/Masonry

Facility: Entire Estimate

URS

Washington Division
Date: 7/17/2008

11:31AM

Priced By: ADC

Office: Denver

Product Line: Gov
Estimate: FFC

HIP Option

Job No: $\quad 27989-325 \quad$ Rev No: 0

\begin{tabular}{|c|c|c|c|c|c|c|c|c|c|c|c|c|}
\hline \multirow[b]{2}{*}{ Account No. } & \multirow[b]{2}{*}{ Description } & \multirow[b]{2}{*}{ Qty } & \multirow[b]{2}{*}{ UM } & \multirow{2}{*}{$\begin{array}{l}\text { Lab. } \\
\text { Fact. }\end{array}$} & \multicolumn{2}{|c|}{ Workhours } & \multirow[b]{2}{*}{ Mat'l Unit } & \multirow[b]{2}{*}{ Subs Unit } & \multicolumn{4}{|c|}{ Totals } \\
\hline & & & & & \begin{tabular}{|l} 
Per Unit \\
\end{tabular} & Total & & & Labor & Material & Subs & Total \\
\hline
\end{tabular}

$\begin{array}{lrl}\text { Elevated Slab on Metal Deck- by Volume HVAC Filter Flo } \\ \text { Formwork } & \mathbf{3 . 2 2} & \mathrm{SF} / \mathrm{CY} \\ \text { Rebar } & \mathbf{1 4 1 . 9 0} & \mathrm{LB} / \mathrm{CY} \\ \text { Embedments } & \mathbf{1 1 . 4 3} & \mathrm{LB} / \mathrm{CY}\end{array}$

\begin{tabular}{|c|c|c|c|c|c|c|c|c|c|c|}
\hline Total & $\begin{array}{l}\text { Elevated Slab on Metal Deck- by Volume HVAC } \\
\text { Filter Floor }\end{array}$ & $86 \mathrm{CY}$ & 1.20 & 9.46 & 815 & $\$ 80.72$ & $\begin{array}{r}\$ 65,766 \\
\$ 763.83 / C Y\end{array}$ & $\begin{array}{c}\$ 25,229 \\
\$ 293.02\end{array}$ & $\begin{array}{c}\$ 0 \\
\$ 0.00 / \mathrm{CY}\end{array}$ & $\$ 90,995$ \\
\hline
\end{tabular}

Elevated Slab on Metal Deck- by Volume Security BIdg Roof

$\begin{array}{ll}043202 & \text { REBAR @ ELEV SLAB ON DECK } \\ 043203 & \text { FORMWORK@ELEV SLAB ON DECK } \\ 043204 & \text { EMBEDS @ ELEV SLAB ON DECK } \\ 043208 & \text { 4000PSI@ESOMD PUMPED } \\ 043209 & \text { FINISHING,CURE,HRDNR@ESOMD } \\ 043211 & \text { GROUT @ ESOMD }\end{array}$

$\begin{array}{rrrrr}1 & \text { ton } 1.20 & 24.00 & 30.40 & \$ 92.37 \\ 57 & \text { sqft } 1.20 & 0.48 & 27.58 & \$ 75.57 \\ 204 & \mathrm{lb} 1.20 & 0.07 & 14.69 & \$ 92.37 \\ 18 & \text { cuyd } 1.20 & 2.74 & 48.84 & \$ 76.74 \\ 918 & \text { sqft } 1.20 & 0.04 & 33.05 & \$ 76.74 \\ 6 & \text { cuft } 1.20 & 2.26 & 14.36 & \$ 76.74\end{array}$
Elevated Slab on Metal Deck- by Volume Security BIdg Roof
$\begin{array}{lrl}\text { Formwork } & \mathbf{3 . 2 2} & \mathrm{SF} / \mathrm{CY} \\ \text { Rebar } & \mathbf{1 4 1 . 9 0} & \mathrm{LB} / \mathrm{CY}\end{array}$
$11.43 \mathrm{LB} / \mathrm{CY}$
Embedments

\begin{tabular}{rr|} 
Wkhr/CY & $\mathbf{9 . 4 6}$ \\
Total/CY & $\mathbf{\$ 1 , 0 5 6 . 8 5}$ \\
\hline
\end{tabular}

\begin{tabular}{|c|c|c|c|c|c|c|c|c|c|}
\hline Total & $\begin{array}{l}\text { Elevated Slab on Metal Deck- by Volume Security } \\
\text { Bldg Roof }\end{array}$ & $18 \mathrm{CY}$ & 1.20 & 9.46 & $\$ 80.72$ & $\begin{array}{r}\$ 13,634 \\
\$ 763.83 / C Y\end{array}$ & $\begin{array}{c}\$ 5,230 \\
\$ 293.02 / C Y\end{array}$ & $\begin{array}{c}\$ 0 \\
\$ 0.00 / C Y\end{array}$ & $\$ 18,865$ \\
\hline
\end{tabular}


Client: INL

Project: FFC

URS

Date: 7/17/2008

11:31AM

Location: GREEN FIELD SITE HIP Option

Washington Division

Priced By: ADC

Office: Denver

Facility: Entire Estimate

Job No: $\quad 27989-325 \quad$ Rev No: 0

Product Line: Gov
Estimate: $F F C$

HIP Optio

\begin{tabular}{|c|c|c|c|c|c|c|c|c|c|c|}
\hline \multirow[b]{2}{*}{ Account No. } & \multirow[b]{2}{*}{ Description } & \multirow[b]{2}{*}{ Qty } & \multirow{2}{*}{\multicolumn{2}{|c|}{$\begin{array}{l}\text { Lab. } \\
\text { Fact. }\end{array}$}} & & khours & \multirow[b]{2}{*}{ Mat'l Unit } & \multirow[b]{2}{*}{ Subs Unit } & \multicolumn{2}{|c|}{ Totals } \\
\hline & & & & & Per Unit & Total & & & Labor & Material \\
\hline
\end{tabular}

Fence/Fence lighting Pole

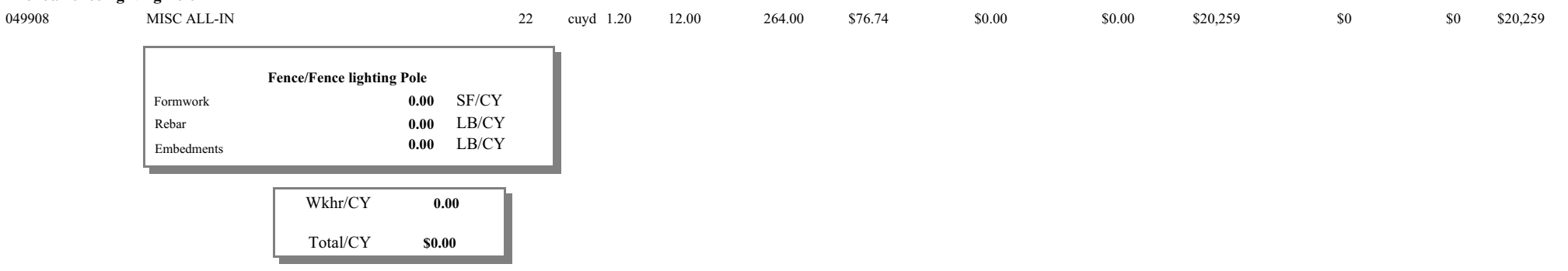

\begin{tabular}{|c|c|c|c|c|c|c|c|c|c|c|c|}
\hline Total & Fence/Fence lighting Pole & $\begin{array}{ll}0 & \mathrm{CY}\end{array}$ & 1.20 & 0.00 & 264 & $\$ 76.74$ & $\begin{array}{r}\$ 20,259 \\
\$ 0.00 / C Y\end{array}$ & $\$ 0.00$ & $\begin{array}{l}\$ 0 \\
\text { /CY }\end{array}$ & $\begin{array}{c}\$ 0 \\
\$ 0.00 / \mathrm{CY}\end{array}$ & $\$ 20,259$ \\
\hline
\end{tabular}

$\begin{array}{ll}\text { Mat (@Grade) - By Volume Hip Fdn } \\ 041102 & \text { REBAR @ MATS } \\ 041103 & \text { FORMWORK 2.5 Ft. Thick@ MATS } \\ 041104 & \text { EMBEDS @ MATS } \\ 041108 & \text { 4000PSI@MATS PUMPED } \\ 041109 & \text { FINISHING @ MATS } \\ 041105 & \text { ANCHOR BOLTS @ MATS } \\ 041109 & \text { FINE GRADE @ MAT } \\ 041106 & \text { WATERSTOP @ MATS } \\ 041111 & \text { GROUT @ MATS }\end{array}$

$\begin{array}{rrrr}10 & \text { ton } & 1.20 & 24.00 \\ 447 & \text { sqft } & 1.20 & 0.48 \\ 298 & \text { lb } & 1.20 & 0.06 \\ 156 & \text { cuyd } & 1.20 & 1.80 \\ 1,678 & \text { sqft } & 1.20 & 0.02 \\ 88 & \text { lb } & 1.20 & 0.24 \\ 1,678 & \text { sqft } & 1.20 & 0.04 \\ 18 & \text { Inft } & 1.20 & 0.07 \\ 3 & \text { cuft } & 1.20 & 4.80\end{array}$

$\begin{array}{rr}237.80 & \$ 92.37 \\ 214.56 & \$ 75.57 \\ 17.88 & \$ 76.74 \\ 281.61 & \$ 76.74 \\ 40.27 & \$ 76.74 \\ 21.01 & \$ 76.74 \\ 60.40 & \$ 75.57 \\ 1.31 & \$ 75.57 \\ 13.11 & \$ 76.74\end{array}$

$\$ 800.00$
$\$ 3.00$
$\$ 2.50$
$\$ 65.00$
$\$ 0.01$
$\$ 1.40$
$\$ 0.03$
$\$ 5.00$
$\$ 60.00$

$\$ 0.00$
$\$ 0.00$
$\$ 0.00$
$\$ 0.00$
$\$ 0.00$
$\$ 0.00$
$\$ 0.00$
$\$ 0.00$
$\$ 0.00$

$\$ 21,965$
$\$ 16,214$
$\$ 1,372$
$\$ 21,611$
$\$ 3,090$
$\$ 1,612$
$\$ 4,564$
$\$ 99$
$\$ 1,006$

$\$ 7,927$
$\$ 1,341$
$\$ 745$
$\$ 10,169$
$\$ 17$
$\$ 123$
$\$ 50$
$\$ 91$
$\$ 164$

$\begin{array}{lr}\$ 0 & \$ 29,892 \\ \$ 0 & \$ 17,555 \\ \$ 0 & \$ 2,117 \\ \$ 0 & \$ 31,780 \\ \$ 0 & \$ 3,107 \\ \$ 0 & \$ 1,734 \\ \$ 0 & \$ 4,615 \\ \$ 0 & \$ 191 \\ \$ 0 & \$ 1,170\end{array}$


Client: INL

Project: FFC

URS

Washington Division
Date: 7/17/2008

$11: 31 \mathrm{AM}$ Priced By: ADC

Office: Denver

Product Line: Gov
Estimate: FFC

Account: 04 Concrete/Masonry

Job No: $\quad 27989-325 \quad$ Rev No: 0

\begin{tabular}{|c|c|c|c|c|c|c|c|c|c|c|c|c|}
\hline \multirow[b]{2}{*}{ Account No. } & \multirow[b]{2}{*}{ Description } & \multirow[b]{2}{*}{ Qty } & \multirow[b]{2}{*}{ UM } & \multirow{2}{*}{$\begin{array}{l}\text { Lab. } \\
\text { Fact. }\end{array}$} & \multicolumn{2}{|c|}{ Workhours } & \multirow[b]{2}{*}{ Mat'l Unit } & \multirow[b]{2}{*}{ Subs Unit } & \multicolumn{4}{|c|}{ Totals } \\
\hline & & & & & \begin{tabular}{|l} 
Per Unit \\
\end{tabular} & Total & & & Labor & Material & Subs & Total \\
\hline
\end{tabular}

Mat (@Grade) - By Volume Hip Fdn

$\begin{array}{lrl}\text { Formwork } & \mathbf{2 . 8 6} & \mathrm{SF} / \mathrm{CY} \\ \text { Rebar } & \mathbf{1 2 6 . 6 7} & \mathrm{LB} / \mathrm{CY}\end{array}$

$\begin{array}{lrl}\text { Rebar } & \mathbf{1 2 6 . 6 7} & \mathrm{LB} / \mathrm{CY} \\ \text { Embedments } & \mathbf{1 . 9 0} & \mathrm{LB} / \mathrm{CY}\end{array}$

\begin{tabular}{rr|} 
Wkhr/CY & $\mathbf{5 . 6 8}$ \\
Total/CY & $\mathbf{\$ 5 8 9 . 0 7}$
\end{tabular}

\begin{tabular}{|c|c|c|c|c|c|c|c|c|c|c|}
\hline Total & Mat (@Grade) - By Volume Hip Fdn & $156 \mathrm{CY}$ & 1.20 & 5.68 & 888 & $\$ 80.56$ & $\begin{array}{r}\$ 71,534 \\
\$ 457.23 / C Y\end{array}$ & $\begin{array}{c}\$ 20,627 \\
\$ 131.84 / C Y\end{array}$ & $\begin{array}{c}\$ 0 \\
\$ 0.00 / \mathrm{CY}\end{array}$ & $\$ 92,160$ \\
\hline
\end{tabular}

$\begin{array}{ll}\text { Mat (@Grade) - By Volume HIP Fdn 18" Thk } \\ 041102 & \text { REBAR@ MATS } \\ 041103 & \text { FORMWORK 1.5 Ft. Thick@ MATS } \\ 041104 & \text { EMBEDS @ MATS } \\ 041108 & \text { 4000PSI@MATS PUMPED } \\ 041109 & \text { FINISHING @ MATS } \\ 041105 & \text { ANCHOR BOLTS @ MATS } \\ 041109 & \text { FINE GRADE @ MAT } \\ 041106 & \text { WATERSTOP @ MATS } \\ 041111 & \text { GROUT @ MATS }\end{array}$

$\begin{array}{rrrrrr}13 & \text { ton } 1.20 & 24.00 & 301.64 & \$ 92.37 \\ 567 & \text { sqft } & 1.20 & 0.48 & 272.16 & \$ 75.57 \\ 378 & \text { lb } & 1.20 & 0.06 & 22.68 & \$ 76.74 \\ 198 & \text { cuyd } & 1.20 & 1.80 & 357.21 & \$ 76.74 \\ 3,400 & \text { sqft } 1.20 & 0.02 & 81.60 & \$ 76.74 \\ 111 & \text { lb } & 1.20 & 0.24 & 26.64 & \$ 76.74 \\ 3,400 & \text { sqft } & 1.20 & 0.04 & 122.40 & \$ 75.57 \\ 23 & \text { Inft } 1.20 & 0.07 & 1.67 & \$ 75.57 \\ 3 & \text { cuft } & 1.20 & 4.80 & 16.63 & \$ 76.74\end{array}$

$\$ 800.00$
$\$ 3.00$
$\$ 2.50$
$\$ 65.00$
$\$ 0.01$
$\$ 1.40$
$\$ 0.03$
$\$ 5.00$
$\$ 60.00$

$\begin{array}{lrr}\$ 0.00 & \$ 27,862 & \$ 10,055 \\ \$ 0.00 & \$ 20,567 & \$ 1,701 \\ \$ 0.00 & \$ 1,740 & \$ 945 \\ \$ 0.00 & \$ 27,412 & \$ 12,899 \\ \$ 0.00 & \$ 6,262 & \$ 34 \\ \$ 0.00 & \$ 2,045 & \$ 155 \\ \$ 0.00 & \$ 9,250 & \$ 102 \\ \$ 0.00 & \$ 126 & \$ 116 \\ \$ 0.00 & \$ 1,276 & \$ 208\end{array}$

$\begin{array}{lr}\$ 0 & \$ 37,917 \\ \$ 0 & \$ 22,268 \\ \$ 0 & \$ 2,685 \\ \$ 0 & \$ 40,311 \\ \$ 0 & \$ 6,296 \\ \$ 0 & \$ 2,200 \\ \$ 0 & \$ 9,352 \\ \$ 0 & \$ 242 \\ \$ 0 & \$ 1,484\end{array}$ 
Client: INL

Project: FFC

URS

Date: 7/17/2008

11:31AM

Location: GREEN FIELD SITE HIP Option

Washington Division

Priced By: ADC

Office: Denver

Facility: Entire Estimate

Job No: $\quad 27989-325 \quad$ Rev No: 0

Product Line: Gov
Estimate: FFC

HIP Option

\begin{tabular}{|c|c|c|c|c|c|c|c|c|c|c|}
\hline \multirow[b]{2}{*}{ Account No. } & \multirow[b]{2}{*}{ Description } & \multirow[b]{2}{*}{ Qty } & \multirow{2}{*}{\multicolumn{2}{|c|}{$\begin{array}{l}\text { Lab. } \\
\text { Fact. }\end{array}$}} & & rkhours & \multirow[b]{2}{*}{ Mat'l Unit } & \multirow[b]{2}{*}{ Subs Unit } & \multicolumn{2}{|c|}{ Totals } \\
\hline & & & & & Per Unit & Total & & & Labor & Material \\
\hline
\end{tabular}

Formwork $\quad \mathbf{2 . 8 6} \mathrm{SF} / \mathrm{CY}$

$\begin{array}{lll}\text { Rebar } & \mathbf{1 2 6 . 6 7} & \text { LB/CY }\end{array}$

$\begin{array}{lrl}\text { Embedments } & \mathbf{1 . 9 0} & \mathrm{LB} / \mathrm{CY}\end{array}$

$\begin{array}{rr}\text { Wkhr/CY } & \mathbf{6 . 0 6} \\ \text { Total/CY } & \$ 618.57\end{array}$

\begin{tabular}{|c|c|c|c|c|c|c|c|c|c|c|}
\hline Total & Mat (@Grade) - By Volume HIP Fdn 18" Thk & $198 \mathrm{CY}$ & 1.20 & 6.06 & 1,203 & $\$ 80.27$ & $\begin{array}{r}\$ 96,540 \\
\$ 486.47 / C Y\end{array}$ & $\begin{array}{c}\$ 26,215 \\
\$ 132.10 / C Y\end{array}$ & $\begin{array}{c}\$ 0 \\
\$ 0.00 / \mathrm{CY}\end{array}$ & $\$ 122,755$ \\
\hline
\end{tabular}

$\begin{array}{ll}\text { Mat (@Grade) - By Volume Under Process Areas } \\ 041102 & \text { REBAR @ MATS } \\ 041103 & \text { FORMWORK 1.5 Ft. Thick @ MATS } \\ 041104 & \text { EMBEDS @ MATS } \\ 041108 & \text { 4000PSI@MATS PUMPED } \\ 041109 & \text { FINISHING @ MATS } \\ 041105 & \text { ANCHOR BOLTS @ MATS } \\ 041109 & \text { FINE GRADE @ MAT } \\ 041106 & \text { WATERSTOP @ MATS } \\ 041111 & \text { GROUT @ MATS }\end{array}$

$\begin{array}{rrrrrr}283 & \text { ton } 1.20 & 24.00 & 6,792.58 & \$ 92.37 \\ 12,768 & \text { sqft } & 1.20 & 0.48 & 6,128.64 & \$ 75.57 \\ 8,512 & \text { lb } & 1.20 & 0.06 & 510.72 & \$ 76.74 \\ 4,469 & \text { ruyd } 1.20 & 1.80 & 8,043.84 & \$ 76.74 \\ 76,565 & \text { sqft } 1.20 & 0.02 & 1,837.57 & \$ 76.74 \\ 2,500 & \text { lb } & 1.20 & 0.24 & 599.99 & \$ 76.74 \\ 76,565 & \text { sqft } 1.20 & 0.04 & 2,756.36 & \$ 75.57 \\ 521 & \text { Inft } 1.20 & 0.07 & 37.54 & \$ 75.57 \\ 78 & \text { cuft } & 1.20 & 4.80 & 374.40 & \$ 76.74\end{array}$

$\$ 1,362.08$
$\$ 3.00$
$\$ 2.50$
$\$ 136.24$
$\$ 0.01$
$\$ 1.40$
$\$ 0.03$
$\$ 5.00$
$\$ 60.00$

$\$ 0.00$
$\$ 0.00$
$\$ 0.00$
$\$ 0.00$
$\$ 0.00$
$\$ 0.00$
$\$ 0.00$
$\$ 0.00$
$\$ 0.00$

$\$ 627,417$

$\$ 463,138$

$\$ 385,501$

$\$ 38,304$

$\$ 617,278 \quad \$ 608,829$

$\$ 141,014 \quad \$ 766$

$\$ 46,043 \quad \$ 3,500$

$\$ 208,296 \quad \$ 2,297$

$\$ 2,837 \quad \$ 2,607$

$\$ 28,731$

$\$ 4,680$

$\begin{array}{lr}\$ 0 & \$ 1,012,918 \\ \$ 0 & \$ 501,442 \\ \$ 0 & \$ 60,472 \\ \$ 0 & \$ 1,226,108 \\ \$ 0 & \$ 141,779 \\ \$ 0 & \$ 49,543 \\ \$ 0 & \$ 210,593 \\ \$ 0 & \$ 5,444 \\ \$ 0 & \$ 33,411\end{array}$


Client: INL

Project: FFC

Location: GREEN FIELD SITE HIP Option

Account: 04 Concrete/Masonry

Facility: Entire Estimate

URS

Washington Division
Date: 7/17/2008

11:31AM Priced By: ADC

Office: Denver

Product Line: Gov
Estimate: FFC

\begin{tabular}{|c|c|c|c|c|c|c|c|c|c|c|c|c|c|}
\hline \multirow[b]{2}{*}{ Account No. } & \multirow[b]{2}{*}{ Description } & \multirow[b]{2}{*}{ Qty } & \multirow[b]{2}{*}{ UM } & \multirow{2}{*}{$\begin{array}{l}\text { Lab. } \\
\text { Fact. }\end{array}$} & \multicolumn{3}{|c|}{ Workhours } & \multirow[b]{2}{*}{ Mat'l Unit } & \multirow[b]{2}{*}{ Subs Unit } & \multicolumn{4}{|c|}{ Totals } \\
\hline & & & & & Per Unit & Total & Rate & & & Labor & Material & Subs & Total \\
\hline
\end{tabular}

\begin{tabular}{|c|c|c|c|}
\hline \multicolumn{4}{|c|}{ Mat (@Grade) - By Volume Under Process Areas } \\
\hline \multirow{3}{*}{\multicolumn{2}{|c|}{$\begin{array}{l}\text { Formwork } \\
\text { Rebar } \\
\text { Embedments }\end{array}$}} & 2.86 & $\mathrm{SF} / \mathrm{CY}$ \\
\hline & & 126.67 & $\mathrm{LB} / \mathrm{CY}$ \\
\hline & & 1.90 & $\mathrm{LB} / \mathrm{CY}$ \\
\hline & $\mathrm{Wkhr} / \mathrm{CY}$ & 6. & \\
\hline & Total/CY & $\$ 725$. & \\
\hline
\end{tabular}

\begin{tabular}{|c|c|c|c|c|c|c|c|c|c|}
\hline Total & Mat (@Grade) - By Volume Under Process Areas & $4,469 \quad \mathrm{CY}$ & 1.20 & 6.06 & 27,082 & $\$ 80.27$ & $\begin{array}{r}\$ 2,173,946 \\
\$ 486.47 / \mathrm{CY}\end{array}$ & $\begin{array}{c}\$ 1,067,764 \\
\$ 238.94 \quad / C Y\end{array}$ & 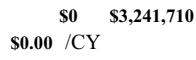 \\
\hline
\end{tabular}

\begin{tabular}{|c|c|c|c|c|}
\hline \multicolumn{5}{|c|}{ Roofing Fire water Collection Tank Sump } \\
\hline \multirow[t]{7}{*}{042505} & PRECAST AI & & & \\
\hline & \multicolumn{4}{|c|}{ Roofing Fire water Collection Tank Sump } \\
\hline & Formwork & & 0.00 & $\mathrm{SF} / \mathrm{CY}$ \\
\hline & Rebar & & 0.00 & $\mathrm{LB} / \mathrm{CY}$ \\
\hline & Embedments & & 0.00 & $\mathrm{LB} / \mathrm{CY}$ \\
\hline & & Wkhr/CY & & 00 \\
\hline & & Total/CY & & \\
\hline
\end{tabular}

\begin{tabular}{|c|c|c|c|c|c|c|c|c|c|}
\hline Total & Roofing Fire water Collection Tank Sump & $0 \mathrm{CY}$ & 1.20 & 0.00 & $\$ 76.74$ & $\begin{array}{r}\$ 2,199 \\
\$ 0.00 / C Y\end{array}$ & $\begin{array}{c}\$ 4,050 \\
\$ 0.00 / C Y\end{array}$ & $\begin{array}{c}\text { so } \\
\text { s0.00 }\end{array}$ & $\$ 6,249$ \\
\hline
\end{tabular}

\section{SUBSTATION SITE WORK \& FOUNDATIONS}

041808 4000PSI@PIERS PUMPED

$4 \quad$ cuyd $1.20 \quad 9.60 \quad 38.40 \quad \$ 76.74$

$\$ 200.00$

$\$ 0.00$

$\$ 2,947$

$\$ 800$

$\$ 0 \quad \$ 3,747$ 
Client: INL

Project: FFC

Location: GREEN FIELD SITE HIP Option

Account: 04 Concrete/Masonry

Facility: Entire Estimate

URS

Washington Division
Date: 7/17/2008

$11: 31 \mathrm{AM}$ Priced By: ADC

Office: Denver

Product Line: Gov
Estimate: FFC

$$
\text { HIP Option }
$$

Job No:

27989-325 Rev No: 0

\begin{tabular}{|c|c|c|c|c|c|c|c|c|c|c|}
\hline \multirow[b]{2}{*}{ Account No. } & \multirow[b]{2}{*}{ Description } & \multirow[b]{2}{*}{ Qty } & \multirow{2}{*}{\multicolumn{2}{|c|}{$\begin{array}{l}\text { Lab. } \\
\text { Fact. }\end{array}$}} & & rkhours & \multirow[b]{2}{*}{ Mat'l Unit } & \multirow[b]{2}{*}{ Subs Unit } & \multicolumn{2}{|c|}{ Totals } \\
\hline & & & & & Per Unit & Total & & & Labor & Material \\
\hline
\end{tabular}

Wall by Volume Greater than $8^{\prime}$ high

61.10 $\mathrm{SF} / \mathrm{CY}$

$\begin{array}{lrl}\text { Rebar } & \mathbf{1 3 5 . 2 4} & \mathrm{LB} / \mathrm{CY} \\ \text { Embedments } & \mathbf{1 2 . 0 6} & \mathrm{LB} / \mathrm{CY}\end{array}$

Embedments

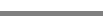

$\begin{array}{lr}\text { Wkhr/CY } & \mathbf{3 5 . 4 9} \\ \text { Total/CY } & \$ 3,077.04\end{array}$

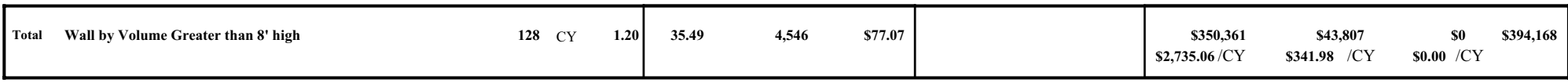

Wall by Volume Greater than 8' high Fire Water Collection Tank

$\begin{array}{ll}040902 & \text { REBAR @ WALLS } \\ 040903 & \text { FORMWORK @ WALLS OVER } 8 \\ 040904 & \text { EMBEDS @ WALLS } \\ 040908 & \text { 4000PSI@WALLS PUMPED } \\ 040909 & \text { FINISHING @ WALLS } \\ 040905 & \text { ANCHOR BOLTS @ WALLS } \\ 040906 & \text { WATERSTOP @ WALL } \\ 040911 & \text { GROUT @ WALLS }\end{array}$

$\begin{array}{rrrrrr}2 & \text { ton } 1.20 & 24.00 & 49.50 & \$ 92.37 \\ 1,958 & \text { sqft } 1.20 & 0.48 & 939.89 & \$ 75.57 \\ 317 & \text { lb } 1.20 & 0.06 & 18.99 & \$ 92.37 \\ 26 & \text { cuyd } 1.20 & 1.80 & 47.25 & \$ 76.74 \\ 1,632 & \text { sqft } 1.20 & 0.02 & 39.16 & \$ 76.74 \\ 65 & \text { lb } 1.20 & 0.24 & 15.70 & \$ 92.37 \\ 19 & \text { Inft } 1.20 & 0.07 & 1.39 & \$ 76.74 \\ 1 & \text { cuft } 1.20 & 4.80 & 3.74 & \$ 76.74\end{array}$

$\$ 1,362.08$
$\$ 3.00$
$\$ 2.50$
$\$ 136.24$
$\$ 0.01$
$\$ 1.40$
$\$ 5.00$
$\$ 60.00$

$\begin{array}{lr}\$ 0.00 & \$ 4,572 \\ \$ 0.00 & \$ 71,027 \\ \$ 0.00 & \$ 1,754 \\ \$ 0.00 & \$ 3,626 \\ \$ 0.00 & \$ 3,005 \\ \$ 0.00 & \$ 1,450 \\ \$ 0.00 & \$ 106 \\ \$ 0.00 & \$ 287\end{array}$

$\$ 2,809$
$\$ 5,874$
$\$ 791$
$\$ 3,576$
$\$ 16$
$\$ 92$
$\$ 96$
$\$ 47$

$\$ 0 \quad \$ 7,382$

$\$ 0 \quad \$ 76,901$

$\$ 0 \quad \$ 2,545$

$\$ 3,022$

$\$ 0 \quad \$ 1,542$

$\$ 0 \quad \$ 203$

WATERSTOP @ WA
GROUT@WALLS

cuft 1.20

$\$ 60.00$

$\$ 106$
$\$ 287$

$\$ 0$ 
Client: INL

Project: FFC

URS

Date: 7/17/2008

11:31AM

Location: GREEN FIELD SITE HIP Option

Washington Division

Priced By: ADC

Office: Denver

Facility: Entire Estimate

Job No: $\quad 27989-325 \quad$ Rev No: 0

Product Line: Gov
Estimate: FFC

HIP Option

\begin{tabular}{|c|c|c|c|c|c|c|c|c|c|c|}
\hline \multirow[b]{2}{*}{ Account No. } & \multirow[b]{2}{*}{ Description } & \multirow[b]{2}{*}{ Qty } & \multirow{2}{*}{\multicolumn{2}{|c|}{$\begin{array}{l}\text { Lab. } \\
\text { Fact. }\end{array}$}} & & rkhours & \multirow[b]{2}{*}{ Mat'l Unit } & \multirow[b]{2}{*}{ Subs Unit } & \multicolumn{2}{|c|}{ Totals } \\
\hline & & & & & Per Unit & Total & & & Labor & Material \\
\hline
\end{tabular}

Wall by Volume Greater than $8^{\prime}$ high Fire Water Collection

$\begin{array}{lrrl}\text { Formwork } & \text { Tank } & \mathbf{7 4 . 5 9} & \mathrm{SF} / \mathrm{CY} \\ \text { Rebar } & & \mathbf{1 5 7 . 1 4} & \mathrm{LB} / \mathrm{CY}\end{array}$

$\begin{array}{lll}\text { Embedments } & \mathbf{1 2 . 0 6} \mathrm{LB} / \mathrm{CY}\end{array}$

$\mathrm{Wkhr} / \mathrm{CY} \quad \mathbf{4 2 . 5 0}$

Total/CY $\quad \$ 3,776.39$

\begin{tabular}{|c|c|c|c|c|c|c|c|c|c|c|}
\hline Total & $\begin{array}{l}\text { Wall by Volume Greater than } 8^{\prime} \text { high Fire Water } \\
\text { Collection Tank }\end{array}$ & $26 \mathrm{CY}$ & 1.20 & 42.50 & 1,116 & $\$ 76.93$ & $\begin{array}{r}\$ 85,828 \\
\$ 3,269.64 / C Y\end{array}$ & $\begin{array}{c}\$ 13,302 \\
\$ 506.75 / C Y\end{array}$ & 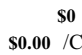 & $\$ 99,130$ \\
\hline
\end{tabular}

Wall by Volume Greater than 8' high Radiography Area

$\begin{array}{ll}040902 & \text { REBAR @ WALLS } \\ 040903 & \text { FORMWORK @ WALLS OVER } 8 \\ 040904 & \text { EMBEDS @ WALLS } \\ 040908 & \text { 4000PSI@WALLS PUMPED } \\ 040909 & \text { FINISHING @ WALLS } \\ 040905 & \text { ANCHOR BOLTS @ WALLS } \\ 040906 & \text { WATERSTOP @ WALL } \\ 040911 & \text { GROUT @ WALLS }\end{array}$

$\begin{array}{rrrrrr}39 & \text { ton } 1.20 & 24.00 & 935.55 & \$ 92.37 \\ 51,030 & \text { sqft } & 1.20 & 0.48 & 24,494.40 & \$ 75.57 \\ 5,982 & \text { lb } & 1.20 & 0.06 & 358.91 & \$ 92.37 \\ 496 & \text { cuyd } & 1.20 & 1.80 & 893.03 & \$ 76.74 \\ 51,030 & \text { sqft } & 1.20 & 0.02 & 1,224.72 & \$ 76.74 \\ 1,236 & \text { lb } & 1.20 & 0.24 & 296.72 & \$ 92.37 \\ 364 & \text { Inft } & 1.20 & 0.07 & 26.20 & \$ 76.74 \\ 15 & \text { cuft } 1.20 & 4.80 & 70.76 & \$ 76.74\end{array}$

$\$ 1,362.08$
$\$ 3.00$
$\$ 2.50$
$\$ 136.24$
$\$ 0.01$
$\$ 1.40$
$\$ 5.00$
$\$ 60.00$

$\$ 0.00$
$\$ 0.00$
$\$ 0.00$
$\$ 0.00$
$\$ 0.00$
$\$ 0.00$

$\$ 86,415$

$\$ 1,851,028$

$\$ 53,096$

$\$ 153,090$

$\$ 33,152 \quad \$ 14,955$

$\$ 68,530$

$\$ 93,984$

$\$ 5,430$

$\$ 1,819$

$\$ 885$ 
Client: INL

Project: FFC

URS

Washington Division

Account: 04 Concrete/Masonry

Facility: Entire Estimate

HIP Option

Rev No: 0

Workhours

Qty UM Lab.

Description

\begin{tabular}{|lll}
\hline Per Unit $\quad$ Total $\quad$ Rate & Matl Unit
\end{tabular}

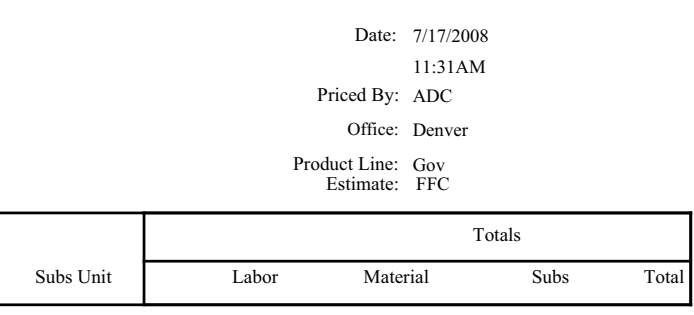

\begin{tabular}{lrl}
\multicolumn{1}{c}{ Wall by Volume Greater than $\mathbf{8}^{\prime}$} & \multicolumn{3}{c}{ high Radiography Area } \\
Formwork & $\mathbf{1 0 2 . 8 6}$ & $\mathrm{SF} / \mathrm{CY}$ \\
Rebar & $\mathbf{1 5 7 . 1 4}$ & $\mathrm{LB} / \mathrm{CY}$ \\
Embedments & $\mathbf{1 2 . 0 6}$ & $\mathrm{LB} / \mathrm{CY}$ \\
\hline
\end{tabular}

\begin{tabular}{rr|} 
Wkhr/CY & $\mathbf{5 7 . 0 4}$ \\
Total/CY & $\mathbf{\$ 4 , 9 6 1 . 7 2}$ \\
\hline
\end{tabular}

\begin{tabular}{l|l}
1.20 & 57.04 \\
\hline
\end{tabular}

28,300

$\$ 76.61$

$\$ 2,167,957$
$\$ 4,369.78 / C Y$

$\$ 293,677$

$\$ 591.94 / C Y$

\$0 $\$ 2,461,634$ $\$ 4,369.78 / C$ 
Client: INL

Project: FFC

URS

Washington Division
Date: $7 / 17 / 2008$

11:31AM Priced By: ADC

Office: Denver

Product Line: Gov
Estimate: FFC

Account: 05 Structual Steel/Platework

Job No: $\quad 27989-325 \quad$ Rev No: 0

\begin{tabular}{|c|c|c|c|c|c|c|c|c|c|c|c|c|c|c|}
\hline \multirow[b]{2}{*}{ Account No. } & \multirow{2}{*}{\multicolumn{2}{|c|}{ Description }} & \multirow[b]{2}{*}{ Qty } & \multirow[b]{2}{*}{ UM } & \multirow{2}{*}{$\begin{array}{l}\text { Lab. } \\
\text { Fact. }\end{array}$} & \multicolumn{3}{|c|}{ Workhours } & \multirow[b]{2}{*}{ Mat'l Unit } & \multirow[b]{2}{*}{ Subs Unit } & \multicolumn{4}{|c|}{ Totals } \\
\hline & & & & & & Per Unit & Total & Rate & & & Labor & Material & Subs & Total \\
\hline \multicolumn{15}{|c|}{05 Structural Steel by Class } \\
\hline Structural Steel: S & ub Total (incl xLight - $x$ & Hvy) & 45 & tons & 1.20 & 27.05 & 1,223 & $\$ 91.25$ & $\$ 4,445$ & & $\$ 111,558$ & $\$ 200,896$ & $\$ 0$ & $\$ 312,455$ \\
\hline \multicolumn{3}{|c|}{$05 \times x 03 * *$ Light Steel $-5-20 \# / L F$} & 15 & tons & 1.20 & 33.30 & 509 & $\$ 91.25$ & $\$ 5,564$ & & $\$ 46,489$ & $\$ 85,134$ & $\$ 0$ & $\$ 131,623$ \\
\hline \multicolumn{15}{|c|}{ Light Steel - 5-20\#/LF } \\
\hline & $\$ 8,603$ & Total Cost/Ton & & & & & & & & & & & & \\
\hline \multirow[t]{3}{*}{$05 \times x 04 * *$} & Medium Steel - 20-40\#/L & & \multirow[t]{3}{*}{30} & \multirow[t]{3}{*}{ tons } & \multirow[t]{3}{*}{1.20} & \multirow[t]{3}{*}{23.85} & \multirow[t]{3}{*}{713} & \multirow[t]{3}{*}{$\$ 91.25$} & \multirow[t]{3}{*}{$\$ 3,872$} & & \multirow[t]{3}{*}{$\$ 65,069$} & \multirow[t]{3}{*}{$\$ 115,762$} & \multirow[t]{3}{*}{$\$ 0$} & \multirow[t]{3}{*}{$\$ 180,831$} \\
\hline & Mediun & 1 Steel - 20-40\#/LF & & & & & & & & & & & & \\
\hline & $\$ 6,048$ & Total Cost/Ton & & & & & & & & & & & & \\
\hline \multicolumn{3}{|c|}{ Minor Struct Steel : Sub Total (incl xLight - xHvy) } & 5 & tons & 1.20 & 48.00 & 254 & $\$ 91.25$ & $\$ 6,454$ & & $\$ 23,147$ & $\$ 34,111$ & \$0 & $\$ 57,258$ \\
\hline \multirow{5}{*}{$\begin{array}{l}05 \times x 03 * * \\
05 \times x 04 * *\end{array}$} & Light Steel - 5-20\#/LF & & 2 & tons & 1.20 & 48.00 & 110 & $\$ 91.25$ & $\$ 6,454$ & & $\$ 10,052$ & $\$ 14,812$ & $\$ 0$ & $\$ 24,864$ \\
\hline & Medium Steel - 20-40\#/L & & 3 & tons & 1.20 & 48.00 & 144 & $\$ 91.25$ & $\$ 6,454$ & & $\$ 13,096$ & $\$ 19,298$ & $\$ 0$ & $\$ 32,394$ \\
\hline & \multicolumn{5}{|c|}{ Minor Struct Steel : Sub Total (incl xLight - xHvy) } & & & & & & & & & \\
\hline & $\begin{array}{r}48.00 \\
\$ 6,454\end{array}$ & $\begin{array}{l}\text { Workhours/Ton } \\
\text { Material Cost/Ton }\end{array}$ & & & & & & & & & & & & \\
\hline & $\$ 10,834$ & Total Cost/Ton & & & & & & & & & & & & \\
\hline
\end{tabular}

Decking and Flooring

9,545

463

$\$ 42,209$

$\$ 22,197$

$\$ 0 \$ 64,406$

\begin{tabular}{|ll|}
\hline & \multicolumn{1}{c|}{ Overall Ratios: } \\
38.40 & Workhour $/$ Ton \\
$\$ 5,095$ & Material Cost $/$ Ton \\
$\$ 8,599$ & Total Cost $/$ Ton \\
\hline
\end{tabular}

Grand Total: Srructural Steel/ Platework

50 tons 1.20

38.40

1,939

$\$ 91.25$

$\$ 176,914$

$\$ 257,204$

$\$ 0$

$\$ 434,119$ 
Client: INL

Project: FFC

URS

Washington Division

Job No: $\quad 27989-325 \quad$ Rev No: 0

Account: 05 Structual Steel/Plater

Facility: Entire Estimate

(a)

$\begin{array}{llll} & & & \\ \text { Lab. }\end{array}$

Qty UM Fact.

Per Unit

Workhours

Total
Total

Total Rate

Rate

\section{$\%$ of total $\%$ of commodity}

$100.00 \%$
Light Steel - 5-20\#/LF
Medium Steel - 20-40\#/LF

0505 Decking and Flooring

$$
\$ 7,323
$$

Total Cost/Ton
Th,323

$0501 \quad$ Building Steel

$\begin{array}{llll}50 & \text { tons } & 1.20 & 29.24\end{array}$

18 tons $1.20 \quad 35.22$

$\$ 91.25$

857

$\$ 91.25$

$\$ 91.25$

$\$ 5,680$

$\$ 4,106$

463

$\$ 91.25$

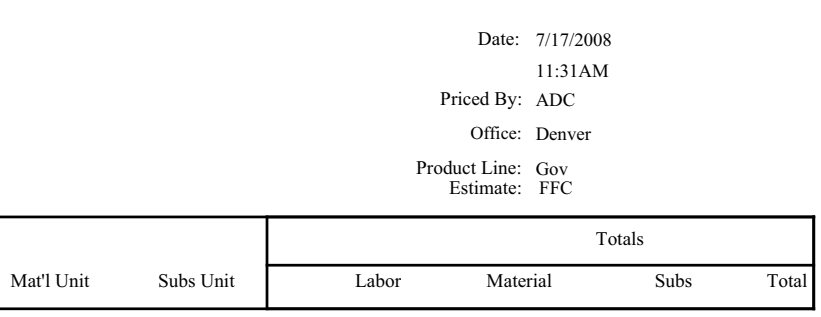


Client: INL

Project: FFC

Location: GREEN FIELD SITE HIP Option

URS

Date: $7 / 17 / 2008$

11:31AM

Account: 05 Structual Steel/Platework

Washington Division

Priced By: ADC

Office: Denver

Facility: Entire Estimate

Job No: $\quad 27989-325 \quad$ Rev No: 0

Educt Line: Gov
Estimate: FFC

\begin{tabular}{|c|c|c|c|c|c|c|c|c|c|c|c|c|c|}
\hline \multirow[b]{2}{*}{ Account No. } & \multirow[b]{2}{*}{ Description } & \multirow[b]{2}{*}{ Qty } & \multirow[b]{2}{*}{ UM } & \multirow{2}{*}{$\begin{array}{l}\text { Lab. } \\
\text { Fact. }\end{array}$} & \multicolumn{3}{|c|}{ Workhours } & \multirow[b]{2}{*}{ Mat'l Unit } & \multirow[b]{2}{*}{ Subs Unit } & \multicolumn{4}{|c|}{ Totals } \\
\hline & & & & & Per Unit & Total & Rate & & & Labor & Material & Subs & Total \\
\hline \multicolumn{2}{|c|}{20 \#/Inft Light Crane Steel-Painted } & 4.60 & ton & & & 162 & 91.25 & & & $\$ 14,782$ & $\$ 26,130$ & \$o & $\$ 40,912$ \\
\hline 050103 & LIGHT STEEL-PAINTED-Crane Support & 4.00 & ton & 1.20 & 33.30 & 133.20 & $\$ 91.25$ & $\$ 5,564.34$ & & $\$ 12,154$ & $\$ 22,257$ & $\$ 0$ & $\$ 34,411$ \\
\hline 050103 & $\begin{array}{l}\text { Minor Struct Steel (Girts, Purlins, Clips, } \\
\text { Gussets, stiffeners, etc) }\end{array}$ & 0.60 & ton & 1.20 & 48.00 & 28.80 & $\$ 91.25$ & $\$ 6,454.22$ & & $\$ 2,628$ & $\$ 3,873$ & $\$ 0$ & $\$ 6,500$ \\
\hline \multicolumn{2}{|c|}{$20 \# /$ Inft Light Crane Steel-Painted 25 ton cranes } & 5.75 & ton & & & 203 & 91.25 & & & $\$ 18,477$ & $\$ 32,662$ & $\$ 0$ & $\$ 51,140$ \\
\hline 050103 & LIGHT STEEL-PAINTED-Crane Support & 5.00 & ton & 1.20 & 33.30 & 166.50 & $\$ 91.25$ & $\$ 5,564.34$ & & $\$ 15,193$ & $\$ 27,822$ & $\$ 0$ & $\$ 43,014$ \\
\hline 050103 & $\begin{array}{l}\text { Minor Struct Steel (Girts, Purlins, Clips, } \\
\text { Gussets, stiffeners, etc) }\end{array}$ & 0.75 & ton & 1.20 & 48.00 & 36.00 & $\$ 91.25$ & $\$ 6,454.22$ & & $\$ 3,285$ & $\$ 4,841$ & $\$ 0$ & $\$ 8,126$ \\
\hline \multicolumn{2}{|c|}{$\begin{array}{l}\text { ¿20 \#/Inft Light Enclosure Support Steel-Painted Interior } \\
\text { Enclosure }\end{array}$} & 6.90 & ton & & & 243 & 91.25 & & & $\$ 22,173$ & $\$ 39,195$ & $\$ 0$ & $\$ 61,368$ \\
\hline 050103 & $\begin{array}{l}\text { LIGHT STEEL-PAINTED-BLDG Siding } \\
\text { Support }\end{array}$ & 6.00 & ton & 1.20 & 33.30 & 199.80 & $\$ 91.25$ & $\$ 5,564.34$ & & $\$ 18,231$ & $\$ 33,386$ & $\$ 0$ & $\$ 51,617$ \\
\hline 050103 & $\begin{array}{l}\text { Minor Struct Steel (Girts, Purlins, Clips, } \\
\text { Gussets, stiffeners, etc) }\end{array}$ & 0.90 & ton & 1.20 & 48.00 & 43.20 & $\$ 91.25$ & $\$ 6,454.22$ & & $\$ 3,942$ & $\$ 5,809$ & $\$ 0$ & $\$ 9,751$ \\
\hline \multicolumn{2}{|c|}{$20 \# /$ Inft Light Equip Suppt Steel-Painted Hoist Support HIP } & 0.35 & ton & & & 12 & 91.25 & & & $\$ 1,109$ & $\$ 1,960$ & \$o & $\$ 3,068$ \\
\hline 050103 & $\begin{array}{l}\text { LIGHT STEEL-PAINTED-EQUIP } \\
\text { SUPPORT }\end{array}$ & 0.30 & ton & 1.20 & 33.30 & 9.99 & $\$ 91.25$ & $\$ 5,564.34$ & & $\$ 912$ & $\$ 1,669$ & $\$ 0$ & $\$ 2,581$ \\
\hline 050103 & $\begin{array}{l}\text { Minor Struct Steel (Girts, Purlins, Clips, } \\
\text { Gussets, stiffeners, etc) }\end{array}$ & 0.05 & ton & 1.20 & 48.00 & 2.16 & $\$ 91.25$ & $\$ 6,454.22$ & & $\$ 197$ & $\$ 290$ & $\$ 0$ & $\$ 488$ \\
\hline \multicolumn{2}{|c|}{20 - 40 \#/Inft Medium Crane Steel-Painted } & 8.80 & ton & & & 229 & 91.25 & & & $\$ 20,914$ & $\$ 36,136$ & \$0 & $\$ 57,050$ \\
\hline 050104 & $\begin{array}{l}\text { MEDIUM STEEL-PAINTED-Crane } \\
\text { Support }\end{array}$ & 8.00 & ton & 1.20 & 23.85 & 190.80 & $\$ 91.25$ & $\$ 3,871.64$ & & $\$ 17,410$ & $\$ 30,973$ & $\$ 0$ & $\$ 48,383$ \\
\hline 050104 & $\begin{array}{l}\text { Minor Struct Steel (Girts, Purlins, Clips, } \\
\text { Gussets, stiffeners, etc) }\end{array}$ & 0.80 & ton & 1.20 & 48.00 & 38.40 & $\$ 91.25$ & $\$ 6,454.22$ & & $\$ 3,504$ & $\$ 5,163$ & $\$ 0$ & $\$ 8,667$ \\
\hline \multicolumn{2}{|c|}{0 - 40 \#/Inft Medium Crane Steel-Painted 25 ton cranes } & 22.00 & ton & & & 573 & 91.25 & & & $\$ 52,284$ & $\$ 90,341$ & \$o & $\$ 142,625$ \\
\hline 050104 & $\begin{array}{l}\text { MEDIUM STEEL-PAINTED-Crane } \\
\text { Support }\end{array}$ & 20.00 & ton & 1.20 & 23.85 & 477.00 & $\$ 91.25$ & $\$ 3,871.64$ & & $\$ 43,524$ & $\$ 77,433$ & $\$ 0$ & $\$ 120,957$ \\
\hline 050104 & $\begin{array}{l}\text { Minor Struct Steel (Girts, Purlins, Clips, } \\
\text { Gussets, stiffeners, etc) }\end{array}$ & 2.00 & ton & 1.20 & 48.00 & 96.00 & $\$ 91.25$ & $\$ 6,454.22$ & & $\$ 8,760$ & $\$ 12,908$ & $\$ 0$ & $\$ 21,668$ \\
\hline \multicolumn{2}{|c|}{20 - $40 \# /$ Inft Medium Equip Suppt Steel-Painted Hoist Support } & 2.09 & ton & & & 54 & 91.25 & & & $\$ 4,967$ & $\$ 8,582$ & \$o & $\$ 13,549$ \\
\hline 050104 & $\begin{array}{l}\text { MEDIUM STEEL-PAINTED-EQUIP } \\
\text { SUPPORT }\end{array}$ & 1.90 & ton & 1.20 & 23.85 & 45.32 & $\$ 91.25$ & $\$ 3,871.64$ & & $\$ 4,135$ & $\$ 7,356$ & $\$ 0$ & $\$ 11,491$ \\
\hline 050104 & $\begin{array}{l}\text { Minor Struct Steel (Girts, Purlins, Clips, } \\
\text { Gussets, stiffeners, etc) }\end{array}$ & 0.19 & ton & 1.20 & 48.00 & 9.12 & $\$ 91.25$ & $\$ 6,454.22$ & & $\$ 832$ & $\$ 1,226$ & $\$ 0$ & $\$ 2,058$ \\
\hline \multicolumn{2}{|c|}{ levated Slab on Metal Deck- by Volume } & 0.00 & ton & & & 233 & 91.25 & & & $\$ 21,286$ & $\$ 10,206$ & $\$ 0$ & $\$ 31,492$ \\
\hline 050503 & FLOOR DECK & $4,860.00$ & sqft & 1.20 & 0.05 & 233.28 & $\$ 91.25$ & $\$ 2.10$ & & $\$ 21,286$ & $\$ 10,206$ & $\$ 0$ & $\$ 31,492$ \\
\hline \multicolumn{2}{|c|}{ Elevated Slab on Metal Deck- by Volume HVAC Filter Floor } & 0.00 & ton & & & 213 & 91.25 & & & $\$ 19,394$ & $\$ 9,299$ & $\$ 0$ & $\$ 28,693$ \\
\hline 050503 & FLOOR DECK & $4,428.00$ & sqft & 1.20 & 0.05 & 212.54 & $\$ 91.25$ & $\$ 2.10$ & & $\$ 19,394$ & $\$ 9,299$ & $\$ 0$ & $\$ 28,693$ \\
\hline \multicolumn{2}{|c|}{ Elevated Slab on Metal Deck- by Volume Security BIdg Roof } & 0.00 & ton & & & 3 & 91.25 & & & $\$ 252$ & $\$ 121$ & $\$ 0$ & $\$ 372$ \\
\hline 050503 & FLOOR DECK & 57.46 & sqft & 1.20 & 0.05 & 2.76 & $\$ 91.25$ & $\$ 2.10$ & & $\$ 252$ & $\$ 121$ & $\$ 0$ & $\$ 372$ \\
\hline \multicolumn{2}{|l|}{ Grating HIP Area } & 0.00 & ton & & & 14 & 91.25 & & & $\$ 1,277$ & $\$ 2,572$ & \$0 & $\$ 3,849$ \\
\hline 050505 & METAL GRATING $11 / 4$ " Over Trenches & 200.00 & sqft & & 0.07 & 14.00 & $\$ 91.25$ & $\$ 12.86$ & & $\$ 1,277$ & $\$ 2,572$ & $\$ 0$ & $\$ 3,849$ \\
\hline
\end{tabular}


Client: INL

Project: FFC

URS

Washington Division

REEN FIELD SITE HIP Optio

Account: 05 Structual Steel/Platework

Facility: Entire Estimate

HIP Option

Job No:

27989-325

Rev No: 0
Date: 7/17/2008

11:31AM

Priced By: ADC

Office: Denver

Product Line: Gov
Estimate: FFC

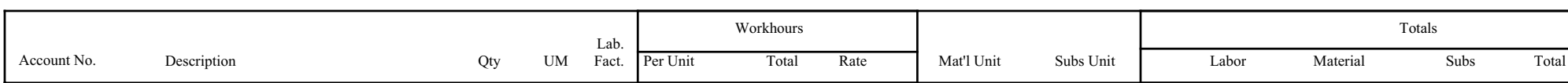

\begin{tabular}{|c|c|c|}
\hline \multirow[t]{4}{*}{ Steel Ratios: } & Enti & Estimate \\
\hline & 38.40 & Workhours/Ton \\
\hline & $\$ 5,095$ & Material Cost/Ton \\
\hline & $\$ 8,599$ & Total Cost/Ton \\
\hline
\end{tabular}

\begin{tabular}{|c|c|c|c|c|c|c|c|c|c|c|}
\hline Totals & $\begin{array}{l}\text { Structual Steel/Platework } \\
\text { Entire Estimate }\end{array}$ & 50 Tons & 38.40 & 1,939 & $\$ 91.25$ & $5,094.67$ & $\$ 176,914$ & $\$ 257,204$ & $\$ 0$ & $\$ 434,119$ \\
\hline
\end{tabular}


Client: INL

Project: FFC

Location: GREEN FIELD SITE HIP Option

Account: 06 Process Equipment

Facility: Entire Estimate

URS

Washington Division
Date: 7/17/2008

11:31AM

Priced By: ADC

Office: Denver

Product Line: Gov
Estimate: FFC

\begin{tabular}{|c|c|c|c|c|c|c|c|c|c|c|c|c|c|}
\hline \multirow[b]{2}{*}{ Account No. } & \multirow[b]{2}{*}{ Description } & \multirow[b]{2}{*}{ Qty } & \multirow[b]{2}{*}{ UM } & \multirow{2}{*}{$\begin{array}{l}\text { Lab. } \\
\text { Fact. }\end{array}$} & \multicolumn{3}{|c|}{ Workhours } & \multirow[b]{2}{*}{ Mat'l Unit } & \multirow[b]{2}{*}{ Subs Unit } & \multicolumn{4}{|c|}{ Totals } \\
\hline & & & & & Per Unit & Total & Rate & & & Labo & Material & & Total \\
\hline \multicolumn{2}{|l|}{ Swaging Item D } & & & & & 346 & 88.63 & & & $\$ 30,629$ & $\$ 1,743,000$ & \$o & $\$ 1,773,629$ \\
\hline 060101 & $\begin{array}{l}\text { Process Equipment-PROCESS EQ } \\
\text {-Swaging Equipment }\end{array}$ & 2 & each & 1.20 & 172.80 & 345.60 & $\$ 88.63$ & $\$ 800,000.00$ & & $\$ 30,629$ & $\$ 1,600,000$ & $\$ 0$ & $\$ 1,630,629$ \\
\hline 060199 & $\begin{array}{l}\text { Process Equipment-DESIGN } \\
\text { DEVELOPMENT ALLOW (DDA) }\end{array}$ & 0.04 & $\%$ & & & & & $\$ 1,600,000.00$ & & $\$ 0$ & $\$ 64,000$ & $\$ 0$ & $\$ 64,000$ \\
\hline 068900 & Process Equipment-VENDOR REPS & 10.00 & days & & & & & $\$ 1,500.00$ & & $\$ 0$ & $\$ 15,000$ & $\$ 0$ & $\$ 15,000$ \\
\hline 069900 & Process Equipment-BID CONDITIONING & 0.04 & $\%$ & & & & & $\$ 1,600,000.00$ & & $\$ 0$ & $\$ 64,000$ & $\$ 0$ & $\$ 64,000$ \\
\hline \multicolumn{2}{|c|}{ ATR Milling Item $\mathbf{J}$} & & & & & 130 & 88.63 & & & $\$ 11,486$ & $\$ 332,700$ & $\$ 0$ & $\$ 344,186$ \\
\hline 060101 & $\begin{array}{l}\text { Process Equipment-PROCESS EQ Milling } \\
\text { Model VR } 11\end{array}$ & 1 & each & 1.20 & 129.60 & 129.60 & $\$ 88.63$ & $\$ 300,000.00$ & & $\$ 11,486$ & $\$ 300,000$ & $\$ 0$ & $\$ 311,486$ \\
\hline 060199 & $\begin{array}{l}\text { Process Equipment-DESIGN } \\
\text { DEVELOPMENT ALLOW (DDA) }\end{array}$ & 0.04 & $\%$ & & & & & $\$ 300,000.00$ & & $\$ 0$ & $\$ 13,200$ & $\$ 0$ & $\$ 13,200$ \\
\hline 068900 & Process Equipment-VENDOR REPS & 5.00 & days & & & & & $\$ 1,500.00$ & & $\$ 0$ & $\$ 7,500$ & $\$ 0$ & $\$ 7,500$ \\
\hline 069900 & Process Equipment-BID CONDITIONING & 0.04 & $\%$ & & & & & $\$ 300,000.00$ & & $\$ 0$ & $\$ 12,000$ & $\$ 0$ & $\$ 12,000$ \\
\hline \multicolumn{2}{|l|}{ Autoclave Item P } & & & & & 19 & 88.63 & & & $\$ 1,702$ & $\$ 81,600$ & $\$ 0$ & $\$ 83,302$ \\
\hline 060126 & $\begin{array}{l}\text { Process Equipment-PROCESS EQ } \\
\text { Autoclave Item P }\end{array}$ & 1 & each & 1.20 & 19.20 & 19.20 & $\$ 88.63$ & $\$ 80,000.00$ & & $\$ 1,702$ & $\$ 80,000$ & $\$ 0$ & $\$ 81,702$ \\
\hline 060199 & $\begin{array}{l}\text { Process Equipment-DESIGN } \\
\text { DEVELOPMENT ALLOW (DDA) }\end{array}$ & 0.02 & $\%$ & 1.20 & & & & $\$ 80,000.00$ & & $\$ 0$ & $\$ 1,600$ & $\$ 0$ & $\$ 1,600$ \\
\hline \multicolumn{2}{|c|}{ Conv, Elev, \& Process Handling } & & & & & 230 & 88.63 & & & $\$ 20,419$ & $\$ 64,500$ & \$0 & $\$ 84,919$ \\
\hline 060116 & $\begin{array}{l}\text { Casting Area-PROCESS EQ Mtl Pass Thru } \\
\text { Casting \& Shop to Storage }\end{array}$ & 2 & each & 1.20 & 115.20 & 230.40 & $\$ 88.63$ & $\$ 30,000.00$ & & $\$ 20,419$ & $\$ 60,000$ & $\$ 0$ & $\$ 80,419$ \\
\hline 060199 & $\begin{array}{l}\text { Casting Area-DESIGN DEVELOPMENT } \\
\text { ALLOW (DDA) }\end{array}$ & 0.05 & $\%$ & & & & & $\$ 30,000.00$ & & $\$ 0$ & $\$ 1,500$ & $\$ 0$ & $\$ 1,500$ \\
\hline 068900 & Casting Area-VENDOR REPS & 2.00 & days & & & & & $\$ 1,500.00$ & & $\$ 0$ & $\$ 3,000$ & $\$ 0$ & $\$ 3,000$ \\
\hline \multicolumn{2}{|c|}{ Element Wash Item N } & & & & & 38 & 88.63 & & & $\$ 3,403$ & $\$ 21,600$ & \$o & $\$ 25,003$ \\
\hline 060101 & $\begin{array}{l}\text { Process Equipment-PROCESS EQ Element } \\
\text { Wash }\end{array}$ & 1 & each & 1.20 & 38.40 & 38.40 & $\$ 88.63$ & $\$ 20,000.00$ & & $\$ 3,403$ & $\$ 20,000$ & $\$ 0$ & $\$ 23,403$ \\
\hline 060199 & $\begin{array}{l}\text { Process Equipment-DESIGN } \\
\text { DEVELOPMENT ALLOW (DDA) }\end{array}$ & 0.04 & $\%$ & & & & & $\$ 20,000.00$ & & $\$ 0$ & $\$ 800$ & $\$ 0$ & $\$ 800$ \\
\hline 069900 & Process Equipment-BID CONDITIONING & 0.04 & $\%$ & & & & & $\$ 20,000.00$ & & $\$ 0$ & $\$ 800$ & $\$ 0$ & $\$ 800$ \\
\hline \multicolumn{2}{|c|}{ Firm Elev, \& Process Handling Motion Table } & & & & & 346 & 88.63 & & & $\$ 30,629$ & $\$ 313,500$ & \$o & $\$ 344,129$ \\
\hline 060116 & $\begin{array}{l}\text { Process Equipment-PROCESS EQ Motion } \\
\text { Table }\end{array}$ & 2 & each & 1.20 & 172.80 & 345.60 & $\$ 88.63$ & $\$ 150,000.00$ & & $\$ 30,629$ & $\$ 300,000$ & $\$ 0$ & $\$ 330,629$ \\
\hline 060199 & $\begin{array}{l}\text { Process Equipment-DESIGN } \\
\text { DEVELOPMENT ALLOW (DDA) }\end{array}$ & 0.05 & $\%$ & & & & & $\$ 150,000.00$ & & $\$ 0$ & $\$ 7,500$ & $\$ 0$ & $\$ 7,500$ \\
\hline 068900 & Process Equipment-VENDOR REPS & 4.00 & days & & & & & $\$ 1,500.00$ & & $\$ 0$ & $\$ 6,000$ & $\$ 0$ & $\$ 6,000$ \\
\hline \multicolumn{2}{|c|}{ Firm Heat Transfer Eq RF Heating \& Cooling Coils } & & & & & 288 & 88.63 & & & $\$ 25,524$ & $\$ 2,225,500$ & $\$ 0$ & $\$ 2,251,024$ \\
\hline 060126 & $\begin{array}{l}\text { Casting Area-PROCESS EQ RF Heating \& } \\
\text { Cooling Coils }\end{array}$ & 2 & each & 1.20 & 144.00 & 288.00 & $\$ 88.63$ & $\$ 1,000,000.00$ & & $\$ 25,524$ & $\$ 2,000,000$ & $\$ 0$ & $\$ 2,025,524$ \\
\hline 060126 & Casting Area-PROCESS EQ Crucibles & 20 & each & & & & & $\$ 2,500.00$ & & $\$ 0$ & $\$ 50,000$ & $\$ 0$ & $\$ 50,000$ \\
\hline 060126 & Casting Area-PROCESS EQ Casting Molds & 32 & each & & & & & $\$ 4,000.00$ & & $\$ 0$ & $\$ 128,000$ & $\$ 0$ & $\$ 128,000$ \\
\hline
\end{tabular}


Client: INL

Project: FFC

Location: GREEN FIELD SITE HIP Option

Account: 06 Process Equipment

Facility: Entire Estimate

URS

Washington Division
Date: 7/17/2008

11:31AM

Priced By: ADC

Office: Denver

Product Line: Gov
Estimate: FFC

\begin{tabular}{|c|c|c|c|c|c|c|c|c|c|c|c|c|c|}
\hline \multirow[b]{2}{*}{ Account No. } & \multirow[b]{2}{*}{ Description } & \multirow[b]{2}{*}{ Qty } & \multirow[b]{2}{*}{ UM } & \multirow{2}{*}{$\begin{array}{l}\text { Lab. } \\
\text { Fact. }\end{array}$} & \multicolumn{3}{|c|}{ Workhours } & \multirow[b]{2}{*}{ Mat'l Unit } & \multirow[b]{2}{*}{ Subs Unit } & \multicolumn{4}{|c|}{ Totals } \\
\hline & & & & & Per Unit & Total & Rate & & & Labor & Material & Subs & Total \\
\hline 060199 & $\begin{array}{l}\text { Casting Area-DESIGN DEVELOPMENT } \\
\text { ALLOW (DDA) }\end{array}$ & 0.02 & $\%$ & & & & & $\$ 2,000,000.00$ & & $\$ 0$ & $\$ 40,000$ & $\$ 0$ & $\$ 40,000$ \\
\hline 068900 & Casting Area-VENDOR REPS & 5.00 & days & & & & & $\$ 1,500.00$ & & $\$ 0$ & $\$ 7,500$ & $\$ 0$ & $\$ 7,500$ \\
\hline \multicolumn{2}{|c|}{ irm Price-Al Cladding Cleaning Station Item 29} & & & & & 38 & $\mathbf{8 8 . 6 3}$ & & & $\$ 3,403$ & $\$ 108,000$ & $\$ 0$ & $\$ 111,403$ \\
\hline 060101 & $\begin{array}{l}\text { Process Equipment-PROCESS EQ Al } \\
\text { Cladding Cleaning Station }\end{array}$ & 1 & each & 1.20 & 38.40 & 38.40 & $\$ 88.63$ & $\$ 100,000.00$ & & $\$ 3,403$ & $\$ 100,000$ & $\$ 0$ & $\$ 103,403$ \\
\hline 060199 & $\begin{array}{l}\text { Process Equipment-DESIGN } \\
\text { DEVELOPMENT ALLOW (DDA) }\end{array}$ & 0.04 & $\%$ & & & & & $\$ 100,000.00$ & & $\$ 0$ & $\$ 4,000$ & $\$ 0$ & $\$ 4,000$ \\
\hline 069900 & Process Equipment-BID CONDITIONING & 0.04 & $\%$ & & & & & $\$ 100,000.00$ & & $\$ 0$ & $\$ 4,000$ & $\$ 0$ & $\$ 4,000$ \\
\hline \multicolumn{2}{|c|}{ irm Price-AL Cladding Washing Item 27} & & & & & 19 & 88.63 & & & $\$ 1,702$ & $\$ 10,800$ & \$o & $\$ 12,502$ \\
\hline 060101 & $\begin{array}{l}\text { Process Equipment-PROCESS EQ } \\
\text { Washing Station }\end{array}$ & 1 & each & 1.20 & 19.20 & 19.20 & $\$ 88.63$ & $\$ 10,000.00$ & & $\$ 1,702$ & $\$ 10,000$ & $\$ 0$ & $\$ 11,702$ \\
\hline 060199 & $\begin{array}{l}\text { Process Equipment-DESIGN } \\
\text { DEVELOPMENT ALLOW (DDA) }\end{array}$ & 0.04 & $\%$ & & & & & $\$ 10,000.00$ & & $\$ 0$ & $\$ 400$ & $\$ 0$ & $\$ 400$ \\
\hline 069900 & Process Equipment-BID CONDITIONING & 0.04 & $\%$ & & & & & $\$ 10,000.00$ & & $\$ 0$ & $\$ 400$ & $\$ 0$ & $\$ 400$ \\
\hline \multicolumn{2}{|c|}{ irm Price-Annealing Furnace 24} & & & & & 43 & 88.63 & & & $\$ 3,829$ & $\$ 40,800$ & \$o & $\$ 44,629$ \\
\hline 060126 & $\begin{array}{l}\text { Process Equipment-PROCESS EQ } \\
\text { Annealing Furnace }\end{array}$ & 1 & each & 1.20 & 43.20 & 43.20 & $\$ 88.63$ & $\$ 40,000.00$ & & $\$ 3,829$ & $\$ 40,000$ & $\$ 0$ & $\$ 43,829$ \\
\hline 060199 & $\begin{array}{l}\text { Process Equipment-DESIGN } \\
\text { DEVELOPMENT ALLOW (DDA) }\end{array}$ & 0.02 & $\%$ & & & & & $\$ 40,000.00$ & & $\$ 0$ & $\$ 800$ & $\$ 0$ & $\$ 800$ \\
\hline \multicolumn{2}{|c|}{ irm Price-Argon Supply \& Distrib Sys } & & & & & 38 & $\mathbf{8 8 . 6 3}$ & & & $\$ 3,403$ & $\$ 42,500$ & $\$ 0$ & $\$ 45,903$ \\
\hline 060150 & $\begin{array}{l}\text { Casting Area-PROCESS EQ Argon } \\
\text { Recycle System }\end{array}$ & 1 & each & 1.20 & 38.40 & 38.40 & $\$ 88.63$ & $\$ 40,000.00$ & & $\$ 3,403$ & $\$ 40,000$ & $\$ 0$ & $\$ 43,403$ \\
\hline 060199 & $\begin{array}{l}\text { Casting Area-DESIGN DEVELOPMENT } \\
\text { ALLOW (DDA) }\end{array}$ & 0.03 & $\%$ & 1.20 & & & & $\$ 40,000.00$ & & $\$ 0$ & $\$ 1,000$ & $\$ 0$ & $\$ 1,000$ \\
\hline 068900 & Casting Area-VENDOR REPS & 1.00 & days & 1.20 & & & & $\$ 1,500.00$ & & $\$ 0$ & $\$ 1,500$ & $\$ 0$ & $\$ 1,500$ \\
\hline \multicolumn{2}{|c|}{ irm Price-Argon Supply \& Distrib Sys for HIP Sys } & & & & & 38 & 88.63 & & & $\$ 3,403$ & $\$ 27,125$ & \$o & $\$ 30,528$ \\
\hline 060150 & $\begin{array}{l}\text { HIP Area-PROCESS EQ Argon Recycle } \\
\text { System }\end{array}$ & 1 & each & 1.20 & 38.40 & 38.40 & $\$ 88.63$ & $\$ 25,000.00$ & & $\$ 3,403$ & $\$ 25,000$ & $\$ 0$ & $\$ 28,403$ \\
\hline 060199 & $\begin{array}{l}\text { HIP Area-DESIGN DEVELOPMENT } \\
\text { ALLOW (DDA) SAMPLE-FIRM PRICE } \\
\text { MATERIAL }\end{array}$ & 0.03 & $\%$ & 1.20 & & & & $\$ 25,000.00$ & & $\$ 0$ & $\$ 625$ & $\$ 0$ & $\$ 625$ \\
\hline 068900 & $\begin{array}{l}\text { HIP Area-VENDOR REPS SAMPLE-FIRM } \\
\text { PRICE MATERIAL }\end{array}$ & 1.00 & days & 1.20 & & & & $\$ 1,500.00$ & & $\$ 0$ & $\$ 1,500$ & $\$ 0$ & $\$ 1,500$ \\
\hline \multicolumn{2}{|c|}{ irm Price-Canning Machines Item 33} & & & & & 77 & 88.63 & & & $\$ 6,806$ & $\$ 165,000$ & \$0 & $\$ 171,806$ \\
\hline 060101 & $\begin{array}{l}\text { Process Equipment-PROCESS EQ Canning } \\
\text { Machine } 33\end{array}$ & 1 & each & 1.20 & 76.80 & 76.80 & $\$ 88.63$ & $\$ 150,000.00$ & & $\$ 6,806$ & $\$ 150,000$ & $\$ 0$ & $\$ 156,806$ \\
\hline 060199 & $\begin{array}{l}\text { Process Equipment-DESIGN } \\
\text { DEVELOPMENT ALLOW (DDA) }\end{array}$ & 0.04 & $\%$ & & & & & $\$ 150,000.00$ & & $\$ 0$ & $\$ 6,000$ & $\$ 0$ & $\$ 6,000$ \\
\hline 068900 & Process Equipment-VENDOR REPS & 2.00 & days & & & & & $\$ 1,500.00$ & & $\$ 0$ & $\$ 3,000$ & $\$ 0$ & $\$ 3,000$ \\
\hline 069900 & Process Equipment-BID CONDITIONING & 0.04 & $\%$ & & & & & $\$ 150,000.00$ & & $\$ 0$ & $\$ 6,000$ & $\$ 0$ & $\$ 6,000$ \\
\hline \multicolumn{3}{|c|}{ irm Price-Conv, Cranes 25 ton Cap Units } & & & & 192 & 88.63 & & & $\$ 17,016$ & $\$ 136,620$ & \$0 & $\$ 153,636$ \\
\hline 060116 & Mechanical Equipment-PROCESS EQ & 2 & each & 1.20 & 96.00 & 192.00 & $\$ 88.63$ & $\$ 62,200.00$ & & $\$ 17,016$ & $\$ 124,400$ & $\$ 0$ & $\$ 141,416$ \\
\hline
\end{tabular}


Client: INL

Project: FFC

Location: GREEN FIELD SITE HIP Option

Account: 06 Process Equipment

Facility: Entire Estimate

URS

Washington Division
Date: 7/17/2008

11:31AM

Priced By: ADC

Office: Denver

Product Line: Gov
Estimate: FFC

\begin{tabular}{|c|c|c|c|c|c|c|c|c|c|c|c|c|c|}
\hline \multirow[b]{2}{*}{ Account No. } & \multirow[b]{2}{*}{ Description } & \multirow[b]{2}{*}{ Qty } & \multirow[b]{2}{*}{ UM } & \multirow{2}{*}{$\begin{array}{l}\text { Lab. } \\
\text { Fact. }\end{array}$} & \multicolumn{3}{|c|}{ Workhours } & \multirow[b]{2}{*}{ Mat'l Unit } & \multirow[b]{2}{*}{ Subs Unit } & \multicolumn{4}{|c|}{ Totals } \\
\hline & & & & & Per Unit & Total & 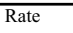 & & & Labor & Material & Subs & Total \\
\hline 060199 & $\begin{array}{l}\text { Mechanical Equipment-DESIGN } \\
\text { DEVELOPMENT ALLOW (DDA) }\end{array}$ & 0.05 & $\%$ & & & & & $\$ 124,400.00$ & & $\$ 0$ & $\$ 6,220$ & $\$ 0$ & $\$ 6,220$ \\
\hline 068900 & Mechanical Equipment-VENDOR REPS & 4.00 & days & & & & & $\$ 1,500.00$ & & $\$ 0$ & $\$ 6,000$ & $\$ 0$ & $\$ 6,000$ \\
\hline m Price-Conv, & nes 5.0 ton Cap Monorail 2Units & & & & & 77 & 88.63 & & & $\$ 6,806$ & $\$ 39,900$ & \$0 & $\$ 46,706$ \\
\hline 060116 & $\begin{array}{l}\text { Mechanical Equipment-PROCESS EQ } \\
\text { Cranes } 5.0 \text { ton cal Monorail Type }\end{array}$ & 2 & each & 1.20 & 38.40 & 76.80 & $\$ 88.63$ & $\$ 18,000.00$ & & $\$ 6,806$ & $\$ 36,000$ & $\$ 0$ & $\$ 42,806$ \\
\hline 060199 & $\begin{array}{l}\text { Mechanical Equipment-DESIGN } \\
\text { DEVELOPMENT ALLOW (DDA) }\end{array}$ & 0.05 & $\%$ & 1.20 & & & & $\$ 18,000.00$ & & $\$ 0$ & $\$ 900$ & $\$ 0$ & $\$ 900$ \\
\hline 068900 & Mechanical Equipment-VENDOR REPS & 2.00 & days & 1.20 & & & & $\$ 1,500.00$ & & $\$ 0$ & $\$ 3,000$ & $\$ 0$ & $\$ 3,000$ \\
\hline m Price-Conv, $\mathrm{E}$ & , \& Process Handling & & & & & 115 & 88.63 & & & $\$ 10,210$ & $\$ 34,500$ & \$o & $\$ 44,710$ \\
\hline 060116 & $\begin{array}{l}\text { HIP Area-PROCESS EQ Mtl Pass Thru to } \\
\text { HIP Process }\end{array}$ & 1 & each & 1.20 & 115.20 & 115.20 & $\$ 88.63$ & $\$ 30,000.00$ & & $\$ 10,210$ & $\$ 30,000$ & $\$ 0$ & $\$ 40,210$ \\
\hline 060199 & $\begin{array}{l}\text { HIP Area-DESIGN DEVELOPMENT } \\
\text { ALLOW (DDA) SAMPLE-FIRM PRICE } \\
\text { MATERIAL }\end{array}$ & 0.05 & $\%$ & 1.20 & & & & $\$ 30,000.00$ & & $\$ 0$ & $\$ 1,500$ & $\$ 0$ & $\$ 1,500$ \\
\hline 068900 & $\begin{array}{l}\text { HIP Area-VENDOR REPS SAMPLE-FIRM } \\
\text { PRICE MATERIAL }\end{array}$ & 2.00 & days & 1.20 & & & & $\$ 1,500.00$ & & $\$ 0$ & $\$ 3,000$ & $\$ 0$ & $\$ 3,000$ \\
\hline m Price-EB Can & ssy Station HIP Area Item 1 & & & & & 19 & 88.63 & & & $\$ 1,702$ & $\$ 13,400$ & \$o & $\$ 15,102$ \\
\hline 060101 & $\begin{array}{l}\text { HIP Area-PROCESS EQ Canning Machine } \\
33\end{array}$ & 1 & each & 1.20 & 19.20 & 19.20 & $\$ 88.63$ & $\$ 10,000.00$ & & $\$ 1,702$ & $\$ 10,000$ & $\$ 0$ & $\$ 11,702$ \\
\hline 060199 & $\begin{array}{l}\text { HIP Area-DESIGN DEVELOPMENT } \\
\text { ALLOW (DDA) SAMPLE-FIRM PRICE } \\
\text { MATERIAL }\end{array}$ & 0.04 & $\%$ & & & & & $\$ 10,000.00$ & & $\$ 0$ & $\$ 400$ & $\$ 0$ & $\$ 400$ \\
\hline 068900 & $\begin{array}{l}\text { HIP Area-VENDOR REPS SAMPLE-FIRM } \\
\text { PRICE MATERIAL }\end{array}$ & 2.00 & days & & & & & $\$ 1,500.00$ & & $\$ 0$ & $\$ 3,000$ & $\$ 0$ & $\$ 3,000$ \\
\hline m Price-Foil Cle & ing Station Item 30 & & & & & 38 & 88.63 & & & $\$ 3,403$ & $\$ 108,000$ & $\$ 0$ & $\$ 111,403$ \\
\hline 060101 & $\begin{array}{l}\text { Process Equipment-PROCESS EQ Al } \\
\text { Cladding Cleaning Station }\end{array}$ & 1 & each & 1.20 & 38.40 & 38.40 & $\$ 88.63$ & $\$ 100,000.00$ & & $\$ 3,403$ & $\$ 100,000$ & $\$ 0$ & $\$ 103,403$ \\
\hline 060199 & $\begin{array}{l}\text { Process Equipment-DESIGN } \\
\text { DEVELOPMENT ALLOW (DDA) }\end{array}$ & 0.04 & $\%$ & & & & & $\$ 100,000.00$ & & $\$ 0$ & $\$ 4,000$ & $\$ 0$ & $\$ 4,000$ \\
\hline 069900 & Process Equipment-BID CONDITIONING & 0.04 & $\%$ & & & & & $\$ 100,000.00$ & & $\$ 0$ & $\$ 4,000$ & $\$ 0$ & $\$ 4,000$ \\
\hline m Price-Hoist $\mathrm{M}$ & orail 25 Ton cap & & & & & 48 & 88.63 & & & $\$ 4,254$ & $\$ 68,700$ & $\$ 0$ & $\$ 72,954$ \\
\hline 060116 & $\begin{array}{l}\text { HIP Area-PROCESS EQ SAMPLE-FIRM } \\
\text { PRICE MATERIAL }\end{array}$ & 1 & each & 1.20 & 48.00 & 48.00 & $\$ 88.63$ & $\$ 64,000.00$ & & $\$ 4,254$ & $\$ 64,000$ & $\$ 0$ & $\$ 68,254$ \\
\hline 060199 & $\begin{array}{l}\text { HIP Area-DESIGN DEVELOPMENT } \\
\text { ALLOW (DDA) SAMPLE-FIRM PRICE } \\
\text { MATERIAL }\end{array}$ & 0.05 & $\%$ & 1.20 & & & & $\$ 64,000.00$ & & $\$ 0$ & $\$ 3,200$ & $\$ 0$ & $\$ 3,200$ \\
\hline 068900 & $\begin{array}{l}\text { HIP Area-VENDOR REPS SAMPLE-FIRM } \\
\text { PRICE MATERIAL }\end{array}$ & 1.00 & days & 1.20 & & & & $\$ 1,500.00$ & & $\$ 0$ & $\$ 1,500$ & $\$ 0$ & $\$ 1,500$ \\
\hline m Price-Hydrau & Press Item 20 & & & & & 29 & 88.63 & & & $\$ 2,552$ & $\$ 34,500$ & $\$ 0$ & $\$ 37,052$ \\
\hline 060101 & $\begin{array}{l}\text { Process Equipment-PROCESS EQ } \\
\text { Hydraulic Press } 20\end{array}$ & 1 & each & 1.20 & 28.80 & 28.80 & $\$ 88.63$ & $\$ 25,000.00$ & & $\$ 2,552$ & $\$ 25,000$ & $\$ 0$ & $\$ 27,552$ \\
\hline 060199 & $\begin{array}{l}\text { Process Equipment-DESIGN } \\
\text { DEVELOPMENT ALLOW (DDA) }\end{array}$ & 0.04 & $\%$ & & & & & $\$ 25,000.00$ & & $\$ 0$ & $\$ 1,000$ & $\$ 0$ & $\$ 1,000$ \\
\hline 068900 & Process Equipment-VENDOR REPS & 5.00 & days & & & & & $\$ 1,500.00$ & & $\$ 0$ & $\$ 7,500$ & $\$ 0$ & $\$ 7,500$ \\
\hline 069900 & Process Equipment-BID CONDITIONING & 0.04 & $\%$ & & & & & $\$ 25,000.00$ & & $\$ 0$ & $\$ 1,000$ & $\$ 0$ & $\$ 1,000$ \\
\hline
\end{tabular}


Client: INL

Project: FFC

Location: GREEN FIELD SITE HIP Option

Account: 06 Process Equipment

URS

Date: $7 / 17 / 2008$

11:31AM

Washington Division

Priced By: ADC

Office: Denver

Facility: Entire Estimate

Job No: $\quad 27989-325 \quad$ Rev No: 0

Product Line: Gov
Estimate: FFC

\begin{tabular}{|c|c|c|c|c|c|c|c|c|c|c|c|c|c|}
\hline \multirow[b]{2}{*}{ Account No. } & \multirow[b]{2}{*}{ Description } & \multirow[b]{2}{*}{ Qty } & \multirow[b]{2}{*}{ UM } & \multirow{2}{*}{$\begin{array}{l}\text { Lab. } \\
\text { Fact. }\end{array}$} & \multicolumn{3}{|c|}{ Workhours } & \multirow[b]{2}{*}{ Mat'l Unit } & \multirow[b]{2}{*}{ Subs Unit } & \multicolumn{4}{|c|}{ Totals } \\
\hline & & & & & Per Unit & Total & Rate & & & Labor & Material & Subs & Total \\
\hline \multicolumn{2}{|c|}{ irm Price-Hydraulic Shear 23} & & & & & 154 & 88.63 & & & $\$ 13,613$ & $\$ 120,000$ & \$0 & $\$ 133,613$ \\
\hline 060101 & $\begin{array}{l}\text { Process Equipment-PROCESS EQ } \\
\text { Hydraulic Shear }\end{array}$ & 4 & each & 1.20 & 38.40 & 153.60 & $\$ 88.63$ & $\$ 25,000.00$ & & $\$ 13,613$ & $\$ 100,000$ & $\$ 0$ & $\$ 113,613$ \\
\hline 060199 & $\begin{array}{l}\text { Process Equipment-DESIGN } \\
\text { DEVELOPMENT ALLOW (DDA) }\end{array}$ & 0.04 & $\%$ & & & & & $\$ 100,000.00$ & & $\$ 0$ & $\$ 4,000$ & $\$ 0$ & $\$ 4,000$ \\
\hline 068900 & $\begin{array}{l}\text { Process Equipment-VENDOR REPS } \\
\text { SAMPLE-FIRM PRICE MATERIAL }\end{array}$ & 8.00 & days & & & & & $\$ 1,500.00$ & & $\$ 0$ & $\$ 12,000$ & $\$ 0$ & $\$ 12,000$ \\
\hline 069900 & Process Equipment-BID CONDITIONING & 0.04 & $\%$ & & & & & $\$ 100,000.00$ & & $\$ 0$ & $\$ 4,000$ & $\$ 0$ & $\$ 4,000$ \\
\hline \multicolumn{2}{|c|}{ irm Price-Ingot Cleaning Station Item 32} & & & & & 38 & 88.63 & & & $\$ 3,403$ & $\$ 86,400$ & $\$ 0$ & $\$ 89,803$ \\
\hline 060101 & $\begin{array}{l}\text { Process Equipment-PROCESS EQ Ingot } \\
\text { Cleaning Station }\end{array}$ & 1 & each & 1.20 & 38.40 & 38.40 & $\$ 88.63$ & $\$ 80,000.00$ & & $\$ 3,403$ & $\$ 80,000$ & $\$ 0$ & $\$ 83,403$ \\
\hline 060199 & $\begin{array}{l}\text { Process Equipment-DESIGN } \\
\text { DEVELOPMENT ALLOW (DDA) }\end{array}$ & 0.04 & $\%$ & & & & & $\$ 80,000.00$ & & $\$ 0$ & $\$ 3,200$ & $\$ 0$ & $\$ 3,200$ \\
\hline 069900 & Process Equipment-BID CONDITIONING & 0.04 & $\%$ & & & & & $\$ 80,000.00$ & & $\$ 0$ & $\$ 3,200$ & $\$ 0$ & $\$ 3,200$ \\
\hline \multicolumn{2}{|c|}{ irm Price-Inspection Tables Item K } & & & & & 96 & 88.63 & & & $\$ 8,508$ & $\$ 17,280$ & $\$ 0$ & $\$ 25,788$ \\
\hline 060101 & $\begin{array}{l}\text { Process Equipment-PROCESS EQ } \\
\text { Inspection Tables }\end{array}$ & 5 & each & 1.20 & 19.20 & 96.00 & $\$ 88.63$ & $\$ 3,200.00$ & & $\$ 8,508$ & $\$ 16,000$ & $\$ 0$ & $\$ 24,508$ \\
\hline 060199 & $\begin{array}{l}\text { Process Equipment-DESIGN } \\
\text { DEVELOPMENT ALLOW (DDA) }\end{array}$ & 0.04 & $\%$ & 1.20 & & & & $\$ 16,000.00$ & & $\$ 0$ & $\$ 640$ & $\$ 0$ & $\$ 640$ \\
\hline 069900 & Process Equipment-BID CONDITIONING & 0.04 & $\%$ & 1.20 & & & & $\$ 16,000.00$ & & $\$ 0$ & $\$ 640$ & $\$ 0$ & $\$ 640$ \\
\hline \multicolumn{2}{|c|}{ irm Price-Jib Cranes } & & & & & 58 & 88.63 & & & $\$ 5,105$ & $\$ 32,250$ & \$o & $\$ 37,355$ \\
\hline 060116 & Casting Area-PROCESS EQ Jib Cranes & 2 & each & 1.20 & 28.80 & 57.60 & $\$ 88.63$ & $\$ 15,000.00$ & & $\$ 5,105$ & $\$ 30,000$ & $\$ 0$ & $\$ 35,105$ \\
\hline 060199 & $\begin{array}{l}\text { Casting Area-DESIGN DEVELOPMENT } \\
\text { ALLOW (DDA) }\end{array}$ & 0.05 & $\%$ & & & & & $\$ 15,000.00$ & & $\$ 0$ & $\$ 750$ & $\$ 0$ & $\$ 750$ \\
\hline 068900 & Casting Area-VENDOR REPS & 1.00 & days & & & & & $\$ 1,500.00$ & & $\$ 0$ & $\$ 1,500$ & $\$ 0$ & $\$ 1,500$ \\
\hline \multicolumn{2}{|c|}{ irm Price-Marking Station Item 28} & & & & & 19 & 88.63 & & & $\$ 1,702$ & $\$ 12,960$ & \$0 & $\$ 14,662$ \\
\hline 060101 & $\begin{array}{l}\text { Process Equipment-PROCESS EQ } \\
\text { Marking Station }\end{array}$ & 1 & each & 1.20 & 19.20 & 19.20 & $\$ 88.63$ & $\$ 12,000.00$ & & $\$ 1,702$ & $\$ 12,000$ & $\$ 0$ & $\$ 13,702$ \\
\hline 060199 & $\begin{array}{l}\text { Process Equipment-DESIGN } \\
\text { DEVELOPMENT ALLOW (DDA) }\end{array}$ & 0.04 & $\%$ & & & & & $\$ 12,000.00$ & & $\$ 0$ & $\$ 480$ & $\$ 0$ & $\$ 480$ \\
\hline 069900 & Process Equipment-BID CONDITIONING & 0.04 & $\%$ & & & & & $\$ 12,000.00$ & & $\$ 0$ & $\$ 480$ & $\$ 0$ & $\$ 480$ \\
\hline \multicolumn{2}{|c|}{ irm Price-Milling Machines Salt Removal Item 31} & & & & & 43 & 88.63 & & & $\$ 3,829$ & $\$ 54,000$ & $\$ 0$ & $\$ 57,829$ \\
\hline 060101 & $\begin{array}{l}\text { Process Equipment-PROCESS EQ Milling } \\
\text { Machines } 31\end{array}$ & 1 & each & 1.20 & 43.20 & 43.20 & $\$ 88.63$ & $\$ 50,000.00$ & & $\$ 3,829$ & $\$ 50,000$ & $\$ 0$ & $\$ 53,829$ \\
\hline 060199 & $\begin{array}{l}\text { Process Equipment-DESIGN } \\
\text { DEVELOPMENT ALLOW (DDA) }\end{array}$ & 0.04 & $\%$ & & & & & $\$ 50,000.00$ & & $\$ 0$ & $\$ 2,000$ & $\$ 0$ & $\$ 2,000$ \\
\hline 069900 & Process Equipment-BID CONDITIONING & 0.04 & $\%$ & & & & & $\$ 50,000.00$ & & $\$ 0$ & $\$ 2,000$ & $\$ 0$ & $\$ 2,000$ \\
\hline \multicolumn{2}{|c|}{ irm Price-Milling Machines Surface Finish 21} & & & & & 480 & 88.63 & & & $\$ 42,540$ & $\$ 979,500$ & \$o & $\$ 1,022,040$ \\
\hline 060101 & $\begin{array}{l}\text { Process Equipment-PROCESS EQ Milling } \\
\text { Machines } 21\end{array}$ & 5 & each & 1.20 & 96.00 & 480.00 & $\$ 88.63$ & $\$ 180,000.00$ & & $\$ 42,540$ & $\$ 900,000$ & $\$ 0$ & $\$ 942,540$ \\
\hline 060199 & $\begin{array}{l}\text { Process Equipment-DESIGN } \\
\text { DEVELOPMENT ALLOW (DDA) }\end{array}$ & 0.04 & $\%$ & & & & & $\$ 900,000.00$ & & $\$ 0$ & $\$ 36,000$ & $\$ 0$ & $\$ 36,000$ \\
\hline 068900 & Process Equipment-VENDOR REPS & 5.00 & days & & & & & $\$ 1,500.00$ & & $\$ 0$ & $\$ 7,500$ & $\$ 0$ & $\$ 7,500$ \\
\hline
\end{tabular}


Client: INL

Project: FFC

Location: GREEN FIELD SITE HIP Option

Account: 06 Process Equipment

Facility: Entire Estimate

URS

Washington Division
Date: 7/17/2008

11:31AM

Priced By: ADC

Office: Denver

Product Line: Gov
Estimate: FFC

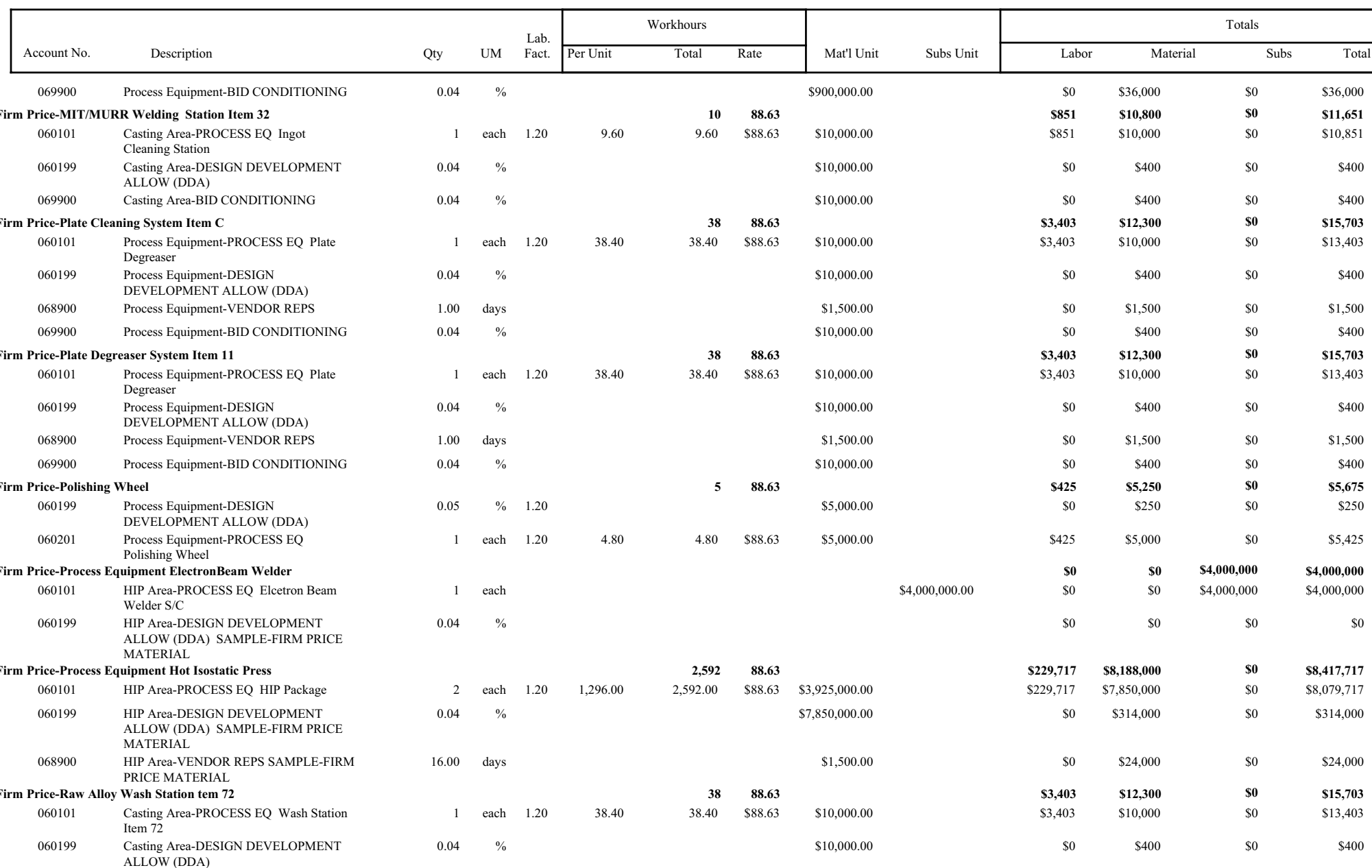


Client: INL

Project: FFC

Location: GREEN FIELD SITE HIP Option

Account: 06 Process Equipment

Facility: Entire Estimate

URS

Washington Division
Date: 7/17/2008

11:31AM

Priced By: ADC

Office: Denver

Product Line: Gov
Estimate: FFC

\begin{tabular}{|c|c|c|c|c|c|c|c|c|c|c|c|c|c|}
\hline \multirow[b]{2}{*}{ Account No. } & \multirow[b]{2}{*}{ Description } & \multirow[b]{2}{*}{ Qty } & \multirow[b]{2}{*}{ UM } & \multirow{2}{*}{$\begin{array}{l}\text { Lab. } \\
\text { Fact. }\end{array}$} & \multicolumn{3}{|c|}{ Workhours } & \multirow[b]{2}{*}{ Mat'l Unit } & \multirow[b]{2}{*}{ Subs Unit } & \multicolumn{4}{|c|}{ Totals } \\
\hline & & & & & Per Unit & Total & Rate & & & Labor & Material & Subs & Total \\
\hline 068900 & Casting Area-VENDOR REPS & 1.00 & days & & & & & $\$ 1,500.00$ & & $\$ 0$ & $\$ 1,500$ & $\$ 0$ & $\$ 1,500$ \\
\hline 069900 & Casting Area-BID CONDITIONING & 0.04 & $\%$ & & & & & $\$ 10,000.00$ & & $\$ 0$ & $\$ 400$ & $\$ 0$ & $\$ 400$ \\
\hline \multicolumn{2}{|c|}{ Firm Price-Rolling Mill w/Tunnel Furnace 25} & & & & & 144 & $\mathbf{8 8 . 6 3}$ & & & $\$ 12,762$ & $\$ 1,087,500$ & $\$ 0$ & $\$ 1,100,262$ \\
\hline 060101 & $\begin{array}{l}\text { Process Equipment-PROCESS EQ Rolling } \\
\text { Mill W/Tunnel Furnace }\end{array}$ & 1 & each & 1.20 & 144.00 & 144.00 & $\$ 88.63$ & $\$ 1,000,000.00$ & & $\$ 12,762$ & $\$ 1,000,000$ & $\$ 0$ & $\$ 1,012,762$ \\
\hline 060199 & $\begin{array}{l}\text { Process Equipment-DESIGN } \\
\text { DEVELOPMENT ALLOW (DDA) }\end{array}$ & 0.04 & $\%$ & & & & & $\$ 1,000,000.00$ & & $\$ 0$ & $\$ 40,000$ & $\$ 0$ & $\$ 40,000$ \\
\hline 068900 & Process Equipment-VENDOR REPS & 5.00 & days & & & & & $\$ 1,500.00$ & & $\$ 0$ & $\$ 7,500$ & $\$ 0$ & $\$ 7,500$ \\
\hline 069900 & Process Equipment-BID CONDITIONING & 0.04 & $\%$ & & & & & $\$ 1,000,000.00$ & & $\$ 0$ & $\$ 40,000$ & $\$ 0$ & $\$ 40,000$ \\
\hline m Price-Stack & & & & & & 336 & 88.63 & & & $\$ 29,778$ & $\$ 19,438$ & $\$ 0$ & $\$ 49,216$ \\
\hline 060161 & $\begin{array}{l}\text { Bldg All In-PROCESS EQ Stack } 70^{\prime \prime} \\
\text { diameter by } 80^{\prime} \text { high }\end{array}$ & 1 & each & 1.20 & 336.00 & 336.00 & $\$ 88.63$ & $\$ 17,500.00$ & & $\$ 29,778$ & $\$ 17,500$ & $\$ 0$ & $\$ 47,278$ \\
\hline 060199 & $\begin{array}{l}\text { Bldg All In-DESIGN DEVELOPMENT } \\
\text { ALLOW (DDA) }\end{array}$ & 0.03 & $\%$ & & & & & $\$ 17,500.00$ & & $\$ 0$ & $\$ 438$ & $\$ 0$ & $\$ 438$ \\
\hline 068900 & Bldg All In-VENDOR REPS & 1.00 & days & & & & & $\$ 1,500.00$ & & $\$ 0$ & $\$ 1,500$ & $\$ 0$ & $\$ 1,500$ \\
\hline \multicolumn{2}{|c|}{ Firm Price-Tanks \& Platework Fire Water Collection Crit tanks } & & & & & 19 & 88.63 & & & $\$ 1,702$ & $\$ 21,900$ & $\$ 0$ & $\$ 23,602$ \\
\hline 060161 & $\begin{array}{l}\text { Fire Water Collection-PROCESS EQ } \\
\text { Pencil Tank Assembly }\end{array}$ & 1 & each & 1.20 & 19.20 & 19.20 & $\$ 88.63$ & $\$ 20,000.00$ & & $\$ 1,702$ & $\$ 20,000$ & $\$ 0$ & $\$ 21,702$ \\
\hline 060199 & $\begin{array}{l}\text { Fire Water Collection-DESIGN } \\
\text { DEVELOPMENT ALLOW (DDA) }\end{array}$ & 0.02 & $\%$ & & & & & $\$ 20,000.00$ & & $\$ 0$ & $\$ 400$ & $\$ 0$ & $\$ 400$ \\
\hline 068900 & Fire Water Collection-VENDOR REPS & 1.00 & days & & & & & $\$ 1,500.00$ & & $\$ 0$ & $\$ 1,500$ & $\$ 0$ & $\$ 1,500$ \\
\hline \multicolumn{2}{|c|}{ Firm Price-Tanks \& Platework Fire Water Collection Tank } & & & & & 19 & 88.63 & & & $\$ 1,702$ & $\$ 11,016$ & $\$ 0$ & $\$ 12,718$ \\
\hline 060161 & $\begin{array}{l}\text { Fire Water Collection-PROCESS EQ Tank } \\
\text { Single Walled Plastic }\end{array}$ & 1 & each & 1.20 & 19.20 & 19.20 & $\$ 88.63$ & $\$ 10,800.00$ & & $\$ 1,702$ & $\$ 10,800$ & $\$ 0$ & $\$ 12,502$ \\
\hline 060199 & $\begin{array}{l}\text { Fire Water Collection-DESIGN } \\
\text { DEVELOPMENT ALLOW (DDA) }\end{array}$ & 0.02 & $\%$ & & & & & $\$ 10,800.00$ & & $\$ 0$ & $\$ 216$ & $\$ 0$ & $\$ 216$ \\
\hline \multicolumn{2}{|c|}{ Forklifts and Power PalletTruck Elec } & & & & & 10 & 88.63 & & & $\$ 851$ & $\$ 87,750$ & $\$ 0$ & $\$ 88,601$ \\
\hline 069900 & $\begin{array}{l}\text { Mechanical Equipment- Front End Loader } \\
6000 \mathrm{lb} \text { cap }\end{array}$ & 3 & each & 1.20 & 2.40 & 7.20 & $\$ 88.63$ & $\$ 28,000.00$ & & $\$ 638$ & $\$ 84,000$ & $\$ 0$ & $\$ 84,638$ \\
\hline 069900 & Mechanical Equipment-Pallet Truck & 1 & each & 1.20 & 2.40 & 2.40 & $\$ 88.63$ & $\$ 3,750.00$ & & $\$ 213$ & $\$ 3,750$ & $\$ 0$ & $\$ 3,963$ \\
\hline \multicolumn{2}{|c|}{ Heat Transfer Eq Curcible Tilter } & & & & & 12 & 88.63 & & & $\$ 1,064$ & $\$ 11,700$ & $\$ 0$ & $\$ 12,764$ \\
\hline 060126 & $\begin{array}{l}\text { Casting Area-PROCESS EQ Crucible } \\
\text { Tilter }\end{array}$ & 1 & each & 1.20 & 12.00 & 12.00 & $\$ 88.63$ & $\$ 10,000.00$ & & $\$ 1,064$ & $\$ 10,000$ & $\$ 0$ & $\$ 11,064$ \\
\hline 060199 & $\begin{array}{l}\text { Casting Area-DESIGN DEVELOPMENT } \\
\text { ALLOW (DDA) }\end{array}$ & 0.02 & $\%$ & & & & & $\$ 10,000.00$ & & $\$ 0$ & $\$ 200$ & $\$ 0$ & $\$ 200$ \\
\hline 068900 & Casting Area-VENDOR REPS & 1.00 & days & & & & & $\$ 1,500.00$ & & $\$ 0$ & $\$ 1,500$ & $\$ 0$ & $\$ 1,500$ \\
\hline PA Filters and & ir Handling Sys for Fuel Fabrication Area & & & & & 4,920 & 88.63 & & & $\$ 436,037$ & $\$ 154,017$ & $\$ 2,654,150$ & $\$ 3,244,204$ \\
\hline 060129 & $\begin{array}{l}\text { Mechanical Equipment-HVAC } \\
\text { EQUIPMENT Process Area Subcontract } \\
\text { furnish \& install ( } 38900 \text { sqft) }\end{array}$ & 1 & each & & & & & & $\$ 2,654,150.00$ & $\$ 0$ & $\$ 0$ & $\$ 2,654,150$ & $\$ 2,654,150$ \\
\hline 060129 & $\begin{array}{l}\text { Mechanical Equipment-HVAC } \\
\text { EQUIPMENT Process Area HEPA Filter } \\
\text { Install Craft Support }\end{array}$ & 1 & each & 1.20 & 372.00 & 372.00 & $\$ 88.63$ & & & $\$ 32,969$ & $\$ 0$ & $\$ 0$ & $\$ 32,969$ \\
\hline
\end{tabular}


Client: INL

Project: FFC

Location: GREEN FIELD SITE HIP Option

Account: 06 Process Equipment

Facility: Entire Estimate
URS

Washington Division
Date: $7 / 17 / 2008$

11:31AM

Priced By: ADC

Office: Denver

Product Line:
Estimate:

Job No: $\quad 27989-325 \quad$ Rev No: 0

\begin{tabular}{c|c} 
Total \\
\cline { 2 - 3 }
\end{tabular}

\begin{tabular}{|lrr|lr|} 
& & & \multicolumn{2}{|c|}{ Workhours } \\
\cline { 3 - 4 } Account No. & Description & Qty $\quad$ UM & Fabt. & Total Rate
\end{tabular}

060129 Mechanical Equipment-HVAC EQUIPMENT Process Area craft labor support (38900 sqft)

HEPA Filters and Air Handling Sys for HIP Area

060129 HIP Area-HVAC EQUIPMENT Process

Area craft labor support (4200 sqft)

HIP Area-HVAC EQUIPMENT Process

Area Subcontract furnish \& install (4200

sqft)

060129 HIP Area-HVAC EQUIPMENT Process

Area HEPA Filter Install Craft Support

(HIP)

Instron Item $O$

060101 Casting Area-PROCESS EQ Pull Testor

060199 Casting Area-DESIGN DEVELOPMENT ALLOW (DDA)

$069900 \quad$ Casting Area-BID CONDITIONING

NIST/HIFR Welding Item $G$

060101 Process Equipment-PROCESS EQ Welding Station

$060199 \quad$ Process Equipment-DESIGN DEVELOPMENT ALLOW (DDA)

$068900 \quad$ Process Equipment-VENDOR REPS

$069900 \quad$ Process Equipment-BID CONDITIONING

Nitric Acid Wash System (incl Piping)

$060101 \quad$ Mechanical Equipment-Nitric Acid Wash System

Platework Crucible Cleaning Station Glovebox Item 39

$060161 \quad$ Casting Area-PROCESS EQ Crucible

Cleaning Station Glovebox Item 39

060199 Casting Area-DESIGN DEVELOPMENT ALLOW (DDA)

$068900 \quad$ Casting Area-VENDOR REPS

Platework Crucible Loading Station Item 38

060161 Casting Area-PROCESS EQ Crucible

Loading Station Item 38

060199 Casting Area-DESIGN DEVELOPMENT ALLOW (DDA)

$068900 \quad$ Casting Area-VENDOR REPS

Platework Entry Glovebox

060161 Casting Area-PROCESS EQ Casting Mold Entry Glovebox

060199 Casting Area-DESIGN DEVELOPMENT ALLOW (DDA)

$068900 \quad$ Casting Area-VENDOR REPS

$\begin{array}{llllll}1 & \text { each } & 1.20 & 4,548.00 & 4,548.00 & \$ 88.63\end{array}$

\begin{tabular}{|c|c|c|c|c|c|}
\hline & & & 494 & 88.63 & \\
\hline each & 1.20 & 456.00 & 456.00 & $\$ 88.63$ & $\$ 154,017.00$ \\
\hline
\end{tabular}

1 each

$1 \quad$ each $\quad 1.20 \quad 38.40 \quad 38.40 \quad \$ 88.63$

$\begin{array}{rrr}48 & 88.63\end{array}$

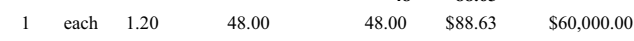

$0.04 \%$

$0.04 \%$

$1 \quad$ each $\quad 1.20 \quad 48.00$

$0.00 \quad \%$

1.00 days

$0.04 \%$

$1 \quad$ each $\quad 1.20 \quad 93.60$

$1 \quad$ each $\quad 1.20 \quad 19.20$

$0.04 \%$

2.00 days

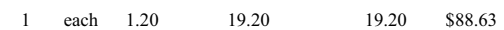

$0.04 \%$

2.00 days

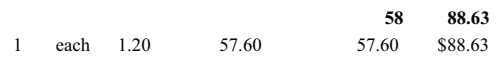

$0.04 \%$

2.00 days $\begin{array}{lr}48 & 88.63\end{array}$

$\$ 60,000.00$

$\$ 60,000.00$

$\$ 100,000.00$

$\$ 100,000.00$

$\$ 1,500.00$

$\$ 100,000.00$

$\begin{array}{ll}94 & 88.63\end{array}$

$93.60 \quad \$ 88.63$

$\begin{array}{rr}19 & 88.63 \\ 19\end{array}$

$\$ 469.00$

$\$ 50,000.00$

$\$ 50,000.00$

$\$ 1,500.00$

$\$ 30,000.00$

$\$ 30,000.00$

$\$ 1,500.00$

$\$ 50,000.00$

$\$ 50,000.00$

$\$ 1,500.00$
Subs Unit

$\$ 403,068$

$\$ 154,017$

aterial

Subs

Tota

$\begin{array}{rrrrr} & \$ 43,816 & \$ 154,017 & \$ 285,600 & \$ \mathbf{4 8 3 , 4 3 3} \\ & \$ 40,413 & \$ 154,017 & \$ 0 & \$ 194,430 \\ \$ 285,600.00 & \$ 0 & \$ 0 & \$ 285,600 & \$ 285,600\end{array}$

$\$ 3,403 \quad \$ 0 \quad \$ 0 \quad \$ 3,403$

\begin{tabular}{|c|c|}
\hline & $\$ 64,800$ \\
\hline
\end{tabular}

$\$ 4,254 \quad \$ 60,000 \quad \$ 0 \quad \$ 64,254$

$\$ 0 \quad \$ 2,400 \quad \$ 0 \quad \$ 2,400$

$\begin{array}{llll}\$ 0 & \$ 2,400 & \$ 0 & \$ 2,400\end{array}$

$\$ 4,254 \$ \$ 105,900$

$\$ 110,154$

$\$ 4,254 \quad \$ 100,000 \quad \$ 0 \quad \$ 104,254$

$\$ 0 \quad \$ 400 \quad \$ 0 \quad \$ 400$

$\begin{array}{llll}\$ 0 & \$ 1,500 & \$ 0 & \$ 1,500\end{array}$

$\$ 0 \quad \$ 4,000 \quad \$ 0 \quad \$ 4,000$

$\begin{array}{llll}\$ 8,295 & \$ 469 & \$ 0 & \$ 8,764\end{array}$

$\begin{array}{llll}\$ 8,295 & \$ 469 & \$ 0 & \$ 8,764 \\ \$ 8,295 & \$ 469 & \$ 0 & \$ 8,764\end{array}$

$\$ 1,702 \quad \$ 55,000 \quad \$ 0 \quad \$ 56,702$

$\$ 1,702 \quad \$ 50,000 \quad \$ 0 \quad \$ 51,702$

$\begin{array}{llll}\$ 0 & \$ 2,000 & \$ 0 & \$ 2,000\end{array}$

$\$ 0 \quad \$ 3,000 \quad \$ 0 \quad \$ 3,000$

\begin{tabular}{|c|c|}
\hline$\$ 1,702$ & $\$ 34,200$ \\
\hline
\end{tabular}

$\$ 1,702 \quad \$ 30,000 \quad \$ 0 \quad \$ 31,702$

$\$ 0 \quad \$ 1,200 \quad \$ 0 \quad \$ 1,200$

$\$ 0 \quad \$ 3,000 \quad \$ 0 \quad \$ 3,000$

$\$ 0 \quad \$ 60,105$

$\begin{array}{llll}\mathbf{\$ 5 , 1 0 5} & \$ \mathbf{5 5 , 0 0 0} & \mathbf{\$ 0} & \mathbf{\$ 6 0 , 1 0 5} \\ \$ 5,105 & \$ 50,000 & \$ 0 & \$ 55,105\end{array}$

$\$ 0 \quad \$ 2,000 \quad \$ 0 \quad \$ 2,000$

$\$ 0 \quad \$ 3,000 \quad \$ 0 \quad \$ 3,000$

Page 36 of 69 
Client: INL

Project: FFC

Location: GREEN FIELD SITE HIP Option

Account: 06 Process Equipment

Facility: Entire Estimate

URS

Washington Division
Date: 7/17/2008

11:31AM

Priced By: ADC

Office: Denver

Product Line: Gov
Estimate: FFC

\begin{tabular}{|c|c|c|c|c|c|c|c|c|c|c|c|c|c|}
\hline \multirow[b]{2}{*}{ Account No. } & \multirow[b]{2}{*}{ Description } & \multirow[b]{2}{*}{ Qty } & \multirow[b]{2}{*}{ UM } & \multirow{2}{*}{$\begin{array}{l}\text { Lab. } \\
\text { Fact. }\end{array}$} & \multicolumn{3}{|c|}{ Workhours } & \multirow[b]{2}{*}{ Mat'l Unit } & \multirow[b]{2}{*}{ Subs Unit } & \multicolumn{4}{|c|}{ Totals } \\
\hline & & & & & Per Unit & Total & Rate & & & Labor & Material & Subs & Total \\
\hline \multicolumn{2}{|c|}{ Platework Exit Glovebox } & & & & & 58 & 88.63 & & & $\$ 5,105$ & $\$ 55,000$ & \$o & $\$ 60,105$ \\
\hline 060161 & $\begin{array}{l}\text { Casting Area-PROCESS EQ Casting Mold } \\
\text { Exit Glovebox }\end{array}$ & 1 & each & 1.20 & 57.60 & 57.60 & $\$ 88.63$ & $\$ 50,000.00$ & & $\$ 5,105$ & $\$ 50,000$ & $\$ 0$ & $\$ 55,105$ \\
\hline 060199 & $\begin{array}{l}\text { Casting Area-DESIGN DEVELOPMENT } \\
\text { ALLOW (DDA) }\end{array}$ & 0.04 & $\%$ & & & & & $\$ 50,000.00$ & & $\$ 0$ & $\$ 2,000$ & $\$ 0$ & $\$ 2,000$ \\
\hline 068900 & Casting Area-VENDOR REPS & 2.00 & days & & & & & $\$ 1,500.00$ & & $\$ 0$ & $\$ 3,000$ & $\$ 0$ & $\$ 3,000$ \\
\hline \multicolumn{2}{|c|}{ Platework Removal Station Glovebox Item 37} & & & & & 19 & 88.63 & & & $\$ 1,702$ & $\$ 13,400$ & \$o & $\$ 15,102$ \\
\hline 060161 & $\begin{array}{l}\text { Casting Area-PROCESS EQ Removal } \\
\text { Glovebox Item } 37\end{array}$ & 1 & each & 1.20 & 19.20 & 19.20 & $\$ 88.63$ & $\$ 10,000.00$ & & $\$ 1,702$ & $\$ 10,000$ & $\$ 0$ & $\$ 11,702$ \\
\hline 060199 & $\begin{array}{l}\text { Casting Area-DESIGN DEVELOPMENT } \\
\text { ALLOW (DDA) }\end{array}$ & 0.04 & $\%$ & & & & & $\$ 10,000.00$ & & $\$ 0$ & $\$ 400$ & $\$ 0$ & $\$ 400$ \\
\hline 068900 & Casting Area-VENDOR REPS & 2.00 & days & & & & & $\$ 1,500.00$ & & $\$ 0$ & $\$ 3,000$ & $\$ 0$ & $\$ 3,000$ \\
\hline \multicolumn{2}{|c|}{ Process Equipment Channel Probe Item B } & & & & & 115 & 88.63 & & & $\$ 10,220$ & $\$ 871,500$ & so & $\$ 881,720$ \\
\hline 060101 & $\begin{array}{l}\text { Process Equipment-PROCESS EQ Channel } \\
\text { Probe }\end{array}$ & 1 & each & 1.20 & 115.32 & 115.32 & $\$ 88.63$ & $\$ 800,000.00$ & & $\$ 10,220$ & $\$ 800,000$ & $\$ 0$ & $\$ 810,220$ \\
\hline 060199 & $\begin{array}{l}\text { Process Equipment-DESIGN } \\
\text { DEVELOPMENT ALLOW (DDA) }\end{array}$ & 0.04 & $\%$ & 1.20 & & & & $\$ 800,000.00$ & & $\$ 0$ & $\$ 32,000$ & $\$ 0$ & $\$ 32,000$ \\
\hline 068900 & Process Equipment-VENDOR REPS & 5.00 & days & 1.20 & & & & $\$ 1,500.00$ & & $\$ 0$ & $\$ 7,500$ & $\$ 0$ & $\$ 7,500$ \\
\hline 069900 & Process Equipment-BID CONDITIONING & 0.04 & $\%$ & 1.20 & & & & $\$ 800,000.00$ & & $\$ 0$ & $\$ 32,000$ & $\$ 0$ & $\$ 32,000$ \\
\hline \multicolumn{2}{|c|}{ Process Equipment HIFR Lathe Item H } & & & & & 67 & 88.63 & & & $\$ 5,962$ & $\$ 118,320$ & \$0 & $\$ 124,282$ \\
\hline 060101 & $\begin{array}{l}\text { Process Equipment-PROCESS EQ-Lathe } \\
\text { Model SL } 40\end{array}$ & 1 & each & 1.20 & 67.27 & 67.27 & $\$ 88.63$ & $\$ 105,000.00$ & & $\$ 5,962$ & $\$ 105,000$ & $\$ 0$ & $\$ 110,962$ \\
\hline 060199 & $\begin{array}{l}\text { Process Equipment-DESIGN } \\
\text { DEVELOPMENT ALLOW (DDA) }\end{array}$ & 0.04 & $\%$ & 1.20 & & & & $\$ 105,000.00$ & & $\$ 0$ & $\$ 4,620$ & $\$ 0$ & $\$ 4,620$ \\
\hline 068900 & Process Equipment-VENDOR REPS & 3.00 & days & 1.20 & & & & $\$ 1,500.00$ & & $\$ 0$ & $\$ 4,500$ & $\$ 0$ & $\$ 4,500$ \\
\hline 069900 & Process Equipment-BID CONDITIONING & 0.04 & $\%$ & 1.20 & & & & $\$ 105,000.00$ & & $\$ 0$ & $\$ 4,200$ & $\$ 0$ & $\$ 4,200$ \\
\hline \multicolumn{2}{|c|}{ Process Equipment MIT Milling Item I } & & & & & 77 & 88.63 & & & $\$ 6,806$ & $\$ 121,320$ & \$o & $\$ 128,126$ \\
\hline 060101 & $\begin{array}{l}\text { Process Equipment-PROCESS EQ-MIlling } \\
\text { Mode IVR } 8\end{array}$ & 1 & each & 1.20 & 76.80 & 76.80 & $\$ 88.63$ & $\$ 105,000.00$ & & $\$ 6,806$ & $\$ 105,000$ & $\$ 0$ & $\$ 111,806$ \\
\hline 060199 & $\begin{array}{l}\text { Process Equipment-DESIGN } \\
\text { DEVELOPMENT ALLOW (DDA) }\end{array}$ & 0.04 & $\%$ & & & & & $\$ 105,000.00$ & & $\$ 0$ & $\$ 4,620$ & $\$ 0$ & $\$ 4,620$ \\
\hline 068900 & Process Equipment-VENDOR REPS & 5.00 & days & & & & & $\$ 1,500.00$ & & $\$ 0$ & $\$ 7,500$ & $\$ 0$ & $\$ 7,500$ \\
\hline 069900 & Process Equipment-BID CONDITIONING & 0.04 & $\%$ & & & & & $\$ 105,000.00$ & & $\$ 0$ & $\$ 4,200$ & $\$ 0$ & $\$ 4,200$ \\
\hline \multicolumn{2}{|c|}{ Roller Levelers Item 19} & & & & & 48 & 88.63 & & & $\$ 4,254$ & $\$ 40,800$ & \$o & $\$ 45,054$ \\
\hline 060101 & $\begin{array}{l}\text { Process Equipment-PROCESS EQ Roller } \\
\text { Levelers }\end{array}$ & 1 & each & 1.20 & 48.00 & 48.00 & $\$ 88.63$ & $\$ 35,000.00$ & & $\$ 4,254$ & $\$ 35,000$ & $\$ 0$ & $\$ 39,254$ \\
\hline 060199 & $\begin{array}{l}\text { Process Equipment-DESIGN } \\
\text { DEVELOPMENT ALLOW (DDA) }\end{array}$ & 0.04 & $\%$ & 1.20 & & & & $\$ 35,000.00$ & & $\$ 0$ & $\$ 1,400$ & $\$ 0$ & $\$ 1,400$ \\
\hline 068900 & Process Equipment-VENDOR REPS & 2.00 & days & 1.20 & & & & $\$ 1,500.00$ & & $\$ 0$ & $\$ 3,000$ & $\$ 0$ & $\$ 3,000$ \\
\hline 069900 & Process Equipment-BID CONDITIONING & 0.04 & $\%$ & 1.20 & & & & $\$ 35,000.00$ & & $\$ 0$ & $\$ 1,400$ & $\$ 0$ & $\$ 1,400$ \\
\hline \multicolumn{2}{|c|}{ Salt Bath with Loading Equipment \& Rolling Mill Item 26} & & & & & 130 & 88.63 & & & $\$ 11,486$ & $\$ 532,500$ & \$0 & $\$ 543,986$ \\
\hline 060116 & $\begin{array}{l}\text { Process Equipment-PROCESS EQ Loading } \\
\text { Equipment }\end{array}$ & 1 & each & 1.20 & 129.60 & 129.60 & $\$ 88.63$ & $\$ 500,000.00$ & & $\$ 11,486$ & $\$ 500,000$ & $\$ 0$ & $\$ 511,486$ \\
\hline
\end{tabular}


Client: INL

Project: FFC

URS

Washington Division

Account: 06 Process Equipm

Facility: Entire Estimate

HIP Option

Job No:

Rev No: 0

Workhours

Workhours

$\begin{array}{llll} & & & \text { Lab. } \\ \text { Account No. Description } & \text { Qty } & \text { UM Fact. }\end{array}$

Per Unit

Rate

Process Equipment-DESIGN

DEVELOPMENT ALLOW (DDA)

$\%$

068900

Process Equipment-VENDOR REPS

5.00 days

$\%$

$\$ 500,000.00$
$\$ 1,500.00$

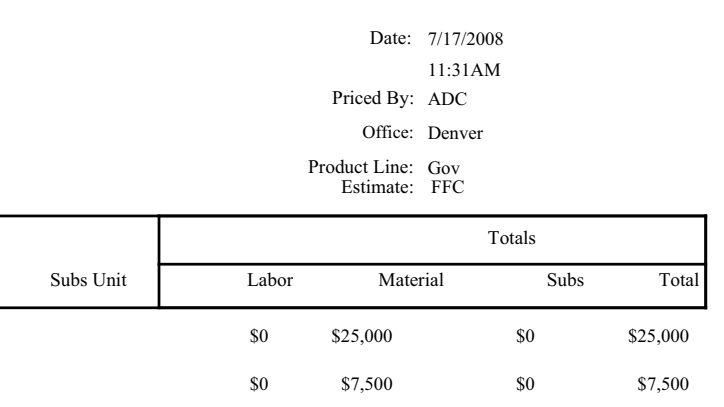

Totals 06-Process Equipment

Entire Estimate

$\$ 1,110,740$

$\$ 18,771,132$

$\$ 6,939,750$

$\$ 26,821,622$ 
Client: INL

Project: FFC

URS

Date: 7/17/2008

$11: 31 \mathrm{AM}$

Location: GREEN FIELD SITE HIP Option

Washington Division

Priced By: ADC

Office: Denver

Account: $11 \quad$ Piping
Facility: Entire Estimate

Job No: $\quad 27989-325 \quad$ Rev No: 0

Product Line: Gov
Estimate: FFC

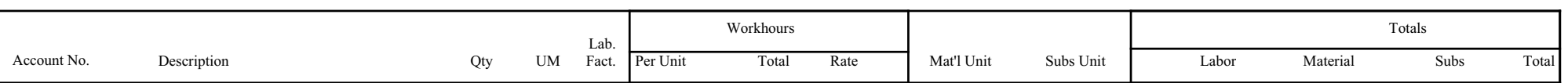

11.01 PIPE BY METALLURGY

$11^{* * * * * *} 1 \mathrm{X} \quad$ Carbon Steel

Small Bore

$11^{* * * * * *} 2 \mathrm{X} \quad$ Stainless Steel

Small Bore

$11 * * * * * * 5 X \quad$ Plastic/Fiberglass

Large Bore

$11^{* * * * * *} 61(62,63)$ Iron

Large Bore

$1103^{* * * *} \quad$ Specialities

$1104 * * * * \quad$ Hangers \& Supports

$11^{* * * * * *} \quad$ Unspecified Metallurgy

110116 Out of Spec - Pipe

110102

110102

110121

WEIGHTED Compressed Air

WEIGHTED Instrument Air

Out of Spec. - Unions

OVERALL PIPING ALLOWANCE-A/G

OVERALL PIPING ALLOWANCE-A/G

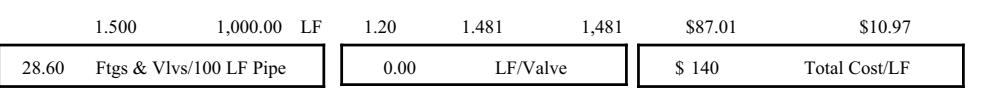

\begin{tabular}{|c|c|c|c|c|c|c|}
\hline & 1.000 & $300.00 \quad$ LF & 1.20 & 2.219 & $\$ 87.01$ & $\$ 55.50$ \\
\hline 63.33 & Ftgs \& & LF Pipe & 3.49 & LF/Valve & $\$ 249$ & Total Cost/LF \\
\hline
\end{tabular}

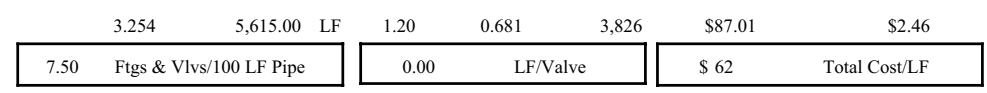

0.000

$\begin{array}{lll}0 & \text { LF } & 1.20\end{array}$

556

$\$ 62$

Total Cost/LF

\begin{tabular}{|c|c|c|c|c|c|c|c|}
\hline & $1,800.00$ & $\operatorname{lnft}$ & & & & $\$ 87.01$ & \\
\hline $\begin{array}{l}\text { WANCE-A/G } \\
\text { Air }\end{array}$ & $1,844.00$ & $\operatorname{lnft}$ & & 1.96 & $3,606.86$ & $\$ 87.01$ & $\$ 12.05$ \\
\hline \multirow{2}{*}{$\begin{array}{l}\text { WII } \\
\text { ir }\end{array}$} & $3,211.00$ & $\operatorname{lnft}$ & & 1.96 & $6,280.72$ & $\$ 87.01$ & $\$ 16.43$ \\
\hline & 4.00 & each & & & & $\$ 87.01$ & \\
\hline 0.06 & Ftgs \& Vlvs/100 LF Pipe & & 0.00 & & & $\$ 136$ & Total Cost/LF \\
\hline
\end{tabular}

\begin{tabular}{|rl|}
\hline \multicolumn{2}{|c|}{ Overall Ratios } \\
6.86 & Ftgs \& Vlvs/100 LF Pipe \\
105.92 & LF/Valve \\
1.38 & Workhours/LF \\
$\$ 131.60$ & Total Cost/LF \\
\hline
\end{tabular}

\begin{tabular}{|c|c|c|c|c|c|c|c|c|c|c|}
\hline \multirow[t]{2}{*}{ Grand Total: 11: Piping } & \multirow[t]{2}{*}{1.458} & \multirow[t]{2}{*}{13,770} & \multirow{2}{*}{\multicolumn{2}{|c|}{ LF $\quad 1.20$}} & \multirow[t]{2}{*}{1.38} & \multirow[t]{2}{*}{19,008} & \multirow[t]{2}{*}{$\$ 87.01$} & $\$ 1,653,839$ & $\$ 158,279$ & so $\$ 1,812,118$ \\
\hline & & & & & & & & $\$ 120.10 / \mathrm{LF}$ & $\$ 11.49 / \mathrm{LF}$ & $\$ 0.00 / \mathrm{LF}$ \\
\hline
\end{tabular}

Page 39 of 69 
Client: INL

Project: FFC

URS

Date: 7/17/2008

11:31AM

Location: GREEN FIELD SITE HIP Option

Washington Division

Priced By: ADC

ccount: 11 Piping

Job No: $\quad 27989-325 \quad$ Rev No: 0

Office: Denver

Facility: Entire Estimate

Product Line: Gov
Estimate: FFC

HIP Option

1

Mat'l U

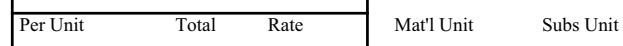

Esting:

Account No. Description Qty UM Lab.

$1.380-19007.82$

\begin{tabular}{|c|c|c|c|c|c|c|}
\hline 1.458 & $13,770.00$ & 1.20 & 1.380 & $19,007.82$ & $\$ 87.01$ & $\$ 11.49$ \\
\hline 6.86 & Ftgs \& & Vlvs/100 LF & 105.92 & LF/Valve & $\$ 131.60$ & Total Cost/LF \\
\hline
\end{tabular}

$\$ 0.00$

$\$ 1,653,839$

$\$ 158,279$

$\$ 0 \quad \$ 1,812,118$

11.02 PIPE BY SERVICE SYSTEM

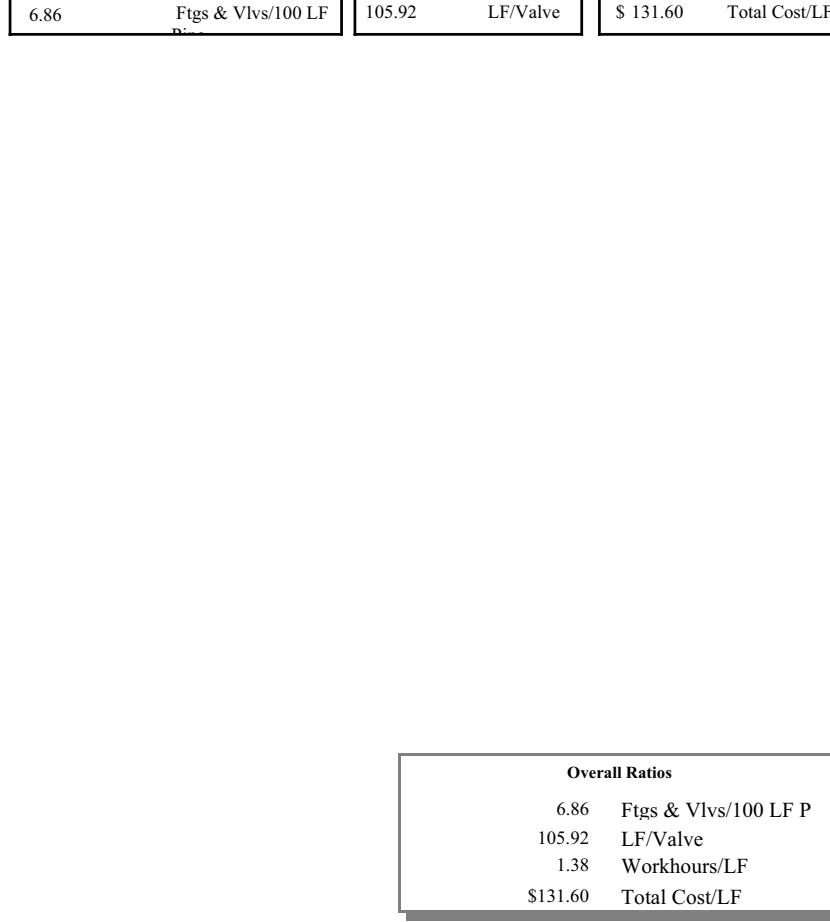

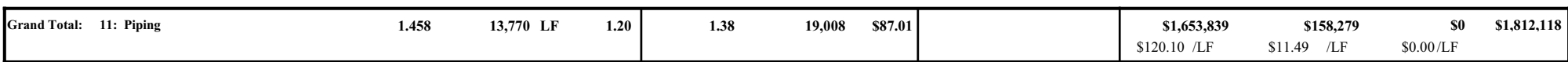

Page 40 of 69 
Client: INL

Project: FFC

Location: GREEN FIELD SITE HIP Option

Account: 11 Piping

Facility: Entire Estimate
URS

Washington Division
Date: 7/17/2008

11:31AM

Priced By: ADC

Office: Denver

Product Line:
Estimate:

Job No: $\quad 27989-325 \quad$ Rev No: 0

\begin{tabular}{|c|c|c|c|c|c|c|c|c|c|c|c|c|c|}
\hline \multirow[b]{2}{*}{ Account No. } & & \multirow[b]{2}{*}{ Qty } & \multirow[b]{2}{*}{ UM } & \multirow{2}{*}{$\begin{array}{l}\text { Lab. } \\
\text { Fact. }\end{array}$} & \multicolumn{3}{|c|}{ Workhours } & \multirow[b]{2}{*}{ Mat'l Unit } & \multirow[b]{2}{*}{ Subs Unit } & \multicolumn{4}{|c|}{ Totals } \\
\hline & & & & & \begin{tabular}{|l} 
Per Unit \\
\end{tabular} & Total & Rate & & & Labor & Material & Subs & Total \\
\hline \multicolumn{14}{|l|}{ 11.04 PIPE BY ASSEMBLY - SUMMARY } \\
\hline Bldg Plumbing & & 0.00 & LF & 1.20 & 0.00 & 19 & $\$ 87.01$ & $\$ 0.00$ & $\$ 0.00$ & $\$ 1,671$ & $\$ 3,930$ & $\$ 0$ & $\$ 5,601$ \\
\hline $\begin{array}{l}\text { Carbon Steel ( } 400 \text { s Specs) Plumbing } \\
\text { Water Pipe ( } 50 \text { lnft per fixture)L }\end{array}$ & 1.500 & $1,000.00$ & LF & 1.20 & 1.91 & 1,908 & $\$ 87.01$ & $\$ 17.05$ & $\$ 0.00$ & $\$ 166,043$ & $\$ 17,047$ & $\$ 0$ & $\$ 183,090$ \\
\hline Plastic (800s) Fire Protection Loop & & $1,800.00$ & LF & 1.20 & 0.89 & 1,608 & $\$ 87.01$ & $\$ 10.41$ & $\$ 0.00$ & $\$ 139,889$ & $\$ 18,746$ & $\$ 0$ & $\$ 158,635$ \\
\hline $\begin{array}{l}\text { Plastic (800s) Floor Drain Pipe (100 lnft } \\
\text { per floor drain) }\end{array}$ & 3.000 & $2,400.00$ & LF & 1.20 & 0.72 & 1,723 & $\$ 87.01$ & $\$ 3.83$ & $\$ 0.00$ & $\$ 149,933$ & $\$ 9,180$ & $\$ 0$ & $\$ 159,113$ \\
\hline $\begin{array}{l}\text { Plastic (800s) Floor Drain Pipe (100 lnft } \\
\text { per floor drain) HIP Area }\end{array}$ & 3.000 & 200.00 & LF & 1.20 & 0.72 & 145 & $\$ 87.01$ & $\$ 3.72$ & $\$ 0.00$ & $\$ 12,613$ & $\$ 743$ & $\$ 0$ & $\$ 13,356$ \\
\hline $\begin{array}{l}\text { Plastic (800s) Outside Sanitary Sewer } \\
\text { Line }\end{array}$ & 6.000 & 475.00 & LF & 1.20 & 1.14 & 540 & $\$ 87.01$ & $\$ 9.30$ & $\$ 0.00$ & $\$ 46,985$ & $\$ 4,416$ & $\$ 0$ & $\$ 51,400$ \\
\hline $\begin{array}{l}\text { Plastic ( } 800 \mathrm{~s}) \text { Roof drain pipe ( } 70 \mathrm{lnft} \text { per } \\
\text { roof drain) }\end{array}$ & 3.000 & $1,400.00$ & LF & 1.20 & 0.70 & 986 & $\$ 87.01$ & $\$ 3.79$ & $\$ 0.00$ & $\$ 85,762$ & $\$ 5,304$ & $\$ 0$ & $\$ 91,066$ \\
\hline $\begin{array}{l}\text { Plastic (800s) Roof drain pipe( } 70 \mathrm{lnft} \text { per } \\
\text { roof drain) HIP AREA }\end{array}$ & 3.000 & 140.00 & LF & 1.20 & 0.73 & 103 & $\$ 87.01$ & $\$ 4.09$ & $\$ 0.00$ & $\$ 8,948$ & $\$ 573$ & $\$ 0$ & $\$ 9,521$ \\
\hline $\begin{array}{l}\text { Plastic (800s) Sanitary Sewer piping ( } 50 \\
\text { lnft per fixture) }\end{array}$ & 3.000 & $1,000.00$ & LF & 1.20 & 1.54 & 1,538 & $\$ 87.01$ & $\$ 9.20$ & $\$ 0.00$ & $\$ 133,801$ & $\$ 9,204$ & $\$ 0$ & $\$ 143,005$ \\
\hline $\begin{array}{l}\text { Stainless Steel (700s) Argon Supply } \\
\text { Piping }\end{array}$ & 1.000 & 150.00 & LF & 1.20 & 1.83 & 275 & $\$ 87.01$ & $\$ 47.21$ & $\$ 0.00$ & $\$ 23,946$ & $\$ 7,081$ & $\$ 0$ & $\$ 31,028$ \\
\hline $\begin{array}{l}\text { Stainless Steel (700s) Argon Supply } \\
\text { Piping for HIP Sys }\end{array}$ & 1.000 & 150.00 & LF & 1.20 & 1.83 & 275 & $\$ 87.01$ & $\$ 47.21$ & $\$ 0.00$ & $\$ 23,946$ & $\$ 7,081$ & $\$ 0$ & $\$ 31,028$ \\
\hline Utilities & & $5,055.00$ & LF & 1.20 & 1.96 & 9,888 & $\$ 87.01$ & $\$ 14.83$ & $\$ 0.00$ & $\$ 860,302$ & $\$ 74,973$ & $\$ 0$ & $\$ 935,275$ \\
\hline
\end{tabular}

Grand Total: 11: Piping

1.458

$13,770 \quad$ LF $\quad 1.20$

1.38

$19,008 \quad \$ 87.01$

$\$ 158,279$

$\$ 120.10 / \mathrm{LF} \quad \$ 11.49 / \mathrm{LF} \quad \$ 0.00 / \mathrm{LF}$

$\$ 120.10 / \mathrm{LF} \quad \$ 11.49 / \mathrm{LF} \quad \$ 0.00 / \mathrm{LF}$

$\$ 0 \$ 1,812,118$ 
Client: INL

Project: FFC

URS

Date: 7/17/2008

11:31AM

Location: GREEN FIELD SITE HIP Option

Washington Division

Priced By: ADC

Account: 11 Piping

Office: Denver

Facility: Entire Estimate

Job No: $\quad 27989-325 \quad$ Rev No: 0

$\begin{aligned} \text { Product Line: } & \text { Gov } \\ \text { Estimate: } & \text { FFC }\end{aligned}$

HIP Optio

Job No: 27989-325 Rev No: 0

\begin{tabular}{|c|c|c|c|c|c|c|c|c|c|c|c|c|c|}
\hline \multirow[b]{2}{*}{ Account No. } & \multirow[b]{2}{*}{ Description } & \multirow[b]{2}{*}{ Qty } & \multirow[b]{2}{*}{ UM } & \multirow{2}{*}{$\begin{array}{l}\text { Lab. } \\
\text { Fact. }\end{array}$} & \multicolumn{3}{|c|}{ Workhours } & \multirow[b]{2}{*}{ Mat'l Unit } & \multirow[b]{2}{*}{ Subs Unit } & \multicolumn{4}{|c|}{ Totals } \\
\hline & & & & & \begin{tabular}{|l|} 
Per Unit \\
\end{tabular} & Total & Rate & & & Labor & Material & Subs & Total \\
\hline
\end{tabular}

\subsection{PIPE BY COMMODITY}

$\begin{array}{ll}1101 & \text { Pipe, Fittings, Welds, and Shopcosts } \\ 1102 & \text { Valves } \\ 1103 & \text { Specialties } \\ 1104 & \text { Hangers \& Supports }\end{array}$

$\begin{array}{rllll}13,770.00 & \text { lnft } & 1.46 & 1.20 & 1.133 \\ 130.00 & \text { ea } & & 1.20 & 6.266 \\ & & & 1.20 & 9.600 \\ 684.00 & \text { ea } & & 1.20 & 3.761\end{array}$

$\begin{array}{rlr}15,601 & \$ 87.01 & \$ 7.66 \\ 815 & \$ 87.01 & \$ 173.19 \\ 19 & \$ 87.01 & \$ 1,965.00 \\ 2,573 & \$ 87.01 & \$ 38.59\end{array}$

$\begin{array}{rr}\$ 1,357,440 & \$ 105,442 \\ \$ 70,874 & \$ 22,514 \\ \$ 1,671 & \$ 3,930 \\ \$ 223,855 & \$ 26,393\end{array}$

$\$ 1,462,882$

$\$ 93,388$

$\$ 5,601$

$\$ 250,248$

\begin{tabular}{|c|c|c|c|c|c|c|c|c|}
\hline & & & & $\begin{array}{l}\text { Overall Ratios } \\
6.86 \text { Ftgs \& Vlvs/100 LF Pipe } \\
105.92 \text { LF / Valve } \\
1.38 \text { Workhours/LF } \\
\$ 131.60 \text { Total Cost/LF }\end{array}$ & & & & \\
\hline Grand Total: 11: Piping & $\begin{array}{ll}13,770 \quad \mathrm{LI} \\
\end{array}$ & $\begin{array}{ll}1.458 & 1.20\end{array}$ & 1.38 & $19,008 \quad \$ 87.01$ & $\begin{array}{c}\mathbf{\$ 1 , 6 5 3 , 8 3 9} \\
\$ 120.10 / \mathrm{LF}\end{array}$ & $\begin{array}{l}\$ 158,279 \\
\$ 11.49 / \mathrm{LF}\end{array}$ & $\begin{array}{c}\text { So } \\
\$ 0.00 / \mathrm{LF}\end{array}$ & $\mathbf{\$ 1 , 8 1 2 , 1 1 8}$ \\
\hline
\end{tabular}




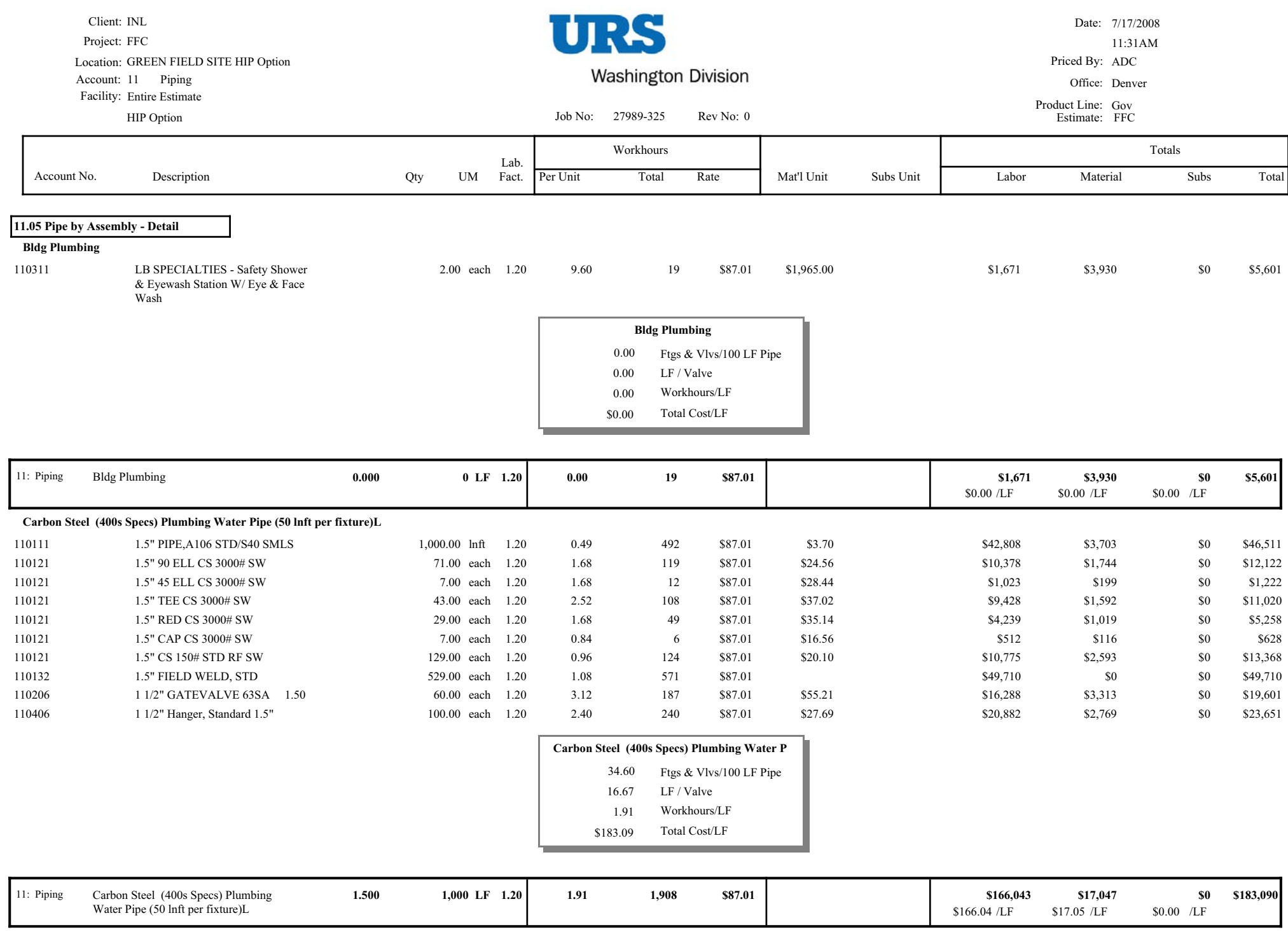


Client: INL

Project: FFC

Location: GREEN FIELD SITE HIP Option

Account: 11 Piping

Facility: Entire Estimate

URS

Washington Division
Date: 7/17/2008

11:31AM

Priced By: ADC

Office: Denver

Product Line: Gov
Estimate: FFC

\begin{tabular}{|c|c|c|c|c|c|c|c|c|c|c|c|c|c|}
\hline \multirow[b]{2}{*}{ Account No. } & \multirow[b]{2}{*}{ Description } & \multirow[b]{2}{*}{ Qty } & \multirow[b]{2}{*}{ UM } & \multirow{2}{*}{$\begin{array}{l}\text { Lab. } \\
\text { Fact. }\end{array}$} & \multicolumn{3}{|c|}{ Workhours } & \multirow[b]{2}{*}{ Mat'l Unit } & \multirow[b]{2}{*}{ Subs Unit } & \multicolumn{4}{|c|}{ Totals } \\
\hline & & & & & \begin{tabular}{|l} 
Per Unit \\
\end{tabular} & Total & Rate & & & Labor & Material & Subs & Total \\
\hline \multicolumn{14}{|c|}{ Plastic (800s) Fire Protection Loop } \\
\hline 110116 & Out of Spec - Pipe & $1,800.00$ & lnft & 1.20 & & & $\$ 87.01$ & & & $\$ 0$ & $\$ 0$ & $\$ 0$ & $\$ 0$ \\
\hline 110126 & 8" 90 ELL S80 PVC SW & 28.00 & 0 each & 1.20 & 4.08 & 114 & $\$ 87.01$ & $\$ 64.30$ & & $\$ 9,940$ & $\$ 1,800$ & $\$ 0$ & $\$ 11,740$ \\
\hline 110126 & 8" TEE S80 PVC SW & 8.00 & 0 each & 1.20 & 6.24 & 50 & $\$ 87.01$ & $\$ 90.98$ & & $\$ 4,343$ & $\$ 728$ & $\$ 0$ & $\$ 5,071$ \\
\hline 10126 & 8" FLG SCH 80 SW PVC & 50.00 & 0 each & 1.20 & 2.04 & 102 & $\$ 87.01$ & $\$ 38.44$ & & $\$ 8,875$ & $\$ 1,922$ & $\$ 0$ & $\$ 10,797$ \\
\hline 110126 & $\begin{array}{l}8 " \text { SM. INLINE Tie into existing fire } \\
\text { line } \quad 8^{\prime \prime}\end{array}$ & 1.00 & 0 each & 1.20 & 16.44 & 16 & $\$ 87.01$ & & & $\$ 1,430$ & $\$ 0$ & $\$ 0$ & $\$ 1,430$ \\
\hline 110126 & 8" LG. INLINE Hydrants $\quad 8 "$ & 6.00 & 0 each & 1.20 & 20.52 & 123 & $\$ 87.01$ & & & $\$ 10,712$ & $\$ 0$ & $\$ 0$ & $\$ 10,712$ \\
\hline 110137 & 8" PLAST FIELD WELD & 140.00 & 0 each & 1.20 & 2.52 & 353 & $\$ 87.01$ & $\$ 2.00$ & & $\$ 30,697$ & $\$ 280$ & $\$ 0$ & $\$ 30,977$ \\
\hline 110146 & 8" 150\# BOLT-UP,PLAST & 25.00 & 0 each & 1.20 & 3.60 & 90 & $\$ 87.01$ & & & $\$ 7,831$ & $\$ 0$ & $\$ 0$ & $\$ 7,831$ \\
\hline 110211 & 8" GATEVALVE 11FA 8.00 & 12.00 & 0 each & 1.20 & 20.52 & 246 & $\$ 87.01$ & $\$ 572.25$ & & $\$ 21,425$ & $\$ 6,867$ & $\$ 0$ & $\$ 28,292$ \\
\hline 10411 & 8" Supports, Standard 8" & 95.00 & 0 each & 1.20 & 5.40 & 513 & $\$ 87.01$ & $\$ 75.25$ & & $\$ 44,635$ & $\$ 7,149$ & $\$ 0$ & $\$ 51,784$ \\
\hline
\end{tabular}

Plastic (800s) Fire Protection Loop

5.44 Ftgs \& Vlvs/100 LF Pipe

$150.00 \quad \mathrm{LF} /$ Valve

0.89 Workhours/LF

$\$ 88.13$ Total Cost/LF

\begin{tabular}{|c|c|c|c|c|c|c|c|c|c|c|c|c|c|c|}
\hline 11: Piping & Plastic (800s) Fire Protection Loop & 0.000 & 1,800 & $\mathbf{L F}$ & 1.20 & 0.89 & 1,608 & $\$ 87.01$ & & $\begin{array}{l}\mathbf{\$ 1 3 9 , 8 8 9} \\
\$ 77.72 / L F\end{array}$ & $\begin{array}{l}\mathbf{\$ 1 8 , 7 4 6} \\
\$ 10.41 / \mathrm{LF}\end{array}$ & $\$ 0.00$ & $\begin{array}{c}\mathbf{\$} 0 \\
/ \mathrm{LF}\end{array}$ & $\$ 158,635$ \\
\hline \multicolumn{15}{|c|}{ Plastic (800s) Floor Drain Pipe (100 Inft per floor drain) } \\
\hline 110116 & 3" PIPE, SCH 40 PVC & & $2,400.00 \ln$ & $\operatorname{lnft}$ & 1.20 & 0.31 & 749 & $\$ 87.01$ & $\$ 0.98$ & $\$ 65,152$ & $\$ 2,352$ & & $\$ 0$ & $\$ 67,504$ \\
\hline 110126 & 3" 90 ELL S40 PVC SW & & $24.00 \mathrm{e}$ & each & 1.20 & 1.56 & 37 & $\$ 87.01$ & $\$ 4.39$ & $\$ 3,258$ & $\$ 105$ & & $\$ 0$ & $\$ 3,363$ \\
\hline 110126 & 3" 45 ELL S40 PVC SW & & $12.00 \mathrm{e}$ & each & 1.20 & & & $\$ 87.01$ & $\$ 5.70$ & so & $\$ 68$ & & $\$ 0$ & $\$ 68$ \\
\hline 110126 & 3" WYE S40 PVC SW & & $24.00 \mathrm{e}$ & each & 1.20 & 2.28 & 55 & $\$ 87.01$ & $\$ 7.03$ & $\$ 4,761$ & $\$ 169$ & & $\$ 0$ & $\$ 4,930$ \\
\hline 110137 & 3" PLAST FIELD WELD & & $144.00 \mathrm{e}$ & each & 1.20 & 0.96 & 138 & $\$ 87.01$ & & $\$ 12,028$ & $\$ 0$ & & $\$ 0$ & $\$ 12,028$ \\
\hline 110411 & 3" Supports, Standard 3" & & $200.00 \mathrm{e}$ & each & 1.20 & 3.72 & 744 & $\$ 87.01$ & $\$ 32.43$ & $\$ 64,734$ & $\$ 6,486$ & & $\$ 0$ & $\$ 71,220$ \\
\hline
\end{tabular}


Client: INL

Project: FFC

Location: GREEN FIELD SITE HIP Option

Account: 11 Piping

Facility: Entire Estimate

URS

Washington Division
Date: 7/17/2008

11:31AM

Priced By: ADC

Office: Denver

Product Line: Gov
Estimate: FFC

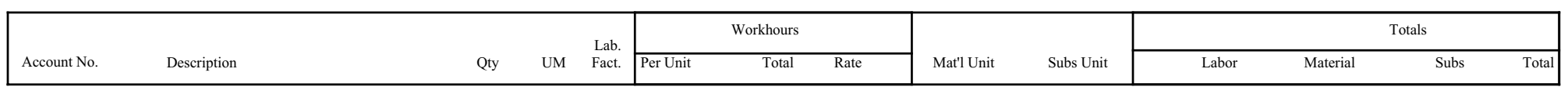

Plastic (800s) Floor Drain Pipe (100 lnft per fl

$2.50 \quad$ Ftgs \& Vlvs/100 LF Pipe

0.00 LF/Valve

0.72 Workhours/LF

$\$ 66.30$ Total Cost/LF

\begin{tabular}{|c|c|c|c|c|c|c|c|c|c|c|c|c|c|c|}
\hline 11: Piping & $\begin{array}{l}\text { Plastic (800s) Floor Drain Pipe (100 lnft } \\
\text { per floor drain) }\end{array}$ & 3.000 & 2,400 & LF & 1.20 & 0.72 & 1,723 & $\$ 87.01$ & & $\begin{array}{l}\$ 149,933 \\
\$ 62.47 / L F\end{array}$ & $\begin{array}{r}\$ 9, \mathbf{1 8 0} \\
\$ 3.83 / \mathrm{LF}\end{array}$ & $\$ 0.00$ & $\begin{array}{l}\mathbf{S 0} \\
/ \mathrm{LF}\end{array}$ & $\$ 159,113$ \\
\hline \multicolumn{15}{|c|}{ Plastic (800s) Floor Drain Pipe (100 Inft per floor drain) HIP Area } \\
\hline 110116 & 3" PIPE, SCH 40 PVC & & 200.00 & $\operatorname{lnft}$ & 1.20 & 0.31 & 62 & $\$ 87.01$ & $\$ 0.98$ & $\$ 5,429$ & $\$ 196$ & & $\$ 0$ & $\$ 5,625$ \\
\hline 110126 & 3" 90 ELL S40 PVC SW & & 2.00 & each & 1.20 & 1.56 & 3 & $\$ 87.01$ & $\$ 4.39$ & $\$ 271$ & $\$ 9$ & & $\$ 0$ & $\$ 280$ \\
\hline 110126 & 3" 45 ELL S40 PVC SW & & 1.00 & each & 1.20 & & & $\$ 87.01$ & $\$ 5.70$ & so & $\$ 6$ & & $\$ 0$ & $\$ 6$ \\
\hline 110126 & 3" WYE S40 PVC SW & & 2.00 & each & 1.20 & 2.28 & 5 & $\$ 87.01$ & $\$ 7.03$ & $\$ 397$ & $\$ 14$ & & $\$ 0$ & $\$ 411$ \\
\hline 110137 & 3" PLAST FIELD WELD & & 16.00 & each & 1.20 & 0.96 & 15 & $\$ 87.01$ & & $\$ 1,336$ & $\$ 0$ & & $\$ 0$ & $\$ 1,336$ \\
\hline 110411 & 3" Supports, Standard 3" & & 16.00 & each & 1.20 & 3.72 & 60 & $\$ 87.01$ & $\$ 32.43$ & $\$ 5,179$ & $\$ 519$ & & $\$ 0$ & $\$ 5,698$ \\
\hline
\end{tabular}

Plastic (800s) Floor Drain Pipe (100 lnft per fl

$2.50 \quad$ Ftgs \& Vlvs/100 LF Pipe

$0.00 \mathrm{LF} /$ Valve

0.72 Workhours/LF

$\$ 66.78$ Total Cost/LF

\begin{tabular}{|c|c|c|c|c|c|c|c|c|c|c|c|c|c|c|}
\hline 11: Piping & $\begin{array}{l}\text { Plastic (800s) Floor Drain Pipe (100 lnft } \\
\text { per floor drain) HIP Area }\end{array}$ & 3.000 & & $0 \mathbf{L F}$ & 1.20 & 0.72 & 145 & $\$ 87.01$ & & $\begin{array}{c}\$ 12,613 \\
\$ 63.06 / L F\end{array}$ & $\begin{array}{ll} & \$ 743 \\
\$ 3.72 / L F & \end{array}$ & $\$ 0.00$ & $\begin{array}{l}\mathbf{S 0} \\
/ \mathrm{LF}\end{array}$ & $\$ 13,356$ \\
\hline \multicolumn{15}{|c|}{ Plastic (800s) Outside Sanitary Sewer Line } \\
\hline 110116 & 6" PIPE, SCH 40 PVC & & 475.00 & $\operatorname{lnft}$ & 1.20 & 0.46 & 217 & $\$ 87.01$ & $\$ 2.56$ & $\$ 18,846$ & $\$ 1,216$ & & $\$ 0$ & $\$ 20,062$ \\
\hline 110126 & 6" 90 ELL S40 PVC SW & & 6.00 & each & 1.20 & 3.12 & 19 & $\$ 87.01$ & $\$ 24.97$ & $\$ 1,629$ & $\$ 150$ & & $\$ 0$ & $\$ 1,779$ \\
\hline 110126 & 6" 45 ELL S40 PVC SW & & 2.00 & each & 1.20 & & & $\$ 87.01$ & $\$ 25.26$ & $\$ 0$ & $\$ 51$ & & $\$ 0$ & $\$ 51$ \\
\hline 110126 & 6" TEE S40 PVC SW & & 1.00 & each & 1.20 & 4.68 & 5 & $\$ 87.01$ & $\$ 39.23$ & $\$ 407$ & $\$ 39$ & & $\$ 0$ & $\$ 446$ \\
\hline 110126 & 6" RED S40 PVC SW & & 1.00 & each & 1.20 & 3.12 & 3 & $\$ 87.01$ & $\$ 11.80$ & $\$ 271$ & $\$ 12$ & & $\$ 0$ & $\$ 283$ \\
\hline 110121 & Out of Spec. - Unions & & 4.00 & each & 1.20 & & & $\$ 87.01$ & & \$0 & $\$ 0$ & & $\$ 0$ & $\$ 0$ \\
\hline 110126 & 6" FLG SCH 40 SW PVC & & 31.00 & each & 1.20 & 1.56 & 48 & $\$ 87.01$ & $\$ 19.79$ & $\$ 4,208$ & $\$ 613$ & & $\$ 0$ & $\$ 4,821$ \\
\hline 110126 & 6" LG. INLINE Tie In to Existing & & 1.00 & each & 1.20 & 14.28 & 14 & $\$ 87.01$ & & $\$ 1,242$ & $\$ 0$ & & $\$ 0$ & $\$ 1,242$ \\
\hline
\end{tabular}




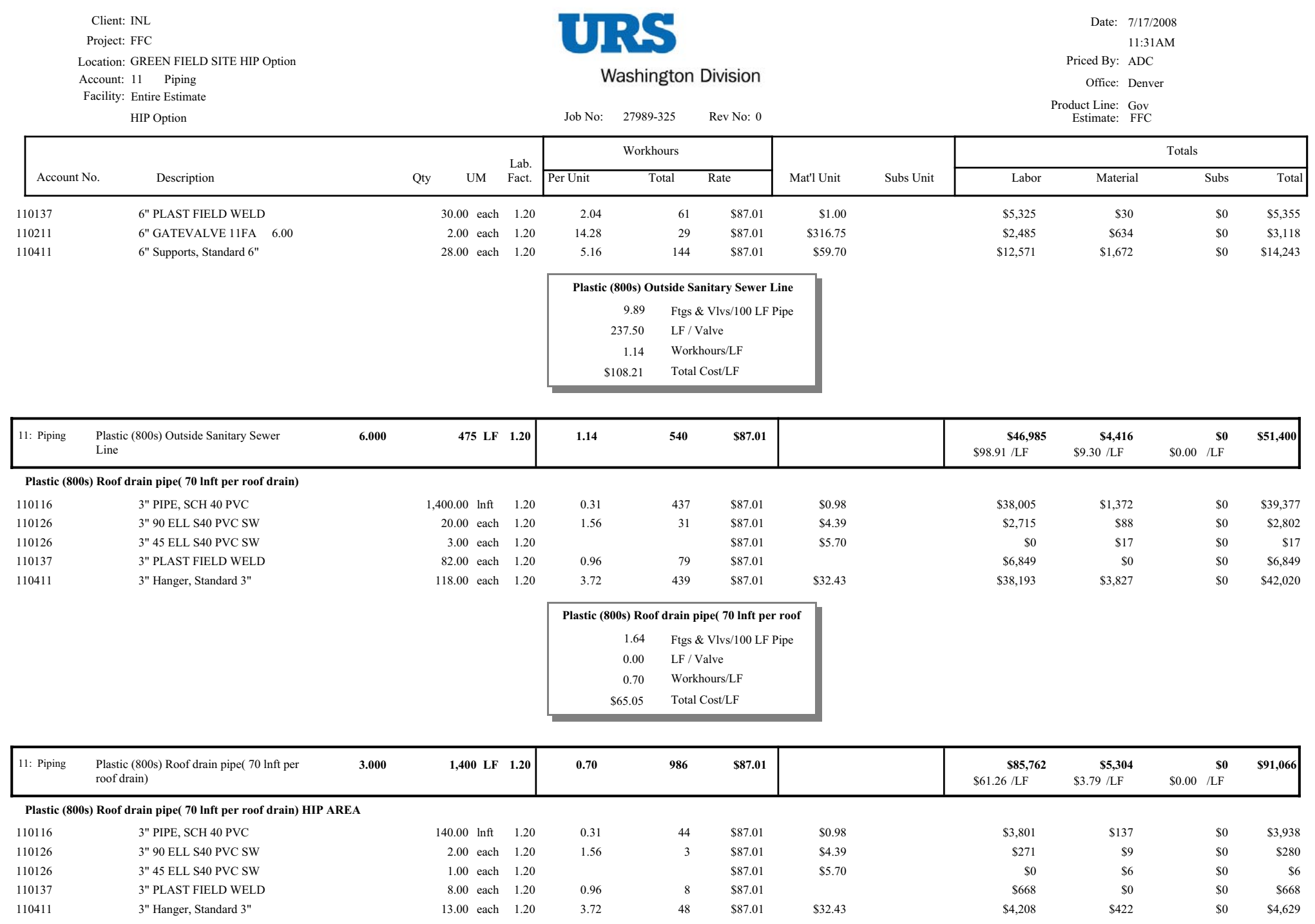


Client: INL

Project: FFC

Location: GREEN FIELD SITE HIP Option

Account: 11 Piping

Facility: Entire Estimate

URS

Washington Division
Date: $7 / 17 / 2008$

11:31AM

Priced By: ADC

Office: Denver

Product Line: Gov
Estimate: FFC

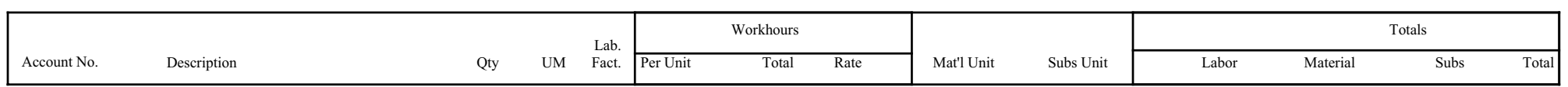

Plastic (800s) Roof drain pipe( 70 lnft per roof

2.14 Ftgs \& Vlvs/100 LF Pipe

0.00 LF/Valve

0.73 Workhours/LF

$\$ 68.01 \quad$ Total Cost/LF

\begin{tabular}{|c|c|c|c|c|c|c|c|c|c|c|c|c|c|c|}
\hline 11: Piping & $\begin{array}{l}\text { Plastic }(800 \mathrm{~s}) \text { Roof drain pipe( } 70 \mathrm{lnft} \text { per } \\
\text { roof drain) HIP AREA }\end{array}$ & 3.000 & & o LF & 1.20 & 0.73 & 103 & $\$ 87.01$ & & $\begin{array}{l}\mathbf{\$ 8 , 9 4 8} \\
\$ 63.91 / \mathrm{LF}\end{array}$ & $\begin{array}{l}\$ 573 \\
\$ 4.09 / \mathrm{LF}\end{array}$ & $\$ 0.00 /$ & $\begin{array}{l}\$ 0 \\
/ \mathrm{LF}\end{array}$ & $\$ 9,521$ \\
\hline \multicolumn{15}{|c|}{ Plastic (800s) Sanitary Sewer piping (50 Inft per fixture) } \\
\hline 110116 & 3" PIPE, SCH 40 PVC & & $1,000.00$ & $\operatorname{lnft}$ & 1.20 & 0.31 & 312 & $\$ 87.01$ & $\$ 0.98$ & $\$ 27,147$ & $\$ 980$ & & $\$ 0$ & $\$ 28,127$ \\
\hline 110126 & 3" 90 ELL S40 PVC SW & & 50.00 & each & 1.20 & 1.56 & 78 & $\$ 87.01$ & $\$ 4.39$ & $\$ 6,787$ & $\$ 220$ & & $\$ 0$ & $\$ 7,006$ \\
\hline 110126 & 3" 45 ELL S40 PVC SW & & 6.00 & each & 1.20 & & & $\$ 87.01$ & $\$ 5.70$ & so & $\$ 34$ & & $\$ 0$ & $\$ 34$ \\
\hline 110126 & 3" TEE S40 PVC SW & & 30.00 & each & 1.20 & 2.28 & 68 & $\$ 87.01$ & $\$ 7.03$ & $\$ 5,951$ & $\$ 211$ & & $\$ 0$ & $\$ 6,162$ \\
\hline 110126 & 3" RED S40 PVC SW & & 20.00 & each & 1.20 & 1.56 & 31 & $\$ 87.01$ & $\$ 2.13$ & $\$ 2,715$ & $\$ 43$ & & $\$ 0$ & $\$ 2,757$ \\
\hline 110126 & 3" CAP S40 PVC SW & & 6.00 & each & 1.20 & 0.84 & 5 & $\$ 87.01$ & $\$ 2.15$ & $\$ 439$ & $\$ 13$ & & $\$ 0$ & $\$ 451$ \\
\hline 110126 & 3" FLG SCH 40 SW PVC & & 91.00 & each & 1.20 & 0.84 & 76 & $\$ 87.01$ & $\$ 9.95$ & $\$ 6,651$ & $\$ 905$ & & $\$ 0$ & $\$ 7,556$ \\
\hline 110137 & 3" PLAST FIELD WELD & & 389.00 & each & 1.20 & 0.96 & 373 & $\$ 87.01$ & & $\$ 32,492$ & $\$ 0$ & & $\$ 0$ & $\$ 32,492$ \\
\hline 110211 & 3" GATEVALVE 11FA $\quad 3.00$ & & 30.00 & each & 1.20 & 9.36 & 281 & $\$ 87.01$ & $\$ 135.80$ & $\$ 24,432$ & $\$ 4,074$ & & $\$ 0$ & $\$ 28,506$ \\
\hline 110411 & 3" Hanger, Standard 3" & & 84.00 & each & 1.20 & 3.72 & 312 & $\$ 87.01$ & $\$ 32.43$ & $\$ 27,188$ & $\$ 2,724$ & & $\$ 0$ & $\$ 29,912$ \\
\hline
\end{tabular}

Plastic (800s) Sanitary Sewer piping (50 Inft pe

23.30 Ftgs \& Vlvs/100 LF Pipe

$33.33 \mathrm{LF} /$ Valve

1.54 Workhours/LF

$\$ 143.01 \quad$ Total Cost/LF

\begin{tabular}{|c|c|c|c|c|c|c|c|c|c|c|c|c|c|c|}
\hline 11: Piping & $\begin{array}{l}\text { Plastic (800s) Sanitary Sewer piping ( } 50 \\
\text { Inft per fixture) }\end{array}$ & 3.000 & 1,000 & 0 LF & 1.20 & 1.54 & 1,538 & $\$ 87.01$ & & $\begin{array}{l}\mathbf{\$ 1 3 3 , 8 0 1} \\
\$ 133.80 / \mathrm{LF}\end{array}$ & $\begin{array}{l}\mathbf{\$ 9 , 2 0 4} \\
\$ 9.20 / \mathrm{LF}\end{array}$ & $\$ 0.00$ & $\begin{array}{l}\$ 0 \\
/ L F\end{array}$ & $\$ 143,005$ \\
\hline \multicolumn{15}{|c|}{ Stainless Steel (700s) Argon Supply Piping } \\
\hline 110111 & 1" PIPE,S40 316L SMLS & & 150.00 & $\operatorname{lnft}$ & 1.20 & 0.49 & 74 & $\$ 87.01$ & $\$ 6.28$ & $\$ 6,421$ & $\$ 941$ & & $\$ 0$ & $\$ 7,363$ \\
\hline 110121 & 1" 90 ELL 316L 3000\# SW & & 13.00 & each & 1.20 & 0.96 & 12 & $\$ 87.01$ & $\$ 16.39$ & $\$ 1,086$ & $\$ 213$ & & $\$ 0$ & $\$ 1,299$ \\
\hline 110121 & 1" 45 ELL 316L 3000\# SW & & 1.00 & each & 1.20 & 0.96 & 1 & $\$ 87.01$ & $\$ 21.01$ & $\$ 84$ & $\$ 21$ & & $\$ 0$ & $\$ 105$ \\
\hline 110121 & 1" TEE 316L 3000\# SW & & 8.00 & each & 1.20 & 1.44 & 12 & $\$ 87.01$ & $\$ 21.70$ & $\$ 1,002$ & $\$ 174$ & & $\$ 0$ & $\$ 1,176$ \\
\hline 110121 & 1" RED 316L 3000\# SW & & 5.00 & each & 1.20 & 0.96 & 5 & $\$ 87.01$ & $\$ 11.56$ & $\$ 418$ & $\$ 58$ & & $\$ 0$ & $\$ 475$ \\
\hline
\end{tabular}


Client: INL

Project: FFC

Location: GREEN FIELD SITE HIP Option

Account: 11 Piping

Facility: Entire Estimate

URS

Washington Division
Date: $7 / 17 / 2008$

11:31AM

Priced By: ADC

Office: Denver

Product Line: Gov
Estimate: FFC

\begin{tabular}{|c|c|c|c|c|c|c|c|c|c|c|c|c|c|}
\hline \multirow[b]{2}{*}{ Account No. } & \multirow[b]{2}{*}{ Description } & \multirow[b]{2}{*}{ Qty } & \multirow[b]{2}{*}{ UM } & \multirow{2}{*}{$\begin{array}{l}\text { Lab. } \\
\text { Fact. }\end{array}$} & \multicolumn{3}{|c|}{ Workhours } & \multirow[b]{2}{*}{ Mat'l Unit } & \multirow[b]{2}{*}{ Subs Unit } & \multicolumn{4}{|c|}{ Totals } \\
\hline & & & & & Per Unit & Total & Rate & & & Labor & Material & Subs & Total \\
\hline 110121 & 1" CAP 316L 3000\# SW & & 1.00 each & 1.20 & 0.48 & 0 & $\$ 87.01$ & $\$ 7.66$ & & $\$ 42$ & $\$ 8$ & $\$ 0$ & $\$ 49$ \\
\hline 110121 & 1" SS 316L 600\# RF SW & & 24.00 each & 1.20 & 0.48 & 12 & $\$ 87.01$ & $\$ 60.00$ & & $\$ 1,002$ & $\$ 1,440$ & $\$ 0$ & $\$ 2,442$ \\
\hline 110132 & 1" FIELD WELD, SCH 40 & & 95.00 each & 1.20 & 0.92 & 88 & $\$ 87.01$ & & & $\$ 7,638$ & $\$ 0$ & $\$ 0$ & $\$ 7,638$ \\
\hline 110206 & 1" GATEVALVE 73SA 1.00 & & 13.00 each & 1.20 & 2.76 & 36 & $\$ 87.01$ & $\$ 293.35$ & & $\$ 3,122$ & $\$ 3,814$ & $\$ 0$ & $\$ 6,935$ \\
\hline 110406 & 1" Hanger, Standard 1" & & 15.00 each & 1.20 & 2.40 & 36 & $\$ 87.01$ & $\$ 27.53$ & & $\$ 3,132$ & $\$ 413$ & $\$ 0$ & $\$ 3,545$ \\
\hline
\end{tabular}

Stainless Steel (700s) Argon Supply Piping

43.33 Ftgs \& Vlvs/100 LF Pipe

$11.54 \mathrm{LF} /$ Valve

1.83 Workhours/LF

$\$ 206.85$ Total Cost/LF

Cost

\begin{tabular}{|c|c|c|c|c|c|c|c|c|c|c|c|c|c|c|}
\hline 11: Piping & $\begin{array}{l}\text { Stainless Steel (700s) Argon Supply } \\
\text { Piping }\end{array}$ & 1.000 & & $0 \mathbf{L F}$ & 1.20 & 1.83 & 275 & $\$ 87.01$ & & $\begin{array}{l}\text { \$23,946 } \\
\$ 159.64 / \mathrm{LF}\end{array}$ & $\begin{array}{l}\$ \mathbf{\$ 7 , 0 8 1} \\
\$ 47.21 / \mathrm{LF}\end{array}$ & $\$ 0.00$ & $\begin{array}{l}\mathbf{s 0} \\
/ \mathrm{LF}\end{array}$ & $\$ 31,028$ \\
\hline \multicolumn{15}{|c|}{ Stainless Steel (700s) Argon Supply Piping for HIP Sys } \\
\hline 110111 & 1" PIPE,S40 316L SMLS & & 150.00 & $\operatorname{lnft}$ & 1.20 & 0.49 & 74 & $\$ 87.01$ & $\$ 6.28$ & $\$ 6,421$ & $\$ 941$ & & $\$ 0$ & $\$ 7,363$ \\
\hline 110121 & 1" 90 ELL 316L 3000\# SW & & 13.00 & each & 1.20 & 0.96 & 12 & $\$ 87.01$ & $\$ 16.39$ & $\$ 1,086$ & $\$ 213$ & & $\$ 0$ & $\$ 1,299$ \\
\hline 110121 & 1" 45 ELL 316L 3000\# SW & & 1.00 & each & 1.20 & 0.96 & 1 & $\$ 87.01$ & $\$ 21.01$ & $\$ 84$ & $\$ 21$ & & $\$ 0$ & $\$ 105$ \\
\hline 110121 & 1" TEE 316L 3000\# SW & & 8.00 & each & 1.20 & 1.44 & 12 & $\$ 87.01$ & $\$ 21.70$ & $\$ 1,002$ & $\$ 174$ & & $\$ 0$ & $\$ 1,176$ \\
\hline 110121 & 1" RED 316L 3000\# SW & & 5.00 & each & 1.20 & 0.96 & 5 & $\$ 87.01$ & $\$ 11.56$ & $\$ 418$ & $\$ 58$ & & $\$ 0$ & $\$ 475$ \\
\hline 110121 & 1" CAP 316L 3000\# SW & & 1.00 & each & 1.20 & 0.48 & 0 & $\$ 87.01$ & $\$ 7.66$ & $\$ 42$ & $\$ 8$ & & $\$ 0$ & $\$ 49$ \\
\hline 110121 & 1" SS 316L 600\# RF SW & & 24.00 & each & 1.20 & 0.48 & 12 & $\$ 87.01$ & $\$ 60.00$ & $\$ 1,002$ & $\$ 1,440$ & & $\$ 0$ & $\$ 2,442$ \\
\hline 110132 & 1" FIELD WELD, SCH 40 & & 95.00 & each & 1.20 & 0.92 & 88 & $\$ 87.01$ & & $\$ 7,638$ & $\$ 0$ & & $\$ 0$ & $\$ 7,638$ \\
\hline 110206 & 1" GATEVALVE 73SA 1.00 & & 13.00 & each & 1.20 & 2.76 & 36 & $\$ 87.01$ & $\$ 293.35$ & $\$ 3,122$ & $\$ 3,814$ & & $\$ 0$ & $\$ 6,935$ \\
\hline 110406 & 1" Hanger, Standard 1" & & 15.00 & each & 1.20 & 2.40 & 36 & $\$ 87.01$ & $\$ 27.53$ & $\$ 3,132$ & $\$ 413$ & & $\$ 0$ & $\$ 3,545$ \\
\hline
\end{tabular}

Stainless Steel (700s) Argon Supply Piping for

43.33 Ftgs \& Vlvs/100 LF Pipe

$11.54 \mathrm{LF} /$ Valve

1.83 Workhours/LF

$\$ 206.85$ Total Cost $/ \mathrm{LF}$

\begin{tabular}{|c|c|c|c|c|c|c|c|c|c|c|c|}
\hline 11: Piping & $\begin{array}{l}\text { Stainless Steel (700s) Argon Supply } \\
\text { Piping for HIP Sys }\end{array}$ & 1.000 & $\begin{array}{lll}150 & \text { LF } & 1.20\end{array}$ & 1.83 & 275 & $\$ 87.01$ & $\begin{array}{l}\mathbf{\$ 2 3 , 9 4 6} \\
\$ 159.64 / \mathrm{LF}\end{array}$ & $\begin{array}{r}\mathbf{\$ 7 , 0 8 1} \\
\$ 47.21 / \mathrm{LF}\end{array}$ & $\$ 0.00$ & $\begin{array}{l}\text { so } \\
/ \mathrm{LF}\end{array}$ & $\$ 31,028$ \\
\hline
\end{tabular}

Utilities 
Client: INL

Project: FFC
URS

Washington Division
Date: 7/17/2008

11:31AM Priced By: ADC

Office: Denver

Product Line: Gov
Estimate: FFC

Job No: $\quad 27989-325 \quad$ Rev No: 0

Etimate: FFC

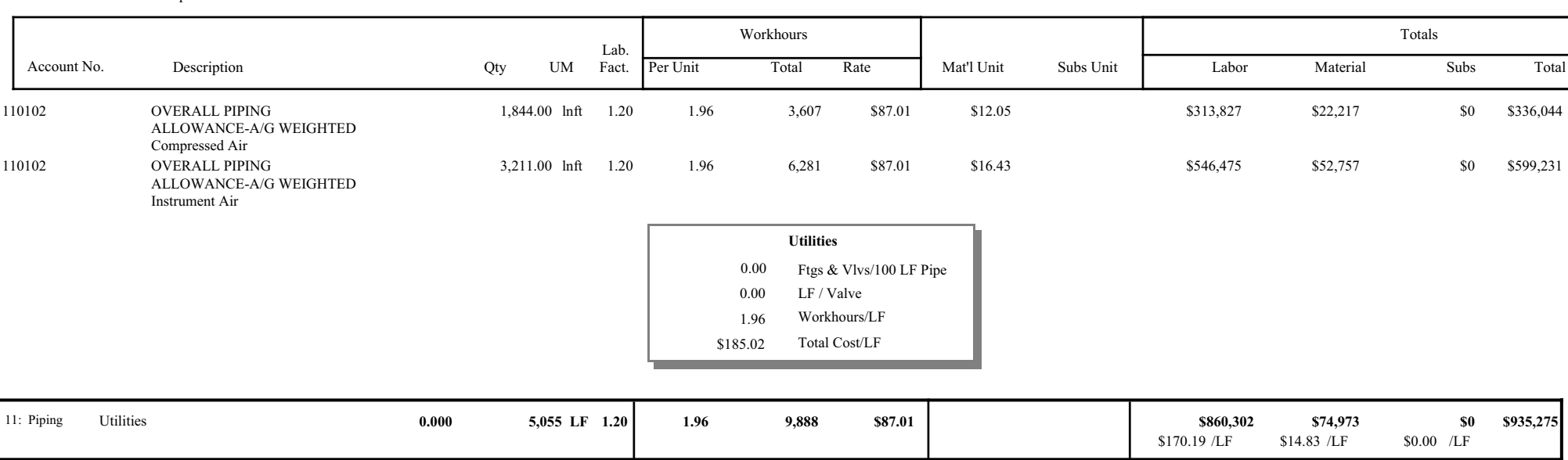

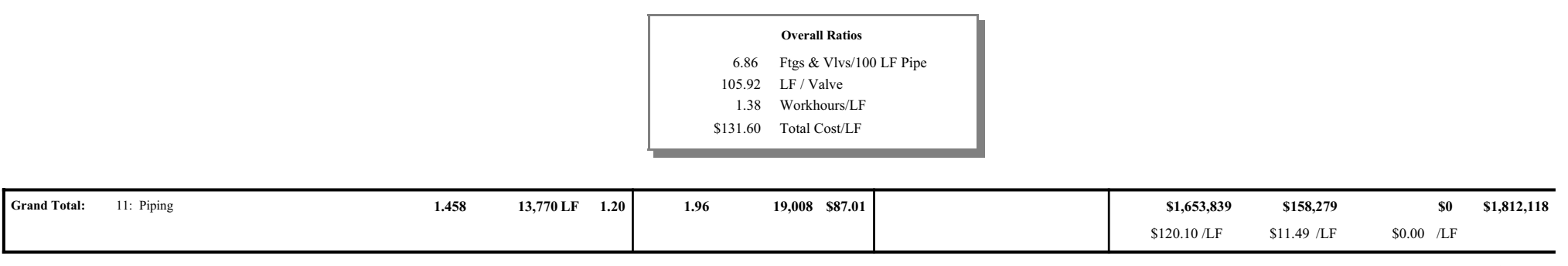




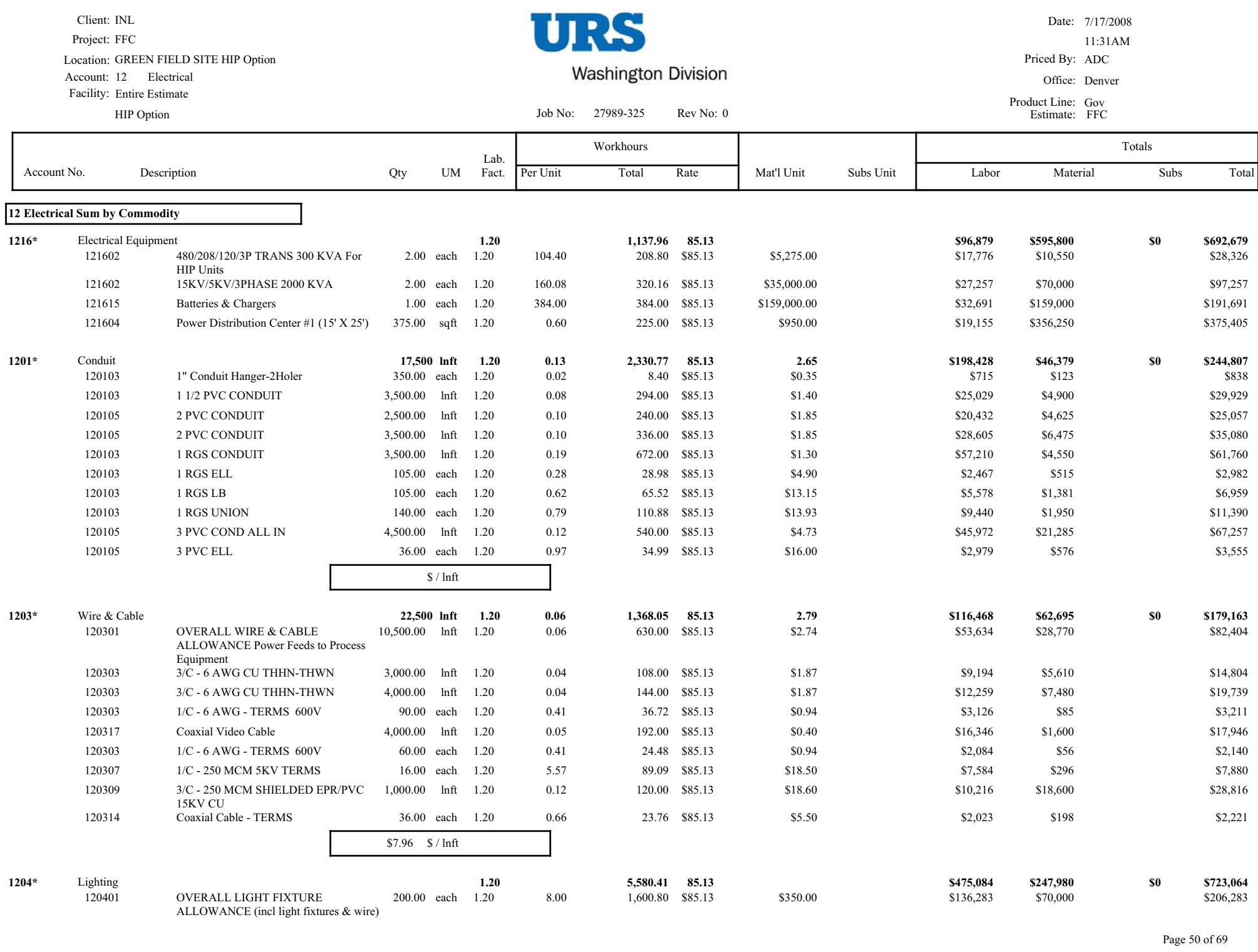




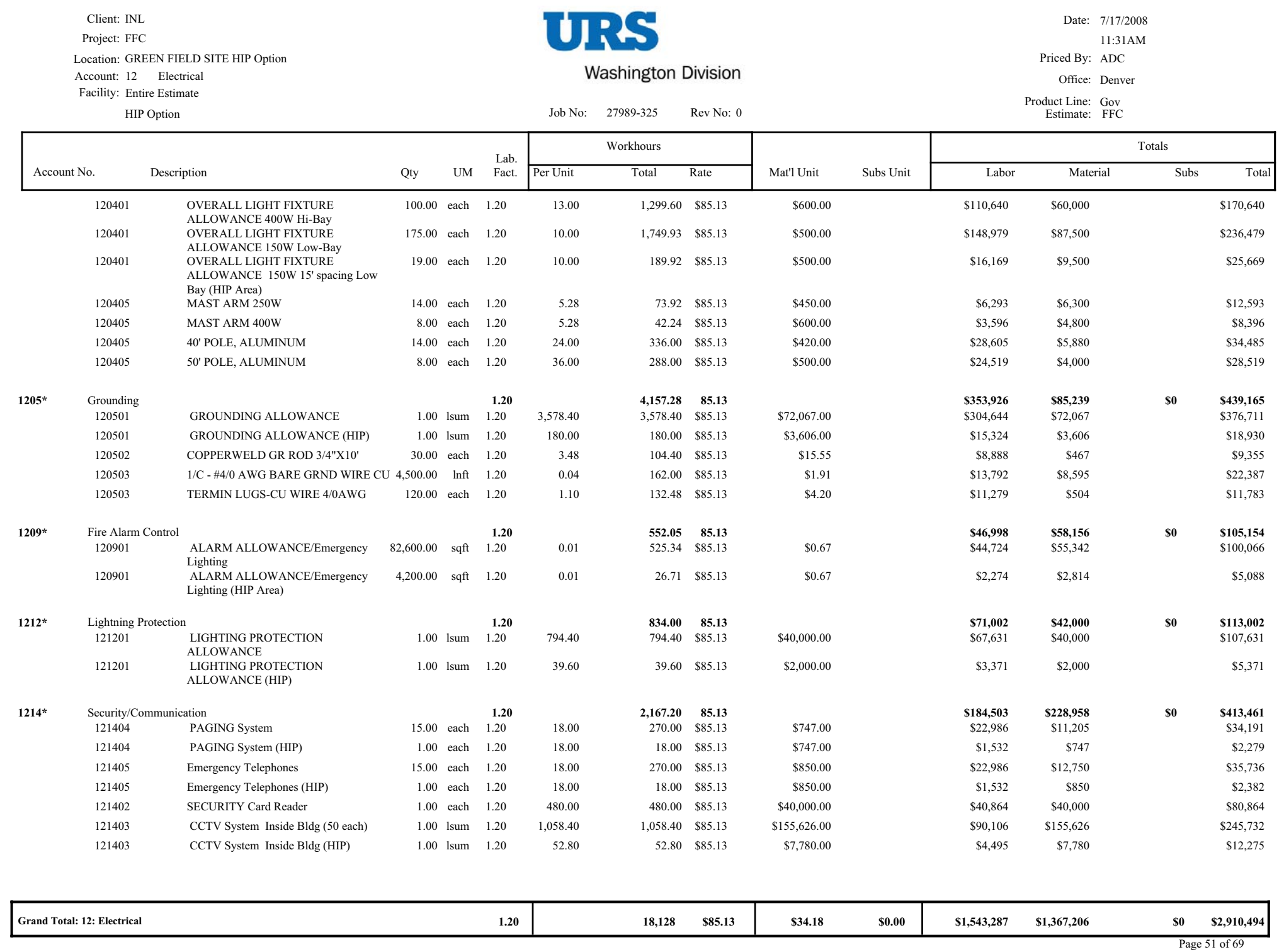


Client: INL

Project: FFC
URS

Washington Division

Job No: $\quad 27989-325 \quad$ Rev No: 0

Workhours

Qty UM Fact.

Per Unit
Date: 7/17/2008

11:31AM

Priced By: ADC

Office: Denver

$\begin{aligned} \text { Product Line: } & \text { Gov } \\ \text { Estimate: } & \text { FFC }\end{aligned}$

Account No.

Description

12.01 Electrical Equipment

Elec. Equip.-Transformers

15KV/5KV/3PHASE $2000 \mathrm{KVA}$

Elec. Equip.-Transformers HIP Area

121602 480/208/120/3P TRANS 300 KVA For HIP Units

Electrical Equipment

$121615 \quad$ Batteries \& Chargers

ELECTRICAL SUBSTATION

121604

Power Distribution Center \#1 (15' X 25')

$\begin{array}{rrrrrrrr}2 & \text { each } & 1.20 & 160.08 & 320.16 & \mathbf{3 8 5 . 1 3} & \\ & & & & \mathbf{2 0 9} & \mathbf{\$ 8 5 . 1 3} & 35,000.00 \\ 2 & \text { each } & 1.20 & 104.40 & 208.80 & 85.13 & 5,275.00 \\ & & & & \mathbf{3 8 4} & \mathbf{\$ 8 5 . 1 3} & \\ 1 & \text { each } & 1.20 & 384.00 & 384.00 & 85.13 & 159,000.00 \\ & & & & \mathbf{2 2 5} & \mathbf{\$ 8 5 . 1 3} & \\ 375 & \text { sqft } & 1.20 & 0.60 & 225.00 & 85.13 & 950.00\end{array}$

\begin{tabular}{l|l|} 
Mat'l Unit $\quad$ Subs Unit & \\
&
\end{tabular}

$\$ 27,257$

$\$ 70,000$

$\$ 27,257 \quad \$ 70,000$

$\$ 17,776 \quad \$ 10,550$

$\$ 17,776 \quad \$ 10,550$

$\$ 32,691$

$\mathbf{\$ 1 9 , 1 5 5} \quad \mathbf{\$ 3 5 6 , 2 5 0}$

$\$ 19,155 \quad \$ 356,250$

\begin{tabular}{|c|c|c|c|}
\hline \multicolumn{4}{|c|}{ Totals } \\
\hline Labor & Material & Subs & Total \\
\hline
\end{tabular}


Client: INL

Project: FFC

URS

Date: 7/17/2008

11:31AM

Location: GREEN FIELD SITE HIP Option

Washington Division

Priced By: ADC

Office: Denver

Facility: Entire Estimate

Job No: $\quad 27989-325 \quad$ Rev No: 0

Product Line: Gov
Estimate: FFC

HIP Optio

Workous

\begin{tabular}{|c|c|c|c|c|}
\hline \multirow[b]{2}{*}{ Mat'l Unit } & \multirow[b]{2}{*}{ Subs Unit } & \multicolumn{3}{|c|}{ Totals } \\
\hline & & Labor & Material & Total \\
\hline
\end{tabular}

Account No.

Description

Qty UM Fact.

Per Unit Total Rate

$3,500.00 \quad \mathbf{L F}$

$\begin{array}{ll}3,500.00 & \text { lnft } \\ 3,500.00 & \text { LF }\end{array}$

$\begin{array}{llll}3,500.00 \quad \text { LF } & 1.20 & 0.10\end{array}$

$3,500.00$

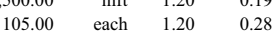

105.00 each $1.20 \quad 0.62$

$\begin{array}{llll}140.00 & \text { each } & 1.20 & 0.79 \\ 350.00 & \text { each } & 1.20 & 0.02\end{array}$

$4,500.00 \quad$ LF

$\begin{array}{llll}4,500.00 & \text { Inft } & 1.20 & 0.12\end{array}$

$\begin{array}{rrrr} & & & \\ 36.00 & \text { each } & 1.20 & 0.12 \\ & & 1.20 & 0.97\end{array}$

36.00
$2,500.00$

$\begin{array}{llll}2,500.00 & \text { Inft } & 1.20 & 0.10\end{array}$

$3,500.00$

$\begin{array}{llll}3,500.00 & \text { lnft } & 1.20 & 0.08\end{array}$

$\begin{aligned} \mathbf{3 3 6} & \\ 336.00 & \$ 85.13 \\ \mathbf{8 8 6} & \\ 672.00 & \$ 85.13 \\ 28.98 & \$ 85.13 \\ 65.52 & \$ 85.13 \\ 110.88 & \$ 85.13 \\ 8.40 & \$ 85.13 \\ \mathbf{5 7 5} & \\ 540.00 & \$ 85.13 \\ 34.99 & \$ 85.13 \\ \mathbf{2 4 0} & \\ 240.00 & \$ 85.13 \\ \mathbf{2 9 4} & \\ 294.00 & \$ 85.13\end{aligned}$

$\$ 1.85$

$\$ 1.30$
$\$ 4.90$
$\$ 13.15$
$\$ 13.93$
$\$ 0.35$

$\$ 4.73$
$\$ 16.00$

$\$ 1.85$
$\$ 1.40$

28,605

$\$ 28,605$

6,475
$\$ 6,475$

$\$ 6,475$

75,410

$\$ 57,210$

$\$ 2,467$

$\$ 5,578$

$\$ 9,440$
$\$ 715$

48,951

$\$ 45,972$

$\$ 45,972$
$\$ 2,979$

20,432

$\$ 20,432$
$\mathbf{2 5 , 0 2 9}$

1 1/2 PVC CONDUIT

$\$ 85.13$

$\$ 1.40$

$\$ 25,029$

$\$ 4,550$

$\$ 4,550$
$\$ 515$

$\$ 1,381$

$\$ 1,950$

$\$ 123$

21,861

$\$ 21,285$

$\$ 576$

4,625
$\$ 4,625$

$\$, 900$
44,96

$\$ 4,900$

$\mathbf{0}$
$\$ 0$
$\mathbf{0}$
$\$ 0$
$\$ 0$
$\$ 0$
$\$ 0$
$\$ 0$
$\mathbf{0}$
$\$ 0$
$\$ 0$
$\mathbf{0}$
$\$ 0$
$\mathbf{0}$
$\$ 0$

$\mathbf{3 5 , 0 8 0}$

$\$ 35,080$

83,928

$\$ 61,760$

$\$ 2,982$

$\$ 6,959$

$\$ 11,390$

$\$ 838$
$\mathbf{7 0 , 8 1 2}$

$\$ 67,257$

$\$ 3,555$

$\mathbf{2 5 , 0 5 7}$

$\$ 25,057$

29,929

$\$ 29,929$

$\$ 2.65$ Material Cost/LF

$\$ 13.99$ Total Cost/LF

Grand Total:

$17,500 \quad$ LF

$2,331 \quad \$ 85.13$

$\$ 198,428$

$\$ 46,379$

$\$ 2.65 / \mathrm{LF}$

$\begin{array}{ll}\$ 0 & \$ 244,807\end{array}$

$\$ 11.34 / \mathrm{LF}$ $\$ 0.00 / \mathrm{LF}$

Page 53 of 69 
Client: INL

Project: FFC

Location: GREEN FIELD SITE HIP Option

Account: 12 Electrical

URS

Date: 7/17/2008

11:31AM

Washington Division

Priced By: ADC

Office: Denver

Facility: Entire Estimate

Job No: $\quad 27989-325 \quad$ Rev No: 0

Product Line: Gov
Estimate: FFC

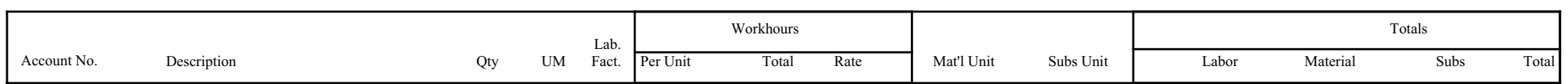

12.03 Electrical Wire/Cable

$\begin{array}{ll}120303 & \text { AREA FENCE LIGHTING CABLE } \\ 120303 & \text { 3/C - 6 AWG CU THHN-THWN } \\ & \text { 1/C - 6 AWG - TERMS 600V } \\ & \text { Power Feeds Process Area } \\ 120301 & \text { OVERALL WIRE \& CABLE ALLOWANCE } \\ & \text { Power Feeds to Process Equipment } \\ & \text { ROADWAY LIGHTING CABLE } \\ 120303 & \text { 3/C - 6 AWG CU THHN-THWN } \\ 120303 & \text { 1/C - 6 AWG - TERMS 600V } \\ & \text { SECURITY CAMERA CABLE } \\ 120314 & \text { Coaxial Cable - TERMS } \\ 120317 & \text { Coaxial Video Cable } \\ & \text { Wire \& Cable-Medium Voltage (5 \& 15kV) } \\ 120307 & \text { 1/C - 250 MCM 5KV TERMS } \\ 120309 & \text { 3/C - 250 MCM SHIELDED EPR/PVC 15KV } \\ & \text { CU }\end{array}$

$\begin{array}{rcccr}4,000 & \text { Inft } & & & \mathbf{1 6 8} \\ 4,000 & \text { Inft } & 1.20 & 0.04 & 144.00 \\ 60 & \text { ach } & 1.20 & 0.41 & 24.48 \\ 10,500 & \text { Inft } & & & \mathbf{6 3 0} \\ 10,500 & \text { Inft } & 1.20 & 0.06 & 630.00 \\ & & & & \mathbf{1 4 5} \\ 3,000 & \text { Inft } & & & 108.00 \\ 3,000 & \text { Inft } & 1.20 & 0.04 & 36.72 \\ 90 & \text { ach } & 1.20 & 0.41 & \mathbf{2 1 6} \\ 4,000 & \text { Inft } & & & 23.76 \\ 36 & \text { ach } & 1.20 & 0.66 & 192.00 \\ 4,000 & \text { Inft } & 1.20 & 0.05 & \mathbf{2 0 9} \\ 1,000 & \text { Inft } & & & 89.09 \\ 16 & \text { ach } & 1.20 & 5.57 & 120.00 \\ 1,000 & \text { Inft } & 1.20 & 0.12 & \end{array}$

85.13

85.13
85.13

85.13

85.13

1.87
0.94

85.13

85.13
85.13

85.13
85.13

85.13

85.13

85.13

85.13
85.13

85.13
85.13

2.74

1.87

1.87
0.94

0.94

5.50
0.40

18.50

18.50
18.60

$\mathbf{1 4 , 3 4 3}$
$\$ 12,259$
$\$ 2,084$
$\$ \mathbf{5 3 , 6 3 4}$
$\$ 53,634$
$\mathbf{\$ 1 2 , 3 2 1}$
$\$ 9,194$
$\$ 3,126$
$\mathbf{1 8 , 3 6 9}$
$\$ 2,023$
$\$ 16,346$
$\$ \mathbf{1 7 , 8 0 1}$
$\$ 7,584$
$\$ 10,216$

$\$ 7,536$

$\$ 7,480$

$\$ 7,480$
$\$ 56$

$\mathbf{\$ 2 8 , 7 7 0}$

$\$ 28,770$

$\$ 5,695$

$\$ 5,610$

$\$ 85$

$\mathbf{\$ 1 , 7 9 8}$

$\$ 198$

$\$ 1,600$

$\$ 18,896$
$\$ 296$

$\$ 18,600$

$\begin{array}{lr} & \\ \mathbf{\$ 0} & \mathbf{\$ 2 1 , 8 8 0} \\ \$ 0 & \$ 19,739 \\ \$ 0 & \$ 2,140 \\ \mathbf{\$ 0} & \mathbf{\$ 2 2 , 4 0 4} \\ \$ 0 & \$ 82,404 \\ & \\ \mathbf{\$ 0} & \mathbf{\$ 1 8 , 0 1 5} \\ \$ 0 & \$ 14,804 \\ \$ 0 & \$ 3,211 \\ \mathbf{\$ 0} & \mathbf{\$ 2 0 , 1 6 7} \\ \$ 0 & \$ 2,221 \\ \$ 0 & \$ 17,946 \\ \mathbf{\$ 0} & \mathbf{\$ 3 6 , 6 9 7} \\ \$ 0 & \$ 7,880 \\ \$ 0 & \$ 28,816\end{array}$

\begin{tabular}{|c|c|c|c|c|c|c|c|c|c|}
\hline & & & & $\begin{array}{r}\text { Overa } \\
0.06 \\
\$ 2.79 \\
\$ 7.96\end{array}$ & $\begin{array}{l}\text { I Ratios: } \\
\text { Workhours/LF } \\
\text { Material Cost/LF } \\
\text { Total Cost/LF }\end{array}$ & & & & \\
\hline Grand Total: Wire \& Cable & $22,500 \quad$ Inft & 1.20 & 0.06 & 85.13 & & $\$ 116,468$ & $\$ 62,695$ & \$0 & $\$ 179,163$ \\
\hline
\end{tabular}


Client: INL

Project: FFC

URS

Date: 7/17/2008

11:31AM

Location: GREEN FIELD SITE HIP Option

Washington Division

Priced By: ADC

Office: Denver

Facility: Entire Estimate

Product Line: Gov HIP Option

Job No: $\quad 27989-325 \quad$ Rev No: 0

Estimate: FFC

\begin{tabular}{|c|c|c|c|c|c|c|c|c|c|c|c|c|}
\hline \multirow[b]{2}{*}{ Account No. } & \multirow[b]{2}{*}{ Description } & \multirow[b]{2}{*}{ Qty } & \multirow[b]{2}{*}{ UM } & \multirow{2}{*}{$\begin{array}{l}\text { Lab. } \\
\text { Fact. }\end{array}$} & \multicolumn{2}{|c|}{ Workhours } & \multirow[b]{2}{*}{ Mat'l Unit } & \multirow[b]{2}{*}{ Subs Unit } & \multicolumn{4}{|c|}{ Totals } \\
\hline & & & & & Per Unit & Total & & & Labor & Material & Subs & Total \\
\hline
\end{tabular}

\section{3a Electical Wire/Cable Summary}

High Voltage

Cable

Terms

Ratio (LF / Term)

Cable + Terms (per LF wire)

$\begin{array}{rrrrr}4.4 \% & 1,000 & \text { LF } & 0.12 & 120 \\ 0 & \text { EA } & 0.00 & 0\end{array}$

0.00

Medium Voltage

Cable

Terms

Ratio (LF / Term)

Cable + Terms (per LF wire)

$0.12 \quad 120 \quad$ Per LF of Wire

Low Voltage / Control

Cable

Terms

Ratio (LF / Term)

Cable + Terms (per LF wire)

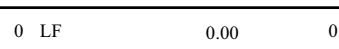

$0.0 \%$

$5.57 \quad 89$

0.00

0.00

$00 \quad 89 \quad$ Per LF of Wire

MIsc Wire/Cable

\begin{tabular}{|c|c|c|c|c|c|}
\hline Cable & $64.4 \%$ & $14,500 \quad$ LF & 0.06 & 822 & \\
\hline Terms & & $0 \quad$ EA & 0.00 & 0 & \\
\hline Ratio (LF / Term) & & 0.00 & & & \\
\hline Cable + Terms (per LF wire) & & & 0.06 & 822 & Per LF of Wire \\
\hline
\end{tabular}

Overall

\begin{tabular}{|lrrrrrr}
\hline Cable & $100.0 \%$ & 22,500 LF & 0.05 & 1,194 & \\
Terms & & 202 EA & 0.86 & 174 & \\
Ratio (LF / Term) & & 111.39 & & & & \\
Cable + Terms & & & 0.06 & 1,368 & Per LF of Wire \\
\hline
\end{tabular}


Client: INL

Project: FFC
URS

Washington Division
Date: 7/17/2008

$11: 31 \mathrm{AM}$

Priced By: ADC

Office: Denver

Product Line:
Estimate:

Job No: $\quad 27989-325 \quad$ Rev No: 0

\begin{tabular}{|c|c|c|c|c|}
\hline \multirow[b]{2}{*}{ Mat'l Unit } & \multirow[b]{2}{*}{ Subs Unit } & \multicolumn{3}{|c|}{ Totals } \\
\hline & & Labor & Material & Subs \\
\hline
\end{tabular}

2.04 Electrical Lightin

AREA FENCE LIGHTING

$120405 \quad$ MAST ARM $400 \mathrm{~W}$

120405 50' POLE, ALUMINUM

Finishes Office Area

$120401 \quad$ OVERALL LIGHT FIXTURE ALLOWANCE

(incl light fixtures \& wire)

Finishes Process Area

OVERALL LIGHT FIXTURE ALLOWANCE

400W Hi-Bay

OVERALL LIGHT FIXTURE ALLOWANCE
150W Low-Bay

150W Low-Bay
OVERALL LIGHT FIXTURE ALLOWANCE

150 W 15' spacing Low Bay (HIP Area)

ROADWAY LIGHTING

MAST ARM $250 \mathrm{~W}$

40' POLE, ALUMINUM

Qty UM Fact.

Per Unit

$\begin{array}{rrrrrrr}8.00 & \text { each } & 1.20 & 5.28 & 42.24 & \$ 85.13 & \\ 8.00 & \text { each } & 1.20 & 36.00 & 288.00 & \$ 85.13 & \$ 500.00 \\ & & & & 1,601 & \$ 85.13 & \\ 200.00 & \text { each } & 1.20 & 8.00 & 1,600.80 & \$ 85.13 & \$ 350.00 \\ & & & & 3,239 & \$ 85.13 & \\ 100.00 & \text { each } & 1.20 & 13.00 & 1,299.60 & \$ 85.13 & \$ 600.00 \\ 175.00 & \text { each } & 1.20 & 10.00 & 1,749.93 & \$ 85.13 & \$ 500.00 \\ 19.00 & \text { each } & 1.20 & 10.00 & 189.92 & \$ 85.13 & \$ 500.00 \\ & & & & & & \\ 14.00 & \text { each } & 1.20 & 5.28 & 73.92 & \$ 85.13 & \$ 450.00 \\ 14.00 & \text { each } & 1.20 & 24.00 & 336.00 & \$ 85.13 & \$ 420.00\end{array}$

$\begin{array}{rr}\$ 28,115 & \$ 8,800 \\ \$ 3,596 & \$ 4,800 \\ \$ 24,519 & \$ 4,000 \\ \$ 136,283 & \$ 70,000 \\ \$ 136,283 & \$ 70,000 \\ \$ 275,788 & \$ 157,000 \\ \$ 110,640 & \$ 60,000 \\ \$ 148,979 & \$ 87,500 \\ \$ 16,169 & \$ 9,500 \\ \$ 34,898 & \$ 12,180 \\ \$ 6,293 & \$ 6,300 \\ \$ 28,605 & \$ 5,880\end{array}$

\$0 $\$ 36,915$

$\$ 0 \quad \$ 8,396$

$\$ 0 \quad \$ 28,519$

$\$ 206,283$

$\$ 206,283$

$\$ 432,788$

$\$ 0 \$ 170,640$

$\$ 0 \$ 236,479$

$\$ 0 \quad \$ 25,669$

120405

$\$ 28,605$
$\$ 47,078$

$\begin{array}{ll}\$ 0 & \$ 12,593 \\ \$ 0 & \$ 34,485\end{array}$ 
Client: INL

Project: FFC

Location: GREEN FIELD SITE HIP Option

Account: 12 Electrical

URS

Date: 7/17/2008

11:31AM

Washington Division

Priced By: ADC

Office: Denver

Facility: Entire Estimate

Job No: $\quad 27989-325 \quad$ Rev No: 0

Product Line: Gov
Estimate: FFC

\begin{tabular}{|c|c|c|c|c|c|c|c|c|c|c|}
\hline \multirow[b]{2}{*}{ Account No. } & \multirow[b]{2}{*}{ Description } & \multirow[b]{2}{*}{ Qty } & \multirow[b]{2}{*}{ UM } & \multirow{2}{*}{$\begin{array}{l}\text { Lab. } \\
\text { Fact. }\end{array}$} & & rkhours & \multirow[b]{2}{*}{ Mat'l Unit } & \multirow[b]{2}{*}{ Subs Unit } & \multicolumn{2}{|c|}{ Totals } \\
\hline & & & & & Per Unit & Total & & & Labor & Material \\
\hline
\end{tabular}

\section{Electrical Other}

\begin{tabular}{|c|c|}
\hline \multicolumn{2}{|r|}{ Electrical Systems } \\
\hline 120501 & GROUNDING ALLOWANCE \\
\hline \multirow[t]{2}{*}{120501} & GROUNDING ALLOWANCE (HIP) \\
\hline & FENCE GROUND CABLE \\
\hline 120503 & 1/C - \#4/0 AWG BARE GRND WIRE CU \\
\hline \multirow[t]{2}{*}{120503} & TERMIN LUGS-CU WIRE 4/0AWG \\
\hline & FENCE GROUND RODS \\
\hline 120502 & COPPERWELD GR ROD 3/4"X10' \\
\hline \multirow[t]{2}{*}{$1205^{*}$} & Grounding \\
\hline & Alarm Systems \\
\hline 120901 & ALARM ALLOWANCE/Emergency Lighting \\
\hline 120901 & $\begin{array}{l}\text { ALARM ALLOWANCE/Emergency Lighting } \\
\text { (HIP Area) }\end{array}$ \\
\hline \multirow[t]{2}{*}{$1209^{*}$} & Fire Alarm Control \\
\hline & Electrical Systems \\
\hline 121201 & LIGHTING PROTECTION ALLOWANCE \\
\hline 121201 & $\begin{array}{l}\text { LIGHTING PROTECTION ALLOWANCE } \\
\text { (HIP) }\end{array}$ \\
\hline \multirow[t]{2}{*}{$1212^{*}$} & Lightning Protection \\
\hline & Access System \\
\hline \multirow[t]{2}{*}{121402} & SECURITY Card Reader \\
\hline & Electrical Systems \\
\hline 121403 & CCTV System Inside Bldg (50 each) \\
\hline 121403 & CCTV System Inside Bldg (HIP) \\
\hline 121404 & PAGING System \\
\hline 121404 & PAGING System (HIP) \\
\hline 121405 & Emergency Telephones \\
\hline 121405 & Emergency Telephones (HIP) \\
\hline 1214 * & Security/Communication \\
\hline
\end{tabular}

$\begin{array}{rrrr}1.00 & \text { lsum } & 1.20 & 3,578.40 \\ 1.00 & \text { lsum } & 1.20 & 180.00 \\ & & & \\ 4,500.00 & \text { Inft } & 1.20 & 0.04 \\ 120.00 & \text { each } & 1.20 & 1.10 \\ & & & \\ 30.00 & \text { each } & 1.20 & 3.48 \\ \mathbf{4 , 5 0 0 . 0 0} & \text { Inft } & \mathbf{1 . 2 0} & \\ & & & \\ 82,600.00 & \text { sqft } & 1.20 & 0.01 \\ 4,200.00 & \text { sqft } & 1.20 & 0.01 \\ & & & \\ \mathbf{0 . 0 0} & \text { Inft } & \mathbf{1 . 2 0} & \\ & & & \\ 1.00 & \text { lsum } & 1.20 & 794.40 \\ 1.00 & \text { lsum } & 1.20 & 39.60 \\ & & & \\ \mathbf{0 . 0 0} & \text { Inft } & \mathbf{1 . 2 0} & \\ & & & \\ 1.00 & \text { each } & 1.20 & 480.00 \\ & & & \\ 1.00 & \text { lsum } & 1.20 & 1,058.40 \\ 1.00 & \text { lsum } & 1.20 & 52.80 \\ 15.00 & \text { each } & 1.20 & 18.00 \\ 1.00 & \text { each } & 1.20 & 18.00 \\ 15.00 & \text { each } & 1.20 & 18.00 \\ 1.00 & \text { each } & 1.20 & 18.00 \\ \mathbf{0 . 0 0} & \text { Inft } & \mathbf{1 . 2 0} & \\ & & & \end{array}$

$\begin{array}{rcr}\mathbf{3 , 7 5 8} & \$ 85.13 & \\ 3,578.40 & \$ 85.13 & \$ 72,067.00 \\ 180.00 & \$ 85.13 & \$ 3,606.00 \\ \mathbf{2 9 4} & \$ 85.13 & \\ 162.00 & \$ 85.13 & \$ 1.91 \\ 132.48 & \$ 85.13 & \$ 4.20 \\ \mathbf{1 0 4} & \$ 85.13 & \\ 104.40 & \$ 85.13 & \$ 15.55 \\ \mathbf{4 , 1 5 7} & \mathbf{\$ 8 5 . 1 3} & \\ \mathbf{5 5 2} & \$ 85.13 & \\ 525.34 & \$ 85.13 & \$ 0.67 \\ 26.71 & \$ 85.13 & \\ & & \\ \mathbf{5 5 2} & \$ \mathbf{8 5 . 1 3} & \\ \mathbf{8 3 4} & \$ 85.13 & \\ 794.40 & \$ 85.13 & \$ 40,000.00 \\ 39.60 & \$ 85.13 & \$ 2,000.00 \\ & & \\ \mathbf{8 3 4} & \mathbf{\$ 8 5 . 1 3} & \\ \mathbf{4 8 0} & \$ 85.13 & \\ 480.00 & \$ 85.13 & \$ 40,000.00 \\ \mathbf{1 , 6 8 7} & \$ 85.13 & \\ 1,058.40 & \$ 85.13 & \$ 155,626.00 \\ 52.80 & \$ 85.13 & \$ 7,780.00 \\ 270.00 & \$ 85.13 & \$ 747.00 \\ 18.00 & \$ 85.13 & \$ 747.00 \\ 270.00 & \$ 85.13 & \$ 850.00 \\ 18.00 & \$ 85.13 & \$ 850.00 \\ \mathbf{2}, 137 & \$ 85.13 & \end{array}$

\begin{tabular}{|c|c|}
\hline$\$ 319,968$ & $\$ 75,673$ \\
\hline$\$ 304,644$ & $\begin{array}{r}\$ 72,067 \\
\$ 3,606\end{array}$ \\
\hline$\$ 15,324$ & $\$ 3,606$ \\
\hline$\$ 25,070$ & $\$ 9,099$ \\
\hline$\$ 13,792$ & $\$ 8,595$ \\
\hline$\$ 11,279$ & $\$ 504$ \\
\hline$\$ 8,888$ & $\$ 467$ \\
\hline$\$ 8,888$ & $\$ 467$ \\
\hline$\$ 353,926$ & $\$ 85,239$ \\
\hline$\$ 46,998$ & $\$ 58,156$ \\
\hline$\$ 44,724$ & $\$ 55,342$ \\
\hline$\$ 2,274$ & $\$ 2,814$ \\
\hline$\$ 46,998$ & $\$ 58,156$ \\
\hline$\$ 71,002$ & $\$ 42,000$ \\
\hline$\$ 67,631$ & $\$ 40,000$ \\
\hline$\$ 3,371$ & $\$ 2,000$ \\
\hline$\$ 71,002$ & $\$ 42,000$ \\
\hline$\$ 40,864$ & $\$ 40,000$ \\
\hline$\$ 40,864$ & $\$ 40,000$ \\
\hline$\$ 143,638$ & $\$ 188,958$ \\
\hline$\$ 90,106$ & $\$ 155,626$ \\
\hline$\$ 4,495$ & $\$ 7,780$ \\
\hline$\$ 22,986$ & $\$ 11,205$ \\
\hline$\$ 1,532$ & $\$ 747$ \\
\hline$\$ 22,986$ & $\$ 12,750$ \\
\hline$\$ 1,532$ & $\$ 850$ \\
\hline$\$ 184,503$ & $\$ 228,958$ \\
\hline
\end{tabular}

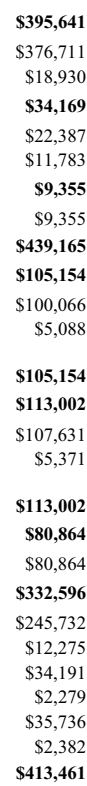

Grand Total: 12.xx Electrical Other Systems

1.20

7,711

$\$ 85.13$

$\$ 656,429$

$\$ 414,353$

so

\$o $\$ \$ 1,070,781$ 
Client: INL

Project: FFC

URS

Washington Division

Job No: $\quad 27989-325 \quad$ Rev No: 0

Product Line:
Estimate:
FFC

Lab.

\begin{tabular}{|l|l|}
\hline \multicolumn{2}{|c|}{ Workhours } \\
Mat'l Unit
\end{tabular}

9.00 ea

1.20
1.20
1.20
1.20

1.20
1.20
1.20

1.20
$64 \quad \$ 89.78$

713
589.78

$555 \$ \$ 89.78$

$163 \$ 85.13$
Date: $7 / 17 / 2008$

11:31AM Priced By: ADC

Office: Denver

\begin{tabular}{|l|llll|}
\multirow{3}{*}{ Subs Unit } & \multicolumn{4}{|c|}{ Totals } \\
\cline { 2 - 5 } & Labor & Material & Subs & Total \\
\cline { 2 - 5 }
\end{tabular}

$\$ 5,764 \quad \$ 17,880$

$\$ 63,994 \quad \$ 4,947,140$

$\$ 49,795 \quad \$ 4,101$

$\$ 13,894 \quad \$ 20,400$
$\$ 0 \$ 23,644$

$\$ 0 \quad \$ 5,011,134$

$\$ 0 \quad \$ 53,896$

$\$ 0 \$ 34,294$ 
Project: FFC

Location: GREEN FIELD SITE HIP Option

Account: 13 Instrumentation

Facility: Entire Estimate

URS

Washington Division
Date: 7/17/2008

11:31AM

Priced By: ADC

Office: Denver

Product Line: Gov
Estimate: FFC

\begin{tabular}{|c|c|c|c|c|c|c|c|c|c|c|c|c|c|}
\hline \multirow[b]{2}{*}{ Account No. } & \multirow[b]{2}{*}{ Description } & \multirow[b]{2}{*}{ Qty } & \multirow[b]{2}{*}{ UM } & \multirow{2}{*}{$\begin{array}{l}\text { Lab. } \\
\text { Fact. }\end{array}$} & \multicolumn{3}{|c|}{ Workhours } & \multirow[b]{2}{*}{ Mat'l Unit } & \multirow[b]{2}{*}{ Subs Unit } & \multicolumn{4}{|c|}{ Totals } \\
\hline & & & & & Per Unit & Total & Rate & & & Labor & Material & & Total \\
\hline \multicolumn{2}{|l|}{ Analyzer- w/ Bulks } & & & & & 138 & 89.78 & & & $\$ 12,368$ & $\$ 105,428$ & \$o & $\$ 117,796$ \\
\hline 130404 & 1/4" BALL VALVE WHITEY 43 & 1 & each & 1.20 & 0.48 & 0.48 & $\$ 89.78$ & $\$ 29.67$ & & $\$ 43$ & $\$ 30$ & $\$ 0$ & $\$ 73$ \\
\hline 130404 & 1/4" BALL VALVE WHITEY 43 & 1 & each & 1.20 & 0.48 & 0.48 & $\$ 89.78$ & $\$ 29.67$ & & $\$ 43$ & $\$ 30$ & $\$ 0$ & $\$ 73$ \\
\hline 130703 & 3/8" TUBING (SS) & 150.00 & $\operatorname{lnft}$ & 1.20 & 0.22 & 32.40 & $\$ 89.78$ & $\$ 1.23$ & & $\$ 2,909$ & $\$ 185$ & $\$ 0$ & $\$ 3,093$ \\
\hline 130703 & 3/8" TUBING (SS) & 150.00 & $\operatorname{lnft}$ & 1.20 & 0.22 & 32.40 & $\$ 89.78$ & $\$ 1.23$ & & $\$ 2,909$ & $\$ 185$ & $\$ 0$ & $\$ 3,093$ \\
\hline 131007 & Determinator O N ANALYZER & 1 & each & 1.20 & 36.00 & 36.00 & $\$ 89.78$ & $\$ 80,000.00$ & & $\$ 3,232$ & $\$ 80,000$ & $\$ 0$ & $\$ 83,232$ \\
\hline 131007 & Determinator C S ANALYZER & 1 & each & 1.20 & 36.00 & 36.00 & $\$ 89.78$ & $\$ 25,000.00$ & & $\$ 3,232$ & $\$ 25,000$ & $\$ 0$ & $\$ 28,232$ \\
\hline \multicolumn{2}{|l|}{ Body Scanners } & & & & & 58 & 89.78 & & & $\$ 5,171$ & $\$ 180,000$ & \$o & $\$ 185,171$ \\
\hline 131007 & Body Scanner & 3 & each & 1.20 & 19.20 & 57.60 & $\$ 89.78$ & $\$ 60,000.00$ & & $\$ 5,171$ & $\$ 180,000$ & $\$ 0$ & $\$ 185,171$ \\
\hline \multicolumn{2}{|c|}{ Field Instr Allow - w/ Bulks } & & & & & 3 & 89.78 & & & $\$ 231$ & $\$ 2,000$ & $\$ 0$ & $\$ 2,231$ \\
\hline 130101 & Scale & 1 & each & 1.20 & 2.57 & 2.57 & $\$ 89.78$ & $\$ 2,000.00$ & & $\$ 231$ & $\$ 2,000$ & $\$ 0$ & $\$ 2,231$ \\
\hline \multicolumn{2}{|c|}{ Field Instr Allow - w/ Bulks Fire Water Collection } & & & & & 208 & 89.78 & & & $\$ 18,634$ & $\$ 17,142$ & \$o & $\$ 35,776$ \\
\hline 130101 & $\begin{array}{l}\text { OVERALL INSTRUMENTS } \\
\text { ALLOWANCE }\end{array}$ & 8 & each & 1.20 & 7.70 & 61.63 & $\$ 89.78$ & $\$ 1,985.00$ & & $\$ 5,533$ & $\$ 15,880$ & $\$ 0$ & $\$ 21,413$ \\
\hline 130404 & 1/4" BALL VALVE WHITEY 43 & 16 & each & 1.20 & 0.48 & 7.68 & $\$ 89.78$ & $\$ 29.67$ & & $\$ 690$ & $\$ 475$ & $\$ 0$ & $\$ 1,164$ \\
\hline 130703 & 3/8" TUBING (SS) & 640.00 & $\operatorname{lnft}$ & 1.20 & 0.22 & 138.24 & $\$ 89.78$ & $\$ 1.23$ & & $\$ 12,411$ & $\$ 787$ & $\$ 0$ & $\$ 13,198$ \\
\hline \multicolumn{2}{|c|}{ Fluorscope Analyzer- w/ Bulks Item 17} & & & & & 81 & 89.78 & & & $\$ 7,304$ & $\$ 200,244$ & $\$ 0$ & $\$ 207,548$ \\
\hline 130404 & 1/4" BALL VALVE WHITEY 43 & 2 & each & 1.20 & 0.48 & 0.96 & $\$ 89.78$ & $\$ 29.67$ & & $\$ 86$ & $\$ 59$ & $\$ 0$ & $\$ 146$ \\
\hline 130703 & 3/8" TUBING (SS) cooling water & 150.00 & $\operatorname{lnft}$ & 1.20 & 0.22 & 32.40 & $\$ 89.78$ & $\$ 1.23$ & & $\$ 2,909$ & $\$ 185$ & $\$ 0$ & $\$ 3,093$ \\
\hline 131007 & Fluoroscope DO & 1 & each & 1.20 & 48.00 & 48.00 & $\$ 89.78$ & $\$ 200,000.00$ & & $\$ 4,309$ & $\$ 200,000$ & $\$ 0$ & $\$ 204,309$ \\
\hline \multicolumn{2}{|c|}{ Gamma Scanner-w/ Bulks } & & & & & 124 & 89.78 & & & $\$ 11,161$ & $\$ 1,000,488$ & $\$ 0$ & $\$ 1,011,649$ \\
\hline 130404 & 1/4" BALL VALVE WHITEY 43 & 4 & each & 1.20 & 0.48 & 1.92 & $\$ 89.78$ & $\$ 29.67$ & & $\$ 172$ & $\$ 119$ & $\$ 0$ & $\$ 291$ \\
\hline 130703 & 3/8" TUBING (SS) & 300.00 & $\operatorname{lnft}$ & 1.20 & 0.22 & 64.80 & $\$ 89.78$ & $\$ 1.23$ & & $\$ 5,818$ & $\$ 369$ & $\$ 0$ & $\$ 6,187$ \\
\hline 131007 & Gamma Scanner DO & 1 & each & 1.20 & 57.60 & 57.60 & $\$ 89.78$ & $\$ 1,000,000.00$ & & $\$ 5,171$ & $\$ 1,000,000$ & $\$ 0$ & $\$ 1,005,171$ \\
\hline \multicolumn{2}{|c|}{ Gamma Spectroscopy (Element) M } & & & & & 36 & 89.78 & & & $\$ 3,232$ & $\$ 80,000$ & $\$ 0$ & $\$ 83,232$ \\
\hline 131007 & Gamm Spectroscopy Item M & 1 & each & 1.20 & 36.00 & 36.00 & $\$ 89.78$ & $\$ 80,000.00$ & & $\$ 3,232$ & $\$ 80,000$ & $\$ 0$ & $\$ 83,232$ \\
\hline \multicolumn{2}{|c|}{ Gamma Spectroscopy (PLate) w/ Bulks Item L } & & & & & 81 & 89.78 & & & $\$ 7,261$ & $\$ 200,214$ & \$o & $\$ 207,475$ \\
\hline 130404 & 1/4" BALL VALVE WHITEY 43 & 1 & each & 1.20 & 0.48 & 0.48 & $\$ 89.78$ & $\$ 29.67$ & & $\$ 43$ & $\$ 30$ & $\$ 0$ & $\$ 73$ \\
\hline 130703 & 3/8" TUBING (SS) & 150.00 & $\operatorname{lnft}$ & 1.20 & 0.22 & 32.40 & $\$ 89.78$ & $\$ 1.23$ & & $\$ 2,909$ & $\$ 185$ & $\$ 0$ & $\$ 3,093$ \\
\hline 131007 & Gamma Spectroscopy & 1 & each & 1.20 & 48.00 & 48.00 & $\$ 89.78$ & $\$ 200,000.00$ & & $\$ 4,309$ & $\$ 200,000$ & $\$ 0$ & $\$ 204,309$ \\
\hline \multicolumn{2}{|c|}{ Hand \& Foot Monitor } & & & & & 72 & 89.78 & & & $\$ 6,464$ & $\$ 145,000$ & $\$ 0$ & $\$ 151,464$ \\
\hline 131007 & Hand \& Foot Monitor & 5 & each & 1.20 & 14.40 & 72.00 & $\$ 89.78$ & $\$ 29,000.00$ & & $\$ 6,464$ & $\$ 145,000$ & $\$ 0$ & $\$ 151,464$ \\
\hline \multicolumn{2}{|c|}{ Hand \& Foot Monitor HIP Area } & & & & & 72 & 89.78 & & & $\$ 6,464$ & $\$ 145,000$ & \$o & $\$ 151,464$ \\
\hline 131007 & Hand \& Foot Monitor HIP Area & 5 & each & 1.20 & 14.40 & 72.00 & $\$ 89.78$ & $\$ 29,000.00$ & & $\$ 6,464$ & $\$ 145,000$ & $\$ 0$ & $\$ 151,464$ \\
\hline \multicolumn{2}{|c|}{ Mass Spectrometer- w/ Bulks } & & & & & 62 & 89.78 & & & $\$ 5,581$ & $\$ 250,244$ & \$o & $\$ 255,824$ \\
\hline 130404 & 1/4" BALL VALVE WHITEY 43 & 2 & each & 1.20 & 0.48 & 0.96 & $\$ 89.78$ & $\$ 29.67$ & & $\$ 86$ & $\$ 59$ & $\$ 0$ & $\$ 146$ \\
\hline
\end{tabular}


Client: INL

Project: FFC

Location: GREEN FIELD SITE HIP Option

Account: 13 Instrumentation

Facility: Entire Estimate
URS

Washington Division
Date: 7/17/2008

$11: 31 \mathrm{AM}$

Priced By: ADC

Office: Denver

Product Line: Gov
Estimate: FFC

Job No: $\quad 27989-325 \quad$ Rev No: 0

Esting

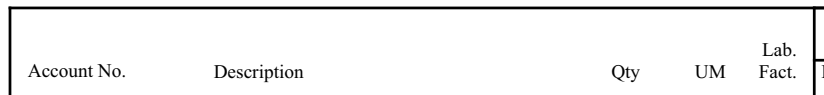

\begin{tabular}{|lll}
\hline Per Unit $\quad$ Total & Rate & Mat'l Unit
\end{tabular}

Subs Unit

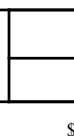

130703 3/8" TUBING (SS)

131007 Mass Spectrometer Hook-up \& Test

$150.00 \quad \operatorname{lnft} \quad 1.20$

1 each 1.20

Optical Microscope

131007 Microscope $1000 \mathrm{X}$ w/Camera \& computer

Radiography Analyzer- w/ Bulks Item 16

$130404 \quad 1 / 4$ " BALL VALVE WHITEY 43

$130703 \quad 3 / 8$ " TUBING (SS) water \& gas

131007 ANALYZER DO

SECURITY CAMERA

139900 FENCE SECURITY CAMERA (mount on

fence lighting poles)

$130404 \quad 1 / 4 "$ BALL VALVE WHITEY 43

$130703 \quad 3 / 8$ " TUBING (SS)

131007 ANALYZER DO
1 each $\quad 1.20 \quad 9.60$

4 each 1.20

$400.00 \quad \operatorname{lnft} \quad 1.20$

2 each 1.20

17 each 1.20

$400.00 \quad \operatorname{lnft} \quad 1.20$

2 each 1.20

57.60
4 each 1.20

$32.40 \quad \$ 89.78$

$28.80-\$ 89.78$

$9.60 \quad \$ 89.78$

$\begin{array}{ll}184 & \mathbf{8 9 . 7 8}\end{array}$

$\begin{array}{ll}1.92 & \$ 89.78\end{array}$

$86.40 \quad \$ 89.78$

$96.00 \quad \$ 89.78$

$\begin{array}{ll}163 & 85.13\end{array}$

$163.20 \quad \$ 85.13$

$\begin{array}{ll}204 & 89.78\end{array}$

\begin{tabular}{l}
$1.92 \quad \$ 89.78$ \\
\hline 86.40
\end{tabular}

$86.40 \quad \$ 89.78$

115.20 $\begin{array}{ll}10 & 89.78\end{array}$

0.22

$\$ 1.23$

$\$ 250,000.00$

$\$ 2,140.00$

$\$ 29.67$

$\$ 1.23$

$\$ 320,000.00$

$\$ 1,200.00$

$\$ 29.67$

$\$ 1.23$

$\$ 89.78 \quad \$ 1,000,000.00$

$\$ 1.23$
$\$ 00.00$
$\$ 0.00$
$\$ 29.67$
$\$ 1.23$

$\$ 0.00$
$\$ 1.23$
$\$ 00.67$

(2)

(2)

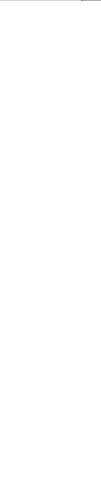

\begin{tabular}{|c|c|c|c|}
\hline & Анатитат & & \\
\hline$\$ 2,909$ & $\$ 185$ & $\$ 0$ & $\$ 3,093$ \\
\hline$\$ 2,586$ & $\$ 250,000$ & $\$ 0$ & $\$ 252,586$ \\
\hline$\$ 862$ & $\$ 2,140$ & $\$ 0$ & $\$ 3,002$ \\
\hline$\$ 862$ & $\$ 2,140$ & $\$ 0$ & $\$ 3,002$ \\
\hline$\$ 16,548$ & $\$ 640,611$ & \$0 & $\$ 657,159$ \\
\hline$\$ 172$ & $\$ 119$ & $\$ 0$ & $\$ 291$ \\
\hline$\$ 7,757$ & $\$ 492$ & $\$ 0$ & $\$ 8,249$ \\
\hline$\$ 8,619$ & $\$ 640,000$ & $\$ 0$ & $\$ 648,619$ \\
\hline$\$ 13,894$ & $\$ 20,400$ & \$0 & $\$ 34,294$ \\
\hline$\$ 13,894$ & $\$ 20,400$ & $\$ 0$ & $\$ 34,294$ \\
\hline$\$ 18,272$ & $\$ 2,000,611$ & \$0 & $\$ 2,018,882$ \\
\hline$\$ 172$ & $\$ 119$ & $\$ 0$ & $\$ 291$ \\
\hline$\$ 7,757$ & $\$ 492$ & $\$ 0$ & $\$ 8,249$ \\
\hline$\$ 10,343$ & $\$ 2,000,000$ & $\$ 0$ & $\$ 2,010,343$ \\
\hline
\end{tabular}

\begin{tabular}{|c|c|c|c|c|c|c|c|}
\hline Totals & $\begin{array}{l}\text { 13-Instrumentation } \\
\text { Entire Estimate }\end{array}$ & $\begin{array}{l}9 \quad \text { each } \\
\text { Field Inst Count }\end{array}$ & $1,495 \quad \$ 89.27$ & $\$ 133,447$ & $\$ 4,989,521$ & \$0 & $\$ 5,122,968$ \\
\hline
\end{tabular}


Project: FFC

Location: GREEN FIELD SITE HIP Option

Account: 16 Buildings

Facility: Entire Estimate

URS

Washington Division

Date: $7 / 17 / 2008$

11:31AM

Priced By: ADC

Office: Denver

Product Line:
Estimate:

Job No: $\quad 27989-325 \quad$ Rev No: 0

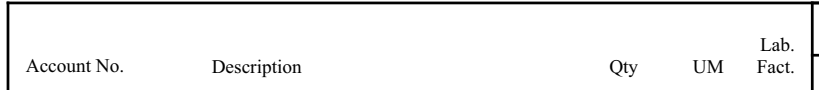

Bldg Furnishings

161101 SPECIALTIES-LOCKERS

161103 OFFICE FURNISHINGS

\section{Bldg Plumbing}

160403 SERVICE SINK,PE ON CI,WALL

SERVICE SINK,PE ON CI,WALL
HUNG,22"x18" (Janitor Closet)
WC (Incl Fixture, Water, Vent \& Drain

WC (In
Pipe)

URINAL

SHOWER,STALL,BAKED

ENAM,TERRAZZO RECEPTOR,30" Inc

Warer, Vent \& Drain Pipe

160403
160403

Safety Showers W/Eye wash Incl Waste

Vent \& Drain Pipe

160403 LAVATORY W/TRIM WALL HUNG PE ON CI,18"x15"

Bldg Specialties

160501 ALLOWANCE Fire Protection (Wet Pipe

ALLOWANCE Fire Protection (Wet Pipe
Sprinkler Sys)
ALLOWANCE Fire Protection (Wet Pipe

Sprinkler Sys) (HIP Area)

CERAMIC TILE Floors Locker \&

Bathrooms 6" by 6" Thin Set

CERAMIC TILE Floors Locker \&

161101 SPECIALTIES-Building Elevator 2 stop
$161102 \quad$ SPECIALTIES-ALLOWANCE Furniture Cubicles

161199 SPECIALTIES-OTHER Shelving Cans \& Fuel

Building

$160100 \quad$ Building Incl Insul Siding, Roofing, \& Support Steel

Building Incl Insul Siding, Roofing, \& Support Steel (HIP Area)

CMU-12" wide Compressor, MCC Mech \& Elec Maint Rooms

160203 CONC BLK,REG WT.,HOLLOW,12"THK W/PERLITE INSUL CORE FILL

Doors \& Windows

160302 HOLLOW METAL,18GA. STEEL,NO LABEL,3'-0"x 7'-0" Interior

160302 HOLLOW METAL,18GA. STEEL,NO

LABEL, 3'-0" $x$ 7'-0" Exterio

HOLLOW METAL,18GA. STEEL,NO

LABEL,DBL., 6'-0" $77^{\prime}-0$ "

\begin{tabular}{rrr}
36 & each & 1.20 \\
24 & each & 1.20 \\
1 & each & 1.20 \\
10 & each & 1.20 \\
4 & each & 1.20 \\
2 & each & 1.20 \\
& & \\
2 & each & 1.20 \\
10 & each & 1.20 \\
& & \\
$82,600.00$ & sqft & 1.20 \\
$4,200.00$ & sqft & 1.20 \\
$1,275.00$ & sqft & 1.20 \\
\hline 265.00 & Inft & 1.20 \\
1.00 & lsum & 1.20 \\
1.00 & lsum & 1.20 \\
1.00 & Isum & 1.20 \\
& & \\
$82,500.00$ & sqft & 1.20 \\
$4,200.00$ & sqft & 1.20 \\
& & \\
$15,350.00$ & sqft & 1.20 \\
& & \\
20 & each & 1.20 \\
7 & each & 1.20 \\
4 & each & 1.20 \\
\hline & & \\
& & \\
& & \\
& & \\
&
\end{tabular}

Per Unit

1

Mat'l Unit

\begin{tabular}{c|c}
\multirow{2}{*}{ Subs Unit } & \\
\cline { 2 - 3 } &
\end{tabular}

\begin{tabular}{|l}
\hline Labor \\
\hline
\end{tabular}

$\begin{array}{rrr} & \mathbf{1 4 4} & \mathbf{8 0 . 4 1} \\ 2.40 & 86.40 & \$ 80.41\end{array}$

$\$ 8.40 \quad \$ 80.41 \quad \$ 267.82$

$57.60 \quad \$ 80.41$

$\$ 1,000.00$

$\$ 265.00$

$\$ 11,580$

$\$ 6,948$

$\$ 4,632$

$\$ 1,424.90$

$\$ 1,000.00$

$\$ 700.00$

$\$ 1,245.30$

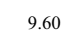

$19.20 \quad \$ 80.41$

$\$ 1,970.00$

$\$ 930.40$

$\mathbf{3 , 5 7 8} \quad \mathbf{8 2 . 1 6}$

$\begin{array}{rrr} & \mathbf{3 , 5 7 8} & \mathbf{8 2 . 1 6} \\ 0.03 & 2,478.00 & \$ 80.41\end{array}$

$\$ 1.25$

$126.00 \quad \$ 80.41$

$\$ 1.25$

$122.40 \quad \$ 80.41$

$\$ 3.36$

$\$ 3.61$

$54.70 \$ 80.41$

0.21

$192.00 \quad \$ 80.41$

$28.80 \quad \$ 80.41$

$\$ 15,500.00$

$\$ 58,650.00$

$$
28.80
$$

$576.00 \quad \$ 91.25$

$\$ 100,000.00$

$\begin{array}{rr}17,479 & 80.41\end{array}$

$\begin{array}{rr}\mathbf{1 7 , 4 7 9} & \mathbf{8 0 . 4 1} \\ 16,632.00 & \$ 80.41\end{array}$

$\$ 11.06$

846.72

0.20

$846.72 \$ 80.41$

$\$ 11.06$

4 each 1.20
Totals

Material

\begin{tabular}{|c|c|c|}
\hline$\$ 33,642$ & $\$ 6,360$ & $\$ 51,581$ \\
\hline$\$ 9,642$ & $\$ 0$ & $\$ 16,589$ \\
\hline$\$ 24,000$ & $\$ 6,360$ & $\$ 34,992$ \\
\hline$\$ 3,940$ & $\$ 26,020$ & $\$ 31,503$ \\
\hline$\$ 0$ & $\$ 1,425$ & $\$ 1,425$ \\
\hline$\$ 0$ & $\$ 10,000$ & $\$ 10,000$ \\
\hline$\$ 0$ & $\$ 2,800$ & $\$ 2,800$ \\
\hline$\$ 0$ & $\$ 2,491$ & $\$ 2,491$ \\
\hline$\$ 3,940$ & $\$ 0$ & $\$ 5,484$ \\
\hline$\$ 0$ & $\$ 9,304$ & $\$ 9,304$ \\
\hline$\$ 287,891$ & $\$ 6,006$ & $\$ 587,851$ \\
\hline$\$ 103,250$ & $\$ 0$ & $\$ 302,518$ \\
\hline$\$ 5,250$ & $\$ 0$ & $\$ 15,382$ \\
\hline$\$ 4,284$ & $\$ 4,973$ & $\$ 19,099$ \\
\hline$\$ 957$ & $\$ 1,034$ & $\$ 6,389$ \\
\hline$\$ 15,500$ & $\$ 0$ & $\$ 30,940$ \\
\hline$\$ 58,650$ & $\$ 0$ & $\$ 60,966$ \\
\hline$\$ 100,000$ & $\$ 0$ & $\$ 152,558$ \\
\hline$\$ 958,989$ & \$0 & $\$ 2,364,534$ \\
\hline$\$ 912,533$ & $\$ 0$ & $\$ 2,249,989$ \\
\hline$\$ 46,456$ & $\$ 0$ & $\$ 114,545$ \\
\hline so & $\$ 290,738$ & $\$ 290,738$ \\
\hline$\$ 0$ & $\$ 290,738$ & $\$ 290,738$ \\
\hline \$0 & $\$ 56,619$ & $\$ 56,619$ \\
\hline$\$ 0$ & $\$ 18,706$ & $\$ 18,706$ \\
\hline$\$ 0$ & $\$ 6,547$ & $\$ 6,547$ \\
\hline$\$ 0$ & $\$ 7,560$ & $\$ 7,560$ \\
\hline
\end{tabular}


Client: INL

Project: FFC

Location: GREEN FIELD SITE HIP Option

Account: 16 Buildings

Facility: Entire Estimate

URS

Washington Division
Date: 7/17/2008

11:31AM

Priced By: ADC

Office: Denver

Product Line: Gov
Estimate: FFC

\begin{tabular}{|c|c|c|c|c|c|c|c|c|c|c|c|c|c|}
\hline \multirow[b]{2}{*}{ Account No. } & \multirow[b]{2}{*}{ Description } & \multirow[b]{2}{*}{ Qty } & \multirow[b]{2}{*}{$\mathrm{UM}$} & \multirow{2}{*}{$\begin{array}{l}\text { Lab. } \\
\text { Fact. }\end{array}$} & \multicolumn{3}{|c|}{ Workhours } & \multirow[b]{2}{*}{ Mat'l Unit } & \multirow[b]{2}{*}{ Subs Unit } & \multicolumn{4}{|c|}{ Totals } \\
\hline & & & & & \begin{tabular}{|l|l} 
Per Unit \\
\end{tabular} & Total & Rate & & & Labor & Material & Subs & Total \\
\hline 160302 & $\begin{array}{l}\text { HOLLOW METAL, 18GA. STEEL,A } \\
\text { LABEL,DBL.,6'-0"x7'-0" (Furnace Area) }\end{array}$ & 1 & each & 1.20 & & & & & $\$ 2,145.50$ & $\$ 0$ & $\$ 0$ & $\$ 2,146$ & $\$ 2,146$ \\
\hline 160302 & sample - Wood Doors & 20 & each & 1.20 & & & & & $\$ 1,083.00$ & $\$ 0$ & $\$ 0$ & $\$ 21,660$ & $\$ 21,660$ \\
\hline inishes & & & & & & 3,511 & 51.59 & & & $\$ 181,139$ & $\$ 37,660$ & $\$ 106,745$ & $\$ 325,544$ \\
\hline 160402 & Roof Drain 3" Diameter & 20 & each & 1.20 & 1.60 & 31.92 & $\$ 80.41$ & $\$ 207.00$ & & $\$ 2,567$ & $\$ 4,140$ & $\$ 0$ & $\$ 6,707$ \\
\hline 160402 & 3" Floor Drains W/P Trap & 24 & each & 1.20 & 2.96 & 71.14 & $\$ 80.41$ & $\$ 220.50$ & & $\$ 5,720$ & $\$ 5,292$ & $\$ 0$ & $\$ 11,012$ \\
\hline 161004 & PAINTING Sealer on Concrete Floor & $37,746.50$ & sqft & 1.20 & 0.00 & 139.81 & $\$ 50.69$ & $\$ 0.05$ & & $\$ 7,087$ & $\$ 2,073$ & $\$ 0$ & $\$ 9,160$ \\
\hline 161004 & PAINTING Epoxy Paint Coating Floor & $41,140.00$ & sqft & 1.20 & 0.00 & 152.37 & $\$ 54.00$ & $\$ 0.04$ & & $\$ 8,229$ & $\$ 1,784$ & $\$ 0$ & $\$ 10,013$ \\
\hline 161004 & $\begin{array}{l}\text { VCT Lunch/Break Room 12" by } 12 \text { " by } \\
3 / 32 \text { " Embossed }\end{array}$ & $1,350.00$ & sqft & 1.20 & 0.02 & 25.92 & $\$ 80.41$ & $\$ 1.18$ & $\$ 1.11$ & $\$ 2,084$ & $\$ 1,593$ & $\$ 1,499$ & $\$ 5,176$ \\
\hline 161004 & CARPET Office Areas & $9,600.00$ & sqft & 1.20 & & & & & $\$ 3.25$ & $\$ 0$ & $\$ 0$ & $\$ 31,200$ & $\$ 31,200$ \\
\hline 161004 & Rubber Base & $2,259.40$ & $\operatorname{lnft}$ & 1.20 & & & & & $\$ 1.61$ & $\$ 0$ & $\$ 0$ & $\$ 3,631$ & $\$ 3,631$ \\
\hline 161004 & Resilient Flooring & $4,052.40$ & $\operatorname{lnft}$ & 1.20 & 0.11 & 435.23 & $\$ 54.00$ & $\$ 0.57$ & & $\$ 23,504$ & $\$ 2,301$ & $\$ 0$ & $\$ 25,805$ \\
\hline 161006 & PAINTING Sealer on Concrete & $8,606.40$ & sqft & 1.20 & 0.02 & 173.19 & $\$ 49.70$ & $\$ 0.16$ & & $\$ 8,607$ & $\$ 1,368$ & $\$ 0$ & $\$ 9,976$ \\
\hline 161006 & PAINTING Epoxy Paint on Drywall & $40,667.00$ & sqft & 1.20 & 0.02 & 818.38 & $\$ 49.70$ & $\$ 0.16$ & & $\$ 40,672$ & $\$ 6,466$ & $\$ 0$ & $\$ 47,137$ \\
\hline 161006 & PAINTING Epoxy Paint on Concrete & $26,877.40$ & sqft & 1.20 & 0.02 & 540.88 & $\$ 49.70$ & $\$ 0.16$ & & $\$ 26,880$ & $\$ 4,273$ & $\$ 0$ & $\$ 31,154$ \\
\hline 161006 & PAINTING Epoxy Paint on CMU & $3,533.20$ & sqft & 1.20 & 0.02 & 71.10 & $\$ 49.70$ & $\$ 0.16$ & & $\$ 3,534$ & $\$ 562$ & $\$ 0$ & $\$ 4,095$ \\
\hline 161006 & PAINTING Latex Paint on Concrete & $8,382.00$ & sqft & 1.20 & 0.02 & 168.68 & $\$ 49.70$ & $\$ 0.16$ & & $\$ 8,383$ & $\$ 1,333$ & $\$ 0$ & $\$ 9,716$ \\
\hline 161006 & PAINTING Latex Paint on CMU & $1,993.20$ & sqft & 1.20 & 0.02 & 40.11 & $\$ 49.70$ & $\$ 0.16$ & & $\$ 1,993$ & $\$ 317$ & $\$ 0$ & $\$ 2,310$ \\
\hline 161006 & PAINTING Latex Paint on Drywall & $38,135.90$ & sqft & 1.20 & 0.01 & 411.97 & $\$ 49.70$ & $\$ 0.07$ & & $\$ 20,474$ & $\$ 2,756$ & $\$ 0$ & $\$ 23,230$ \\
\hline 161006 & PAINTING Decon coating on Concrete & $21,401.60$ & sqft & 1.20 & 0.02 & 430.68 & $\$ 49.70$ & $\$ 0.16$ & & $\$ 21,404$ & $\$ 3,403$ & $\$ 0$ & $\$ 24,807$ \\
\hline 161006 & $\begin{array}{l}\text { GYPSUM BD PARTITION ( } 5 / 8 \text { " Gypsum } \\
\text { both sides) Office Areas }\end{array}$ & $7,000.00$ & sqft & 1.20 & & & & & $\$ 5.38$ & $\$ 0$ & $\$ 0$ & $\$ 37,660$ & $\$ 37,660$ \\
\hline 161006 & $\begin{array}{l}\text { GYPSUM BD PARTITION ( } 5 / 8 \text { " Gypsum } \\
\text { both sides) Lab, Programmer's \& } \\
\text { supervisor'sO }\end{array}$ & $1,200.00$ & sqft & 1.20 & & & & & $\$ 5.38$ & $\$ 0$ & $\$ 0$ & $\$ 6,456$ & $\$ 6,456$ \\
\hline 161006 & $\begin{array}{l}\text { GYPSUM BD PARTITION ( } 5 / 8 \text { " Gypsum } \\
\text { ONE sides) Office Areas }\end{array}$ & $6,000.00$ & sqft & 1.20 & & & & & $\$ 3.55$ & $\$ 0$ & $\$ 0$ & $\$ 21,300$ & $\$ 21,300$ \\
\hline 161103 & TOILET ACCESSORIES Partitions & 10 & each & 1.20 & & & & & $\$ 500.00$ & $\$ 0$ & $\$ 0$ & $\$ 5,000$ & $\$ 5,000$ \\
\hline ire Protection & & & & & & 452 & 80.41 & & & $\$ 36,377$ & $\$ 2,775$ & \$o & $\$ 39,152$ \\
\hline 160501 & $\begin{array}{l}\text { Fire Protection Allowance Fire Line From } \\
\text { Blgd Exterior to Sprinkler Sys }\end{array}$ & 287.00 & $\operatorname{lnft}$ & 1.20 & 1.58 & 452.37 & $\$ 80.41$ & $\$ 9.67$ & & $\$ 36,377$ & $\$ 2,775$ & $\$ 0$ & $\$ 39,152$ \\
\hline VAC Equipment & & & & & & 3,523 & 80.41 & & & $\$ 283,269$ & $\$ 209,502$ & $\$ 0$ & $\$ 492,771$ \\
\hline 160901 & $\begin{array}{l}\text { ALLOWANCE Process Area Non } \\
\text { Radiological }\end{array}$ & $30,900.00$ & sqft & 1.20 & 0.11 & $3,522.60$ & $\$ 80.41$ & $\$ 6.78$ & & $\$ 283,269$ & $\$ 209,502$ & $\$ 0$ & $\$ 492,771$ \\
\hline VAC Equipment S & andard Hvac Office area & & & & & 1,359 & 80.41 & & & $\$ 109,255$ & $\$ 81,396$ & \$o & $\$ 190,651$ \\
\hline 160901 & Office area & $10,200.00$ & sqft & 1.20 & 0.13 & $1,358.64$ & $\$ 80.41$ & $\$ 7.98$ & & $\$ 109,255$ & $\$ 81,396$ & $\$ 0$ & $\$ 190,651$ \\
\hline terior Finish & & & & & & 431 & 80.41 & & & $\$ 34,652$ & $\$ 32,832$ & \$o & $\$ 67,484$ \\
\hline
\end{tabular}




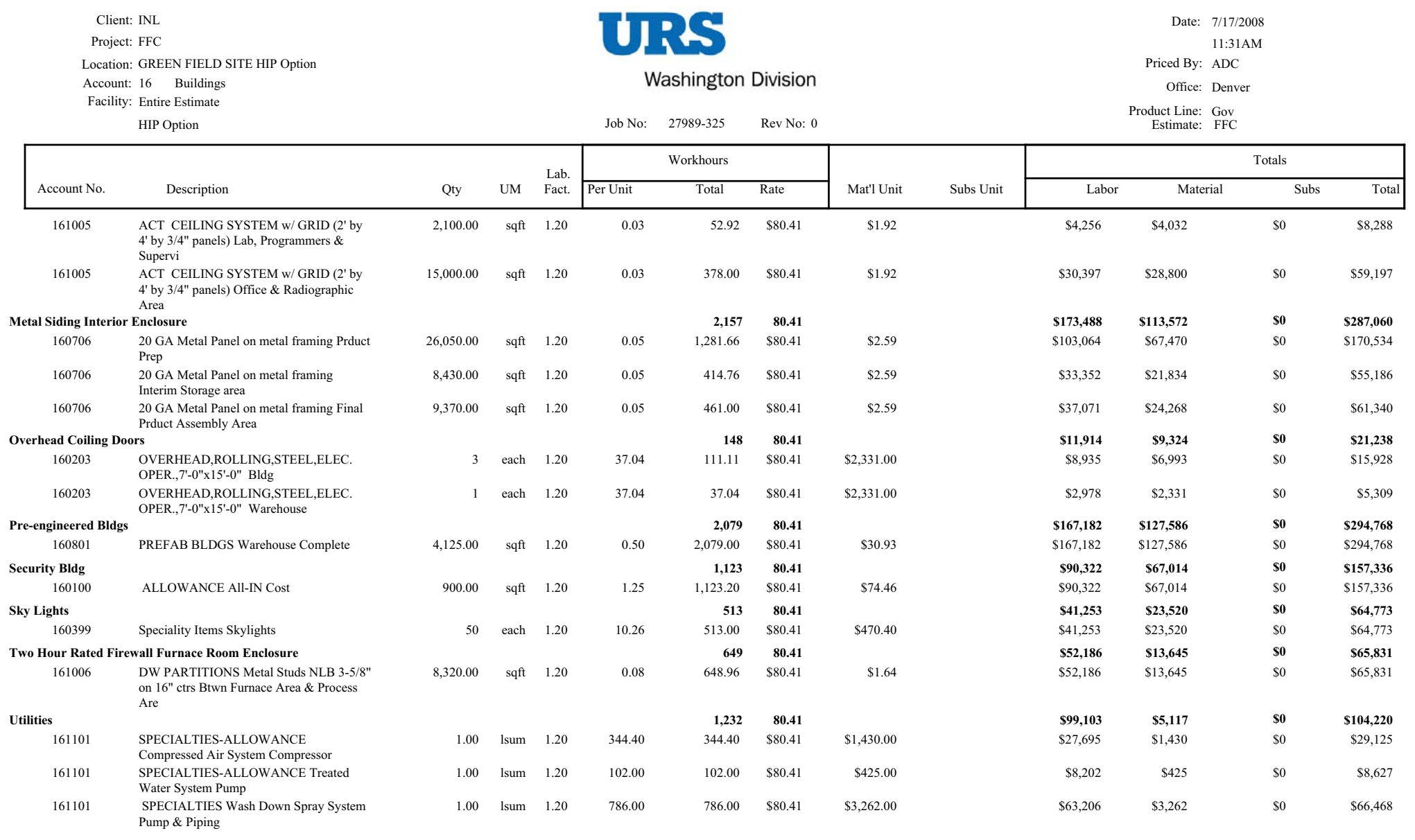

\begin{tabular}{|c|c|c|c|c|c|c|c|}
\hline Totals & $\begin{array}{l}\text { 16-Buildings } \\
\text { Entire Estimate }\end{array}$ & 38,398 & $\$ 77.94$ & $\$ 2,992,762$ & $\$ 2,008,403$ & $\$ 492,487$ & $\$ 5,493,652$ \\
\hline
\end{tabular}


Client: INL

Project: FFC

URS

Date: 7/17/2008

11:31AM

Location: GREEN FIELD SITE HIP Option

Washington Division

Priced By: ADC

ccount: 21 Spare Part

Washington Division

Office: Denver

Facility: Entire Estimate

Job No: $\quad 27989-325 \quad$ Rev No: 0

Product Line: Gov
Estimate: FFC

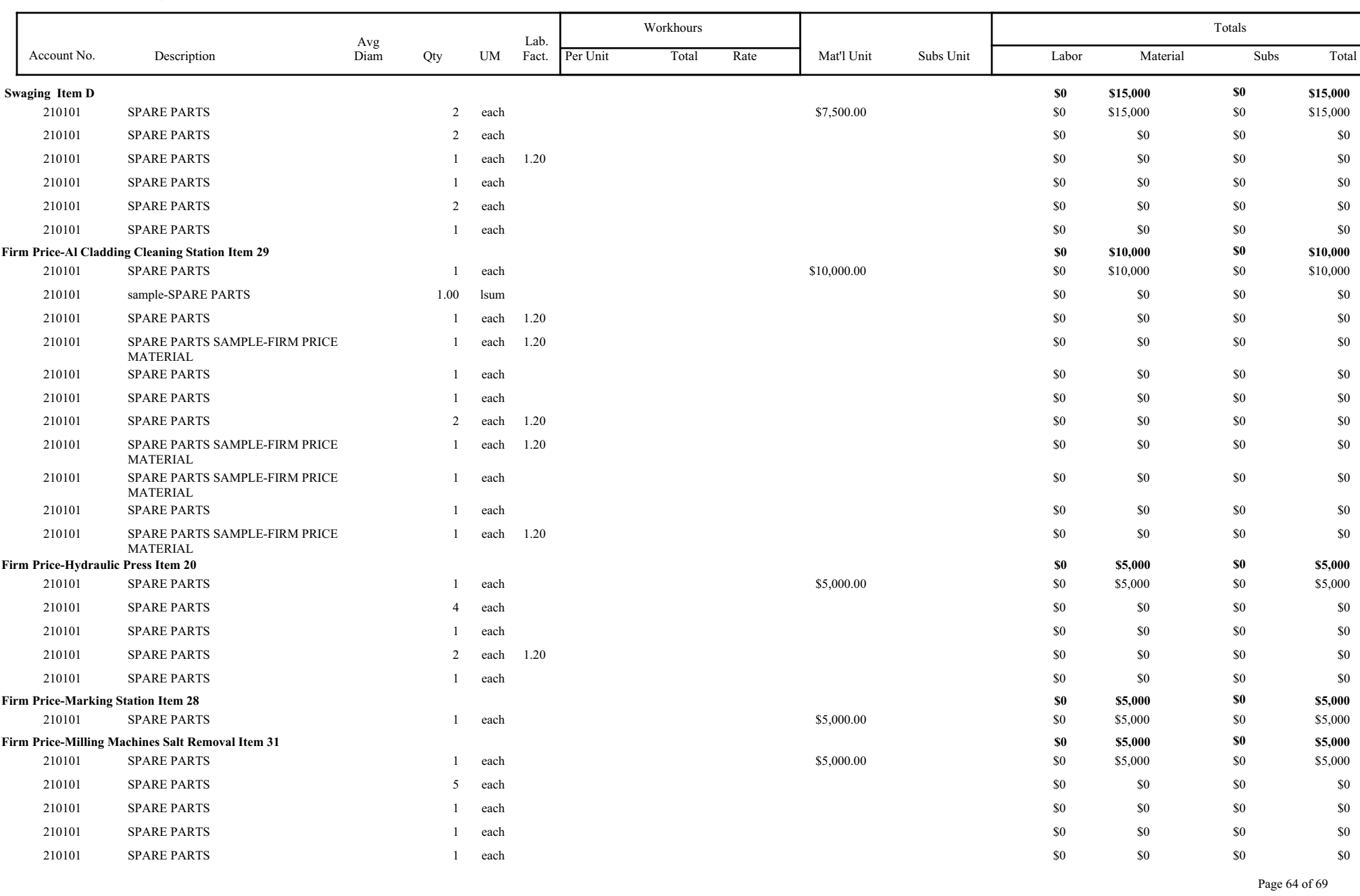


Client: INL

Project: FFC

URS

Washington Division

Date: 7/17/2008

$11: 31 \mathrm{AM}$

GREEN FIELD SITE HIP Option

Priced By: ADC

Office: Denver

Facility: Entire Estimate

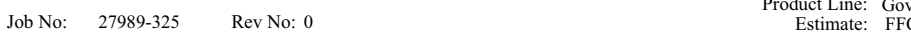

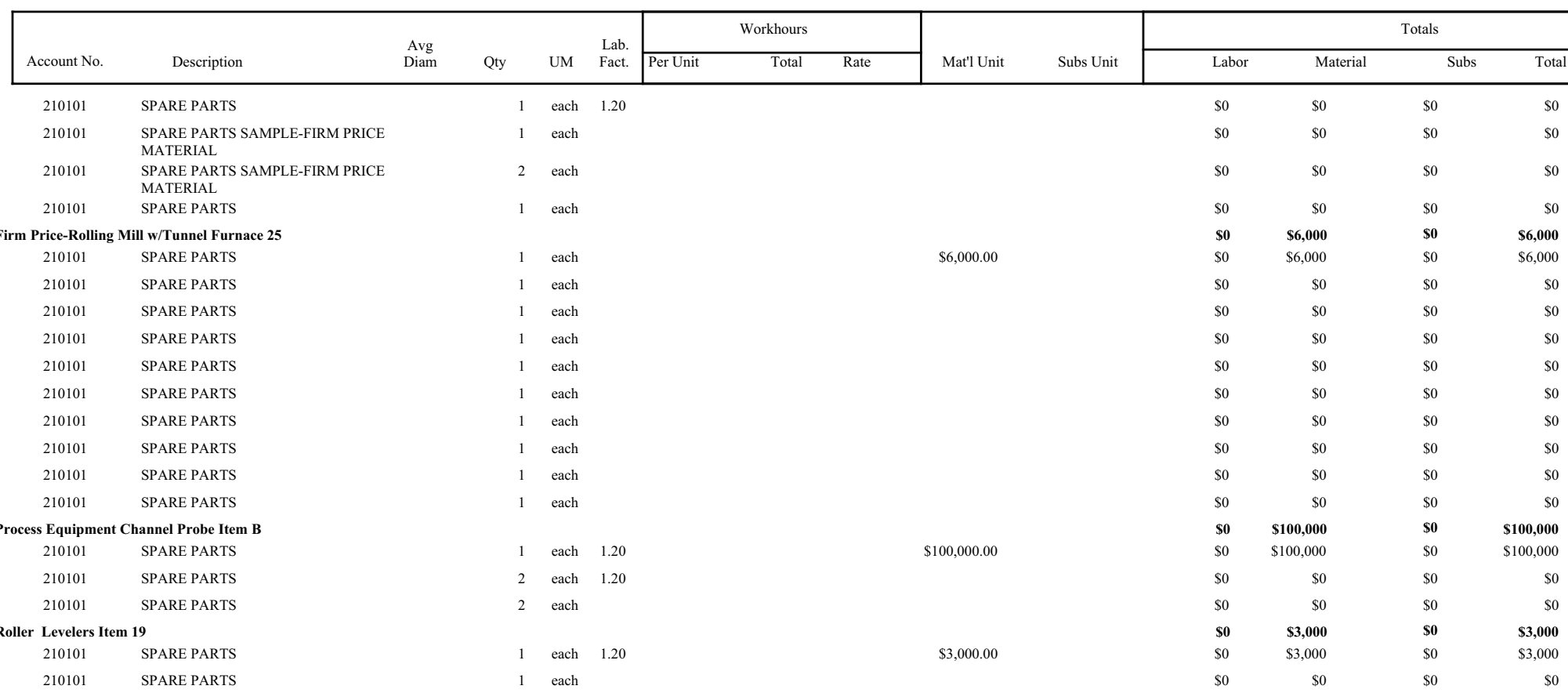

Totals 21-Spare Parts

$0 \quad \$ 0.00$

(1)


Client: INL

Project: FFC

URS

Date: 7/17/2008

$11: 31 \mathrm{AM}$

Location: GREEN FIELD SITE HIP Option

Washington Division

Priced By: ADC

ccount: 22 Freight

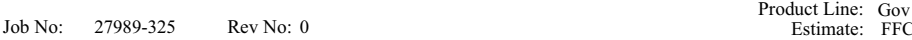

Office: Denver

Facility: Entire Estimate

$\begin{array}{llll} & & & \\ \text { Account No. } & \text { Description } & \text { Qty } & \text { UM } \\ \text { Fact. }\end{array}$

\begin{tabular}{|cc|} 
& Workhours \\
\hline Per Unit $\quad$ Total Rate
\end{tabular}

Mat'l Unit

Firm Price-EB Can Assy Station HIP Area Item 1

220100 BID CONDITIONING SAMPLE-NON

FIRM PRICE MATERIAL

220100 BID CONDITIONING SAMPLE-NON

FIRM PRICE MATERIAL

Firm Price-Process Equipment Hot Isostatic Press

220100 BID CONDITIONING SAMPLE-NON
FIRM PRICE MATERIAL

$0.04 \%$

$\$ 10,000.00$

$0.04 \%$

$0.04 \quad \%$

$\$ 7,850,000.00$

Subs Unit

Estimate: FFC

\begin{tabular}{|c|c|c|c|}
\hline & \multicolumn{3}{|c|}{ Totals } \\
\hline Labor & Material & Subs & Total \\
\hline so & $\$ 400$ & \$0 & $\$ 400$ \\
\hline$\$ 0$ & $\$ 400$ & $\$ 0$ & $\$ 400$ \\
\hline$\$ 0$ & $\$ 0$ & $\$ 0$ & $\$ 0$ \\
\hline so & $\$ 314,000$ & $\$ 0$ & $\$ 314,000$ \\
\hline$\$ 0$ & $\$ 314,000$ & $\$ 0$ & $\$ 314,000$ \\
\hline
\end{tabular}

(1)

$\mid$


Client: INL

Project: FFC

URS

Washington Division

Job No: $\quad 27989-325 \quad$ Rev No: 0

HIP Option

\begin{tabular}{|c|c|c|c|c|c|c|c|c|c|c|c|c|c|}
\hline \multirow[b]{2}{*}{ Account No. } & \multirow[b]{2}{*}{ Description } & \multirow[b]{2}{*}{ Qty } & \multirow[b]{2}{*}{ UM } & \multirow{2}{*}{$\begin{array}{l}\text { Lab. } \\
\text { Fact. }\end{array}$} & \multicolumn{3}{|c|}{ Workhours } & \multirow[b]{2}{*}{ Mat'l Unit } & \multirow[b]{2}{*}{ Subs Unit } & \multicolumn{4}{|c|}{ Totals } \\
\hline & & & & & Per Unit & Total & Rate & & & Labor & Material & & Total \\
\hline \multicolumn{2}{|c|}{ Construction Management - URS } & & & & & 20,984 & 70.40 & & & $\$ 1,477,274$ & \$0 & $\$ 238,220$ & $\$ 1,715,494$ \\
\hline 310101 & Site Manager & 24.00 & mnth & & 172.00 & $4,128.00$ & $\$ 95.00$ & & $\$ 2,150.00$ & $\$ 392,160$ & $\$ 0$ & $\$ 51,600$ & $\$ 443,760$ \\
\hline 310603 & Project Secretary (Local) & 24.00 & mnth & & 172.00 & $4,128.00$ & $\$ 38.20$ & & $\$ 1,720.00$ & $\$ 157,690$ & $\$ 0$ & $\$ 41,280$ & $\$ 198,970$ \\
\hline 310901 & Safety Professional & 24.00 & mnth & & 172.00 & $4,128.00$ & $\$ 81.00$ & & $\$ 2,150.00$ & $\$ 334,368$ & $\$ 0$ & $\$ 51,600$ & $\$ 385,968$ \\
\hline 310901 & Superientendant Elec/I\& C (local) & 14.00 & mnth & & 172.00 & $2,408.00$ & $\$ 65.00$ & & $\$ 1,720.00$ & $\$ 156,520$ & $\$ 0$ & $\$ 24,080$ & $\$ 180,600$ \\
\hline 310901 & Project Controls & 18.00 & mnth & & 172.00 & $3,096.00$ & $\$ 76.00$ & & $\$ 2,150.00$ & $\$ 235,296$ & $\$ 0$ & $\$ 38,700$ & $\$ 273,996$ \\
\hline 310901 & Superientendant Civil/Structural (local) & 8.00 & mnth & & 172.00 & $1,376.00$ & $\$ 65.00$ & & $\$ 1,720.00$ & $\$ 89,440$ & $\$ 0$ & $\$ 13,760$ & $\$ 103,200$ \\
\hline 310901 & Superientendant Piping/Mech (local) & 4.00 & mnth & & 172.00 & 688.00 & $\$ 65.00$ & & $\$ 1,720.00$ & $\$ 44,720$ & $\$ 0$ & $\$ 6,880$ & $\$ 51,600$ \\
\hline 310901 & Superientendant Elec/I\& C (local) & 6.00 & mnth & & 172.00 & $1,032.00$ & $\$ 65.00$ & & $\$ 1,720.00$ & $\$ 67,080$ & $\$ 0$ & $\$ 10,320$ & $\$ 77,400$ \\
\hline
\end{tabular}

Date: 7/17/2008

11:31AM Priced By: ADC

Office: Denver

Product Line: Gov
Estimate: FFC 
Client: INL

Project: FFC

URS

Washington Division

Account: 61 Home Office Services

Facility: Entire Estimate

HIP Option

Job No:

Rev No: 0

Product Line: Gov
Estimate: FFC

Account No.

$\begin{array}{llll}\text { Account No. Description } & \text { Qty } & \text { UM Fact. }\end{array}$

All-In Home Office at $105 \mathrm{~S} / \mathrm{hr}$

$610000 \quad$ ALL IN HOME OFFICE w/ Overhead \&

Per Unit

\begin{tabular}{ll|l} 
Total Rate & Mat'l Unit
\end{tabular}

1.00

$\mathbf{8 5 , 7 1 4} \quad \mathbf{1 0 5 . 0 0}$

$85,714.00 \quad \$ 105.00$

Subs Unit

Date: $7 / 17 / 2008$

11:31AM

Priced By: ADC

Office: Denver

$$
\text { other costs }
$$

.


Client: INL

Project: FFC

Location: GREEN FIELD SITE HIP Option

Account:

URS

Washington Division

Job No: $27989-325 \quad$ Rev No: 0
Date: 7/17/2008

11:31AM

Priced By: ADC

Office: Denver

$\begin{aligned} \text { Product Line: } & \text { Gov } \\ \text { Estimate: } & \text { FFC }\end{aligned}$

\begin{tabular}{|c|c|c|c|c|c|c|c|c|c|c|c|c|}
\hline \multirow[b]{2}{*}{ Account No. } & \multirow[b]{2}{*}{ Description } & \multirow[b]{2}{*}{ Qty } & \multirow[b]{2}{*}{ UM } & \multirow{2}{*}{$\begin{array}{l}\text { Lab. } \\
\text { Fact. }\end{array}$} & \multicolumn{3}{|c|}{ Workhours } & \multirow[b]{2}{*}{ Mat'l Unit } & \multirow[b]{2}{*}{ Subs Unit } & \multicolumn{3}{|c|}{ Totals } \\
\hline & & & & & Per Unit & Total & Rate & & & Labor & Material & Subs \\
\hline
\end{tabular}

\begin{tabular}{|c|c|c|c|c|c|c|}
\hline Grand Total: Entire Estimate & 300,809 & 87.11 & $\$ 26,202,011$ & $\$ 30,226,546$ & $\$ 7,716,607$ & $\$ 64,145,165$ \\
\hline
\end{tabular}

